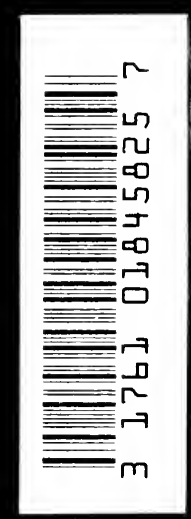



:

. 


$$
\text { . }
$$




\section{ELEMENTARY PARTICLES AND COSMIC RAYS}




\title{
Elementary Particles and
}

\section{Cosmic Rays}

\author{
by \\ ALLADI RAMAKRISHNAN \\ Director, Institute of Mathematical Sciences, \\ Madras, India
}

A Pergamon Press Book

THE MACMILLAN COMPANY

NEW YORK

1962 
This book is distributed by

THE MACMILLAN COMPANY - NEW YORK pursuant to a special arrangement with PERGAMON PRESS LIMITED

Oxford, England

\section{Copyright}

(C)

1962

Pergamon Press Ltd.

Library of Congress Card Number 61-1114

\section{ERINDALE COLLEGE LIBRARY}


To the memory of my revered father

$$
\text { "ALLADI" }
$$

one of the principal architects of the Indian Constitution

"His was a life not just lived

but a life charged with humanity and intellect." 


\section{CONTENTS}

PART ONE

\section{ELEMENTARY PARTICLES}

Prefatory Note

xiii

Chapter I. Single Particle Wave Functions and Wave Equations 3

1. Basic Postulates of Wave Mechanics 3

Concept of wave function and the Schrödinger equation 3

The principle of complementarity $\quad 4$

$\begin{array}{ll}\text { The principle of superposition } & 9\end{array}$

2. Single Particle Wave Equations

Spin and Pauli's equation

$\begin{array}{ll}\text { Relativistic requirements and the Klein-Gordon equation } & 18\end{array}$

$\begin{array}{ll}\text { The Dirac equation } & 21\end{array}$

Solutions of the Dirac equation for a free particle $\quad 24$

$\begin{array}{ll}\text { Wave equations for proton and neutron } & 29\end{array}$

Wave equation for the meson $\quad 33$

Wave equation for the neutrino $\quad 35$

3. Wave Mechanics of the Photon 38

$\begin{array}{ll}\text { Wave equation for the photon } & 38\end{array}$

$\begin{array}{ll}\text { Schrödinger equation of the photon in momentum space } & 39\end{array}$

$\begin{array}{ll}\text { Energy of the electromagnetic field } & 40\end{array}$

Momentum of the photon field 41

Wave function in configuration space $\quad 41$

Plane wave solutions $\quad 41$

$\begin{array}{ll}\text { Angular momentum and spin of the photon } & 42\end{array}$

$\begin{array}{ll}\text { Spin wave functions } & 43\end{array}$

Angular momentum eigenfunctions $\quad 44$

$\begin{array}{ll}\text { Parity eigenfunctions } & \mathbf{4 5}\end{array}$

Expansion of the electric and magnetic fields in terms of the photon eigenfunctions

Potentials and their expansions in terms of the eigenfunctions of angular momentum and parity

Chapter II. Quantum Electrodynamics

1. Perturbation Expansions and Kernel Functions $\quad 52$

Old fashioned perturbation theory $\quad \mathbf{5 5}$

$\begin{array}{ll}\text { Kernel function formalism } & 63\end{array}$ 
2. Perturbation Theory of the Dirac Electron $\quad 65$

Feynman kernel and its momentum representation $\quad 65$

$\begin{array}{ll}\text { Calculation of matrix elements } & 69\end{array}$

3. Applications to Electrodynamics $\quad 74$

$\begin{array}{ll}\text { Compton effect } & \mathbf{7 5}\end{array}$

Two photon pair annihilation $\quad=\quad 79$

$\begin{array}{ll}\text { Bremsstrahlung } & 81\end{array}$

$\begin{array}{ll}\text { Pair production } & 86\end{array}$

$\begin{array}{ll}\text { Electron-electron interaction } & 90\end{array}$

4. Self Energy and Renormalization $\quad 95$

Self energy of the electron $\quad 97$

$\begin{array}{lr}\text { Self energy of the photon } & 99\end{array}$

$\begin{array}{ll}\text { The vertex part } & 104\end{array}$

The anomalous magnetic moment of the electron $\quad 105$

$\begin{array}{ll}\text { The Lamb shift } & 107\end{array}$

$\begin{array}{ll}\text { The removal of divergences from a general graph } & 109\end{array}$

Chapter III. Formalism of Quantum Field Theory

1. The State Vector and Field Operators 112

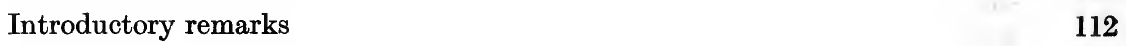

$\begin{array}{ll}\text { Perturbation expansion in field theory } & 117\end{array}$

2. Normal Products and Wick's Theorem 126

Proof of Wick's theorem $\quad 130$

3. Representation of Interactions in Field Theory 132

4. The Heisenberg Representation and Operators in Field Theory 133

The Heisenberg representation 133

Interaction of fermion and electromagnetic fields $\quad 137$

$\begin{array}{ll}\text { Propagators in field theory } & 139\end{array}$

5. Transformation and General Principles of Invariance 143

$\begin{array}{ll}\text { General considerations } & 143\end{array}$

$\begin{array}{ll}\text { Particular transformations } & 147\end{array}$

$\begin{array}{ll}\text { Continuous transformations } & 160\end{array}$

Chapter IV. Pion Physics

$\begin{array}{ll}\text { 1. Introduction } & 167\end{array}$

Properties of the $\pi$-meson $\quad 167$

The resonances in the low energy pion-nucleon interaction $\quad 170$

2. Earlier Attempts and Theories 172

$\begin{array}{ll}\text { Weak coupling theory } & 172\end{array}$

$\begin{array}{ll}\text { Strong coupling theory } & \mathbf{1 7 5}\end{array}$

$\begin{array}{ll}\text { Phenomenological theories } & \mathbf{1 7 6}\end{array}$ 
3. Other Theories not involving Expansion in terms of a Coupling Constant

Heitler's theory of radiation damping

The Tamm-Dancoff approximation

4. The Chew-Low Formalism

The Kemmer Hamiltonian

Pion-nucleon scattering

The theory of photo-meson production

Chapter V. Non-Perturbative Approach to Quantum Field Theory

Single variable dispersion relations

The Mandelstam representation

Chapter VI. Strange Particles and Their Interactions

1. Strange particles

Introduction

Isotopic spin and strangeness

2. Decays of $K$ Particles

The $\tau^{+}-\theta^{+}$puzzle

Neutral $K$ decay

The $|\Delta I|=1 / 2$ rule

Chapter VII. Weak Interactions

1. Weak Interactions

Tests for invariances under $C, P, T$

Violation of $P$ and $C$ invariances in weak interactions

2. Universal Fermi Interaction

3. Some Recent Work on the Theory of Weak Interactions

Chapter VIII. Strong Interactions and Strange Particles

1. $K$-particle Interactions

$K^{+}$-interactions

$K^{-}$-interactions

2. Hyperons and Hyperfragments 294

$\begin{array}{ll}\text { Production of hyperons } & 294\end{array}$

$\begin{array}{ll}\text { Hyperon interactions } & 296\end{array}$

$\begin{array}{ll}\text { Hyperfragments } & 297\end{array}$

3. Relative Parity between Strange Particles 
Chapter IX. Symmetries and Strong Interactions

1. Introduction

2. Models of Strong Interactions

Global and cosmic symmetries

Doublet approximation

Pais model

Salam-Polkinghorne model

Feynman's model

3. Compound Models

4. Space-Time Properties and Internal Symmetries of Strong Interactions 353

Heisenberg's non-linear theory of elementary particles

PART TWO

\section{COSMIC RAYS}

Chapter X. Primary Cosmic Radiation

1. Primary Cosmic Radiation

The mass spectrum

Energy spectrum and spatial distribution

2. Time Variation of Cosmic Rays $\quad 372$

The diurnal variation $\quad 373$

Variations at the source of cosmic radiation $\quad \mathbf{3 7 3}$

$\begin{array}{ll}\text { Modulation effects } & \mathbf{3 7 5}\end{array}$

$\begin{array}{lr}\text { Prehistoric intensity variations } & \mathbf{3 7 6}\end{array}$

Chapter XI. Geomagnetic Effects

1. Motion of a Charged Particle in the Field of a Magnetic Dipole 377 $\begin{array}{ll}\text { Equations of motion } & \mathbf{3 7 7}\end{array}$

2. Stormer and Main Cones $\quad 380$

3. Deviations from the Stormer and Main Cones 383

4. Latitude Effect $\quad 392$

Chapter XII. Interactions of Cosmic Rays With Matter

1. General Description 393

2. $\mu$-meson Interactions in Cosmic Rays $\quad 396$

Classical electromagnetic interactions: ionization $\quad 397$

$\begin{array}{ll}\text { Collisions involving free electrons } & \mathbf{3 9 7}\end{array}$

$\begin{array}{ll}\text { Photoproduction } & 398\end{array}$ 
Heisenberg's theory of multiple production $\quad 401$

L.O.W. method $\quad 402$

Fermi's statistical theory of multiple meson production $\quad 403$

Some applications of Fermi's theory $\quad 411$

Extension of Fermi's theory by Landau and Pomeranchuk 415

$\begin{array}{ll}\text { A covariant formulation of the statistical theory } & 420\end{array}$

Bhabha's theory $\quad 421$

Isobar and fireball models $\quad 422$

Chapter XIII. Cascade Processes $\quad 424$

1. Introduction $\quad 424$

Longitudinal development of showers $\quad 424$

Lateral spread of showers $\quad \mathbf{4 2 4}$

2. One-Dimensional Cascades $\quad 425$

General formulation $\quad \mathbf{4 2 5}$

$\begin{array}{ll}\text { Method of product density functions } & \mathbf{4 2 7}\end{array}$

$\begin{array}{lr}\text { Cascades involving only one type of particle } & 429\end{array}$

Electron-photon cascades $\quad 432$

Alternative approach to the cascade theory $\quad 441$

Further refinements to the cascade theory $\quad 443$

$\begin{array}{ll}\text { Discussion of numerical results } & 447\end{array}$

Intranuclear cascades $\quad \mathbf{4 5 4}$

3. Interactions at Extremely High Energies $\quad 457$

$\begin{array}{ll}\text { Longitudinal developments of jets } & 457\end{array}$

The lateral spread of the extensive air showers (EAS) 461

4. Energy Balance of Cosmic Radiation $\quad 469$

$\begin{array}{ll}\text { The } \mu \text {-mesonic component } & \mathbf{4 7 0}\end{array}$

The electron-photon component $\quad 474$

The nucleonic component $\quad \mathbf{4 7 5}$

Chapter XiV. Origin of Cosmic Radiation

$\begin{array}{ll}\text { 1. Theories of Intermediate Mechanism } & 480\end{array}$

Swann's mechanism $\quad 481$

Fermi's mechanism of acceleration of particles $\quad \mathbf{4 8 5}$

2. Sources of Cosmic Radiation $\quad 489$

Solar contribution to cosmic radiation $\quad 490$

Galactic sources of cosmic rays $\quad 492$

\section{APPENDICES}

1. Dimensions and Units 498

2. Composition of Angular Momenta $\quad 499$

Addition of two angular momenta-Clebsch-Gordan coefficients $\quad 499$

Computation of Clebsch-Gordan coefficients $\quad 501$ 
Symmetry properties of the Clebsch-Gordan coefficients and the Wigner 3-j symbol

Rotation matrices

The coupling of three angular momenta, Racah coefficients and the Wigner $6-j$ symbol

The 9-j symbol

3. Algebra of the $\gamma$-Matrices

4. The Density of States

5. Relativistic Transformations

6. Invariant Functions

7. Quantization of the Electromagnetic Field

8. A Re-examination of the Equivalence of the Feynman and $S$-matrix Formalisms

A new viewpoint regarding the kernel function formalism of Feynman

Derivation of the Feynman expansion from the $S$-matrix formalism

9. On the Connection between Spin and Statistics

Hermitian spinzero field

Hermitian spin one-half field

Non-Hermitian fields

10. Phase Shift Analysis of Scattering

11. Numerical Results on Cascade Theory

Tables I-IX

12. Resonance States of Strongly Interacting Particles

The $\pi-\pi$ resonance

The $3 \pi$ resonances

The $K-\pi$ resonance

The $\Lambda-\pi$ resonance

The $\Sigma-\pi$ resonance

The $\bar{K}-\pi$ resonance

13. Gauge Theories of Elementary Particles

14. The Principle of Equivalence for all Strongly Interacting Particles 


\section{PREFATORY NOTE}

When Professor Heitler invited me a few years ago to write a book on cosmic ray theory, I accepted it with a modest purpose in view - to present a theoretical account of various phenomena associated with cosmic radiation. Since such phenomena included interactions of elementary particles and their production at the place of their origin and in their passage through matter, it was thought necessary to start the book with an introduction dealing with quantum mechanical collision processes. The writing of this introduction slowly inveigled me into the fascinating domain of fundamental physics and the scope of the book gradually became enlarged. The introduction thereby stretched to two-thirds the span of the book till the role of the introductory and substantial parts became reversed. It now professes to be a book of elementary particles, their interactions being described through cosmic ray phenomena.

The main stimulus for such a transformation came through my close association with leading physicists abroad during my stay at the Yukawa Hall, Japan, at the kind invitation of Professor Yukawa under the auspices of the Asia Foundation, and the following visit to the various centres of fundamental research in experimental and theoretical physics in the United States and Europe. My next visit to the United States, again with the assistance of the Asia Foundation, and my stay at the Institute for Advanced Study where I enjoyed the hospitality of Professor Oppenheimer brought me almost "face to face" with some of the creators of modern physics, especially at the time when the prediction and demonstration of nonconservation of parity was attracting the attention of physicists throughout the world. While of course by the end of my visit to Princeton I was desirous of shifting the emphasis of the book to elementary particles, I would not have undertaken the task but for the willing and enthusiastic co-operation of Mr. N. R. Ranganathan, one of the most promising young members of the theoretical physics group at Madras. Other members of the group also convinced me not merely of the necessity of writing a book with an essentially physical approach to problems involving complex and formal mathematical methods but also of its possibility.

It is a pleasure to acknowledge in some detail the help rendered to me by my young students in the writing of this book; Mr. P. Rajagopal in regard to the sections on single particle wave functions and wave equations, Mr. A. P. Balachandran on self energy and renormalization, Dr. R. Vasudevan and Miss T. K. Radha on Feynman processes, Mr. G. Ramachandran on primary cosmic radiation, Dr. S. K. Srinivasan and Miss Thunga on geomagnetic effects, Mr. N. R. Ranganathan on new particles, Mr. K. Venkatesan on meson pro- 
cesses and the Heisenberg representation, Miss S. Indumathi on cascade processes, Miss G. Bhamathi on hyperfragments and Mr. V. Devanathan on angular momentum.

Four of my students, Mr. A. P. Balachandran, Miss S. Indumathi, Miss G. Bhamathi and Miss R. Thunga scrutinized the manuscript with critical care and I enjoyed the frank and free discussions we had on the subject matter of each chapter. I am particularly grateful to Miss T. K. Radha and Mr. P. Rajagopal for undertaking the arduous and responsible task of carefully preparing the manuscript with equations, graphs and tables for publication, and my assistant Mr. E. T. Nambi Iyengar for typing the book, a task rendered rather tiresome in view of the constant changes that had to be made before publication.

The tenor and style of the book have naturally been determined by the particular background of my scientific education under my great teachers Professor H. J. Bhabha who first initiated me into theoretical physics and Professor M. S. Bartlett who taught me the methods of probability and stochastic theory. From both of them I have attempted to learn one valuable lesson-that it is advisable for a scientist, particularly a theoretical physicist, to combine initiative with caution. This book is the product of such an effort. In presenting established results and well-known theories I have taken care to adhere closely to the treatments of the authors themselves. In doing this, I had to make demands on the generosity of the authors, in particular, Professors Yang, Feynman, P.T.Matthews, Jauch, Chew and Källen and their publishers who have without hesitation permitted me to draw freely from their work. I am grateful to them, to the Office of the Technical Services and to the Addison-Wesley Publishers for such kind permission.

A rather detailed description of strange particle interactions is given in two chapters to illustrate the complexity of the maze which is puzzling even the most creative minds in physics today. The nature of this challenge can be realized only by the wild and desperate attempts that are being made to discern "a plan behind the maze". Part I concludes with a summary of such current ideas and shifting speculations.

The exposition in the rest of the book, particularly relating to the theory of scattering and perturbation expansions in Part I and Cascade Theory in Part II, is essentially influenced by my leanings towards the theory of probability and the mathematical methods associated with it. The kernel function formalism of Feynman and its relation to field theory have been described from a point of view and in a sequence different from the conventional; the old wine has been placed in new bottles mainly as a tribute to the delectable vintage which has become the stock-in-trade of the theoretical physicist today.

Cascade theory has been presented in a portmanteau form made possible only by recent methods and techniques developed in stochastic theory; the description of geomagnetic effects has been condensed without omitting the intricate 
details which alone can reveal the exquisite beauty of the geometrical pattern of the motion of charged particles in a magnetic field.

Like the postscript of a letter, some of the appendices deal with my own ideas which I was hesitant to include in the main body of the book as they relate to theories which are considered too well established to require criticism. While I am aware that a "blue-blooded" theoretical physicist will be reluctant to accept the intrusion of "commonplace" probability theory, I am encouraged to discuss the role of probability in quantum mechanics since it seems to have engaged the active attention of Professor Feynman, one of the creators of modern quantum electrodynamics. I have interpreted many of his casual remarks in his papers to imply that all has not been said on the Pauli principle.

I became more conscious of my shortcomings after the completion of the book than at its commencement. I have not attempted to lead the reader but preferred to walk by his side to the base of the pyramid of modern physics which is too mighty for single minds, save the most gifted, even for contemplation.

I do not know whether it is wisdom or impertinence to write a book on the theory of elementary particles and cosmic rays at a time when modern physics is offering its greatest challenge since the birth of relativity and quantum mechanics. But from a humbler pedagogic point of view, I could not have chosen a more propitious occasion for its publication, to meet, in a small measure at least, the needs of scientific education in my country. This book is just the projection of the newly awakened hopes and aspirations of the young scientific community in my home town and university and its nascent interest in mathematical sciences. It is gratifying that the efforts of this enterprising group have attracted the sympathetic attention of Professor Niels Bohr and Professor Abdus Salam who convinced me that the writing of this book was a pedagogic necessity in my country especially at the inception of advanced scientific effort on an organized scale.

The writing of any text book is considered a drudgery but to me it has been a most profitable and exciting experience, made more so because of the peculiar and exhilarating circumstance that every chapter of this book was discussed at informal meetings with my students in my family home in an atmosphere of leisure and comfort. On this occasion I wish to express my gratitude to the distinguished Vice-Chancellor of our University, Dr. A. L. Mudaliar, who was kind enough to recognize the work of this group and to the farsighted Finance Minister, Mr. C. Subrahmaniam for the creation of the Institute of Mathematical Sciences to provide adequate facilities for future effort.

It has been a particular pleasure and privilege to me that Professor Heitler, one of the leading theoretical physicists in Europe, has been the main sponsor of this book. The manuscript was revised in the light of his detailed comments given with such amiable candour and friendly interest, and I cannot adequately express my gratitude to him for his kindness to the point of leniency in allowing me so much time for the preparation of the book and latitude in enlarging its scope. 
I am grateful to the Pergamon Press for undertaking the publication of this work and to Captain I. R. Maxwell, its Managing Director, for tolerating the almost inexcusable delay in submitting the manuscript. The delay has placed a greater responsibility on me since this book will have to deserve to be a sequel to two important works published by the Pergamon Press on theoretical astrophysics and experimental work on elementary particles. Therefore, before committing it to publication I was anxious to present its contents through lectures, and such an opportunity arose when Professors A. Mercier and S. Flügge invited me to Berne and Marburg respectively. I shall consider my efforts worthwhile if this book helps a new entrant to realize the depth and extent of the great stir that is taking place in physical science today.

Alladi Ramakrishnan

'Ekamra Nivas', 27 Luz Church Road, Madras-4, India. 


\section{Part ONE \\ ELEMENTARY PARTI CLES}

"A mighty maze, but not without a plan"

A. Pope 
. 


\section{SINGLE PARTICLE WAVE FUNCTIONS ${ }^{1}$ AND WAVE EQUATIONS}

\section{BASIC POSTULATES OF WAVE MECHANICS}

\section{Concept of the wave function and the Schrödinger equation}

Is CLASSICAL physics an elementary particle in the absence of interaction is characterized only by one "intrinsic" attribute, its mass"2. Other properties are ascribed when we consider its interaction with fields of force. For example, charge can be regarded as a constant characteristic of the interaction of the particle with an electromagnetic field. Dynamical attributes like energy and momentum are not "intrinsic" in the sense of mass and charge. The variations of these dynamical attributes with time and the relations between them are given by the equations of motion.

In quantum mechanies a free or interacting particle is described by what is known as its wave function which is a function of its "intrinsic" attributes and the dynamical variables. Let us ignore for the moment the concept of spin which is of course an intrinsic quantum mechanical attribute. It is customary to represent the particle by a complex function $\psi$ of its spatial and temporal coordinates $(x, y, z, t)$ or by a complex function $\varphi$ of its momentum and time coordinates $\left(p_{x}, p_{y}, p_{z}, t\right)$. These wave functions in configuration and momentum spaces $^{3}$ have a probabilistic interpretation. $\psi^{*} \psi \mathrm{d}^{3} x$ represents the probability

1 W. PAuli, Handbuch" der Physik, Vol. 5, Springer-Verlag (1958).

2 In this point of view we take mass as the rest energy of the particle and not as the constant characteristic of the interaction with the gravitational field. The question of the origin of this rest energy is of course fundamental and attempts have been made to interpret this energy as arising due to the interaction with the self field.

3 The following notation will be used as regards space-time points $x, y, z, t: x$ denotes the space-time point with components $x, y, z$ and $t . x$ is a space point with components $x, y, z$. In a corresponding manner a four vector $\alpha$ has components $\alpha_{x}, \alpha_{y}, \alpha_{z}$ and $\alpha_{t}$ and $\alpha$ represents a three-vector with $\alpha_{x}, \alpha_{y}, \alpha_{z}$ as its components. The scalar product of two three-vectors $c$ and $\beta$ is:

$$
\boldsymbol{c} \cdot \boldsymbol{\beta}=\alpha_{x} \beta_{x}+\alpha_{y} \beta_{y}+\alpha_{z} \beta_{z}
$$

but for four-vectors we define the scalar product as

We also use the notation

$$
\alpha \cdot \beta=\alpha_{\iota} \beta_{t}-\alpha_{x} \beta_{x}-\alpha_{y} \beta_{y}-\alpha_{z} \beta_{z} .
$$

$$
\mathrm{d}^{4} x=\mathrm{d} x \mathrm{~d} y \mathrm{~d} z \mathrm{~d} t \quad \text { and } \quad \mathrm{d}^{3} x=\mathrm{d} x \mathrm{~d} y \mathrm{~d} z .
$$

Under normal circumstances, indices 1, 2, are used to distinguish between two four-vectors and not for their components. Sometimes it is more convenient to use $1,2,3,4$ instead of $x$, $1 *$ 
that the particle is found in the volume element $\mathrm{d}^{3} x$ while $\varphi^{*} \varphi \mathrm{d}^{3} p$ represents the probability that the particle lies in the momentum volume element $\mathrm{d}^{3} p$. The quantum mechanical description of a particle in terms of its wave function is based on two fundamental principles, those of complementarity and superposition.

\section{The principle of complementarity}

Without going into any discussions on the epistemological basis of complementarity, the principle in relation to wave functions can be stated as follows.

A particle can be described either by its wave function in configuration space or in momentum space, but not by a function of both momentum and spatial coordinates ${ }^{1}$. In the simpler language of probability, this means that we can either get the probability distribution in configuration space or in momentum space but not the joint distribution in both the spaces as will be explained presently. The statement regarding the amplitudes $\psi$ and $\varphi$ is deeper and more fundamental than regarding probability distributions.

The principle of complementarity is based on the fundamental postulate that $\varphi$ and $\psi$ are connected by the relation

$$
\psi(\boldsymbol{x}, t)=(2 \pi \hbar)^{-3 / 2} \int_{\boldsymbol{p}} \varphi(\boldsymbol{p}, t) \exp \left(\frac{i \boldsymbol{p} \cdot \boldsymbol{x}}{\hbar}\right) \mathrm{d}^{3} p,
$$

or equivalently by

$$
\dot{\varphi}(\boldsymbol{p}, t)=(2 \pi \hbar)^{-3 / 2} \int_{\boldsymbol{x}} \psi(\boldsymbol{x}, t) \exp \left(-\frac{i \boldsymbol{p} \cdot \boldsymbol{x}}{\hbar}\right) \mathrm{d}^{3} x .
$$

This implies that if $\lambda_{x}, \lambda_{y}$ and $\lambda_{z}$ are the wavelengths associated with the $x, y$ and $z$ directions for the wave function with spatial dependence of the type $\mathrm{e}^{+i \boldsymbol{p} \cdot \boldsymbol{x} / \hbar}$,

i.e.

$$
\lambda_{x}=\frac{h}{p_{x}} ; \quad \lambda_{y}=\frac{h}{p_{y}} \quad \text { and } \quad \lambda_{z}=\frac{h}{p_{z}}
$$

then it is an eigenstate of the operators $-i \hbar \frac{\partial}{\partial x},-i \hbar \frac{\partial}{\partial y},-i \hbar \frac{\partial}{\partial z}$ with eigenvalues $p_{x}, p_{y}$ and $p_{z}$ respectively.

$y, z, t$ to denote the components of a four-vector. This is usually done when we wish to sum over the four components $x, y, z, t$ and we then use a dummy index $i$, the summation extending from 1 to 4 . However the distinction between the two different modes in the use of the indices will be apparent from the context.

Except in the first chapter we shall set $\hbar=1, c=1$, i.e. we work in natural units. The conversion table for transforming natural units into usual units is given in the appendix.

1 There are some who believe that it is possible to define a function $\Phi(p, x)$ such that $\Phi^{*} \Phi \mathrm{d}^{3} p \mathrm{~d}^{3} x$ represents the joint probability that the particle lies in the spatial volume $\mathrm{d}^{3} x$ and the momentum volume $\mathrm{d}^{3} p$, i.e. $\psi^{*} \psi$ and $\varphi^{*} \varphi$ are marginal probability distributions. But in such a case, the relationship between $\Phi, \psi$ and $\varphi$ is obscure and it is not clear whether the principle of complementarity as envisaged in equations (1) and (2) will be consistent with the existence of $\Phi(p, x)$. 
In classical theory, given the field with which the particle interacts, all the dynamical variables are determined by the equations of motion. In particular, the spatial and momentum coordinates of the particles can be determined uniquely. In quantum mechanics, the particle is described only in terms of the wave function $\psi$ or $\varphi$. We can thus speak only of the mean values of the dynamical variables of the particle. For instance, the mean value of the $x$ coordinate can be obtained from $\psi$ as ${ }^{1}$

$$
\bar{x}=\int \psi^{*} x \psi \mathrm{d}^{3} x .
$$

Since the particle can be represented either by $\psi$ or $\varphi$ it is possible to get the mean value of $x$ from $\varphi$ functions also as

$$
\begin{aligned}
\bar{x} & =(2 \pi \hbar)^{-3 / 2} \int \psi^{*} x \mathrm{~d}^{3} x \int \varphi(\boldsymbol{p}) \exp \left(\frac{i \boldsymbol{p} \cdot \boldsymbol{x}}{\hbar}\right) \mathrm{d}^{3} p \\
& =(2 \pi \hbar)^{-3 / 2} \int \psi^{*} \mathrm{~d}^{3} x \int i \hbar \frac{\partial}{\partial p_{x}}[\varphi(\boldsymbol{p})] \exp \left(\frac{i \boldsymbol{p} \cdot \boldsymbol{x}}{\hbar}\right) \mathrm{d}^{3} p \\
& =(2 \pi \hbar)^{-3 / 2} \int i \hbar-\frac{\partial}{\partial p_{x}}[\varphi(\boldsymbol{p})] \mathrm{d}^{3} p \int \psi^{*} \exp \left(\frac{i \boldsymbol{p} \cdot \boldsymbol{x}}{\hbar}\right) \mathrm{d}^{3} x \\
& =\int \varphi^{*}(\boldsymbol{p}) i \hbar \frac{\partial}{\partial p_{x}}[\varphi(\boldsymbol{p})] \mathrm{d}^{3} p .
\end{aligned}
$$

Therefore to obtain the mean value of the dynamical variable $x$ we have to insert the operator $i \hbar \frac{\partial}{\partial p_{x}}$ between $\varphi^{*}$ and $\varphi$ if we are dealing with wave functions in momentum space. The same considerations apply to the coordinates $y$ and $z$ and to any dynamical variable $\alpha$ which is a function only of the coordinates $x, y$ and $z$. To obtain the mean value of $\alpha$ when the wave function is represented in momentum space we have to express $\alpha$ in terms of $x, y$ and $z$ and replace them by operators, insert $\alpha$ between $\varphi^{*}$ and $\varphi$ and integrate over momentum space.

Conversely if we wish to find the mean value of the momentum, it is given by

$$
\overline{p_{x}}=\int_{p} \varphi^{*} p_{x} \varphi \mathrm{d}^{3} p .
$$

But if we work in configuration space

$$
\overline{p_{x}}=\int \psi^{*}\left(-i \hbar \frac{\partial}{\partial x}\right) \psi \mathrm{d}^{3} x
$$

i.e. we have to represent $p_{x}$ by the operator $-i \hbar \partial / \partial x$. The representation of $p$ by an operator when working in configuration space and $x$ by an operator when working in momentum space is equivalent to writing the commutation rules for momentum and space coordinates as follows:

$$
\left[x, p_{x}\right]=\left[y, p_{y}\right]=\left[z, p_{z}\right]=i \hbar
$$

1 We adopt the convention that the symbols $\int$ and $\Sigma$ imply, except when otherwise stated explicity, integration and summation over the entire permissible range. 
All other pairs of dynamical variables taken from $p_{x}, p_{y}, p_{z}, x, y$ and $z$ commute with each other. If a dynamical variable $\beta$ is a function of $p$ only, its mean value can be obtained directly from the $\varphi$ function. In configuration space, we have to represent $p$ by an operator in $\beta(p)$. Speaking quite generally, if two dynamical variables are canonically conjugate in the classical sense like the space and momentum coordinates, one of them is to be represented by an operator when we work with the wave function in the space of the other variable.

The question now arises as to how we can determine the mean values of functions of both the space and momentum coordinates. Examples of such functions which play a dominant role are the Lagrangian and the Hamiltonian. It is obvious that we should express such functions in terms of both space and momentum coordinates, and replace the momenta by operators in configuration space or replace the spatial coordinates by operators in momentum space. The coordinate canonically conjugate to the Hamiltonian $H$ is $t$ and hence $H$ can also be represented by the operator $i \hbar \frac{\partial}{\partial t}$. Equivalence of this operator with that of the Hamiltonian expressed in terms of space and momentum coordinates, gives the equation of motion

$$
i \hbar \frac{\partial \psi}{\partial t}=H \psi
$$

known as the Schrödinger equation representing the temporal evolution of the wave function $\psi$. The wave function is considered stationary if the only time dependence of $\psi$ is of the form $\mathrm{e}^{-i E t / \hbar}$, i.e. $\psi$ can be written as ${ }^{1}$

$$
\psi(x, y, z, t)=\mathrm{e}^{-i E t / \hbar} \psi(x, y, z)
$$

where $E$ is a constant presently to be identified with energy. In such a case, substituting for $\psi$ in the equation of motion, we get

$$
H \psi(\boldsymbol{x})=E \psi(\boldsymbol{x})
$$

where $\psi$ satisfies the time independent Schrödinger equation. This leads to the problem of finding the eigenvalues $E_{n}$ and the eigenfuntions $\psi_{n}$ corresponding to the Hamiltonian operator $H$. The eigenvalue $E_{n}$ of $H$ is the energy of the particle if the particle is in a state $\psi_{n}$. The eigenstate of energy can also be the eigenstate of the momentum operator and such an eigenfunction will have space and time dependence of the type $\mathrm{e}^{-i p \cdot x / \hbar}=\mathrm{e}^{(-i E t+i \boldsymbol{p} \cdot \boldsymbol{x}) / \hbar}$ corresponding to a frequency $v$ with $h v=E$.

If the particle is in interaction with external fields, the interaction is represented through the inclusion in the Hamiltonian of terms corresponding to these fields and stationary solutions may exist under suitable circumstances for particles in such fields. In such a case the particle is supposed to be bound to the external field and the corresponding wave function $\psi_{n}$ represents the bound state of the

${ }^{1}$ For convenience we may use the same symbol to denote different functions if they can be distinguished from the context or through the parameters in brackets and consequently no confusion is likely to arise. 
particle corresponding to the energy $E_{n}$. For example, in the case of the hydrogen atom the Coulomb field of the nucleus $V(r)$ can be included in the Hamiltonian. In such a case energy is a function of both space and momentum variables and the eigenstate of energy is not an eigenstate of space and momentum operators separately. This implies that the wave function which is an eigenstate of energy is not of the exponential type in the configuration or momentum space. Defining the quantity

$$
\boldsymbol{j}=\frac{\hbar}{2 i m}\left(\psi^{*} \nabla \psi-\psi \nabla \psi^{*}\right)
$$

we find that it satisfies the continuity equation

$$
\frac{\partial \varrho}{\partial t}+\operatorname{div} \boldsymbol{j}=0
$$

where $\varrho=\psi^{*} \psi \cdot \boldsymbol{j}$ can therefore be identified with the current. If we do not include any term representing the interaction then the Hamiltonian corresponds to that of a free particle and energy is connected with momentum by the relation

$$
E^{2}=p^{2} c^{2}+m^{2} c^{4}
$$

in the relativistic case and by

$$
E=p^{2} / 2 m
$$

in the nonrelativistic case. The choice of the Hamiltonian and the wave function must be such that

$$
\psi(x, y, z, t)=\psi(x, y, z) \mathrm{e}^{-i E_{p} t / \hbar} \quad \text { and } \quad H \psi(x, y, z)=E \psi(x, y, z)
$$

are satisfied. In the present discussion we have considered $\psi$ to be a complex function and in such a case the Hamiltonian in momentum space for a free nonrelativistic particle is $p^{2} / 2 m$. On the other hand if we postulate that the wave function is represented by a set of $\psi$ 's, the choice of the Hamiltonian depends on the energy-momentum relation and on what properties of the free particle we want to include in $\psi$. For example, if we wish to include spin in the nonrelativistic case by defining a two component wave function, then $H$ will involve $2 \times 2$ matrices in a manner as to yield the energy of the system when operating on $\psi$. If we wish however to satisfy the demands of relativity and spin then $\psi$ must have four components and $H$ consequently will involve $4 \times 4$ matrices suitably chosen to give the energy when operating on $\psi$.

The equation of motion (8) need not have stationary solutions. In fact this is the starting point of collision or scattering theory where the interaction is such that we do not obtain bound state solutions. In such a case the Hamiltonian is split into two parts $H_{0}$ and $H^{\prime}$ and the evolution of the system from $t=-\infty$ to $t=+\infty$ is studied under the assumption that $H_{0}$ is time independent and $H^{\prime}$ is such that it vanishes at $t=-\infty$ and $t=+\infty$. We are then interested in transitions from eigenstates of $H_{0}$ at $t=-\infty$ to those at $t=+\infty$.

\footnotetext{
${ }^{1} \nabla$ is the gradient operator.
} 
Having included the exponential time dependence in a free particle wave function, we can define a transform for $\psi(x, y, z, t)$ as

$$
A(\boldsymbol{p}, t)=(2 \pi h)^{-3 / 2} \int \psi(\boldsymbol{x}, t) \mathrm{e}^{-(i / \hbar)\left(\boldsymbol{p} \cdot \boldsymbol{x}-E_{p} t\right)} \mathrm{d}^{3} x
$$

where $E_{p}$ is the relativistic energy of the free particle with

$$
E_{p}^{2}=\boldsymbol{p}^{2} c^{2}+m^{2} c^{4}
$$

We immediately recognize that

$$
\varphi(\boldsymbol{p}, t)=A(\boldsymbol{p}, t) \mathrm{e}^{-i E t / \hbar} .
$$

In terms of $\boldsymbol{A}(\boldsymbol{p}, t)$ the inverse relation can be written as

$$
\psi(\boldsymbol{x}, t)=(2 \pi \hbar)^{-3 / 2} \int A(\boldsymbol{p}, t) \mathrm{e}^{+(i / \hbar)\left(\boldsymbol{p} \cdot \boldsymbol{x}-E_{p} t\right)} \mathrm{d}^{3} p .
$$

A particular case of the wave function is that of a free particle with a definite momentum and energy $\boldsymbol{p}_{0}, E_{0}$. The wave function in configuration space would then be represented by

$$
\psi=\mathrm{e}^{(i / \hbar)\left(p_{0} \cdot x-E_{0} t\right)} .
$$

Its momentum representation is given by

$$
\varphi(\boldsymbol{p}, t)=\delta\left(p_{x}-p_{0 x}\right) \delta\left(p_{y}-p_{0 y}\right) \delta\left(p_{z}-p_{0 z}\right) \mathrm{e}^{-i E_{0} t / \hbar} .
$$

Let us now calculate the mean and mean square deviations of $x$ and $p$ corresponding to the general wave function $\psi$. The Fourier relationship between $\varphi$ and $\psi$ forces upon us, as a necessary mathematical consequence, a relation between these two deviations which is known as the uncertainty principle. We first calculate

and

$$
\overline{p^{2}}=\int p^{2} \varphi^{*} \varphi \mathrm{d}^{3} p=\hbar^{2} \int \frac{\partial \psi^{*}}{\partial x} \frac{\partial \psi}{\partial x} \mathrm{~d}^{3} x
$$

If

$$
\begin{gathered}
\overline{x^{2}}=\int x^{2} \psi^{*} \psi \mathrm{d}^{3} x=\hbar^{2} \int \frac{\partial \varphi^{*}}{\partial p} \cdot \frac{\partial \varphi}{\partial p} \mathrm{~d}^{3} p \\
\left(\overline{x-\bar{x})^{2}}=(\Delta x)^{2} ; \quad \bar{p}^{2}=\overline{(p-\bar{p})^{2}}=(\Delta p)^{2} .\right.
\end{gathered}
$$

Working, without loss of generality, in a system in which $\bar{p}=0, \bar{x}=0$ so that

Let us consider

$$
(\Delta p)^{2}=p^{2} ; \quad(\Delta x)^{2}=x^{2}
$$

i.e.

$$
D=\left|\frac{x}{2 \bar{x}^{2}} \psi+\frac{\partial \psi}{\partial x}\right|^{2} \geqq 0,
$$

$$
\begin{array}{r}
D=\frac{x^{2}}{4\left(\overline{x^{2}}\right)^{2}} \psi \psi^{*}+\frac{x}{2 \overline{x^{2}}}\left(\psi \frac{\partial \psi^{*}}{\partial x}+\psi^{*} \frac{\partial \psi}{\partial x}\right)+\frac{\partial \psi}{\partial x} \frac{\partial \psi^{*}}{\partial x} \\
=\frac{1}{4}\left(\frac{x}{\overline{x^{2}}}\right)^{2} \psi \psi^{*}+\frac{1}{2} \frac{\partial}{\partial x}\left(\frac{x}{\overline{x^{2}}} \psi \psi^{*}\right)-\frac{1}{2} \frac{1}{\overline{x^{2}}} \psi \psi^{*}+\frac{\partial \psi}{\partial x} \frac{\partial \psi^{*}}{\partial x} \\
=\frac{1}{4} \frac{1}{\left(\overline{x^{2}}\right)^{2}}\left[x^{2}-2 \overline{x^{2}}\right] \psi \psi^{*}+\frac{1}{2} \frac{\partial}{\partial x}\left(\frac{x}{\overline{x^{2}}} \psi \psi^{*}\right)+\frac{\partial \psi}{\partial x} \frac{\partial \psi^{*}}{\partial x}
\end{array}
$$


Also

$$
\int D \mathrm{~d}^{3} x=\frac{1}{\hbar^{2}} \bar{p}^{2}-\frac{1}{4 \overline{x^{2}}} \geqq 0
$$

and

$$
\overline{p^{2}} \overline{x^{2}}=(\Delta p)^{2}(\Delta x)^{2} \geqq \frac{\hbar^{2}}{4}
$$

More generally such a relationship exists for all canonically conjugate variables. The other physically interesting relationship is between energy and time. The meaning of $\Delta E$, the uncertainty in energy, is obvious since energy is a function of momentum and space and its fluctuation can be computed from the wave function. However the physical interpretation of $\Delta t$ is not so obvious since the wave function is defined at a particular time and till now we have not adverted to $t$ as a parameter with a probability distribution. For the non-relativistic particle

$$
E=p^{2} / 2 m=\frac{1}{2} m v^{2} ; \quad \Delta E=m \Delta v
$$

(where $v$ is the velocity) and hence from the uncertainty principle

$$
\Delta p \cdot \Delta x \simeq \hbar
$$

we can write $\Delta E \cdot \Delta t \simeq \hbar$ where $\Delta t=\frac{\Delta x}{v}$ is the time taken for the particle to travel a distance equal to its spatial extension $\Delta x$. This is equivalent to saying that it is the time for the creation of the particle provided we identify a wave pulse of spread $\Delta x$ with the particle itself. The energy $E$ of the particle is then to be attributed not to any particular time but only to a time point lying anywhere in the interval $\Delta t$, the error involved in the energy being $\Delta E$.

\section{The principle of superposition ${ }^{\mathrm{I}}$}

The wave functions $\psi$ and $\varphi$ expressed in configuration and momentum space respectively are representations of the same state of the system. It has naturally been found convenient to conceive of the state of the system without any reference to the particular representation and interpret the wave functions $\psi$ and $\varphi$ for example, as projections of the state in the configuration and momentum spaces. The dynamical variables are operators, their representations depending on the space in which we wish to describe the states. Since the eigenvalues of these operators are real, we require the operators to be hermitian. A set of states for example can be obtained as the eigenstates of a suitably chosen operator $\underline{\alpha}$. If $\alpha$ is an eigenvalue of $\underline{\alpha}$, it will be independent of the representation of the operator $\underline{\alpha}$. The eigenstate can be symbolically represented by the "ket" vector $|\alpha\rangle^{2}$

i.e.

$$
\underline{\alpha}|\alpha\rangle=\alpha|\alpha\rangle \text {. }
$$

1 P. A. M. Dirac, The Principles of Quantum Mechanics, Oxford University Press, London (1947).

2 P. T. Matthews, Lectures delivered at the University of Rochester on "The Relativistic Quantum Theory of Flementary Particle Interactions" (1957). 
A complete system of eigenvectors is obtained by taking all the eigenvectors corresponding to all the eigenvalues. Defining the "bra" vector $\langle\alpha|$ as the hermitian conjugate of the ket vector $|\alpha\rangle$, the orthonormality relation can then be written as

$$
\left\langle\alpha^{\prime} \mid \alpha^{\prime \prime}\right\rangle=\delta_{\alpha^{\prime} \alpha^{\prime \prime}}
$$

where $\delta_{\alpha^{\prime} \alpha^{\prime \prime}}$ is the Kronecker delta function if $\alpha^{\prime}$ and $\alpha^{\prime \prime}$ belong to a discrete spectrum and is the Dirac delta function if $\alpha^{\prime}$ and $\alpha^{\prime \prime}$ belong to a continuous spectrum. The completeness relationship is expressed by

$$
\int_{\alpha^{\prime}}\left|\alpha^{\prime}\right\rangle\left\langle\alpha^{\prime}\right|=1
$$

where $\int_{a^{\prime}}$ is a summation or an integration over the variable $\alpha^{\prime}$.

The principle of superposition of states: Any given state $|\psi\rangle$ of a system can be expressed as a superposition of a complete set of eigenstates, i.e. $|\psi\rangle$ can be written as :

$$
|\psi\rangle=\sum_{k} c_{k}\left|\alpha_{k}\right\rangle
$$

$|\psi\rangle$ may be the eigenfunction of an operator other than $\underline{\alpha}$ but we are not concerned with it here. $\mathrm{c}_{k}$ is the amplitude for obtaining the eigenvalue $\alpha_{k}$ in a state $|\psi\rangle$.

$$
c_{k}=\left\langle\alpha_{k} \mid \psi\right\rangle \text {. }
$$

In quantum mechanical problems we deal with a $|\psi\rangle$ which can be expressed as $F|i\rangle$ where $F$ is an operator and $|i\rangle$ the eigenstate corresponding to another operator $G$. If $F$ and $G$ commute $F|i\rangle$ is an eigenstate of $G$ and hence the projection of $F|i\rangle$ on any state other than $|i\rangle$ is zero. However if $F$ does not commute with $G$ then the projection of $F|i\rangle$ on a state other than $|i\rangle$ say $|f\rangle$ is non-zero, i.e.

$$
\langle f|F| i\rangle=c_{f} \neq 0 .
$$

This is also called the matrix element of $F$ between $|i\rangle$ and $|f\rangle$. From the orthonormality relations we have $c_{k}^{*} c_{k}$ to be the probability of obtaining the eigenvalue $\alpha_{k}$ in the state $\psi$. In particular the amplitude that a single particle has momentum $p$ is $\langle p \mid \psi\rangle$, identified to be $\varphi(p)$ and the corresponding amplitude that it is at $\boldsymbol{x}$ is $\langle x \mid \psi\rangle$ equal to $\psi(x)$. Thus we have the amplitude for a particle to be at $\boldsymbol{x}$ when it has a momentum $p$ to be $\langle x \mid p\rangle^{1}$. This is equal to $(2 \pi \hbar)^{-3 / 2} \mathrm{e}^{i \boldsymbol{p} \cdot \boldsymbol{x} / \hbar}$. From this it follows that

$$
\begin{aligned}
\psi(x)=\langle x \mid \psi\rangle & =\int\langle x \mid p\rangle\langle p \mid \psi\rangle \mathrm{d}^{3} p \\
& =(2 \pi \hbar)^{-3 / 2} \int \mathrm{e}^{i \boldsymbol{p} \cdot \boldsymbol{x} / \hbar}\langle p \mid \psi\rangle \mathrm{d}^{3} p \\
& =(2 \pi \hbar)^{-3 / 2} \int \mathrm{e}^{i \boldsymbol{p} \cdot \boldsymbol{x} / \hbar} \varphi(p) \mathrm{d}^{3} p .
\end{aligned}
$$

${ }^{1}\langle x \mid p\rangle$ represents the wave function of a particle of momentum $p$. This implies a normalization corresponding to $\frac{1}{(2 \pi)^{3}}$ particles per unit volume or one particle in a volume of $(2 \pi)^{3}$ units. Later in dealing with collision processes, we will be requiring a normalization of one particle per unit volume and hence $\langle x \mid p\rangle$ will be taken to be $\mathrm{e}^{\boldsymbol{i} \boldsymbol{p} \cdot \boldsymbol{x}}$. 
Similarly

$$
\begin{aligned}
\varphi(p)=\langle p \mid \psi\rangle & =\int\langle p \mid x\rangle\langle x \mid \psi\rangle \mathrm{d}^{3} x \\
& =(2 \pi \hbar)^{-3 / 2} \int \mathrm{e}^{-i \boldsymbol{p} \cdot \boldsymbol{x} / \hbar}\langle x \mid \psi\rangle \mathrm{d}^{3} x \\
& =(2 \pi \hbar)^{-3 / 2} \int \mathrm{e}^{-i \boldsymbol{p} \cdot \boldsymbol{x} / \hbar} \psi(x) \mathrm{d}^{3} x .
\end{aligned}
$$

The probabilistic interpretation combined with the hermiticity of the operators implies that by making a unitary transformation upon a state all equations stay invariant, or equivalently theunitary transform of an eigenstate is still an eigenstate with the same eigenvalue. Let $U$ be any unitary operator which takes $\alpha$ to $\hat{\alpha}$, i.e.

$$
U \underline{\alpha} U^{-1}=\underline{\hat{\alpha}} .
$$

Conservation of probability requires $U U^{+}=1$. We have

Put

$$
\begin{gathered}
\underline{\alpha}|\alpha\rangle=\alpha|\alpha\rangle \\
U \underline{\alpha} U^{-1} U|\alpha\rangle=\alpha U|\alpha\rangle .
\end{gathered}
$$

which gives

$$
U|\alpha\rangle=|\hat{\alpha}\rangle
$$

$$
\hat{\alpha}|\hat{\alpha}\rangle=\alpha|\hat{\alpha}\rangle \text {. }
$$

When the unitary operator $U$ differs from unity by an infinitesimal quantity we can set

Thus

$$
U=1-i \varepsilon F
$$

$$
U^{+}=1+i \varepsilon F
$$

The unitarity condition on $U$ requires $F$ to be hermitian. Under this infinitesimal unitary transformation an arbitrary operator $\underline{\alpha}$ goes into

$$
\begin{aligned}
\hat{\alpha}=U \underline{\alpha} U^{-1} & =(1-i \varepsilon F) \underline{\alpha}(1+i \varepsilon F) \\
& =\underline{\alpha}-i \varepsilon[F, \alpha]
\end{aligned}
$$

or

$$
\hat{\alpha}-\underline{\alpha}=-i \varepsilon[F, \underline{\alpha}] .
$$

The above equation of motion is also true in classical dynamics if the commutator is interpreted as the Poisson bracket and $U$ the generating function of a contact transformation.

The principle of complementarity includes in a strict sense the principle of superposition. The postulate that $\varphi(\boldsymbol{p})$ is a Fourier transform of $\psi(\boldsymbol{x})$ implies that $\varphi(\boldsymbol{p})$ is a superposition of eigenstates of momentum with amplitude $\psi(\boldsymbol{x})$. It is customary to work with a complete set of free particle states which are chosen to be the eigenstates of momentum or equivalently energy.

While the square of the modulus of the amplitude has a probabilistic interpretation it is important to note that a knowledge of $c_{k}^{*} c_{k}$ for all $k$ is insufficient to describe the state $\psi$. This is merely another way of saying that the principle 
of superposition in quantum mechanics applies to wave functions and not to their moduli. The mean value of any dynamical quantity for the state $\psi$ is

$$
\langle\psi|A| \psi\rangle=\sum_{m, n} c_{n} c_{m}^{*}\langle m|A| n\rangle
$$

with

$$
\langle m|A| n\rangle=\left\langle\alpha_{m}|A| \alpha_{n}\right\rangle
$$

i.e. we should know all the amplitudes $c_{m}$ or equivalently, coefficients not merely, for the case $m=n$ but for all $m$ and $n$. The terms $c_{m}^{*} c_{n}$ are called the elements of the density matrix $\varrho$, the trace of $\varrho$ being unity. The expectation value of $A$ by the above equation is

$$
\langle\psi|A| \psi\rangle=T_{r}[\varrho A]
$$

where the elements of $[A]$ are given by

$$
A_{m n}=\langle m|A| n\rangle \text {. }
$$

In the quantum mechanical description of single particles, it is also possible to include attributes like spin which can be imbedded in the free particle wave functions. Other properties like charge can be ascribed as has been stated at the outset only when we consider the particle under interaction with external fields. The concept of interaction in dealing with elementary particles in a wave function formalism is derived through the inclusion of suitable interaction terms representing classical fields in the Hamiltonian. This results either in solutions which are eigenstates of this Hamiltonian representing bound states or transitions of various orders between eigenstates of the free particle Hamiltonian. In a more rigorous sense, it is difficult to distinguish between properties imbedded in the free particle wave function and those described through interaction. For example, mass which is included in the free particle wave function is interpreted as a "self-energy effect" due to interaction with a field associated with the particle itself. Similarly charge can be introduced in the free particle wave function using an isotopic spin formalism by increasing the components of the wave function in a suitable manner. In fact, new techniques have been developed to describe particles which are identical as regards a certain class of interactions but behave differently under other interactions. This leads to the more difficult and unsolved problem of the meaning of an elementary particle and its properties, as will be discussed in detail in later chapters.

If we were to require the annihilation or creation of a particle through interaction, the wave function formalism would be unsuitable and a field-theoretic description involving an operator calculus is forced upon us. In this formalism, even the transition of the same elementary particle from one state to another is treated as an annihilation of the one and the creation of the other. We shall in the next few chapters be concerned only with single particle wave functions and the concept of interaction as introduced through the perturbation theory.

Besides introducing attributes like spin and isotopic spin in the wave function one important mathematical requirement will be imposed, viz. the equations of 
motion should be covariant under Lorentz transformations. This is also physically reasonable since the laws of physics should be describable in the same manner in all inertial frames of reference. The immediate conseqence of the application of the principle of relativity is the possibility of the existence of negative energy states in view of the quadratic relation between energy and momentum. Thus another restriction has to be imposed on the physical grounds that such negative energy particles do not exist. This is taken care of by a suitable interpretation of these states. Particles which exist in positive energy states continue to do so in the absence of interaction. If the interaction implies the absorption of a negative energy particle then this absorption is interpreted as the emission of a positive energy antiparticle, the antiparticle being defined by this interpretation itself. If the particle and antiparticle are identical as in the case of photons, we just take the emission of a negative energy photon to be the absorption of a positive energy photon. In perturbation theory absorption should succeed emission and hence the emission of a negative energy photon should succeed the absorption.

There is however a very important principle governing the behaviour of an assembly of similar particles known as the Pauli principle, which states that it is impossible to find two particles in the same state. Particles which obey this rule are called fermions while the rest are called bosons. In a field theoretic formalism, the Pauli principle is introduced through commutation relations between field operators associated with the particle. In a wave function formalism it cannot be introduced in the description of a single free particle. However the statistical behaviour in an assembly of similar particles which can be described in the wave function formalism when the total number of particles is not changing, depends upon whether the particles are bosons or fermions. In the case of fermions, the requirement that no two particles should occupy the same state can be satisfied by choosing the wave function to be a Slater determinant

$$
\Psi=\frac{1}{\sqrt{k} !}\left|\begin{array}{cccc}
\psi_{1}^{(1)} & \psi_{1}^{(2)} & \cdots & \psi_{1}^{(\bar{k})} \\
\psi_{2}^{(1)} & \psi_{2}^{(2)} & \cdots & \psi_{2}^{(k)} \\
\vdots & \vdots & & \vdots \\
\psi_{k}^{(1)} & \psi_{k}^{(2)} & \cdots & \psi_{k}^{(k)}
\end{array}\right|
$$

where $\psi_{j}^{(i)}$ represents the $i$ th particle in an assembly of $k$ particles, in the $j$ th state. This implies that the wave function should be antisymmetric under an exchange of two particles. One of the most important applications of the Pauli principle is the suitable interpretation of negative energy states of fermions through the postulate of an unobservable "sea" of negative energy particles and the consequent introduction of the concept of the antiparticle.

When dealing with interactions of elementary particles, a single particle formalism with the inclusion of negative energy states therefore leads to the creation and annihilation of particles and antiparticles and necessitates the use of field-theoretic methods. But the single particle formalism can still be retained by the Feynman interpretation of negative energy states by a suitable prescription 
for tracing the sequence of events when considering the transitions of various orders in an interaction.

Thus the theory of elementary particles is presented in the following order. We first give a systematic account of free particle wave functions of various elementary particles with the corresponding attributes imbedded suitably in the wave function subject to the demands of relativity. Interaction is then described through perturbation theory for a non-relativistic system and then by introducing the concept of negative energy the Feynman formalism is dealt with in great detail. This leads naturally to the field-theoretic description of interactions.

\section{SINGLE PARTICLE WAVE EQUATIONS}

\section{Spin and Pauli's equation}

We have till now considered the wave function of a particle without introducing the concepts of spin and the requirements of relativity. We shall first deal with the concept of spin, one of the intrinsic attributes of a fundamental particle.

Before we introduce the concept of spin, we shall discuss the nature of the dynamical variable representing angular momentum ${ }^{1}$. In classical mechanics, the components of angular momentum associated with a particle at the point $x, y, z$, are given by

$$
M_{x}=y p_{z}-z p_{y} ; \quad M_{y}=z p_{x}-x p_{z} ; \quad M_{z}=x p_{y}-y p_{x} .
$$

In wave mechanics where the concept of a point particle is replaced by that of a wave function, the corresponding angular momentum operators are constructed by replacing $p_{x}, p_{y}$ and $p_{z}$ by the operators $-i \frac{\partial}{\partial x},-i \frac{\partial}{\partial y}$ and $-i \frac{\partial}{\partial z}$ respectively when the wave function is expressed in configuration space. Using the well-known commutation relations for the components of position and linear momentum of a particle we can readily derive the commutation rules for the components of the angular momentum operator which read

$$
\left[M_{x}, M_{y}\right]=i M_{z} ; \quad\left[M_{y}, M_{z}\right]=i M_{x} ; \quad\left[M_{z}, M_{x}\right]=i M_{\nu} .
$$

The square of the total angular momentum is

$$
M^{2}=M_{x}^{2}+M_{y}^{2}+M_{z}^{2} .
$$

As can be seen easily, this operator commutes with $M_{x}, M_{y}$ and $M_{z}$.

Now if we define the non-hermitian operators $M_{+}$and $M_{-}$as

$$
M_{+}=M_{x}+i M_{y} ; \quad M_{-}=M_{x}-i M_{y}
$$

they obey the commutation relations

$$
\begin{aligned}
& {\left[M^{2}, M_{ \pm}\right]=0 \quad\left[M_{z}, M_{+}\right]=M_{+}} \\
& {\left[M_{z}, M_{-}\right]=-M_{-} ; \quad\left[M_{+}, M_{-}\right]=2 M_{z} .}
\end{aligned}
$$

1 G. Wentzel, Lectures delivered at the Tata Institute of Fundamental Research, Bombay, on "Special Topics in Quantum Mechanics" (1956). 
Since $M^{2}$ an $\mathrm{d} M_{z}$ commute, we can choose a wave function which is an eigenfunction of both $M^{2}$ and $M_{z}$ simultaneously and let us represent the eigenvalue of these operators by $M_{j}^{2}$ and $m$. As we are dealing with the simultaneous eigenfunction of $M^{2}$ and $M_{z}$, we can characterize such an eigenstate by two quantum numbers $j$ and $m, j$ being related to $M_{j}^{2}$. Just from the use of the commutation relations, we can obtain the values of $\boldsymbol{M}_{j}^{2}$ and the possible values of $m$. We shall show that $M_{j}^{2}=j(j+1)$ and that $m$ can assume $(2 j+1)$ values from $-j$ to $+j$, $j$ being positive. Taking the matrix element of $M^{2}$ and $M_{z}$ between two states $j m$ and $j^{\prime} m^{\prime}$ we have

$$
\begin{aligned}
& \left\langle j^{\prime} m^{\prime}\left|M^{2}\right| j m\right\rangle=M_{j}^{2} \delta_{j j^{\prime}} \delta_{m m^{\prime}} \\
& \left\langle j^{\prime} m^{\prime}\left|M_{z}\right| j m\right\rangle=m \delta_{j j^{\prime}} \delta_{m m^{\prime}} .
\end{aligned}
$$

From the commutation relation

$$
\left[M^{2}, M_{+}\right]=0
$$

we obtain the condition

$$
\left\langle j^{\prime} m^{\prime}\left|M_{+}\right| j m\right\rangle\left(M_{j^{\prime}}^{2}-M_{j}^{2}\right)=0
$$

which implies that the matrix element of $M_{+}$exists only for $j=j^{\prime}$.

The commutation relation

leads to

$$
\left[M_{z}, M_{+}\right]=M_{+}
$$

i.e.

$$
\left(m^{\prime}-m\right)\left\langle m^{\prime}\left|M_{+}\right| m\right\rangle=\left\langle m^{\prime}\left|M_{+}\right| m\right\rangle,
$$

$$
\left(m^{\prime}-m-1\right)\left\langle m^{\prime}\left|M_{+}\right| m\right\rangle=0
$$

which means that the operator $M_{+}$switches the state $m$ to $m+1$, and similarly it can be shown that the operator $M_{-}$switches the state $m$ to $m-1$. It is to be noted that the various values of $m$ differ from the previous one by unity.

Finally it can be shown that the commutation relation

$$
\left[M_{+}, M_{-}\right]-2 M_{z}=0 \text {, }
$$

leads to a difference equation from which the entire spectrum of eigenvalues of $M^{2}$ and $M_{z}$ can be abstracted. From the above equation we have

$$
\sum_{m^{\prime \prime}}\left\langle j m\left|M_{+}\right| j m^{\prime \prime}\right\rangle\left\langle j m^{\prime \prime}\left|M_{-}\right| j m^{\prime}\right\rangle-\left\langle j m\left|M_{-}\right| j m^{\prime \prime}\right\rangle\left\langle j m^{\prime \prime}\left|M_{+}\right| j m^{\prime}\right\rangle=2 m \delta_{m m^{\prime}}
$$

Noting that $\left\langle m\left|M_{+}\right| m^{\prime \prime}\right\rangle$ is the complex conjugate of $\left\langle m^{\prime \prime}\left|M_{-}\right| m\right\rangle$ and writing for convenience $\left\langle j m\left|M_{+}\right| j m-1\right\rangle=N_{j m}$, we have

$$
\left|N_{j m}\right|^{2}-\left|N_{j m+1}\right|^{2}=2 m \text {. }
$$

The solution of the above difference equation is given by

$$
\left|N_{j m}\right|^{2}=C_{j}-m(m-1)
$$

Now two numbers $m_{2}$ and $m_{1}$ with $m_{1}>m_{2}$ can be found such that

$$
\begin{gathered}
\left|N_{j m+1}\right|^{2}=0 \\
\text { for } m \geqq m_{1} \text { or } \quad m<m_{2}
\end{gathered}
$$


i.e. the permissible values of $m$ range from $m_{2}$ to $m_{1}$ and no eigenstates exist with $m>m_{1}$ or $m<m_{2}$. The above equation is equivalent to writing

$$
\left|N_{j} m_{1}+1\right|^{2}=0
$$

and

$$
\left|N_{j m_{2}}\right|^{2}=0
$$

(i.e.) $m_{1}+1$ and $m_{2}$ are the roots of the equation

$$
\left|N_{j m}\right|^{2}=0
$$

or

$$
-C_{j}+m(m-1)=\left(m-m_{2}\right)\left(m-m_{1}-1\right) .
$$

Equating coefficients of same powers of $m$ on both sides, we obtain

and

$$
m_{2}=-m_{1} ; C_{j}=m_{1}\left(m_{1}+1\right)
$$

$$
\left|N_{j m}\right|^{2}=C_{j}-m(m-1)=m_{1}\left(m_{1}+1\right)-m(m-1) .
$$

Writing $m_{1}=j$ we have

$$
\left|N_{j m}\right|^{2}=j(j+1)-m(m-1) .
$$

Now $m$ can take the values

$$
=-j,-j+1, \ldots j-1, j
$$

i.e. $2 j$ must be an integer or $j$ may be an integer or half integer.

Experimental evidence necessitated the assumption of an intrinsic angular momentum to some fundamental particles, i.e. an angular momentum which is an inseparable attribute like the mass or charge. The square of this angular momentum for an electron is known to be $\frac{3}{4}$ which should be identified with $j=\frac{1}{2}$ since we know that the total angular momentum operator $M^{2}$ has the eigenvalue $j(j+1)$. Hence $m$ can take the values $\pm \frac{1}{2}$. The operators $M_{x}$ and $M_{y}$ connect $m=\frac{1}{2}$ and $m=-\frac{1}{2}$ state and vice versa while $M_{z}$ connects $m= \pm \frac{1}{2}$ with $m= \pm \frac{1}{2}$ respectively.

Since "spin" is attributed to a particle and cannot be traced to its spatial distribution it is clear that we cannot use differential operators like $-i \frac{\partial}{\partial x}$ as we did in the case of angular momentum.

Assuming that the commutation relationships define completely the nature of the angular momentum operators and that these operators must connect states of eigenvalues $\pm \frac{1}{2}$ it is possible to convince ourselves that the above requirements can be satisfied if the spin states are represented in the two component form as $\left[\begin{array}{c}\psi_{1} \\ 0\end{array}\right]$ and $\left[\begin{array}{c}0 \\ \psi_{2}\end{array}\right]$, the eigenvectors corresponding to the eigenvalues $\pm \frac{1}{2}$ respectively and the angular momentum operators are represented by the matrices, ${ }^{1}$

$$
\sigma_{x}=\left[\begin{array}{ll}
0 & 1 \\
1 & 0
\end{array}\right] ; \quad \sigma_{y}=\left[\begin{array}{cc}
0 & -i \\
i & 0
\end{array}\right] ; \quad \sigma_{z}=\left[\begin{array}{cc}
1 & 0 \\
0 & -1
\end{array}\right]
$$

1 W. PAuli, Handbuch der Physik, Vol. 5, Springer-Verlag (1958). 
It can be easily verified that these matrices satisfy the same commutation relations as the components of angular momentum and also that these matrices connect the states referred to as before. Any spin state of the system is a linear combination of $\left[\begin{array}{c}\psi_{1} \\ 0\end{array}\right]$ and $\left[\begin{array}{c}0 \\ \psi_{2}\end{array}\right]$. Thus the Schrödinger equation for a particle of spin $\frac{1}{2}$ should be

$$
i \frac{\partial \psi}{\partial t}=H \psi
$$

where $H$ will involve the $2 \times 2$ spin matrices in a manner that the angular momentum operator commutes with it. Pauli showed that such a Hamiltonian can be obtained by replacing $\boldsymbol{x}^{2}$ in the Schrödinger equation by the matrix $(\sigma \cdot \boldsymbol{x})^{2}$. Pauli's equation reads:

$$
W \psi=\frac{1}{2 m}(\sigma \cdot \pi)^{2} \psi+V \psi
$$

where $\boldsymbol{\pi}=(\boldsymbol{p}-e \boldsymbol{A})$ and $V=e \varphi$ and $\boldsymbol{W}$ is the kinetic energy where $\boldsymbol{A}$ and $\varphi$ are the vector and scalar potentials of the electromagnetic field in which the particle is moving. Using the general formula that if $\boldsymbol{B}$ and $\boldsymbol{C}$ are any 3-dimensional vectors

$$
(\boldsymbol{\sigma} \cdot \boldsymbol{B})(\boldsymbol{\sigma} \cdot \boldsymbol{C})=(\boldsymbol{B} \cdot \boldsymbol{C})+i(\boldsymbol{\sigma} \cdot \boldsymbol{B} \times \boldsymbol{C})
$$

(in our case $\boldsymbol{B}=\boldsymbol{C}=\boldsymbol{p}-e \boldsymbol{A}$ ) and remembering that curl $\boldsymbol{A}=\boldsymbol{H}$ (where $\boldsymbol{H}$ is the magnetic field), we can write Pauli's equation in the form

$$
W \psi=\frac{1}{2 m}\left[\pi^{2}+e \boldsymbol{\sigma} \cdot \boldsymbol{H}\right] \psi+V \psi .
$$

The Hamiltonian given by (24) is similar to the classical Hamiltonian for an electron in a field except for the term $\frac{e}{2 m} \boldsymbol{\sigma} \cdot \boldsymbol{H}$, which arises only in quantum theory.

This additional energy can be attributed to an intrinsic magnetic moment of the electron given by $\frac{e}{2 m} \sigma$, since an electron which has a spin angular momentum $\frac{1}{2}$ in a particular direction will have a magnetic moment in the same direction. The magnetic moment associated with orbital angular momentum $l$ is $\frac{e l}{2 m}$ or $l$ Bohr magnetons. The ratio of magnetic moment in units of Bohr magnetons to the angular momentum is called the gyromagnetic ratio and in the case of spin it is twice that associated with orbital angular momentum, i.e. the magnetic moment is not given by $\frac{e s}{2 m}$ but by $\frac{e}{2 m} g s$. For the electron $g$, the gyromagnetic ratio is approximately equal to 2 since the magnetic moment is found to be approximately one Bohr magneton. For a free particle (24) reduces, if we retain the first form of the equation, to

$$
W \psi=\frac{1}{2 m}(\boldsymbol{\sigma} \cdot \boldsymbol{p})^{2} \psi .
$$

EPCR 2 
Expanding the right hand side, we note that the commutation relations for $\sigma_{x}, \sigma_{y}, \sigma_{z}$ are just the conditions for the above equation to reduce to the form of Schrödinger equation remembering of course that $\psi$ has two components. In the free particle case each of these components satisfies the Schrödinger equation while equation (25) is satisfied only by the two component $\psi$. It can be verified that only the square of the sum of the orbital and spin angular momenta commutes with the Hamiltonian and not of each of them separately. In this way, the concept of spin is deduced from the equation postulated first.

It would be worth emphasizing the peculiar features of angular momentum in quantum mechanics as contrasted with angular momentum in classical mechanics. In the latter case the angular momentum of a particle is represented by a vector $\boldsymbol{M}$. But such a vector representation for the total angular momentum is not possible in quantum mechanics for the following reason. If we are dealing with a particle in a definite state of total angular momentum, i.e. represented by an eigenstate of $M^{2}$, it is possible to choose a state which is an eigenstate of both the operator corresponding to the total angular momentum $M^{2}$ and the operator corresponding to its component in any specified direction (which, without loss of generality, can be chosen to be the $z$-direction), but this function is not an eigenstate of $M_{x}$ or $M_{y}$ but only of $M_{x}^{2}+M_{y}^{2}$. This implies that we cannot represent the total angular momentum by a vector as we do in classical mechanics, since if we did so, the three components would be determined and this contradicts the above hypothesis.

Later in dealing with general principles of invariance of equations of motion of fundamental particles we shall show how the concept of angular momentum and spin arise from the transformation properties under space rotation.

\section{Relativistic requirements and the Klein-Gordon equation}

We shall now turn to the demands of relativity which can be stated in the simplest terms as follows. According to the principle of relativity, the laws of the fundamental particles should be the same in all systems of reference moving with uniform velocity with respect to each other. Thus the equations which express these laws should be covariant under Lorentz transformations. The Schrödinger equation of a free particle which we obtained as the quantum mechanical analogue of the classical non-relativistic equation connecting energy and momentum is obviously not invariant under the Lorentz transformation since it is of second order in spatial coordinates and of first order in the time coordinate.

To obtain a relativistically invariant equation, we start from the classical relativistic relation between the energy and momentum of a free particle

$$
E^{2}=p^{2}+m^{2} \quad \text { or } \quad p_{\mu}^{2}=m^{2} .
$$

In quantum mechanics the corresponding equation for the wave function $\psi$ in configuration space is given by

$$
\left[p_{\mu}^{2}-m^{2}\right] \psi=0
$$

where

$$
p_{4}=i \frac{\partial}{\partial t} \quad \text { and } \quad p_{i}=-i \nabla_{i}
$$


This equation known as the Klein-Gordon equation was first discovered by Schrödinger. It satisfies the demands of relativity but does not include the concept of spin. In the presence of an electromagnetic field described by the 4-vector potential $A_{\mu}$ the 4 -vector $p_{\mu}$ is replaced by $\pi_{\mu}=p_{\mu}-e A_{\mu}$. Defining the 'current' $\boldsymbol{j}$ as

$$
\boldsymbol{j}=\frac{1}{2 i m}\left\{\psi^{*} \nabla \psi-\psi \nabla \psi^{*}\right\}
$$

the charge density $\varrho$ which should satisfy the continuity equation along with $\boldsymbol{j}$ is given by

$$
\varrho=\frac{i}{2 m}\left\{\psi^{*} \frac{\partial \psi}{\partial t}-\psi \frac{\partial \psi^{*}}{\partial t}\right\} .
$$

It is to be noted that while in the case of the non-relativistic Schrödinger equation $\varrho$, defined as $\psi^{*} \psi$, satisfied the continuity equation, in this case it is only the $\varrho$ defined as above that satisfies such an equation. Since the Klein-Gordon equation is of second order in the time derivative, its solution can be obtained only by specifying two initial conditions, i.e. $\psi$ and $\frac{\partial \psi}{\partial t}$ at some time $t$. As these can be chosen independently, $\varrho$ may assume positive as well as negative values. This defect was realised even when the equation was originally proposed by Schrödinger. Later Pauli and Weisskopf ${ }^{\mathbf{1}}$ were able to interpret the Klein-Gordon equation as a field equation and $\varrho$ as the difference between the densities of two types of particles with opposite charges. This interpretation can be understood in the wave function formalism by rewriting the Klein-Gordon equation in the following manner ${ }^{2}$. It is well known that a second order equation in $\psi$ is equivalent to two simultaneous first order equations in two independent variables $\psi$ and $\frac{\partial \psi}{\partial t}$ or more generally in two independent linear combinations of $\psi$ and $\frac{\partial \psi}{\partial t}$. In particular defining $\Phi$ and $\chi$ by

$$
\Phi=\frac{1}{\sqrt{ } 2}\left(\psi+\frac{\partial \psi}{\partial t}\right), \quad \chi=\frac{1}{\sqrt{ } / 2}\left(\psi-\frac{\partial \psi}{\partial t}\right)
$$

we see that $\Phi$ and $\chi$ satisfy

$$
\begin{aligned}
& i \frac{\partial \Phi}{\partial t}=\frac{1}{2 m}\left[\frac{\nabla}{i}-e \boldsymbol{A}\right]^{2}(\Phi+\chi)+(e \varphi+m) \Phi \\
& i \frac{\partial \chi}{\partial t}=\frac{-1}{2 m}\left[\frac{V}{i}-e \boldsymbol{A}\right]^{2}(\Phi+\chi)+(e \varphi-m) \psi .
\end{aligned}
$$

The above set of equations can be written in the matrix form

$$
i \frac{\partial \Psi}{\partial t}=H \Psi
$$

1. W. Pautli and V. Weisskopf, Helv. Phys. Acta 7,709 (1934).

2 H. Feshbach and F. Villars, Rev. Mod. Phys. 30, 24 (1958). 
where

$$
\Psi=\left[\begin{array}{l}
\Phi \\
\chi
\end{array}\right] ; H=\left(\sigma_{z}+i \sigma_{y}\right) \frac{1}{2 m}(\boldsymbol{p}-e \boldsymbol{A})^{2}+m \sigma_{z}+e \varphi
$$

and $\sigma_{x}, \sigma_{y}, \sigma_{z}$ are the Pauli spin matrices given by equation (20). $\Psi$ is the two component wave function which satisfies the Klein-Gordon equation in the first order form. In terms of this two component wave function $\Psi$ we define $\varrho$ as

$$
\varrho=\Psi * \sigma_{z} \Psi=\Phi * \Phi-\chi^{*} \chi .
$$

Note that we have introduced the matrix $\sigma_{z}$ in the expression for $\varrho$ and that $\varrho$ is the difference of two positive-definite quantities. Defining another two component wave function $\Psi_{c}=\left[\begin{array}{l}\chi^{*} \\ \Phi^{*}\end{array}\right]$ we find that $\Psi_{c}$ satisfies

$$
i \frac{\partial \Psi_{c}}{\partial t}=H(-e) \Psi_{c}
$$

where $H(-e)$ is the Hamiltonian (34) with $e$ replaced by $-e$. We therefore recognize $\Psi_{c}$ to be the charge conjugate wave function of $\Psi$. $\Phi$ and $\chi$ are determined if we are given $\Psi$ and $\Psi_{c}$. The density $\varrho$ can be defined in terms of $\Psi$ and $\Psi_{c}$ as:

$$
\varrho=\frac{1}{2}\left[\Psi^{*} \sigma_{z} \Psi-\Psi_{c}^{*} \sigma_{z} \Psi_{c}\right]=\Phi^{*} \Phi-\chi^{*} \chi,
$$

i.e. as the difference of two densities corresponding to particles of opposite charges.

For a given momentum $\boldsymbol{p}$ we can write the free particle solution of the KleinGordon equation as

$$
\Psi=\Psi_{0}(p) \mathrm{e}^{i\left(\boldsymbol{p} \cdot \boldsymbol{x}-p_{4} t\right)}=\left[\begin{array}{l}
\Phi_{0}(p) \\
\chi_{0}(p)
\end{array}\right] \mathrm{e}^{i\left(\boldsymbol{p} \cdot \boldsymbol{x}-p_{4} t\right)}
$$

where $p_{4}$ is the eigenvalue of $H$. Feeding this solution in equation (33) we have

$$
\begin{aligned}
\left(p_{4}-m\right) \Phi_{0} & =\left(p^{2} / 2 m\right)\left(\Phi_{0}+\chi_{0}\right) \\
\left(p_{4}+m\right) \chi_{0} & =\left(-p^{2} / 2 m\right)\left(\Phi_{0}+\chi_{0}\right) .
\end{aligned}
$$

The characteristic equation for $p_{4}$ is $p_{4}^{2}=\left(p^{2}+m^{2}\right)=E_{p}^{2}$. Thus for a given value of $\boldsymbol{p}$ there are two solutions corresponding to the two values $\pm E_{p}$ of $p_{4}$ where $E_{p}=+V p^{2}+m^{2}$. The associated solutions are:

$$
\begin{aligned}
& p_{4}=+E_{p} ; \quad \Psi_{0}^{+}(p)=\left[\begin{array}{c}
\Phi_{0}^{+} \\
\chi_{0}^{+}
\end{array}\right]=\left[\begin{array}{l}
\frac{E_{p}+m}{2 \gamma m E_{p}} \\
\frac{m-E_{p}}{2 \gamma / m E_{p}}
\end{array}\right] \\
& \varrho=\Psi_{0}^{+*} \Psi_{0}^{+}=1 \\
& p_{4}=-E_{p}, \quad \Psi^{0}(p)=\left[\begin{array}{c}
\Phi_{0}^{-} \\
\chi_{0}^{-}
\end{array}\right]=\left[\begin{array}{c}
\frac{m-E_{p}}{2 \gamma / m E_{p}} \\
\frac{E_{p}+m}{2 \sqrt{ } E_{p}}
\end{array}\right] \\
& \varrho=\Psi_{0}^{-* *} \Psi_{0}^{-}=-1 .
\end{aligned}
$$

We recognise that $\Psi^{-}(p)=\Psi_{c}^{+}(p)$, i.e. the negative energy solution $\Psi^{-}$is just the charge conjugate solution $\Psi_{c}$ of the positive energy. 


\section{The Dirac equation ${ }^{1}$}

We have seen that when the non-relativistic Schrödinger equation is generalized to meet the demands of relativity, it becomes an equation of the second order in the differential coefficient with respect to time. This brings in its wake the difficulty of the probability density becoming negative, a characteristic feature of the second order (in time) equations. Such an equation can be reduced to a first order equation with two component wave functions. On the other hand, if we ignore relativity but take into account the two possible spin states of a spin half particle, we are led to a first order (in time) two component equation.

To satisfy the demands of both relativity and spin it is necessary either to generalize the Pauli equation to satisfy the relativistic requirements or the two component first order form of the Klein-Gordon equation to include spin. The first procedure is easier and is outlined below. This will be followed by a formal derivation of the Dirac equation from "first principles".

We shall adopt a derivation due to Feynman ${ }^{2}$ which consists in writing a second order relativistic equation including spin and expressing it in the first order form which is the Dirac equation. The Pauli equation was obtained by replacing the term $\pi^{2}$ in the Schrödinger Hamiltonian by $(\sigma \cdot \pi)^{2}$. Thus including spin in the second order Klein-Gordon equation we expect to have

where

$$
\left\{\pi_{4}^{2}-(\sigma \cdot \pi)^{2}\right\} \psi=m^{2} \psi
$$

$$
\pi_{\mu}=p_{\mu}-e A_{\mu} .
$$

It is to be noted that $\psi$ now has two components. The second order form of the Dirac equation which can be reduced to the familiar first order form with four components should correctly be written as

Defining $\chi$ by

$$
\left(\pi_{4}+\boldsymbol{\sigma} \cdot \boldsymbol{\tau}\right)\left(\pi_{4}-\boldsymbol{\sigma} \cdot \boldsymbol{\tau}\right) \psi=m^{2} \psi
$$

we have

$$
\left(\pi_{4}+\boldsymbol{\sigma} \cdot \boldsymbol{x}\right) \psi=m \chi
$$

$$
\left(\pi_{4}-\boldsymbol{\sigma} \cdot \boldsymbol{x}\right) \chi=m \psi \text {. }
$$

Writing $\psi_{a}=\psi+\chi$ and $\psi_{b}=\chi-\psi$ the above pair of equations reduces to

$$
\left(\gamma_{4} \pi_{4}-\boldsymbol{\gamma} \cdot \boldsymbol{\tau}\right) \psi=m \psi
$$

where $\gamma_{\mu}(\mu=1,2,3,4)$ are the $4 \times 4$ Dirac matrices defined as follows:

$$
\gamma_{4}=\left[\begin{array}{cc}
I & 0 \\
0 & -I
\end{array}\right] ; \quad \gamma=\left[\begin{array}{cc}
0 & \sigma \\
-\sigma & 0
\end{array}\right] \quad \text { and } \quad \psi=\left[\begin{array}{l}
\psi_{a} \\
\psi_{b}
\end{array}\right] \text {. }
$$

This is the well known form of the Dirac equation.

${ }^{1}$ P. A. M. Drrac, Principles of Quantum Mechanics, Oxford University Press, London (1947).

2 R. P. Feynman, Lectures delivered at the California Institute of Technology, on "Quantum Electrodynamics" (1953).

${ }^{3}(\mathbf{4 5})$ is distinct from (44) since $\pi_{4}$ does not commute with $\sigma \cdot \boldsymbol{\pi}$. 
If we adopt a similar procedure to include spin in the two component KleinGordon equation, we encounter the square root of the operator $\pi^{2}$ in place of $\pi$ so that we cannot form a scalar product like $\boldsymbol{\sigma} \cdot \boldsymbol{\pi}$. Thus it seems that the derivation of the Dirac equation is not so obvious in this approach.

For the sake of completeness we shall now give the formal derivation of the Dirac equation ${ }^{1}$. The most general linear equation of the first order in space and time derivatives satisfied by an $N$ component wave function $(N \times 1$ matrix $)$ $\psi$ can be written as

$$
\frac{\partial \psi}{\partial x_{4}}+\sum_{k=1}^{3} \alpha_{k} \frac{\partial \psi}{\partial x_{k}}+i m \beta \psi=0
$$

where $\alpha_{1}, \alpha_{2}, \alpha_{3}$ and $\beta$ are $N \times N$ matrices whose elements are independent of space time coordinates. We shall now obtain the conditions satisfied by these matrices merely by imposing the quantum mechanical requirements on $\psi$. We define the probability density $\varrho$ as $\psi^{*} \psi$ where $\psi^{*}$ the hermitian adjoint of $\psi$, is a row matrix. In order that $\varrho$ is always positive-definite and satisfies the equation of continuity, we must have $\alpha^{\prime}$ s and $\beta$ hermitian, i.e.

$$
\left(\alpha_{k}\right)^{\dagger}=\alpha_{k} ; \quad \beta^{\dagger}=\beta
$$

If we now require that the second order form of the Dirac equation should correspond to the Klein-Gordon equation, we obtain the following properties of the $\alpha^{\prime}$ s and $\beta$. where

(i) $\alpha_{i} \alpha_{l}+\alpha_{l} \alpha_{i}=2 \delta_{i l}$

(ii) $\alpha_{k} \beta+\beta \alpha_{k}=0$ and

$$
\begin{array}{ll}
\delta_{i l}=0, & i \neq l \\
\delta_{i l}=1, & i=l
\end{array}
$$

(iii) $\left(\alpha_{k}\right)^{2}=(\beta)^{2}=1$.

Using these relations we shall show that $N$ must be even. We have from (ii)

$$
\alpha_{k} \beta=-\beta \alpha_{k}
$$

Taking the trace on both sides we have:

$$
\begin{aligned}
\operatorname{Tr}\left(\alpha_{k} \beta\right) & =-\operatorname{Tr}\left(\beta \alpha_{k}\right) \\
\operatorname{Tr}(\beta) & =-\operatorname{Tr}\left[\left(\alpha_{k}\right)^{-1} \beta \alpha_{k}\right] \\
& =-\operatorname{Tr}\left[\alpha_{k}\left(\alpha_{k}\right)^{-1} \beta\right]=-\operatorname{Tr} \beta=0 .
\end{aligned}
$$

If we choose the $\beta$ matrix to be diagonal, since $\beta^{2}=1$ its diagonal elements are +1 or -1 . As $\operatorname{Tr}(\beta)=0, \beta$ must have an even number of diagonal elements, i.e. $\beta$ is even. The $\alpha_{k}$ 's must also be even as they are of the same dimensions as $\beta$. It can be shown that there are only sixteen linearly independent matrices obeying

1 S. Schweber, H. A. Bethe and F. De Hoffmann, Mesons and Fields, Vol. I, Row, Peterson, Evanston, Illinois (1955). 
the above relations. Consequently it is enough if $N=4$ as we can choose sixteen linearly independent $4 \times 4$ matrices.

To cast the Dirac equation in a form more symmetric in space and time we write

$$
\beta=\gamma_{4} ; \quad \beta \alpha_{k}=\gamma_{k}(k=1,2,3) \quad \text { or } \quad \beta \alpha=\gamma .
$$

On this definition, the $\gamma_{k}$ are anti-hermitian, i.e. $\left(\gamma_{k}\right)^{\dagger}=-\gamma_{k}$ with $\left(\gamma_{k}\right)^{2}=-1$ while $\gamma_{4}$ is hermitian with $\gamma_{4}^{2}=+1$. As before we have

$$
\left(\gamma_{\mu} \gamma_{\nu}+\gamma_{\nu} \gamma_{\mu}\right)=2 \delta_{\mu \nu}
$$

These commutation rules suffice to define the Dirac equation. However it will be convenient to represent the $\gamma$-matrices explicitly the standard representation of which has been given earlier. The Dirac equation now reads:

$$
\left\{-i\left(\gamma_{4} \frac{\partial}{\partial x_{4}}+\gamma \cdot \nabla\right)+m\right\} \psi=0 \text {. }
$$

In accordance with Feynman's notation if $A$ be any 4-vector we define $\mathrm{A}$ as:

$$
\mathrm{A}=\gamma_{4} A_{4}-\gamma \cdot \boldsymbol{A}=\gamma_{\mu} A_{\mu} .
$$

Defining the 4-dimensional $\boldsymbol{\nabla}$ as

$$
\nabla_{x, y, z}=\frac{-\partial}{\partial x}, \frac{-\partial}{\partial y}, \frac{-\partial}{\partial z} ; \quad \nabla_{t}=\frac{\partial}{\partial x_{1}}
$$

the Dirac equation reads

$$
(i \nabla-m) \psi=0
$$

Replacing $\frac{i \partial}{\partial x_{\mu}}$ by $p_{\mu}$ the equation becomes

$$
(\mathrm{P}-m) \psi=0, \quad \mathrm{P}=\gamma_{\mu} p_{\mu} .
$$

Defining $\bar{\psi}$ the adjoint wave function by $\bar{\psi}=\psi^{\dagger} \gamma_{4}$, we find that $\bar{\psi}$ satisfies $\bar{\psi}(\mathrm{p}-m)=0$. The current-density 4-vector is now:

$$
(\varrho, j)=j_{\mu}=\bar{\psi} \gamma_{\mu} \psi
$$

Before discussing the solutions of the Dirac equation let us advert to one important transformation property of the equation under a Lorentz transformation. If we define the Lorentz transformation by $x^{\prime}=L x$ then the wave function in the new frame is not obtained by merely replacing $x$ by $L^{-1} x^{\prime}$ but also by operating a matrix $S$ on $\Phi\left(L^{-1} x^{\prime}\right)$, i.e. $\Phi^{\prime}\left(x^{\prime}\right)=S \Phi\left(L^{-1} x^{\prime}\right) . \Phi^{\prime}$ satisfies the same equation as $\Phi$ the differential coefficients now being with respect to $x^{\prime}$ instead of $x$ and this matrix $S$ is defined by

$$
S \gamma_{\mu} S^{-1}=\sum_{v} L_{\mu \nu} \gamma_{v}
$$

where $L_{\mu \nu}$ are the elements of the matrix $L$. We shall continue this discussion in the chapter III, section 4.

${ }^{1}$ Here $\delta_{\mu \nu}=0 \mu \neq v, \delta_{11}=\delta_{22}=\delta_{33}=-1, \delta_{14}=1$. This should be distinguished from $\delta_{i l}$ in (52). 
Solutions of the Dirac equation for a free particle

From now on we will confine ourselves to the solutions of the Dirac equation for a free particle

$$
(\mathrm{P}-m) \psi=0 \text {. }
$$

In analogy with the free particle solution of the Schrödinger equation let us assume the solution for a given momentum $\boldsymbol{p}$ to be of the form

i.e.

$$
\psi=u(p) \mathrm{e}^{-i p \cdot x}
$$

$$
\left[\begin{array}{l}
\psi_{1} \\
\psi_{2} \\
\psi_{3} \\
\psi_{4}
\end{array}\right]=\left[\begin{array}{l}
u_{1} \\
u_{2} \\
u_{3} \\
u_{4}
\end{array}\right] \mathrm{e}^{-i p \cdot x}
$$

where the coefficient $p_{4}$ of $t$ and the eigen vector $u(p)$ are to be determined from the condition that $\psi$ has to satisfy the Dirac equation. The equation for the Dirac spinor $u$ is obtained by feeding the solution in equation (63):

$$
\mathrm{P} \psi=m \psi ; \quad \mathrm{P} u=m u .
$$

We can rewrite the Dirac equation in the form

where

$$
H u=p_{4} u \text {. }
$$

$$
H=(\boldsymbol{\alpha} \cdot \boldsymbol{p}+\beta m)
$$

$p_{4}$ is the eigenvalue of $(\boldsymbol{\alpha} \cdot \boldsymbol{p}+\beta m)$ and the characteristic equation is $p_{4}^{2}=p^{2}+m^{2}$. Thus $p_{4}$ can be identified with the energy which can take positive or negative values $\pm E$ for a given value of $\boldsymbol{p}$. To obtain the eigenvector corresponding to an eigenvalue we must solve the following set of homogeneous equations

$$
\begin{aligned}
& (E-m) u_{1}-p_{z} u_{3}-\left(p_{x}-i p_{y}\right) u_{4}=0 \\
& (E-m) u_{2}-\left(p_{x}+i p_{y}\right) u_{3}+p_{z} u_{4}=0 \\
& p_{z} u_{1}+\left(p_{x}-i p_{y}\right) u_{2}-(E+m) u_{3}=0 \\
& \left(p_{x}+i p_{y}\right) u_{1}-p_{z} u_{2}-(E+m) u_{4}=0
\end{aligned}
$$

We can choose any two of the four components $u_{1}, u_{2}, u_{3}$ and $u_{4}$ arbitrarily, the other two being determined from two of the four equations. The condition that the solutions from the two sets are the same is just that $E^{2}=p^{2}+m^{2}$. There are two forms in which the two independent solutions can be obtained.

\section{Form $A$.}

Taking $E=V p^{2}+m^{2}$ we arrive at two independent solutions $u(1)$ and $u(2)$ by setting $u_{1}=E+m, u_{2}=0$ and $u_{1}=0, u_{2}=E+m$

$$
\frac{1}{V E+m}\left[\begin{array}{c}
u(1) A \\
E+m \\
0 \\
p_{z} \\
p_{x}+i p_{y}
\end{array}\right] ; \quad \frac{1}{\sqrt{E}+m}\left[\begin{array}{c}
u(2) A \\
0 \\
E+m \\
p_{x}-i p_{y} \\
-p_{z}
\end{array}\right] .
$$


The factor $\frac{1}{\sqrt{E}+m}$ is chosen on the basis of a normalization corresponding to $2 E$ electrons per unit volume.

\section{Form $B$.}

Alternatively we could have chosen $u_{3}=(E-m), u_{4}=0$ and $u_{3}=0, u_{4}=E-m$ in which case the solutions read:

$$
\frac{1}{V E-m}\left[\begin{array}{c}
u(1) B \\
p_{z} \\
p_{x}+i p_{y} \\
E-m \\
0
\end{array}\right] ; \quad \frac{1}{\sqrt{E-m}}\left[\begin{array}{c}
u(2) B \\
p_{x}-i p_{y} \\
-p_{z} \\
0 \\
E-m
\end{array}\right] .
$$

The above solutions ${ }^{1}$ are valid even for negative energy and in such a case it is convenient to write $E$ as $-|E|$. But it is to be remembered that the normalization corresponds to $2 E$ electrons per unit volume and this is negative when the energy is negative.

However if we normalize the solutions such that there are $2|E|$ electrons per unit volume, the positive energy solutions are the same while the negative energy solutions have to be written as

$$
\begin{aligned}
& \frac{1}{V E-m}\left[\begin{array}{c}
v(1) A \\
-E+m \\
0 \\
p_{z} \\
p_{x}+i p_{y}
\end{array}\right] ; \quad \frac{1}{\sqrt{ }-m-m}\left[\begin{array}{c}
v(2) A \\
0 \\
-E+m \\
p_{x}-i p_{y} \\
-p_{z}
\end{array}\right] \\
& v \text { (1) } B \\
& v(2) B \\
& \frac{1}{\sqrt{E}+m}\left[\begin{array}{c}
p_{z} \\
p_{x}+i p_{y} \\
-E-m \\
0
\end{array}\right] ; \quad \frac{1}{\sqrt{E}+m}\left[\begin{array}{c}
p_{x}-i p_{y} \\
-p_{z} \\
0 \\
-E-m
\end{array}\right] .
\end{aligned}
$$

It is convenient to choose $u(1) A$ and $u(2) A$ as the positive energy solutions and $v(1) B$ and $v(2) B$ as the negative energy solutions as in the rest system $u(1) B$, $u(2) B, v(1) A$ and $v(2) A$ vanish.

Thus any solution for a positive energy $E$ is a linear combination of $u(1) A$ and $u(2) A$ while that for a negative energy $-|E|$ is a combination of $v(1) B$ and $v(2) B$. To determine the solution in any particular case it is obvious that the mere specification of energy is not enough. There must be some physical quantity represented by an operator, the eigenvectors of which must be unique linear combinations of these solutions. ${ }^{2}$ The eigenvectors of this operator must

\footnotetext{
1 The $B$ solutions can be obtained by premultiplying the $A$ solutions by $i \gamma_{5}$ and replacing $m$ by $-m$. This is generally known as the $\gamma_{5},-m$ invariance of the Dirac equation.

2 R. P. Feynman, Lectures delivered at the California Institute of Technology, on "Quantum Electrodynamics" (1953).
} 
correspond to different eigen values for a given energy. This operator must commute with $H$.

In the rest system of the electron the positive energy solutions $u$ (1) $A$ and $u(2) A$ degenerate to

$$
\left[\begin{array}{l}
1 \\
0 \\
0 \\
0
\end{array}\right] \text { and }\left[\begin{array}{l}
0 \\
1 \\
0 \\
0
\end{array}\right] \text { and } v(2) B \text { are }\left[\begin{array}{l}
0 \\
0 \\
1 \\
0
\end{array}\right] \text { and }\left[\begin{array}{l}
0 \\
0 \\
0 \\
1
\end{array}\right] \text {. }
$$

The first two components of the positive energy solutions are the eigenvectors of the $\sigma_{z}$ operator with eigenvalues +1 and -1 respectively. Similarly the last two components of $v(1) B$ and $v(2) B$ are eigenvectors with eigenvalues \pm 1 . Hence the spin operator $\sigma$ should be $\left[\begin{array}{ll}\sigma & 0 \\ 0 & \sigma\end{array}\right]$ where $\sigma^{\prime}$ s are the $2 \times 2$ Pauli spiri operators.

The operator $\boldsymbol{\sigma} \cdot \boldsymbol{p}$ commutes with $H$ and any eigenvector with components $u_{1}, u_{2}, u_{3}$ and $u_{4}$ such that

$$
\frac{u_{1}}{u_{2}}=\frac{u_{3}}{u_{4}}=\frac{p_{x}-i p_{y}}{p-p_{z}}
$$

is an eigenvector of $\frac{\boldsymbol{\sigma} \cdot \boldsymbol{p}}{|p|}$ with eigenvalue +1 .

Thus neglecting normalization factors the combination

$$
\left(p_{x}-i p_{y}\right) u(1) A+\left(p-p_{z}\right) u(2) A=u^{\prime}(1)
$$

is an eigenvector of both $H$ and $\frac{\boldsymbol{\sigma} \cdot \boldsymbol{p}}{|p|}$ corresponding to the eigenvalues $+E$ and +1 respectively. The combination

$$
\left(p_{x}-i p_{y}\right) u(1) A+\left(-p-p_{z}\right) u(2) A=u^{\prime}(2)
$$

corresponds to $+E$ and $-1 . \frac{\boldsymbol{\sigma} \cdot \boldsymbol{p}}{|p|}$ is the projection of the spin in the direction of motion. The specification of the amplitudes of the spin in and opposite to the direction of momentum therefore determines the wave function corresponding to $+E$. If the amplitudes are $a$ and $b$ in and opposite to the direction of motion, then the wave function corresponding to $+E$ is given by

$$
a u^{\prime}(1)+b u^{\prime}(2)=c u(1) A+d u(2) A
$$

$c$ and $d$ being given by

$$
c=(a+b)\left(p_{x}-i p_{y}\right) ; \quad d=a\left(p-p_{z}\right)-b\left(p+p_{z}\right)
$$

If $\boldsymbol{k}_{0}$ is a unit vector it should be noted that the operator $\sigma \cdot \boldsymbol{k}_{0}$ will not commute 
with $H$ if $|p| \neq 0$ and $\boldsymbol{k}_{0}$ is not in the direction of $\boldsymbol{p}$. In the rest system of the particle the wave function $(75)$ reduces to

$$
c\left[\begin{array}{c}
E+m \\
0 \\
0 \\
0
\end{array}\right]+d\left[\begin{array}{c}
0 \\
E+m \\
0 \\
0
\end{array}\right]
$$

It is possible to find a spatial vector $\boldsymbol{k}_{0}$ with direction cosines $\left(l_{0}, m_{0}, n_{0}\right)$ such that the above solution is an eigenvector of $\sigma \cdot \boldsymbol{k}_{0}$ with eigenvalue +1 . This implies $\frac{c}{d}=\frac{l_{0}-i m_{0}}{1-n_{0}}$, i.e. the projection of spin in the direction $\left(l_{0}, m_{0}, n_{0}\right)$ is +1 . Note that (77) is not an eigenvector of $\boldsymbol{\sigma} \cdot \boldsymbol{k}$ if $\boldsymbol{k}$ is different from $\boldsymbol{k}_{\mathbf{0}}$. $\boldsymbol{k}$ will be different from $\boldsymbol{k}_{0}$ unless $a=1$ and $b=0$. Summarizing we write the wave function of the particle with momentum $\boldsymbol{p}$, energy $E$ and with the direction of spin in the rest system defined by the direction cosines $\left(l_{0}, m_{0}, n_{0}\right)$ as

$$
\left(l_{0}-i m_{0}\right) u(1) A+\left(1-n_{0}\right) u(2) A
$$

\section{Particular cases:}

(1) In the case when $\left(l_{0}, m_{0}, n_{0}\right)$ is coincident with $\pm z$ axis, the above method fails, but it is easy to see that the wave functions in these cases are $u$ (1) $A$ and $u(2) A$ since, operating $\sigma_{z}$ on the wave functions in the rest system corresponding to $u(1) A$ and $u(2) A$, we obtain the eigenvalues \pm 1 , i.e. $u(1) A$ and $u(2) A$ are solutions corresponding to spin along $\pm z$ in the rest system, noting of course that there is no meaning in operating $\sigma_{z}$ on $u(1) A$ and $u(2) A$ when $|p| \neq 0$, i.e. the system in which the particle is moving.

(2) Expressing $u(1) A$ as a linear combination of $u^{\prime}(1)$ and $u^{\prime}(2)$ we note that $u$ (1) $A$ corresponds to spin with amplitudes

$$
a=\frac{1}{2 p} \frac{p+p_{z}}{p_{x}-i p_{y}} ; \quad b=\frac{1}{2 p} \frac{p-p_{z}}{p_{x}-i p_{y}}
$$

in and opposite the directions of motion.

(3) If

$$
p_{x}=p_{y}=0, \quad p=p_{z}
$$

it is not possible to obtain $u^{\prime}(1)$ from $u(1) A$ and $u(2) A$ just from the equations $(73,74)$. But it is easy to recognize that $u^{\prime}(1)$ is identical with $u(1) A$ since $\sigma_{z}$ operating on $u(1) A$ yields +1 . In this case, the spin is in the direction of momentum which coincides with the direction of motion.

(4) The solutions $u(1) A$ and $u(2) A$ with $p_{z}=0$ represent a particle moving in the $(x, y)$ plane with spin in the $\pm z$ direction in the rest system of the particle.

(5) It is interesting to compare $u^{\prime}(1)$ and $u^{\prime}(2) . u^{\prime}(2)$ can be obtained by just reversing all the signs of momenta in $u^{\prime}(1)$. This implies that if in one system of reference we have a wave function $u^{\prime}(1)$ and we observe this wave function 
in a system in which the momenta are reversed, the wave function in the new system is $u^{\prime}(2)$. This is as should be expected since spin is an axial vector while $\boldsymbol{p}$ is polar and their relative orientations get reversed under inversion.

In analogy with light we can introduce the concept of polarization. A particle is longitudinally polarized if its spin is in or opposite to the direction of motion. If in one system of coordinates the momentum is in the positive $z$ direction, since the velocity of the particle with finite mass is less than the velocity of light it is possible to find a system of coordinates in which the direction of momentum is in the $-z$ direction. But if the spin is in the $+z$ direction in the first system it is still in the $+z$ direction in the other. If the longitudinal polarization is such that the spin and momentum are in the same direction, we call the particle righthanded; if the direction of momentum and spin are opposite we call it left-handed. The concept of handedness is not relativistically invariant for a particle with finite mass since for a particle with spin in the direction of motion in one frame it is possible to choose a frame in which the direction of spin is opposite to that of momentum.

In collision problems where we have a free incident particle which is scattered by a scattering centre and moves out ultimately as a free outgoing particle, it is customary to choose the incident particle to have its $p_{z}=p_{y}=0$ but $p_{x} \neq 0$ and for the outgoing particle its $p_{z}=0$ but $p_{x} \neq 0, p_{y} \neq 0$, i.e. we choose the plane of the directions of the ingoing and outgoing particles as the $x y$-plane.

We will conclude this dicussion about the direction of spin with some remarks on transverse polarization. We have stated that it is only for the $\frac{\boldsymbol{\sigma} \cdot \boldsymbol{p}}{|p|}$ operator we can obtain a wave function which yields the value \pm 1 . If we are interested in the component of spin in a direction $\boldsymbol{k}$ not coincident with $\boldsymbol{p}$ we can only obtain average values since $\boldsymbol{\sigma} \cdot \boldsymbol{k}$ does not commute with the Hamiltonian if $\boldsymbol{k}$ is not in the same direction as $\boldsymbol{p}$. In particular if $\boldsymbol{p}$ is in the $z$-direction we can ask for the average value of the operator $\sigma_{x}$ or $\sigma_{y}$ and if this average value is nonzero we can say the particle is "transversely polarized".

Till now we discussed the meaning of the two solutions corresponding to the positive value of energy. We now turn to the solutions corresponding to negative energy. As we had mentioned earlier the negative energy solutions $v(1) A$ and $v(2) A$ obtained by replacing $E$ by $-E$ in the positive energy solution vanish in the rest system. But the solutions $v(1) B$ and $v(2) B$ have a similar interpretation as $u(1) A$ and $u(2) A$. In the rest system they correspond to spin along the $\pm z$ directions. All other considerations apply to these negative energy solutions. Note that the solutions $v(1) B$ and $v(2) B$ can be obtained from $u(1) A$ and $u(2) A$ by operating the matrix $-i \gamma_{4} \gamma_{5}$,

$$
\left[\begin{array}{cccc}
0 & 0 & 1 & 0 \\
0 & 0 & 0 & 1 \\
-1 & 0 & 0 & 0 \\
0 & -1 & 0 & 0
\end{array}\right]
$$


on them ${ }^{1}$. The physical interpretation of the negative energy solution is as follows:

The positive energies form a continuum from $+m$ to $\infty$ and the negative energies, if accepted, form another continuum from $-m$ to $-\infty$. $E$ cannot take a value between $+m$ and $-m$. The meaning of the negative energies is at first obscure and devoid of physical content. For, as we shall see later, one of the consequences of inclusion of negative energy states is that if a particle is initially in a positive energy state and if a perturbation is applied to it for a finite duration then there is a finite probability for the particle to be in a negative energy state. This is contrary to physical fact since we know that positive energy particles always remain in positive energy states even after a perturbation is applied.

To overcome this difficulty, Dirac postulated that all the negative energy states are normally filled. Thus a vacuum is a state when all the negative energy states are filled with electrons. This postulate will be valid and useful only if Pauli's exclusion principle is invoked. Once Pauli's exclusion principle is assumed, it is clear that an electron in a positive energy state cannot make a transition to a negative energy state if these states are full. However if a "hole" is produced by the transition of one of the electrons with energy $-E$, momentum $\boldsymbol{p}$ and charge $-e$ (due to interaction with a field) to a positive energy state, the "hole" manifests itself as a positron of charge $+e$ momentum $-\boldsymbol{p}$ and energy $+E$. In other words, a free positron of momentum $-\boldsymbol{p}$ and energy $+E$ has the same wave function as a free electron of momentum $\boldsymbol{p}$ and energy $-E$, i.e.

$$
u_{+}(-\boldsymbol{p}, E)=u_{-}(\boldsymbol{p},-E) .
$$

There is another way of dealing with negative energy states which is equivalen to that of Dirac's hole theory. It consists in extending the kernel function approach to the Dirac equation. This was first formulated by Feynman and since his formulation is now accepted as the most convenient way to deal with electrodynamic processes (and meson theory to some extent), we shall develop the perturbation theory for Dirac's equation in Feynman's approach.

Before dealing with perturbation theory we shall discuss the wave equations of other elementary particles keeping in mind that the only valid wave equation known is the Dirac equation for the electron. However it is found useful under restricted conditions to represent other elementary particles through wave equations.

\section{Wave equations for the proton and the neutron}

Protons and neutrons, like the electrons obey the Pauli principle, have spin $\frac{1}{2}$ and are assumed to be described by the Dirac equation. On this assumption it would merely imply that the mass of the electron has to be replaced by the mass of the proton or the neutron in the equation. One of the main objections to this

${ }^{1}$ In the non-relativistic case when $p=0, E=m$ it can be verified that the effect of multiplying the Dirac $u$ solutions by $-i \gamma_{4} \gamma_{5}$ is the same as that of operating $i \gamma_{5}$. Hence it is usually stated that $\gamma_{5}$ connects the positive and negative energy solutions. 
simple extension of the Dirac theory to nucleons is that it yields for the magnetic moment of the proton and-the neutron the values 1 and 0 nuclear magnetons while the observed experimental values are 2.78 and -1.94 nuclear magnetons ${ }^{1}$ respectively. One of the important consequences of the Dirac theory is the prediction of anti-protons and anti-neutrons. As in the hole theory of the electrons the negative energy states are assumed to be filled and as a result, antiprotons can be produced or annihilated only along with protons. Similar considerations apply to anti-neutrons. These are the well known phenomena of pair production and pair annihilation which have been first observed in the case of electrons.

The essential difference between the nucleons and the electrons lies in that there exists between nucleons strong forces of very short range of non-electromagnetic character. It is postulated that nuclear forces are independent of the charge of the nucleon and are the same between any two nucleons provided their spin and orbital momentum states are the same. This is described by the introduction of the concept of isotopic spin and the description of the neutron and proton in the isotopic spin formalism. ${ }^{2}$ The only role which charge plays is in determining the possible states as are permissible by the Pauli principle.

Assuming charge independence we can speak of only one particle, the nucleon, which is supposed to exist in one of two states, the proton and the neutron, characterized by a charge of 1 and $0 .^{3}$ This immediately suggests the correspondence with the spin formalism where a particle can exist in one of the two spin states $\frac{1}{2}$ and $-\frac{1}{2}$. In analogy with this, we can characterize the proton state by a quantum number

$$
I_{z}=\frac{1}{2}
$$

and the neutron state by

$$
I_{z}=-\frac{1}{2}
$$

Though we do not attribute to this quantum number any dynamical significance as in the case of spin $^{4}$ it is clear that it must be linearly related to the charge of the particle. It is introduced merely to help us to compute the number of permissible states of a system of nucleons. The charge, in units of $e$, of the nucleon is related to $I_{z}$ by

$$
q=I_{z}+\frac{1}{2}
$$

1 In analogy with the Bohr magneton the nuclear magneton is defined as $\frac{e}{2 M}$.

2 B. Cassen and E. U. Condon, Phys. Rev. 50, 846 (1936).

${ }^{3}$ H. A. Bethe and P. De Hoffmann, Mesons and Fields, Volume II, Row Peterson, Evanston, Illinois (1955).

4 The analogy between spin and isotopic spin is purely formal. See Foldy, Phys. Rev. 93, 1395 (1954). In the case of spin, though it is possible to obtain a simultaneous eigenfunction of only the total spin and its $z$-component, the other two components represent the same physical quantity, the angular momentum in the corresponding directions. However in the case of isotopic spin it is only the $z$ component that is related to charge and the other two components have no physical meaning. As regards conservation it is the total, i.e. the spin together with the orbital angular momentum that is conserved in a quantum mechanical system. But charge $Q$ and hence $I_{z}$ is conserved independent of $I$. 
Thus the wave function of a nucleon can be supposed to have two components $\eta_{n}$ and $\eta_{p}$ and can be written as the linear combination of the wave functions $\eta_{p}$ and $\eta_{n}$ given by

$$
\eta_{p}=\left[\begin{array}{l}
1 \\
0
\end{array}\right] \quad \eta_{n}=\left[\begin{array}{l}
0 \\
1
\end{array}\right]
$$

In our discussion on spin, we have emphasized that $\pm \frac{1}{2}$ are the possible values of the $z$-component of the spin. The spin matrix $\sigma_{z}$ operating upon the wave function yields the eigenvalue of the $z$-component of spin. $\sigma_{x}$ and $\sigma_{y}$ are matrices such that the eigenvalue of the operator $\sigma_{x}^{2}+\sigma_{y}^{2}+\sigma_{z}^{2}$ yields the total spin momentum of the particle.

Having introduced the two component formalism to represent the state of the nucleon, we can proceed on similar lines as outlined above. We introduce the isotopic spin operator $\tau_{z}$ which yields the eigenvalue $I_{z}= \pm \frac{1}{2}$ and the operator can be represented by the matrix $\sigma_{z}$. This implies that we can also conceive of the operators $\tau_{x}, \tau_{y}$ represented by the matrices $\sigma_{x}, \sigma_{y}$. By analogy with spin the eigenvalue of $\tau_{x}^{2}+\tau_{y}^{2}+\tau_{z}^{2}$ is $I(I+1)$ where $I=\frac{1}{2}$ for a system containing a single nucleon. $I$ is the total isotopic spin quantum number and $I_{z}$ the quantum number corresponding to the $z$-component of the isotopic spin which is related to the charge. From $\tau_{x}$ and $\tau_{y}$ we can form $\tau_{+}=\frac{1}{2}\left(\tau_{x}+i \tau_{y}\right)$ and $\tau_{-}=\frac{1}{2}\left(\tau_{x}-i \tau_{y}\right)$ which have interesting physical interpretations

$$
\tau_{+} \eta_{n}=\eta_{p} \quad \tau_{-} \eta_{p}=\eta_{N},
$$

i.e. $\tau_{+}$transforms a neutron into a proton, $\tau_{-}$transforms a proton into a neutron.

If we have a system of nucleons ${ }^{1}$, the $z$-component of the total isotopic spin is the sum of the $z$-components of each particle:

$$
t_{z}=\frac{1}{2} \sum_{i} \tau_{i z}
$$

Similarly $t_{y}$ and $t_{x}$ are defined. If we now define the total isotopic spin operator $t$ by

$$
t^{2}=t_{x}^{2}+t_{y}^{2}+t_{z}^{2}
$$

in complete analogy with spin, the eigenvalue of $t^{2}$ is given by $T(T+1)$. $T$ being the total isotopic spin quantum number of the system of nucleons. $T$ is obtained by the usual vector addition of the isotopic spins of individual nucleons each of which has magnitude $\frac{1}{2}$ with the condition that the composition should be such that it is non-negative. Hence $T$ has integral eigenvalues if the number of nucleons $A$ is even while $T$ is half-integral if $A$ is odd. The maximum possible value of $T$ is obviously $A / 2$.

Since $M_{T}$ is the eigenvalue of the $z$ component of $t$ and $T$ is the quantum number of total isotopic spin, we have for any given $T$

$$
M_{T}=T, T-1, \ldots,-T .
$$

${ }^{1}$ We use the symbol $t$ for the isotopic spin operator of a system of particles and $\tau$ for a single particle, their eigenvalues being denoted by $T$ and $I$ respectively. 
The charge of the system is related to $M_{T}$ by the relation $Z=M_{T}+\frac{1}{2} A$. Thus the charge of a nucleus defines only $M_{T}$ and not $T$. Conversely if $A$ and $Z$ (hence $M_{T}$ ) are given, the $T$ values of the nucleus are

$$
\left|M_{T}\right|=\left|Z-\frac{1}{2} A\right|,\left|M_{T}\right|+1, \ldots, \frac{1}{2} A
$$

To fix our ideas, let us consider the case of a two-nucleon system: the $p-p$, $n-n$ and $p-n$ systems. We list below the $M_{T}$ and $T$ values of these systems and also their charge wave functions with their symmetries.

\begin{tabular}{|c|c|c|c|c|}
\hline System & $M_{T}$ & $T$ & $\begin{array}{l}\text { Charge wave function } \\
\qquad \chi_{T}, M_{T}\end{array}$ & $\mid \begin{array}{c}\text { Symmetry } \\
\text { of charge } \\
\text { wave function }\end{array}$ \\
\hline$p-p$ & 1 & 1 & $\chi_{1,1}=\eta_{p}^{(1)} \eta_{p}^{(2)}$ & $S$ \\
\hline$n-n$ & -1 & 1 & $\chi_{1,-1}=\eta_{n}^{(1)} \eta_{n}^{(2)}$ & $S$ \\
\hline$p-n$ & 0 & 1 & $\chi_{1,0}=\frac{1}{\gamma^{2}}\left[\eta_{p}^{(1)} \eta_{n}^{(2)}+\eta_{p}^{(2)} \eta_{n}^{(1)}\right]$ & $S$ \\
\hline$p-n$ & 0 & 0 & $\chi_{0,0}=\frac{1}{\sqrt{ } 2}\left[\eta_{p}^{(1)} \eta_{n}^{(2)}-\eta_{p}^{(2)} \eta_{n}^{(1)}\right]$ & $A$ \\
\hline
\end{tabular}

Of these, the first three are charge symmetric with respect to interchange of particles 1 and 2 whereas the last changes sign. Now the Pauli principle states the wave function must be anti-symmetric under the exchange of all coordinates, i.e. space, spin and isotopic spin. Thus if the charge wave function is one of the first three listed, the space-spin wave function is anti-symmetric while it is symmetric if the charge wave function is of the fourth type.

The postulate of charge independence of nuclear forces states that the forces do not depend upon the charge of the nucleons provided the space-spin state of the system is the same. Therefore, the energy levels, scattering phase shifts etc. are the same for states involving the same space-spin state multiplied by any of the first three charge wave functions $\chi_{1,1}, \chi_{1,-1}, \chi_{1,0}$. The last one $\chi_{0,0}$ is antisymmetric in the charge wave function and has to be multiplied by a symmetric space-spin wave function and hence it will behave quite differently. Thus we see that the $p-n$ system with symmetric charge wave function behaves like $p-p$ and $n-n$ systems and quite differently from $p-n$ system with anti-symmetric charge wave function. Hence the behaviour of the system is determined by the symmetry of the charge wave function. We also note that the symmetric charge wave functions $\chi_{1,1}, \chi_{1,0}, \chi_{1,-1}$ have the same isotopic spin 1 and form a triplet in charge space with $M_{T}=1,0,-1$ respectively. The anti-symmetric charge wave function $\chi_{0,0}$ has the total isotopic spin 0 . Thus we arrive at the important result that the behaviour of the system is determined only by $T$ and not by $M_{T}$. 


\section{Wave equation for the meson}

The Klein-Gordon equation was obtained merely by imposing the relativistic requirements on a particle described by a one component wave function, i.e. a particle of zero spin. The neutral meson is one such particle. Historically the meson was predicted by Yukawa ${ }^{1}$ in attempting to understand the nature of the nuclear forces. Since by a nucleon is meant both a neutron and a proton, Yukawa's theory included positive, negative and neutral mesons. Thus it became necessary to introduce the isotopic spin formalism into the Klein-Gordon equation.

Since field theory (to be discussed later) is almost as old as wave mechanics, Yukawa's original paper was written in field theoretic formalism. In view of the "epoch making idea that the introduction of the ever unknown new field enables us to account for the nature of the nuclear forces" we shall summarize the results of the celebrated paper in wave mechanical formalism by a suitable adaptation to fit in with our discussion of the wave functions of single particles.

Yukawa introduced a field of force to describe the interaction between the nucleons in close analogy with the electromagnetic field which describes the interaction between charged particles. The scalar potential of the electromagnetic field satisfies the equation

$$
\left(\nabla^{2}-\frac{\partial^{2}}{\partial t^{2}}\right) U=0
$$

Substituting $U=V(r) \mathrm{e}^{i \omega t}$ we have

$$
\left\{D^{2}+\omega^{2}\right\} V=0
$$

This has a solution $V(r)=\frac{1}{r} \mathrm{e}^{i \omega r}$ where $r=\left(x^{2}+y^{2}+z^{2}\right)^{1 / 2}$. Leaving aside the oscillatory part, $\frac{1}{r}$ is the spherically symmetric static solution for the potential, valid for all $r$ except $r=0$. The potential between the nucleons can be defined in analogy with this static potential; however it should not be of the Coulomb type but should decrease more rapidly with distance. Yukawa required a potential of the type

$$
\pm g^{2} \frac{\mathrm{e}^{-\mu r}}{r}
$$

where $g$ has the dimensions of charge and $\mu$ that of inverse length. This function $(90 \mathrm{a})$ is a static solution in the same sense as above of the equation

$$
\left\{\nabla^{2}-\frac{\partial^{2}}{\partial t^{2}}-\lambda^{2}\right\} U(x, y, z, t)=0
$$

with $\mu$ given by $\mu^{2}=\lambda^{2}-\omega^{2}$ provided $\left.\lambda\right\rangle|\omega|$. However when $\lambda\langle|\omega|, \mu$ will be purely imaginary. The corresponding solution is a spherically symmetric wave as in the case of the electromagnetic field. In such a case (91) can be treated

1 H. Yukawa, Proc. Phys. Math. Soc., Japan 17, 48 (1935). 
as a free particle equation as in the case of the photon field, the source being taken to be at an infinite distance. The static solution of the inhomogeneous equation

$$
\left(\nabla^{2}-\mu^{2}\right) U(x, y, z)=-4 \pi g f(x, y, z)
$$

is obtained according to the standard theory of partial differential equations as

$$
\int g f\left(x^{\prime} y^{\prime} z^{\prime}\right) \frac{\mathrm{e}^{-\mu\left|\boldsymbol{r}^{\prime}-\boldsymbol{r}\right|}}{\left|\boldsymbol{r}^{\prime}-\boldsymbol{r}\right|} \mathrm{d}^{3} \gamma^{\prime}
$$

where $g f(x, y, z)$ is interpreted as the "source distribution function" of the potential. If we have a point source at $r^{\prime}=0$, we have merely to write

$$
f\left(x^{\prime}, y^{\prime}, z^{\prime}\right)=\delta\left(x^{\prime}\right) \delta\left(y^{\prime}\right) \delta\left(z^{\prime}\right)
$$

and the solution reduces to $g \frac{\mathrm{e}^{-\mu r}}{r}$. If $U$ were to represent the meson field the source function should be the nucleon density. If we wish to envisage positive, negative and neutral mesons, the isotopic spin formalism should be suitably imbedded in the nucleon density. If $\psi$ is the two-component nucleon wave function (i.e. in isotopic spin formalism) we can convince ourselves that the source function for the positive meson potential should be

$$
4 \pi g \bar{\psi} \tau_{-} \psi
$$

which merely expresses that the proton wave function is taken over to the neutron state. The corresponding source functions for the negative and neutral mesons are

$$
4 \pi g \bar{\psi} \tau_{+} \psi, 4 \pi g \bar{\psi} \tau_{3} \psi
$$

Thus we have as on the right hand side

$$
\left\{\nabla^{2}-\frac{\partial^{2}}{\partial t^{2}}-\lambda^{2}\right\} U_{i}=-4 \pi g \bar{\psi} \tau_{i} \psi
$$

$i=-,+, 3$ corresponding to positive, negative and neutral mesons. Since $\tau_{-}=\tilde{\tau}_{+}$ the wave function $U_{-}$for the negative meson is $\tilde{U}_{+}$. This is in conformity with wave mechanics where the charge conjugate solution is obtained simply by complex conjugation. For the neutral meson $\tau_{3}=\tilde{\tau}_{3}$ and obviously $U_{3}=\tilde{U}_{3}$.

We disregard for the moment that the nucleon has spin. Since no correct relativistic theory exists for heavy particles we assume that the simple nonrelativistic ${ }^{1}$ equation for $\psi$ neglecting spin can be written as

$$
\begin{array}{r}
{\left[\left\{\frac{1}{4}\left(\frac{1-\tau_{3}}{M_{n}}+\frac{1+\tau_{3}}{M_{p}}\right) \nabla^{2}+i \frac{\partial}{\partial t}-\frac{1-\tau_{3}}{2} M_{n}-\frac{1+\tau_{3}}{2} M_{p}\right\}\right.} \\
\left.+g\left(U_{-} \tau_{-}+U_{+} \tau_{+}\right)\right] \psi=0
\end{array}
$$

1 It is to be noted that by non-relativistic, we only mean that the velocity of the nucleon is negligible but we take the relativistic expression for the energy. 
corresponding to the Hamiltonian

$H=\left[\frac{1-\tau_{3}}{4 M_{n}}+\frac{1+\tau_{3}}{4 M_{p}}\right] p^{2}+\frac{1-\tau_{3}}{2} M_{n}+\frac{1+\tau_{3}}{2} M_{p}+g\left(U_{-} \tau_{-}+U_{+} \tau_{+}\right)$.

Each term of the Hamiltonian is self-explanatory. Consider now a transition from a proton state of energy $\omega_{p}$ to a neutron state of energy $\omega_{n}$. These states can be expressed as

$$
\psi_{n}=u(x, y, z) \mathrm{e}^{-i \omega_{n} t} ; \quad \psi_{p}=v(x, y, z) \mathrm{e}^{-i \omega_{p} t} .
$$

Substituting in (97) we have

$$
\left\{\nabla^{2}-\frac{\partial^{2}}{\partial t^{2}}-\lambda^{2}\right\} U_{+}=-4 \pi g \tilde{u} v \mathrm{e}^{-i t\left(\omega_{p}-\omega_{n}\right)}
$$

Put $U_{+}=U_{+}^{\prime}(x, y, z) \mathrm{e}^{i \omega t}$ where $\omega=\left(\omega_{n}-\omega_{p}\right)$. We have

Integrating,

$$
\left\{\nabla^{2}-\left(\lambda^{2}-\omega^{2}\right)\right\} U_{+}^{\prime}=-4 \pi g \tilde{u} v \text {. }
$$

$$
U_{+}^{\prime}(r)=g \iiint \frac{\mathrm{e}^{-\mu\left|\boldsymbol{r}-\boldsymbol{r}^{\prime}\right|}}{\left|\boldsymbol{r}-\boldsymbol{r}^{\prime}\right|} \tilde{u}\left(\boldsymbol{r}^{\prime}\right) v\left(\boldsymbol{r}^{\prime}\right) \mathrm{d} \boldsymbol{r}^{\prime}
$$

where as before we have set $\mu=V \lambda^{2}-\omega^{2}$.

(i) When $\lambda>\omega, \mu$ is real and depends on $\left|\omega_{n}-\omega_{p}\right|$. This shows that the range of the interaction between a neutron and a proton increases with $\left|\omega_{n}-\omega_{p}\right|$.

(ii) When $\lambda<|\omega|, \mu$ is imaginary and we interpret this wave as that corresponding to the quanta of the field. This implies that a quantum of energy greater than $\lambda$ can be emitted in a proton to neutron transition provided $\left|\omega_{n}-\omega_{p}\right|>\lambda$.

One simple question can be raised in regard to our analogy between the electromagnetic and the meson fields. In the meson field we have made the statement that if $\lambda^{2}<\omega^{2}, \mu$ becomes imaginary and therefore we do not get the potential solutions. But $\lambda=0$ should correspond to the photon field and in this case though $\mu$ is imaginary we interpreted $\frac{1}{r}$ as the static solution. This interpretation should not be carried to the case of the meson field when $\mu$ is imaginary as the physical situation is different from the photon field. When enough energy is given to the nucleon system, i.e. if $\omega^{2}>\lambda^{2}$, the meson goes well out of the very short range of the potential field and can therefore be considered free. In the electromagnetic case the situation is much more complex and the static and radiation fields have to be considered together in any description since the static Coulomb field has infinite range.

\section{Wave equation for the neutrino}

It will be interesting to study the Dirac equation in the limit as the mass $m$ tends to zero. For this purpose it is convenient to go back to the derivation of the Dirac equation through the relativistic generalization of the Pauli equation. We have

$$
\begin{aligned}
& \left(\pi_{4}-\boldsymbol{\sigma} \cdot \boldsymbol{x}\right) \varphi=m \psi \\
& \left(\pi_{4}+\boldsymbol{\sigma} \cdot \boldsymbol{\tau}\right) \chi=m \varphi
\end{aligned} \quad \text { where } \quad \pi_{4}=i \frac{\partial}{\partial t}-e A_{4} ; \quad \boldsymbol{x}=\boldsymbol{p}-e \boldsymbol{A}
$$


and $\varphi$ and $\chi$ are two component wave functions. The four component Dirac wave function was identified to be $\left[\begin{array}{l}\varphi+\chi \\ \varphi-\chi\end{array}\right]$. If we let $m \rightarrow 0$ we notice that $\varphi$ and $\chi$ are uncoupled and should represent the wave equation of particles with spin $\frac{1}{2}$ and mass zero. Such a particle had indeed been postulated by Pauli in order to explain the continuous spectrum of $\beta$ particles emitted by radioactive nuclei. It was a theoretical necessity that such a particle, called the neutrino should have mass zero, spin half and neutral charge, for energy, angular momentum and charge conservations respectively.

The limit $m \rightarrow 0$ in the above equation gives rise to an interesting feature not present in the Dirac case. If mass is finite as in the Dirac case, an eigenfunction of $\boldsymbol{\sigma} \cdot \boldsymbol{p}$ need not be an eigenfunction of the Hamiltonian $H$, i.e. it can be a linear combination of eigenfunctions of $H$ corresponding to positive and negative energies. Thus we can have electrons in positive and negative energy states having their spins both parallel or antiparallel to their momenta. If we set $m$ equal to zero then $H_{\alpha} \boldsymbol{\sigma} \cdot \boldsymbol{p}$ and the eigenfunctions of $\boldsymbol{\sigma} \cdot \boldsymbol{p}$ corresponding to the two eigenvalues \pm 1 (spin parallel or antiparallel to the momentum, i.e. positive or negative helicity) are also the eigenfunctions of the Hamiltonian with positive and negative eigenvalues of energy. But still the question remains as to what helicity should be attributed to the positive and negative energies respectively. In analogy with the Dirac hole theory if the antineutrino is to be identified as a hole in the negative energy sea of neutrinos, the antineutrino has the same helicity as the negative energy neutrino or opposite to that of the neutrino. Experiments have decided in favour of negative helicity for the neutrino and positive helicity for the anti-neutrino.

A positive energy neutrino with spin anti-parallel to the momentum under spatial reflection would have spin parallel to momentum which is a forbidden state for a neutrino with positive energy. This is precisely the reason why the two component theory of the neutrino, first discussed by $\mathrm{Weyl}^{\mathbf{1}}$ was rejected. Since it has been found that neutrinos are produced in weak interactions which do not conserve parity, the possible use of the two component theory was proposed and considered by Lee and Yang, ${ }^{2}$ Landau $^{3}$ and Salam. ${ }^{4}$ It is also seen that the theory is not invariant under charge conjugation.

The two component formalism can also be derived directly from the four component Dirac equation as follows:

Consider the Dirac equation

$$
\left(-i \gamma_{\mu} \frac{\partial}{\partial x_{\mu}}+m\right) \psi=0
$$

1 H. WeYl, Z. f. Phys. อ6, 330 (1929).

2 T. D. LeE and C. N. YANG, Elementary Particles and Weak Interactions, Office of Technical Services, Washington, D. C., 25.

3 L. Landau, Nucl. Phys. 3, 127 (1957).

4 A. Salam, Nuovo Cim.5, 229 (1957). 
The charge conjugate of this equation is

$$
\left(-i \gamma_{\mu} \frac{\partial}{\partial x_{\mu}}+m\right) C \psi^{T}=0
$$

where $C$ is defined through $C^{-1} \gamma_{\mu} C=-\gamma_{\mu}^{T}, C^{T}=-C, C^{\dagger}=C^{-1}$.

The 4-component $\psi=\left[\begin{array}{l}\varphi+\chi \\ \varphi-\chi\end{array}\right]$ is a solution of the Dirac equation of first order but $\gamma_{5} \psi$ is not a solution unless $m=0$. But if we take the second order form of the Dirac equation

$$
\left(+i \gamma_{\mu} \frac{\partial}{\partial x_{\mu}}+m\right)\left(i \gamma_{\mu} \frac{\partial}{\partial x_{\mu}}-m\right) \psi_{D}=0
$$

then $\gamma_{5} \psi_{D}$ is also a solution since $\gamma_{5}$ commutes with the operator on the left-hand side. Defining

$$
\psi_{ \pm}=\frac{1}{2}\left(1 \pm \gamma_{5}\right) \psi ; \quad \gamma_{ \pm}^{\mu}=\frac{1}{2}\left(1 \pm \gamma_{5}\right) \gamma_{\mu}
$$

we note that $\psi_{+}$and $\psi_{-}$are not solutions of the Dirac equation since $\left(1 \pm \gamma_{5}\right)$ does not commute with the Dirac Hamiltonian. $\psi_{+}$and $\psi_{-}$are charge conjugate solutions and they satisfy

$$
\begin{aligned}
& -i \gamma_{-}^{\mu} \frac{\partial}{\partial x_{\mu}} \psi_{+}+m \psi_{-}=0 \\
& -i \gamma_{+}^{\mu} \frac{\partial}{\partial x_{\mu}} \psi_{-}+m \psi_{+}=0 .
\end{aligned}
$$

$\psi_{+}$and $\psi_{-}$can be identified in terms of $\varphi$ and $\chi$ as

$$
\psi_{+}=\left[\begin{array}{c}
\varphi \\
\varphi
\end{array}\right] \text { and } \psi_{-}=\left[\begin{array}{c}
\psi \\
-\psi
\end{array}\right] \text {. }
$$

$\psi_{+}$and $\psi_{-}$have eigenvalues \pm 1 with respect to $\gamma_{5}$ and are known as the positive and negative chiral states respectively, the eigenvalue being termed the chirality of the state. Thus $\varphi$ and $\chi$ might as well be chosen as the wave functions of the neutrino and the anti-neutrino respectively.

The covariance of these equations under proper Lorentz transformations is easily demonstrated by defining the transformation matrix $S$ which connects the wave function $\varphi$ to its transform $\varphi^{\prime}$, i.e.

$$
\varphi^{\prime}\left(x^{\prime}\right)=S \varphi(x) \text {. }
$$

For a rotation of the axis through $\theta$ around the spatial direction $\boldsymbol{q}$, $S=\exp \left(\frac{1}{2} i \theta \sigma \cdot \boldsymbol{q}\right)$. For a Lorentz transformation corresponding to a velocity $V$ in the direction $\boldsymbol{q}, S=\exp \left(\frac{1}{2} V \boldsymbol{\sigma} \cdot \boldsymbol{q}\right)$. All other proper transformations can be generated from these two fundamental transformations.

When we consider discrete transformations such as space reflection, we find that the equation (114) is no longer covariant in the sense that we are unable to find an $S$ such that $\varphi^{\prime}\left(x^{\prime}\right)=S \varphi(x)$. For in order to have covariance we need for the operator $\sigma$

$$
S \sigma S^{-1}=-\sigma \text {. }
$$


However $\sigma$ and $-\sigma$ satisfy different commutation relations. Hence no unitary transformation is possible. However if we modify the definition of covariance under spatial reflection to imply

$$
\varphi^{\prime}\left(x^{\prime}\right)=S \varphi^{*}(x)
$$

then it is possible ${ }^{1}$ to find an $S$ which satisfies such a relation. Since $\sigma_{i}^{*}$ and $\sigma_{i}$ have the same commutation relations it is possible to find a matrix $A$ such that

$$
A \sigma_{i}^{*} A^{-1}=-\sigma_{i} \text {. }
$$

From the properties of $\sigma$ matrices it follows that

$$
A=A^{-1}=A^{*}
$$

$S$ can be now identified as

$$
S=\varepsilon A
$$

where $\varepsilon$ is imaginary. The purpose of using the auxiliary matrix $A$ is to draw attention to the fact that in the case of time reflection $S$ can be written as

where $\mu$ is real.

$$
S=\mu A
$$

It is interesting to note that this modified definition is the one used to prove the covariance of Maxwell's equations under spatial reflection. Thus we can conclude from this point of view that equation (114) is covariant under the full Lorentz group. In other words, the presence or absence of the mass term does not affect the covariance of the free Dirac equation and hence also the interactions formed from such wave functions.

\section{WAVE MECHANICS OF THE PHOTON}

\section{Wave equation for the photon ${ }^{2}$}

Our discussion of single particle equations will be complete only if we include the photon as an elementary particle of mass zero and discuss its equation of motion from the point of view of wave functions. While in the case of particles like the electron the wave function is postulated, in the case of the photon the electromagnetic field associated with it is already represented in configuration space through the Maxwell equations. Thus if the photon were to be described through a Schrödinger equation the latter should be related to the Maxwell equations. It turns out that despite the fact that the electromagnetic field variables $\boldsymbol{E}$ and $\boldsymbol{H}$, or equivalently $\boldsymbol{A}$ and $A_{4}$ are functions of space and time there is no meaning in speaking of a wave function of a photon in configuration space $^{3}$. However we can by taking a suitable combination of the momentum

1 K. M. Case, Phys. Rev. 107, 307 (1957).

2 This section is based on Quantum Electrodynamics, Part I, by A. I. Akhiezer and V. B. Berestetsky (English translation) Office of Technical Services, Washington, D.C., 25. 3 That is if we require a probability interpretation as defined in chapter $\mathbf{I}$. 
transforms of $\boldsymbol{E}$ and $\boldsymbol{H}$ obtain a wave function for the photon in momentum representation which satisfies a Schrödinger equation. Thus we start by writing the Maxwell equations in momentum space and derive the Schrödinger equation of the photon therefrom. In this procedure we realise that the very familiar Maxwell field has a complicated structure and the wave function formalism of the photon has peculiar features not met with in the case of other elementary particles. We realise that the quantum mechanics of the photon should necessarily be relativistic $a b$ initio. We shall first study the photon, i.e. the electromagnetic field in the absence of charges. So we take the Lorentz-invariant free-field Maxwell equations and obtain the wave function for the photon.

\section{Schrödinger equation of the photon in momentum space}

The Maxwell field is completely specified by the distribution of the electric and magnetic vectors in space and time. These vectors $\boldsymbol{E}(\boldsymbol{x}, t)$ and $\boldsymbol{H}(\boldsymbol{x}, t)$ satisfy the coupled equations

$$
\begin{aligned}
\operatorname{curl} \boldsymbol{E}=-\frac{\partial \boldsymbol{H}}{\partial t} & \operatorname{div} \boldsymbol{H}=0 \\
\operatorname{curl} \boldsymbol{H}=\frac{\partial \boldsymbol{E}}{\partial t} & \operatorname{div} \boldsymbol{E}=0 .
\end{aligned}
$$

Since the charge density $\varrho$ is assumed to be zero everywhere (i.e. $\operatorname{div} \boldsymbol{E}=0$ ) we are dealing with a radiation field and thus only with photons.

Taking the Fourier transforms ${ }^{1}$ of $\boldsymbol{E}(\boldsymbol{x})$ and $\boldsymbol{H}(\boldsymbol{x})$ as $\boldsymbol{E}(\boldsymbol{k})$ and $\boldsymbol{H}(\boldsymbol{k})$ defined by

$$
\boldsymbol{E}(\boldsymbol{k})=\frac{1}{(2 \pi)^{3}} \int \boldsymbol{E}(\boldsymbol{x}) \mathrm{e}^{-i \boldsymbol{k} \cdot \boldsymbol{x}} \mathrm{d}^{3} x ; \quad \boldsymbol{H}(\boldsymbol{k})=\frac{1}{(2 \pi)^{3}} \int \boldsymbol{H}(\boldsymbol{x}) \mathrm{e}^{-i \boldsymbol{k} \cdot \boldsymbol{x}} \mathrm{d}^{3} x
$$

we can write the Maxwell's equations in momentum space as

$$
\begin{aligned}
\dot{\boldsymbol{H}}(\boldsymbol{k}) & =-i[\boldsymbol{k} \times \boldsymbol{E}(\boldsymbol{k})], & \dot{\boldsymbol{E}}(\boldsymbol{k}) & =i[\boldsymbol{k} \times \boldsymbol{H}(\boldsymbol{k})] \\
\boldsymbol{k} \cdot \boldsymbol{E}(\boldsymbol{k}) & =0, & \boldsymbol{k} \cdot \boldsymbol{H}(\boldsymbol{k}) & =0 .
\end{aligned}
$$

Further we see that $\boldsymbol{H}(\boldsymbol{k}) \doteq \frac{i[\boldsymbol{k} \times \dot{\boldsymbol{E}}(\boldsymbol{k})]}{k^{2}}$ and the reality condition on $\boldsymbol{E}$ and $\boldsymbol{H}$ imposes the relationships

$$
\boldsymbol{E}(-\boldsymbol{k})=\boldsymbol{E}^{*}(\boldsymbol{k}) \text { and } \boldsymbol{H}(-\boldsymbol{k})=\boldsymbol{H}^{*}(\boldsymbol{k}) .
$$

To arrive at the wave equation we now define a function $\boldsymbol{f}(\boldsymbol{k})$ by:

$$
\begin{aligned}
& \boldsymbol{E}(\boldsymbol{k})=N(k)\left[\boldsymbol{f}(\boldsymbol{k})+\boldsymbol{f}^{*}(-\boldsymbol{k})\right] \\
& \dot{\boldsymbol{E}}(k)=-i k N(k)\left[\boldsymbol{f}(\boldsymbol{k})-\boldsymbol{f}^{*}(-\boldsymbol{k})\right]
\end{aligned}
$$

1 In defining these transforms we are adopting natural units. Otherwise we should use $k / \hbar$ instead of $k$ and here the Fourier transform is defined with a factor $(2 \pi)^{3}$ unlike those in chapter I, sec. 1. $k$ being used for $|\boldsymbol{k}|$ where convenient. 
where $N(k)$ the normalizing factor turns out to be equal to $/ k / 4 \pi^{3 / 2}$ and the function $f$ satisfies the transversality condition:

$$
\boldsymbol{k} \cdot \boldsymbol{f}(\boldsymbol{k})=0
$$

Substituting for $\boldsymbol{E}(\boldsymbol{k})$ and $\dot{\boldsymbol{E}}(\boldsymbol{k})$ in terms of $\boldsymbol{f}(\boldsymbol{k})$ in the Maxwell's equation

$$
\left[\frac{\partial^{2}}{\partial t^{2}}+k^{2}\right] \boldsymbol{E}(\boldsymbol{k})=0
$$

we obtain the following equations for $\boldsymbol{f}(\boldsymbol{k})$ and its conjugate

$$
i \frac{\partial \boldsymbol{f}(\boldsymbol{k})}{\partial t}=k \boldsymbol{f}(\boldsymbol{k}), \quad-i \frac{\partial \boldsymbol{f}^{*}(\boldsymbol{k})}{\partial t}=k \boldsymbol{f}^{*}(\boldsymbol{k})
$$

Since $k$ is the energy of the photon in our chosen unit, the above has the form of the Schrödinger equation for the photon in momentum space if the energy operator $\underline{\omega}$ of the photon in $\boldsymbol{k}$ space is defined as the operator of multiplication by the wave number $k$. Thus we identify $\boldsymbol{f}(\boldsymbol{k})$ to be the wave function of the photon in momentum space. To verify this we show that the energy defined through the electric and magnetic fields is identical with that derived from the Schrödinger equation.

\section{Energy of the electromagnetic field}

The energy of the electromagnetic field is given by the usual expression quadratic in $\boldsymbol{E}$ and $\boldsymbol{H}$ as

$$
\bar{\omega}=\frac{1}{2} \int\left(\boldsymbol{E}^{2}+\boldsymbol{H}^{2}\right) \mathrm{d}^{3} x .
$$

Using the "wave function" of the photon we can calculate the average value of the energy as

$$
\bar{\omega}=\int \boldsymbol{f}^{*}(\boldsymbol{k}) \underline{\omega} \boldsymbol{f}(\boldsymbol{k}) \mathrm{d}^{3} k .
$$

To show the equivalence of equations (9) and (10) we write field vectors in terms of their momentum transforms :

$$
\bar{\omega}=\frac{1}{2} \int\left[\boldsymbol{E}(\boldsymbol{k}) \boldsymbol{E}\left(\boldsymbol{k}^{\prime}\right)+\boldsymbol{H}(\boldsymbol{k}) \boldsymbol{H}\left(\boldsymbol{k}^{\prime}\right)\right] \mathrm{e}^{i\left(\boldsymbol{k}+\boldsymbol{k}^{\prime}\right) \cdot \boldsymbol{x}} \mathrm{d}^{3} k \mathrm{~d}^{3} k^{\prime} \mathrm{d}^{3} x .
$$

This when expressed in terms of $\boldsymbol{f}(\boldsymbol{k})$ and $\boldsymbol{f}^{*}(\boldsymbol{k})$ reduces to

$$
\bar{\omega}=16 \pi^{3} \int N^{2} f^{*}(\boldsymbol{k}) \boldsymbol{f}(\boldsymbol{k}) \mathrm{d}^{3} k
$$

and since $N$ has been chosen as $/ k / 4 \pi^{3 / 2}$ we see that

$$
\bar{\omega}=\int \boldsymbol{f}^{*}(\boldsymbol{k}) k \boldsymbol{f}(\boldsymbol{k}) \mathrm{d}^{3} k=\int \boldsymbol{f}^{*}(\boldsymbol{k}) \underline{\omega} \boldsymbol{j}(\boldsymbol{k}) \mathrm{d}^{3} k .
$$

If we take a monochromatic solution of equation (8)

$$
\boldsymbol{f}(\boldsymbol{k})=\boldsymbol{f}_{\mathbf{0}}(\boldsymbol{k}) \mathrm{e}^{-i p t}
$$


corresponding to one of the energy eigenvalues $k=p$ for the $\underline{\omega}$ operator we find that $\bar{\omega}=p \int \boldsymbol{f}^{*}(\boldsymbol{k}) \boldsymbol{f}(\boldsymbol{k}) \mathrm{d}^{3} k=p$ if the wave function $\boldsymbol{f}$ satisfies the normalization condition ${ }^{1}$

$$
\int \boldsymbol{f}^{*}(\boldsymbol{k}) \boldsymbol{f}(\boldsymbol{k}) \mathrm{d}^{3} k=1
$$

\section{Yomentum of the photon field}

The momentum of the electromagnetic field in terms of $\boldsymbol{E}$ and $\boldsymbol{H}$ is given by the integral

$$
\overline{\boldsymbol{p}}=\int[\boldsymbol{E} \times \boldsymbol{H}] \mathrm{d}^{3} x,
$$

This integral on substitution in terms of the functions $\boldsymbol{f}(\boldsymbol{k})$ is found to be

$$
\begin{gathered}
(2 \pi)^{3} \cdot \int N^{2} \cdot\left\{\boldsymbol{f}^{*}(\boldsymbol{k}) \boldsymbol{f}(\boldsymbol{k})-\boldsymbol{f}^{*}(-\boldsymbol{k}) \boldsymbol{f}(-\boldsymbol{k})+\boldsymbol{f}^{*}(-\boldsymbol{k}) \boldsymbol{f}^{*}(\boldsymbol{k})\right. \\
-\boldsymbol{f}(\boldsymbol{k}) \boldsymbol{f}(-\boldsymbol{k})\} \frac{\boldsymbol{k}}{k} \mathrm{~d}^{3} k \\
\overline{\boldsymbol{p}}=\int \boldsymbol{f}^{*}(\boldsymbol{k}) \boldsymbol{k} f(\boldsymbol{k}) \mathrm{d}^{3} k .
\end{gathered}
$$

Thus the momentum of the field is given as the quantum mechanical average of the photon momentum operator $\boldsymbol{k}$.

\section{Wave function in configuration space}

Though it may appear possible for us to go over to the wave function of the photon in configuration space by taking $\boldsymbol{f}(\boldsymbol{x})$ the Fourier inverse of the function $\boldsymbol{f}(\boldsymbol{k})$, we realise that such a representation is not possilbe.

Due to the normalization condition on $\boldsymbol{f}(\boldsymbol{k})$ we know that $\boldsymbol{f}^{*}(\boldsymbol{k}) \boldsymbol{f}(\boldsymbol{k})$ is the probability density of the photon in $\boldsymbol{k}$-space. But the normalization condition in configuration space

$$
\int \boldsymbol{f}^{*}(\boldsymbol{x}) \boldsymbol{f}(\boldsymbol{x}) \mathrm{d}^{3} x=1
$$

cannot lead to the interpretation that $\boldsymbol{f}^{*}(\boldsymbol{x}) \boldsymbol{f}(\boldsymbol{x})$ is the probability of finding a photon at $\boldsymbol{x}$. This is because the presence of the photon is detected by its interaction with charges which is determined by the values of $\boldsymbol{E}(\boldsymbol{x})$ and $\boldsymbol{H}(\boldsymbol{x})$ at a given point. From equation (5) we see that due to the presence of $\gamma k$ in $N, \boldsymbol{E}(\boldsymbol{x})$ and $\boldsymbol{f}(\boldsymbol{x})$ are not connected by a local but by an integral relationship. Hence the probability density for a photon to be at a point in space does not exist.

\section{Plane wave solutions}

The photon wave function $\boldsymbol{f}(\boldsymbol{k})$ in momentum space satisfies the Schrödinger equation (8) and transversality condition (6). Considering a single photon with a definite momentum $\boldsymbol{k}=\boldsymbol{p}$, we write the photon wave function as

$$
\boldsymbol{f}_{i \mu}=i \boldsymbol{e}_{\mu} \mathrm{e}^{-i p t} \frac{H[\boldsymbol{k}+\boldsymbol{p}+\Delta \boldsymbol{p}] H[\boldsymbol{k}-(\boldsymbol{p}-\Delta \boldsymbol{p})]}{(\Delta \boldsymbol{p})^{\frac{1}{2}}}
$$

1 The normalization implies that we are interpreting the electromagnetic field as being associated with one photon, $\boldsymbol{f}(\boldsymbol{k})$ being the amplitude for the photon having a momentum $\boldsymbol{k}$. 
where $\boldsymbol{e}_{\mu}$ is the polarization vector with modulus unity and is perpendicular to the momentum vector $\boldsymbol{p}$ and $H(\boldsymbol{x})$ is the Heaviside unit function defined by

$$
\begin{aligned}
H(x) & =1 \text { for } x>0 \\
& =0 \text { for } x<0 .
\end{aligned}
$$

The product of the Heaviside functions is chosen to express the fact that the photon momentum lies between $\boldsymbol{p}$ and $\boldsymbol{p}+\Delta \boldsymbol{p}$. The normalization condition of a single photon in the momentum range $\Delta \boldsymbol{p}$ necessitates the term $(\Delta \boldsymbol{p})^{1 / 2}$ in the denominator. For a given $\boldsymbol{p}$, two linear polarizations or two circular polarizations are possible, denoted by the values 1, 2 for the index $\mu$. Taking $\boldsymbol{p}$ to be along the $z$-axis they can be represented by either

$$
\left.\begin{array}{r}
e_{1 x}=1 ; e_{1 y}=0 ; e_{1 z}=0 \\
e_{2 x}=0 ; e_{2 y}=1 ; e_{2 z}=0
\end{array}\right\} \text { linear polarization. }
$$

Thus in either case $\boldsymbol{e}_{1} \cdot \boldsymbol{e}_{2}=\mathbf{0}$ and the $\boldsymbol{f}_{p \mu}$ solutions form a complete orthonormal set for the given set of photon quantum numbers $p_{x}, p_{y}, p_{z}$ and $\mu$. Any arbitrary function $\boldsymbol{f}(\boldsymbol{k})$ can be expanded in terms of this set $\boldsymbol{f}_{p \mu}$

$$
\boldsymbol{f}(\boldsymbol{k})=\sum_{p \mu} c^{p \mu} \boldsymbol{f}_{p \mu}
$$

where

$$
c^{p \mu}=\int \boldsymbol{f} \boldsymbol{f}_{p \mu}^{*} \mathrm{~d}^{3} k .
$$

\section{Angular momentum and spin of the photon}

In a manner similar to the above we proceed to identify the expectation value of the photon angular momentum in the state $\boldsymbol{f}(\boldsymbol{k})$ with the angular momentum of the field.

The angular momentum in terms of $\boldsymbol{E}$ and $\boldsymbol{H}$ of the field is

$$
\overline{\boldsymbol{M}}=\int \boldsymbol{x} \times(\boldsymbol{E} \times \boldsymbol{H}) \mathrm{d}^{3} x .
$$

In terms of the transforms $\boldsymbol{E}(\boldsymbol{k})$ and $\boldsymbol{H}(\boldsymbol{k})$ this becomes

$$
\overline{\boldsymbol{M}}=\int \boldsymbol{x} \times\left[\boldsymbol{E}(\boldsymbol{k}) \times \boldsymbol{H}\left(\boldsymbol{k}^{\prime}\right)\right] \mathrm{e}^{i\left(\boldsymbol{k}+\boldsymbol{k}^{\prime}\right) \cdot x} \mathrm{~d}^{3} k \mathrm{~d}^{3} k^{\prime} \mathrm{d}^{3} x .
$$

Substituting in terms of $\boldsymbol{f}(\boldsymbol{k})$ and after considerable calculation we arrive at

$$
\overline{\boldsymbol{M}}=\int \mathrm{d}^{3} k\left\{-i\left[\boldsymbol{k} \times \nabla\left(\boldsymbol{f}_{c}^{*} \cdot \boldsymbol{f}\right)\right]-i\left[\boldsymbol{f}^{*} \times \boldsymbol{f}\right]\right\}
$$

Where the index $c$ indicates that in performing the differentiation $\boldsymbol{f}_{c}$ is treated as a constant or its components

$$
\bar{M}_{\alpha}=\int \mathrm{d}^{3} k\left\{f_{\beta}^{*}[-i \boldsymbol{k} \times \bar{V}]_{x} f_{\beta}-i e_{\alpha \beta \gamma} f_{\beta}^{*} f_{\gamma}\right\}
$$


where $e_{\alpha \beta \gamma}$ is the anti-symmetric unit tensor of rank three. Defining an operator $\boldsymbol{S}$ such that

$$
\mathrm{S}_{\alpha} f_{\beta}=-i e_{x \beta \gamma} f_{\gamma}
$$

whereby any cross product $[\boldsymbol{g} \times \boldsymbol{f}]$ can be written as

$$
[\boldsymbol{g} \times \boldsymbol{f}]_{x}=\boldsymbol{g}\left(i S_{\alpha}\right) \boldsymbol{f}
$$

we can write the angular momentum of the field as

$$
\overline{\boldsymbol{M}}=\int f_{\alpha}^{*}[-i \boldsymbol{k} \times \boldsymbol{r}+\boldsymbol{S}] f_{\alpha} \mathrm{d} \boldsymbol{k}
$$

Thus we see that the quantum mechanical average of the operator $[-i \boldsymbol{k} \times \boldsymbol{V}+\boldsymbol{S}]$ vields the angular momentum of the photon field.

It is shown later that the angular momentum operator

$$
\boldsymbol{M}=i \boldsymbol{J}=-i[\boldsymbol{k} \times \boldsymbol{D}]+\boldsymbol{S}
$$

corresponds to an infinitesimal rotation operator $\boldsymbol{J}$ of a vector field. This operator $\boldsymbol{M}$ can be shown to obey the commutation relations

$$
\begin{aligned}
& M_{x} M_{y}-M_{y} M_{x}=i M_{z} \\
& M_{x} M^{2}-M^{2} M_{x}=0
\end{aligned}
$$

and since $M^{2}$ commutes with the energy operator and has eigenvalues $j(j+1)$ and since $M_{z}$ can be shown to have $(2 j+1)$ eigenvalues ranging from $-j$ to $j$, the photon states with definite values of $\omega, M^{2}$, and $M_{z}$ exist with corresponding quantum numbers $p, j$ and $M$. We shall obtain the wave functions of these states.

\section{Spin wave functions}

From the above discussion we see that a rotation operator for a vector field involves two terms, one of the type

$$
\boldsymbol{L}=-i[\boldsymbol{k} \times \boldsymbol{D}]
$$

called the angular momentum operator (in momentum representation) and a spin operator $\boldsymbol{S}$ defined by (29). Hence the photon wave function should be dependent on $\boldsymbol{k}$ its momentum and another spin variable $\alpha ; \boldsymbol{L}$ operates only on the $\boldsymbol{k}$ space and $\boldsymbol{S}$ only on the spin space.

It can be seen that the operator $L^{2}$ has eigenfunctions corresponding to eigenvalues $l(l+1)$ and the operator $L_{z}$ has $(2 l+1)$ eigenvalues, i.e.

$$
\begin{aligned}
& L^{2} \varphi_{l, m}=l(l+1) \varphi_{l, m} \\
& L_{z} \varphi_{l, m}=m \varphi_{l, m}
\end{aligned}
$$

where $\varphi_{l m}$ are the usual spherical functions

$$
\varphi_{l, m}=a(k) Y_{l, m}^{(\boldsymbol{n})}: \quad \boldsymbol{n}=\frac{\boldsymbol{l}}{k}
$$


$a(k)$ and $Y_{l m}$ being normalised suitably. That is

$$
\int_{0}^{\infty} a^{*}(k) a(k) \mathrm{k}^{2} \mathrm{~d} k=1 \quad \text { and } \quad \int Y_{l^{\prime} m^{\prime}}^{*(n)} Y_{l m}^{(n)} \mathrm{d} 0=\delta_{l l^{\prime}} \delta_{m m^{\prime}}
$$

The operator $\boldsymbol{S}$ consists of $3 \times 3$ matrices with corresponding eigenfunctions $\chi_{s \mu}(\alpha)$ which satisfy the equations

$$
\begin{aligned}
& S^{2} \psi_{s \mu}=s(s+1) \chi_{s \mu} \\
& S_{z} \chi_{s \mu}=\mu \chi_{s \mu}
\end{aligned}
$$

From the form of the spin matrices we realise that a vector field is a spin 1 field with eigenvalues $s$ equal to 1 and $\mu=0, \pm 1$. The spin eigenfunctions $\chi_{0}, \chi_{ \pm 1}$ are also given below

$$
\begin{gathered}
S_{x}=\left[\begin{array}{ccc}
0 & 0 & 0 \\
0 & 0 & -i \\
0 & i & 0
\end{array}\right] ; \quad S_{y}=\left[\begin{array}{ccc}
0 & 0 & i \\
0 & 0 & 0 \\
-i & 0 & 0
\end{array}\right] ; \quad S_{z}=\left[\begin{array}{ccc}
0 & -i & 0 \\
i & 0 & 0 \\
0 & 0 & 0
\end{array}\right] . \\
S^{2}=\left[\begin{array}{ccc}
2 & 0 & 0 \\
0 & 2 & 0 \\
0 & 0 & 2
\end{array}\right] ; \quad \chi_{0}=\left[\begin{array}{l}
0 \\
0 \\
1
\end{array}\right] ; \quad \chi_{1}=-\frac{1}{\sqrt{ } 2}\left[\begin{array}{c}
1 \\
i \\
0
\end{array}\right] ; \quad \chi_{-1}=\frac{1}{\sqrt{2}}\left[\begin{array}{c}
1 \\
-i \\
0
\end{array}\right] .
\end{gathered}
$$

The spin eigenfunctions $\chi_{\mu}-s$ are orthogonal and they form a basic set in terms of which any vector $f$ can be resolved

i.e.

$$
\boldsymbol{f}=\sum_{\mu=-1}^{+1} f_{\mu} \boldsymbol{\chi}_{\mu}
$$

$$
f_{0}=f_{z} ; \quad f_{ \pm}=\mp \frac{1}{\sqrt{ } 2}\left[f_{x} \mp i f_{y}\right]
$$

We may choose the components of the polarization vector $\boldsymbol{e}$ to be identical with $\chi_{\mu}$. The two possible polarizations correspond exactly to the two values of the spin projection $\mu= \pm 1$. If $\boldsymbol{p}$ the momentum of the photon is taken along the $z$-axis, the transversality condition excludes the state $\chi_{0}$. Hence $\chi_{1}$ and $\chi_{-1}$ are the allowed spin states where $\mu=1$ corresponds to right circular polarization and $\mu=-1$ to left circular polarization.

\section{Angular momentum eigenfunctions}

If $\boldsymbol{f}_{j M}(\boldsymbol{k}, \alpha)$ are the simultaneous eigenvectors of $M^{2}$ and $M_{z}$

$$
\begin{aligned}
& M^{2} \boldsymbol{f}_{j M}(\boldsymbol{k}, \alpha)=j(j+1) \boldsymbol{f}_{j M}(\boldsymbol{k}, \alpha) \\
& M_{z} \boldsymbol{f}_{j M}(\boldsymbol{k}, \alpha)=\boldsymbol{M} \boldsymbol{f}_{j M}(\boldsymbol{k}, \alpha) .
\end{aligned}
$$

Since the spin of the photon is equal to 1 , the orbital angular momentum eigenvalue $l$ for a given $j$ can only be

$$
l=j \quad \text { or } \quad l=j \pm 1(j \neq 0) .
$$

${ }^{1} \mathrm{~d} 0$ indicates differentials of the angular variables. 
Hence the wave functions $\boldsymbol{f}_{j l M}$ are got by the superposition of the products of orbital and spin functions $\varphi_{l, m}$ and $\chi_{s \mu}$ subject to the rule for the projection values of $m$ and $\mu$

$$
m+\mu=M \text {. }
$$

Hence the total angular momentum wave function

$$
\boldsymbol{f}_{j l M I}(\boldsymbol{k}, \alpha)=\sum_{m+\mu=M} C(l, s, j ; m \mu M) \varphi_{l m}(\boldsymbol{k}) \boldsymbol{\chi}_{\delta \mu}(\alpha)
$$

where the $C$ 's are the usual Clebsch-Gordon coefficients. For a given value of $l$ and $M$ these coefficients will form an orthogonal matrix. We can decompose $\boldsymbol{f}_{j l M}$ in terms of the spherical basis $\chi_{\mu}$ and obtain the components of vector $\boldsymbol{f}_{j l M}$ as

$$
f_{j l M}^{\mu}=a C(l, s, j ; M-\mu, \mu, M) Y_{l M-\mu}=a(k) Y_{j l M}^{\mu}
$$

where $\mathfrak{Y}_{j l M}^{\mu}$ is called the spherical vector function. Since $\boldsymbol{f}_{j l M}$ form an orthonormal set expressed by the condition

$$
\int \boldsymbol{f}_{j l M}^{*} \boldsymbol{f}_{j^{\prime} l^{\prime} M M^{\prime}} \mathrm{d}^{3} k=\delta_{j j^{\prime}} \delta_{l l^{\prime}} \delta_{M M N^{\prime}}
$$

it follows that the spherical vector functions are an orthonormal set

$$
\int \mathfrak{Y}_{j l M}^{*} \mathfrak{Y}_{j^{\prime} l^{\prime} M^{\prime}} \mathrm{d} 0=\delta_{j j^{\prime}} \delta_{l l^{\prime}} \delta_{M M M^{\prime}}
$$

\section{Parity eigenfunctions}

The $f_{j M}$ functions can be expressed as a linear combination of the three $f_{j l M}$ functions which span the space completely. Ignoring the radial functions we write

$$
\boldsymbol{f}_{j M}=\sum_{l=j-1}^{l=j+1} \varrho_{l} \mathfrak{Y}_{j l M}
$$

Since $\boldsymbol{f}_{j \boldsymbol{M}}$ should satisfy the transversality condition

$$
\boldsymbol{f}_{j M} \cdot \boldsymbol{n}=0
$$

where $\boldsymbol{n}$ is a unit vector in the direction of momentum there are only two independent photon states $\boldsymbol{f}_{j M}^{\lambda}$ with given quantum numbers $j$ and $M$ where the allowed values of $\lambda$ are $\lambda=0$ and $\lambda=+1$. These are the two parity eigenfunctions for given $j$ and $M$.

To obtain these $\boldsymbol{f}_{j M}^{(\lambda)}$ 's explicitly, we combine the three $\mathfrak{9}_{j l M}$ vectors in $k$ space to give three mutually perpendicular spherical vectors $\mathfrak{Y}_{j M}^{(-1)}$ which is longitudinal and $\mathfrak{Y}_{j M}^{(1)}$ and $\mathfrak{Y}_{j M}^{(0)}$ which are transverse. Remembering that the unit vector in the spherical basis has components

$$
n_{0}=\cos \theta ; \quad n_{ \pm 1}=\mp \frac{1}{\sqrt{2}} \sin \theta \mathrm{e}^{ \pm i}
$$

we obtain the longitudinal vector $\mathfrak{Y}_{j M}^{(-1)}$ as a combination of $\mathfrak{Y}_{j, j-1, M}$ and $\mathfrak{Y}_{j, j+1, M}$

$$
\mathfrak{Y}_{j M}^{(-1)}=\sqrt{\frac{j}{2 j+1}} \mathfrak{Y}_{j, j-1, M}-\sqrt{\frac{j+1}{2 j+1}} \cdot \mathfrak{Y}_{j j+1 M} \text {. }
$$


The other two desired transverse spherical vectors are seen to be

and

$$
\mathfrak{Y}_{j M}^{(0)}=\mathfrak{Y}_{j j M}
$$

$$
i \mathfrak{Y}_{j M}^{(1)}=\left[n \times \mathfrak{Y}_{j M}^{(0)}\right] \text { or } \mathfrak{Y}_{j M}^{(1)}=\sqrt{\frac{j}{2 j+1}} \mathfrak{Y}_{j, j+1, M}+\sqrt{\frac{j+1}{2 j+1}} \mathfrak{Y}_{j, j-1, M} \text {. }
$$

These $\mathfrak{Y}_{j M}^{\lambda}$ are normalized and are orthogonal in the same way as $\mathfrak{Y}_{j l M}$ and we have

$$
\int \mathfrak{Y}_{j M}^{\lambda *} \mathfrak{Y}_{j^{\prime} M^{\prime}}^{\lambda^{\prime}} \mathrm{d} 0=\delta_{j j^{\prime}} \delta_{M M^{\prime}} \delta_{\lambda \lambda^{\prime}}
$$

The photon eigenfunction for an angular momentum eigen state with given $j$ and $M$ is

$$
f_{j M}=\varrho_{1} \mathfrak{Y}_{j M}^{(1)}+\varrho_{0} \mathfrak{Y}_{j M}^{(0)}
$$

where $\varrho_{1}$ and $\varrho_{0}$ are arbitrary. This degeneracy in $\boldsymbol{f}_{j \boldsymbol{M}}$ can be split by the application of the parity operator $P$ defined by

$$
P \boldsymbol{f}(\boldsymbol{k})= \pm \boldsymbol{f}\left(\boldsymbol{k}^{\prime}\right)= \pm \boldsymbol{f}(-\boldsymbol{k})
$$

We know that the spherical functions $Y_{l m}(\boldsymbol{k})$ have a parity $(-1)^{l}$ and hence the spherical vector functions $\mathfrak{Y}_{j l n}(\boldsymbol{n})$ have a parity $(-1)^{l+1}$

$$
P \mathfrak{Y}_{j l M}(\boldsymbol{n})=(-1)^{l+1} \mathfrak{Y}_{j l M}(\boldsymbol{n})
$$

For transverse spherical vectors we have

$$
\begin{aligned}
& P \mathfrak{Y}_{j M}^{(1)}=(-1)^{j} \mathfrak{Y}_{j M}^{(1)} \\
& P \mathfrak{Y}_{j M}^{0}=(-1)^{j+1} \mathfrak{Y}_{j M}^{(0)}
\end{aligned}
$$

Hence the photon states can be given by the $\mathfrak{Y}_{j M}^{\lambda}(\boldsymbol{n})$ functions uniquely. $\lambda=1$ is called an electric state and $\lambda=0$ is called a magnetic state. It can be seen that the parity states of $\boldsymbol{f}$ correspond to the parity of the $\boldsymbol{H}$ field of the radiation which is $(-1)^{j}$ for the electric state and $(-1)^{j+1}$ for the magnetic state. These names "electric" and "magnetic" states correspond to the fact that the emission of the photon in these states arises from the electric and magnetic moment respectively of the system of charges. For a photon with a given energy value $|\boldsymbol{p}|$ in the small range $\mathrm{d}|\boldsymbol{p}|$, the radial function is

$$
a(k)=c \mathrm{e}^{-i p t} H[|\boldsymbol{k}|+(|\boldsymbol{p}|+\mathrm{d}|\boldsymbol{p}|)] H[|\boldsymbol{k}|-(|\boldsymbol{p}|-\mathrm{d}|\boldsymbol{p}|)]
$$

and since $a(k)$ is normalized to unity

$$
c=i / V p^{2} \mathrm{~d} p
$$

Hence the photon state characterized uniquely by the four quantum numbers corresponding to the energy $p$, total angular momentum $j$ and its projection $M$ and parity $\lambda$ is given by the eigenfunction

$$
\boldsymbol{f}_{p j M \lambda}=\frac{i}{V p^{2} \mathrm{~d} p} \mathfrak{Y}_{j M}^{\lambda} \mathrm{e}^{-i p t} H[k+(p+\mathrm{d} p)] H[k-(p-\mathrm{d} p)] .
$$


It is to be noted that when $\lambda=1$ the spherical vector function $\mathfrak{Y}_{j M}^{(1)}$ is a linear combination of two $\mathfrak{Y}_{j l M}$ functions with different $l$ values and when $\lambda=0$, the total angular momentum is equal to the orbital angular momentum. This explains the well-known statement that it is not possible to divide the angular momentum of the photon into an orbital and spin part.

A photon which has total angular momentum value $j=0$ has only one spherical vector function corresponding to it namely $\mathfrak{Y}_{0,1,0}$ which is according to the equations (56) a longitudinal spherical vector. A spherically symmetric vector field has total angular momentum equal to zero and can be represented only by a longitudinal photon which does not exist.

Thus the wave function of the photon in an arbitrary state $\boldsymbol{f}$ can be expanded in a series of eigenfunctions as

$$
\boldsymbol{f}=\sum_{p j \boldsymbol{M} \lambda} C^{p j \boldsymbol{M \lambda}} \boldsymbol{f}_{p j \boldsymbol{M \lambda}}
$$

and the expansion coefficients are obtained by

$$
C^{p j M \lambda}=\int f f_{p j M \lambda}^{*} \mathrm{~d}^{3} k
$$

\section{Expansion of the electric and magnetic fields in terms of the photon eigenfunctions}

We can express the electric and magnetic fields $\boldsymbol{E}(\boldsymbol{x})$ and $\boldsymbol{H}(\boldsymbol{x})$ in terms of the eigenfunctions $\boldsymbol{f}_{p j \boldsymbol{M} \lambda}$ of the photon as

$$
\begin{aligned}
& \boldsymbol{E}_{p j M \lambda}(\boldsymbol{k})=\frac{i}{4 \pi^{3 / 2} V p d p}\left(\mathfrak{Y}_{j M}^{(\lambda)} \mathrm{e}^{-i p t}-\mathfrak{Y}_{j M}^{(\lambda)} * \mathrm{e}^{i p t}\right) \\
& \boldsymbol{H}_{p j M \lambda}(\boldsymbol{k})=\frac{-1}{4 \pi^{2 / 2} / p d p}\left(\mathfrak{Y}_{j M}^{(1-\lambda)} \mathrm{e}^{-i p t}+\mathfrak{Y}_{j M}^{(1-\lambda)} \mathrm{e}^{i p t}\right)
\end{aligned}
$$

Using the well-known expansion of $\mathrm{e}^{i \boldsymbol{k} \cdot \boldsymbol{x}}$ in spherical functions

$$
\mathrm{e}^{i \boldsymbol{k} \cdot \boldsymbol{x}}=\sum g_{l}(k x) Y_{l m}^{*}\left(\frac{\boldsymbol{k}}{k}\right) Y_{l m}\left(\frac{\boldsymbol{x}}{x}\right)
$$

we obtain integrals of the type

$$
\int \mathfrak{Y}_{l M}^{(\lambda)}\left(\frac{\boldsymbol{k}}{k}\right) \mathrm{e}^{i \boldsymbol{k} \cdot \boldsymbol{x}} \mathrm{d} o=g_{l}(k x) \mathfrak{Y}_{j l M}\left(\frac{\boldsymbol{x}}{x}\right)
$$

where $g_{l}(k x)$ are related to Bessel functions $J_{l+1 / 2}(k x)$ by

$$
g_{l}(k x)=(2 \pi)^{3 / 2} i^{l} \frac{T_{l+1 / 2}(k x)}{1 / k x} .
$$

Expressing $\boldsymbol{E}(\boldsymbol{x})$ and $\boldsymbol{H}(\boldsymbol{x})$ as

$$
\begin{aligned}
& \boldsymbol{E}(\boldsymbol{x})=\boldsymbol{\mathfrak { E }}(\boldsymbol{x})+\mathfrak{E}^{*}(\boldsymbol{x}) \\
& \boldsymbol{H}(\boldsymbol{x})=\boldsymbol{\mathfrak { y }}(\boldsymbol{x})+\mathbf{5}^{*}(\boldsymbol{x})
\end{aligned}
$$


we obtain for the electric state, i.e. $\lambda=1$ the following values of $\boldsymbol{E}(\boldsymbol{x})$ and $\boldsymbol{\mathfrak { y }}(\boldsymbol{x})$ in terms of the $\mathfrak{Y}_{j l M}(\boldsymbol{x})$ and $g_{l}(k x)$ functions.

$\mathfrak{\mathbf { E }}_{p, j, M,+1}=\frac{i V p^{3} \mathrm{~d} p}{4 \pi^{3 / 2}}\left[\sqrt{\frac{j}{2 j+1}} g_{j+1}(p x) \mathfrak{Y}_{j j+\mathbf{1} M}+\sqrt{\frac{j+1}{2 j+1}} g_{j-1} \mathfrak{Y}_{j j-1 M}\right] \mathrm{e}^{-i p t}$

$\mathfrak{\mathfrak { y }}_{p, j, M,+1}=\frac{-V p^{3} \mathrm{~d} p}{4 \pi^{3 / 2}}\left[g_{j}(p x) \mathfrak{Y}_{j j M} \mathrm{e}^{-i p t}\right]$

For the magnetic state we have $\lambda=0$

$$
\begin{aligned}
& \mathfrak{\mathfrak { E }}_{p, j, M, 0}=\frac{i V p^{3} \mathrm{~d} p}{4 \pi^{3 / 2}} g_{i}(p x) \mathfrak{Y}_{j j M} \mathrm{e}^{-i p t} \\
& \mathfrak{\mathfrak { Y }}_{p, j, M, 0}=\frac{-V p^{3} \mathrm{~d} p}{4 \pi^{3 / 2}}\left[\sqrt{\frac{j}{2 j+1}} g_{j+1} \mathfrak{Y}_{j j+1 M}+\sqrt{\frac{j+1}{2 j+1}} g_{j-1} \mathfrak{Y}_{j j-1 M}\right] \mathrm{e}^{-i p t} .
\end{aligned}
$$

The normalization implies that there is a single photon in the energy range $p$ and $p+\mathrm{d} p$. It is clear that the $\mathfrak{\mathfrak { Y }}$ field in electric state and the $\mathbb{C}$ field in magnetic state are represented by the $\mathfrak{Y}_{j M}^{(0)}=\mathfrak{Y}_{j j M}$ functions which are transverse vectors in $r$ space also. Hence

$$
\boldsymbol{H}_{p, j, M,+1} \cdot \boldsymbol{x}=\boldsymbol{E}_{p, j, M, 0} \cdot \boldsymbol{x}=0 .
$$

The electric field in the electric state and the magnetic field in the magnetic state can be split into transverse and longitudinal vectors, i.e. $\mathfrak{Y}_{j M}^{(1)}$ functions and $\mathfrak{Y}_{j M}^{(-1)}$ functions respectively

$$
\begin{aligned}
\mathfrak{\mathbf { E }}_{p, j, M,+1}=\frac{i V p^{3} \mathrm{~d} p}{4 \pi^{3 / 2}}\left[\frac{V j(j+1)}{2 j+1}\left(g_{j-1}-g_{j+1}\right) \mathfrak{Y}_{j M}^{(-1)}+\right. \\
\left.+\left(\frac{j}{2 j+1} g_{j+1}+\frac{j+1}{2 j+1} g_{j-1}\right) \mathfrak{Y}_{j M}^{(1)}\right] \mathrm{e}^{-i p t} \\
\mathfrak{\mathbf { S }}_{p, j, M, 0}=i \mathfrak{\mathbf { E }}_{p, j, M,+1} .
\end{aligned}
$$

At large distances, i.e. for $p x \gg 1$ the first term, i.e. the radial component vanishes since $g_{j+1} \sim g_{j-1}$ and the field becomes in the radiation zone,

$$
\mathfrak{C}_{p, j, M,+1}=-i \mathfrak{\mathbf { s }}_{p, j, M, 0} \approx \frac{i^{j+1}}{V \pi} p^{3} \mathrm{~d} p \mathfrak{Y}_{j M}^{(1)} \frac{\cos \left(p x-\pi-\frac{\pi j}{2}\right)}{x} \mathrm{e}^{-i p t}
$$

Thus it is possible to express any electromagnetic field in the form

$$
\mathfrak{E}=\sum_{p j M \lambda} C^{p j M \lambda} \mathfrak{C}_{p j M \lambda}
$$

Potentials and their expansions in terms of the eigenfunctions of angular momentum and parity

Since it is possible to specify an electromagnetic field by the vector potential $\boldsymbol{A}$ and the scalar potential $A_{4}$ instead of the electric and magnetic fields, we shall conclude this chapter by expressing $\boldsymbol{A}$ and $A_{4}$ in terms of the eigenfunctions of parity and angular momentum. 
For a given electromagnetic field the potentials $\boldsymbol{A}$ and $A_{4}$ are defined by the equations

$$
\boldsymbol{E}=-\frac{\partial \boldsymbol{A}}{\partial t}-\nabla A_{4} ; \quad \boldsymbol{H}=\operatorname{curl} \boldsymbol{A} .
$$

The above equation (86) can be written in terms of $\boldsymbol{A}(\boldsymbol{k})$ and $A_{4}(\boldsymbol{k})$ the Fourier transforms of $\boldsymbol{A}(\boldsymbol{x}), A_{4}(\boldsymbol{x})$ as well as in terms of $\boldsymbol{E}(\boldsymbol{k})$ and $\boldsymbol{H}(\boldsymbol{k})$ as

$$
\begin{gathered}
\boldsymbol{E}(\boldsymbol{k})=-\dot{\boldsymbol{A}}(\boldsymbol{k})-i \boldsymbol{k} A_{4}(\boldsymbol{k}) \\
\boldsymbol{H}(\boldsymbol{k})=i(\boldsymbol{k} \times \boldsymbol{A}(\boldsymbol{k})) .
\end{gathered}
$$

Splitting $\boldsymbol{A}$ into transverse and longitudinal parts

with

$$
\boldsymbol{A}=\boldsymbol{B}+\boldsymbol{n} \varphi \quad\left(\boldsymbol{n}=\frac{\boldsymbol{k}}{k}\right)
$$

$$
\boldsymbol{k} \cdot \boldsymbol{B}(\boldsymbol{k})=0
$$

the transversality condition $\boldsymbol{n} \cdot \boldsymbol{E}=0$ yields the relation

$$
i k A_{4}+\dot{\varphi}=0 \text {. }
$$

It is easy to see by equation (3) that $\boldsymbol{B}$ and $\dot{\boldsymbol{B}}$ are related to $\boldsymbol{E}$ and $\dot{\boldsymbol{E}}$ as

$$
\boldsymbol{B}=\frac{1}{k^{2}} \dot{\boldsymbol{E}} ; \quad \dot{\boldsymbol{B}}=-\boldsymbol{E} .
$$

Hence $\boldsymbol{B}$ and $\dot{\boldsymbol{B}}$ can be put in terms of photon wave functions $\boldsymbol{f}$ and $\boldsymbol{f}^{*}$ as

$$
\begin{aligned}
& \boldsymbol{B}(\boldsymbol{k})=\frac{-i}{4 \pi^{3 / 2} / k}\left[\boldsymbol{f}(\boldsymbol{k})-\boldsymbol{f}^{*}(-\boldsymbol{k})\right] \\
& \dot{\boldsymbol{B}}(\boldsymbol{k})=\frac{-\bigvee k}{4 \pi^{3 / 2}}\left[\boldsymbol{f}(\boldsymbol{k})+\boldsymbol{f}^{*}(-\boldsymbol{k})\right]
\end{aligned}
$$

The gauge invariance condition according to which $\boldsymbol{E}$ and $\boldsymbol{H}$ do not change if $\frac{\partial \Lambda}{\partial t}$ is added to $A_{4}$ and $\nabla \Lambda$ is added to $\boldsymbol{A}$ is expressed by the equation already given,

$$
i k A_{4}+\dot{\varphi}=0
$$

Similarly the subsidiary Lorentz condition for the potentials

$$
\operatorname{div} \boldsymbol{A}+\frac{\partial A_{4}}{\partial t}=0
$$

gives another relation for $A_{4}$ and $\varphi$,

$$
i k \varphi+\dot{A_{4}}=0 .
$$

EPCR 4 
Note that equations $(90,94)$ are just like the equations connecting $\boldsymbol{E}$ and $\boldsymbol{H}$. Hence if $A_{4}(\boldsymbol{k})$ is real, we can introduce complex scalar functions $f_{0}(\boldsymbol{k})$ given by

$$
\begin{aligned}
& A_{4}(\boldsymbol{k})=\frac{1}{4 \pi^{3 / 2} / \sqrt{k}}\left[f_{0}(\boldsymbol{k})+f_{0}^{*}(-\boldsymbol{k})\right] \\
& \dot{A}_{4}(\boldsymbol{k})=\frac{-i \bigvee k}{4 \pi^{3 / 2}}\left[f_{0}(\boldsymbol{k})-f_{0}^{*}(-\boldsymbol{k})\right]
\end{aligned}
$$

and $f_{0}$ satisfies the Schrödinger equation

$$
i \frac{\partial f_{0}}{\partial t}=k f_{0}
$$

So we obtain for $\boldsymbol{A}(\boldsymbol{k})$

where

$$
\boldsymbol{A}(\boldsymbol{k})=\frac{-i}{4 \pi^{3 / 2} \sqrt{k}}\left(\boldsymbol{h}(\boldsymbol{k})-\boldsymbol{h}^{*}(\boldsymbol{k})\right)
$$

$$
\boldsymbol{h}(\boldsymbol{k})=\boldsymbol{f}(\boldsymbol{k})+i \boldsymbol{n} f_{0}(\boldsymbol{k}) .
$$

The expectation value of the photon energy can be written as

$$
\bar{\omega}=\int k \boldsymbol{f}^{*} \boldsymbol{f} \mathrm{d}^{3} k=\int k\left(\boldsymbol{h}^{*} \boldsymbol{h}-f_{0}^{*} f_{0}\right) \mathrm{d}^{3} k .
$$

Similarly the expectation values of linear momentum and angular momentum are given by

$$
\overline{\boldsymbol{p}}=\int \boldsymbol{k}\left(\boldsymbol{h}^{*} \boldsymbol{h}-f_{0}^{*} f_{0}\right) \mathrm{d}^{3} k ; \quad \overline{\boldsymbol{M}}=\int\left(h_{\alpha}^{*} \boldsymbol{M} h_{\alpha}-f_{0}^{*} \boldsymbol{L} f_{0}\right) d^{3} k
$$

where $\boldsymbol{M}$ is the total angular momentum operator and $\boldsymbol{L}$ the orbital angular momentum operator.

If we want to obtain the potentials corresponding to the angular momentum and linear momentum eigenstates of the photon, we write down the angular momentum and linear momentum eigenfunctions of $f_{0}$ and $\boldsymbol{h}$,

and

$$
f_{0 p}=c \frac{i}{\sqrt{\Delta \boldsymbol{p}}} \mathrm{e}^{-i k t} H[\boldsymbol{k}+(\boldsymbol{p}+\Delta \boldsymbol{p})] H[\boldsymbol{k}-(\boldsymbol{p}-\Delta \boldsymbol{p})]
$$

$$
\boldsymbol{h}_{p \mu}=\frac{i \mathrm{e}^{-i k t}}{V \Delta \boldsymbol{p}}\left(\boldsymbol{e}_{\mu}+i \boldsymbol{c} \boldsymbol{n}\right)_{!}^{!} H[\boldsymbol{k}+(\boldsymbol{p}+\Delta \boldsymbol{p})] H[\boldsymbol{k}-(\boldsymbol{p}-\Delta \boldsymbol{p})],
$$

where $c$ is an arbitrary constant.

For the functions $f_{0}$ the angular momentum operator is only the orbital momentum and the eigenfunctions $f_{0}$ for angular momentum $j$ and its component $M$ are

$$
f_{0 i M}=\frac{i c_{d}^{?}}{\sqrt{p^{2} \Delta p}} Y_{j M} \mathrm{e}^{-i k t} H[k+(p+\Delta p)] H[k-(p-\Delta p)
$$

and the parity of $f_{0 j M}=(-1)^{j}$, i.e. that of an electric state. Hence for the magnetic state $f_{0 j M}$ vanishes, i.e.

$$
f_{0 j M 0}=0 \text { and } \boldsymbol{h}_{j M 0}=\boldsymbol{f}_{j M 0}
$$


For the electric state

$$
\boldsymbol{h}_{p j M_{,}+1}=\frac{i}{\sqrt{ } p^{2} \Delta p}\left(\bigcup_{j M}^{(1)}+i c \boldsymbol{n} Y_{j M}\right) \mathrm{e}^{-i k t} H[k+(p+\Delta p)] H[k-(p-\Delta p)] \text {. }
$$

The choice of the constant $c$ determines $\boldsymbol{h}$ and if $c=i] \frac{j}{j+1}, \boldsymbol{h}$ is proportional to the spherical function $\mathfrak{Y}_{j, j-1 . M}$ :

$$
\begin{aligned}
h_{p j \boldsymbol{M}+1} & =\frac{i}{V p^{2} \Delta p} \sqrt{\frac{2 j+1}{j+1}} \mathfrak{9}_{j, j-1, M} \mathrm{e}^{-i k t} H[k+(p+\Delta p)] H[k-(p-\Delta p)] \\
f_{0 p j M,+1} & =\sqrt{\frac{j}{j+1}} \frac{1}{\sqrt{p^{2} \Delta p}} Y_{j M} \mathrm{e}^{-i k t} H[k+p+\Delta p] H[k-(p-\Delta p)] .
\end{aligned}
$$

From these eigenfunctions $\boldsymbol{h}$ and $f_{0}$, we can find the potentials $\boldsymbol{A}$ and $A_{0}$ if we set

$$
\begin{aligned}
\boldsymbol{A}(\boldsymbol{r}) & =\mathfrak{P}(\boldsymbol{r})+\mathfrak{A}^{*}(\boldsymbol{r}) \\
A_{4}(\boldsymbol{r}) & =\mathfrak{A}_{4}(\boldsymbol{r})+\mathfrak{A}_{4}^{*}(\boldsymbol{r})
\end{aligned}
$$

We determine the potentials for the plane waves:

$$
\begin{aligned}
\mathfrak{A}_{p \mu}(\boldsymbol{x}) & \left.=\frac{1}{4 \pi^{3 / 2}}\right] / \frac{\Delta \boldsymbol{p}}{p}\left[\boldsymbol{e}_{\mu}+\frac{c \boldsymbol{p}}{p \mid}\right] \mathrm{e}^{i\left(\boldsymbol{p} \cdot \boldsymbol{x}-p_{4} t\right)} \\
\mathfrak{U}_{4 p \mu}(\boldsymbol{x}) & \left.=\frac{1}{4 \pi^{3 / 2}}\right] \frac{\Delta \boldsymbol{p}}{p}\left[c \mathrm{e}^{i\left(\tilde{p} \cdot \boldsymbol{x}-p_{4} t\right)}\right]
\end{aligned}
$$

From the spherical wave solution, we obtain for $\mathfrak{P}_{p j M}$ and $\mathfrak{A}_{+p j M}$ the following expressions if we set $c=-\sqrt{\frac{j}{j+1}}$ :

For the electric state

$$
\begin{aligned}
\mathfrak{F}_{p j M+1} & =\frac{V p \Delta p}{4 \pi^{3 / 2}} \sqrt{\frac{2 j+1}{j+1} g_{j-1}} \mathfrak{9}_{j j-1 M} \mathrm{e}^{-i p t} \\
\mathfrak{A}_{4 p j M,+1} & =-\sqrt{\frac{j}{j+1}} \frac{V p \Delta p}{4 \pi^{3 / 2}} g_{j} Y_{j M} \mathrm{e}^{-i p t}
\end{aligned}
$$

and for the magnetic state

for arbitrary $c$.

$$
\begin{aligned}
\mathfrak{2}_{p j M 0} & =\frac{V p \Delta p}{4 \pi^{3 / 2}} g_{j} \mathfrak{Y}_{j M}^{(0)} \mathrm{e}^{-i p t} \\
\mathfrak{A}_{4 p j M 0} & =0
\end{aligned}
$$




\section{QUANTUM ELECTRODYNAMICS}

\section{PERTURBATION EXPANSIONS AND KERNEL FUNCTIONS1}

We shall now examine how time dependence other than that of the exponential type enters into the wave function. Let us consider a particle in the stationary state corresponding to a time independent Hamiltonian $H_{0}$ and let the eigenfunctions of $H_{0}$ be $\varphi_{n}(x) \mathrm{e}^{-i E_{n} t}$ where $E_{n}$ represents the eigenvalue of $H_{0}$. By time independence we also imply that $H_{0}$ exists for all time, i.e. from $t=-\infty$ to $t=+\infty$. If a particle is found in any one of these eigenstates, it will continue to do so for all time. Time dependence other than that of the exponential type can occur if the wave function $\psi$ satisfies the Schrödinger equation

$$
i \frac{\partial \psi(t)}{\partial t}=H(t) \psi(t)
$$

where $H$ is time dependent. In any physical process we deal with the Hamiltonian $H(t)$ such that $H \rightarrow H_{0}$ as $t \rightarrow+\infty$ and $t \rightarrow-\infty$. This implies that if we can write

$$
H=H_{0}+H^{\prime}
$$

where $H^{\prime}$ is called the perturbing Hamiltonian, $H^{\prime} \rightarrow 0$ as $t \rightarrow+\infty$ and $t \rightarrow-\infty$. But if we wish to insist that $H^{\prime}$ should be in operation for an infinite period of time the above distinction between $H^{\prime}$ and $H$ can still be kept, provided we set

$$
\begin{array}{ll}
H^{\prime} \equiv 0 & \text { for } t<T_{0} \\
H^{\prime} \equiv H^{\prime}(t) & \text { for } T_{0}<t<T \\
H^{\prime} \equiv 0 & \text { for } t>T
\end{array}
$$

and let $T$ and $T_{0}$ tend to $+\infty$ and $-\infty$ respectively. Even if $H^{\prime}$ does not vary with time in the interval $T_{0}<t<T$ it is still in a strict sense time dependent since we have assumed $H^{\prime}$ to vanish outside this interval. We shall obtain the solution of (1) quite generally by expressing the wave function at $T$ in terms of the wave function at $T_{0}$ and later discuss the limiting process as $T$ and $T_{0}$ tend to $+\infty$ and $-\infty$ respectively.

1 R. P. Feynman, Phys. Rev. 76, 749. See also for example, the semi-expository paper by Alladi Ramakrishnan and R. Vasudevan on "Perturbation expansions and Kernel functions associated with single particle wave function", Proceedings of the Summer School of Theoretical Physics, Mussoorie, India (1959). 
If we assume that $\varphi_{n}$ 's form a complete orthonormal set it is clear that $\psi(x)$ can be expanded in terms of $\varphi_{n}$ 's as

$$
\begin{aligned}
\psi(x) & =\sum_{n} b_{n}(t) \varphi_{n}(\boldsymbol{x}) \mathrm{e}^{-i E_{n} t} \\
& =\sum_{n} b_{n}(t) \psi_{n}(x)=\sum_{n} c_{n}(t) \varphi_{n}(\boldsymbol{x})^{1} .
\end{aligned}
$$

We note that $c_{n}(t)$ and $b_{n}(t)$ satisfy the following equations

$$
\begin{aligned}
& i \frac{\mathrm{d} c_{n}(t)}{\mathrm{d} t}=\sum_{m} H_{n m}^{\prime} c_{m}(t)+E_{n} c_{n}(t) \\
& i \frac{\mathrm{d} b_{n}(t)}{\mathrm{d} t}=\sum_{m} H_{n m}^{\prime}(t) b_{m}(t)
\end{aligned}
$$

where

and

$$
H_{n m}^{\prime}=\int \varphi_{n}^{*}(\boldsymbol{x}) H^{\prime} \varphi_{m}(\boldsymbol{x}) \mathrm{d}^{3} x
$$

This implies that

$$
H_{n m}^{\prime}(t)=\int \psi_{n}^{*}(x) H^{\prime} \psi_{m}(x) \mathrm{d}^{3} x
$$

since

$$
\begin{aligned}
H_{n m}^{\prime}(t) & =H_{n m}^{\prime} \mathrm{e}^{i\left(E_{n}-E_{m}\right) t} \\
& =\mathrm{e}^{i H_{0} t} H_{n m}^{\prime} \mathrm{e}^{-i H_{0} t}
\end{aligned}
$$

The equation for $c(t)$ can be put in the vector matrix form as

$$
i \frac{\mathrm{d} \boldsymbol{c}(t)}{\mathrm{d} t}=\left[H^{\prime}\right] \boldsymbol{c}(t)+\left[H_{0}\right] \boldsymbol{c}(t)
$$

where $\boldsymbol{c}(t)$ is a column vector with typical elements $c_{n}(t),\left[H^{\prime}\right]$ and $\left[H_{0}\right]$ are matrices with elements $H_{n m}^{\prime}$ and $E_{n} \delta_{m n}$ respectively. We write the vector matrix equation for $\boldsymbol{b}(t)$ below as we are going to discuss it in detail.

$$
i \frac{\mathrm{d} \boldsymbol{b}(t)}{\mathrm{d} t}=\left[H^{\prime}(t)\right] \boldsymbol{b}(t)
$$

where $\left[H^{\prime}(t)\right]$ is the matrix with typical elements $H_{n m}^{\prime}(t)$. If we work with $b(t)^{\prime}$ s instead of $c(t)$ 's we need only the matrix representation of the perturbing Hamlitonian $H^{\prime}$ remembering of course the relation between $H_{n m}^{\prime}(t)$ and $H_{n m}^{\prime}$. When discussing the field theoretic formalism we will be able to identify $\boldsymbol{c}(t)$ and $\boldsymbol{b}(t)$ as the two representations of the same states called the Schrödinger and the interaction representations. We shall not go into this nomenclature any further now, but proceed to discuss the equation for $\boldsymbol{b}(t)$.

The calculation of the coefficients $b_{n}(t)$ constitutes the main problem in any quantum mechanical collision process. There are at present two main methods of calculation: (1954).

${ }^{1}$ W. HeItLen, Quantum Theory of Radiation, p. 139, Oxford University Press, London 
(i) The old fashioned perturbation theory relating to single particles,

(ii) The kernel function formalism of Feynman.

We are concerned only with wave functions of a single particle with $b_{n}(t)$ representing the amplitude for the particle to be found in a state $n$. It is to be noted that only transitions between positive energy states are envisaged here. Later we will be using the same equation with a generalized interpretation for the Hamiltonian so as to include systems of many particles. This corresponds to including the negative energy states in the kernel function formalism and its consequent modification by Feynman'.

By a solution of the equation (13), we mean that given $\boldsymbol{b}\left(t_{0}\right)$ we have to determine $\boldsymbol{b}(t)$ for $t>t_{0}$. From elementary iteration we obtain

$$
\boldsymbol{b}(t)=\left[U\left(t, t_{0}\right)\right] \boldsymbol{b}\left(t_{0}\right)
$$

where $U\left(t, t_{0}\right)$ is the expansion

$$
\begin{gathered}
U\left(t_{1}, t_{0}\right)=1+(-i) \int_{t_{0}}^{t} H^{\prime}\left(\tau_{1}\right) \mathrm{d} \tau_{1}+(-i)^{2} \int_{t_{0}}^{t} \mathrm{~d} \tau_{2} \int_{t_{0}}^{\tau_{2}} \mathrm{~d} \tau_{1} H^{\prime}\left(\tau_{2}\right) H^{\prime}\left(\tau_{1}\right)+\cdots \\
\cdots+(-i)^{k} \int_{t_{0}}^{t} \mathrm{~d} \tau_{k} \int_{t_{0}}^{\tau_{k}} \mathrm{~d} \tau_{k-1} \cdots \int_{t_{0}}^{\tau_{2}} \mathrm{~d} \tau_{1} H^{\prime}\left(\tau_{k}\right) \cdots H^{\prime}\left(\tau_{1}\right)+\cdots
\end{gathered}
$$

The physically interesting case is obtained by setting $t=T$ and $t_{0}=T_{0}$, i.e. the times corresponding to "switch off" and "switch on" of the perturbation and letting $T$ and $T_{0}$ tend to $+\infty$ and $-\infty$ respectively. In the above integration we have ordered the time parameters in the following way

$$
t>\tau_{k}>\tau_{k-1}>\ldots>\tau_{1}>t_{0}
$$

In a physical problem since we are interested in computing the amplitude of transition from a definite intial state to a definite final state, we assume that all the elements of $\boldsymbol{b}\left(t_{0}\right)$ are zero except a specified $b_{i}\left(t_{0}\right)$ which is set equal to unity. Denoting $b_{j}(t)$ the typical element of $\boldsymbol{b}(t)$ for obvious reasons as $b_{f i}(t)$ we can write

with

$$
b_{f i}(t)=\langle f|S| i\rangle=\sum_{n}\left\langle f\left|S^{(n)}\right| i\right\rangle
$$

and

$$
S=\lim _{\substack{t \\=\\ t_{0}=T_{0} \rightarrow+\infty}} U\left(t, t_{0}\right)
$$

$$
S^{(n)}=(-i)^{n} \int_{t_{0}}^{t} \mathrm{~d} \tau_{n} \int_{t_{0}}^{\tau_{n-1}} \mathrm{~d} \tau_{n-1} \ldots \int_{t_{0}}^{\tau_{2}} \mathrm{~d} \tau_{1} H^{\prime}\left(\tau_{n}\right) H^{\prime}\left(\tau_{n-1}\right) \ldots H^{\prime}\left(\tau_{1}\right)
$$

where for the present we have adopted the notation that $|i\rangle$ is the vector $\boldsymbol{b}$ with all its elements zero except $b_{i}$ which is set equal to unity and $|f\rangle$ is a vector with all its elements zero except $b_{f}$ which is set equal to unity. By

1 W. Heitler, Quintum Theory of Radiation, Oxford University Press, London (1954). 
definition each element of $\left[H^{\prime}(\tau)\right]$ is an integral over space and therefore we define $R_{n m}(\boldsymbol{x}, t)$ as

so that

$$
R_{n m}(\boldsymbol{x}, t)=\psi_{n}^{*}(x) H^{\prime}(x) \psi_{m}(x)
$$

$$
H_{n m}^{\prime}(t)=\int R_{n m}(x) \mathrm{d}^{3} x .
$$

Thus we write the expansion as

$$
\begin{aligned}
\langle f|S| i\rangle=\boldsymbol{b}(t)[ & 1+(-i) \int_{-\infty}^{+\infty} \mathrm{d}^{3} x_{1} \int_{t_{0}}^{t}\left[R\left(x_{1}\right)\right] \mathrm{d} \tau_{1}+ \\
& \cdots+(-i)^{k} \int_{-\infty}^{\infty} \mathrm{d}^{3} x_{k} \int_{-\infty}^{\infty} \mathrm{d}^{3} x_{k-1} \cdots \int_{-\infty}^{\infty} \mathrm{d}^{3} x_{1} \int_{t_{0}}^{t} \mathrm{~d} \tau_{k} \int_{t_{0}}^{\tau_{k}} \mathrm{~d} \tau_{k-1} \cdots \int_{t_{0}}^{\tau_{2}} \mathrm{~d} \tau \\
& {\left.\left[R\left(x_{k}\right)\right]\left[R\left(x_{k-1}\right)\right] \cdots\left[R\left(x_{1}\right)\right] \boldsymbol{b}\left(t_{0}\right)+\cdots\right] }
\end{aligned}
$$

with

$$
b_{k}\left(t_{0}\right)=0 \quad \text { if } \quad h \neq i, b_{k}(t)=0 \quad \text { if } \quad k \neq f .
$$

The difference between the old-fashioned perturbation theory and the kernel function formalism is just the following: In the former we perform the space integration first and the time integration subsequently while in the four dimensional formalism, the integration is performed over all the four variables simultaneously.

\section{Old fashioned perturbation theory}

We shall obtain the probability per unit time that a particle makes a transition from the state $i$ to the state $f$ under a perturbation $H^{\prime}$. Taking the first order term in the expansion we have

$$
\left\langle f\left|S^{(1)}\right| i\right\rangle=\lim _{\substack{t \rightarrow+\infty \\ t_{0} \rightarrow-\infty}}(-i) \int_{t_{0}}^{t} \mathrm{~d} \tau_{1} \int_{-\infty}^{\infty} \mathrm{d}^{3} x_{1} \psi_{f}^{*}\left(x_{1}\right) H^{\prime}\left(x_{1}\right) \psi_{i}\left(x_{1}\right) .
$$

Assuming $\psi_{f}$ and $\psi_{i}$ are free particle eigenstates of energy we can write them as

$$
\psi_{f}=\varphi_{f} \mathrm{e}^{-i E_{p_{f}} t}, \quad \psi_{i}=\varphi_{i} \mathrm{e}^{-i E_{p_{i}} t}
$$

where $\boldsymbol{p}_{\boldsymbol{i}}$ and $\boldsymbol{p}_{f}$ are initial and final momenta of the particle. Let us also assume that $H^{\prime}(x)$ can be written as $H^{\prime}(x) \mathrm{e}^{ \pm i q_{4} t}$, i.e. using $-i q_{4} t$ in the exponent when the interaction imparts an energy $q_{4}$ and $+i q_{4} t$ when it takes away an energy $q_{4}$ from the particle. Thus we write

where

$$
b_{f i}=H_{f i}^{\prime}(-i) \int_{t_{0}}^{t} \mathrm{e}^{-i E_{p j} t} \mathrm{e}^{ \pm i q_{4} t} \mathrm{e}^{i E_{p i} t} \mathrm{~d} t
$$

$$
H_{f i}^{\prime}=\int \varphi_{f}^{*}(\boldsymbol{x}) H^{\prime}(\boldsymbol{x}) \varphi_{i}(\boldsymbol{x}) \mathrm{d}^{3} x
$$


i.e. we are performing the space integration first and subsequently the time integration. For performing the space integration, we first notice that the space dependence of $\varphi_{i}$ and $\varphi_{f}$ occur only through the factors $\mathrm{e}^{i \boldsymbol{p}_{\boldsymbol{i}} \cdot \boldsymbol{x}}$ and $\mathrm{e}^{\boldsymbol{i} \boldsymbol{p}_{f} \cdot \boldsymbol{x}}$ respectively. Let us assume therefore that

i.e.

$$
\varphi_{i}=\mathrm{e}^{+\boldsymbol{i}_{\boldsymbol{i}} \cdot \boldsymbol{x}}, \quad H^{\prime}(\boldsymbol{x})=\mathrm{e}^{+i \boldsymbol{q} \cdot \boldsymbol{x}} ; \quad \varphi_{f}=\mathrm{e}^{+i \boldsymbol{p}_{f} \cdot \boldsymbol{x}},
$$

$$
\psi_{i}=\mathrm{e}^{-i p_{i} \cdot x}, \quad H^{\prime}(x)=\mathrm{e}^{-i q \cdot x} ; \quad \psi_{f}=\mathrm{e}^{-i p_{f} \cdot x},
$$

ignoring the normalization factors which are not relevant for the present discussion. The fact that we take $H^{\prime}(x)=\mathrm{e}^{-i q \cdot x}$ implies the absorption of four momentum $q$ with $q_{4}$ the energy positive (i.e. we are considering a first order electrodynamic interaction of an incident photon and an electron). If on the other hand, we are considering a first order emission (i.e. when there is only an incoming electron) we write $H^{\prime}(x)=\mathrm{e}^{i q \cdot x}$. Considering the case of absorption and integrating with respect to space in $H_{f i}^{\prime}$ we obtain as a factor the $\delta$-function $(2 \pi)^{3} \delta\left(\boldsymbol{p}_{f}-\boldsymbol{p}_{i}-\boldsymbol{q}\right)$ and with respect to time we obtain the factor $2 \pi \delta\left(E_{p_{f}}-E_{q}-E_{p_{i}}\right)$. This implies that the matrix element will vanish unless

$$
\boldsymbol{p}_{f}=\boldsymbol{p}_{i}+\boldsymbol{q} \text { and } E_{p_{f}}=E_{q}+E_{p_{i}} \text {. }
$$

It is easy to see that the second condition, i.e. the energy conservation, cannot be satisfied if we assume the first. In other words if we add the two four momenta $p_{i}$ and $q$ we obtain a four momentum which does not satisfy the energy momentum relation. Thus there cannot be a first order process in which an electron can absorb a photon. In a similar manner we find that there cannot be a first order emission of a photon also.

However if the time component $q_{4}$ of $q$ or more generally of $H^{\prime}(t)$ is zero, the energy momentum relation for $p_{i}+q$ still holds. An electromagnetic potential which is only a function of space and is independent of time can be written as

$$
\begin{aligned}
A(x) & =\int a(q) \delta\left(q_{4}\right) \mathrm{e}^{-i q \cdot x} \mathrm{~d}^{4} q \\
& =\int a(\boldsymbol{q}) \mathrm{e}^{i \boldsymbol{q} \cdot x} \mathrm{~d}^{3} q .
\end{aligned}
$$

This implies that a potential is a superposition of time indpendent waves with $q_{4}=0$ and in the interaction the particle can acquire a momentum $\boldsymbol{q}$ with an amplitude $a(\boldsymbol{q})$. Given $p_{f}$ and $p_{i}$ we can choose a $q$ such that $\boldsymbol{p}_{f}-\boldsymbol{p}_{i}=\boldsymbol{q}$ (with $q_{4}=0$ ) i.e. a first order scattering in an electromagnetic potential is possible. Taking $\psi_{i}$ and $\psi_{f}$ as given in equation (26) and $H^{\prime}$ as $A(x)$ the matrix element for the first order process will be proportional to $a(q)$.

At this stage it is necessary to explain the meaning of the initial and final states in the old-fashioned perturbation theory. In the case of absorption, the initial system consists of an electron and a photon and the initial state $i$ refers to this system. The final system is just the electron and the final state refers to it. The energy of the initial system is therefore given by $E_{i}=E_{q}+E_{p_{i}}$ and the energy of the final system is $E_{f}=E_{p_{f}}$. If on the other hand, there is an emission, the 
initial system consists of just an electron and the final system of an electron and a photon. In such a case we have

$$
E_{i}=E_{p_{i}} \text { and } E_{f}=E_{p_{f}}+E_{q} .
$$

In the above discussion about energy-momentum conservation we have represented the eigenstates of energy by simple wave functions of the type $\mathrm{e}^{-i p \cdot x}$. The arguments are valid where the wave function of a particle has more than one component (as in the case of spinors) since for our argument it suffices that any eigenfunction of energy must be written in the form

$$
\psi_{i}(t)=\varphi_{i} \mathrm{e}^{-i E_{p} t},
$$

i.e. the only dependence on time is through $\mathrm{e}^{-i E_{p} t}$. It will be convenient to write the first order matrix element as

$$
\left\langle f\left|S^{(1)}\right| i\right\rangle=-2 \pi i \delta\left(E_{f}-E_{i}\right) H_{f i}
$$

In a wave function formalism dealing with the interaction of an electron with an electromagnetic field the perturbation term itself contains the wave function of the photon (or the electromagnetic field) which is part of the system before the interaction if the perturbation represents an absorption, and is part of the system after the interaction when it represents an emission. If we use the notation $|i\rangle$ for the initial system it cannot be strictly identified with $\boldsymbol{b}\left(t_{0}\right)$ with all except its $i$ th element zero, for the latter represents the state of the electron. This difficulty will not arise as we shall see in a field theoretical formalism where the state at $t$ refers to the entire system at $t$ and the interaction represents operators which cause transitions in these states. In the Feynman formalism, the states always refer to the electron and the inequality of the energy between the states before and after the interaction is imbedded in the concept of the virtual state of the electron.

Thus for the sake of uniformity of notation with the field theoretical formalism we shall write $\langle f|S| i\rangle$ for $b_{f i}$ and $\left\langle f\left|H^{\prime}\right| i\right\rangle$ for $H_{f i}^{\prime}$. Thus

$$
\left\langle f\left|S^{(1)}\right| i\right\rangle=-\left\langle f\left|H^{\prime}\right| i\right\rangle 2 \pi i \delta\left(E_{f}-E_{i}\right) .
$$

When the time component of $H^{\prime}(t)$ is not zero, the first order interaction vanishes and we take the second order interaction

$$
\begin{aligned}
&\left\langle f\left|S^{(2)}\right| i\right\rangle=(-i)^{2} \sum_{m} \int_{t_{0}}^{t} \mathrm{~d} \tau_{2} \int_{t_{0}}^{\tau_{2}} \mathrm{~d} \tau_{1} \int_{-\infty}^{\infty} \mathrm{d}^{3} x_{2} \int_{-\infty}^{\infty} \mathrm{d}^{3} x_{1} \psi_{f}^{*}\left(x_{2}\right) H^{\prime}\left(x_{2}\right) \psi_{m}\left(x_{2}\right) \\
&=\left.(-i)^{2} \sum_{m}\langle f| x_{1}\right) H^{\prime}\left(x_{1}\right) \psi_{i}\left(x_{1}\right) \\
& \int_{t_{0}}^{t} \mathrm{~d} \tau_{2} \int_{t_{0}}^{\tau_{2}} \mathrm{e}^{-i\left(E_{m}-E_{f}\right) \tau_{2}-i\left(E_{i}-E_{m}\right) \tau_{1}, \mathrm{~d} \tau_{1},} \\
& t_{0} \rightarrow-\infty, \quad t \rightarrow+\infty
\end{aligned}
$$


the summation over $m$ implying summation (or integration) over all possible intermediate states.

In the case of the first order matrix element we have made use of the lemma

$$
\lim _{\substack{t \rightarrow+\infty \\ t_{0} \rightarrow-\infty}} \int_{t_{0}}^{t} \mathrm{e}^{i\left(E_{f}-E_{i}\right) \tau} \mathrm{d} \tau=2 \pi \delta\left(E_{f}-E_{i}\right) .
$$

In the second order matrix element we find that we have first to integrate with respect to the intermediate variable $\tau_{1}$ between the limits $\tau_{2}$ and $t_{0}$ and let only $t_{0}$ tend to $-\infty$. To evaluate this integral we adopt the following device. We multiply the integrand by $\mathrm{e}^{\varepsilon \tau_{1}}$ where $\varepsilon$ is a positive infinitesimal quantity which can be made as small as we please, i.e. $\varepsilon \rightarrow 0$. Keeping $\varepsilon$ non-zero and performing the integration we evaluate the integral as

$$
\lim _{\varepsilon \rightarrow 0} \frac{\mathrm{e}^{i\left(E_{m}-E_{i}\right) \tau_{2}}}{-i\left(E_{i}-E_{m}+i \varepsilon\right)} .
$$

The second integration over $\tau_{2}$ yields the delta function $\delta\left(E_{f}-E_{i}\right)$ i.e. $f$ should be on the energy shell. It is not necessary however that $E_{m}$ should be equal to $E_{i}$, i.e. the summation over $E_{m}$ will include values both off and on the energy shell $E=E_{i}$. The separation into these two kinds of intermediate states will by made presently. Writing $\psi_{i}\left(x_{1}\right)$ and $\psi_{f}\left(x_{2}\right)$ as $\mathrm{e}^{-i p_{i} \cdot x_{1}}$ and $\mathrm{e}^{-i p_{f} \cdot x_{2}}$ respectively and taking $H^{\prime}\left(x_{1}\right)=\mathrm{e}^{-i q_{1} \cdot x_{1}} ; H^{\prime}\left(x_{2}\right)=\mathrm{e}^{i q_{2} \cdot x_{2}}$ corresponding to the absorption and emission of a photon, we have

$$
\begin{aligned}
\left\langle f\left|S^{(2)}\right| i\right\rangle= & (-i)^{2} \sum_{m} \int_{t_{0}}^{t} \mathrm{~d} \tau_{2} \int_{t_{0}}^{\tau_{2}} \mathrm{~d} \tau_{1} \int_{-\infty}^{\infty} \mathrm{d}^{3} x_{1} \int_{-\infty}^{\infty} \mathrm{d}^{3} x_{2} \mathrm{e}^{i p_{j} \cdot x_{1}} \mathrm{e}^{i q_{2} \cdot x_{2}} \\
& \mathrm{e}^{-i p_{m} \cdot x_{2}} \mathrm{e}^{i p_{m} \cdot x_{1}} \mathrm{e}^{-i q_{1} \cdot x_{1}} \mathrm{e}^{-i p_{i} \cdot x_{1}}
\end{aligned}
$$

as

$$
t \rightarrow+\infty t_{0} \rightarrow-\infty
$$

where each of the four vectors $p$ and $q$ satisfy the correct energy momentum relation. The space integration leads to the conservation of momentum (i.e.)

$$
\boldsymbol{p}_{m}=\boldsymbol{p}_{i}+\boldsymbol{q}_{1} ; \boldsymbol{p}_{f}+\boldsymbol{q}_{2}=\boldsymbol{p}_{m} .
$$

As mentioned before, the initial system in this case consists of an electron of four momentum $p_{i}$ and a photon of four momentum $q_{1}$, (i.e.) the energy of the initial system is given by

$$
E_{i}=E_{p_{i}}+E_{q_{1}}
$$

The intermediate state is that of a single electron with momentum $\boldsymbol{p}_{\boldsymbol{i}}+\boldsymbol{q}_{\mathbf{1}}$ and energy $E_{m}=E_{p_{i}+q_{1}}$. The final state is that of an electron of four momentum $p_{f}$ and a photon of four momentum $q_{2}$ and has energy $E_{f}=E_{p_{f}}+E q_{2}$. While $E_{f}=E_{i}$ the energy $E_{m}$ of the intermediate state is not equal to $E_{i}$ or $E_{f}$, i.e. energy is not conserved in the intermediate state though momentum is conserved.

In any physical process, when we consider the absorption of a real photon of four momentum $q_{1}$, it is obvious that this should form part of the initial system 
and therefore $q_{1}$ and $p_{i}$ are given. Hence for a required $p_{f}$ we find $q_{2}$ is determined and hence the summation over the intermediate state implies no summation over values of $q_{1}$ or $q_{2}$. However since we are interested only in the emission of a photon $q_{2}$ and the absorption of a photon $q_{1}$ but not in their relative sequence, we could have taken the emission of the photon $q_{2}$ at $x_{1}$ and the absorption of the photon $q_{1}$ at $x_{2}$. In this case the initial system is an electron of four momentum $p_{i}$ and a photon of four momentum $q_{1}$. The intermediate state refers to three particles viz., the electron of momentum $\boldsymbol{p}_{i}-\boldsymbol{q}_{2}$ and energy $E_{p^{\prime}{ }_{m}}=E_{\left(p_{i}-q_{2}\right)}$ a photon of four momentum $q_{1}$ and a photon of four momentum $q_{2}$. The final state consists of an electron of four momentum $p_{f}$ and a photon of four momentum $q_{2}$. In this case we have the contribution

$$
\lim _{\substack{t \rightarrow+\infty \\ t_{0} \rightarrow-\infty}}(-i)^{2} \sum_{m} H_{f m}^{\prime} H_{m i}^{\prime} \int_{t_{0}}^{t} \mathrm{~d} \tau_{2} \int_{t_{0}}^{\tau_{2}} \mathrm{~d} \tau_{1} \mathrm{e}^{i\left(E_{f}-E_{i}\right) \tau_{2}} \mathrm{e}^{i\left(E_{i}-E_{m}\right)\left(\tau_{2}-\tau_{1}\right)}
$$

with

$$
\begin{gathered}
E_{f}=E_{p_{f}}+E_{q_{2}} ; E_{i}=E_{p_{i}}+E_{q_{1}} \\
E_{m}=E_{p_{i}-q_{2}}+E_{q_{1}}+E_{q_{2}} .
\end{gathered}
$$

$\left\langle f\left|S^{(2)}\right| i\right\rangle$ gets contribution from both these cases and hence we write the matrix element as the sum over all possible intermediate states.

Thus we have here only two possible intermediate states corresponding to the two possible sequences in which the absorption and emission can occur. More generally the summation should be interpreted as including summations or integrations over various parameters characterizing the intermediate states.

The above considerations can be generalized in exactly the same manner to the $m$ th order process. We write the matrix element in such a case as

$$
\begin{aligned}
\left\langle f\left|S^{(n)}\right| i\right\rangle= & -2 \pi i \delta\left(E_{f}-E_{i}\right) \\
& \sum \frac{\left\langle f\left|H^{\prime}\right| m_{1}\right\rangle\left\langle m_{1}\left|H^{\prime}\right| m_{2}\right\rangle \cdots\left\langle m_{n-1}\left|H^{\prime}\right| i\right\rangle}{\left(E_{i}-E_{m_{1}}+i \varepsilon\right)\left(E_{i}-E_{m_{2}}+i \varepsilon\right) \ldots\left(E_{i}-E_{m_{n-1}}+i \varepsilon\right)}
\end{aligned}
$$

Defining $\left\langle f\left|T^{n}\right| i\right\rangle$ through

and writing

we note that

$$
\left\langle f\left|S^{(n)}\right| i\right\rangle=-2 \pi i \delta\left(E_{f}-E_{i}\right)\left\langle f\left|T^{(n)}\right| i\right\rangle
$$

$$
T=\Sigma T^{(n)}
$$

or

where

In the definition of

$$
S=1-2 \pi i \delta\left(E_{f}-E_{i}\right) T
$$

$$
S=1+\boldsymbol{T}
$$

$$
\boldsymbol{T}=-2 \pi i \delta\left(E_{f}-E_{i}\right) T
$$

$$
\left\langle f\left|S^{(n)}\right| i\right\rangle \quad \text { or } \quad\left\langle f\left|T^{(n)}\right| i\right\rangle
$$

we have stated that the intermediate states have to be summed over states both off and on the energy shell, i.e. it is implied that if we take any typical factor 
$E_{i}-E_{m}+i \varepsilon$ in the denominator and replace $E_{m}-i \varepsilon$ by a complex variable, we are integrating over the real axis passing through the singularity at $E_{i}$ (when $\varepsilon \rightarrow 0$ ). This is equivalent to writing

$$
\frac{1}{E_{i}-E_{m}+i \varepsilon}=\frac{P}{E_{i}-E_{m}}-i \pi \delta\left(E_{i}-E_{m}\right) .
$$

In the first part the integration over $m$ has to be understood in the sense of Cauchy-principle value, i.e. omitting the contribution from the immediate neighbourhood of $E_{i}$. The second part represents the contribution from the integration along a small semi-circle round the singularity $E_{i}$ when the radius tends to zero. In other words, in the first part the energy $E_{m}$ is off the energy shell while in the second term it is on the shell. We give here a very simple argument by which a relation can be established between $\langle f|T| i\rangle$ and the part $\langle f|K| i\rangle$ which does not involve an intermediate state on the energy shell.

The matrix element $\langle f|T| i\rangle$ must be a sum of two mutually exclusive parts: one involving no intermediate state on the energy shell, the other involving at least one intermediate state on the energy shell. The first is by definition $\langle f|K| i\rangle$. In computing the second let $\left|f_{\alpha}\right\rangle$ be the "last" among the intermediate states on the energy shell in following the sequence from $|i\rangle$ to $|f\rangle$ i.e. the state on the energy shell next to the final state itself. We can speak of such a sequence even in the strictest sense before performing the time integration. The transition from $|i\rangle$ to $\left|f_{\alpha}\right\rangle$ proceeds through $T$ while that from $\left|f_{\alpha}\right\rangle$ to $|f\rangle$ proceeds through $K$. Thus we can immediately write

$$
\langle f|T| i\rangle=\langle f|K| i\rangle-i \pi \int_{f_{\alpha}}\left\langle f|K| f_{\alpha}\right\rangle\left\langle f_{\alpha}|T| i\right\rangle
$$

the factor $i \pi$ occuring due to integration over the $\delta$-function part (since $\left|f_{\alpha}\right\rangle$ is on the energy shell) of $1 /\left(E_{i}-E_{f_{\alpha}}+i \varepsilon\right)$. The above relation can also be obtained by a systematic substitution for each $1 /\left(E_{i}-E_{m}+i \varepsilon\right)$ in terms of the off and on the energy shell components and suitable rearrangements.

We shall now obtain a similar integral equation for $\langle m|T| i\rangle$ where $|m\rangle$ (unlike $|f\rangle$ ) is not necessarily on the energy shell $E_{i}$. The above equation in principle is valid when we replace $|f\rangle$ by $|m\rangle$ but as it is customary to define the $K$-matrix connecting states on the energy shell, we shall obtain $\langle m|T| i\rangle$ in terms of $\langle l|H| k\rangle$ where $|l\rangle$ and $|k\rangle$ are not necessarily on the energy shell. We use the following argument.

Either (i) a transition occurs directly from $|i\rangle$ to $|m\rangle$ and the amplitude for this is $\langle m|H| i\rangle$ or (ii) there is at least one intermediate state. Let the "last" intermediate state (next to $m$ ) be $|l\rangle$. The transition.from $|l\rangle$ to $|m\rangle$ is through $H^{\prime}$ and from $|i\rangle$ to $|l\rangle$ through $T$. Thus we have

$$
\begin{aligned}
\langle m|T| i\rangle= & \left\langle m\left|H^{\prime}\right| i\right\rangle+\sum \frac{1}{E_{i}-E_{l}+i \varepsilon}\left\langle m\left|H^{\prime}\right| l\right\rangle\langle l|T| i\rangle \\
= & \left\langle m\left|H^{\prime}\right| i\right\rangle+\sum_{l} \frac{\left\langle m\left|H^{\prime}\right| l\right\rangle\langle l|T| i\rangle}{E_{i}-E_{l}} \\
& -i \pi \int_{f^{\prime}}\left\langle m\left|H^{1}\right| f^{\prime}\right\rangle\left\langle f^{\prime}|T| i\right\rangle
\end{aligned}
$$


where if the "last" intermediate state is on the energy shell we denote it by $\left|f^{\prime}\right\rangle$ or else by $|l\rangle$.

The connection between matrix elements of the type $\langle f|T| i\rangle$ where $f$ is on the energy shell $E_{i}$ and $\langle m|T| i\rangle$. where $|m\rangle$ is not necessarily on the shell $E_{i}$ can also be seen by a mere examination of the expansion $\langle f|T| i\rangle$. Taking the expression for $\left\langle f\left|T^{(n)}\right| i\right\rangle$ we can write it as

$$
\begin{aligned}
& \left\langle f\left|T^{(n)}\right| i\right\rangle=\sum_{m} \frac{\left\langle f\left|H^{\prime}\right| m\right\rangle\left\langle m\left|T^{(n-1)}\right| i\right\rangle}{\left(E_{i}-E_{m}+i \varepsilon\right)}(n>1) \\
& \left\langle f\left|T^{(1)}\right| i\right\rangle=\left\langle f\left|H^{\prime}\right| i\right\rangle
\end{aligned}
$$

or

$$
\begin{aligned}
\langle f|T| i\rangle & =\left\langle f\left|H^{\prime}\right| i\right\rangle+\sum_{m} \frac{\left\langle f\left|H^{\prime}\right| m\right\rangle\langle m|T| i\rangle}{E_{i}-E_{m}+i \varepsilon} \\
& =\left\langle f\left|H^{\prime}\right| \psi_{i}^{(+)}\right\rangle
\end{aligned}
$$

where we define

$$
\psi_{i}^{(+)}=\sum_{m} \frac{|m\rangle\langle m|T| i\rangle}{E_{i}-E_{m}+i \varepsilon}+|i\rangle .
$$

We thus have

and recalling that

$$
T|i\rangle=H \psi_{i}^{(+)}
$$

$$
T|i\rangle=H^{1}|i\rangle+H^{1} \frac{1}{E-H_{0}+i \varepsilon} T|i\rangle
$$

we can write

$$
\psi_{i}^{(+)}=|i\rangle+\frac{1}{E_{i}-H_{0}+i \varepsilon} H^{1} \psi_{i}^{(+)}
$$

We can also establish the connection between the matrix elements $\langle m|T| i\rangle$ and $\langle f|T| i\rangle$ in another manner by just observing that we can also write

$$
\left\langle f\left|T^{n}\right| i\right\rangle=\sum_{m} \frac{\left\langle f\left|T^{(n-1)}\right| m\right\rangle\left\langle m\left|H^{\prime}\right| i\right\rangle}{E_{i}-E_{m}+i \varepsilon}
$$

with

$$
\left\langle f\left|T^{1}\right| i\right\rangle=\left\langle f\left|H^{\prime}\right| i\right\rangle
$$

or

$$
\begin{aligned}
\langle f|T| i\rangle & =\left\langle f\left|H^{1}\right| i\right\rangle+\sum_{m} \frac{\langle f|T| m\rangle\left\langle m\left|H^{\prime}\right| i\right\rangle}{E_{i}-E_{m}+i \varepsilon} \\
& =\left\langle\psi_{f}^{(-)}\left|H^{\prime}\right| i\right\rangle
\end{aligned}
$$

where

$$
\left\langle\psi_{f}^{(-)}\right|=\sum_{m} \frac{\langle f|T| m\rangle\langle m|}{E_{f}-E_{m}+i \varepsilon}+\langle f|,
$$

i.e.

$$
\begin{aligned}
\left|\psi_{f}^{(-)}\right\rangle & =|f\rangle+\sum_{m} \frac{\langle m|T| f\rangle|m\rangle}{E_{f}-E_{m}-i \varepsilon} \\
& =|f\rangle+\frac{1}{E_{f}-H_{0}-i \varepsilon} H^{\prime}\left|\psi_{f}^{(-)}\right\rangle .
\end{aligned}
$$


We shall now examine the meaning of $\psi^{(+)}$and $\psi^{(-)}$solutions in greater detail. This we can do by remembering that the matrix element $\langle f|S| i\rangle$ on the energy shell was computed by first obtaining $b_{f i}\left(t, t_{0}\right)$ as an infinite series expansion for finite $t$ and $t_{0}$ and passing to the limit as $t \rightarrow \infty$ and $t_{0} \rightarrow-\infty$. For applying this limiting process it is only natural to assume $t>t_{0}$ and obtain $b_{f i}\left(t, t_{0}\right)$ as the element of the vector $\boldsymbol{b}(t)$ satisfying (13) with the "initial" condition that $\boldsymbol{b}\left(t_{0}\right)$ is a vector with all elements zero except the $i$-th which is unity and represents the amplitude for the system being in $|i\rangle$. But we know that the solution for $\boldsymbol{b}(t)$ is meaningful even for $t<t_{0}$ or in other words writing $\boldsymbol{b}(t)=U\left(t, t_{0}\right) \boldsymbol{b}\left(t_{0}\right)$ the transformation matrix $U\left(t, t_{0}\right)$ exists for $t \gtrless t_{0}$ as has been elaborated earlier.

It is found convenient to divide the solution for $\boldsymbol{b}(t)$ into two classes.

$$
\begin{aligned}
& \boldsymbol{b}^{+}(t) \text { given } \boldsymbol{b}\left(t_{0}\right) \text { when } t>t_{0}, t_{0} \rightarrow-\infty \\
& \boldsymbol{b}^{-}(t) \text { given } \boldsymbol{b}\left(t_{0}\right) \text { when } t<t_{0}, t_{0} \rightarrow+\infty .
\end{aligned}
$$

We now write

$$
\begin{aligned}
& \langle f|S| i\rangle=\lim _{\substack{t \rightarrow+\infty \\
t_{0} \rightarrow-\infty}}\left\langle f\left|U\left(t, t_{0}\right)\right| i\right\rangle \\
& \lim _{\substack{t \rightarrow+\infty \\
t_{0} \rightarrow-\infty}}\left\langle f\left|U\left(t, t_{1}\right) U\left(t_{1}, t_{0}\right)\right| i\right\rangle \\
& \lim _{\substack{t \rightarrow+\infty \\
t_{0} \rightarrow-\infty}}\left\langle U\left(t_{1}^{\prime}, t\right) f\left|U\left(t_{1} t_{0}\right)\right| i\right\rangle \\
& =b_{f}^{(-) \dagger}(t) b_{i}^{(+)}(t)
\end{aligned}
$$

where we have used the lemma

$$
U^{\dagger}\left(t_{1}, t\right)=U^{-1}\left(t_{1}, t\right)=U\left(t, t_{1}\right)
$$

noting that in the above definition of both $\boldsymbol{b}^{(+)}$and $\boldsymbol{b}^{(-)}, t$ is kept finite and this has the consequence that the elements of $\boldsymbol{b}_{i}^{(+)}(t)$ are non-zero whether they are off or on the energy shell $E_{i}$ (similarly for $\boldsymbol{b}_{f}^{(-)}(t)$ on the shell $E_{f}$ ). When $t$ is finite in the integral expansion for $\boldsymbol{b}_{i}(t)$ the last integration is performed from $-\infty$ to $t$ hence yielding the factor $\frac{\mathrm{e}^{-i\left(E_{i}-E_{m}\right) t}}{E_{i}-E_{m}+i \varepsilon}$. If we make $t \rightarrow \infty$ we should replace it by $\delta\left(E_{m}-E_{i}\right)$. If we set $t=0$ we note that $\boldsymbol{b}_{i}^{(+)}(0)$ is identical with the $\psi_{i}^{(+)}$ we have defined before. In a similar manner we identify $\boldsymbol{b}_{f}^{(-)}(0)$ with $\psi_{f}^{(-)}$. Thus $\langle f|S| i\rangle$ is also expressed in the form

$$
\langle f|S| i\rangle=\left\langle\psi_{f}^{(-)} \mid \psi_{i}^{(+)}\right\rangle .
$$

We have now to convince ourselves that the above equation is consistent with (52). That this is so, follows directly from the equation (51) for $\psi_{i}^{(+)}$. For if we substitute for $\psi_{i}^{(+)}$in $\left\langle f\left|H^{\prime}\right| \psi_{i}^{(+)}\right\rangle$successively by the expression (56) we obtain the above. 


\section{Kernel function formalism}

We shall now write the integral expansion form of $\langle f|S| i\rangle$ using the kernel function formalism.

In the expression for $\langle f|S| i\rangle$ given above we replace $R_{n m}$ by

$$
\psi_{n}^{*} H^{\prime} \psi_{m}
$$

and a typical term of the expansion (say the $(n+1)$-th) becomes

$$
\begin{gathered}
\lim _{\substack{t \rightarrow+\infty \\
t_{0} \rightarrow-\infty}}(-i)^{n} \int_{-\infty}^{+\infty} \mathrm{d}^{3} x_{n} \ldots \int_{-\infty}^{\infty} \mathrm{d}^{3} x_{1} \int_{t_{0}}^{t} \mathrm{~d} \tau_{n} \ldots \int_{t_{0}}^{\tau_{2}} \mathrm{~d} \tau_{1} \\
\sum \psi_{f}^{*}\left(\boldsymbol{x}_{n}, \tau_{n}\right) H^{\prime}\left(\tau_{n}\right) \psi_{n}\left(\boldsymbol{x}_{n}, \tau_{n}\right) \ldots \\
\ldots \psi_{m}^{*}\left(\boldsymbol{x}_{1} \tau_{1}\right) H^{\prime}\left(\tau_{1}\right) \psi_{i}\left(\boldsymbol{x}_{1} \tau_{1}\right)
\end{gathered}
$$

where $\tau_{i}$ are ordered from $t_{0}$ to $t$. If we define the function

$$
K(2,1)=\sum_{m} \psi_{m}(2) \psi_{m}^{*}(1)
$$

where (1) and (2) refer to the space time points $\left(x_{1}, \tau_{1}\right)$ and $\left(\boldsymbol{x}_{2}, \tau_{2}\right)$, the above expression is written as

$$
\begin{gathered}
\lim _{\substack{t \rightarrow+\infty \\
t_{0} \rightarrow-\infty}}(-i)^{n} \int_{t_{0}}^{t} \mathrm{~d} \tau_{n} \ldots \int_{t_{0}}^{\tau_{2}} \mathrm{~d} \tau_{1} \int_{-\infty}^{\infty} \mathrm{d}^{3} x_{n} \ldots \int_{-\infty}^{\infty} \mathrm{d}^{3} x_{1} \\
\psi_{f}^{*}(n) H^{\prime}(n) K(n, n-1) H^{\prime}(n-1) \ldots H^{\prime}(2) K(2,1) H^{\prime}(1) \psi_{i}(1) .
\end{gathered}
$$

$K(2,1)$ as defined in $(68)$ is valid for all points and satisfies the homogeneous equation

$$
i \frac{\partial}{\partial t_{2}} K(2,1)-H(2) K(2,1)=0
$$

but in the expansion, since time is ordered $K(2,1)$ occurs with $t_{2}>t_{1}$. We can remove the restriction on time ordering if we define a kernel $K_{0}(2,1)$ which is such that $K_{0}(2,1) \equiv K(2,1)$ for $t_{2}>t_{1}$ and $K_{0}(2,1)=0$ if $t_{2}<t_{1} . K_{0}$ then satisfies the inhomogenous equation

$$
i \frac{\partial}{\partial t_{2}} K_{0}(2,1)-H(2) K_{0}(2,1)=\delta(2,1) .
$$

But there is nothing in the Schrödinger equation itself to imply that its solution should not be valid for $t_{2}<t_{1}$. If in our formulation we take care to order our time in the integral, it is immaterial whether we take $K(2,1)$ or $K_{0}(2,1)$. The physical interpretation of the terms in the expansion can be had if we imagine that the perturbation acts at space-time points $1,2, \ldots$, and the "motion" of the particle is represented by a propagator in between. These four-dimensional "vertex" points at which perturbations act are integrated out. Thus $K_{0}$ is the 
same as Feynman's kernel, the important difference being that our kernel $K$ obeys the homogenous equation.

This formalism of perturbation theory is called non-relativistic though all the expressions used here are four dimensional. This is perhaps due to the fact that the $\psi$ functions used here are solutions of the non-relativistic equations. We shall be discussing the kernel function formalism for the Dirac equation in the next section.

We have till now given the prescription for the modes of calculation of $\langle f|S| i\rangle$ and hence the probability of transition from a state $i$ to a state $f$ in the interval $t-t_{0}$ as $t \rightarrow+\infty$ and $t_{0} \rightarrow-\infty$. But the physically interesting quantity is the transition per unit time into a small interval $\Delta E$ in the neighbourhood of this final state $E_{f}$. If we take the density of final states to be $\varrho(E) \mathrm{d} E$ then the transition probability for the infinite interval is given by

$$
\omega=|\langle f|S| i\rangle|^{2} \varrho(E) \mathrm{d} E .
$$

We have seen that $\langle f|S| i\rangle$ can be written as

$$
\left.\langle f|S| i\rangle=-2 \pi i \delta\left(E_{f}-E_{i}\right)<f_{\|}|T| i\right\rangle,
$$

i.e. we have separated out the delta function singularity. Thus

$$
|\langle f|S| i\rangle|^{2}=\left.4 \pi^{2}\left\{\delta\left(E_{f}-E_{i}\right)\right\}^{2}\langle f|T| i\rangle\right|^{2}
$$

and the transition probability is given by

$$
\omega=4 \pi^{2}\left\{\delta\left(E_{f}-E_{i}\right)\right\}^{2}\langle f|T| i\rangle \varrho(E) \mathrm{d} E .
$$

We can interpert the term $\left\{\delta\left(E_{f}-E_{i}\right)\right\}^{2}$ as follows :

$$
\begin{aligned}
\left\{\delta\left(E_{f}-E_{i}\right)\right\}^{2} & =\delta\left(E_{f}-E_{i}\right) \lim _{t-t_{0} \rightarrow \infty} \frac{1}{2 \pi} \int_{t_{0}}^{t} \mathrm{e}^{i\left(E_{i}-E_{f}\right)\left(t-t_{0}\right)} \mathrm{d}\left(t-t_{0}\right) \\
& =\frac{\delta\left(E_{f}-E_{i}\right)}{2 \pi} \lim _{t-t_{0} \rightarrow \infty} \int_{t_{0}}^{t} \mathrm{~d} \tau \\
& =\frac{\delta\left(E_{f}-E_{i}\right)}{2 \pi}\left(t-t_{0}\right) \lim t-t_{0} \rightarrow \infty
\end{aligned}
$$

Thus the transition probability during the time interval $t-t_{0}$ as $t \rightarrow+\infty$ and $t_{0} \rightarrow-\infty$ is infinite. However what we are interested in is the transition probability per unit time which is given by

$$
\frac{|\langle f|S| i\rangle|^{2} f(E) \mathrm{d} E}{t-t_{0}}=2 \pi \delta\left(E_{f}-E_{i}\right)|\langle f|T| i\rangle|^{2} \varrho(E) \mathrm{d} E .
$$




\section{PERTURBATION THEORY OF THE DIRAC ELECTRON'}

\section{Feynman kernel and its momentum representation}

We shall discuss a perturbation method of obtaining the solution of the Dirac equation

$$
i \frac{\partial \psi}{\partial t}=\left(H_{0}+H^{\prime}\right) \psi
$$

where $H_{0}$ is the free particle Dirac Hamiltonian $(\boldsymbol{\alpha} \cdot \boldsymbol{p}+\beta m)$ and $H^{\prime}$ is the interaction term which we are going to treat as a perturbation. If the interaction term is due to an electromagnetic field, $H^{\prime}$ is identified with $e(\boldsymbol{\alpha} \cdot \boldsymbol{A}+\beta \varphi)$ where $\boldsymbol{A}$ and $\varphi$ are the vector and scalar potentials of the electromagnetic field. Alternatively the Dirac equation for a free particle perturbed by an electromagnetic field can be written as

We identify

$$
\begin{gathered}
(\mathrm{p}-e \mathrm{~A}-m) \psi=0 . \\
H^{\prime}=e \beta \mathrm{A} .
\end{gathered}
$$

The extension of the kernel function formalism to the Dirac equation is made in the first instance by replacing the single component wave function by the Dirac wave function and representing the interaction term $H^{\prime}$ by $e \beta \mathrm{A}$. Since it is customary to use for the interaction $e \mathbf{A}$, the matrix $\beta$ is attached to the kernel which is defined as:

$$
\begin{gathered}
K_{0}(2,1)=\sum_{m} \psi_{m}(2) \psi_{m}^{\dagger}(1) \beta=\sum_{m, E 〉 0} \psi_{m}^{(2)} \bar{\psi}_{m}(1) \text { for } t_{2}>t_{1} \\
=0 \text { for } t_{2}<t_{1}
\end{gathered}
$$

where the sum $m$ extends over both positive and negative energy eigenstates. The kernel satisfies the inhomogeneous equation

$$
(i \nabla-m) K_{0}(2,1)=i \delta^{4}(2,1)
$$

where $\delta^{4}(2,1)$ inplies a Dirac delta function in both space and time variables. The kernel $K_{0}(2,1)$ is a $4 \times 4$ matrix since the $\psi$ 's have four components. Such a definition of the kernel leads to a difficulty not met with in the Schrödinger case. This arises from the fact that the Dirac equation being relativistic, there exists a negative energy solution corresponding to each positive energy solution. Any free particle solution $\psi(2)$ at time $t_{2}$ can be obtained from the wave function at $t_{1}$,

$$
\psi(2)=\int K_{0}(2,1) \beta \psi(1) \mathrm{d}^{3} x_{1} .
$$

This means that if we have a wave function with positive energy at $t_{1}$, it will be propagated to $t_{2}$ with positive energy. The same applies to an initial negative

1 R. P. Feynman, Phys. Rev. 76, 749 and 769 (1949). See also for example, the semiexpository paper by ALLADI RAMAKRISHNAN and R. VASUDEVAN on "Perturbation Expansions and Kernel functions associated with single particle wave functions", Proceedings of the Summer School of Theoretical Physics, Musoorie, India (1959).

EPOR 5 
energy state. If we postulate only the existence of positive energy particles, the presence of negative energy wave functions in the kernel does not lead to difficulties when there is no perturbation since the particle continues to be in the positive energy state if once it is in a positive energy state. However in the presence of interactions, a transition to a negative energy state can take place and since the negative energy states are also propagated, we now have a finite probability of realising a negative energy state at a later time. This is physically impossible. The way out of this difficulty was found by Feynman who defined a modified kernel as follows:

$$
\begin{aligned}
& K_{F}(2,1)=\sum_{m, E^{\prime}>0} \psi_{m}^{(2)} \psi_{m}^{\dagger(1)} \quad \text { for } \quad t_{2}>t_{1} \\
& =-\sum_{m, E<0} \psi_{m}^{(2)} \psi_{m}^{\dagger(1)} \text { for } t_{2}<t_{1} .
\end{aligned}
$$

This kernel was obtained by Feynman by imposing two requirements:

(1) Only positive energy states are propagated "forward in time".

(2) The modified kernel should be a solution of the inhomogeneous equation. Both these are satisfied if we remove from $K_{0}(2,1)$ the sum $\sum_{m, E<0} \psi_{m}(2) \bar{\psi}_{m}^{(1)}$, the latter being defined both for positive and negative values of $t$ and is therefore a solution of the homogeneous equation, i.e. with $\delta^{4}(2,1)$ replaced by zero. The negative energy states are propagated "backward in time" but the propagator for $t_{2}<t_{1}$ is obtained not merely by going over from positive to negative energy but by adding a minus sign. This minus sign is closely connected with the Pauli principle as we shall see presently.

We shall now establish the correspondence between negative energy states occurring in the kernel function and the positron formalism of Dirac. As already mentioned in our discussion of the Dirac equation it was postulated by Dirac that all negative energy states are filled and Pauli's exclusion principle forbids a first order transition of a positive energy electron to a negative energy state. 'The vacuum with no observable charge is therefore to be interpreted as the negative energy "sea" of electrons. However if due to a perturbation, one of the electrons in the negative energy state $(-E, \boldsymbol{p})$ jumps to a positive energy state $\left(+E^{\prime}, \boldsymbol{p}^{\prime}\right)$ the hole created by the negative energy electron is to be identified with a positron of positive energy $+E$ and momentum $-\boldsymbol{p}$. Thus in the Dirac theory we deal with only positive energy particles, i.e. electrons and positrons and the above transition amounts to the creation of a pair. The first order process is not physically realisable due to the requirement of simultaneous conservation of energy and momentum. However a second order process is possible if we assume that this hole is occupied by the incident positive energy electron. To describe this in the perturbation expansion we should envisage states with electrons and positrons with their numbers changing at every vertex where the perturbation acts. Since time has to be ordered in the expansion, the above second order process is characterized by a perturbation at $t_{1}$ where a pair is created and at $t_{2}\left(t_{2}>t_{1}\right)$ where the positron is annihilated along with the incident electron. Thus for times 
$t<t_{1}$ we have a single electron, for $t$ between $t_{1}$ and $t_{2}$ a pair, i.e. an electron and a positron besides the incident electron and for $t>t_{2}$ one electron which was created at $t_{1}$ along with the positron. If we insist on the time ordering in the perturbation it is obvious that we cannot directly take over our formalism of single particle wave functions and we will be constrained to use the theory of quantized fields to be described later.

However the use of the kernel function $K_{F}$ of Feynman in the perturbation expansion takes into account all these while retaining the single particle formalism. If at a vertex $(k)$, the electron jumps from a positive energy state to a negative energy state, it is propagated by the kernel $K_{F}$ to the vertex $(k+1)$ with $t_{k+1}<t_{k}$. The transition to a negative energy state at $t_{k}$ corresponds in the Dirac theory to the destruction of a positron which must have been created earlier at $t_{k+1}<t_{k}$. The propagation of a negative energy electron to an earlier point $t_{k+1}$ is equivalent to the propagation of a positive energy electron from $t_{k+1}$ to $t_{k}$. This is realised by noting that the term $E\left(t_{k}-t_{k+1}\right) \equiv|E|\left(t_{k+1}-t_{k}\right)$ if $E$ is negative, the spinors with negative energy electrons being interpreted as the spinors of the positive energy positron. In a somewhat picturesque manner we can say that "an electron going backward in time looks like a positron going forward in time". If two time points $t_{1}, t_{2}$ are represented by $A$ and $B\left(t_{2}<t_{1}\right)$, the state at $B$ of a free electron starting at $A$ and travelling backward in time to $B$ is identical with the state at $A$ of a positron starting from $B$ and travelling forward to $A$.

The occurrence of the negative sign before the sum over negative energy states in the kernel $K_{F}(2,1)$ for $t_{2}<t_{1}$ meets the demand of the hole theory that the amplitude should have its sign reversed in considering the double scattering of an electron with the creation and annihilation of a positron in the intermediate state since such a scattering amounts to an exchange of the incident electron with an electron in the "sea". Thus the requirement of the Pauli principle is met by the use of the Feynman kernel with the negative sign attached to the sum over the negative energy states.

There is an alternative way of convincing ourselves that $K_{F}(2,1)$ should be the correct form of the kernel based upon arguments in extending perturbation theory to the case when solutions are traced back in differential equations. This leads to a kernel which is identical with $K_{F}$ except for the omission of the negative sign and the ascription of the negative sign to the perturbation at the vertex $t_{2}$ if $t_{2}<t_{1}$. Since Feynman's arguments have been accepted as a part of quantum electrodynamics we need not interrupt our discussion of that formulation by these alternative arguments.

The Feynman kernel in configuration representation has no explicit expression except as a sum of an infinite number of terms. However we shall show that the 4-dimensional transform of the kernel is expressible in an elegant operational form and fortunately it is only the transform that is required in calculations.

To realise the significance of this we first indicate how the free particle kernel in momentum representation is obtained. The free particle eigenfunctions $\psi_{n}(x)$ 
are of the form $u_{n}(\boldsymbol{p}) \mathrm{e}^{-i p \cdot x}$ where $u(\boldsymbol{p})$ is the spinor corresponding to momentum $\boldsymbol{p}$ with positive or negative energy and spin up or down. Then the unperturbed propagator is given by

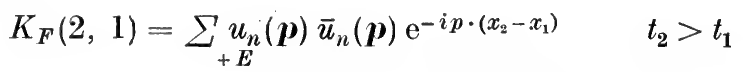

$$
\begin{aligned}
& =-\sum_{-E} u_{n}(\boldsymbol{p}) \bar{u}_{n}(\boldsymbol{p}) \mathrm{e}^{-i p \cdot\left(x_{2}-x_{1}\right)} \quad t_{2}<t_{1} .
\end{aligned}
$$

The summation over $n$ becomes an integral over $p$ (since $E$ and $p$ can take a continuous range of values in the case of a free particle).

$$
\begin{aligned}
K_{F}(2,1) & =\sum_{\text {spin }} \int_{E_{p}>0} \frac{1}{2 E_{p}} u(\boldsymbol{p}) \bar{u}(\boldsymbol{p}) \mathrm{e}^{-i p \cdot x} \mathrm{~d}^{3} p \quad \text { for } \quad t_{2}>t_{1} \\
\text { and } & =-\sum_{\text {spin }} \int_{E_{p}<0} \frac{1}{2 E_{p}} \mathrm{e}^{-i p \cdot x} u(\boldsymbol{p}) \bar{u}(\boldsymbol{p}) \mathrm{d}^{3} p \quad \text { for } \quad t_{2}<t_{1}
\end{aligned}
$$

where we have set $x_{2}-x_{1}=x$ and used the Feynman normalization $\bar{u} u=2 E_{p}$. We note that

$$
\sum_{\text {spin }} u(\boldsymbol{p}) \bar{u}(\boldsymbol{p})=\mathrm{p}+m
$$

for both positive and negative energy spinors.

If we wish to normalize the wave function as $\bar{u}(\boldsymbol{p}) u(\boldsymbol{p})=2\left|E_{p}\right|$ then in the expression for $K_{F}(2,1)$ for $E_{p}<0$ we have to write $2\left|E_{p}\right|$ instead of $2 E_{p}$ in the denominator. But then

$$
\sum_{\text {spin }} u \bar{u}=-(\mathrm{p}+m)
$$

Thus the ratio $\frac{u \bar{u}}{2\left|E_{p}\right|}$ under the normalization $\bar{u} u=2\left|E_{p}\right|$ is identical with the ratio $\frac{u \bar{u}}{2 E_{p}}$ under the normalization $\bar{u} u=2 E_{p}$. We have

Similarly

$$
\begin{aligned}
K_{F}(2,1) & =\int(\mathrm{p}+m) \mathrm{e}^{-i p \cdot x} \frac{1}{2 E_{p}} \frac{\mathrm{d}^{3} p}{(2 \pi)^{3}} \\
& =(i \nabla+m) \int \frac{1}{2 E_{p}} \mathrm{e}^{-i p \cdot x} \frac{\mathrm{d}^{3} p}{(2 \pi)^{3}} \text { for } \begin{array}{r}
t>0 \\
E_{p}>0
\end{array} .
\end{aligned}
$$

$$
K_{F}(2,1)=-(i \nabla+m) \int \frac{1}{2 E_{p}} \mathrm{e}^{-i p \cdot x} \frac{\mathrm{d}^{3} p}{(2 \pi)^{3}} \text { for } \begin{array}{r}
t<0 \\
E_{p}<0
\end{array} .
$$

Equivalently we can write

where

$$
K_{F}(2,1)=(i \nabla+m) \Delta_{F}(x)
$$

$$
\Delta_{F}(x)=\int \frac{1}{2\left|E_{p}\right|} \mathrm{e}^{-i E_{p} t+i \boldsymbol{p} \cdot \boldsymbol{x}} \frac{\mathrm{d}^{3} p}{(2 \pi)^{3}}
$$


for both positive and negative energy and times. Noting that

$$
\frac{1}{E_{p}} \mathrm{e}^{-i E_{p} t}=\lim _{\varepsilon \rightarrow 0} \frac{i}{\pi} \int_{-\infty}^{\infty} \frac{\mathrm{e}^{-i p_{4} t}}{p_{4}^{2}-E_{p}^{2}+i \varepsilon} \mathrm{d} p_{4}
$$

we recognize that

$$
\Delta_{F}(x)=i \int \mathrm{e}^{-i p \cdot x} \frac{1}{p^{2}-m^{2}+i \varepsilon} \frac{\mathrm{d}^{4} p}{(2 \pi)^{4}}
$$

and

$$
K_{F}(2,1)=(i \nabla+m) \Delta_{F}(x)=i \int \mathrm{e}^{-i p \cdot x} \frac{\mathrm{P}+m}{p^{2}-m^{2}} \mathrm{~d}^{4} p .
$$

The momentum transforms $\delta_{F}\left(p^{2}\right)$ and $k_{F}(p)$ of $\Delta_{F}(x)$ and $K_{F}(x)$ are found to be

and

$$
\delta_{F}\left(p^{2}\right)=i /\left(p^{2}-m^{2}\right)
$$

$$
k_{F}(p)=(\mathrm{p}+m) \frac{i}{p^{2}-m^{2}}=\frac{i}{\mathrm{p}-m} .
$$

We could have obtained the same result from the equation

$$
(i \nabla-m) K_{F}(2,1)=i \delta(2,1) .
$$

Momentum representation of $i \nabla$ is $p$ and of $\delta(2,1)$ is unity. Taking the 4-dimensional transform of both sides we have

$$
(\mathrm{p}-m) k_{F}(p)=i \quad \text { or } \quad k_{F}(p)=\frac{i}{\mathrm{P}-m} .
$$

\section{Calculation of matrix elements}

We shall in all the electrodynamic processes consider the perturbed system to be an electron and the perturbation as an electromagnetic field $A$. The perturbed kernel $K_{F}^{A}(2,1)$ will satisfy the equation

$$
\left(i \nabla_{2}-e \mathrm{~A}-m\right) K_{F}^{A}(2,1)=i \delta(2,1) .
$$

This admits a series expansion for $K_{F}^{A}$ in terms of the unperturbed kernel

$$
\begin{aligned}
K_{F}^{A}(2,1) & =K_{F}(2,1)-i e \int K_{F}(2,3) \mathrm{A}(3) K_{F}(3,1) \mathrm{d}(3) \\
& +i^{2} e^{2} \int K_{F}(2,4) \mathrm{A}(4) K_{F}(4,3) \mathrm{A}(3) K_{F}(3,1) \mathrm{d}(3) \mathrm{d}(4)+\cdots
\end{aligned}
$$

In perturbation problems we are only interested in the matrix element $b_{f i}$ of transition from an initial free particle state $\psi_{i}$ to a final free particle state $\psi_{f}$ both being definite momentum states:

where

$$
b_{f i}=\int \bar{\psi}_{f}(2) K_{F}^{A}(2,1) \psi_{i}(1) \mathrm{d}(2) \mathrm{d}(1)
$$

$$
\begin{aligned}
& \psi_{i}(\mathrm{I})=u\left(p_{i}\right) \mathrm{e}^{-i p_{i} \cdot x_{1}} \\
& \bar{\psi}_{f}(2)=\bar{u}\left(p_{f}\right) \mathrm{e}^{+i p_{f} \cdot x_{2}} .
\end{aligned}
$$


Using the expansion for $K_{F}^{A}$, we note that the zeroth order term vanishes as $\psi_{j}$ and $\psi_{i}$ are orthogonal. The transition amplitude to the first order is

$$
(-i) \int \bar{\psi}_{f}(2) \text { e } \mathrm{A}(2) \psi_{i}(1) \mathrm{d}(1) \text {. }
$$

Substituting for $\psi_{i}$ and $\psi_{f}$ and integrating, we can reduce the matrix element to a form in which only the momentum transforms of perturbations and kernels are required. We shall obtain this reduction to the first and second order and generalize the mode of reduction to the $n$th order.

Defining the momentum transform of $\mathrm{A}(x)$ as

$$
\mathrm{a}(q)=\int \mathrm{A}(x) \mathrm{e}^{i q \cdot x} \mathrm{~d}^{\mathbf{4}} x
$$

we obtain the first order matrix element

with

$$
\bar{u}\left(p_{f}\right) \text { a }(q) u\left(p_{i}\right)
$$

$$
p_{f}-p_{i}=q
$$

The first order transition from $p_{i}$ to $p_{f}$ can occur only when the four momentum $p_{f}-p_{i}=q$ has no time component or equivalently a $(q)$ must be the transform of a function of space only, as in the case of the Coulomb potential. The electron changes its momentum alone without picking up any energy from the Coulomb field. If however the perturbation is due to a photon, the first order matrix element vanishes since $p_{f} \neq q+p_{i}$ where we substitute $\mathrm{A}(k)$ for $\left(4 \pi \mathrm{e}^{2}\right)^{1 / 2} \mathrm{e}^{i k \cdot x}$. However the second order matrix element is non-vanishing and is given by

$$
(-i)^{2} \iint \bar{\psi}_{f}(2) \text { e } \mathrm{A}(2) K_{F}(2,1) \text { e A }(1) \psi_{i}(1) \mathrm{d}(1) \mathrm{d}(2)
$$

We now substitute for $\psi_{f}(2)$ and $\psi_{i}(1)$ the free particle wave functions and write the perturbation $A(1)$ as

and the kernel as

$$
\mathrm{A}(1)=\frac{1}{(2 \pi)^{4}} \int \mathrm{a}(q) \mathrm{e}^{-i q \cdot x} \mathrm{~d}^{4} q
$$

$$
\frac{1}{\mathrm{P}-m} \mathrm{e}^{i p \cdot\left(x_{2}-x_{1}\right)} \frac{\mathrm{d}^{4} p}{(2 \pi)^{4}}
$$

Integrating over the space time points (2) and (1) we get

$$
(-i)^{2} \int_{\pi^{\prime}} \bar{u}_{2} \text { e a }\left(q_{2}\right) \frac{1}{p_{1}+q_{1}-m} e \text { a }\left(q_{1}\right) u_{1} \frac{d^{4} q_{1}}{(2 \pi)^{4}} .
$$

When the perturbations are due to photons, we have

$$
\mathrm{a}\left(q_{1}\right)=\mathrm{e} / 4 \pi
$$

and the matrix element is given by

$$
M=\bar{u}_{2} \mathrm{e}_{2} \cdot \frac{1}{\rho_{1}+\mathrm{q}_{1}-m} \mathrm{e}_{1} u_{1} .
$$


When one of the perturbations is a Coulomb potential, then

and

$$
e \mathrm{a}(q)=\frac{4 \pi Z e^{2}}{q^{2}} \delta\left(q_{4}\right)=\mathrm{v}(\boldsymbol{q})
$$

$$
M=(-i)^{2} \int \bar{u}_{2} \mathrm{e} \frac{i}{\mathrm{P}_{1}+\mathrm{q}-m} \mathbf{v}(\boldsymbol{q}) u_{1} \frac{\mathrm{d}^{4} q}{(2 \pi)^{4}} .
$$

When both the perturbations are potentials, we have

$$
M=(-i)^{2} \int \bar{u}_{2} \vee\left(\boldsymbol{q}_{2}\right) \frac{i}{\mathrm{p}_{1}+\mathrm{q}-m} \vee\left(\boldsymbol{q}_{1}\right) u_{1} \frac{\mathrm{d}^{4} q_{1}}{(2 \pi)^{4}} .
$$

The integration over the space-time variables corresponding to the initial and final wave functions puts the terms of the matrix element in the momentum representation with the arguments of the four momenta associated with each term depending upon the initial and final momentum states and the perturbation, i.e. it is not the kernel that occurs in the matrix elements but only its fourdimensional transform. If $p$ is the momentum of an electron which absorbs a photon of momentum $q$, the transform of the propagator with energy-momentum argument $(p+q)$ is $\frac{i}{\mathrm{p}+\mathrm{q}-m}$.

It is interesting to examine what happens if in the Feynman perturbation expansion we integrate with respect to space and time separately, i.e. we wish to obtain an expression for the 4-dimensional transform of the kernel by first integrating with respect to space and then with respect to time. Integrating with respect to space amounts to picking out from the kernel the terms corresponding to momentum $\boldsymbol{p}+\boldsymbol{q}$ and energy $\boldsymbol{E}(\boldsymbol{p}+\boldsymbol{q})$ which can take two values, $\pm|E(\boldsymbol{p}+\boldsymbol{q})|$. When integrating with respect to time from $-\infty$ to $+\infty$, we must use $+|E(\boldsymbol{p}+\boldsymbol{q})|$ for the range 0 to $+\infty$ and $-|E(\boldsymbol{p}+\boldsymbol{q})|$ for the range $-\infty$ to 0 . Performing the integration, we have

$$
\left[\frac{\stackrel{+}{\mathrm{P}}+m}{|\boldsymbol{E}(\boldsymbol{p}+\boldsymbol{q})|(\boldsymbol{E}(\boldsymbol{p})+E(\boldsymbol{q})-|\boldsymbol{E}(\boldsymbol{p}+\boldsymbol{q})|)}-\frac{\overline{\mathrm{P}}+m}{|\boldsymbol{E}(\boldsymbol{p}+\boldsymbol{q})|\{\boldsymbol{E}(\boldsymbol{p})+E(\boldsymbol{q})+|E(\boldsymbol{p}+\boldsymbol{q})|\}}\right]
$$

where $P=p+q$ and $\stackrel{+}{\mathrm{P}}$ implies that we have used $+|E(\boldsymbol{p}+\boldsymbol{q})|$ as the coefficient of $\gamma_{t}$ and $\bar{P}$ implies the use of $-|E(\boldsymbol{p}+\boldsymbol{q})|$ as the coefficient of $\gamma_{t}$.

The matrix element with the various terms in the momentum representation can be written down by describing the processes by what are known as Feynman graphs. The familiarity with Feynman graphs and the ease with which the calculations are made using them have led many physicists to believe that the Feynman formalism is intuitive or is in the nature of an algorithm while the field theoretic approach is rigorous and deductive. The author is of the opinion that as long as in field theory interactions are expressed only through annihilation and creation operators (see later chapter on field theory) and wave functions of single 
particles, the domains of applicability of the Feynman and field theoretic descriptions are co-extensive as far as the perturbation calculations are concerned.

We shall therefore describe this graphical representation in detail. For this purpose, we start with the perturbation expansion with the integrand in configuration representation. For obvious convenience, we can denote the space variables along one axis (say $x$ ) and the time variable along the other (say $y$ ). The integrand in (36) is then represented diagramatically as follows:

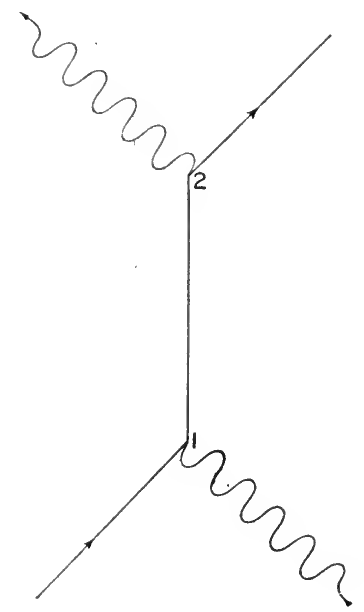

Fig. 1. Feynman diagram of an electron scattered twice.

An electron with wave function $\psi_{i}(1)$ is perturbed at the space-time point (1) by the potential $e A(1)$ and then moves to the space-time point (2). The free particle kernel is represented by the line joining the points (1) and (2). Again at (2) the electron is perturbed by $e \mathrm{~A}(2)$ and emerges with wave function $\psi_{f}(2)$. On integrating with respect to space time variables, the momentum transforms are obtained in the same sequence as in the integrand and therefore we can still represent the matrix element by the same graph writing instead of the kernels and perturbations their corresponding transforms and retaining the indices (1) and (2) for the vertices. We should however remember that the vertices merely indicate the sequence which now bears a one to one correspondence with the space-time points in the integrand. Then the process is described as follows:

The electron with momentum $\mathrm{p}$, and wave function $u_{1}$ moves as a free particle. At point (1) it is scattered by a photon of momentum $q_{1}$. Having absorbed the photon, the electron moves from (1) to (2) as a virtual particle of momentum $p+q_{1}$. It gets scattered again at $(2)$ by the second photon of monentum $q_{2}$. Then it emerges from (2) as a free particle with momentum $p_{2}=p_{1}+q_{1}+q_{2}$.

We see that there is no significance attached to the two axes and it is only the sequence of vertices which is important. This is also implied when we take the 
propagator as $\frac{1}{\mathrm{P}-m}$ which means that time has been integrated out and hence includes double scattering through the creation and annihilation of pairs. However we might wish to specify (i) whether the incoming and outgoing particles are electrons or positrons, (ii) whether the photon is emitted or absorbed at a vertex. Since in the Feynman point of view a positron is interpreted as a negative energy electron travelling back in time, it has become customary even in the momentum representation to maintain the time sense as far as incident and emergent particles are concerned, i.e. the arrows have to be pointed upwards for electrons and downwards for positrons. Similarly, the photon that is absorbed is represented by a line attached to a vertex from below and the photon that is emitted is represented by a line which is attached to a vertex from above.

However if we wish to retain time ordering in a strict sense for the entire process, i.e. including intermediate states we have to do the space integration first and time integration subsequently. In this case only time has a definite meaning while the other axis is necessary only for diagrammatic representation and has no significance as a space axis. Hence we split the propagator $\frac{1}{\mathrm{p}-m}$ as $A_{+}+A_{-}$
where

$$
\begin{aligned}
& A_{+}=\frac{\stackrel{+}{\mathrm{p}}+m}{|E(\boldsymbol{p}+\boldsymbol{q})|[E(\boldsymbol{p})+E(\boldsymbol{q})-|E(\boldsymbol{p}+\boldsymbol{q})|]} \\
& A_{-}=\frac{-(\overline{\mathrm{p}}+m)}{E(\boldsymbol{p}+\boldsymbol{q}) \mid[E(\boldsymbol{p})+E(\boldsymbol{q})+|E(\boldsymbol{p}+\boldsymbol{q})|]}
\end{aligned}
$$

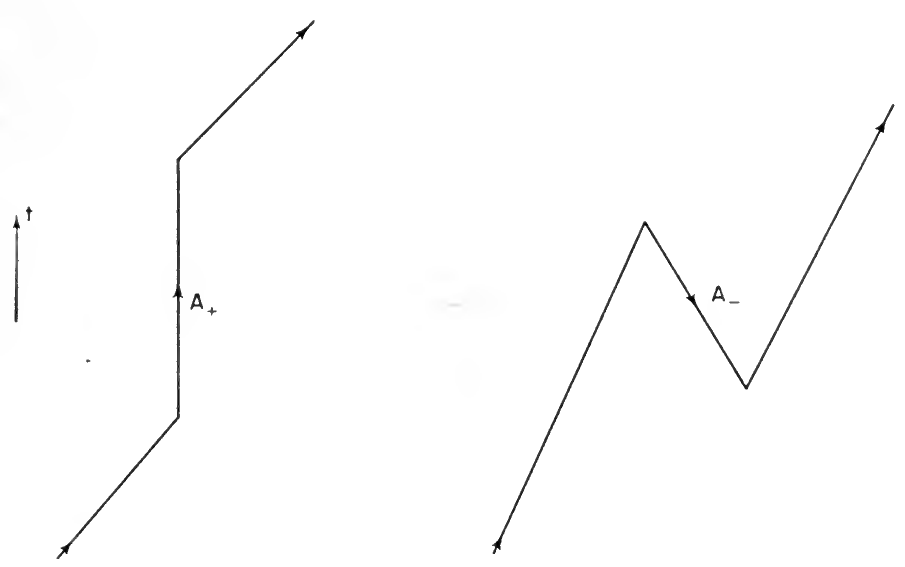

Fig. 2. Diagrammatic representation of propagators $A_{+}$and $A_{-}$.

and the corresponding graphs for $A_{+}$and $A_{-}$are given above (Fig. 2). We have thus seen that the Feynman formalism is essentially characterized by two features: (1) even when pair creation and annihilation of particles are included, electrodynamic processes can still be described through a single particle formalism which 
obviates the use of field theoretic methods and (2) the perturbation expansions are "inherently four-dimensional" and the covariance of the equations is apparent at every stage of the calculations, the integration over space-time variables being performed together. However the elegance of the four-dimensional methods is still maintained even if we integrate the space and time variables separately which leads to the decomposition of the Feynman propagator. This leads to a better understanding of virtual processes. We shall indicate the contribution to the matrix element from transitions to negative energy virtual states in electrodynamic processes which will be considered in the next section.

We shall now summarize the method of writing the matrix element corresponding to a Feynman graph by giving below what are known as the Feynman rules.

(i) An electron in a virtual state of four momentum $p$ contributes an amplitude $i /(\mathrm{p}-m)$.

(ii) The amplitude for picking up a momentum $\boldsymbol{q}$ from a potential is -iea $(q)$ where $a(q)$ is the momentum transform of $A(x)$ representing the potential which is independent of time:

$$
A(x)=\int a(q) \mathrm{e}^{-i q \cdot x} \delta\left(q_{4}\right) \mathrm{d}^{3} q .
$$

In particular when $A_{\mu}=0$ for $\mu=1,2,3$ and $A_{4}=\varphi=\frac{Z e^{2}}{r}$

$$
\mathrm{a}(q)=\frac{4 \pi Z e^{2}}{(\boldsymbol{q} \cdot \boldsymbol{q})} \delta\left(q_{4}\right) \gamma_{t}
$$

A real photon of polarization e contributes the factor $\mathrm{a}(q)=(4 \pi)^{1 / 2} \mathrm{e}$ where $a_{\mu}=(4 \pi)^{1 / 2} e_{\mu}$. This is because the photon wave function is taken to be $4 \pi^{1 / 2} e_{\mu} \mathrm{e}^{i q \cdot x}$. If we have a photon of polarization $e_{\mu}$ we set $e_{\mu}=1$ and $a_{\mu}=(4 \pi)^{1 / 2}$.

(iii) All indeterminate momenta $q_{i}$ are summed over $\mathrm{d}^{4} q_{i} /(2 \pi)^{4}$. The matrix element obtained using the above rules has to be multiplied by the normalization factors $\frac{1}{\sqrt{2} \omega}$ for a photon and $\frac{1}{\sqrt{ } 2 E}$ for a real electron.

\section{APPLICATIONS TO ELECTRODYNAMICS}

We shall now consider the following electromagnetic processes ${ }^{1}$,

(1) Compton effect,

(2) Two photon pair annihilation,

(3) Bremsstrahlung,

(4) Pair creation,

(5) Electron-electron interaction.

The five processes have been chosen in this order for convenience of comparison of the corresponding Feynman diagrams. Besides these there are two other im-

1 R. P. Fexnman, Lectures delivered at the California Institute of Technology, on "Quantum Electrodynamics" (1953). For discussions on the cross-sections, see W. HeITLER, Quantum Theory of Radiation, Oxford University Press, London (1954). 
portant electrodynamic processes-(1) the loss of energy of fast particles by ionisation, (2). loss of energy by magnetic Bremsstrahlung. Since these processes can be satisfactorily considered by the use of semi-classical methods, we shall postpone their discussion to a later stage (see Part II).

\section{Compton effect}

This is the process of scattering of a photon by a free electron. A photon of momentum $q_{1}\left(\boldsymbol{q}_{1}, \omega_{1}\right)$ and polarization $\boldsymbol{e}_{1}$ is incident on an electron which is assumed to be at rest, since in the rest system of the electron the calculation of the cross-section is greatly simplified. The general case when the electron is not at rest can be obtained from this by a Lorentz transformation. In the final state, we have a photon of momentum $q_{2}\left(\boldsymbol{q}_{2}, \omega_{2}\right)$ and polarization $\boldsymbol{e}_{2}$ and an electron of momentum $p_{2}\left(\boldsymbol{p}_{2}, E_{2}\right)$. From the Feynman formalism we can regard the process as follows. The incident photon $q_{1}$ is absorbed by the electron of momentum $p_{1}(0, m)$. Absorbing the photon, the electron propagates itself as $\frac{i}{p_{1}+q_{1}-m}$. Subsequently it emits a photon of momentum $q_{2}$ the electron having a residual momentum $p_{2}$. The conservation of energy and momentum is expressed by the relation

$$
p_{1}+q_{1}=p_{2}+q_{2}
$$

Instead of absorbing the incident photon first, the electron may emit the final photon and subsequently absorb the incident photon. Thus there are two possibilities which are represented by the diagrams below (Fig. 3). To calculate the

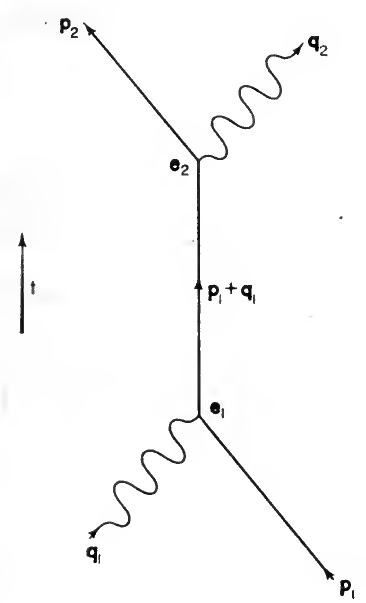

(R)

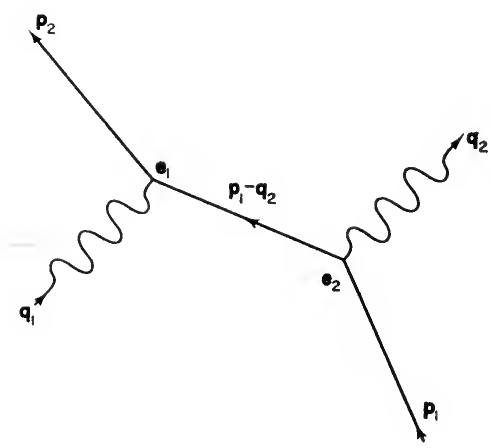

(S)

Fig. 3. Feynman diagrams for Compton effect.

matrix element for the transition from the initial to the final state, we represent the incoming photon by $A_{1 \mu}=\left(4 \pi \mathrm{e}^{2}\right)^{1 / 2} e_{1 \mu} \mathrm{e}^{-i q_{1} \cdot x}$ and the emitted photon by $A_{2 \mu}=\left(4 \pi \mathrm{e}^{2}\right)^{1 / 2} e_{2 \mu} \mathrm{e}^{+i q_{2} \cdot x}$ where the normalization is to $(2 \omega)$ photons per unit 
volume. Further since the photon is polarized perpendicular to the direction of propagation we have

Also

$$
\begin{gathered}
\boldsymbol{e}_{1} \cdot \boldsymbol{q}_{1}=0 ; \quad \boldsymbol{e}_{2} \cdot \boldsymbol{q}_{2}=0 \\
q_{1}^{2}=0 \text { and } q_{2}^{2}=0 .
\end{gathered}
$$

The initial and final state electron wave functions are chosen as

where

$$
\psi_{1}=u_{1} \mathrm{e}^{i p_{1} \cdot x} ; \quad \psi_{2}=u_{2} \mathrm{e}^{-i p_{2} \cdot x}
$$

$$
\begin{aligned}
\mathrm{P}_{1} u_{1} & =m u_{1} ; \quad \mathrm{P}_{2} u_{2}=m u_{2} . \\
p_{1} \cdot p_{1} & =m^{2} ; \quad p_{2} \cdot p_{2}=m^{2} .
\end{aligned}
$$

Since the initial electron is at rest we have

$$
\begin{gathered}
\mathrm{p}_{1}=m \gamma_{t} ; \quad \mathrm{P}_{2}=E_{2} \gamma_{t}-\gamma_{x} p_{2} \cos \varphi+\gamma_{y} p_{2} \sin \varphi . \\
\mathrm{q}_{1}=\omega_{1}\left(\gamma_{t}-\gamma_{x}\right) ; \quad \mathrm{q}_{2}=\omega_{2}\left(\gamma_{t}-\gamma_{x} \cos \theta-\gamma_{y} \sin \theta\right)
\end{gathered}
$$

where $\theta$ is the angle between the outgoing and incoming photon directions (initial photon direction is taken as the $x$ direction) and $2 \pi-\varphi$ is the angle which the recoil electron makes with the $x$ direction. The possible directions of polarizations

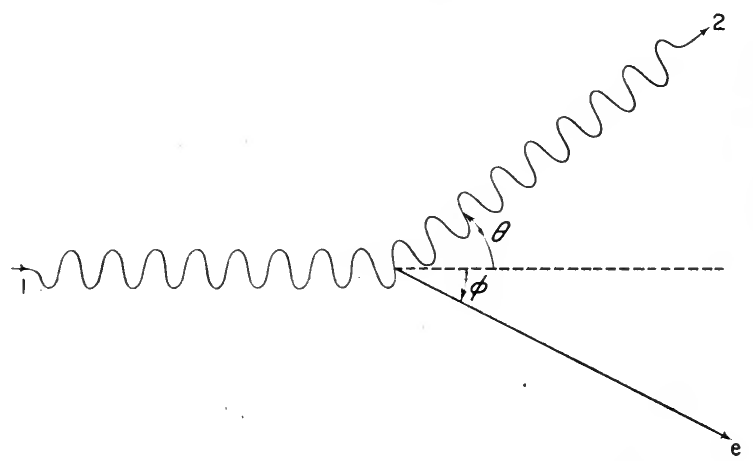

Fig. 4. Schematic diagram for Compton effect.

of the incident photon will be along the $z$ or $y$ directions denoted as "Type $A$ " and "Type $B$ " respectively. We then have

$$
\begin{array}{lll}
(A) \mathrm{e}_{1}=\gamma_{z} & (B) \mathrm{e}_{1}=\gamma_{y} .
\end{array}
$$

Similarly for the outgoing photon we have

$$
\left(A^{\prime}\right) \mathrm{e}_{2}=\gamma_{z} \quad\left(B^{\prime}\right) \mathrm{e}_{2}=\gamma_{y} \cos \theta-\gamma_{x} \sin \theta .
$$

Substituting relations (2) to (7) in (1) we can obtain the formula for Compton shift in frequency as

$$
\frac{m}{\omega_{2}}-\frac{m}{\omega_{1}}=1-\cos \theta
$$


In computing the transition probability the density of final states involved is that of a two particle system. This is given by

$$
=\frac{(2 \pi)^{-3} E_{2} \omega_{2}^{3} \mathrm{~d} \Omega_{\omega_{2}}}{m \omega_{1}} .
$$

Making use of the Feynman rules stated in the previous section, the matrix elements for the diagrams $R$ and $S$ are respectively

and

$$
-i\left(4 \pi e^{2}\right)\left(\bar{u}_{2} \mathrm{e}_{2} \frac{1}{\mathrm{p}_{1}+\mathrm{q}_{1}-m} \mathrm{e}_{1} u_{1}\right)=-i 4 \pi e^{2} \bar{u}_{2} R u_{1}
$$

$$
-i\left(4 \pi e^{2}\right)\left(\bar{u}_{2} \mathrm{e}_{1} \frac{1}{\mathrm{p}_{1}-\mathrm{q}_{2}-m} \mathrm{e}_{2} u_{1}\right)=-i 4 \pi e^{2} \bar{u}_{2} S u_{1} .
$$

Hence the differential cross-section for Compton effect is given by

$$
\mathrm{d} \sigma=\frac{2 \pi}{2 E_{1} 2 E_{2} 2 \omega_{1} 2 \omega_{2}}\left(4 \pi e^{2}\right)^{2}\left|\bar{u}_{2}(R+S) u_{1}\right|^{2} \frac{(2 \pi)^{-3} E_{2} \omega_{2}^{3} \mathrm{~d} \Omega_{\omega_{2}}}{m \omega_{1}}
$$

Making use of the identities

and

$$
\frac{1}{\mathrm{P}-m}=\frac{\mathrm{P}+m}{p^{2}-m^{2}}
$$

$$
\mathrm{ab}=2 a \cdot b-\mathrm{b} \mathrm{a}
$$

and the relations ( 8 and 9 ) stated above, it can be shown that $R$ and $S$ reduce to

$$
R=\frac{\mathrm{e}_{1} \mathrm{q}_{1} \mathrm{e}_{1}}{2 m \omega_{1}} \quad \text { and } \quad S=\frac{\mathrm{e}_{1} \mathrm{q}_{2} \mathrm{e}_{2}}{2 m \omega_{2}} .
$$

The matrix elements corresponding to the different sets of polarisations $A A^{\prime}$, $A B^{\prime}, B A^{\prime}$ and $B B^{\prime}$ can then be simplified. The final results are given below.

\section{Polarization}

$$
\begin{array}{cc}
A A^{\prime} & \frac{\left(\omega_{1}-\omega_{2}\right)^{2}}{\omega_{1} \omega_{2}}+4, \\
A B^{\prime} & \frac{\left(\omega_{1}-\omega_{2}\right)^{2}}{\omega_{1} \omega_{2}}, \\
B A^{\prime} & \frac{\left(\omega_{1}-\omega_{2}\right)^{2}}{\omega_{1} \omega_{2}}, \\
B B^{\prime} & \frac{\left(\omega_{1}-\omega_{2}\right)^{2}}{\omega_{1} \omega_{2}}+4 \cos ^{2} \theta
\end{array}
$$$$
\begin{gathered}
|M|^{2} \\
\frac{\left(\omega_{1}-\omega_{2}\right)^{2}}{\omega_{1} \omega_{2}}+4
\end{gathered}
$$

More generally we can write the matrix element as

$$
|\boldsymbol{M}|^{2}=\frac{\left(\omega_{1}-\omega_{2}\right)^{2}}{\omega_{1} \omega_{2}}+4\left(\boldsymbol{e}_{1} \cdot \boldsymbol{e}_{2}\right)^{2}
$$


The cross-section for scattering with prescribed directions $\boldsymbol{e}_{1}$ and $\boldsymbol{e}_{2}$ of polarization of the incoming and outgoing photons is

$$
\mathrm{d} \sigma=\frac{e^{4}}{4 m^{2}} \frac{\omega_{2}^{2}}{\omega_{1}^{2}} \mathrm{~d} \Omega_{\omega_{2}}\left[\frac{\omega_{2}}{\omega_{1}}+\frac{\omega_{1}}{\omega_{2}}-2+4\left(\boldsymbol{e}_{1} \cdot \boldsymbol{e}_{2}\right)^{2}\right] .
$$

This is the well-known Klein-Nishina formula.

\section{Discussion of the Klein-Nishina formula}

In the non-relativistic limit, $\omega_{1} \ll m$ the electron picks up very little energy and hence $\omega_{1} \approx \omega_{2}$. The Klein-Nishina formula then takes the form

$$
\mathrm{d} \sigma=\frac{e^{4}}{m^{2}}\left(e_{1} \cdot e_{2}\right)^{2} \mathrm{~d} \Omega_{\omega_{2}}
$$

which is the Rayleigh-Thomson scattering cross-section.

To obtain the scattering cross-section independent of polarization we have to sum over the polarizations of the outgoing beam and average over the incoming beam which can be seen in the following way. The total probability for scattering a photon of type $A$ to one of the type $A^{\prime}$ or $B^{\prime}$ is $\left(A A^{\prime}+A B^{\prime}\right)$. Since the incoming beam can be of type $A$ or $B$ with equal probability the cross-section for an unpolarized incoming beam is

$$
\begin{gathered}
\frac{1}{2}\left\{A A^{\prime}+A B^{\prime}\right\}+\frac{1}{2}\left\{B A^{\prime}+B B^{\prime}\right\} \\
=\frac{e^{4}}{2 m^{2}}\left(\frac{\omega_{2}}{\omega_{1}}\right)^{2} \mathrm{~d} \Omega_{\omega_{2}}\left\{\frac{\omega_{2}}{\omega_{1}}+\frac{\omega_{1}}{\omega_{2}}-\sin ^{2} \theta\right\} .
\end{gathered}
$$

On the other hand if the outgoing beam is taken to be of definite polarization, we have, if the incident beam is unpolarized,

$$
\begin{aligned}
\frac{\text { Probability for polarization of type } A^{\prime}}{\text { Probability for polarization of type } B^{\prime}} & =\frac{\frac{1}{2}\left(A A^{\prime}+B A^{\prime}\right)}{\frac{1}{2}\left(A B^{\prime}+B B^{\prime}\right)} \\
& =\frac{\frac{\omega_{1}}{\omega_{2}}+\frac{\omega_{2}}{\omega_{1}}}{\frac{\omega_{2}}{\omega_{1}}+\frac{\omega_{1}}{\omega_{2}}-2 \sin ^{2} \theta}
\end{aligned}
$$

The unscattered radiation $(\theta=0)$ remains unpolarized but the scattered radiation will be polarized to a certain extent. When $\omega_{1} \simeq \omega_{2}$ (i.e. in the lower frequency limit), the incoming unpolarized beam becomes plane polarized, when scattered through $90^{\circ}$. If the incident beam is polarized for large angles the outgoing beam is unpolarized and for small angles it is almost unpolarized as $\omega_{2} / \omega$ is negligible.

To obtain the total scattering cross-section, integration over all angles should be performed. Hence, integrating, the total cross-section is

$$
\frac{\sigma}{\sigma_{0}}=\frac{3}{4}\left\{\frac{1+\gamma}{\gamma^{3}}\left[\frac{(1+\gamma) 2 \gamma}{1+2 \gamma}-\log (1+2 \gamma)\right]+\frac{1}{2 \gamma} \log (1+2 \gamma)-\frac{1+3 \gamma}{(1+2 \gamma)^{2}}\right\}
$$

where

$$
\sigma_{0}=\frac{8 \pi}{3}, \quad \gamma=\frac{\omega_{1}}{m} \text {. }
$$


In the non-relativistic case, $\gamma \ll 1, \sigma=\sigma_{0}$. The right-hand side reduces when expanded in powers of $\gamma$ to

$$
\sigma=\sigma_{0}\left(1-2 \gamma+\frac{26}{5} \gamma^{2}+\cdots\right) .
$$

In the extreme relativistic case

$$
\sigma=\sigma_{0} \frac{3}{\gamma} \frac{m}{\omega_{1}}\left(\log \frac{2 \omega_{1}}{m}+\frac{1}{2}\right) .
$$

Hence for very high energies, the number of quanta scattered decreases with increasing energy of the incident radiation. This is the reason for the increase of penetrating power of $\gamma$-rays with frequency as long as there is no other absorption process such as pair production.

\section{Two photon pair annihilation}

In this process a free electron is annihilated in a collision with a free positron. Conservation of energy and momentum requires that two photons be emitted. In Feynman formalism, this phenomenon is completely analogous to Comptonscattering as the diagrams below show.

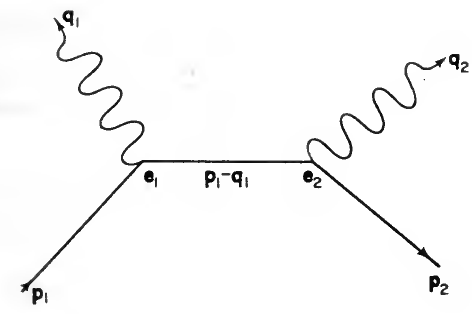

(R)

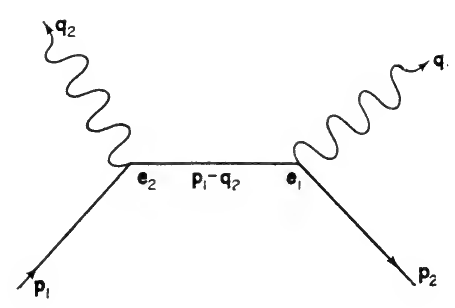

(S)

Fig. 5. Feynman diagrams for two photon pair annihilation.

The diagrams differ from those of Compton effect only in the ordering in time. The direction of $q$, is reversed and the second particle is a positron, i.e. an electron travelling back in time. The electron of momentum $p_{1}\left(\boldsymbol{p}_{1}, E_{1}\right)$ emits a photon of momentum $q_{1}\left(\boldsymbol{q}_{1}, \omega_{1}\right)$ and polarization $\boldsymbol{e}_{1}$ and propagates itself as $\frac{1}{\mathrm{p}_{1}-\boldsymbol{q}_{1}-m}$. It collides with a positron of momentum $p_{2}\left(\boldsymbol{p}_{2}, E_{2}\right)$ resulting in the annihilation of the pair with the emission of a photon of momentum $q_{2}\left(\boldsymbol{q}_{2}, \omega_{2}\right)$. The second diagram is obtained by the interchange of the two photons. The conservation law gives

$$
p_{2}=p_{1}-q_{1}-q_{2}
$$


which is similar to that for Compton effect. Denoting the matrix elements of the two diagrams as $M_{1}$ and $M_{2}$ we have

and

$$
M_{1}=-i 4 \pi e^{2}\left[\bar{u}_{2} \mathrm{e}_{2} \frac{1}{\left(\mathrm{p}_{1}-\mathrm{q}_{1}-m\right)} \mathrm{e}_{1} u_{1}\right]
$$

where

$$
M_{2}=-i 4 \pi e^{2}\left[\bar{u}_{2} \mathrm{e}_{1} \frac{1}{\mathrm{p}_{1}-\mathrm{q}_{2}-m} \mathrm{e}_{2} u_{1}\right]
$$

$$
\mathrm{P}_{1}=\left(E_{-} \gamma_{t}-\boldsymbol{p}_{-} \cdot \boldsymbol{\gamma}\right) \quad \mathrm{p}_{2}=-\left(E_{+} \gamma_{t}-\boldsymbol{p}_{+} \cdot \boldsymbol{\gamma}\right) \text {. }
$$

To calculate the cross-section, we apply the methods as before. The electron is assumed to be at rest while the positron is moving. The density of the two photon final states is given by

$$
\frac{\omega_{1} \omega_{2}}{(2 \pi)^{3}} \frac{\omega_{1}^{2} \mathrm{~d} \Omega_{\omega}}{\omega_{2} \omega_{1}-\boldsymbol{q}_{2} \cdot \boldsymbol{q}_{1}}
$$

From the diagrams it is clear that the matrix elements for pair annihilation are the same as the matrix elements for Compton effect if the sign of $q_{1}$ is changed. In the cross-section this necessitates a change of sign of $\omega_{1}$. Hence the crosssection is

$$
\sigma=\frac{e^{4} \omega_{1}^{2} \mathrm{~d} \Omega_{\omega}}{4 m^{2}\left(E_{+}+m\right)\left|p_{+}\right|}\left\{\frac{\omega_{2}}{\omega_{1}}+\frac{\omega_{1}}{\omega_{2}}+2-4\left(\boldsymbol{e}_{1} \cdot \boldsymbol{e}_{2}\right)^{2}\right\}
$$

Polarization: As in the case of Compton effect, to consider the variation of the cross-section with the polarization, it will be more convenient to work in a coordinate system in which the centre of mass of the electron and positron is at rest. Then the differential cross-section is given by

$$
\begin{gathered}
\mathrm{d} \sigma=\frac{e^{4}}{\gamma p_{+}} \frac{\mathrm{d} \Omega}{E_{+}}\left[\frac{E_{+}^{2}-\left(E_{+}^{2}-2 p_{+}^{2} \cos ^{2} \theta\right)\left(\boldsymbol{e}_{1} \cdot \boldsymbol{e}_{2}\right)^{2}+4\left(\boldsymbol{p}_{+} \cdot \boldsymbol{e}_{1}\right)\left(\boldsymbol{p}_{+} \cdot \boldsymbol{e}_{2}\right)\left(\boldsymbol{e}_{1} \cdot \boldsymbol{e}_{2}\right)}{E_{+}^{2}-p_{+}^{2} \cos ^{2} \theta}\right. \\
\left.-\frac{4\left(\boldsymbol{p}_{+} \boldsymbol{e}_{1}\right)^{2}\left(\boldsymbol{p}_{+} \cdot \boldsymbol{e}_{2}\right)^{2}}{\left(E_{+}^{2}-p_{+}^{2} \cos ^{2} \theta\right)^{2}}\right] .
\end{gathered}
$$

The polarization vectors $\boldsymbol{e}_{1}$ and $\boldsymbol{e}_{2}$ can be so chosen that they are in the $\boldsymbol{p}_{+} \boldsymbol{q}_{1}$ plane or perpendicular to it. In accordance with this, the cross-section will be denoted by $\mathrm{d} \sigma_{||||}$when both photons are polarized in this plane and by $\mathrm{d} \sigma_{\perp||}$ when one of them is polarized in this plane and the other perpendicular to it. When $p_{-}$is very small $\mathrm{d} \sigma_{\perp \perp}=\mathrm{d} \sigma_{||||}=0$ and

$$
\mathrm{d} \sigma_{\perp \|}=\frac{e^{4} \mathrm{~d} \Omega}{\gamma p_{+} m} \quad \ldots .(\text { (non-relativistic case) } \text {. }
$$

Thus the photons are polarized perpendicular to each other. For $E_{0} \gg m$ no polarization effect exists.

Integrating over $\theta(0$ to $\pi)$ and $\varphi$ we obtain the total probability of annihilation. Limits of $\theta$ are 0 to $\pi$ since permutation of the two photons does not lead to a new state. 
The integration yields

where

$$
\sigma=\frac{\pi e^{4}}{4\left(p_{+} E_{+}\right)}\left\{2\left(\beta^{2}-2\right)+\frac{3-\beta^{4}}{\beta} \log \frac{1+\beta}{1-\beta}\right\}
$$

$$
\beta=\frac{p_{+}}{E_{+}}=v_{+}
$$

The above expression represents the cross-section for the annihilation of an electron and positron of equal and opposite momentum $p_{+}$. In the practical case, the electron is at rest. The probability of annihilation in this case is obtained by a suitable Lorentz transformation which gives $\sigma$ as

$$
\sigma=\pi r_{0}^{2} \frac{1}{\gamma+1}\left\{\frac{\gamma^{2}+4 \gamma+1}{\gamma^{2}-1} \log \gamma+V\left(\gamma^{2}-1\right)-\frac{\gamma+3}{\sqrt{ } \gamma^{3}-1}\right\}
$$

where $\gamma=E_{+} / m$ and $E_{+}$and $v_{+}$are the energy and velocity of positron and $r_{0}=e^{2} / m$. The above expression first deduced by Dirac has a maximum for small values of $v_{+}(\gamma \sim 1)$ and for $E_{+} \rightarrow m$ the cross-section diverges. This does not mean that the cross-section will become infinity. The rate of annhilation in a substance with $N$ atoms/unit volume is given by $R=N \sigma Z v_{+}=N Z \pi r_{0}^{2} \sec ^{-1}$ (in the nonrelativistic case). For lead, $R=2 \times 10^{10} \mathrm{sec}^{-1}$. Hence the life of a very slow positron in lead is about $10^{-10}$ secs. For higher energies the cross-section decreases and is given by

$$
\sigma=\pi r_{0}^{2} \frac{m}{E_{+}}\left\{\log \frac{m}{2 E_{+}}-1\right\} \quad \text { (in the extreme relativistic case) }
$$

In a Lorentz frame in which the electron is at rest, generally the two quanta do not have the same frequency. For high energies of the positron in the initial Lorentz system, the two quanta emitted are mainly in the forward and backward directions. After the Lorentz transformation the quantum emitted forward takes up most of the energy of the positron and the second quantum has energy only of the order of $m$. If however the positron has small kinetic energy, both quanta pick up almost equal energies $(\sim m)$ and are emitted in the opposite directions with perpendicular polarizations.

\section{Bremsstrahlung}

An electron traversing in the field of a nucleus is deflected, according to the classical theory, due to the inverse square law force of attraction. Since deflection involves acceleration according to classical electrodynamics, the electron should radiate. This process is called "Bremsstrahlung". In the language of quantum electrodynamics, there exists a definite probability that the incident electron will make a radiative transition to another state with the emission of a photon. Interaction with the field of the nucleus is necessary to conserve EPCR 6 
energy and momentum since it is impossible for a free electron to emit a single photon and make a transition to a real state.

The Feynman diagrams for Bremsstrahlung are given below.

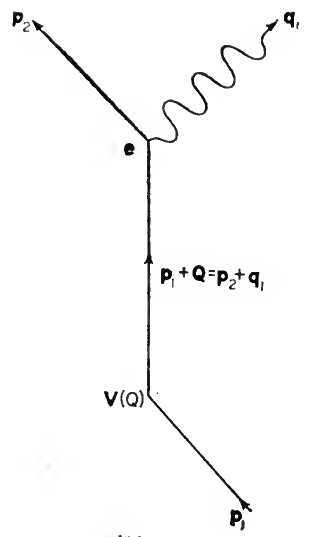

(R)

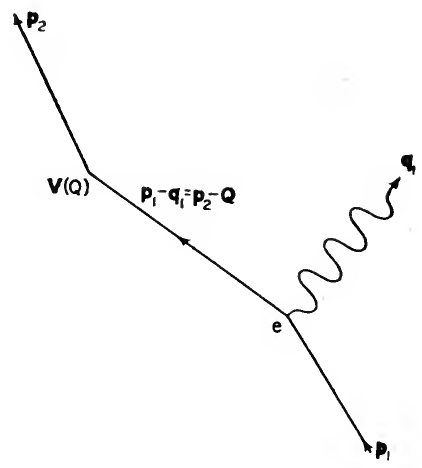

(S)

FIG. 6. Feynman diagrams for Bremsstrahlung.

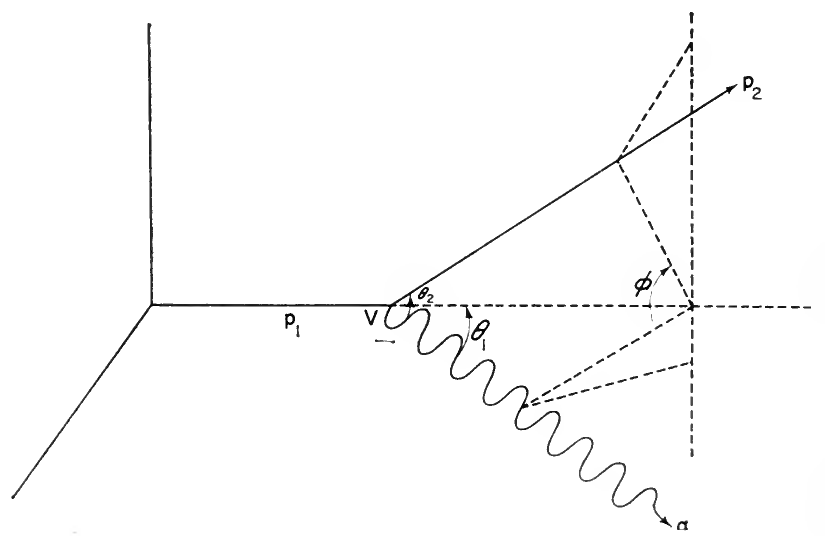

FIG. 7. Schematic diagram for Bremsstrahlung.

It is assumed that the nucleus is so heavy compared to the electron that it is at rest throughout the process. This assumption, as will be shown later, is equivalent to assuming that the electron interacts only with the Coulomb field of the nucleus. The two diagrams describe the following indistinguishable processes.

(1) The electron interacts with the Coulomb field and subsequently emits a photon,

(2) The electron emits a photon and then interacts with the Coulomb field. 
In the first diagram, an electron of momentum $p_{1}$ initially in a state described by the wave function $u_{1} \mathrm{e}^{-i p_{1} \cdot x}$ is scattered by the Coulomb potential, acquiring an additional momentum $\boldsymbol{Q}$. It then moves as $\frac{i}{\mathrm{P}_{1}+\mathrm{Q}-m}$ until it emits a photon of momentum $q$ and polarization $\boldsymbol{e}$. The final state of the electron of momentum $p_{2}$ is $u_{2} \mathrm{e}^{-i p_{2} \cdot x}$. The matrix element corresponding to this diagram is

where

$$
M_{1}=\left(4 \pi e^{2}\right)^{1 / 2}\left[\bar{u}_{2} \mathrm{e} \frac{1}{\mathrm{P}_{1}+\mathrm{Q}-m} \vee(Q) u_{1}\right]
$$

$$
v(Q)=\frac{4 \pi z e^{2}}{Q^{2}} \delta\left(Q_{4}\right) \gamma_{t}=v(Q) \delta\left(Q_{4}\right) \gamma_{t} .
$$

It should be noted that the Coulomb potential $Z e^{2} / r$ is independent of time and hence the Fourier transform of $Z e^{2} / r$ is proportional to $\delta\left(Q_{4}\right)$. This implies that $Q_{4}=0$ for the transition and hence $E_{1}=E_{2}+\omega$. The second diagram can be similarly understood and its matrix element $M_{2}$ is given by

$$
M_{2}=\left(4 \pi e^{2}\right)^{1 / 2}\left[\bar{u}_{2} \vee(Q) \frac{1}{\mathrm{P}_{2}-\mathrm{Q}-m} \mathrm{e} u_{1}\right]
$$

The energy momentum conservation requires

$$
\mathrm{P}_{2}=\mathrm{P}_{1}+\mathrm{Q}-\mathrm{q} \text {. }
$$

The density of final states in this case corresponds to that of a three particle system consisting of the nucleus, electron and photon. Since the nucleus is considered to be infinitely heavy, the expression for the density of states can be reduced to

$$
\varrho=(2 \pi)^{-6} E_{2} p_{2} \mathrm{~d} \Omega_{2} \omega^{2} \mathrm{~d} \omega \mathrm{d} \Omega_{\omega} .
$$

The outgoing photon can be polarized in any direction and the incoming and outgoing electron states each have two possible spin states. Hence after summing over the photon and the outgoing electron polarizations and averaging over the initial polarizations of the electron, we have the following differential crosssection

$$
\begin{gathered}
\mathrm{d} \sigma=\frac{1}{2 \pi}\left(\frac{Z e^{2}}{Q^{2}}\right)^{2} \frac{1}{137} \frac{\mathrm{d} \omega}{\omega} \frac{p_{2}}{p_{1}} \sin \theta_{2} \mathrm{~d} \theta_{2} \sin \theta_{1} \mathrm{~d} \theta_{1} \mathrm{~d} \varphi \\
{\left[\left\{\frac{p_{2}^{2} \sin ^{2} \theta_{2}\left(4 E_{1}^{2}-Q^{2}\right)}{\left(E_{2}-p_{2} \cos \theta_{2}\right)^{2}}+\frac{p_{1}^{2} \sin ^{2} \theta_{1}\left(4 E_{2}^{2}-Q^{2}\right)}{\left(E_{1}-p_{1} \cos \theta_{1}\right)^{2}}\right\}\right.} \\
\left.-\frac{2 p_{1} p_{2} \sin \theta_{1} \sin \theta_{2} \cos \varphi\left(4 E_{1} E_{2}-Q^{2}+2 \omega^{2}\right)-2 \omega^{2}\left(p_{2}^{2} \sin ^{2} \theta_{2}+p_{1}^{2} \sin ^{2} \theta_{1}\right)}{\left(E_{2}-p_{2} \cos \theta_{2}\right)\left(E_{1}-p_{1} \cos \theta_{1}\right)}\right]
\end{gathered}
$$

where $Z e$ is the charge of the deflecting nucleus, $p_{1}, E_{1}$ the initial momentum and energy of the electron, $p_{2}, E_{2}$ the final momentum and energy of the electron and $\omega$ the frequency of the emitted photon. $Q$ is the total momentum transmitted to the nucleus $=\left(\boldsymbol{p}_{1}-\boldsymbol{p}_{2}-\boldsymbol{q}\right), \theta_{1}$, the angle between $\boldsymbol{q}$ and $\boldsymbol{p}_{1}, \theta_{2}$ the angle between $\boldsymbol{q}$ and $\boldsymbol{p}_{2}, \varphi$ the angle between the planes $\left(\boldsymbol{q} \boldsymbol{p}_{1}\right)$ and $\left(\boldsymbol{q} \boldsymbol{p}_{2}\right)$. 
When the photon energy is small compared to the rest mass of the electron, the matrix element reduces to the form

$$
M=\left(4 \pi e^{2}\right)^{1 / 2} v(Q)\left[\frac{\boldsymbol{e} \cdot \boldsymbol{p}_{1}}{\boldsymbol{q} \cdot \boldsymbol{p}_{1}}-\frac{\boldsymbol{e} \cdot \boldsymbol{p}_{\mathbf{2}}}{\boldsymbol{q} \cdot \boldsymbol{p}_{2}}\right] \gamma_{t}
$$

The differential cross-section for photon emission then becomes

$$
\mathrm{d} \sigma=\frac{1}{v}\left[\frac{2 \pi}{2 E_{1} 2 E_{2}}|v(Q)|^{2} \frac{E_{2} p_{2} \mathrm{~d} \Omega_{2}}{(2 \pi)^{3}}\right]\left[\frac{e^{2} \mathrm{~d} \omega \mathrm{d} \Omega_{\omega}}{\pi \omega}\left(\frac{\boldsymbol{p}_{2} \cdot \boldsymbol{e}}{\boldsymbol{p}_{2} \cdot \boldsymbol{q} / \omega}-\frac{\boldsymbol{p}_{1} \cdot \boldsymbol{e}}{\boldsymbol{p}_{1} \cdot \boldsymbol{q} / \omega}\right)^{2}\right]
$$

which can be easily interpreted. The first bracket represents the transition probability for elastic scattering while the second bracket gives the probability of emission of a photon in the frequency interval $\mathrm{d} \omega$ and in the solid angle $\mathrm{d} \Omega_{\omega}$ if there is elastic scattering from momentum $\boldsymbol{p}_{1}$ to $\boldsymbol{p}_{2}$.

The total cross-section for the emission of a quantum with energy between $\omega$ and $\omega+\mathrm{d} \omega$ is obtained by integrating $\mathrm{d} \sigma$ over all angles. Usually the crosssection is represented as a function of $\omega /\left(E_{1}-m\right), m$ being the rest energy of the electron and $E_{1}-m$ its initial kinetic energy. The total cross-section is given by

$$
\begin{aligned}
\sigma_{\omega} \mathrm{d}\left(\frac{\omega}{E_{1}-m}\right)= & \int \mathrm{d} \sigma \mathrm{d} \Omega_{2} \mathrm{~d} \Omega_{\omega} \\
= & A \frac{\mathrm{d} \omega}{\omega} \frac{p_{2}}{p_{1}}\left\{\frac{4}{3}-2 E_{1} E_{2} \frac{p_{2}^{2}+p_{1}^{2}}{p_{2}^{2} p_{1}^{2}}+m^{2}\left(\frac{\varepsilon_{1} E_{2}}{p_{1}^{3}}+\frac{\varepsilon_{2} E_{1}}{p_{2}^{3}}-\frac{\varepsilon_{1} \varepsilon_{2}}{p_{1} p_{2}}\right)\right. \\
& +L\left[\frac{8}{3} \frac{E_{1}}{p_{1}} \frac{E_{2}}{p_{2}}+\frac{\omega^{2}}{p_{1}^{3} p_{2}^{3}}\left(E_{1}^{2}+E_{2}^{2}+p_{1}^{2} p_{2}^{2}\right)\right] \\
& \left.+\frac{m^{2} \omega}{2 p_{1} p_{2}}\left(\frac{E_{1} E_{2}+p_{1}^{2}}{p_{1}^{3}} \varepsilon_{1}-\frac{E_{1} E_{2}+p_{2}^{2}}{p_{2}^{3}} \varepsilon_{2}+\frac{2 \omega E_{1} E_{2}}{p_{2}^{2} p_{1}^{2}}\right)\right\}
\end{aligned}
$$

where

$$
\begin{aligned}
L & =\log \frac{p_{1}^{2}+p_{1} p_{2}-E_{1} \omega}{p_{1}^{2}-p_{1} p_{2}-E_{1} \omega}=2 \log \frac{E_{1} E_{2}+p_{1} p_{2}-m^{2}}{m \omega} \\
\varepsilon_{1} & =\log \frac{E_{1}+p_{1}}{E_{1}-p_{1}}=2 \log \frac{E_{1}+p_{1}}{m} ; \quad \varepsilon_{2}=2 \log \frac{E_{2}+p_{2}}{m}
\end{aligned}
$$

and

$$
A=\frac{Z^{2} r_{0}^{2}}{137}
$$

Discussion of the cross-section

1. As in the case of the production of continous $X$-ray beams, where primary energies are small, i.e. in the non-relativistic region, $E_{1} \simeq E_{2} \simeq m$ we can neglect $p_{2}$ and $\omega$ compared to $m$. The differential cross-section is then given by

$$
\begin{aligned}
\mathrm{d} \sigma= & \frac{2 Z^{2} e^{4}}{\pi 137} \frac{\mathrm{d} \omega}{\omega} \frac{p_{2}}{p_{1}} \frac{\sin \theta_{2} \mathrm{~d} \theta_{2} \sin \theta_{1} \mathrm{~d} \theta_{1} \mathrm{~d} \varphi}{Q^{4}} \\
& \left\{p_{2}^{2} \sin ^{2} \theta_{2}+p_{1}^{2} \sin ^{2} \theta_{1}-2 p_{1} \cdot p_{2} \sin \theta_{1} \sin \theta_{2} \cos \varphi\right\} .
\end{aligned}
$$


For a given angle between $p_{1}$ and $p_{2}$, the maximum intensity of emitted radiation is perpendicular to the plane of motion of the electron. This corresponds to the classical theory where there is maximum emission perpendicular to the plane of acceleration. The intensity in a certain direction $\theta_{1}$ is obtained by integrating the above expression over all directions of $p_{2}$. The total cross-section for emission of the quantum $\omega$ is given by

$$
\begin{aligned}
\sigma_{\omega} \mathrm{d}\left(\frac{\omega}{T_{0}}\right) & =A \frac{16}{3} \frac{\mathrm{d} \omega}{\omega} \frac{m^{2}}{p_{1}^{2}} \log \frac{p_{1}+p_{2}}{p_{1}-p_{2}} \\
& =A \frac{8}{3} \mathrm{~d}\left(\frac{\omega}{T_{0}}\right) \frac{m}{\omega} \log \left\{\frac{\left(V_{0}+V T_{0}-\omega\right)^{2}}{\omega}\right\}
\end{aligned}
$$

where $T_{0}=E_{1}-m=p_{1}^{2} / 2 m$, the initial kinetic energy of the electron. At the limit $\omega=p_{1}^{2} / 2 m, \sigma_{\omega}$ appears to vanish but this result is true only in the case of a pure Coulomb field and when Born approximation is valid.

2. For a low energy electron, the Born approximation is no longer valid. Sommerfeld has computed the correction factor

$$
\frac{\xi_{1}}{\xi_{2}} \times \frac{1-\mathrm{e}^{-2 \pi \xi_{1}}}{1-\mathrm{e}^{-2 \pi \xi_{2}}} ; \xi_{2}=\frac{Z e^{2}}{v_{2}}, \quad \xi_{1}=\frac{Z e^{2}}{v_{1}}
$$

which has to be applied to the total cross-section $v_{1}$ and $v_{2}$ being the velocities before and ofter collision. This correction contributes for large values of $\xi$, and the correction becomes appreciable for heavy elements.

3. The charge distribution of the outer electrons of an atom produces a screening of the Coulomb field of the nucleus which is appreciable for high primary energies. The impact parameter is

$$
r_{\max }=\frac{1}{Q_{\min }}=\frac{1}{m} \frac{2 E_{1} E_{2}}{m \omega} \sim \lambda_{0} \frac{E_{1} E_{2}}{m \omega}
$$

(i.e. a minimum value of $Q$ leads to a large increase in the cross-section) and for a given ratio of $\frac{\omega}{E_{2}}$, the higher the primary energy the larger is $r_{\max }$. If $\omega \sim E_{2}, r_{\max } \simeq$ radius of the $K$ shell for $E_{1} \sim \frac{137 m}{Z}$. Thus for high energies screening of the Coulomb field leads to a decrease in the cross-section. Assuming the value obtained from the Thomas-Fermi model for the atomic radius,

$$
a \sim a_{0} Z^{-1 / 3} \sim 137 \lambda_{0} Z^{-1 / 3}
$$

where $a_{0}$ is the Bohr radius of the hydrogen atom and if $r_{\max }$ is large compared to $a$ (i.e. in the case of complete screening) we may replace $r_{\max }$ by ' $a$ ' and the cross-section thus obtained will be of the right order. In the case of complete screening the formula becomes

$$
\sigma_{\omega} \mathrm{d}\left(\frac{\omega}{E}\right)=2 A \frac{\mathrm{d} \omega}{\omega} \frac{E_{2}}{E_{1}}\left\{\left[\frac{E_{1}^{2}+E_{2}^{2}}{E_{1} E_{2}}-\frac{2}{3}\right] 2 \log \left(183 Z^{-1 / 3}\right)+\frac{2}{9}\right\}
$$

for $\frac{E_{1} E_{\mathbf{2}}}{m \omega} \gg \frac{a}{\lambda_{0}}$. 
4. In the extreme relativistic case ${ }^{1}\left(E_{1} E_{2} \gg m\right)$ and for large angles $\left(\theta_{1} \gg \frac{m}{E_{1}}\right)$ the intensity of radiation emitted in the energy interval $\mathrm{d} \omega$ at $\omega$ in a solid angle $\mathrm{d} \Omega_{\omega}$ at an angle $\theta$ is proportional to $\omega \mathrm{d} \omega_{\sigma}$ and is given by

where

$$
\omega \mathrm{d} \omega_{\sigma}=E_{1} A \mathrm{~d}\left(\frac{\omega}{E_{1}}\right) \mathrm{d} \Omega_{\omega}\left(\frac{m}{E_{1}}\right)^{2} \frac{1}{4 \pi} T
$$

$$
\begin{aligned}
& A=Z^{2} / 137\left(e^{2} / m^{2}\right)^{2} \\
& T=\left(1+\gamma^{2}\right) \log \frac{2 E_{1}}{m}\left[\frac{\cot ^{2} \theta / 2}{\sin ^{2} \theta / 2}\right]+g(\gamma, \theta)
\end{aligned}
$$

and $\gamma=E_{2} / E_{1}$. In $T$ the angular dependence has been separated out as $g(\gamma, \theta)$ which depends only on the ratios of energy, from the other term depending directly on the absolute value of $E_{1}$. Screening can be neglected only if

$$
\theta \gg\left\{\left[\frac{2 m E_{2}}{\omega E_{1}}\right]\left[\frac{Z^{1 / 3}}{137}\right]\right\}^{1 / 2} \text {. }
$$

For zero angle scattering, i.e. for radiation in the forward direction, the crosssection is given by

$$
\omega \mathrm{d} \omega_{\sigma ; \theta_{=0}}=E_{1} A \mathrm{~d} \Omega_{\omega} \mathrm{d}\left(\frac{\omega}{E_{1}}\right) \frac{4}{\pi}\left\{\frac{E_{1}^{2}+E_{2}^{2}}{m^{2}} \log \frac{2 E_{1} E_{2}}{m \omega}-\frac{\left(E_{1}+E_{2}\right)^{2}}{2 m^{2}}\right\}
$$

with the restriction

$$
E_{1}, E_{2} \gg m \text { and } \frac{2 E_{1} E_{2}}{m \omega} \ll 137 Z^{-1 / 3} .
$$

\section{Pair production}

A photon of energy greater than $2 m$ can create an electron-positron pair when passing through the field of a nucleus. The presence of a nucleus is necessary to conserve energy and momentum.

There are two ways in which this process can happen.

(1) The incoming photon creates a pair and subsequently the electron interacts with the field of the nucleus.

(2) The photon creates a pair and the positron interacts with the field of the nucleus. As in Bremsstrahlung, we assume that the nucleus is stationary. The Feynman diagrams are as shown below. Comparing these diagrams with those of Bremsstrahlung we find that the diagrams look alike except for the ordering in time. For example in the diagram $(R)$ the particle of momentum $p_{1}$ is scattered by Coulomb potential and then emits the photon. However when the ordering in time is taken into account, we observe that

1 P. V. C. Hough, Phys. Rev. 74, 81 (1948). 
(1) $P_{1}$ is a positron, i.e. an electron travelling backward in time,

(2) The photon $\mathrm{q}$ is absorbed.

Hence the Bremsstrahlung matrix elements can be used for this process if $p_{1}$ is replaced by $-p_{+}$(where $p_{+}$is the positron momentum) and $q$ by $-q_{-}{ }^{1}$. The density of final states here is different since the particles in the final state are a positron

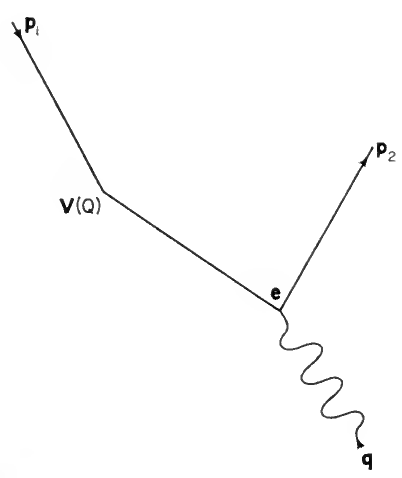

$(R)$

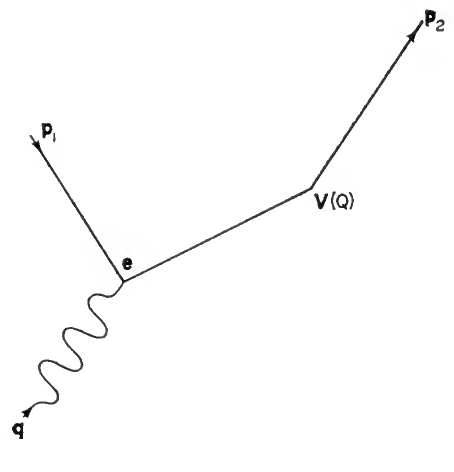

(S)

Fig. 8. Feynman diagrams for pair production by a photon in Coloumb field.

and an electron. It should be noticed that the density of final states in this case corresponds to a three-particle system as in the case of Bremsstrahlung. Thus the differential cross-section is given by

$$
\begin{aligned}
\mathrm{d} \sigma= & -\frac{1}{2 \pi}\left(\frac{Z e^{2}}{Q^{2}}\right)^{2} \frac{1}{137} \frac{p_{+} p_{-} \sin \theta_{+} \mathrm{d} \theta_{+} \sin \theta_{-} \mathrm{d} \theta_{-} \mathrm{d} \varphi \mathrm{d} E_{+}}{\omega^{3}} \\
& {\left[\frac{p_{-}^{2} \cdot \sin ^{2} \theta_{-} \cdot\left(4 E_{+}^{2}-Q^{2}\right)}{\left(E_{-}-p_{-} \cos \theta_{-}\right)^{2}}+\frac{p_{+}^{2} \cdot \sin ^{2} \theta_{+} \cdot\left(4 E_{-}^{2}-Q^{2}\right)}{\left(E_{+}-p_{+} \cos \theta_{+}\right)^{2}}\right.} \\
& +\frac{2 p_{+} p_{-} \sin \theta_{+} \sin \theta_{-} \cos \varphi \cdot\left(4 E_{+} E_{-}+Q^{2}-2 \omega^{2}\right)}{\left(E_{-}-p_{-} \cos \theta_{-}\right)\left(E_{+}-p_{+} \cos \theta_{+}\right)} \\
& \left.-\frac{2 \omega^{2}\left(p_{-}^{2} \sin ^{2} \theta_{-}+p_{+}^{2} \sin ^{2} \theta_{+}\right)}{\left(E_{-}-p_{-} \cos \theta\right)\left(E_{+}-p_{+} \cos \theta_{+}\right)}\right]
\end{aligned}
$$

where $p_{-}$and $p_{+}$are the momenta of the electron and the positron, $E_{-}$and $E_{+}$ their energies, $\theta_{-}$and $\theta_{+}$the angles which the directions of the electron and positron make with the direction of the photon, $\varphi$ is the angle between the electronphoton plane and positron-photon plane and $\boldsymbol{Q}^{2}=\left(\boldsymbol{q}-\boldsymbol{p}_{+}-\boldsymbol{p}_{-}\right)^{2}$. This formula

1 This, as well as the fact mentioned earlier that the matrix elements for Compton scattering and the two-photon pair annihilation are connected, are special cases of what is known as the "substitution law" which is discussed in more detail in Chapter V. See also J. M. JAUCH and F. RoHrLich, The Theory of Photons and Electrons, Addison-Wesley (1955), p.161. 
has been obtained from the corresponding one for Bremsstrahlung by the following substitutions :

$p_{-}$for $p_{2},-p_{+}$for $p_{1}, E_{-}$for $E_{2},-E_{+}$for $E_{1},-\theta_{-}$for $\theta_{2},-\theta_{+}$for $\theta_{1}$ and $-\omega$ for $\omega$.

Integration over the angles gives the cross-section. In the extreme relativistic case, where all energies are large compared to the rest energy of the electron, the total cross-section becomes

$$
\sigma_{E_{+}} \mathrm{d} E_{+}=4 A \mathrm{~d} E_{+} \frac{E_{+}^{2}+E_{-}^{2}+2 / 3 E_{+} E_{-}}{\omega^{3}}\left(\log \frac{2 E_{+} E_{-}}{\omega m}-\frac{1}{2}\right) .
$$

The limits of validity

The use of Born approximation demands that

$$
\frac{2 \pi Z e^{2}}{v_{+}} \ll 1, \frac{2 \pi Z e^{2}}{v_{-}} \ll 1
$$

i.e. the velocities of both electrons must be large and $Z$ small. If $v_{+}, v_{-} \ll$ velocity of light, Born approximation can no longer be applied and the Sommerfeld form factor $\frac{2 \pi \xi_{+} \xi_{-}}{\left(\mathrm{e}^{2 \pi \xi_{+}}-1\right)\left(1-\mathrm{e}^{-2 \pi \xi_{-}}\right)}$is introduced and this produces an asymmetry in the expression. The positron is repelled by the nucleus and the electron attracted. The probability for pair creation gets small for small $p_{+}$and large for small $p_{-}$. A similar correction has to be made in the relativistic case for heavy elements (i.e. large $Z$ values).

(2) The charge distribution of the outer electrons produces a screening of the Coulomb field of the nucleus. This effect is appreciable only at high primary energy. As in the case of Bremsstrahlung, the expression is valid for $\frac{2 E_{+} E_{-}}{\omega m} \ll 137 Z^{-1 / 3}$. (3) When $E_{+}, E_{-}>m$, and for large angles, the limit is given by

$$
\mathrm{d} \sigma_{-}=A \mathrm{~d}\left(\frac{E_{-}}{\omega}\right) \mathrm{d} \Omega_{-}\left(\frac{m}{\omega_{2}}\right) \frac{1}{4 \pi} S
$$

where $A=\frac{Z^{2}}{137}\left(\frac{e^{2}}{m}\right)^{2}$ and $S$ contains the angular variation. The above is the probability per nucleus of pair production in a solid angle $\mathrm{d} \Omega$ at an angle $\theta\left(=\theta_{-}\right)$and in the energy interval $d E_{-}$at energy $E_{-}$and $S=(1+\gamma)^{2} \log \frac{2 \omega}{m}\left(\frac{\cot ^{2} \theta / 2}{\sin ^{2} \theta / 2}\right)+f(r, \theta)$. It can be seen that in $S$, we have separated the term depending on $E_{-}$directly, the other term $f(r, \theta)$ depending only on $E_{-} / \omega$ and $\gamma=\frac{E_{+}}{E_{-}}$. This is valid only when $E_{+}, E_{-} \gg m$ and $\theta \gg \frac{m}{E_{-}}$.

Momentum transfer in a production process is large enough for screening to 
be neglected, provided $\theta \gg\left(\frac{2 m E_{+}}{p E_{-}} \frac{Z^{1 / s}}{137}\right)^{1 / 2}$. Hence the limitation on $\theta$ is of the same magnitude of $\frac{2 E_{+} E_{-}}{\omega m} \sim 137 Z^{-1 / 3}$ which is the boundary region where screening becomes important in the single differential cross-section. Extension of (55) to smaller angles by exact integration is valid only at energies for which screening is unimportant in the single differential cross-section. At high energies, the small angle formula requires numerical integration as the form factor for the atom is introduced. But for zero angle scattering at high energies, there is no azimuthal dependence of triple differential cross-section. The cross-section (unscreened) is given by

$$
\left(\mathrm{d} \sigma_{-}\right)_{\theta=0}=A \mathrm{~d} \Omega_{-} \mathrm{d}\left(\frac{E_{-}}{\omega}\right) \frac{4}{\pi} \frac{E_{-}^{2}}{\omega^{2}}\left[\frac{E_{+}^{2}+E_{-}^{2}}{2 m^{2}} \log \frac{E_{+} E_{-}}{\omega m}+\frac{\left(E_{+}+E_{-}\right)^{2}}{2 m^{2}}\right]
$$

the limitations being

$$
E_{+}, E_{-} \gg m \text { and } \quad \frac{2 E_{+} E_{-}}{\omega m} \ll 137 Z^{-1 / 3} .
$$

Thus the average angle into which the electrons are emitted is of the order of $\theta \sim \frac{m c^{2}}{\omega}$ and for smaller energies the concentration in the forward direction is less marked. For large momentum changes, there is a modification of the Coloumb field resulting from the finite extent of the nucleus which will give an appreciable decrease in the cross-section.

The use of the decomposed propagator helps us to study the relative contributions of transitions through positive and negative intermediate states to the matrix element in electrodynamic processes discussed above. Calculations on the Compton effect lead ${ }^{\mathbf{1}}$ to the apparently paradoxical result that the negative energy intermediate states give almost the entire contribution even if the initial electron is non-relativistic while in the extreme relativistic case contributions arise from transitions to both positive and negative energy intermediate states. It is conventionally accepted that when the initial particles are non-relativistic the energy denominators become large for negative energy states which therefore do not contribute. This may lead to erroneous results since as in the Compton effect the numerators may also become large a circumstance which can be revealed only by the use of the decomposed propagator. Since in the case of annihilation and creation of pairs the non-relativistic limit is not possible, contributions are made both by positive and negative energy intermediate states. In Bremsstrahlung, in the non-relativistic limit for the outgoing photon the positive energy intermediate state contributes to a larger extent.

1 Alladi Ramakrishnan, T. K. Radha and R. Thunga, Proc. Ind. Acad. Sci., A, 52, 228 (1960). 


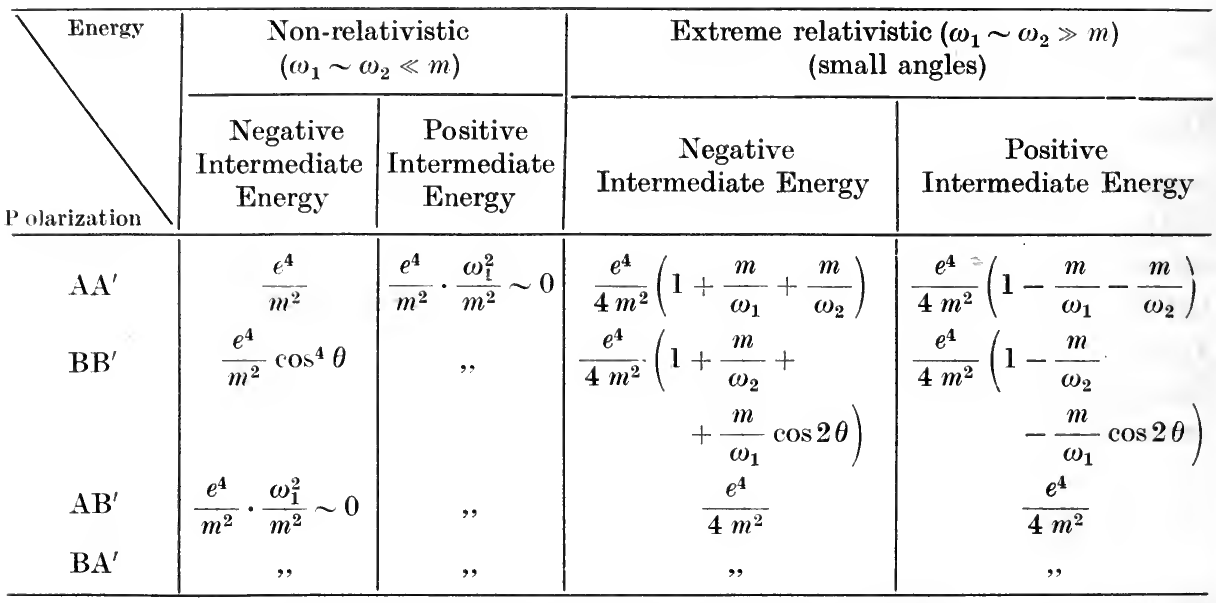

The differential cross-sections for Compton-scattering

\section{Electron-electron interaction}

The quantum electrodynamic processes we have considered till now relate to the scattering of an electron in an electromagnetic field which may be either due to a photon or a potential of a heavy stationary nucleus. We shall now deal with the interaction between two charged particles, in particular two electrons.

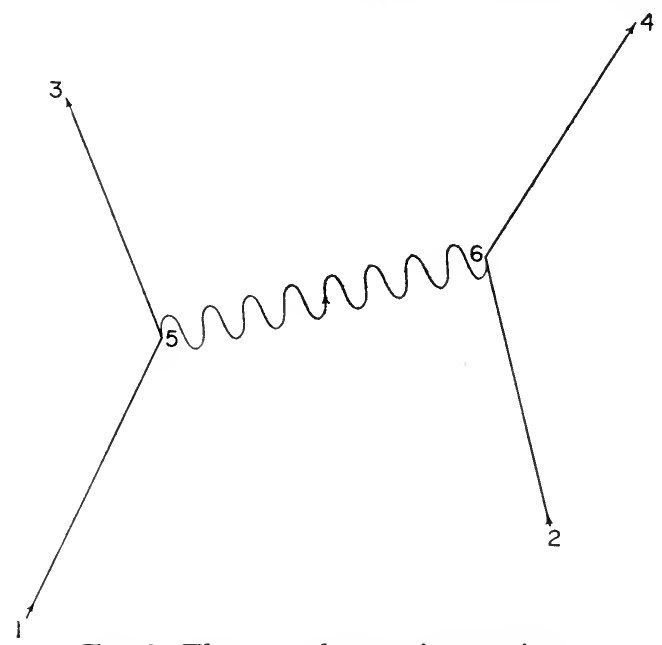

Fig. 9. Electron-electron interaction.

Even if the electrons do not interact we have to deal with a system of two particles which obey the Pauli principle. Hence we define a kernel for a system of two free Fermi particles representing the amplitude that the particles at space-time points 1 and 2 move to 3 and 4. Obviously the kernel should be written in the form

$$
K_{F}(4,3 ; 2,1)=K_{F}^{a}(3,1) K_{F}^{b}(4,2)-K_{F}^{a}(4,1) K_{F}^{b}(3,2)
$$


where for convenience we have labelled the electron at 1 as ' $a$ ' and that at 2 as ' $b$ '. Hereafter we shall consider only one of the two terms comprising $K_{F}$ ' remembering that the total amplitude can always be obtained by interchanging the proper space-time variables, making the corresponding changes in sign and summing the amplitudes so obtained.

Assuming now an interaction defined by a potential between the two particles, we are first tempted to write the perturbed kernel in the first order approximation as $\begin{aligned} K_{F}^{A}(4,3 ; 2,1) & =K_{F}(4,3 ; 2,1)-i \int K_{F}(4,3 ; 5,6) V(5,6) K_{F}(6,5 ; 2,1) \mathrm{d}(5) \mathrm{d}(6) \\ & =K_{F}(4,3 ; 2,1)-K_{F}^{1}(4,3 ; 2,1) .\end{aligned}$

If we assume only a space dependent potential $V(5,6)$ it should contain the factor $\delta\left(t_{6}-t_{5}\right)$ (i.e. the interaction is instantaneous). In the case of electrons we write $V(5,6)=\frac{e^{2}}{\left(r_{5}-r_{6}\right)} \delta\left(t_{5}-t_{6}\right)$. However if the potential associated with the interral $(5,6)$ is not instantaneous but is required to satisfy the demands of relativity, we must replace $\delta\left(t_{5}-t_{6}\right)$ by

$$
\delta\left(t_{5}-t_{6}-\overline{r_{5}-r_{6}}\right)+\delta\left(t_{5}-t_{6}+\overline{r_{5}-r_{6}}\right)=\delta(t-r)+\delta(t+r) .
$$

This implies that $\left|t_{5}-t_{6}\right|$ is the time interval for the potential to travel with the velocity of light. But this form of the $\delta$ function is not quite correct. Its Fourier transform contains both positive and negative frequencies. Since it is natural to identify any function occurring between $K_{F}(4,3 ; 6,5)$ and $K_{F}(6,5 ; 2,1)$ as the propagator of a photon the transform of this propagator should contain only positive frequencies. We choose instead of $\frac{1}{r}[\delta(t-r)+\delta(t+r)]$

$$
\frac{1}{r}\left[\delta_{+}(t-r)+\delta_{+}(t+r)\right]=2 \delta_{F}\left(t^{2}-r^{2}\right)=2 \delta_{F}\left(s^{2}\right)
$$

where

$$
\delta_{F}\left(s^{2}\right)=\int_{0}^{\infty} \mathrm{e}^{-i \omega s^{2}} \frac{\mathrm{d} \omega}{\pi} .
$$

The unperturbed kernel can be written as

$$
K_{F}(4,3 ; 2,1)=\iint K_{\dot{F}}(4,3 ; 6,5) K_{F}(6,5 ; 2,1) \mathrm{d}(6) \mathrm{d}(5) .
$$

From (62) we get the first order term of the perturbed kernel to be

$$
K_{F}^{1}=-i e^{2} \int K_{F}(4,3 ; 6,5) \delta_{F}\left(s^{2}\right) K_{F}(6,5 ; 2,1) \mathrm{d}(5) \mathrm{d}(6) .
$$

However even this modification is insufficient since it implies that as far as the electrons are concerned, they are unaffected by the exchange of a photon, the propagator of which is given by $\delta_{+}\left(s^{2}\right)$. To include the effect on the electron we first note that the unperturbed kernel can be written in the equivalent form (considering only one of the terms in (61))

$$
K_{F}=\int K_{F^{\prime}}(4,6) K_{F}(6,2) K_{F}(3,5) K_{F}(5,1) \mathrm{d}(5) \mathrm{d}(6) .
$$


In this picture we are concerned with the amplitude for the electron ' $a$ ' at 1 going to 3 through 5 and (b) at (2) going to (4) through (6). In the perturbed case this amplitude should be altered due to the emission of the photon. This is achieved not merely by the addition of $\delta_{F}\left(s^{2}\right)$ but also by inserting the matrix $\gamma_{\mu}$ in between the kernels of each electron

$$
\begin{gathered}
K_{F}^{1}(3,4 ; 1,2)=-i e^{2} \int K_{F^{\prime}}(4,6) \gamma_{\mu} K_{F^{\prime}}(6,2) \delta_{F^{\prime}}\left(s^{2}\right) K_{F}(3,5) \gamma_{\mu} \\
K_{F}(5,1) \mathrm{d}(5) \mathrm{d}(6) .
\end{gathered}
$$

We are led to the choice of $\gamma_{\mu}$ on using the following argument. Since in classical electrodynamics there is also an interaction through the vector potential, the complete interaction should be $\left[1-\left(V_{5} \cdot V_{6}\right)\right] \delta_{F}\left(s_{56}^{2}\right)$ or in the relativistic case

$$
\left(1-\alpha_{a} \cdot \alpha_{b}\right) \delta_{F}\left(s_{56}^{2}\right)=\beta_{a} \beta_{b} \gamma_{a \mu} \gamma_{b \mu} \delta_{F}\left(s^{2}\right)
$$

where the suffices $a$ and $b$ indicate that the quantities "belong" to the particles $a$ and $b$ respectively. Hence we have for electrons obeying the Dirac equation

$$
\begin{array}{r}
K_{F}^{1}(3,4 ; 1,2)=-i e^{2} \int K_{F}^{a}(3,5) K_{F}^{b}(4,6) \gamma_{a_{\mu}} \gamma_{b_{\mu}} \\
\delta_{F}\left(s^{2}\right) K_{F}^{a}(5,1) K_{F}^{b}(6,2) \mathrm{d}(5) \mathrm{d}(6)
\end{array}
$$

where $\gamma_{a \mu}$ and $\gamma_{b \mu}$ are the Dirac matrices applying to the spinors corresponding to the particles $a$ and $b$ respectively. We can drop the suffices $a$ and $b$ if we write the kernel in the form (68).

The expression (70) for the perturbed kernel can be derived rigorously if we assume that the two electrons are perturbed by a photon field. The amplitude that the electron ' $a$ ' goes from 1 to 5 , emits a photon of polarization $e_{\mu}$ with a momentum $\boldsymbol{k}$ at $t_{5}$, and then goes to 3 , is

$$
\left[K_{F}(3,5) e_{\mu} \gamma_{\mu} / \frac{4 \pi e^{2}}{2 k_{14}} \mathrm{e}^{-i k_{1} \cdot r_{5}} \mathrm{e}^{i k_{14} t_{5}} K_{F}(5,1)\right]_{a} .
$$

Similarly the amplitude that the electron ' $b$ ' goes from 2 to 6 , absorbs a photon of polarization $e_{\eta}$ and momentum $\boldsymbol{k}_{2}$ at $t_{6}$ and then goes to 4 , is

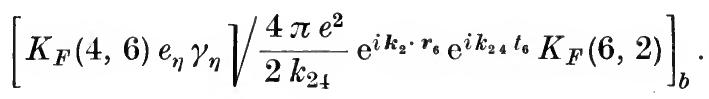

If there has to be an interaction between the two electrons ' $a$ ' and ' $b$ ', then ' $b$ ' must absorb the same photon emitted by ' $a$ '. This is guaranteed if the amplitude for both these processes to happen is taken as the product of the two amplitudes (71) and (72) with $k_{1} \equiv k_{2}, \eta \equiv \mu$. The total amplitude for the exchange of any photon between 5 and 6 is obtained by assuming all the polarization vectors to be unity and summing over photon polarization and momentum and integrating over $t_{5}$ and $t_{6}$. Thus the amplitude is

$$
\begin{gathered}
4 \pi e^{2} \sum_{u} \int \mathrm{e}^{-i k \cdot\left(r_{5}-r_{6}\right)} \mathrm{e}^{i k_{4}\left(t_{5}-t_{6}\right)}\left[K_{F}(3,5) \gamma_{\mu} K_{F}(5,1)\right]_{a} \\
{\left[K_{F}(4,6) \gamma_{\mu} K_{F}(6,2)\right]_{b} \frac{1}{2 k_{4}} \frac{\mathrm{d}^{3} k}{(2 \pi)^{3}} \mathrm{~d} t_{5} \mathrm{~d} t_{6} \text { for } t_{6}>t_{5} .}
\end{gathered}
$$


When $t_{6}<t_{5}$ the amplitude is obtained by reversing the signs of the exponents. We have only a three dimensional integration as the fourth component $k_{4}$ of the four vector, $k$ is related to $\boldsymbol{k}$. As in the case of the electron the summation over all the momenta (with equal weight) gives the propagator $\delta_{F}\left(s^{2}\right)$. Thus

$$
\delta_{F}\left(s^{2}\right)=4 \pi \int \mathrm{e}^{-k \cdot\left(\boldsymbol{r}_{5}-\boldsymbol{r}_{6}\right)} \mathrm{e}^{i k_{4}\left(t_{5}-t_{6}\right)} \frac{1}{(2 \pi)^{3}} \frac{\mathrm{d}^{3} k}{2 k_{4}} .
$$

We can convert the above into a four dimensional integral using the lemma

$$
\mathrm{e}^{-i k_{4}|t|}=\underset{\varepsilon \rightarrow 0}{L} \int_{-\infty}^{\infty} \frac{2 i k_{4}}{\left(\omega^{2}-k_{4}^{2}+i \varepsilon\right)} \mathrm{e}^{-i \omega t} \frac{\mathrm{d} \omega}{2 \pi}
$$

Thus $\delta_{+}\left(s^{2}\right)$ is the inverse of

$$
4 \pi(-i) \int_{-\infty}^{\infty} \frac{\mathrm{e}^{i k \cdot\left(x_{5}-x_{6}\right)}}{k_{4}^{2}-\boldsymbol{k}^{2}+i \varepsilon} \mathrm{d}^{4} k=4 \pi(-i) \int \frac{\mathrm{e}^{i k \cdot\left(x_{5}-x_{6}\right)}}{k^{2}+i \varepsilon} \mathrm{d}^{4} k .
$$

Note that $k_{4}$ and $\boldsymbol{k}$ do not have the energy-momentum relationship though they form a four vector. Thus the momentum representation of $\delta_{F}\left(s^{2}\right)$ is $(-i) 4 \pi / k^{2}$.

It may be intriguing that in this derivation we assumed that the perturbation of the kernel acts through a real photon. But it should be remembered that the summation over all these photon functions yielded the kernel of the photon and that the final expression is only a kernel. But in the case of interaction between two electrons we remind ourselves that we require the amplitude for transitions between states of real electrons. These amplitudes are obtained by multiplying the perturbed kernel by wave functions and their conjugates corresponding to the incident (at 1,2) and emergent (at 3,4) particles and integrating over the points $1,2,3,4$, and are non-vanishing only if the momentum of the photon is equal to the difference between the four momenta of the electrons i.e. $q$ can represent a virtual photon.

We shall make a digression and discuss the propagator of a photon in comparison with that of an electron. Examining the definition of $\delta_{F}\left(s^{2}\right)$ we note that we have taken the energy of a photon $k_{4}$ to be always positive. When $\left.t=t_{5}-t_{6}\right)$ is negative, we have replaced $k_{4}$ by $-k_{4}$ only in the exponent. But we can also replace the term $2 k_{4}$ in the denominator by $-2 k_{4}$ and attach a minus sign to the integrand. Then we immediately recognise that when $t_{6}<t_{5}$ we can interpret the absorption of the positive energy photon at $t_{5}$ as the emission of a negative energy photon at $t_{5}$ and in a similar way the emission of a positive energy photon at $t_{6}$ as the absorption of a negative energy photon at $t_{6}$. Thus we can always ascribe only emission of photons (both of positive and negative energy) to the vertex $t_{5}$ and absorption of photons at the vertex $t_{6}$. This implies the propagation of a positive energy photon for $t_{5}<t_{6}$ which is "forward" in time and the propagation of a negative energy photon for $t_{5}>t_{6}$ which is "backward" in time. Thus the procedure is identical with that of the electron except that we do not have a term arising from the spinors and the mass $m$ is zero in this case. 
In this point of view the minus sign occurs before the sum over negative energies just as in the case of the electron propagator. We have earlier argued that this minus sign is intimately related to the Pauli principle which has to be invoked in dealing with a second order scattering of electron involving the creation of a virtual pair. But we know that photons are bosons and the occurrence of minus sign may seem perplexing. The question also arises why the postulate of a negative energy sea has not to be invoked even in the boson case to explain the negative sign. Perhaps the answer may be in that we always assume an interaction which is bilinear in the fermion field variables while linear in the boson field variables. The bilinear nature implies that the destruction of a negative energy particle which is equivalent to the creation of a positive energy antiparticle should be associated with the creation of a positive energy particle. Thus the propagator of a negative energy electron from $\mathrm{A}$ to $\mathrm{B}$ can be associated with the destruction of a real electron at $A$ and creation of a real electron at $B$ and the negative sign plays a role in the statistics of these electrons. A similar circumstance does not arise in the case of the photon because of the linearity of the interaction in the photon field.

We shall now obtain the matrix element for electron-electron scattering. The two diagrams corresponding to this process are

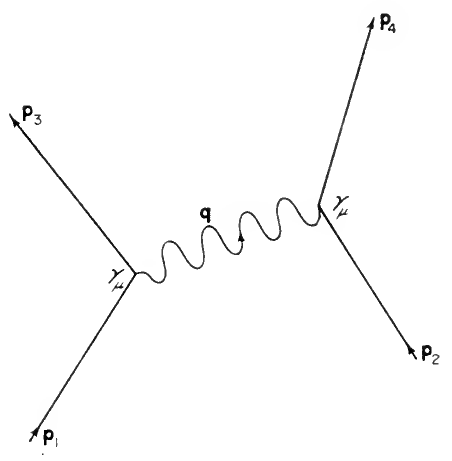

$\mathbf{q}=\mathbf{p}_{3}-\mathbf{p}_{1}=\mathbf{p}_{2}-\mathbf{p}_{4}$

(R)

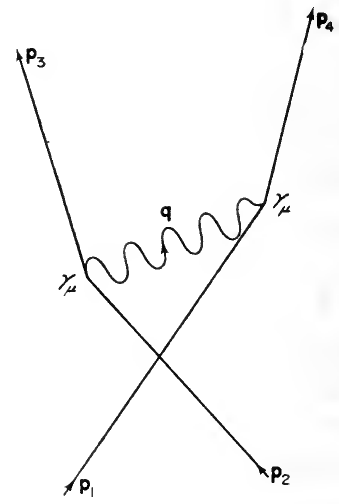

$q=p_{2}-p_{3}=p_{4}-p_{1}$

(S)

FIG. 10. Feynman diagrams for the electron-electron scattering.

The amplitude for the process is given by

$$
e^{2} \sum_{\mu} \int\left[K_{F}(3,5) \gamma_{\mu} K_{F}(5,1)\right]_{a} \frac{4 \pi}{q^{2}}\left[K_{F}(4,6) \gamma_{\mu} K_{F}(6,2)\right]_{b} \frac{\mathrm{d}^{4} q}{(2 \pi)^{4}} \mathrm{~d}(5) \mathrm{d}(6) \text {. }
$$

The states at 1, 2, 3 and 4 are free particle states represented by plane waves. In momentum representation the spinor parts of the brackets are $\bar{u}_{3} \gamma_{\mu} u_{1}$ and 
$\bar{u}_{4} \gamma_{\mu} u_{2}$. Integration over (5) and (6) gives conservation of four-momenta. Thus the matrix element $M$ is given by

$$
M=4 \pi e^{2}\left[\frac{\left(\bar{u}_{4} \gamma_{\mu} u_{2}\right)\left(\bar{u}_{3} \gamma_{\mu} u_{1}\right)}{\left(\mathrm{P}_{3}-\mathrm{P}_{1}\right)^{2}}-\frac{\left(\bar{u}_{4} \gamma_{\mu} u_{1}\right)\left(\bar{u}_{3} \gamma_{\mu} u_{2}\right)}{\left(\mathrm{P}_{4}-\mathrm{P}_{1}\right)^{2}}\right] .
$$

The first and second terms come from $R$ and $S$ respectively. The summation. over $\mu$ is implied. We shall now show that this is exactly equivalent to an instantaneous Coulomb interaction and exchange of transverse photons. We choose one of the space directions parallel to the photon momentum $\boldsymbol{q}$ and the other two transverse to it. We can now split the matrix element as

We note that

$$
\begin{aligned}
\frac{M}{4 \pi e^{2}}=\left(\bar{u}_{4} \gamma_{t} u_{2}\right) \frac{1}{q^{2}}\left(\bar{u}_{3} \gamma_{t} u_{1}\right) & -\left(\bar{u}_{4} \gamma_{Q} u_{2}\right) \frac{1}{q^{2}}\left(\bar{u}_{3} \gamma_{Q} u_{1}\right) \\
& -\sum_{1,2 t r}\left(\bar{u}_{4} \gamma_{t r} u_{2}\right) \frac{1}{q^{2}}\left(\bar{u}_{3} \gamma_{t r} u_{1}\right) .
\end{aligned}
$$

Substituting

$$
\bar{u}_{4} \mathrm{q} u_{2}=\bar{u}_{4}\left(\mathrm{p}_{2}-\mathrm{P}_{4}\right) u_{2}=m\left(\bar{u}_{2} u_{4}-\bar{u}_{4} u_{2}\right)=0
$$

$$
\begin{aligned}
\frac{M}{4 \pi e^{2}} & =\left(\bar{u}_{4} \gamma_{t} u_{2}\right) \frac{1}{q^{2}}\left(1-q_{4}^{2} / Q^{2}\right)\left(\bar{u}_{3} \gamma_{t} u_{1}\right)-\sum_{1,2 t r}\left(\bar{u}_{4} \gamma_{t r} u_{2}\right) \frac{1}{q^{2}}\left(\bar{u}_{3} \gamma_{t r} u_{1}\right) \\
& =-\left(\bar{u}_{4} \gamma_{t} u_{2}\right) \frac{1}{Q^{2}}\left(\bar{u}_{3} \gamma_{t} u_{1}\right)-\sum_{1.2 t r}\left(\bar{u}_{4} \gamma_{t r} u_{2}\right) \frac{1}{q^{2}}\left(\bar{u}_{3} \gamma_{t r} u_{1}\right) .
\end{aligned}
$$

The Coloumb potential which has only spatial dependence $A_{4}(|\boldsymbol{r}|)\left(\boldsymbol{A}=0 ; \varphi=Z e^{2} / r\right)$ is the time-like component of the four vector $A$. Thus

$$
\mathrm{A}(r)=\gamma_{t} Z e^{2} / r
$$

Taking the Fourier transform we have

$$
\mathrm{a}(q)=\int \mathrm{A}(r) e^{-i q \cdot x} \mathrm{~d}^{4} q=\gamma_{t} 4 \pi Z e^{2} \delta\left(q_{4}\right) \frac{1}{Q_{2}}
$$

$\delta\left(q_{4}\right)$ occurs due to the time independence of A. ${ }^{1}$

\section{SELF-ENERGYAND RENORMALIZATION}

Once we have envisaged intermediate states where energy and momentum need not be simultaneously conserved, it is clear that we have to take into consideration processes of the following type: A free electron emits a virtual photon

1 It is interesting to consider the problem when the masses of the charged particles are taken as different. In such a case the Pauli principle need not be invoked and in the limit when one of the masses becomes infinite it can be shown that the interaction reduces to that of a pure potential. Recoil corrections can also be studied as in the case of Bremsstrahlung. See Alladi Ramakrishnan, S. Indumathi and G. Bhamathi, Proc. Ind. Acad. Sci., A 53, 206 (1961). 
which it subsequently reabsorbs (this is usually termed interaction with its own radiation field) or a photon creates virtual pairs which subsequently annihilate themselves creating the photon. These are called self-energy processes and we shall consider them below in detail.

A free electron even in the absence of an external electromagnetic field can emit a virtual photon and reabsorb it. The free electron kernel we till now considered is a two-point function which does not include this process. On the other hand, the kernel representing the amplitude of going from one space time point to another when the emission and absorption of photons in between in the Feynman sense is taken into account is the propagator perturbed by the self-field and indeed should be considered the propagator of a physical particle in the absence of an external field. The perturbed propagator for the photon is obtained by the inclusion of the process of creation of a pair by the photon and the annihilation of that pair with the creation of the photon. In a similar manner, the vertex operator has to be modified by the emission and reabsorption of a photon across the vertex.

To obtain the kernels and the vertex operator of the physical particle, we start with the kernel of the bare particle defined by its mass and wave function and use the standard techniques of Feynman theory. Since any free particle kernel is defined through its mass and wave function, the modification in the kernel can be interpreted as changes in mass and wave function. The mass of the physical particle is identified with the mass we observe in the real world. This principle of identifying the attributes of the physical particle with the observable attributes is known as the principle of renormalization. The importance of recognizing this is essentially due to the fact that if the physical mass is to be treated as the bare mass plus a correction arising from the self interaction, the correction turns out to be an infinite quantity. The principle of renormalization asserts that it is after the addition of this infinite quantity that we obtain the finite observed mass of the physical particle. Since the self-energy process involves an emission and an absorption of a photon and therefore contains a factor $e^{2}$ along with the wave function, instead of treating the wave function as modified due to these processes we can take the charge to be modified. Likewise, the modifications in the vertex operator and photon propagator can also be interpreted as suitable modifications in the charge and the principle of renormalization applied to the charge.

It may seem at first sight that the principle of renormalization is merely a justification for taking the observed values for the mass and charge. However it will be shown that it leads to observable effects which are not predicted if these self-energy processes are not taken into account. This is because even after the renormalizations, there exist finite corrections depending on the nature of the process considered. The computation of these corrections can be carried out by the regularization of the infinite integrals which means expressing the infinite integrals as the limits of integrals which can be evaluated and which 
involve an auxiliary parameter with respect to which the limiting process is defined ${ }^{1}$.

We shall now consider these radiative processes in detail ${ }^{2}$.

\section{Self-energy of the electron}

We shall consider the perturbed kernel $K_{F}^{\prime}(2,1)$ to the lowest order of a particle going from 1 to 2 emitting a photon at 3 and absorbing it at 4 . We have

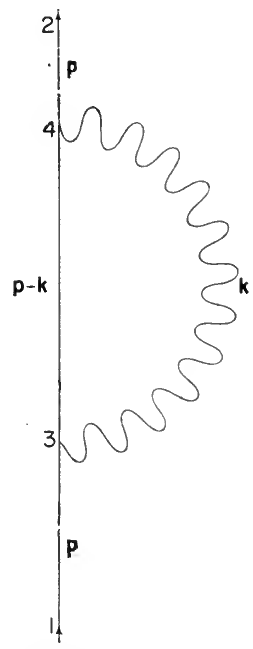

Fig. 11. Feynman diagram for the self energy of an electron.

$$
K_{F}^{\prime}(2,1)=K_{F^{\prime}}(2,1)-i e^{2} \int K_{F}(2,4) \gamma_{\mu} K_{F}(4,3) \gamma_{\mu} K_{F}(3,1) \delta_{F}\left(s_{4,3}^{2}\right) \mathrm{d}(4) \mathrm{d}(3)
$$

or in momentum representation,

where

$$
S_{F}^{\prime}(p)=S_{F}(p)+S_{F}(p) \sum(p) S_{F}(p)
$$

$$
S_{F}(p)=1 /(p-m)
$$

and

$$
\Sigma(p)=-\frac{i e^{2}}{\left(4 \pi^{3}\right)} \int \gamma_{\mu} \frac{\mathrm{l}}{\mathrm{P}-\mathrm{k}-m} \gamma_{\mu} \frac{1}{k^{2}} \mathrm{~d}^{4} k .
$$

$\sum(p)$ can be written in the form

$$
\sum(p)=A+(\mathrm{p}-m) B+(\mathrm{p}-m)^{2} \Sigma_{c}(p)
$$

1 W. Pauli and F. Villars, Rev. Mod. Phys. 21, 434 (1949).

2 In this section we follow closely the treatment of J. M. JAUCH and F. RoHrLICH, The Theory of Photons and Electrons, Addison-Wesley, Cambridge, Mass. (1955). See also F. J. Dyson, Phys. Rev. 75, 486 and 1736 (1949); R. P. Feynman, Phys. Rev. 76, 769 (1949); R. P. Feynman, Lectures delivered at the California Institute of Technology on Quantum Electrodynamics (1953).

EPCR 7 
where

$$
\begin{aligned}
& A=\frac{e^{2}}{2 \pi} m\left[\frac{3}{2} \frac{1}{i \pi^{2}} \int \frac{\mathrm{d}^{4} k}{\left(k^{2}-m^{2}\right)^{2}}+\frac{9}{4}\right] \\
& B=\frac{-e^{2}}{4 \pi}\left[\frac{1}{i \pi^{2}} \int \frac{\mathrm{d}^{4} k}{\left(k^{2}-m^{2}\right)^{2}}-4 \int_{0}^{1} \frac{\mathrm{d} x}{x}+\frac{11}{2}\right]
\end{aligned}
$$

and

with

$$
\begin{aligned}
\sum_{c}(p)= & \frac{e^{2}}{2 \pi m}\left\{\frac{1}{2(1-\varrho)}\left(1-\frac{2-3 \varrho}{1-\varrho} \log \varrho\right)\right. \\
& -\frac{\mathrm{P}+m}{2 m}\left[\frac{1}{2 \varrho(1-\varrho)}\left(-2-\varrho+\frac{4-8 \varrho+\varrho^{2}}{1-\varrho} \log \varrho\right)\right. \\
& \left.\left.+\frac{2}{\varrho} \int_{0}^{1} \mathrm{~d} x\left(\frac{1}{x}-x\right)\right]\right\}
\end{aligned}
$$

$$
\underline{Q}=-\frac{\left(p^{2}-m^{2}\right)}{m^{2}} \text {. }
$$

The integrals $A$ and $B$ are logarithmically divergent and this presented a major obstacle to quantum electrodynamics for many years till the renormalization procedure was adopted. To regularize the integrals Feynman introduced a cut-off factor $\lambda$ such that the photon propagator instead of being $\frac{1}{k^{2}}$ is taken as $\frac{1}{k^{2}}-\frac{1}{k^{2}-\lambda^{2}}$. where $\lambda \rightarrow \infty$. In such a case $A$ and $B$ are obtained as

$$
\begin{aligned}
& A=\frac{3 e^{2}}{2 \pi} m\left[\log \frac{\lambda}{m}+\frac{1}{4}\right] \\
& B=\frac{-e^{2}}{4 \pi}\left[\left(2 \log \frac{\lambda}{m}-1\right)-4 \int_{0}^{1} \frac{\mathrm{d} x}{x}+\frac{11}{2}\right] .
\end{aligned}
$$

To interpret the term $A$, we consider the expansion

$$
S_{F}(p, m+\delta m)=\frac{1}{\mathrm{P}-m}+\frac{1}{\mathrm{P}-m} \delta m \frac{1}{\mathrm{P}-m}+\cdots .
$$

Comparing this expansion with that of $\Sigma(p)$ we identify $A$ to be $\delta m$ which can be considered as the correction to the bare mass $m$. Since $A$ is infinite, we invoke the principle of renormalization and identify $m+\delta m$ to be $m_{0}$, the observed mass which is finite. The perturbed propagator $S_{F}^{\prime}(p, m)$ is now expressed in terms of the physically significant $m_{0}$ as

$$
\begin{aligned}
S_{F}^{\prime}(p, m)=S_{F}\left(p, m_{0}\right) & +S_{F}\left(p, m_{0}\right)\left[(A-\delta m)+\left(\mathrm{p}-m_{0}\right) B\right. \\
& \left.+\left(\mathrm{p}-m_{0}\right)^{2} \sum_{c}(p)\right] S_{F}\left(p, m_{0}\right)^{1} .
\end{aligned}
$$

The above prescription for writing $S_{F}^{\prime}(p, m)$ can be stated in the following manner.

\footnotetext{
1 In writing this, we must remember that $\delta m$ occurs with a factor $e^{2}$ and terms of the order $e^{4}$ and higher are neglected.
} 
A theory using the bare mass and including self action is equivalent to a theory using the observed mass plus self action minus $\delta m$ computed as a correction to the bare mass $m$ of a free particle.

The remaining terms in the expansion of $S_{F}^{\prime}(p, m)$ can be written as $(1+B)$ $\left[S_{F}\left(p, m_{0}\right)+\Sigma_{c}\left(p, m_{0}\right)\right]$ to order $e^{2}$. Since the propagator $S_{F}(p)$ is the transform of the kernel $\sum_{n} \psi_{n}(2) \bar{\psi}^{n}(1), S_{F}^{\prime}(p, m)$ can be considered as the corresponding transform of a kernel with a re-defined wave function. (in momentum space)

$$
\psi_{n}^{\prime}=(1+B)^{1 / 2} \psi_{n} .
$$

Since these wave functions occur in association with the charge $e$ in any perturbation expansion, we can retain the form of $\psi$ by attaching the factor $(1+B)^{1 / 2}$ to the unrenormalized charge $e$. This may be shown in the following way. Consider an $n$th order diagram in any quantum electrodynamic process containing $\mathscr{E}_{e}$ external electron lines and $\mathscr{E}_{i}$ internal electron lines. There are two electron lines coming into each corner or the number of electron line endings is $2 n$. But each internal electron line has two endings and each external electron line one ending. Thus

$$
2 n=2 \mathscr{E}_{i}+\mathscr{E}_{e} \quad \text { or } \quad \mathscr{E}_{i}=n-\frac{\mathscr{E}_{e}}{2} .
$$

The second order radiative corrections are taken into account by replacing every $S_{F}(p)$ by $(1+B)\left[S_{F^{\prime}}(p)+\sum_{c}(p)\right]$, every $\psi$ by $(1+B)^{1 / 2} \psi$ and every $\bar{\psi}$ by $(1+B)^{1 / 2} \bar{\psi}$ in the original diagram. There are $\left(n-\frac{\mathscr{E}_{e}}{2}\right) S_{F}(p)^{\prime} \mathrm{s}, \frac{\mathscr{E}_{e}}{2} \psi^{\prime}$ 's and $\frac{\mathscr{E}_{e}}{2} \bar{\psi}$ 's originally. Thus the whole matrix element is modified by finite terms only and the infinite terms can be grouped together into a multiplicative factor of the form

$$
(1+B)^{n-\frac{\mathscr{E} e}{2}}(1+B)^{\frac{\mathscr{E}_{e}}{2}}=(1+B)^{n}
$$

Since there are exactly $n$ e's occuring in the matrix element corresponding to the $n$ corners, we can eliminate $(1+B)^{n}$ by defining the renormalized charge as

$$
e_{0}=(1+B) e \text {. }
$$

Replacement of every $e$ by $\frac{e_{0}}{1+B}$ results in the cancellation of the infinite factor.

We are finally left with a finite matrix element which takes into account the second order radiative corrections. However, to complete the renormalization of charge to this order, we have to take into account other processes which will be considered presently.

\section{Self-energy of the photon ${ }^{1}$}

In discussing the photon propagator, we shall consider the creation of an electron positron pair by the photon and the subsequent annihilation of the pair with the creation of the photon. The photon propagator which includes the creation at

1 J. M. JAUCh and F. RohrLich, loc. cit.; J. Schwinger, Phys. Rev. 74, 1439 (1948); G. Wentzel, Phys. Rev. 74, 1070 (1948). 
vertex 1 and the annihilation at vertex 2 is the perturbed photon propagator to the lowest order and is given by ${ }^{1}$

where

$$
D_{F \mu \nu}^{\prime}(k)=\delta_{\mu \nu} D_{F^{\prime}}(k)+D_{F}(k) \Pi_{\mu \nu}(k) D_{F^{\prime}}(k)
$$

and

$$
\begin{gathered}
D_{F}(k)=\frac{1}{k^{2}} \\
\Pi_{\mu \nu}(k)=-\frac{i e^{2}}{4 \pi^{3}} \operatorname{Spur} \int \gamma_{\mu} \frac{1}{\mathrm{P}-m} \gamma_{v} \frac{1}{\mathrm{P}-\mathrm{k}-m} \mathrm{~d}^{4} p .
\end{gathered}
$$

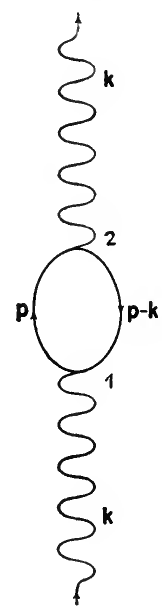

Fra. 12. Feynman diagram for the self energy of a photon.

The integral is quadratically divergent. However, we can reduce the degree of divergence by invoking the principle of gauge invariance. The photon propagator $D_{F^{\prime}}(k)$ is bilinear in $a_{\mu}^{2}$ so that if we make the gauge transformation

in

$$
a_{\mu}(k) \rightarrow a_{\mu}(k)+k_{\mu} \chi(k)
$$

we get new terms of the form

$$
D_{F}(k) \Pi_{\mu v}(k) D_{F}(k)
$$

$$
a_{\mu} \Pi_{\mu \nu} k_{\nu}^{\prime} \chi+\chi k_{\mu} \Pi_{\mu \nu} a_{\nu}+\chi^{2} k_{\mu} k_{\nu} \Pi_{\mu \nu}
$$

1 Earlier in this chapter we denoted the photon propagator by $D_{F}$. In a strict sense it should be written as $\delta_{\mu \nu} D_{F}(k)$, the coefficient $\delta_{\mu \nu}$ implying that $\mu=\nu$ when we introduce $\gamma_{\mu}$ and $\gamma_{v}$ at the vertices representing emission and absorption. In modifying this propagator by virtual processes of annihilation and creation of pairs, we are constrained to introduce two indices $\mu$ and $\nu$ since we are treating quite generally the $\gamma_{\mu}$ interactions at the vertices. It will be proved that $\Pi_{\mu \nu}(k)$ will be of the form $\delta_{\mu \nu} \Pi\left(k^{2}\right)$.

${ }^{2} a_{\mu}(k)$ represents the amplitude for a photon to have a momentum $k$ and polarization $\mu$, i.e. it is the momentum transform of $A_{\mu}(x)$. In studying the electron-electron interaction through the exchange of a photon at the two vertices, we had set the magnitude of $a_{\mu}(k)$ equal to unity apart from a factor of $\gamma / 4 \pi / 2 \omega$ and that is why only $\gamma_{\mu}$ occurred at the vertices. However since we wish to introduce transformations we have to use general $a_{\mu}$ 's along with the subsidiary condition. 
We therefore require

$$
k_{\mu} \Pi_{\mu \nu}(k)=0 \quad \Pi_{\mu \nu}(k) k_{\nu}=0
$$

to maintain gauge invariance.

Since $\Pi_{\mu \nu}(k)$ is a second rank tensor and depends only on $k_{\mu}$, it can be written in the form

$$
\Pi_{\mu \nu}(k)=C\left(k^{2}\right) k_{\mu} k_{\nu}+D\left(k^{2}\right) \delta_{\mu \nu} .
$$

Therefore from (15) we get

$$
k^{2} C\left(k^{2}\right)+D\left(k^{2}\right)=0
$$

or

or

$$
\Pi_{\mu \nu}(k)=\left(k_{\mu} k_{v}-k^{2} \delta_{\mu_{v}}\right) C\left(k^{2}\right)
$$

and

$$
\sum_{\mu \nu} \delta_{\mu \nu} \Pi_{\mu \nu}(k) \equiv \Pi_{\mu}^{\mu}(k)=-3 k^{2} C\left(k^{2}\right)
$$

$$
\Pi_{\mu}^{\mu}(0)=0 .
$$

However $\Pi_{\mu}^{\mu}(0)$ when evaluated from (13) turns out to be quadratically divergent. Hence we subtract the term out from (13) in order to preserve gauge invariance. The resultant integral can be evaluated and is only logarithmically divergent.

It may be noted however ${ }^{1}$ that by treating the photon as a neutral vector meson of mass $\varkappa$, the term $\Pi_{\mu}^{\mu}(0)$ can be identified as a mass term and eliminated by mass renormalization. Finally, the limiting process as $x \rightarrow 0$ can be adopted to obtain a gauge invariant expression identical with the one got by our procedure.

To return to our discussion, $C\left(k^{2}\right)$ can be written as

$$
C\left(k^{2}\right)=C(0)-k^{2} \Pi_{c}\left(k^{2}\right)
$$

where $C(0)$ is logarithmically divergent while $\Pi_{c}\left(k^{2}\right)$ is convergent. $C(0)$ and $\Pi_{c}\left(k^{2}\right)$ are given by

and

$$
C(0)=\frac{e^{2}}{3 \pi}\left[\frac{1}{i \pi^{2}} \int \frac{\mathrm{d}^{4} k}{\left(k^{2}-m^{2}\right)^{2}}+\frac{5}{6}\right]
$$

for small $k^{2} / 4 m^{2}$.

$$
k^{2} \Pi_{c}\left(k^{2}\right) \approx \frac{e^{2}}{\pi}\left[\frac{1}{15} \frac{k^{2}}{m^{2}}+\ldots\right]
$$

If now $\Pi_{\mu \nu}(k)$ is inserted into an external photon line, the $k_{\mu} k_{v}$ term cannot contribute since $k_{\mu} a_{\mu}=0$ for a free photon. Suppose however that the insertion is into an internal photon line. Then corresponding to each such diagram we can obtain two new diagrams by omitting the self-energy part as shown in Fig. 13. Each of these new diagrams gives terms of the form $A \gamma_{\mu} a_{\mu} B$ where $A$ and $B$ are contributions from the rest of the diagram. Invariance under the gauge transformation $a_{\mu} \rightarrow a_{\mu}+k_{\mu} \chi(k)$ requires

$$
\sum A \gamma_{\mu} k_{\mu} B=0 .
$$

1 P. T. Matthews, Phys. Rev. 76, 1657 (1949); F. Coester, Phys. Rev. 83, 798 (1951) 
The summation is over all the different diagrams corresponding to the different places of insertion of $I_{\mu \nu}(k)$.
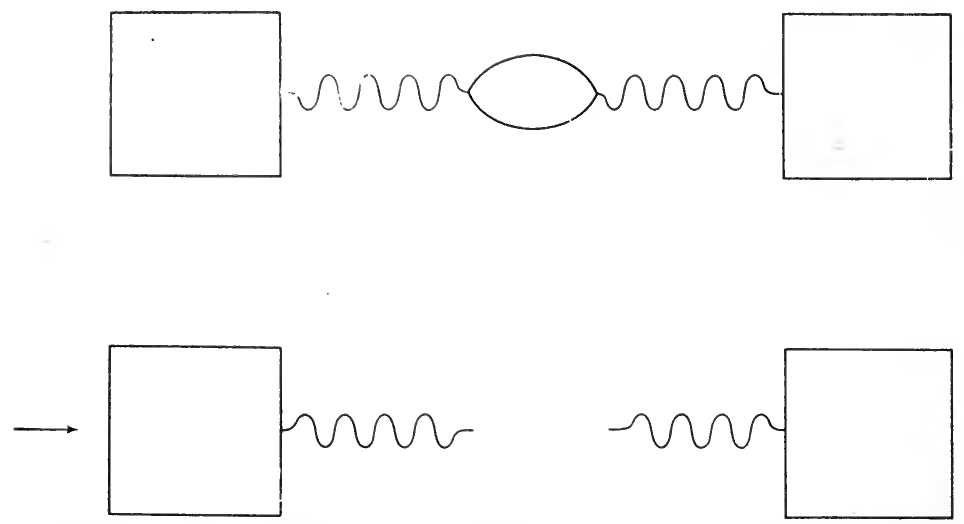

Fig. 13. Inclusion and omission of the self energy part in an internal photon line.

Now the original set of diagrams furnishes an expression of the form

functions similar to $A$ and $B$ )

$$
\sum A \gamma_{\mu} B \frac{1}{k^{2}} \Pi_{\mu \nu}(k) \frac{1}{k^{2}} C \gamma_{\nu} D \quad \text { (where } C \text { and } D \text { are }
$$

$$
\begin{aligned}
= & \sum A \mathrm{k} B \frac{1}{k^{2}} C\left(k^{2}\right) \frac{1}{k^{2}} C \mathrm{k} D \\
& +\sum A \gamma_{\mu} B \frac{1}{k^{2}}\left[-k^{2} \delta_{\mu \nu}\left(C(0)-k^{2} \Pi_{c}\left(k^{2}\right)\right)\right] \frac{1}{k^{2}} C \gamma_{\nu} D .
\end{aligned}
$$

The first term on the right-hand side has been shown to vanish. Thus $\Pi_{\mu \nu}(k)$ reduces to

$$
\Pi_{\mu v}(k)=-k^{2} C(0) \delta_{\mu \nu}+k^{4} \Pi_{C}\left(k^{2}\right) \delta_{\mu \nu}
$$

Thus the modified photon propagator is given by

where

$$
D_{F^{\prime}}^{\prime}(k)=(1-C) D_{F_{0}}(k) \quad \text { to order } e^{2}
$$

$$
\begin{gathered}
D_{F^{\prime}}^{\prime}(k) \delta_{\mu \nu}=D_{F^{\prime} \mu \nu}^{\prime}(k) \\
D_{F_{0}}(k)=D_{F}(k)+\Pi_{c}\left(k^{2}\right)
\end{gathered}
$$

and $D_{F_{0}}(k)$ is the renormalized photon propagator. We also obtain the modified wave function for free photons as

$$
a_{\mu}^{\prime}(k)=(1-C)^{1 / 2} a_{\mu}(k) .
$$

As we have mentioned earlier, the modifying factor $(1-C)^{1 / 2}$ can be carried over to the charge since with each vertex a factor $e$ is associated and the number of vertices is equal to the number of times the factor $(1-C)^{1 / 2}$ occurs in any diagram. This can be shown by the following simple argument. 
Consider an $n$-th order diagram having $P_{e}$ external and $P_{i}$ internal photon lines. Since one photon line ends at each corner, the number of photon line endings will be equal to the number of corners. Each $P_{i}$ has two endings while each $P^{e}$ one ending. Thus $2 P_{i}+P_{e}=n$ or $P_{i}=\frac{n-P_{e}}{2}$. The modified matrix element is got by replacing $D_{F}(k)$ by $D_{F}^{\prime}(k), a_{\mu}(k)$ by $a_{\mu}^{\prime}(k)$ and $a_{\mu}^{\dagger}(k)$ by $a_{\mu}^{\dagger}(k)$ in the original diagram. Thus the infinite constants can be grouped together as

$$
(1-C)^{\frac{n-P_{e}}{2}}(1-C)^{\frac{P_{e}}{2}}=(1-C)^{n / 2}
$$

while the rest of the matrix element is modified by finite terms only. The infinite factor can as before be removed by the renormalization of charge by defining $e_{0}$ by $e_{0}=(1-C)^{1 / 2} e, e_{0}$ being the renormalized charge. The final result thus contains only finite and observable quantities.

We have seen above how the photon propagator has to be modified if we include virtual annihilation and creation of pairs. Inclusion of this possibility implies that we can always consider even in a vacuum such processes to occur and the amplitude $c$ for a vacuum remaining a vacuum is obtained by considering such diagrams as Fig.14.
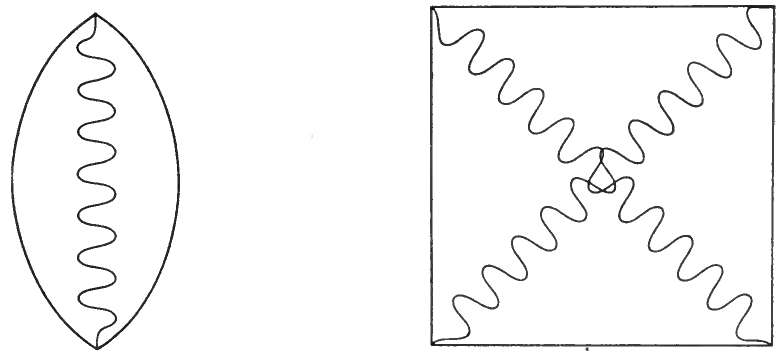

Fig. 14. Feynman diagrams representing vacuum processes.

Since in the absence of an external field the probability $|c|^{2}$ for a vacuum remaining a vacuum is unity, $|c|^{2}=\mathrm{e}^{i \alpha}$ where $\alpha$ is real. In any process involving real particles, we have to include these vacuum diagrams from which no external lines emerge. Each of the vacuum diagrams is divergent and this implies that the phase factor $\alpha$ is infinite. However this is going to be of no consequence since the only effect of such an inclusion is to multiply the real process by a numerical phase factor which is just the amplitude for a vacuum remaining a vacuum. The modification of all real processes by a common phase factor can have no observable effect and hence we need not consider these diagrams at all. In fact we shall see in the definition of the propagator in field theory that this factor is cancelled by dividing any amplitude by the amplitude of a vacuum remaining a vacuum. 
An external electromagnetic field will be modified by the inclusion of the processes of virtual pair creation. This is known as the vacuum polarization effect due to the external field, the word "polarization" being used evidently to denote that when we have an external field it also implies a polarization of the medium due to the creation of virtual pairs

The vertex part $^{1}$

The diagram corresponding to the second order radiative correction to the vertex operator $\gamma_{\mu}$ is shown in Fig. 15.

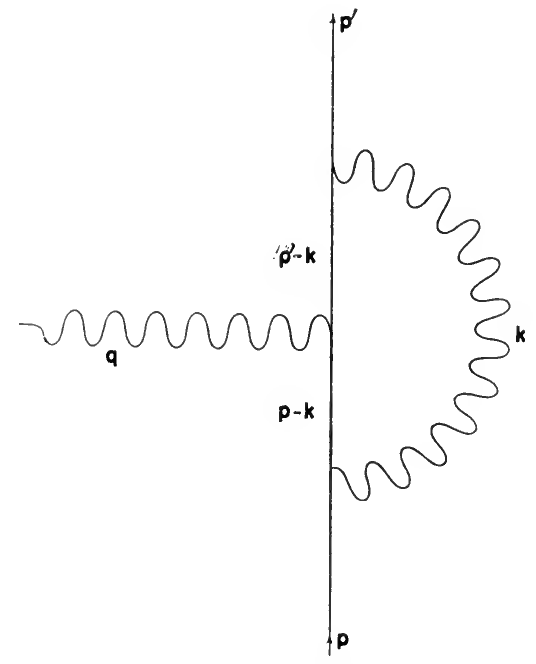

FIG. 15. Second order radiative correction to the vertex operator.

The inclusion of this correction replaces the vertex operator $\gamma_{\mu}$ by

where

$$
\Gamma_{\mu}=\gamma_{\mu}+\Lambda_{\mu}\left(p^{\prime}, p\right)
$$

$$
\Lambda_{\mu}\left(p^{\prime}, p\right)=-\frac{i e^{2}}{4 \pi^{3}} \int \gamma_{\lambda} \cdot \frac{1}{\mathrm{p}^{\prime}-\mathrm{k}-m} \cdot \gamma_{\mu} \cdot \frac{1}{\mathrm{p}-\mathrm{k}-m} \gamma_{\lambda} \frac{\mathrm{d}^{4} k}{k^{2}} .
$$

The integral is logarithmically divergent and can be treated in a way similar to $\sum(p)$. The final result may be written as

$$
\Lambda_{\mu}\left(p^{\prime}, p\right)=L \gamma_{\mu}+\Lambda_{\mu c}\left(p^{\prime}, p\right) .
$$

$\Lambda_{\mu c}\left(p^{\prime}, p\right)$ is convergent and is uniquely defined by the condition $\Lambda_{\mu c}\left(p^{\prime}, p\right)=0$ for $\mathrm{P}=\mathrm{P}^{\prime}=m$ (i.e. they correspond to free particles and are equal in magnitude). $L$ is a logarithmically divergent integral and is given by

$$
L=\frac{e^{2}}{4 \pi}\left[\frac{1}{i \pi^{2}} \int \frac{\mathrm{d}^{4} k}{\left(k^{2}-m^{2}\right)^{2}}-4 \int_{0}^{1} \frac{\mathrm{d} x}{x}+\frac{11}{2}\right]
$$

\footnotetext{
1 J. M. JAUCh and F. Rohrlich, loc. cit; R. P. Feymman, Phys. Rev. 76, 769 (1949).
} 
which is recognized to be equal to $-B$. This result is a special case of a general identity due to Ward $^{1}$.

The general form of $\Lambda_{\mu c}\left(p^{\prime} p\right)$ is quite complicated. However when $p^{\prime}$ and $p$ correspond to free particles, the regularized expression for it may be shown to be

$$
\begin{aligned}
& \Lambda_{\mu c}\left(p^{\prime} p\right)=-\frac{i e^{2}}{2 \pi}\left[2\left(\log \frac{m}{\lambda_{\min }}-1\right)\left(1-\frac{2 \theta}{\tan 2 \theta}\right)+\theta \tan \theta\right. \\
& \left.+\frac{4}{\tan 2 \theta} \int_{0}^{\theta} \alpha \tan \alpha \mathrm{d} \alpha\right] \gamma_{\mu}-i \frac{e^{2}}{2 \pi}\left[\frac{1}{4 m}\left(\gamma_{\mu} \mathrm{q}-\mathrm{q} \gamma_{\mu}\right) \frac{2 \theta}{\sin 2 \theta}\right]
\end{aligned}
$$

where $\lambda_{\min }$ is a small mass attributed to the photon as an infra-red cut-off by replacing $k^{2}$ by $k^{2}-\lambda_{\text {min }}^{2}$ in the denominator of the integrand. This is done in order to remove the singularity of the integrand at $k^{2}=0 . \theta$ is given by the relation $4 m^{2} \sin ^{2} \theta=q^{2}$. We may thus write the modified vertex operator as

$$
\Gamma_{\mu}\left(p^{\prime} p\right)=(1+L)\left(\gamma_{\mu}+\Lambda_{\mu c}\left(p^{\prime} p\right)\right)
$$

to order $e^{2}$. Since exactly one $e$ occurs at each corner, we can remove the infinite factor $(1+L)$ by defining the renormalized charge $e_{0}$ by $e_{0}=(1+L) e$.

We thus see that if we take into account all the three charge renormalizations, the final $e_{0}$ will be

$$
e_{0}=(1+B)(1+L)(1-C)^{1 / 2} e=(1-1 / 2 C) e
$$

to order $e^{2}$ since $B=-L$.

We shall now give two examples where the corrections due to the above processes have been worked out, viz. the radiative corrections to the magnetic moment of the electron (which gives rise to its anomalous magnetic moment) and the Lamb shift.

\section{The anomalous magnetic moment of the electron ${ }^{2}$}

The basic process in which the magnetic moment term makes its appearance is the vertex diagram without radiative corrections. Figures 16 and 17 give the second order radiative corrections to this diagram. The diagrams (a) and $\left(a^{\prime}\right)$ contribute only to the mass and wave function renormalizations since the radiative corrections occur only in the external lines. The corresponding charge renormalization is spurious cancelling with that arising from(b). (c) gives a true charge renormalization and a vacuum polarization effect, but since this is something that modifies the external potential and not the properties of the electron it cannot contribute to the magnetic moment. (b) makes a finite

1 J. C. WARD, Phys. Rev. 78, 182 (1950).

2 J. Schwinger, Phys. Rev. 73, 416 (1948) and 76, 790 (1949); R. Karplus and N. M. Kroll, Phys. Rev. 77, 536 (1950). 
contribution to the magnetic moment of the electron. The total contribution to the matrix element from the first three diagrams can be shown to be (cf. Eq.(34))

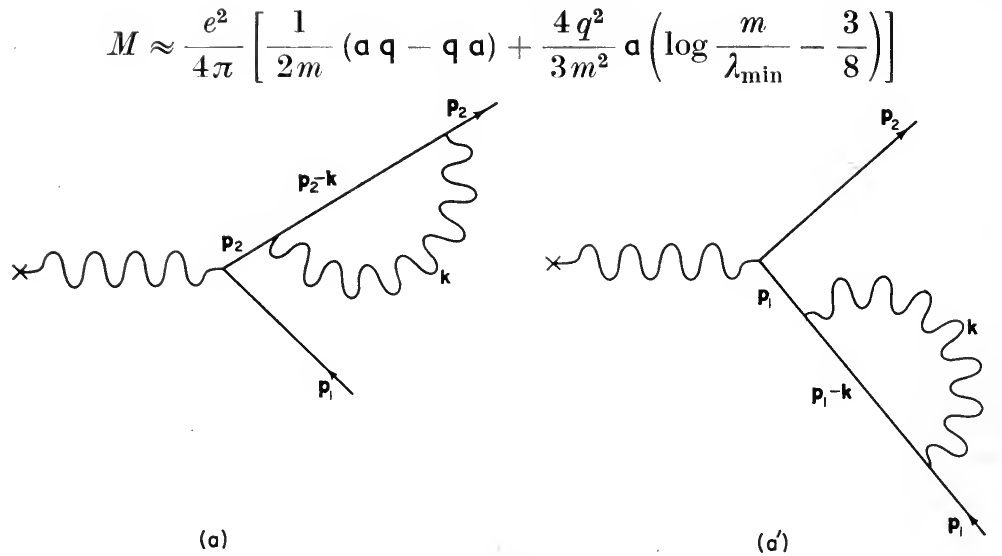

FIG. 16. Electron self-energy corrections.

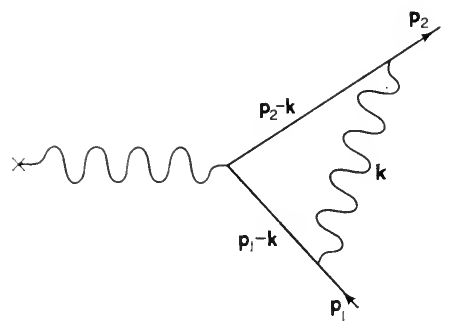

(a)

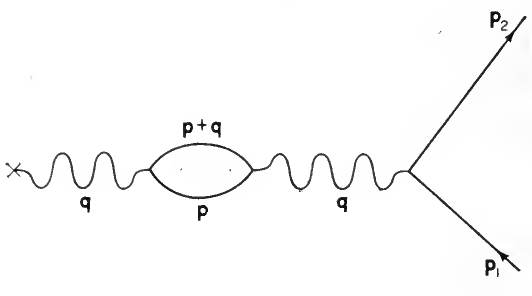

(b)

Fig. 17. (a) Radiative correction to the vertex;

(b) Vacuum polarization diagram.

when $q=p_{2}-p_{1}$ is small. The above $M$ will thus modify the Coulomb scattering and we will now show that the first term in $M$ will give rise to an additional magnetic moment. We have

$$
\mathrm{aq}-\mathrm{qa}=\gamma_{\mu} \gamma_{\nu}\left(a_{\mu} q_{\nu}-q_{\mu} a_{\nu}\right)
$$

which becomes in coordinate representation

$$
\gamma_{\mu} \gamma_{\nu}\left(\nabla_{\nu} A_{\mu}-\nabla_{\mu} A_{\nu}\right)=-\gamma_{\mu} \gamma_{\nu} F_{\mu \nu}
$$

where $F_{\mu \nu}$ is the electromagnetic field tensor. But the effect of a particle having a magnetic moment $\mu$ is to subtract a potential $\mu \gamma_{\mu} \gamma_{\nu} F_{\mu \nu}$ from the ordinary potential $\mathrm{A}=\gamma_{\mu} A_{\mu}$ appearing in the Dirac equation. This is precisely what the first term of $M$ does, so that it is interpreted as a correction to the magnetic moment. It follows that

$$
\mu_{\text {electron }}=\frac{e}{2 m}\left(1+\frac{e^{2}}{2 \pi}\right) \text {. }
$$


Karplus and Kroll have calculated the corrections to the magnetic moment to the fourth order in $e$. Their final result is

$$
\mu_{\text {electron }}=1 \cdot 0011454 \frac{e}{2 m}
$$

a value which has been experimentally verified.

\section{The Lamb shift ${ }^{1}$}

According to the Dirac theory, the $2{ }^{2} S_{1 / 2}$ and $2{ }^{2} P_{1 / 2}$ levels of hydrogen should be coincident. However when the radiative corrections are taken into account, the levels get separated by $1057.19 \pm 0.2 \mathrm{Mc} / \mathrm{sec}$. Precise experiments have in fact confirmed this level shift and the agreement between theory and experiment is at present up to $+0.22+0.23 \mathrm{Mc} / \mathrm{sec}$. Thus this provides one of the most accurate confirmations of the correctness of the renormalization techniques developed in quantum electrodynamics.

In order to proceed with the calculations in this case, we must first derive an expression for the bound state electron propagator. For this, we note that if $A_{\mu}^{e}(x)$ is the external potential due to the nucleus, the bound state wave function satisfies the equation

$$
\left(i \nabla-e \mathbf{A}^{e}\right) \psi-m \psi=0 .
$$

We define the Fourier transform of $A_{\mu}^{e}(x)$ through

$$
A_{\mu}^{e}(x)=\frac{1}{(2 \pi)^{3}} \int A_{\mu}^{e}(\boldsymbol{q}) \delta\left(q_{4}\right) \mathrm{e}^{i q \cdot x} \mathrm{~d}^{4} q
$$

Then, if the electron is scattered from the state $p$ to the state $p^{\prime}$ due to this potential, the electron propagator will satisfy the equation

$$
\left(\mathrm{p}^{\prime}-m\right) S_{\boldsymbol{F}}^{e}\left(p^{\prime}, p\right)=\frac{e}{(\mathbf{2 \pi})^{3}} \gamma_{\mu} \int A_{\mu}^{e}\left(\boldsymbol{p}^{\prime}-\boldsymbol{p}^{\prime \prime}\right) S_{F}^{e}\left(p^{\prime \prime}, p\right) \mathrm{d}^{3} p^{\prime \prime}+i \delta\left(\boldsymbol{p}^{\prime}-\boldsymbol{p}\right) .
$$

Since we are considering Coulomb scattering the fourth components of $p^{\prime}$ and $p$ are the same here. The integral equation implies that we are considering $S_{F}^{e}\left(p^{\prime}, p\right)$ as the solution of equation (44) when $A_{\mu}^{e}(x)$ is not treated as a perturbation, or equivalently, the solution is taken to all orders. However, the radiation field is treated as a perturbation.

The above equation may be symbolically written as

$$
\left(\mathrm{P}-\frac{e \mathrm{~A}^{e}}{(2 \pi)^{3}}-m\right) S_{F}^{e}=i \text {. }
$$

1 W. E. Lamb, Jr. and R. E. Retherford, Phys. Rev. 72, 241 (1947); S. Triebwasser. E. S. DAYhoff and W. E. Lamb, JR., Phys. Rev. 89, 98 and 106 (1953); H. A. Bethe, Phys, Rev. 72, 339 (1947); N. M. Kroll and W. E. Lamb, Phys. Rev. 75, 388 (1949); J. B. French and V. F. Weisskopf, Phys. Rev. 75, 1240 (1949); R. P. Feynman, Phys. Rev. 74, 1430 (1948); M. Baranger, H. A. Bethe and R. P. Feynman, Phys. Rev. 92, 482 (1953); R. KarPlus, A. Klein and J. Schwinger, Phys. Rev. 86, 288 (1952); H. M. Fried and D. R. Yennie, Phys. Rev. 112, 1391 (1958). See also J. C. Gunn, Rep. Progr. Phys. 1S, 127 (1955) whose treatment we follow here. 
The main contributions if radiative corrections are included arise from two types of graphs. One is the self-energy part. Here it is the correction due to the emission and absorption of a photon to a bound state propagator, represented by a double line in the diagram. The second is the vacuum polarization graph. These are illustrated to order $e^{2}$ below:

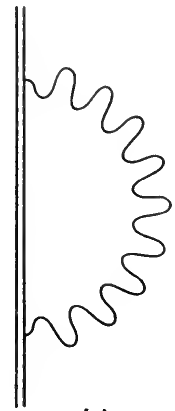

(a)

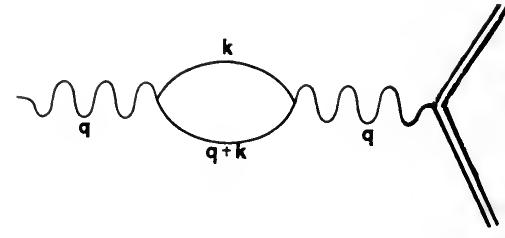

(b)

Fig. 18. Feynman diagrams for Lamb shift.

(a) Self-energy diagram for the bound electron.

(b) Vacuum polarization diagram.

The contribution due to the first type of graph will be

$$
\begin{gathered}
\left(a\left|S^{(2)}\right| a\right)=-\frac{i e^{2}}{4 \pi^{3}} \iiint \frac{1}{k^{2}} \bar{\varphi}_{a}\left(\boldsymbol{p}^{\prime}\right) \gamma_{\mu} S_{F}^{e}\left(\boldsymbol{p}^{\prime}-\boldsymbol{k}, \boldsymbol{p}-\boldsymbol{k}, E_{a}-k_{4}\right) \\
\times \gamma_{\mu} \varphi_{a}(\boldsymbol{p}) \mathrm{d}^{4} k \mathrm{~d}^{3} p^{\prime} \mathrm{d}^{3} p .
\end{gathered}
$$

Here $\varphi_{a}$ 's are the Coulomb wave functions of the state under consideration and may be written as

$$
\varphi_{a}(x)=\varphi_{\alpha}(\boldsymbol{x}) \mathrm{e}^{-i E_{a} t}, \quad \varphi_{a}(\boldsymbol{p})=\frac{1}{(2 \pi)^{3 / 2}} \int \varphi_{a}(\boldsymbol{x}) \mathrm{e}^{-i \boldsymbol{p} \cdot \boldsymbol{x}} \mathrm{d}^{3} x .
$$

If the total contribution due to this diagram is denoted by $M^{(2)}$, then, since the interaction Hamiltonian makes a contribution of the form $-i\left(a\left|H_{I}\right| a\right)$ to the second order matrix element, the level shift $\Delta E_{a}$ of the state $a$ is given by

$$
\begin{gathered}
\Delta E_{a}=\operatorname{Re} i M^{(2)}=e^{2} / 4 \pi^{3} \\
\times \iiint \frac{1}{k^{2}} \bar{\varphi}_{a}\left(\boldsymbol{p}^{\prime}\right) \gamma_{\mu} S_{F}^{e}\left(\boldsymbol{p}^{\prime}-\boldsymbol{k}, \boldsymbol{p}-\boldsymbol{k}, E_{a}-k_{a}\right) \gamma_{\mu} \varphi_{a}(\boldsymbol{p}) \mathrm{d}^{4} k \mathrm{~d}^{3} p \mathrm{~d}^{3} p^{\prime} .
\end{gathered}
$$

It can be shown that here also renormalization can be carried out using the free particle counter-terms. One can thus obtain a final convergent result.

By a similar procedure the contribution from the second graph can be calculated. The matrix element in this case will in effect replace $A_{\mu}^{e}(q)$ by $A_{\mu}^{e \prime}(q)$ where

$$
A_{\mu}^{e^{\prime}}(q)=-\frac{i e^{2}}{4 \pi^{3}} \frac{1}{q^{2}} \int \mathrm{d}^{4} k \quad \text { Spur } \quad\left[\gamma_{\mu} S_{F^{\prime}}(k) \gamma_{\nu} S_{F}(k+q) A_{v}^{e}(q)\right] .
$$


The charge renormalization is carried through as before and the level shift calculated. The total contribution from the two graphs is a very good approximation to the observed value. Note that the main shift comes from a non-relativistic contribution, applying only to the $2 S$ state and is of the order of $\alpha(Z \alpha)^{4} \log Z \alpha$ where $Z$ is the nuclear charge. The corresponding relativistic contribution is considerably smaller being of the order of $\alpha(Z \alpha)^{4}$.

\section{The removal of divergences from a general $\operatorname{graph}^{1}$}

We shall now consider the methods of removing the divergences from a general $n$-th order graph. There may appear in the $S$-matrix three distinct types of infinities which may be classified as follows:

(i) The ultra-violet divergences which arise because of the integrand not vanishing rapidly enough at large momenta;

(ii) Singularities caused by the coincidence of two or more poles of the integrand;

(iii) The infra-red divergences caused by the presence of a factor $\frac{1}{p^{2}}$ in the integrand. This is only a spurious divergence since it vanishes when all the diagrams contributing to a particular process to the given order are considered ${ }^{2}$.

The renormalization procedure is devised to handle only the first type of divergence. Any divergence of this type is removed if a sufficient number of integration variables (i.e. internal momenta) are kept fixed. This is equivalent to cutting the corresponding internal lines thereby making them equivalent to a pair of external lines. A divergent graph is called "primitive" if whenever one of the 4-momentum vectors in its integrand is held fixed, the integration over the remaining variables is convergent.

We now define the "dimension" $D$ of an integral of a function with respect to a variable as equal to the highest power of the variable in the numerator minus the highest power of the variable in the denominator, each differential inside the integral contributing unity to the power of the numerator. It is clear then that $D$ should be less than zero for the integral to be convergent. To calculate $D$ we note first that integration over each internal electron line is of the form $\int \frac{1}{\mathrm{p}-m} \mathrm{~d}^{4} p$ and contributes three powers to $D$. Similarly each internal photon line contributes two powers to $D$. There are altogether $n \delta$-functions, but one of these merely expresses the overall momentum conservation while each of the others contributes a power -4 to $D$. Integration with respect to a $\delta$-function just amounts to stating that the integral is equal to the integrand

1 F. J. Dyson, Phys. Rev. 75, 486, 1736 (1949).

2 J. M. JAUCH, and F. Rohrlich, Helv. Phys. Acta 27, 613 (1954); F. Rohrlich, Phys. Rev. 98, 181 (1955); F. Bloch and A. Nordsieck, Phys. Rev. 52, 54 (1937); W. Braunbeck and E. Wernmann, Z. Phys. 110, 360 (1938); W. Pauli and M. Fierz, Nuovo Cim. 15, 167 (1938); R. J. Glauber, Phys. Rev. 84, 395 (1951); W. Thirring and B. Touchek, Phil. Mag. 42, 244 (1951). See also G. Morpurgo, Nuovo Cim. Suppl., 109 (1951). 
with parametric values corresponding to the singularity of the $\delta$-function. Since we have generally assumed that integration contributes +4 to $D$ we have to assume that the $\delta$-function contributes -4 . But the $\delta$-function expressing the over all energy momentum conservation is not integrated and therefore it does not contribute to the power. Thus we have

$$
D=3 F_{i}+2 B_{i}-4(n-1)
$$

where $n$ is the number of vertices, $F_{i}$ the number of internal fermion lines and $B_{i}$ the number of internal boson lines. To express $D$ in terms of the number of external lines, we note the two relations we have already derived, viz.

$$
\begin{aligned}
& 2 F_{i}+F_{e}=2 n, \\
& 2 B_{i}+B_{e}=n .
\end{aligned}
$$

Therefore

$$
D=4-3 / 2 F_{e}-B_{e} .
$$

It follows that the graph will be convergent if

$$
D<0 \text { or } 3 / 2 F_{e}+B_{e}>4 \text {. }
$$

Thus the important fact emerges that the degree of divergence is independent of the order of the diagram.

We wish now to give a consistent prescription for the removal of the infinities from the graphs of any order in the $S$-matrix. For this it is convenient to use the following terminology. A graph is called improper if it can be separated into two distinct graphs by cutting an electron or a photon line. A proper graph is one which is not improper. Thus an improper graph can be split into proper graphs and renormalization carried out separately for each such part. We will therefore be concerned only with proper graphs. The part of a diagram which is connected to the rest by two photon or electron lines is called the self energy part. The part which is connected to the rest of the diagram by a pair of electron lines and a photon line is the vertex part. The graph obtained from any graph $G$ by replacing all the electron self energy parts by electron lines, the photon self energy parts by photon lines and the vertex parts by simple vertices (two electron lines and a photon line) i.e. by removing the insertions on the lines and at various corners is called the skeleton of $G$. A graph which is its own skeleton is called "irreducible". Thus a "reducible" graph of any order can be built up by inserting in the lines and vertices of its skeleton self energy and vertex parts of requisite orders in all possible ways. All such reducible graphs may be divided into three classes:

(i) graphs in which no two insertions overlap,

(ii) graphs in which each insertion is completely contained within the next,

(iii) graphs in which insertions overlap.

For the first type of diagram, renormalizations can be carried out for each separate insertion by the methods already outlined. For the second type we may successively remove the divergences from each insertion starting with the innermost 
and ending with the divergence arising from $G$ itself. For the third type however, the method is more complicated. Consider for instance in Fig. 19 the fourth order electron self-energy part:

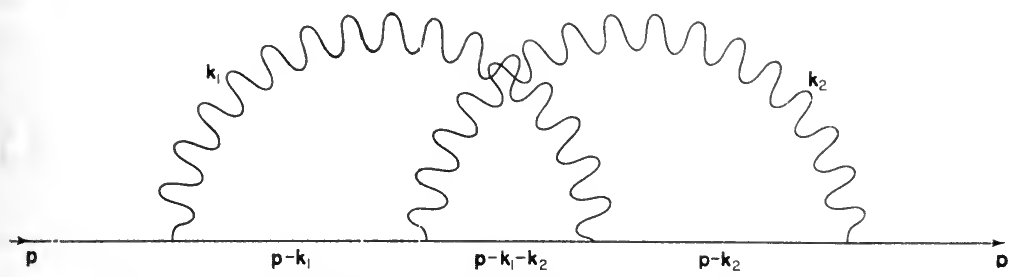

FIG. 19. Fourth order self energy diagram with overlapping photon lines.

The corresponding matrix element is

$$
\begin{aligned}
& -\frac{e^{4}}{\left(4 \pi^{3}\right)^{2}} \iint \mathrm{d}^{4} k_{1} \mathrm{~d}^{4} k_{2} \frac{1}{k_{1}^{2}} \gamma_{\mu} \frac{\mathrm{P}-\mathrm{k}_{1}+m}{\left(p-k_{1}\right)^{2}-m^{2}} \times \\
& \gamma_{\nu} \frac{\mathrm{P}-\mathrm{k}_{1}-\mathrm{k}_{2}+m}{\left(p-k_{2}-k_{1}\right)^{2}-m^{2}} \gamma_{\mu} \frac{\mathrm{P}-\mathrm{k}_{2}+m}{\left(p-k_{2}\right)^{2}-m^{2}} \gamma_{\nu} \frac{1}{k_{2}^{2}} .
\end{aligned}
$$

Keeping either $k_{1}$ or $k_{2}$ fixed and integrating over the other leads to a logarithmic divergence, while the double integral diverges linearly. The procedure to remove the divergence here has been given by Salam ${ }^{1}$ and is as follows.

Consider for simplicity an electron self-energy graph with three integration variables $t_{1}, t_{2}$ and $t_{3}$. First keep $t_{2}$ and $t_{3}$ fixed and substract the divergence for integration over $t_{1}$. This divergent term is obtained by setting in those factors in which $t_{1}$ occurs, the other integration variables $t_{2}$ and $t_{3}$ equal to zero and the external momenta equal to their corresponding free particle values, the factors not involving $t_{1}$ being left unchanged. This is done in turn for integrations over $t_{2}$ and $t_{3}$ also. The next step is to substract the divergences for integrations over the pairs of variables $t_{i} t_{j}(i, j=1,2,3)$ from the integrand obtained after subtracting the divergences for $t_{1}, t_{2}$ and $t_{3}$ the method being similar to that for $t_{i}$. We finally repeat the procedure for integration over $t_{1} t_{2} t_{3}$, the divergent terms being separated out using Dyson's prescription. The resultant integral after all these subtractions is convergent for any of the integrations $t_{i}, t_{i} t_{j}$ or $t_{1} t_{2} t_{3}$ and is the finite correction to the electron propagator which we set out to evaluate. The procedure outlined here has also been generalized to any arbitrary diagram involving $n$ variables by Salam.

1 A. Salam, Phys. Rev. 82, 217 (1951). 


\section{FORMALISM OF QUANTUM FIELD THEORY}

\section{THE STATEVECTOR AND FIELD OPERATORS}

\section{Introductory remarks}

WE HAVE till now been confining our attention to the equations of single particles and perturbation theories of the wave functions relating to these particles. The systems containing many particles were described in terms of single particle wave functions when the total number of particles was assumed to be constant. Such a description cannot include the concept of creation and annihilation of particles unless such creation and annihilation are treated as scattering in the Feynman formalism.

A systematic attempt to deal with a multiplicity of particles is the more sophisticated field theory ${ }^{1}$. The aim of field theory is to enlarge the scope of quantum mechanics beyond the treatment of single particle wave equations. We know that the interaction between two fundamental particles like electrons can be understood through the exchange of photons, both real and virtual. One of the effects of interaction is, for example, a change in the momentum of the interacting particles. If the momentum of a particle changes from $p$ to $p^{\prime}$ the process may be viewed as the destruction of a particle of momentum $p$ and the creation of a particle of momentum $p^{\prime}$. Viewed in this manner a system of fundamental particles may be described by a state vector involving the occupation numbers of particles in different states characterized by the values of dynamical variables. If there is no interaction between these particles, the occupation numbers do not change. The effect of the interaction is to make these occupation numbers vary with time. Our object will now be to study the main features and properties of these state vectors and thereby prove the necessity for the introduction of field operators which operating on these state vectors yield the occupation numbers and their mode of variation. The essential peculiarity of field theory can be summarized in a naïve manner as follows. While in the single particle formalism we deal with the wave function $\psi$, in field theory we have to deal

1 Excellent introductions to Quantum Field Theory are given in G. Wentzel, The Quantum Theory of Fields, Interscience (1946); S. S. SchWEBER, H. A. BETHE and F. DE Hoffmann, loc. cit., J. M. J $\mathrm{AUCH}$ and F. RoHRLich, The Theory of Photons and Electrons, Addison-Wesley (1955); N. N. Bogoliubov and D. V. SHIRkov, Introduction to the Theory of Quantized Fields, Interscience (1959). 
with two types of quantities, $\psi$ the field operator ${ }^{1}$ and $\Psi$ the state vector. $\Psi$ bears a correspondence to wave function $\psi$ in that both are state vectors while the field operators also bear correspondence to the wave function since they have "equations of motion" similar to single particle wave equations. That is why a difficulty is felt as to the best manner of introducing the two fundamental quantities, the field operators $\psi$ and the state vector $\Psi$, as an extension of the single particle formalism. Fermi ${ }^{2}$ for example introduces the field operators in dircet analogy with $\psi$ while Bethe and Schweber describe $\Psi$ in analogy with $\psi$. The deductive approach will be to develop the state vector $\Psi$ in analogy with the single particle wave function and deduce the operators which satisfy suitable commutation relations. In the absence of interactions, the number of particles present in a field is a constant of motion and this situation can be described by the Schrödinger equation ${ }^{3}$

$$
\begin{gathered}
\left\{\sum_{k=1}^{n} H\left(x_{k}\right)\right\} \Phi\left(x_{1}, x_{2}, \cdots, x_{n} ; t\right)=i \frac{\partial \Phi}{\partial t}\left(x_{1}, \cdots, x_{n} ; t\right) \\
H\left(x_{k}\right)=-\frac{1}{2 m} \nabla_{k}^{2}+V\left(x_{k}\right)
\end{gathered}
$$

where $\Phi$ is the wave function (in configuration space) of an $n$ particle system and $\left\{\boldsymbol{x}_{k}\right\}$ the spatial coordinates of the aggregate and $H$ the Hamiltonian of the system which is a function of the aggregate $\left\{x_{k}\right\}$ and operators associated with it. For example if the particles are moving under a common potential $V, H$ can be written as

$$
H\left[\left\{\boldsymbol{x}_{k}\right\}\right]=\sum_{k} H\left(\boldsymbol{x}_{k}\right)=\sum_{k}\left[\frac{-1}{2 m} \boldsymbol{V}_{k}^{2}+V\left(\boldsymbol{x}_{k}\right)\right] .
$$

$\Psi$ can be expanded in terms of properly symmetrized functions $\Phi$ as

$$
\Psi=\sum_{\left\{n_{i}\right\}} c_{n}\left(\left\{n_{i}\right\}, t\right) \Phi_{\left\{n_{i}\right\}}^{n}
$$

where $\Phi_{\left\{n_{i}\right\}}^{n}$ is the configuration representation of a state corresponding to an aggregate of particles $\left\{n_{i}\right\}$ with $n_{i}$ particles in the $i$-th state $(i=1,2, \ldots)$ the total number of particles being $n$. $c$ represents the amplitude for the members of the aggregate $\left\{n_{i}\right\}$ being in their respective states. The symbol $\sum$ denotes integration or summation according as the states form a continuous or a discrete set.

The Schrödinger equation satisfied by the wave function $\Phi$ can only be used when the total number of particles is fixed since the Hamiltonian which is a function of the coordinates of the particles can be described only if the total number is specified. However $\Psi$ as described by (4) by very definition need not

1 It is now an accepted notation to use the same symbol for the wave function of a single particle and the field operator.

2 E. Fermi, Elementary Particles, Yale University Press (1952).

3 S. S. Schweber, H. A. Bethe and F. De Hofrman, Mesons and Fields, Vol. I, pp. 145, Row Peterson and Co. (1956).

FPCR 8 
be confined to the case of a fixed number of particles since $n$ can vary in $\Phi_{n}$. In such a case if we wish to write a Schrödinger equation in $\Psi$ similar to (1), then $H$ cannot be just described through a function of a fixed number of variables but in terms of what are known as field operators. We shall take up the discussion of the expansion in a rather pedagogic manner but in a way which leads us most naturally to the operator calculus of field theory. We first realize that the states that are usually referred to are definite momentum states of an assembly of non-interacting particles. The $\Phi_{\left\{n_{i}\right\}}^{n}$ is the configuration representation of a stationary state corresponding to the specified momenta of a fixed number $n$ of free non-interacting particles, stationary in the sense that the only time dependence is of the form $\mathrm{e}^{-i E t}$ where $E$ is the total energy of the non-interacting system. Any other time dependence of $\Psi$ is imbedded in $c$. The state vector $\Psi$, assuming interaction, is the linear combination of such $\Phi$ 's. $c$ in such a case can be more explicitly written as $c\left(n_{1} p_{1}, n_{2} p_{2} \ldots\right)$, which is therefore the many particle analogue of the decomposition of a single particle wave function into free particle wave functions. Taking the single particle case

$$
\psi(x)=\int c(p) \mathrm{e}^{-i p x} \mathrm{~d}^{3} p
$$

$\mathrm{e}^{-i p \cdot x}$ is the configuration representation of a single particle of momentum $p$, i.e. it is a degenerate or trivial form of the $\Phi$ function. $c(p)$ the Fourier transform of $\psi(x)$ represents the amplitude that a single particle has momentum $p$ or what is the same it is the amplitude in the "occupation number space". Therefore it is the degenerate or trivial form of the general $c$-function with $n_{1}=1, p_{1}==p$ and the rest of the $n_{i}=0$. Thus we realise that the amplitude in the occupation number space is nothing but the momentum representation of $\Psi$. Since the number of particles is greater than one, we have assumed the possibility of more than one particle in a single state. It is to be noted that in the many particle case, $\Psi$ in configuration space is the linear combination of the wave functions $\Phi_{\left\{n_{i}\right\}}^{n}$, each wave function for a particular number of particles having as its arguments the corresponding number of space variables. How then are we to make the transformation into the momentum space for such a $\Psi$ ?

The peculiarity of the situation can be very easily illustrated even in the elementary theory of functions. If $f_{1}(x)$ is a function of a single variable $x$, its Fourier transform is defined as

$$
\varphi_{1}(p)=\int f_{1}(x) \mathrm{e}^{i p x} \mathrm{~d} x .
$$

If $f_{2}(x, y)$ is a function of two variables $x$ and $y$ its Fourier transform is given by

$$
\varphi_{2}(p, q)=\int f_{2}(x, y) \mathrm{e}^{i p x+i q y} \mathrm{~d} x \mathrm{~d} y .
$$

Then what is the Fourier representation of

$$
F=f_{1}(x)+f_{2}(x, y) \text {. }
$$

Two possibilities arise. If we treat $F$ as a function of both the variables $x$ and $y$ we have to write the double transformation as $\varphi_{1}(p) \delta(q)+\varphi_{2}(p, q)$. The intro- 
duction of the $\delta$-function when the number of variables is very large becomes very clumsy. To avoid this we can take $\varphi_{1}(p)+\varphi_{2}(p, q)$ as the transform of $F^{\prime}$ with the specific understanding that to obtain the inverse of $F$ in space representation, we must take the corresponding inverses of $\varphi_{1}$ and $\varphi_{2}$ separately and add them. Adopting this convention, $\varphi_{1}(p)+\varphi_{2}(p, q)$ is the Fourier representation of $F$. This is exactly the procedure we adopt in the theory of wave functions and therefore identify $\sum c$ or equivalently $c$ as the momentum representation of $\Psi$. The $c$ 's are referred to as state amplitudes and $\Psi$ as the state vector. This completes the analogy with the single particle wave function (see chapter I).

In any quantum mechanical problem we shall be interested in the rate of variation of the state vector $\Psi$. In the one-particle formalism, this is given by the Schrödinger equation and we would like $\Psi$ to obey a similar equation. However in the many particle case $\Psi$ is a linear combination of $c$ 's and $\varphi$ 's and $\partial \Psi / \partial t$ cannot be expressed only in terms of $\Psi$. But we could still insist upon the form $\frac{i \partial \Psi}{\partial t}=H \Psi$ provided the operation of $H$ on $\Psi$ represents the required linear combination, i.e. $H$ contains operators which connect the $\Phi_{\left\{n_{i}\right\}}^{n}$ s. These are just the annihilation and creation operators and the entire formalism of field theory is built round them to such an extent that it has become conventional to start with these operator and the commutation relations associated with them as postulates on which the quantum field theory is based. (It is noteworthy that workers in the field do not seem to adopt a uniform sequence of ideas and postulates though every one is agreed upon the methods of calculation and convinced of the equivalence of the different approaches.) Even in regard to the definition of these operators, no uniform procedure is followed. For example, the annihilation and creation operators $a_{i}$ and $a_{i}^{\dagger}$ are defined as follows:

$$
\begin{gathered}
a_{i} \Phi_{n_{1} n_{2} \cdots n_{i}}^{n}\left(x_{1}, x_{2}, \cdots, x_{n}\right)=V n_{i} \Phi_{n_{1} n_{2} \cdots, n_{i}-1}^{n-1}\left(x_{1}, x_{2}, \cdots, x_{n-1}\right) \\
a_{i}^{\dagger} \Phi_{n_{1} n_{2} \cdots n_{i}}^{n}\left(x_{1}, x_{2}, \cdots, x_{n}\right)=V n_{i}+1 \Phi_{n_{1} n_{2} \cdots n_{i}+1}^{n+1}\left(x_{1}, x_{2}, \cdots, x_{n+1}\right) .
\end{gathered}
$$

On the other hand, they are also defined with respect to $c$ as:

$$
\begin{gathered}
a_{i}^{\dagger} c_{n}\left(n_{1}, n_{2}, \cdots, n_{i}, \cdots\right) \rightarrow \bigvee / n_{i}+1 c_{n+1}\left(n_{1}, n_{2}, \cdots, n_{i}+1, \cdots\right) . \\
a_{i} c_{n}\left(n_{1}, n_{2}, \ldots, n_{i}, \ldots\right) \rightarrow \bigvee n_{i} c_{n-1}\left(n_{1}, n_{2}, \ldots, n_{i}-1, \ldots\right) .
\end{gathered}
$$

To understand the equivalence of the two definitions let us ask the more comprehensive question: what is the effect of operating the creation and annihilation operators on any $\Psi$, where $\Psi$ represents the linear combination of $\Phi_{n}$ 's, the coefficients $c$ being the amplitudes for the various occupation states?

Now $a_{k}^{\dagger} \Psi$ naturally differs from $\Psi$ and should imply the additional information that there is one more particle with momentum $k$. To put it in a more mathematical form we suitably apply the above definitions $(9)$ and $(9 \mathrm{a}, \mathrm{b})$. To obtain $a_{k}^{\dagger} \Psi$ if we just replace every $\Phi^{n}$ by $\gamma n_{k}+1 \Phi^{n+1}$ in $\Psi$ the coefficient $c_{n}$ will be attached to $\Phi^{n+1}$ which is an inconsistent notation though it is meaningful to make the $8 *$ 
statement that the coefficient of $\Phi^{n+1}$ in the expansion of $a_{k}^{\dagger} \Phi$ is $V n_{k}+1 c_{n}$. To get over this difficulty we write

where

$$
a_{k}^{+} \sum_{n} c_{n} \Phi^{n}=\sum_{n} c_{n}^{\prime} \Phi^{n}
$$

$$
c_{n+1}^{\prime}\left(k_{1}, k_{2}, \cdots, k_{n+1}\right)=\bigvee n_{k}+1 c_{n}\left(k_{1}, k_{2}, \cdots, k_{n}\right) \delta_{k, k_{n+1}} .
$$

$\delta$ being the Kronecker delta function of the discrete parameter $k$. We realize that $a_{k}^{\dagger}$ 's operate both an $\Phi_{n}$ and $c$ and change $\Phi^{n}$ to $\Phi^{n+1}$ and $c_{n}$ to $c_{n+1}^{\prime}$. If we take in particular

$$
\Psi=\Phi_{\left\{n_{i}\right\}}^{n},
$$

i.e. all the coefficients $c$ are zero except $c_{\left\{n_{i}\right\}}^{n}$ corresponding to $\Phi_{\left\{n_{i}\right\}}^{n}$ which is unity, then operating $\boldsymbol{a}^{\dagger}$ on $\Psi$

$$
a_{k}^{\dagger} \Psi=a_{k}^{\dagger} \Phi^{n}=c_{n+1}^{\prime}\left(k_{1} \cdots k_{n}, k\right) \Phi_{\left\{n_{i}\right\}}^{n+1}=1 n_{k}+1 \Phi^{n+1} .
$$

Hence in this case it is enough if we define the operation of $a$ on $\Phi$ 's .

From the definition of annihilation and creation operators through $(9 \mathrm{a})$ and (9b) it follows that

$$
\left.\begin{array}{l}
{\left[a_{\alpha}, a_{\beta}\right]=\left[a_{\alpha}^{\dagger}, a_{\beta}^{\dagger}\right]=0} \\
{\left[a_{\alpha}, a_{\beta}^{\dagger}\right]=\delta_{\alpha \beta}}
\end{array}\right\}
$$

which are the commutation relations for bosons. If we define the operators not through (9), (9a) and (9b) but by the equation

$$
\left.\begin{array}{l}
a_{i}^{\dagger} c_{n}\left(n_{1}, n_{2}, \ldots, n_{i}, \ldots\right)=V\left(1-n_{i}\right) c_{n+1}\left(n_{1}, n_{2}, \ldots,\left(1-n_{i}\right), \ldots\right) \\
a_{i} c_{n}\left(n_{1}, n_{2}, \ldots, n_{\imath}, \ldots\right)=V n_{i} \cdot c_{n-1}\left(n_{1}, n_{2}, \ldots,\left(1-n_{i}\right), \ldots\right)
\end{array}\right\}
$$

we get the anticommutation relations for fermions:

$$
\left.\begin{array}{l}
{\left[a_{\alpha}, a_{\beta}\right]_{+}=\left[a_{\alpha}^{\dagger}, a_{\beta}^{\dagger}\right]_{+}=0} \\
{\left[a_{\alpha}, a_{\beta}^{\dagger}\right]_{+}=\delta_{\alpha \beta} .}
\end{array}\right\}
$$

Thus we have introduced the concept of the state vector in configuration and momentum spaces when we have a changing number of particles and thereby the annihilation and creation operators. We have yet to arrive at the form of the Hamiltonian and the definition of field operators. Before doing this it will be convenient to develop perturbation expansions for the Schrödinger equation, and by putting these expansions into correspondence with the wave function formalism we arrive at the definition of field operators. In adopting this procedure we have completely reversed the order of the conventional presentation of ideas mainly to emphasize how a field-theoretic description is forced upon us in the collision theory of many particle systems.

1 Here the arguments in the brackets refer to the individual momenta of the particles; grouping the momenta yields the occupation numbers of $(9 a)$. 
We shall show in the succeeding chapter that in writing the generalized Schrödinger equation the state vector should be in momentum representation, i.e. described by $c(t)$ and hence we deliberately use $c(t)$ instead of $\Psi(t)$ as is customary. In dealing with a single particle system $c(t)$ becomes identical with $b(t) \mathrm{e}^{-i E t}$ used in chapter II.

\section{Perturbation expansion in field theory}

We are concerned with the solution of the generalized Schrödinger equation

$$
i \frac{\partial c(t)}{\partial t}=H(t) c(t)
$$

representing the variation of the state vector $c(t)$ with time $t$ and $H(t)$ is the Hamiltonian operator the form and structure of which depends upon the "representation of $c "$. The Schrödinger equation expresses a relationship between states separated by infinitesimal intervals of $t$, i.e. between $t$ and $t+\Delta, \Delta$ being a positive or negative infinitesimal quantity. By solution we mean the establishment of a relationship between $c\left(t_{2}\right)$ and $c\left(t_{1}\right), t_{2}-t_{1}$ being finite. It will be convenient for us to express such a relation in terms of sums and integrals. The integral expansion can then be extended to include descriptions of state, the temporal variation of which cannot be expressed in terms of a differential equation with respect to $t$. In such cases, we take the integral relationship to be fundamental and in any quantum mechanical process we compute $c\left(t_{2}\right)$ given $c\left(t_{1}\right)$ under particular conditions and limiting processes. It is customary to decompose $H$ as

$$
H(t)=H_{0}+H^{\prime}(t),
$$

where $H_{0}$ is independent of time and $H^{\prime}(t)$ is the part which is treated as a perturbation and may in general be time-dependent. The Schrödinger equation is written in the form

$$
c(t+\Delta)=\left\{1+(-i)\left[H_{0}+H^{\prime}(t)\right] \Delta\right\} c(t)+0\left(\Delta^{2}\right),
$$

i.e. given $c(t), c(t+\Delta)$ is obtained by operating the infinitesimal operator $\left\{1+(-i)\left[H_{0}+H^{\prime}(t)\right] \Delta\right\}$ on $c(t)$ and therefore to obtain the solution $c\left(t_{2}\right)$ in terms of $c\left(t_{1}\right)$ for $t_{2}>t_{1}$ we iterate this operation which yields the integral expansion

where

$$
c\left(t_{2}\right)=U_{s}\left(t_{2}, t_{1}\right) c\left(t_{1}\right)
$$

$$
\begin{aligned}
U_{8}\left(t_{2}, t_{1}\right)= & \mathrm{e}^{-i H_{0}\left(t_{2}-t_{1}\right)}+(-i) \int_{t_{1}}^{t_{2}} \mathrm{e}^{-i H_{0}\left(t_{2}-\tau\right)} H^{\prime}(\tau) \mathrm{e}^{-i H_{0}\left(\tau-t_{1}\right)} \mathrm{d} \tau \\
& +(-i)^{2} \int_{t_{1}}^{t_{2}} \mathrm{~d} \tau_{2} \int_{t_{1}}^{\tau_{2}} \mathrm{e}^{-i H_{0}\left(t_{2}-\tau_{2}\right)} H^{\prime}\left(\tau_{2}\right) \mathrm{e}^{-i H_{0}\left(\tau_{2}-\tau_{1}\right)} H^{\prime}\left(\tau_{1}\right) \mathrm{e}^{-i H_{0} \tau_{1}} \mathrm{~d} \tau_{1} . \\
& +\cdots
\end{aligned}
$$

We can also write

$$
U_{s}\left(t_{2}, t_{1}\right)=\mathrm{e}^{-i H_{0} t_{2}} U_{I}\left(t_{2}, t_{1}\right) \mathrm{e}^{i H_{0} t_{1}},
$$


where

$$
U_{I}\left(t_{2}, t_{1}\right)=1+(-i) \int_{t_{1}}^{t_{2}} H_{I}^{\prime}(\tau) \mathrm{d} \tau+(-i)^{2} \int_{t_{1}}^{t_{2}} \mathrm{~d} \tau_{1} \int_{t_{1}}^{\tau_{2}} \mathrm{~d} \tau_{1}
$$

$$
H_{I}^{\prime}\left(\tau_{2}\right) H_{I}^{\prime}\left(\tau_{1}\right)+\cdots
$$

Defining $b(t)$ through

we note that

$$
c(t)=\mathrm{e}^{-H_{0} t} b(t)
$$

$$
b\left(t_{2}\right)=U_{I}\left(t_{2}, t_{1}\right) b\left(t_{1}\right) .
$$

The state vector can be represented either by $b(t)$ or $c(t) . b(t)$ is called the interaction representation and $c(t)$ the Schrödinger representation of the state, both being described in the momentum space.

Without splitting $H(t)$ we can write $U_{8}\left(t_{2}, t_{1}\right)$ as

$$
\begin{aligned}
U_{\delta}\left(t_{2}, t_{1}\right)=1 & +(-i) \int_{t_{1}}^{t_{2}} H(\tau) \mathrm{d} \tau+(-i)^{2} \int_{t_{1}}^{t_{2}} \mathrm{~d} \tau_{2} \int_{t_{1}}^{\tau_{2}} \mathrm{~d} \tau_{1} H\left(\tau_{2}\right) H\left(\tau_{1}\right) \\
& +\cdots+(-i)^{n} \int_{t_{1}}^{t_{2}} \mathrm{~d} \tau_{n} \int_{t_{1}}^{\tau_{n}} \mathrm{~d} \tau_{n-1} \cdots \int_{t_{1}}^{\tau_{2}} \mathrm{~d} \tau_{1} H\left(\tau_{n}\right) \cdots H\left(\tau_{1}\right)+\cdots
\end{aligned}
$$

The most important feature of this expansion is that the time points $\tau_{n}, \ldots, \tau_{1}$ in the integrand of $n$-th order, when $t_{2}>t_{1}$, are ordered such that

$$
t_{2}>\tau_{n}>\tau_{n-1} \cdots>\tau_{1}>t_{1} .
$$

The infinitesimal form of the equation is also valid if $\Delta$ is negative, or equivalently we can write

$$
c(t-\Delta)=[1-(-i) H(t) \Delta] c(t)
$$

$\triangle$ being positive. This immediately implies that we can equally well obtain $c\left(t_{2}\right)$ in terms of $c\left(t_{1}\right)$ even if $t_{2}<t_{1}$. We obtain $u\left(t_{2}, t_{1}\right)$ for $t_{2}<t_{1}$ as

$$
\begin{aligned}
U_{s}\left(t_{2}, t_{1}\right)=1 & +(-i)(-1) \int_{t_{2}}^{t_{1}} H(\tau) \mathrm{d} \tau+(-1)^{2}(-i)^{2} \int_{t_{2}}^{t_{1}} \int_{t_{2}}^{\tau_{1}} H\left(\tau_{2}\right) H\left(\tau_{1}\right) \mathrm{d} \tau_{1} \mathrm{~d} \tau_{2} \\
& +\cdots+(-1)^{n}(-i)^{n} \int_{t_{2}}^{t_{1}} \int_{t_{2}}^{\tau_{1}} \cdots \int_{t_{2}}^{\tau_{n-1}} H\left(\tau_{n}\right) \cdots H\left(\tau_{1}\right) \mathrm{d} \tau_{1} \cdots \mathrm{d} \tau_{n}+\cdots
\end{aligned}
$$

where $t_{2}<\tau_{n}<\tau_{n-1} \cdots<t_{1}$. It is to be noted that since we chose $\Delta$ to be positive, the integration is from the lower limit $t_{2}$ to the upper limit $t_{1}$. Instead of having $(-1)^{n}$ outside the integral, we can ascribe a factor $(-1)$ to each $H(\tau)$. Thus we see that we have to use different forms of integral expansions for $t_{2}>t_{1}$ and for $t_{2}<t_{1}$. On the other hand if we wish to retain the same form, i.e. have the integration performed from $t_{1}$ to $t_{2}$ whether $t_{2} \gtrless t_{1}$ we can drop the factor $(-1)^{n}$ and order the variables $\tau_{1} \ldots \tau_{n}$ in the direction $t_{1}$ to $t_{2}$. This mode of ordering implies that if

and if

$$
t_{2}>t_{1}, t_{1}<\tau_{1}<\tau_{2} \ldots<\tau_{n}<t_{2}
$$

$$
t_{2}<t_{1}, t_{2}<\tau_{n}<\tau_{n-1} \ldots<\tau_{1}<t_{1} .
$$


From this point of view it is evident that

and

$$
\begin{gathered}
U_{s}\left(t_{2}, t_{1}\right)=U_{s}\left(t_{2}, t_{0}\right) u\left(t_{0}, t_{1}\right) \\
U_{s}\left(t_{1}, t_{1}\right)=1=U_{s}\left(t_{1}, t_{0}\right) u_{s}\left(t_{0}, t_{1}\right) \\
U_{s}\left(t_{1}, t_{0}\right)=U_{s}^{-1}\left(t_{0}, t_{1}\right) .
\end{gathered}
$$

We note that such an ordering of the $c$ variables is an essential consequence of the differential equation, or put more emphatically if $c$ satisfies a differential equation, the time variables in $U_{s}\left(t_{2}, t_{1}\right)$ are to be ordered from $t_{1}$ to $t_{2}$. Since we shall be requiring integration of the variables from $t_{1}$ to $t_{2}$ as $t_{1} \rightarrow-\infty$ and $t_{2} \rightarrow+\infty$ the ordering demands

$$
\infty>\tau_{n}>\tau_{n-1} \ldots>\tau_{1}>-\infty .
$$

Such an ordering is called the $P$ ordering. We can remove the restriction on the ordering provided we can introduce an operator which operating on the product $H\left(\tau_{n}\right) \ldots H\left(\tau_{1}\right)$ orders the terms in a manner such that the $\tau$ parameter decreases from left to right. $U_{s}\left(t_{2}, t_{1}\right)$ for $t_{2}>t_{1}$ can then be written as

$$
\begin{gathered}
U_{8}\left(t_{2}, t_{1}\right)=\mathrm{e}^{-i H_{0}\left(t_{2}-t_{1}\right)}+(-i) \int_{t_{1}}^{t_{2}} P\left[\mathrm{e}^{-i H_{0}\left(t_{2}-\tau\right)} H^{\prime}(\tau) \mathrm{e}^{-i H\left(\tau-t_{1}\right)} \mathrm{d} \tau_{1}\right. \\
\left.+\cdots+\frac{(-i)^{n}}{n !}\right] \int_{t_{1}}^{t_{2}} \cdots \int_{t_{1}}^{t_{2}} P[\cdots] \mathrm{d} \tau_{1} \cdots \mathrm{d} \tau_{n}+\cdots
\end{gathered}
$$

In any collision process we are interested in the matrix element $b_{f i}\left(t_{2}, t_{1}\right)$ of transition from a given initial state $i$ at $t_{1}$ to a definite final state $f$ at $t_{2}$ as as $t_{2} \rightarrow+\infty$ and $t_{1} \rightarrow-\infty$. The general final state $b\left(t_{2}\right)$ is

$$
b\left(t_{2}\right)=\sum_{f} b_{f i}\left(t_{2}, t_{1}\right) b_{i}\left(t_{1}\right) .
$$

It is customary to write $b_{f i}$ as

$$
\begin{gathered}
b_{i i}=b_{f i}\left(t_{2}, t_{1}\right) \\
L t t_{2} \rightarrow+\infty \\
t_{1} \rightarrow-\infty .
\end{gathered}
$$

$b_{f i}$ is the projection of the state $b(t)$ at $t_{2}$ on the required final state $f$. The method of obtaining the matrix element $b_{f i}$ depends on the way in which the integral expansion is computed.

We shall first interpret the term $\mathrm{e}^{-i H_{0} t_{2}} b\left(t_{1}\right)$ with $b_{j}\left(t_{1}\right)=0$ for all $j \neq i$ and $b_{i}\left(t_{1}\right)=1$ and thus independent of $t . c_{i}\left(t_{1}\right)$ is the momentum representation of the initial system of particles which can be written as

$$
c_{i}\left(t_{1}\right)=b_{i}\left(t_{1}\right) \mathrm{e}^{-i E_{0} t_{1}}=\mathrm{e}^{-i E_{0} t_{1}} .
$$

where $E_{0}$ is the total energy of the initial system. If the initial state consists of a system of free particles, $b\left(t_{1}\right)$, as $t_{1} \rightarrow-\infty$, can be written as a sequence of creation operators independent of time operating on a vacuum, i.e.

$$
=(\text { a sequence of creation operators })\rangle_{0} \text {. }
$$


We shall call such a state $|i\rangle$. A similar interpretation is given to the final state $f \mid$. As before it can be expressed as a sequence of destruction operators independent of time operating on a vacuum,

$$
\text { i.e. }\langle f|=\langle\text { (a sequence of destruction operators). }
$$

Since the matrix element $b_{f i}$ can now be written as

$$
\begin{aligned}
b_{f i} & =\left\langle f\left|\mathrm{e}^{+i H_{0} t_{2}} U_{8}\left(t_{2}, t_{1}\right) \mathrm{e}^{-i H_{0} t_{1}}\right| i\right\rangle \\
& =\left\langle f\left|U_{I}\left(t_{2}, t_{1}\right)\right| i\right\rangle \\
& =\langle f|S| i\rangle
\end{aligned}
$$

where

$$
S=\operatorname{Lt}_{t_{2} \rightarrow+\infty, t_{1} \rightarrow-\infty} U_{I}\left(t_{2}, t_{1}\right)
$$

and is known as the $S$-matrix introduced by Heisenberg. For convenience we write $S=\sum_{n=0}^{\infty} S^{(n)}$ where $S^{(n)}$ is the $n$-th order matrix element. Now we have reached a stage when we can identify $H_{I}$ in terms of annihilation and creation operators and wave functions of single particles. We shall do so for the system of fermions and bosons and their interactions. For this purpose we shall take only the first order matrix element, i.e.

$$
\left\langle f\left|S^{(1)}\right| i\right\rangle=(-i)\left\langle f\left|\int H_{I}^{\prime}(\tau) \mathrm{d} \tau\right| i\right\rangle .
$$

For simplicity we shall consider electron scattering in the non-relativistic case, i.e. when we postulate that the initial state consists of one electron and the interaction is such that there is no annihilation or creation of positrons. We shall also assume that the photon field is classical and is represented for simplicity by (ignoring normalization and polarization factors) .

$$
\mathrm{e}^{-i q \cdot x}
$$

It is clear that if we choose

$$
H_{I}^{\prime}(t)=\int\left[\sum_{k} a_{k}^{\dagger} \bar{\psi}_{k}(x)\right]\left[\sum_{k^{\prime}} a_{k^{\prime}} \psi_{k^{\prime}}(x)\right] \mathrm{e}^{-i q \cdot x} \mathrm{~d}^{2} x,
$$

substitution of this and $\left|a_{i}^{\dagger}\right\rangle_{0}$ for $|i\rangle$ and $\left|a_{f}^{\dagger}\right\rangle_{0}$ for $|f\rangle$, with the use of the result

$$
\begin{aligned}
\left.a_{k}^{\dagger} a_{k^{\prime}}\right\rangle & =0 \text { if } k \neq k^{\prime} \\
& =1 \text { for } k=k^{\prime},
\end{aligned}
$$

yields exactly the perturbation expansions of chapter II. $H_{I}^{\prime}(\tau)$ can therefore be written as

where

$$
\begin{gathered}
H^{\prime}(\tau)=\int \bar{\psi}(x) \psi(x) \mathrm{e}^{-i q \cdot x} \mathrm{~d}^{3} x, \\
\psi(x)=\sum_{k^{\prime}} a_{k^{\prime}} \psi_{k^{\prime}}(x) \\
\bar{\psi}(x)=\sum_{k} a_{k}^{\dagger} \bar{\psi}_{k}(x)
\end{gathered}
$$


$\psi(x)$ is then called the field variable associated with the fermion. The summation is over all $\boldsymbol{k}$ but only positive energy.

However this treatment is incomplete since we have not taken into account the negative energy states in the definition of $\psi(x)^{1}$. More generally we can define

$$
\begin{aligned}
& \psi(x)=\left[\sum_{k}^{+} b(\boldsymbol{k}) \psi_{\boldsymbol{k}}(x)+\sum_{k}^{-} b(-\boldsymbol{k}) \psi_{-\boldsymbol{k}}(x)\right] \\
& \bar{\psi}(x)=\left[\sum_{k}^{+} b^{\dagger}(\boldsymbol{k}) \bar{\psi}_{k}(x)+\sum_{k}^{-} b^{\dagger}(-\boldsymbol{k}) \bar{\psi}_{-k}(x)\right],
\end{aligned}
$$

where + denotes summation over positive energies and - the summation over negative energies. Since there cannot be negative energy particles, we have to interpret the annihilation operator $b(-\boldsymbol{k})$ of a particle of negative energy as the creation operator of an antiparticle with positive energy; and creation operator $b^{\dagger}(-\boldsymbol{k})$ of a particle with negative energy as the destruction of an anti-particle with positive energy

and

$$
b_{-E_{k}}(-\boldsymbol{k})=d_{+E_{k}}^{\dagger}(\boldsymbol{k})
$$

$$
b_{-E_{k}}^{\dagger}(-\boldsymbol{k})=d_{+E_{k}}(\boldsymbol{k})
$$

Thus we define a field operator associated with a fermion field as

$$
\begin{array}{r}
\psi(x)=\sum_{k} \sum_{r=1} \stackrel{b}{r}_{r}(\boldsymbol{k}) u^{r}(\boldsymbol{k}) \mathrm{e}^{-i k \cdot x}+d_{r}^{\dagger}(\boldsymbol{k}) v_{n}(\boldsymbol{k}) \mathrm{e}^{+i k \cdot x} \\
\bar{\psi}(x)=\sum_{k} \sum_{r=1}^{2} b_{r}^{\dagger}(k) \bar{u}^{r}(k) \mathrm{e}^{i k \cdot x}+d_{r}(\boldsymbol{k}) \bar{v}^{r}(\boldsymbol{k}) \mathrm{e}^{-i k \cdot x}
\end{array}
$$

or more precisely in the limit of continuous $p$ values including normalization factors and summation over spin states

$$
\psi(x)=\frac{1}{(2 \pi)^{3 / 2}} \int\left(\frac{m}{E_{k}}\right)^{1 / 2} \sum_{r=1}^{2}\left\{b_{r}(\boldsymbol{k}) u^{r}(\boldsymbol{k}) \mathrm{e}^{-i k \cdot x}+d_{r}^{\dagger}(\boldsymbol{k}) v^{r}(\boldsymbol{k}) \mathrm{e}^{i k \cdot x}\right\} \mathrm{d}^{3} k
$$

and

$$
\bar{\psi}(x)=\frac{1}{(2 \pi)^{3 / 2}} \int\left(\frac{m}{E_{k}}\right)^{1 / 2} \sum_{r=1}^{2}\left\{b_{r}^{\dagger}(\boldsymbol{k}) \bar{u}^{r}(\boldsymbol{k}) \mathrm{e}^{+i k \cdot x}+d_{r}(\boldsymbol{k}) \bar{v}^{r}(\boldsymbol{k}) \mathrm{e}^{-i k \cdot x}\right\} \mathrm{d}^{3} k
$$

the normalization being $\bar{u} u=1$ and $\bar{v} v=-1$.

Therefore the bilinear interaction term represents the following four processes envisaged in the Feynman theory:

1 Till now we were using $a^{\dagger}(\boldsymbol{k})$ and $a(\boldsymbol{k})$ for the creation and annihilation operators as the discussion was general. As we are now going to consider the case of the electron we shall use $b(\boldsymbol{k}), b^{\dagger}(\boldsymbol{k})$ and $d(\boldsymbol{k}), d^{\dagger}(\boldsymbol{k})$ for the particle and anti particle operators respectively. 
Photon emission with

(1) $b_{k}^{\dagger} b_{k^{\prime}}$-electron scattering

(2) $d_{k}^{\dagger}-d_{k^{\prime}}$-positron scattering

(3) $b_{k}^{\dagger} d_{k_{i}}^{\dagger}$-pair creation

(4) $b_{k} d_{k^{\prime}}$-pair annihilation

More precisely the electron-photon interaction is given by

$$
H_{I}^{\prime}(t)=(4 \pi)^{\frac{1}{2}} e \int \mathrm{d}^{3} x \bar{\psi}(\boldsymbol{x}, t) \Gamma \psi(\boldsymbol{x}, t) \varphi(\boldsymbol{x}, t)
$$

where $T=\gamma_{\mu}$, and $\varphi(x)=A_{\mu}(x)$ is the photon field.

Any $\psi_{k}(\boldsymbol{x}, t)$ can be written as

$$
\psi_{k}(\boldsymbol{x}, t)=\psi_{k}(\boldsymbol{x}) \mathrm{e}^{-i \omega_{k} t} .
$$

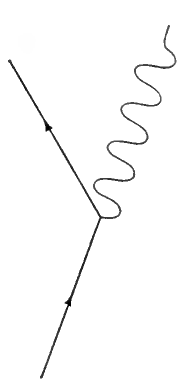

(I)

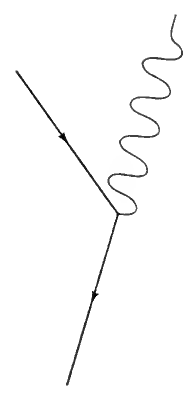

(2)

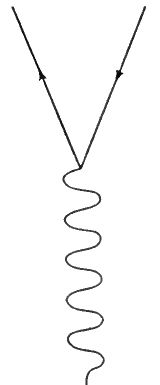

(3)

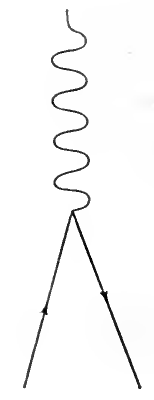

(4)

Fig. 20. (1) $a a^{\dagger}$ : electron scattering;

(2) $b b^{\dagger}$ : positron scattering;

(3) $a^{\dagger} b^{\dagger}$ : pair creation;

(4) $a b$ : pair annihilation.

This is equivalent to writing the operator $\psi(x, t)$ as

where

$$
\psi(\boldsymbol{x}, t)=\mathrm{e}^{+i H_{0} t} \psi(\boldsymbol{x}) \mathrm{e}^{-i H_{0} t},
$$

This implies that

$$
\psi(\boldsymbol{x})=\sum_{k} a_{k} \psi_{k}(\boldsymbol{x})
$$

$$
a_{k} \mathrm{e}^{-i \omega_{k} t}=\mathrm{e}^{i H_{0} t} a_{k} \mathrm{e}^{-i H_{0} t}
$$

That this is so is easily seen by the following argument. $\mathrm{e}^{-i H_{0} t}$ operating upon the state vector in momentum representation yields the factor $\mathrm{e}^{-i E_{i} t}$ where $E_{i}$ is the energy of the system. Removing this numerical factor we now operate $\mathrm{e}^{i H_{0} t}$ on a state vector containing one particle less corresponding to momentum $\boldsymbol{k}$, since $a_{k}$ has operated on the state vector. Hence we get the factor $\mathrm{e}^{+i\left(E_{i}-\omega_{k}\right) t}$ so that we get finally $a_{k} \mathrm{e}^{-i \omega_{k} t}$ which is considered as the interaction representation of the operator $a_{k}$. In (38) we have expressed $H_{I}^{\prime}(t)$ in terms of the creation 
and annihilation operators and single particle wave functions. In a similar manner it is possible to identify $H^{\prime}$. For,

where

$$
H_{I}^{\prime}(t)=\mathrm{e}^{i H_{0} t} H^{\prime} \mathrm{e}^{-i H_{0} t},
$$

$$
H^{\prime}=\int \bar{\psi}(\boldsymbol{x}) \psi(\boldsymbol{x}) \varphi(\boldsymbol{x}) \mathrm{d}^{3} x .
$$

Substituting for $H^{\prime}$ in (31) we just obtain the expansion $U_{s}\left(t_{2}, t_{1}\right)$ and the correct relationship between $U_{s}\left(t_{2}, t_{1}\right)$ and $U_{I}\left(t_{2}, t_{1}\right)$.

Now we wish to work with the interaction Hamiltonian $H_{I}^{\prime}(\boldsymbol{x})$ in computing matrix elements. Operating $\mathrm{e}^{-i H_{0} \tau}$ on $|i\rangle$ yields the term $\mathrm{e}^{-i E_{i} \tau}$. But when $H^{\prime}$ acts on $|i\rangle$, we get a new system of particles depending on the structure of the interaction. The total momentum of the new system of particles is identical with that before the operation of $H^{\prime}$. However the energy of the new system corresponding to the total momentum is different from the energy of the initial system for the obvious reason that we are dealing with a different system of particles. Hence the operation of $\mathrm{e}^{i H_{0} \tau}$ on the new state yields $\mathrm{e}^{i E_{1} \tau}$ where the value $E_{1}$ will depend upon the type of particles in the system. If we perform the integration over $\tau$ we obtain the energy denominator $\frac{1}{E_{1}-E_{i}}$. The above procedure can be obviously extended to all orders and we are led to the old fashioned perturbation theory. This method of computing the matrix element is based on the use of the Schrödinger representation.

On the other hand, if we work with $H_{I}^{\prime}(x)$ and use the commutation rules we will arrive at the Feynman expansion as shall be demonstrated in the succeeding chapters.

Thus we have introduced the concept of field operators by demonstrating the necessity of expressing the interaction Hamiltonian in terms of annihilation and creation operators and single particle wave functions. Our final task is to identify $H_{0}$ which should be expressed in terms of field operators such that $H_{0}$ operating on a system of particles should yield its energy. $H_{0}$ for a fermion field may then be written as

$$
H_{0}=\int \mathrm{d}^{3} x \bar{\psi}(\boldsymbol{x})(-i \gamma \cdot \boldsymbol{V}+m) \psi(\boldsymbol{x}),
$$

where $\bar{\psi}(\boldsymbol{x})$ and $\psi(\boldsymbol{x})$ have already been defined and $\boldsymbol{\gamma}$ are the well-known Dirac matrices.

\section{Fermion fields}

The field variable associated with each point $\boldsymbol{x}$ is given by

$$
\psi(\boldsymbol{x})=\frac{1}{(2 \pi)^{3 / 2}} \int\left(\frac{m}{E_{p}}\right)^{1 / 2}\left[\sum_{r=1}^{2} b_{r}(\boldsymbol{p}) u^{r}(\boldsymbol{p}) \mathrm{e}^{-i \boldsymbol{p} \cdot \boldsymbol{x}}+d_{r}^{\dagger}(\boldsymbol{p}) v^{r}(p) \mathrm{e}^{+i \boldsymbol{p} \cdot \boldsymbol{x}}\right] \mathrm{d}^{3} p,
$$

where $b, b^{\dagger}$ and $d, d^{\dagger}$ are the annihilation and creation operators of the particles and anti-particles respectively. $u^{r}(\boldsymbol{p})$ is the positive energy spinor and $v^{r}(p)$ the negative energy spinor with negative momentum - $\boldsymbol{p}$. Note that $\psi(x)$ is a destruc- 
tion operator for particles and a creation operator for antiparticles. In the Schrödinger representation the commutation relations are given by

and

$$
\left[\psi(\boldsymbol{x}), \psi\left(\boldsymbol{x}^{\prime}\right)\right]_{+}=\left[\psi^{\dagger}(\boldsymbol{x}), \psi^{\dagger}\left(\boldsymbol{x}^{\prime}\right)\right]_{+}=\mathbf{0}
$$

$$
\left[\psi(\boldsymbol{x}), \psi^{\dagger}\left(\boldsymbol{x}^{\prime}\right)\right]_{+}=\delta\left(\boldsymbol{x}-\boldsymbol{x}^{\prime}\right) .
$$

The expression for the field operator in the interaction representation is given by

$$
\psi_{I}(x)=\frac{1}{(2 \pi)^{3 / 2}} \int \mathrm{d}^{3} p\left(\frac{m}{E_{p}}\right)^{1 / 2} \sum_{r=1}^{2}\left\{b_{r}(\boldsymbol{p}) u^{r}(\boldsymbol{p}) \mathrm{e}^{-i p \cdot x}+d_{r}^{\dagger}(\boldsymbol{p}) v^{r}(\boldsymbol{p}) \mathrm{e}^{+i p \cdot x}\right\} .
$$

and $\quad \bar{\psi}_{I}(x)=\frac{1}{(2 \pi)^{3 / 2}} \int \mathrm{d}^{3} p\left(\frac{m}{E_{p}}\right)^{1 / 2} \sum_{r=1}^{2}\left\{b_{r}^{\dagger}(\boldsymbol{p}) \bar{u}^{r}(\boldsymbol{p}) \mathrm{e}^{i p \cdot x}+d_{r}(\boldsymbol{p}) \bar{v}^{r}(\boldsymbol{p}) \mathrm{e}^{-i p \cdot x}\right\}$.

The anticommutation rules are

$$
\left[\psi_{x}(x), \bar{\psi}_{\beta}\left(x^{\prime}\right)\right]_{+}=(-\nabla+i m)_{\dot{\alpha} \beta} \Delta\left(x-x^{\prime} ; m\right)=-i S_{\alpha \beta}\left(x-x^{\prime}\right),
$$

where $\Delta(x, m)$ is given by (16) with $m$ in the place of $\mu$ and $\alpha, \beta$ represent the components of a spinor. $S_{\alpha \beta}$ is an element of a matrix $S$ where $S$ is given by

$$
S\left(x-x^{\prime}\right)=-(i \nabla+m) \Delta\left(x-x^{\prime} ; m\right) .
$$

It is easily verified that

$$
(i \nabla-m) S\left(x-x^{\prime}\right)=: 0 .
$$

We notice that $\psi(x)$ and $\bar{\psi}(x)$ can be split up into positive and negative frequency parts which correspond to destruction and creation operators. The anticommutation relations between these can be easily written down as

and

$$
\begin{aligned}
& {\left[\psi^{(+)}(x), \psi^{(-)}\left(x^{\prime}\right)\right]_{+}=\left[\bar{\psi}^{(-)}(x), \bar{\psi}^{(+)}\left(x^{\prime}\right)\right]_{+}=0} \\
& {\left[\bar{\psi}^{(+)}(x), \psi^{(+)}\left(x^{\prime}\right)\right]_{+}=\left[\bar{\psi}^{(-)}(x), \psi^{(-)}\left(x^{\prime}\right)\right]_{+}=0}
\end{aligned}
$$

$$
\begin{aligned}
& {\left[\psi_{\alpha}^{(+)}(x), \bar{\psi}_{\beta}^{(+)}\left(x^{\prime}\right)\right]_{+}=-i S_{\alpha \beta}^{(+)}\left(x-x^{\prime}\right)} \\
& {\left[\psi_{\alpha}^{(-)}(x), \bar{\psi}_{\beta}^{(+)}\left(x^{\prime}\right)\right]_{+}=-i S_{\alpha \beta}^{(-)}\left(x-x^{\prime}\right) .}
\end{aligned}
$$

Real scalar (neutral) field

Let $\varphi(\boldsymbol{x})$ represent the field variable associated with every point $\boldsymbol{x}$ in space. Now for a real scalar field $\varphi=\varphi^{*}$ and hence $a_{k}=a_{k}^{\ddagger}$. Thus we have

$$
\varphi(x)=\frac{1}{(2 \pi)^{3 / 2}} \sum_{k} \frac{1}{\sqrt{2} \omega(k)}\left[a_{k} \mathrm{e}^{i \boldsymbol{k} \cdot \boldsymbol{x}}+a_{k}^{\dagger} \mathrm{e}^{-i \boldsymbol{k} \cdot \boldsymbol{x}}\right] .
$$

In this form $\varphi(\boldsymbol{x})$ clearly satisfies the physical requirement, namely, the creation and annihilation are only of particles of positive energy. Similarly

$$
\pi(\boldsymbol{x})=\frac{1}{(2 \pi)^{3 / 2}} \sum_{k}\left[\frac{\omega_{k}}{2}\right]^{1 / 2} i\left[a_{k}^{\dagger} \mathrm{e}^{-i \boldsymbol{k} \cdot \boldsymbol{x}}-a_{k} \mathrm{e}^{+i \boldsymbol{k} \cdot \boldsymbol{x}}\right] .
$$


Thus we obtain the commutation relations

and

$$
\left[\varphi(\boldsymbol{x}), \varphi\left(\boldsymbol{x}^{\prime}\right)\right]=\left[\pi(\boldsymbol{x}), \pi\left(\boldsymbol{x}^{\prime}\right)\right]=0
$$

$$
\left[\varphi(\boldsymbol{x}), \pi\left(\boldsymbol{x}^{\prime}\right)\right]=i \delta\left(\boldsymbol{x}-\boldsymbol{x}^{\prime}\right) .
$$

In the interaction representation

$$
\begin{aligned}
\varphi_{I}(x) & =\frac{1}{(2 \pi)^{3 / 2}} \int \frac{\mathrm{d}^{3} k}{\sqrt{2} \omega_{k}}\left\{a_{k} \mathrm{e}^{i\left(k \cdot x-\omega_{k} t\right)}+a_{k}^{\dagger} \mathrm{e}^{-i\left(k \cdot x-\omega_{k} t\right)}\right\} \\
& =\frac{1}{(2 \pi)^{3 / 2}} \int \frac{\mathrm{d}^{3} k}{\sqrt{2} \omega_{k}}\left\{a_{k} \mathrm{e}^{-i k \cdot x}+a_{k}^{\dagger} \mathrm{e}^{i k \cdot x}\right\} .
\end{aligned}
$$

Note that $\omega_{k}$ is always taken as positive and thus the frequency dependence of creation and annihilation operators are different. The commutation relations in the interaction representation can easily be worked out using the commutation relations satisfied by $a(\boldsymbol{k})$ and $a^{\dagger}(\boldsymbol{k})$. Thus

where

$$
\begin{aligned}
{\left[\varphi_{I}(x), \varphi_{I}\left(x^{\prime}\right)\right]=} & \frac{1}{2(2 \pi)^{3}} \int \frac{\mathrm{d}^{3} k}{\sqrt{\omega_{k}}} \int \frac{\mathrm{d}^{3} k^{\prime}}{\sqrt{\omega_{k^{\prime}}}}\left\{\left[a_{k}, a_{k^{\prime}}^{\dagger}\right] \mathrm{e}^{i\left(k^{\prime} \cdot x^{\prime}-k \cdot x\right)}\right. \\
& \left.+\left[a_{k}^{\dagger}, a_{\boldsymbol{k}^{\prime}}\right] \mathrm{e}^{-i\left(k^{\prime} \cdot x^{\prime}-k \cdot x\right)}\right\} \\
= & \frac{1}{2(2 \pi)^{3}} \int_{k_{4}>0} \frac{\mathrm{d}^{3} k}{k_{4}}\left[\mathrm{e}^{-i k \cdot\left(x-x^{\prime}\right)}-\mathrm{e}^{i k \cdot\left(x-x^{\prime}\right)}\right] \\
= & i \Delta\left(x-x^{\prime}\right),
\end{aligned}
$$

$$
\Delta\left(x-x^{\prime}\right)=\frac{-i}{2} \frac{1}{(2 \pi)^{3}} \int_{k_{4}>0} \frac{\mathrm{d}^{3} k}{k_{4}}\left\{\mathrm{e}^{-i k \cdot\left(x-x^{\prime}\right)}-\mathrm{e}^{i k \cdot\left(x-x^{\prime}\right)}\right\} .
$$

This function $\Delta\left(x-x^{\prime}\right)$ is invariant under translations and homogeneous Lorentz transformations. ( $\frac{\mathrm{d}^{3} k}{k_{4}}$ is an invariant quantity under Lorentz transformation.) In order to make the covariance more explicit, we remove the restriction on $k_{4}$ by using

Thus

$$
\delta\left(k^{2}-\mu^{2}\right)=\frac{1}{2\left|k_{4}\right|}\left\{\delta\left(k_{4}+\omega_{k}\right)+\delta\left(k_{4}-\omega_{k}\right)\right\}
$$

$$
\Delta\left(x-x^{\prime}\right)=\frac{-i}{(2 \pi)^{3}} \int_{-\infty}^{\infty} \mathrm{d}^{4} k \mathrm{e}^{-i k\left(x-x^{\prime}\right)} \delta\left(k^{2}-\mu^{2}\right) \varepsilon(k)
$$

where $\varepsilon(k)=\frac{k_{4}}{\left|k_{4}^{\prime}\right|}$ and has an invariant meaning. We further note that the commutator will vanish for equal times which also means that the commutator will 
vanish for space-like separations between the two points $x$ and $x^{\prime}$. Now $\varphi_{\mathrm{I}}(x)$ can be split up into $a$ positive and a negative frequency part, i.e.

where

$$
\varphi_{\mathrm{I}}(x)=\varphi^{+}(x)+\varphi^{-}(x),
$$

and

$$
\varphi^{(+)}(x)=\frac{1}{(2 \pi)^{3 / 2}} \int_{k_{4}>0} \frac{\mathrm{d}^{3} k}{\sqrt{2} \omega_{k}}-a_{k} \mathrm{e}^{-i k \cdot x}
$$

$$
\varphi^{(-)}(x)=\frac{1}{(2 \pi)^{3 / 2}} \int_{k_{4}>0} \frac{\mathrm{d}^{3} k}{\sqrt{2 \omega_{k}}} a_{k}^{\dagger} \mathrm{e}^{i k \cdot x} .
$$

The following relations are then easily obtained.

where

$$
\begin{aligned}
& {\left[\varphi^{(+)}(x), \varphi^{(+)}\left(x^{\prime}\right)\right]=\left[\varphi^{(-)}(x), \varphi^{(-)}\left(x^{\prime}\right)\right]=0} \\
& {\left[\varphi^{(+)}(x), \varphi^{(-)}\left(x^{\prime}\right)\right]=i \Delta^{(+)}\left(x-x^{\prime}\right)} \\
& {\left[\varphi^{(-)}(x), \varphi^{(+)}\left(x^{\prime}\right)\right]=i \Delta^{(-)}\left(x-x^{\prime}\right),}
\end{aligned}
$$

$$
\Delta^{(+)}\left(x-x^{\prime}\right)=\frac{-i}{2(2 \pi)^{3}} \int_{k_{4}>0} \frac{\mathrm{d}^{3} k}{k_{4}} \mathrm{e}^{-i k \cdot\left(x-x^{\prime}\right)}
$$

and

$$
\Delta^{(-)}\left(x-x^{\prime}\right)=\frac{i}{2(2 \pi)^{3}} \int_{k_{4}>0} \frac{\mathrm{d}^{3} k}{k_{4}} \mathrm{e}^{+i k \cdot\left(x-x^{\prime}\right)} .
$$

Defining

$$
\Delta\left(x-x^{\prime}\right)=\Delta^{(+)}\left(x-x^{\prime}\right)+\Delta^{(-)}\left(x-x^{\prime}\right)
$$

and

$$
-i \Delta^{(1)}\left(x-x^{\prime}\right)=\Delta^{(+)}\left(x-x^{\prime}\right)-\Delta^{(-)}\left(x-x^{\prime}\right)
$$

it can be seen that $\Delta^{(1)}\left(x--x^{\prime}\right)$ is the vacuum expectation value of

$$
\left[\varphi(x), \varphi\left(x^{\prime}\right)\right]_{+}=\varphi(x) \varphi\left(x^{\prime}\right)+\varphi\left(x^{\prime}\right) \varphi(x) .
$$

In the above consideration, we have assumed that the field is described by a real field variable $\varphi$. However to describe charged bosons, the field variable has to be complex. But the quantization procedure is essentially the same. Two types of creation and annihilation operators are defined $a_{k}^{\dagger}, b_{k}^{\dagger}$ and $a_{k}, b_{k}$ for positively and negatively charged particles. The total charge operator in terms of creation and annihilation operators is

$$
Q=\frac{1}{2} e\left\{a_{k}^{\dagger} a_{k}+a_{k} a_{k}^{\dagger}-b_{k}^{\dagger} b_{k}-b_{k} b_{k}^{\dagger}\right\}
$$

\section{NORMAL PRODUCTS ANDWICK'S THEOREM}

We shall now describe the method of obtaining the matrix element $\langle f|S| i\rangle$ from a given initial state $|i\rangle$ to a final state $|f\rangle$ using the $S$-matrix expansion given before. We have already explained why the states $|i\rangle$ and $|f\rangle$ should be expressed as creation operators operating on a bare vacuum and the field operators 
in the $S$-matrix in the interaction representation. The initial state is represented by $|i\rangle$ and the final state by $\langle f|$, the latter therefore being expressed as a sequence of annihilation operators operating on the vaccum to the left, i.e. on \langle| . Our method should be such as to yield exactly the same matrix element as is obtained through the Feynman formalism. That is, for example in electrodynamic processes, it should be expressed in terms of the momentum representation of the incident and emergent particles and of the propagators corresponding to intermediate states. The integrands in the $S$-matrix can be expressed through the field operators and each field operator consists of two terms, one containing annihilation operators and the other creation operators. The calculation of matrix elements consists in rearranging the terms in such a manner as to reduce them to a product of single particle propagators in configuration space and the wave function corresponding to the incident and emergent particles. The integration with respect to space and time variables puts the propagators in momentum representation and the desired matrix element is thereby obtained.

A single particle propagator connecting two space time points implies the creation of a particle at one point and annihilation at the other and thus is identified in the rearrangement if a creation operator with the wave function associated with it is placed to the right of an annihilation operator with the corresponding complex conjugated wave function. The only sequence of terms which therefore does not contain a propagator is one which contains only creation operators or only annihilation operators or all the annihilation operators to the right of the creation operators. Such a sequence is said to be in the "normal product" form. Thus it is clear that given any sequence of terms, they can be rearranged and expressed as a product of propagators and a normal product. Remembering that $|i\rangle$ and $\langle f|$ are represented as creation and annihilation operators acting on the vacuum, it is easy to see that the normal product has terms with annihilation operators to the right, and these taken together with $|i\rangle$ on the right represent the incident particles; the terms with creation operators in the normal product taken with $\langle f|$ on the left represent emergent particles. The incoming and emergent particles are represented by external lines in the Feynman diagram. It is quite obvious that the expectation value of a normal product between vacuum states is zero since an annihilation operator acting on a vacuum yields zero. We now proceed to demonstrate the mode of reduction of the $S$-matrix into propagators and normal products.

For this purpose we have to introduce the Wick's chronological operator $T$ and the normal product operator $N$ as follows:

$$
\begin{aligned}
T(\psi(x) \psi(y)) & =+\psi(x) \psi(y), x_{4}>y_{4} \\
& =-\psi(y) \psi(x), x_{4}<y_{4}
\end{aligned}
$$

for fermions. Using the commutation relations for the $\psi$ 's, i.e. $[\psi, \psi]_{+}=0$ we have for all times

$$
T(\psi(x) \psi(y))=\psi(x) \psi(y) .
$$


Similarly we have

For bosons

$$
\begin{aligned}
T(\psi(x) \bar{\psi}(y)) & =+\psi(x) \bar{\psi}(y), x_{4}>y_{4} \\
& =-\bar{\psi}(y) \psi(x), y_{4}>x_{4} .
\end{aligned}
$$

$$
\begin{aligned}
T(\varphi(x) \varphi(y)) & =\varphi(x) \varphi(y), \quad x_{4}>y_{4} \\
& =+\varphi(y) \varphi(x), \quad y_{4}>x_{4} .
\end{aligned}
$$

Thus the $T$-operator is identical with the Dyson $P$ operator with the addition of a minus sign in the case of an interchange of fermion field operators.

The normal product operator $N$ is defined as

and

$$
\begin{aligned}
N\left(\psi^{(+)}(x) \bar{\psi}^{(-)}(x)\right) & =-N\left(\bar{\psi}^{(-)}(y) \psi^{(+)}(x)\right) \\
& =-\left(\bar{\psi}^{(-)}(y) \psi^{(+)}(x)\right)
\end{aligned}
$$

for fermions. For bosons, we have

$$
\begin{aligned}
N\left(\psi^{(+)}(x) \psi^{(+)}(y)\right) & =-N\left(\psi^{(+)}(y) \psi^{(+)}(x)\right) \\
& =\psi^{(+)}(x) \psi^{(+)}(y)=-\psi^{(+)}(y) \psi^{(+)}(x)
\end{aligned}
$$

The $S$-matrix is given by

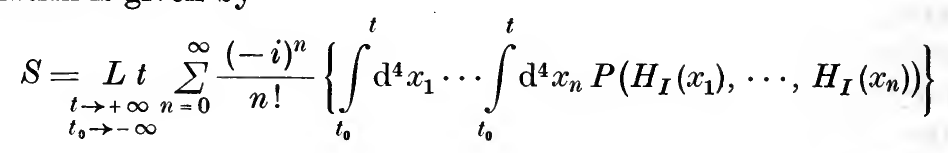

where $H_{I}\left(x_{n}\right)=\bar{\psi}\left(x_{n}\right) \mathrm{A}\left(x_{n}\right) \psi\left(x_{n}\right)$ in quantum electrodynamics. We note that there is an ambiguity in the ordering of operators in the term corresponding to a vertex. To remove this ambiguity we shall examine the meaning of the term,

$$
\bar{\psi}(x) \psi(x)=\left[\begin{array}{r}
\bar{\psi}^{(+)}(x) \psi^{(+)}(x) \\
+\bar{\psi}^{(+)}(x) \psi^{(-)}(x) \\
+\bar{\psi}^{(-)}(x) \psi^{(+)}(x) \\
+\bar{\psi}^{(-)}(x) \psi^{(-)}(x)
\end{array}\right]
$$

Only one of these terms has the creation operator to the right of the annihilation operator. Treating $\bar{\psi}^{(+)}(x) \psi^{(-)}(x)$ as the limit of $\bar{\psi}^{(+)}(y) \psi^{(-)}(x)$ as $y \rightarrow x$, i.e. with $y_{4}-x_{4} \rightarrow+0$ we note that the vacuum expectation value of $\bar{\psi}^{(+)}(x) \psi^{(-)}(x)$ is the limit of a propagator which is infinite. On the other hand, if we put the annihilation operator to the right of the creation operator, it will not represent the limit of a propagator. Therefore assuming the interaction to be represented in this form would imply that we are taking it in the normal product form. This difficulty of the vacuum expectation value of the current becoming infinite and the necessity for the modified expression were realized by Heisenberg ${ }^{1}$ as early as 1934 .

1 Heisenberg, W., Z. Phys., 90, 209 (1934); 92, 692 (1934). 
Thus taking the current in the normal product form as

$$
j_{\mu}(x)=\mathrm{e} N\left(\bar{\psi}(x) \gamma_{\mu} \psi(x)\right)
$$

we can write the $S$-matrix as

$$
\begin{aligned}
S=\sum_{n=0}^{\alpha} \frac{(-i e)^{n}}{n !} \int_{-\infty}^{\infty} \mathrm{d}^{4} x_{1} \cdots & \int_{-\infty}^{\infty} \mathrm{d}^{4} x_{n} . \\
& T\left\{N\left(\bar{\psi}\left(x_{1}\right) \mathrm{A}\left(x_{1}\right) \psi\left(x_{1}\right)\right) \cdots N\left(\bar{\psi}\left(x_{n}\right) \mathrm{A}\left(x_{n}\right) \psi\left(x_{n}\right)\right)\right\} .
\end{aligned}
$$

Since $A_{\mu}(x)$ commutes with $\bar{\psi}(x) \gamma_{\mu} \psi(x)$ the relative ordering of $A_{\mu}(x)$ and $\bar{\psi}(x) \gamma_{\mu} \psi(x)$ is irrelevant and hence we have included the $A_{\mu}$ also in the normal product.

We shall first prove that a $T$-product for two operators can be uniquely decomposed into a normal product and a propagator. We have

$$
T\left(\psi_{\alpha}(x) \bar{\psi}_{\beta}(y)-N\left(\psi_{\alpha}(x) \bar{\psi}_{\beta}(y)\right) \quad \text { for } \quad x_{\mathbf{4}}>y_{4}\right.
$$

$=T\left[\begin{array}{r}\psi_{\alpha}^{+}(x) \bar{\psi}_{\beta}^{+}(y) \\ +\psi_{\alpha}^{+}(x) \bar{\psi}_{\beta}^{-}(y) \\ +\psi_{\alpha}^{-}(x) \bar{\psi}_{\beta}^{+}(y) \\ +\psi_{\alpha}^{-}(x) \bar{\psi}_{\bar{\beta}}^{-}(y)\end{array}\right]-N\left[\begin{array}{r}\psi_{\alpha}^{+}(x) \bar{\psi}_{\beta}^{+}(y) \\ +\psi_{\alpha}^{+}(x) \bar{\psi}_{\beta}^{-}(y) \\ +\psi_{\alpha}^{-}(x) \psi_{\beta}^{+}(y) \\ +\psi_{\alpha}^{-}(x) \bar{\psi}_{\beta}^{-}(x)\end{array}\right]$

$=\left[\begin{array}{r}\psi_{\alpha}^{+}(x) \bar{\psi}_{\beta}^{+}(y) \\ +\psi_{\alpha}^{+}(x) \bar{\psi}_{\beta}^{-}(y) \\ +\psi_{\alpha}^{-}(x) \bar{\psi}_{\beta}^{+}(y) \\ +\psi_{\alpha}^{-}(x) \bar{\psi}_{\beta}^{-}(y)\end{array}\right]-\left[\begin{array}{r}\psi_{\alpha}^{+}(x) \bar{\psi}_{\beta}^{+}(y) \\ -\psi_{\beta}^{-}(y) \bar{\psi}_{\alpha}^{+}(x) \\ +\psi_{\alpha}^{-}(x) \bar{\psi}_{\beta}^{+}(y) \\ +\psi_{\alpha}^{-}(x) \bar{\psi}_{\beta}^{-}(y)\end{array}\right]=\left[\psi_{\alpha}^{+}(x), \bar{\psi}_{\beta}^{-}(y)\right]_{+}=-i S_{\alpha \beta}^{+}(x-y)$.

For $y_{4}>x_{4}$

$$
\begin{aligned}
& T\left(\psi_{\alpha}(x) \bar{\psi}_{\beta}(y)\right)-N\left(\psi_{\alpha}(x) \bar{\psi}_{\beta}(y)\right)=-T\left(\bar{\psi}_{\beta}(y) \psi_{\alpha}(x)\right)-N\left(\psi_{\alpha}(x) \bar{\psi}_{\beta}(y)\right) . \\
&=-\left[\begin{array}{r}
\bar{\psi}_{\beta}^{+}(y) \psi_{\alpha}^{+}(x) \\
+\bar{\psi}_{\beta}^{+}(y) \psi_{\alpha}^{-}(x) \\
+\bar{\psi}_{\beta}^{-}(y) \psi_{\alpha}^{+}(x) \\
+\bar{\psi}_{\beta}^{-}(y) \psi_{\alpha}^{-}(x)
\end{array}\right]-\left[\begin{array}{r}
\psi_{\alpha}^{+}(x) \bar{\psi}_{\beta}^{+}(y) \\
+\psi_{\alpha}^{-}(x) \bar{\psi}_{\beta}^{+}(y) \\
-\bar{\psi}_{\beta}^{-}(y) \psi_{\alpha}^{+}(x) \\
+\psi_{\alpha}^{-}(x) \bar{\psi}_{\beta}^{-}(y)
\end{array}\right] \\
&=-\left[\bar{\psi}_{\beta}^{+}(y), \psi_{\alpha}^{+}(x)\right]_{+}-\left[\bar{\psi}_{\beta}^{+}(y), \psi_{\alpha}^{-}(x)\right]_{+} \\
&-\left[\bar{\psi}_{\beta}^{-}(y), \psi_{\alpha}^{-}(x)\right]_{+} \\
&-\left[\bar{\psi}_{\beta}^{+}(y), \psi_{\alpha}^{-}(x)\right]_{+} \\
&=+i S_{\alpha \beta}^{-}(x-y) .
\end{aligned}
$$

Thus for all times we have

$$
T\left(\psi_{\alpha}(x) \bar{\psi}_{\beta}(y)\right)-N\left(\psi_{\alpha}(x) \bar{\psi}_{\beta}(y)\right)=-1 / 2 S_{F \alpha \beta}(x-y) .
$$

EPCR 9 
The difference of the $T$ product and the $N$ product is defined as the contraction $\psi_{\alpha}(x) \bar{\psi}_{\dot{\beta}}(y)$. This has been shown to be the propagator. Similarly defining the contraction for bosons as $\varphi^{*}(x) \varphi^{*}(y)$, we can show that

$$
\begin{aligned}
\varphi^{*}(x) \varphi^{*}(y) & =(i) \Delta^{+}(x-y) \quad \text { for } \quad x_{4}>y_{4} \\
& =(-i) \Delta^{-}(x-y) \quad \text { for } \quad y_{4}>x_{4} \\
& =\frac{1}{2} \Delta_{F}(x-y) \quad \text { for all times. }
\end{aligned}
$$

We must now prove that by the application of this rule the entire $T$ product of a number of operators can be decomposed into normal products and propagators. This is known as Wick's theorem which states that a $T$-product can be decomposed into a unique sum of normal products of the form:

$$
\begin{aligned}
T(U V \ldots, X Y Z)= & N(U V \ldots, X Y Z)+N\left(U^{\bullet} V^{\bullet} \ldots, X Y Z\right) \\
& +\cdots+N\left(U^{\bullet} V^{\bullet} \ldots, X^{\bullet} Y Z^{\bullet}\right)+\ldots
\end{aligned}
$$

where the following notation has been used; the first term represents the normal product of all the operators, the second, the normal product of operators in which any one pair has been contracted, the third in which two pairs are contracted, and so on until all possible combinations are exhausted. The proof follows from induction.

\section{Proof of Wick's theorem}

To prove the theorem by induction, we note that it is true in the case of a single factor and also for two factors as in the latter case it merely gives the definition of the contraction of two operators in terms of the $T$ and $N$ products (15). Let us assume that it holds for $n$ factors. Multiplying the left-hand side of (19) on the right by an operator $\Omega$ labelled with a time earlier than any other factor, we have

$$
T(U V \ldots X Y Z) \Omega=T(U V \ldots X Y Z \Omega)
$$

The terms on the right-hand side of (19) will be of the form

$$
N(U V \ldots X Y Z) \Omega \text {. }
$$

Now if we are able to decompose each one of these in the following way,

$$
\begin{aligned}
N(U V \ldots X Y Z) \Omega & =N(U V \ldots X Y Z \Omega) \\
& +N\left(U V \ldots X Y Z^{\bullet} \Omega^{\circ}\right) \\
& +N\left(U V \ldots X Y^{\bullet} Z \Omega^{\circ}\right) \\
& +\ldots \\
& +N\left(U^{\bullet} V \ldots X Y Z \Omega^{\circ}\right)
\end{aligned}
$$

then the sum of the terms on the right-hand side so obtained will evidently correspond to the right-hand side of (19) written for $n+1$ factors, if $\Omega$ is earlier than $U V \ldots X Y Z$. (This restriction is however removed once $\Omega$ is inside the $T$ and $N$ products since these remain by definition unchanged for the same re- 
grouping of factors on both sides of the equation.) Thus we will have proved Wick's theorem if we prove (21). For this purpose, we again have to take recourse to induction. (21) is true for a single factor in $N$ since then it is merely the definition of contraction of two operators given earlier. Let us assume now that it is true for $n$ factors, that $\Omega$ is a creation operator and that all the operators $U V \ldots X Y Z$ are annihilation operators. If $\Omega$ is an annihilation operator and $U V \ldots X Y Z$ contain both creation and annihilation operators, then (21) would become a trivial identity since the contraction of two annihilation operators is zero as also that of an annihilation operator whose time label is earlier than that of the creation operator with which it is contracted. Further if $\Omega$ is a creation operator as we have assumed, we may add any number of creation operators on the left of all the factors inside the $N$ products on both sides of equation (21) and the theorem would still be valid since the contraction of two creation operators is zero. Thus our assumption that $\Omega$ is a creation operator earlier than that of any of the annihilation operators $U V \ldots X Y Z$ is sufficient for our purpose. Multiplying (21) by an annihilation operator $D$ which has a time later than $\Omega$ on the left, we get

$$
\begin{aligned}
& D N(U V \ldots X Y Z) \Omega=N\left(D U V \ldots X Y Z^{*} \Omega^{*}\right)+\ldots+N\left(D U^{*} V \ldots X Y Z \Omega^{*}\right) \\
& \qquad \quad+D N(U V \ldots X Y Z \Omega) . \\
& \text { Further since }
\end{aligned}
$$

$$
D N(U V \ldots X Y Z \Omega)=\delta_{P} D \Omega U \ldots X Y Z
$$

$\left(\delta_{P}\right.$ being the sign of the permutation for fermion factors) and

$$
D \Omega=T(D \Omega)=D^{\bullet} \Omega^{\bullet}+N(D \Omega)=D^{\bullet} \Omega^{\bullet}+\delta_{Q} \Omega D,
$$

(where $\delta_{Q}= \pm 1$ respectively for boson and fermion factors)

$$
\begin{gathered}
D \Omega U V \ldots X Y Z=D^{\bullet} \Omega^{*} U V \ldots X Y Z+\delta_{Q} \Omega D U V \ldots X Y Z \\
=\delta_{P} N\left(D^{\bullet} U V \ldots X Y Z \Omega .\right)+\delta_{Q} \delta_{P} N(D U V \ldots Y Z \Omega),
\end{gathered}
$$

so that we have finally

$$
\begin{aligned}
& D N(U V \cdots X Y Z \Omega)=\delta_{p} D \Omega U V \cdots X Y Z \\
& \quad=\delta_{P}^{2} N\left(D^{\bullet} U V \cdots X Y Z \Omega \cdot\right)+\delta_{Q}^{2} \delta_{P}^{2} N^{\prime}(D U V \cdots X Y Z \Omega) .
\end{aligned}
$$

Noting that $\delta_{Q}^{2}=\delta_{P}^{2}=1$, we see that the theorem (21) is true for $n+1$ factors and hence by induction is true in general.

A mixed $T$ product is defined to be a $T$ product in which normal products occur. We have such mixed products in the terms of the $S$-matrix. Wick's ${ }^{1}$ extension of his theorem to these products states that they can also be decomposed into a unique sum of normal products as in (19) provided we do not contract factors which are already in normal product form.

To prove the theorem we first observe that the factors inside each of the normal products occurring in the mixed products of the $S$-matrix expansion bear the same time label as mentioned earlier. Let us split each of the operators $\psi, \bar{\psi}$

1 G. C. WICK, loc. cit. 
and $A$ occuring in the mixed $T$ product into creation and annihilation operators and consider the mixed $T$ product

$$
T(N(A B C) \ldots N(X Y Z)),
$$

where $A B C, X Y Z$ are either creation or annihilation operators, $A B C$ having the same time label and similarly $X Y Z$. We can think of it as the limit of $T(A B C \ldots$ $X Y Z$ ), where the time label of the creation operators amongst $A B C \ldots X Y Z$ is assumed to be later than that of the destruction operators by an infinitesimal amount. We can now apply the theorem (19) and find that all contractions amongst the operators $A B C \ldots$ etc. vanish (since the creation operators have a later time than the annihilation operators) which is what the theorem states.

\section{REPRESENTATION OF INTERACTIONS IN FIELD THEORY}

We have seen how the interaction between particles in the field theoretic formalism is expressed through the inclusion of an interaction term in the Lagrangian or Hamiltonian density. This term representing the interaction density at a space-time point $x$ consists of a product of field operators corresponding to the same space-time point $x$ (neglecting for the moment, numerical factors, differential operators and matrices) or in other words the interaction is local and represents the creation and annihilation of particles at the vertex $x$. The interaction between two particles when they are separated by a finite distance is interpreted as due to the action of a mediating field, the quanta of which are exchanged between the two particles, i.e. emitted by one and absorbed by the other. In this picture, the basic interaction is only between the particle and the quanta and is therefore local. The advantage of postulating such local interactions lies in the fact that the interaction term in the Lagrangian can be made relativistically invariant. This condition and other considerations of symmetry and invariance impose restrictions on the nature of the coupling between the various fields. The strength of the interaction is measured by the magnitude of a numerical multiplicative factor $g$, the coupling constant.

It is customary to divide interactions into two groups according to the nature of the coupling between the fields: (1) the direct coupling in which the field operators occur as such, and (2) the coupling in which the derivatives of the field operator occur. The actual interaction term should be hermitian and should have definite transformation properties which necessitates the introduction of suitable matrices.

Direct couplings: Since the only known particles are either bosons or fermions, the most general term for the interaction (ignoring the $\gamma$-matrices, the choice of which depend on the transformation properties of the fields) is.

$$
g(\bar{\psi} \psi)^{F} \varphi^{B}
$$

where $F$ and $B$ denote the number of fermions and bosons at a single vertex. The fermion field $\psi$ occurs in a bilinear manner since fermions are emitted or absorbed only in pairs. The coupling constant $g$ giving the strength of the 
interaction should preferably be a dimensionless number. In a general type of interaction this need not necessarily be true. To determine the dimensions of $g$, we find that since

$$
\begin{gathered}
{\left[m^{2} \varphi^{2}\right]=L^{-4} \text { and }[m \bar{\psi} \psi]=L^{-4}} \\
{[\varphi]=L^{-1} \text { and }[\psi]=L^{-3 / 2}} \\
(\text { in units of } \hbar=c=1) \\
{[g] L^{-B-3 F}=L^{-4}} \\
\text { i.e. } \quad[g]=L^{-4+B+3 F} .
\end{gathered}
$$

we have

The tables give the dimensions of $g$ for various processes and the invariant interaction term for the various transformation properties of the $\varphi$ field. The combinations of the $\gamma$-matrices for direct and derivative couplings are different due to the introduction of the differential operator. The coupling constant $f$ in the latter case has to be divided by a mass $\mu$ to make it dimensionless.

\begin{tabular}{c|c|c|c}
\hline Symbol & $B$ & $F$ & $-4+B+3 F$ \\
\hline $\bar{\psi} \psi \varphi$ & 1 & 1 & 0 \\
$\varphi^{4}$ & 4 & 0 & 0 \\
$\varphi^{3}$ & 3 & 0 & -1 \\
$\bar{\psi} \psi \varphi^{2}$ & 2 & 1 & +1 \\
$\left(\bar{\psi}_{\dot{c}}^{\cdot} \psi\right)^{2}$ & 0 & 2 & 2 \\
$(\bar{\psi} \psi)^{2} \varphi$ & 1 & 2 & 3 \\
\hline
\end{tabular}

\begin{tabular}{l|c|c}
\hline \multirow{2}{*}{$\begin{array}{c}\text { Transformation } \\
\text { of } \varphi \text { field }\end{array}$} & \multicolumn{2}{|c}{ The invariant interaction term } \\
\cline { 2 - 3 } & Direct coupling & Derivative coupling \\
\hline Scalar & $g \bar{\psi} \psi \varphi$ & $\frac{f}{\mu} \bar{\psi} \gamma_{\nu} \psi \frac{\partial \varphi}{\partial \varkappa_{\nu}}$ \\
Pseudoscalar & $g \bar{\psi} \gamma_{5} \psi \varphi$ & $\frac{i f}{\mu} \bar{\psi} \gamma_{5} \gamma_{\nu} \psi \frac{\partial \psi^{\prime}}{\partial \varkappa_{\nu}}$ \\
Vector & $\sum_{\mu} g \bar{\psi} \gamma_{\mu} \psi \varphi_{\mu}$ & $\frac{f}{\mu} \bar{\psi}\left(\gamma_{\mu} \gamma_{\nu}-\gamma_{\nu} \gamma_{\mu}\right) \psi F_{\mu \nu}$ \\
Pseudovector & $\sum_{\mu} i g \bar{\psi} \gamma_{5} \gamma_{\mu} \psi \varphi_{\mu}$ & \\
\hline
\end{tabular}

\section{THE HEISENBERG REPRESENTATION AND OPERATORS IN FIELD THEORY}

\section{The Heisenberg representation ${ }^{1}$}

In the Schrödinger representation, the spatial coordinates are treated on a different footing from the time coordinate, whereby the manifest relativistic invariance of the theory is lost. In many cases however it is useful to employ

1 This chapter is largely based on KaLLEN's article in Handbuch der Physik, Vol. 5, Part I, Springer-Verlag (1958). Since it is impossible to improve upon the terseness and accuracy of Kallen's style, the author has attempted to adhere strictly to his sequence of ideas. Here we are using the symbol $\psi(t)$ instead of $c(t)$ as we should in conformity with the earlier notations of the previous chapter. However we prefer in this chapter to retain $\psi(t)$ since we are following closely Kallen's treatment, in which the notation $\psi(t)$ has been adopted. 
the relativistic invariance to prove general theorems and under certain circumstances it may be necessary to use such invariance considerations to give a meaning to quantities which are mathematically undefined. In such cases it is more suitable to use the Heisenberg representation in which there is complete symmetry in all the four coordinates.

The Heisenberg representation of a state vector $\Psi_{H}$ is obtained from the Schrödinger representation $\Psi_{S}$ by the transformation

$$
\Psi_{H}(t)=\mathrm{e}^{i H t} \Psi_{S}(t)
$$

where $H$ is the total Hamiltonian. Since the Schrödinger equation for $\Psi_{S}(t)$ has the solution $\Psi_{S}(t)=\mathrm{e}^{-i H t} \Psi(0)$, we recognize

$$
\Psi_{H}(t)=\Psi_{S}(0)
$$

for all $t$; i.e. the Heisenberg state vector is independent of time and coincides with the Schrödinger state vector at $t=0$. Hence it obeys no equation of motion. However, the Heisenberg representation $F_{H}(t)$ of an operator $F_{S}$ in the Schrödinger representation is

$$
F_{H}(t)=\mathrm{e}^{i H t} F_{S} \mathrm{e}^{-i H t} .
$$

Differentiating both sides with respect to $t$, we have

$$
\frac{\partial F_{H}}{\partial t}=i\left[H, F_{H}\right]
$$

which can be interpreted as the equation of motion of the operator $F_{H}$. In particular if we choose the operator $F$ to be the Hamiltonian $H$, we find that the Hamiltonians in both the representations are identical and time independent.

The definition of the transformation implies the choice of zero as the point where the two representations coincide. More generally, any point can be chosen for such coincidence and in collision problems it has become customary to choose a point which is made to tend to $-\infty$. This limiting process obviously introduces mathematical difficulties but the physical interpretation is made easier if the two representations are made to coincide "initially," and as has been shown in the chapter on the formal theory of scattering, it is convenient to interpret "initial" as corresponding to the point $t_{1}=-\infty$.

It can be readily established that if we set up a field theory derived from a Lagrangian density function of the type ${ }^{1} L\left(\varphi_{\alpha}(x), \frac{\partial \varphi_{\alpha}(x)}{\partial x}\right)$, then the Heisenberg equations of motion for the field operators $\varphi_{\alpha}(x)$ and the conjugate momentum operators $\pi_{\alpha}(x)$ are identical with the equation defining $\pi_{\alpha}(x)$ in terms of $\varphi_{\alpha}(x)$, i.e.

$$
\pi_{\alpha}(x)=\frac{\partial L}{\partial\left(\frac{\partial \varphi_{\alpha}(x)}{\partial x_{4}}\right)}
$$

${ }^{1}$ From now on in this chapter we drop the suffix $H$ since we will be working only in the Heisenberg representation unless otherwise specified. 
and the Lagrangian equation of motion

$$
\sum_{\mu=1}^{4} \frac{\partial}{\partial x_{\mu}} \frac{\partial L}{\partial\left(\frac{\partial \varphi_{\alpha}(x)}{\partial x_{\mu}}\right)}=\frac{\partial L}{\partial \varphi_{\alpha}(x)}
$$

respectively, provided the latter is considered as an operator equation and we impose the canonical commutation relations

$$
\begin{aligned}
& {\left[\pi_{\alpha}(x), \varphi_{\beta}\left(x^{\prime}\right)\right]=-i \delta_{\alpha \beta} \delta\left(\boldsymbol{x}-\boldsymbol{x}^{\prime}\right)} \\
& {\left[\boldsymbol{\pi}_{\alpha}(x), \pi_{\beta}\left(x^{\prime}\right)\right]=\left[\varphi_{\alpha}(x), \varphi_{\beta}\left(x^{\prime}\right)\right]=0,}
\end{aligned}
$$

which are considered postulates of the theory. Further if as in classical theory, the three spatial components of the momentum are defined by the equation

$$
P_{k}=-\int \mathrm{d}^{3} x \sum_{\alpha} \pi_{\alpha}(x) \frac{\partial \varphi_{\alpha}(x)}{\partial x_{k}}
$$

then it follows for any operator $F(x)$ constructed from $\varphi_{\alpha}(x)$ and $\pi_{\alpha}(x)$ that

$$
i\left[P_{k}, F^{\prime}(x)\right]=\frac{\partial F(x)}{\partial x_{k}} .
$$

This, together with equation (4) forms the complete system of equations for the operators in the Heisenberg representation. If we set $P_{4}=H$, then the four equations can be written as

$$
i\left[P_{\mu}, F^{\prime}(x)\right]=\frac{\partial F(x)}{\partial x_{\mu}} .
$$

$P_{\mu}$ is called the space-time displacement operator for obvious reasons.

The operator equation is equivalent to stating that the matrix element between two states of $i\left[P_{\mu}, F(x)\right]$ is equal to the differential coefficient with respect to the variable $x_{\mu}$ of the matrix element of $F(x)$ between the two states. In particular, if we choose the two states $|a\rangle$ and $|b\rangle$ with definite four-momenta $p_{\mu}^{(a)}$ and $p_{\mu}^{(b)}$ respectively, we get

$$
i\left\langle a\left|\left[P_{\mu}(x), F^{\prime}(x)\right]\right| b\right\rangle=i\left(p_{\mu}^{(a)}-p_{\mu}^{(b)}\right)\langle a|F(x)| b\rangle=\frac{\partial}{\partial x_{\mu}}\langle a|F(x)| b\rangle
$$

the solution of which is

$$
\left\langle a\left|F^{\prime}(x)\right| b\right\rangle=\left\langle a\left|F^{\prime}(0)\right| b\right\rangle \mathrm{e}^{i \sum_{\mu}\left(p_{\mu}^{(a)}-p_{\mu}^{(b)}\right) x_{\mu} .}
$$

Thus we obtain a relationship between the matrix elements of the operators $F^{\prime}(x)$ and $F(0)$ or more generally $F\left(x_{1}\right)$ and $F\left(x_{2}\right)$ respectively. This principle of translational invariance of a Heisenberg operator is used extensively in calculations.

We shall now study the problem of the interaction of the electromagnetic field with a given classical current distribution $j_{\mu}(x)$ as this will enable us to introduce certain concepts and definitions. From the Lagrangian for this problem

$$
L=-\frac{1}{4} F_{\mu \nu} F_{\mu \nu}-\frac{1}{2} \frac{\partial A_{\nu}}{\partial x_{\nu}} \frac{\partial A_{\lambda}}{\partial x_{\lambda}}+A_{\mu} j_{\mu},
$$


where the first two terms of the right-hand side form the Fermi Lagrangian for the free electromagnetic field and the last term represents the interaction Lagrangian, we obtain the equations of motion for the field operators $A_{\mu}$ as

$$
A_{\mu}(x)=j_{\mu}(x)
$$

and the Hamiltonian operator as

$$
H=1 / 2 \int \mathrm{d}^{3} x\left\{\frac{\partial A_{\mu}}{\partial x_{4}} \frac{\partial A_{\mu}}{\partial x_{4}}+\frac{\partial A_{\mu}}{\partial x_{k}} \frac{\partial A_{\mu}}{\partial x_{k}}\right\}-\int \mathrm{d}^{3} x j_{\mu}(x) A_{\mu}(x) .
$$

The solution $A_{\mu}(x)$ can be written in the form

$$
A_{\mu}(x)=A_{\mu}^{(0)}(x)+\int D_{R}\left(x-x^{\prime}\right) j_{\mu}\left(x^{\prime}\right) \mathrm{d} x^{\prime},
$$

where $A_{\mu}^{(0)}(x)$ is just the initial value of $A_{\mu}(x)$, i.e. at $t=-\infty$, and it is assumed that $j_{\mu}(x)$ vanishes as $t \rightarrow-\infty$ in such a manner that the integral in (16) exists ${ }^{1}$. $A_{u}^{(0)}(x)$ satisfies the homogeneous equation

$$
\square A_{\mu}^{(0)}(x)=0 .
$$

Since the integral is a $c$-number, $A_{\mu}^{(0)}(x)$ satisfies the same commutation relations as $A^{\mu}(x)$ :

$$
\begin{aligned}
{\left[A_{\mu}^{(0)}(x), A_{\nu}^{(0)}\left(x^{\prime}\right)\right] } & =-i \delta_{\mu \nu} D\left(x^{\prime}-x\right) \\
\left\langle 0\left|\left\{A_{\mu}^{(0)}(x), A_{\nu}^{(0)}\left(x^{\prime}\right)\right\}\right|^{0}\right\rangle & =\delta_{\mu \nu} D^{1}\left(x^{\prime}-x\right) .
\end{aligned}
$$

$A_{\mu}^{(0)}(x)$ is called the "incoming" field. On calculating the energy with the above solution and assuming that the current vanishes also at $t \rightarrow+\infty$, it can be shown that

$$
\lim _{t \rightarrow-\infty} H=H^{0}\left(A_{\mu}^{(0)}\right) .
$$

If we choose the state vector at $t=-\infty$ to be the eigenfunction of $H_{0}\left(A_{k}^{0}\right)$, the state vector has particles corresponding to the "incoming" free particles. Since we have assumed that $j_{\mu}(x)$ vanishes at $t=+\infty$, we can also write the solution in the form

$$
A_{\mu}(x)=\bar{A}_{\mu}^{(0)}(x)+\int D_{A}\left(x-x^{\prime}\right) j_{\mu}\left(x^{\prime}\right) \mathrm{d} x^{\prime}
$$

where the operator $\bar{A}_{\mu}^{(0)}(x)$ corresponds to the value of $A_{\mu}(x)$ at $t=+\infty$ and also satisfies the homogeneous equation

$$
\bar{A}_{\mu}^{(0)}(x)=0 .
$$

As for $H_{0}$, if it is expressed in terms of $\bar{A}_{\mu}^{(0)}(x)$, as $t \rightarrow+\infty$ the eigen functions of this would have free particles corresponding to the "outgoing" state. Since we are interested only in transitions, the state vectors for the "incoming" and "outgoing" cases would be different. From their very definitions, the relation between the incoming and outgoing fields is given by

$$
A_{\mu}^{(0)}(x)-\bar{A}_{\mu}^{(0)}(x)=\int D\left(x-x^{\prime}\right) j_{\mu}\left(x^{\prime}\right) \mathrm{d} x^{\prime} .
$$

1 For an alternative definition, see chapter II, section 1. 
In the above discussion, we have assumed $j_{\mu}$ to be a classical current but when we are interested in a quantized charged fermion field, then $j_{\mu}(x)$ will have to be expressed in terms of fermion field operators.

\section{Interaction of fermion and electromagnetic fields}

We write the Lagrangian for this system as

$$
L=L_{\psi}+L_{A}+L_{\text {int }}
$$

where $L_{\psi}$ and $L_{A}$ are the free field Lagrangians for the two fields and $L_{\text {int }}$ is given by,

$$
L_{\text {int }}=A_{\mu}(x) j_{\mu}(x)
$$

the fermion current in this case being the commutator, $\frac{e}{2}\left[\bar{\psi}, \gamma_{\mu} \psi\right]$.

The corresponding equations of motion are

$$
\begin{gathered}
\left(-i \gamma \frac{\partial}{\partial x}+m\right) \psi(x)=e \gamma A(x) \psi(x)^{1} \\
\square A_{\mu}(x)=\frac{e}{2}\left[\bar{\psi}(x), \gamma_{\mu} \psi(x)\right] \equiv j_{\mu}(x) .
\end{gathered}
$$

Since the term $L_{\text {int }}$ does not contain the time derivative of the field operators, the canonical momenta will be the same function of the dynamical variables as before and the commutation relations for equal times can be written down immediately:

$$
\begin{aligned}
{\left[A_{\mu}(x), A_{\nu}\left(x^{\prime}\right)\right]_{x_{4}=x_{4}^{\prime}} } & =\left[\frac{\partial A_{\mu}^{(x)}}{\partial x_{4}}, \frac{\partial A_{\nu}^{\left(x^{\prime}\right)}}{\partial x_{4}^{\prime}}\right]_{x_{4}=x_{4}^{\prime}}=0 \\
{\left[\frac{\partial A_{\mu}(x)}{\partial x_{4}}, A_{\nu}\left(x^{\prime}\right)\right]_{x_{4}=x_{4}^{\prime}} } & =i \delta_{\mu \nu} \delta\left(\boldsymbol{x}-\boldsymbol{x}^{\prime}\right) \\
\left\{\psi(x), \psi\left(x^{\prime}\right)\right\}_{x_{4}=x_{4}^{\prime}} & =\left\{\bar{\psi}(x), \bar{\psi}\left(x^{\prime}\right)\right\}_{x_{4}=x_{4}^{\prime}}=0 \\
\left\{\bar{\psi}(x), \psi\left(x^{\prime}\right)\right\}_{x_{4}=x_{4}^{\prime}} & =\gamma_{4} \delta\left(\boldsymbol{x}-\boldsymbol{x}^{\prime}\right) \\
{\left[A_{\mu}(x), \psi\left(x^{\prime}\right)\right]_{x_{4}=x_{4}^{\prime}} } & =\left[\frac{\partial A_{\mu}^{(x)}}{\partial x_{4}}, \psi\left(x^{\prime}\right)\right]_{x_{4}=x_{4}^{\prime}}=0 \\
{\left[A_{\mu}(x), \bar{\psi}\left(x^{\prime}\right)\right]_{x_{4}-x_{4}^{\prime}} } & =\left[\frac{\partial A_{\mu}(x)}{\partial x_{4}}, \bar{\psi}\left(x^{\prime}\right)\right]_{x_{4}=x_{4}^{\prime}}=0 .
\end{aligned}
$$

While the commutator for arbitrary times for free fields can be obtained from the commutator at equal times, this cannot be done if we include the interaction since the commutators do not satisfy simple equations of motion. The differentiaI equations of motion can be transformed into corresponding integral equations

$$
\begin{aligned}
\psi(x) & =\psi^{(0)}(x)-\int S_{R}\left(x-x^{\prime}\right) \text { e } \gamma A\left(x^{\prime}\right) \psi\left(x^{\prime}\right) \mathrm{d} x^{\prime} \\
A_{\mu}(x) & =A_{\mu}^{(0)}(x)+\int D_{R}\left(x-x^{\prime}\right) \frac{e}{2}\left[\bar{\psi}\left(x^{\prime}\right), \gamma_{\mu} \psi\left(x^{\prime}\right)\right] \mathrm{d} x^{\prime}
\end{aligned}
$$

\footnotetext{
1 Here we are using rationalized units of charge, $\frac{e^{2}}{4 \pi}=1 / 137^{\circ}$
} 
$\psi^{(0)}, A^{(0)}$ and $\bar{\psi}^{(0)}, \bar{A}^{(0)}$ are identified with the "in" and "out" fields respectively. It is to be noted that in the previous case, since we had assumed a classical current $j_{\mu},(16)$ represented a solution for $A_{\mu}(x)$ while in this case $A_{\mu}$ and $\psi$ satisfy coupled integral equations which are yet to be solved. The solution is not very easy and suitable perturbation methods should be devised. It is interesting to note that the perturbation theory here applies to operators while in the Schrödinger and interaction representations we were applying it to the state vectors. The most obvious way of applying perturbation theory to such integral equations is to write $\psi(x)$ and $A(x)$ as

$$
\begin{aligned}
\psi(x) & =\psi^{(0)}(x)+e \psi^{(1)}(x)+e^{2} \psi^{(2)}(x)+\ldots \\
A_{\mu}(x) & =A_{\mu}^{(0)}(x)+e A_{\mu}^{(1)}(x)+e^{2} A_{\mu}^{(2)}(x)+\cdots
\end{aligned}
$$

By substituting these in the equations of motion, we obtain the recurrence relations for the different approximations as

$$
\begin{aligned}
& \psi^{n+1}(x)=-1 / 2 \int S_{R}\left(x-x^{\prime}\right) \gamma_{\nu} \sum_{m=0}^{n}\left\{A_{\nu}^{(m)}(x)^{\prime}, \psi^{(n-m)}\left(x^{\prime}\right)\right\} \mathrm{d} x^{\prime} \\
& A_{\mu}^{n+1}(x)=1 / 2 \int D_{R}\left(x-x^{\prime}\right) \sum_{m=0}^{n}\left[\bar{\psi}^{(m)}\left(x^{\prime}\right), \gamma_{\mu} \psi^{(n-m)}\left(x^{\prime}\right)\right] \mathrm{d} x^{\prime} .
\end{aligned}
$$

(In the first of these expressions, we have symmetrized the right hand side which is permissible since the operator $A_{\mu}(x)$ and $\psi(x)$ or $\bar{\psi}(x)$ commute for equal times.) In particular we have

$$
\begin{aligned}
\psi^{(1)}(x) & =\int S_{R}\left(x-x^{\prime}\right) \gamma A^{(0)}\left(x^{\prime}\right) \psi^{0}\left(x^{\prime}\right) \mathrm{d} x^{\prime} \\
\bar{\psi}^{(1)}(x) & =\int \bar{\psi}^{(0)}\left(x^{\prime}\right) \gamma A^{0}\left(x^{\prime}\right) S_{A}\left(x-x^{\prime}\right) \mathrm{d} x^{\prime} \\
A^{(1)}(x) & =\frac{1}{2} \int D_{R}\left(x-x^{\prime}\right)\left[\bar{\psi}^{(0)}\left(x^{\prime}\right), \gamma_{\mu} \psi^{(0)}\left(x^{\prime}\right)\right] \mathrm{d} x^{\prime} .
\end{aligned}
$$

The general expression for $\psi(x)$ or $A_{\mu}(x)$ is very complicated. We must note the occurrence even in the first approximation $\psi^{(1)}(x)$ of the product $A_{\mu}^{(0)}(x) \psi^{0}(x)$ which implies transition from the vacuum to a two electron state

$$
\langle 0|\psi(x)| q, k\rangle=e \int S_{R}\left(x-x^{\prime}\right) \gamma_{v}\left\langle 0\left|A_{v}^{(0)}\left(x^{\prime}\right)\right| k\right\rangle\left\langle 0\left|\psi^{(0)}\left(x^{\prime}\right)\right| q\right\rangle \mathrm{d} x^{\prime}+\cdots
$$

This is a peculiar feature of coupled fields which is not present in the case of free fields where the operators applied on the vacuum produce only states with one particle. Matrix elements for products of operators can be simplified by introducing intermediate states and summing over them. For example,

$$
\left\langle 0\left|\psi^{(0)}(x)\left\{A_{v_{1}}^{(0)}(x), A_{\nu_{2}}^{(0)}\left(x^{\prime}\right)\right\}\right| q\right\rangle=\sum_{z}\left\langle 0\left|\psi^{(0)}(x)\right| z\right\rangle\left\langle z\left|\left\{A_{v_{1}}^{(0)}(x), A_{v_{2}}^{(0)}\left(x^{\prime}\right)\right\}\right| q\right\rangle .
$$

The nature and number of the intermediate state are restricted by the nature of the operators. (In the above example, the matrix element has a non-vanishing value only if the intermediate state is a one-electron state.) 
We know that the "in" and "out" fields satisfy the same canonical commutation relations. Hence there must exist a unitary matrix $S$ such that ${ }^{1}$

$$
\begin{gathered}
y^{\text {out }}(x)=S^{-1} \psi^{\text {in }}(x) S \\
A_{\mu}^{\text {out }}(x)=S^{-1} A_{\mu}^{\text {in }}(x) S \\
S^{\dagger} S=S S^{\dagger}=1 .
\end{gathered}
$$

If we assume as before that the state vector at $t=-\infty$ is an eigen function of $H^{0}$ (in) and at $t=+\infty$ that of $H^{0}$ (out) where

$$
H^{(0)}\left(A^{\text {(out) }}, \psi^{(\text {out })}\right)=S^{-1} H^{(0)}\left(A^{(\text {in) }}, \psi^{(\text {in) })} S\right.
$$

then it is clear that the probability that the state $|n\rangle$ goes over to $\left|n^{\prime}\right\rangle$ by the interaction is given by

$$
\left.\left\langle n^{\prime}|S| n\right\rangle\right|^{2}
$$

This merely implies that the transformation matrix $S$ is just the $S$ matrix of collision theory with the operators in the Heisenberg representation. In calculations, it has been found more convenient to work with the $S$ matrix in the interaction representation.

\section{Propagators in field theory}

In the foregoing methods of computation, we have used the momentum representation of the initial and final states since we are interested only in the matrix elements for transition between definite momentum states. If we wish to use the configuration representation for the initial and final states, then we are constrained to use a kernel function connecting the space-time points associated with the wave functions of the initial and final particle systems and integrate out over these space-time points. This process should be identical with that using initial and final states in momentum representations and the $S$-matrix expansion. In defining propagators in field theory it is necessary to remember the corresponding concept in the Feynman formalism where we were dealing with propagators of single particles, and the propagators of systems of particles were defined in terms of these, as for example the two particle propagator in the presence of an interaction or the modified propagator of a single particle assuming interaction (self field). In field theory however we can only speak of propagators connecting space-time points at which the particles are annihilated and those at which particles are created.

Before obtaining a general expression for the kernel or propagator let us take the case when the initial system consists, say, of a fermion and a boson of

1 C. N. Yang and D. Feldman, Phys. Rev. 79, 972 (1950). 
momenta $p$ and $k$ respectively and is represented by $\left.a^{\dagger}(k) b^{\dagger}(p)\right\rangle_{0}$. We first recognize that

$$
\left.a^{\dagger}(k) b^{\dagger}(p)\right\rangle_{0}=A \int \bar{\psi}(x) \varphi(x) u(p) \mathrm{e}^{-i(p \cdot x+k \cdot y)} \mathrm{d} x \mathrm{~d} y
$$

where $\bar{\psi}$ and $\varphi$ represent the fermion and boson field operators respectively and $A$ the normalization factor. Similarly for the final system we can write

$$
\left\langle b\left(p^{\prime}\right) a\left(k^{\prime}\right)=A \int \psi\left(x^{\prime}\right) \varphi\left(y^{\prime}\right) \bar{u}\left(p^{\prime}\right) \mathrm{e}^{+i\left(p^{\prime} \cdot x^{\prime}+k^{\prime} \cdot y^{\prime}\right)} \mathrm{d}^{4} x^{\prime} \mathrm{d}^{4} y^{\prime} .\right.
$$

In operating the $S$-matrix on the state $\left.a^{\dagger}(k) b^{\dagger}(p)\right\rangle_{0}$ the annihilations of the particles with momentum $p$ and $k$ may occur at any one of the vertices in the $n$-th order term of the $S$-matrix expansion. This circumstance is taken into account when using the wave functions in configuration representation by defining a propagator as

$$
G\left(x^{\prime}, x, y^{\prime}, y\right)=\frac{1}{c}\left\langle T\left(\psi\left(x^{\prime}\right) \varphi\left(y^{\prime}\right) S \bar{\psi}(x) \varphi(y)\right)\right\rangle_{0}
$$

where $S$ implies the $S$-matrix expansion and the $T$-operator operates only after explicitly writing the $S$-matrix expansion, i.e. on the entire aggregate of terms $\psi\left(x^{\prime}\right) \varphi\left(y^{\prime}\right) \bar{\psi}(x) \varphi(y)$ and the terms in the integrand of the $n$-th order term of the $S$-matrix. $c$ is the normalization factor defined as ${ }_{0}\langle S\rangle_{0}$.

Thus integrating with respect to the space-time points corresponding to the arguments of the operators of the propagator, i.e. of the wave functions of the initial and final systems, merely implies taking the momentum transform of the propagator with arguments corresponding to the momenta of the initial and final states. Thus the propagator is a four-point function involving two fermion and two boson lines and therefore can be used for obtaining transitions not merely from an initial fermion plus boson state to a final fermion plus boson state but also for two other processes, viz. fermion + anti-fermion $\rightarrow 2$ boson states or vice versa. (This is implied in the use of the operators $\psi$ and $\bar{\psi}$ in the propagator.) Thus the matrix element for all these processes is obtained from the same Green's function by integrating over suitable initial and final wave functions. ${ }^{1}$ This circumstance imposes certain conditions on the matrix element when viewed as the boundary value of an analytic function. This is the starting point of the theory of dispersion relations which will be discussed in a later chapter.

We define the function $G\left(x^{\prime}, x\right)$ as:

$$
G\left(x^{\prime}, x\right)=\sum_{n=0}^{\infty} \frac{(-i)^{n}}{n !} \int_{-\infty}^{\infty} \mathrm{d} x_{1} \cdots \int_{-\infty}^{\infty} \mathrm{d} x_{n} \frac{{ }_{0}\left\langle T\left(\psi\left(x^{\prime}\right) \bar{\psi}(x) H^{\text {int }}\left(x_{1}\right) \cdots H^{\text {int }}\left(x_{n}\right)\right)\right\rangle_{0}}{{ }_{0}\left\langle T\left(H^{\text {int }}\left(x_{1}\right) \cdots H^{\text {int }}\left(x_{n}\right)\right)\right\rangle_{0}}
$$

We shall examine the meaning of the individual terms in the expansion of $G\left(x^{\prime}, x\right)$ and the normalization factor $c$ in terms of Feynman diagrams. At every vertex corresponding to a $H^{\text {int }}(x)$ there is only one photon line and as $G$ represents

1 See for the derivation of a differential equation for $G\left(x, x^{\prime}\right)$, J. Schwinger, Proc. Nat. Acad. Sci. 37, 452 (1951). See also N. N. Bogoliubov and D. V. Shirkov, loc. cit., pp. 416. 
only two electron lines, it is obvious that the number of vertices should be even. The numerator is thus given upto the fourth order as:

$$
\left.\begin{array}{lr}
\left\langle T\left(\psi\left(x^{\prime}\right) \bar{\psi}(x)\right)\right\rangle_{0} & \text { (zeroth order), } \\
\left\langle T\left(\psi\left(x^{\prime}\right) \bar{\psi}(x) H^{\text {int }}\left(x_{1}\right) H^{\text {int }}\left(x_{2}\right)\right)\right\rangle_{0} & \text { (second order) } \\
\text { and }\left\langle T\left(\psi\left(x^{\prime}\right) \bar{\psi}(x) H^{\text {int }}\left(x_{1}\right) H^{\text {int }}\left(x_{2}\right) H^{\text {int }}\left(x_{3}\right) H^{\text {int }}\left(x_{4}\right)\right)\right\rangle_{0} & \text { (fourth order) }
\end{array}\right\}
$$

the last two being represented by Figs. 21 a and 21 b respectively.
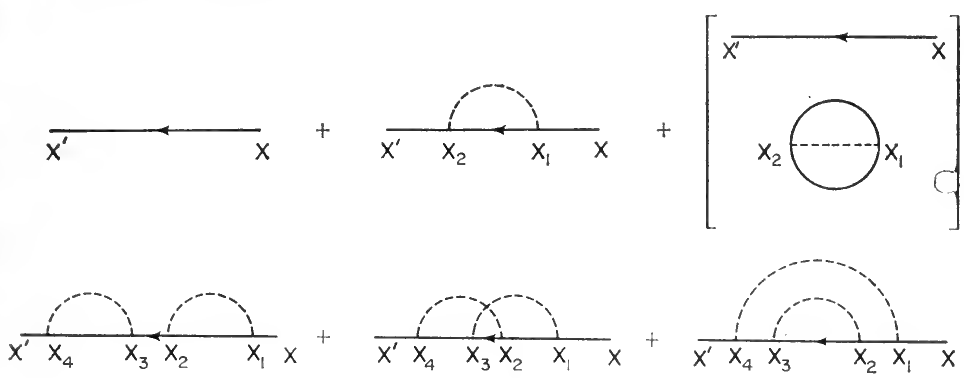

a
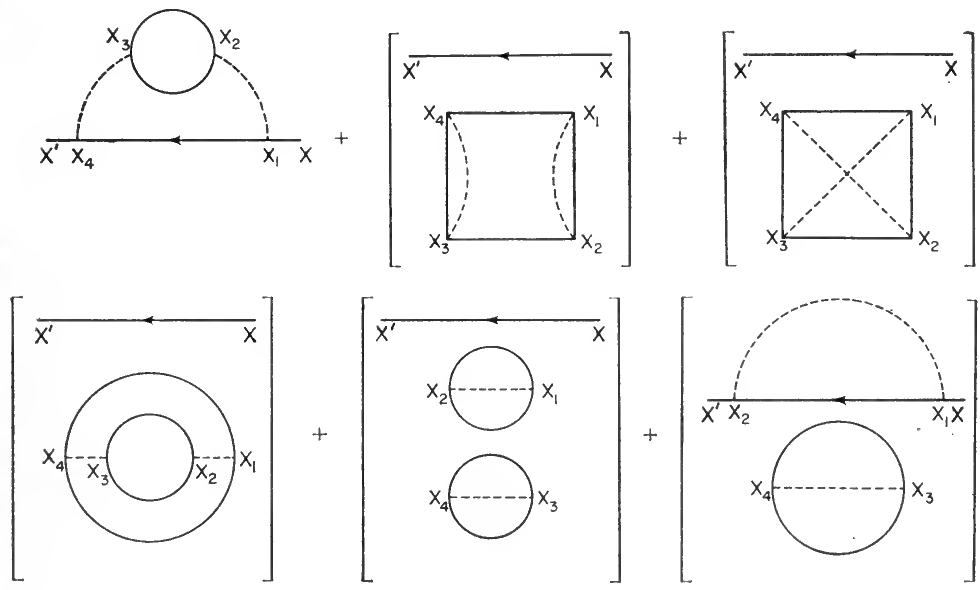

b

FIG. 21. Diagrammatic representation of the numerator.

The terms within brackets are vacuum to vacuum transitions through virtual creation and annihilation of pairs. The denominator which is ${ }_{0}\langle S\rangle_{0}$ is given up to the fourth order as

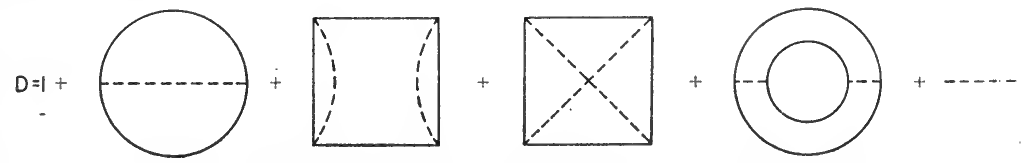

FrG. 22. Diagrammatic representation of the denominator. 
i.e. a sum over all possible vacuum fluctuation terms. It is to be noted that the vacuum cannot be connected to any other state by the $S$-matrix. This implies that if we do not have any initial particle, there will not be any emergent ones, i.e. the vacuum is an eigenstate of the $S$-matrix. The unitarity of the $S$-matrix requires the eigenvalue to be unimodular so that we can write $\left.S\rangle_{0}=\mathrm{e}^{i \alpha}\right\rangle_{0}$ or $D=\mathrm{e}^{i \alpha}$ as $_{0}\langle 1\rangle_{0}=1$. Thus by separating out the bubble terms in the numerator as a multiplicative factor $e^{i \alpha}$ which cancels with the denominator, we have $G\left(x^{\prime}, x\right)=S_{F}^{\prime}$ the propagator for the electron in the presence of its self-field.

More generally we can define a propagator connecting $N$ points such that $\dot{N}=2 n+m_{1}+m_{2}, 2 n$ points corresponding to the functions $\psi\left(x_{1}\right), \psi\left(x_{2}\right) \ldots \psi\left(x_{n}\right)$ and $\bar{\psi}\left(x_{1}\right) \ldots \bar{\psi}\left(x_{n}\right), m_{1}$ corresponding to $\varphi\left(y_{1}\right), \varphi\left(y_{2}\right) \ldots \varphi\left(y_{m_{1}}\right)$ and $m_{2}$ to $\varphi\left(y_{1}^{\prime}\right) \ldots \varphi\left(y_{m_{2}}^{\prime}\right)$ inside the time ordered product which also contains the $H^{\text {int }}\left(x_{1}\right) \ldots H^{\text {int }}\left(x_{n}\right)$ term. This propagator is used to obtain matrix elements from initial to final states where every $\psi(x)$ occurring in the propagator corresponds to either an incident electron or an emergent positron and $\bar{\psi}(x)$ to an emergent electron or an incident positron. In collision problems, usually the incident particles are only two in number while the emergent particles can be any number, the restriction being only due to conservation laws. In particular we can write the propagator involving four external lines (two fermion and two boson lines) as in (51). In quantum electrodynamics and quantum mesodynamics the form of the interaction Hamiltonian of the Yukawa type is

$$
\begin{aligned}
H^{\text {int }} & =\dot{e} \bar{\psi}(x) \gamma_{\mu} \psi(x) A_{\mu}(x) \\
H^{\text {int }} & =g \bar{\psi}(x) \gamma_{5} \tau_{i} \psi(x) \varphi_{i}(x) .
\end{aligned}
$$

We shall now explicitly obtain the rules for using the above propagator in calculating matrix elements. These can be carried over without any difficulty for propagators involving more than four external lines.

The method described in the previous chapter consisted in taking the expectation value of the $S$-matrix between the initial and final states which were described by creation and annihilation operators operating on the vacuum. In propagator formalism, we have, instead of the creation operator corresponding to each member of the initial system, field operators. Since these involve space-time points their position in the time ordering is determined by the $P$ operator; which means the time point for an interaction vertex could be earlier than that of a field operator. In the previous picture we started with incident bare particles and the interaction was supposed to be switched on and switched off, the duration of the interaction being assumed to tend to $\infty$. Here since the field operators are taken along with the interaction terms, we are assuming that the interaction is operative all the time and therefore we are dealing with the propagator of physical particles.

We should note an interesting feature of the momentum transform of the propagator. The annihilation of the initial electron at a vertex may be followed by the creation of the same electron at another vertex if the two vertices are connected by the emission and the absorption of a photon (electron self-energy 
diagram). But the existence of the matrix element implies that the initial particles should not occur in the final state. Thus they will be finally annihilated at some vertex after which we do not take the self-energy processes into consideration. Thus we should expect the momentum transform of the propagator to be of the form

$$
\gamma\left(p^{\prime}, p ; k^{\prime}, k=\Delta_{F}^{-1}\left(k^{\prime}\right) S_{F}^{-1}\left(p^{\prime}\right) G\left(p^{\prime}, p ; k^{\prime}, k\right) S_{F}^{-1}(p) \Delta_{F}^{-1}(k)\right.
$$

and since we are interested only in transitions from real physical initial states to real physical final states, we write the matrix elements as:

$$
\bar{u}\left(p^{\prime}\right) \gamma\left(p^{\prime}, p ; k^{\prime}, k\right) u(p) \text {. }
$$

We shall now establish a relationship between the propagators in terms of interaction and Heisenberg operators. For this purpose, we recall that if we take the time at which the two representations coincide to be $t_{0}$, then the state vectors and operators in the two representations are related by

and

$$
\left|\Phi_{I}(t)\right\rangle=U\left(t, t_{0}\right)\left|\Phi_{H}\left(t_{0}\right)\right\rangle
$$

$$
O_{H}(t)=U^{-1}\left(t, t_{0} O_{I}(t) U\left(t, t_{0}\right)\right.
$$

The propagator in the interaction representation is

$$
\begin{aligned}
& G\left(x_{1}^{\prime} x_{2}^{\prime} \cdots ; x_{1}^{0}, x_{2}^{0} \cdots\right) \\
= & \sum_{n=0}^{\infty} \frac{(-i)^{n}}{n !} \int_{-\infty}^{\infty} \cdots \int_{-\infty}^{\infty} \mathrm{d} x_{1} \cdots \mathrm{d} x_{n}\left\langle T\left(\psi_{I}\left(x_{1}^{\prime}\right) \cdots \bar{\psi}_{I}\left(x_{n}^{0}\right) H_{I}^{\prime}\left(x_{1}\right) \cdots H_{I}^{\prime}\left(x_{n}\right)\right)\right\rangle_{0} \\
= & \left\langle T\left(U(+\infty,-\infty) \psi_{I}\left(x_{1}^{\prime}\right) \cdots \psi_{I}\left(x_{n}^{0}\right)\right)\right\rangle_{0} \\
= & \left\langle T\left(U\left(+\infty, t_{0}\right) U\left(t_{0}, \tau_{1}^{\prime}\right) \psi_{I}\left(x_{1}^{\prime}\right) \cdots U\left(t_{0}, \tau_{n}^{0}\right) \psi_{I}\left(x_{n}^{0}\right) U\left(\tau_{n}^{0}, t_{0}\right) U\left(t_{0},-\infty\right)\right)\right\rangle_{0} \\
= & \left(\psi_{0}, T\left(\psi_{H}\left(x_{1}^{\prime}\right) \cdots \psi_{H}\left(x_{n}^{0}\right)\right) \psi_{0}\right),
\end{aligned}
$$

where $\psi_{0}$ represents the "true" or physical vacuum and where all possible time orderings of the operators have been taken into account. The last line represents the propagator in the Heisenberg representation.

\section{TRANSFORMA'TIONSANDGENERALPRINCIPIES O F INVA RIA N C F ${ }^{1}$}

\section{General considerations}

So far, in developing quantum field theory, we have assumed the equation of motion to be the generalization of the Schrödinger equation which states simply that the rate of change of the state vector with time is obtained by the Hamiltonian

1 This section is based mostly on lectures given by P. T. MatThews at the University of Rochester, 1957 on "The Relativistic Quantum Theory of Elementary Particle Interactions." See also N. Kemmer, J. C. Polkinghorne and D. L. Pursey, "Invariance in Elementary Particle Physics", Rep. Progr. Phys. XXII, 368 (1959). 
operating on the state vector. The formal method of obtaining the equation of motion is to begin with a Lagrangian function and impose on it the principle of least action. If we denote by $L\left(\varphi_{\alpha}(x), \varphi_{\alpha}^{\mu}(x)\right)$ the Lagrangian density for any field where $\varphi_{\alpha}(x)$ and $\varphi_{\alpha}^{\mu}(x)$ are the field variables and their first order space or time derivatives, then the equation of motion is obtained by the variational principle (as in classical mechanics) that $\int \mathscr{L} \mathrm{d} t$ should be an extremum (where $\mathscr{L}$ is the total Lagrangian) or

$$
\delta \int \mathscr{L} \mathrm{d} t=0 \quad \text { or } \quad \delta \int L \mathrm{~d}^{4} x=0
$$

where $L$ is the Lagrangian density.

This leads to the equation of motion

$$
\frac{\partial L}{\partial \varphi_{\alpha}}-\frac{\partial}{\partial x_{\mu}} \frac{\partial L}{\partial \varphi_{\alpha}^{\mu}}=0 .
$$

Since we assume that $L$ does not contain derivatives higher than the first order, the equation of motion will be at most of the second order. We also note that there is a certain arbitrariness in the choice of a Lagrangian, i.e. we can always add a four-divergence of the type $\frac{\partial \Lambda_{v}}{\partial x_{\mu}} \partial \lambda_{\alpha},\left(\varphi_{\alpha} \varphi_{\alpha}^{\mu}\right)$ and yet obtain the same equation of motion. The integral $\int \mathrm{d}^{3} x \frac{\partial \Lambda_{\nu}}{\partial x_{\mu}}\left(\varphi_{\alpha}, \varphi_{\alpha}^{\mu}\right)$ can be converted into a 3 -dimensional surface integral by the Gauss theorem so that its variation vanishes identically. Although the two Lagrangians $L$ and $L^{\prime}$ give rise to the same equations of motion, expressions for physical observables such as charge, current etc. will be different and hence the effect of an interaction with an external field will also be different.

We can now proceed in analogy with classical mechanics and define the Hamiltonian density as

$$
H=\sum_{\alpha} \pi_{\alpha} \varphi_{\alpha}^{0}-L
$$

where

$$
\pi_{\alpha}=\frac{\partial L}{\partial \varphi_{\mu}^{0}} \quad \text { and } \quad \varphi_{\alpha}^{0}=\frac{\partial \varphi_{\alpha}}{\partial t} .
$$

We can define also a canonical energy-momentum tensor by

$$
T_{\mu \nu}=\sum_{\alpha} \varphi_{\alpha}^{\mu} \frac{\partial L}{\partial \varphi_{\alpha}^{\nu}}-L \delta_{\mu \nu}
$$

with $T_{44}$ and $T_{\mu 4}$ corresponding to the energy and momentum of the field which are constants of motion. Defining

$$
M_{\mu \nu}=\int \mathrm{d}^{3} x\left(x_{\mu} T_{\nu 4}-x_{\nu} T_{\mu 4}\right)
$$

the space part of which corresponds to angular momentum we find that if angular momentum is to be a constant of motion, the necessary condition is that $T_{\mu \nu}$ should be symmetric ${ }^{1}$, i.e.

$$
T_{\mu \nu}=T_{\nu \mu} .
$$

1 F. J. Belinfante, Physica 6, 887 (1939); see also G. Wentzel, loc. cit. 
The choice of the Lagrangian is based on the important criterion that it should yield the desired equations of motion. Since in many cases the correct equations of motion are not known, physicists have been guided in the choice of the Lagrangian by certain general principles of invariance under various transformations. These principles are becoming increasingly important especially in dealing with the interactions of strange particles (hyperons and $K$-mesons) as we do not know the equations of motion of most of these elementary particles. We summarize these principles in the following sections.

We shall now proceed to describe in a rather detailed manner the meaning of invariance with respect to a transformation. For this purpose, it will be convenient to discuss at first transformations of space-time points. If a space-time point $P$ has coordinates $x$ in one system and if we define the Lorentz transformation $x^{\prime}=[A] x$, we imply that in the transformed system the "same physical" point $P$ has coordinates $x^{\prime}$. With every point $P$ let us associate a physical quantity $f(P)$ which is a function of $P$. In terms of the variable $x$, let it be defined as $\varphi(x)$. If from the physical nature of the problem the value of a physical quantity does not depend upon the coordinate system, then

$$
f(P)=\varphi(x)=\varphi\left(A^{-1} x^{\prime}\right)=\varphi^{\prime}\left(x^{\prime}\right) .
$$

We have merely replaced $x$ in terms of $x^{\prime}$ and obtained the physical quantity as a function of $x^{\prime}$. This procedure implies the invariance of the physical quantity under the transformation $A$. More generally, the physical quantity associated with $x$ may not be independent of the transformation. For example, if it is represented by a set of functions $\varphi_{\alpha}(x)(\alpha=1,2, \ldots)$ as in the case of a spinor or a vector, then in the transformed coordinate system we may have quite-generally

$$
\varphi_{\alpha}^{\prime}\left(x^{\prime}\right)=\sum_{\beta} S_{\alpha \beta} \varphi_{\beta}(x)=\sum_{\beta} S_{\alpha \beta} \varphi_{\beta}\left(A^{-1} x^{\prime}\right),
$$

where $\varphi$ and $\varphi^{\prime}$ are column vectors and $S$ is a matrix. To obtain $\varphi^{\prime}$ we have not only replaced $x$ in terms of $x^{\prime}$ but operated $S$ on $\varphi$. The variation in the value of the physical quantity at the same physical point due to the transformation is through the operation of $S$. A trivial example of $\varphi$ is the position vector of the point $P$.

In equation (7) we have compared the values of the functions at the same physical point and hence the arguments $x^{\prime}$ and $x$ are not equal. If we wish to compare the values of the functions for the same parametric values we merely write

$$
\varphi^{\prime}(l)=\sum_{\beta} S_{\alpha \beta}\left\{\varphi_{\beta}(l)+\left(\frac{\partial \varphi_{\beta}}{\partial x_{\nu}}\right)_{x=l} \varepsilon_{\mu \nu}\right\},
$$

where $l$ is used to denote the parametric value of $x$ and the infinitesimal $\varepsilon_{\mu \nu}^{\prime}$ is defined through the relation

$$
x_{\mu}^{\prime}=\sum_{\nu}\left(\delta_{\mu \nu}+\varepsilon_{\mu \nu}\right) x_{\nu} .
$$

Thus we note that there are two contributions to the difference between the functional values corresponding to points in the two coordinate systems which EPCR 10 
have the same parametric value. One is due to the difference in the values of the coordinates when referred to same coordinate system and the other due to $S$. It is convenient to call $S$ the matrix of intrinsic variation. If we are considering the same physical point, there is no need for such separation because the only change is due to intrinsic variations.

We shall now generalize the concept of invariance. Let $\varphi(x)$ satisfy certain equations involving $x$. If $\varphi^{\prime}\left(x^{\prime}\right)$ satisfies the same equation involving $x^{\prime}$, then the equation for $\varphi$ is covariant. The Dirac equation is covariant under Lorentz transformation and this is demonstrated by proving that it is always possible to find a matrix $S$ such that

$$
\psi^{\prime}\left(x^{\prime}\right)=S \psi(x)
$$

when $\psi$ and $\psi^{\prime}$ are the wave functions in the two Lorentz frames connected $x^{\prime} \rightarrow L x$, $L$ being the Lorentz matrix and $\psi$ and $\psi^{\prime}$ are assumed to satisfy the same equation. $S$ is completely defined by the equation

$$
S^{-1} \gamma_{\mu} S=\sum_{\nu} a_{\mu \nu} \gamma_{\nu}
$$

It is not necessary that the physical quantity should be represented by a set of functions for $S$ to exist. Even if it is defined by one function $\varphi(x)$, quite generally $\varphi^{\prime}\left(x^{\prime}\right)=S \varphi(x)$. In physics we usually deal with functions with $S= \pm 1$ under the transformation $\boldsymbol{x}^{\prime}=-\boldsymbol{x}$, i.e. space reflection. When $S=+\boldsymbol{1}$ it is called a scalar function and when $S=-1$ we call it a pseudoscalar function. Scalar functions are easily cited while pseudoscalar functions can be arrived at for example as follows.

The scallar product of two vector fields associated with space is a scalar function. But the scalar product of a polar vector and an axial vector is a pseudoscalar. The most obvious example of a pseudoscalar is the scalar product of linear momentum and angular momentum.

The relation between $\varphi^{\prime}\left(x^{\prime}\right)$ and $\varphi(x)$ can also be expressed by a transformation $U$, i.e.

If we require that

$$
\varphi^{\prime}\left(x^{\prime}\right)=U^{-1} \varphi(x) U=S \varphi(x)
$$

$$
U^{-1} L[\varphi(x)] U=L[\varphi(x)]
$$

$U$ must commute with $L[\varphi(x)]$, i.e.

$$
[L, U]=0 .
$$

If $U$ can be written as $\mathrm{e}^{-i F \alpha}$ where $\alpha$ is a continuous parameter, then the change in $\varphi$ for an infinitesimal change $\delta \alpha$ of $\alpha$ is given by

$$
i \delta \varphi=[F, \varphi] \delta \alpha ; \quad i \frac{\partial \varphi}{\partial \alpha}=[F, \varphi]
$$

If the Lagrangian is invariant under $U$, then it should commute with both $F$ and $U$. Hence the Hamiltonian should also commute with $F$ and $U$. Since the 
Hamiltonian is a constant of motion, $F$ is also a constant of motion. The eigenvalues of $F$ are observables and should be real; hence $F$ should be hermitian and $U$ unitary. Thus the invariance with respect to the unitary transformation has generated and conserved quantity $f$, the eigenvalue of $F$.

The transformations can be broadly divided into two categories,

(a) Continuous transformations, e.g. Lorentz transformation, gauge transformations, etc.

(b) Discrete transformations, e.g. when $A$ the operator of transformation applied twice becomes an identity operator, i.e. $A^{2}=+1$. The eigenvalues of the operator are \pm 1 as for example these of space-reflection, charge conjugation and time reversal. We will now enumerate some well known transformations and the corresponding conserved quantities.

\begin{tabular}{l|l|l}
\hline \multicolumn{1}{c|}{ Type of transformation } & \multicolumn{1}{|c}{ Conserved quantity } & \multicolumn{1}{|c}{$\begin{array}{c}\text { Remark about } \\
\text { invariance }\end{array}$} \\
\hline $\begin{array}{l}\text { (a) Translation in four- } \\
\text { dimensional space }\end{array}$ & $\begin{array}{l}\text { Energy-momentum } \\
\text { four-vector. } \\
\text { (b) Space rotation }\end{array}$ & Angular momentum \\
(c) Space inversion & Parity & $\begin{array}{l}\text { Exact } \\
\text { Approximate }\end{array}$ \\
(d) Time inversion & - & - \\
(e) Charge conjugation & Number of particles minus & Approximate \\
(f) Gauge & Charge and current & Exact \\
(g) Baryon Gauge & Heavy particle number & Exact \\
(h) Rotations in 3-dimensional & Total isotopic spin $I$. & Approximate \\
isotopic spin space. & $I_{3}$-projection of $I$ along & Approximate \\
(i) Rotation about third axis in & third axis & \\
\hline
\end{tabular}

We shall discuss the various types of transformations one by one.

\section{Particular transformations}

\section{Translation in four-dimensional space}

If the Hamiltonian is invariant for infinitesimal translation of space-time, i.e. $x_{v}^{\prime}=x_{v}+\delta x_{v}$ where $\delta x_{v}$ is an infinitesimal 4 -vector, then

$$
\delta H=\sum_{v} \frac{\partial H}{\partial x_{v}} \delta x_{v}=0 .
$$

Since $\delta x_{v}$ is arbitrary

$$
\frac{\partial H}{\partial x_{v}}=0 \quad \text { or } \quad\left[\frac{\partial}{\partial x_{v}}, H\right]=0
$$

i.e. the four-momentum vector $\frac{\partial}{\partial x_{v}}$ commutes with the Hamiltonian and it
is a constant of motion. $10 *$ 


\section{Space rotation}

Consider now an infinitesimal rotation $\omega$ of the coordinate system about the origin. Its components $\omega_{1}, \omega_{2}, \omega_{3}$ represent rotations about $x, y$ and $z$ axes respectively, i.e. $\boldsymbol{r}^{\prime}=\boldsymbol{r}+\boldsymbol{\omega} \times \boldsymbol{r}$. The corresponding momentum vector will become

$$
\boldsymbol{p}^{\prime}=\boldsymbol{p}+\boldsymbol{\omega} \times \boldsymbol{p} \text {. }
$$

The change in the Hamiltonian, $\delta H$, should vanish.

$$
\begin{aligned}
\delta H= & (\boldsymbol{\omega} \times \boldsymbol{r})_{\mu} \cdot \frac{\partial H}{\partial x_{\mu}}+(\boldsymbol{\omega} \times \boldsymbol{p})_{\mu} \cdot \frac{\partial H}{\partial p_{\mu}}=0 \\
& \omega\left\{\left(\boldsymbol{r} \times \frac{\partial H}{\partial \boldsymbol{r}}\right)+\left(\boldsymbol{p} \times \frac{\partial H}{\partial \boldsymbol{p}}\right)\right\}=0 .
\end{aligned}
$$

Noting that $\boldsymbol{l}=\boldsymbol{r} \times \boldsymbol{p}$ where $\boldsymbol{l}$ is the orbital angular momentum we see that $\boldsymbol{\omega} \cdot \boldsymbol{l}=0$, i.e. $\boldsymbol{l}$ is a constant of motion.

\section{Lorentz transformation}

We have already referred to the Lorentz transformation properties of single particle Dirac equations. However the following observation is necessary when we replace the $c$-number wave functions by $q$-numbers, i.e. operators. The Lagrangian density should be properly symmetrized or antisymmetrized to remove the unobservable singularities. This will be illustrated for the Dirac field. The free Lagrangian density for the Dirac field is ${ }^{1}$

$$
\begin{aligned}
-L & =+\bar{\psi}\left(\gamma_{\mu} \frac{\partial}{\partial x_{\mu}}+m\right) \psi \\
& =\psi^{\dagger} \gamma_{4}\left(\gamma_{\mu} \frac{\partial}{\partial x_{\mu}}+m\right) \psi \\
& =\psi^{\dagger} u \psi \quad \text { where } \quad u=\left\{\gamma_{4}\left(\gamma_{\mu} \frac{\partial}{\partial x_{\mu}}+m\right)\right\} \\
& =\sum_{\alpha \beta} \psi_{a}^{\dagger} u_{\alpha \beta} \psi_{\beta}=\sum_{\alpha \beta} u_{\alpha \beta} \psi_{\alpha}^{\dagger} \psi_{\beta} \\
& =\sum_{\alpha \beta} u_{\alpha \beta}\left(\bar{\psi} \gamma_{4}\right)_{\alpha} \psi_{\beta} \\
& =\sum u_{\alpha \beta} \bar{\psi}_{\nu} \psi_{\beta}\left(\gamma_{4}\right)_{v \alpha}=\sum_{\alpha \beta \nu} u_{\alpha \beta}\left[\frac{1}{2}\left(\bar{\psi}_{\nu} \psi_{\beta}-\psi_{\beta} \bar{\psi}_{\nu}\right)+\frac{1}{2}\left(\bar{\psi}_{\nu} \psi_{\beta}+\psi_{\beta} \bar{\psi}_{\nu}\right)\right]\left(\gamma_{4}\right)_{\nu \alpha} .
\end{aligned}
$$

The second term is a singular $c$-number

$$
u_{\alpha \beta}\left(\bar{\psi}_{\nu} \psi_{\beta}+\psi_{\beta} \bar{\psi}_{\nu}\right)\left(\gamma_{4}\right)_{\nu \alpha}=i\left(u_{\alpha \beta} S_{\nu \beta}^{(x)}\right)_{x=0}\left(\gamma_{4}\right)_{\nu \alpha}
$$

and is to be discarded because it will cause merely a constant (though infinite) shift of the eigenvalue. Therefore

$$
\begin{aligned}
-L & =\frac{1}{2} \sum_{\alpha \beta} u_{\alpha \beta}\left(\bar{\psi}_{\nu} \psi_{\beta}-\psi_{\beta} \bar{\psi}_{\nu}\right)\left(\gamma_{4}\right)_{\nu \alpha} . \\
& =\frac{1}{2}\left[\bar{\psi} \gamma_{4} u \psi-\psi u^{T} \gamma_{4}^{T} \bar{\psi}\right] \\
& =\frac{1}{2}\left[\psi^{\dagger}, u \psi\right] .
\end{aligned}
$$

${ }^{1} \mathrm{It}$ is to be noted that in this section we use the metrie $\delta_{\mu \nu}=1$ for $\mu=v=1, \cdots 4$ and $\delta_{\mu \nu}=0$ for $\mu \neq \nu$. The $\gamma_{j}(j=1, \cdots 3)$ matrices used here are $-i$ times the Feynman $\gamma_{j-8}$ while $\gamma_{4}$ is the same. 
We shall now consider the three important discrete transformations-space inversion $P$, charge conjugation $C$ and time inversion $T$. In doing this for $P$ and $C$ we shall adopt the procedure of assuming the transformations for the field variable and obtain the transformations for annihilation and creation operators directly therefrom. For the $T$-operation the procedure is a little more complicated, since it is not sufficient to define the operation for a single operator but it is necessary to define it with respect to a sequence of operators from physical arguments.

\section{Space inversion}

Complex scalar and pseudoscalar fields: Let $P$ represent the operation of space reflection,

$$
\text { i.e. } P: \boldsymbol{x} \rightarrow \boldsymbol{x}^{\prime}=-\boldsymbol{x} \text { and } P: x_{4} \rightarrow x_{4}^{\prime}=x_{4} \text {. }
$$

The complex field variable $\varphi(x)$ transforms as

$$
P \varphi(x) P^{-1}=\varphi^{\prime}\left(x^{\prime}\right)=\eta_{p} \varphi(x)=\eta_{p} \varphi\left(-\boldsymbol{x}^{\prime}, x_{4}\right),
$$

where $\eta_{p}$ can be complex. If we assume that reflection performed twice is equivalent to an identity transformation,

$$
\eta_{p}^{2}=+1, \text { i.e. } \eta_{p}= \pm 1 \text {. }
$$

$\eta_{p}=+1$ if $\varphi(x)$ is a scalar field and $\eta_{p}=-1$ if $\varphi(x)$ is a pseudoscalar field. $\eta_{p}$ is called the parity quantum number. From the transformation properties of $\varphi(x)$ we can deduce those of the annihilation and creation operators in the expansion of $\varphi(x)$. Omitting normalization factors, the Fourier decomposition of $\varphi(x)$ is

Then we have

$$
\varphi\left(\boldsymbol{x}, x_{4}\right)=\sum_{\boldsymbol{k}}\left[a(\boldsymbol{k}) \mathrm{e}^{i\left(\boldsymbol{k} \cdot \boldsymbol{x}-k_{4} x_{4}\right)}+b^{\dagger}(\boldsymbol{k}) \mathrm{e}^{-i\left(\boldsymbol{k} \cdot x-k_{4} x_{4}\right)}\right] .
$$

$$
\begin{aligned}
P \varphi(x) P^{-1} & =\sum_{k}\left[P a(\boldsymbol{k}) P^{-1} \mathrm{e}^{-i\left(k \cdot x^{\prime}\right)}+P b^{\dagger}(\boldsymbol{k}) P^{-1} \mathrm{e}^{-i k \cdot x^{\prime}}\right] \\
& =\eta_{p} \varphi\left(-\boldsymbol{x}^{\prime}, x_{4}\right)=\sum_{k^{\prime}}\left[\eta_{p} \boldsymbol{a}(\boldsymbol{k}) \mathrm{e}^{i\left(-\boldsymbol{k} \cdot \boldsymbol{x}^{\prime}-k_{4} x_{4}^{\prime}\right)}+\eta_{p} b^{\dagger}(\boldsymbol{k}) \mathrm{e}^{-i\left(-\boldsymbol{k} \cdot \boldsymbol{x}^{\prime}-k_{4} x_{4}^{\prime}\right)}\right] \\
& =\sum_{\boldsymbol{k}} \eta_{p} a(-\boldsymbol{k}) \mathrm{e}^{i\left(\boldsymbol{k} \cdot \boldsymbol{x}^{\prime}-k_{4} x_{4}\right)}+\eta_{p} b^{\dagger}(-\boldsymbol{k}) \mathrm{e}^{-i\left(\boldsymbol{k} \cdot \boldsymbol{x}^{\prime}-k_{4} x_{4}^{\prime}\right)}
\end{aligned}
$$

therefore

$$
\begin{gathered}
P a(\boldsymbol{k}) P^{-1}=\eta_{p} a(-\boldsymbol{k}) . \\
P b^{\dagger}(\boldsymbol{k}) P^{-1}=\eta_{p} b^{\dagger}(-\boldsymbol{k}) .
\end{gathered}
$$

Similarly we have

$$
P a^{\dagger}(\boldsymbol{k}) P^{-1}=\eta_{p} a^{\dagger}(-\boldsymbol{k}) ; \quad P b(\boldsymbol{k}) P^{-1}=\eta_{p} b(-\boldsymbol{k}) .
$$

To understand the meaning of these equations consider the one particle state $\left.a^{\dagger}(\boldsymbol{k})\right\rangle_{0}$. Making the natural assumption that the vacuum state has even parity, i.e. $P>_{0}=>_{0}$ we get

$$
\left.\left.\left.P a^{\dagger}(\boldsymbol{k})\right\rangle_{0}=P a^{\dagger}(\boldsymbol{k}) P^{-1} P\right\rangle_{0}=\eta_{p} a^{\dagger}(-\boldsymbol{k})\right\rangle_{0} .
$$


The parity operation changes the one particle state with momentum $\boldsymbol{k}$ to a one particle state with momentum $-\boldsymbol{k}$. If $\eta_{p}=+1$ the particle has even intrinsic parity and if $\eta_{p}=-1$ it has odd intrinsic parity. If we expand $\varphi(x)$ not in terms of free particle wave functions as above but in terms of the eigenfunctions of the angular momentum operator we have

where

$$
\varphi(x)=\left[\sum_{n} a_{n} u_{n}(x)+b_{n}^{\dagger} u_{n}^{*}(x)\right],
$$

$$
u_{n}(x)=\int \frac{1}{l^{\prime 2} k_{4}} / \frac{k}{r} J_{l+1 / 2}(k r) Y_{l m}(\theta, \varphi) \mathrm{d}^{3} k .
$$

$l=0,1,2 \ldots$ denote $S, P, D \ldots$ states.

Proceeding as before we can show that a particle having an even intrinsic parity (i.e. a scalar particle) existing in a state of angular momentum $l, m$ has a parity $(-1)^{l}$ (i.e. the parity of $Y_{l m}$ ), but for a pseudoscalar particle (i.e. odd intrinsic parity) the parity is $(-1)^{l+1}$.

Electromagnetic field: The electromagnetic field is a four vector $A_{\mu}\left(\boldsymbol{A}, A_{4}\right)$ and it transforms under space reflection $P$ as

$$
\begin{aligned}
P \boldsymbol{A}_{k}\left(\boldsymbol{x}, x_{4}\right) P^{-1} & =-A_{k}\left(-\boldsymbol{x}, x_{4}\right) \quad k=1,2,3 \\
P A_{4} P^{-1} & =A_{4}\left(-\boldsymbol{x}, x_{4}\right) .
\end{aligned}
$$

These properties follow from the fact that the magnetic field $\boldsymbol{H}$ is an axial vector and the electric field $\boldsymbol{E}$ is a polar vector. Since for a pure radiation field $A_{4}=0$ the photon has intrinsic parity $-1 .^{1}$

Spinor field: Applying space inversion we have

$$
P \psi(x) P^{-1}=\psi_{p}\left(x^{\prime}\right)=S_{p} \psi(x) .
$$

$S_{p}$ is so chosen that the free Lagrangian density for a spinor field is left invariant. The choice $S_{p}=\eta_{p} \gamma_{4}$ where $\eta_{p}$ is a complex number of modulus unity is sufficient. Reflecting a coordinate system twice is equivalent to a rotation through an angle $2 \pi$. Under this rotation a vector gets reflected twice whereas a spinor only once. So a rotation through $4 \pi$ is necessary so that the spinor is restored to its original state. Hence

$$
\eta_{p}^{2}= \pm 1, \text { i.e. } \eta_{p}= \pm 1, \pm i
$$

Yang and Tiomno ${ }^{2}$ have classified the four kinds of spinor fields as $A, B, C$ and $D$ according as $\eta_{p}=+i,-i,+1$ and -1 respectively.

As in the case of the boson field, we can prove, making use of the expansion for $\psi(x)$ and $\bar{\psi}(x)$ in terms of annihilation and creation operators and noting $\gamma_{4} u_{i}(\boldsymbol{k})=u_{i}(-\boldsymbol{k}), \gamma_{4} v_{i}(\boldsymbol{k})=-v_{i}(-\boldsymbol{k})$ that

$$
\begin{aligned}
& P b_{r}(\boldsymbol{k}) P^{-1}=\eta_{p} b_{r}(-\boldsymbol{k}) \\
& P d_{r}^{\dagger}(\boldsymbol{k}) P^{-1}=-\eta_{p} d_{r}^{\dagger}(-\boldsymbol{k}) .
\end{aligned}
$$

1 This result is consistent with the classical theory of the electromagnetic field.

2 C. N. Yang and J. Tromno, Phys. Rev. 79, 495 (1950). F. GürsEY has adopted this result to establish a connection between strangeness and parity. See Phys. Rev. Lett. 1, 98 (1958). 
On applying the $P$ operator to the one particle state we find that

$$
\begin{aligned}
& \left.\left.P b_{r}^{\dagger}(\boldsymbol{k})\right\rangle_{0}=\eta_{p} b_{r}^{\dagger}(-\boldsymbol{k})\right\rangle_{0} \\
& \left.\left.P d_{r}^{\dagger}(\boldsymbol{k})\right\rangle_{0}=-\eta_{p}, d_{r}^{\dagger}(-\boldsymbol{k})\right\rangle_{0} .
\end{aligned}
$$

For the case $\eta_{p}= \pm 1$, the anti-fermion has a parity and momentum opposite to that of a fermion.

Having determined the transformation properties of $\bar{\psi}$ and $\psi$ it is easy to study the transformation properties of bilinear covariants $\bar{\psi} \Omega_{i} \psi$ where $\Omega_{i}$ 's consist only of the $\gamma$-matrices. Under space reflection $P$ we have

$$
P\left(\bar{\psi} \Omega_{i} \psi\right) P^{-1}=P \bar{\psi} P^{-1} \Omega_{i} P \psi P^{-1}=\bar{\psi} \gamma_{4} \Omega_{i} \gamma_{4} \psi .
$$

In the table below are listed the properties of the covariants.

\begin{tabular}{c|c|c}
\hline$\Omega_{i}$ & $\gamma_{4} \Omega_{i} \gamma_{4}$ & Remark \\
\hline 1 & 1 & Scalar \\
$i \gamma_{\mu}$ & $-i \gamma_{\mu} ; \mu=1,2,3$ & Vector \\
& $i \gamma_{4}$ & \\
$\frac{i}{2}\left(\gamma_{\mu} \gamma_{\nu}-\gamma_{\nu} \gamma_{\mu}\right)$ & $\frac{i}{2}\left(\gamma_{\mu} \gamma_{\nu}-\gamma_{\nu} \gamma_{\mu}\right) ; \mu, \nu=1,2,3$ & Tensor \\
& $-\frac{i}{2}\left(\gamma_{\mu} \gamma_{\nu}-\gamma_{\nu} \gamma_{\mu}\right) ;$ either $\mu$ or $v=4$ & \\
$i \gamma_{\mu} \gamma_{5}$ & $+i \gamma_{\mu} \gamma_{5} ; \mu=1,2,3$ & Pseudovector \\
$i \gamma_{5}$ & $-i \gamma_{4} \gamma_{5}$ & Pseudoscalar \\
\hline
\end{tabular}

It should be noted that these transformations will also depend upon the types of spinors $\bar{\psi}$ and $\psi$. Demanding that the interaction of a spinor field with a boson field should be a scalar, we can write down the proper interactions immediately from the transformation properties of the boson field using the table. For example consider a pseudoscalar field $\varphi$ interacting with the Dirac field $\psi$ with $\eta_{p}=+1$. Then the interaction will have the form $i g \bar{\psi} \gamma_{5} \psi \varphi$. This is the famous Yukawa interaction.

\section{Charge conjugation}

We can define an operator $U_{c}$ which transforms the field $\varphi$ into its hermitian conjugate as

$$
U_{c} \varphi(x) U_{c}^{-1}=\eta_{c} \varphi^{\dagger}(x)
$$

This as we shall see is equivalent to changing a particle to its anti-particle. If the particle is charged its anti-particle has the opposite charge. However even for neutral particles, anti-particles exist and the operation $U_{c}$ which switches the particle to its anti-particle and vice versa should strictly be called particle-anti-particle conjugation rather than charge conjugation.

We shall now consider the effect of this operation on various fields. 
Complex fields: Consider a non-hermitian spinless field (scalar or pseudoscalar) $\varphi$ which is expanded as

$$
\varphi(x)=\sum_{k}\left\{a(k) \mathrm{e}^{i k \cdot x}+b^{\dagger}(k) \mathrm{e}^{-i k \cdot x}\right\} .
$$

Performing the charge conjugation operation on $\varphi$ we have

$$
\begin{aligned}
U_{c} \varphi(x) U_{c}^{-1} & =\sum_{k}\left[U_{c} a(\boldsymbol{k}) U_{c}^{-1} \mathrm{e}^{i k \cdot x}+U_{c} b^{\dagger}(\boldsymbol{k}) U_{c}^{-1} \mathrm{e}^{-i k \cdot x}\right] \\
\eta_{c} \varphi^{\dagger}(x) & =\eta_{c} \sum_{k}\left[a^{\dagger}(k) \mathrm{e}^{-i k \cdot x}+b(\boldsymbol{k}) \mathrm{e}^{i k \cdot x}\right]
\end{aligned}
$$

Since

$$
U_{c} \varphi(x) U_{c}^{-1}=\eta_{c} \varphi^{\dagger}(x)
$$

we get

$$
U_{c} a(\boldsymbol{k}) U_{c}^{-1}=\eta_{c} b(\boldsymbol{k})
$$

and

$$
U_{c} b^{\dagger}(\boldsymbol{k}) U_{c}^{-1}=\eta_{c} a^{\dagger}(\boldsymbol{k}) \text {. }
$$

For the complex field $\varphi \neq \varphi^{\dagger}$ and therefore the transformed field variable is different from the original one. Thus a charged particle state will not be an eigen state of $U_{c}$.

Neutral fields: For a neutral field (scalar or pseudoscalar) $\varphi=\varphi^{\dagger}$ and hence $b_{k}$ and $b_{k}^{\dagger}$ are identical with $a_{k}$ and $a_{k}^{\dagger}$ respectively. A neutral particle state will be an eigenstate of $U_{c}$ and hence $\eta_{c}=\eta_{c}^{*}$ and is real. Since $\eta_{c}^{2}=1, \eta_{c}= \pm 1$, i.e. we have two eigenstates of $U_{c}$ corresponding to $\eta_{c}=+1$ and $\eta_{c}=-1$ respectively. We shall make use of the possibility of the two eigenstates of $U_{c}$ while disscusing $K^{0}$ and $\bar{K}^{0}$.

Spinor field: As before we write

$$
U_{c} \psi(x) U_{c}^{-1}=S \psi^{\dagger}(x)=\psi_{c}
$$

where $S=\eta_{c} C^{*}$ with the $C$ matrix satisfying the properties

$$
C \gamma C^{-1}=\gamma^{*}, C^{*}=C^{-1} \text { and } C^{-1}=-\bar{C} .
$$

Further we have

Thus

$$
u^{*}(\boldsymbol{p})=C v(\boldsymbol{p}) ; \quad u(\boldsymbol{p})=C^{*} v^{*}(\boldsymbol{p})
$$

But

$$
U_{c} \psi(x) U_{c}^{-1}=\sum_{p} \sum_{r}\left[b_{r}^{\dagger}(\boldsymbol{p}) c^{*} u_{r}(\boldsymbol{p}) \mathrm{e}^{-i p \cdot x}+d_{r}(\boldsymbol{p}) c^{*} v_{r}(\boldsymbol{p}) \mathrm{e}^{i p \cdot x}\right.
$$

where

$$
\psi_{c}(x)=\sum_{p} \sum_{r}\left[b_{r}^{c}(\boldsymbol{p}) u_{r}(\boldsymbol{p}) \mathrm{e}^{i p \cdot x}+d_{r}^{c \dagger}(\boldsymbol{p}) v_{r}(\boldsymbol{p}) \mathrm{e}^{-i p \cdot x}\right.
$$

Hence we require

$$
b_{r}^{c}=U_{c} b_{r} U_{c}^{-1} \quad \text { etc. }
$$

$$
\begin{gathered}
U_{c} b_{r}(\boldsymbol{p}) U_{c}^{-1}=\eta_{c} d_{r}^{\dagger}(\boldsymbol{p}) \\
U_{c} d_{r}(\boldsymbol{p}) U_{c}^{-1}=\eta_{c} b_{r}^{\dagger}(\boldsymbol{p}) .
\end{gathered}
$$

1 Actually here $\psi^{\dagger}$ should be written as a column. i.e. the $\psi^{\dagger}$ is here actually $\left(\psi^{\dagger}\right)^{T}$. 
Electromagnetic field: The transformation properties of the photon field $A_{\mu}$ under $U_{c}$ can be obtained by demanding that the interaction Hamiltonian of the electron-photon fields should be invariant under this transformation. This implies that

$$
U_{c} A_{u} U_{c}^{-1}=-A_{u} .
$$

The transformation properties of $\psi$ and $\bar{\psi}$ determine the corresponding transformation properties $[\psi, \Omega \psi]$ which are listed below:

\begin{tabular}{c|c|c}
\hline$i$ & $\Omega_{i}$ & $\eta_{c}^{(i)}$ \\
\hline $\mathrm{S}$ & 1 & 1 \\
$\mathrm{~V}$ & $i \gamma_{\mu}$ & -1 \\
$\mathrm{~T}$ & $\frac{i}{2}\left(\gamma_{\mu} \gamma_{\nu}-\gamma_{\nu} \gamma_{\mu}\right)$ & -1 \\
$\mathrm{~A}$ & $i \gamma_{\mu} \gamma_{5}$ & +1 \\
$\mathrm{P}$ & $i \gamma_{5}$ & +1 \\
\hline
\end{tabular}

The transformation properties of bilinear covariants can be extended to more than one such covariant. A typical example is that of the $\beta$-decay interaction given by

$$
H_{\beta}=\sum_{i} g_{i}\left(\bar{\psi}_{a} \Omega_{i} \psi_{b}\right)\left(\bar{\psi}_{c} \Omega_{i} \psi_{d}\right)+h \cdot c .
$$

where $a, b, c$ and $d$ represent the four fermion fields. Invariance of $H_{\beta}$ under charge conjugation requires that $g_{i}$ 's should be real.

Let us consider the Yuksawa interaction of a boson with a fermion field. If we require it to be invariant under charge conjugation, we can identify the transformation factor $\eta_{c}$ (i.e. charge conjugation parity) for the boson fields defined by

$$
U_{c} \varphi U_{c}^{-1}=\eta_{c}^{(i)} \varphi .
$$

For convenience of reference we tabulate them below:

\begin{tabular}{l|c|r}
\hline Boson field & $i$ & $\eta_{c}^{(i)}$ \\
\hline Neutral scalar or pseudoscalar & S, P & 1 \\
Photon & V & -1 \\
Vector meson & V & -1 \\
Axial vector meson & A & 1 \\
\hline
\end{tabular}

In order to realize the use of this transformation we cite some applications.

(a) If the interaction is left invariant under $U_{c}$ so also will be the $S$-matrix, i.e.

$$
\langle f|S| i\rangle=\left\langle f\left|U_{c}^{-1} S U_{c}\right| i\right\rangle=\left\langle f^{c}|S| i^{c}\right\rangle .
$$

which means for example the scattering of $\pi^{+}$from protons is identical with $\pi^{-}$ from antiprotons. 
(b) We will prove a very important result known as Furry's theorem ${ }^{1}$ in quantum electrodynamics. Consider a process having an odd number of external photon lines. Then the propagator in the momentum representation is

$$
\begin{aligned}
G\left(p_{1}, p_{2}, \ldots, p_{n}\right)=\int \mathrm{e}^{i\left(p_{1} x_{1}+p_{2} x_{2}+\cdots p_{n} x_{n}\right)} \mathrm{d} x_{1} \mathrm{~d} x_{2} \ldots, \mathrm{d} x_{n} \\
\\
\left\langle T\left(A_{\mu}\left(x_{1}\right) A_{v}\left(x_{2}\right) \ldots A_{\lambda}\left(x_{n}\right)\right)\right\rangle .
\end{aligned}
$$

The operators $A_{\mu}$ are in Heisenberg representation.

Applying $U_{c}$ we see

$$
\begin{aligned}
G^{c}\left(p_{1}, p_{2}, \ldots, p_{n}\right) & =\int \mathrm{e}^{i\left(p_{1} x_{1}+p_{2} x_{2} \cdots+p_{n} x_{n}\right)}\left\langle T\left(A_{\mu}^{c}\left(x_{1}\right) \ldots, A_{\lambda}^{c}\left(x_{n}\right)\right)\right\rangle \\
& =(-1)^{n} G\left(p_{1}, \ldots, p_{n}\right) .
\end{aligned}
$$

Since $G^{c} \equiv-G, G=0$ if $n$ is odd. Thus no real process can occur with an odd number of external photons.

\section{Time reflection}

We would expect that the time reflection $T$ in analogy with that of space reflection should imply $t \rightarrow t^{\prime}=-t$. We shall see that this definition is inadequate and requires an additional prescription which is forced upon us by both physical and mathematical considerations. We are led to two types of time reversals strong and weak, $T_{s}$ and $T_{w}$ respectively.

Assuming that the $T$ operation corresponds to going over from $t$ to $-t$, we proceed as in the case of space reflection and consider a scalar field variable which becomes under $T$ :

$$
T \varphi(x) T^{-1}=\varphi^{\prime}\left(\boldsymbol{x}, t^{\prime}\right)=\varphi(\boldsymbol{x}, t)=\varphi\left(+\boldsymbol{x}, t^{\prime}\right) .
$$

Expanding $\varphi(\boldsymbol{x}, t)$ in terms of creation and annihilation operators we have

$$
\begin{aligned}
\varphi(x) & =\sum_{k}\left[a(\boldsymbol{k}) \mathrm{e}^{i k \cdot x}+b^{\dagger}(\boldsymbol{k}) \mathrm{e}^{-i k \cdot x}\right] \\
T \varphi(x) T^{-1} & =\sum_{k}\left[T a(\boldsymbol{k}) T^{-1} \mathrm{e}^{-i\left(k \cdot x+k_{4} t\right)}+\sum_{k} T b^{\dagger}(\boldsymbol{k}) T^{-1} \mathrm{e}^{i\left(\boldsymbol{k} \cdot \boldsymbol{x}+k_{4} t\right)}\right] .
\end{aligned}
$$

Thus we have

$$
\begin{aligned}
& T a(\boldsymbol{k}) T^{-1}=b^{\dagger}(-\boldsymbol{k}) \quad \text { or } \quad T a^{\dagger}(\boldsymbol{k}) T^{-1}=b(-\boldsymbol{k}) \\
& T b^{\dagger}(\boldsymbol{k}) T^{-1}=a(-\boldsymbol{k}) \quad \text { or } \quad T b(+\boldsymbol{k}) T^{-1}=a^{\dagger}(-\boldsymbol{k}),
\end{aligned}
$$

i.e. creation operators of particles of momentum $\boldsymbol{k}$ become the annihilation operator of antiparticles of momentum - $\boldsymbol{k}$. This time reflection with the inclusion of a phase factor $\eta$ is known as the strong time reflection $T_{s}{ }^{2}$ under which the particle and anti-particle are interchanged. However we can define another time reflection called the weak time reflection $T_{w}$ known also as Wigner's ${ }^{3}$ time reversal. Under $T_{w}$ we have

$$
T_{u} \varphi(x) T_{\omega}^{-1}=\varphi^{\prime}\left(\boldsymbol{x}, t^{\prime}\right)=\eta \varphi^{\dagger}(\boldsymbol{x}, t) .
$$

1 W. H. Furry, Phys. Rev.51, 125 (1937). This theorem can equally well be applied to $\pi^{0}$ decaying into two photons, see C. N. YANG, Phys. Rev. 77, 242 (1956).

2 J. Schwinger, Phys. Rev. 82, 914 (1951).

3 E. P. Wigner, Gottinger Nachr., 546 (1932); Proc. Nat. Acad. Sci. 38, 449 (1952). 
Proceeding as in the above, we arrive at the following relations for ereation and annihilation operators

$$
T_{w} a^{\dagger}(\boldsymbol{k}) T_{w}^{-1}=\eta a(-\boldsymbol{k}) ; \quad T_{w} b^{\dagger}(\boldsymbol{k}) T_{w}^{-1}=\eta b(-\boldsymbol{k}) .
$$

The striking feature of $T_{w}$ is that the particle and anti-particle nature is not disturbed. The two time reflections are related by

$$
T_{w}=C T_{s} .
$$

Since in both the cases, the ereation operators become annihilation operators, it is physically reasonable to expect that under time reversal we should also change the order of events, i.e. the initial state becomes the final state and vice versa. But this description implies that in the case of operators, it is not just enough to reverse the time, i.e.

$$
T \alpha(t) T^{-1}=\alpha^{\prime}\left(t^{\prime}\right)
$$

but we have also to impose an additional rule in the ease of a product of operators $\alpha(t) \beta(t)$. Precisely we have

$$
T \alpha(t) \beta(t) T^{-1}=\beta^{\prime}\left(t^{\prime}\right) \alpha^{\prime}\left(t^{\prime}\right),
$$

i.e. we reverse the order of the product of operators. The mathematical consequence of this precsription is the preservation of commutation which is otherwise not possible.

Let us apply $T$ to $q(t)$ and $p(t)$, i.e. coordinate and momentum operators

$$
T q(t) T^{-1}=q^{\prime}\left(t^{\prime}\right)=q(t) ; T p(t) T^{-1}=p^{\prime}(t)=-p(t) .
$$

The commutation relation between $q^{\prime}\left(t^{\prime}\right), p^{\prime}\left(t^{\prime}\right)$ is

$$
\begin{aligned}
{\left[q^{\prime}\left(t^{\prime}\right), p^{\prime}\left(t^{\prime}\right)\right] } & =[q(t),-p(t)]=-[q(t), p(t)] \\
& \neq[q(t), p(t)] .
\end{aligned}
$$

Hence it is not invariant under the transformation $T$ and hence $T$ is not unitary. However accepting the prescription of the reversal of order we find

$$
\begin{aligned}
T[q(t), p(t)] T^{-1} & =\left[p^{\prime}\left(t^{\prime}\right), q^{\prime}\left(t^{\prime}\right)\right]=-[p(t), q(t)] \\
& =[q(t), p(t)] .
\end{aligned}
$$

Under time reversal the state vectors transform as follows:

$$
\begin{gathered}
T|\psi\rangle=\left\langle\psi^{\prime}\right| . \\
\langle\psi| T^{\dagger}=\left\langle\psi\left|T^{-1}=\right| \psi^{\prime}\right\rangle .
\end{gathered}
$$

We shall now show that $T_{w}$ is equivalent to complex conjugation followed by a unitary transformation. Consider the time dependent Schrödinger equation

$$
i \frac{\partial \psi(t)}{\partial t}=H \psi(t)
$$

If we now change $t$ to $-t$, we find

$$
-i \frac{\partial}{\partial t} \psi^{\prime}\left(t^{\prime}\right)=H^{\prime} \psi^{\prime}\left(t^{\prime}\right)
$$


where $\psi^{\prime}\left(t^{\prime}\right)=\psi(-t)$ and $H^{\prime}$ is obtained from $H$ by replacing $\boldsymbol{p}$ by $-\boldsymbol{p}$ and reversing the order of events. This reversal is of no consequence if $H$ is a symmetric function of $p$ and $q$. Taking the complex conjugate of the above equation, we find that $\psi^{*}$ satisfies the same equation as $\psi$ provided

$$
H^{\prime *}=T H T^{-1}=H \text {. }
$$

Thus we find that

$$
\psi^{\prime}\left(\boldsymbol{x}, t^{\prime}\right)=K \psi^{*}(\boldsymbol{x}, t),
$$

i.e. the time reversed wave function is obtained by a unitary transformation and a complex conjugation (i). Thus the operator for time reversal can be expressed as

$$
T_{w}=K \times \text { (complex conjugation). }
$$

From the transformation properties of $q$ and $p$ it follows that the angular momentum operators $J_{z}$ and $J^{2}$ should transform as

$$
T_{w} J_{z} T_{w}^{-1}=-J_{z}
$$

or $\left[J_{z}, T_{w}\right]_{+}=0$, i.e. $J_{z}$ anti-commutes with $T_{w}$. But

or

$$
T_{w} J^{2} T_{w}^{-1}=T_{w} J T_{w}^{-1} T_{w} J T_{w}^{-1}=J^{2}
$$

$$
\left[J^{2}, T_{w}\right]=0 \text {. }
$$

Hence the eigenvalue of $J^{2}$, i.e. $j(j+1)$ will be a constant of motion while that of $J_{z}$ will not be a "good" quantum number. ${ }^{1}$ These considerations apply equally well to spin operator $\sigma$.

Consider a state with total angular momentum $j$ and $z$-component $m$. We have

$$
J_{z} T_{w}|j m\rangle=-T_{w} J_{z}|j m\rangle=-m T_{w}|j m\rangle,
$$

i.e. the state $T_{w}|j m\rangle$ is an eigenstate of $J_{z}$ with eigenvalue $-m$. However $T_{w}|j m\rangle$ is still an eigenstate of $J^{2}$ with eigenvalue $j(j+1)$. Therefore $T_{w}|j m\rangle=c_{j m}\langle j,-m|$ where $c_{j m}$ depends on $j$ and $m$ with $\left|c_{j m}\right|^{2}=1(i e) c_{j m}= \pm 1$.

We will deduce a theorem analogous to the principle of detailed balance widely used in statistical mechanics. ${ }^{2}$ Let us assume that the $S$-matrix is left invariant under $T_{w}$ i.e.

$$
\begin{aligned}
S & =T_{w}^{-1} S T_{w}=\left\langle\boldsymbol{k} r_{1}, \boldsymbol{k}_{2} r_{2}, \ldots|S| \boldsymbol{k}_{1}^{\prime}, r_{1}^{\prime}, \boldsymbol{k}_{2}^{\prime} r_{2}^{\prime}, \ldots\right\rangle \\
& = \pm\left\langle-\boldsymbol{k}_{1}^{\prime}-r_{1}^{\prime},-\boldsymbol{k}_{2}^{\prime}-r_{2}^{\prime}, \ldots|S|-\boldsymbol{k}_{1}-r_{1},-\boldsymbol{k}_{2}-r_{2}, \ldots\right\rangle
\end{aligned}
$$

where we have described the initial and final system by the momenta $\boldsymbol{k}$ and spin $r$ of the various particles. However this result is not identical to the theorem of detailed balance which demands that

$\left|\left\langle\boldsymbol{k}_{1} r_{1}, \boldsymbol{k}_{2} r_{2}, \ldots|S| \boldsymbol{k}_{1}^{\prime} r_{1}^{\prime}, \boldsymbol{k}_{2}^{\prime} r_{2}^{\prime}, \ldots\right\rangle\right|^{2}=\left|\left\langle\boldsymbol{k}_{1}^{\prime} r_{1}^{\prime}, k_{2}^{\prime} r_{2}^{\prime}, \ldots|S| \boldsymbol{k}_{1} r_{1}, \boldsymbol{k}_{2} r_{2}, \ldots\right\rangle\right|^{2}$.

1 From this result it can be shown that there cannot be an observable which connects states of integral and half integral spin. See G. C. Wick, A. S. Wightman and E. P.Wigner, Phys. Rev. 88, 101 (1952); Kemmer et. al., Rep. Progr. Phys. 23, 400 (1959).

2 J. Hamilton and H. W. Peng, Proc. Roy. Ir. Acad. 49, 197 (1944); F. Coester, Phys. Rev. 84, 1259 (1951). 
But since we know that $\boldsymbol{k}$ becomes - $\boldsymbol{k}$ under $P$, if we also assume that $P S P^{-1}=S$, then (81) becomes

$$
\begin{aligned}
\left\langle\boldsymbol{k}, r_{1}, \boldsymbol{k}_{2} r_{2}, \ldots|S| \boldsymbol{k}_{1}^{\prime} r_{1}^{\prime},\right. & \left.\boldsymbol{k}_{2}^{\prime} r_{2}^{\prime}, \ldots\right\rangle \\
& = \pm\left\langle\boldsymbol{k}_{1}^{\prime}-r_{1}^{\prime} ; \boldsymbol{k}_{2}^{\prime}-r_{2}^{\prime}, \ldots|S| \boldsymbol{k}_{1}-r_{1}, \boldsymbol{k}_{2}-r_{2}, \ldots\right\rangle
\end{aligned}
$$

where we have space-reflected the states. This still differs from (81a) in that the spin states are different. We can remove the dependence on spin states by summing over all possible spin states. Thus

$$
\sum_{\text {Spin }}\left|\left\langle\boldsymbol{k}_{1}, \boldsymbol{k}_{2}, \ldots|S| \boldsymbol{k}_{1}^{\prime}, \boldsymbol{k}_{2}^{\prime}, \ldots\right\rangle\right|^{2}=\sum_{\text {spin }}\left|\left\langle\boldsymbol{k}_{1}^{\prime}, \boldsymbol{k}_{2}^{\prime}, \ldots|S| \boldsymbol{k}_{1}, \boldsymbol{k}_{2} \ldots\right\rangle\right|^{2}
$$

Imposing rotational invariance on $S$, the matrix element cannot depend upon the $m$ value. Then we can obtain a detailed balancing theorem for transitions between states of the same total angular momentum quantum number $j$ :

$$
\langle\beta j m|S| \alpha j m\rangle=\langle\alpha j-m|S| \beta j-m\rangle
$$

where $\alpha$ and $\beta$ are some other quantum numbers necessary to identify the "channels". This result is very useful in the study of nuclear reactions.

Another interesting application of the principle of invariance under time reversal is the following. Defining a reaction matrix $K$ through

$$
S=\frac{1+\frac{1}{2} i K}{1-\frac{1}{2} i K} \quad \text { or } \quad T=\frac{i K}{1-\frac{i}{2} K}
$$

the unitarity of $S$ implies the hermiticity of $K$. If $S$ is invariant under $T_{w}$ and rotation, $K$ is real. The importance of the $K$ matrix is that it describes the scattering process in terms of a minimum number of real parameters. Using (85) we can show that

$$
t=\frac{k}{1-i \pi k},
$$

where $t$ and $k$ are sub-matrices of $T$ and $K$ respectively containing the matrix elements which are diagonal in $E, J, J_{z}$ but differ with respect to other quantum numbers necessary to denote the various final channels if available.

In the case of single channel if we define $K$ in terms of a single real parameter which we can take to be the phase shift $\delta$, i.e.

$$
k=\frac{1}{\pi} \tan \delta
$$

we have

$$
t=\frac{1}{\pi} \mathrm{e}^{i \delta} \sin \delta .
$$

If we consider the following two channel reaction

$$
\begin{array}{rlrl}
\pi^{+}+n & \rightleftarrows \pi^{+}+n & & \text { (elastic scattering) } \\
& \rightleftarrows \gamma+p & & \text { (radiative capture or } \\
& & \text { photo production of mesons) }
\end{array}
$$


we can characterize the $K$ matrix with the real parameters $a(\gamma p \rightarrow \gamma p)$, $b\left(\pi^{+}+n \rightleftarrows \gamma+p\right)$ and $\tan \delta\left(\pi^{+}+n \rightarrow \pi^{+}+n\right)$. Since there is only little elastic $\gamma p$ scattering, we can set $a=0$. We can show that $t$ is given by

$$
t=\frac{1}{\pi}\left[\begin{array}{cc}
0 & b \mathrm{e}^{i \delta} \cos \delta \\
b \mathrm{e}^{i \delta} \cos \delta & \mathrm{e}^{i \delta} \sin \delta
\end{array}\right] .
$$

The importance of this result lies in that the photo production amplitude involves the same phase shift as $\pi^{+}+n$ scattering. ${ }^{1}$ The $K$ matrix is particularly useful when there are many final channels both elastic and inelastic as for example in $K^{-}-p$ or $K^{-}-n$ interactions. $^{2}$

Strong reflection $R_{s}$ or $C P T\left(T=T_{w}\right)$

We will now study the effect of strong reflection, i.e. reflection of both space and time coordinates. This is defined as

$$
R_{s} x_{\mu}=x_{\mu}^{\prime}=-x_{\mu} .
$$

The effect of $R_{s}$ on a scalar field $\varphi(x)$ is given by

$$
R_{s} \varphi(x) R_{s}^{-1}=\varphi^{\prime}\left(x^{\prime}\right)=\eta_{s} \varphi(x) .
$$

By familiar arguments we now obtain by comparing the coefficients of $e^{i k \cdot x}$ suitably in $\eta_{s} \varphi(x)$ and $\varphi^{\prime}\left(x^{\prime}\right)$

$$
\begin{aligned}
R_{8} a(\boldsymbol{k}) R_{s}^{-1} & =\eta_{s} b^{\dagger}(\boldsymbol{k}) \\
R_{s} b^{\dagger}(\boldsymbol{k}) R_{8}^{-1} & =\eta_{s} a(\boldsymbol{k}) .
\end{aligned}
$$

Thus an initial state of a single particle with momentum $\boldsymbol{k}$ is transformed into a final state of an anti-particle of momentum $\boldsymbol{k}$.

In obtaining the transformation for operators, we notice that we have combined $P$ with strong time reflection as defined before.

The spinor field transforms as

$$
\begin{aligned}
& R_{s} \psi(x) R_{s}^{-1}=\psi^{\prime}\left(x^{\prime}\right)=i \eta_{s} \gamma_{5} \psi(x) \\
& R_{s} \bar{\psi}(x) R_{s}^{-1}=i \eta_{s}^{*} \gamma_{5}^{T} \bar{\psi}(x) .
\end{aligned}
$$

The corresponding rules for the annihilation and creation operators are

$$
\begin{aligned}
& R_{s} a_{r}(\boldsymbol{k}) R_{s}^{-1}=(-r) \eta_{s} b_{-r}^{\dagger}(\boldsymbol{k}) \\
& R_{s} b_{r}^{\dagger}(\boldsymbol{k}) R_{s}^{-1}=(-r) \eta_{s} a_{-r}^{\dagger}(\boldsymbol{k}) .
\end{aligned}
$$

Therefore an initial state of single particle with momentum $\boldsymbol{k}$ and spin $r$ is transformed to a final state of an antiparticle state with momentum $\boldsymbol{k}$ and spin $-r$, i.e.

$$
R_{s}|\boldsymbol{k}, r\rangle=(-1)^{r} \eta_{s}\left\langle_{\boldsymbol{k}},-r\right|
$$

1 M. Gell-Mann and K.M. Watson, Ann. Rev.Nucl. Sci.4, 219 (1954); G. Takeda, Phys. Rev. 101. 1547 (1956).

${ }^{2}$ M. H. Ross and G. L. Shaw, Phys. Rev. 115, 1773 (1959). For an interesting alternative formulation for describing inelastic scattering, see P. T. Matthews and A. Salam, Nuovo Cim. 13, 381 (1959). 
It is interesting to see the effect of $C P T$ on a state

Thus

$$
\begin{aligned}
T|\boldsymbol{k}, r\rangle & =\langle-\boldsymbol{k},-r| \\
P\langle-\boldsymbol{k},-r| & =\langle\boldsymbol{k},-r| \\
C\langle\boldsymbol{k},-r| & =\langle\boldsymbol{k},-r| .
\end{aligned}
$$

and $R_{s}$ is equivalent to $C P T$.

If we now define Schwinger's time reflection ${ }^{1}$ as

$$
T_{s}=R_{s} P \text {, }
$$

it is equal to $C T$, i.e. to performing charge conjugation on the time reversed state. This is equivalent to the strong time reversal previously defined. $T_{s}$ switches an initial particle state with momentum $\boldsymbol{k}$ spin $r$ to a final anti-particle of momentum - $\boldsymbol{k}$ spin $-r$.

Following Matthews and Feldman ${ }^{2}$, we will prove an important result called the $C P T$ theorem or the Pauli-Lüders theorem. ${ }^{3}$ The theorem states that any physical theory invariant under proper orthochronous Lorentz transformation with the usual relation between spin and statistics is automatically invariant under strong reflection or $C P T{ }^{4}$ We wish to stress that while a proper Lorentz transformation is a continuous transformation, $C P T$ is a discrete transformation and hence any result connecting these different transformations is very remarkable.

The propagator of a particle with incoming and outgoing momenta $q$ and $p$ is given by

$$
G_{\alpha \beta}\left(p, q, k_{i}\right)=\int \mathrm{e}^{-i(p x-q u \cdots)}\left\langle T\left(\psi_{x}(x) \bar{\psi}_{\beta}(y) \cdots\right)\right\rangle \mathrm{d}^{4} x \mathrm{~d}^{4} y,
$$

where $\alpha, \beta$ specify the states in more detail and $k_{i}$ denote collectively the incoming and outgoing momenta of other kinds of particles. Performing $R_{s}$ on this gives

$$
\int \mathrm{e}^{+i(p x-q y \cdots)}\left(i \gamma_{5}\right)_{\alpha \varrho}\left\langle T\left(\bar{\psi}_{\lambda}(y) \psi_{\varrho}(x)\right)\right\rangle\left(i \gamma_{5}\right)_{\lambda \beta} \mathrm{d}^{4} x \mathrm{~d}^{4} y
$$

where we have changed the integration variables $x \rightarrow-x$ and $y \rightarrow-y$. Using the anti-commutability (or Fermi statistics) of $\psi$ field we can write $\bar{\psi}(y) \psi_{\alpha}(x)$ $=-\psi_{\alpha}(x) \bar{\psi}_{\beta}(y)$. Notice however that this sign will cancel with $(-i)^{2}$. Thus

$$
G_{\alpha \beta}\left(p_{1}, q_{1}, k_{i}\right)=\gamma_{5} G_{\alpha \beta}\left(-p_{1},-q_{1},-k_{i}\right) \gamma_{5} .
$$

In order that $G_{i}$ be covariant, it should involve $p, q, k_{i}$ in the form $\mathrm{p}, \mathrm{q}, \mathrm{k}_{i}$ etc. Hence we have

since

$$
\gamma_{5} G_{\alpha \beta}\left(-\mathrm{p},-\mathrm{q},-\mathrm{k}_{i}\right)=G_{\alpha \beta}\left(\mathrm{p} \cdot \mathrm{q}, \mathrm{k}_{i}\right)
$$

1 J. SCHWINGER, loc. cit.

2 G. Feldman and P. T. Matthews, Phys. Rev. 10:, 421 (1956).

3 G. Luders, Kgl. Danske Vidensk. 28, 5 (1954); Ann. Phys. 2, 1 (1957); W. Pauli, Viels Bohr and the Development of Physics, Pergamon (1950).

4 Invariance under $C P T$ implies that there exists a state for the anti-particles corresponding to each possible state of the particle with the difference that the space and time are inverted. 
Thus the propagator is left invariant under $R_{s}$ or $C P T$ and the theorem is proved. For convenience we will collect all the different transformation properties of the scalar and spinor fields and exhibit in a table.

\begin{tabular}{|c|c|c|c|c|c|}
\hline Field & $\begin{array}{c}P \\
\boldsymbol{x}^{\prime}=-\boldsymbol{x} \\
x_{4}^{\prime}=x_{4}\end{array}$ & $\mid \begin{array}{c}U_{c} \\
\text { particle } \rightleftarrows \\
\text { anti-particle }\end{array}$ & $\begin{array}{c}R_{\delta} \\
x^{\prime}=-x\end{array}$ & $\begin{array}{c}R_{w} \\
R_{w}=R_{s} U_{c}\end{array}$ & $\begin{array}{c}T_{w} \\
T_{w w}=R_{w} \cdot P\end{array}$ \\
\hline $\begin{array}{c}\varphi \\
\text { scalar } \\
\text { or } \\
\text { pseudoscalar }\end{array}$ & $\begin{array}{c}\varphi_{p}=\eta_{p} \varphi(-x) \\
\varphi_{p}^{\dagger}=\eta_{p}{ }^{*} \varphi^{\dagger}(-x) \\
\eta_{p}{ }^{2}=1 \\
\eta_{p}=1 \text { for } \mathrm{s} \\
=-1 \text { for p.s. }\end{array}$ & $\begin{array}{c}\varphi_{c}=\eta_{c} \varphi \dagger \\
\varphi_{c}^{\dagger}=\eta_{c}^{*} \varphi \\
\left|\eta_{c}\right|^{2}=1 \\
\text { for charged } \\
\eta_{c}^{2}=1 \\
\text { for neutral }\end{array}$ & $\begin{array}{c}\varphi_{s}=\eta_{s} \varphi(-x) \\
\varphi_{s}^{+}=\eta_{s}^{*} \varphi^{\dagger}(-x) \\
\pi_{s}=-\eta_{s} \pi(-x) \\
\pi_{s}^{\dagger}=-\eta_{s}^{*} \pi^{\dagger}(-x) \\
\eta_{s}^{2}=1\end{array}$ & $\begin{array}{c}\varphi_{w}=\eta_{w} \varphi \dagger(-x) \\
\varphi_{w^{\dagger}}=\eta_{w^{*}}^{*} \varphi(-x) \\
\eta_{w}=\eta_{c} \eta_{s}\end{array}$ & $\begin{array}{c}\varphi_{T}=\eta_{T} \varphi^{\dagger}\left(-x_{4}\right) \\
\varphi_{T^{\dagger}}=\eta_{T^{*}}^{*} \varphi\left(-x_{4}\right)\end{array}$ \\
\hline $\begin{array}{c}\psi \\
\text { spinor }\end{array}$ & $\begin{array}{c}\psi_{p}=\eta_{p} \gamma_{4} \psi(-\boldsymbol{x}) \\
\psi_{p}^{\dagger}=\eta_{p}{ }^{*} \psi(-x) \gamma_{4} \\
\bar{\psi}_{p}=\eta_{p}^{*} \bar{\psi}(-x) \gamma_{4} \\
\eta_{p}{ }^{2}= \pm 1 \\
\eta_{p}=i \\
\text { for A type }\end{array}$ & $\begin{array}{c}\psi_{c}=\eta_{c} c^{*} \psi^{\dagger} \\
\psi_{c}^{\dagger}=\eta_{c}^{*} \psi c \\
\bar{\psi}_{c}=\eta_{c}^{*} \psi c \gamma_{a} \\
\left|\eta_{c}\right|^{2}=1\end{array}$ & $\left\{\begin{array}{c}\psi_{s}=i \eta_{s} \gamma_{5} \psi(-x) \\
\psi_{s}^{\dagger}=-i \eta_{s}^{*} \psi^{\dagger}(-x) \gamma_{5} \\
\bar{\psi}_{s}=i \eta_{s}^{*} \bar{\psi}(-x) \gamma_{5} \\
\eta_{s}^{2}= \pm 1\end{array}\right.$ & $\begin{array}{c}\psi_{w}=i \eta_{w} \gamma_{5} c^{*} \varphi^{\dagger}(-x) \\
\psi_{w}^{\dagger}=-i \eta_{w}^{*} \psi(-x) c \gamma_{5} \\
\bar{\psi}_{w}=i \eta_{w^{*}}^{*} \psi(-x) c \gamma_{\downarrow} \gamma_{5} \\
\eta_{w}=\eta_{c} \eta_{s}\end{array}$ & 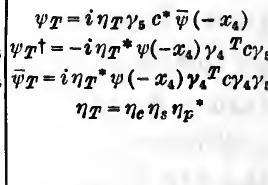 \\
\hline
\end{tabular}

\section{Continuous transformations}

The continuous transformations we have till now studied are those dealing with space-time coordinates. More generally we can define a continuous transformation with respect to a single parameter or a set of parameters, the spacetime transformations being particular cases of these. In discussing this, we shall first take up the case of a single parameter other than space-time. We shall denote the field operator $\varphi(x)$ as $\varphi(x, \lambda)$ and ask how $\varphi(x, \lambda)$ transforms when the parameter $\lambda$ is changed to $\lambda+\mathrm{d} \lambda$ without $x$ being affected. We can write

$$
\varphi(x, \lambda+\mathrm{d} \lambda)=(1+T \mathrm{~d} \lambda) \varphi(x, \lambda)
$$

where $T$ is a matrix of transformation when $\varphi(x, \lambda)$ has more than one component but is a complex number for a scalar $\varphi(x, \lambda)$. Suppressing the parameter $\lambda$, we write

$$
\varphi^{\prime}(x)=(1+T \mathrm{~d} \lambda) \varphi(x)
$$

or in terms of the components of $\varphi$

$$
\varphi_{\alpha}^{\prime}(x)=\left(\delta_{\alpha \beta}+\mathrm{d} \lambda T_{\alpha \beta}\right) \varphi_{\beta}(x) .
$$

If we assume that $\varphi_{\alpha}^{\prime}(x)=u^{-1} \varphi_{\alpha}(x) u$, a relation between $u$ and $T$ can be established as follows.

$$
\text { Setting } \quad u=1-i F \mathrm{~d} \lambda \text {, }
$$

where $F$ is also defined equally, if $u$ is unitary, $F$ will be Hermitian.

$$
\begin{gathered}
\varphi_{\alpha}^{\prime}=(1+i F \mathrm{~d} \lambda) \varphi_{\alpha}(1-i F \mathrm{~d} \lambda) \\
=\varphi_{\alpha}+i\left[F, \varphi_{\alpha}\right] \mathrm{d} \lambda . \\
T \varphi=i[F, \varphi] .
\end{gathered}
$$


The solution for $F$ can be recognized to be

$$
F=i \int \pi_{\alpha}(x) T_{\alpha \beta} \varphi_{\beta}(x) \mathrm{d}^{3} x .
$$

For finite variation of $\lambda$, we can write

$$
\varphi^{\prime}(x)=\mathrm{e}^{i T \lambda} \varphi(x)
$$

If the Lagrangian density $L$ is invariant under such a transformation, we see that $F$ will be a constant of motion. Defining,

$$
f_{\mu}(x)=\frac{\partial L}{\partial\left(\frac{\partial \varphi_{\alpha}(x)}{\partial x_{\mu}}\right)} \cdot T_{\alpha \beta} \varphi_{\beta}(x),
$$

this leads to the equation of continuity

$$
\sum_{\mu} \frac{\partial f_{\mu}}{\partial x_{\mu}}=0
$$

for $f_{\mu}$, the vectorial operator corresponding to $F$ which is consequently conserved.

We shall now consider a few applications of the above result.

The complex scalar field

Consider a non-Hermitian field $\varphi=\varphi_{1}+i \varphi_{2}$ where $\varphi_{1}$ and $\varphi_{2}$ are real fields. Putting $T_{\alpha \beta}=i \varepsilon \delta_{\alpha \beta}$ the conserved operator $F$ is

$$
F=i \varepsilon \int\left[\pi_{\alpha}(x) \varphi_{\alpha}(x)-\pi_{\alpha}^{\dagger}(x) \varphi_{\alpha}^{\dagger}(x)\right]
$$

Utilizing the expression for $\varphi_{\alpha}(x)$ and $\pi_{\alpha}(x)$, we get

$$
F=\varepsilon\left(n_{+}-n_{-}\right)
$$

where $n_{+}$and $n_{-}$are total numbers of particles and anti-particles in the field. Choosing the constant $\varepsilon$ properly, we can identify $F$ with the total charge of the field, i.e.

$$
Q=\varepsilon\left(n_{+}-n_{-}\right) \text {. }
$$

Calculating the corresponding vectorial quantity $f_{\mu}$, making use of the Lagrangian for the scalar field, we have

$$
f_{\mu}=-i \varepsilon\left[\frac{\partial \varphi^{\dagger}}{\partial x_{\mu}} \varphi-\frac{\partial \varphi}{\partial x_{\mu}} \varphi^{\dagger}\right]
$$

which we identify as the current.

The spinor field

In a similar way we calculate $f_{\mu}$ for a spinor field

$$
f_{\mu}=-\frac{\varepsilon}{2}\left[\bar{\psi}, i \gamma_{\mu} \psi\right]
$$

which is the current for Dirac field.

EPCR 11 
Thus imposing a phase transformation $\varphi \rightarrow \mathrm{e}^{i \varepsilon} \varphi$ leads to the existence of charge and current and invariance under the transformation assures the continuity equation. Hence this gauge is more appropriately termed the "charge gauge".

\section{The electromagnetic field ${ }^{1}$}

We know from classical electromagnetic theory that the electromagnetic field equations are invariant under the gauge transformations (first kind)

$$
A_{\mu}(x) \rightarrow A_{\mu}^{\prime}(x)=A_{\mu}(x)+\frac{\partial \chi(x)}{\partial x_{\mu}}
$$

$\chi(x)$ is arbitrary to the extent that it satisfies

$$
\square \chi(x)=0 \text {. }
$$

This transformation is a gauge transformation of the first kind as distinct from the gauge transformation of the second kind defined by extending the charge gauge, i.e.

$$
\varphi(x) \rightarrow \varphi^{\prime}(x)=\mathrm{e}^{i \varepsilon \lambda(x)} \varphi(x) .
$$

We find a remarkable feature now, that on demanding invariance of the total free Lagrangian density $L$ under both the gauge transformations, we are led to a unique interaction of the charged fields with the elctromagnetic field. We first notice that a term of the type $\left(\frac{\partial}{\partial x_{\mu}}-i \varepsilon A_{\mu}\right) \varphi$ stays invariant under both these
transformations, i.e.

$$
\begin{aligned}
\left(\frac{\partial}{\partial x_{\mu}}-i \varepsilon A_{\mu}^{\prime}\right) \varphi^{\prime}(x) & =\left\{\frac{\partial}{\partial x_{\mu}}-i \varepsilon\left(A_{\mu}+\frac{\partial \chi(x)}{\partial x}\right)\right\} \mathrm{e}^{i \varepsilon \chi(x)} \varphi(x) \\
& =\mathrm{e}^{i \varepsilon \chi}\left(\frac{\partial}{\partial x_{\mu}}-i \varepsilon A_{\mu}\right) \varphi(x) .
\end{aligned}
$$

However remembering that for the Lagrangian of the charged fields' (whose field variables are necessarily complex) to be hermitian, only combinations $\varphi^{\dagger} \varphi$ or $\frac{\partial \varphi^{\dagger}}{\partial x_{\mu}} \frac{\partial \varphi}{\partial x_{\mu}}$ can occur, we can easily convince ourselves that the exponential phase factor will always cancel. This suggests the prescription that in the free field Lagrangian we should replace $\partial / \partial x_{\mu}$ by $\frac{\partial}{\partial x_{\mu}}-i \varepsilon A_{\mu}$ and thereby obtain the Lagrangian in the presence of the field. We shall indicate this procedure in the case of the scalar field (charged),

$$
L=-\left[\frac{\partial \varphi^{\dagger}}{\partial x_{\mu}} \frac{\partial \varphi}{\partial x_{\mu}}+\mu^{2} \varphi^{\dagger} \varphi\right]
$$

1 C. N. Yang and R. L. Mills, Phys. Rev. 96, 191 (1954); R. Utiyama, Phys. Rev. 101, 1957 (1956). These papers deal with the general gauge transformations which are space dependent. From this theory it is possible to deduce the existence of the electromagnetic field. 
Applying our prescription and after a little rearrangement of terms, we get

where

$$
L_{F}=L-j_{\mu} A_{\mu}+\varepsilon^{2} A_{\mu}^{2} \varphi^{\dagger} \varphi
$$

$$
j_{\mu}=-i \varepsilon\left(\varphi^{\dagger} \frac{\partial \varphi}{\partial x_{\mu}}-\varphi \frac{\partial \varphi^{\dagger}}{\partial x_{\mu}}\right) .
$$

The last two terms represent the interaction Lagrangian $L_{\text {int }}$. (Figs. 23 a and 23 b.) It is interesting to verify the interaction of the electron with an external electromagnetic field by this method.

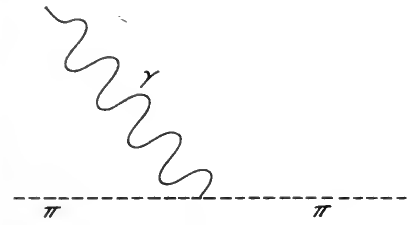

(a)

Fig. 23. (a) Current term;

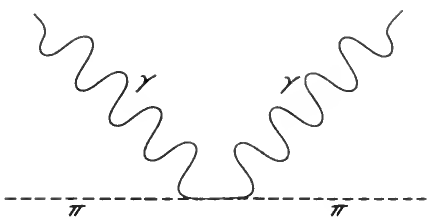

(b)

(b) "Catastrophic" term.

The spin operator

We shall now discuss continuous transformation associated with space-time. We recall the intrinsic variation of the fields under space-time rotations. An infinitesimal rotation of space-time is a particular case of the Lorentz transformation. The change in the fields due to this transformation can be split into two parts, the change connected with coordinate dependence and that connected with an intrinsic variation. We have already argued that $S$ is the matrix for intrinsic variation. Interpreting it as a gauge transformation we will obtain the spin operator.

Consider a change in the field at the same physical point $P$ under a space-time rotation. The coordinates $x_{\mu}^{\prime}$ in the new system after rotation are related to the coordinates $x_{\mu}$ in the original system by

$$
x_{\mu}^{\prime}=\sum_{\nu}\left(\delta_{\mu \nu}+\varepsilon_{\mu \nu}\right) x_{\nu}
$$

where $\varepsilon_{\mu \nu}$ is the Lorentz transformation matrix.

However for a non-scalar field with more than one component we can write

$$
\varphi_{\alpha}^{\prime}\left(x^{\prime}\right)=\sum_{\beta} S_{\alpha \beta} \varphi_{\beta}(x)=\sum_{\beta} S_{\alpha \beta} \varphi_{\beta}\left(x\left(x^{\prime}\right)\right) .
$$

The change in the functional form is obtained by not merely writing $x$ in terms of $x^{\prime}$ but also by operating $S_{\alpha \beta}$. If we now define

$$
S_{\alpha \beta}=\delta_{\alpha \beta}+\frac{1}{2} \sum_{\alpha \beta}^{\mu \nu} \varepsilon_{\mu \nu},
$$

where $\sum_{\alpha \beta}^{\mu \nu}$ is an operator defined by the above equations, then considering the intrinsic variation alone, the new $\varphi$ can be written as

$$
\varphi_{\alpha}^{\prime}\left(x^{\prime}\right)=\sum_{\beta}\left(\delta_{\alpha \beta}+\frac{1}{2} \sum_{\alpha \beta}^{\mu v} \varepsilon_{\mu \nu}\right) \varphi_{\beta}(x) .
$$


From (108) the corresponding observable is

$$
s_{\mu v}=\int \pi_{\alpha}(x) \sum_{\alpha \beta}^{\mu v} \varphi_{\beta}(x) \mathrm{d}^{3} x .
$$

We will now evaluate $s_{\mu \nu}$ for various fields. We have shown that under a Lorentz transformation $\psi(x)$ becomes $\psi^{\prime}\left(x^{\prime}\right)$ with $\psi^{\prime}\left(x^{\prime}\right)=S \psi(x)$ and the condition for covariance is

Substituting

$$
S^{-1} \gamma_{\mu} S=\sum_{\nu} a_{\mu \nu} \gamma_{\nu}
$$

we find

$$
S=1+\frac{1}{2} \sum^{\mu v} \varepsilon_{\mu \nu}
$$

$$
\Sigma^{\mu \nu}=\frac{1}{2} \gamma_{\mu} \gamma_{\nu}=\frac{1}{4}\left(\gamma_{\mu} \gamma_{\nu}-\gamma_{\nu} \gamma_{\mu}\right)
$$

Using the explicit representations of $\gamma_{\mu}, \mathrm{s}$,

$$
\Sigma^{\mu \nu}=\frac{i}{2} \sigma_{\lambda}, \quad \text { where } \quad \mu, \nu, \lambda=1,2,3
$$

Now

$$
s_{\mu \nu}=\frac{1}{2} \int \psi^{\dagger}(x) \sigma_{\mu \nu} \psi(x) \mathrm{d}^{3} x,
$$

which becomes in the non-relativistic limit (only large components of $\psi$ ),

$$
s=-\frac{1}{2} \int \psi^{\dagger}(x) \sigma \psi(x) \mathrm{d}^{3} x .
$$

Thus we have obtained the well known Pauli representations of spin $\sigma$ in the non-relativistic region.

Since by definition the scalar field transforms as

$$
\varphi^{\prime}\left(x^{\prime}\right)=\varphi(x),
$$

we note there is no intrinsic change for scalar or pseudoscalar field and hence their quanta are spinless. A vector field $\varphi_{\mu}$ transforms as the coordinates by definition, i.e.

We recognize that

$$
\varphi_{\mu}^{\prime}\left(x^{\prime}\right)=\sum_{\nu}\left(\delta_{\mu v}+\varepsilon_{\mu v}\right) \varphi_{\nu}(x) .
$$

Setting

$$
\sum_{\alpha \beta}^{\mu v}=\left(\delta_{\mu \alpha} \delta_{v \beta}-\delta_{\mu \beta} \delta_{v \alpha}\right) .
$$

$$
s_{\mu \nu}=s_{\lambda}(\lambda, \mu, v \quad \text { cyclic } 1,2,3) \text {, }
$$

we obtain

$$
\boldsymbol{s}=\int(\boldsymbol{\pi} \times \boldsymbol{\varphi}) \mathrm{d}^{3} x .
$$

We can identify $s_{1}, s_{2}, s_{3}$ to be the well known matrices for spin 1 (see chapter II·3). Thus the vector field has spin 1 .

Since we have already established the correspondence between spin and isotopic spin formalisms, it is clear that the above considerations can be carried over to rotations in isotopic spin space. Thus the nucleon field has two compo- 
nents (2 component Pauli spinor) i.e. proton and neutron and hence its isotopic spin vector is

$$
\boldsymbol{I}=-\frac{1}{2} \int \psi^{\dagger} \boldsymbol{\tau} \psi \mathrm{d}^{3} x
$$

where $\boldsymbol{\tau}$ is identical with $\sigma$. In particular for a rotation around the third axis

$$
I_{3}=-\frac{1}{2} \int \psi^{\dagger} \tau_{3} \psi \mathrm{d}^{3} x
$$

and will be conserved. The nucleon is thus an isospinor. Similarly we can treat the meson field which has three components (corresponding to two charged particles and a neutral particle $\pi^{ \pm}, \pi^{0}$ ) as a vector and obtain

$$
\boldsymbol{I}=\int(\boldsymbol{x} \times \boldsymbol{\varphi}) \mathrm{d}^{3} x .
$$

The isotopic spin accordingly is 1 , the $\pi$-meson is therefore an isovector. We are already aware that the importance of isotopic spin arises only when one introduces charge independence. Since charge independence of the interaction is equivalent to invariance of the interaction under any rotation in charge space the interaction should be a scalar in charge space. The scalar product of two vectors is obviously an invariant and hence we form the scalar product of the isotopic spin vector and the meson field which itself is an isovector. Thus the charge independent Yukawa interaction for a pseudoscalar meson field is

$$
=g \bar{\psi}\left(\tau_{1} \varphi_{1}+\tau_{2} \varphi_{2}\right) \psi+g \bar{\psi} \tau_{3} \varphi_{3} \psi
$$

or in terms of the observable $\varphi_{+}, \varphi_{-}, \varphi_{0}$

$$
=\gamma^{2} G \bar{\psi} \tau_{-} \varphi_{-} \psi+\gamma^{2} G \bar{\psi} \tau_{+} \varphi_{+} \psi+G \bar{\psi} \tau_{3} \varphi_{3} \psi
$$

which is the Kemmer Hamiltonian to be discussed later. Here

$$
\varphi_{+}=\frac{\varphi_{1}+i \varphi_{2}}{V^{2}} \quad \varphi_{-}=\frac{\varphi_{1}-i \varphi_{2}}{V^{2}} .
$$

We note that neutral mesons are oppositely coupled to the neutron and the proton, i.e. $G_{p}=-G_{n}$. From the expression it is also clear that the probability for emission or absorption of a charged meson is $(/ 2)^{2}$, i.e. twice the probability for the emission or absorption of a neutral meson.

The interaction of the charged particle with the electromagnetic field depends upon the charge and hence on $I_{3}$. The interaction is of the type

where

$$
H_{e \cdot m}=\int j_{\mu} A_{\mu} \mathrm{d}^{3} x
$$

$$
j_{\mu}=\frac{1}{2} e\left[\bar{\psi} \gamma_{\mu}\left(1+\tau_{3}\right) \psi\right],
$$

the term $\left(1+\tau_{3}\right)$ ensures that the neutron has no interaction with the electromagnetic field. The interaction behaves like the third component for rotations in the isotopic spin space. Thus though the interaction remains invariant under rotations about the third axis, i.e. it will conserve $I_{3}$ and hence the charge, it will 
not remain invariant under arbitrary rotations in charge space. Thus the total $I$-spin will not be a good quantum number.

Charge symmetry was originally introduced in nuclear forces to denote that the nuclear forces between two neutrons is the same as that between two protons. The operation implied here is changing a proton to a neutron or vice versa and charge symmetry is a consequence of invariance under this operation. By rotating through $\theta=\pi$ about the second axis in charge space we can perform this operation which we will denote by $T_{2}(\pi)$. Because of the identity in the operator relations between angular momentum and isotopic spin, this operator for a spinor is $\mathrm{e}^{i \tau_{2} \pi}$ and for $\varphi$ is $\mathrm{e}^{i s_{2} \pi}$ where

$$
s_{1}=\left[\begin{array}{ccc}
0 & 0 & 0 \\
0 & 0 & -i \\
0 & i & 0
\end{array}\right] ; \quad s_{2}=\left[\begin{array}{ccc}
0 & 0 & i \\
0 & 0 & 0 \\
i & 0 & 0
\end{array}\right] \quad s_{3}=\left[\begin{array}{ccc}
0 & -\sqrt{2} i & 0 \\
1 / 2 i & 0 & 0 \\
0 & 0 & 0
\end{array}\right] .
$$

Lee and Yang ${ }^{1}$ have defined an extended charge conjugation, i.e. $G$ operator, which is a product of charge symmetry and charge conjugation

Thus

$$
G=T_{2}(\pi) U_{c}
$$

and

$$
G\left[\begin{array}{l}
p \\
n
\end{array}\right] G^{-1}=\left[\begin{array}{l}
\bar{n} \\
\bar{p}
\end{array}\right]
$$

$$
G \varphi G^{-1}=-\varphi \text {. }
$$

$G$ anti-commutes with the heavy particle number operator, i.e. $\{G, N\}_{+}=0$. Also $[G S]_{+}=0, G^{2}=(-1)^{N+S},[G, I]=0$. It can be seen that $G$ is equivalent to reflection in isotopic spin space for pions. If interactions are invariant under $G$ then a state with even number of pions cannot go into odd number of pions.

1 T. D. LeE and C. N. YANG, Nuovo Cim. 3, 749 (1956).

${ }^{2}$ S. Weinberg, Phys. Rev. 112, 1375 (1958). 


\section{PION PHYSICS}

\section{I N'TR O D U CTI O N}

THE STUDY of quantum electrodynamics is considered so satisfactory that it has led to the belief that it has reached a state of completeness. This belief is perhaps well-grounded because of the validity of the perturbation expansion and the high accuracy attainable by taking only a few terms of the expansion in powers of $e^{2}$. The chief characteristic of the electromagnetic interaction is that it is linear in the photon field variable which means that only a single photon can be emitted or absorbed at a vertex. The concept of the basic interaction merely consists in assuming creation and annihilation of particles at a vertex, the number of such vertices being very small. The question arises whether these concepts can be carried over to the study of interactions of other types, e.g. the Yukawa interaction. It was, in fact, application of such considerations to the problem of nuclear forces that led Yukawa to predict the existence of the meson. ${ }^{1}$ But the situation here is rendered difficult since (as it turns out to be the case), the validity of the perturbation expansion is questionable and even granting it, the number of terms of the expansion to be taken which will lead to results in agreement with experiment is not known.

The various attempts to develop a meson theory can be broadly classified as follows:

(1) The weak-coupling approximation in which Feynman's methods in quantum electrodynamics are applied to meson problems.

(2) The strong coupling theory of Wentzel, Heisenberg and others.

(3) Phenomenological theories.

(4) Other theoretical methods for the treatment of strong interactions of which we shall be considering only Chew's approach in detail. But before discussing these various approaches, it would be interesting to study

(i) the properties of the meson, and

(ii) certain striking features of the meson-nucleon interaction in the low energy region.

\section{Properties of the $\boldsymbol{x}$-meson}

The existence of a particle with mass approximately 300 times that of the electron was postulated by Yukawa in an attempt to explain the nuclear forces of very short range and great strength as arising due to the exchange of such

1 H. Yukawa, Proc. Phys. Math. Soc. Japan 17, 48 (1935). 
a particle between the nucleons. Since the proton is positively charged and the neutron uncharged three types of particles can be exchanged, positively, neutrally and negatively charged particles, $\pi^{+}, \pi^{0}$ and $\pi^{-}$respectively. Their existence was confirmed experimentally in cosmic rays $^{1}$ and later they were copiously produced in the laboratory in nucleon-nucleus collisions in high energy accelerators. The fact that $\pi^{+}$and $\pi^{-}$are singly charged is verified by reactions like

$$
\begin{aligned}
& \pi^{-}+p \rightarrow n+\gamma \\
& \pi^{+}+D \rightarrow 2 p .
\end{aligned}
$$

Both the charged and neutral pions are unstable particles with lifetimes which can be determined by studying their decays. The charged pion decays into the lighter particle, the $\mu$-meson and a neutrino or an electron and a neutrino

$$
\begin{aligned}
& \pi^{+} \rightarrow \mu^{+}+v \\
& \pi^{-} \rightarrow \mu^{-}+\bar{v} \\
& \pi^{+} \rightarrow e^{+}+v \\
& \pi^{-} \rightarrow e^{-}+\bar{v} .
\end{aligned}
$$

The $\mu$-mesons unlike the $\pi$ do not have strong interactions with matter but decay according to the scheme

$$
\mu^{ \pm} \rightarrow e^{ \pm}+v+\bar{v}
$$

Originally the $\mu$-meson was mistaken to be the Yukawa particle but since it was found that it did not interact with the nuclei of matter, further search led to the discovery of the $\pi$-meson, through its decay into the $\mu$-meson, in experiments in cosmic ray process. $(2 \mathrm{~b})$ has a branching ratio of $(1.2 \pm 0.3) \times 10^{-4}$ as compared to a value $\sim 100$ for process $(2 \mathrm{a})$. The lifetime of the charged pion is therefore determined by process $2(\mathrm{a})$ and is found to be $(2.55 \pm 0.05) \times 10^{-8}$ sec.

The interaction which causes the decay is "weak" compared to the electromagnetic and more so with respect to the "strong" nuclear reactions and has been the subject of considerable theoretical study, as we shall see later (chapter VII). Process (2a) is understood as going through two virtual processes-a strong process in which the pion goes over into a nucleon-anti-nucleon pair and a weak one in which this pair is connected to the final system of a $\mu$-meson and a neutrino by a Fermi interaction. On this view point, the small branching ratio of $(2 \mathrm{~b})$ is explicable and a satisfactory quantitative explanation of this problem has been given recently (see, chapter VII).

The neutral pion decays very fast by means of the electromagnetic processes

$$
\begin{aligned}
& \pi^{0} \rightarrow \gamma^{+} \gamma \\
& \pi^{0} \rightarrow e^{+}+e^{-}+\gamma .
\end{aligned}
$$

The branching ratios for processes $(4 \mathrm{a})$ and $(4 \mathrm{~b})$ are 98.8 and 1.2 respectively. It is through the observation of the secondary electron-positron pair produced

1 C. M. G. LatTes et al., Nature Lond. 160, 453, 486 (1947). 
by the $\gamma$-rays in reaction 4 (a) or the pair produced in reaction 4 (b) that the lifetime of the neutral pion is determined. It is estimated to be less than $4 \times 10^{-16} \mathrm{sec}$. Here again the decay process is understood to go in two stages, a strong reaction followed by an electromagnetic one.

The masses of $\pi^{+}$and $\tau^{-}$have been found to be equal, within experimental errors, the value being $\sim 273.3 m_{e}$ (where $m_{e}$ is the mass of the electron). The existence of $\pi^{0}$ is inferred from its decay into two $\gamma$-rays which can be observed by the electron-positron pairs they produce as mentioned earlier. The direct determination of the mass of $\pi^{0}$ is obviously difficult. An indirect, but accurate measurement is based on the reaction

$$
\pi^{-}+p \rightarrow n+\pi^{0}
$$

where the mass difference between $\pi^{-}$and $\pi^{0}$ is measured. Neglecting the kinetic energy of $\pi^{-}$in comparison with the neutron proton mass difference, a simple calculation yields the $\pi^{0}-\pi^{-}$mass difference which is determined by observing the energy distribution of the $\gamma$-rays. This small difference of about $5 \mathrm{MeV}$ is amplified by Doppler effect into a large energy spread for the $\gamma$-rays of about $30 \mathrm{MeV}$.

The spin of the charged pion can be deduced from the reaction

$$
p+p \leftrightarrow D+\pi^{+}
$$

By the principle of detailed balance the forward and backward reactions differ only by statistical weights and it can be shown that

$$
\frac{\sigma\left(\text { production of } \pi^{+}\right)}{\sigma\left(\text { absorption of } \pi^{+}\right)}=\frac{3(2 S+1) k^{2}}{2 K^{2}}
$$

where $k$ and $K$ are the meson and proton momenta respectively in the c.m. system and $S$ is the spin of the pion. Experiments performed at the same energy and scattering angle in both cases give a value $S=0$.

The experimentally observed fact that the neutral pion decays into two photons (and not three) immediately determines the spin of the $\pi^{0}$. If we assume the $\pi^{0}$ to have a spin equal to 1 , then in its rest system, its wave function must transform like a vector which determines the spin direction. The final wave function of the two photons involves three vectors $\varepsilon_{1}$ and $\varepsilon_{2}$ the two polarizations, and $\boldsymbol{k}$ the relative momentum. The wave function must be bilinear in $\varepsilon_{1}$ and $\varepsilon_{2}$ and symmetric with respect to the interchange of the two photons (since they are bosons). Further, because of the transversality condition we have $\varepsilon_{1} \cdot \boldsymbol{k}=\varepsilon_{2} \cdot \boldsymbol{k}=0$. Now the only vectors bilinear in $\varepsilon_{1}$ and $\varepsilon_{2}$ that we can construct are:

(i) $\varepsilon_{1} \times \varepsilon_{2}$

(ii) $\left(\varepsilon_{1} \cdot \varepsilon_{2}\right) \boldsymbol{k}$

(iii) $\boldsymbol{k} \times\left(\varepsilon_{1} \times \varepsilon_{2}\right)=\varepsilon_{1}\left(\boldsymbol{k} \cdot \varepsilon_{2}\right)-\varepsilon_{2}\left(\boldsymbol{k} \cdot \varepsilon_{1}\right)$

(i) and (ii) are excluded since both change sign when the photons are interchanged and (iii) vanishes identically because of the transversality condition. Thus we find that a particle with spin 1 can never decay into two photons. 
If, on the other hand, the $\pi^{0}$ has zero spin, its wave function is scalar or pseudoscalar depending on its parity. We can now form two possible final wave functions

(iv) $\varepsilon_{1} \cdot \varepsilon_{2}$

(v) $\left(\varepsilon_{1} \times \varepsilon_{2}\right) \cdot \boldsymbol{k}$

This decay is thus possible and if we exclude spins greater than one (which is also necessitated on grounds of renormalizability) we arrive at the conclusion that the $\pi^{0}$ has zero spin.

Under a space reflection $\boldsymbol{k}$ will change sign. Hence (iv) is a scalar and (v) is a pseudoscalar. This shows that the decay photons of a scalar meson have parallel polarizations while a pseudoscalar pion decays into photons which are perpendicularly polarized. Thus a determination of the polarization of the decay photons determines the intrinsic parity of $\pi^{0}$ which is found to be odd (with respect to the nucleon).

The invariance of the interaction of the pion with nucleons under the parity operation (space reflection) can be made to yield the parity of the $\pi^{-}$-meson with respect to the nucleon (the parity of which is assumed to be even). Let us consider the process

$$
\pi^{-}+D \rightarrow n+n
$$

We know that the processes

$$
\pi^{+}+D \leftrightarrow p+p
$$

have the same matrix element, by the principle of detailed balance and therefore, as before, the ratio of cross-sections will contain a weight factor $(2 S+1)=1$ since $S=0$ for the pion. The capture of $\pi^{-}$takes place mainly from the $1 S$ orbit since the $1 S$ wave function of the $\pi^{-}$is almost "inside" the nucleus. Thus the initial system in 9 (a) has $J=1$ and its parity is the same as that of the relative parity of $\pi^{-}$with respect to the nucleons. The only state of the two neutrons with $J=1$ (which obeys the Pauli principle) is the $3 P_{1}$ state which has odd parity. Assuming that $n$ and $p$ have even relative parity, we can deduce from parity conservation that $\pi^{-}$-meson has odd (intrinsic) parity. By charge independence, $\pi^{+}$and $\pi^{0}$ should also have odd intrinsic parity.

\section{The resonances in the low energy pion-nucleon interaction}

We next consider the most striking feature of the pion-nucleon interaction as well as that of the interaction of the electromagnetic field with the pion-nucleon system, viz. the occurrence of resonances in the total cross-sections for the scattering of pions by nucleons and the photo production of mesons from nucleons. The interaction of charged mesons with protons can proceed in the following ways

$$
\begin{aligned}
& \pi^{+}+p \rightarrow \pi^{+}+p \\
& \pi^{-}+p \rightarrow \pi^{-}+p \\
& \pi^{-}+p \rightarrow \pi^{0}+n \\
& \pi^{-}+p \rightarrow n+\gamma
\end{aligned}
$$


We shall not deal with (IV) as its cross-section is known to be very low. For processes (I) to (III), experiments show that in the region around $100 \mathrm{MeV}$ (kinetic energy of the pion in the c.m.s.) there is a rapid rise in the total crosssections culminating in a dominant peak at about $200 \mathrm{MeV}$, indicating a resonance. The association of a 33 state (where the Fermi notation has been used, the first number referring to twice the total isotopic spin and the second to twice the total angular momentum of the state) with this resonance can be argued out on the basis of the odd intrinsic parity of the pion and the requirements of charge independence. Recent experiments on the scattering of $\pi^{-}$and $\pi^{+}$by hydrogen at energies from 400 to $1200 \mathrm{MeV}$ reveal (see Fig. 24) that the $\pi^{+}$crosssection falls off above the 33 resonance and has a minimum at about $650 \mathrm{MeV}$ and then steadily rises ${ }^{1}$. The $\pi^{-}$cross-section, on the other hand shows two clear peaks at $600-650 \mathrm{MeV}$ and at about $900 \mathrm{MeV}$. This fact and the large polarization of the recoil protons observed at $700 \mathrm{MeV}$ (which means a strong interference between states of opposite parity) lead one to characterize the first of these states as a 13 state, the parity of this state being odd (a $D$-state). A further maximum has also been observed at about $1.5 \mathrm{BeV}$ in the $\pi^{+}$cross-section. The differential elastic coss-section throghout the energy range considered is symmetric about $\theta=90^{\circ}$, having peaks in both the forward and backward directions.

The data on the two photo-production processes

$$
\gamma+p \rightarrow\left\{\begin{array}{l}
\pi^{+}+n \\
\pi^{0}+p
\end{array}\right.
$$

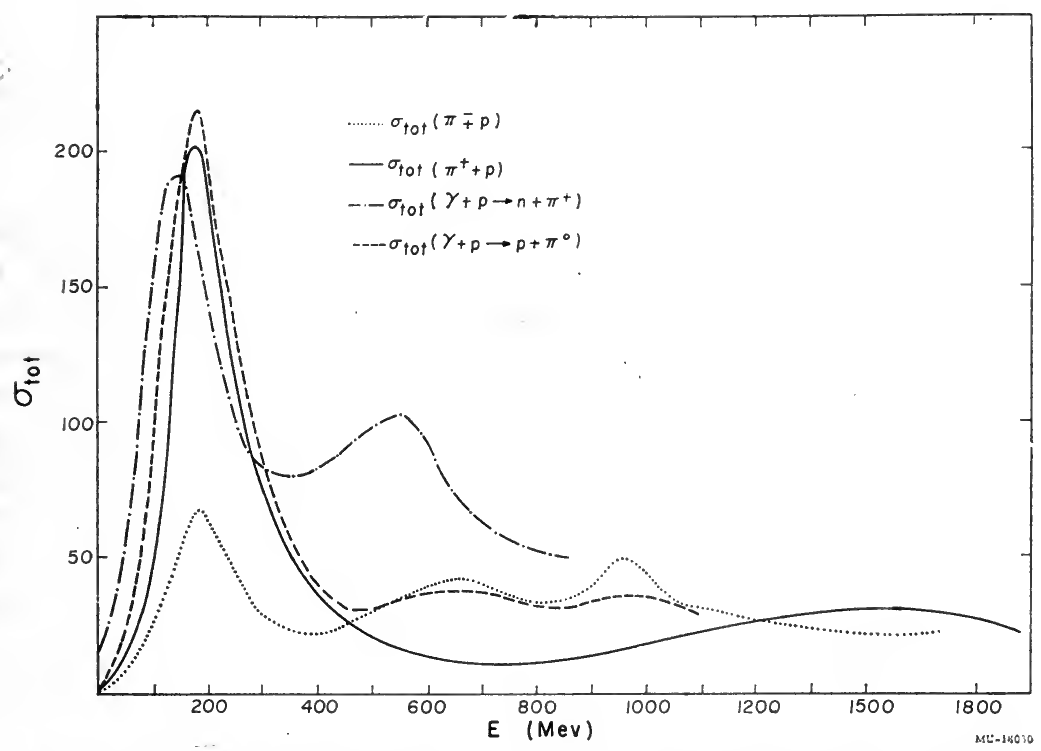

Fig. 24. Total cross-section for the scattering and photo production of pions.

1 R. F. PeIerls, Phys. Rev. 118, 325 (1960). 
are given in Fig. (24). In addition to the (33) resonance, there is a second maximum at an energy somewhat below that corresponding to the peak in $\pi^{-}$scattering and apparently rising towards a third peak at $1100 \mathrm{MeV}$ photon energy. The total cross-section for production of $\pi^{0}$ has a small second peak at an energy corresponding to the scattering peak and another small peak at about $1100 \mathrm{MeV}$.

The above are only approximate representations of the experimental data, some of which, particularly at higher energies, have poor statistical accuracy. Thus we can roughly summarize the different resonant states (considered as the excited states of the nucleon) in the following table.

\begin{tabular}{c|c|c|c}
\hline Energy (MeV) & $I$ & $J$ & $\mathrm{P}$ (= parity) \\
\hline 1300 & $3 / 2$ & $5 / 2$ & $?$ \\
900 & $1 / 2$ & $5 / 2$ & $?$ \\
600 & $1 / 2$ & $3 / 2$ & - \\
200 & $3 / 2$ & $3 / 2$ & + \\
0 & $1 / 2$ & $1 / 2$ & + \\
\hline
\end{tabular}

As we shall see presently there is a good theoretical explanation for the (33) resonance, the shape and position of which can be fitted with the experimental data by the introduction of two parameters. The same can hardly be said of the other resonances. Some attempts have been made to explain the second and third resonances on the basis of the existence of resonances in the pion-pion interaction $^{1}$ (see below). We shall be considering only the (33) resonance.

\section{EARLIER ATTEMPTS AND THEORIES}

\section{Weak coupling theory ${ }^{2}$}

This theory is a simple minded extension of Feynman's methods which lead to such excellent experimental agreement in electromagnetic processes, to the interaction of the mesons with the nucleons. As mentioned earlier because of the largeness of the coupling constant (in this case $g^{2} \approx 15$ ), we do not expect to get results agreeing with the experiments. The lowest order Feynman diagrams for the scattering processes (I) to (III) (equation (10), sec. 1) are shown in Fig. (25). It is readily seen that since a proton can emit but not absorb a $\pi^{+}$and similarly a neutron can absorb but not emit a $\pi^{+}$, only diagram 25 (a) contributes to the process (I) (equation (10), sec. 1) and only diagram 25 (b) to the process (II). Both the diagrams contribute to the charge-exchange scattering process (III). Evaluating the matrix elements in the usual way, squaring, averaging over the spin of the initial nucleon and summing over the spin of the final nucleon, the differential cross-section in the laboratory system can be found. We give the

1 See e.g. K. ItTabashi et al., I.N.S. Report 14.

2 See R. E. Marshak, Meson Physics, McGraw-Hill, New York (1952). 
results for only the total cross-section which can be obtained by integrating the differential cross-sections. For the extreme relativistic case and for pseudoscalar coupling between the meson and the nucleon we get

$$
\begin{aligned}
\sigma_{I} & =\frac{\pi g^{4}}{2 m \varepsilon_{1}} \log \left(\frac{2 \varepsilon_{1}}{m}\right) \\
\sigma_{I I} & =\frac{\pi g^{4}}{4 m \varepsilon_{1}} \\
\sigma_{I I I} & =\frac{\pi g^{2} g_{p}^{2}}{2 m \varepsilon_{1}} \log \left(\frac{2 \varepsilon_{1}}{m}\right) .
\end{aligned}
$$

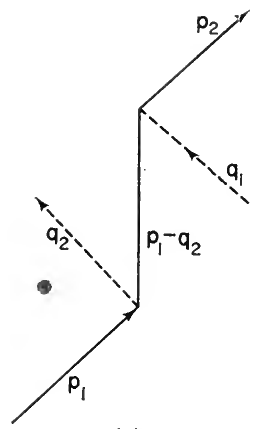

(a)

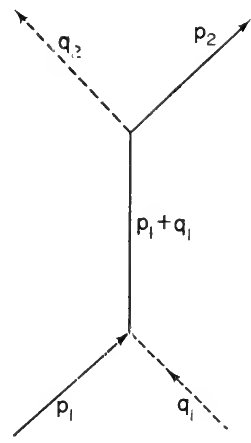

(b)

Fig. 25. Meson-nucleon scattering.

For pseudovector coupling the expressions are

$$
\begin{aligned}
\sigma_{I} & =\pi\left(\frac{\dot{f}}{\mu}\right)^{4} m \varepsilon_{1} \\
\sigma_{I I} & =\frac{\pi f^{4} m \varepsilon_{1}}{\mu^{4}} \\
\sigma_{I I I} & =\frac{\pi f^{2}\left(f_{n}-f_{p}\right)^{2} m \varepsilon_{1}}{\mu^{4}} .
\end{aligned}
$$

Here $g$ and $f$ are the pseudoscalar and pseudovector coupling constants of the charged pion with the nucleon $g_{n}$ and $g_{p}$, and $f_{n}$ and $f_{p}$ are the coupling constants of the $\pi^{0}$ with the neutron and proton respectively. $\mu$ is the mass and $\varepsilon_{1}$ the energy of the incident pion and $m$ the nucleon mass. The pseudoscalar coupling gives an essentially constant cross-section as the energy of the pion increases while the pseudovector coupling gives a continuous increase of cross-section with energy, both of which are in contradiction with experiment.

The ratio between the $\pi^{+}$and $\pi^{-}$direct scattering is about 1.5 for $p s$ coupling and 1 for $p v$ coupling at $50-100 \mathrm{MeV}$. Experimentally, the ratio is about 5 at $60 \mathrm{MeV}$. Further the ratio of the charge exchange to direct scattering is not correctly predicted by the weak coupling theory for either coupling. 
Turning now to the application of the weak coupling theory to the photo production of a meson from a nucleon, we note that the following types of diagrams enter the calculation:

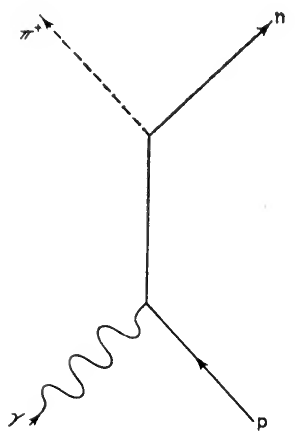

(a)

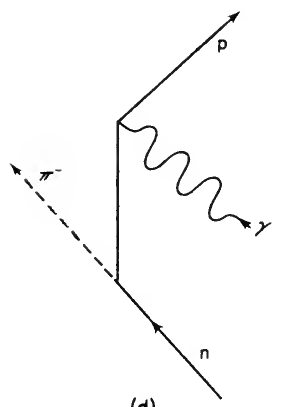

(d)

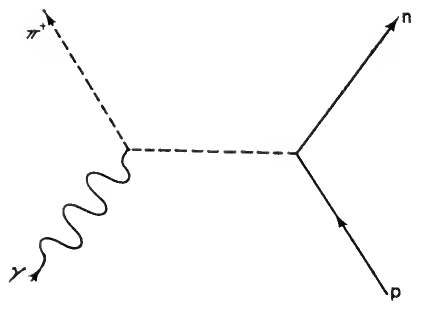

(b)

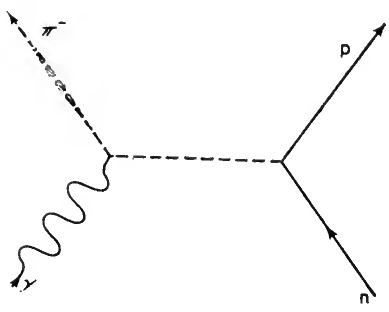

(e)

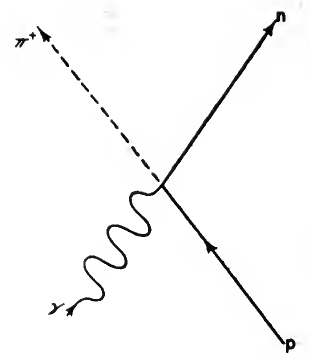

(c)

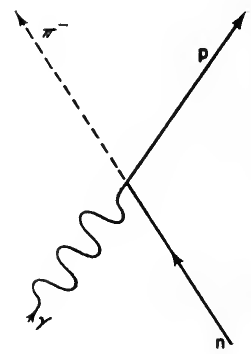

(f)

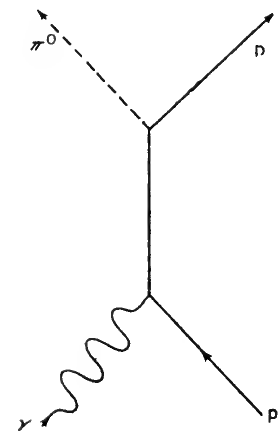

(g)

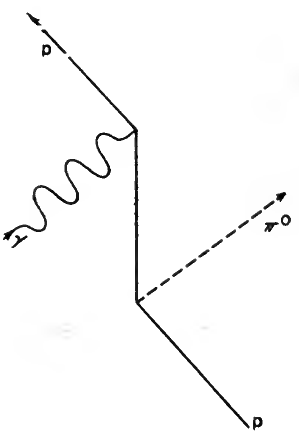

(h)

FIG. 26. Photo production of pions. 
Figs. 26 (a) to (h), contain the relevant diagrams for the production of $\pi^{+}, \pi^{-}$and $\pi^{0}$ as indicated. (The anomalous magnetic moments of the nucleons are neglected.) For obvious reasons, Figs. (a), (d), (g) and (h) may be called "shaking off" transitions, Figs. (b) and (e) "photoelectric" transitions and Figs. (c) and (f) "catastrophic" transitions. It may be noticed that neither the "photoelectric" nor the "catastrophic" transition is present in the case of $\pi^{0}$ production since it is neutral. At moderate energies the two contributions from the two diagrams (g) and (h) just about cancel each other so that we should expect a small cross-section for $\pi^{0}$ production. Also $\pi^{0}$ should not be produced from neutrons. Both these results disagree with experiment. At about $300 \mathrm{MeV}$ the cross-sections for neutral and charged mesons are of the same order of magnitude (Fig. 24), the peak for $\pi^{0}$ being in fact slightly higher. Kaplon ${ }^{1}$ tried to improve this state of affairs by including the interaction of the anomalous magnetic moment of the nucleon with the photon. He did get a better yield for $\pi^{0}$ but the angular distributions of the mesons predicted by the earlier weak coupling theory as well as Kaplon's improved version are found to be in disagreement with experiment. Thus, as expected from the beginning, the weak coupling theory is not able to give correct results for meson interactions.

\section{Strong coupling theory}

Realizing the large value of the coupling constant even during the early development of the meson theory, Heisenberg ${ }^{2}$, Wentzel ${ }^{3}$ and others ${ }^{4}$ assumed that many virtual mesons will be closely associated with the nucleon (unlike in the weak coupling theory where only a single meson is so associated) and therefore it is permissible to treat the nucleon classically. At that time, the classical approach was the only one possible as the technique of renormalization and the consequent. solution of the self-energy problems were not available. The only way then to avoid the difficulties due to divergences was to postulate a fixed extended source. Meson scattering was considered as a small perturbation of the intrinsic field of the nucleon. The calculation of the cross-section using the strong limit $\left[\right.$ viz. $\frac{1}{a} \gg \mu$ with the meson energy $\omega \ll \frac{1}{a}$, where $\frac{1}{a}=\frac{2}{\pi} \int|v(k)|^{2} \mathrm{~d} k$ and $v(k)$ is the Fourier transform of the source distribution function] leads to a resonance denominator $\frac{1}{\left[1-\left(\frac{\omega}{\varepsilon}\right)^{2}\right]^{2}+\left(\frac{2 a k^{3}}{\varepsilon \omega}\right)^{2}}$ in the sense that at $\omega=\varepsilon$ the cross-section becomes a maximum. Here $\varepsilon=\frac{6 \pi a \mu^{2}}{f_{r}^{02}}$ where $f_{r}^{0}$ is the pseudovector rationalized unrenormalized

1 M. F. Kaplon, Phys. Rev. 83, 712 (1951).

2 W. Heisenberg, Z. Phys. 113, 61 (1939).

${ }^{3}$ G. Wentzel, Helv. Phys. Acta 13, 269 (1940).

4 W. Pauli, Meson Theory of Nuclear Forces, Interscience Publishers, New York (1946). 
coupling constant. Thus the strong coupling theory predicts the characteristic resonance in the meson-nucleon scattering. But the most important feature of the theory is the prediction of excited or "isobaric" states of the nucleon the first of which will be the $I=J=3 / 2$ state. The ground state i.e. the state of the single nucleon has $J=1 / 2$. The excitation energy is

$$
E=2 \varepsilon(J+3 / 2)(J-1 / 2) .
$$

Now if the isobar is to be stable against the emission of a meson, for the $J=3 / 2$ state we should have $6 \varepsilon<\mu$ whereas for resonance $\varepsilon>\mu$. Thus the existence of a resonance rules out the possibility of a bound (stable) isobaric state.

Though the strong coupling theory predicts one of the most striking features of low energy pion-nucleon interaction it can be objected to on the grounds that (i) the treatment of the meson cloud classically is not permissible since the number of mesons of moderate momentum is really not large (because of the small value of the pseudovector coupling constant $f^{2} \approx 0.1$ ) (ii) the absence of renormalization, which eliminates self energy effects which in turn play a decisive role in the strong coupling theory is a serious defect and (iii) the theory cannot handle problems of the production of additional mesons. We shall later see that Chew's theory which also uses the concept of a fixed extended source meets all these objections.

\section{Phenomenological theories}

It has been emphasized that perturbation methods are inapplicable to interactions involving strong forces which exist in the case of the meson-nucleon system. Therefore, phenomenological theories were attempted to explain the main features of meson-nucleon interactions. A particularly successful one is Brueckner's theory of meson-nucleon scattering and photo-meson production which satisfactorily explains the main features at low energies. ${ }^{1}$

Brueckner made the important assumption that the scattering amplitude depends only on the total isotopic spin $I$ of the meson-nucleon system and not on $I_{z}$. On this assumption the scattering process is completely described by the two amplitudes $a\left(\frac{3}{2}\right)$ and $a\left(\frac{1}{2}\right)$ corresponding to the two isotopic spin states of the meson-nucleon system, namely, $I=3 / 2$ and $I=1 / 2$. Of course, the scattering depends also on the angular momentum quantum number $J$ which we shall consider a little later. For the present, we shall analyse the dependence of scattering on the isotopic spin state of the system.

In any process in which the scattering or the collision cross-section depends only on the isotopic spin states, the general procedure for calculating the crosssection is as follows. The initial system of particles is to be expressed as a linear combination of definite isotopic spin states since the amplitudes of the scattering

1 K. A.Brueckner, Phys. Rev. 80, 106 (1952); also "Mesons and fields" Bethe and de Hoffmann, see Vol. II, Row Peterson (1955). 
are known for the isotopic spin states. This linear combination of isotopic spin states is re-expressed in terms of the possible final systems of particles. This final system of particles may in general be different from the incident system. Thus the amplitude of scattering for any particular process is expressed in terms of the total isotopic spin scattering amplitudes (see equations 4-6).

The $\pi^{+}-p$ system $\left(I_{z}=3 / 2\right)$ can exist only in the $I=3 / 2$ state; the corresponding normalized wave function is given by $\eta_{p} \pi^{+}$. However the $\pi^{-}+p$ or $\pi^{0}+n$ system for which $I_{z}=-1 / 2$ can exist both in the $I=3 / 2$ and $I=1 / 2$. states.

$$
\begin{aligned}
\eta_{p} \pi^{-} & =V^{1 / 3} \Phi(3 / 2)+V^{2} /{ }_{3} \Phi(1 / 2) \\
\eta_{n} \pi^{0} & =V^{2} /{ }_{3} \Phi(3 / 2)+V^{1} /{ }_{3} \Phi(1 / 2) .
\end{aligned}
$$

Conversely the two isotopic spin states $\Phi(3 / 2)$ and $\Phi\left(\frac{1}{2}\right)$ can be expressed as linear combinations of the wave functions of $\left(p+\pi^{-}\right)$and $\left(n+\pi^{0}\right)$ systems.

$$
\begin{aligned}
& \Phi\left({ }^{3} /{ }_{2}\right)=V^{1} /{ }_{3} \eta_{p} \pi^{-}+V^{2} /{ }_{3} \eta_{n} \pi^{0} \\
& \Phi\left({ }^{1} / 2\right)=V^{2} /{ }_{3} \eta_{p} \pi^{-}-V^{1} /{ }_{3} \eta_{n} \pi^{0}
\end{aligned}
$$

Let $a\left({ }^{3} / 2\right)$ and $a(1 / 2)$ be the scattering amplitudes for the two isotopic spín states $I=3 / 2$ and $I=1 / 2$. Then the amplitudes for the processes (I), (II) and (III) (section 1 equation 10) are given by

$$
\begin{aligned}
& p \pi^{+} \rightarrow p \pi^{+1 / 3} a(3 / 2) \\
& p \pi^{-} \rightarrow p \pi^{-1 / 3}[a(3 / 2)+2 a(1 / 2)] \\
& p \pi^{-} \rightarrow n \pi^{01 / 3} V^{2} / 3[a(3 / 2)-a(1 / 2)] .
\end{aligned}
$$

We shall now consider the cross-sections of the above processes under three particular assumptions.

(a) Let

Then

(b) Let

Then

(c) Let

Then

$$
\begin{aligned}
a(1 / 2)=0, & a(3 / 2) \neq 0 \\
\sigma_{I}: \sigma_{I I}: \sigma_{I I I} & =9: 1: 2 \\
a(1 / 2) & =a(3 / 2) \\
\sigma_{I}: \sigma_{I I}: \sigma_{I I I} & =1: 1: 0 \\
a(1 / 2) & \neq 0, \quad a(3 / 2)=0
\end{aligned}
$$

$$
\sigma_{I}: \sigma_{I I}: \sigma_{I I I}=0: 2: 1
$$

The experimental values at $120 \mathrm{MeV}$ and $144 \mathrm{MeV}$ closely conform to the ratio $9: 1: 2 .^{1}$ Thus we are led to the conclusion that at least in the $100-150 \mathrm{MeV}$ range, the scattering takes place predominantly in the $I=3 / 2$ state.

For a complete analysis of scattering we have to consider, besides the isotopic spin states, the angular momentum states of the pion-nucleon system. In the low (1954).

1 H. L. Anderson et al., Phys. Rev. 91, 155 (1953); J. Orear et al., Phys. Rev. 95, 624 EPCR 12 
energy region, it is reasonable to suppose that the mesons are scattered mainly in the $S$ and $P$ states. Since there are two isotopic spin states $I=1 / 2$ and $I=3 / 2$ and three angular momentum states $S_{1 / 2}, P_{1 / 2}$ and $P_{3 / 2}$ for the pion-nucleon system, the low energy scattering can be completely described in terms of six scattering amplitudes or alternatively by six phase shifts. We shall denote the $S$-wave phase shifts for $I=3 / 2$ and $I=1 / 2$ states by $\delta_{3}$ and $\delta_{1}$ and the $p$ wave phase-shifts by $\delta_{33}, \delta_{31}, \delta_{13}$, and $\delta_{11}$.

In the case of scattering of $\pi^{+}$by protons, only the isotopic spin state $I=3 / 2$ is involved. The total cross-section increases with energy and shows a resonance at about $200 \mathrm{MeV}$. This indicates that one angular momentum state predominates over all the other states. The measured angular distribution of $\pi^{+}$ which can be expressed in the form

$$
\frac{\mathrm{d} \sigma}{\mathrm{d} \Omega}=A+B \cos \theta+C \cos ^{2} \theta
$$

has been found to have a large term proportional to $\cos ^{2} \theta$. In order to obtain such a term from a single state $J$ we must have $j \geqq 1$. In this case only $P_{3 / 2}$ is such a state. The other states also contribute though not to the same extent. The $\cos \theta$ term indicates an interference of $S$ - and $P$-waves.

The differential cross-sections for the three processes involving scattering by protons can be written as

$$
\begin{aligned}
& \frac{\mathrm{d} \sigma}{\mathrm{d} \Omega}\left(\pi^{+} \rightarrow \pi^{+}\right)=A_{+}+B_{+} \cos \theta+C_{+} \cos ^{2} \theta \\
& \frac{\mathrm{d} \sigma}{\mathrm{d} \Omega}\left(\pi^{-} \rightarrow \pi^{-}\right)=A_{-}+B_{-} \cos \theta+C_{-} \cos ^{2} \theta \\
& \frac{\mathrm{d} \sigma}{\mathrm{d} \Omega}\left(\pi^{-} \rightarrow \pi^{0}\right)=A_{0}+B_{0} \cos \theta+C_{0} \cos ^{2} \theta
\end{aligned}
$$

$A, B, C$ can be expressed in terms of the phase shifts $\delta_{3}, \delta_{1}, \delta_{33}, \delta_{31}, \delta_{13}$, and $\delta_{11}$. From the phase shift analysis, Fermi ${ }^{1}$ noticed that the absolute sign of the phase shifts is not fixed. What is determined is only the relative sign. If the signs of all the phase shifts were to be reversed, even then we get the computed crosssection. The absolute sign of the phase shifts can be determined from very low energy scattering data since in this region, there is an interference of nuclear scattering with Coulomb scattering, the absolute sign of which is known. ${ }^{2}$

Besides the ambiguity of the sign of the phase shifts, there exists another ambiguity which was first observed by Yang. ${ }^{3}$ This corresponds to the reversal of the sign of $\left(\delta_{31}-\delta_{33}\right)$ and also fits the experimental data equally well. We give

1 E. Fermi et al., Phys. Rev. 95, 1581 (1954).

2 J. Orear, Phys. Rev. 96, 1417 (1954).

3 C. N. YANG (Private communication to E. Fermi), see Bethe et al., loc. cit., p. 72. 
below the Fermi and Yang solutions of the meson-proton scattering phase shifts in degrees.

Fermi solution

\begin{tabular}{l|rr|rr|rr}
\hline Energy & \multicolumn{2}{|c|}{$S$} & \multicolumn{2}{|c|}{$P_{3 / 2}$} & \multicolumn{2}{c}{$P_{1 / 2}$} \\
\cline { 2 - 7 } MeV & $I=3 / 2$ & $1 / 2$ & $3 / 2$ & $1 / 2$ & $3 / 2$ & $1 / 2$ \\
& $\delta_{3}$ & $\delta_{1}$ & $\delta_{33}$ & $\delta_{13}$ & $\delta_{31}$ & $\delta_{11}$ \\
\hline 120 & -15.2 & 9.0 & 29.6 & 3.9 & 1.8 & -2.8 \\
135 & -14.0 & 10.3 & 37.9 & 2.0 & 5.4 & -4.6 \\
\hline
\end{tabular}

Yang solution

\begin{tabular}{l|rr|rr|rr}
\hline 120 & -15.4 & 9.1 & 12.9 & -1.4 & 38.6 & 3.8 \\
135 & -14.2 & 10.4 & 17.2 & -2.9 & 49.3 & 5.6 \\
\hline
\end{tabular}

The Fermi solution gives a large phase shift for $\delta_{33}$ which as we have seen earlier, is responsible for the resonance in $\pi^{+}-p$ scattering. On the other hand the Yang solution gives a large scattering in the $P_{1 / 2}$ state and there is no way of explaining such a large phase shift for $\delta_{31}$. The Yang solution is less reasonable and can be ruled out experimentally by measuring the polarization of the recoil nucleon. The Fermi-Yang ambiguity can also be resolved theoretically by the application of dispersion relations. ${ }^{1}$ The pion-proton scattering cross-section is of the form $(A+B \cos \theta)^{2}+C \sin ^{2} \theta$ where the $\sin ^{2} \theta$ term corresponds to spin-flip, while the other term corresponds to no-spin flip. For forward scattering, $\sin ^{2} \theta=0$ and the dispersion relation will have no term corresponding to spin-flip. If we differentiate the dispersion relation with respect to the scattering angle to obtain the rate of change of amplitude in the forward direction, then it is sensitive to spin-flip. Further, the derivative dispersion relation will not involve $S$-wave phase shifts so that the scattering amplitude should be a function of only the energy and the $P$-wave phase-shifts. It can be written as a term proportional to $f^{2}$ plus a rapidly convergent integral. In a graphical representation, while the Fermi phase-shifts form a linear plot (as required by theory) and intersect the axis at a point giving a value $f^{2}=0.1$ for the pseudovector coupling constant (see next section) which is in excellent agreement with the value obtained by other methods, the Yang phase shifts lead to an erratic behaviour with a negative value for $f^{2}$. Thus the application of derivative dispersion relations rules out quite conclusively the Yang set of phase-shifts. ${ }^{2}$

1 W. C. Davidon and M. L. Goldberger, Phys. Rev. 104, 1119 (1956). See also chapter V.

2 Minami (Progr. Theor. Phys.11, 213 (1954), found a further ambiguity in assigning phase shifts which can be stated as follows: If for a given $I$ and $J$ of the pion-nucleon state, we interchange the phase shifts for $l=J+\frac{1}{2}$ and $l^{\prime}=J-\frac{1}{2}$, the differential cross-sections are unaltered which means that the dominance of the $P_{3 / 2}$ state may well be the dominance of the $D_{3 / 2}$ state. Dispersion-theoretic considerations rule out the Minami set of phase shifts also. 


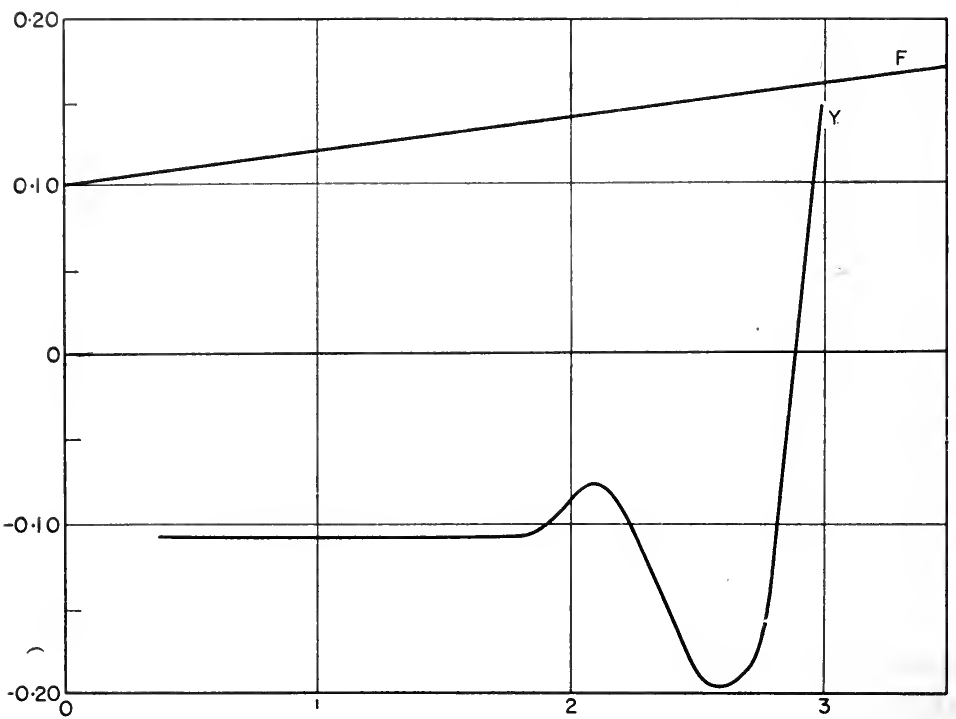

FIG. 27. Fermi(F) vs. Yang(Y) solution-application of dispersion relations.

\section{OTHER THEORIES NOT INVOLVING EXPANSION IN TERMS OF A COUPLING CONSTANT}

We now come to a consideration of certain approximation methods based on quantum mechanical or field theoretic considerations which do not involve expansion in terms of a coupling constant. We shall be considering the ChewLow formalism in detail, but before doing this we shall look briefly into two other methods which have been used, namely, Heitler's theory of radiation damping ${ }^{1}$ and the Tamm-Dancoff method ${ }^{2}$ both of which can be considered generalizations of the wave-mechanical theory of level-widths developed by Weisskopf and Wigner ${ }^{3}$.

\section{Heitler's theory of radiation damping}

We have already given a derivation of Heitler's integral equation (chapter III, sec. 1), which we rewrite here for convenience

$$
\langle A|T| 0\rangle=\langle A|K| 0\rangle+i \pi \int_{B}\langle A|K| B\rangle\langle B|T| 0\rangle
$$

1 W. Heitler, Proc. Camb. Phil. Soc. 37, 291 (1941); W. Heitler and H. W. Peng, ibid. 38, 296 (1942). M. L. Goldberger, Phys. Rev. 84, 929 (1951). W. Pauli, Meson Theory of Nuclear Forces. Interscience Publishers, New York (1946), Chapter IV.

${ }^{2}$ I. TAMm, J. Phys. (U.S.S.R.) 9, 449 (1945). S. M. DAncoff, Phys. Rev. 78, 382 (1950).

3 V. Weisskopf and E. Wigner, Z. Phys. 63, 54 (1930); 65, 18 (1930). 
where all the matrices are defined only between states on the energy shell. Also

$$
\langle l|K| m\rangle=\sum_{n=1}\left\langle l\left|H_{\mathrm{int}}^{(n)}\right| m\right\rangle
$$

with the understanding that all the intermediate states involved are off the energy shell (which is taken care of by taking only the principal values in the integrations over the intermediate state energies). The convergence necessary for obtaining equation (1) can be achieved by giving an extension to the target particle (nucleon) which however means that one has to lose the relativistic invariance of the theory (as is the case for the Chew-Low theory). Heitler uses instead a "correspondence argument" based on analogy with quantum electrodynamics which states that in the sum $\langle A|K| B\rangle$ all terms beyond the lowest non-vanishing term shall be omitted. The motivation for this is that the matrix elements of the higher orders generally diverge. Heitler's rule will mean that, if for example, the initial state $|0\rangle$ consists of a single physical nucleon with no meson, the only matrix that need be taken in the expansion of $\langle B|K| 0\rangle$ is $\left\langle B\left|H_{I}\right| 0\right\rangle$ if $|B\rangle$ represents a state containing a nucleon plus a meson and if $H_{I}$ is the usual Yukawa type of coupling (absorption or emission of a single meson at a vertex). If on the other hand both the initial and final states contain a nucleon and a meson (meson scattering), then obviously the first order term will be zero and $\langle B|K| 0\rangle$ has to be approximated by the second order term. It can be proved that Heitler's procedure is relativistically invariant. Further, for any $K$, the unitarity of the $S$-matrix is maintained which is an argument in favour of the theory since one does not obtain obviously meaningless cross-sections which increase indefinitely with energy. The theory using this approximation is called the theory of radiation damping since the inclusion of the second term in equation (1) produces the effect of true radiation damping in the classical sense of reactions due to real processes (e.g. the reaction of an emitted photon on an accelerated eletron). Omission of this second (damping) term leads to the results of the usual perturbation theory.

Let us consider as an application of the theory the scattering of a meson from a nucleon. Choosing for simplicity the extreme non-relativistic case (no-recoil limit for the nucleon) and using pseudovector coupling, we can write the lowest non-vanishing term of $\left\langle\boldsymbol{k}|K| \boldsymbol{k}_{\mathbf{0}}\right\rangle$ as:

$$
\left\langle\boldsymbol{k}|K| \boldsymbol{k}_{0}\right\rangle=\frac{f^{2}}{2 \mu^{2} \omega} \boldsymbol{\sigma} \cdot \boldsymbol{k}_{0} \sigma \cdot \boldsymbol{k} .
$$

Here $\boldsymbol{k}_{0}$ and $\boldsymbol{k}$ are the initial and final meson momenta, $\mu$ and $\omega$ the meson mass and energy respectively and $f$ is the pseudovector coupling constant. The Heitler equation will be

$$
\begin{array}{r}
\left\langle\boldsymbol{k}|T| \boldsymbol{k}_{0}\right\rangle=\frac{f^{2}}{2 \mu^{2} \omega} \boldsymbol{\sigma} \cdot \boldsymbol{k}_{0} \sigma \cdot \boldsymbol{k}+i\left(\frac{f^{2} k_{0}}{16 \pi^{2} \mu^{2} \omega}\right) \\
\int \mathrm{d} \Omega^{\prime} \sigma \cdot \boldsymbol{k}^{\prime} \sigma \cdot \boldsymbol{k}\left\langle\boldsymbol{k}^{\prime}|T| \boldsymbol{k}_{0}\right\rangle .
\end{array}
$$


The solution of this equation is found by noting that the only scalars that can be formed from $\boldsymbol{k}_{\mathbf{0}}, \boldsymbol{k}$ and $\sigma$ are $\boldsymbol{k}_{\mathbf{0}} \cdot \boldsymbol{k}$ and $\sigma \cdot\left(\boldsymbol{k}_{\mathbf{0}} \times \boldsymbol{k}\right)$. The solution must therefore be a linear combination of these. Actually the solution turns out to be

$$
\left\langle\boldsymbol{k}|T| \boldsymbol{k}_{0}\right\rangle=\frac{f^{2}}{2 \mu^{2} \omega} \frac{(1+2 i x) \boldsymbol{k}_{0} \cdot \boldsymbol{k}+i \boldsymbol{\sigma} \cdot \boldsymbol{k}_{0} \times \boldsymbol{k}}{1-i x+2 x^{2}}
$$

where $x=\frac{f^{2}}{4 \pi} \frac{k_{0}^{3}}{3 \mu^{2} \omega}$. The cross-section is

$$
\frac{\mathrm{d} \sigma}{\mathrm{d} \Omega}=\frac{f^{2}}{4 \pi} \frac{1}{\mu^{2}} \frac{k_{0}^{4}}{\mu^{2} \omega^{2}} \frac{\left[1+4 x^{2} \cos ^{2} \theta\right]}{\left(1+2 x^{2}\right)^{2}+x^{2}} .
$$

For very large $x$, the cross-section is

$$
\frac{\mathrm{d} \sigma}{\mathrm{d} \Omega}=\frac{36}{k_{0}^{2}} \cos ^{2} \theta
$$

showing the characteristic $P$-wave angular distribution of the scattered mesons.

Heitler's procedure also predicts the (3.3) resonance at the correct value of the pion kinetic energy $(\sim 200 \mathrm{MeV})$, but gives too high a cross-section for ordinary and charge exchange scattering of $\pi^{-}$. Further there are cases in which the neglect of the higher order terms in $K$ is simply incorrect. The most prominent case is in the problem of electron scattering taking into account radiative corrections where it has been shown ${ }^{1}$ that the inclusion of virtual photons in $K$ removes the discrepancies found in the simpler treatment. Blatt ${ }^{2}$ has shown that for a few simple problems the neglect of higher order terms would appear to be numerically unjustified, since the neglected terms are larger than those retained.

\section{The Tamm-Dancoff approximation}

In this the state vector of the system considered is expanded in terms of complete sets of "bare" particle states which are eigenfunctions of the free field Hamiltonian, the expansion coefficients being interpreted as the probability for finding a definite number of bare particles in the state, i.e.

$$
\begin{gathered}
\psi(\boldsymbol{p} \cdots)=c(\boldsymbol{p}) \Phi_{0}+\frac{1}{\sqrt{1} !} \int \mathrm{d}^{3} k_{1} c\left(\boldsymbol{p} ; \boldsymbol{k}_{1}\right) a^{\dagger}\left(\boldsymbol{k}_{1}\right) \Phi_{0} \\
+\frac{1}{\sqrt{2} !} \iint \mathrm{d}^{3} k_{1} \mathrm{~d}^{3} k_{2} c\left(\boldsymbol{p} ; \boldsymbol{k}_{1}, \boldsymbol{k}_{2}\right) a^{\dagger}\left(\boldsymbol{k}_{1}\right) a^{\dagger}\left(\boldsymbol{k}_{2}\right) \Phi_{0}
\end{gathered}
$$

where $\Phi_{0}$ is the free particle meson vacuum, $\boldsymbol{p}$ the nucleon momentum and $a^{\dagger}(\boldsymbol{k})$ and $a(\boldsymbol{k})$ are the creation and annihilation operators of a meson of momen-

1 T. Sebe, Progr. Theoret. Phys. 3, 304 (1948).

2 J. B. Blatt, Phys. Rev. 72, 466 (1947). 
tum $\boldsymbol{k}$. Writing the expansion in the form $\sum_{n} C_{n} \Phi_{n}$ where $H_{0} \Phi_{n}=E_{n} \Phi_{n}$ and substituting it in the Schrödinger equation

we get

$$
\left(H_{0}+H_{\mathrm{I}}\right) \psi=E \psi
$$

or

$$
\begin{gathered}
\sum_{n} C_{n}\left(\Phi_{m},\left(H_{0}+H_{I}\right) \Phi_{n}\right)=E \sum_{n}\left(\Phi_{m}, \Phi_{n}\right) C_{n} \\
=E_{m} C_{m}+\sum_{n}\left(\Phi_{m}, H_{I} \Phi_{n}\right) C_{n}=E C_{m} \\
\left(E-E_{m}\right) C_{m}=\sum_{n}\left(\Phi_{m}, H_{I} \Phi_{n}\right) C_{n} .
\end{gathered}
$$

If we consider $H_{\mathrm{I}}$ to be linear in the meson field, this represents an infinite system of coupled equations, a typical one of which connects the amplitude for $n$ mesons with those for $n-1$ or $n+1$ mesons. To make a solution of this set possible, the number $n$ of the particles is restricted so that the set of equations is broken off arbitrarily.

The rate of convergence of the above procedure is still an unsolved problem, the expectation being that better approximations can be had by taking an increasingly larger number of particles. The computation involved in the solution of the integral equations for the simplest problems are prohibitive. ${ }^{1}$ But the chief objection against the approximation is that the equations are unrenormalizable in higher approximations. The approximation assumes that those components of the state vector containing more than a certain number of bare mesons are negligibly small, an approximation which is known to be completely false for the experimental value of the coupling constant.

\section{THE CHEW-LOW FORM A L IS M ${ }^{2}$}

\section{The Kemmer Hamiltonian}

The Yukawa interaction was discussed in the chapter on single particle wave functions and their equations. In that formulation, only the wave functions of the nucleons occurred and $U$ represented the potential field of the meson. If this interaction were to be represented in the field-theoretic form we need to include the concept of emission and absorption of charged and neutral mesons with the creation and annihilation of the corresponding nucleons.

Kemmer $^{3}$ postulated a symmetric theory of meson-nucleon interactions introducing the isotopic spin formalism to include the emission and absorption of all

1 F. J. Dyson et al. Phys. Rev. 95, 1644 (1954).

2 This section is essentially based upon Chew's article (see below) on pion scattering and photoproduction. The important papers and reviews on the subject are: G. F. CHew, Phys. Rev. 89, 591 (1953); 94, 1748, 1755 (1954); 95, 1669 (1954); also his article "Theory of Pion scattering and photo production" in the Handbuch der Physik, Springer-Verlag, Vol. 43 (1959), which contains an extensive bibliography on the subject. G. C. Wick: Rev. Mod. Phys. 27, 339 (1955); F. E. Low, Phys. Rev. 97, 1392 (1955); also Rev. Mod. Phys. 29, 216 (1957); G. F. Chew and F. E. Low, Phys. Rev. 101, 1570, 1579 (1956).

3 N. Kemmer, Proc. Camb. Phil. Soc. 34, 354 (1938). 
the three types of mesons. The interaction Hamiltonian which should be a scalar in isotopic spin space was written as

$$
H_{\mathrm{I}}=g \bar{\psi} \Gamma\left(\sqrt{ } 2 \tau_{-} \varphi+\sqrt{ } 2 \tau_{+} \varphi^{*}+\tau_{3} \varphi_{3}\right) \psi .
$$

This corresponds to a proper combination of the three distinct equations which we wrote in the wave function formalism, the weights $V_{2}$ being attached to $\tau_{+}$ and $\tau_{-}$. The choice of these weights will be apparent if we express $\tau_{+}$and $\tau_{-}$in terms of $\tau_{1}$ and $\tau_{2}$ in which case the Hamiltonian reads:

$$
H_{\mathrm{I}}=g \bar{\psi} \Gamma\left(\tau_{1} \varphi_{1}+\tau_{2} \varphi_{2}+\tau_{3} \varphi_{3}\right) \psi
$$

The meson variable $\varphi=\frac{1}{\sqrt{ } / 2}\left(\varphi_{1}+i \varphi_{2}\right)$ when expressed in terms of creation and destruction operators represents the destruction of a $\pi^{-}$or the creation of a $\pi^{+}$. Similarly $\varphi^{*}=\frac{1}{\sqrt{ } 2}\left(\varphi_{1}-i \varphi_{2}\right)$ represents the creation of a $\pi^{-}$or destruction of a $\pi^{+}$ while $\varphi_{3}$ represents the creation or destruction of a $\pi^{0}$. Though the symmetry of the Hamiltonian is apparent only when it is written in terms of $\varphi_{1}, \varphi_{2}, \varphi_{3}$, physical interpretation is possible only if it is expressed in terms of $\varphi, \varphi^{*}$ and $\varphi_{3}$. We give here the Feynman diagrams corresponding to all the possible processes obtained by operating (1) on a nucleon wave function (ignoring the isotopic spin operator $\tau_{i}$ ).

If $\varphi_{1}, \varphi_{2}$ and $\varphi_{3}$ can be considered as a 3 -vector in the same charge space as $\left(\tau_{1}, \tau_{2}, \tau_{3}\right)$ then the sum $\sum_{i=1}^{3} \tau_{i} \varphi_{i}$ can be considered as the scalar product $\boldsymbol{\tau} \cdot \boldsymbol{\varphi}$. For this to exist there must be a hermitian operator $\boldsymbol{T}$ which generates unitary transformations of $\varphi$ corresponding to infinitesimal rotations in isotopic spin space. Taking the component $T_{3}$, we have for a real infinitesimal $\varepsilon$,

$$
\mathrm{e}^{i \varepsilon T_{3}} \varphi_{1} \mathrm{e}^{-i \varepsilon T_{3}} \approx \varphi_{1}+i \varepsilon\left[T_{3}, \varphi\right]
$$

with two similar relations for $\varphi_{2}$ and $\varphi_{3}$. If this transformation is to represent a rotation by $\varepsilon$ about the 3 -axis, we must have

$$
i\left[T_{3}, \varphi_{1}\right]=-\varphi_{2} ; i\left[T_{3}, \varphi_{2}\right]=\varphi_{1} ; i\left[\dot{T}_{3}, \varphi\right]=0 .
$$

These conditions are satisfied by choosing

$$
T_{3}=\int \mathrm{d}^{3} r\left[\Phi_{1}(\boldsymbol{r}) \pi_{2}(\boldsymbol{r})-\pi_{1}(\boldsymbol{r}) \Phi_{2}(\boldsymbol{r})\right]
$$

where $\pi_{i}$ is the field momentum conjugate to $\varphi_{i}$ and is connected with it by the commutation rule

Thus we can write

$$
\left[\pi_{i}(r), \varphi_{j}\left(r^{\prime}\right)\right]=-i \delta\left(r-r^{\prime}\right) \delta_{i j} .
$$

$$
\boldsymbol{T}=\int \mathrm{d} r(\boldsymbol{\varphi} \times \boldsymbol{x}) .
$$



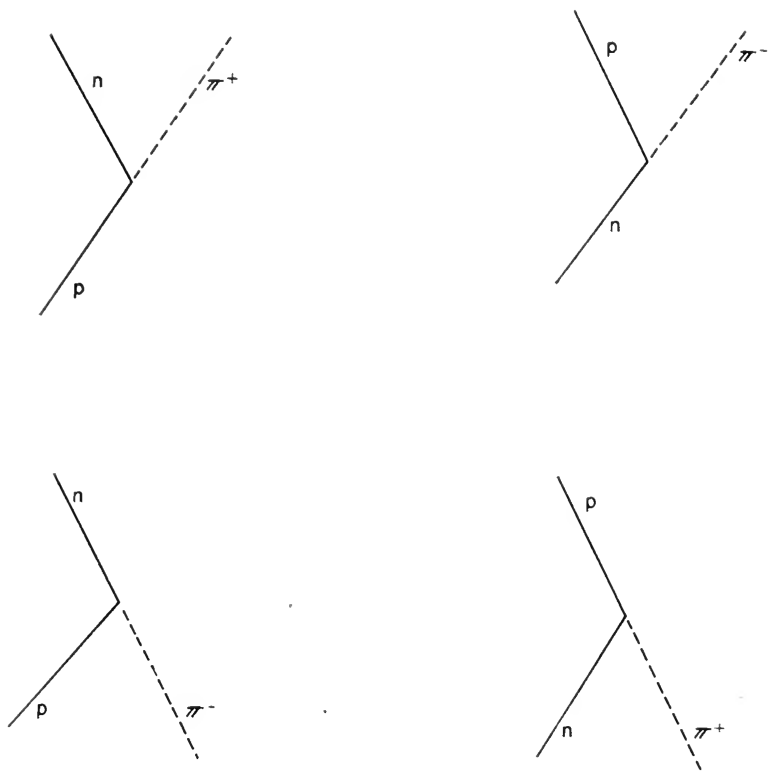

a
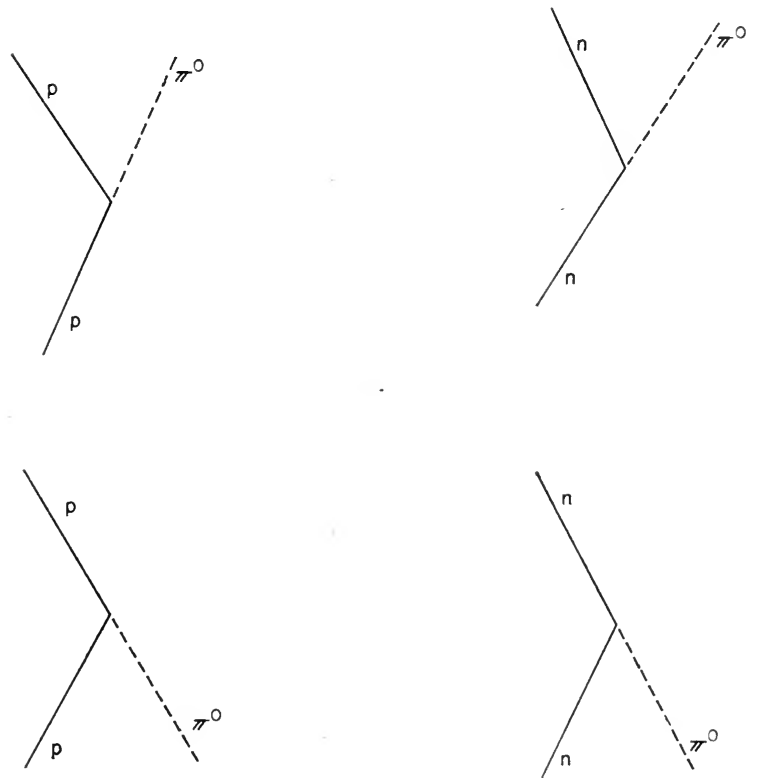

b

Fig. 28. Diagrams representing the meson-nucleon interaction. For simplicity, diagrams involving anti-nucleons are not shown. 
The rotations of the nucleon isotopic spin vector $\boldsymbol{\tau}$ are generated by $\boldsymbol{\tau} / \mathbf{2}$ itself because of the commutation rule $\boldsymbol{\tau} \times \boldsymbol{\tau}=2 i \boldsymbol{\tau}$. It can be proved that $\boldsymbol{I}=\frac{\boldsymbol{\tau}}{2}+\boldsymbol{T}$ commutes with the Kemmer interaction $\sum_{i} \varphi_{i} \tau_{i} . I_{z}=\frac{\tau_{z}}{2}+T_{z}$ is the total charge of the pion-nucleon system and hence commutes with the charge-conserving Kemmer interaction. We can show that $I_{x}$ and $I_{y}$ also commute with $\sum_{i} \varphi_{i} \tau_{i}$. Thus the Kemmer interaction is invariant under transformations generated by $\boldsymbol{I}$ so that $\boldsymbol{\tau} \cdot \boldsymbol{\varphi}$ is a true scalar product. $\boldsymbol{I}$ also commutes with the kinetic energy part of the Hamiltonian and is called the total isotopic spin operator. The eigenvalue of $I^{2}$ is a constant of motion.

The Kemmer interaction is the only one of the Yukawa type (linear in the meson field) which is invariant under all rotations in charge space, i.e. is a true scalar in charge space.

\section{Pion-nucleon scattering ${ }^{1}$}

The complete interaction Hamiltonian density for the interaction of a pseudoscalar meson with the nucleon is given by the expression

$$
\begin{aligned}
H_{I} & =g \bar{\psi}(x) \gamma_{5} \tau_{i} \psi(x) \varphi_{i}(x)+\delta m \bar{\psi}(x) \psi(x) \\
& +\frac{1}{2} \delta \mu^{2} \varphi_{i}(x) \varphi_{i}(x)-\frac{1}{4} \lambda\left[\varphi_{i}(x) \varphi_{i}(x)\right]^{2} .
\end{aligned}
$$

The first term on the right is the Kemmer Hamiltonian. The second and third terms are the counter terms for the proper masses of the nucleon and the meson respectively. The last term arises as follows: due to the absence of gauge invariance (since the meson mass is different from zero) the diagram with four external meson lines leads, in contrast to quantum electrodynamics to a divergence. In order to compensate for this we have to introduce a counter term which is precisely the last term. $\lambda$ is the coupling constant of this direct pion-pion interaction which is expected to become important at high energies. As not much is known about this interaction, we can omit the last term from further consideration. ${ }^{2}$

1 F. E. Low, Phys. Rev. 97, 1392 (1955).

2 Recently, however, experimental evidence has appeared which would seem to point to the existence of a strong pion-pion interaction. Evidence for such an interaction has been found for example in the analysis of single pion production data in $\pi-p$ collisions by $\mathrm{F}$. Bonsignori and F. Selleri (Nuovo Cim. 15, 465 (1960)) and by I. Derado (Nuovo Cim. 15, 853 $(1960))$. Further, Frazer and Fulco have shown that the isotopic vector part of the nucleon form factors may be satisfactorily fitted if one assumes a $\pi-\pi$ scattering resonance in the $J=1, I=1$ state (W. R. Frazer and J. R. Fulco; Phys. Rev. Lett. 2, 365 (1959); UCRL 8880 ). Using the Mandelstam representation (see next chapter), Chew and Mandelstam have found, on assuming that there is no stable bound state of the pion-pion system, that the limits for the pion-pion coupling constant, $\lambda$ are given by $-0.36 \leqq \lambda \leqq 0.3$. (G. F. CHEw and S. Mandelstam, Phys. Rev., 119, 467 (1960)). Recent calculations such as for instance the study of pion production in pion-nucleon collisions and photoproduction of two and three mesons [S. K. Srinivasan and K. Venkatesan, Nucl. Phys. (to be published)] seem to indicate that $\lambda$ does lie within this range. 
The $S$-matrix element for the scattering of a meson of momentum $q_{1}$ and isotopic spin index $\alpha$ to a state of momentum of $q_{2}$ and isotopic spin index $\beta$ while the nucleon changes its momentum from $p_{1}$ to $p_{2}$ can be written as

$$
\begin{gathered}
\left\langle p_{2} ; q_{2} \beta|S| p_{1} ; q_{1} \alpha\right\rangle=\sum_{n=0}^{\infty} \frac{(-i)^{n}}{n !} \int \mathrm{d} x_{1} \mathrm{~d} x_{2} \ldots \mathrm{d} x_{n} \\
\times\left(\Phi_{p_{2}}, a_{q_{2}} T\left(H_{I}\left(x_{1}\right) \ldots H_{I}\left(x_{n}\right)\right) a_{q_{1}}^{\dagger} \Phi_{p_{1}}\right)
\end{gathered}
$$

where all the quantities are in the interaction representation. $a_{q}$ and $a_{q}^{+}$are the annihilation and creation operators of a meson respectively and $\Phi_{p}$ represents the state containing a "bare" nucleon of momentum $p$. Commuting the operator $a_{q_{2}}$ to the right and the operator $a_{q_{1}}^{\dagger}$ to the left and using the relevant commutation rules, we get

$$
\begin{gathered}
\left\langle p_{2} ; q_{2} \beta|S| p_{1} ; q_{1} \alpha\right\rangle=\frac{(-i)^{2}}{\sqrt{4} \omega_{1} \omega_{2}} \int \mathrm{d} x \mathrm{~d} y \mathrm{e}^{-i q_{1} x+i q_{2} y} \\
\sum_{n=0}^{\infty} \frac{(-i)^{n}}{n !} \int \mathrm{d} x_{1} \mathrm{~d} x_{2} \cdots \mathrm{d} x_{n}\left(\Phi_{p_{2}}, T\left(H_{I}\left(x_{1}\right) \cdots H_{I}\left(x_{n}\right) j_{\beta}(y) j_{\alpha}(x)\right) \Phi_{p_{1}}\right)
\end{gathered}
$$

where $\omega_{1}$ and $\omega_{2}$ are the energies of the initial and final mesons and $j_{i}(x)$ is the current operator in the interaction representation and is given by

$$
j_{i}^{(x)}=g \bar{\psi}(x) \gamma_{5} \tau_{i} \psi(x)+\delta \mu^{2} \varphi_{i}(x) .
$$

We now use the general relationship, based on the Gell-Mann-Low ${ }^{1}$ theorem, between the matrix elements in the interaction and Heisenberg representations

$$
\begin{gathered}
\sum_{n=0}^{\infty} \frac{(-i)^{n}}{n !} \int \mathrm{d} x_{1} \ldots \mathrm{d} x_{n}\left(\Phi_{p_{2}}, T\left(H_{I}\left(x_{1}\right) \ldots H_{I}\left(x_{n}\right) j_{\beta}(y) j_{\alpha}(x) \Phi_{p_{1}}\right),\right. \\
=\left(\Psi_{p_{2}}, T\left(\dot{j}_{\beta}(y) \dot{j}_{\alpha}(x)\right) \Psi_{p_{1}}\right)^{2}
\end{gathered}
$$

where all the quantities on the right hand side are in the Heisenberg representation, the $\psi_{p}$ 's being exact single particle energy-momentum eigenstates of the total Hamiltonian. (We demonstrated such an equivalence in the case of the propagators in the two representations, in chapter III, section 3.) Thus we can write

$$
\begin{gathered}
\left\langle p_{2} ; q_{3} \beta|S| p_{1} ; q_{1} \alpha\right\rangle=\frac{-1}{\sqrt{4} \omega_{1} \omega_{2}} \int \mathrm{d} x \mathrm{~d} y \\
\mathrm{e}^{-i q_{1} \cdot x+i q_{2} y}\left\langle p_{2}\left|T\left(\dot{\mathrm{j}}_{\beta}(y) \dot{\mathrm{\alpha}}_{\alpha}(x)\right)\right| p_{1}\right\rangle .
\end{gathered}
$$

Using the translational invariance theorem for the Heisenberg operators

$$
\dot{j}_{\alpha}(x)=\mathrm{e}^{i P x} \mathrm{j}_{\alpha}(0) \mathrm{e}^{-i P x}
$$

and performing the $x$-integration first, we get the right hand side to be equal to

$$
\frac{\dot{-} \delta\left(p_{2}+q_{2}-p_{1}-q_{1}\right)}{\sqrt{4} \omega_{1} \omega_{2}} \int \mathrm{d} y \mathrm{e}^{i q_{2} y}\left\langle p_{2}\left|T\left(\dot{\mathrm{i}}_{\beta}(y) \dot{\mathrm{\lambda}}_{\alpha}(0)\right)\right| p_{1}\right\rangle .
$$

1 M. Gell-Mann and F. E. Low, Phys. Rev. 84, 350 (1951).

2 This result can also be arrived at using the approach of Lehmann, SYManziK and ZrmmerMANN (see chapter V) which however is built on more general premises and does not involve the concept of a specific interaction. 
Expanding in terms of a complete set of "incoming" states and doing the $y$-integration, we finally get

$$
\begin{aligned}
\left\langle p_{2} ; q_{2} \beta|T| p_{1} ; q_{1} \alpha\right\rangle & \\
= & -\frac{1}{\sqrt{4} \omega_{1} \omega_{2}}\left[\sum_{m} \frac{\left\langle p_{2}\left|\dot{j}_{\beta}(0)\right| m\right\rangle\left\langle m\left|\dot{j}_{\alpha}(0)\right| p_{1}\right\rangle}{E_{m}-p_{20}-\omega_{2}-i \varepsilon}\right. \\
& \left.+\sum_{m} \frac{\left\langle p_{2}\left|\dot{j}_{\alpha}(0)\right| m\right\rangle\left\langle m\left|\dot{j}_{\beta}(0)\right| p_{1}\right\rangle}{E_{m}+\omega_{2}-p_{10}-i \varepsilon}\right]
\end{aligned}
$$

where the " $T$ " matrix is defined as the corresponding $S$-matrix with $i$ times the deltafunction factor for over-all energy momentum left out. This is the Low equation for scattering which is exact and the properties of which we shall be presently studying in the fixed source limit. If we retain terms involving more than one meson in the intermediate states, this equation will become a very complicated system of coupled non-linear integral equations. But if we take the inelastic cross-sections to be small compared to the elastic ones, we can make the "TammDancoff" approximation of retaining only the intermediate states containing (i) a single physical nucleon and (ii) a physical nucleon plus one meson. We then obtain an integral equation for the scattering amplitude, the inhomogeneous term of which will turn out to be, as we shall see presently, the renormalized Born approximation.

Next we shall derive an expression for the vertex function $\left\langle p_{2}|\mathrm{i}(x)| p_{1}\right\rangle$. Using translationąl invariance, we have

$$
\begin{aligned}
\left\langle p_{2}|\mathrm{j}(x)| p_{1}\right\rangle & =\left\langle p_{2}\left|\left(\square^{2}+\mu^{2}\right) \varphi(x)\right| p_{1}\right\rangle \\
& =\left[-\left(p_{2}-p_{1}\right)^{2}+\mu^{2}\right] \cdot\left\langle p_{2}|\varphi(0)| p_{1}\right\rangle \mathrm{e}^{i\left(p_{2}-p_{1}\right) x} \\
& =\left[-\left(p_{2}-p_{1}\right)^{2}+\mu^{2}\right]\left(\Phi_{p_{2}}, T(S \varphi(0)), \Phi_{p_{1}}\right) \mathrm{e}^{i\left(p_{2}-p_{1}\right) x}
\end{aligned}
$$

where the last step is obtained by going back to the interaction representation, $S$ being the $S$-matrix. If we take the lowest order term in $S$, namely $-i \int H_{I}\left(x_{1}\right) \mathrm{d} x_{1}$ and use the anticommutation rules for the spinor field variables and the fact that the vacuum expectation value of $T\left(\varphi\left(x_{1}\right) \varphi(0)\right)$ is the meson propagator we get for the lowest order matrix element

$$
\left[-\left(p_{2}-p_{1}\right)^{2}+\mu^{2}\right] g \tau \bar{u}\left(p_{i}\right) \gamma_{5} u\left(p_{1}\right) \Delta_{F}\left(p_{2}-p_{1}\right) \mathrm{e}^{i\left(p_{2}-p_{1}\right) x} .
$$

The complete matrix element (17) is obtained by replacing $\Delta_{F}$ by $\Delta_{F}^{\prime}, \gamma_{5}$ by $\Gamma_{s c}$ and $g$ and $\varphi(x)$ by the corresponding renormalized quantities.

We can write the vertex function as

$$
\begin{aligned}
\left\langle p_{2}\left|\mathrm{j}_{\alpha}(0)\right| p_{1}\right\rangle & =\left[-\left(p_{2}-p_{1}\right)^{2}+\mu^{2}\right] \\
& \times g_{r} \Delta_{F}^{\prime}\left(p_{2}-p_{1}\right) \bar{u}\left(p_{2}\right) \Gamma_{s c}\left(p_{2}, p_{1}\right) u\left(p_{1}\right) \tau_{\alpha} .
\end{aligned}
$$

As such it is practically useless for calculation since the matrix $\gamma_{5}$ leads to a coupling between nucleon and anti-nucleon states much stronger than that between ordinary nucleon states so that the intermediate states will contain an abundance of virtual nucleon-anti-nucleon pairs making calculations extremely 
complicated. If however $\left[(\Delta p)^{2} / m^{2}\right] \ll 1$ then $\left[(\Delta p)^{2}+\mu^{2}\right] \Delta_{F}^{\prime} \simeq 1$. Therefore in the non-relativistic region we have

$$
\left\langle p_{\mathbf{2}}\left|\mathrm{i}_{\alpha}(0)\right| p_{\mathbf{1}}\right\rangle=\frac{i g_{r} \tau_{\alpha} \sigma \cdot \Delta \boldsymbol{p}}{2 m}
$$

where $m$ is the nucleon mass. Using the equivalence theorem ${ }^{1}$ for pseudoscalar and pseudovector couplings ${ }^{2}$

we finally get

$$
\frac{g_{r}}{2 m}=f_{r}
$$

$$
\left\langle p_{2}\left|\mathrm{i}_{\alpha}(0)\right| p_{1}\right\rangle=i f_{r} \tau_{\alpha} \sigma \cdot \boldsymbol{q}
$$

which will represent an emission or an absorption of a meson. $f_{r}$ is the renormalized rationalized pseudovector coupling constant.

The presence of the meson momentum in the matrix element indicates that integrals over all virtual pions will diverge. The obvious way to remove this divergence is to cut off virtual pions of high momenta. Chew ${ }^{3}$ realized that this could be done by fixing the nucleon and assuming the interaction to be nonlocal and effective within a small region which gives the "spread" of the nucleon. The larger the spread the smaller the momenta of the virtual mesons to be taken account of. With a momentum cut-off factor $v(q)$ the vertex function now reads:

$$
\left\langle p_{2}\left|\dot{j}_{\alpha}(0)\right| p_{1}\right\rangle=i f_{r} \tau_{\alpha} v(q) \boldsymbol{\sigma} \cdot \boldsymbol{q} .
$$

The right hand side has to be considered a matrix in the spin and isospin space of the nucleon.

We notice that

$$
\left\langle p_{2}\left|\dot{\mathrm{j}}_{\alpha}(0)\right| p_{1}\right\rangle=\left\langle p_{1}\left|\dot{\mathrm{i}}_{\alpha}(0)\right| p_{2}\right\rangle^{\dagger} \text {. }
$$

Further since we are taking the static limit for the nucleon we can take $p_{20}=p_{10}$ $=$ the rest energy of the nucleon as the ground state energy and set it equal to zero. We now define the operator

and

$$
V_{q}=\frac{j_{\alpha}(0)}{\sqrt{ } 2 \omega_{q}}=\frac{i f_{r} \tau_{\alpha} v(q) \boldsymbol{\sigma} \cdot \boldsymbol{q}}{\sqrt{ } 2 \omega_{q}}
$$

$$
T_{q}(m)=\left\langle m\left|V_{q}\right| p\right\rangle
$$

where $|p\rangle$ denotes a single nucleon state. Using the notation $T_{1}(2)$ for the $T$ matrix for a meson of momentum $q_{1}$ to go to a state with momentum $q_{2}$, we can now write the Low equation as

$$
T_{1}(2)=-\sum_{m}\left[\frac{T_{2}^{\dagger}(m) T_{1}(m)}{E_{m}-\omega_{2}-i \varepsilon}+\frac{T_{1}^{\dagger}(m) T_{2}(m)}{E_{m}+\omega_{2}}\right]
$$

1 See, e.g. Bethe and DE Hoffmann, Mesons and fields, Vol. I. Appendix (Row Peterson (1955)).

${ }^{2}$ Here and in the following, we assume the pion mass to be equal to 1.

${ }^{3}$ See, e.g. G. F. Chew, "Theory of pion scattering and photo production", Handbuch der Physik (Springer-Verlag). Loc. cit. 
where $m$ now refers to the meson degrees of freedom and the $i \varepsilon$ has been omitted in the denominator of the second term since it is not a "resonant" denominator. The matrix has the following properties:

(a) It satisfies the unitarity condition

$$
T_{1}^{\dagger}(1)-T_{1}(2)=2 \pi i \sum_{m} T_{2}^{\dagger}(m) T_{1}(m) \delta\left(E_{m}-\omega\right) .
$$

(b) It obeys the "crossing" theorem. To show this and for future convenience we define a hermitian matrix function $t_{21}(z)$ of a complex variable $z=\omega_{2}+i \varepsilon$ :

with

$$
t_{21}(Z)=-\sum_{m}\left\{\frac{T_{2}^{\dagger}(m) T_{1}(m)}{E_{m}-z}+\frac{T_{1}^{\dagger}(m) T_{2}(m)}{E_{m}+z}\right\}
$$

We note that

$$
\operatorname{Lim}_{\varepsilon \rightarrow 0} t_{21}(z)=T_{1}(2) \text {. }
$$

or equivalently

$$
\begin{gathered}
t_{21}^{\dagger}\left(z^{*}\right)=t_{21}(-z) \\
t_{12}(z)=t_{21}(-z)
\end{gathered}
$$

which is called the "crossing" theorem and expresses the fact that for every Feynman diagram representing meson scattering, there must exist another which is got from the first by exchanging the incoming and outgoing meson lines.

(c) $t_{21}(z)$ has a pole at the origin with the residue

$$
R_{21}=T_{2}^{\dagger}(0) T_{1}(0)-T_{1}^{\dagger}(0) T_{2}(0)=-\left[V_{2}, V_{1}\right] .
$$

which is determined by the single parameter $f_{r}$. Thus we see that the inhomogeneous term of Low's equation is merely the renormalized Born approximation as already mentioned.

(d) As $z \rightarrow \infty, t_{21}(z)$ behaves as $1 / z$.

(e) The other singularities of $t_{21}(z)$ are also located on the real axis and are branch points corresponding to $z= \pm 1, \pm 2 \ldots$ etc. with cuts to $\pm \infty$ respectively.

Chew and Low ${ }^{1}$ have shown that the above conditions completely determine $t_{21}(z)$ so that if we could get a function having the above properties it would be a solution of the Low equation. It is convenient to connect $t_{21}(z)$ with the experimentally observed phase shifts of the four $P$-states of the meson-nucleon system.

We can write

$$
t_{21}(z)=-\frac{4 \pi v\left(q_{1}\right) v\left(q_{2}\right)}{\sqrt{4} \omega_{1} \omega_{2}} \sum_{\alpha=1}^{4} P_{\alpha}(2,1) h_{\alpha}(z)
$$

where $P_{\alpha}$ 's are the projection operators for the four states $(33,31,13,11)$ which can be written down as follows. $t_{21}(z)$ depends linearly on $\boldsymbol{q}_{1}$ and $\boldsymbol{q}_{2}$ and since it must be a scalar its most general form is a linear combination of, $\boldsymbol{q}_{2} \cdot \boldsymbol{q}_{1}$ and $\sigma \cdot\left(\boldsymbol{q}_{2} \times \boldsymbol{q}_{1}\right)$. If we now form the operators

and

$$
\begin{aligned}
& J_{1 / 2}(2,1)=\boldsymbol{q}_{2} \cdot \boldsymbol{q}_{1}+i \sigma \cdot\left(\boldsymbol{q}_{2} \times \boldsymbol{q}_{1}\right) \\
& J_{3 / 2}(2,1)=2 \boldsymbol{q}_{2} \cdot \boldsymbol{q}_{1}-i \sigma \cdot\left(\boldsymbol{q}_{2} \times \boldsymbol{q}_{1}\right)
\end{aligned}
$$

1 G. F. CHew and F. E. Low, Phys. Rev. 101, 1570 (1956). 
they can be shown to project out the states of total angular momentum $1 / 2$ and $3 / 2$ respectively. As regards the isotopic spin projection operators, we note that if we define the matrix

then

$$
R_{q_{1} q_{2}}=\tau_{q_{1}} \tau_{q_{2}}
$$

$$
\left(R^{2}\right)_{q_{1} q_{2}}=\sum_{q} \tau_{q_{1}} \tau_{q} \tau_{q} \tau_{q_{2}}=3(R)_{q_{1} q_{2}}
$$

or

$$
R(R-3)=0
$$

so that $R$ has the eigenvalues 0 or 3 . Further, for the expectation value of $R$ for the state $\left|P \pi^{+}\right\rangle$which is a pure $3 / 2$ isotopic spin state, we have, since

$$
\begin{aligned}
&\left|P \pi^{+}\right\rangle=\left|I=3 / 2 ; \quad I_{z}=3 / 2\right\rangle=\frac{1}{\sqrt{2}}\left|P\left(\varphi_{1}+i \varphi_{2}\right)\right\rangle \\
&\left\langle P \pi^{+}|R| P \pi^{+}\right\rangle=\frac{1}{2}\left\langle P\left(\varphi_{1}-i \varphi_{2}\right)|R|\left(\varphi_{1}+i \varphi_{2}\right)\right\rangle \\
&=\frac{1}{2}\left\langle P\left|R_{11}-i R_{21}+i R_{12}+R_{22}\right| P\right\rangle \\
&=\frac{1}{2}\left\langle P\left|2-2 \tau_{3}\right| P\right\rangle \\
&=\frac{1}{2}\left[2\left(\langle P \mid P\rangle-\left\langle P\left|\tau_{3}\right| P\right\rangle\right)\right]=0 \\
& R|I=3 / 2\rangle=0
\end{aligned}
$$

and therefore

$$
R|I=1 / 2\rangle=3|I=1 / 2\rangle \text {. }
$$

Since we can write

$$
\begin{aligned}
\tau_{q_{1}} \tau_{q_{2}} & =\frac{1}{2}\left(\tau_{q_{1}} \tau_{q_{2}}+\tau_{q_{2}} \tau_{q_{1}}\right)+\frac{1}{2}\left(\tau_{q_{1}} \tau_{q_{2}}-\tau_{q_{2}} \tau_{q_{1}}\right) \\
& =\delta_{q_{1} q_{2}}+\frac{1}{2}\left[\tau_{q_{1}}, \tau_{q_{2}}\right]
\end{aligned}
$$

we have finally the projection operators for the $3 / 2$ and $1 / 2$ states respectively as

and

$$
I_{3 / 2}(2,1)=\frac{2}{3} \delta_{q_{2} q_{1}}-\frac{1}{3} \frac{\left[\tau_{q_{2}}, \tau_{q_{1}}\right]}{2}
$$

$$
I_{1 / 2}(2,1)=\frac{1}{3} \delta_{q_{2} q_{1}}+\frac{1}{3} \frac{\left[\tau_{q_{2}}, \tau_{q_{1}}\right]}{2} \text {. }
$$

Combining the spin and isospin projection operators we finally get the four $P$-state projection operators

$$
\begin{aligned}
& P_{11}(2,1)=I_{1 / 2}(2,1) J_{1 / 2}(2,1) \\
& P_{13}(2,1)=I_{1 / 2}(2,1) J_{3 / 2}(2,1) \\
& P_{31}(2,1)=I_{3 / 2}(2,1) J_{1 / 2}(2,1) \\
& P_{33}(2,1)=I_{3 / 2}(2,1) J_{3 / 2}(2,1) .
\end{aligned}
$$

The functions $h_{\alpha}(z)$ have the same singularities as the function $t_{21}(z)$. Since there is complete symmetry between the spin and isotopic spin angular momenta in the cut-off theory, we need deal with only the three functions:

$$
h_{1}=h_{11} ; \quad h_{2}=h_{13}=h_{31} ; \quad h_{3}=h_{33} .
$$


A comparison with the conventional expression for the scattering amplitude in terms of the phase shifts shows that

$$
\operatorname{Lim}_{z \rightarrow 0} h_{\alpha}(z)=\frac{\mathrm{e}^{i \delta_{\alpha}} \sin \delta_{\alpha}}{q^{3} v^{2}(q)},
$$

This is also consistent with the unitary condition (equation 28) which gives the relation

$$
\operatorname{Im} h_{\alpha}(z)=q^{3} v^{2}(q)\left|h_{\alpha}\left(\omega_{q}\right)\right|^{2} .
$$

The zero-energy limit of $h_{\alpha}(z)$ is given by

$$
h_{\alpha}(z)=\frac{2}{3} \frac{f^{2}}{z}\left[\begin{array}{l}
-4 \\
-1 \\
-2
\end{array}\right]=\frac{\lambda_{\alpha}}{z}
$$

where $f^{2}=\frac{f_{r}^{2}}{4 \pi}$ is the renormalized, unrationalized coupling constant. Thus we get the first important prediction of the Yukawa theory namely that the four $P$-wave phase shifts, in the zero-energy limit, depend on a single parameter $f^{2}$.

To remove the pole at the origin, we can define a new function

$$
g_{\alpha}(z)=\frac{\lambda_{\alpha}}{z}\left[h_{\alpha}(z)\right]^{-1}
$$

It obeys the crossing relation

$$
\frac{1}{g_{\alpha}(z)}=\sum_{\beta} B_{\alpha \beta} \frac{1}{g_{\beta}(-z)}
$$

where $B$ is a reflection matrix; i.e. $B^{2}=1$.

Explicitly

$$
B=\frac{1}{9}\left[\begin{array}{rrr}
-1 & 2 & 8 \\
8 & -7 & 8 \\
8 & 2 & -1
\end{array}\right] \text {. }
$$

The unitarity condition on it is

Also

$$
\operatorname{Im} g_{\alpha}\left(\omega_{q}\right)=-\frac{\lambda_{\alpha}}{\omega_{q}} q^{3} v^{2}(q)
$$

$$
\operatorname{Re} g_{\alpha}\left(\omega_{q}\right)=\frac{\lambda_{\alpha}}{\omega_{q}} q^{3} v^{2}(q) \cot \delta_{\alpha}\left(\omega_{q}\right)
$$

which is important because it gives the real part of $g_{\alpha}$ in terms of the phase shifts which are experimentally determinable.

If the function $h_{\alpha}(z)$ has no zeros in the entire complex plane, the function $g_{\alpha}(z)$ has no singularities other than branch points with cuts to $\pm \infty$. We can then write

$$
g_{\alpha}(z)=1-\frac{z}{\pi} \int_{1}^{\infty} \mathrm{d} \omega_{q}\left[\frac{F_{\alpha}\left(\omega_{q}\right)}{\omega_{q}-z}+\frac{G_{\alpha}\left(\omega_{q}\right)}{\omega_{q}+z}\right]
$$


where the functions $F_{\alpha}$ and $G_{\alpha}$ are determined by the unitarity condition and crossing relation respectively. Substituting the value for $F_{\alpha}$ we get finally

$$
g_{\alpha}(z)=1-\frac{z}{\pi} \int_{1}^{\infty} \mathrm{d} \omega_{q} \frac{q^{3} v^{2}(q)}{\omega^{2}(q)}\left\{\frac{\lambda_{\alpha}}{\omega_{q}-z}+\frac{H_{\alpha}\left(\omega_{q}\right)}{\omega_{q}+z}\right\}
$$

where

$$
H_{\alpha}\left(\omega_{q}\right)=\frac{\omega_{q}^{2} G_{\alpha}\left(\omega_{q}\right)}{q^{3} v^{2}(q)}
$$

$H_{\alpha}$ is so chosen that $g_{\alpha}$ satisfies the crossing relation. Chew and Low got a closed form for the solution only on simplifying the matrix $B$ to the form

$$
B^{\mathrm{I}}=\left[\begin{array}{rrr}
0 & 0 & 1 \\
1 & -1 & 1 \\
1 & 0 & 0
\end{array}\right]
$$

The result is

$$
\begin{aligned}
& g_{1}^{\mathrm{I}}(z)=1+\frac{2}{3} f^{2} \frac{z}{\pi} \int_{1}^{\infty} \mathrm{d} \omega_{q} \frac{q^{3} v^{2}(q)}{\omega_{q}^{2}}\left\{\frac{4}{\omega_{q}-z}+\frac{2}{\omega_{q}+z}\right\} \\
& g_{3}^{\mathrm{I}}(z)=1-\frac{2}{3} f^{2} \frac{z}{\pi} \int_{1}^{\infty} \mathrm{d} \omega_{q} \frac{q^{3} v^{2}(q)}{\omega_{q}^{2}}\left\{\frac{2}{\omega_{q}-z}+\frac{4}{\omega_{q}+z}\right\} .
\end{aligned}
$$

We notice that $g_{2}^{\mathrm{I}}(z)$ is not determined. ${ }^{1}$ However these solutions are in excellent agreement with the ones got from the rigorous dispersion relations. ${ }^{2}$

The form of the real part of the function $g_{\alpha}(z)$ (53) suggests by analogy to the corresponding situation in nucleon-nucleon scattering theory, that we can try an "effective range" treatment of the present problem. From (53) and (55) we have

$$
\begin{aligned}
\operatorname{Re} g_{\alpha}(z) & =\frac{\lambda_{\alpha} q^{3} v^{2}(q)}{\omega_{q}} \cot \delta_{\alpha}\left(\omega_{q}\right) \\
& =1-\omega_{q}\left\{\frac{\lambda_{\alpha}}{\pi} P \int \frac{\mathrm{d} \omega_{q}}{\omega_{q}} \frac{q^{3} v^{2} \cdot(q)}{\left(\omega_{q}-\omega\right)}+\frac{P}{\pi} \int \frac{\mathrm{d} \omega_{q}}{\omega_{q}^{2}} \frac{q^{3} v^{2}(q)}{\left(\omega_{q}+\omega\right)} H_{\alpha}\left(\omega_{q}\right)\right.
\end{aligned}
$$

1 Castillejo et al. (Phys. Rev. 101, 453 (1956) have pointed out that if we add to $g_{\alpha}^{\prime}(z)$ an arbitrary number of terms of the forms $z\left[\frac{c_{\alpha}}{\omega_{0}-z}+\frac{d_{\alpha}}{\omega_{0}+z}\right]$ where $\omega_{0}, c_{\alpha}$ and $d_{\alpha}$ are constants, the last two satisfying the crossing relation $c_{1}=-d_{3}, c_{3}=-d_{1}$ the new functions will satisfy the one-meson Low equation. It can be shown that the solutions given above without these extra terms are unique in that they correspond to the analytic continuation of the power series in $f^{2}$ for the functions $h_{\alpha}^{\prime}(z)$ or $g_{\alpha}^{\prime}(z)$.

2 The solutions are also in agreement with the numerical solution of G. SALzMan and F. Salzman, Phys. Rev. 108, 1619 (1951).

EPCR 13 
where $P$ denotes the Cauchy principal value. The effective range approximation consists in neglecting the dependence of $\operatorname{Re} g_{\alpha}\left(\omega_{q}\right)$ on $\omega_{q}$ for values of $\omega_{q} \ll \omega_{\max }$, i.e. we can write

$$
\frac{\lambda_{\alpha} q^{3} \cot \delta_{\alpha}}{\omega_{q}} \approx\left[1-\omega_{q} r_{\alpha}^{\mathbf{I}}\left(\omega_{q}\right)\right]
$$

where $r_{\lambda}^{\mathrm{I}}\left(\omega_{q}\right)$ is almost a constant for small $\omega_{q}$ and is given by

$$
r_{\alpha}^{\mathrm{I}}=\left[\begin{array}{r}
-1 \\
0 \\
1
\end{array}\right] r_{3}^{\mathrm{I}} ; r_{3}^{\mathrm{I}}=\frac{1}{3 f^{2}} \frac{1}{4 \pi^{2}} \int_{1}^{\infty} \mathrm{d} \omega_{q} \frac{\frac{5}{2} \sigma_{3}+\sigma_{1}+\sigma_{2}}{\omega_{q} q v^{2}(q)}
$$

where the $\sigma$ 's are the total cross-sections for the states corresponding to the suffixes. This linear dependence of the known quantity $\lambda_{\alpha} q^{3} \cot \delta_{\alpha} / \omega_{q}$ on the meson energy $\omega_{q}$ has been verified experimentally for the (33) phase shift. The intercept at zero energy, $\lambda_{3}^{-1}=\left(\frac{3}{4}\right) f^{2}$ gives a value of $f^{2}=0.08$.

We notice that the (33) state effective range $r_{3}^{\mathrm{I}}$ is positive so that there should be a resonance at an energy $\omega_{q} \doteq \frac{1}{r_{3}^{\mathrm{I}}}$ which is when $\delta_{33}=90^{\circ}$. This is an excellent agreement with the well-known resonance in scattering at $200 \mathrm{MeV}$ incident energy of the pion, if we take energy cut-off to be $\omega_{\max } \approx 6 \mu$.

The above effective range approximation which implies that an expansion of $r_{x}(\omega)$ in powers of $\omega$ has a radius of convergence $\omega \geqq \omega_{\max }$ should not be correct because there are singularities in the form of branch points at $z= \pm 1$. But actually the contributions from the non-analytic parts are small compared to the remainder of $g_{\alpha}(z)$ which has no other singularity in the low-energy region.

Finally we may mention that independent investigations of the relativistic theory of meson scattering by means of the dispersion relations generally uphold the validity of the above results in the low energy region.

\section{The theory of photo-meson production ${ }^{1}$}

Chew's cut-off theory has been successfully applied to the problem of production of a meson of momentum $q$, by a photon of momentum $k$ impinging on a static nucleon. The matrix element for the process is

$$
H_{k}(q)=\left(\psi_{q}^{(-)}, \int \mathrm{d} v\left(\mathbf{j} \cdot \boldsymbol{A}_{k}\right)_{i}^{*} \psi_{0}\right)
$$

where $\mathbf{i}$ the current density operator of the matter system is determined by the gauge-transformations of the field variables. ${ }^{2}$ This leads to the conventional

1 G. F. CHEw, "The theory of pion scattering and photo production", Handbuch der Physik Springer-Verlag loc. cit. G. F. CHew and F. E. Low, Phys. Rev. 101, 1579 (1956)

2 This means that we have to replace the $\nabla$ operator wherever it occurs in the Hamiltonian by $\nabla-e A$. The interaction term in the $\gamma_{5}$ theory (i.e. with pseudoscalar coupling) does not obviously contribute to the current density operator while the corresponding term in the static theory does, because of the presence of the gradient. 
division of $\mathbf{j}$ into three parts corresponding to the "meson current", "nucleon current" and the "interaction current". But in Chew's treatment, as we want to lay emphasis on the difference between $\psi_{0}$ the single physical nucleon state and the excited states, which contain one or more real mesons, the current density operator is split as

$$
\mathbf{j}=\mathbf{j}_{N}+\mathbf{j}_{\pi} .
$$

where $\mathbf{j}_{N}$ is defined to be independent of the meson variables, but has the same matrix elements as the total current operator between the single physical nucleon states $\psi_{0} \cdot \mathbf{j}_{x}$ is defined negatively as $\mathbf{j}-\mathbf{j}_{N}$ and has the property

$$
\begin{aligned}
\left(\psi_{0}, \dot{\mathfrak{j}}_{\imath t} \psi_{0}\right) & =0 \\
{\left[a_{q}, \mathbf{j}_{\pi}\right] } & =\left[a_{q}, \mathbf{i}\right] ;\left[a_{q}^{\dagger}, \mathbf{j}_{\pi}\right]=\left[a_{q}^{\dagger}, \mathbf{j}\right]
\end{aligned}
$$

Thus it has matrix elements connecting $\psi_{0}$ to only states containing one or more real mesons. The matrix element for the interaction of a slow-moving photon with a fixed nucleon can be written as

$$
\left(\psi_{0}^{\beta}, \int \dot{j} \cdot \boldsymbol{A} \mathrm{d} r \psi_{0}^{\alpha}\right)=\left(u^{\beta},-\mu_{r} \sigma \cdot \Delta \times \boldsymbol{A} u^{\alpha}\right)
$$

where $u^{\beta, \alpha}$ are "bare" nucleon wave functions and $\mu_{r}$ the static magnctic moment of the nucleon which can be written as $\mu_{r}=\left[\frac{\mu_{p}+\mu_{n}}{2}+\frac{\mu_{p}-\mu_{n}}{2} \tau_{3}\right] \frac{e_{r}}{2 m}$ where $\mu_{p}=2.78$ and $\mu_{n}=-1.91$ are the magnetic moments of the proton and neutron respectively. For large photon momentum $k$ however we have to write

$$
\mu_{r}\left(k^{2}\right)=\frac{e_{r}}{2 m}\left[\frac{\mu_{p}+\mu_{n}}{2} F_{s}\left(k^{2}\right)+\frac{\left(\mu_{p}-\mu_{n}\right)}{2} \tau_{3} F_{v}\left(k^{2}\right)\right]
$$

where $F_{s}\left(k^{2}\right)$ and $F_{v}\left(k^{2}\right)$ are the magnetic moment form factors similar to the ones used in electron scattering theories. We have a further division of $\mathbf{j}_{N}$ into an isotopic vector part $\mathbf{j}_{v}$ involving the isotopic spin matrix $\tau_{3}$ and an isotopic scalar part $\mathbf{j}_{\delta}$. The matrix element for the former is given by

$$
\begin{aligned}
H_{k}^{v}(q) & =\left(\psi_{q}^{(-)}, \frac{A \tau_{3}}{(2 k)^{1 / 2}} i \sigma \cdot(\boldsymbol{k} \times \lambda) F_{v}\left(k^{2}\right) \psi_{0}\right) \\
& =\frac{e_{r}}{f_{r}} \frac{\mu_{p}-\mu_{n}}{4 m} \frac{F_{v}\left(k^{2}\right)}{v\left(q^{\prime}\right)}\left(\frac{\omega_{q^{\prime}}}{r}\right)^{1 / 2} T_{q^{\prime}}(q) .
\end{aligned}
$$

Here $\boldsymbol{\lambda}$ is the polarization vector of the photon and

$$
A=\frac{\left(\mu_{p}-\mu_{n}\right)}{2} \frac{e_{r}}{2 m}
$$

where $v\left(q^{\prime}\right)$ and $T^{q^{\prime}}(q)$ denote the same quantities as in the section on scattering. This simple result which means that $\dot{\mathfrak{j}}_{v}$ generates neutral mesons of momentum $\boldsymbol{q}^{\prime}=(\boldsymbol{k} \times \boldsymbol{\lambda})$ which are then scattered by the nucleon, does not follow from the 
general theory of scattering, but is a chance result. The matrix element due to $\mathbf{j}_{s}$ is not so simply related to the scattering amplitude. It is given by

$$
\begin{aligned}
H_{k}^{(s)}(q) & =\left(\psi_{q}^{(-)} ; \int \mathrm{d} v \mathbf{j}_{s} \cdot \boldsymbol{A}_{k} \psi_{0}\right) \\
& =-\sum_{n}\left\{\frac{T_{q}^{+}(n) H_{k}^{(s)}(n)}{E_{n}-\omega_{q}-i \varepsilon}+\frac{H_{k}^{(s) \dagger}(n) T_{q}(n)}{E_{n}+\omega_{q}}\right\}
\end{aligned}
$$

which is the Low equation for photo-meson production. This part turns out to be small compared to the previous one as it has a factor $\mu_{p}+\mu_{n} \approx 0.9$ nuclear magnetons whereas $\mu_{p}-\mu_{n}=4.7$.

Finally the operator $\dot{j}_{\pi}$ gives the matrix element

where

$$
H_{k}^{\pi}(q)=H_{k}^{(0)}(q)-\sum_{n}\left\{\frac{T_{q}^{\dagger}(n) H_{k}^{(\pi)}(n)}{E_{n}-\omega_{q}-i \varepsilon}+\frac{H_{k}^{(\pi) \dagger}(n) T_{q}(n)}{E_{n}+\omega_{q}}\right\}
$$

$$
H_{k}^{(0)}(q)=\frac{i e_{r} f_{r}}{\left[4 \omega_{q} k\right]^{1 / 2}} \frac{\left[\tau_{q} \tau_{3}-\tau_{3} \tau_{q}\right]}{2}\left[\sigma \cdot \lambda-\frac{2 \sigma \cdot(\boldsymbol{q}-\boldsymbol{k})(\boldsymbol{q} \cdot \lambda)}{(\boldsymbol{q}-\boldsymbol{k})^{2}+1}\right] .
$$

"The first term of $H_{k}^{(0)}(q)$ is a pure $S$-wave production term which depends only on the parameter $f_{r}$, a result known as the Kroll-Ruderman theorem. ${ }^{1}$ The value for $f^{2}=f_{r}^{2} / 4 \pi$ thus got is 0.074 in satisfactory agreement with the value got from scattering.

Predominant contributions to pion photoproduction comes from the terms $H_{k}^{(v)}(q)$ and $H_{k}^{(0)}(q)$. As $H_{k}^{(0)}(q)$ vanishes for the case of $\pi^{0}$ production we can directly test the isotopic vector part $H_{k}^{(v)}(q)$ which makes the major contribution to the neutral $P$-wave amplitude. Using the measured total cross-section of $\pi^{0}$ at a photon energy of $325 \mathrm{MeV}$ where there is resonance, the value of $c_{0}=\frac{\mu_{p}-\mu_{n}}{4 m} \frac{e}{f}$ is found to be $0.05 \pm 0.003$ which compares with the theoretical value $c_{0}=0.06$. Amplitudes of the form $H_{k}^{(v)}(q)$ lead to the characteristic $\left(2+3 \sin ^{2} \theta\right)$ dependence of the differential cross-section on the angle between the photon direction and the direction of the emitted meson. Further, the energy dependence for the total cross-section is correctly reproduced.

The total cross-section for charged mesons is very closely the contribution from $H_{k}^{(v)}(q)$ which is $P$-wave, and the first term of $H_{k}^{(0)}(q)$ which is $S$-wave. Hence if we substract the $S$-wave part from the measured total cross-section at $325 \mathrm{MeV}$ photon laboratory energy we may test the $P$-wave part of the charged meson production predicted by $H_{k}^{(v)}(q)$. The result is a value of 0.046 for $c_{0}$. Further, the interference of $H_{k}^{(v)}(q)$ and the $S$-wave part of $H_{k}^{(0)}(q)$ should produce preferentially backward positive photo-pions, and such an asymmetry is actually observed.

The problem of the production of a pair of mesons by a photon has also been studied using the Chew-Low method. ${ }^{2}$ A good agreement is obtained with the experimental cross-sections available.

1 N. M. Kroll and M. Ruderman, Phys. Rev. 93, 233 (1954).

2 See, e.g. R. E. Cutkosky and F. Zachariasen, Phys. Rev. 103, 1108 (1957). S. K. Srinivasan and K. Venkatesan, Nucl. Phys. 12, 418 (1959). 
Finally we may mention that Chew's method has been applied to other problems like the anomalous magnetic moment of the nucleon, the theory of nuclear forces and the scattering of $K$-mesons. Miyazawa's ${ }^{1}$ calculations give meson contributions of \pm 2 nuclear magnetons (approximately) to the proton and neutron anamalous magnetic moments respectively. A nuclear potential with a repulsive core was obtained (Gartenhaus) ${ }^{2}$ by using this theory. The width of the core was found to depend on the energy cut-off and the coupling constant. This potential has been used recently as the starting point for further work in the problem of nuclear forces. ${ }^{3}$ It may also be mentioned that the use of Chew-Low amplitudes to the problem of scattering and photoproduction of pions from deuterons ${ }^{4}$ yields results in good agreement with experiments in the low energy region.

1 H. Miyazawa, Phys. Rev. 104, 1741 (1956).

2 S. Gartenhaus, Phys. Rev. 100, 900 (1955).

3 P. S. Signell and R. E. Marshak, Phys. Rev. 109, 692 (1958).

4 V. Devanathan and G. Ramachandran, Nucl. Phys. 23, 312(1961). A. Ramakrishnan, V. Devanathan and G. Ramachandran, Nucl. Phys. 24, 163 (1961). A. Ramakrishnan, V. Devanathan and K. Venkatesan, Nucl. Phys. (in press). 


\section{NON-PERTURBATIVE APPROACH TO QUANTUM FIELD THEORY}

WE HAVE seen in an earlier chapter that in quantum electrodynamics the prescriptions for mass and charge renormalizations are sufficient for obtaining finite results for all observable quantities in every approximation of the perturbation expansion of the $S$-matrix. If we could further show that the series so obtained is convergent, we would have a satisfactory theory. Investigations of certain simplified models of a quantized field theory have shown however that for these models the series actually diverges even after renormalization has been carried out and hence we can expect a similar situation in quantum electrodynamics also. ${ }^{1}$ Hence it has been found desirable to develop a procedure for renormalization of mass and charge without recourse to perturbation expansions. Kallen ${ }^{2}$ has carried out such an investigation and found that if quantum electrodynamics is a consistent theory it contains at least one infinite renormalization constant.

The situation is much worse in meson theory where the meson-nucleon coupling constant $g^{2}$ is not small $\left(g^{2} \approx 15\right)$ and hence a power series expansion in terms of it is bound to be invalid.

Lehmann, Symanzik and Zimmermann ${ }^{3}$ made a non-perturbative approach to the problem of strongly coupled fields using only renormalized quantities. The main merit of their approach is that they do not make use of any specific theory of interaction as given by a Hamiltonian but base their theory on certain postulates which are of quite general validity. These postulates which refer to the field operators are the following:

(1) The field operators shall have correct transformation properties under the inhomogeneous Lorentz transformations, both proper and improper, such that the theory is invariant with respect to such transformations.

(2) They obey the principle of microscopic causality. The relativistic statement of causality is that events at different space-time points cannot affect each other unless they can be connected by a light signal. Events at points having a spacelike separation cannot affect each other. Since in quantum mechanics, the non-

1 C. A. Hurst, Proc. Camb. Phil. Soc. 48, 625 (1952). W. Thirring, Helv. Phys. Acta 26, 33 (1953). A. Petermann, Phys. Rev. 89, 1160 (1953). Arch. Sci. Phys. Nat. 6, 5 (1953).

2 G. Kallen, "Quantum Electrodynamics", Handbuch der Physik, Vol. 5, Part I, SpringerVerlag (1958).

3 H. Lehmann, K. SymanziK and W. Zimmermane, Nuovo Cim. 1, 205 (1955). See also H. Lehmann, Nuovo Cim., Supplement series X, 14, No. 1, 153 (1959). 
interference between the simultaneous measurement of two dynamical variables is expressed by the vanishing of the commutator between them, the principle of microscopic causality is imbedded in the statement that the commutator (anti-commutator in the case of fermions) ${ }^{1}$ of two Heisenberg operators taken at two space-time points should vanish for space-like separation of the two points, i.e.

$$
[A(x), A(y)]=0,(x-y)^{2}>0 .
$$

(3) The field operators satisfy the asymptotic condition, viz. that the field operator $A(x)$ shall go over for $x_{4} \rightarrow-\infty$ and $x_{4} \rightarrow+\infty$ into the free field operators $A_{\text {in }}(x)$ and $A_{\text {out }}(x)$ respectively which obey the free field equations but with the physical masses of the particles appearing in them. ${ }^{2}$

The asymptotic condition restricts the theory to only such local fields which do not allow bound states. Only the creation, annihilation or the scattering of particles are envisaged. To formulate the asymptotic condition suitably, we expand the operator $A(x)$ using a discrete set of wave functions $f_{\alpha}(x)$

$$
A(x)=\sum_{\alpha}\left\{f_{\alpha}(x) A^{\alpha}(x)+f_{\alpha}^{*}(x) A^{\alpha \dagger}(x)\right\}
$$

where the $f_{\alpha}(x)$ satisfy the free field equation

$$
\left(\square-\mu^{2}\right) f(x)=0 \quad \text { and } \quad-i \int\left\{f \frac{\partial f^{*}}{\partial x_{4}}-f^{*} \frac{\partial f}{\partial x_{4}}\right\} \mathrm{d}^{3} x=1 .
$$

From (2) we have

$$
\begin{aligned}
A_{\alpha}(x) & =i \int \mathrm{d}^{3} x A(x) \cdot \frac{\overleftrightarrow{\partial}}{\partial x_{4}} f_{\alpha}(x) \\
& =i \int \mathrm{d}^{3} x\left\{A(x) \frac{\partial}{\partial x_{4}} f_{a}(x)-\frac{\partial}{\partial x_{4}} A(x) f_{\alpha}(x)\right\}
\end{aligned}
$$

with $A_{\text {in }}^{\alpha}(x)$ and $A_{\text {out }}^{\alpha}(x)$ defined correspondingly. The asymptotic condition for the field operators can now be written in the form

$$
\lim _{x_{4} \rightarrow \pm \infty}\left\langle\Phi\left|A^{\alpha}\left(x_{4}\right)\right| \Psi\right\rangle=\left\langle\Phi\left|A_{\left(\begin{array}{c}
\text { out } \\
\text { in }
\end{array}\right)}^{\alpha}\right| \Psi\right\rangle
$$

for arbitrary $\Phi$ and $\Psi$.

Hence

and

$$
\left\langle 0 \mid A(x) \underset{\text { out }}{\mid \alpha_{\text {in }}}\right\rangle=\left\langle 0\left|A_{\text {in }}(x)\right| \underset{\text { out }}{\alpha_{\text {in }}}\right\rangle=f_{\alpha}(x)
$$

$$
\langle 0|A(x)| 0\rangle=\left\langle 0\left|A_{\text {in }}(x)\right| 0\right\rangle=0
$$

1 At first sight this looks as if fermion fields were acausal but the apparent contradiction is removed by noting that the fermion field operators always appear in bilinear combinations, for all observables. Two such combinations taken at different space-time points can be shown to commute with each other.

2 See chapter III, where however the point of view is taken that the Heisenberg fields in the remote past and future coincide with the in and out fields only up to certain constant multiplicative factors (the renormalization constants).

3 In this chapter we again revert to the metrix, $\delta_{11}=\delta_{22}=\delta_{33}=-\delta_{44}=1$ to be in conformity with the literature on the subject. 
Using the asymptotic condition, the scattering matrix element can be reduced to the vacuum expectation value of products of Heisenberg operators and this reduction is necessary since we can impose the causality condition only on the field operators. Consider the scattering of a particle ${ }^{1}$ from a state of momentum $k$ to a state of momentum $k^{\prime}$ while the target, changes its momentum from $p$ to $p^{\prime}$. The $S$ matrix element for the process is given by

$$
\begin{aligned}
\left\langle p^{\prime} k_{\text {in }}|S| p k_{\text {in }}\right\rangle= & \left\langle p^{\prime} k_{\text {out }}^{\prime} \mid p k_{\text {in }}\right\rangle=\left\langle p^{\prime}\left|a_{\text {out }}^{k^{\prime}}\right| p k_{\text {in }}\right\rangle \\
= & \lim _{x_{4} \rightarrow+\infty}\left\langle p^{\prime}\left|A\left(\boldsymbol{k}^{\prime}, x_{4}\right)\right| p k_{\text {in }}\right\rangle \\
= & \int_{-\infty}^{\infty} \mathrm{d} x_{4} \frac{\partial}{\partial x_{4}}\left\langle p^{\prime}\left|A\left(\boldsymbol{k}^{\prime}, x_{4}\right)\right| p k\right\rangle+ \\
& +\lim _{x_{4} \rightarrow-\infty}\left\langle p^{\prime}\left|A\left(\boldsymbol{k}^{\prime}, x_{4}\right)\right| p k_{\text {in }}\right\rangle,
\end{aligned}
$$

where $a_{\left(\begin{array}{c}\text { out } \\ \text { in }\end{array}\right)}\left(k^{\prime}\right)$ is the annihilation operator for an outgoing (incoming) particle with the commutation relations

and

$$
\begin{gathered}
{\left[a_{\left(\begin{array}{c}
\text { in } \\
\text { out }
\end{array}\right)}(k), a_{\left(\begin{array}{c}
\text { in } \\
\text { out }
\end{array}\right)}^{\dagger}\left(k^{\prime}\right)\right]=2 k_{4} \delta\left(\boldsymbol{k}-\boldsymbol{k}^{\prime}\right)} \\
{\left[a_{\left(\begin{array}{c}
\text { in } \\
\text { out }
\end{array}\right)}(k), a_{\left(\begin{array}{c}
\text { in } \\
\text { out }
\end{array}\right)}\left(k^{\prime}\right)\right]=0}
\end{gathered}
$$

Now

The first term becomes

$$
a_{\text {in }}(k)=A_{\text {in }}(k) ; \quad a_{\text {in }}^{\dagger}(k)=A_{\text {in }}(-k) \quad \text { for } \quad k_{4}>0 .
$$

$$
\left\langle p^{\prime}\left|a_{\text {in }}\left(k^{\prime}\right)\right| p k_{\text {in }}\right\rangle=\left\langle p^{\prime} k_{\text {in }}^{\prime} \mid p k_{\text {in }}\right\rangle \text {. }
$$

$$
\begin{aligned}
& i \int \mathrm{d}^{3} x \frac{\partial}{\partial x_{4}}\left\{\left\langle p^{\prime}|A(x)| p k_{\mathrm{in}}\right\rangle \frac{\overleftrightarrow{\partial}}{\partial x_{4}} \cdot \frac{\exp \left[i k^{\prime} x\right]}{(2 \pi)^{3 / 2}}\right\} \\
= & i_{0} \int \mathrm{d}^{4} x \frac{\exp \left[-i k^{\prime} x\right]}{(2 \pi)^{3 / 2}}\left[\square_{x}-\mu^{2}\right]\left\langle p^{\prime}|A(x)| p k_{\mathrm{in}}\right\rangle
\end{aligned}
$$

after partial integration with respect to the space coordinates. The matrix element in the integrand can be evaluated by again applying the asymptotic condition

$$
\begin{aligned}
\left\langle p^{\prime}|A(x)| p k_{\mathrm{in}}\right\rangle= & \left\langle p^{\prime}\left|A(x) a_{\mathrm{in}}^{\dagger}(k)\right| p\right\rangle \\
= & \lim _{y_{4} \rightarrow-\infty}\left\langle p^{\prime}\left|A(x) A^{\dagger}\left(k, y_{4}\right)\right| p\right\rangle \\
= & \lim _{y_{4} \rightarrow-\infty}\left\langle p^{\prime}\left|T\left\{A(x) A^{\dagger}\left(\boldsymbol{k}, y_{4}\right)\right\}\right| p\right\rangle \\
= & -\int \mathrm{d} y_{4} \frac{\partial}{\partial y_{4}}\left\langle p^{\prime}\left|T\left\{A(x) A^{\dagger}\left(k, y_{4}\right)\right\}\right| p\right\rangle+ \\
& +\lim _{y_{4} \rightarrow+\infty}\left\langle p^{\prime}\right| T\left\{A(x) A^{\dagger}\left(k, y_{4}\right)|p\rangle\right.
\end{aligned}
$$

1 Throughout this chapter, we assume both the target and scattered particles are scalar unless otherwise stated.

2 Notice the difference in normalization between this and of the creation and annihilation operators defined in chapter III. 
The last term gives

$$
\left.\left\langle p^{\prime}\left|a_{\text {out }}^{\dagger}(k) A(x)\right| p\right\rangle=2 k_{4} \delta\left(\boldsymbol{p}^{\prime}-\boldsymbol{k}\right)<0|A(x)| p\right\rangle
$$

which vanishes when $\left(\square_{x}-\mu^{2}\right)$ operates on it. The other term leads to

Hence

$$
i \int \mathrm{d}^{4} y \frac{\exp (i k y)}{(2 \pi)^{3 / 2}}\left(\square_{y}-\mu^{2}\right)\left\langle p^{\prime}|T\{A(x) A(y)\}| p\right\rangle \text {. }
$$

$$
\begin{aligned}
& \left\langle p k_{\text {out }}^{\prime} \mid p k_{\text {in }}\right\rangle-\left\langle p^{\prime} k_{\text {in }}^{\prime r} \mid p k_{\text {in }}\right\rangle \\
& =\frac{1}{(2 \pi)^{3}} \int \mathrm{d}^{4} x \mathrm{~d}^{4} y \exp -\left[i k^{\prime} x-i k y\right] \\
& \left(\square_{x}-\mu^{2}\right)\left(\square_{y}-\mu^{2}\right)\left\langle p^{\prime}|T\{A(x) A(y)\}| p\right\rangle .
\end{aligned}
$$

To express the scattering amplitude in terms of the retarded product defined by

$$
R(A(x) A(y))=-i \theta\left(x_{4}-y_{4}\right)[A(x), A(y)]
$$

where $\theta\left(x_{4}-y_{4}\right)$ is the Heaviside function, we again start from (13)

$$
\begin{aligned}
\left\langle p^{\prime}|A(x)| p k_{\text {in }}\right\rangle= & \left\langle p^{\prime}\left|A(x) a_{\text {in }}^{\dagger}(k)\right| p\right\rangle=\left\langle p^{\prime}\left|\left[A(x), a_{\text {in }}^{\dagger}(k)\right]\right| p\right\rangle+ \\
& +\left\langle p^{\prime}\left|a_{\text {in }}^{\dagger}(k) A(x)\right| p\right\rangle .
\end{aligned}
$$

The last term gives $2 k_{4} \delta\left(\overrightarrow{\boldsymbol{p}}^{\prime}-\boldsymbol{k}\right)\left\langle 0|A(x)| p^{\prime}\right\rangle$ which again vanishes on applying $\left(\square x-\mu^{2}\right)$. Hence we obtain

$$
\left\langle p^{\prime}\left|\left[A(x), a_{\text {in }}^{\dagger}(k)\right]\right| p\right\rangle=\lim _{y_{4} \rightarrow-\infty}\left\langle p^{\prime}\left|\left[A(x) ; A^{*}\left(\boldsymbol{k}, y_{4}\right)\right]\right| p\right\rangle .
$$

There is no boundary term for $y_{4} \rightarrow+\infty$ since the retarded product vanishes if $y_{4}>x_{4}$. Proceeding as before we get

$$
\begin{aligned}
\left\langle p^{\prime} k_{\text {out }}^{\prime} \mid p k_{\text {in }}\right\rangle= & \left\langle p^{\prime} k_{\text {in }}^{\prime}|S| p k_{\text {in }}^{\prime}\right\rangle \\
= & \frac{-i}{(2 \pi)^{3}} \int \mathrm{d}^{4} x \mathrm{~d}^{4} y \exp -\left[i k^{\prime} x-i k y\right] \times \\
& \times\left(\square_{x}-\mu^{2}\right)\left(\square_{y}-\mu^{2}\right)\left\langle p^{\prime}|R(A(x) A(y))| p\right\rangle .
\end{aligned}
$$

We can continue to take particles out of the state vectors and finally reduce the scattering matrix to the vacuum expectation values of time-ordered or retarded products of Heisenberg operators.

Though the scattering element can be expressed in terms of either time-ordered or retarded products, it turns out that the Feynman amplitude (namely, that which involves the $T$ products) does not have such convenient analytic properties as the retarded amplitude (which is expressed in terms of the retarded products) since the latter is the Fourier transform of a function vanishing outside the forward light cone which, as we shall see presently, enables us to write dispersion relations between the real (dispersive) and imaginary (absorptive) parts of the scattering amplitude. But before discussing the general mathematical and physical premises from which such relations can be derived we shall outline the derivation of the dispersion relations for the forward scattering of a meson by a nucleon, due to 
Goldberger $^{1}$ who established such relations as algebraic identities between the absorptive and dispersive parts of the retarded amplitude, the division being made according to whether real or virtual intermediate states are involved, i.e. whether or not conservation laws are satisfied in the intermediate as well in the initial and final states.

To realise that there should exist a relationship between the absorptive and dispersive parts, we can adopt the following general argument. ${ }^{2}$ The absorptive part of the forward scattering amplitude at a particular energy arises through the real intermediate states and can be found directly from a knowledge of the total cross-section for incident particles of that energy by using the optical theorem. On the other hand, the dispersive part arises from virtual intermediate states the energies of which are not equal to the initial energy. Such intermediate states contribute to the absorptive part for an initial state with the corresponding energy. This energy can take any value and therefore the dispersive part should be an integral, over all energies, of the total cross-section. This is precisely the dispersion relation.

Consider the forward scattering of a boson of four momentum $q$ and isospin index $\beta$ which is scattered into a state with four-momentum $k$ and isospin index $\alpha$ while the target nucleon goes from the state $(p, \lambda)$ to the state $\left(p^{\prime}, \lambda^{\prime}\right)$ where $p$ and $p^{\prime}$ are the four momenta and $\lambda$ and $\lambda^{\prime}$ represent the spin and isospin variables of the initial and final nucleon respectively. The retarded amplitude can be written as

where

$$
M_{\alpha \beta}\left(\omega, \lambda^{\prime} \lambda\right)=D_{\alpha \beta}\left(\omega \lambda^{\prime} \lambda\right)+i \varepsilon(\omega) A_{\alpha \beta}\left(\omega \lambda^{\prime} \lambda\right)
$$

$$
\begin{aligned}
D_{\alpha \beta}\left(\omega ; \lambda^{\prime} \lambda\right)= & \frac{i}{2} \int \mathrm{d}^{4} x \mathrm{e}^{i \omega x_{4}} \cos (\boldsymbol{k} \cdot \boldsymbol{x})<p^{\prime} \lambda^{\prime} \mid \varepsilon\left(x_{4}\right)\left[\mathrm{j}_{\alpha}(x) \dot{j}_{\beta}(0)\right]- \\
& -2 \delta\left(x_{4}\right)\left[\dot{\mathrm{j}}_{\alpha}(x), \dot{\varphi}_{\beta}(0)\right]|p \lambda\rangle
\end{aligned}
$$

and

$$
A_{\alpha \beta}\left(\omega ; \lambda^{\prime} \lambda\right)=\frac{1}{2} \int \mathrm{d}^{4} x \mathrm{e}^{i \omega x_{4}} \cos (\boldsymbol{k} \cdot \boldsymbol{x}) \cdot\left\langle p^{\prime} \lambda^{\prime}\left|\left[\mathrm{j}_{\alpha}(x), \mathrm{j}_{\beta}(0)\right]\right| p \lambda\right\rangle
$$

where $\varepsilon\left(x_{4}\right)$ is the sign function which is connected to the step function in the retarded product by $\theta\left(x_{4}\right)=\frac{\left[1+\varepsilon\left(x_{4}\right)\right]}{2}$. The $j$ 's are the usual meson current operators in the Heisenberg representation. Splitting $D$ and $A$ further into parts symmetric and anti-symmetric under interchange of $\alpha$ and $\beta$ (denoted respectively by the superscripts 1 and 2) and introducing a complete set of intermediate states, it has been demonstrated by Goldberger that

$$
\begin{aligned}
I_{\alpha \beta}\left(\omega_{1} \omega_{0} \lambda^{\prime} \lambda\right)= & \frac{\omega^{2}-\omega_{0}^{2}}{\pi} P \int_{-\infty}^{\infty} \mathrm{d} \omega^{\prime} \frac{A_{\alpha \beta}^{(1)}\left(\omega^{\prime} \lambda^{\prime} \lambda\right)+i A_{\alpha \beta}^{(2)}\left(\omega^{\prime} \lambda^{\prime} \lambda\right)}{\left(\omega^{\prime}-\omega_{0}\right)\left(\omega^{\prime 2}-\omega^{2}\right)} \\
= & D_{\alpha \beta}^{(1)}\left(\omega \lambda^{\prime} \lambda\right)-D_{\alpha \beta}^{(1)}\left(\omega_{0} \lambda^{\prime} \lambda\right)+ \\
& +i\left[\left(\frac{\omega_{0}}{\omega}\right) D_{\alpha \beta}^{(2)}\left(\omega \lambda^{\prime} \lambda\right)-D_{\alpha \beta}^{(2)}\left(\omega_{0} \lambda^{\prime} \lambda\right)\right] .
\end{aligned}
$$

1 M. L. Goldberger, Phys. Rev. 99, 979 (1955).

2 J. S. Toll, Phys. Rev. 104, 1760 (1956). 
The generalized dispersion relations (appropriate for the scattering of particles with mass) followed from this; but as this derivation is not considered rigorous, we shall proceed to a study of dispersion relations from a more deductive point of view based on certain general principles and prescriptions.

\section{Single variable dispersion relations}

Single variable dispersion relations connect the real and imaginary parts of the scattering amplitude considered as a function of a complex variable which can be either the energy or the momentum transfer. They can be derived from the causality condition which imposes certain restrictions on the scattering matrix elements from which the dispersions follow either by the application of the Cauchy integral theorem or by a theorem due to Titchmarsh. Here we start from Cauchy's theorem which states that an analytic function $f(z)$ of the complex variable $z$ can be represented as an integral over a closed contour $C$ enclosing the region $R$ within which $f(z)$ is analytic.

$$
\begin{aligned}
\oint \frac{f\left(z^{\prime}\right)}{z^{\prime}-z} \mathrm{~d} z^{\prime} & =2 \pi i f(z) \text { if } z \text { lies inside } C \\
& =0 \quad \text { if } z \text { lies outside } C .
\end{aligned}
$$

If $z$ lies on the contour, $f(z)$ can be represented by the principal value integral

$$
f(z)=\frac{1}{\pi i} P \oint \frac{f\left(z^{\prime}\right)}{z^{\prime}-z} \mathrm{~d} z^{\prime}
$$

If $z$ lies on the real axis, the contour can be chosen to be the real axis itself and a semicircle with infinite radius in the upper half-plane. If the contribution to the integral from this semicircle vanishes as the radius tends to $\infty$, we obtain

$$
f(z)=\frac{P}{i \pi} \int_{-\infty}^{\infty} \frac{f\left(z^{\prime}\right)}{z^{\prime}-z} \mathrm{~d} z^{\prime} .
$$

This immediately implies, on taking the real and imaginary parts on both sides

$$
\operatorname{Re} f(z)=\frac{1}{\pi} P \int_{-\infty}^{\infty} \frac{\operatorname{Im} f\left(z^{\prime}\right)}{\left(z^{\prime}-z\right)} \mathrm{d} z^{\prime} .
$$

which is exactly the dispersion relations if $f(z)$ represents the scattering amplitude as a function of energy or momentum transfer. Thus the validity of this relation depends upon the analyticity of the function $f(z)$ in the upper half-plane and the vanishing of the contribution from the semicircle, i.e. we have to carry out an investigation of the analytic properties of the scattering amplitudes and the possibility of an analytic continuation of these into the upper half-plane. If such a continuation is possible and the resulting function $f(E)$ as a function of energy $E$, falls off not slower than a constant $(|E|)^{-1}$ as $|E| \rightarrow \infty$ we see that the contribution 
from the infinite semicircle over the real axis (taking the contour to be the real axis and the infinite semicircle in the upper half-plane) will be zero and hence we will have

or

$$
\begin{gathered}
f(E)=\frac{P}{\pi i} \int_{-\infty}^{+\infty} \frac{f\left(E^{\prime}\right)}{E^{\prime}-E} \mathrm{~d} E^{\prime} \\
\operatorname{Re} f(E)=\frac{P}{\pi} \int_{-\infty}^{+\infty} \frac{\operatorname{Im} f\left(E^{\prime}\right)}{E^{\prime}-E} \mathrm{~d} E^{\prime}
\end{gathered}
$$

which will be the required dispersion relation. But we cannot always expect the scattering amplitude to be sufficiently bounded. If $f(E)$ has a pole of $n$-th order at $\infty$, then $\frac{f(E)}{\left(E-E_{0}\right)^{n+1}}$ will be an analytic function in the upper half-plane and we get instead

$$
\begin{aligned}
\operatorname{Re} f(E)= & \frac{\left(E-E_{0}\right)^{n+1}}{\pi} P \int_{-\infty}^{\infty} \frac{\operatorname{Im} f\left(E^{\prime}\right) \mathrm{d} E^{\prime}}{\left(E^{\prime}-E\right)\left(E^{\prime}-E_{0}\right)^{n+1}}+ \\
& +\operatorname{Re} f\left(E_{0}\right)+\cdots+\frac{\operatorname{Re} f^{n}\left(E_{0}\right)\left(E-E_{0}\right)^{n}}{n !} \quad\left(-\infty<E, E_{0}<\infty\right),
\end{aligned}
$$

where $f^{(n)}$ denotes the $n$-th derivative of $f\left(E_{0}\right)$. From (28) we can immediately write down the dispersion relations for any process provided we know the analytic behaviour of the scattering matrix element as a function of energy. The analyticity of $\frac{f(E)}{\left(E-E_{0}\right)^{n+1}}$ at $\infty$ introduces a pole of order $n+1$ at $E_{0}$. Using the properties of $f(E)$, the integration from $-\infty$ to $\infty$ can be replaced by one from 0 to $\infty$ over the entire range of energy from 0 to $\infty$. Since particles of finite mass cannot take energies less than the rest energy, the above equation when applied to particles with finite mass will involve integration both over the physical region (where the energy is $>$ the rest energy) and the unphysical region (where the energy $E$ lies in the interval, $0<E<$ rest energy). Of course we are confining our statements to scattering processes and the situation is more complicated when we discuss processes involving more particles. As an example, let us consider the forward scattering of light taking $f(E)$ to be the refractive index of light in an absorbing medium. Setting in (28) $n=0, E_{0}=0$ (since the photon has no mass) and $\operatorname{Im} f\left(E^{\prime}\right)=-\operatorname{Im} f\left(-E^{\prime}\right)$ we get the Kramers-Kronig ${ }^{1}$ formula

$$
\operatorname{Re}[n(\omega)-n(0)]=\frac{2}{\pi} P \int_{0}^{\infty} \frac{\omega^{2} \operatorname{Im} n\left(\omega^{\prime}\right)}{\omega^{\prime}\left(\omega^{\prime 2}-\omega^{2}\right)} \mathrm{d} \omega^{\prime} .
$$

${ }^{1}$ H. A. Kramers, Atti. Cong. Inter. Fisic. Comt. 2, 545 (1927). R. Kronig, J. Opt. Soc. Amer. 12, 547 (1926). 
As another example, consider the case of the forward scattering of a neutral pion by a nucleon for which Karplus and Ruderman ${ }^{1}$ assume that the scattering amplitude, $f(E)$ is an analytic function in the upper half-plane and that its pole at infinity is of an order not higher than the first. Since the neutral meson field is real, we have $\operatorname{Im} f(-E)=\operatorname{Im} f(E)$. Also for forward scattering we can use the optical theorem $\operatorname{Im} f(E)=\frac{k}{4 \pi} \sigma$ where $k$ is the momentum of the meson and $\sigma$ the total scattering cross-section. Hence setting $n=1$ in (28), changing over to momentum as the variable and excluding the non-observable region $0 \leqq E \leqq \mu$ we get

$\operatorname{Re}\left[f(|\boldsymbol{k}|)-f\left(\left|\boldsymbol{k}_{0}\right|\right)\right]=\frac{1}{(2 \pi)^{2}} \int_{0}^{\infty} \sigma\left(k^{\prime}\right) \mathrm{d} k^{\prime} \times\left\{\frac{|\boldsymbol{k}|^{2}}{\left|\boldsymbol{k}^{\prime}\right|^{2}-|\boldsymbol{k}|^{2}}-\frac{\left|\boldsymbol{k}_{0}\right|^{2}}{\left|\boldsymbol{k}^{\prime}\right|^{2}-\left|\boldsymbol{k}_{0}\right|^{2}}\right\}$,

which is the dispersion relation for the forward scattering of a neutral pion by a nucleon.

The derivation of relations of the type (29) and (30) depends on the possibility of continuing the scattering matrix element $f(E)$ into the upper half plane and this is related to the principle of causality which therefore forms the physical basis of dispersion relations. To realise this let us suppose that $f(E)$ can be written as

$$
f(E)=\int_{-\infty}^{\infty} f(t) \mathrm{e}^{i E t} \mathrm{~d} t
$$

The condition of causality is imposed on $f(t)$ by specifying that $f(t)=0$ for $t<0$. Then

$$
\oint \frac{f\left(E^{\prime}\right)}{E^{\prime}-E} \mathrm{~d} E^{\prime}=\int_{-\infty}^{\infty} f(t) \mathrm{d} t \oint \frac{\dot{e}^{i E^{\prime} t}}{E^{\prime}-E} \mathrm{~d} E^{\prime}
$$

For $t>0$, the convergence of the integral is assured since when we pass to the upper half-plane, $f(E)$ will acquire the factor $\exp (-t|\operatorname{Im} E|)$. For $t<0$, the integral vanishes because of the causality condition. If on the other hand $f(t)$ vanishes only for $t<-a$ where a is some "elementary length" then by replacing $t$ by $t-a$ in the integrand of $f(E)$ we shall have

$$
f(E)=\mathrm{e}^{-i a E} f_{1}(E)
$$

where the function $f_{1}(E)$ will have no essential singularity at infinity while the factor $\mathrm{e}^{-i a E}$ will have such a singularity when $E \rightarrow \infty$. Hence if we wish to write a dispersion relation for this case we must multiply $f(E)$ by $\exp (i \alpha E)$ where $\alpha \geqq a$. Thus an experimental observation of a deviation from the usual type of dispersion relation will provide evidence for the necessity of a non-local theory.

1 R. Karplus and M. A. Ruderman, Phys. Rev. 98, 771 (1955). 
Goldberger's relations discussed earlier were found to hold in the physical region of energies but his expressions diverge for $E<\mu$. Oehme ${ }^{1}$ returned to the study of the analytical properties of the scattering amplitude and succeeded in carrying out the analytic continuation of the amplitude into the upper halfplane by assuming its quadratic integrability. This however is too restrictive an assumption for physical amplitudes in which improper functions such as the $\delta$-function can possibly occur. Recently however rigorous proofs of dispersion relations have been given for the case of the forward scattering of a boson with mass by Symanzik ${ }^{2}$ and for the scattering of such a particle at any angle by Bogoliubov ${ }^{3}$ et al. and Bremmerman et al. ${ }^{4}$ who use theorems in complex variable theory for their proofs. The difficulty involved in the latter approach can be seen from the following.

According to a theorem due to Wightman, Hall and Bargmann ${ }^{5}$ if a function $f\left(q_{1}, \ldots, q_{n}\right)$ of $n$-four vectors $q_{i}, \ldots, q_{n}$ is an analytic function of the variables $q_{i}$ and is invariant under both proper and improper Lorentz transformations, then $f\left(q_{1}, \ldots, q_{n}\right)$ is a function of the scalar products $q_{j}, q_{k}$ and is analytic in the corresponding domain of the variables $q_{j} \cdot q_{k}$ which will be $\frac{n(n-1)}{2}$ in number.

For the case of scattering of one particle by another, there will be four vectors and six scalar products involved. Functions of single complex variable are simple in that there exists for every domain $D$ a function $f(z)$ which is analytic only in that domain and hence cannot be continued outside $D$. However for functions of more than one complex variable any arbitrary domain will not in general be the domain of analyticity for an analytical function and there exists the possibility that all functions of many complex variables which are analytic in $D$ can be analytically continued simultaneously into larger domains. The largest such domain in which the class of functions are analytic is called the envelope of holomorphy $E(D)$ of the domain $D$. The problem of obtaining this is very difficult. Bremmermann et al. however succeeded in computing the holomorphy for a suitable sub-domain of the six complex variables involved in scattering and were also able to demonstrate enough analytic properties of the scattering amplitude to be able to prove the dispersion relations for $\pi-N$ scattering and for several other cases with restrictions on the momentum transfer or on the ratio of the masses of the scattering particle and target.

There is however another approach to the problem - that of obtaining an integral representation for the scattering amplitudes for real values of the arguments.

1 R. Oenue, Phys. Rev. 97, 1392 (1955).

2 K. Symanzik, Phys. Rev. 105, 743 (1957).

${ }^{3}$ See Introduction to Theory of Quantized Fields by N. N. Bogoliubov and D. V. Shrrkov, Interscience, New York (1959). Bogoliubov avoids the explicit use of the theory of several complex variables by employing parametrizations and using distribution methods.

4 Bremmermann, Oehme and Taylor, Phys. Rev. 109, 2178 (1958).

5 Wightman, Hall and Bargmann, K. Danske, Vidensk. Mat. Fys. Medd. 31, No. 5, (1957). 
The advantage of this approach is that the analytic continuation to complex values becomes trivial and the analytic properties of the scattering amplitude are readily apparent. 'This approach which would thus be the converse of the previous one, was used by Dyson ${ }^{1}$ who solved the problem of constructing a general integral representation for a scattering matrix element and by Lehmann ${ }^{2}$ who used the Dyson representation for studying the analytic properties of the scattering matrix element. We shall summarize the important steps and the results obtained.

The main objects of study are the vacuum expectation values of products of field operators whose structures are to be determined from the following general principles:

(1) Transformation properties of the operators under Lorentz transformations,

(2) The causality condition,

(3) The asymptotic condition,

(4) The absence of negative energy states,

(5) The spectral condition which states that all the physical states of the universe form a complete set of basic vectors in the quantum mechanical sense, each state being associated with a total energy-momentum vector which is positive time-like.

The integral representation for the two-fold vacuum expectation values of operators is easily derived, e.g.

can be written as

$$
\left\langle\varphi(x) \varphi\left(x^{\prime}\right)\right\rangle_{0}=\Delta^{(+\prime)}\left(x-x^{\prime}\right)
$$

$$
\Delta^{(+)^{\prime}}\left(x-x^{\prime}\right)=\frac{-i}{(2 \pi)^{3}} \int \theta\left(k_{4}\right) P\left(k^{2}\right) \mathrm{e}^{-i k\left(x-x^{\prime}\right)} \mathrm{d}^{4} k .
$$

The prime on $\Delta$ denotes that the operators are in the Heisenberg representation.

Here

$$
P\left(k^{2}\right)=(2 \pi)^{3} \sum_{n}\langle 0|\varphi(0)| n\rangle\langle n|\varphi(0)| 0\rangle .
$$

On using the integral representation for the free field function, $\Delta^{(+)}\left(x-x^{\prime}\right)$ we obtain the integral representation

$$
\Delta^{(+)^{\prime}}\left(x-x^{\prime}\right)=\int_{0}^{\infty} P\left(k^{2}\right) \Delta^{(+)}\left(x-x^{\prime}, k^{2}\right) \mathrm{d} k^{2} .
$$

The problem of finding an integral representation for the vacuum expectation value of products of three or more operators is difficult and has not been solved completely. Dyson however succeeded in solving the problem of an integral representation for the function

$$
f(x)=\langle Q|[A(x / 2), B(-x / 2)]| P\rangle
$$

1 F. J. Dyson, Phys. Rev. 110, 1460 (1958). See also V. Ya. Fainberg, JETP 36 (9), 1066 (1959).

2 H. Lehmann, Nuovo Cim. 10, 578 (1958). 
which is immediately connected with the matrix element of a scattering process. He showed that if the function $f(x)$ has the following properties:

$$
\begin{aligned}
& f(x)=0 \quad x^{2}>0 \quad \text { (causality condition) } \\
& f(q)=\int \mathrm{e}^{i q x} f(x) \mathrm{d}^{4} x=0 \text { unless } \\
& \left(\frac{P+Q}{2}+q\right)^{2}=l_{1}^{2} \geqq m_{1}^{2} ; \quad \frac{P_{4}+Q_{4}}{2}+q_{4} \geqq 0 \\
& \left.\left(\frac{P+Q}{2}-q\right)^{2}=l_{2}^{2} \geqq m_{2}^{2} ; \quad \frac{P_{4}+Q_{4}}{2}-q_{4} \geqq 0\right\} \text {, }
\end{aligned}
$$

or

where $m_{1}$ and $m_{2}$ are the smallest masses of the intermediate states for which

and

$$
\left\langle Q|A(0)| l_{1}\right\rangle \neq 0 ;\left\langle l_{1}|B(0)| P\right\rangle \neq 0
$$

$$
\left\langle Q|B(0)| l_{2}\right\rangle \neq 0 ;\left\langle l_{2}|A(0)| P\right\rangle \neq 0
$$

respectively, then $f(q)$ can be represented by

$$
f(q)=\int_{0}^{\infty} \mathrm{d} \chi^{2} \int \mathrm{d}^{4} u \Phi\left(\chi^{2}, u\right) \varepsilon(q-u) \delta\left[(q-u)^{2}-\chi^{2}\right]
$$

if and only if $\varphi\left(\chi^{2}, u\right)$ vanishes everywhere except for the region

$$
\begin{aligned}
\chi \geqq \max & \left\{0, m_{1}-\left|\frac{P+Q}{2}+u\right| ; \quad m_{2}-\left|\frac{P+Q}{2}-u\right|\right\} \\
& \left(\frac{P+Q}{2} \pm u\right)^{2} \geqq 0 .
\end{aligned}
$$

For the retarded product, this leads to the representation

$$
f_{R}(q)=-\frac{1}{2 \pi} \int \mathrm{d}^{4} u \int \mathrm{d} \chi^{2} \cdot \frac{\varphi\left(u, \chi^{2}\right)}{(q-u)^{2}-\chi^{2}} .
$$

Lehmann used the above integral representation for deriving the region of analyticity of the scattering amplitude and the restrictions on the momentum transfer for proving the dispersion relations for the scattering process

$$
p+k \rightarrow p^{\prime}+k^{\prime}
$$

the scattering amplitude for which is given by

where

$$
T=-\int \mathrm{d}^{4} x \exp \left[\frac{i\left(k+k^{\prime}\right) \cdot x}{2}\right]\left\langle p^{\prime}\left|R^{\prime}\left\{A\left(\frac{x}{2}\right) A\left(-\frac{x}{2}\right)\right\}\right| p\right\rangle
$$

$$
R^{\prime}\{A(x / 2) A(-x / 2)\}=\theta\left(x_{4}\right)[\mathrm{j}(x / 2) \mathrm{j}(-x / 2)] .
$$

This is defined not only on the energy shell $k^{2}=k^{2}=\mu^{2}$ but for arbitrary real vectors $k, k^{\prime}$. The variables we can choose are

$$
W^{2}=(p+k)^{2} ; \quad \Delta^{2}=\frac{-\left(k-k^{\prime}\right)^{2}}{4} ; \quad \xi=k^{2}=k^{\prime 2},
$$


where the first two correspond to the square of the total energy and the negative of the square of the momentum transfer in the centre of the mass system respectively. Instead of $W^{2}$, we can also use the variable

$$
\omega=\frac{\left(p+p^{\prime}\right)\left(k+k^{\prime}\right)}{2 \sqrt{\left(p+p^{\prime}\right)^{2}}},
$$

which is connected to $W^{2}$ by

$$
W^{2}=2 \omega \sqrt{\Delta^{2}+m^{2}}+2 \Delta^{2}+m^{2}+k^{2} .
$$

Consider the expression

$$
\begin{aligned}
M & =\int \mathrm{d}^{4} x \exp \left[\frac{i\left(k+k^{\prime}\right) x}{2}\right]\left\langle p^{\prime}\left|\mathrm{i}\left({ }^{x} / 2\right) \mathrm{j}(-x / 2)\right| p\right\rangle \\
& =(2 \pi)^{4}\left\langle p^{\prime}|\mathrm{j}(0)| p+k\right\rangle\langle p+k|\mathrm{j}(0)| p\rangle,
\end{aligned}
$$

which is related to the imaginary part of the scattering amplitude by

$$
\operatorname{Im} T\left(\omega, \Delta^{2}, \xi\right)=\frac{1}{2}\left\{M\left(\omega, \Delta^{2}, \xi\right)-M\left(-\omega, \Delta^{2}, \xi\right)\right\},
$$

where $M\left(-\omega, \Delta^{2}, \xi\right)$ can be obtained from $M\left(\omega, \Delta^{2}, \xi\right)$ by the transformation

$$
k \rightarrow-k^{\prime}, k^{\prime} \rightarrow-k, p \rightarrow p^{\prime}, p^{\prime} \rightarrow p .
$$

(Such a relation between $M(+\omega)$ and $M(-\omega)$ is essential for converting the integration over the negative energies into one over the positive energies only.) In the Breit frame for the target particles in which $\boldsymbol{p}+\boldsymbol{p}^{\prime}=0$ the $T$-matrix can be written as

$$
\begin{gathered}
T\left(\omega, \Delta^{2}, \xi\right)=-\int \mathrm{d}^{4} x \exp \left[i \omega x_{4}-i \boldsymbol{e} \cdot \boldsymbol{x} \cdot \gamma / \omega^{2}-\Delta^{2}-\xi\right] \\
\left\langle-\boldsymbol{p}\left|R^{\prime}\left\{A\left({ }^{x} / 2\right) A(-x / 2)\right\}\right| \boldsymbol{p}\right\rangle .
\end{gathered}
$$

where $\boldsymbol{e}$ is the unit vector perpendicular to $\boldsymbol{p}$. All the $\omega$ dependence of $T$ lies in the exponential. Set

$$
\xi=\xi_{1}+i \xi_{2} ; \quad \omega=\omega_{1}+i \omega_{2} .
$$

Then (54) defines an analytic function of the complex variables $\omega$ and $\xi$ regular in

$$
\begin{gathered}
\omega_{2}>0, \quad \omega_{1}^{2}>\xi_{1}+\Delta^{2} ; \quad \operatorname{Im} \omega>\operatorname{Im} \gamma / \omega^{2}-\Delta^{2}-\xi \\
\text { or } \quad 2 \omega_{2}\left(\omega_{1}-V \omega_{1}^{2}-\xi^{2}-\Delta^{2}\right)<\xi_{2}<2 \omega_{2}\left(\omega_{1}+\gamma \omega_{1}^{2}-\xi_{1}-\Delta^{2}\right) .
\end{gathered}
$$

If we chose $\xi=\xi_{1}<-\Delta^{2}$ then $T$ is regular in $\operatorname{Im} \omega>0$ and if $T$ is sufficiently bounded we have the relation

$$
\begin{aligned}
T\left(\omega, \Delta^{2}, \xi_{1}\right) & =\frac{1}{\pi} \int_{-\infty}^{\infty} \mathrm{d} \omega \frac{\operatorname{Im} T\left(\omega^{\prime}, \Delta^{2}, \xi_{1}\right)}{\omega^{1}-\omega} \\
& =\frac{1}{\pi} \int_{-\infty}^{\infty} \mathrm{d} \omega^{\prime} M\left(\omega^{\prime}, \Delta^{2}, \xi_{1}\right)\left\{\frac{1}{\omega^{\prime}-\omega}+\frac{1}{\omega^{\prime}+\omega}\right\} .
\end{aligned}
$$

(A term $i \varepsilon$ is understood in the denominator.) 
Lehmann next shows that this relation can be continued analytically to the mass shell $\xi=\mu^{2}$. Further from (51) and the corresponding definition of $M\left(-W, \Delta^{2}, \xi\right)$ one notes that $M\left(W, \Delta^{2}, \xi\right)$ has a pole for $W^{2}=(p+k)^{2}=m^{2}$ and is zero unless $(p+k)^{2} \geqq(m+\mu)^{2} . M\left(-W, \Delta^{2}, \xi\right)$ has a pole for $\bar{\omega}^{2}=m^{2}$ $=(p-k)^{2}$ and is zero unless $\bar{W}^{2}>(m+\mu)^{2}$. The contributions from these can be shown to be given by

$$
\begin{gathered}
M\left(\omega, \Delta^{2}, \xi=g^{2}(\xi) \delta\left(W^{2}-m^{2}\right)\right. \\
M\left(-\omega, \Delta^{2}, \xi\right)=g^{2}(\xi) \delta\left(W^{2}-m^{2}\right),
\end{gathered}
$$

where $g^{2}$ is apart from constant factors the conventional coupling constant. Changing back from $\omega$ to $W^{2}$ as the energy variable, we get finally the dispersion relation for the scattering process

$$
\begin{aligned}
& \operatorname{Re} T\left(W^{2}, \Delta^{2}\right)=g^{2}\left[\frac{1}{W^{2}-4 \Delta^{2}-2 \mu^{2}-m^{2}}-\frac{1}{W^{2}-m^{2}}\right] \\
& +\frac{P}{2 \pi} \int_{(m+\mu)^{2}}^{\infty} \mathrm{d} W^{\prime 2} M\left(W^{\prime 2}, \Delta^{2}, \xi\right)\left[\frac{1}{W^{\prime 2}-W^{2}}+\frac{1}{W^{\prime 2}-4 \Delta^{2}-2\left(m^{2}+\mu^{2}\right)+W^{2}}\right]
\end{aligned}
$$

To obtain the region of validity of the dispersion relation we have to study the analyticity properties of $M$. Using Dyson's integral representation for the matrix elements of the retarded products appearing in $M$ it can be proved that for the validity of the dispersion relation, the momentum transfer must be restricted by the inequality

$$
\Delta^{2} \leqq \Delta_{\max }^{2}=K^{2}+\frac{\left(m_{1}^{2}-\mu^{2}\right)\left(m_{2}^{2}-m^{2}\right)}{W^{2}-\left(m_{1}-m_{2}\right)^{2}},
$$

where $K$ is the particle momentum in the centre of the mass system and is given by

$$
K^{2}=\frac{\left(W^{2}+m^{2}-\xi\right)^{2}-4 m^{2} W^{2}}{4 W^{2}} .
$$

For the case of pion-nucleon scattering it can be shown ${ }^{1}$ that

Hence

$$
m_{1}=3 \mu, m_{2}=m+\mu \text { and we have } W \geqq m+\mu .
$$

$$
\Delta^{2} \leqq \frac{8}{3} \mu^{2} \frac{2 m+\mu}{2 m-\mu},
$$

which means that the dispersion relations for the scattering amplitude as a function of energy can be proved rigorously for fixed momentum transfer.

For nucleon-nucleon scattering, however, for which $\mu=m=$ nucleon mass, $m_{1}=m+m_{\pi}, m_{2}=m_{\pi}$ and $W \geqq 2 \mu$ we have

$$
\Lambda^{2}<\Delta_{\max }^{2}=m_{\pi}^{2}-m^{2}+\frac{\left(2 m+m_{\pi}\right)^{2}}{4},
$$

1 See e.g. N. N. Bogoliubov and D. V. Shinkov, Introduction to the Theory of Quantized Fields. Interscience Publishers, New York (1959) chap. IX. 
which is positive only if

$$
m_{\pi} \geqq\left(\gamma^{2}-1\right) m .
$$

Thus even for forward scattering, a dispersion relation cannot be proved in this case.

Dispersion relations for other quantities besides scattering amplitudes have been studied. The following table due to Goldberger ${ }^{1}$ summarizes the present situation regarding the dispersion relations for various processes and vertex functions.

(a) Proved relations

\begin{tabular}{|c|c|c|}
\hline $\begin{array}{c}\text { Process } \\
k+p \rightarrow k^{\prime}+p^{\prime}\end{array}$ & $\begin{array}{c}\text { Limitation on invariant } \\
\text { momentum transfer } \\
\Delta^{2} \equiv-\frac{1}{4}\left\{\left(\boldsymbol{k}-\boldsymbol{k}^{\prime}\right)^{2}-\left(k_{0}-k_{0}^{\prime}\right)^{2}\right\}\end{array}$ & $\begin{array}{l}\text { Continuation of the } \\
\text { imaginary part into } \\
\text { the unphysical region } \\
\text { by convergent partial } \\
\text { wave expansion }\end{array}$ \\
\hline$\pi+N \rightarrow \pi+N$ & $\Delta_{\max }^{2}=\frac{8 \mu^{2}}{3} \frac{2 m+\mu}{2 m-\mu}$ & $0 \leqq \Delta^{2} \leqq \Delta_{\max }^{2}$ \\
\hline$\pi+\pi \rightarrow \pi+\pi$ & $\Delta_{\max }^{2}=7 \mu^{2}$ & $0 \leqq \Delta^{2}<\Delta_{\max }^{2}$ \\
\hline$\gamma+N \rightarrow \gamma+N$ & $\begin{aligned} \mathcal{A}_{\max }^{2}=\mu^{2} & \left\{\frac{(2 m+\mu)^{2}}{(4 m+\mu)^{2}}\right. \\
& \left.+\frac{2 m+\mu}{m}\right\}\end{aligned}$ & $0 \leqq \Delta^{2}<\Delta_{\max }^{2}$ \\
\hline $\begin{array}{c}\gamma+N \rightarrow \pi+N \\
e+N \rightarrow e+\pi+N\end{array}$ & $\begin{array}{c}\Delta_{\max }^{2} \sim 3 \mu^{2} \\
\Delta_{\max }^{2}=F(\gamma) ; \gamma=k_{4}^{2}-\boldsymbol{k}^{2} \\
F\left(-9 \mu^{2}\right) \sim 6 \mu^{2}\end{array}$ & $\begin{array}{c}\Delta_{t h}^{2} \leqq \Delta^{2}<\Delta_{\max }^{2} \\
\Delta_{t h}^{2}=\frac{m}{m+\mu} \cdot \frac{\mu^{2}-\gamma}{4}\end{array}$ \\
\hline
\end{tabular}

(b) Some unproved relations

\begin{tabular}{c|c|c}
\hline Process & Mass restrictions; $\Delta^{2}=0$ & $\begin{array}{c}\text { Perturbation theory } \\
\text { (every finite order) }\end{array}$ \\
\hline$N+N \rightarrow N+N$ & $\mu>(/ 2-1) m$ & proved for $\Delta^{2}<^{\mu^{2} / 4}$ \\
\hline$K+N \rightarrow K+N$ & $\begin{array}{c}\text { Complicated; not ful- } \\
\text { filled by narrow margin }\end{array}$ & \\
\hline$x+\mathrm{D} \rightarrow \pi+D$ & $\varepsilon>\frac{\mu}{3} ; M_{D}=2 m-\varepsilon$ & \\
\hline
\end{tabular}

1 M. L. Goldberger, International Conference on High Energy Physics, CERN (1958), Ed. B. Feretti, pp. 208, 209.

2 It may appear odd that one should try to prove dispersion relations in perturbation theory when the aim of the approach is to get away from it, but it is expected that a study of the analyticity properties of the individual terms of the perturbation series may serve as a guide for the more complicated situation.

${ }^{3}$ Numerical calculation gives the condition $M \geqq 8.573$, which again renders a proof impossible in this case. 
(c) Vertex functions

\begin{tabular}{|c|c|c|c|}
\hline ' Vertex & $\begin{array}{c}\text { Mass } \\
\text { restrictions }\end{array}$ & Remarks & $\begin{array}{l}\text { Perturbation theory } \\
\text { (finite order) }\end{array}$ \\
\hline $\begin{array}{c}\left\langle N\left|\square A_{\mu}\right| N\right\rangle \\
\left\langle N\left|\left(\square+\mu^{2}\right) \varphi_{\pi}\right| N\right\rangle\end{array}$ & $\begin{array}{l}\mu>(/ 2-1) m \\
\mu>\left(\gamma^{2}-1\right) M\end{array}$ & $\begin{array}{l}\text { Representations } \\
\text { do not follow } \\
\text { from causality } \\
\text { and spectrum } \\
\text { alone }\end{array}$ & $\begin{array}{l}\text { Proved } \\
\text { Proved }\end{array}$ \\
\hline $\begin{array}{c}\left\langle N\left|\left(\gamma \frac{\partial}{\partial x}+m\right)\right| N\right\rangle \\
\left\langle\pi\left(\square+\mu^{2}\right) \varphi_{\pi} \mid \tau\right\rangle\end{array}$ & $\begin{array}{l}\text { Proved } \\
\text { Proved }\end{array}$ & & . \\
\hline
\end{tabular}

Lehmann has also proved that the real and imaginary parts of the scattering amplitude as a function of the momentum transfer (or equivalently the angle of scattering in the c.m.s.) are analytic within certain ellipses which include the physical region. The region of analyticity for the imaginary part is greater than that for the real part and is given by an ellipse with foci at \pm 1 and with semimajor axis

$$
Z_{0}=\left\{1+\frac{2\left(m_{1}^{2}-m^{2}\right)\left(m_{2}^{2}-\mu^{2}\right)}{K^{2}\left[W^{2}-\left(m_{1}-m_{2}\right)^{2}\right]}\right\}^{1 / 2}
$$

This is a particular case of a more general representation due to Mandelstam (see next section). We conclude this section with some applications of the dispersion relations with energy as the variable.

(1) A truncation of the dispersion relations for meson-nucleon interactions (scattering and photoproduction of mesons) on the basis of the dominance of the $(3 / 2,3 / 2)$ resonance, whereby high energy phenomena are excluded from consideration, leads to approximations which can be considered a relativistic generalization of Chew's static model but which are superior in that they satisfy Lorentz and gauge invariance and also take recoil corrections into account.

(2) The use of phase-shift analysis for the reduction of experimental data on meson-nucleon scattering does not lead to a unique determination of the phase angles but yields a number of possible sets of these angles. The dispersion relations give a number of additional equations which enable us to choose the correct system of phase angles as mentioned earlier.

(3) The dispersion relations provide a possibility for an experimental test of the existence of an elementary length.

(4) The dispersion relations lead to a precise definition of the pion-nucleon coupling constant which appears as the residue of poles in the pion-nucleon and nucleon-nucleon scattering amplitudes (see the next section). 


\section{The Mandelstam representation ${ }^{1}$}

It was mentioned in the last section that proofs for dispersion relations for scattering processes can be given only for restricted values of the momentum transfer and the imaginary part of the scattering amplitude is analytic within a certain ellipse in the complex momentum transfer plane. But this latter region does not extend much further than the limitation already present in the derivations of the dispersion relations. Mandelstam has made an intuitively appealing conjecture that the scattering amplitude is an analytic function of both energy and momentum transfer variables with singularities only on the real axis of these two variables. This leads to a simple integral representation of the scattering amplitude. The representation has been verified up to fourth order in perturbation theory. ${ }^{2}$

Let us start by noting the important consequences of the "substitution law" of perturbation theory. Consider the following processes in which pions and nucleons can take part:

$$
\left.\begin{array}{l}
\pi+\pi \leftrightarrow \pi+\pi \\
\pi+\pi \leftrightarrow N+\bar{N} \\
\pi+N \leftrightarrow \pi+N \text { or } \pi+\bar{N} \leftrightarrow \pi+\bar{N} \\
N+N \leftrightarrow N+N \\
N+\bar{N} \leftrightarrow N+\bar{N}
\end{array}\right\} .
$$

These ean be represented diagrammatically by Figures 29 (a), (b) and (c), if we observe the convention that all incoming particles have a positive time-like four-

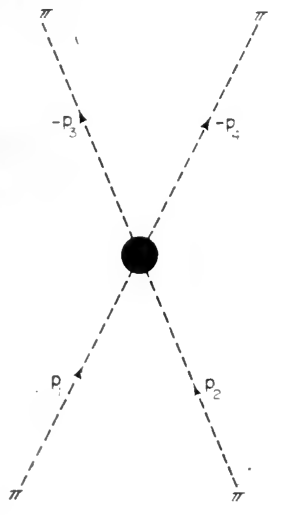

(a)

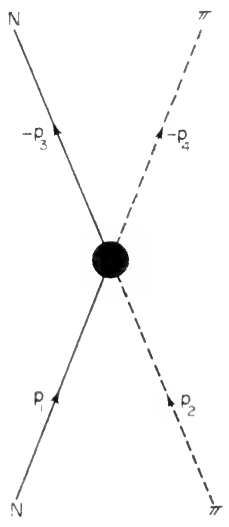

(b)

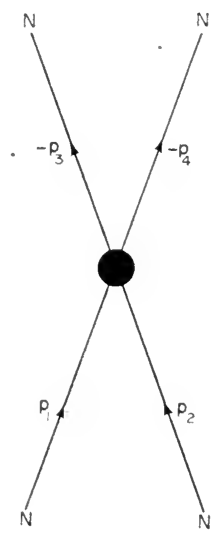

(c)

Fig. 29. Graphs for the pion-nucleon system.

1 S. Mandelstan, Phys. Rev. 112, 1344 (1958); 115, 1741, 1752 (1959). T. W. B. Kibble, Phys. Rev. 117, 1159 (1960); see also G. F. Chew, Ann. Rev. Nucl. Sci. 9, 29 (1959).

${ }^{2}$ Eden has given a proof of the Mandelstam representation valid for all orders of perturbation theory: R. J. Eden, Phys. Rev. Lett. 5, 213 (1960); see also R. J. Eden et al., Phys. Rev. 122, 307 (1961). 
momentum and that going opposite to the direction of an arrow means changing particle to antiparticle. Figure 29 (b) includes both the processes (2) and (3) while 29 (c) includs processes (4) and (5) so that processes (1) to (5) are really different manifestations of three fundamental matrix elements represented by Figures 29 (a), (b) and (c).

For two incoming and two outgoing particles, we can construct two invariants corresponding to the total energy and angle of scattering in the c.m.s. Define three invariants

with

$$
\left.\begin{array}{rl}
s_{1}=\left(p_{1}+p_{4}\right)^{2} & =\left(p_{2}+p_{3}\right)^{2} \\
s_{2}=\left(p_{2}+p_{4}\right)^{2} & =\left(p_{1}+p_{3}\right)^{2} \\
s_{3}=\left(p_{3}+p_{4}\right)^{2} & =\left(p_{1}+p_{2}\right)^{2} \\
s_{1}+s_{2}+s_{3} & =\sum_{i=1}^{4} m_{i}^{2}=K .
\end{array}\right\}
$$

Hence only two of the three $s$ variables are independent. Each is the square of the total energy $W^{2}$. in the c.m.s. for a particular pairing or "channel" of incoming and outgoing particles. (For each diagram there will be three channels.) For instance if $p_{1}$ and $p_{2}$ correspond to the incoming particles and $-p_{3}$ and $-p_{4}$ to the outgoing particles (process I) then the total energy is $V s_{3} ; s_{2}$ will be the negative of the square of the momentum transfer, $\Delta^{2}$, while $s$, in general may involve both $W^{2}$ and $\Delta^{2}$. For processes II and III in which the incoming particles are 1 and 4 and 1 and 3 respectively the total energies will be $V s_{1}$ and $V s_{2}$.

The condition for a physical process to occur may be expressed in terms of $s_{1}, s_{2}$ and $s_{3}$. In order that one of the physical processes (I, II or III) may be energetically possible for a given set of values of the scalar products $p_{i} p_{j}$, we must have

$$
\pm p_{i} p_{j}>m_{i} m_{j} \text {. }
$$

The positive sign is to be taken if the particles $i$ and $j$ are both incoming or both outgoing and the negative sign if it is otherwise. Further the requirement that the scattering angle be real can be stated in the form ${ }^{1}$

$$
\left|\begin{array}{lll}
p_{1}^{2} & p_{1} \cdot p_{2} & p_{1} \cdot p_{3} \\
p_{2} \cdot p_{1} & p_{2}^{2} & p_{2} \cdot p_{3} \\
p_{3} \cdot p_{1} & p_{3} \cdot p_{2} & p_{3}^{2}
\end{array}\right|>0
$$

In terms of the variables $s_{1}, s_{2}$ and $s_{3},(70 \mathrm{a})$ and $(70 \mathrm{~b})$ can be written as

and

$$
\begin{gathered}
s_{3}>\left(m_{1}+m_{2}\right)^{2} \text { or } s_{3}<\left(m_{1}-m_{2}\right)^{2} \text { etc. } \\
s_{1} s_{2} s_{3}>\left(s_{1}+s_{2}+s_{3}\right)^{2}\left(a s_{3}+b s_{2}+c s_{1}\right)
\end{gathered}
$$

respectively. In (72) the dimensionless constants $a, b, c$ are given by

$$
\left.\begin{array}{rl}
K^{3} a & =\left(m_{1}^{2} m_{2}^{2}-m_{3}^{2} m_{4}^{2}\right)\left(m_{1}^{2}+m_{2}^{2}-m_{3}^{2}-m_{4}^{2}\right) \\
K^{3} b & =\left(m_{1}^{2} m_{3}^{2}-m_{2}^{2} m_{4}^{2}\right)\left(m_{1}^{2}+m_{3}^{2}-m_{2}^{2}-m_{4}^{2}\right) \\
K^{3} c & =\left(m_{1}^{2} m_{4}^{2}-m_{2}^{2} m_{3}^{2}\right)\left(m_{1}^{2}+m_{4}^{2}-m_{2}^{2}-m_{3}^{2}\right)
\end{array}\right\} .
$$

1) T. Kibble, Phys. Rev. 117, 1159 (1960). 
The variables $s_{1}, s_{2}$ and $s_{3}$ may now be considered as homogeneous coordinates in a plane in which the line at infinity is $s_{1}+s_{2}+s_{3}=0$. The region (72) is bounded by a cubic curve in this plane, the asymptotes of which are $s_{1}=0, s_{2}=0$ and $s_{3}=0$. The curve intersects its asymptotes on the line $a s_{3}+b s_{2}+c s_{1}=0$. The bounding lines of the region defined by (71) are tangential to the curve the shape of which is given in Fig. 30. If we assume that $m_{1} \geqq m_{2} \geqq m_{3} \geqq m_{4}$

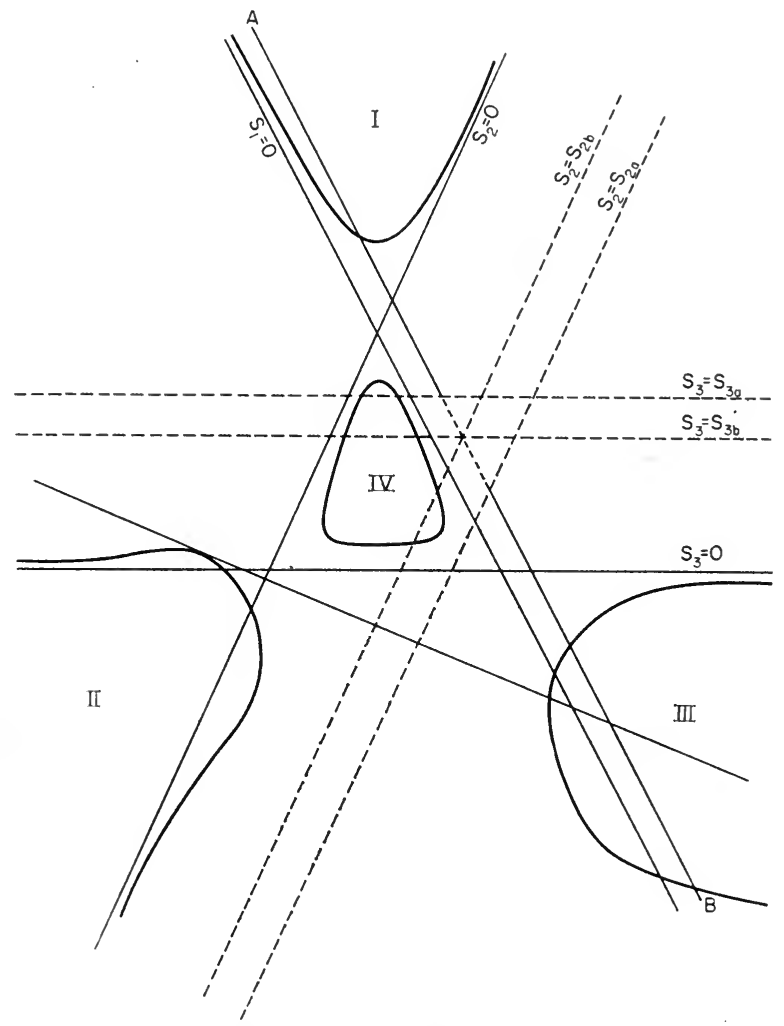

FIg. 30. The physical regions.

(which is satisfied in practice) we have $a \geqq b \geqq c$. $a$ and $b$ are positive while $c$ may have both signs. The regions I, II and III are the physical regions for the three scattering processes (the region IV, corresponding to the decay process $1 \rightarrow \overline{2}+3+4$ can occur only if $m_{1}>m_{2}+m_{3}+m_{4}$. We shall not be considering it here). We note that the entire boundary of each of the regions I, II and III is a part of the cubic curve.

Now we can conjecture from the substitution rule as follows: A single analytic function describes all three channels contained in the same diagram (Fig. 29). The physical amplitude for the process when 1 and 2 are the incoming particles is the boundary value of an analytic function, as the variable $s_{3}$ approaches the positive 
real axis from above in its physical energy range with one of the other two variables held fixed at a physical value in the momentum transfer range. The amplitude for the cases when 1 and 3 and 1 and 4 are considered as incoming particles are got from corresponding limits on the same function taken with the variables $s_{2}$ or $s_{1}$ respectively.

To derive the Mandelstam representation from this fundamental assumption we can restate the latter in the form; There exists a function $A\left(z_{1}, z_{2}, z_{3}\right)$ of the three complex variables (only two of which are independent), which is analytic except in the following regions.

$$
\begin{array}{lllll}
z_{1}=\alpha_{1} & \text { or } & z_{1}(\text { real })>a_{1} & \text { for all } & z_{2} \\
z_{2}=\alpha_{2} & \text { or } & z_{2} \text { (real) }>a_{2} & \text { for all } & z_{3} \\
z_{3}=\alpha_{3} & \text { or } & z_{3} \text { (real) }>a_{3} & \text { for all } & z_{1} .
\end{array}
$$

All the $\alpha$ 's and $a$ 's are real and positive and correspond respectively to poles and branch points for the three interactions. Thus in the case of $\pi-N$ scattering, for instance, we have

$$
a_{1}=\alpha_{3}=M^{2} ; \quad a_{1}=a_{3}=(M+\mu)^{2} ; \quad a_{2}=4 \mu^{2}
$$

but there is no isolated pole in $z_{2}$.

To obtain the Mandelstam representation, we have to assume further that

$$
a_{1}+a_{2}+a_{3}>K
$$

which is evidently fulfilled in the $\pi-N$ case. We will write down the dispersion relations for the function $A\left(z_{1}, z_{2}\right)$ assuming that it is well-behaved at infinity. Let us fix the variable $z_{2}$ (we assume for definiteness that $\operatorname{Im}\left(z_{2}\right)>0$ ) and consider $A\left(z_{1}, z_{2}\right)$ as a function of $z_{1}$ alone. An application of the Cauchy theorem gives the dispersion relation.

$$
\left.\begin{array}{rl}
A\left(z_{1}, z_{2}\right) & =\frac{h_{1}\left(z_{2}\right)}{\alpha_{1}-z_{1}}+\frac{h_{3}\left(z_{2}\right)}{\left(K-z_{2}-\alpha_{3}\right)-z_{1}} \\
& +\frac{1}{\pi} \int_{a_{1}}^{\infty} \frac{A_{1}\left(x_{1}^{\prime}, z_{2}\right)}{x_{1}^{\prime}-z_{1}} \mathrm{~d} x_{1}^{\prime}-\frac{1}{\pi} \int_{a_{3}}^{\infty} \frac{A_{3}\left(x_{3}^{\prime}, z_{2}\right) \mathrm{d} x_{3}^{\prime}}{\left(K-z_{2}-x_{3}^{\prime}\right)-z_{1}} \cdot
\end{array}\right\}
$$

(The cuts will not meet each other since $\operatorname{Im} z_{2} \neq 0$ ). The functions $A_{1}$ and $A_{3}$ are the jumps across the cuts of the function $A$. For example

$$
A_{1}\left(x_{1}, z_{2}\right)=\frac{1}{2 i} \lim _{\epsilon \rightarrow 0}\left[A\left(x_{1}+i \varepsilon, z_{2}\right)-A\left(x_{1}-i \varepsilon, z_{2}\right)\right] \text {. }
$$

$h_{1}$ and $h_{3}$ are the residues of the function $A$ at the poles $\alpha_{1}$ and $K-z_{2}-\alpha_{3}$. To simplify the notation we can include the contributions from the poles 
into the integrals. For this purpose we replace the spectral functions $A_{1}$ and $A_{3}$ by

$$
\left.\begin{array}{l}
A_{1}\left(x_{1}^{\prime}, z_{2}\right) \rightarrow \delta\left(x_{1}^{\prime}-\alpha_{1}\right) h_{1}\left(z_{2}\right)+A_{1}\left(x_{1}^{\prime}, z_{2}\right) \\
A_{3}\left(x_{3}^{\prime}, z_{2}\right) \rightarrow-\delta\left(x_{3}^{\prime}-\alpha_{3}\right) h_{3}\left(z_{2}\right)+A_{3}\left(x_{3}^{\prime}, z_{2}\right)
\end{array}\right\}
$$

so that we have

$$
A_{1}\left(z_{1}, z_{2}\right)=\frac{1}{\pi} \int_{\alpha_{1}}^{\infty} \frac{A_{1}\left(x_{1}^{\prime}, z_{2}\right)}{x_{1}^{\prime}-z_{1}} \mathrm{~d} x_{1}^{\prime}-\frac{1}{\pi} \int_{\alpha_{3}}^{\infty} \frac{A_{3}\left(x_{3}^{\prime}, z_{2}\right)}{\left(K-z_{2}-x_{3}^{\prime}\right)-z_{1}} \mathrm{~d} x_{3}^{\prime} .
$$

For real values of $z_{1}$ appearing in (77) both cuts in the $z_{2}$ plane are along the real axis. The condition (75) guarantees that the two cuts in the $z_{2}$ plane do not meet (as $x_{1}>a_{1}$ ). Using (77) we obtain

$$
\left.\begin{array}{rl}
A_{1}\left(x_{1}^{\prime}, z_{2}\right)= & \frac{1}{\pi} \int_{\alpha_{3}}^{\infty} \frac{A_{23}\left(x_{1}^{\prime}, x_{2}^{\prime}\right)}{x_{2}^{\prime}-z_{2}} \mathrm{~d} x_{2}^{\prime} \\
& -\frac{1}{\pi} \int_{x_{3}}^{\infty} \frac{A_{12}\left(x_{1}^{\prime}, x_{3}^{\prime}\right)}{\left(K-x_{1}^{\prime}-x_{3}^{\prime}\right)-z_{2}} \mathrm{~d} x_{3}^{\prime}
\end{array}\right\}
$$

where $A_{23}$ and $A_{12}$ are again jumps (barring the residues at the poles) across the cuts.

$$
A_{23}\left(x_{1}, x_{2}\right)=\frac{1}{2 i} \lim _{\epsilon \rightarrow 0}\left[A_{1}\left(x_{1}, x_{2}+i \varepsilon\right)-A_{1}\left(x_{1}, x_{2}-i \varepsilon\right)\right] \text {. }
$$

From (77) and (81) we have, for $x_{1}>a_{1}, x_{2}>a_{2}$

$$
\left.\begin{array}{rl}
A_{23}\left(x_{1}, x_{2}\right)= & -\frac{1}{4} \lim _{\varepsilon, \eta \rightarrow 0}\left[A\left(x_{1}+i \varepsilon, x_{2}+i \eta\right)\right. \\
& +A\left(x_{1}-i \varepsilon, x_{2}-i \eta\right) \\
& -A\left(x_{1}+i \varepsilon, x_{2}-i \eta\right) \\
& \left.-A\left(x_{1}-i \varepsilon, x_{2}+i \eta\right)\right]
\end{array}\right\}
$$

A similar representation can be written for $A_{3}$

$$
\left.\begin{array}{rl}
A_{3}\left(x_{3}^{\prime}, z_{2}\right) & =\frac{1}{\pi} \int_{\alpha_{2}}^{\infty} \frac{\mathrm{d} x_{2}^{\prime} A_{13}\left(x_{2}^{\prime}, x_{3}^{\prime}\right)}{x_{2}^{\prime}-z_{2}} \\
& -\frac{1}{\pi} \int_{x_{1}}^{\infty} \frac{\mathrm{d} x_{1}^{\prime} A_{12}\left(x_{3}^{\prime}, x_{1}^{\prime}\right)}{\left(K-x_{1}^{\prime}-x_{3}^{\prime}\right)-z_{2}}
\end{array}\right\}
$$

Using the identity

$$
\frac{1}{K-x_{1}-x_{3}-z_{2}}\left[\frac{1}{x_{3}-z_{1}}+\frac{1}{x_{3}-z_{3}}\right]=-\frac{1}{\left(x_{1}-z_{1}\right)\left(x_{3}-z_{3}\right)}
$$


and combining with (79) and (80), we finally get on changing over to the $s$-variables, the Mandelstam representation

$$
\begin{aligned}
A\left(s_{1}, s_{2}, s_{3}\right)= & \frac{1}{\pi^{2}} \iint \mathrm{d} s_{1}^{\prime} \mathrm{d} s_{2}^{\prime} \frac{A_{23}\left(s_{1}^{\prime} s_{2}^{\prime}\right)}{\left(s_{1}^{\prime}-s_{1}\right)\left(s_{2}^{\prime}-s_{2}\right)} \\
& +\frac{1}{\pi^{2}} \iint \mathrm{d} s_{1}^{\prime} \mathrm{d} s_{3}^{\prime} \frac{A_{12}\left(s_{1}^{\prime}, s_{3}^{\prime}\right)}{\left(s_{1}^{\prime}-s_{1}\right)\left(s_{3}^{\prime}-s_{3}\right)} \\
& +\frac{1}{\pi^{2}} \iint \mathrm{d} s_{3}^{\prime} \mathrm{d} s_{3}^{\prime} \frac{A_{13}\left(s_{3}^{\prime}, s_{2}^{\prime}\right)}{\left(s_{2}^{\prime}-s_{2}\right)\left(s_{3}^{\prime}-s_{3}\right)},
\end{aligned}
$$

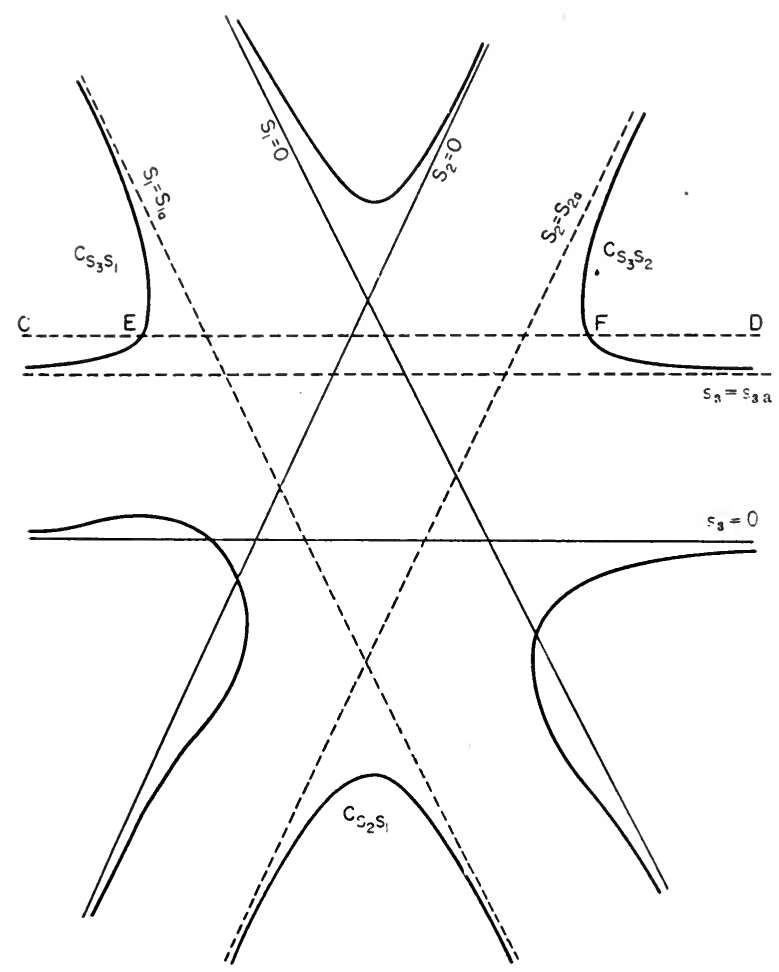

FIG. 31. The boundaries of the region in which the spectral functions are non-zero.

where the weight functions $A_{i j}\left(s_{i}^{\prime}, s_{j}^{\prime}\right)$ are real and the integral in each $s^{\prime}$ variable goes over the region of positive real axis extending to infinity. (In the denominator a small negative imaginary part is to be understood.) It is essential to specify the region where the spectral functions differ from zero. Mandelstam has given the precise boundaries for different processes on the basis of unitarity and perturbation theoretic arguments (see Fig. 31). $C_{\delta_{3} s_{2}}, C_{8_{3} s_{1}}$ and $C_{8_{2} \delta_{1}}$ are the boundaries. $s_{3 a}, s_{2 a}$ and $s_{1 a}$ are the lower limits of the corresponding variables, $s_{3}, s_{2}$ and $s_{1}$ occurring in equation (85). 
If poles occur in the scattering amplitude, their contributions must be added to (85). Holding one $s$ variable fixed at a real value outside its energy range and carrying out one of the two integrations in (85), we arrive at the ordinary singlevariable dispersion relations,

$$
\begin{aligned}
& A=\frac{1}{\pi} \int \mathrm{d} s_{3}^{\prime} \frac{A_{1}\left(s_{1}, s_{2}, s_{3}^{\prime}\right)}{s_{3}^{\prime}-s_{3}}+\frac{1}{\pi} \int \mathrm{d} s_{1}^{\prime} \frac{A_{2}\left(s_{1}^{\prime}, s_{2}, s_{3}\right)}{s_{1}^{\prime}-s_{1}} \\
& A=\frac{1}{\pi} \int \mathrm{d} s_{3}^{\prime} \frac{A_{1}\left(s_{1}, s_{2}, s_{3}^{\prime}\right)}{s_{3}^{\prime}-s_{3}}+\frac{1}{\pi} \int \mathrm{d} s_{2}^{\prime} \frac{A_{3}\left(s_{1}, s_{2}^{\prime}, s_{3}\right)}{s_{2}^{\prime}-s_{2}} \\
& A=\frac{1}{\pi} \int \mathrm{d} s_{1}^{\prime} \frac{A_{2}\left(s_{1}^{\prime}, s_{2}, s_{3}\right)}{s_{1}^{\prime}-s_{1}}+\frac{1}{\pi} \int \mathrm{d} s_{2}^{\prime} \frac{A_{3}\left(s_{1}, s_{2}^{\prime}, s_{3}\right)}{s_{2}^{\prime}-s_{2}},
\end{aligned}
$$

For this there should be the corresponding relations for the absorptive parts of the amplitude $A_{1}, A_{2}$ and $A_{3}$ for processes I, II and III respectively

$$
\left.\begin{array}{l}
A_{1}=\frac{1}{\pi} \int \mathrm{d} s_{2}^{\prime} \frac{A_{13}\left(s_{3}^{\prime} s_{2}^{\prime}\right)}{s_{2}^{\prime}-s_{2}}+\frac{1}{\pi} \int \mathrm{d} s_{1}^{\prime} \frac{A_{12}\left(s_{1}^{\prime} s_{3}^{\prime}\right)}{s_{1}^{\prime}-s_{1}} \\
A_{2}=\frac{1}{\pi} \int \mathrm{d} s_{2}^{\prime} \frac{A_{23}\left(s_{1}^{\prime} s_{2}^{\prime}\right)}{s_{2}^{\prime}-s_{2}}+\frac{1}{\pi} \int \mathrm{d} s_{3}^{\prime} \frac{A_{12}\left(s_{1}^{\prime} s_{3}^{\prime}\right)}{s_{3}^{\prime}-s_{3}} \\
A_{3}=\frac{1}{\pi} \int \mathrm{d} s_{1}^{\prime} \frac{A_{23}\left(s_{2}^{\prime}, s_{1}^{\prime}\right)}{s_{1}^{\prime}-s_{1}}+\frac{1}{\pi} \int \mathrm{d} s_{3}^{\prime} \frac{A_{13}\left(s_{3}^{\prime}, s_{2}^{\prime}\right)}{s_{3}^{\prime}-s_{3}}
\end{array}\right\} .
$$

Equations (85), (86) and (87) imply a number of important properties:

(1) The ordinary dispersion relations hold for arbitrary values of momentum transfer.

(2) The absorptive parts satisfy dispersion relations as functions of momentum transfer for fixed energy, i.e. they are functions of momentum transfer, regular except for cuts along parts of the real axis. This is a much more general result than the ellipses of Lehmann.

(3) Analytical properties of the partial waves as a function of energy follow. The partial waves are always boundary values of analytic functions which are regular except for cuts. The dispersion relations for fixed angular momentum transfers are particularly powerful because they allow a simple incorporation of the unitarity of the $S$-matrix in the problem.

(4) One of the most important applications of the Mandelstam representation consists in extrapolating scattering amplitudes to poles in the unphysical region with the aim of determining the parities of particles and the coupling constants. It was mentioned in the last section that the residue of the pole in the amplitude for pion-nucleon scattering for fixed momentum transfer gives, apart from constant factors, the pion-nucleon coupling constant. We can expect poles in the scattering amplitudes for other processes. Perturbation theory suggests a broad rule for locating the poles. If the two incoming and the two outgoing particles in any of the three channels of a diagram can be "connected" by a stable single particle state of mass, $m_{0}$ (by which is meant that the initial and final two-particle 
states can both assume the same quantum numbers as the single particle) then there will be a pole when the $s$-variable corresponding to the square of the fourmomentum in this channel is equal to $m_{0}^{2}$. The poles, though lying on the real axis can never lie in the physical region since then the single particle could decay via strong interactions into either of the two-particle states to which it is coupled. Applying the above criterion there should be no poles in the pion-pion scattering amplitude if electro-magnetic effects are neglected since a two-pion state has quantum numbers different from any known particle except a photon. The twonucleon channel has three poles, one from each channel. The $2 N$-channel gives rise to a pole at $s_{3}=M_{D}^{2}$ corresponding to the deuteron state while the $N-\bar{N}$ channels give rise to poles at $s_{1}=\mu^{2}$ and $s_{2}=\mu^{2}$ both corresponding to the single pion state. Chew ${ }^{1}$ has proposed a way of determining the pion-nucleon coupling constant from the residue of the pole arising from the single pion intermediate state. The difficulty in such a determination with the energy as the variable is the existence of extensive non-physical contributions to the dispersion relations which make practical applications difficult. But Chew has conjectured by considering the Feynman diagrams for the process that if the scattering amplitude is considered as a function of the momentum transfer $\Delta^{2}$ at fixed energy then there is probably a pole of residue $g^{2}$ (the same coupling constant as in the case of the pion-nucleon interaction) located at $\Delta^{2}=-\mu^{2}$ where $\mu$ is the pion rest mass. Since $\cos \theta=1-\frac{2 \Delta^{2}}{k^{2}}$ we see that though the point $\Delta^{2}=-\mu^{2}$ is always unphysical corresponding to $\cos \theta=1+\frac{2 \mu^{2}}{k^{2}}>1$ this point comes nearer to the physical region as $k^{2}$ increases. For practical reasons the region of very high energy is not suitable and so we must consider the possibility of bridging by analytic continuation the appreciable gap between the physical region and the position of the pole. Chew has proposed such an extrapolation procedure which can be justified using the Mandelstam dispersion relations. From an inspection of (85) it follows that if the fixed variable $s_{3}$ is in the physical energy range then if the remaining two variables $s_{1}$ and $s_{2}$ are expressed in terms of $\cos \theta$, all the singularities in the complex plane are outside the physical interval. Further the nearest branch points lie beyond any poles that occur. Thus a simple polynomial extrapolation can be made from the physical region.

Finally we may mention that a much more ambitious proposal for applying the Mandelstam representation has been made ${ }^{2}$ which consists in combining it with the unitary relations to obtain a dynamical theory of low energy pionnueleon phenomena.

1 G. F. Chew, Phys. Rev. 112, 1380 (1958).

2 G. F. Chew and S. Mandelstam, Phys. Rev. 119, 467, 478 (1960). 


\section{STRANGE PARTICLES AND THEIR INTERACTIONS}

\section{STRANGE PAR'TICLES ${ }^{1}$}

\section{Introduction}

IVE HAVE till now considered the interactions of the following elementary particles: the photon, electron, positron, neutron, proton, $\pi$-meson, $\mu$-meson and the neutrino. In investigations on cosmic rays, new elementary particles were discovered which were later produced artificially in high energy accelerators.

Before we discuss the nature of the interactions involving these new particles, it will be interesting to attempt a definition of an "elementary particle". It is rather curious that no such definition can be given in terms of their intrinsic attributes though they are certainly characterized by them. In the absence of interactions, the only intrinsic attributes of a particle are its mass and spin. In interaction, new attributes can be revealed namely charge, parity, strangehess and isotopic spin. The lifetime of a particle can also be treated as an attribute as the concept of lifetime involves that of an interaction between the particle and its decay product. There does not seem to be any specific criterion for the values which these attributes can take. Masses of the known elementary particles range from 0 (photon) to 2642 times the electronic mass (the $\Xi$ particle). There is no specific limit for the spin, though on present evidence it is firmly believed that the elementary particles have spin $0, \frac{1}{2}$ or 1 . The lifetime of known elementary particles range from $10^{-20} \mathrm{sec}$ to $\infty$. Thus it looks as if there can be no well defined criterion for "elementarity" though we are quite clear that atoms and molecules cannot be called elementary particles. The definition is made still more difficult as there are some theorists who consider certain elementary particles as composite particles, i.e. compound or bound states of other elementary particles. We shall be content with defining an elementary particle as one whose properties cannot be better understood by breaking it into sub-components.

We shall now discuss how the various intrinsic attributes are assigned to the new particles through a knowledge of their interactions. The new particles are the following:

$$
\Lambda^{0}, \Sigma^{+}, \Sigma^{0}, \Sigma^{-}, \quad \Xi^{0}, \Xi^{-}, K^{+}, K^{0}, \bar{K}^{0}, K^{-} .
$$

The known interactions involving their production and decay are given below.

${ }^{1} \mathrm{~K}$ mesons and hyperons, their strong and weak interactions by R. H. Dalitz, Rep . Progr. Phys. 20, 163 (1957). Also Hyperons and heavy mesons, systematics and decay by M. Gell-Mann and A. H. Rosenfeld, Ann. Rev. Nucl. Sci. 7, 407 (1957). 
Some typical production reactions and decay schemes of strange particles ${ }^{*}$

\begin{tabular}{|c|c|c|c|}
\hline $\begin{array}{l}\text { Name of } \\
\text { the particle }\end{array}$ & Production reaction & Decay scheme & Lifetimes in $\sec ^{b}$ \\
\hline $\boldsymbol{K}^{+}$ & $\begin{array}{l}\tau^{-}+p \rightarrow \Sigma^{-}+K^{+} \\
\tau^{+}+p \rightarrow \Sigma^{+}+K^{+}\end{array}$ & $\begin{aligned} K^{+} & \rightarrow \pi^{+}+\pi^{0} \\
& \rightarrow \pi^{+}+\pi^{+}+\pi^{-} \\
& \rightarrow \mu^{+}+\nu \\
& \rightarrow \pi^{0}+\mu^{+}+\nu \\
& \rightarrow \pi^{0}+e^{+}+\nu .\end{aligned}$ & $1.224 \pm 0.013 \times 10^{-3}$ \\
\hline$K^{-}$ & $\begin{aligned} \pi^{-}+p & \rightarrow n+K^{+}+K^{-} \\
\bar{p} & +p \rightarrow K^{+}+K^{-}\end{aligned}$ & $\begin{array}{l}\text { The decay modes can be ob- } \\
\text { tained from those of } K^{+} \text {by } \\
\text { changing the charge and } \\
\text { replacing } \nu \text { by } \bar{v} \text {. }\end{array}$ & 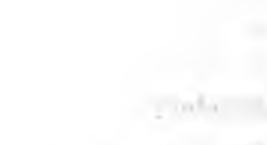 \\
\hline$K^{0}$ & $\begin{aligned} x^{-}+p & \rightarrow \Sigma^{0}+K^{0} \\
& \rightarrow \Lambda^{0}+K^{0}\end{aligned}$ & $\begin{aligned} K_{1}^{0} & \rightarrow \pi^{+}+\pi^{-} \\
& \rightarrow \pi^{0}+\pi^{0} \\
& \rightarrow \pi^{+}+\pi^{-}+\pi^{0}(\text { rare }) \\
& \rightarrow \pi^{\mp}+\mu \pm+\nu(\bar{v}) \\
& \rightarrow \pi^{\mp}+e^{ \pm}+\nu(\bar{v})\end{aligned}$ & $1.00 \pm 0.04 \times 10^{-10}$ \\
\hline$h^{0}$ & $\begin{array}{c}\tau^{-}+p \rightarrow n+K^{0}+\overline{K^{0}} \\
\bar{p}+p \rightarrow K^{0}+\bar{K}^{0}\end{array}$ & $\begin{aligned} K_{2}^{0} & \rightarrow \pi^{+}+\pi^{-}+\pi^{0} \\
& \rightarrow 3 \pi^{0} \\
& \rightarrow \pi^{\mp}+\mu \pm+\nu(\bar{v}) \\
& \rightarrow \pi^{\mp}+e \pm+v(\bar{v})\end{aligned}$ & $6.1_{-1.1}^{+1.6} \times 10^{-8}$ \\
\hline $.1^{3}$ & $\begin{aligned} K^{-}+p & \rightarrow \Lambda^{0}+\pi^{0} \\
\Sigma^{-}+p & \rightarrow \Lambda^{0}+n \\
p+p & \rightarrow K^{+}+\Lambda^{1}+p \\
p+n & \rightarrow K^{0}+\Lambda^{0}+p \\
& \rightarrow K^{+}+\Lambda^{0}+n \\
p+\bar{p} & \rightarrow \Lambda^{0}+\bar{\Lambda}^{0}\end{aligned}$ & $\begin{aligned} \Lambda^{0} & \rightarrow p+\pi^{-} \\
& \rightarrow \pi^{0}+n \\
& \rightarrow p+\mu^{-}+\bar{v}(?) \\
& \rightarrow p+e^{-}+\bar{v}(?)\end{aligned}$ & $2.51 \pm 0.09 \times 10^{-10}$ \\
\hline$\Sigma^{j}$ & $\begin{aligned} K^{-}+p & \rightarrow \Sigma^{0}+\pi^{0} \\
p+p & \rightarrow K^{+}+\Sigma^{0}+p \\
p+n & \rightarrow K^{0}+\Sigma^{0}+p \\
& \rightarrow K^{+}+\Sigma^{0}+n \\
p+\bar{p} & \rightarrow \Sigma^{0}+\bar{\Sigma}^{0}\end{aligned}$ & $\Sigma^{\nu} \rightarrow A^{0}+\gamma$ & $<0.1 \times 10^{-10}$ \\
\hline$\Sigma+$ & $\begin{aligned} K^{-}+p & \rightarrow \Sigma^{+}+\pi^{-} \\
p+p & \rightarrow K^{0}+\Sigma^{+}+p \\
& \rightarrow K^{+}+\Sigma^{+}+n \\
p+\bar{p} & \rightarrow \Sigma^{+}+\bar{\Sigma}^{+}\end{aligned}$ & $\begin{aligned} \Sigma^{+} & \rightarrow p+\pi^{0} \\
& \rightarrow n+\pi^{+}\end{aligned}$ & $0.81_{-0.05}^{+0.06} \times 10^{-10}$ \\
\hline$\Sigma-$ & $\begin{array}{l}K^{-}+p \rightarrow \Sigma^{-}+\pi^{+} \\
p+n \rightarrow K^{+}+\Sigma^{-}+p \\
p+\bar{p} \rightarrow \Sigma^{-}+\bar{\Sigma}^{-}\end{array}$ & $\Sigma^{-} \rightarrow n+\pi^{-}$ & $1.61 \pm 0.09 \times 10^{-10}$ \\
\hline$\equiv$ & $x^{-}+p \rightarrow \Xi^{-}+K^{+}+K^{0}$ & $\Xi^{-} \rightarrow \Lambda^{0}+\pi^{-}$ & $1.3_{-0.3}^{+0.4} \times 10^{-10}$ \\
\hline$\Xi^{0}$ & $x^{-}+p \rightarrow \Xi^{0}+K^{0}+K^{0}$ & $\Xi^{0} \rightarrow \Lambda^{0}+\pi^{0}$ & $1.5 \times 10^{-10}$ \\
\hline
\end{tabular}

a (1) Though we have not written down $\pi-p$ collision reactions for hyperons, they must also be treated as production reactions for hyperons. In a similar way the $N-N$ collisions also produce $K$-mesons.

(2) We have only written reactions involving $p$. We can also write $\pi-N$ collisions and $K-p$ absorption reactions.

${ }^{b}$ G. A. Sxow and M. M. Shapiro. Rev. Mod. Phys. 33, 231 (1961). 
The most striking feature of the interactions involving the new particles is their copious production which is irreconcilable with their long lifetimes. In order to understand this contradiction, Pais proposed the idea of "associated production" i.e. the hyperons and $K$-mesons are always produced in association while the particles decay separately. Thus the interactions involving production may be considered different from those involving decay, the former being assumed to be strong while the latter is weak. The strength of the interactions involving production is of the same order of magnitude as that of the meson-nucleon interaction while the decay interactions are found to be much weaker than the electromagnetic interactions. The various interactions are characterized not only by their strength but by other characteristics as well.

Before dealing with these interactions in detail and comparing them with the known interactions discussed before, it will be convenient to classify the elementary particle interactions as follows:

(i) Strong interactions: This group includes the forces responsible for the production and scattering of nucleons, pions, hyperons and $K$-mesons. It is characterized by a coupling constant of the order of magnitude unity and the conservation of $Q, I$ and $I_{z}$, the charge, the total isotopic spin and its $z$-component respectively.

(ii) Electromagnetic interactions: These are characterized by the coupling constant $\frac{e^{2}}{\hbar c}=1 / 137$ and the conservation of $Q$ and $I_{z}$.

(iii) Weak interactions ${ }^{1}$ : These are characterized by the coupling constant $y^{2} / \hbar c \sim 10^{-14}$ and the conservation of $Q$.

(iv) Gravitational interactions: The gravitational interaction is characterized by a coupling constant $\frac{G^{2} m^{2}}{\hbar c}=2 \times 10^{-39}$ where $G$ is Newton's gravitational constant and $m$ chosen to be the mass of the proton. The gravitational interaction will not be further discussed.

\section{Isotopic spin and strangeness}

We now make a physically satisfying assumption that no elementary particle can have multiple charge, i.e. all the elementary particles are either neutral or singly charged. The relationship between isotopic spin and charge is given in the case of nucleons by

$$
Q=I_{z}+1 / 2
$$

and in the case of mesons by

$$
Q=I_{z}
$$

1 The lifetimes of weak decays however have a very wide range from $0.75 \times 10^{-10}$ sec to $10^{3} \mathrm{sec}$. The universal weak interaction can explain this very sensitive dependence of the lifetime for decays on available energy (115 $\mathrm{MeV}$ for $\Sigma^{+}$and $0.75 \mathrm{MeV}$ for neutron). 
More generally we can write the relationship between $Q^{1}$ and $I_{z}$ of any elementary particle as follows:

$$
Q=I_{z}+\gamma / 2 \text {. }
$$

The total charge $Q$ and the total $I_{z}$ of a system of elementary particles are additive and thus the relation between them should be linear. ${ }^{2}$ But to convince ourselves that in the relation between $Q$ and $I_{z}$ the coefficient of $I_{z}$ should be unity we adopt the following argument. Consider the strong interaction involving pions and nucleons

$$
\text { pions }+ \text { nucleons } \rightarrow A+B .
$$

'Two pion-nucleon systems differing in $I_{z}$ (and not in $\gamma$ ) by unity will also differ in charge by unity. Since charge is conserved, the corresponding reaction products in the two cases will differ by unity in their charge. Let the relation between $Q$ and $I_{z}$ for systems $A$ and $B$ be written as:

$$
Q_{A}=\alpha_{A} I_{z}^{A}+\frac{\gamma}{2} ; \quad Q_{B}=\alpha_{B} I_{z}^{B}+\frac{\gamma}{2} .
$$

Since $I_{z}$ is also conserved, we have $\delta I_{z}=\delta Q$, i.e.

$$
\delta I_{z}=\delta I_{z}^{A}+\delta I_{z}^{B}=\alpha_{A} \delta I_{z}^{A}+\alpha_{B} \delta I_{z}^{B} .
$$

This should be true for all values of $\delta Q$. Hence

$$
\alpha_{A}=\alpha_{B}=1 \text {. }
$$

Since we have barred the existence of multiply charged particles, the $I$ value of single particles is limited to $0,1 / 2$ and 1 . Since there are no charged particles of about the same mass as $\Lambda^{0}$, we can assume:

Consider the reaction

$$
I\left(\Delta^{0}\right)=0 ; \quad I_{z}\left(\Lambda^{0}\right)=0 .
$$

$$
\pi^{-}+p \rightarrow \Lambda^{0}+K^{0}
$$

The left-hand side can have $I=1 / 2$ or $3 / 2$. The right-hand side should have the same value of $I$ and $I_{z}$ due to conservation of $I$ in strong interactions. Therefore, from (7) we conclude

$$
I\left(K^{0}\right)=1 / 2 ; \quad I_{z}\left(K^{0}\right)=-1 / 2 .
$$

1 By $Q$ is here meant the ratio of the amount of charge to charge of electron and this is an integral number for all particles. The question arises whether we could assign such integral charge quantum numbers to the various particles but conceive of a difference in the amount of charge say between a proton and an electron as in some cosmological theories where a slight difference is postulated. Would this imply that the amount of charge is not conserved in processes like $\beta$ decay though the charge as an integral number as defined by (3) is conserved; or would it be possible to observe processes like $e^{-} \rightarrow \gamma+\gamma$ and $e^{-} \rightarrow 2 \nu+\bar{\nu}$ whose lifetime is of the order of $10^{19}$ years? (Marsha and Sudershan, Elementary Particles, Interscience 1960).

2 See also "Elementary Particles and Weak Interactions" by T. D. LEE and C. N. YANG, Lectures at Brookhaven National Laboratory, p. 5 (1957). We have to assume that $\gamma$ is independent of $I_{z}$. 
From the above discussion, the corresponding particle with $I_{z}=1 / 2$ must be the positively charged particle $K^{+}$, i.e. $K^{+}, K^{0}$ form an isotopic doublet. By applying the charge conjugation operator to $K^{+}, K^{0}$, we can generate the antiparticles $K^{-}$and $\bar{K}^{0}$ with

$$
I\left(\bar{K}^{0}\right)=1 / 2 ; \quad I\left(K^{-}\right)=1 / 2 ; \quad I_{z}\left(\bar{K}^{0}\right)=1 / 2 ; I_{z}\left(K^{-}\right)=-1 / 2 .
$$

We have thus uniquely determined the $I$ spin of the $K$ particle. The pairs $K^{+}$, $K^{0}$ and $\bar{K}^{0}, K^{-}$are, like nucleons, spinors in isotopic spin space.

Consider the reaction

$$
\pi^{-}+p \rightarrow \Sigma^{-}+K^{+}
$$

Conservation of $I_{z}$ on both sides leads to

$$
I_{z}\left(\Sigma^{-}\right)=-1 ; I\left(\Sigma^{-}\right)=1 \text {. }
$$

Thus $\Sigma^{+}, \Sigma^{0}, \Sigma^{-}$should form an isotopic triplet, i.e. $\Sigma$ particles transform like a vector in charge space like $\pi$-mesons. As regards the cascade particle $\Xi$, from the fact that $\Xi$ is produced along with two neutral $K$-mesons in $\pi-N$ collisions, we deduce that $\Xi^{-}$corresponds to $I_{z}=-1 / 2$ of an isotopic doublet. Thus $I(\Xi)=1 / 2$;

$$
I_{z}\left(\Xi^{0}\right)=1 / 2 \text {. }
$$

Having assigned $I_{z}=0$ for $A^{0}$, we are inevitably led to the consequence that a reaction which conserves charge need not conserve $I_{z}$. For, consider the reaction

$$
n+n \rightarrow \Lambda^{0}+\Lambda^{0} \text {. }
$$

which conserves charge but not $I_{z}$ since $I_{z}=-1$ for the L.H.S. and 0 for the R.H.S. But we know that $Q$ and $I_{z}$ are related by a linear relation $Q=I_{z}+\frac{Y}{2}$. Since the above reaction conserves the baryon number $N$, it is clear that $Y$ cannot be just identified with $N^{\prime}$. The way out of this difficulty was suggested by Nishijima and Gell-Mann ${ }^{2}$ by defining a new quantum number $S$ with

$$
Y=N+S
$$

each particle being assigned a particular $S$ value. Thus conservation of $Q$ implies only conservation of $I_{z}+\frac{S}{2}$ together and not separately. The calculation of $S$ from (15) is easy and we collect the $I, I_{z}, Y, S$ values in a table of reference.

1 If baryon number is absolutely conserved there should be no physical process by which the proton can decay into lighter particles. The search for this has set a lower limit of $3 \times 10^{24}$ years for proton decay by any mode.

2 M. Gell-Mann, Phys. Rev. 92, 833 (1953); Nuovo Cim. Suppl.2, 848 (1956). T. Nakano and K. Nishisma, Progr. Theoret. Phys. 10, 581 (1953). K. Nishijima, Progr. Theoret. Phys. 13, 285 (1955).

EPCR 15 


\begin{tabular}{c|c|r|r|r|r|r}
\hline Particle & $I$ & $I_{z}$ & $Q$ & $N$ & $Y=N+S$ & $Y-N=S$ \\
\hline$\Xi^{0}$ & $1 / 2$ & $1 / 2$ & 0 & 1 & -1 & -2 \\
$\Xi$ & $1 / 2$ & $-1 / 2$ & -1 & 1 & -1 & -2 \\
$\Sigma^{+}$ & 1 & 1 & 1 & 1 & 0 & -1 \\
$\Sigma^{0}$ & 1 & 0 & 0 & 1 & 0 & -1 \\
$\vdots$ & 1 & -1 & -1 & 1 & 0 & -1 \\
$I^{0}$ & 0 & 0 & 0 & 1 & 0 & -1 \\
$p$ & $1 / 2$ & $1 / 2$ & 1 & 1 & 1 & 0 \\
$\prime \prime$ & $1 / 2$ & $-1 / 2$ & 0 & 1 & 1 & 0 \\
$K^{+}$ & $1 / 2$ & $1 / 2$ & 1 & 0 & 1 & 1 \\
$K^{0}$ & $1 / 2$ & $1 / 2$ & 0 & 0 & 1 & 1 \\
$K^{0}$ & $1 / 2$ & $1 / 2$ & 0 & 0 & -1 & -1 \\
$K^{+}=K^{-}$ & $1 / 2$ & $-1 / 2$ & -1 & 0 & -1 & -1 \\
$\pi^{+}$ & 1 & 1 & 1 & 0 & 0 & 0 \\
$\pi^{0}$ & 1 & 0 & 0 & 0 & 0 & 0 \\
$\pi^{-}$ & 1 & -1 & -1 & 0 & 0 & 0 \\
\hline
\end{tabular}

There exists an interesting explanation for the strangeness quantum number. ${ }^{1}$ We have already estab! ished that the conservation of charge can be interpreted as a consequence of invariance under a gauge transformation. We may similarly introduce a baryon gauge transformation applicable to nucleons and hyperons, i.e. the baryon field $\psi$ is replaced by $\mathrm{e}^{i \chi_{N}} \psi$ where $\chi_{N}$ is a $c$-number. As before we can easily see that the existence of such a transformation will lead to baryon charge $N$, the conservation of which follows from the invariance of the Lagrangian under such a transformation.

Observing that nucleons and $\left(K^{+}, K^{0}\right)$ are iso-spinors, we introduce another gauge transformation for these, i.e. $\psi \rightarrow \mathrm{e}^{i \chi_{Y}} \psi$. For $\left(\Xi^{-}, \Xi^{0}\right)$ which is also an isospinor we postulate the transformation $\psi \rightarrow \mathrm{e}^{i \chi_{F}} \psi$. This gauge is called the "hypercharge gauge". The conserved quantity due to this transformation is called the hypercharge $Y$. The particles $p, n, K^{+}, K^{0}$ are called isofermions, $\Xi^{-} \Xi^{0}$ and the anti-particles of isofermions are called anti-isofermions. In any reaction the number of isofermions minus the number of anti-isofermions will be a constant of motion. Similarly the number of baryons minus the number of anti-baryons will remain a constant. The importance of these two gauges is revealed through the interesting relation between strangeness $S$, the hypercharge $Y$ and the baryon number $N$,

$$
S=Y-N .
$$

It should be noted that the fermion or boson character of a particle and its isofermion or isoboson character are entirely independent. The names "isofermion" or "isoboson" do not imply anything about the statistics obeyed by the particle.

We have already remarked on the existence of four $K$-particles $K^{+}, K^{0}$ and $\bar{K}^{0}, K^{-}$ as a pair of iso-doublets (see however p. 221). $\left(K^{-}, \bar{K}^{0}\right)$ is the anti-pair of $\left(K^{+}, K^{0}\right)$.

1 B. D'Espagnat and J. Prentki, Nucl. Phys. 1, 33 (1956). See also. "Some Theoretical Aspects of the Strong Interactions of the New Particles" by B. D'Espagnat and J. Préntkr, Progr. Elementary Particle and Cosmic Ray Physics 4, 1 (1958). 
It is interesting to note that though $K^{0}$ is a neutral particle it has a distinct anti-particle as contrasted with the case of $\pi^{0}$ which is its own anti-particle. This is due the fact that the strangeness of $K^{0}$ is +1 while that of $\pi^{0}$ is zero. ${ }^{1}$ This feature has some interesting consequences which will be discussed below. We can show that $K^{0}$ and $\bar{K}^{0}$ cannot be identical or in other words, $K$ particles cannot form an isotopic triplet like the $\pi$-meson. Consider the virtual reaction

This implies

$$
n \leftrightarrow \Lambda^{0}+K^{0} .
$$

If $K^{0} \equiv \bar{K}^{0}$, then $n+K^{0} \leftrightarrow \Lambda^{0}$ is allowed.

Thus

$$
n+n \rightarrow \Lambda^{0}+K^{0}+n \rightarrow \Lambda^{0}+\Lambda^{0},
$$

a reaction which has not been observed in spite of its low threshold.

We will now list some of the results which follow as natural consequences of the concept of strangeness. It is clear that if we require the conservation of $Q, I_{z}$ and $N$ for strong and electromagnetic interactions, then we obtain the selection rule $\Delta S=0$ (i.e. strangeness is conserved). We have already mentioned that electromagnetic interactions violate the conservation of total isotopic spin.

If we consider collisions of pions and nucleons the initial system has $S=0$ and the conservation of $S$ clearly requires the production of a $K$ particle $(S=+1)$ whenever a hyperon $(S=-1)$ is produced. This feature in the production of strange particles is called "associated production" and was originally invoked by Pais in order to reconcile the copious production with the long lifetimes of the strange particles. We can equally understand the higher threshold for the production of $K^{-}$in $\pi-N$ or $N-N$ collisions as contrasted with $K^{+}$, for $K^{-}$can only be produced along with $K^{+}$as there are no hyperons with $S=+1$. Associated production with antihyperons for $K^{-}$is not possible in $\left(\pi^{-}-p\right)$ collisions as it implies the violation of baryon conservation. If we now turn to the reaction of $K^{+}$particles with baryons, we find that $K^{+}$particles of moderate energy interacting with nucleons can only be elastically scattered due to the absence of hyperons with positive $S$. Since $S=-1$ for the hyperons, the $K^{-}$particle can undergo a variety of interactions with nucleons at the same energy besides elastic scattering. In a similar way, the interaction of the $\Lambda^{0}$ particle with a nucleon can lead only to elastic scattering while the $\Sigma$ particle will interact rapidly with neutron or a proton at all energies transforming to a $\Lambda^{0}$ particle with the release of approximately $80 \mathrm{MeV}$ of kinetic energy $\left(\simeq \Sigma-\Lambda^{0}\right.$ mass difference) i.e.

$$
\Sigma^{-}+p \rightarrow \Lambda^{0}+n \text {. }
$$

All the particles, i.e. hyperons, $K$ - and $\pi$-mesons, remain stable whell only strong and electromagnetic interactions are present with three exceptions namely the decays of $\pi^{0}, \Sigma^{0}, \bar{\Sigma}^{0}$. Decays like $n \rightarrow p+\pi^{-}, \Lambda^{0} \rightarrow p+K^{-}$or $\Sigma^{+} \rightarrow \Lambda^{0}+\pi^{+}$are forbidden

1 It is to be noted that even though neutron has a distinct anti-particle, it cannot go into an anti-neutron because baryon number conservation is valid even in weak interactions. 
by conservation of energy though they conserve strangeness and hence they can only be virtual processes. The rule $\Delta S=0$ prevents the energetically possible decays like $\Lambda^{0} \rightarrow p+\pi^{-}$or $K^{+} \rightarrow \pi^{+}+\pi^{0}$ through strong interaction.

The $\pi^{0}$ meson has zero charge and zero strangeness and thus the transition of the $\pi^{0}$ to the vacuum can occur with the emission of $\gamma$-rays, i.e. $\pi^{0} \rightarrow \gamma+\gamma$. It is to be noticed that this reaction requires a change of isotopic spin by one unit which is accomplished by the electromagnetic coupling. Since the electromagnetic interaction is proportional to the charge, it is linear in $I_{z}$ and hence it transforms in the isotopic spin space like a scalar plus the $z$ component of a vector. If we treat the electromagnetic coupling as a perturbation, whenever it occurs it can change the total isotopic spin either by zero or by one unit, i.e. $|\Delta I|=0$ or 1 . Turning to $\Sigma^{0}$ we find that the transition $\Sigma^{0} \rightarrow \Lambda^{0}+\gamma$ satisfies $\Delta Q=0, \Delta S=0$, $|\Delta \boldsymbol{I}|=1$. Hence the transition will take place through electromagnetic coupling. A rough theoretical estimate of lifetime can be obtained if we assume a magnetic dipole transition between particles of $\operatorname{spin} 1 / 2\left(\Sigma^{0}\right.$ and $\left.\Lambda^{0}\right)$ with a transition magnetic moment approximately equal to the neutron magnetic moment. We then have lifetime of $\Sigma^{0} \simeq 5 \times 10^{-20}$ sec. Thus $\Sigma^{0}$ undergoes a decay too rapid to be observed directly. As a result of this rapid radiative decay, $\Sigma^{0}$ will rarely undergo the slow pion decay $\Sigma^{0} \rightarrow p+\pi^{-}+110 \mathrm{MeV}$.

All the baryons, anti-baryons and mesons which are stable under strong and electromagnetic interactions decay with finite lifetimes into other particles. The interactions which cause these decays are called "weak interactions" because the coupling strength for these interactions is of the order $10^{-14}$. The strange particles which are produced in strong interactions should decay very rapidly, i.e. they should have a lifetime $\sim \frac{\hbar}{m_{\pi} c^{2}} \sim 10^{-23}$ sec. However lifetimes of these particles range mostly from $10^{-8}$ to $10^{-10} \mathrm{sec}$. It is this unexpectedly long life time of the strange particles that caused theoretical physicists to look for a selection rule which prevents rapid decay of strange particles. It was in a search for an explanation of these seemingly irreconcilable facts that Nishijima and Gell-Mann ${ }^{\mathbf{1}}$ postulated the strangeness quantum number. On the basis of this scheme of assignment of strangeness $S$, the weak interactions violate conservation of $S$ as is seen from the table (p. 226) i.e. $\Delta S= \pm 1$. The decay $\Xi^{-} \rightarrow n+\pi^{-}$which is energetically more favourable than the observed decay $\Lambda^{0}+\pi^{-}$is perhaps forbidden because the decay requires a change of strangeness by two units. Recent developments indicate that all the weak interactions violate also the symmetry under parity and charge conjugation operation (to be discussed later). ${ }^{2}$ Thus the study of strange particle interactions divides itself into two classes,

(1) Strong interactions involving productions,

(2) Weak interactions involving decays.

1 M. Gell-Mavx, Phys. Rev. 92, 833 (1953); Nuovo Cim. Suppl.2, 848 (1956). K. NishiJima, Progr. Theoret. Phys. 13, 285 (1955).

2 See chapter $V$, section 2. 
Surprising as it may seem, it is the attempt at a theory of weak interactions that has led to some definite and interesting conclusions, while the theory of strong interaction is yet in a primitive and speculative stage. We shall first deal with the decays of $K$ particles, the study of which led to the postulate of nonconservation of parity with its far reaching consequences. We shall then examine in some detail the theory of weak interactions particularly on the basis of a universal coupling between four fermions.

By considering the strangeness quantum number we have been able to classify interactions but it does not lead to any detailed predictions of cross-sections or quantitative relationships between various processes. However the assumption of charge independence for interactions involving strange particles will give rise to many quantitative relationships between various interactions connecting given multiplets. Various equalities and inequalities resulting from charge independence in elementary processes can be derived. ${ }^{1}$ We will now discuss a few examples of these relations relating to the capture of $K$ particles by nucleons.

Consider the production of $\Sigma$ particles on capture of $K$ by nucleons. ${ }^{2}$ The initial $K^{-}+p$ system has two particles each with $I=1 / 2$ and since the total $I_{z}=0$, the system will be a mixture of $I=0$ and $I=1$ states. Let $a_{0}$ and $a_{1}$ denote the reaction amplitudes for states $I=0$ and $I=1$. In the final state we require $I=0$ or 1 and we should decompose a $\Sigma+\pi$ system into $I=1$ and $I=0$ states for charge independence requires that $I$ should be a constant of motion during the reaction, i.e. the reaction amplitudes depend only on total $I$. Hence the final $\Sigma+\pi$ state will be

$$
\begin{aligned}
& \frac{1}{\sqrt{2}}\left[a_{0} \Phi_{0,0}(\Sigma \pi)+a_{1} \Phi_{1,0}(\Sigma \pi]\right. \\
& =\frac{1}{\sqrt{2}}\left[\left(\frac{a_{0}}{\sqrt{3}}+\frac{a_{1}}{\sqrt{2}}\right) U_{+}(\Sigma) U_{-}(\pi)-\frac{a_{0}}{\sqrt{3}} U_{0}(\Sigma) U_{0}(\pi)\right. \\
& \left.\quad+\left(\frac{a_{0}}{\sqrt{3}}-\frac{a_{1}}{\sqrt{2}}\right) U_{-}(\Sigma) U_{+}(\pi)\right]
\end{aligned}
$$

where we have made use of the necessary Clebsch-Gordon coefficients to get the various substates arising from the composition of two $I=1$ particles (cf. Appendix 2). Thus $\left(K^{-}+p\right)$ reaction will lead to the following ratio for the production of charged and neutral $\Sigma$ 's

$$
\left(\Sigma^{+} \pi^{-}\right):\left(\Sigma^{0} \pi^{0}\right):\left(\Sigma^{-} \pi^{+}\right)=\frac{\left|\gamma / 2 a_{0}+\right| /\left.3 a_{1}\right|^{2}}{12}: \frac{a_{0}^{2}}{6}: \frac{|| 2 a_{0}-\left|/ 3 a_{1}\right|^{2}}{12} .
$$

Remembering that $a_{0}$ and $a_{1}$ are generally complex, charge independence provides us with the inequality

$$
\sqrt{ } n\left(\Sigma^{+}\right)+\gamma n\left(\Sigma^{-}\right) \geqq 2 / n\left(\Sigma^{0}\right) \geqq\left|\gamma n\left(\Sigma^{+}\right)-\gamma n\left(\Sigma^{-}\right)\right|:
$$

where $n(\Sigma)$ denote the number of $\Sigma$ particles of specified charge.

1 D. Feldian, Phys. Rev. 103, 254 (1956).

2 K. M. CASe, R. Karplus and C. N. YANG, Phys. Rev. 101, 358 (1956). 
The capture of $K^{\prime}$ - by neutrons can go only through $I=1$ state since $I_{z}=-1$ and hence the final $\Sigma+\pi$ state is

Thus we have

$$
a_{1} \Phi_{1,-1}(\Sigma \pi)=\frac{a_{1}}{1 / 2}\left[U_{-}(\Sigma) U_{0}(\pi)-U_{0}(\Sigma) U_{-}(\pi)\right] .
$$

$$
\left(\Sigma^{-} \pi^{0}\right):\left(\Sigma^{0} \pi^{-}\right)=1 / 2 a_{1}^{2}: 1 / 2 a_{1}^{2} .
$$

If $\mathrm{K}^{-}$is incident on $\mathrm{He}^{4}$ or $\mathrm{C}^{12}$, it interacts with the neutron or the proton with equal probability. The number of $\Sigma$ particles of given charge can be obtained by averaging the probabilities given in (22) and (25) and we thus have

$$
n\left(\Sigma^{+}\right)+n\left(\Sigma^{-}\right) \geqq 2 n \Sigma^{0},
$$

a relation independent of the values of $a_{0}$ or $a_{1}$. The difficulty of identifying $\Sigma^{0}$ renders verification of even this simple relation by experiment difficult. One of the simplest predictions is due to Lee ${ }^{1}$ who has discussed the absorption of $K^{-}$ particles by $\mathrm{He}^{4}$ nuclei. We note that since $\mathrm{He}^{4}$ nucleus has $I=0$, the initial system has an isotopic spin $I=1 / 2$ and thus there is no mixture of states in the initial system. We can deduce that the branching ratios between reactions (a) and (b) should be as $2: 1$

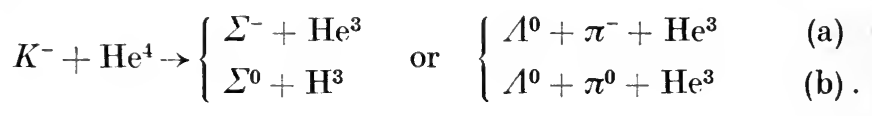

Experimental confirmation of such relations will strengthen the postulate of charge independence in interactions involving the strange particles. ${ }^{2}$

\section{DECAYS OF K.PARTICLES ${ }^{3}$}

Charged as well as neutral $K$-mesons decay by a variety of modes and the multiplicity of decay modes initially created quite a confusion in the identification of the particles. At the beginning, each physically different mode of decay was phenomenologically characterized as a different particle. It is now well established that all

1 T. D. LeE, Phys. Rev. 99, 337 (1955).

2 Sakurai first noted [see Phys. Rev. 107, 908 (1957)) that if charge independence is valid for the reactions $\pi^{+}+p \rightarrow \Sigma^{+}+K^{+} ; \pi^{-}+p \rightarrow \Sigma^{0}+K^{0}$ and $\pi^{-}+p \rightarrow \Sigma^{-}+K^{+}$the relation among the differential cross-sections for $\Sigma^{+}, \Sigma^{0}$ production is $\left[2 \mathrm{~d} \sigma\left(\Sigma^{0}\right)\right]_{2}^{1} \leqq\left[\mathrm{~d} \sigma\left(\Sigma^{+}\right)\right]_{2}^{\frac{1}{2}}$ $+\left[\mathrm{d} \sigma\left(\Sigma^{-}\right)\right]^{\frac{1}{2}}$. Earlier experiment by Brows et al. (Phys. Rev. 107, $\left.906(1957)\right)$ indicated that this relation seemed to break down for the backward produced $\Sigma$ 's. However a recent experiment by Crawford et al. (Phys. Rev. Lett. 3, 394 (1959)) has verified that this relation holds good. Their results also show that this relation is more an equality and that most of the $\Sigma^{0}$ and $\Sigma^{-}$are produced in $I=1 / 2$ state.

${ }^{3}$ For complete information on experimental aspects, see the Study of Elementary Particles by the Photographic Method by C. F. Powell, P. H. Fowlen and D. H. Perkins, Pergamon Press (1959). 
the different mesons have the same mass and lifetime. We list in the table below the available information on the decay modes of charged $K^{+}$. Corresponding decay modes exist for the $K^{-}$, suitable changes being made in the charges of the

\begin{tabular}{c|c|c}
\hline Name & Decay scheme & $\begin{array}{c}\text { Branching ratio } \\
\text { (per cent) }\end{array}$ \\
\hline$\theta^{+}$ & $\pi^{0}+\pi^{+}+220 \mathrm{MeV}$ & 25 \\
$\tau$ & $\pi^{+}+\pi^{-}+\pi^{+}+75 \mathrm{MeV}$ & 6 \\
$\tau$ & $\pi^{+}+\pi^{0}+\pi^{0}+84 \mathrm{MeV}$ & 2 \\
$K_{\mu 2}$ & $\mu+v+368 \mathrm{MeV}$ & 58 \\
$K_{\mu 3}$ & $\mu^{+}+\pi^{0}+v+253 \mathrm{MeV}$ & 5 \\
$K_{e 3}$ & $e^{+}+\pi^{0}+v+358 \mathrm{MeV}$ & 4 \\
\hline
\end{tabular}

decay products. The most frequent mode in the decay of charged $K$ is $K_{\mu 2}$, i.e. $K^{+}\left(K^{-}\right) \rightarrow \mu^{+}\left(\mu^{-}\right)+\nu(\bar{v})$. The analogous decay mode of $K$ into an electron and a neutrino, i.e. $K^{+}\left(K^{-}\right) \rightarrow e^{+}\left(e^{-}\right)+v(\bar{v})$ (i.e. $\left.K_{e 2}\right)$ seems to be rare or absent. Experiments on $K^{+}$stopping in emulsion with emission of $e^{+}$indicate that the ratio $\frac{e^{+}+v}{\mu^{+}+v}$ is probably $>0.1$ per cent. The existence or absence of $K_{e 2}$ decay mode has important implications as we shall see later when we discuss the Universal Fermi interaction. It is to be noted that there is no $K$ decay which involves neutrino pair emission as in the case of $\mu$ decay.

\section{The $\tau^{+}-\theta^{+}$puzzle $^{1}$}

The idea that the various decay modes of $K^{+}$particles empirically denoted by different names like $\tau^{+}, \theta^{+}$etc., are merely manifestations of a single particle, brought in its wake a puzzle which troubled theoretical physicists for more than a year. If $\theta^{+}$and $\tau^{+}$are the same, their intrinsic parity should also be identical and if the conservation of parity is considered valid, then since the $2 \pi$ and $3 \pi$ decay modes indicate that the parities of $\theta^{+}$and $\tau^{+}$are different, they cannot be the same particle. However if $\theta^{+}$and $\tau^{+}$are different particles, there is no explicable reason why their masses and lifetimes should be identical. If the postulate of conservation of parity is given up the puzzle of course does not exist. In view of the fundamental importance of the study of the two decay modes which led Lee and Yang to question the conservation of parity in weak interactions, we give a detailed discussion of the analysis by Dalitz. This is based on the assumption that parity is conserved in decay interactions.

If the $\theta^{+}$particle has spin $j$, since we are considering its decay at rest, it is clear that the relative motion of the two pions must posses an orbital angular momentum $j$ since the pions are spinless. Assuming parity to be conserved in

1 See R. H. Dalitz, loc. cit., pp. 195. 
the decay, this decay will be allowed only if the $\theta^{+}$particle has an intrinsic parity $(1)$ given by

$$
\omega=(-1)^{j} .
$$

Thus the $\theta^{+}$particle can have the spin-parity assignments $\left(0^{+}\right)\left(1^{-}\right)\left(2^{+}\right)\left(3^{-}\right) .^{1}$

All the three pions in the $\tau^{+}$decay mode may be followed to the end of their range and this allows us to establish fully the configuration of the decay. The event has the well-known $Q$ value $\sim 75.1 \mathrm{MeV} .^{2}$ This information along with the assumption of the conservation of momentum helps us to fix the configuration of the decay exactly even if only two of the three pions are observed. The configuration is conveniently specified by two momenta; $\boldsymbol{p}$ the momentum of the $\pi^{-}$in the initial rest system of $\tau^{+}$and $\boldsymbol{q}$ the momentum of one of the two $\pi^{+}$in their centre of mass system. We have the relation (see Appendix 5).

$$
q=\frac{1}{2}\left(M_{\tau}^{2}-3 m_{\pi}^{2}-2 M_{\tau} E_{p}\right)^{1 / 2}
$$

where $E_{p}=\left(m_{\tau}^{2}+p^{2}\right)^{1 / 2}$ is the total $\pi^{-}$energy. If $\theta$ denotes the angle between $\boldsymbol{p}$ and $\boldsymbol{q}, \cos \theta$ is related to the observed momenta by the expression

$$
\cos \theta=\frac{p_{1}^{2}-p_{2}^{2}}{p q}\left\{1-\left(\frac{p}{M_{\tau}-E_{p}}\right)^{2}\right\}^{-1 / 2}=\frac{\omega_{1}-\omega_{2}}{p} \frac{\omega_{q}}{q}
$$

$\boldsymbol{p}_{1}, \omega_{1}$ and $\boldsymbol{p}_{2}, \omega_{2}$ being the observed momenta and energies of the two $\pi^{+}$.

In order to discuss the spin-parity assignments of $\tau^{+}$, let us denote by $L$ and $l$, the orbital angular momenta associated with the linear momenta $\boldsymbol{p}, \boldsymbol{q}$ respectively. The two positive pions are identical Bose particles and hence they must be described by a wave function which is symmetric under the interchange of the two particles. This is possible only if their relative motion has even parity and so $l$ must be even. Taking into account the odd intrinsic parity of the three pions, conservation of parity in $\tau^{+}$decay requires that the intrinsic parity $\omega$ of $\tau^{+}$be

$$
\omega=-(-1)^{L} \text {. }
$$

We note therefore that the intrinsic parity is determined by the orbital angular momentum of the odd negative pion. Further if $\tau^{+}$has a spin $j$, then from the usual vectorial addition of angular momenta, we must have

$$
\boldsymbol{J}=\boldsymbol{L}+\boldsymbol{l} .
$$

If $j=0$, then $L=l$ i.e. $L$ is even and the parity of $\tau^{+}$is necessarily negative. Thus for zero spin $\tau \neq \theta$. For higher values of $j$, we can make the following statements.

(a) If there exists an $S$ wave $\pi^{-}$meson, its parity is odd and its spin is even. Since $L=0, \omega=1$ and $j=l$ and is even. Thus the probability density for $\tau^{+}$

1 Associating $\theta^{+}$with $\theta^{0}$ which decays into $2 \pi^{0}$, we can conclude that $\theta^{+}$will have only even spin since $2 \pi^{0}$ can have only even orbital angular momentum.

${ }^{2}$ By $Q$ value we refer, as usual, to the kinetic energy available for the particles in the final state. 
decay events must vanish for the decay configuration in which the $\pi^{-}$meson is at rest, unless the $\tau^{+}$parity is odd and its spin is even. It should be noted that for even spin $\theta$ has even parity.

(b) If the two $\pi^{+}$mesons have a relative $S$-wave motion, i.e. $l=0$ then, $\omega=-(-1)^{L}=-(-1)^{j}$. This implies that if $\tau^{+} \equiv \theta^{+}$the probability density for $\tau^{+}$decay events must vanish for the decay configuration in which $\pi^{-}$meson has its maximum energy.

(c) A configuration in which three pions have parallel momenta, i.e. $\cos \theta=1$ cannot result from $\tau^{+}$decay if its spin-parity relation is the same as that of $\theta^{+}$. The total parity of this "linear" configuration is necessarily $-(-1)^{j}$ and hence the probability density for $\tau^{+}$decay events must vanish for a "linear" configuration if $\tau^{+} \equiv \theta^{+}$.

These three situations provide an essential basis to distinguish between various spin-parity possibilities for the $\tau$ particle and also between $\tau$ and $\theta$. The probability for $\tau$ decay configurations consists essentially of two factors,

(a) The phase space density $\varrho$ given by

$$
\varrho \mathrm{d} E_{p} \mathrm{~d}(\cos \theta)=\left\{\frac{p q}{M_{\tau}^{2}+m_{\pi}^{2}-2 M_{\tau} E_{p}}\right\}^{1 / 2} \mathrm{~d} E_{p} \mathrm{~d}(\cos \theta) .
$$

(b) The probability density $\varphi$ which is the square of the amplitude $S(m)$ for this decay configuration averaged over $\tau$-spin orientations, i.e.

$$
\varphi=\frac{1}{2 j+1} \sum_{m}|S(m)|^{2} .
$$

Plotting the observed decay events with $t=E_{p}-m_{\pi}$ and $\cos \theta$, it was found that the events are uniformly distributed indicating no strong dependence either on $t$ or $\cos \theta$ as should be the case if $\tau^{+} \equiv \theta^{+}$. Figs. (32) and (33) show the distri-

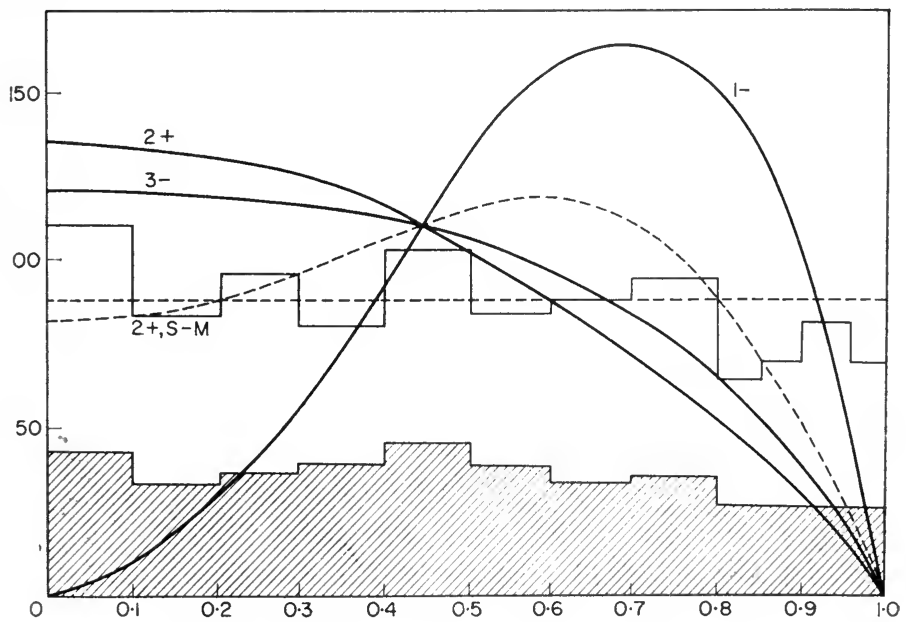

FrG. 32. Distribution of $\tau^{+}$decay events in $\cos \theta$. 


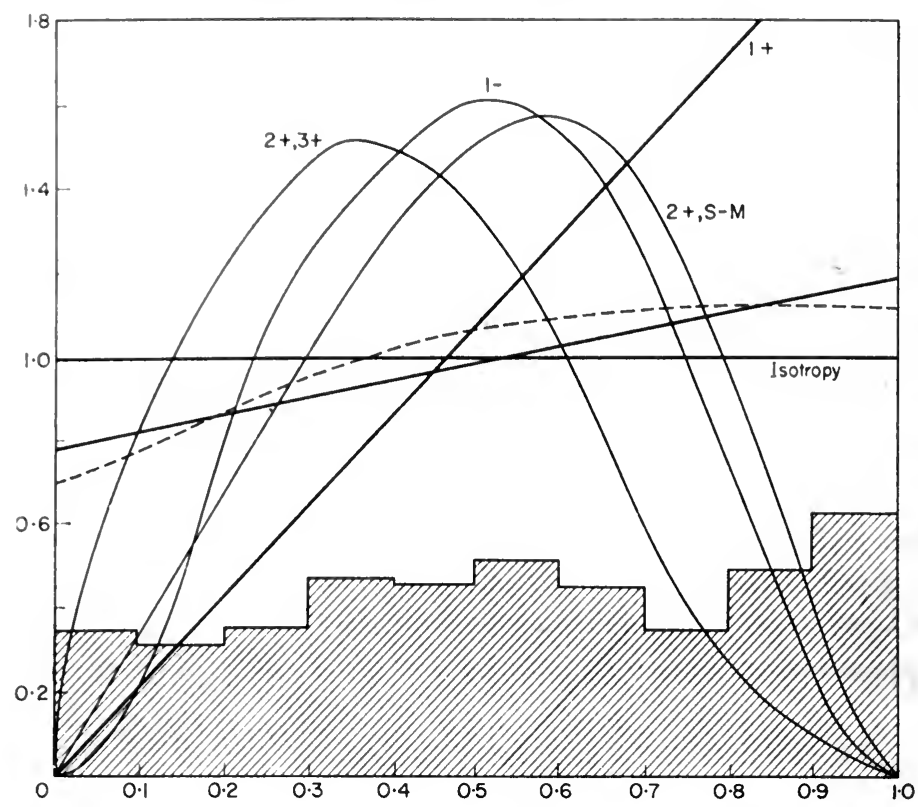

FIG. 33. Distribution of $\tau^{+}$decay events as a function of $\pi^{-}$kinetic energy obtained from 892 observed events; the hatched histogram refers to events of Baldo-Ceolin et al. only. The dotted curve shows the spin $\Sigma^{+}$distribution of Marshak and Sudarshan. The dashed curve is the prediction on Schwinger's model.

butions of events plotted against $\pi^{-}$energies and $\cos \theta$ separately. From angular momentum considerations alone the decay of $\tau^{+}$meson from a state with spin component $m$ can be described by

$$
S(m)=\sum_{L l} d_{L l}\left(p^{2} q^{2}\right) \sum_{m_{L}+m_{l}=m} C\left(L l j ; m_{L}, m_{l}\right) Y_{L}^{m_{L}}\left(\cos \theta_{p}\right) Y_{l}^{m_{l}}\left(\cos \theta_{q}\right),
$$

where $C$ 's are the Wigner coefficients (see Appendix 2). The $d_{l}$ are scalar coefficients and are functions of $p^{2}$ only.

We will consider whether there exists any spin-parity possibility for which both $2 \pi$ and $3 \pi$ decay are permissible. The distributions for different configurations for the various spin-parity assignments have been worked out by Dalitz ${ }^{1}$, Fabri $^{2}$ and others. From Figs. (32) and (33), we immediately neglect the case of $1^{-}$ as it disagrees with the data in all respects. Spin-parity $2^{+}$represents the lowest possible value for which $\tau$ and $\theta$ can be identical. The configurations $(0,0),(1,2)$ do not at all agree with experimental data. Marshak and Sudarshan ${ }^{3}$ have investigated the effect of including the configuration $(3,2)$. Though the inclusion improves the agreement, $\boldsymbol{\tau}^{-}$energy distribution proves to be a difficulty. To get

1 R. H. Dalitz, Phil. Mag. 44, 1068 (1953); Phys. Rev. 94, 1046 (1954).

2 E. Fabri, Nuovo Cim. 11, 479 (1954).

${ }^{3}$ R. E. Marshak, Proc. Roch. Conf. on High Energy Physics, 1956. 
the high probability observed for very fast $\pi^{-}$-mesons, it will be necessary to postulate a resonance near zero energy in the $D$-wave for two $\pi^{+}$-mesons and this requires a $\pi^{+}-\pi^{+}$interaction which has no experimental justification ${ }^{1}$. Still there are a large number of slow $\pi$-mesons. Higher spins will slow down $\tau^{+}$ decay because of their large centrifugal barrier and further in these cases we have to explain the absence of the radiative decay of $\tau$,

$$
\tau^{+} \lambda_{\pi^{+}+e^{+}+e^{-}}
$$

which will be just forbidden if $\tau$ 's are spinless. Teucher, Thirring and Winzeler ${ }^{2}$ have suggested a search for angular correlations in the spatial orientations of $\tau^{+}$ decay with respect to the production plane which should exist "barring accidents". For, any polarization of the $\tau^{+}$particle when it is produced will be reflected in the distribution of the $\pi^{-}$-decay. Orear et al. ${ }^{3}$ have reported no significant deviations from isotropy but this may be due to the fact that the production process do not polarize the $K$ particle as they are spinless.

To sum up, the statistical data on $\tau$ decay configurations is consistent with spin-parity assignment $0^{-}$for $\tau, 0^{+}$for $\theta$. Therefore $\tau$ and $\theta$ are distinct particles as long as parity conservation is assumed. Dalitz ${ }^{4}$ has also pointed out the sym-

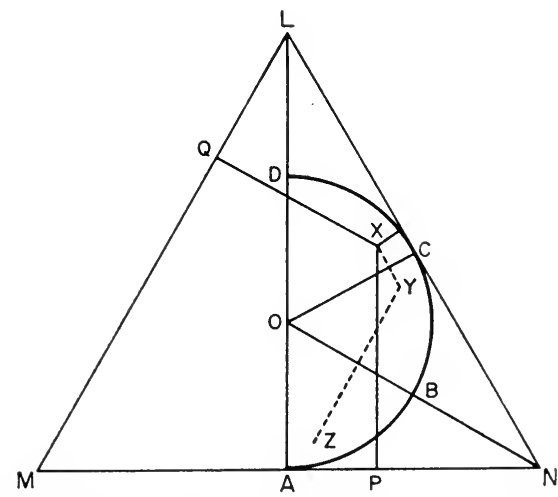

Fig. 34. Triangular plot for representation of $\tau^{+}$decay events.

metry between the three pions irrespective of their charge in the $\tau^{+}$decay. In Fig. 34, $X P, X Q$, and $X R$ are the perpendiculars from $X$ to the sides of an equi-

1 The departure of the $\tau^{+}$-decay spectrum from the one due to phase space amounting to a $25 \%$ variation of the matrix element over the $\pi^{+}$or $\pi^{-}$energy has been attributed to the final state $\pi-\pi$ interaction. See the Report of the Tenth Annual Conference on High Energy Physics, Rochester, pp. 518 et. seq.

${ }^{2}$ M. Teucher, W. Thirring and H. Winzeler, Nuovo Cim. 1, 733 (1955).

3 J. Orear, G. Harris and O. S. TAYlor, Phys. Rev. 102. 1676 (1956).

+ R. H. Dalitz, Proc. Phys. Soc. A 69, 527 (1956). 
lateral triangle $L, U N$ and are proportional to $\pi^{-}$and $\pi^{+}$kinetic energies respectively. Momentum conservation confines the point $X$ to the inscribed semi-circle within the triangle. Fabri ${ }^{1}$ has shown that these boundaries are considerably distorted if relativistic effects are taken into account. The lines $O B$ and $O C$ divide this allowed region into three equal parts, $Y$ and $Z$ obtained by reflecting $X$ in $O C$ and then $Y$ in $O B$, which represent configurations with the same pion energies but a different distribution of charges. There is good experimental evidence for the strong symmetry among the three pions.

Pais ${ }^{2}$ pointed out the possibility for an alternative $3 \pi$ decay called the $\tau^{\prime}$ mode, i.e. $\tau^{ \pm} \rightarrow \pi^{ \pm}+\pi^{0}+\pi^{0}$. The maximum energy allowed for a changed $\pi$ meson is $55 \mathrm{MeV}$ and the $Q$ value is $86 \mathrm{MeV}$. The statistically most significant value for the ratio of $\frac{\tau^{\prime}}{\tau}$ is given by

$$
\frac{\tau^{\prime}}{\tau}=0.332 \pm 0.067
$$

The $\theta-\tau$ puzzle does not exist if the postulate of parity conservation is given up. Before this far reaching development took place, there had been quite a few suggestions to solve the $\theta-\tau$ puzzle still assuming that parity is conserved in weak interactions.

Lee and Orear ${ }^{3}$ attempted to explain the apparent equality of lifetime of $\tau$ and $\theta$ as due to a cascade process. They assumed that $\tau$ and $\theta$ are different particles with two different lifetimes $10^{-8}$ and $10^{-10}$ sec. Besides the various decay modes, they suggested that $\tau$ decays into $\theta$ by emitting $\gamma$-radiation, i.e.

$$
\tau^{+} \rightarrow \theta^{+}+\gamma \text {. }
$$

If we assume that $\tau$ and $\theta$ are spinless, i.e. $\left(0^{-}\right)$and $\left(0^{+}\right)$, then we require emissions of two photons, i.e.

$$
\tau^{+} \rightarrow \theta^{+}+\gamma+\gamma
$$

The secondary $\theta^{+}$will give rise to $K_{\pi 2}$ events. As the lifetime of $\theta^{+}$is smaller compared to that of $\tau$, any directly produced $\theta^{+}$will decay very close to the target and would not contribute to the $K_{\pi 2}$ events. Thus the lifetime of the $K_{\pi 2}$ events would necessarily equal the lifetime for $3 \pi$ decay since the observed $K_{\pi 2}$ decays are mostly due to the secondary $\theta^{+}$. It is also clear that mass measurements by momentum-range method will be appropriate only in the case of the $\tau^{+}$particle. In order to give a radiative lifetime of $10^{-8} \mathrm{sec}$. for $\tau^{+}$and to have the correct branching ratio to explain $K_{\pi 2}$ decay, it is found that a mass difference $\left(m_{\tau^{+}}-m_{\theta^{+}}\right) \simeq 10 m_{e}$ is needed. Further the search for the $\gamma$-ray by Alvarez ${ }^{4}$ proved futile. This proposal is now given up.

1 E. FABRI, loc. cit.

2 A. Pais, Phys. Rev. 86, 663 (1952).

3 T. D. Lee and J. Orear, Phys. Rev. 100, 932 (1955).

${ }^{4}$ L. Alvarez, Proc. 6th Rochester Conf., Interscience, New York (1956). 
Lee and Yang ${ }^{1}$ put forward a proposal to explain the mass degeneracy of $\tau$ and $\theta$ based on the "parity-doublet" hypothesis. They argued that just as there is a mass degeneracy between proton and neutron (i.e. states of different charges) there can exist a mass degeneracy between states of different parities. If $C_{p}$ is the operator that switches $\tau$ into $\theta$ and vice versa (i.e. switches the parity) $C_{p}$ is called parity conjugation in analogy with charge conjugation.

$$
C_{p}|\tau\rangle=|\theta\rangle ; \quad C_{p}|\theta\rangle=|\tau\rangle .
$$

The approximate mass degeneracy between $\tau$ and $\theta$ follows if we postulate that the strong part of the Hamiltonian commutes with $C_{p}$,

$$
\text { i.e. }\left[C_{p}, H\right]=0 .
$$

This implies that under strong interactions alone $m_{\tau}=m_{\theta}$. If we consider a reaction like

$$
\pi^{-}+p \rightarrow \Lambda^{0}+\theta^{0}
$$

and operate $C_{p}$ on it, then it follows that $\Lambda^{0}$ must be a parity doublet which we shall call by $\Lambda_{1}^{0}$ and $\Lambda_{2}^{0}$. Under $C_{p}$

becomes

$$
\pi^{-}+p \rightarrow \Lambda_{1}^{0}+\theta^{0}
$$

$$
\pi^{-}+p \rightarrow \Lambda_{2}^{0}+\tau^{0} .
$$

Thus it is necessary that all the hyperons should be parity doublets. We have assumed that pions and nucleons are unaffected by $C_{p}$. A definite prediction of this theory is that for $\pi-N$ or $N-N$ collisions, the production cross-section and angular distribution for a given strange particle must be equal to those for the corresponding parity conjugate particle. Thus it follows that $\tau / \theta$ ratio at production is necessarily unity. Treiman ${ }^{2}$ has studied the effect of parity doublet theory in hyperfragments and Lee, Yang ${ }^{3}$ and Gatto ${ }^{4}$ have pointed out some interesting interference effects whose observation would indicate the existence of a parity doublet. As such there is no direct empirical evidence which requires the existence of hyperon parity doublets.

\section{Neutral $\boldsymbol{K}$ decay}

We have already noted that the existence of a neutral $K^{0}$ particle with a quite distinct antiparticle $\bar{K}^{0}$ has some interesting consequences which were first studied by Gell-Mann and Pais. ${ }^{5}$ In strong and electromagnetic interactions owing to conservation of strangeness, $K^{0}$ and $\bar{K}^{0}$ cannot be connected even by virtual processes. However the situation is different in decay interactions in which

1 T. D. Lee and C. N. YANG, Phys. Rev. 102, 290 (1956).

2 S. B. Treiman, Phys. Rev. 104, 1475 (1956).

3 T. D. LeE and C. N. YANG, Phys. Rev. 104, 822 (1956).

* R. Gatto, Nuovo Cim. 4, 197 (1956).

5 M. Gell-Manx and A. PaIs, Phys. Rev. 97, 1387 (1955). In this paper they studied this effect assuming charge conjugation invariance. 
strangeness is not a good quantum number and is not conserved ${ }^{\mathbf{1}}$. We instead consider the quantum number $C P$ as we expect weak interactions to remain invariant under $C P$. We can form suitable linear combinations of $K^{0}$ and $\bar{K}^{0}$ which are eigen states of $C P$. Noting that since $K^{0}$ and $\bar{K}^{0}$ are antiparticles, these two go into each other under $C P$. if we define

$$
\begin{aligned}
& \left.K_{1}^{0}\right\rangle=\frac{1}{l^{2}}\left|K^{0}\right\rangle+\frac{1}{\sqrt{2}}\left|\bar{K}^{0}\right\rangle \\
& \left.K_{2}^{0}\right\rangle=\frac{1}{i V^{2}}\left|K^{0}\right\rangle-\frac{1}{i \sqrt{2}}\left|\bar{K}^{0}\right\rangle
\end{aligned}
$$

we find that $\left|K_{1}^{0}\right\rangle$ and $\left|K_{2}^{0}\right\rangle$ are eigen states of $C P$ with eigenvalues +1 and -1 respectively.

Since $C P$ is conserved in weak interactions, in decay processes we can treat $K_{1}^{0}$ and $K_{2}^{0}$ as basic particles with their own modes of decay and consider the decays of $K^{0}$ and $\bar{K}^{0}$ as a consequence of their being linear combinations of $K_{1}^{0}$ and $K_{2}^{0}$. We note the important feature, that the lifetimes of $K_{1}^{0}$ and $K_{2}^{0}$ will in general be different since conservation of $C P$ during decay makes available certain modes to $K_{1}^{0}$ which are forbidden to $K_{2}^{0}$. $K_{2}^{0}$ cannot decay into a $2 \pi$ system with $C P=+1$. The state $\left[\psi\left(\mu^{-}+\bar{v}+\pi^{+}\right)-\psi\left(\mu^{+}+v+\pi^{-}\right)\right]$corresponding to $C P=-1$ is a possible final state for the decay of $K_{2}^{0}$ and comparison with $K_{\mu 3}^{+}$suggests that $K_{2}^{0}$ can have a lifetime exceeding that of $K_{1}^{0}$ by a factor of $10^{3}$. Thus we are led to predict the existence of two neutral particles, one short-lived and another longlived.

Suppose we generate a beam of $K$-mesons by the reactions $\pi^{-}+p \rightarrow \Lambda^{0}+K^{0}$. We note from (18) and (19) that half of them are $K_{1}^{0}$ and the other half $K_{2}^{0}$. After a lapse of time $\sim 10^{-9}$ sec. most of the $K_{1}^{0}$ would have decayed into $2 \pi$ while very few of the $K_{2}^{0}$ would have decayed. The "stale" beam consists mostly of $K_{2}^{0}$. Since $K_{2}^{0}$ is a "mixture" state of strangeness, the "stale" beam when allowed to

1 It is an interesting question under what circumstances we can consider linear combination of two different particle fields as representing the field of a new particle; more so when we wish to have a linear combination of particles and antiparticles. In discussing this it is convenient to divide 'antiparticles' into three classes:

1. Particles and antiparticles are not identical and the number of particles minus antiparticles is conserved, as in the case of fermions which can be baryons or leptons.

2. Antiparticles are different from particles but the conservation law is not valid as in the case of bosons $K^{0}, \overline{K^{0}}, \pi^{+}, \pi^{-}$and $K^{+}, K^{-}$.

3. Particle and antiparticle are identical as in the case of $\pi^{0}$ and $\gamma^{\prime}$

Since in (2) and (3) the conservation law is not applicable, particles can be absorbed or emitted singly while in the case of fermions the interaction has to be bilinear. Linear combinations of fermions and antifermions are meaningless in view of such conservation. In (3) it is trivial though the photon can of course be treated as a linear combination of right and left circularly polarized photons. Since conservation of charge is absolute, there is no meaning in speaking of combinations of the charged $\pi$-mesons. So the only meaningful combination is for the $K$ particles and this is possible since the decay channels of $K^{0}$ and $\bar{K}^{0}$ are identical, but linear combinations could be so chosen that the channels can be apportioned in a mutually exclusive manner. 
interact with matter will produce interactions characteristic both of $S=+1$ and $S=-1$. This behaviour of the "stale beam" is to be contrasted with that of the "fresh beam" of $K$-mesons all of which had strangeness +1 .

Since in weak interactions only $K_{1}^{0}$ and $K_{2}^{0}$ are meaningful, it is necessary to consider $K^{0}$ and $\bar{K}^{0}$ as linear combinations of $K_{1}^{0}$ and $K_{2}^{0}$. In strong interactions however, $K^{0}$ and $\bar{K}^{0}$ retain their respective identities. This is quite obvious from the fact that we can have a "fresh beam" of $K^{0}$ or $\bar{K}^{0}$ at the time of production.

We give here the decay modes of $K_{1}^{0}$ and $K_{2}^{0}$.

In analogy with $\tau^{+}$, the $K_{1}^{0}$ and $K_{2}^{0}$ particles which decay into three mesons are also called $\tau_{1}^{0}$ and $\tau_{2}^{0}$ respectively. But the $\theta^{0}$ decay will be similar to the $\tau^{0}$ decay. The $3 \pi^{0}$ decay of $\tau_{2}^{0}$ corresponds to $C P=-1$ and the $\pi^{+}$mode to $C P=-1$. If we denote by $l$ the angular momentum of $\pi^{+}$and $\pi^{-}$in their centre of mass system and $L$ the angular momentum of $\pi^{0}$ in the $\tau^{0}$ rest system for the $\tau_{2}^{0}$ particle (corresponding to $K_{2}^{0}$ ) we have $l=L=0,2, \ldots K_{1}^{0}$ will rarely decay through this mode because of phase space considerations. However for the $\tau_{1}^{0}$ particle (cor-

\begin{tabular}{c|c|c|c}
\hline Name & Decay mode & $\begin{array}{c}\text { Branching ratio } \\
\text { (per cent) }\end{array}$ & Lifetime \\
\hline$K_{1}^{0}\left(\theta_{1}^{0}\right)$ & $K_{1}^{0} \rightarrow \pi^{+}+\pi^{-}$ & 86.6 & $(1.00 \pm 0.038) \times 10^{-19} \mathrm{sec}$ \\
$K_{1}^{0}\left(\theta_{1}^{0}\right)$ & $K_{1}^{0} \rightarrow 2 \pi^{0}$ & 14.6 & \\
$\tau_{1}^{0}$ & $K_{1}^{0} \rightarrow \pi^{+}+\pi^{-}+\pi^{0}$ & very rare & \\
$\tau_{2}^{0}$ & $K_{2}^{0} \rightarrow \pi^{+}+\pi^{-}+\pi^{0}$ & 30 & \\
& $\rightarrow 3 \pi^{0}$ & & $\left(6.1_{-1.1}^{+1.6}\right) \times 10^{-8} \mathrm{sec}$ \\
$K_{2}^{0}\left(K_{e 3}\right)$ & $K_{2}^{0} \rightarrow e^{+}+v+\pi^{\mp}$ & 30 & \\
$K_{2}^{0}\left(K_{\mu 3}\right)$ & $K_{2}^{0} \rightarrow \mu^{ \pm}+v+\pi^{\mp}$ & 30 & \\
\hline
\end{tabular}

responding to $\left.K_{1}^{0}\right) L=l=1,3, \ldots$ are allowed and hence the outgoing pions face centrifugal barriers owing to their angular momentum even in the lowest configuration. Since $3 \pi^{0}$ has $C P=-1$, only $K_{2}^{0}$ can decay into this mode while $K_{1}^{0}$ cannot undergo decay through this. ${ }^{1}$ For $K_{1}^{0}$ even the $3 \pi$ mode is less important because of phase space considerations. The existence of the long lived $K_{2}^{0}$ particle has been established by experiments. ${ }^{2}$

We will discuss now two interesting phenomena which depend upon considering $K^{0}$ and $\vec{K}^{0}$ as a linear combination of $K_{1}^{0}$ and $K_{2}^{0}{ }^{3}$ From the above discussion it is clear that at the time of production, i.e. $t=0$ the wave function describing the $K^{0}$ particle has the form

$$
\psi\left(K^{0}, 0\right)=\frac{1}{\sqrt{ } 2}\left[\psi\left(K_{1}^{0}, 0\right)+i \psi\left(K_{2}^{0}, 0\right)\right] .
$$

1 See A. PaIs, Proc. 7th Rochester Conference, Interscience, New York (1957).

2 K. Lande, E. T. Booth, J. Impeduglia, L. M. Lederman and W. Chinowski, Phys. Rev. 102, 1901 (1956).

${ }^{3}$ A. Pais and O. Piccioni, Phys. Rev. 100, 1487 (1955). 
This state changes with time due to the phase changes arising from the difference in energy of $K_{1}^{0}$ and $K_{2}^{0}$ and the difference in decay lifetimes. Thus at a later time $t$, the wave function becomes

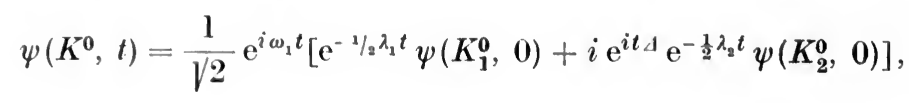

where

$$
\omega_{1}=V m_{1}^{2}+p^{2} ; \quad \Delta=\omega_{2}-\omega_{1} \simeq \frac{m_{1} \delta m}{\omega_{1}}
$$

and $\lambda_{1}$ and $\lambda_{2}$ are the decay constants for $K_{1}^{0}$ and $K_{2}^{0}$ respectively. From the interpretation of Gell-Mann ${ }^{1}$ we know that after a flight time long compared with $10^{-10}$ sec we will be left with a pure $K_{2}^{0}$ beam. Since $K_{2}^{0}$ contains the $\bar{K}^{0}$ component with $S=-1$ which was absent in the initial $K^{0}$ beam there is a possibility of observing hyperons if the $K_{2}^{0}$ beam, at distances far from the primary target, is allowed to interact with nuclei and this will provide a convincing proof for the existence of $K_{2}^{0}$. Supposing this secondary target is thick enough to remove a considerable amount of $\bar{K}^{0}$ from $K_{2}^{0}$, the $K^{0}$ which interacts little with the nuclei will pass on beyond this target and thus there is a regeneration of the shortlived $K^{0}$ far from the previous target. We can then observe the characteristic $2 \pi$ decay of $K_{1}^{0}$ component of this secondary $K^{0}$ beam. We shall now consider this process in a quantitative way. Denoting the wave function at time $t$ by

where

$$
\begin{aligned}
{\left[a(t) \psi\left(K^{0}\right)\right.} & \left.+\bar{a}(t) \psi\left(\bar{K}^{0}\right)\right] \mathrm{e}^{i \omega_{1} t} \\
& =\left[a_{1}(t) \psi\left(K_{1}^{0}\right)+a_{2}(t) \psi\left(K_{2}^{0}\right)\right] \mathrm{e}^{i w t},
\end{aligned}
$$

$$
a_{1}(t)=\frac{a(t)+\bar{a}(t)}{\gamma^{2}} ; \quad a_{2}(t)=\frac{a(t)-\bar{a}(t)}{i \sqrt{2}}
$$

we arrive at the following differential equations for amplitudes $a_{1}(t)$ and $a_{2}(t)$

$$
\begin{aligned}
\frac{\mathrm{d} a_{1}(t)}{\mathrm{d} t}= & \left(-\frac{1}{2 \tau}-\frac{1}{4 \tau_{a}}+\frac{1}{4 \tau_{s}}\right) a_{1}(t)+i\left(\frac{1}{4 \tau_{a}}-\frac{1}{4 \tau_{s}}\right) a_{2}(t) \\
= & \alpha a_{1}(t)+i \lambda a_{2}(t) . \\
\frac{\mathrm{d} a_{2}(t)}{\mathrm{d} t}= & -i\left(\frac{1}{4 \tau_{a}}-\frac{1}{4 \tau_{s}}\right) a_{1}(t)+\left(i \Delta-\frac{1}{4 \tau_{a}}-\frac{1}{4 \tau_{s}}\right) a_{2}(t) \\
& -i \lambda a_{1}(t)+\beta a_{2}(t),
\end{aligned}
$$

where $\tau_{s}$ and $\tau_{a}$ denote the mean time of flight for $K^{0}$ before scattering and for $K^{0}$ before nuclear absorption respectively, $\tau=1 / \lambda_{1}$ being the mean lifetime for $K_{1}^{0}$ decay. Initially we start with a pure $K^{0}$ beam and do not take into account nuclear absorption, i.e. after a lapse of time of the order $10^{-10} \mathrm{sec}$ we have a $K^{\mathbf{0}}$ beam. We shall use the above equations to study what happens to this $K^{0}$ beam of

1 M. Gell-Mann and A. Pais, loc. cit. 
velocity $v$ when passing through a nuclear matter of thickness $d=v t$. Thus at $t=0, a_{1}(0)=0, a_{2}(0)=A$. Then

$$
\begin{aligned}
& a_{1}(t)=-i \lambda A\left[\frac{\mathrm{e}^{-\mu_{2} t}-\mathrm{e}^{-\mu_{1} t}}{\mu_{2}-\mu_{1}}\right] \\
& a_{2}(t)=A \frac{\left[\left(\alpha+\mu_{2}\right) \mathrm{e}^{-\mu_{2} t}-\left(\alpha+\mu_{1}\right) \mathrm{e}^{-\mu_{1} i}\right\rfloor}{\mu_{2}-\mu_{1}},
\end{aligned}
$$

where

$$
\mu_{2}, \mu_{1}=\frac{1}{2}\left[-(\alpha+\beta) \pm V /\left\{4 \lambda^{2}+(\alpha-\beta)^{2}\right]\right.
$$

The absorption curve for $K_{2}^{0}$ is given by the ratio of the final intensity to the initial one,

$$
\frac{I(d)}{l(0)}=\frac{\left|a_{2}\left(\frac{d}{v}\right)\right|^{2}}{\left|a_{2}(0)\right|^{2}} .
$$

Serber ${ }^{1}$ has pointed out that if $\delta m$ is large relative to the $K_{1}^{0}$ lifetime width, nuclear absorptions leading to hyperons can occur within the material rather close to the primary target. In this case, the wave function oscillates between $\psi\left(K^{0}\right)$ and $\psi\left(\bar{K}^{0}\right)$ and if the oscillatory variation of these reactions with distance can be measured, this should allow an estimate of $\delta m$ despite its smallness.

It is not yet clear whether $K_{1}^{0}$ can decay into a pion and two leptons. Assuming that both $K_{1}^{0}$ and $K_{2}^{0}$ can have such a decay mode with similar rates, we can discuss some interesting effects due to interference between $K_{1}^{0}$ and $K_{2}^{0}{ }^{2}$ It is expected that these decays will have a complicated time dependence rather than the usual exponential one. The observation of such effects will directly confirm the theoretical prediction of $K_{1}^{0}$ and $K_{2}^{0}$. Considering only the decay $(\pi e v)$ of a neutral $K$ particle for $K_{1}^{0}$, the final state is

$$
\psi\left(K_{1}^{0}\right) \rightarrow a_{1}\left[\psi\left(e^{+}+\nu+\pi^{-}\right)+\psi\left(e^{-}+\bar{v}+\pi^{+}\right)\right]
$$

where $a_{1}$ denotes the decay amplitude for the process while for $K_{2}^{0}$

$$
\psi\left(K_{2}^{0}\right) \rightarrow a_{2}\left[\psi\left(e^{+}+\nu+\pi^{-}\right)-\psi\left(e^{-}+\bar{v}+\pi^{+}\right)\right] .
$$

Starting with an initial $K^{0}$ state we have at a later time a state described by the wave function $\psi(t)$, the quantity $\left|\left\langle K^{0} \mid \psi(t)\right\rangle\right|^{2}$ giving the fraction of the original $K^{0}$ remaining in the beam after time $t$, while $\left|\left\langle\bar{K}^{0} \mid \psi(t)\right\rangle\right|^{2}$ gives the fraction of the original $K^{0}$ turned into $\bar{K}^{0}$. The sum of these fractions keeps decreasing with increasing $t$ as the mesons decay. The rates at which $\left(e^{-}+\bar{v}+\pi^{+}\right)$and

1 R. Serber and A. Pais, Phys. Rev. 99, 1551 (1955); K. M. Case, Phys. Rev. 103, 1449 (1956). M. L. Good, Phys. Rev. 106, 591 (1957). The most convincing experiments concerning the existence of $K_{2}^{0}$ are the observation of hyper fragments in emulsion stacks located near a target exposed to $\mathrm{K}^{+}$-meson beam. This production of hyperfragments must be obviously due to the following process :

$K^{+} \rightarrow K^{0}$ (charge exchange scattering), followed by Pais-Piccioni oscillations producing $K^{0}$, giving rise to hyperfragments.

2 S. B. Treiman and R. G. Sachs, Phys. Rer. 103, 1545 (1956).

EPCR 16 
$\left(e^{+}-\boldsymbol{\nu}+\boldsymbol{\tau}^{-}\right)$decays occur at time $\boldsymbol{t}$ are proportional to the squares of the amplitudes of these two states in (31 and 32). Thus we have.

$$
\begin{aligned}
& R\left(e^{+}+v+\pi^{-}\right)=\frac{1}{2}\left\{a_{1}^{2} \mathrm{e}^{-\lambda_{1} t}+a_{2}^{2} \mathrm{e}^{-\lambda_{2} t}+2 a_{1} a_{2} \cos (t \Delta) \mathrm{e}^{-\left(\lambda_{1}+\lambda_{2}\right) t / 2}\right\} \\
& R\left(e^{-}+\bar{v}+\pi^{+}\right)=\frac{1}{2}\left\{a_{1}^{2} \mathrm{e}^{-\lambda_{1} t}+a_{2}^{2} \mathrm{e}^{-\lambda_{2} t}-2 a_{1} a_{2} \cos (t \Delta) \mathrm{e}^{-\left(\lambda_{1}+\lambda_{2}\right) t_{2}}\right\} .
\end{aligned}
$$

It is to be noted that these expressions contain the interference terms with an exponentially diminishing factor and an oscillatory factor depending upon the energy difference between $K_{1}^{0}$ and $K_{2}^{0}$. The total rate of decay into $(\pi e v)$ states follows the exponential law corresponding to mixed $K_{1}^{0}$ and $K_{2}^{0}$, i.e.

$$
R\left(e^{+}+\nu+\pi^{-}\right)+R\left(e^{-}+\bar{\nu}+\pi^{+}\right)=a_{1}^{2} \mathrm{e}^{-\lambda_{1} t}+a_{2}^{2} \mathrm{e}^{-\lambda_{2} t} .
$$

The special case $a_{1}=a_{2}$ is of interest, for then $K^{0}$ can decay only to $e^{+}+\nu+\pi^{-}$ and $\bar{K}^{0}$ only to $\left(e^{-}+\bar{v}+\pi^{+}\right)$. If we start with a pure $K^{0}$ beam, then near the origin $R\left(e^{-}+\bar{v}+\tau^{+}\right)$must be zero. However as time proceeds, the beam acquires the $\vec{K}^{0}$ component and the strength is measured by the appearance of $R\left(e^{-}+\bar{v}+\pi^{+}\right)$. The time dependence of the electron and positron decay modes in this case for two values of energy difference $\Delta=\frac{m_{1} \delta m}{\omega_{1}}$ are shown in the figure.

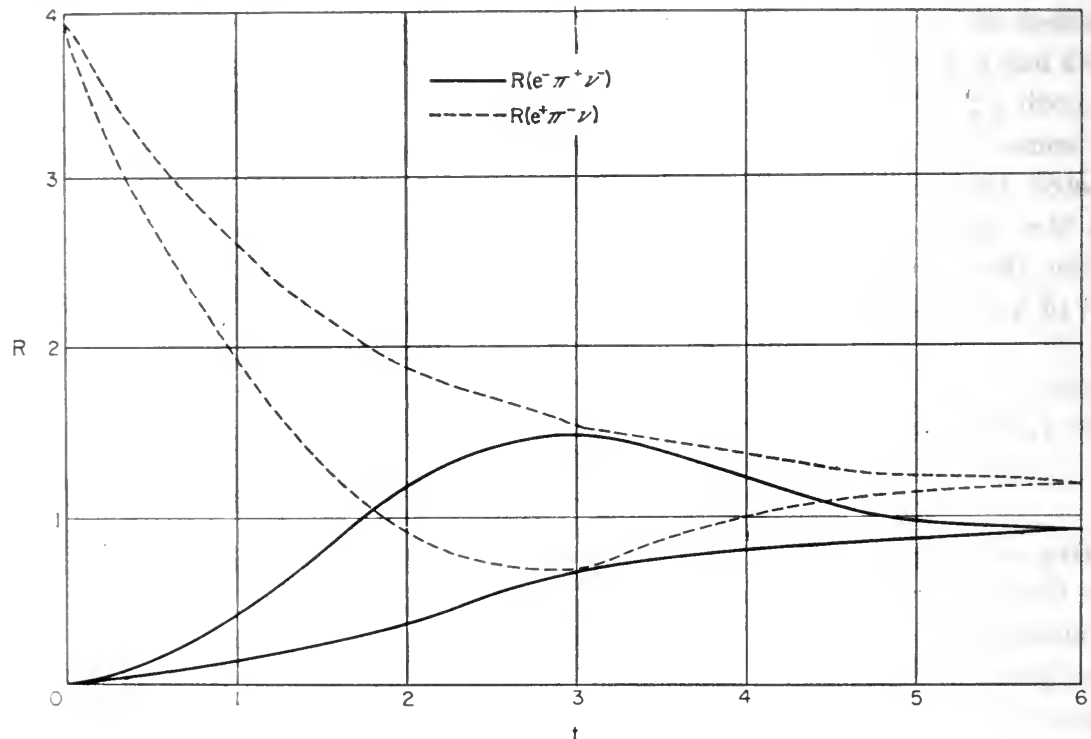

FIG. 35. The decay rate of the $K^{0}$-particle into the modes $e^{+} \nu \pi^{-}$and $e^{-} \bar{\nu} \pi^{+}$as a function of time under assumptions $a_{1}=a_{2}$ for two values of the energy difference. It is assumed that $\lambda_{2} \ll \lambda_{1}$.

Exactly similar effects can also occur when $K^{0}$ decays into final states $\left(\mu^{+}+\nu+\tau^{-}\right)$and $\left(\mu^{-}+\bar{\nu}+\pi^{+}\right)$. A time dependence of $\frac{e^{+}}{e^{-}}$or $\frac{\mu^{+}}{\mu}$ ratio in the decay of neutral beam can be absent if this ratio equals unity, i.e. $a_{1}=0$ or $a_{2}=0$. 
It might also be due to the energy difference $\Delta$ being so large that the oscillatory terms do not give observable effects.

In the above discussion, we have assumed that $C P$ invariance is obtained which also implies through the $C P T$ theorem that the interactions are invariant under time reversal. Lee, Yang and Oehme ${ }^{1}$ have discussed the situation when time reversal invariance fails. They show that we can still obtain $K_{1}^{0}$ and $K_{2}^{0}$ (which are mixtures of $K^{0}$ and $\bar{K}^{0}$ states) with different lifetimes. They also point out that a charge asymmetry between $\left(e^{+}+\pi^{-}+\nu\right)$ and $\left(e^{-}+\pi^{+}+\bar{v}\right)$ modes is possible for the $K_{2}^{0}$ beam.

\section{$|\Delta I|=\frac{1}{2}$ rule}

A phenomenological rule was proposed to explain the lifetime and branching ratio of the strange particle decays by postulating that the decay interaction Hamiltonian transforms like a spinor of $\operatorname{rank} 1 / 2$ in the isotopic spin space. ${ }^{2}$ Consequently the isotopic spin of the initial and the final state will differ by $1 / 2$, i.e.

$$
\left|\boldsymbol{I}_{f}-\boldsymbol{I}_{i}\right|=|\Delta \boldsymbol{I}|=\frac{1}{2}
$$

This rule is termed the $|\Delta \boldsymbol{I}|=1 / 2$ rule and can also be interpreted as follows. ${ }^{3.4}$ In the decay of a particle an additional particle called the "spurion" is emitted or absorbed that carries no energy or momentum or charge but does carry an isotopic spin $1 / 2$ and whatever the $I_{z}$ component is, that is needed to conserve $I_{z}$. With the inclusion of the spurion, the "violation rule" $|\Delta \boldsymbol{I}|=1 / 2$ can be dropped and we are allowed to assume that $\boldsymbol{I}$ is conserved in the decays. This rule has quite an amount of success in explaining the branching ratios between different modes of decays of hyperons as well.

Let us discuss the utility of this rule regarding $K^{ \pm}$and $K^{0}$ decays. Application of rule $|\Delta \boldsymbol{I}|=\frac{1}{2}$ to $K$ decay requires that the final state must have $I=0$ or $I=1$ since $I(K)=\frac{1}{2}$. When a spinless particle decays into two $\pi$ mesons (i.e. $\theta$ mode), the two pions are in the $S$ state. Because $\pi$ 's are bosons, their complete wave function must be symmetric. We know that the relative orbital motion is also symmetric and thus their isotopic spin wave function must be symmetric. Thus the final state having $I=1$ is excluded. However $K^{ \pm}$cannot decay into $I=0$

1 T. D. Lee, C. N. Yang and R. Oenme, Phys. Rev. 105̃, 1671 (1957).

2 M. Gell-Mann and A. PaIs, Proc. Int. Conference on High Energy Physics, Pergamon, London (1955).

3 Due to G. Wentzel, see p. 24. M. Gell-Mann and A. Rosenfeld, loc. cit.

4 It is shown by SALAM and WARD (Phys. Rev. Lett., 5, 390 (1960)) that conventional field theory contains a natural realization of "spurions". The vacuum expectation value $\left\langle K_{1}\right\rangle_{0}$ is non-zero and is proportional to $f_{w}$ where $f_{w} K_{1} \pi^{+} \pi^{-}$is the weak interaction Hamiltonian causing the decay $K_{1} \rightarrow \pi^{+}+\pi^{-}$. Thus even the isotopic spin conserving $T$ product of operators such as $\Lambda N^{+} \boldsymbol{\tau} \pi K$ has a non-zero matrix element for the decay $\Lambda \rightarrow N+\pi$ through $\Lambda N^{+} \boldsymbol{\tau} \boldsymbol{\pi}\langle K\rangle_{0}$. Thus $\langle K\rangle_{0}$ gives a realization for the spurion. Parity non-conservation is explained by postulating a $K^{\prime}$-meson of opposite (strong) parity to $K$ with $\left\langle K^{\prime}\right\rangle_{0} \neq 0$. 
since their final state has $I_{z}= \pm 1$ while $K^{0}$ can decay into $I=0$ state. $K^{ \pm}$decay can occur only through the violation of the rule $|\Delta \boldsymbol{I}|=1 / 2$. Perhaps electromagnetic corrections to the rule are responsible for the decay. At any rate the rule explains the rapid decay of $K^{0}$ into $2 \pi$ state as compared with $K^{ \pm}$.

We obtain as a second result that the ratio of $K_{1}^{0}$ decay into two modes

should be $1 / 3$ i.e.

$$
\begin{aligned}
K_{1}^{0} & \rightarrow \pi^{0}+\pi^{0} \\
& \rightarrow \pi^{+}+\pi^{-}
\end{aligned}
$$

$$
f=\frac{K_{1}^{0} \rightarrow 2 \pi^{0}}{K_{1}^{0} \rightarrow 2 \pi}=1 / 3 .
$$

At present there are these experimental values for the ratio, viz. $0.30 \pm 0.06$ and $0.14 \pm 0.06 .^{1}$ We have already noted that the ratio of $\frac{\tau^{\prime}}{\tau}=0.332 \pm 0.067$ For a pseudoscalar $\tau, S$ wave emission of three pions is possible and since pions are bosons, the final isotopic spin state must be symmetrical. Let us try to predict the ratio by the $|\Delta \boldsymbol{I}|=1 / 2$ rule. Since the final state carries a unit charge $I_{z}=+1$, $I=1$ is the only possible final state. Then the ratio of squares of the matrix elements $\left|M\left(\pi^{+}+2 \pi^{0}\right)\right|^{2}:\left|M\left(\pi^{-}+2 \pi^{+}\right)\right|^{2}$ is determined to be $1: 4$. Remember. ing however that the ratio of $Q$ values for the two processes is 84:75 the phase space ratio becomes $1.3: 1$. Thus the branching ratio should be $1.3: 4.325$, a result quite consistent with the experimental ratio. If we now consider " $\tau$ " decays of neutral $K$-mesons, we note that there are two possible charge states $3 \pi^{0}$ and $\pi^{+}+\pi^{-}+\pi^{0}$. Assuming $C P$ invariance $3 \pi^{0}$ has $C P=-1$ and hence is allowed for $K_{2}^{0}$ but is forbidden for $K_{1}^{0}$. Of course we have assumed that $K$ is spinless. Without a knowledge of the wave function we cannot decide $C P$ parity of $\pi^{+}+\pi^{-}+\pi^{0}$ state. Experimentally the fraction of decays into $3 \pi$ is quite appreciable. Assuming that the final $3 \pi$ state is totally symmetric, Gell-Mann ${ }^{2}$ finds that $|\Delta \boldsymbol{I}|=1 / 2$ rule requires that the total rate of $K_{2}^{0} \rightarrow 3 \pi$ should equal the total rate of $K^{+} \rightarrow 3 \pi$ while the ratio of

$$
\frac{K_{2}^{0} \rightarrow 3 \pi^{0}}{K_{2}^{0} \rightarrow \pi^{+}+\pi^{-}+\pi^{0}}=\frac{3}{2}
$$

From the above application of $|\Delta \boldsymbol{I}|=1 / 2$ rule to $K^{+}$decay, we find it fails to explain correctly the $2 \pi$ mode of $K^{+}$. Gell-Mann ${ }^{3}$ has put forth some proposals assuming the $|\Delta \boldsymbol{I}|=1 / 2$ rule to be approximately valid. To obtain a non-zero decay rate for $K^{+} \rightarrow \pi^{+}+\pi^{0}$ he proposes that contributions from $|\Delta \boldsymbol{I}|=3 / 2$ and possibly from $|\Delta \boldsymbol{I}|=5 / 2$ should be taken into account. Denoting the complex

${ }^{1}$ See "Strange Particle Decays", Report by D. A. Glaser, International Conference on High Energy Physics at Kiev, 1959, and Crawford et al., Phys. Rev. Letters 2, 266 (1959).

2 M. Gell-Mann and A. H. Rosenfeld, loc. cit.

${ }^{3}$ M. Gell-Mans, Nuovo Cim. 5), 76 (1957). 
amplitudes by $\varepsilon_{3}$ and $\varepsilon_{5}$ respectively relative to that for $|\Delta \boldsymbol{I}|=1 / 2$, the ratio of $2 \pi$ decay mode for $K^{+}$and $K^{0}$ and the ratio $f$ becomes

$$
\begin{array}{r}
\frac{K^{+} \rightarrow 2 \pi}{K^{0} \rightarrow 2 \pi}=\frac{1}{3} \frac{\left|3 / 2 \varepsilon_{3}-\varepsilon_{5}\right|^{2}}{1+\left|\varepsilon_{3}+\varepsilon_{5}\right|^{2}}=\gamma . \\
\quad f=\frac{1}{3} \frac{|1-| 2\left(\varepsilon_{3}+\left.\varepsilon_{5}\right|^{2}\right.}{1+\left|\varepsilon_{3}+\varepsilon_{5}\right|^{2}}
\end{array}
$$

On setting $\gamma=1 / 500$ as indicated by experiment, Gell-Mann finds that $f=0.14$ can be made to fit with $\varepsilon_{3} \simeq 12$ per cent; $\varepsilon_{5} \simeq 11$ per cent or with $\varepsilon_{3} \simeq 6$ per cent and $\varepsilon_{5}=17$ per cent. It seems improbable that electro-magnetic effects can give rise to such large corrections. Thus we can conclude that $|\Delta \boldsymbol{I}|=1 / 2$ is only approximately valid even for weak interactions and we can expect violations to the order of ten per cent. It is not clear whether the $|\Delta \boldsymbol{I}|=1 / 2$ rule can be extended to other decay modes involving leptons, of course assuming the leptons to possess zero isotopic spin. Sudarshan et al. ${ }^{1}$ have suggested that the strangeness violating non-leptonic current should transform like an isospinor. We will discuss the consequences of this later.

1 E. C. G. Sudarshan, S. Okubo, R. E. Marshak and S. Weinberg, Phys. Rev. 112, 665 (1958). 


\section{WEAK INTERACTIONS}

\section{WEAK INTERACTIONS ${ }^{1}$}

WE REMARKED carlier that the $\tau-\theta$ puzzle does not exist if we accept the violation of parity conservation in decay interactions. Lee and Yang ${ }^{2}$ first made this suggestion and proposed a large number of experiments which can test the hypothesis of non-conservation of parity in decays. As we shall see presently experiments have overwhelmingly supported this hypothesis. Since from the celebrated theorem of Pauli and Luders ${ }^{3}$ any interaction should be invariant under the combined operations of parity $(P)$, charge conjugation $(C)$ and time reversal $(T)$, and as parity is not conserved, at least one of the operations $C$ and $T$ should be violated. It is believed from theoretical arguments and experimental evidence that $C$ is violated along with $P$.

Weak interactions responsible for decays can be broadly divided into two classes:

(a) Decays involving leptons,

(b) Decays not involving leptons. i.e. the particle decays only into strongly interacting particles. Such a classification is made since as we shall see later it is possible to explain features like parity non-conservation in the leptonic decays by the two-component theory of the neutrino, an explanation which cannot be applied to the non-leptonic decays. For the latter we further seem to have a selection rule $|\Delta \boldsymbol{I}|=1 / 2$. Despite these seemingly different characteristics, the coupling constants for these two classes are remarkably identical. Of course, we do not know how to calculate the various decays since we do not have a complete knowledge of how the various fields are coupled though, as we shall see presently, recent developments indicate a universal form of interaction. Even in the absence of a knowledge of detailed interactions, we can estimate the coupling constant by the simple formula

$$
\tau=2 \pi G^{2}\left(\frac{1}{R^{2}}\right) \varrho_{E}
$$

1 Part of this chapter closely follows the discussion on Weak Interactions in T. D. LEE and C. N. YANG's "Elementary Particles and Weak Interactions". - Lectures given at the Brookhaven National Laboratory, U.S.A. (1957).

2 T. D. Lee and C. N. YANG, Phys. Rev. 104, 254 (1956).

3 W. Pauli's particle in Niels Bohr and the Development of Physics, Pergamon, London 1955; see also G. Iuders, Kgl. Danske Vidensk. 28, No. 5 (1954). 
where $\tau$ is the lifetime of the particle, $\varrho_{E}$ is the number of final states per unit energy, and $R$ the characteristic length of these decays which can be crudely estimated from the unbalance in energy by means of the uncertainty principle. We have listed below the estimates of $G^{2}$ for a number of decays belonging to both classes. For simplicity we take $R=\frac{\hbar}{m_{\pi} c}$.

\begin{tabular}{c|c|c}
\hline Decays & Lifetime (sec) & $G^{2} \times 10^{14}$ \\
\hline$\Lambda^{0} \rightarrow p+\pi^{-}$ & $3 \times 10^{-10}$ & 1.6 \\
$\Sigma^{-} \rightarrow n+\pi^{-}$ & $1.6 \times 10^{-10}$ & 1.2 \\
$K_{1}^{0} \rightarrow 2 \pi$ & $0.95 \times 10^{-10}$ & 2.8 \\
$\pi^{ \pm} \rightarrow \mu^{ \pm}+v$ & $2.6 \times 10^{-8}$ & 0.3 \\
$\mu^{ \pm} \rightarrow e^{ \pm}+v+\bar{v}$ & $2.2 \times 10^{-6}$ & 2.0 \\
$K^{ \pm} \rightarrow \mu^{ \pm}+v$ & $1.2 \times 10^{-8}$ & 0.02 \\
\hline
\end{tabular}

Despite the wide variation in lifetimes of the particles, the coupling constants $G^{2}$ seem to be identical. This remarkable feature supports the surmise of a common origin for all weak interactions.

Decays involving leptons can be further classified as follows:

(i) Those involving only leptons, e.g. $\mu^{ \pm} \rightarrow e^{ \pm}+v+\bar{v}$.

(ii) those which involve no change of strangeness $\Delta S=0$, e.g. $n \rightarrow p+e^{-}+\bar{v}$, and (iii) Those involving change of strangeness $\Delta S=1$, e.g. $\Lambda \rightarrow p+e^{-}+\bar{v}$.

The non-leptonic decays like $\Lambda \rightarrow p+\pi^{-}$always involve change of strangeness. All the weak interactions in the current-current picture can be generated from the four typical interactions by the introduction of a strong vertex, e.g., $\pi$ gets replaced by $N \bar{N}, K$ by $\bar{\Lambda} N$ etc., and also by replacing an electron by a $\mu$-meson.

Before discussing the theory of weak interactions we shall first outline in a general way how observational quantities can reveal invariance or non-invariance under symmetry operations like $P$ and $T$, and apply such considerations to particular cases like the $\beta$ decay.

\section{Tests for invariance under $\boldsymbol{C}, \boldsymbol{P}, \boldsymbol{T}$}

(a) Parity: If the state of a quantum mechanical system is not invariant under parity, it implies that the eigenstate of the Hamiltonian is not an eigenstate of the parity operator (i.e. a state $\psi$ is a linear combination $a \psi_{+}+b \psi_{-}$where $\psi_{+}$ and $\psi_{-}$are even and odd parity eigenstates. Let a quantum mechanical system $A$ decay to a state $B$. Parity non-conservation implies that if $A$ is an eigenstate of parity, $B$ is not. In the double scattering of protons $^{1}$ a beam of incoming pro-

1 O. Chamberlain, E. Ségrè, R. Tripp, C. Wiegand and T. Ypsilantis, Phys. Rev. 93, 1430 (1954). 
tons with momentum $\boldsymbol{p}_{1}$ is scattered by a target into a state with momentum $\boldsymbol{p}_{2}$, which is further scattered by a second target into momentum $\boldsymbol{p}_{3}$. The crosssection for double scattering should be independent of $\boldsymbol{p}_{3} \cdot\left(\boldsymbol{p}_{1} \times \boldsymbol{p}_{2}\right)$ which is a pseudoscalar (see below). The measurement reveals no dependence on $\boldsymbol{p}_{\mathbf{3}} \cdot\left(\boldsymbol{p}_{\mathbf{1}} \times \boldsymbol{p}_{\mathbf{2}}\right)$ and this supports the conclusion that parity is conscrved in strong interactions. Experimental evidence indicates that parity is conserved by nuclear forces down to one part in $10^{7}$ in intensity. ${ }^{1}$ This is based on the study of the yield of the highestenergy group of $\alpha$ particles in the reaction

$$
p+\mathrm{F}^{19} \rightarrow \mathrm{O}^{16}+\alpha .
$$

The spin and parity of $\mathrm{O}^{16}$ and $\alpha$-particles are both $0^{+}$. The reaction can have a resonance corresponding to a known $\mathrm{1}^{+}$state of $\mathrm{Ne}^{20}$ if parity is not conserved. No such resonance was found. The conservation of parity in electromagnetic interactions is established experimentally to about one part in $10^{6}$ in intensity by the validity of selection rules in atomic transitions.

In any decay process we will have particles emerging in various directions with different energies and we normally measure the distribution in intensity, i.e. the number of particles emerging at a given angle. This distribution function can depend on many quantities that can be formed from the momenta and spins of the interacting particles. However the symmetry properties of the interaction responsible for the decay in question will limit the dependence of the distribution function only to certain of these. If, for example, an interaction is invariant under rotation, then it is clear that the distribution function should contain only quantities which are also invariant under rotation. In a similar manner the distribution function can depend on pseudoscalar quantities only when the interaction is not invariant under $P$. It is convenient to discuss the $\beta$ decay interaction since it is one of those weak interactions which has been already studied experimentally and theoretically though not with the object of testing the postulate of parity conservation.

Admitting non-conservation of parity the most general interaction Hamiltonian for $\beta$ decay is

$$
H_{\text {int }}=\sum_{i}\left(\bar{\psi}_{p} 0_{i} \psi_{n}\right)\left\{\left(C_{i} \bar{\psi}_{e} 0_{i} \psi_{v}\right)+\left(C_{i}^{\prime} \bar{\psi}_{e} 0_{i} \gamma_{5} \psi_{v}\right)\right\}
$$

where $i=S, T, V, A, P$ denotes the well known bilinear covariants of the Dirac spinors, i.e. scalar, tensor, vector, axial vector and pseudoscalar, $C_{i}$ and $C_{i}^{\prime}$ being the ten complex coupling constants. The second term is pseudoscalar while the first term is the "old" interaction Hamiltonian and is scalar. The square of any typical matrix element has the form

$$
\mid M^{2}=\left(\sum_{i j} f_{i j} C_{i}^{*} C_{j}+c \cdot c\right)+\left(\sum f_{i j}^{\prime} C_{i}^{\prime *} C_{j}^{\prime}+c \cdot c\right)+\left(\sum g_{i j} C_{i}^{*} C_{j}^{\prime}+c \cdot c\right),
$$

1 N. TAnner, Phys. Rev. 107, 1203 (1957). Also D. H. Wilkinson, Phys. Rev. 109, 1603, 1610, 1614 (1958); R. HaAs, L. B. LeIPUner and R. K.Adair, Phys. Rev. 116, 1221 (1959). 
where $f_{i j}, f_{i j}^{\prime}$ and $g_{i j}$ are functions of the momenta and spins of the particles. It is important to note that any transformation which leaves the $H_{\text {int }}$ invariant will leave (3) invariant. As has been shown earlier both $\psi_{\nu}$ and $\gamma_{5} \psi_{\nu}$ are solutions of the Dirac equation for the neutrino.

(a) Let us make the transformation

$$
\psi_{v} \rightarrow \gamma_{5} \psi_{v} ; C_{i} \rightarrow C_{i}^{\prime} \text { and } C_{i}^{\prime} \rightarrow C_{i} .
$$

This leaves $H_{\text {int }}$ invariant. Hence it follows that

$$
f_{i j}=f_{i j}^{\prime} .
$$

(b) $C_{i} \rightarrow C_{i} ; C_{i}^{\prime} \rightarrow-C_{i}^{\prime}$ with space inversion. This transformation also leaves $H_{\text {int }}$ invariant. Note that the minus sign acquired by the second term in the Hamiltonian under $P$ is compensated by the minus sign of $C_{i}^{\prime}$. Since under $P$ we have $\boldsymbol{r} \rightarrow-\boldsymbol{r}, \boldsymbol{p} \rightarrow-\boldsymbol{p}$ and spin $\mathbf{s} \rightarrow \boldsymbol{s}$, we can deduce that

$$
\begin{aligned}
& f_{i j}(-\boldsymbol{p}, \boldsymbol{s} \ldots)=f_{i j}(+\boldsymbol{p}, \boldsymbol{s} \ldots) \\
& f_{i j}^{\prime}(-\boldsymbol{p}, \boldsymbol{s} \ldots)=f_{i j}^{\prime}(+\boldsymbol{p}, \mathbf{s} \ldots)
\end{aligned}
$$

and

$$
g_{i j}(-\boldsymbol{p}, \boldsymbol{s} \ldots)=-g_{i j}(+\boldsymbol{p}, \boldsymbol{s} \ldots) .
$$

It has thus been shown that $f_{i j}$ and $f_{i j}^{\prime}$ are scalars while the $g_{i j}$ 's are pseudoscalars. Since only the term containing $g_{i j}$ involves the interference of both the scalar and pseudoscalar parts of $H_{\text {int }}$, in order to test the question of parity conservation we must measure a pseudoscalar quantity. Thus if in the $\beta$ decay from oriented nuclei, the angular distribution of the $\beta$ particles depends on the pseudoscalar $\boldsymbol{j} \cdot \boldsymbol{p}_{e}$ where $\boldsymbol{j}$ is the polarization of the oriented nuclei and $\boldsymbol{p}_{e}$ the momentum of the $\beta$ particle, it implies an asymmetry of this distribution about the direction of the spin of the oriented nuclei and this asymmetry can be detected by experiment and will constitute a proof for the violation of parity conservation. ${ }^{1}$ It can be noted here that the measurement of any scalar quantity in the new $H_{\text {int }}$ is equivalent to working with the "old" parity-conserving $H_{\text {int }}$ provided we replace $C_{i}^{*} C_{j}$ in the old formulae by $\left(C_{i}^{*} C_{j}+C_{i}^{\prime *} C_{j}^{\prime}\right)$ which essentially means only a redefinition of the constants $C_{i}$.

Recalling the $C P T$ theorem discussed earlier, we deduce that if weak interactions fail to be invariant under $P$, then it implies that the interaction must violate at least one of the other invariances under $C$ or $T$. It might be argued that equality of masses and lifetimes in the decays of charge-conjugate particles like $\pi^{ \pm}, \mu^{ \pm}$have already established that charge-conjugation invariance is valid in weak decays. However Lee, Yang and Oehme ${ }^{2}$ pointed out that the equality of masses and lifetimes for a particle and its anti-particle follows directly from proper Lorentz invariance and the $C P T$ theorem whether separate conservation.

1 T. D. Lee and C. N. YANG, Phys. Rev. 104, 254 (1956).

2 T. D. Lee, R. Oehne and C. N. Yang, Phys. Rev. 106, 340 (1957). 
of $C, P$ and $T$ is obtained or not. This theorem is true only in the absence of any final state interactions. ${ }^{1,2}$

(b) Time reversal: We shall now discuss the consequences of invariance or non-invariance under time reversal. Let $H_{\omega}$ be the weak-interaction Hamiltonian which causes the decay $A \rightarrow B$. Writing $H_{\omega}$ in the form $\sum C_{i} H_{i}$ such that under $T$ we have

$$
T H_{\omega} T^{-1}=\Sigma C_{i}^{*} H_{i}^{*} \quad \text { and } \quad T H_{i} T^{-1}=H_{i},
$$

we note that if the $C_{i}$ 's are real or at least all the $C_{i}$ 's have the same phase then time reversal invariance holds. Thus to prove non-invariance under time reversal we must measure the dependence of the distribution function on quantities whose mean values depend on the relative phases between these $C_{i}$ 's. For simplicity we shall assume that there exist no final-state strong interactions between the decay products. If free particle states of momenta $\boldsymbol{p}_{1}, \boldsymbol{p}_{\mathbf{2}} \ldots$ and spin $\boldsymbol{s}_{1}, \boldsymbol{s}_{2}$ are denoted by $|\boldsymbol{p}, \boldsymbol{s}\rangle$, then the final state $B$ ean be written as

$$
\begin{aligned}
\psi & =\sum_{\boldsymbol{p}, \boldsymbol{s}}\left\langle\boldsymbol{p}, \boldsymbol{s}\left|H_{\omega}\right| A\right\rangle|\boldsymbol{p}, \boldsymbol{s}\rangle \\
& =\sum_{i} C_{i} M_{i}(\boldsymbol{p}, \boldsymbol{s})|\boldsymbol{p}, \boldsymbol{s}\rangle,
\end{aligned}
$$

where

We know that

$$
\begin{gathered}
M_{i}(\boldsymbol{p}, \boldsymbol{s})=\left\langle\boldsymbol{p}, \boldsymbol{s}\left|H_{i}\right| A\right\rangle . \\
T|\boldsymbol{p}, \boldsymbol{s}\rangle=\langle-\boldsymbol{p},-\boldsymbol{s}| \\
M_{i}^{*}(\boldsymbol{p}, \boldsymbol{s})=M_{i}(-\boldsymbol{p},-\boldsymbol{s}) .
\end{gathered}
$$

1 The proofs of these statements will now be indicated (see G. Luders and B. Zumino, Phys. Rev. 106, 385 (1957). T. D. LeE and C. N. Y ANG, Phys. Rev. 105, 1671 (1957)). That masses of particles and antiparticles are equal easily follows on noting that $C P T$ is a unitary transformation and hence the eigenvalues, in particular mass, will not be altered under such a transformation provided the Hamiltonian is left invariant under $C P T$. We recall that particle and antiparticle states go over into each other under $C P T$.

To prove the life time equality, consider the decay $A \rightarrow B$ and $\bar{A} \rightarrow \bar{B}$. Let us put the weak interaction part $H \omega$ as

with

$$
\begin{aligned}
& H_{\omega}=H_{+}+H_{-} \\
& H_{ \pm}=\frac{1}{2}\left[H_{\omega} \pm P H_{\omega} P^{-1}\right]
\end{aligned} .
$$

where $P H_{ \pm} P^{-1}= \pm H_{ \pm}$. Also let us denote the final state as $|B\rangle=\left|B_{+}\right\rangle+\left|B_{-}\right\rangle$. Applying $C P T$ theorem and using rotational invariance we can show

and

$$
\left\langle B_{+}\left|H_{+}\right| A\right\rangle^{*}=+\left\langle\bar{B}_{+}\left|H_{+}\right| \bar{A}\right\rangle
$$

$$
\left\langle B_{-}\left|H_{-}\right| A\right\rangle^{*}=-\left\langle\bar{B}_{-}\left|H_{-}\right| \bar{A}\right\rangle
$$

Since the two matrix elements differ in phase by $\pi / 2$, there can be no interference between the two matrix elements. Thus the lifetimes $\tau_{A}$ and $\tau_{\dot{A}}$ are

$$
\begin{aligned}
\tau_{A} & \propto \Sigma\left|\left\langle B_{+}\left|H_{+}\right| A\right\rangle\right|^{2}+\left|\left\langle B_{-}\left|H_{-}\right| A\right\rangle\right|^{2} \\
\tau_{\bar{A}} & \propto \Sigma\left|\left\langle\vec{B}^{-}\left|H^{-}\right| \bar{A}\right\rangle\right|^{2}+\left.\left|\overline{\langle} B_{-}\right| H_{-}|A\rangle\right|^{2} .
\end{aligned}
$$

(Thus $\tau_{A}=\tau_{\bar{A}}$. However if final state interaction is present, then (3) and (4) are no longer true cf. our discussion of invariances with final state interaction. Hence the lifetimes of particles and antiparticles are not identical).

2 See S. Oкuво, Phys. Rev. 109, 984 (1958). 
The mean value of any observable 0 in the final state will be given by

where

$$
\langle\psi|0| \psi\rangle=\sum_{i j} C_{i}^{*} C_{j} 0_{i j},
$$

We note that

$$
0_{i j}=\sum_{p, s} M_{i}^{*}(\boldsymbol{p}, \mathbf{s}) M_{j}(\boldsymbol{p}, \mathbf{s})\langle\boldsymbol{p}, \mathbf{s}|0| \boldsymbol{p}, \mathbf{s}\rangle \text {. }
$$

$$
0_{i j}^{*}=0_{j i} .
$$

If we divide the observables into even and odd functions, i.e. $0_{+}$and $0_{-}$, then under $T$ we have

$$
\left\langle\boldsymbol{p}, \boldsymbol{s}\left|0_{ \pm}\right| \boldsymbol{p}, \boldsymbol{s}\right\rangle= \pm\left\langle-\boldsymbol{p},-\boldsymbol{s}\left|0_{ \pm}\right|-\boldsymbol{p},-\boldsymbol{s}\right\rangle,
$$

where

$$
T 0_{ \pm} T^{-1}= \pm 0_{ \pm}
$$

From (16) and (17), we deduce that

and

$$
\left(0_{+}\right)_{i j}=\left(0_{+}\right)_{j i}=\text { real }
$$

Thus we see that the mean values of $0_{ \pm}$are related to the real and imaginary parts of products of $C_{i}^{*} C_{j}$.

$$
\begin{aligned}
& \left\langle\psi\left|0_{+}\right| \psi\right\rangle=\sum\left(0_{+}\right)_{i j}\left(C_{i}^{*} C_{j}+C_{i} C_{j}^{*}\right) \\
& \left\langle\psi\left|0_{-}\right| \psi\right\rangle=\sum\left(0_{-}\right)_{i j}\left(C_{i}^{*} C_{j}-C_{i} C_{j}^{*}\right) .
\end{aligned}
$$

Remembering that the imaginary parts of $C_{i}^{*} C_{j}$ exist only when they have relative phases, we find that the measurement of a dependence on $0_{-}$will constitute a proof for non-invariance under time reversal in the absence of final state interactions.

The assumption of the absence of final state interaction is only an ideal one, for there always exists some form of final state interaction in the decays such as Coulomb interaction in the $\beta$ decay between the $\beta$ particles and the recoil nucleus. The free particle solutions will no longer be eigensolutions of the strong part of the Hamiltonian. It is well-known that the exact solution will consist asymptotically of a plane wave and incoming spherical waves. Under time reversal this solution will correspond to a plane wave of opposite momentum plus outgoing spherical waves. We also know from elementary scattering theory in quantum mechanics that the asymptotic solution differs from the plane wave by a phase shift depending on the interactions. Let us denote the eigenstate of $H_{\text {strong }}$ by $|B\rangle$. The final state $\psi$ will be

$$
\psi=\sum_{B, \boldsymbol{p}, \boldsymbol{s}}\left\langle\boldsymbol{p}, \boldsymbol{s} \mid B^{\text {out }}\right\rangle\left\langle B^{\text {out }}\left|H_{\omega}\right| A\right\rangle|\boldsymbol{p}, \boldsymbol{s}\rangle .
$$

The state $\left|B^{\text {out }}\right\rangle$ is the outgoing part of the exact solution $|B\rangle$. Let us denote the phase factor of $\left|B^{\text {out }}\right\rangle$ by $\mathrm{e}^{-i \eta_{B}}$ where $\eta_{B}$ is the phase shift due to the final state interactions. Then we can write $|\psi\rangle$ as

where

$$
|\psi\rangle=\sum C_{i} \mathrm{e}^{i \eta_{B}} M_{i B}(\boldsymbol{p}, \boldsymbol{s})|\boldsymbol{p}, \boldsymbol{s}\rangle,
$$

$$
M_{i B}^{*}(p, s)=M_{i B}(-p,-s)
$$


P'roceeding as before, we find that

$$
\begin{aligned}
\left\langle\psi\left|0_{+}\right| \psi\right\rangle=\Sigma & \Sigma\left(0_{+}\right)_{i B, j B}\left[\left(C_{i}^{*} C_{j}+C_{i} C_{j}^{*}\right) \cos \left(\eta_{B}-\eta_{B^{\prime}}\right)\right. \\
& \left.+i\left(C_{i}^{*} C_{j}-C_{i} C_{j}^{*}\right) \sin \left(\eta_{B}-\eta_{B^{\prime}}\right)\right] \\
\left\langle\psi\left|0_{-}\right| \psi\right\rangle=\Sigma & \Sigma\left(0_{-}\right)_{i B, j B}\left[\left(C_{i}^{*} C_{j}-C_{i} C_{j}^{*}\right) \cos \left(\eta_{B}-\eta_{B^{\prime}}\right)\right. \\
& \left.+i\left(C_{i}^{*} C_{j}+C_{i} C_{j}^{*}\right) \sin \left(\eta_{B}-\eta_{B^{\prime}}\right)\right] .
\end{aligned}
$$

'The important modification due to the presence of final state interactions is that both $0_{+}$and $0_{-}$depend on the imaginary parts of $C_{i}^{*} C_{j}$ and hence measurements of a dependence on either $0_{+}$or $0_{-}$will serve as proof for the violation of time reversal invariance. We shall conclude this general discussion with the following observations.

Let us write an interaction Hamiltonian $H_{\omega}$ as

$$
H_{\omega}=H_{1}+H_{2} \text {. }
$$

where $H_{1}$ and $H_{2}$ commute and anticommute with $P$ respectively, i.e. $H_{1}$ conserves parity while $\mathrm{H}_{2}$ does not. Let us also write

$$
H_{1}=\sum_{i}\left(C_{1}\right)_{i} H_{i} \text { and } H_{2}=\sum_{i}\left(C_{2}\right)_{i} H_{i} .
$$

If time reversal holds for both, then $\left(C_{1}\right)_{i}$ and $\left(C_{2}\right)_{i}$ must all be real. From $C P T$ theorem it follows that for charge conjugation symmetry to hold, all $\left(C_{1}\right)_{i}$ must be real while all $\left(C_{2}\right)_{i}$ must be imaginary. Thus $\left(C_{1}\right)_{i}$ and $\left(C_{2}\right)_{i}$ differ in phase by $\pi / 2$ and hence there can be no interference between the two parts of the Hamiltonian.

This proves the very important result that if time reversal invariance holds for an interaction then the effects due to parity violation can be observed only if the charge conjugation invariance is equally violated. ${ }^{1}$ In the table below are presented results which follow from the above discussion.

\begin{tabular}{l|c|c|c|c}
\hline \multicolumn{1}{c|}{ Observable } & Example & $\begin{array}{c}\text { Mean value } \\
\text { involves }\end{array}$ & $\begin{array}{c}\text { Violates } \\
\text { invariance } \\
\text { of }\end{array}$ & $\begin{array}{c}\text { Invariant } \\
\text { under }\end{array}$ \\
\hline 0. Scalar & $\boldsymbol{p}_{1} \cdot \boldsymbol{p}_{2}$ & $\operatorname{Re} C_{i}^{*} C_{j}$ & - & $P, C, T$ \\
0+ Scalar & $\left(\boldsymbol{p}_{e} \cdot \boldsymbol{p}_{\boldsymbol{v}}\right)$ & $\operatorname{Im} C_{i}^{*} C_{j}$ & $C, T$ & $P$ \\
0. Scalar & $\boldsymbol{\sigma} \cdot\left(\boldsymbol{p}_{1} \times \boldsymbol{p}_{2}\right)$ & $\operatorname{Im} C_{i}^{*} C_{j}$ & $C, T$ & $P$ \\
0. Scalar & $\boldsymbol{\sigma} \cdot\left(\boldsymbol{p}_{1} \times \boldsymbol{p}_{2}\right)$ & $\operatorname{Re} C_{i}^{*} C_{j}$ & - & $P, C, T$ \\
0+ Pseudoscalar & $(\boldsymbol{\sigma} \cdot \boldsymbol{p})$ & $\operatorname{Re} C_{i}^{*} C_{j}$ & $P, C$ & $T$ \\
0. Pseudoscalar & $(\boldsymbol{\sigma} \cdot \boldsymbol{p})$ & $\operatorname{Im} C_{i}^{*} C_{j}$ & $P, T$ & $C$ \\
0. Pseudoscalar & $\left(\boldsymbol{p}_{1} \cdot\left(\boldsymbol{p}_{2} \times \boldsymbol{p}_{3}\right)\right)$ & $\operatorname{Im} C_{i}^{*} C_{j}$ & $P, T$ & $C$ \\
0. Pseudoscalar & $\left(\boldsymbol{p}_{1} \cdot\left(\boldsymbol{p}_{2} \times \boldsymbol{p}_{3}\right)\right)$ & $\operatorname{Re} C_{i}^{*} C_{j}$ & $P, C$ & $T$ \\
\hline
\end{tabular}

(c) Charge Conjugation: In view of the invariance under the combined operations $C P T$ the conclusions regarding the invariance under $C$ can be drawn from those

1 T. D. Lee, C. N. Yang and R. Oenue, Phys. Rev. 106, 340 (1957). Note that this theorem holds only in the absence of final state interaction. 
regarding $P$ and $T$. Landau, ${ }^{1}$ has postulated that weak interactions remain invariant under $C P$ or in other words time reversal invariance holds. Invariance under $C P$ guarantees that a mirror reflection of a state of particles is always a possible state of the corresponding antiparticles. For example, if the $\beta$ particles emitted in the decay of a radioactive nucleus have a particular angular distribution, then the same angular distribution should exist for $\beta^{+}$ particles emitted in the decay of the corresponding antinucleus. It is interesting to note that in this case it is not possible to define a right-handedness from $\beta$ decay alone unless we can distinguish matter from anti-matter. The results of all experiments so far have upheld this invariance.

\section{Violation of $\boldsymbol{P}$ and $\boldsymbol{C}$ invariance in weak interactions}

Having outlined the general consequences of the three operations $C P T$ we shall now discuss in some detail the experimental evidence for the violation of invariance under $P$ and $C .^{2}$ The first demonstration of the violation of parity conservation came from the now famous experiment due to $\mathrm{Wu}$ et $a l^{3} \mathrm{On}$ the suggestion of Lee and Yang, they studied the asymmetry in the angular distribution of $\beta^{-}$particles emitted from oriented $\mathrm{Co}^{60}$ radioactive nuclei. The reaction is

$$
\mathrm{Co}^{60} \rightarrow \mathrm{Ni}^{60}+e^{-}+\bar{v} \quad J=5 \rightarrow J=4, \Delta J=1(N O),
$$

which obeys the Gamow-Teller selection rule. Hence we denote the nuclear matrix element by $M_{G \cdot T}$. Similarly the matrix element corresponding to the Fermi selection rule $(\Delta J=0(N O)$ and $0 \rightarrow 0)$ will be denoted by $M_{F}$. Two gamma rays are emitted when the exited $\mathrm{Ni}^{60}$ reaches its ground state. The anisotropy of gamma rays was used as a measure of the polarization of $\mathrm{Co}^{60}$ nuclei. The electrons were found to be emitted preferentially in a direction antiparallel to nuclear orientation, i.e. the angular distribution $f(\theta)$ of the radial vector, $\boldsymbol{p}_{\boldsymbol{e}}$ (the momentum of the electron) was asymmetric about the axial vector $\boldsymbol{J}$ (the direction of polarization of the nucleus) where $\theta$ is the angle between them. This implies

$$
f(\theta) \neq f(\pi-\theta) \text {. }
$$

Or equivalently $F(\theta)$ the angular distribution on space inversion is different from $f(\theta)$ since

$$
F(\theta)=f(\pi-\theta) \text {. }
$$

Lee and Yang $^{5}$ have shown that $f(\theta)$ is of the form $f(\theta)=1+\alpha \cos \theta$ with $\alpha=\beta \frac{\left\langle\boldsymbol{J}_{z}\right\rangle}{J}$ for $J \rightarrow J-1$,

1 L. Landau, Nucl. Phys. 3, 127 (1957).

2 See Proceedings of Rehovoth Conference on Nuclear Structure, North Holland (1958). Also Rev. Mod. Phys. 31 (1958).

3 C. S. Wu, E. Aybler, R. W. Hayward, D. D. Hoppes and R. P. Hudson, Phys. Rev. 105,1413 (1957).

4 T. D. Lee and C. N. YANG, Phys. Rev. 104, 254 (1956),

5 T. D. LEE and C. N. YANG, loc. cit. 
where

and

$$
\begin{aligned}
\beta=\operatorname{Re}\left[\left[ \pm\left(C_{T} C_{T}^{\prime *}\right.\right.\right. & \left.\left.-C_{A} C_{A}^{\prime *}\right)+\frac{Z e^{2}}{h c p}\left(C_{A} C_{T^{*}}^{\prime}+C_{A}^{\prime} C_{T^{*}}\right)\right] \\
& \times \frac{v_{e}}{c} \frac{2}{\xi+\frac{\xi b}{W}}\left|M_{G T}\right|^{2}
\end{aligned}
$$

$$
\xi=\left\{\left|C_{T}\right|^{2}+\left|C_{A}\right|^{2}+\left|C_{T}^{\prime}\right|^{2}+\left|C_{A}^{\prime}\right|^{2}\right\}\left|M_{G T}\right|^{2} .
$$

$\left\{\left.M_{G T}\right|^{2}\right.$ represents the square of the nuclear matrix elements. Many authors ${ }^{1}$ have calculated a for other transitions. For $J \rightarrow J+1$,

$$
\alpha=-\beta \frac{J_{z}}{J+1} .
$$

For

with

$$
J \rightarrow J, \quad \alpha=\frac{\beta J_{z}}{J(J+1)}+\beta^{\prime} \frac{J_{z}}{\gamma / J(J+1)}
$$

$$
\begin{aligned}
\beta^{\prime}=\operatorname{Re} & {\left[\left(C_{S}^{*} C_{T}+C_{S}^{\prime} * C_{T}-C_{V}^{*} C_{A}^{\prime}-C_{V}^{\prime} C_{A}^{*}\right)\right.} \\
& \left. \pm \frac{i Z e^{2}}{p}\left(C_{S}^{*} C_{A}^{\prime}+C_{S}^{\prime} * C_{A}-C_{V}^{*} C_{T}^{\prime}-C_{V}^{\prime} * c_{T}\right)\right] \times \underline{v_{e}} \frac{2 M_{F} M_{G T}}{\xi+\frac{\xi b}{W}} .
\end{aligned}
$$

In all these equations the plus sign corresponds to $e^{-}$emission while the minus sign corresponds to $e^{+}$emission. In deriving (33 to 36$)$ it has been assumed that the nuclear matrix elements $M_{F}$ and $M_{G T}$ are real as a consequence of the invariance

under time reversal.
It was found by $\mathrm{Wu}$ et al. that $\beta \simeq-0.7$ for $\frac{v_{e}}{c} \simeq 0.65$. For $\mathrm{Ni}^{60}, \frac{Z e^{2}}{\hbar}=0.2$. and the second term has an upper limit 0.23 and hence can be neglected. Thus the negative asymmetry can be explained by either assuming $C_{T} \simeq-C_{T}^{\prime}$ or $C_{A} \simeq+C_{A}^{\prime}$. We shall discuss below this important question of coupling in $\beta$ decay. From the table we also see that the observation of this asymmetry establishes that charge conjugation invariance also does not hold. From the expression for $\alpha$ given by (35) we see, that there exist interference terms between $F$ and $G T$ transitions. While the asymmetry is quite consistent with a pure $G T$ transition for $\mathrm{Co}^{60}$, it provides no evidence for interference in $\mathrm{Co}^{58}$. However for $\mathrm{Sc}^{46}$ the asymmetry value can be explained only by full interference. ${ }^{2}$

It is possible to prove parity violation if experiments are performed even with unpolarized nuclei. It is sufficient if we observe the recoil nuclei to be partially polarized since in such a case the state of polarization of the recoil nuclei relative

${ }^{1}$ R. B. Curtis and R. R. Lewis, Phys. Rev. 107, 1387 (1957); J. D. JACKson, S. B. TreiMan and H. W. Wyld Jr., Phys. Rev. 106, 517 (1957); M. Morita and R.S.Morita, Phys. Rev. 107, 1316 (1957).

2 See C. S. Wu, Proceedings of the Rehovoth Conference on Nuclear Structure, p. 346, North Holland (1958).

* Here $b \xi=2 \gamma \operatorname{Re}\left[C_{T}^{*} C_{A}+C_{T}^{\prime *} C_{A}^{\prime}\right]\left|M_{G T}\right|^{2}$ and $\gamma=\left[1-Z^{2} e^{4}\right]^{\frac{1}{2}}$. 
to the direction of the electron momentum is not invariant under space inversion. The partial polarization of recoiling nuclei can be detected through the polarization of the $\gamma$-rays emitted by these nuclei which depends on the angle between the direction of the $\gamma$-ray and $\boldsymbol{p}_{e}$. The angular distribution of polarized $\gamma$-rays as has been calculated by Alder, Stech and Winter, ${ }^{1}$ is of the form

$$
I(\theta)=1 \pm A \frac{v}{c} \cos \theta,
$$

where the plus sign stands for right circular and the minus for left circular polarization.

The circular polarization of the $\gamma$-ray can be detected by utilizing the dependence of the cross-section for Compton-scattering of polarized electrons on the state of circular polarization of the incident $\gamma$-ray. ${ }^{2}$ The conclusions from the experiments on the polarization of $\gamma$-rays are consistent with those based on $\beta$ asymmetry.

Many experiments have been suggested to test the invariance under time reversal. The $Z$-dependent term in the asymmetry can be used but the contribution of this term is very small. From the table we see that tests for time reversal invariance can be made by looking for the dependence on triple scalar products in the $\beta$-decay distribution functions. For example, the electron neutrino correlation from oriented nuclei $\boldsymbol{J} \cdot\left(\boldsymbol{p}_{e} \times \boldsymbol{q}_{v}\right)$ was suggested by Jackson et al. ${ }^{3}$ The coefficient of this term is proportional to

$$
\operatorname{Im}\left(C_{S} C_{T}^{*}+C_{S}^{\prime} C_{T}^{*}-C_{V} C_{A !}^{*}-C_{V}^{\prime} C_{A}^{*}\right)\left|M_{F}\right|\left|M_{G T}\right| .
$$

Experiments on the electron-neutrino correlation from oriented neutrons ${ }^{4}$ carried out indicate that the coefficient has a value $0.02 \pm 0.28$ which can be interpreted as confirming time reversal invariance for $\beta$ decays. Another method has been suggested by Lewis and Curtis ${ }^{5}$ which depends on the interference between $T$ and $S$ or $A$ and $V$ in the first-forbidden $\beta$ decays. This term gives rise to a transverse polarization of the $\beta$-particles from unoriented nuclei which can be studied through the term $\left(\boldsymbol{\sigma} \cdot\left(\boldsymbol{p}_{e} \times \boldsymbol{k}_{v}\right)\right)\left(\boldsymbol{p}_{e} \cdot \boldsymbol{k}_{v}\right)$. A third method is to measure $\beta-v$ correlations from polarized nuclei, the quantity measured being $\boldsymbol{J}_{n} \cdot\left(\boldsymbol{p}_{e}^{\prime} \times \boldsymbol{k}_{\nu}\right)$. The coefficient depends on the product $\left|M_{F}\right| M_{G T} \mid$ and the effect of this term will be small if the value $\left|M_{F^{\prime}}\right| M_{G T} \mid$ is very nearly zero. In fact, the experiment on $\mathrm{Co}^{58}$ has indicated that the effect of this term is not present. But in view of the uncertainties in the interpretation of $\beta$ asymmetry data from $\mathrm{Co}^{58}$ experiment nothing conclusive can be drawn from the absence of this term. The experiments performed so far, while lacking in precision, still give no evidence of any spectacular violation of time reversal invariance. Thus we can conclude that $\beta$ decay processes are left invariant under $C P$ as postulated by Landau and others. ${ }^{6}$

1 K. Alder, B. Stech and A. Winter, Phys. Rev. 107, 728 (1957).

2 S. P. Guhst and L. A. Page, Phys. Rev. 92, 970 (1953).

3 J. D. Jackson, S. B. Treiman and H.W. Wyld, Phys. Rev. 106, 517 (1957).

4 Burgy et al. Phys. Rev. 107, 731 (1957).

5 R. B. Curtis and R. R. Lewis, loc. cit.

6 L. Landau, loc. cit. 
Suggested Experiments for testing non-invariance under $T$ in decay experiments

\begin{tabular}{|c|c|c|c|c|}
\hline $\begin{array}{c}\text { The "pseudoscalar" } \\
\text { quantity (under } T \text { ) }\end{array}$ & Observation & $\begin{array}{l}\text { The couplings } \\
\text { between which } \\
\text { interference } \\
\text { occurs in the } \\
\text { coefficient }\end{array}$ & Remarks & Authors \\
\hline $\boldsymbol{J} \cdot(\boldsymbol{\mu}, \times(\boldsymbol{y})$ & $\begin{array}{l}\beta-v \text { correlation } \\
\text { in the decay of po- } \\
\text { larized neutrons. }\end{array}$ & $S . T$ & $\begin{array}{l}\text { Since }\left|C_{S}\right|^{2} \text { and } \\
\left|C_{T}\right|^{2} \text { are known } \\
\text { to be large and } \\
\text { if } \operatorname{Im} C_{S} \text { and } \operatorname{Im} C_{T} \\
\text { are also large, the } \\
\text { effect may be easy } \\
\text { to detect. }\end{array}$ & $\begin{array}{l}\text { JACKSON } \\
\text { et al. }\end{array}$ \\
\hline$\sigma \cdot\left(\boldsymbol{\mu}_{0} \times \boldsymbol{y}_{\mathrm{r}}\right)$ & $\begin{array}{l}\text { Polarization of } \\
\text { electrons in the } \\
\beta \text { decay of non- } \\
\text { oriented nuclei, } \\
\text { detected by Mott } \\
\text { scattering. }\end{array}$ & $S, V$ and $A, T$ & $\begin{array}{l}\text { If }\left|C_{A}\right|^{2} \text { and }\left|C_{T}\right|^{2} \\
\text { are small, the } \\
\text { effect may be dif- } \\
\text { ficult to detect. }\end{array}$ & $\begin{array}{l}\text { JACKSON } \\
\text { et al. }\end{array}$ \\
\hline$\sigma \cdot\left(. J \times \boldsymbol{l}_{0}\right)$ & $\begin{array}{l}\text { Polarization of } \\
\text { electrons in the } \\
\beta \text { decay of orient- } \\
\text { ed nuclei, detect- } \\
\text { ed by Mott } \\
\text { scattering. }\end{array}$ & $\begin{array}{l}S, A, V, T \\
\text { and } T A\end{array}$ & $\begin{array}{l}\text { The effect is } \\
\text { small and is hence } \\
\text { difficult to de- } \\
\text { tect. }\end{array}$ & $\begin{array}{l}\text { JACKSON } \\
\text { et al. }\end{array}$ \\
\hline.$J \cdot\left(p_{e} \times[\boldsymbol{k})(. J \cdot k)^{n}\right.$ & $\begin{array}{l}\beta-\gamma \text { correlations } \\
\text { in the decay of } \\
\text { polarized nuclei. }\end{array}$ & $\begin{array}{l}T, S \\
\text { and } \\
A, V\end{array}$ & & $\begin{array}{l}\text { Morita } \\
\text { and } \\
\text { Morita }\end{array}$ \\
\hline$\sigma \cdot\left(p_{e} \times k\right)\left(p_{e} \cdot k\right)$ & $\begin{array}{l}\text { Correlation be- } \\
\text { tween the trans- } \\
\text { verse polarization } \\
\text { of } \beta \text { particles and } \\
\text { electron and } \\
\text { photon momenta }\end{array}$ & $S, T$ & $\begin{array}{l}\text { This method was } \\
\text { later found er- } \\
\text { roneous since the } \\
\text { tránsverse polari- } \\
\text { zation may exist } \\
\text { due to the finite } \\
\text { size of the nucleus. }\end{array}$ & $\begin{array}{l}\text { CurTis } \\
\text { and } \\
\text { LEwis }\end{array}$ \\
\hline$\sigma \cdot\left(. J_{\mu} \times \mu_{e}\right)$ & $\begin{array}{l}\text { Polarization of } \\
\text { electrons in the } \\
\beta \text { decay of polariz- } \\
\text { ed } \mu \text {-mesons de- } \\
\text { tected by Mott } \\
\text { scattering }\end{array}$ & $A, V$ & $\begin{array}{l}\text { If } C_{V} \approx C_{A}, \text { then } \\
\text { the effect will be } \\
\text { difficult to detect. }\end{array}$ & Kotani \\
\hline
\end{tabular}

It is easy to see that the use of such right- and left-handed particles and antiparticles naturally fits in with the non-conservation of parity and charge conjugation. For under $P$, we invert the momentum of a neutrino but not its spin direction and thus a right-handed neutrino will become a left-handed neutrino which is a forbidden state for the neutrino. Thus the theory violates invariance 
under $P$. Since $C$ only changes the particle into its anti-particle without changing its spin or momentum, we see that a right-handed neutrino will become under $C$, a right-handed anti-neutrino which is again forbidden. Hence the theory is not invariant under $C$. However the theory is left invariant under $C P$.

We have already remarked that the negative asymmetry in the $\mathrm{Co}^{60}$ experiment is compatible with $C_{i} \simeq \pm C_{i}^{\prime}$ with - for $T, S$ and + for $A$ and $V$. If an antineutrino is emitted along with $\beta^{-}$, it should be left-handed. Consider the transition in $\mathrm{Co}^{60}$ where $T_{z}=5 \rightarrow T_{z}=4, \Delta T_{z}=-1 .{ }^{1}$ If both the electrons and the anti-neutrinos are emitted predominantly parallel to each other in the $-z$ direction there is conservation of angular momentum since both are left-handed particles. Of course, this negative asymmetry can be explained also with different assumptions regarding $C_{i}^{\prime}$ 's which will be clear from the following discussion on the longitudinal polarization of $\beta$-particles in the two-component theory. ${ }^{2}$

To see how the $\beta$-particles acquire longitudinal polarization, let us consider the coupling between the neutrino and the electron fields given by

$$
\bar{\psi}_{e} O_{i}\left(1-\gamma_{5}\right) \psi_{v} \text {. }
$$

Use of commutation relations gives us

$$
\begin{aligned}
O_{i} \gamma_{5}= \pm \gamma_{5} O_{i} \text { with } & + \text { for } i=S, P, T \\
& - \text { for } i=A, V .
\end{aligned}
$$

Thus we obtain

$$
\bar{\psi}_{e}\left(1 \mp \gamma_{5}\right) O_{i} \psi_{v} \text {. }
$$

For highly relativistic electrons the mass can be neglected and as for the neutrino we have 100 per cent right-handed electrons for $V$ and $A$ and 100 per cent lefthanded electrons for $S, T$ and $P$. The positron, being the anti-particle, will possess the opposite handedness. It is important to notice that the handedness of the $\beta$ particle depends both on the handedness of the neutrino and the type of $\beta$ coupling. However we can measure experimentally the longitudinal polarization of the $\beta$ particles irrespective of such a knowledge and this information can be used as a tool to reveal the type of the coupling responsible for the $\beta$ decay. For $\beta$-particles moving with velocity $v$, we can easily show that the helicity $(\boldsymbol{\sigma} \cdot \boldsymbol{p})$ will be equal $\pm v / c$ with + for $V$ and $A$, and - for $S, T$ and $P$.

A large number of experiments have been performed to measure the longitudinal polarization of $\beta^{+}$particles emitted in the pure Gamow-Teller, pure Fermi, mixed and forbidden transitions. ${ }^{3}$ Generally an electrostatic deflector converts the initial longitudinal polarization of the particles into transverse polarization which is then detected by the left-right asymmetry due to spin-orbit forces in the ordinary

1 T. D. Lee and C. N. YANG, 105, 1671 (1957); A. SalaM, Nuovo Cim. o, 297 (1957); and

L. Landau, Nuclear Physics 3, 127 (1957).

2 T. D. LeE and C. N. YANG, Elementary Particles and Weak Interactions, 47, Brookhaven National Laboratory (1957).

3 See "Measurements of Helicity" by Lee Grodzins, Progress in Nuclear Physics, Vol. 7, p. 165, Pergamon (1959).

EPCR 17 
Coulomb scattering. ${ }^{1}$ Other methods devised to measure the longitudinal polarization are based on the fact that the Moller scattering cross-section depends sensitively on the polarization of the scattering electrons. ${ }^{2}$ The high energy bremsstrahlung of such polarized electrons inherits the polarization and the observation of the circular polarization of such $\gamma$-rays constitutes another method for estimating the longitudinal polarization. ${ }^{3}$ For positrons, Page and Heinberg ${ }^{4}$ in their experiments exploit the spin-dependent features in the two-photon annihilation in flight of a polarized positron with a polarized electron (available in magnetized iron). With an accuracy of 10 per cent all data are consistent with a longitudinal polarization of the maximum amount $\pm v / c$ for $\beta^{+}$emission. This implies the following choice of coupling constants and handedness of the neutrino

$$
\begin{aligned}
& C_{S}=-C_{S}^{\prime} ; C_{T}=-C_{T}^{\prime} \text { for a right-handed neutrino } \\
& C_{V}=C_{V}^{\prime} ; \quad C_{A}=C_{A}^{\prime} \quad \text { for a left-handed neutrino. }
\end{aligned}
$$

A very ingenious cxperiment due to Goldhaber et al. ${ }^{5}$ indicates that the neutrino is left-handed. In this experiment the nucleus with spin $T=0\left(\mathbf{E u}^{152}\right)$ decays by a $K$ electron capture to an excited nucleus $B$ with $T=1\left(\mathrm{Sm}^{\mathbf{1 5 2}}\right)$ emitting a ncutrino. If the neutrino is emitted along the $z$-axis, then the $\tau_{z}$ value of $B$ is -1 or 0 for positive helicity of the neutrino and +1 or 0 for negative helicity of the neutrino. The recoiling nucleus $B$ reaches the ground state by the emission of a $\gamma$-ray. In either case, the helicity of the $\gamma$-ray emitted along the $-z$ axis is the same as that of the neutrino. The preferential detection of $\gamma$-rays emitted along the recoil direction is accomplished by use of resonance fluorescence detection which is sensitive only to those photons whose Doppler shift due to the motion of the excited recoil nucleus almost compensates for the sharing of the transition energy between the. $\gamma$-ray and the final nucleus. Thus the measurement of the circular polarization of the $\gamma$-rays which are resonantly scattered by the nucleus $B$ yields directly the helicity of the neutrino. The experiment shows that the neutrino is left-handed.

From the above discussion of the longitudinal polarization and the handedness of the neutrino we can assume that the $\beta$ decay proceeds through a linear combination of $V$ and $A$. The experiments on $\beta$ decay from polarized neutrons indicates that this linear combination is $V-A$. It is interesting to note that this conclusion is in agreement with $(\beta-\gamma)$ correlation experiments ${ }^{6}$ performed recently.

1 H. Frauenfelder et al., Phys. Rev. 106, 386 (1957).

2 A. M. Bixcer, Phye. Rev. 107, 1434 and 1467 (1957). See also G. W. Ford and C. J. MulLIs, Phys. Rev. 108, 477 (1957).

3 M. Goldhaber, L. Grodzins and A. W. Sunyar, Phys. Rev. 106, 826 (1957); K. W. Irvoy, Phys. Rev. 106, 820 (1927); K. W. McVoy and F. J. Dyson, Phys. Rev. 106, 1360 (1957).

4 L. A. Page and M. Heinberg, Phys. Rev. 106, 1220 (1957).

5 M. Goldhaber, M Grodzins and A. W. Sunyar, Phys. Rev. 109, 1015 (1958).

${ }^{6}$ See especially The Veutrino by James S. Allen, ch. 5, Princeton (1958). 
Some Experiments on $\beta$-decay

\begin{tabular}{|c|c|c|c|c|c|c|}
\hline $\begin{array}{c}\text { Radio } \\
\text { active } \\
\text { nuclides }\end{array}$ & Transitions & $\begin{array}{l}\text { Type of } \\
\text { inter- } \\
\text { action }\end{array}$ & Observation & $\begin{array}{c}\text { Depend- } \\
\text { ence } \\
\text { on the } \\
\text { pseudoscalar }\end{array}$ & $\begin{array}{c}\text { Result of } \\
\text { observation }\end{array}$ & Authors \\
\hline $\mathrm{Co}^{80}$ & $\begin{array}{c}5^{+}-4^{+} \\
\beta^{-}\end{array}$ & Pure $G T$ & $\begin{array}{l}\text { Asymmetry } \\
\text { from polarized } \\
\text { nuclei }\end{array}$ & $\left(\boldsymbol{J} \cdot \boldsymbol{r}_{\boldsymbol{e}}\right)$ & $-1 \pm 20 p . c$. & $\begin{array}{l}\text { C. S. Wu } \\
\text { et al. }\end{array}$ \\
\hline $\mathrm{Co}^{58}$ & $\begin{array}{c}2^{+}-2^{+} \\
\beta^{+}\end{array}$ & Mixed & , & , & $+\cdot 325 \pm \cdot 047$ & $\begin{array}{l}\text { E. Ambler } \\
\text { et al. }\end{array}$ \\
\hline${ }^{\circ} n^{1}$ & $\frac{1}{2}_{\beta^{-}}^{+\frac{1}{2}^{+}}$ & $\frac{\left|M_{F}\right|^{2}}{\left|M_{G T}\right|^{2}}=\frac{1}{3}$ &. & , & $-0 \cdot 37 \pm \cdot 11$ & $\begin{array}{l}\text { M. T. Burgy } \\
\text { et al. }\end{array}$ \\
\hline $\mathrm{Na}^{22}$ & $\begin{array}{c}3^{+}-2^{+}-0^{+} \\
\beta^{+} \gamma\end{array}$ & Pure $G T$ & $\begin{array}{l}\quad \beta-\gamma \\
\text { (circularly } \\
\text { polarized) } \\
\text { correlation }\end{array}$ & $\sigma \cdot\left(p_{e} \times k\right)$ & $0 \cdot 39 \pm \cdot 08$ & H. Schopper \\
\hline $\mathrm{Co}^{60}$ & $\begin{array}{c}5^{+}-4^{+}-2^{+}-0^{+} \\
\beta^{-} \gamma \gamma \gamma\end{array}$ & Pure $G T$ & . $\quad$, & ", & $-0 \cdot 41 \pm \cdot 07$ & $\begin{array}{c}\text { A. H. Wapstra } \\
\text { et al. }\end{array}$ \\
\hline 西 & $\frac{2^{-}-0^{+}}{\beta^{-}}$ & $G T$ & $\begin{array}{l}\text { Longitudinal } \\
\text { polarization } \\
\text { of } \beta \text { detectend } \\
\text { by (1) Mott, } \\
\text { (2) Moller scat- } \\
\text { tering and cir- } \\
\text { cular polarization } \\
\text { forward Brems- } \\
\text { strahlung. }\end{array}$ & , & $-v / c$ & \\
\hline $\mathrm{Cl}^{34}$ & $\begin{array}{c}0^{+}-0^{+} \\
\beta^{+}\end{array}$ & Fermi & $\begin{array}{l}\text { Bremsstrahlung } \\
\text { or annihilation } \\
\text { radiation. }\end{array}$ & & $+v / c$ & $\begin{array}{l}\text { M. Deutsch } \\
\text { et al. }\end{array}$ \\
\hline
\end{tabular}

It was suggested by Lee and Yang ${ }^{1}$ in their original paper that the $\pi-\mu-e$ chain can be used to test parity non-conservation. From the two-component neutrino theory it immediately follows that $\mu$-mesons obtained from the decay of $\pi^{ \pm}$should be longitudinally polarized along their direction of motion. Hence in the subsequent decay of the $\mu$-meson into electron and $\nu-\bar{v}$ pairs, the electron will have an asymmetric angular distribution if parity violation exists.

The $\mu$ decay process can be described by an interaction Hamiltonian based on the two component theory of the neutrino

$$
H_{\text {int }}=\sum_{V, A} f_{i}\left(\bar{\psi}_{e} 0_{i} \psi_{\mu}\right)\left(\bar{\psi}_{v} 0_{i} \psi_{\nu}\right)+h \cdot c,
$$

where $\psi_{\nu} \rightarrow \frac{1}{2}\left(1+\gamma_{5}\right) \psi_{\nu}$ corresponds to left-handed neutrino. The sum is over $V$ and $A$ as the other covariants $S, T$ and $P$ vanish identically due to commutation properties. $^{2}$ Note that this result is independent of our knowledge about nuclear decay $\mu^{ \pm}$can decay as

$$
\begin{aligned}
\mu^{ \pm} & \rightarrow e^{ \pm}+v+\bar{v} \\
& \rightarrow e^{ \pm}+2 \bar{v} \\
& \rightarrow e^{ \pm}+2 v .
\end{aligned}
$$

1 T. D. Lee and C. N.YANG, Phys. Rev. 104, 254 (1956); Phys. Rev. 105, 1671 (1957).

2 This result is valid only in the two component theory. If we use four component theory, by a Fierz transformation, the interaction can be made to contain all the covariants instead of just $A$ and $V$. 
Michel ${ }^{1}$ showed that the energy spectrum of the decay electrons has the form

$$
N(x) \mathrm{d} x=4 x^{2}\left[3(1-x)+\frac{2}{3} \varrho(4 x-3)\right] \mathrm{d} x,
$$

where $x$ is the electron energy in units of its maximum value. $\varrho$ is called Michel's parameter which has a different value for each of the three schemes of decay: $\varrho=0.75$ for $(43 a)$ while $\varrho=0$ for $(43 b)$ and $(43 \mathrm{c})$. Experiments indicate that $\varrho=.68 \pm 0.02$. Thus $(43 a)$ is the only allowed decay scheme and this has a bearing on the principle of conservation of leptons to be discussed below. The angular distribution of the decay electron from polarized $\mu$-meson averaged over the energy distribution is

$$
\left\langle\frac{\mathrm{d} \sigma}{\mathrm{d} \Omega}\right\rangle_{E}=\frac{1}{4 \pi}\left(1 \pm \frac{\xi}{3} \cos \theta\right),
$$

where $\theta$ is the angle between $\boldsymbol{p}_{e}$ and the spin of the $\mu$-meson and

$$
\xi=\frac{f_{V} f_{A}^{*}+f_{A} f_{V}^{*}}{\left|f_{V}\right|^{2}+\left|f_{A}\right|^{2}} .
$$

The upper sign corresponds to $\mu^{-}$decay while the lower sign refers to $\mu^{+}$decay. This angular distribution was confirmed in an ingenious experiment due to Garwin et al..$^{2} \mathrm{~A}$ beam of $85 \mathrm{MeV} \pi^{+}$mesons was stopped in a carbon absorber. Some of the $\mu^{+}$from the decay of $\pi^{+}$mesons came to rest in a carbon target at a short distance from the absorber. The $\beta$ decay of $\mu$ was detected by electron telescopes which recorded electrons with energies $>25 \mathrm{MeV}$. It was so arranged that electrons will be recorded in an interval between 0.75 and $2 \mu$ sec. after $\mu$ had come to rest. To record the entire angular distribution of electrons, the $\mu$-mesons were caused to precess by means of a small vertical magnetic field. The various experiments yield a value

$$
|\xi|=0.87 \pm 0.13
$$

and show that electrons are emitted preferentially backwards relative to the line of flight of the $\mu$-meson. The sign of $\xi$ cannot be known from this experiment as it depends on the helicity of the $\mu$-meson acquired when $\pi$ decayed. The helicity of $\mu$ is intimately related to the principle of conservation of leptons. However if we can measure the helicity of the decay electrons from randomly oriented $\mu$-mesons we can find the sign of $\xi$ (cf. longitudinal polarization in nuclear $\beta$ decay). The longitudinal polarization of the decay electron is $\varrho= \pm \xi \frac{v}{c} \simeq \pm \xi$ the negative sign referring to $\mu^{+}$decay. Experiments have shown that the posi-

${ }^{1}$ L. Michel, Nature, Lond. 153, 959 (1949); Proc. Phys. Soc. 63 A, 514 (1950); Phys. Rev. S6, 814 (1952).

2 R. L. Garwin, L. M. Lederman and L. M. Weinrich, Phys. Rev. 105, 1415 (1957). 
trons from $\mu^{+}$decay are right-handed indicating that $\xi$ is negative. ${ }^{1}$ This implies that $f_{A} \simeq-f_{V}$ as seen from (45a) which in other words means that the interaction is $V-A$ which is in remarkable agreement with the tentative conclusion arrived at after the discussion of nuclear $\beta$ decay experiments. Attempts have been made to improve the agreement between theoretical and experimental value of $\varrho$ through the inclusion of non-local interaction in $\mu$ decay. ${ }^{2}$ The effects of a radiative correction to the electron energy spectrum arising in the $\mu$ decay have also been - discussed. ${ }^{3}$

In the discussion of the $\mu^{ \pm}$decay we have in dicated the need for a principle of conservation of leptons. ${ }^{4}$ By the mere use of the two component theory we cannot decide whether the $\beta$ decay is

$$
\begin{aligned}
n & \rightarrow p+e^{-}+v \\
& \rightarrow p+e^{-}+\bar{v} .
\end{aligned}
$$

By choosing different handedness for $v$ and $\bar{v}$ and also correspondingly different couplings we can explain the present experimental data ( $\mathrm{Co}^{60}$ experiments etc.). However if we allow both the processes to have equal amplitudes then there will be no asymmetry in the $\mathrm{Co}^{60}$ experiment. We thus find it necessary to invoke the principle of conservation of leptons. This principle is analogous to the principle of conservation of baryons and implies that the number of leptons minus the number of anti-leptons should be conserved in any process. But this law is obviously incomplete without specifying which particles are leptons and which are anti-leptons. Let us assign a leptonic charge -1 to $e^{+}, \mu^{+}$and $\bar{v}$ (anti-leptons) and a leptonic charge +1 to $e^{-}, \nu, \mu^{-}$. The baryons and bosons have leptonic charge 0 . An immediate consequence of this is that $\mu^{+}$decay can proceed only through (43a).

Pion decay offers an interesting test case for this principle. Consider $\pi^{+}$decay. This principle demands

$$
\pi^{+} \rightarrow \mu^{+}+\nu
$$

Conservation of angular momentum requires that $\mu^{+}$be left-handed as the neutrinos. ${ }^{5}$ When $\mu$ decays, we know that the high energy positrons emerge in the backward direction relative to the $\mu^{+}$meson's line of flight. Conservation of angular momentum requires that positrons should be right-handed as was found from experiment. Thus with the assumption of $V-A$ interaction, we find there is

1 G. Culligan et al., Nature, Lond. 180, 751 (1957).

2 T. D. Lee and C. N. YANG, Phys. Rev. 10S, 1611 (1957).

3 T. Kinoshita and A. Siruin, Phys. Rev. 106, 1110 (1957); Phys. Rev. 107, 593 (1957).

4 E. Konopinski and H. M. Mahmoud, Phys. Rev. 92, 1145 (1953); see also T. D. LeE and C. N. YANG, Phys. Rev. 105, 1671 (1956).

5 It is interesting to note that though leptons are left-handed antileptons are right-handed. particles we can never detect handedness of $\mu^{+}$from $\pi^{+}$decay as the conservation of linear momentum requires $\mu^{+}$to be left-handed. We recall that the handedness is an invariant quantity under Lorentz transformations only for mass zero particles, i.e. for particles moving with the velocity of light. 
conservation of leptonic charge in the $\pi$ decay. It may be noted here that if the neutrino were assumed to be right-handed, we will have violation of the principle of conservation of leptons in the $\pi$ decay.

'The conservation of leptons has another confirmation from the double and inverse $\beta$ processes. These two processes can be used to test which of the two descriptions of the neutrino namely the Majorana ${ }^{1}(v \equiv \bar{v})$ or the Dirac $(v \neq \bar{v})$ is correct. The double $\beta$ decay as the name indicates refers to a phenomenon by which a nucleus changes its charge by two units by $\beta$ decay. If the neutrinos are of the Dirac type, then the processes are

followed by

$$
n \rightarrow p+e^{-}+\bar{v}
$$

$$
n \rightarrow p+e^{-}+\boldsymbol{v} \text {. }
$$

Thus, in Dirac's picture there should be two neutrinos and two $\beta$ particles. However in Majorana's picture $\nu \equiv \bar{v}$ and we can envisage a process involving a virtual emission and absorption of a neutrino. Thus the two neutrons change into two protons with the emission of two electrons, i.e.

$$
\begin{aligned}
n & \rightarrow p+e^{-}+\bar{v} \\
n+v & \rightarrow p+e^{-} .
\end{aligned}
$$

In contrast with the continuous energy spectrum of electrons emitted with Dirac's neutrinos, the sum of the kinetic energies of the two electrons should be a sharp line spectrum for the Majorana neutrino. It is clear that the Majorana view of identical $v$ and $\bar{v}$ violates the principle of conservation of leptonic charge while Dirac's view upholds it. As there is very little evidence for the presence of double $\beta$ decay, we can conclude that this confirms the principle of the conservation of leptons.

The very difficult inverse $\beta$ reactions have also been experimentally studied. ${ }^{1}$ If $\nu$ and $\bar{v}$ are distinct, the following inverse $\beta$ processes are possible

$$
\begin{aligned}
& \bar{v}+p \rightarrow n+e^{+} \\
& v+n \rightarrow p+e^{-} .
\end{aligned}
$$

while if $\nu$ and $\bar{v}$ are identical, the following are also possible

$$
\begin{aligned}
& v+p \rightarrow n+e^{+} \\
& \bar{v}+n \rightarrow p+e^{-} .
\end{aligned}
$$

The cross-section for such processes can be estimated by perturbation theory and they are of extremely small value $\left(\sim 10^{-44} \mathrm{~cm}^{2}\right)$. If the principle of conserration of leptonic charge is correct, the nuclear reaction pile should be a source of anti-neutrinos. Considering the inverse reaction ${ }^{2}$

$$
{ }_{17} \mathrm{Cl}^{37}+\bar{\nu} \rightarrow{ }_{18} \mathrm{Al}^{37}+e^{-}
$$

\footnotetext{
1 See chapter VII of The Neutrino by JAMes S. Allex. loc. cit.

2 R. Davies, Phys. Rev. 97, 766 (1957).
} 
we find that if the Dirac description of neutrino is correct, these anti-neutrinos should not disintegrate $\mathrm{Cl}^{37}$. Experiments have been performed and an upper limit of $9 \times 10^{-45} \mathrm{~cm}^{2}$ was obtained for the reaction cross-section. Cowan et al. ${ }^{1}$ have measured the cross-section averaged over neutrino spectrum and find $\bar{\sigma}_{\exp }=4.0 \pm 1.5$ $\times 10^{-44} \mathrm{~cm}^{2}$, in close agreement with the theoretical value $6 \times 10^{-44} \mathrm{~cm}^{2}$. In calculating this we have assumed that the statistical weight factor is 2 corresponding to spin $1 / 2$. However if the "two-component" neutrino theory is correct, we find the interesting situation that all the anti-neutrinos emerging from the reactor have only one spin state in which they can be absorbed. It is to be noted however that the neutrino flux is independent of two or four components. Thus the "spin weight" becomes 1 and hence the cross-section gets doubled. This means the experimental value of neutrino absorption found out by Cowan et al. is smaller by a factor as the theoretical cross-section is $12 \times 10^{-44} \mathrm{~cm}^{2}$. At any rate, the negative results of Davies ${ }^{2}$ can be taken to confirm the principle of conservation of leptons.

Pauli $^{3}$ has pointed out that conservation of leptons is more complicated than what the above discussion implies. For even as in parity conserving $\beta$ decays, we can have violation to any degree (instead of "yes" or "no") of the conservation of lepton number in a $\beta$ interaction of the form

$$
H_{\mathrm{int}}=\sum_{i}\left(\bar{\psi}_{p} 0_{i} \psi_{n}\right)\left[C_{I}\left(\bar{\psi}_{e} 0_{i} \psi_{\nu}\right)+C_{I I}\left(\bar{\psi}_{e} 0_{i} \psi_{\nu}^{C}\right)\right]+h \cdot c .
$$

Conservation of lepton number implies that either $C_{I}$ or $C_{I I}$ vanishes, and maximum violation occurs when $\left|C_{I}\right|=\left|C_{I I}\right|$. It is interesting to compare the same situation when there is violation of parity. Allowing such violation in the interaction, we get in all four constants $C_{I}, C_{I}^{\prime}, C_{I I}$ and $C_{I I}^{\prime}$, two for the parityconserving part and two for the parity non-conserving part. Only experiments can decide the presence or absence of such constants. It has been pointed out by Case $^{4}$ that violation of parity and occurrence of double $\beta$ decay anticommute with each other, which implies that if there is maximum violation of parity there is very little of double decay. According to Case, ${ }^{5}$ mere use of the two component theory will not cause violation of parity but the specific form of the weak interaction in that the $\psi_{\nu}$ should occur only multiplied by $\left(1-\gamma_{5}\right)$ or $\left(1+\gamma_{5}\right)$ causes violation of parity. Physically this means that only one spin state of the Majorana neutrino is operative in emission or absorption. Thus if only left-handed neutrinos are emitted, only right-handed neutrinos (or anti-neutrinos as we call them) can be absorbed to produce the same reaction.

The decays of strange particles offer possibilities of testing parity violation. Among the various modes of decay of the $K$ particle the $K_{\mu 2}$ decay is very important

1 C. L. Cowan et al., Science, 124, 103 (1956).

2 R. Davies, loc. cit.

3 W. Pauli, Nuovo Cim. 6, 204 (1957); D. L. Pursey, Nuovo Cim. 6, 266 (1957).

4 K. M. Case, Phys. Rev. 107, 307 (1957); J. Serpe, Physica 18, 295 (1952); J. A. Mclennen, Phys. Rev. 106, 821 (1957).

5 K. M. CASE, ibid. 
in that it tests not only violation of $P$ and $C$ but also tests the principle of conservation of leptons. It has been found that the electrons from the subsequent $\mu$-meson decay have the same distribution as in $\pi$ decay, as it should be.

We now refer to discussions by Lee and $\mathrm{Yang}^{1}$ on the study of parity violation through $A^{0}$ and $\Sigma \pm 0$ deeay. Consider $A^{0}$ produced through $\left(\pi^{-}-p\right)$ collisions

$$
\pi^{-}+p \rightarrow \Lambda^{0}+K^{0} \text {. }
$$

If in the production both $S$ and $P$ waves are important, then there arises the possibility that $\Lambda^{0}$ will be polarized, the polarization direction being given by $\left(\boldsymbol{p}_{\tau}^{\text {inc }} \times \boldsymbol{p}_{.1}\right)$, i.e. perpendicular to the plane of production, if strong interactions conserve parity. 'The decay $\pi$ 's in the subsequent decay of $\Lambda^{0}$ can have an asymmetrical angular distribution about the direction $\left(\boldsymbol{p}_{\pi}^{\text {inc }} \times \boldsymbol{p}_{\Lambda}\right)$ as in the $\beta$ decay from oriented nuclei. This means that there will be an "up-down" asymmetry about the plane of production. In the centre of mass system of $\Lambda^{0}$ and $K^{0}$ the asymmetry is given by (assuming that $\Lambda^{0}$ has spin $1 / 2$ and $K^{0}$ has spin zero)

$$
\frac{\mathrm{d} \omega}{\mathrm{d} \Omega} \sim 1+\alpha\left\langle\boldsymbol{\sigma}_{\Lambda}\right\rangle \frac{\boldsymbol{p}_{\boldsymbol{\pi}}^{\text {out }}}{\left|\boldsymbol{p}_{\boldsymbol{r}}^{\text {out }}\right|} .
$$

$\left.\sigma_{.1}\right\rangle$ is the average polarization of $\Lambda^{0}, \boldsymbol{p}_{\pi}^{\text {out }}$ the momentum of the outgoing pion and $\alpha$ the asymmetry parameter. If $\Lambda^{0}$ has spin $1 / 2$, parity violation allows the emission of both $S$ and $P$ wave pions and if $A$ and $B$ denote the relative amplitudes for $S$ and $P$ wave emission $\alpha=\frac{-2 \operatorname{Re} A B^{*}}{|A|^{2}+|B|^{2}}$. A large up down asymmetry in the $A^{0}$ decay has been found, ${ }^{2}$ i.e.

$$
\alpha\left|\left\langle\sigma_{\Lambda}\right\rangle\right|=0.44 \pm 0.11 \text {. }
$$

Similar experiments on $\Sigma^{-}$production and its decay have shown that very little asymmetry exists. ${ }^{3}$

We shall now discuss the application of the $|\Delta \boldsymbol{I}|=1 / 2$ rule to hyperon decays. For $\Lambda^{0}$ decay, the final pion and nucleon should possess $I=1 / 2$. From the isotopic wave function of the combined $\pi-N$ system corresponding to $I=1 / 2$,

1 T. D. LeE and C. N. YANG, Phys. Rev. 104, 254 (1956); 105, 1671 (1957).

2 F. Eisler et al., Phys. Rev. 108, 1353 (1957); F. S. Crawford et al., Phys. Rev. 108, 1102 (1957); Bold et al., Phys. Rev. Lett. 1356 (1958); R. Gatto (see Phys. Rev. 108, 1103 (1957)) has shown using the $\pi-p$ scattering phase shifts at $37 \mathrm{MeV}$ that $\alpha \leqq 0.18 \pm 0.02$ for charge conjugation invariance to hold good.

Lee and Yang (see Phys. Rev. 109, $1755(1958)$ ) have pointed how the spin of $\Lambda^{0}$ can be determined from the large asymmetry of its decay products. They have shown that if $\langle\xi\rangle$ denotes the average value of the component of $\boldsymbol{p}_{\pi}$ along the normal to the production plane (in units of $100 \mathrm{MeV} / \mathrm{c}$ )

$$
-\frac{1}{2 j+2} \leqq\langle\xi\rangle \leqq \frac{1}{2 j+2}
$$

where $j$ is the spin of $\Lambda^{0}$. The large asymmetry indicates $j=1 / 2$ and $|\alpha|$ now equals $3\langle\xi\rangle$. It has aiso been pointed out that $|\alpha|$ uniquely depends on the spin of $\Lambda^{0}$ if we study the decay of completely polarized $\Lambda^{0}$, i.e. we set $\left|\boldsymbol{P}_{\Lambda^{0}}\right|=1$.

3 Cool et al., Phys. Rev. 114, 912 (1959). 
we find that the $\pi^{-}+p$ configuration is present with the probability ${ }^{2} / 3$. Thus the fraction of charged decays of $\Lambda^{0}$ as contrasted with its neutral decay mode $\Lambda^{0} \rightarrow \pi^{0}+n$ is given by ${ }^{2} / 3$. This is in very good agreement with the reported experimental value of $0.65 . \pm 0.05$.

$$
\begin{aligned}
& \Sigma^{+} \text {can decay into two channels, i.e. } \\
& \begin{aligned}
\Sigma^{+} & \rightarrow p+\pi^{0} \\
& \rightarrow n+\pi^{-}
\end{aligned}
\end{aligned}
$$

while $\Sigma^{-}$can decay into $n+\pi^{-}$only. $|\Delta \boldsymbol{I}|=1 / 2$ rule for $\Sigma^{+1}$ decay allows both $\boldsymbol{I}=1 / 2$ and $\boldsymbol{I}=3 / 2$ for the final pion and nucleon states in arbitrary proportion. Gatto and others have estimated the fraction of $\Sigma^{+}$decays into $n+\pi^{+}$system using the pion-nucleon scattering phase shifts. $\Sigma^{-}$decays necessarily into the $I=3 / 2$ state. With the $|\Delta \boldsymbol{I}|=1 / 2$ rule, it may be shown that a relationship exists between this transition and $\Sigma^{+}$transition.

Denoting by $A^{+}, A^{-}, A^{0}$ the amplitudes of decay into $n \pi^{+}, n \pi^{-}$and $p \pi^{0}$ respectively, we have the remarkable relation

$$
A^{0}=\frac{A^{-}-A^{+}}{\sqrt{ } 2} .
$$

If time reversal invariance holds then we can show that these decay amplitudes will involve pion-nucleon scattering phase shifts due to the final state interactions but the amplitude can still be treated as real numbers since the phase shifts will be small at these energies. Remembering that parity non-conservation allows both $S$ and $P$ waves for each amplitude, we can treat with Gell-Mann ${ }^{2}$ each amplitude as a vector $\boldsymbol{A}$ with $A^{S}$ and $A^{P}$ as the $x$ and $y$ components. The decay rates will be

$$
\Omega^{+}=\left|\boldsymbol{A}^{+}\right|^{2} ; \quad \Omega^{-}=\left|\boldsymbol{A}^{-}\right|^{2} ; \quad \Omega^{0}=\left|\boldsymbol{A}^{0}\right|^{2}=\frac{\left.\left|\boldsymbol{A}_{\mid}^{+\mid 2}+\right| \boldsymbol{A}^{-}\right|^{2}}{2}-\boldsymbol{A}^{+} \cdot \boldsymbol{A}^{-} .
$$

However we know from experiments that

Thus we have

$$
\frac{\tau_{\Sigma^{+}}}{\tau_{\Sigma^{-}}} \approx 1 / 2 ; \quad \Sigma^{+} \rightarrow \frac{n \pi^{+}}{p \pi^{0}} \sim 1 \quad(0.96 \pm 0.06)
$$

and the interesting result

$$
\Omega^{+} \approx \Omega^{-}-\Omega^{0}
$$

$$
\boldsymbol{A}^{+} \cdot \boldsymbol{A}^{-}=0 \quad\left|\boldsymbol{A}^{+}\right|=\left|\boldsymbol{A}^{-}\right|^{2}
$$

i.e. the vectors $\boldsymbol{A}^{+}$and $\boldsymbol{A}^{-}$are orthogonal to each other and are of equal length. We can express the various amplitudes as follows.

$$
\begin{aligned}
& A^{+S}=c \cos \beta ; \quad A^{+P}=c \sin \beta \\
& A^{-S}=-c \sin \beta ; \quad A^{-P}=c \cos \beta
\end{aligned}
$$

1 R. Gatto, Nuovo Cim. 3, 318 (1956); B. D'Espagnat and J. Prentki, Nuovo Cim. 3, 1045 (1956); M. Kawaguchi and K. Nishijima, Progr. Theoret. Phys. 15, 182 (1956).

2 M. Gell-MANN and A. H. Rosenfeld, loc. cit., p. 206. 
and

$$
A^{0 s}=\frac{-c(\cos \beta+\sin \beta)}{1 / 2} ; \quad A^{0 P}=\frac{c(\cos \beta-\sin \beta)}{1 / 2} .
$$

If $\alpha$ is the "up-down" asymmetry parameter discussed before in the $\Lambda$ decay the $\alpha$ 's for the three $\Sigma$ decays are

$$
\begin{aligned}
& n \pi^{+}: \alpha^{+}=-\sin 2 \beta \\
& n \pi^{-}: \alpha^{-}=\sin 2 \beta \\
& p \pi^{0}: \alpha^{0}=\cos 2 \beta
\end{aligned}
$$

where $c$ and $\beta$ are arbitrary parameters. Thus $|\Delta I|=1 / 2$ rule along with $C P$ invariance predicts a definite relationship between the three asymmetry parameters. Recent experiments ${ }^{1}$ on up-down asymmetry of $\Sigma^{+}$and $\Sigma^{-}$-decays indicate that

$$
\begin{aligned}
& \alpha^{0} P\left(\Sigma^{+}\right) \neq 0 \\
& \alpha^{+} P\left(\Sigma^{+}\right)=0 \\
& \alpha^{-} P\left(\Sigma^{-}\right)=0 .
\end{aligned}
$$

Of course we must remember that we can measure only $\alpha P(\Sigma)$ where $P(\Sigma)$ is the polarization of the $\Sigma$ particles when they are produced. These results very clearly indicate that $P\left(\Sigma^{+}\right) \neq 0$ and that $\alpha^{+} \approx 0$. Thus $\beta$ is $\approx 0$ or $\pi / 2$. It then follows that

$$
\alpha^{+} \approx 0 ; \quad \alpha^{0} \approx \pm 1 ; \quad \alpha^{-} \approx 0 .
$$

The absence of asymmetry in $\Sigma^{-}$decay may also be due to the fact that $\Sigma^{-}$- are not polarizedwhen they are produced. At any rate the predictions of the $|\Delta I|=1 / 2$ rule seem to be consistent with the experimental results. If we can measure the longitudinal polarization of the protons emitted in $\Sigma^{0}$ and $\Lambda^{0}$ decays, then we can check whether there exists any relation between the asymmetries $\alpha^{0}$ and $\alpha_{\Lambda}^{+}$ which should have opposite signs under the doublet approximation of baryons.

Finally applying the $|\Delta \boldsymbol{I}|=1 / 2$ rule for $\Xi^{-}$and $\Xi^{0}$ decays, we find that $\tau_{\Xi-} / \tau_{\Xi^{0}}=1 / 2$ for the ratio of average lifetimes of these hyperons. However if the transition is a pure $|\Delta \boldsymbol{I}|=3 / 2$ transition, this ratio is 2 . This ratio has not been verified.

\section{UN I VERSAL FERMI INTERACTION}

Long before the recent developments in weak interactions Wheeler and Tiomno ${ }^{2}$ had noticed the remarkable fact that the coupling constants in the three processes, viz. $\beta$ decay, $\mu$ capture and $\mu$ decay are almost equal. Since all the particles involved in these processes are only fermions, this equality suggested the attractive posssibility of a Universal Fermi Interaction (UFI) which can be represented by the triangle below (usually referred to as Puppi's ${ }^{3}$ triangle).

1 Cool et al., loc. cit.

2 J. Thomno and J. A. Wheeler, Rev. Mod. Phys. 21, 144, 153 (1949).

${ }^{3}$ G. Puppi, Nuovo Cim. 5, 587 (1948). 
The three sides of the triangle $C, E$ and $D$ refer respectively to $\beta$ decay, $\mu$ decay and $\mu$ capture. By UFI it was not only meant that the coupling strengths should be the same but also the form of the interaction. Though it was known that $\mu$ decay should proceed through $A$ and $V$ coupling, the "old" electronneutrino angular correlation experiments gave conflicting evidence regarding

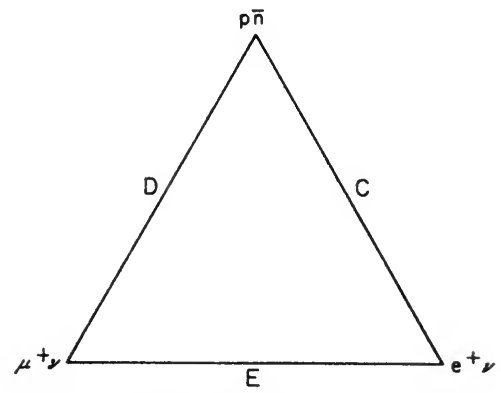

Frg. 36. (a) Puppi triangle.

the couplings responsible for $\beta$ decay. The evidence prior to the recent experiments on the longitudinal polarization of $\beta$ particles emitted in $\beta$ decay was interpreted to support the view that only $S$ and $T$ coupling induced $\beta$ decay. Under these circumstances, no definite progress could be made regarding the validity of UFI. However the experiments on the longitudinal polarization of $\beta$ particles have shown that the couplings present in $\beta$ decay are $A$ and $V$ and thus the Universal Fermi Interaction was taken to be a valid hypothesis. We shall show presently that the UFI can be derived from more general grounds consistent with the new experimental facts on weak interactions, viz. parity non-conservation and violation of invariance under charge conjugation.

Before we take this up for discussion let us consider the possibility of the modification of Puppi's triangle to accommodate other weak processes like the decays of $K$ particles and hyperons. ${ }^{1}$ Other processes involving fermions in Puppi's triangle can be obtained by reversing the reaction arrow and replacing the particle by its antiparticle when the particle is transferred from one side of the arrow to the other. Thus we obtain from the $\beta$ decay of a neutron, the $K$ electron capture by a proton in a nucleus

$$
e^{-}+p \rightarrow n+v
$$

and from the $\beta$ decay of a proton in a nucleus, the process

$$
\bar{\nu}+p \rightarrow n+e^{+} .
$$

The latter can be used to detect the anti-neutrino. It is clear that Puppi's triangle can never account for the decays of strange particles. Dallaporta and Gell-Mann ${ }^{1}$

1 N. Dallaporta, Nuovo Cim.1, 962 (1953); M. Gell-Mans, Proc. 6th Rochester Conference. Interscience, New York (1956). 
have independently discussed the tetrahedron representation of the four-fermion interactions shown below.

The tetrahedron was obtained by adding to the triangle the vertex containing pairs like $p \bar{\Lambda}, p \bar{\Sigma} 0$ etc. into which $K^{+}$can decay and including in the $p \bar{n}$ vertex pairs like $\Sigma^{+} \bar{\Lambda}, 1 \bar{\Sigma}$ - into which $\pi^{+}$can decay. The two arrows represent virtual dissociation. We can easily verify that the couplings between different vertices
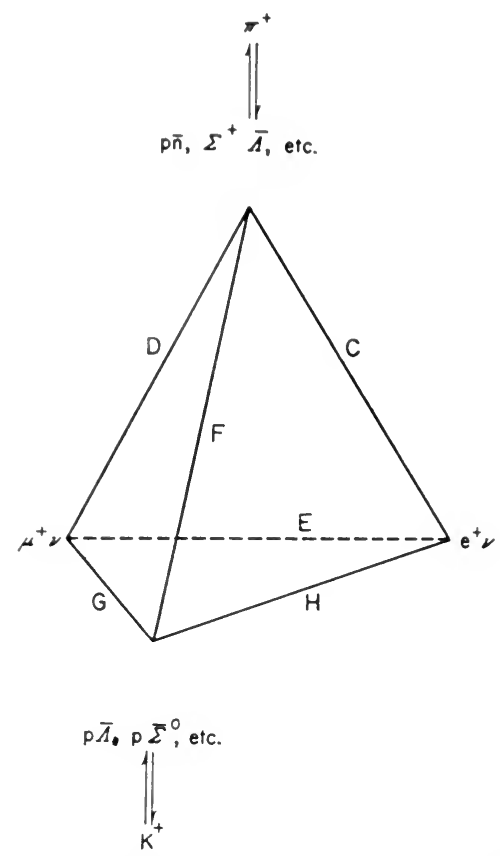

FIG. 36. (b) Tetrahedron representation of four fermion interaction of positively charged pairs.

account for all the various decay modes of hyperons, $K$-mesons and pions. For example, the pionic decay modes of $K$-mesons are described by the side $F$ of the tetrahedron. In this picture we can have only the following decays of neutral $K$-mesons, i.e.

$$
\begin{aligned}
& \bar{K}^{0} \rightarrow e^{-}+\bar{v}+\pi^{+} \text {or } \mu^{-}+\bar{v}+\pi^{+} \\
& K^{0} \rightarrow e^{+}+\nu+\pi^{-} \text {or } \mu^{+}+\nu+\pi^{-} .
\end{aligned}
$$

The consequences of this in the decays of $K_{1}^{0}$ and $K_{2}^{0}$ have been discussed earlier (see p. 224). The tetrahedron scheme also prohibits the processes $\mu^{ \pm} \rightarrow e^{ \pm}-e^{ \pm}-e^{\mp} ; \mu^{+}\left(\mu^{-}\right) \rightarrow e^{+}\left(e^{-}\right)+\nu(\bar{v})$ which are known to be vary rare or absent. If we wish to include a Fermi interaction between pairs of particles of total strangeness -1 such as $\left(\Sigma^{+}, \bar{r}\right)$ etc. a fifth vertex can be 
introduced to account for the leptonic decay modes of hyperons as has been pointed out by Gell-Mann. ${ }^{1}$

We have seen that the "two-component" neutrino theory was quite succesful in explaining the violation of parity and charge conjugation invariance in decays involving neutrinos and we know that a two-component theory is equivalent to multiplying the neutrino spinor by $\left(1 \pm \gamma_{5}\right)$. The violation of $C$ and $P$ invariance in the decay processes not involving neutrinos can also be explained provided we can convince ourselves that we should insert $\left(1 \pm \gamma_{5}\right)$ before the proper fields. However we recall that the zero mass of the neutrino played an important role in obtaining the factor $\left(1 \pm \gamma_{5}\right)$ and arguments applicable to the neutrino cannot be used in the case of particles with mass. Recently Marshak and Sudarshan $^{2}$ and independently Feynman and Gell-Mann ${ }^{3}$ have advanced arguments for attaching the factor $\left(1 \pm \gamma_{5}\right)$ to all the fermion fields in the quadrilinear Fermi interaction. The arguments are apparently different but are actually equivalent and we here present the theory of Feynman and Gell-Mann ${ }^{3}$ in some detail. When the Dirac equation for a particle of mass $m$ is written as

$$
(i \nabla-m) \psi=0
$$

it is of the first degree with $\psi$ having four components. However the KleinGordon equation describing a spin zero particle is of the second degree and relates to a one component wave function. Thus it should be possible to obtain a "two component" spinor equation for particles with spin $1 / 2$ by raising the degree of the equation. To arrive at this let us define a $\chi$ with four components by

$$
\psi=\frac{1}{m}(i \nabla+m) \chi
$$

$\chi$ satisfies the second degree equation

$$
(i \nabla)^{2} \chi=m^{2} \chi
$$

It is important to note that any solution of the Dirac equation will be a solution of the second degree equation while any solution of the second degree equation need not be a solution of the Dirac equation. While $\gamma_{5}{ }^{4}$ does not commute with $i \Delta$, it does so with $(i \nabla)^{2}$. Hence we can find $\chi$ 's which satisfy the second degree equation as well as the relation $\gamma_{5} \chi= \pm \chi$. This is easily done, for

$$
\begin{gathered}
\frac{1}{2}\left(1+\gamma_{5}\right) \psi=\chi ; \frac{1}{2}\left(1-\gamma_{5}\right) \psi=\chi^{\prime} . \\
\gamma_{5} \chi=\chi ; \gamma_{5} \chi^{\prime}=-\chi^{\prime} .
\end{gathered}
$$

'M. Gell-Mann and A. H. Rosenfeld, loc. cit.

2 E. C. G. Sudarshan and R. E. Marshak, Phys. Rev. 109, 1860 (1958).

3 R. P. Fexnman and M. Gell-Mann, Phys. Rev. 109, 193 (1953); also J. J. Sakurai, Nuovo Cim. 7, 649 (1958).

4 The $\gamma_{5}$ used in this section corresponds to the $i \gamma_{5}$ used in the earlier part of the book. 
If we write $\psi^{\prime}=\left(\begin{array}{l}a \\ b\end{array}\right)$ where $a$ and $b$ are two-component spinors, from the usual representation of Dirac matrices we find

and

$$
\frac{1}{2}\left(1+\gamma_{5}\right)\left(\begin{array}{l}
a \\
b
\end{array}\right)=\left(\begin{array}{l}
a+b \\
a+b
\end{array}\right)=\left(\begin{array}{l}
\varphi \\
\varphi
\end{array}\right)=\chi .
$$

$$
\frac{1}{2}\left(1-\gamma_{5}\right)\left(\begin{array}{l}
a \\
b
\end{array}\right)=\left(\begin{array}{l}
a-b \\
b-a
\end{array}\right)=\left[\begin{array}{r}
\Phi \\
-\Phi
\end{array}\right]=\chi^{\prime}
$$

We thus notice that $\chi$ and $\chi^{\prime}$ are made up of two identical two-component spinors and from the equation obeyed by $\chi$ it is clear that both $\varphi$ and $\Phi$ obey the same equation. ${ }^{1}$ Feynman and Gell-Mann ${ }^{2}$ argued that there was no particular reason why we should couple the solutions of the first order Dirac equation and not those of the second order. Taking $\varphi$ to be fundamental it was postulated that the fermion fields $\psi$ should be replaced by $\Phi=\frac{1}{2}\left(1+\gamma_{5}\right) \psi$, the solution of the second order equation. This implies that we replace $\psi$ by $\frac{1}{2}\left(1+\gamma_{5}\right) \psi$ and $\overline{4}$ by $\overline{4}, \frac{\left(1-\gamma_{5}\right)}{2}$. Using the properties of the Dirac matrices, we find that

$$
\begin{aligned}
\left(1-\gamma_{5}\right) 0_{i}\left(1+\gamma_{5}\right) & =0 \text { for } i=S, T, P . \\
= & 20_{i}\left(1+\gamma_{5}\right) \text { for } i=A, V .
\end{aligned}
$$

Thus the $\beta$ decay interaction takes the form

$$
\frac{1}{2} G\left(\bar{\psi}_{n} \gamma_{\mu} a \psi_{p}\right)\left(\bar{\psi}_{e} \gamma_{\mu} a \psi_{\nu}\right)
$$

where $a=\left(1+\gamma_{5}\right)$ and $G$ is a single coupling constant for both $V$ and $A$ and the coupling is $V-A .^{3}$ From the recent experiments on the asymmetry of decay electrons in the $\pi-\mu-e$ decay chain, we have already concluded that the above interaction should be present in $\mu$ decay. We have also shown that this coupling will predict the correct handedness of the particles in processes like the $\beta$ decay and $K$ capture.

For example, this theory predicts that the neutrino is always left-handed, which was confirmed by Goldhaber's ${ }^{4}$ experiment.

In contrast to the two-component theory of the neutrino, which can explain the $C$ and $P$ violation only in processes involving neutrinos, the above theory can explain the violation of $C$ and $P$ in any weak decay. Already in the tetrahedron scheme we have indicated how all the weak processes can be thought of as arising from four-fermion interactions. For this we have to envisage new

1 For electrodynamics in the "two-component" form, see L. M. Brown, Phys. Rev. 111, 957 (1958). Also G. Marx, Nuclear Physics 9, 337 (1958).

2 R. P. Feynman and M. Gell-Mans, loc. cit.

${ }^{3}$ If we were to use right-handed neutrino, i.e. $\left(1-\gamma_{5}\right) \psi_{\nu}$ we would have obtained $V+A$ coupling which does not agree with experiment.

+ M. Goldhaber, L. W. Grodzixs and A. W. Sunyar. loc. cit. 
couplings like $\left(\bar{\Lambda}^{0} p\right)(\bar{v} \mu),\left(\bar{\Lambda}^{0} p\right)(\bar{v} e),\left(\bar{\Lambda}^{0} p\right)(p \bar{n})$ as in the tetrahedron scheme. The parity violation in the pion decay mode of $K^{+}$can be envisaged as follows:

$$
\begin{gathered}
K^{+} \rightarrow \bar{\Lambda}^{0}+p \text { (strong virtual interaction) } \\
\bar{\Lambda}^{0}+p \rightarrow p+\bar{n} \rightarrow(2 \pi \text { or } 3 \pi) \text { (UFI). }
\end{gathered}
$$

In the second step of the reaction violation of parity and charge conjugation invariance takes place.

It is remarkable that the vector coupling constant obtained from the decay of $\mathrm{O}^{14}$ (pure Fermi $\beta$ decay) should give the lifetime of $\mu$ decay correctly. While $\mu$ decay involves only leptons, the nucleon can emit and absorb virtual pions. It is rather puzzling that the vector part of the weak decay coupling constant is not affected by the strong virtual pion interaction. In other words the decay constant need not be renormalized. We refer to a similar feature in electrodynamics, namely, that the charge of a particle, i.e. the coupling constant to electromagnetic field is the same for all the particles. This feature is essentially a consequence of the conservation law for electric charge and current. Feynman and Gell-Mann ${ }^{1}$ have shown how the vector part of the Fermi coupling can be made to have no renormalization.

The field theoretic Lagrangian for the four Fermion processes would include the six terms containing pairs selected from the four bilinears terms $(\bar{\mu} v),(\bar{e} v),(\bar{p} n)$ and $(\bar{p} \Lambda)$ and their hermitian conjugates. (We could of course add to these all the equivalent meson or baryon currents.) If to these twelve terms we add the four terms representing the coupling of each bilinear term to itself then the interaction can be written as $J_{w}^{\dagger} J_{w}$ where

$$
J_{w}=(\bar{v} e)+(\bar{\mu} v)+(\bar{p} n)+(\dot{\bar{p}} \Lambda) .
$$

An interaction of this type is called a current-current interaction, the total current comprising the sum of the individual currents. Such a Lagrangian is possible only if it is permissible to include the self coupling terms. The consequences of inclusion of such squared terms are:

(1) Neutrino-electron scattering arising from $(\bar{v} e)^{\dagger}(\bar{v} e)$. This scattering process can occur even in the absence of the squared term as a second order effect in the weak interaction which however would be far weaker than the interaction due to direct coupling.

(2) Pair production of neutrinos by an electron pair or $\gamma$-rays. The matrix element for the first process vanishes in any local theory ${ }^{2}$. The second process could happen in stars and supernovae. But any process involving neutrinos leads to the loss of neutrino flux and hence of energy and does not lead to equilibrium.

1 R. P. Feynman and M. Gell-Mann, Phys. Rev. 109, 193 (1958); S. S. Gershtein and J. B. Zer'Dovich, J. Expt. Theoret. Phys. \&, 576 (1957). These authors suggested this idea before non-conservation of parity was postulated.

${ }^{2}$ M. Gell-ManN, Phys. Rev. Lett. 6, 70 (1961). 
The eross-sections for these processes are just small enough to leave the present theories of supernovae unaffected.

(3) A short range parity violating nuclear force arisin gout of $(\bar{p} n)^{\dagger}(\bar{p} n)$. The force due to this local coupling will be spread out due to virtual strong interactions.

What we wish to show now is the possibility of not renormalizing the total current, i.e. in the total current at least the veetor part of $J_{v}$ is unaffected by the presence of other interactions. As we have noted above, the currents involving only leptons will not be affected by pion interaetions. However considering the nucleon eurrent and splitting it into vector and axial vector parts, we have

where

$$
J_{N}=\frac{1}{2}\left(J_{N}^{V}+J_{N}^{A}\right)
$$

$$
J_{N}^{V}=\bar{\psi}_{p} \gamma_{\mu} \psi_{n} ; \quad J_{N}^{A}=\bar{\psi}_{p} \gamma_{\mu} \gamma_{5} \psi_{n} .
$$

Using isotopic spin formalism the vector part can be written as

$$
J_{N}^{V}=\frac{1}{2} \bar{\psi} \gamma_{\mu} \tau_{+} \psi
$$

The electric current is given by

$$
J_{N}^{\mathrm{el}}=\bar{\psi} \gamma_{\mu} \frac{\left(1+\tau_{z}\right)}{2} \psi
$$

The isovector part of the electric current is

$$
J_{N}^{\mathrm{el}, V}=\frac{1}{2} \bar{\psi} \gamma_{\mu} \tau_{z} \psi .
$$

Comparing (20) and (22) we see that they can be obtained by different projections of the same isotopic vector. Thus we can expect that the matrix elements of vector part of the electromagnetic and weak interactions should be related; especially since the strong interactions are isotopically invariant, the vector parts of these two interactions should have similar properties. ${ }^{1}$ The term $\frac{1}{2} \bar{\psi} \gamma_{\mu}\left(1+\tau_{z}\right) \psi$ is not conserved unless we add the electrie eurrent of pions and strange particle. In analogy we can argue now that $J_{N}^{V}$ will not be conserved unless we add the pion and strange particle contributions to it. Thus the conserved $J_{N}^{V}$ is

$$
J_{N}^{V}=\bar{\psi} \gamma_{\mu} \varrho_{+} \psi+i\left[\varphi^{*} \varrho_{+} \nabla_{\mu} \varphi-\left(\nabla_{\mu} \varphi^{*}\right) \varrho_{+} \varphi\right]+\cdots
$$

If we substitute this current for the vector part in (17) the veetor coupling constant will not be affected. The newly added terms prediet the transition $\pi^{ \pm} \rightarrow \pi^{0}+v+e^{ \pm}$, a process of low probability. These terms will predict the processes

$K^{0} \rightarrow K^{+}+e^{-}+\nu, \bar{K}_{0} \rightarrow K^{-}+e^{+}+\nu, \Sigma^{-} \rightarrow \Sigma^{0}+e^{-}+\nu$ and $\Xi^{-} \rightarrow \Xi^{0}+e^{-}+\nu$.

1 It is interesting to note that the conservation of vector part of the weak interaction current is automatic in Sakata's model which considers only $p, n$, and $\Lambda^{0}$ as elementary and all the other particles as composite. See "Strange Particle Decays" by L. B. Okun, Ann. Rev. Nucl. Sci. 9, 61 (1959). 
While the first two processes depend only on the vector coupling constants, the remaining two processes can also proceed through axial vector current, the reason being that it is impossible to construct axial vector currents bilinear in meson fields of the same parity and zero spin.

Pursuing the analogy of the vector part of the weak interaction with the electromagnetic interaction Gell-Mann ${ }^{1}$ noticed an important correction to the nuclear matrix element in $\beta$ decay which he called "weak magnetism" corresponding to the magnetic form factor occurring in the nuclear matrix element for electromagnetic interaction. The magnitude of this "weak magnetism" can be precisely estimated if we accept the conserved vector current hypothesis. Then the weak magnetism form factor is equal to the moment form factor of Hofstadter et al. ${ }^{2}$ The effect of weak magnetism can be best estimated if we have $\gamma$ and $\beta$ decay from two initial nuclear states both of them belonging to the same isomultiplet. Such an experiment has been proposed by Gell-Mann utilizing the $\beta^{-}$, $\gamma$ and $\beta^{+}$decay of $\mathrm{B}^{12}, \mathrm{C}^{12 *}$ and $\mathrm{N}^{12}$ respectively which form an isomultiplet. The effect of weak magnetism is considerable in $\mu^{-}$capture $^{3}$

Regarding the axial vector part of $J_{N}$ it is not clear whether there should be any renormalization or not. The present evidence from the decay of free neutrons indicates that the ratio of Gamow-Teller to Fermi coupling constants is $1.19: 0.4{ }^{4}$ It is hoped that departure from unity is due to renormalization. Conservation of axial vector current is not desirable as it forbids $\pi-\mu$ and $\pi-e$ decays. Further in contrast to the case of vector current such a hypothesis does not lead to equality of axial vector coupling constants, i.e. absence of renormalization effects. ${ }^{5}$ It has been noted by Goldberger and Treiman ${ }^{6}$ that this hypothesis gives a larger effective pseudoscalar interaction in $\beta$ decay. The situation concerning strangeness non-conserving current like $\left({\overline{\Lambda^{0}}}^{0} p\right)$ is more complicated. As for the strangeness-conserving current the axial part of this current should not be conserved as it will forbid $K_{\mu 2}$ decay (we have assumed that $K^{+}$is pseudoscalar). Since $\Lambda^{0}$ and $p$ belong to different isomultiplets conservation of the vector part of this current will not lead to the absence of renormalization effects for coupling constants unless "global symmetry" is assumed.

When the Universal Fermi Interaction with $V-A$ coupling was proposed it was faced with what seemed an inexplicable fact that the experimental ratio of the two decay modes of pion $\frac{\pi^{-} \rightarrow e^{-}+v}{\pi^{-} \rightarrow \mu^{-}+v}$ is $<10^{-5}$ while the UFI predicted

1 M. Gell-Mann, Phys. Rev. 111, 362 (1958).

2 R. Hofstadter et al., Phys. Rev. Lett. 6, 290, 293 (1960).

3 Unlike in $\beta$ decay, the momentum transfer is large in this case and hence the effect of weak magnetism will be large. See S. Weinberg, Phys. Rev. Lett. 2. 223 (1959).

${ }^{4}$ M. T. Burgy et al., Phys. Rev. 110, 1214 (1958).

5 For a more complete discussion of this question, see Problems in Theory of Weak Interactions R. Gatto, Fortschritte der Physik, X, 147-181 (1959); see also R. J. Buin-Stoyle, Nuovo Cim. 10, 132 (1958). See also A. P. Balachandran, Nuovo Cim. In press.

6 M. L. Goldberger and S. B. Treiman, Phys. Rev. 110, 1478 (1958). 
a ratio $-10^{-4}$. However recent experiments ${ }^{2}$ have borne out the ratio $\sim 10^{-4}$. The experiment is based on the fact that the electron emitted in the direct $\tau \rightarrow e+v$ decay has a sharp energy $\sim 72 \mathrm{MeV}$ while the electrons emitted in $\tau-\mu-e$ decay chain has a spectrum and the maximum energy is only $52 \mathrm{MeV}$.

According to UFI the pion undergoes a strong virtual interaction and dissociates into any baryon-anti-baryon pair consistent with charge independence and strangeness conservation such as $(p \bar{n}),\left(\Sigma^{+}, \bar{\Lambda}\right)$ etc. the annihilation of the anti-baryon-baryon pair resulting in the production of $\mu, v$ or $e, v$ through UFI.

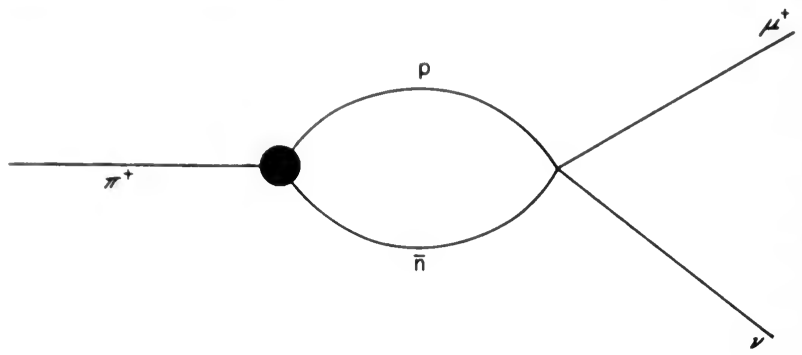

FI:. 37. $\pi-\mu$ decay.

'The black box denotes all possible strong interactions. The pseudoscalar nature of the pion demands that these currents in UFI can only be axial vector. Treating the Yukawa process in the lowest order, Finkelstein and Ruderman ${ }^{3}$ showed that this ratio is $-1.3 \times 10^{-4}$. It is important to notice that in the $\pi-e$ decay mode both the electron and neutrino are relativistic while the $\mu$-meson is not so. Hence the difference in the processes. A similar difficulty existed with the radiative decay of the pion, i.e.

$$
\pi \rightarrow e+\nu+\gamma \text { and } \pi \rightarrow \mu+\nu+\gamma .
$$

Treiman and $W_{y} d^{4}$ have discussed the process under various couplings and find that it has a larger cross-section for tensor coupling. Expressing the ratio of the radiative to the ordinary decay mode by $\varrho^{\prime}$, Cassels ${ }^{5}$ has found that the ordinary pion decay goes through the axial vector coupling. The ratio is much higher for tensor coupling. In the decay of the pion six possible baryon-anti-baryon pairs can occur in the intermediate states and may have interference effects which cannot be estimated with present computational techniques. Attempts have recently been made to apply the theory of dispersion relations to $\pi^{ \pm}$and $\pi^{0}$ decay. Retaining nucleon pair alone in the intermediate state Goldberger and

1 See for a thorough discussion on this difficulty "Forbidden $\pi-\beta$ decay" by R. P. FEYNMAx. International Conference on High Energy Physics at CERN (1958) p. 216, Ed. B. FereTtr.

2 Fazzini et al., Phys. Rev. Lett. 1, 247 (1958); Impeduglia et al., Phys. Rev. Lett. 1, 249 (1958): H. L. Anderson et al., Phys. Rev. Lett. 2, 53 (1959).

${ }^{3}$ M. Ruderian and R. Finkelstein, Phys. Rev. 76, 1458 (1949).

4 S. B. Treiman and H. W. Wyld Jr., Phys. Rev. 106, 1320 (1957).

5 J. M. Cassels et al., Proc. Phys. Soc. A 70, 729 (1957). 
Treiman ${ }^{1}$ have obtained surprisingly good agreement with experimental decay rate for $\pi-\mu$ decay.

The theory of weak interactions developed above is based on the currentcurrent interaction, i.e. $J_{W}^{\dagger} J_{W}$. The current $J_{W}$ consists of these terms

(1) a leptonic current $j_{l}$ consisting of terms $\left(\mu^{-} \bar{v}\right),(e-\bar{v})$

(2) a strangeness-conserving current $j_{s c}$ involving terms like $(\bar{n} p),(\vec{\Lambda} \Lambda)$

(3) a strangeness non-conserving current $j_{s n c}$ containing terms like $\left(\bar{p} \Lambda^{0}\right),\left(\bar{p} \Sigma^{0}\right)$, $\left(\Sigma^{+} \bar{n}\right),\left(\Sigma^{+} \bar{\Xi}^{0}\right)$.

$\mu$ decay is a pure leptonic decay and arises from the coupling of $j_{l}$ with itself. $j_{l}^{\dagger} j_{l}$ will also describe electron-neutrino scattering. $\mu$ capture, $\pi$ decay and $\beta$ decays are described by the various terms arising from $j_{s c}^{\dagger} j_{l}$. Leptonic decays of strange particles are due to the coupling $j_{s n c}^{\dagger} j_{l}$ while the non-leptonic decays of strange particles arise from the interaction term $j_{s n c}^{\dagger} j_{s c}$. An important property of all the terms in the three currents are that they are charged and no term is composed of oppositely charged particles. The absence of neutral currents has not hitherto been explained. ${ }^{2}$ The presence of neutral currents will induce processes like $\mu^{+} \rightarrow e^{+}+e^{-}+e^{+}$which seem to be rare or absent.

An interesting rule has been proposed by Feynman and Gell-Mann ${ }^{3}$ for the terms in $j_{s n c}$ which states that the terms should obey $\frac{\Delta Q}{\Delta S}=+1$. This rule forbids terms like $\left(\bar{\Sigma}^{+}, n\right)$ in which $\frac{\Delta Q}{\Delta S}=-1$ and $\left(\bar{p} \Xi^{0}\right)$ which has $\frac{\Delta Q}{\Delta S}=+1 / 2$ and also involves $\Delta S=+2$. The presence of such terms can be tested as follows. The coupling of $\left(\bar{\Sigma}^{+}, n\right)$ with $\left(e^{-}, v\right)$ will lead to an appreciable $\Sigma^{+} \rightarrow n+e^{+}+v$ which has not been observed so far. ${ }^{4}$ The coupling of $\left(\bar{\Sigma}^{+}, n\right)$ with $\left(\bar{p} \Lambda^{0}\right)$ will lead to weak interactions having $\Delta S= \pm 2$. The terms like $\left(\bar{p} \Xi^{0}\right)$ will induce the decay $\Xi^{0} \rightarrow p+\pi^{-}(\Delta S=+2)$ instead of the observed two-step decay. As Okun and Pontecorvo ${ }^{5,6}$ have pointed out the presence of such weak interactions will lead to a more frequent oscillation $\left(\sim 10^{-16} \mathrm{sec}\right)$ between $K^{0}$ and $\bar{K}^{0}$ instead of the normal oscillation $\left(\sim 10^{-10} \mathrm{sec}\right)$ and the transition $K^{0}-\bar{K}^{0}$ will be proportional to $G$ instead of $G^{2}$ as it would be in the absence of these interactions. The present experimental evidence does not favour rapid oscillation of $K^{0}$ and $\bar{K}^{0}$.

1 M. L. Goldberger and S. B. Treiman, Phys. Rev. 110, 1216, 1478 (1958).

2 See G. Gamba and R. E. Marshak, Proc. Nat. Acad. Sci., 45, 881 (1959). In this paper, the authors suggest an interesting symmetry between $\Lambda-n-p$ and $\mu-e-v$.

3 R. P. Feymman and M. Gell-ManN, loc. cit.

4 J. Leitner et al., Phys. Rev. Lett. 3, 186 (1959).

5 L. B. OKun' and B. Ponteconvo, J. Expt. Theoret. Phys. 5, 1297 (1957).

${ }^{6}$ It is usually argued that since the mass difference between $K_{1}^{0}$ and $K_{2}^{0}$ is small, it can arise only due to a second order weak interaction so that first order $\Delta S=2$ non-leptonic decays should be forbidden. It can however be shown (see S. L. Glashow, Phys. Rev. Lett. 6, 196 (1961)) that even if $\Delta S=2$ decays are allowed only that part of the transition matrix element which is even under charge conjugation contributes to $\delta m$, so that it is only required that part of the interaction Lagrangian even under $C$ be no more than $10^{-5}$ times as strong as the usual $\Delta S=1$ interaction $\delta m$ is the $K_{1}^{0} K_{2}^{0}$ mass difference. 
Earlier we have discussed the $|\boldsymbol{I}|=1 / 2$ rule for strange particle decays. Sudarshan et al. ${ }^{1}$ have attempted to explain this rule by postulating that the $j_{s n c}$ transforms like an isospinor. This transformation property will give for the leptonic decay of strange particles the rule $|\Delta \boldsymbol{I}|=1 / 2$ for the strangeness non-conserving part. A very important prediction of these rules is that the energy spectra of secondary particles, angular distribution and polarization in $K_{\mu 3}$ decays of $K^{+}\left(K^{-}\right)$and $K^{0}\left(\vec{K}^{0}\right)$ mesons should be similar and the total decay rates should be in the ratio $1: 2$. We also have the result that three-body leptonic decay modes of $K_{1}^{0}$ and $K_{2}^{0}$ should have equal probabilities. This implies for the decay rates $\omega$ the relations

$$
\omega\left(K_{1}^{0} \rightarrow \pi^{-}+l^{+}+\nu\right)=\omega\left(K_{2}^{0} \rightarrow \pi^{-}+l^{+}+\nu\right)=\omega\left(K^{+} \rightarrow \pi^{0}+l^{+}+\nu\right)
$$

where $l$ refers to $\mu$ or $e$. Estimates of the lifetime of $K_{2}^{0}$ from the knowledge of $K_{\mu 3}^{+}$and $K_{e 3}^{+}$and $K_{1}^{0}$ decays are in general agreement with present experimental value. Howerer the rule $\frac{\Delta Q}{\Delta S}=+1$ does not yield the $2: 1$ ratio of charged to neutral decays of $K$ which is predicted by $|\boldsymbol{I} \boldsymbol{I}|=1 / 2$ rule when applied to the whole process. Further this rule in the lowest approximation prohibits $\Sigma^{+}$decays while allowing $\Sigma$ - decay. Recent experimental evidence seems to indicate that the probabilities of the three modes are equal and the decays of $\Sigma$ are well described by the $|\Delta I|=1 / 2$ rule. $^{2}$ Since $\Lambda^{0}$ decay seems to obey the rule $|\Delta I|=1 / 2$ in the lowest approximation it is difficult to reconcile $\frac{\Delta Q}{\Delta S}=1$ with the non-leptonic $\Sigma$ decays. It is interesting to note that to obtain $|\Delta I|=1 / 2$ for $\Lambda^{0}$ decay we are forced to include neutral currents like $\bar{n} n$. The observation of $\Sigma^{+} \rightarrow n+e^{+}\left(\mu^{+}\right)+\nu$ will invalidate the rule $\frac{\Delta Q}{\Delta S}=+1$. Only increasing experimental evidence on polarization properties in various non-leptonic decay of strange particles can throw light on these conflicting issues.

Another problem which remains to be solved concerns the $\beta$ decay of hyperons, i.e.

$$
\begin{aligned}
& \Lambda^{0} \rightarrow\left\{\begin{array}{l}
p+e^{-}+\bar{v} \\
p+\mu^{-}+\bar{v}
\end{array}\right. \\
& \Sigma^{-} \rightarrow\left\{\begin{array}{l}
n+e^{-}+\bar{v} \\
n+\mu^{-}+\bar{v} .
\end{array}\right.
\end{aligned}
$$

Assuming renormalization effects to be small we expect that the fractions of the $\beta$ decay of $A$ and $\Sigma^{-}$should be 1.6 and 5.6 per cent respectively. However the present experimental evidence seems to indicate these decays are much less

1 E. C. G. Sudarshax et al., Phys. Rev. 112, 665 (1958). This paper also contains references to their earlier work on the subject.

2 Cool et al., loc. cit. 
frequent and if we take this seriously we are forced to conclude that the $j_{s n c}$ is weaker than the $j_{s c}$ by a factor of $13 .^{1}$

Finally we shall take up the problem of why all the terms in weak interactions should be charged. In analogy with the electromagnetic interaction we can assume that weak interactions are not primary but are caused by an exchange of a charged vector boson $B$ between the two currents. ${ }^{2}$ This will at once explain the absence of neutral currents. Following this argument we conclude that the mass of the boson must be greater than the mass of $K$-meson in order to prevent the latter from undergoing decay through the boson, i.e. $m_{B}>m_{K}$. The lifetime of $B$ is estimated to be $10^{-17} \mathrm{sec}$ and the direct observation of $m_{B}$ is rather difficult. ${ }^{3}$ However if $B$ exists it causes the decay $\mu \rightarrow e+\gamma \cdot{ }^{4}$ It is found that the estimated branching ratio is greater than the experimental value $\sim 2 \times 10^{-5}$. Thus we can conclude that $B$ does not exist. ${ }^{5}$

\section{SOME RECENT WORKON THE THEORY OF WEAK INTERACTIONS}

In our previous discussion, we have referred to a few problems which are yet to be understood theoretically. The following three problems have been explained to some extent by recent work:

(a) The life time of $\pi-\mu$ decay and the surprising validity of the GoldbergerTreiman formula ${ }^{6}$

(b) The up-down asymmetries of $\Lambda$ and $\Sigma$ hyperon decays which suggest a need for an extension of $\gamma_{5}$ invariance in a manner such that left-handed fields occur in $\Lambda$ decay while both right- and left-handed fields occur in $\Sigma$ decays; ${ }^{7}$

(c) The $|\Delta \boldsymbol{I}|=1 / 2$ rule and the need for the inclusion of neutral currents.

We shall summarize here Gürsey's attempts in devising a theory to explain such problems ${ }^{8}$ and make references to similar works of others. Gürsey has

1 R. H. Dalitz, Rev. Mod. Phys. 31, 823 (1959). This volume also contains interesting papers on weak interactions presented at Gatlinburg Conference, 1958.

2 R. P. Fexnman and M. Gell-ManN, loc. cit.

${ }^{3}$ If the intermediate vector boson $B$ has a mass roughly equal to that of $K$, the crosssection for the process

$$
\bar{v}+e^{-} \rightarrow B \rightarrow \bar{v}+\mu^{-}
$$

will show a resonance at $2.5 \times 10^{11} \mathrm{eV}$. The cross-section at resonance is independent of the strength of the interaction and the width is related to the lifetime of $B$ (see T. Kinoshita, Phys. Rev. Lett. 4, 378 (1960)).

4 G. Feinberg, Phys. Rev. 110, 1482 (1958).

5 H. F. Davis et al., Phys. Rev. Lett. 2, 211 (1959); D. Berlyet et al., Phys. Rev. Lett. 2, 357 (1959).

6 M. L. Goldberger and S. B. Treiman, Phys. Rev. 110, 1478 (1958).

7 S. A. Bludman, "Phenomenological analysis of hyperon decay rates and asymmetries", Gatlinburg Conference on Weak Interactions (October, 1958); Bull. Amer. Phys. Soc. 9, 83 (1959); also Phys Rev. 115, 468 (1959).

8 F. Gürsex, Nuovo Cim. 16, 230 (1960); also "On the Structure and Parity of Weak Interaction Currents", preprint, Institute for Advanced Study (1960). 
considered the possibility of a four-dimensional extension $G_{4}$ of the isotopic spin group $G_{3}$, such that $G_{4}$ together with the hypereharge gauge group contains charge independence, strangeness seleetion rules and the empirical symmetries of weak interactions as various subgroups. 'The model treated by him should be regarded as an illustration of the possibility that the symmetries of weak interactions may be essentially contained in those of strong interactions and not as a definite proposal for a theory of elementary particles.

The strong interaction Lagrangian is taken to consist of two parts $L$ and $L^{\prime}$ where $L$ is rigorously invariant under $G_{4}$ and $L^{\prime}$ is invariant only under $G_{3}$ and acts as a perturbation on $L . G_{4}$ invariance implies a doublet approximation $(D A)$ for baryons and $I^{\prime}$ acts as a doublet perturbation $(D P)$. If $G_{4}$ is approximately valid for strong interactions, one may hope that renormalization effects will be small for any interaction invariant under a subgroup of $G_{4}$ and especially for weak interactions. To define $G_{4}$ we first take a strongly interacting system like pions and nucleons. Consider the following transformation of the $\pi-N$ system:

$$
G_{3}^{\prime}: \psi \rightarrow \mathrm{e}^{i \gamma_{s} \tau \cdot \omega} \psi
$$

This does not leave the nucleon equation invariant unless $m=0$. The transformed equation reads:

$$
i \gamma_{\mu} \partial_{\mu} \psi=m \mathrm{e}^{2 i \gamma_{\varepsilon} \boldsymbol{r} \cdot \boldsymbol{a}} \psi
$$

with $\omega-\boldsymbol{a}$ and $\partial_{\mu}=\frac{\partial}{\partial x^{\mu}}$. Following Nishijima ${ }^{1}$, let us identify $\boldsymbol{a}$ with the pion field $\varphi$ :

$$
\boldsymbol{a}=f \boldsymbol{\varphi}=\frac{g \boldsymbol{\varphi}}{2 m}
$$

where $f$ and $g$ are the pseudovector and pseudoscalar coupling constants. We then obtain

$$
i \gamma_{\mu} \partial_{\mu} \psi=m \mathrm{e}^{2 i f \gamma_{5} \tau \cdot \varphi} \psi=m \psi+i g \gamma_{5} \tau \cdot \varphi \psi-\frac{g^{2} \varphi^{2}}{2 m} \psi+\cdots
$$

(2) may be written as

where $^{2}$

$$
\begin{aligned}
& i \gamma_{\mu} \partial_{\mu} \psi_{L}=m \Phi \psi_{R} \\
& i \gamma_{\mu} \partial_{\mu} \psi_{R}=m \Phi \psi_{L}
\end{aligned}
$$

$$
\begin{array}{cc}
\Phi=\mathrm{e}^{2 i f \tau \cdot \varphi}, & \bar{\Phi}=\mathrm{e}^{-2 i f \boldsymbol{\tau} \cdot \boldsymbol{\varphi}} \\
\psi_{L}=\frac{1}{2}\left(1+\gamma_{5}\right) \psi, & \psi_{R}=\frac{1}{2}\left(1-\gamma_{5}\right) \psi
\end{array}
$$

and

$$
\Phi \bar{\Phi}=\bar{\Phi} \Phi=1 \text {. }
$$

Now we may define two three-dimensional commuting rotation groups $G_{3}^{(1)}$ and $G_{3}^{(2)}$ operating on left- and right-handed nueleons independently. Adjoining $G_{3}^{(1)}$ to $G_{3}^{(2)}$, we obtain the four-dimensional rotation group $G_{4}$.

$$
\begin{array}{lll}
G_{3}^{(1)}: \psi_{L} \rightarrow \mathrm{e}^{i \tau \cdot \mu} \psi_{L}, & \Phi \rightarrow \Phi \mathrm{e}^{-i \tau \cdot \mu}, & \psi_{R} \rightarrow \psi_{R} \\
G_{3}^{(?)}: \psi_{R} \rightarrow \mathrm{e}^{i \tau \cdot \nu} \psi_{R}, & \Phi \rightarrow \mathrm{e}^{i \tau \cdot \nu} \Phi & \psi_{L} \rightarrow \psi_{L}
\end{array}
$$

${ }^{1}$ K. Nishijima, Nuovo Cim. 11, 910 (1959).

2 The $\gamma_{5}$ here is equal to $i \gamma_{5}$ of chapter $\mathrm{I}$. 
with

$$
\begin{aligned}
G_{4}: \psi & \rightarrow \exp \left[i\left\{\frac{1+\gamma_{5}}{2} \tau \cdot \mu+\frac{1-\gamma_{5}}{2} \tau \cdot v\right\}\right] \psi \\
\Phi & \rightarrow \mathrm{e}^{i \tau \cdot \nu} \Phi \mathrm{e}^{-i \tau \cdot \mu}
\end{aligned}
$$

The isotopic spin group $G_{3}$ obtained by taking $\boldsymbol{\mu}=\boldsymbol{v}=\omega$ commutes with $C P$, $C$ and $P$ while $G_{3}^{(1)}$ and $G_{3}^{(2)}$ commute only with $C P$ and not with $C$ and $P$.

The pion kinetic energy term in the strong Lagrangian can be made invariant under $G_{4}$ by the substitution

$$
\left(\partial_{\mu} \varphi\right)^{2} \rightarrow-\frac{1}{4} f^{-2} \operatorname{Tr}\left[\left(\partial_{\mu} \Phi\right)\left(\partial_{\mu} \bar{\Phi}\right)\right]^{2}
$$

The first term in the expansion in powers of $f$ is the usual pion kinetic energy while the additional terms represent pion-pion interactions. The pion bare mass term however cannot be made invariant under $G_{4}$ and will consequently act as a $D P$.

We may now assume the following transformation properties of the other fields. For the $\Xi$ field, $\Xi_{L}$ is assumed to transform like $\psi_{R}$ and $\Xi_{R}$ like $\psi_{L}$ so that its interaction with $\varphi$ will be

$$
i \gamma_{\mu} \partial_{\mu} \psi_{\Xi}=m_{\Xi} \exp \left\{-2 i f \gamma_{5} \tau \cdot \dot{\varphi}\right\} \psi_{\Xi} .
$$

We also introduce the following matrices for the $\Lambda$ and $\Sigma$ hyperons:

$$
Y_{L}=\Lambda_{L}+i \boldsymbol{\tau} \cdot \Sigma_{L} ; \quad Y_{R}=\Lambda_{R}+i \tau \cdot \Sigma_{R}
$$

They are assumed to transform under $G_{4}$ as

$$
\begin{aligned}
& Y_{L} \rightarrow \mathrm{e}^{i \tau \cdot \mu} Y_{L} \mathrm{e}^{-i \tau \cdot \mu} \\
& Y_{R} \rightarrow \mathrm{e}^{i \tau \cdot \vartheta} Y_{R} \mathrm{e}^{-i \tau \cdot \nu}
\end{aligned}
$$

so that we have the following equations of motion invariant under $G_{4}$ :

The matrix

$$
\begin{aligned}
& i \gamma_{\mu} \partial_{\mu} Y_{L}=m_{Y} \bar{\Phi} Y_{R} \\
& i \gamma_{\mu} \partial_{\mu} Y_{R}=m_{Y} \Phi Y_{L}
\end{aligned}
$$

transforms as

$$
Y_{L}^{\prime}=\Lambda_{L}-i \boldsymbol{\tau} \cdot \Sigma_{L}
$$

$$
Y_{L}^{\prime} \rightarrow \mathrm{e}^{i \tau \cdot \nu} Y_{L}^{\prime} \mathrm{e}^{-i \tau \cdot \mu}
$$

while $Y_{R}^{\prime}=\Lambda_{R}-i \tau \cdot \Sigma_{R}$ transforms as $Y_{R}$. The $K$-meson doublet is assumed to transform like $\psi_{R}$.

The group $G_{4}$ may be enlarged to $\left(G_{4} \times H\right)$ where $H$ is the hypercharge gauge group defined by

$$
H: \psi \rightarrow \mathrm{e}^{i u} \psi, \quad \Xi \rightarrow \mathrm{e}^{-i u} \Xi, \quad K \rightarrow \mathrm{e}^{i u} K
$$

$\left(G_{4} \times H\right)$ thus admits $H, G_{3}, G_{3}^{(1)}$ and $G_{3}^{(2)}$ as subgroups. When the $D P$ is switched on, $G_{4}$ invariance is lost and the strong interactions are invariant only under $H$ and $G_{3}$. Now since charge must be conserved in all cases, we may enlarge $G_{3}^{(1)}$ and $G_{3}^{(2)}$ to 4-parameter unitary groups which also describe charge conservation. Any interaction invariant under $G_{3}^{(1)}$ or $G_{3}^{(2)}$ alone necessarily violates parity. 
Thus we can have two classes of weak interactions, Class I invariant under $G_{3}^{(1)}$ and Class II under $G_{3}^{(2)}$. First we consider the unitary subgroup $U^{(1)}$ of $\left(G_{4} \times H\right)$ obtained by taking $v_{1}=v_{2}=0, v_{3}=u$. Then under $U^{(1)}$ we have

$$
\begin{array}{rlrl}
U^{(1)}: \psi_{L} & \rightarrow \mathrm{e}^{i u} \mathrm{e}^{i \tau \cdot \mu} \psi_{L}, & & \psi_{R} \rightarrow \mathrm{e}^{i\left(1+\tau_{\mathrm{s}}\right) u} \psi_{R} \\
\Xi_{L} & \rightarrow \mathrm{e}^{-i\left(1+\tau_{\mathrm{s}}\right) u} \Xi_{L}, & \Xi_{R} \rightarrow \mathrm{e}^{-i u} \mathrm{e}^{i \tau \cdot \mu} \Xi_{R} \\
Y_{L} & \rightarrow \mathrm{e}^{i \tau \cdot \mu} Y_{L} \mathrm{e}^{-i \tau_{\mathrm{s}} u}, & Y_{R} \rightarrow \mathrm{e}^{i \tau_{\mathrm{s}} u} Y_{R} \mathrm{e}^{-i \tau_{\mathrm{s}} u} \\
\bar{\Phi} & \rightarrow \mathrm{e}^{i \tau \cdot \bar{\mu} \Phi} \mathrm{e}^{-i \tau_{\mathrm{s}} u}, & & K \rightarrow \mathrm{e}^{i u} \mathrm{e}^{i \tau_{\mathrm{s}} u} K .
\end{array}
$$

Similarly we have another unitary group assoeiated with $G_{3}^{(2)}$, namely,

$$
\begin{array}{rlrl}
U^{(2)}: \psi_{L} & \rightarrow \mathrm{e}^{i\left(1+\tau_{s}\right) u} \psi_{L}, & & \psi_{R} \rightarrow \mathrm{e}^{i u} \mathrm{e}^{i \tau \cdot v} \psi_{R} \\
\Xi_{L} & \rightarrow \mathrm{e}^{-i u} \mathrm{e}^{i \tau \cdot \nu} \Xi_{L}, & \Xi_{R} \rightarrow \mathrm{e}^{-i\left(1-\tau_{s}\right) u} \Xi_{R} \\
Y_{L} \rightarrow \mathrm{e}^{i \tau_{\mathrm{s}} u} Y_{L} \mathrm{e}^{-i \tau \cdot \nu}, & Y_{R} \rightarrow \mathrm{e}^{i \tau \cdot v} Y_{R} \mathrm{e}^{-i \tau \cdot \nu} \\
\bar{\Phi} & \rightarrow \mathrm{e}^{i \tau_{\mathrm{s}} u} \bar{\Phi} \mathrm{e}^{-i \tau \cdot \nu}, & & K \rightarrow \mathrm{e}^{i u} \mathrm{e}^{i \tau \cdot \nu} K .
\end{array}
$$

To include leptons, we may define the transformation properties of the lepton current

as

$$
l_{\mu}=(\bar{e}+\bar{\mu}) \gamma_{\mu} \nu=\frac{1}{2}(\bar{e}+\bar{\mu}) \gamma_{\mu}\left(1+\gamma_{5}\right) \nu
$$

Now we define the matrix

$$
U^{(1)}: l_{\mu} \rightarrow \mathrm{e}^{2 i u} l_{\mu} \text {. }
$$

$$
\theta=\left[\begin{array}{cc}
\bar{K}^{0} & K^{+} \\
-K^{-} & K^{0}
\end{array}\right]=K_{1}^{0}+i \boldsymbol{\tau} \mathbf{K}
$$

with

$$
U^{(1)}: \theta \rightarrow \mathrm{e}^{i \tau_{3} u} \theta \mathrm{e}^{-i \tau_{3} u} \text {. }
$$

If the decay of the $K$-meson into pions is described by Class I interactions, we have the interaction invariant under $U^{(1)}$ as

$$
L_{K}=a G f^{-3} \operatorname{Tr}\left[\left(\Phi \partial_{\mu} \bar{\Phi}\right)\left\{\partial_{\mu} \theta+K_{1}^{0}\left(\Phi \partial_{\mu} \bar{\Phi}\right)\right\}\right]+h \cdot c
$$

where $a$ is a dimensionless coupling constant and $G$ the Fermi constant. Expanding $\Phi$, we have

$$
\begin{aligned}
L_{K} \approx & 4 a G f^{-1}\left[K_{1}^{0}\left(\partial_{\mu} \varphi\right)^{2}+\frac{1}{2} f^{-1}\left(\partial_{\mu} \boldsymbol{K}\right)\left(\partial_{\mu} \varphi\right)\right. \\
& \left.-\left(\partial_{\mu} \boldsymbol{K}\right)\left(\boldsymbol{\varphi} \cdot \partial_{\mu} \varphi\right)+\frac{1}{3} f\left(\partial_{\mu} \boldsymbol{K}\right)\left(\varphi^{2} \partial_{\mu} \varphi-2 \varphi \partial_{\mu} \varphi^{2}\right)\right] .
\end{aligned}
$$

The first and fourth terms of (26) lead respectively to the two pion decay of $K_{1}^{0}$ and the three pion decay of $K_{2}^{0}$ and $K^{+}$. The $|\Delta \boldsymbol{I}|=1 / 2$ rule holds since $L_{K}$ transforms like an isotopic spinor.

The pionic decays of $\Sigma$ and $\Lambda$ may also be described by requiring invariance under $U^{(1)}$. We have

$$
\begin{gathered}
L_{Y}=a^{\prime} G f^{-2} \operatorname{Tr}\left[\left(\bar{\Phi} \partial_{\mu} \Phi\right) Y_{L}\left(\begin{array}{cc}
0 & 0 \\
\bar{p}_{L} & \bar{n}_{L}
\end{array}\right) \gamma_{\mu}\right. \\
\left.+\left(\Phi \partial_{\mu} \bar{\Phi}\right)\left\{Y_{L}^{\prime}\left(\begin{array}{cc}
\bar{n}_{L} & 0 \\
-\bar{p}_{L} & 0
\end{array}\right)+Y_{R}^{\prime}\left(\begin{array}{cc}
0 & 0 \\
\bar{p}_{R} & \bar{n}_{R}
\end{array}\right)+Y_{R}\left(\begin{array}{cc}
\bar{n}_{R} & 0 \\
-\bar{p}_{R} & 0
\end{array}\right)\right\} \gamma_{\mu}\right]+h \cdot c,
\end{gathered}
$$


where $a^{\prime}$ is a dimensionless coupling constant. Expanding $\Phi$ in powers of $f$, we have

$$
\begin{aligned}
& L_{Y} \approx 4 a^{\prime} G f^{-2}\left\{\left(\gamma / 2 \bar{p}_{L} \gamma_{\mu} \Lambda_{L} \partial_{\mu} \varphi^{+}-\bar{n}_{L} \gamma_{\mu} \Lambda_{L} \partial_{\mu} \varphi^{0}\right)\right. \\
&+\left(\bar{n}_{L} \gamma_{\mu} \Sigma_{L}^{0} \partial_{\mu} \varphi^{0}-\sqrt{2} \bar{p}_{R} \gamma_{\mu} \Sigma_{R}^{0} \partial_{\mu} \varphi^{+}\right)+\sqrt{2} \bar{p}_{R} \gamma_{\mu} \Sigma_{R}^{+} \partial_{\mu} \varphi^{0} \\
&+\left(\bar{n}_{L} \gamma_{\mu} \Sigma_{L}^{-}-\bar{n}_{R} \gamma_{\mu} \Sigma_{R}^{-}\right) \partial_{\mu} \varphi^{+}+\left(\bar{n}_{L} \gamma_{\mu} \Sigma_{L}^{+}+\bar{n}_{R} \gamma_{\mu} \Sigma_{R}^{+}\right) \partial_{\mu} \varphi^{-}+h \cdot c .
\end{aligned}
$$

The $|\Delta I|=\frac{1}{2}$ rule is again seen to hold good. Also this gives the $V-A$ interaction for $\Lambda$ decay and for the $\pi^{0}$ decay mode of $\Sigma^{+}$while the $\pi^{+}$decay mode of $\Sigma^{+}$goes through $V$ alone and $\Sigma^{-}$decay goes through $A$ alone ${ }^{1}$.

Now the current generated by rotations associated with parameters $\mu$ is a mixture of vector and axial vector currents and reads

$$
\boldsymbol{j}_{\lambda}=\bar{\psi} \gamma_{\lambda} \frac{1+\gamma_{5}}{2} \boldsymbol{\tau} \psi+\frac{i}{4 f^{2}} \operatorname{Tr}\left(\boldsymbol{\tau} H \partial_{\lambda} H^{+}\right)
$$

where we have replaced $\Phi$ by a more general quantity $H$. The only restrictions on it are that it is unitary and that

$$
H(0)=H^{\prime}(0)=1, \quad H=a\left(\varphi^{2}\right)+b\left(\varphi^{2}\right) i \tau \cdot \varphi .
$$

The current (29) is invariant under $v$ rotations. (29) may be written as

$$
\begin{aligned}
2 \boldsymbol{j}_{\lambda}= & \boldsymbol{j}_{\lambda}^{V}+\boldsymbol{j}_{\lambda}^{A}=\left[\bar{\psi} \gamma_{\lambda} \boldsymbol{\tau} \psi+\boldsymbol{\varphi} \times \partial_{\lambda} \boldsymbol{\varphi}\right] \\
& +\left[\bar{\psi} \gamma_{\lambda} \gamma_{5} \boldsymbol{\tau} \psi+\frac{1}{2 f} \partial_{\lambda} \boldsymbol{\varphi}\right] .
\end{aligned}
$$

$j_{\lambda}^{V}$ is the isotopic spin current and $\boldsymbol{j}_{\lambda}^{A}$ the current conserved in the pseudovector coupling theory. While $\boldsymbol{j}_{\lambda}^{A}$ is conserved only in the limit of vanishing pion mass, $\boldsymbol{j}_{\lambda}^{V}$ is conserved even if $\mu \neq 0$, provided the $p-n$ mass difference is neglected.

Only a charged leptonic current $l_{\mu}^{+}$seems to enter weak interactions. If we assume that $l_{\mu}^{+}$transforms like $j_{\lambda}^{+}$under rotations round the third axis in $\mu$-space a weak Lagrangian invariant under this subgroup is obtained by taking

with

$$
L_{W}=2^{-1 / 2} G\left(j_{\lambda}^{+}+l_{\lambda}^{+}\right)\left(j_{\lambda}^{+}+l_{\lambda}^{+}\right)^{\dagger}
$$

$$
G \cong 10^{-5} m^{-2}, \quad j_{\lambda}^{+}=\bar{n} \gamma_{\lambda}\left(1+\gamma_{5}\right) p ; \quad l_{\lambda}^{+}=(\bar{e}+\bar{\mu}) \gamma_{\lambda}\left(1+\gamma_{5}\right) \nu
$$

1 An attempt has been made by A. Ramakrishnan, A. P. Balachandran and N. G. Deshpande (to be published) to determine the structure and parity of weak interaction currents. It is found that the current-current picture of weak interactions is not valid, i.e. $\Delta Q \mid \Delta S=-1$ leptonic decays are allowed. The current is $V-A$ whenever $A, p$ or $\Xi^{-}$occurs (for both leptonic or non-leptonic decay mode). The $\Sigma-\Sigma$ current is a pure vector and all other baryonic currents are either $V$ or $A$.

The suggestion that the observed $\Sigma$-decay asymmetries arise from decay diagrams involving $\Lambda$ and $\Xi$ has been made by A. P. Balachandran and K. Venkatesan. They use the experimental result (Fowler et al., Phys. Rev. Lett. 6, 134 (1961)) that the asymmetry parameters $\alpha_{A}$ and $\alpha_{\Xi}$ have opposite signs. See A. P. Balachandran and K. Venkatesan, Progr. Theor. Phys. 26-5 (L), 792 (1961). 
It is to be noted that $L_{W}$ contains a direet pion-lepton interaction with a coupling constant $1 / 2 G / 2 f$ corresponding to the limit $\mu \rightarrow 0$. When the finite pion mass is taken into account. this will be a function of $\mu^{2}$, say $c\left(\mu^{2}\right)$ with

$$
c\left(\mu^{2}\right) \cong c(0)=1 / 2 G / 2 f
$$

if $c\left(\mu^{2}\right)$ is a slowly varying function of $\mu^{2}$. In this case $c(0)$ may be regarded as a good approximation to the actual $\pi$-lepton eoupling constant. This is just the Goldberger-Treiman estimate ${ }^{1}$ based on dispersion relation techniques. As long as $\mu$ can be negleeted, the vector and axial vector renormalizations should be the same, namely unity, since otherwise $j_{\lambda}$ will not be a vector under $\mu$ rotations. The deviation of $\frac{-G_{A}}{G_{V}}$ from unity is then ascribed to the finite pion mass that perturbs the 4-dimensional symmetry.

The form factors (and consequently the ratio of the renormalized coupling constants) occurring in weak interactions are easily calculable in the pole approximation. In general we have to calculate the matrix element of the current operator between one nucleon states, pion states etc. This matrix element can be shown to be related to the product of the matrix element of the operator between vacuum and all the allowed intermediate states, and the transition matrix element between these intermediate states and the final state of a nucleon and antinucleon, two pions etc. In principle the conplete set of intermediate states should be taken into account. But in the pole approximation the nearest singularity is expected to dominate. ${ }^{2}$

Earlier in the discussion of $A$ decay, we pointed out that the $|\Delta \boldsymbol{I}|=1 / 2$ rule can be built into the Lagrangians for non-leptonic decays of the strange particles provided we allow neutral currents. The chief difficulty in such an introduction arises from the neutral lepton currents which lead to many unobserved processes like $\mu^{+} \rightarrow e^{+}+e^{-}+e^{+}$and $K^{0} \rightarrow e^{+}+e^{-}$. Recently neutral baryon currents have been included in the weak interaction Lagrangian, neutral lepton currents however being excluded. ${ }^{3}$ As Lee and Yang ${ }^{4}$ have shown, the present experimental evidence is definitely against the existence of the neutral lepton currents. Of course such a view of the structure of weak interaction goes against the notion of the universal Fermi interaction.

With the introduction of the neutral currents, the $j_{s c}$ current will form an isotopic vector while the $j_{s n c}$ current will now form an isotopic spinor. The latter

1 M. L. Goldberger and S. B. Treiman, loc. cit.

2 J. Bernstein, S. Fubini, M. Gell-Mann and W. Thirring (Nuovo Cim. 17, 757 (1960)) have studied the pion decay problem using this approximation and are able to arrive at the Goldberger-Treiman formula for pion decay (M. L. Goldberger and S. B. Treiman, Phys. Rev. 110. 1478 (1958)).

3 S. B. Treimax, Nuovo Cim. 15, 916 (1960).

4 T. D. LEE and C. N. YANG, "Implications of the Intermediate Boson basis of the Weak Interactions: The Existence of a Quartet of Intermediate Bosons and Their Dual Isotopic Spin Transformation Properties", preprint, Institute for Advanced Study (1960). 
will also satisfy the rule $\frac{\Delta S}{\Delta Q}=+1$. The $j_{s c}$ will contain the terms $\bar{N} \boldsymbol{\tau} N$, $\bar{\Xi} \boldsymbol{\tau} \boldsymbol{\Xi}, \boldsymbol{\Sigma} \cdot \Lambda$, and $\Sigma \times \Sigma$. The $j_{s n c}$ will consist of terms like

$\left[\begin{array}{c}S_{-} \\ \sqrt{2} S_{0}\end{array}\right] ;\left[\begin{array}{c}\bar{\Lambda} p \\ -\bar{\Lambda} n\end{array}\right] ;\left[\begin{array}{c}\bar{\Xi}^{-} \Lambda \\ \bar{\Xi}^{0} \Lambda\end{array}\right] ;\left[\begin{array}{c}\sqrt{2} \bar{\Sigma}^{-} n+\bar{\Sigma}^{0} p \\ \overline{\Sigma^{0}} n-\sqrt{2} \Sigma^{+} p\end{array}\right] ;\left[\begin{array}{c}\sqrt{2} \Xi^{0} \Sigma^{+}-\bar{\Xi}^{-} \Sigma^{0} \\ \bar{\Xi}^{0} \Sigma^{0}+\sqrt{2} \bar{\Xi}^{-} \Sigma^{-}\end{array}\right]$,

where $S^{-}$and $S_{0}$ satisfy $\Delta Q=-1$ and $\Delta Q=0$. If we now write the total weak interaction as

$$
H_{\mathrm{int}}^{W}=j_{+} S_{-}+j_{0} S_{0}+h \cdot c,
$$

where $j_{+}$and $j_{0}$ are the charged and neutral parts of the current $j_{s c} ; H_{\text {int }}^{W}$ transforms as an isotopic spinor.

While the Universal Fermi Interaction is able to explain most of the known phenomena relating to weak interactions, it is quite possible that it may be inadequate at high energies and large momentum transfers. This led Lee and Yang $^{1}$ to speculate upon the possibility that weak interactions are transmitted by an intermediate vector boson field. In other words the four fermion vertex splits up into two vertices, each vertex involving two fermions and the boson $W$ which connects the vertices. The splitting of the vertices mounts to introducing non-locality into the four fermion interaction.

Detailed considerations led Lee and Yang to postulate that:

(a) There must exist a $W^{+}$, a $W^{0}$, a $\bar{W}^{0}$ and a $W^{-}$,

(b) Their spin is unity in order to transmit the observed vector and axial vector form of weak interactions,

(c) Their mass must be $>m_{K}$ in order to explain the absence of $K \rightarrow W+\gamma$.

Among the predicted properties are:

(a) The non-locality introduced by $W$ causes the Michel parameter $\varrho$ to deviate from $3 / 4$ by the factor $\frac{1}{3}\left(\frac{m_{\mu}}{m_{W}}\right)^{2}$.

(b) In the non-local interaction $\mu^{+} \rightarrow e^{+}+\bar{\nu}+v$, by coupling the vertices $\mu^{-}+v \rightarrow W^{-}$and $W^{-}+v \rightarrow e^{-}$, which implies in turn that the neutrino emitted in the first vertex is absorbed in the second, the decay $\mu^{-} \rightarrow e^{-}+\gamma$ will be allowed. Since this decay is known to be forbidden in nature the intermediate boson hypothesis can be saved only if it is postulated that the neutrinos coupled to the electron and muon are distinct, so that the absorption of the emitted neutrino is not possible. Divergence difficulties also arise since the theory of vector mesons interacting with photons is not renormalizable.

(c) $W^{+}$can decay rapidly $\left(<10^{-17} \mathrm{sec}\right)$ into pions, leptons or $K$-mesons. Their coupling with the leptons $l$ is given by:

$$
\text { if } \bar{\psi} \gamma_{\lambda}\left(1+\gamma_{5}\right) \psi_{\nu} W_{\lambda}+h \cdot c .
$$

1 See T. D. LeE, Proceedings of the Tenth Annual Conference on High Energy Physics (Rochester), pp. 567 et seq. T. D. LeE and C. N. YANG, loc. cit. 
where the eoupling constant $f$ is given by

$$
f^{2}=\frac{m_{W}^{2}}{\sqrt{2}} G_{V^{\cdot}} \cong \frac{10^{-5}}{l^{2}}\left(\frac{m_{W}}{m_{p}}\right)^{2}
$$

Lee and Yang have also discussed the production cross-section for $W^{+}$through the reaction $v+\mathrm{Fe} \rightarrow W^{+}+\mu^{-}+\mathrm{Fe}$ and estimated it to be $\sim 10^{-35} \mathrm{~cm}^{2}$. This coherent production will dominate at low momentum transfer to the nucleus, i.e. at high energies. The spin and polarization of $W^{+}$can be determined by a study of its decay.

If we admit the validity of the $\Delta I=1 / 2$ postulate for weal interactions, we are constrained to postulate the existence of two more neutral bosons $W^{0}$ and $W^{0}$. The interactions between $W$ and strongly interacting particles should conserve isotopic spin. For such a conservation in the reaction

$$
\Lambda_{0} \rightleftarrows p+W^{-}
$$

the $W^{\text {- }}$ particles must be assumed to have $I_{W}=1 / 2$. Thus the $W$ 's form two doublets like the $K$-mesons. However in the reaction

$$
n \rightleftarrows p+W^{-}
$$

the $W$ particles must have integral isotopic spin. They are thus grouped into a triplet

and a singlet

$$
W^{+}, W_{a}^{0}, W^{-} \quad \text { with } \quad I_{W}=1
$$

$W_{b}^{0}$ with $I_{W}=0$

where

$$
W_{a}^{0}=-\frac{1}{\sqrt{2}}\left(W^{0}+{\overline{W^{0}}}^{0}\right)
$$

and

$$
W_{b}^{0}=\frac{i}{\sqrt{2}}\left(W^{0}-\bar{W}^{0}\right) \text {. }
$$

In view of this dual-isotopic spin property they are called schizons. The schizon can be considered established only if it is possible to devise two experiments other than the dissociation of $\Lambda$ and $n$ requiring the alternate assignments of isotopic spin. The experiments suggested are:

(1) Decay of $W^{+}$

$$
R\left(W^{+} \rightarrow K^{+}+\pi^{0}\right)=\frac{1}{2} R\left(W^{+} \rightarrow K^{0}+\pi^{+}\right)
$$

and

$$
W^{+} \nrightarrow \overrightarrow{K^{0}}+\pi^{+}
$$

corresponding to $I_{\mathrm{W}}=1 / 2$, and

$$
R\left(W^{+} \rightarrow 2 \pi^{+}+\pi^{-}\right) \cong R\left(W^{+} \rightarrow 2 \pi^{0}+\pi^{+}\right)
$$

due to $I_{W}=1 / 2$.

More difficult experiments involving neutrino capture have also been suggested. 


\section{STRONG INTERACTIONS AND STRANGE PARTICLES}

\section{K PARTICLE INTERA C'TIONS ${ }^{1}$}

WE WILL now present the salient features of the experimental work on the interaction of $K^{+}$and $K^{-}$with nucleons and nuclei. The theoretical implications of these interactions especially relating to the determination of the relative parities of strange particles will be discussed later. We have already referred to the interesting phenomena due to the interaction of $K^{\mathbf{0}}$ and $\overline{K^{0}}$ with nuclei. As a consequence of strangeness conservation, $K^{+}$interactions with nuclei cannot lead to hyperon production while $K^{-}$interactions can, a theoretical prediction confirmed by experiment.

\section{$\mathbf{K}^{+}$-interactions}

Scattering of $K^{+}$by free nucleons can be described by the following processes:

$$
\begin{aligned}
K^{+}+p & \rightarrow K^{+}+p \text { (direct scattering) } \\
K^{+}+n & \rightarrow K^{+}+n \text { (direct scattering) } \\
& \rightarrow K^{0}+p \text { (charge exchange) }
\end{aligned}
$$

The first two reactions are the direct scattering of $K^{+}$by protons and neutrons while the last refers to the charge exchange scattering of $K^{+}$by neutrons. $K^{+}+p$ reactions can be studied directly in the hydrogen bubble chamber. However, the features of the $K^{+}+n$ scattering have to be deduced by combining the knowledge of $K^{+}+p$ and $K^{+}$-nucleus scattering, e.g. $K^{+}$interactions with deuterium (bubble chamber) which is the simplest nucleus or with more complex nuclei (emulsions). In studying these $K^{+}$scattering processes, it has been found convenient to classify the events into elastic and inelastic events. An elastic $K^{+}$ scattering event is defined as an event in which there is no visible excitation of the nucleon or nuclei (emulsion) and consequently there is no loss of energy. Actually it is very difficult to establish an energy loss less than $5 \mathrm{MeV}$ from measurements on $K$ particles alone. These elastic events are mostly due to the Coulomb-scattering especially for small angles of deflection while the large angle scatterings are due to $K^{ \pm}$nuclear interactions. By inelastic events we mean those

1 See M. F. Kaplon, International Conference on High Energy Physics at CERN, Edited by B. FerRetti p. 171 (1958). 
in which $K$ particles suffer energy loss and also those in which new particles are produced.

In $\mathrm{K}^{+}+p$ scattering. it has been found that the total cross-section remains constant at $15 \mathrm{mb}$ and the angular distribution is consistent with isotropy. Such an isotropy implies an $S$-wave interaction in the $I=1$ channel since the $K^{+} \quad p$ system can exist only in the $I=1$ state.

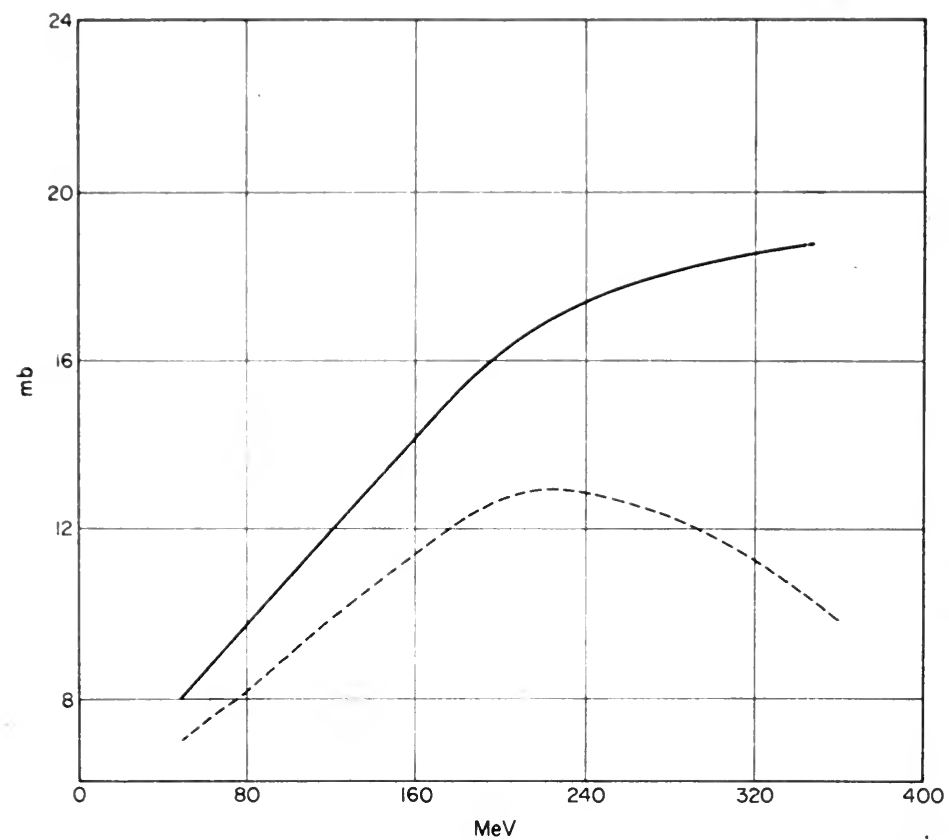

Fis. 38. Collected emulsion data on $K^{+}$scattering by nucleons; corrected cross-sections. The graph shows the total $K^{+}$-nucleon cross-section (full line) and the non-charge exchange cross-section (interrupted line) (CERN).

Study of the inelastic events in $K^{+}+p$ scattering reveals that the relative kinetic energy loss $\frac{\Delta T_{K}}{T_{K}}\left(T_{K}\right.$ is the kinetic energy of $K^{+}$and $\Delta T_{K}$ is the loss in kinetic energy) is small and that this loss is even smaller at lower $T_{K}$. Further the inelastic events are strongly peaked backwards (see Fig. 39), a fact which strongly suggests a repulsive $K^{+}$-nucleon potential. $P$-wave contribution in $K^{+}$-nucleus interaction has also been observed. The total cross-section on heavy pure elements indicates that the cross-section per nucleon may decrease as the mass number $A$ increases.

Measurements on total eross-sections which are a sum of the contributions from both direet and charge exchange scatterings of $K^{+}-n$ have been made by attentuation experiments in water and heavy water the results of which are plotted opposite. 


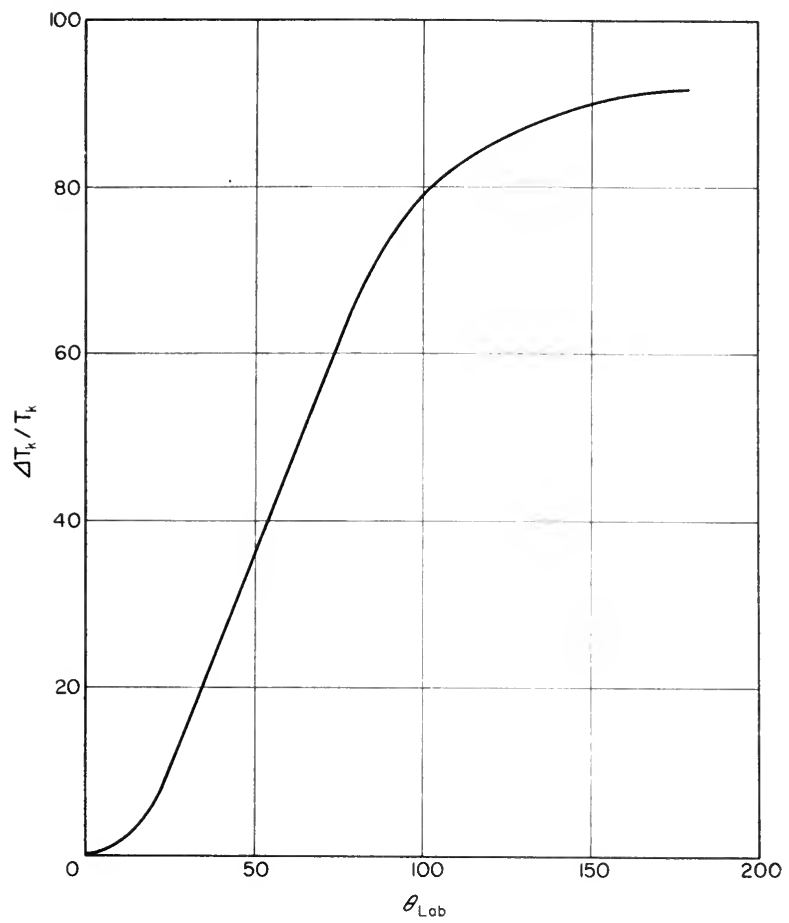

Fia. 39. Inelastic scattering of $K^{+}$mesons by emulsion nuclei. The curve shows the expected relation (in the laboratory system) between inelasticity $\Delta T_{K} / T_{K}$ and $\theta(\mathrm{CERN})$.

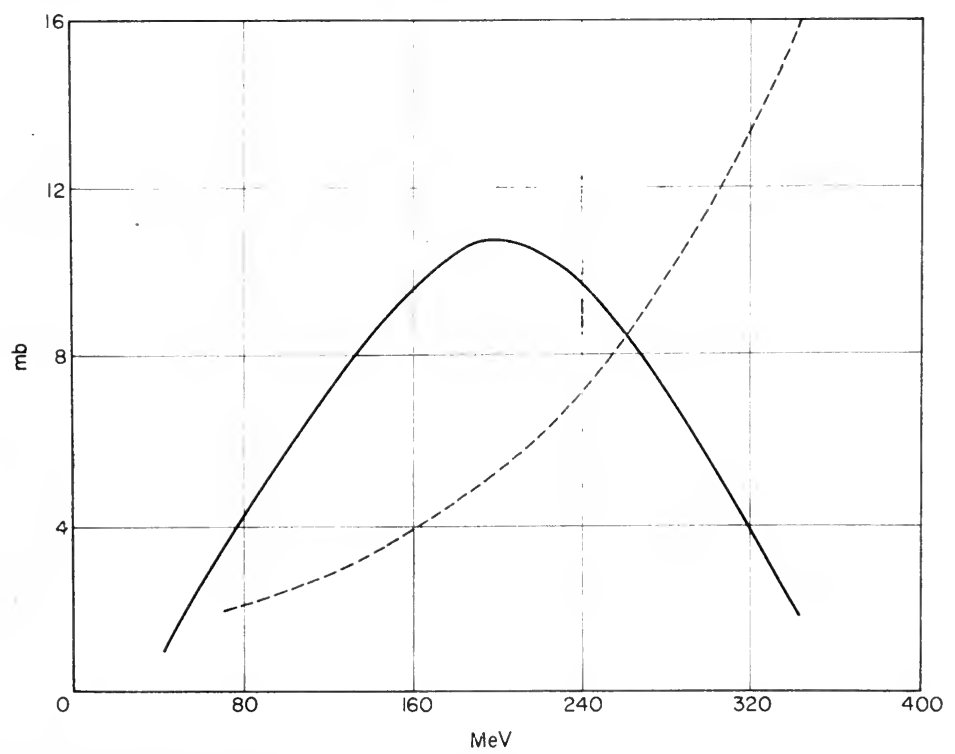

Fig. 40 (a) Graph showing deduced $K^{+}$-neutron cross-sections; non-charge exchange (full line) and charge exchange (interrupted line) (CERN). 
Since the $K^{+}-n$ charge exchange scattering eross-section is known to rise with energy, it is difficult to derive the $K^{+}-n$ elastic scattering cross-section from this. However as noted above, $K^{+}-n$ eross-sections have been obtained by a detailed analysis of $K^{+}$-nueleus inelastic scattering events with the help of the known $K^{+}-p$ scattering results. $K^{+}-n$ elastic scattering rises to a maximum of $10 \mathrm{mb}$ at a bout $200 \mathrm{MeV}$ and falls off at higher energies. The $K^{+}-n$ charge exchange scattering cross-sections have been obtained through counter experiments by subtraction of the data from the interactions of $K^{+}$with $\mathrm{CD}_{2}$ and $\mathrm{CH}_{2}$. Figure $(40 \mathrm{~b})$ below gives the energy dependence of the charge exchange scattering as well as the ratio of the charge exchange to total cross sections for $k^{+}+n$ scattering.

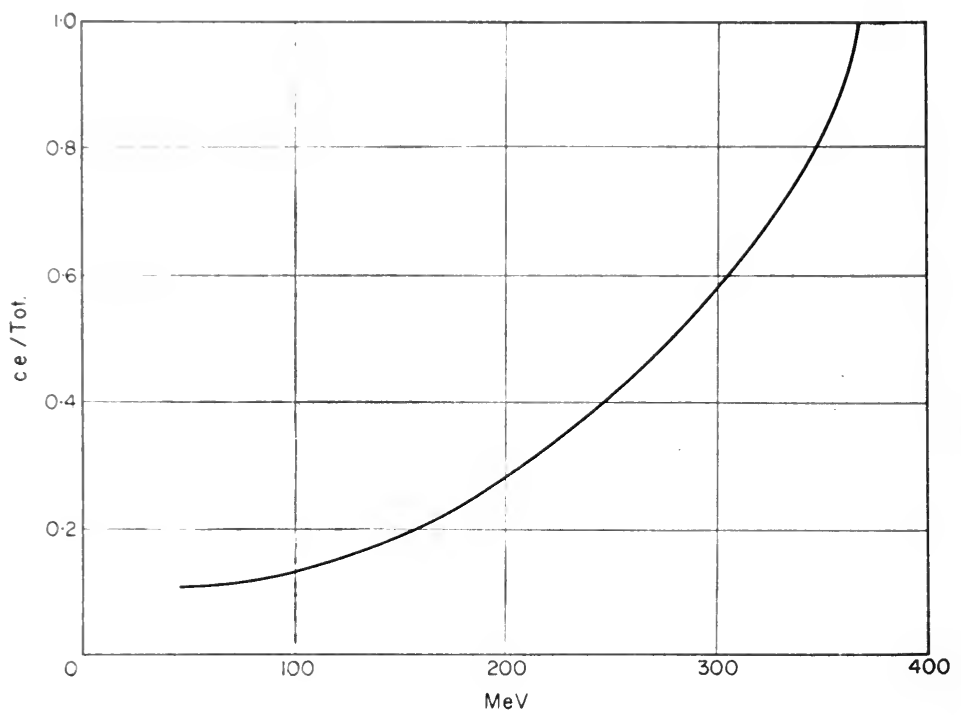

FIG. 40 (b) Energy dependence of charge exchange to total cross-sections for $K^{+}-n$ scattering.

The striking feature of $K^{+}-n$ charge exchange cross-sections is its rise with energy and its $\eta^{3}$ dependence where $\eta$ is the centre-of-mass momentum. The latter result implies a $P$-wave contribution to the $K^{+}-n$ interaction. It is interesting to note that the enhancement in the $K^{+}-n$ charge exchange cross-sections sets in at about the threshold for $\pi$ production in $K-N$ collisions $(\sim 220 \mathrm{MeV}$ for the scattering of a $K^{+}$meson on a free nucleon). Thus from this brief survey of experimental results of $K^{+}-n$ data, we find that at low energies the $I=1$ channel is dominant and only $S$-wave scattering is present. However at higher energies the $I=0$ channel becomes important but the $S$ wave interaction is relatively weak in that channel. A $P$ wave interaction is required by the backward peaking of 
the $K^{+}-n$ cross-section. The decrease in the $K^{+}-n$ elastic cross-section can be understood by an interference of the $P$ waves in the $I=1$ and $I=0$ channels. ${ }^{1}$

\section{$K^{-}$-interactions}

Since the $I_{z}$ values for $K^{-}$and $p$ are respectively $-\frac{1}{2}$ and $\frac{1}{2}$, the scattering of $K^{-}$by protons can proceed through both $I=0$ and $I=1$ channels. We have

$$
\begin{array}{ll}
K^{-}+p \rightarrow K^{-}+p & \text { (direct scattering) } \\
K^{-}+p \rightarrow \overline{K^{0}}+n & \text { (charge exchange) } \\
K^{-}+p \rightarrow Y+\pi & \text { (inelastic or capture). }
\end{array}
$$

However the interactions of $K^{-}$with neutrons proceed through the $I=1$ channel alone. Capture events indicate that $K^{-}$is absorbed by the nucleon after $K^{-}$comes to rest, while inelastic events refer to absorption of $K^{-}$of non-zero momentum.

$$
\begin{array}{ll}
K^{-}+n \rightarrow K^{-}+n & \text { (elastic) } \\
K^{-}+n \rightarrow Y+\pi & \text { (inelastic or capture). }
\end{array}
$$

$K^{-}+p$ elastic scattering has been studied both in the hydrogen bubble chamber and in emulsion. The angular distribution of elastic scattering events is isotropic. The $K^{-}+p$ elastic cross-section rises to about $90 \mathrm{mb}$ at about $120 \mathrm{MeV} / \mathrm{c}$ and then drops to about $40 \mathrm{mb}$.

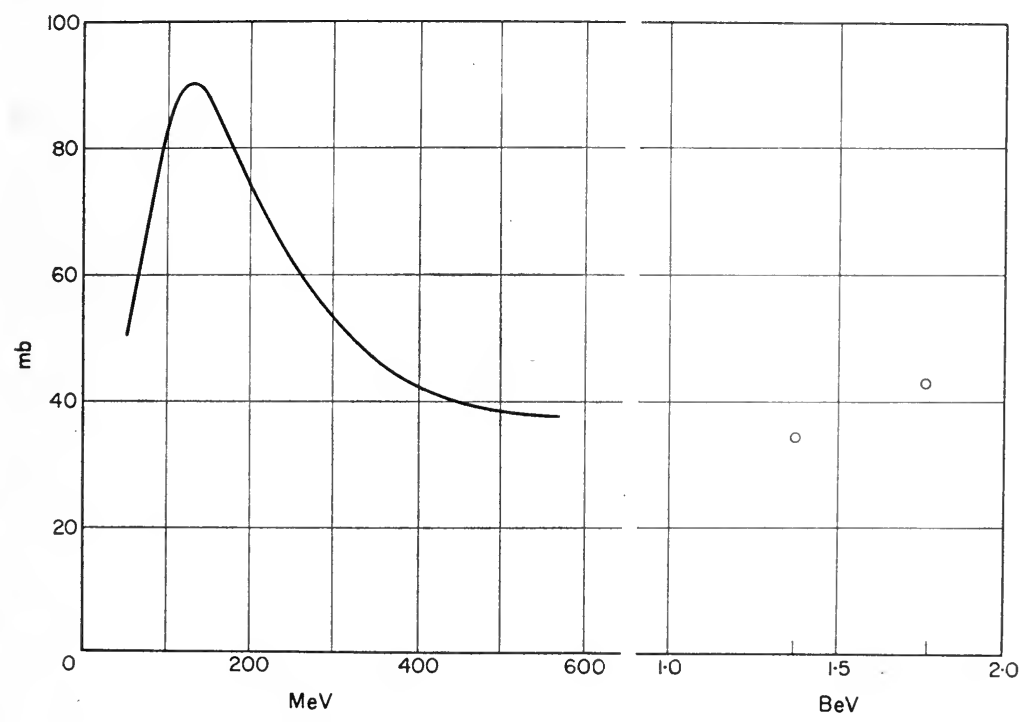

FIG. 41. Energy dependence of $K^{-}-p$ elastic scattering cross-section.

1 See R. H. Dalitz, International Conference on High Energy Physics at CERN, Edited by B. Ferretti p. 187 (1958).

EPCR 19 
Since it has been established that $\overline{K^{0}}$ is heavier than $K^{-}$by about $3.9 \mathrm{MeV}, 1$ the charge exchange scattering of $K^{-}$by protons has a threshold of $89 \mathrm{MeV} / \mathrm{c}$. 'This has been established by observing the charged decay mode of the short lived neutral $\overline{K^{0}}$, i.e. the mode $K_{1}^{0} \rightarrow \pi^{+}+\pi^{-}$which accounts for 34 per cent of the decays of $h^{\circ}$. 'The energy dependence of the cross-section is shown below. Both the direct and charge exchange scattering of $K^{-}$by protons seem to fit well with a zero effective range approximation assuming only $S$ wave interaction. ${ }^{2}$

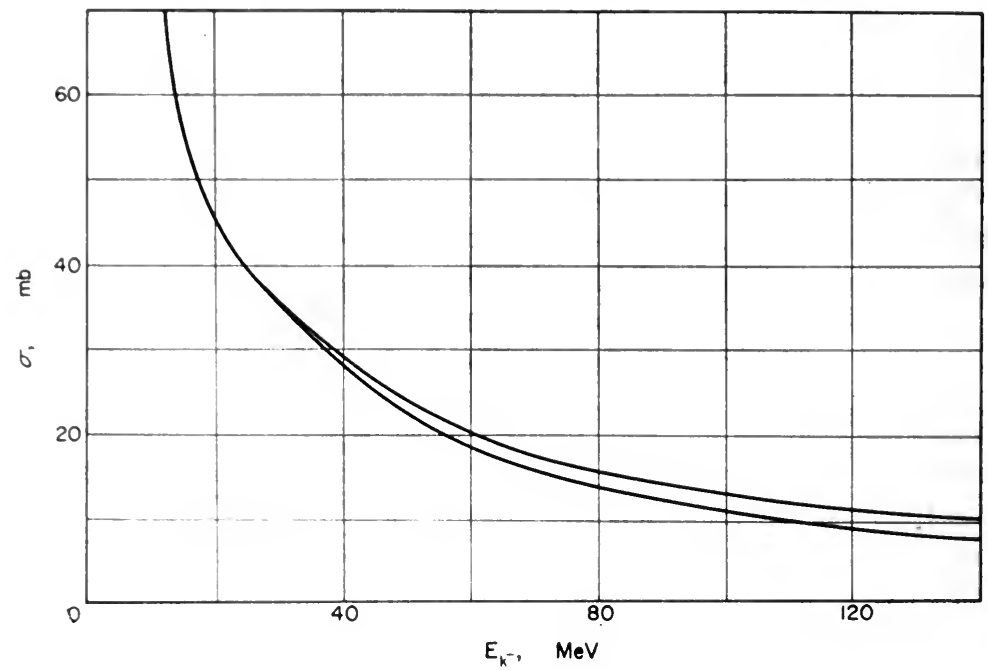

FIG. 42. Combined emulsion data (75 events) for the reactions $K^{-}+p \rightarrow \Sigma \pm+\pi^{\mp}$ (CERN).

The data on $K^{-}-p$ inelastic interactions are given in Fig. 42. At high momenta, the $\Sigma^{+}$production cross-section is about $12 \mathrm{mb}$ and is slightly larger than the production cross-section of $\Sigma^{-}$which is about $8 \mathrm{mb}$. However at low energies the cross-section of $\Sigma^{-}$increases much more rapidly than that of $\Sigma^{+}$. It is interesting to note that at $K^{-}$momentum of $100 \mathrm{MeV} / \mathrm{c}$ the $\Sigma^{-} / \Sigma^{+}$production ratio is $<1$ while for $K^{-}$capture at rest it is 2 . At higher energies the ratio becomes unity. The emulsion data on $\Sigma^{ \pm}$production in $K^{-}-p$ interactions in flight do not seem to support this rapid variation of the $\Sigma^{-} / \Sigma^{+}$ratio. The angular distribution of $\Sigma^{-}$and $\Sigma^{+}$produced is consistent with isotropy. Dalitz ${ }^{3}$ has analysed

${ }^{1}$ A. H. Rosenfeld et al. using charge exchange scattering of $K^{-}$in hydrogen bubble chamber find that $\overline{K^{0}}$ is heavier than $K^{-}$by about $3.70-0.07 \mathrm{MeV}$. See Phys. Rev. Lett. 2,110 (1959). CRAWForD et al. by studying associated production in $\pi^{-}-p$ collisions have shown that $K^{0}$ is heavier than $K^{+}$by about $5 \mathrm{MeV}$. See Phys. Rev. Lett. 2, 112 (1959); Ceolis et al., Nuovo Cim. 13, 818 (1959).

2 J. D. Jacksox, D. G. Ravenhall and H. W. Wyld, Nuovo Cim. 9, 834 (1958). See also R. H. Dalitz, loc. cit., R. H. Dalitz and S. F. Tuan, Ann. Phys. 8, 100 (1959).

3 R. H. Dalitz, loc. cit. Also R. H. Dalitz and S. F. Tuan, loc. cit. 
these inelastic events in terms of complex phase shifts, again assuming an $S$-wave interaction and zero effective range. In the high energy $K^{-}-p$ interactions an event giving rise to the $\Xi$ particle has been observed. The process is

$$
K^{-}+p \rightarrow \Xi^{-}+K^{0}+\pi^{+} \text {. }
$$

In an attempt to observe the cascade particle, $K^{-}$interactions with nuclei in flight have been studied and the following processes observed:

$$
\begin{aligned}
& K^{-}+N \rightarrow Y+\pi+\pi \\
& K^{-}+N+N \rightarrow Y+Y+K^{0} \\
& K^{-}+N \rightarrow K^{-}+N+\pi+\pi .
\end{aligned}
$$

The energy spectrum of the inelastically scattered $K^{-}$ranges from 6 to $270 \mathrm{MeV}$ which is possible because of the frequent productiom of $\pi$ mesons.

As in the case of $K^{+}$-nucleus interactions, the $K^{-}$-nucleus data can be used to yield information on the $K^{-}$-nucleon potential. To this end, optical model analyses of $K^{-}$-nucleus data have been carried out. The imaginary part of the potential is found to be about $10 \mathrm{MeV}$ and the real part negative indicating an attractive potential. Further a study of the inelastic $K^{-}-p$ events reveals a relatively larger kinetic energy loss with $\frac{\Delta T_{K^{-}}}{T_{K}}=0.45$, and angular distribution which is peaked forward. These two strongly suggest an attractive $K^{-}$-nuclear potential.

Application of the principle of charge independence to the $K^{-}-p$ or $K^{-}-n$ capture reactions leads to interesting relations among cross-sections for the production of charged and neutral $\Sigma$ particles. Of particular interest is the production of neutral and charged hyperons $\left(\Sigma\right.$ and $\Lambda$ ) in the capture of $K^{--} p$ by nucleons. The $K^{-}-p$ capture reactions are:

$$
\begin{aligned}
K^{-}+p & \rightarrow \Lambda^{0}+\pi^{0} \\
& \rightarrow \Sigma^{-}+\pi^{+} \\
& \rightarrow \Sigma^{0}+\pi^{0} \\
& \rightarrow \Sigma^{+}+\pi^{-} .
\end{aligned}
$$

Denoting the reaction amplitude by $a_{0}$ and $a_{1}$ for production of $\Sigma$ 's in the $I=0$ and $I=1$ channels, we obtain the relation

$$
\left(\Sigma^{+} \pi^{-}\right):\left(\Sigma^{0} \pi^{0}\right):\left(\Sigma^{-} \pi^{+}\right)=\frac{1}{2}\left|\frac{a_{0}}{\sqrt{3}}+\frac{a_{1}}{\sqrt{ } 2}\right|^{2}:\left|\frac{a_{0}}{6}\right|^{2}:\left|\frac{a_{0}}{\sqrt{3}}-\frac{a_{1}}{V^{2}}\right|^{2} .
$$

Thus by comparison with experimental data, we can only deduce the relative phase between $a_{0}$ and $a_{1}$. Since $\Lambda^{0}$ has $I=0$, the $\Lambda^{0}+\pi^{0}$ system can exist only in the $I=1$ state. Thus if we denote by $b$, the corresponding production amplitude $19 *$ 
the $A^{0}+\pi^{0}$ production cross-section is proportional to $\frac{\left|b^{2}\right|}{2}$. From $K^{-}$captures in hydrogen, it has been found that

$$
\Sigma^{-}: \Sigma^{+}: \Sigma^{0}: \Lambda^{0}=8: 4: 4: 1 \text {. }
$$

To fit with data, the phase between $a_{0}$ and $a_{1}$ is required to be $108^{\circ}$. This ratio, as we shall see later, is inconsistent with the prediction by the "global symmetry" model of strong interactions due to Gell-Mann. Besides the above reactions, there also exist two reactions

$$
\begin{aligned}
K^{-}+p & \rightarrow \Lambda^{0}+\pi^{+}+\pi^{-} \\
& \rightarrow \Lambda^{0}+\pi^{0}+\pi^{0}
\end{aligned}
$$

A study of these can throw light on the relative $K-\Lambda^{0}$ parity (see chapter VII.3). The capture of $K^{-}$by neutrons can lead to the following:

$$
\begin{aligned}
K^{-}+n & \rightarrow \Sigma^{-}+\pi^{0} \\
& \rightarrow \Sigma^{0}+\pi^{-} \\
& \rightarrow \Lambda^{0}+\pi^{-} \\
& \rightarrow \Lambda^{0}+\pi^{-}+\pi^{0} .
\end{aligned}
$$

The last reaction has been observed. The $K^{-}-n$ interactions can proceed only through the $I=1$ channel. We thus find the ratio $\frac{\Sigma^{-}}{\Sigma^{0}}$ for $K^{-}-n$ capture to be 1 . A more interesting relation is obtained if we consider the $K$-capture by a target containing an equal number of protons and neutrons, i.e. deuterium and helium. By adding the $K^{-}-p$ and $K^{-}-n$ production amplitudes, we obtain

$$
N\left(\Sigma^{+}\right)+N\left(\Sigma^{-}\right)=2 N\left(\Sigma^{0}\right)
$$

where $N(\Sigma)$ denotes the number of hyperons produced. This relation seems to be confirmed by the $K^{-}-d$ capture reactions observed in the deuterium bubble chamber. The data from the deuterium bubble chamber are

$$
\begin{aligned}
N\left(\Sigma^{+}+\Sigma^{-}\right) & =150 \pm 8 \\
N\left(\Sigma^{0}\right) & =86 \pm 6
\end{aligned}
$$

Another feature of the $K^{-}-d$ capture is that the $\Sigma^{-}: \Sigma^{+}: \Sigma^{0}: \Lambda^{0}$ value is strikingly different from that in hydrogen ${ }^{1}$ (see table below). In view of the fact calculations based on impulse approximation agree with the experimental data, this difference scems to indicate that the interaction amplitudes are momentum dependent, i.e. the interaction amplitudes are influenced by the Fermi motion within the nucleus.

Capture of $K^{-}$by nuclei has been extensively studied in emulsion nuclei and the data have been given a phenomenological interpretation. One of the main

1 R. D. TRIPP, Int. Conf. on High Energy Physics at CERN p. 184 (1958). 
difficulties in the emulsion work is that the neutral particles cannot be detected and hence the reduction of data requires a complicated correction procedure to infer the number of $\Sigma^{0}, \Lambda^{0}$ and $\pi^{0}$ emitted. There is clear evidence for a multinucleon interaction of $K^{-}$from the $K^{-}$-nucleus capture data. This is in contrast to the usual assumption that $K^{-}$is captured by an individual nucleon inside the nucleus. If hyperon production proceeds through a single nucleon interaction, then the emitted $\Sigma$ hyperons can have a maximum energy of $60 \mathrm{MeV}$ corresponding to an interaction with a single nucleon of Fermi momentum of about $240 \mathrm{MeV} / \mathrm{c}$. However several high energy hyperons have been observed. According to the data, about 40 per cent of the hyperons produced are due to multinucleon interactions. The multi-nucleon interactions are

$$
\begin{aligned}
K^{-}+p+p & \rightarrow \Sigma^{+}+n \\
& \rightarrow \Sigma^{0}+p \\
& \rightarrow \Lambda^{0}+p \\
K^{-}+p+n & \rightarrow \Sigma^{-}+p \\
& \rightarrow \Sigma^{0}+n \\
& \rightarrow \Lambda^{0}+n \\
K^{-}+n+n & \rightarrow \Sigma^{-}+n .
\end{aligned}
$$

Another important feature of the multi-nucleon interaction is that the hyperons are unaccompanied by $\pi$ mesons. ${ }^{1}$

The $\mathrm{K}^{-}$-nucleus data yield some information on the site of the capture. It is found that in $\mathrm{K}^{-}$-capture at rest there is relatively little absorption of the accompanying $\pi$-meson while in interactions in flight the number of $\pi$ 's that emerge from the nucleus is greatly reduced. The implication is that in interactions in flight, the hyperon production takes place much deeper inside the nucleus and thus there is a greater chance of the $\pi$ being absorbed, while $K^{-}$-capture at rest is more of a surface phenomenon. It is also found that there is less of multi-nucleon interaction in captures in flight. These facts can be used to conclude that the correlated pairs of nucleons occur much more frequently at the surface of the nucleus. In the table below are given the various percentages for the production of hyperons through various modes in emulsion, $\mathrm{D}_{2}$ and $\mathrm{H}_{2}$. It is assumed that emulsion nuclei contain an equal number of protons and neutrons. The $K^{+}+n$ data in $\mathrm{H}$ are computed using the charge independence hypothesis. In emulsion data, it is assumed that the multi-nucleon processes are responsible for 30 per cent of the hyperons. It is obvious from the table that the data from elementary $K^{-}-p$ and $K^{-}-n$ interactions from $\mathrm{H}$ do not hold for the $\mathrm{D}_{2}$ and emulsion nuclei.

1 " $K$ - collaboration experiments", Parts I and II, Nuovo Cim. 13, 670 (1959), 14, 315 (1959). 
Hyperon production by $\mathrm{K}^{-}$-capture. ${ }^{1}$

\begin{tabular}{c|c|c|c}
\hline Interaction process & $\begin{array}{c}\text { Hydrogen } \\
\text { bub. ch. }\end{array}$ & $\begin{array}{c}\text { Deuterium } \\
\text { bub. ch. }\end{array}$ & Emulsion \\
\hline$h^{-}+p \rightarrow \Sigma^{-}+\pi^{+}$ & 29.5 & 24.5 & 12.0 \\
$\rightarrow \Sigma^{+}+\pi^{-}$ & 14.8 & 21.6 & 21.5 \\
$\rightarrow \Sigma^{0}+\pi^{0}$ & 14.8 & 20.8 & 12.4 \\
$\rightarrow \Lambda^{0}+\pi^{0}$ & 3.7 & 8.0 & 12.1 \\
$h+n \rightarrow \Sigma^{-}+\pi^{0}$ & $(14.8)$ & 4.6 & 8.9 \\
$\rightarrow \Sigma^{0}+\pi^{-}$ & $(14.8)$ & 4.6 & 8.9 \\
$\rightarrow \Lambda^{0}+\pi^{-}$ & $(7.4)$ & 16.0 & 24.2 \\
$\rightarrow \pi^{-} / \pi^{+}$ratio & $1.75 \pm 1.0$ & $1.75 \pm 0.3$ & $4.5 \pm 0.9$ \\
$\Sigma^{-} / \Sigma^{+}$ratio & $3.2 \pm 1.3$ & $0.82 \pm 0.2$ & $\mathbf{0 . 9 7} \pm 0.3$ \\
\hline
\end{tabular}

It is also noticed that the $\pi^{-} / \pi^{+}$ratio of 4.5 from $K^{-}$-captures at rest is very large compared to the value of 1.5 obtained assuming charge independence. Another interesting feature in the $K^{-}$-captures by nuclei and $\mathrm{D}_{2}$ is the enhancement of $\Lambda^{0}$ production which requires the charge exchange scattering of hyperons as we shall see below.

\section{HYPERONSAND HYPERFRAGMENTS}

\section{Production of hyperons}

The associated production of hyperons and $K$-mesons can be achieved with $\tau-N$ and $N-N$ collisions. The following reactions leading to the production of hyperons have been studied in $\pi-N$ collisions:

Threshold momenta $\mathrm{MeV} / \mathrm{c}$

$$
\begin{aligned}
& \pi^{-}+p \rightarrow \Lambda^{0}+K^{0} \\
& \boldsymbol{\pi}^{-}+p \rightarrow \Sigma^{-}+K^{+} \\
& \pi^{-}+p \rightarrow \Sigma^{0}+K^{0} \\
& \boldsymbol{\pi}^{+}+p \rightarrow \Sigma^{+}+K^{+}
\end{aligned}
$$$$
\sim 900
$$$$
\sim 1050
$$$$
\sim 1045
$$$$
\sim 1040
$$

These interactions have been studied in the range $930-1430 \mathrm{MeV} / \mathrm{c}$ of $\pi^{-}$momentum. Many events with $\Lambda^{0}$ production have been observed. The present experimental results are as follows: ${ }^{2}$

(1) All the observed total cross-sections lie in the interval

$$
0.1 \mathrm{mb}<\sigma<1 \mathrm{mb} \text {. }
$$

(2) The total cross-section for $\Lambda^{0}$ rises from the threshold to a peak of about $0.8 \mathrm{mb}$ near $1100 \mathrm{MeV} / \mathrm{c}$ and the drops again to $0.2-0.3 \mathrm{mb}$ in the interval

1 The table is reproduced from a report prepared by A. C. Melissinos on behalf of the Rochester group. We thank Dr. Sudarshan for making available this report.

2 Report by J. Steinberger, International Conference on High Energy Physics, CERN, Ed. B. Feretti, p. 147 (1958). 
1250-1450 MeV/c. Similar behaviour has not been observed in the $\Sigma^{-}$and $\Sigma^{0}$ cross-sections.

(3) The most remarkable result concerns the angular distributions. It has been noticed even before that while the charged hyperons are peaked forwards, the neutral hyperons are always peaked backwards. In the above experiments it is found that the angular distribution of $\Lambda^{0}$ does not change in the interval of $1000-1450 \mathrm{MeV} / \mathrm{c}$ for $\pi^{-}$momenta. The backward peaking of the $\Sigma^{0}$ is less marked compared to that of the $\Lambda^{0}$.

A very interesting method to study the spin of the hyperon is due to Adair ${ }^{1}$ who suggested that the angular distribution of the decay products of the hyperons produced in the forward or backward directions in the c.m. system of $\pi^{-}-p$ will depend only on the spin of the $\Lambda^{0}$. An assumption essential to the argument is that the spin of the $K^{-}$meson is zero. It is easy to see from conservation of $J$ and $J_{z}$ that for production angle $\theta=0$ or $\pi$ in the c.m. system, $\Lambda^{0}$ will be produced with $m_{z}=\frac{1}{2}$ or $-\frac{1}{2}$ with equal probability and there will be no phase correlation if we perform $\pi^{-}-p$ experiments with unpolarized targets. The component of the angular momentum of the $\Lambda^{0}$ vanishes along $\theta=0$ or $\pi$. Thus for spin $1 / 2, \Lambda^{0}$ will be produced unpolarized and hence the angular distribution of its decay products will be isotropic. If $\Lambda^{0}$ has higher spins like $3 / 2$ or $5 / 2$ it will be produced with polarization since all its spin states are not equally populated ( $\Lambda^{0}$ will still be produced only in the $m_{z}=\frac{1}{2}$ and $-\frac{1}{2}$ states). So the angular distribution of the decay products will be anisotropic and the form for spin $3 / 2$ will be $\left(1+3 \cos ^{2} \theta\right)$. The above considerations can be applied for $\theta=0$ or $\pi$ provided the contribution of higher angular momentum waves (i.e. $m_{l} \neq 0$ ) can be assumed to be very small. Since the hyperons are produced fortunately with forward or backward peaking, recent experiments ${ }^{2}$ indicate that hyperons have spin 1/2.

A few experiments on the production of hyperons by $3 \mathrm{BeV}$ protons incident upon nuclei have been done. The hyperons and mesons were detected by observing the $\gamma$-rays originating from regions several centimeters down stream from the target. The following reactions are possible.

$$
\begin{aligned}
& \left.\begin{array}{rl}
p+p & \rightarrow K^{+}+\Lambda^{0}+p \\
& \rightarrow K^{+}+\Sigma^{0}+p \\
& \rightarrow K^{0}+\Sigma^{+}+p
\end{array}\right\} \\
& \rightarrow K^{+}+\Sigma^{+}+n \text { (not observed) } \\
& \left.\begin{array}{rl}
p+n & \rightarrow K^{0}+\Lambda^{0}+p \\
& \rightarrow K^{0}+\Sigma^{0}+p
\end{array}\right\} \\
& \rightarrow K^{+}+\Sigma^{-}+p \text { (not observed) } \\
& \left.\begin{array}{l}
\rightarrow K^{+}+\Sigma^{0}+n \\
\rightarrow K^{+}+\Lambda^{0}+n
\end{array}\right\}
\end{aligned}
$$

1 R. AdatR, Phys. Rov. 100, 1540 (1955).

2 F. Eisler et al., Nuovo Cim. 7, 222 (1958). 
Making the assumption that there is only one production interaction (i.e. neglecting multiple interactions) it was estimated that the cross-sections are $40 \pm 6 \mu \mathrm{b}$, $210 \pm 30$ and $420 \pm 60 \mu \mathrm{b}$ for (2), (4) and (6) respectively. ${ }^{1}$ The results seem to show that the cross-section for associated production in $p-p$ collisions is significantly smaller than the cross-section in $p-n$ collisions. It has also been found that the cross-sections for the production of $\Sigma^{+}, \Lambda^{0}$ or $K^{0}$ are extremely small for $p-p$ collisions. ${ }^{2}$

\section{Hyperon interactions}

Some events corresponding to the interaction of hyperons at rest and in flight have been observed. Elastic, inelastic and charge exchange scattering events have been identified. There is also the possibility of hyperons forming bound states with nucleons, i.e. the formation of hyperfragments which will be discussed presently.

\section{5--interactions}

$\Sigma$ - capture reactions have been studied in the hydrogen bubble chamber. The reactions are

$$
\begin{aligned}
& \Sigma^{-}+p \rightarrow \Lambda^{0}+n \\
& \Sigma^{-}+p \rightarrow \Sigma^{0}+n .
\end{aligned}
$$

The present data yield a ratio $\frac{\Sigma^{0}}{\Sigma^{0}+\Lambda^{0}}=0.34 . \Sigma$ - captures in $\mathrm{D}$ can proceed as follows:

$$
\begin{aligned}
& \Sigma^{-}+\mathrm{D} \rightarrow \Lambda^{0}+n+n \\
& \Sigma^{-}+\mathrm{D} \rightarrow \Sigma^{0}+n+n .
\end{aligned}
$$

This yields a ratio $\frac{\Sigma^{0}}{\Sigma^{0}+\Lambda^{0}} \simeq 0.037 \pm 0.022 .^{3}$ Fortunately it is easy to distinguish the $\Lambda^{0}$ that are produced directly from the $\Lambda^{0}$ that arise from the decay of the $\Sigma^{0}$ since the latter $A^{0}$ 's have a momentum less than $134 \mathrm{MeV} / \mathrm{c}$. The difference in this ratio for $\mathrm{H}$ and $\mathrm{D}$ can be explained assuming the impulse approximation in the $\Sigma$-D interactions. In order to account for the enhanced $\Lambda^{0}$ production there is no need to invoke two-step $\Sigma^{-}-\mathrm{D}$ interactions such as

$$
\Sigma^{-}+\mathrm{D} \rightarrow \Sigma^{0}+n+n \rightarrow \Lambda^{0}+n+n .
$$

However there is strong evidence for such a two-step process in the $K^{-}-\mathrm{D}$ capture. It is to be noted that this two-step process is essentially a charge exchange scattering of hyperons. As for $\Sigma^{-}-\mathrm{D}$ interactions, it is quite easy to distinguish the directly produced $\Lambda^{0}$ from those produced in the decay of the $\Sigma^{0}$

1 D. Berley and G. B. Colliers, Phys. Rev. 112, 614 (1958).

${ }^{2}$ Cool et al., Phys. Rev. 108, 1048 (1957).

${ }^{3}$ O. Dahl et al., Phys. Rev. Lett. 4, 77 (1960). 
in the $K^{-}-\mathrm{D}$ capture. Under the impulse approximation we can write the $K^{-}-\mathrm{D}$ interaction as

$$
K^{-}+\mathrm{D} \rightarrow\left(K^{-}+n\right)+p \rightarrow \Lambda^{0}+\pi^{-}+p \rightarrow\left(\Sigma^{0}+\pi^{-}\right)+p .
$$

Since hyperon production is a two body reaction, $\pi^{-}$must have a unique momentum smeared out only by the Fermi motion of the nucleon. The $\pi$ 's accompanying the $\Sigma$ have a peak at about $182 \mathrm{MeV} / \mathrm{c}$ indicating the validity of the impulse approximation. However the $\pi$ 's accompanying the $\Lambda^{0}$ exhibit two peaks one at $253 \mathrm{MeV} / \mathrm{c}$ and another at $182 \mathrm{MeV} / \mathrm{c}^{1}$ This interesting phenomenon implies that some of the $\pi^{-}$accompanying the $\Lambda^{0}$ were partners to $\Sigma^{0}$ or $\Sigma^{+}$according to the following reactions which proceed in two steps.

$$
\begin{array}{cc}
\text { I } \quad\left(K^{-}+n\right)+p \rightarrow\left(\Sigma^{0}+\pi^{-}\right)+p \\
\\
\\
\text { II } \left.\quad K^{-}+p\right)+n \rightarrow\left(\Sigma^{+}+\pi^{-}\right)+n \\
K^{-}+\mathrm{D} \rightarrow \Sigma^{0}+\pi^{-}+p \rightarrow \Lambda^{0}+p+\pi^{-} \\
K^{-}+\mathrm{D} \rightarrow \Sigma^{+}+\pi^{-}+n \rightarrow \Lambda^{0}+p+\pi^{-}
\end{array}
$$

The last step refers to the production of $\Lambda^{0}$ by charge or particle exchange of the hyperons.

Very few clear data exist on $\Sigma^{-}-p$ elastic scattering events. It has been estimated that the probability of escape of $\Sigma^{+}, \Sigma^{-}$and $\Sigma^{0}$ from nuclei is 45 per cent corresponding to a mean free path of $0.5 \times 10^{-13} \mathrm{~cm}$ in nuclear matter.

The following events concerning $\Lambda^{0}$ interactions have been observed which correspond to elastic and charge exchange scatterings:

$$
\begin{aligned}
& \Lambda^{0}+p \rightarrow \Lambda_{0}+p \\
& \Lambda^{0}+p \rightarrow \Sigma^{+}+n .
\end{aligned}
$$

The second reaction is exothermic and has a threshold at $635 \mathrm{MeV} / \mathrm{c}$.

\section{Hyperfragments ${ }^{2}$}

We have seen that the strange particles that have been discovered are divided into two classes, the hyperons and the heavy mesons. The hyperons along with the neutron and the proton are called baryons or the heavy particles. It was found interesting to investigate the possibility of the existence of nuclei in which some of the neutrons were replaced by hyperons. Such a speculation was encouraged when a very unusual event was reported by Danysz and Pniewski ${ }^{3}$ in which a nuclear fragment from a cosmic ray star stopped in the emulsion and subsequently disintegrated with an energy release of about 140 to $180 \mathrm{MeV}$. There are two possible explanations for such an event. (1) The fragment might have contained

1 A. FujII and R. E. Marshak, Nuovo Cim. 8, 643 (1958).

2 Parts of this section are based on "Some Properties of Uncommon Forms of Matter", Lectures given by P. Morrison at the University of Alberta, Edmonton, Canada (1957).

${ }^{3}$ M. Danysz and J. Pniewski, Phil. Mag. 44, 348 (1953). 
a $\pi^{-}$in its orbit and after the fragment stopped it could have been absorbed with consequent release of energy. (2) The fragment might have contained a $\Lambda^{0}$ which decayed after the fragment had stopped with a corresponding release of energy. Further experimental work confirmed the second possibility and for such nuclei the name "hyperfragment" or "hypernucleus" was proposed by Goldhaber.

Once such hyperfragments were observed in cosmic ray events, experiments in high energy accelerators with protons of energies of the order of $\mathrm{BeV}$ followed. 'The frequency of occurrence of hyperfragments was found to be of the order of 1 in 1000 for $3 \mathrm{BeV}$ protons and 1 in 500 for $1.5 \mathrm{BeV}$ mesons. A typical cosmic

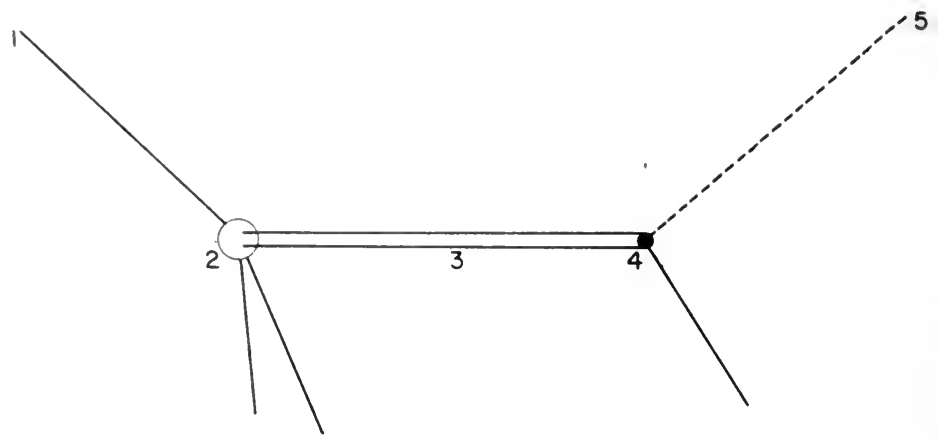

Fra. 43. A typical high-energy primary particle interaction.

ray event involving a hyperfragment can be described as follows. A fast primary particle enters at (1) and suffers a nuclear collision at (2) giving rise to a spray of mesons and some other particles. Among these is one which makes a heavy track (3) and has a short range. It comes to rest at (4) and then disintegrates. The track indicates a nuclear mass but it is not an ordinary nucleus since one of the decay products is a pion (5). Hence we conclude that the track is probably that of a hypernucleus. The observation of a not very heavily charged recoil product together with a proton and a pion and an energy release of about $37 \mathrm{MeV}$ was interpreted as the decay of a $\Lambda^{0}$ in the nucleus.

We shall here consider only light hyperfragments containing $\Lambda$ particles. We should except the simplest hypernucleus to be a $\Lambda^{0}+p$ system. This is analogous to the deuteron and the forces involved are of the same nature and approximately of the same magnitude as the nuclear forces. The system denoted by ${ }_{A} \mathrm{H}^{2}$ if it exists should be easy to observe having a track corresponding to a charge $Z=1$ (Fig. 44). The particle would come to rest and decay into two protons with an energy release of about $30 \mathrm{MeV}$. Such an event has never been observed and hence it is concluded that ${ }_{A} \mathrm{H}^{2}$ does not exist or that the $\left(\Lambda^{0}, p\right)$ system has no bound state. Several events of the type shown in (Fig. 44) have been observed in which all the particles corresponding to the observed tracks have their momenta in the forward direction. Since the initial particle comes to rest before decaying there must be a neutral decay particle in the backward direction due to the conservation 
of momentum. Hence the initial hypernucleus must be $\Lambda^{0}+n+p$, i.e. ${ }_{4} \mathrm{H}^{3}$. In a similar manner, we can analyse other hypernuclei.

Analysis of hypernuclei consists in measuring the ranges of the decay products and balancing the energy and momentum assuming one or two recoil neutrons. In this way the energy of the decay is obtained and since the decay products are also known, the binding energies can be calculated.

The majority of the hypernuclei that have been studied experimentally so far are those with a $\Lambda^{0}$ replacing a nucleon. We know that the $\Lambda^{0}$ has been assigned

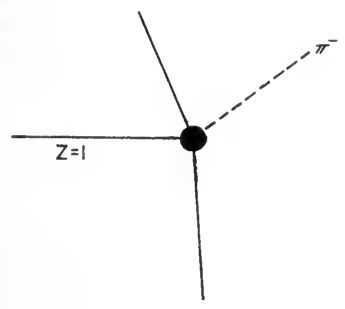

(A)

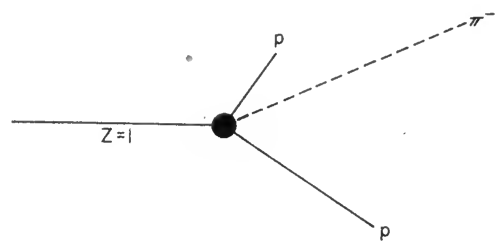

(B)

Fic. 44. A-hyperfragment decay.

the isotopic spin zero since it has no charged counterpart. Its spin is half integral and probably equal to half. We assume that the $\Lambda^{0}$-nucleon forces have a short range and are charge independent. By comparison of the energy levels of the lighter hypernuclei with those of ordinary nuclei it is found that the hypernuclei can be obtained by the simple addition of a $\Lambda^{0}$ to the appropriate nucleus. This means that the $\Lambda^{0}$ is not bound to the nucleons by very strong forces though they may be of the order of nuclear forces. It has been shown by Dalitz ${ }^{1}$ and Downs that the $\Lambda$ nucleon forces should be somewhat weaker than the nucleon-nucleon forces as a consequence of charge independence. The argument is as follows: If the $\Lambda$-nucleon forces are comparable to the nucleon-nucleon forces, then, since the Pauli principle will not be effective for a $\Lambda^{0}$ bound to a nucleus, the binding energy $B_{\Lambda}$ of the $\Lambda^{0}$ should be quite large compared to the corresponding binding energy $B_{n}$ of the neutron for which the Pauli principle provides an additional strong repulsion. But actually $B_{A} \ll B_{n}$ and hence the above conclusion. But this does not imply that the $\Lambda^{0}$ nucleon coupling is weaker than the nucleon-nucleon coupling since $\Lambda^{0}$-nucleon forces are probably of a shorter range and therefore less effective in binding. It is also found that the binding energy tends to increase with the mass number. The facts that the hyperfragments ${ }_{4} \mathrm{H}^{4}$ and ${ }_{A} \mathrm{He}^{5}$ exist and that the binding energy of the $\Lambda^{0}$ particle in ${ }_{A} \mathrm{Be}^{9}$ is greater than the binding energy of the last neutron in $\mathrm{Be}^{9}$ show that the Pauli principle does not apply for a $\Lambda^{0}$ in a nucleus.

1 R. H. Dalitz and B. Downs, Phys. Rev. 110, 958 (1958); 111, 967 (1958). These papers contain references to earlier works on hyperfragments. See also S. Twao, Progr. Theoret. Phys. Japan 13, 111 (1955). 
The low binding energies of the light hypernuelei imply that the $\Lambda^{0}$ spends much of its time outside the nucleus as is shown by the fact that in general the momenta of the $\Lambda^{0}$ (computed from those of $\pi^{-}$and $p$ ) are quite low.

Since the nucleon-mucleon forees are spin dependent it is interesting to investigate whether such spin dependent forces exist for a $\Lambda$-nucleon system. If such forces were to exist, then the $\Lambda$-nucleon system will be in the triplet or the singlet state (assuming the spin of the $\Lambda^{0}$ to be half). A convenient system for such an analysis is the hypernueleus ${ }_{\Lambda} \mathrm{He}^{5}$. The $\Lambda$ particle is loosely bound to the stable a-particle core. Taking up the shell model approach, to find the interaction potential we assume that the total potential is the sum of the average of potentials between the $A^{0}$ particle and the individual nueleons. We then have

$$
V_{A}\left(\boldsymbol{r}_{A}\right)=\sum_{N=1}^{4 \pi} \int V_{\Lambda-N}\left(\boldsymbol{r}_{A}-\boldsymbol{r}_{N}\right) \varrho\left(\boldsymbol{r}_{N}\right) \mathrm{d}^{3} \boldsymbol{r}_{N}
$$

where $V_{A-N}$ is the potential between the $\Lambda^{0}$ and a nucleon and $\varrho\left(r_{N}\right)$ is the nuclear density. Since the forces are assumed to be charge independent, we sum over all the nucleons. Assuming the range of the $\Lambda^{0}$-nueleon forces to be so small that we can write

we have

$$
V_{\Lambda-N}\left(r_{\Lambda}-r_{N}\right)=V_{\Lambda-N} \delta\left(r_{\Lambda}-r_{N}\right)
$$

$$
V_{\Lambda}\left(\boldsymbol{r}_{\Lambda}\right)=\sum_{N=1}^{4}\left(V_{A-N}\right) \varrho\left(\boldsymbol{r}_{A}\right)
$$

These approximations correspond to the situation where the $\Lambda$-particle is mostly outside the nucleus and 'feels' the nuclear forces only when it overlaps the nuclear matter.

With the above form of $V_{A}$ and an appropriate wave function for $\Lambda^{0}$, we can estimate $\langle T\rangle$ and $\langle V\rangle$, the mean kinetic and potential energies. Using the zero range function of the deuteron theory we can match the observed values of the binding energy values with the calculated ones so that we obtain a value for $u_{4}=\sum_{1}^{4} V_{\Lambda N}$. By this method, Dalitz and Downs ${ }^{1}$ have obtained $u_{4} \sim 660 \mathrm{MeV} \mathrm{f}\left(f=1 \times 10^{-13} \mathrm{~cm}\right)$, $Q\left(r_{N}\right)$ being assumed from experimental results on the electron scattering by $\mathbf{H e}^{4}$ nuclei. An overall error of ten to twenty per cent was expected from the nature of the approximation made for the wave function of ${ }_{\Lambda} \mathrm{He}^{5}$. In this case, we consider the interaction of $\Lambda^{0}$ with two nucleons having spins parallel to it and two having spins antiparallel to it. If the spin of the $\Lambda^{0}$ is half, then it can form triplet and singlet states with a nucleon depending on its spin orientation, i.e.,

$$
u_{4}=4\left(\frac{3}{4} u_{t}+1 / 4 u_{s}\right) \quad \text { and } \quad u_{a}=\frac{u_{4}}{4}=165 \mathrm{MeV} f^{3}
$$

where $u_{t}$ is the triplet potential and $u_{\delta}$ the singlet potential.

1 R. H. Dalitz and B. Dowss, loc. cit. 
Let us consider the hypernuclei ${ }_{A} \mathrm{He}^{4}$ and ${ }_{A} \mathrm{H}^{4}$ corresponding to the addition of a $\Lambda^{0}$ to $\mathrm{He}^{3}$ and $\mathrm{H}^{3}$ nuclei respectively. Analyses can be made similar to that of ${ }_{A} \mathrm{He}^{5}$ except that the core consists of a coupled pair of particles of opposite spins and an extra nucleon. The spin of the $\Lambda^{0}$ is either parallel or antiparallel to that of the extra nucleon. One of these orientations is favoured and the interaction of the $\Lambda^{0}$ with the unpaired nucleon in this favoured state is

$$
u_{f}=u_{3}-\frac{1}{2} u_{4}
$$

where $u_{3}$ is the potential of interaction of the $\Lambda^{0}$ with the three nucleons and $\frac{1}{2} u_{4}$ that with a pair.

Assuming the value of $720 \mathrm{MeV} f^{3}$ for $u_{3}$, we obtain $u_{f} \sim 390 \mathrm{MeV} \mathrm{f}$. The large difference between $u_{a}$ and $u_{f}$ shows that the $\Lambda^{0}-N$ forces are spin-dependent. Similar calculations have been carried out for ${ }_{A} \mathrm{Li}^{7}$ giving consistent results. This explains also why ${ }_{A} \mathrm{H}^{3}$ is more tightly bound than ${ }_{A} \mathrm{He}^{3}$. Only in ${ }_{A} \mathrm{H}^{3}$ can both the nucleons have spins in the favoured direction with respect to the $\Lambda^{0}$ spin. But this does not confirm that the spin of the $\Lambda^{0}$ is half and also it has not been possible to find the relative directions of the spins of the $\Lambda^{0}$ and the nucleons.

To determine whether the favoured orientation for a $\Lambda$ nucleon system is a singlet or a triplet state we consider the simplest hypernucleus ${ }_{\Lambda} \mathrm{H}^{3}$. The protonneutron part is assumed to be a deuteron, i.e. in the triplet state and the trial wave function used is

$$
\psi=\mathrm{e}^{-\alpha\left(r_{1}+r_{2}\right)} \mathrm{e}^{-\beta d}
$$

with $\alpha$ and $\beta$ as variational parameters and $\mathrm{e}^{-\beta d}$ the deuteron wave function in the triplet spin state. It is to be noted that we have been able to assume such a wave function since the $\Lambda^{0}$ is loosely bound to the other two nucleons. This approximation will not be valid for a three nucleon system such as $\mathrm{H}^{3}$ or $\mathrm{He}^{3}$. To decide between the two spin orientations consider the cases when:

\section{(A) All spins are parallel}

The binding potentials should satisfy the condition $u_{t}>u_{s}$ and there is only one potential to be defined from the experiment since all pairs are in the triplet state. We have essentially an effective range theory as in the $S$ wave scattering in low energy physics. Solving for the scattering amplitude with different potential shapes and ranges the variation is only \pm 10 per cent. The effective range $\alpha_{t}$ is found to be negative. Hence there is no bound state for the two-body system. The results for thermal scattering cross-sections $\left(\sigma_{t} \sim 1\right.$ barn) are consistent with experimental results. For a Yukawa well the best range is $\sim \hbar / 2 m_{\pi} c$ which is consistent with the idea that the force arises from the exchange of two pions.

(B) The $\Lambda$-spin is opposite to the deuteron spin

The singlet state for the $\Lambda$-nucleon system is favoured here and the potential is not purely $u_{s}$ since the deuteron system is in the triplet state. Hence there is no unique solution and only relative magnitudes can be found. The limiting factor is that the ${ }_{1} H^{2}$ is unbound and hence the range cannot be too small. 
Another method of dealing with this problem is to write down relations between the total volume integrals and the average parameters of two body interactions for the hyperfragments ${ }_{A} \mathrm{H}^{3},{ }_{A} \mathrm{H}^{4},{ }_{A} \mathrm{He}^{4}$ and ${ }_{A} \mathrm{He}^{5}$ for both parallel as well as anti-parallel spin orientations. These relations can be written as:

$$
\begin{aligned}
& u_{2}=T_{A}+\frac{1}{2} u_{t}+3 / 2 u_{s} \\
& u_{3}=T_{A}+\frac{3}{2} u_{t}+\frac{3}{2} u_{s} \\
& u_{4}=T_{A}+3 u_{t}+u_{8} . \\
& u_{2}=T_{A}+2 u_{t} \\
& u_{3}=T_{A}+\frac{5}{2} u_{t}+\frac{1}{2} u_{s} \\
& u_{4}=T_{A}+3 u_{t}+u_{s} !
\end{aligned}
$$

By this analysis it has been found by Sudarshan ${ }^{1}$ that the values of $T_{A}$ obtained from antiparallel spin orientations are consistent with those obtained by other methods, whereas the equations form an inconsistent set for parallel spin orientations and hence have no solution at all. This leads to the conclusion that antiparallel spins are favoured and in particular that the ${ }_{\Lambda} \mathrm{He}^{4}$ spin is zero. This has the important consequence of establishing the $K-\Lambda$ relative parity to be odd since the mesonic absorption of $K^{-}$in $\mathrm{He}^{4}$, i.e. $K^{-}+\mathrm{He}^{4}, \rightarrow{ }_{\Lambda} \mathrm{He}^{4}+\pi^{-}$ has been observed.

So far we have been considering only two-body $\Lambda-N$ forces. Three-body forces may also play an important role due to the longer range. In the simplest case the $\Lambda$ particle in the hyperfragment can exchange two pions with two neighbouring nucleons. In such a case the form of the potential will be

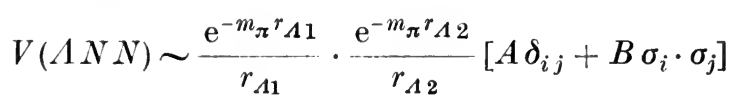

where $r_{\Lambda 1}$ and $r_{A_{2}}$ are the distances between the $\Lambda$ and the nucleons 1 and 2 respectively, and $i$ and $j$ the spin state of the two nucleons and hence that of the intermediate particle (i.e. $\Sigma$ ) at the two vertices. Since $i$ and $j$ can be interchanged the spin-dependent term $B \sigma_{i} \cdot \sigma_{j}$ in the potential drops out. Thus we have

$$
V(\Lambda N N)=\frac{\mathrm{e}^{-m_{\pi} r_{11}}}{r_{\Lambda 1}} \frac{\mathrm{e}^{-m_{\pi} r_{\Lambda 2}}}{r_{\Lambda 2}} \sigma_{1} \cdot \sigma_{2} \tau_{1} \cdot \tau_{2}
$$

as the $\tau-N$ interaction is proportional to $\sigma \cdot \tau$. This is true only for the long range part of the three-body forces. It has the simple property that (i) it does not depend on the spin of the $\Lambda$ (ii) $\sigma_{i} \cdot \sigma_{j} \tau_{i} \cdot \tau_{j}=-3$ for two nucleons in a relative $S$ state.

1 E. C. G. Sudarshan and S. Iwao Phys. Rev. Lett. 4, 140, (1960) 
The binding energies for light hypernuclei have been calculated including three-body forces. It has been suggested that the potential arising from threebody forces is repulsive. This might possibly explain the fact that the potentials $V(A-1)$ of hypernuclei do not increase linearly with the mass number $A$.

\section{Decay modes of hypernuclei}

One of the methods of determining the spin of the $\Lambda^{0}$ is to observe the decay modes of hypernuclei. They are classified into modes in which

(i) a $\pi^{-}$is emitted due to the $\Lambda^{0}$ decay.

(ii) no $\pi^{-}$is emitted. It is assumed that the $\pi^{-}$emitted in the $\Lambda^{0}$ decay is absorbed by a nucleon by a process analogous to the internal conversion process in $\beta$ decay. The internal conversion coefficient $Q$ is defined as the ratio of the number of nonmesonic to mesonic decays.

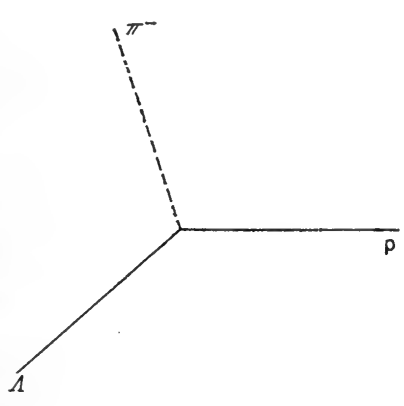

(a)

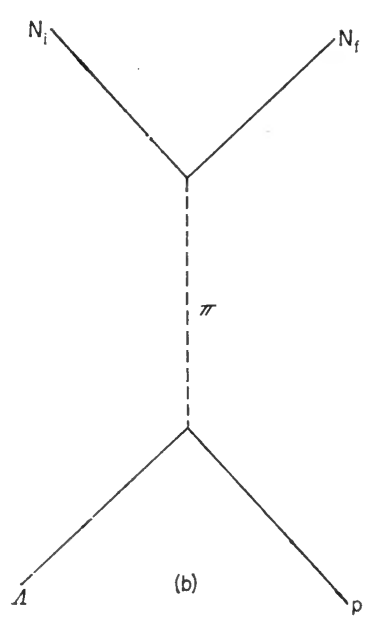

(b)

Fig. 45. Decay modes of $\Lambda$-nuclei

(a) Mesonic decay; (b) Non-mesonic decay.

The experimental results show a smooth transition from the mesonic to the non-mesonic decays. The internal conversion coefficient $Q$ depends strongly on the spin of the $\Lambda^{0}$. Lee and Yang ${ }^{1}$ have given arguments from angular momentum considerations that the spin of the $\Lambda^{0}$ should be $1 / 2$. An approximate method of determining the spin from a rough calculation based on the analysis of the decay of hyperfragments is as follows:

In an electromagnetic field, at large distances (i.e. in the wave zone), the potential has the form $V_{\text {wave }} \sim \frac{\mathrm{e}^{i k r}}{r}$ while in the near-zone $V_{\text {near }} \sim\left(\frac{1}{k r}\right)^{l} \frac{1}{r}$. For higher multipole moments $V_{\text {near }}$ increases very rapidly near the origin. Conversion takes

1 T. D. Lee and C. N. YANG, Phys. Rev. 109, 1755 (1958). 
place by interaction through the potential $\bar{V}_{\text {near }}$ whereas emission takes place only by radiating a wave asymptotically to great distances. The internal conversion coefficient $Q$ is equal to the ratio of the two amplitudes. Carrying this analogy to the pion field we find that the energy required for $\pi^{-}$emission is less than that for internal conversion. The momentum $k_{c}$ for internal conversion is of the order of $450 \mathrm{MeV}$ c and the momentum $k_{0}$ for emission is of the order of $95 \mathrm{MeV} / \mathrm{c}$. For a given $l$ therefore $Q \propto\left(\frac{k_{c}}{k_{0}}\right)^{2 l} \times f(l) \sim(4 \cdot 5)^{2 l}$ where $f(l)$ is the normalization factor. $Q$ also depends on the hypernuelear structure and in the simplest approximation is proportional to the probability of finding $\Lambda$ near the nucleon, i.e. $\left|\psi^{\prime} .1(0)\right|^{2}$. Because of the small binding we can take the wavelength of the hyperon to be large and the wave function for simplicity may be taken as

$$
\psi=\frac{1}{\lambda^{3 / 2}} \frac{\mathrm{e}^{-r / \lambda}}{r / \lambda}
$$

(where $\lambda$ is the Compton wavelength of $\Lambda^{0}$ ).

We find that $Q=\frac{R}{\lambda}$ where $R$ is the nuclear radius, and since we are integrating $\left|\psi_{A}(0)\right|^{2}$ over the nuclear sphere we obtain $\frac{1}{\lambda}=\frac{\hbar}{\left(2 m_{\Lambda} E_{B A}\right)^{1 / 2}}$ with $E_{B A}=$ binding
energy of $A$. Hence we have $Q \propto\left(E_{B A}\right)^{1 / 2}$

For ${ }_{A} \mathrm{He}^{4}$, the comparison of calculated and experimental values of $Q^{-}$(for which $A^{0} \rightarrow p+\tau^{-}$alone is considered) shows that $l=0$ or 1 , i.e. the $\pi^{-}$is emitted as an $S$ or a $P$ wave, higher moments being ruled out.

There are corrections to be made in the above calculations due to (1) all the real mesons produced not escaping because of the probability of internal selfabsorption, and (2) the $\Lambda^{0}$ not being "free" as it is assumed to be. Corrections due to the first effect have been shown to be unimportant. Calculations of these corrections have been done only in a qualitative fashion and there is no quantitative agreement with experimental results.

From these results, Dalitz ${ }^{1}$ has shown that the following possibility exists. Since $S_{p}=1 / 2$ and $S_{\pi}=0$ the final state should correspond to $l=0$ or 1 . The distinction between these gives the relative parities of the $\Lambda^{0}$ and $p$. A mixture of the two states $l=0$ and 1 is also allowed because of the possibility of the nonconservation of parity. Calculations of Karplus and Ruderman ${ }^{2}$ yield

$$
Q^{-}=\frac{Q_{0}^{-}+\varepsilon Q_{1}^{-}}{1+\varepsilon} \quad \text { with } \quad \varepsilon=0.1
$$

$Q_{0}^{-}$corresponds to the case $l=0$ and $Q_{1}^{-}$to $l=1$, the negative sign referring to $\pi^{-}$ emission. Thus the $S$ wave predominates though the $P$ wave is not excluded since

${ }^{1}$ R. H. Dalitz, Phys. Rev. 112, 605 (1958). See also the report by R. H. Dalitz on hyperon-nucleon interactions, International Conference on High Energy Physics, Kiev (1959).

2 R. Karplus and M. Ruderman, Phys. Rev. 102, 247 (1956). 
parity is not conserved. This idea can be checked by considering specific decay modes such as

$$
{ }_{\Lambda} \mathrm{H}^{4} \rightarrow \mathrm{He}^{4}+\pi^{-} \text {. }
$$

If the spin of the hypernucleus is $J$, then $\pi$ has $l=J$ (since the spin of $\mathrm{He}^{4}$ is zero). If $J=1$ is a weak channel for the $\Lambda$-decay this will rarely occur. For the other decay mode ${ }_{\Lambda} \mathrm{H}^{4} \rightarrow \mathrm{H}^{3}+p+\pi^{-}$other possibilities exist. Experimentally ten of the former have been observed as against seven of the latter decay modes. So $\pi^{-}$has a high probability of being emitted in the dominant channel $J=0$ for ${ }_{4} \mathrm{H}^{4}$. Hence the singlet state is a bound state for ${ }_{\Lambda} \mathrm{H}^{4}$. We can also show that $\pi$ is emitted in the $S$ state in the decay ${ }_{\Lambda} \mathrm{H}^{3} \rightarrow \mathrm{He}^{3}+\pi^{-}$. Similar reasonings favour the following conclusions:

(1) The spin of the $\Lambda^{0}, S_{\Lambda}=1 / 2$,

(2) $\Lambda^{0}$ decays with mixed parities, the $S$ state being predominant,

(3) $\Lambda^{0}$ is bound to the nuclei with a favoured singlet orientation.

Lifetimes of hypernuclei have been measured and these can be used to verify our assumptions since the calculation that gives the conversion coefficient should also give the total (conversion + radiation) lifetime.

So far we have not considered hypernuclei having bound hyperons other than a $\Lambda^{0}$ such as $\Sigma^{ \pm, 0}$ or $\Xi^{-}$. The $\Sigma^{\prime}$ 's can decay rapidly by exchanging pions with nucleons without changing their strangeness. Therefore stable nuclei can only be formed by $\Sigma^{+}$particles with protons and $\Sigma^{-}$with neutrons. But the formation of the $\left(\Sigma^{+}, p\right)$ system is difficult because of Coulomb repulsion. The system $\left(\Sigma^{+}, n\right)$ making a collision in flight with a nucleus is believed to have been observed although the interpretation of the event is quite complicated. ${ }^{1}$

Two events have been identified where the disintegration energy is considerably greater than that of the $\Lambda^{0}$ decay. The energy shows that the unstable particle can be bound to the nucleus for times greater than $10^{-11}$ secs. This leads to two possibilities, (i) bound charged hyperon, (ii) bound $K$-particle. In the first case the decay scheme $\Xi^{0} \rightarrow \Lambda^{0}+\pi^{-}$leads to a disintegration energy of about $380 \mathrm{MeV}$ which is oonsiderably greater than that due to the decay of a $A^{0}$ particle. In the second case the energy available from the absorption of a bound $K$ particle is $496 \mathrm{MeV}$.

\section{RELATIVE PARITY BETWEEN STRANGE PAR'TICLES ${ }^{2}$}

In an earlier chapter we merely summarized the experimental data available on the strong interactions of strange particles, particularly those of $K^{+}, K^{-}$and $\Lambda^{0}$. We will now describe the attempts to understand these data from a theroretical study of strong interactions. Since there is no single theory of strong interactions which adequately explains the strange particle phenomena, we shall briefly refer

1 R. H. Dalitz, loc. cit., International Conference on High Energy Physics, Kiev (1959).

2 This section was written in a rather detailed manner at the suggestion of Dr. T. Kotani while he was on a visit to Madras.

EPCR 20 
to the salient features of the various attempts to understand the strong interactions.

The first step in the study of any interaction is to postulate or derive the Lagrangian or Hamiltonian for the interaction between the particles. In order to be able to write down the interaetion Lagrangian for $\pi$-baryon and $K$-baryon interactions we should know the relative parities of the strange particles with respect to the nucleons. We have already seen how through $\pi-d$ reactions $\pi$ is shown to be a pseudoscalar with respect to the nucleon. Since parity is not a conserved quantity in weak interactions the parity of the final system after a decay reaction cannot be equated to that of the initial system. Thus we have to deduce the parity of the strange particles only from the strong and electromagnetic interactions. The parity of a strange particle relative to the nucleon can be determined if we can have a reaction in which one strange particle takes part in the reaction forming part of the initial or final system. However conservation of the strangeness quantum number in the strong and electromagnetic interactions demands that more than one strange particle be involved. We recall that the associated production of strange particles is a typical example. For these interactions only the relative parity of one strange particle with respect to another has a meaning. It is easy to see that we need only the information on the relative parities $p_{K .1}, p_{K \Sigma}, p_{A \Sigma}, p_{K K}$ and $p_{N \Sigma}$. We will discuss now the various proposals made to determine these parities. ${ }^{1}$

Before doing so we shall refer to a difficulty in defining uniquely the relative parities $^{2}$ of the particles, attention to which was originally drawn by Wick, Wightman and Wigner. ${ }^{3}$ Since the parity of a field is essentially a phase factor under $P$. we can define the relative parity between two states only when we can measure the relative phase of the two states, i.e. the two states form a coherent system to which the quantum mechanical principle of superposition of states applies. This in turn implies the existence of an observable which has a nonvanishing matrix element between them. If all the states of a system can be grouped into different sets such that we can find no observable having a non-vanishing matrix element between any state drawn from one set and any state dirawn from another set, a transition between them is forbidden completely, and this is known as a super-selection rule. A typical example of a super-selection rule is provided by the total charge operator, for we cannot conceive of any observable (e.g. total energy and angular momentum. operators) that has a non-zero matrix element between states of different total charge. This feature is a consequence of charge conservation in all the known interactions.

1 See the review article "Some Topics Regarding the Strong Interactions of Strange Particles" by D. Amati and B. Vitale, Fortschr. der Physik 7, 375 (1959). This paper also contains an extensive bibliography on this subject.

2 The parity referred to here is achieved by means of a suitable choice of phase to provide for the invariance of the strong and electromagnetic interactions.

3 G. C. Wick, A.S. Wightman and E. P. Wigner, Phys. Rev. 88, 101 (1952). 
Whenever a super-selection rule exists it is impossible to define the relative parity uniquely and the definition of it becomes a matter of convention. To see this, we recall that charge conservation implies that the interactions are invariant under a phase transformation (i.e. gauge transformations of the first kind). - Invariance under such a phase transformation necessarily means that the phase of the field operators and consequently the parities are unobservable. We wish to remind ourselves that for invariance under phase transformations the interaction Lagrangian should contain only terms like $\varphi^{\dagger} \varphi, \psi^{\dagger} \psi$. Thus the above discussion shows that for a group of particles obeying a gauge transformation, the relative parity can be defined only when we fix arbitrarily the parity of one particle from the group. However for a particle which does not belong to this group we can define a parity which is independent of the convention used for the particles in the group.

Besides charge conservation, we have also baryon, lepton and hypercharge (or strangeness) conservations which also necessarily imply the existence of super-selection rules for the observables we normally use. These conservation laws can lead to another possibility that several gauge groups may exist with common members. In this circumstance we can adopt the following procedure. If $A$ and $B$ are two gauge groups of particles having common members, we can for the purpose of parity consider the two gauge groups $A$ and $B^{\prime}$ where $B^{\prime}$ contains all the particles in the original $A$ and $B$ after removing the common members, for, as a convention, we can adopt an identical phase for the common members of both the groups. Once we arrive at $A$ and $B^{\prime}$ we can adopt the usual procedure. This rearrangement idea can be easily extended to any number of overlapping gauge groups. The particles belonging to an isomultiplet have the same intrinsic parity for they are treated as different components of the same field and thus the relative parities within an isotopic multiplet should be even.

The above discussion implies the following conclusions regarding the intrinsic parities of the elementary particles. The vacuum is assumed to have even parity.

(1) The electromagnetic field can be realised classically and thus the correspondence principle requires that the phase of the electromagnetic field should be so chosen that the classical theory should be left invariant under $P$. Since $\boldsymbol{A}$ is a polar vector, the photon has odd parity.

(2) The intrinsic parity of $\pi^{0}$ can in principle be determined if we can measure the polarizations of the two gamma rays emitted when it decays. ${ }^{1}$

(3) The relative parity between $\Sigma^{0}$ and $\Lambda^{0}$ can be determined by means of the reaction $\Sigma^{0} \rightarrow \Lambda^{0}+\gamma$ (see below).

(4) All the other relative parities can be determined only by making further assumptions. The usual assumption is that $p, n$ and $\Lambda^{0}$ have even relative parity. As noted before charge independence helps us to fix the parities of particles within an isomultiplet. Thus the $\pi^{+}$and $\pi^{-}$parity can be chosen through that of the $\pi^{0}$.

1 L. Wolfenstein and D. G. Ravenhall, Phys. Rev. \$8, 229 (1952). 
(5) The electrons and $\mu$-mesons have only electromagnetic interactions. Due to lepton conservation, the total number of electrons and $\mu$-mesons must be conserved and they must also be separately eonserved to prevent strong $\mu \rightarrow e$ transformations. Thus even this interaction is subject to a superselection rule which allows us to conclude that the $\mu-e$ relative parity and the relative parities of $\mu$ and $e$ with respect to other particles have no physical significance. In all these discussions we have neglected the weak interactions the inclusion of which will render the definition of parity meaningless.

Relative parity $p_{K A}$ and $p_{K \Sigma}$

There exist "in the air" many suggestions to determine $p_{K A}$ and $p_{K \Sigma}$ (which will hereafter be denoted as $p_{K Y}$ where $Y$ can refer to $\Lambda$ or $\Sigma$ ). In what follows the even and odd $p_{K A}$ will correspond to "sealar" and "pseudoscalar" $K$-mesons respectively. Let us now consider these suggestions based upon (a) production, (b) scattering and (c) absorption processes.

(a) Production processes. Associated production by pions in $\mathrm{He}^{4}$ can be studied by the passage of a pion beam through a bubble chamber. We have

$$
\pi^{+}+\mathrm{He}^{4} \rightarrow{ }_{\Lambda} \mathrm{He}^{4}+K^{+} .
$$

As the reaction indicates the produced $\Lambda^{0}$ is absorbed by $\mathrm{He}^{3}$ nucleus to form the hyperfragment ${ }_{\Lambda} \mathrm{He}^{4}$. This process offers a very good indication regarding $p_{K} \boldsymbol{A}$ as was first observed by Sakurai. ${ }^{1}$ Let us assume that ${ }_{1} \mathrm{He}^{4}$ has spin 0 and even parity since the $\Lambda-N$ relative parity is even by assumption. Since $\mathrm{He}^{4}$ has spin 0 and even parity, the parity of the initial system, i.e. $\pi$ and $\mathrm{He}^{4}$ is given by $p=-(-1)^{l}$ where $l$ represents the orbital angular momentum and the total angular momentum equals the orbital angular momentum. From conservation of total angular momentum, it follows that the orbital angular momentum of the final state is also $l$ remembering that ${ }_{\Lambda} \mathrm{He}^{4}$ and $K^{+}$are spinless. If $p_{K \Lambda}$ is even, the parity of the final state will be given by $(-1)^{l}$ in which case the reaction cannot take place. Thus the observation of hyperfragments in $\pi^{+}+\mathrm{He}^{4}$ reaction clearly indicates that $p_{K A}$ is odd, i.e. $K$-meson is pseudoscalar like the $\pi$-meson.

Photoproduction of $K$-mesons is represented by the reactions

$$
\gamma+N \rightarrow K+Y \text {. }
$$

The experiments indicate that the angular distribution of $K^{+}$from $\gamma+p \rightarrow \Lambda^{0}+K^{+}$does not deviate significantly from isotropy though there is a rough indieation of a small peak in the region around $90^{\circ}$. The cross-sections for $\gamma+N \rightarrow \Sigma+K$ and $\gamma+N \rightarrow \Lambda+K$ do not differ very much at comparable energies. We know very little about the photoproduction of $K^{\mathbf{0}}$. Except for the lowest order perturbation ealculations ${ }^{2}$ no theoretical study of the problem has

1 J. Sakurai, Phys. Rev. 107, 1119 (1957).

2 A. FuJil and R. E. Marshak, Phys. Rev. 107, 570 (1957). 
been made so far. However if we assume only $S$ and $P$ waves exist in the final state, we can reduce the number of multipoles that contribute to the reaction. ${ }^{1}$ We give below in a table a list of all the allowed initial and final states.

\begin{tabular}{c|c|c|c}
\hline Multipole & $J$ & $p_{K Y \text { even }}$ & $p_{K Y \text { odd }}$ \\
\hline$E_{1}$ & $1 / 2$ & $P_{1 / 2}$ & $S_{1 / 2}$ \\
$E_{1}$ & $3 / 2$ & $P_{3 / 2}$ & - \\
$M_{1}$ & $1 / 2$ & $S_{1 / 2}$ & $P_{1 / 2}$ \\
$M_{1}$ & $3 / 2$ & - & $P_{3 / 2}$ \\
\hline
\end{tabular}

Here $E_{1}$ and $M_{1}$ refer to the electric and magnetic dipole transitions. As in the case of the photoproduction of $\pi$ 's, the neutral $K^{0}$ can only be produced directly by the nucleons ("shaking off" transitions) and hence they cannot change the orbital angular momentum by direct interaction with the electromagnetic field. Thus for even $p_{K Y}, K^{0}$ will be produced in an $S$ state while for odd $p_{K Y}, K^{0}$ will be in a $P$-state. This feature implies that near the threshold the total cross-section will vary as $p_{k}$ and $p_{k}^{3}$ in the two cases respectively where $p_{k}$ is the momentum of the $K$-meson in the laboratory system while the differential cross-section will be isotropic for even $p_{K \Lambda}$ and will have the form $A+B \cos ^{2} \theta$ for odd $p_{K \Lambda}$. At present very little experimental work has been done on photo- $K^{0}$ production. Further it has been shown that if we take into account the anomalous magnetic moments of the fermions involved in the reaction, the distinction between the features of the angular distribution of scalar and pseudoscalar photoproduction of $K$ becomes less pronounced. ${ }^{2}$ Recently Moravcisk ${ }^{3}$ has adopted the extrapolation procedure (see below the discussion on $p_{K K}$ parity) used to determine the pion-nucleon coupling constant from pion photoproduction ${ }^{4}$ to the photo production of $K$ in order to determine the parity of the $K$-meson. This suggestion depends on the fact that the sign of the residue is positive for pseudoscalar $K$-mesons and negative for scalar $K$-mesons. The analysis of the $K^{+}-\Lambda^{0}$ photoproduction between $100-1000 \mathrm{MeV}$ seems to favour the pseudoscalar $K^{+}$-meson.

Associated production in $\pi-N$ collisions can yield us independent information on $p_{K \Lambda}$ and $p_{K \Sigma}$. Consider the process

$$
\pi^{-}+p \rightarrow \Lambda^{0}+K^{0}
$$

At the threshold the $\Lambda^{0}+K^{0}$ will be produced in the $S$ state and the $P$ state respectively for the two cases of even and odd $p_{K A}$. It is clear that only $P_{\frac{1}{2}}$ and

1 D. Amati and B. Vitale, Nuovo Cim. 6, 395 (1957).

2 M. Kawaguchi and M. J. Moravcisk, Phys. Rev. 107, 563 (1957).

3 M. J. Moravcisk, Phys. Rev. Lett. 2, 171 (1959).

4 J. G. Taylor, M. J. Moraycisk and J. L. Uretsky, Phys. Rev. 113, 689 (1959). 
$S_{1}$ waves of the initial system will be affected by the production in the two cases respectively. Thus we can expeet the $\pi^{-}-p$ seattering to exhibit an anomalous behaviour (i.e. cusp phenomena) at the threshold for $A^{0}$ production. This effect was estimated by Adair ${ }^{1}$ to be 10 per cent of the $\pi^{-}-p$ differential cross-section. We will discuss presently the anomalous behaviour in a process near the threshold of another process in detail when we study the suggestions made to determine $\Sigma-N$ relative parity. Experiments can also help in deciding whether the $S_{\frac{1}{2}}$ or $P_{1_{2}}$ wave is affected. However we know that the $\pi^{-}-p$ cross-section has a

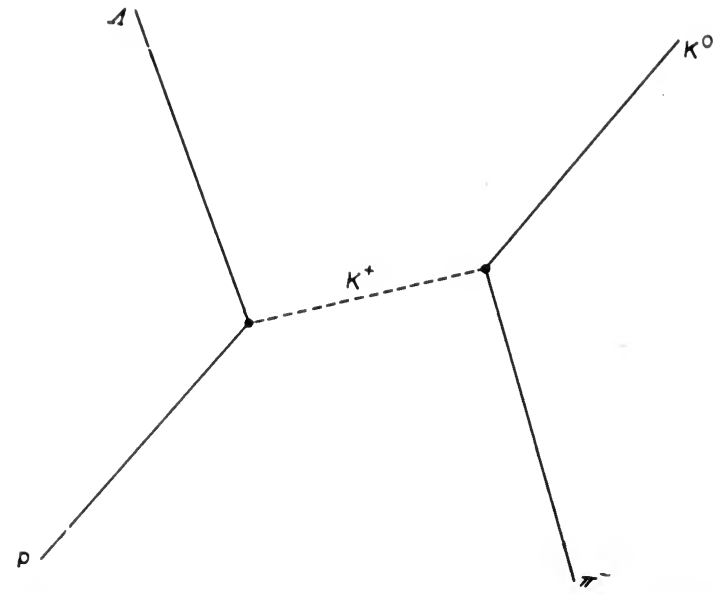

FIG. 46. Associated production in $\pi^{-}-p$ collision involving direct $K-K-\pi$ interaction.

rather broad maximum near $900 \mathrm{MeV}$ and thus there seems to be no anomalous effect.

Another possibility of using this production process has been pointed out and is based on the fact that the angular distribution of the produced hyperon depends on the sign of $p_{K Y^{r}}{ }^{2}$. To realise this, we note that the transition matrix in the c.m. system will have the form

$$
\begin{aligned}
T & =A+B \boldsymbol{\sigma} \cdot\left(\boldsymbol{p}_{\pi} \times \boldsymbol{p}_{K}\right) & & p_{K Y}=\text { odd } \\
& =C \boldsymbol{\sigma} \cdot \boldsymbol{p}_{\pi}+D \boldsymbol{\sigma} \cdot \boldsymbol{p}_{K} & & p_{K Y}=\text { even }
\end{aligned}
$$

where $\boldsymbol{\sigma}$ is the baryon spin operator and $\boldsymbol{p}_{\boldsymbol{\tau}}$ and $\boldsymbol{p}_{\boldsymbol{K}}$ are the momenta of the $\pi$ and $K$ mesons respectively in the c.m. system. The expressions are derived using considerations based on parity conservation. Remembering that $\pi$ is pseudoscalar, $T$ must be scalar for $p_{K Y}=$ odd and pseudoscalar for $p_{K Y}=$ even since $T$ connects

1 R. Adair, Phys. Rev. 111, 632 (1958).

2 D. Amati and B. Vitale, Nuovo Cim. 9, 340 (1958); R. H. Capps, Phys. Rev. 115,736 (1959). 
the $\pi-p$ and $K-\Lambda$ states. $A, B, C$ and $D$ are scalar coefficients and are complex functions of $\boldsymbol{p}_{\pi} \cdot \boldsymbol{p}_{K}$ and the energy $\omega$. We can now adopt Watson's ${ }^{1}$ theorem which expresses the photoproduction amplitude in terms of the pion-nucleon phase shifts provided we can treat the $K-Y$ interaction as weaker than the $\pi-N$ interaction, i.e. we can neglect the final state interaction. However in the energy region where $\Lambda^{0}$ production becomes possible, the phase shift analysis becomes difficult since the phase shifts themselves become complex due to the opening of inelastic channels and also because of the increase in the number of partial waves of angular momentum making significant contributions to the reaction. Using the density matrix formalism we can express the initial polarization in a direction $\boldsymbol{n}$, if $\varrho_{i}$ is the density matrix for the initial state, as

$$
\varrho_{i}(\boldsymbol{n})=S p\left[\boldsymbol{\sigma} \cdot \boldsymbol{n} \varrho_{i}\right],
$$

where $S p[A]$ indicates the spur of the matrix $[A]$. Also we have normalized the initial state such that $S p\left[\varrho_{i}\right]=1$. The density matrix for the final state $\varrho_{f}$ is given as a function of the energy $\omega$ and the production zenith and azimuth angles $\theta$ and $\varphi$ :

$$
\varrho_{f}(\omega, \theta, p)=T(\omega, \theta, \varphi) \varrho_{i} T^{\dagger}(\omega, \theta, \varphi) .
$$

The differential cross-section and final polarization in a direction $\boldsymbol{m}$ are given by

$$
\begin{aligned}
\frac{\mathrm{d} \sigma}{\mathrm{d} \Omega}(\omega, \theta, \varphi) & =S p\left[\varrho_{f}(\omega, \theta, \varphi)\right] \\
\varrho_{f}(\omega, \theta, \varphi) & =\frac{S p\left[\boldsymbol{\sigma} \cdot \boldsymbol{m} \varrho_{f}(\omega, \theta, \varphi)\right]}{S p\left[\varrho_{f}(\omega, \theta, \varphi)\right]} .
\end{aligned}
$$

Using these formulae, we can obtain the differential cross-section and final polarization of $\Lambda^{0}$ whose measurement can serve to distinguish between the two cases of scalar and pseudoscalar $K$. In passing we note that the $\Lambda^{0}$ can be polarized only in a direction normal to the production plane defined by $\boldsymbol{p}_{\pi}$ and $\boldsymbol{p}_{K}$ due to parity conservation. Capps ${ }^{2}$ has noted an interesting situation namely that if the nucleon polarization direction $\boldsymbol{n}$ is perpendicular to the incident $\pi$ beam, then the transverse spin-spin correlation can serve to determine $p_{K A}$. More precisely we have in the forward (or backward) direction

$$
\begin{aligned}
& p_{f}(\boldsymbol{n})=p_{\imath}(\boldsymbol{n}) \quad p_{K \Lambda} \text { odd } \\
& p_{f}(\boldsymbol{n})=-p_{i}(\boldsymbol{n}) \quad p_{K \Lambda} \text { even } .
\end{aligned}
$$

The $\Lambda$ polarization direction may be obtained from the asymmetry in the $\Lambda$ decay, the sign of the asymmetry being known from the experiment of Boldt et al. ${ }^{3}$ Another method of deciding $p_{K A}$ is to perform experiments with polarized

${ }^{1}$ K. M. Watson, Phys. Rev. 95, 228 (1954).

2 R. H. Capps, loc. cit. See also, A. P. Balachandran and N. R. Ranganathan, Nuovo Cim. 16, 1142 (1960).

${ }^{3}$ BoLdt et al., Phys. Rev. Lett. 1, 256 (1958). 
and unpolarized targets. It can be shown that the polarization of $\Lambda^{0}$ when the target is unpolarized is given by the left-right asymmetry in the differential cross-section for $A^{0}$ production resulting from a polarized target, but the sign of the polarization depends on $p_{K A} \cdot{ }^{1}$ The result is given by

$$
\frac{\mathrm{d} \sigma}{\mathrm{d} \Omega}(\theta, \varphi)=\frac{\mathrm{d} \sigma_{0}}{\mathrm{~d} \Omega}(\theta, \varphi)\left[1 \pm p_{i}(\theta, \varphi) p_{0}(\theta, \varphi)\right]
$$

where $\frac{\mathrm{d} \sigma_{0}}{\mathrm{~d} \Omega}$ and $p_{0}$ denote the differential cross-section and final polarization using an unpolarized target while $\frac{\mathrm{d} \sigma}{\mathrm{d} \Omega}$ and $p_{i}$ refer to the differential cross-section obtained when the target has polarization given by $p_{i}$. The plus and minus signs refer to odd and even $p_{K A}$ cases respectively.

Associated production in the $N-N$ collisions can also be analysed using parity and angular momentum conservation. As before for the two $p_{K A}$ cases, we will have different orbital angular momenta for the final states. Thus studying the momentum dependence of the total cross-section and the angular distribution, we can hope to distinguish between the two $p_{K A}$ cases. The present data for this process are so meagre that we will not consider this any further. ${ }^{2}$

(b) Scattering experiments. We shall now study the $K-N$ and $\bar{K}-N$ scatterings with a view to deciding $p_{K A}$. We shall envisage the scattering to occur through the strangeness-conserving Yukawa interactions between $K$-mesons and other baryons. Later when we discuss the symmetries of the strong interactions we shall write them down explicitly. We shall merely indicate what can happen at a vertex, i.e.

$$
N \leftrightarrow \Lambda+K ; \quad N \leftrightarrow \Sigma+K ; \quad \Lambda \leftrightarrow \Xi+K ; \quad \Sigma \leftrightarrow \Xi+K .
$$

In order to see the influence of $p_{K A}$, we observe that for a scalar $K$, the final $A+K$ will be in an $S$ state at the threshold. In a similar way, for a pseudoscalar $K$, it will be in a $P$ state. Thus the corresponding interaction Lagrangian will be different in the two cases. The first will not involve a $\gamma_{5}$ while the second will. A simplified model treating the nucleon as a fixed heavy source and also neglecting the contribution from the virtual $\bar{K}$ and $\pi$ from the intermediate states for the $K-N$ scattering has been studied using the Tamm-Dancoff approximation. ${ }^{3}$ Neglect of the nucleon recoil is certainly unjustifiable in the $K-N$ scattering unlike the $\pi-N$ scattering. The study throws practically no light on $p_{K Y}$.

The dispersion relations technique has been used for the determination of $p_{K \boldsymbol{A}}$ and $p_{K \Sigma}$ and the corresponding coupling constants $g_{K \Lambda}$ and $g_{K \Sigma}$. Disregarding for the moment the difficulty in proving the dispersion relations for $K^{ \pm}-N$ scattering because of the possible breakdown of the necessary relation between

1 S. M. Bilenkil and R. M. Ryndin, J. Expt. Theoret. Phys. 35, 827 (1958).

2 G. Costa and B. T. Feld, Phys. Rev. 109, 606 (1958).

3 D. Amati and B. Vitale, Nuovo Cim. 5, 1533; 6, 261 (1957). C. Ceolin and N. Taffara, Nuovo Cim. 6, 425 (1957). 
$K$ and $\Lambda$ masses, the dispersion relations for the $K$-proton forward scattering can be written down as follows. ${ }^{1}$

$$
\begin{aligned}
D_{+}(\omega)= & p_{K A} \frac{X(\Lambda)}{\omega_{\Lambda}+\omega}+p_{K \Sigma} \frac{X\left(\sum\right)}{\omega_{\Sigma}+\omega} \\
& +\frac{1}{4 \pi^{2}} \int_{m_{K}}^{\infty} k^{\prime} \mathrm{d} \omega^{\prime}\left[\frac{\sigma_{+}\left(\omega^{\prime}\right)}{\omega^{\prime}-\omega}+\frac{\sigma_{-}\left(\omega^{\prime}\right)}{\omega^{\prime}+\omega}\right] \\
& +\frac{1}{\pi} \int_{\omega_{\Lambda \pi}}^{m_{K}} \frac{\mathrm{d} \omega^{\prime} A_{-}\left(\omega^{\prime}\right)}{\omega^{\prime}+\omega} \\
D_{-}(\omega)= & p_{K \Lambda} \frac{X(\Lambda)}{\omega_{\Lambda}-\omega}+p_{K \Sigma} \frac{X(\Sigma)}{\omega_{\Sigma}-\omega} \\
& +\frac{1}{4 \pi^{2}} \int_{m_{K}}^{\infty} k^{\prime} \mathrm{d} \omega^{\prime}\left[\frac{\sigma_{+}\left(\omega^{\prime}\right)}{\omega^{\prime}+\omega}+\frac{\sigma_{-}\left(\omega^{\prime}\right)}{\omega^{\prime}-\omega}\right] \\
& +\frac{1}{\pi} \int_{\omega_{\Lambda \pi}}^{m_{K}} \frac{\mathrm{d} \omega^{\prime} A_{-}\left(\omega^{\prime}\right)}{\omega^{\prime}-\omega}
\end{aligned}
$$

In this expression $D_{ \pm}$denote the real parts of the $K^{ \pm}-p$ forward scattering amplitudes respectively and $A_{-}$denotes the imaginary part of $K^{ \pm}-p$ forward scattering amplitude and $\omega_{i}=\frac{m_{i}^{2}-m_{N}^{2}-m_{K}^{2}}{2 m_{N}}, m_{i}$ being the rest mass of the system $i$ $(i=\Lambda$ or $\Sigma)$. The first two terms on the right hand side are the poles arising from the possible intermediate states, viz. $\Lambda$ and $\Sigma$ particles in the $K^{-}-p$ scattering alone. The interesting point is that the residues at these poles depend on the parities $p_{K A}$ and $p_{K \Sigma}$ and involve the corresponding coupling constants. The expressions for the residues are

$$
\begin{array}{ll}
p_{K \Lambda}=+1 ; & X(\Lambda)=\frac{g_{K \Lambda}^{2}}{4 \pi}\left[\frac{\left(m_{\Lambda}+m_{p}\right)^{2}-m_{K}^{2}}{4 m_{p} m_{\Lambda}}\right] \\
p_{K \Lambda}=-1 ; & X(\Lambda)=\frac{g_{K \Lambda}^{2}}{4 \pi}\left[\frac{m_{K}^{2}-\left(m_{\Lambda}-m_{p}\right)^{2}}{4 m_{p} m_{\Lambda}}\right] .
\end{array}
$$

Similar expressions hold for $\Sigma$ also with $\Sigma$ replacing $\Lambda$. Since in the energy region from $\omega_{\Lambda \pi}\left(<\omega_{\Sigma \pi}\right)$ to $m_{K}$, though unphysical for $K^{ \pm}-p$ scattering we can have $\Lambda-\pi$ or $\Sigma-\pi$ states of positive kinetic energy, we have to integrate $A_{-}$over this region. For $K^{ \pm}-n$ scattering, the dispersion relations have the same form except that $X(\Lambda)=0$ since $K^{-}-n$ is in a pure $T=1$ state. Thus only the $\Sigma$ state can contribute a pole and the $K^{ \pm}-n$ dispersion is related to $p_{K \Sigma}$. Also $X(\Sigma)$ is twice the expression given above.

1 D. Amati and B. Vitale, Nuovo Cim. 7, 190 (1958). 
The behaviour of $A_{-}$in the unphysical region can be quite complicated. $A_{-}$ becomes a finite quantity as $\omega \rightarrow m_{K}$ since for an endothermic reaction the cross section behaves like $\frac{1}{k}$ near the threshold. We can also assert that $A_{-}$has to vanish for $(1)<\omega_{.1 \pi}$. Though in principle an analytic continuation of $A_{-}$from the physical region to the unphysical region should throw light on the behaviour of $A_{-}$ the scattering amplitudes can have cusps in the unphysical region at the threshold for reactions like $\Sigma \tau$ and $A+\pi+\pi^{1}$. It was also pointed out by Salam and Mat thews who have analysed the behaviour of $A_{-}$by means of perturbation theory that $A_{-}$ean go through zero and take negative values in the unphysical region. ${ }^{2}$

The convergence of the integrals over the cross-sections in (11) is not possible if both $\sigma_{+}$and $\sigma_{-}$approach constant values at infinite energy. Goebel ${ }^{3}$ and Matthews and Salam ${ }^{4}$ have suggested the use of the dispersion relations obtained by subtracting (12) from (11). Assuming $p_{K \Sigma}$ to be even, we have at $\omega=m_{K}$

$$
\begin{aligned}
m_{K} & {\left[D_{+}\left(m_{K^{\prime}}\right)-D_{-}\left(m_{K^{\prime}}\right)\right]-\frac{m_{K}^{2}}{4 \pi^{2}} \int_{m_{K}}^{\infty} \frac{\mathrm{d} \omega^{\prime}}{k^{\prime}}\left[\sigma_{+}\left(\omega^{\prime}\right)-\sigma_{-}\left(\omega^{\prime}\right)\right] } \\
& \quad-\frac{m_{K}^{2}}{\pi} \int_{\omega_{A \pi}}^{m_{K}} \frac{\mathrm{d} \omega^{\prime}}{k^{\prime 2}} A_{-}\left(\omega^{\prime}\right) \\
& =2 p_{K \Lambda}\left[X(\Lambda)+X\left(\sum\right)\right] \\
& \simeq 2 \frac{\left(g_{K}^{2} \Lambda+g_{K \Sigma}^{2}\right)}{4 \pi} \quad \text { for scalar } K \text {-meson } \\
& \simeq-2\left[\frac{m_{K}}{2 m_{p}}\right]^{2}\left[\frac{g_{K \Lambda}^{2}+g_{K \Sigma}^{2}}{4 \pi}\right] \text { for pseudoscalar } K \text {-mesons. }
\end{aligned}
$$

It is probable that the integral over the cross-section in (14a) is convergent. In the energy region where we have experimental data, $\sigma_{-}$is always larger than $\sigma_{+}$. Since $K^{-}+p$ has so many channels compared to $K^{+}+p$ we can expect that even at higher energies $\sigma_{-}>\sigma_{+}$. The integral over the cross-section is therefore positive. Further $D_{+}\left(m_{K}\right)$ is known to be negative and small while $D_{-}\left(m_{K}\right)$ is quite large. If $K^{-}-p$ interaction is repulsive then $D_{-}\left(m_{K}\right)$ is negative. We can thus conclude, neglecting the small contribution from the unphysical region, that the $K$-meson is a scalar, for the whole quantity in the left-hand side is positive. However if $K^{--p}$ interaction is attractive as it seems to be, then $D_{-}\left(m_{K}\right)$ is positive and the quantity in the left-hand side depends upon the relative magnitude of $D_{-}\left(m_{K}\right)$ and $D_{+}\left(m_{K}\right)$ and the numerical value of the integral. But it seems that this is more likely to be negative indicating that $K$-meson is probably

1 K. Igr. Progr. Theoret. Phys. 19, 238 (1958).

2 P. T. Matthews and A. Salair, Phys. Rev. 110, 565, 569 (1958); R. H. Dalitz and S. F. Tuan, Ann. Phys. S, 100 (1959).

${ }^{3}$ C. Goebel, Phys. Rev. 100, 572 (1958).

4 P. T. Matthews and A. Salay, loc. cit. (1958). 
pseudoscalar. Igi ${ }^{1}$ has suggested a different type of dispersion relation where the integrals converge rapidly and which is in some measure weighted against contributions from the unphysical region. The dispersion relation has the form

$$
\begin{aligned}
& \omega\left[D_{+}(\omega)-\frac{1}{2}\left(m_{K}+\omega\right) D_{+}\left(m_{K}\right)-\frac{1}{2}\left(m_{K}-\omega\right) D_{-}\left(m_{K}\right)\right] \\
- & \frac{1}{4 \pi i} \int_{m_{K}}^{\infty} \frac{\mathrm{d} \omega^{\prime}}{k^{\prime}}\left[\frac{\sigma_{+}^{-}}{\omega^{\prime}-\omega}+\frac{\sigma_{-}^{-}}{\omega^{\prime}+\omega}\right]-\frac{1}{\pi} \int_{\omega_{\Lambda \pi}}^{m_{K}} \frac{\mathrm{d} \omega^{\prime}}{k^{\prime 2}} \frac{A_{-}\left(\omega^{\prime}\right)}{\omega^{\prime}+\omega} \\
= & -p_{K Y}[X(\Lambda)+X(\Sigma)]
\end{aligned}
$$

The contribution from the unphysical region is estimated by taking a smooth extrapolation of $A_{-}$for $\omega<m_{K}$. Assuming $\sigma_{+}$to be constant it is found that if $D_{-}$is positive, $K$ is pseudoscalar with $\frac{g_{K A}^{2}+{ }_{K \Sigma}^{2}}{4 \pi} \simeq 4$, while if $D_{-}$is negative, $K$ is scalar with $\left(g_{K \Lambda}^{2}+g_{K \Sigma}^{2}\right) / 4 \pi \simeq 0.08$. Assuming that $\sigma_{+}$falls off by a factor of two between $50 \mathrm{MeV}$ and zero energy, the $K$-meson seems to be scalar irrespective of the sign of $D_{-}$for $\frac{g_{K A}^{2}+g_{K}^{2} \Sigma}{4 \pi} \simeq 4$. Recently the dispersion relations have been used to determine the sign and magnitude of $D_{-}\left(m_{K}\right)$ which is sensitive to $p_{K Y}$. Also the low energy behaviour $\sigma^{e l}$ has been used to decide $p_{K Y}$. All these arguments favour only a pseudoscalar $K$-meson assuming $p_{\Lambda \Sigma}$ to be even.

Perhaps with more accurate data on $K^{+}-p$ scattering cross-sections at energies below $50 \mathrm{MeV}$ and also from their angular distributions, we may be able to decide $p_{K \Lambda}$. Further if we have more data from $K^{ \pm}-n$ scattering, we can form an independent conclusion on $p_{K \Sigma}$ which can be also utilized to discuss $K^{ \pm}-p$ dispersion relations.

(c) Absorption processes. Several proposals to decide $p_{K Y}$ from $K^{-}$absorption in hydrogen, deuterium and helium have been put forward. It is obviously very important in studying these processes to have some idea of the atomic orbit from which $K^{-}$-mesons are captured. Recently, Day, Snow and Sucher ${ }^{2}$ have drawn attention to the influence of Stark effect collisions of a highly excited $K^{-}-p$ atom with the neighbouring protons in the liquid hydrogen. They thus obtain the very important result that the capture at rest of $K^{-}$always takes from $S$ orbits, i.e. the relative orbital angular momentum of the $K^{-}-p$ system is always zero. ${ }^{3}$ Similar considerations can be expected to hold for captures at rest of $K^{-}$in $d$.

1 K. Igi, Progr. Theoret. Phys. 20, 403 (1958).

2 T. B. Day, G. A. Snow and J. Sucher, Phys. Rev. Lett. 3, 61 (1959).

3 From the capture at rest of $K^{-}$in protons we can determine the spin of the hyperons. Making use of the fact that capture is from $S$ orbit, the arguments of Adair given for determination of hyperon spin in $\pi^{-}-P$ collisions can be repeated. Thus if there is no anisotropy in the decay products of the hyperon, the spin of the hyperon is $\frac{1}{2}$ (see $\mathrm{S}$. B. Treiman, Phys. Rev. 101, 1217 (1956)). Recent experiments by LeItNer et al. (see Phys. Rev. Lett. 3, 238 (1959)) indicate that the decay distribution does not statistically deviate from isotropy and hence they conclude that spin of $\Sigma^{+}$is $1 / 2$. They also find that there is no violation of 
Let us consider the as yet unobserved reactions

$$
\begin{aligned}
K^{-} p & \rightarrow \Lambda^{\mathbf{0}}+\pi^{+}+\pi^{-} \\
& \rightarrow \Lambda^{\mathbf{0}}+\pi^{\mathbf{0}}+\pi^{\mathbf{0}} .
\end{aligned}
$$

Okun and Pomeranchuk ${ }^{1}$ have estimated from phase space considerations. that these should constitute 2 per cent of all the capture reactions for scalar $K$-mesons. If $K$ were pseudoscalar this reaction is damped due to centrifugal barrier effects for $P$ wave motions necessary in the final state to conserve parity and angular momentum. In this case $A^{0}$ from $(16 \mathrm{~b})$, can be polarized and due to the symmetry of identical boson wave functions the branching ratio will be affected. Similar results obtain for absorption in flights for kinetic energies up to $30 \mathrm{MeV}$ of $\mathrm{K}^{-}$in the labotarory. ${ }^{2}$ We ean also note that the reaction

$$
K^{-}+n \rightarrow \Sigma^{+}+\pi^{-}+\pi^{-},
$$

which can take place for $K^{-}$with the kinetic energy $\sim 80 \mathrm{MeV}$ in the laboratory system will be permitted only if $p_{K} \Sigma$ is even. Odd $p_{\Lambda \Sigma}$ requires $P$ wave motions in the final state which is not possible for the $2 \pi^{-3}$

If $\left(\Sigma^{-} n\right)$ can exist as a bound system, Pais and Treiman ${ }^{4}$ have shown that for the capture of $K^{-}$from an $S$ orbit in deuterium the branching ratio $\frac{\left(\Sigma^{-} n\right) \pi^{+}}{\Sigma^{-}+n+\pi^{+}}$ for a binding energy of $1 \mathrm{MeV}$ or more is possible. Since we know from arguments of Day et al..$^{5}$ that the capture at rest of $K^{-}$takes place only from $S$ orbits, the absence of the reaction

$$
K^{-}+d \rightarrow\left(\Sigma^{-} n\right)+\pi^{+}
$$

can be only due to the $\left(\Sigma^{-} n\right)$ bound state having zero spin and $p_{K} \Sigma$ being odd. Consider the reaction ${ }^{6}$

$$
K^{-}+d \rightarrow Y+N
$$

The fact that $K^{-}$is captured at rest only from $S$ orbits helps us to write down the transition matrix for both scalar and pseudoscalar $K$ cases. Using arguments given earlier in $\pi^{-}-p$ reactions, it has been noticed that the sign of the correlation between the polarization of $Y$ and $N$ depends on $p_{K Y}$ parity as we noted before. However this process oceurs in only about 1 per cent of all the $K^{-}$capture

parity in strong interactions. It is to be noted that any component of the polarization $\boldsymbol{P}_{\Sigma}$ in the production plane is an evidence for parity non-conservation in the strong interaction. CraWford et al. (see Phys. Rev. Lett. 1, 418 (1958)) have arrived at a similar conclusion, i.e. parity is not violated in strong interactions, by an analysis of the decay products of $\Lambda^{0}$ produced in $\pi^{-}+p \rightarrow \Lambda^{0}+K^{0}$ by making various choices of coordinate systems.

1 L. B. Okun and I. YA. Pomeranchuk, J. Expt. Theoret. Phys., 34, 997 (1958).

2 L. Fonda and J. E. Russel, Phys. Rev. Lett. 2, 57 (1959).

${ }^{3}$ D. Avati and B. Vitale. Fortsch. Phys., loc. cit.

4 A. Pais and S. B. Treiman, Phys. Rev. 106, 1106 (1957).

T. B. DAY et al., loc. cit. (1959).

6 A. Sirlin and R. Spitzer, Phys. Rev. Lett. 3, 110 (1959). 
reactions and the determination of polarization is not easy for $n$ and $\Sigma^{-}$, though for $\Lambda^{0}$ its polarization can be estimated from the decay asymmetry.

Finally we refer to an interesting proposal due to Dalitz and Downs ${ }^{1}$ who suggested the possibility of determining $p_{K \Lambda}$ from $K$-absorption in $\mathrm{He}^{4}$. We have

$$
K^{-}+\mathrm{He}^{4} \rightarrow{ }_{\Lambda} \mathrm{He}^{4}+\pi^{-} .
$$

Assuming that ${ }_{4} \mathrm{He}^{4}$ has zero spin and even parity, the reaction is forbidden for even $p_{K \Lambda}$ since $\pi^{-}$is pseudoscalar. For odd $p_{K}$, the reaction can take place. Dalitz $^{2}$ has also given arguments against the existence of an excited state of ${ }_{\Lambda} \mathrm{He}^{4}$ with $J=1$. There is also a high yield of hyperfragments in the $K^{-}$capture by $\mathrm{He}^{4}$. Thus we can conclude that $p_{K \Lambda}$ is odd. Recently Pevsner et al. ${ }^{3}$ have observed ${ }_{\Lambda} \mathrm{He}^{4}$.

Summing up, we find that the dispersion relation approach seems to indicate that pseudoscalar $K$-meson with respect to both the hyperons is more likely. However we need to study the dispersion relations for $K-N$ scattering when the $\Sigma-\Lambda$ system has odd relative parity. If the various proposals also confirm that $K$-meson is pseudoscalar, we have the interesting fact that both the bosons $\pi$ and $K$ are pseudoscalar.

Relative parity $p_{K K}$. As pointed out before, the relative parity $p_{K K}$ of $K^{+}-K^{\mathrm{e}}$ has to be even as long as $K^{+}-K^{0}$ forms an isodoublet. However, as we shall see later, it is possible to describe the $K^{+}$and $K^{0}$ as two different isosinglets (see Pais" "Model of Strong Interactions") in which case $p_{K K}$ can be defined and tests for measuring $p_{K K}$ are necessary. Pais has studied the effect of odd $p_{K K}$ on charge independence in strong interactions and the production and scattering of baryons and $K$-mesons. In the nucleon-anti-nucleon annihilation into the $K$ pair, i.e.

$$
\bar{n}+p \rightarrow K^{+}+\overline{K^{0}}
$$

the sign of $p_{K K}$ will determine the initial angular momentum states.

Recently a new method has become possible to obtain the relative parities and coupling constants for the strange particles using general field theoretic concepts. These methods are essentially a consequence of the Mandelstam representation $^{5}$ of dispersion relations from which it is possible to show that the existence of dispersion relations in the momentum transfer is just like that in the total energy. Chew ${ }^{6}$ first made use of the analyticity properties of the scattering and production amplitudes in the momentum transfer variable to determine the pion-nucleon coupling constant from nucleon-nucleon scattering

1 R. Dalitz and B. Downs, Phys. Rev. 111, 967 (1958).

2 Hyperon-Nucleon Interactions, DaLITz, Kiev (1959).

3 Pevsner et al., Phys. Rev. Lett. 3, 291 (1959). See also, Block et al. Proc. of the Ann. Int. Conf. on High energy Physics at Rochester (1960)., p. 419.

4 A. Pats, Phys. Rev. 110, 574 (1958); 112, 624 (1958); Phys. Rev. Lett. 1, 418 (1958).

5 S. Mandlestam, Phys. Rev. 112, 1344 (1958).

${ }^{6}$ G. F. Chew, Phys. Rev. 112, 1380 (1958). 
(see chapter V). We shall discuss here how the method can be applied to determine the sign of $p_{K K^{*}}{ }^{1}$

Let us eonsider the matrix element $M$ for the associated production process $\tau+p \rightarrow 1^{0}+K^{0}$. $M$ will be a function of the two invariants, the total energy $\omega=\left(p_{p}+p_{\Omega}\right)^{2}$ and the momentum transfer $\Delta^{2}=\left(p_{p}-p_{A}\right)^{2}$. If we study the analytic behaviour of $M\left(\omega, \Delta^{2}\right)$ as a function of $\Delta^{2}$, we should expect a pole arising from an intermediate state composed of a single $K$-meson as shown in Fig. 46.

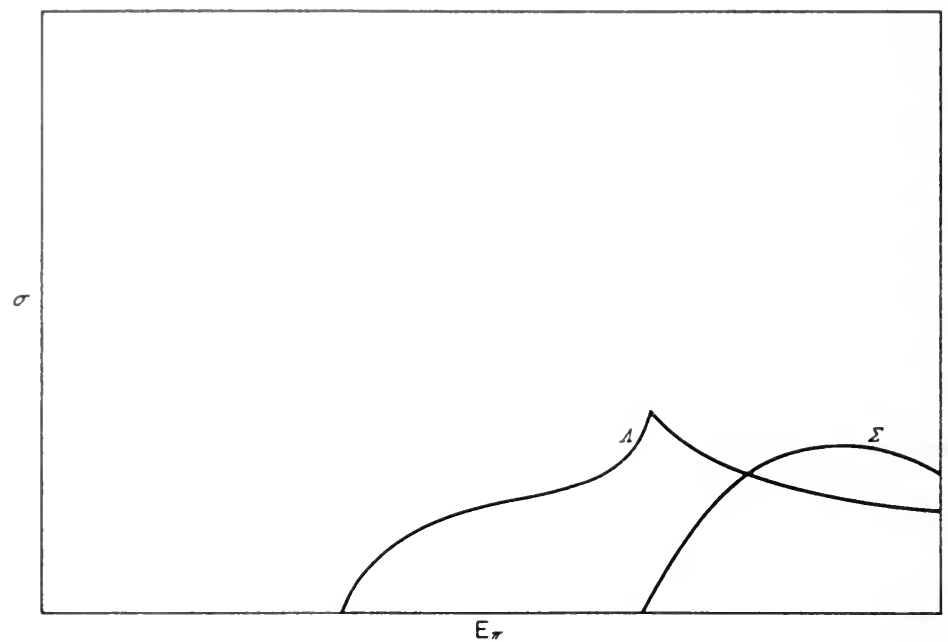

FIG. $47 . \Lambda^{0}$ and $\Sigma^{0}$ production cross-section.

This figure essentially involves a $K^{0}-K^{ \pm} \pi^{-}$interaction which is possible as a strong interaction only if $p_{K K}$ were odd. Thus the residue at this pole should be zero if $p_{K K}$ were even. With this remark, we just state that we can write $M\left(\omega, \Delta^{2}\right)$ as

$$
M\left(\omega, \Delta^{2}\right)=\frac{g(\omega, \cos \theta)}{\lambda-\cos \theta}+f(\omega, \cos \theta)
$$

The first term on the right-hand side is only the renormalized Born term corresponding to the figure so that the function is known. $f$ is a regular function and has no singularity at $\cos \theta=x$. $x$ is given by

$$
\boldsymbol{x}=\frac{2 p_{\pi^{0}} p_{K^{0}}-m_{K}^{2}}{2 \boldsymbol{p}_{\boldsymbol{x}} \mid \boldsymbol{p}_{\boldsymbol{K}} !}
$$

and $\theta$ is the angle between $\boldsymbol{p}_{\pi}$ and $\boldsymbol{p}_{K}$ in the centre of mass system. Since $\alpha$ is $>1$, the pole at $\cos \theta=\alpha$ will lie outside the physical range of $\cos \theta$, viz. $-1 \leqq \cos \theta$

1 J. G. TaYlor, Nucl. Phys. 9, 357 (1959); M. J. Moravicisk, J. G. Taylor and J. L. Uretsky. Phys. Rev. 113, 689 (1959). 
$\leqq 1 .^{1}$ For high $\pi$ energies, $\alpha$ is close to unity. For pion laboratory momenta from 1.3 to $1.4 \mathrm{BeV} / \mathrm{c}$, the range of the values of $\alpha$ is between 1.6 and 1.4. The differential cross-section is proportional to $|\mathbf{M}|^{2}$ and so we can write

$$
\begin{aligned}
\frac{\mathrm{d} \sigma(\omega, \theta)}{\mathrm{d} \Omega}= & K(\omega) \frac{\mid g\left(\omega,\left.\cos \theta\right|^{2}\right.}{(\alpha-\cos \theta)^{2}}+\frac{g(\omega, \cos \theta) f^{*}(\omega, \cos \theta)}{\alpha-\cos \theta} \\
& +|f(\omega, \cos \theta)|^{2}
\end{aligned}
$$

where $K(\omega)$ is a known function of the energy. It is convenient to study the singularity of the function

$$
F(\omega, \cos \theta)=(\alpha-\cos \theta)^{2} \frac{\mathrm{d} \sigma(\omega, \theta)}{\mathrm{d} \Omega} .
$$

We see that

$$
F(\omega, \alpha)=K(\omega)|g(\omega, \alpha)|^{2}
$$

Thus we have been able to separate the term which can be calculated. In order to decide $p_{K K}$, we extrapolate $F(\omega, \cos \theta)$ from the physical region to the unphysical region up to $\cos \theta=\alpha$ and see if a non-zero residue exists which will definitely show that $p_{K K}$ is odd. Further if $F(\omega, \alpha)$ is $>0$, then $p_{K A}$ is even while if $F(\omega, \alpha)<0, p_{K A}$ is odd (see the previous discussion on $K^{ \pm}-p$ scattering using dispersion relations). It is also possible to estimate the coupling constants from the magnitude of the residue. The main difficulty with this method lies in the extrapolation procedure. Recently it has been shown that this method of extrapolation may lead to fallacious results like $\pi^{0}$ being a scalar or the possibility of non-conservation of parity in strong interactions. ${ }^{2}$ If $F(\omega, \cos \theta)$ is linear in $\cos \theta$, we should not conclude that this linearity is caused mainly by the pole term. We should be very cautious in assuming shapes for $F(\omega, \cos \theta)$ for the extrapolation procedure. Possible applications of this procedure to hyperon-nucleon scattering in order to determine hyperon parities and coupling strengths have been discussed to Barshay and Glashow. ${ }^{3}$ Recently Taylor ${ }^{4}$ has discussed in detail what can be gleaned by this method from the processes of associated production by photons on nucleons, by pions on protons and from the absorption and charge-exchange scattering of $K^{ \pm}$on nucleons. He finds that if $p_{K}$ is odd, then strange particle parities may be obtained from all these processes while if $p_{K K}$ is even, only the photoproduction process may be used to determine the parities.

Relative parity $p_{\Lambda \Sigma}$. The role of $p_{\Lambda \Sigma}$ in $K^{ \pm}-N$ scattering has already been discussed where $\Lambda$ or $\Sigma$ are present only in the intermediate states. We shall describe a few methods for determining directly $p_{\Lambda} \Sigma$. A very interesting suggestion

1 There is another pole arising from an intermediate hyperon state which can be made use of only at higher $\pi^{-}$energies.

2 G. Feldman and T. Fultow, Phys. Rev. Lett. 3, 64 (1959).

3 S. Barshay and S. L. Glashow, Phys. Rev. Lett. 2, 371 (1959).

4 J. G. Taylor, Phys. Rev. 116, 768 (1959). 
is due to Baz and Okun ${ }^{1}$ who made use of a theorem in nuclear physies due to Breit and Wigner regarding the behaviour of eross-sections for a reaction near the threshold of another reaction. As the method is quite generally applicable we shall discuss it in some detail. As an example, let us consider the reaction

$$
\pi^{-}+p \rightarrow \Lambda^{0}+K^{0}
$$

near the threshold $\simeq 890 \mathrm{MeV}$ in the laboratory system for the reaction

$$
\pi^{-}+p \rightarrow \Sigma^{0}+K^{0} \text {. }
$$

At this energy range the total cross-section and angular distribution for (27) will exhibit a cusp-like behaviour, the study of which can reveal $p_{A \Sigma}$. Near the threshold it is quite justifiable to assume that $\left(\Sigma^{0} K^{0}\right)$ is produced in an $S$ state. Due to angular momentum conservation it is clear that only the $S$ and $P$ states of $A^{0} K^{0}$ will be affected by the $\Sigma$ production for even or odd $p_{A \Sigma}$ respectively. Also we need only eonsider the $I=1 / 2$ production amplitude since $\Lambda^{0} K^{0}$ system can have only $I=1 / 2$. It is important to observe that since the total cross-section for the production of two neutral particles is proportional to $=|\boldsymbol{k}|$ where $\boldsymbol{k}$ is the wave vect or in the $\Sigma^{0} K^{0}$ eentre of mass system, the creation amplitude will be proportional to $k^{\frac{1}{2}}$. We can thus write the creation amplitude for $\Sigma^{0} K^{0}$ as $m_{i} k^{\frac{1}{2}}$ where $m_{i}$ is an energy independent term (depending only on the initial states). Considering the se two ehannels only, the $S$ matrix ean be written as follows: ${ }^{2}$

$$
S=\left[\begin{array}{llc}
S_{11} & S_{12} & m_{1} k^{\frac{1}{2}} \\
S_{21} & S_{22} & m_{2} k^{\frac{1}{2}} \\
m_{1} k^{\frac{1}{2}} & m_{2} k^{\frac{1}{2}} & X
\end{array}\right],
$$

where $i=1,2$ refer to $\pi^{-}+p$ and $\Lambda^{0}+K^{0}$ systems respectively and $S_{i j}$ refer to the transition amplitudes from the initial state $i$ to the final state $j . X$ describes the elastic seattering of $K^{0}$ by $\Sigma^{0}$ and it is assumed that $X \rightarrow 1$ as $k \rightarrow 0$. We can now write $S_{i j}$ 's as

$$
S_{i j}(k)=s_{i j}+a_{i j} k+0\left(k^{2}\right),
$$

where $s_{i j}$ is the value of $S_{i j}$ at the threshold and $a_{i j}$ are $k$ independent coefficients, at least near the threshold. We can now make use of the condition that $S$ matrix is unitary above the threshold, i.e. $S S^{\dagger}=1$. This leads to

$$
\sum_{l=1}^{2}\left(s_{i l} a_{j l}^{*}+s_{j l} a_{i l}\right)=-m_{i} m_{j}^{*} ; \quad \sum_{l=1}^{2} s_{i l}^{*} m_{l}=-m_{i}^{*} X .
$$

Below the threshold $\Sigma^{0} K^{0}$ channel is closed and $k$ beeomes imaginary, i.e. $k=i|k|$. Remembering that the asymptotic wave functions for $\Sigma^{0} K^{\mathbf{0}}$ should vanish we choose $+i|k|$. Unitarity of $S$ below the threshold leads to

$$
\sum_{l=1}^{2}\left(-s_{i l} a_{j l}^{*}+s_{j l}^{*} a_{i l}\right)=0 .
$$

1 A. I. Baz and L. B. Okun, J. Expt. Theoret. Phys. 8, 526 (1959); R. AdaIR, Phys. Rev. $111,632(1958)$.

2 We are omitting a number of possible channels describing processes like single and double pion production in $\pi^{-}-p$ collisions. 
Also the unitarity at the threshold gives us the condition

$$
\sum_{l=1}^{2} s_{i l} s_{j l}^{*}=\delta_{i j} .
$$

Solving these three equations we obtain

$$
a_{i j}=\frac{1}{2} m_{i} m_{j} .
$$

In terms of partial waves, this result can be expressed as

$$
M_{l}^{\frac{1}{2}}(E)=M_{l}^{\frac{1}{2}}\left(E_{0}\right)+\frac{1}{2} m_{1} m_{2} k,
$$

where $M_{l}^{\frac{1}{2}}(E)$ is the matrix element that describes the creation of $\Lambda^{0}$ and $K^{0}$ with an orbital angular momentum $l$ at an energy $E . E_{0}$ is the threshold energy and $1 / 2$ denotes the total angular momentum available. It is to be noted that only those $M_{l}$ 's having the same quantum numbers as the state of $\Sigma^{0} K^{0}$ will be affected. The differential cross-section $\frac{\mathrm{d} \sigma}{\mathrm{d} \Omega}(\theta, E)$ for $\Lambda^{0}-K^{0}$ production can be expressed as

$$
\frac{\mathrm{d} \sigma_{\Lambda}}{\mathrm{d} \Omega}(\theta, E)=|g(\theta, E)|^{2}+|h(\theta, E)|^{2},
$$

where $g(\theta, E)$ is the non-spin flip amplitude given by

$$
g(\theta, E)=\frac{1}{2 i k_{1}} \sum_{l}\left[(l+1) M_{l}^{l+\frac{1}{2}}+l M_{l}^{l-\frac{1}{2}}\right] P_{l}(\cos \theta)
$$

and $h(\theta, E)$ is the spin-flip amplitude given by

$$
h(\theta, E)=\frac{1}{2 i k_{1}} \sum_{l}\left[M_{l}^{l+\frac{1}{2}}-M_{l}^{l-\frac{1}{2}}\right] P_{l}^{(1)}(\cos \theta) .
$$

Here $k_{1}$ is the wave vector of the colliding $\pi^{-}+p$ system. The polarization of $\Lambda^{0}$ is given by

$$
P_{\Lambda}(\theta, E)=2 \operatorname{Im} \frac{h(\theta, E) g^{*}(\theta, E)}{\frac{\mathrm{d} \sigma_{\Lambda}}{\mathrm{d} \Omega}(\theta, E)} .
$$

For even $p_{A \Sigma}$ only $l=0$ will be affected in (37). Thus

with

$$
\begin{gathered}
\frac{\mathrm{d} \sigma_{\Lambda}}{\mathrm{d} \Omega}(\theta, E)=\frac{\mathrm{d} \sigma_{\Lambda}}{\mathrm{d} \Omega}\left(\theta, E_{0}\right)+\frac{|k|}{k_{1}}\left\{\begin{array}{rr}
-\operatorname{Im} g\left(\theta, E_{0}\right) \alpha^{*} ; & E>E_{0} \\
\operatorname{Re} g\left(\theta, E_{0}\right) \alpha^{*} ; & E<E_{0}
\end{array}\right. \\
P_{\Lambda}(\theta, E)=P_{\Lambda}\left(\theta, E_{0}\right)+\frac{|k|}{k_{1}} \begin{cases}\operatorname{Re} f\left(\theta, E_{0}\right) \alpha^{*} ; & E>E_{0} \\
\operatorname{Im} f\left(\theta, E_{0}\right) \alpha^{*} ; & E<E_{0}\end{cases}
\end{gathered}
$$

$$
f\left(\theta, E_{0}\right)=\frac{h\left(\theta, E_{0}\right)-i P\left(\theta, E_{0}\right) g\left(\theta, E_{0}\right)}{\frac{\mathrm{d} \sigma_{\Lambda}}{\mathrm{d} \Omega}\left(\theta, E_{0}\right)} .
$$

EPCR 21 
For odd $p_{A} \Sigma$, only $l=1$ will be affected in (37). We therefore have

with

$$
\begin{array}{r}
\frac{\mathrm{d} \sigma_{A}(\theta, E)}{\mathrm{d} \Omega}=\frac{\mathrm{d} \sigma_{A}\left(\theta, E_{0}\right)}{\mathrm{d} \Omega}+\frac{|k|}{k_{1}}\left\{\begin{array}{rr}
-\operatorname{Im} l\left(\theta, E_{0}\right) \alpha^{*} ; & E>E_{0} \\
\operatorname{Re} l\left(\theta, E_{0}\right) \alpha^{*} ; & E<E_{0}
\end{array}\right. \\
P_{.1}(\theta . E)=P_{A}\left(\theta, E_{0}\right)+\frac{|k|}{k_{1}} \begin{cases}\operatorname{Re} f_{1}\left(\theta, E_{0}\right) \alpha^{*} ; & E>E_{0} \\
\operatorname{Im} f_{1}\left(\theta, E_{0}\right) \alpha^{*} ; & E<E_{0}\end{cases}
\end{array}
$$

$$
f_{1}\left(\theta, E_{0}\right)=\frac{m\left(\theta, E_{0}\right)-i P\left(\theta, E_{0}\right) l\left(\theta, E_{0}\right)}{\frac{\mathrm{d} \sigma_{A}}{\mathrm{~d} \Omega}\left(\theta, E_{0}\right)}
$$

where

$$
\begin{aligned}
m\left(\theta, E_{0}\right) & =h\left(\theta, E_{0}\right) \cos \theta-g\left(\theta, E_{0}\right) \sin \theta \\
l\left(\theta, E_{0}\right) & =g\left(\theta, E_{0}\right) \cos \theta+h\left(\theta, E_{0}\right) \sin \theta
\end{aligned}
$$

We thus see that near $E_{0}$, the cross-section and polarization are linear functions of $|k|$ and are singular at the threshold. The singularity may be a peak, a dip or a step. The width of the singularity corresponds to $|k| \widetilde{\gtrless} \mu_{\pi}$. If we assume that only $S$ and $P$ waves are present in the $A^{0}$ and $K^{0}$ production, then $g$ and $h$ do not contain higher powers of $\cos \theta$ and $\sin \theta$ than the linear term. We see from (39a) and (40a) that the singularity in the cross-section is determined by $g(\theta)$ for even $p_{\Lambda \Sigma}$ and by $g(\theta) \cos \theta+h(\theta) \sin \theta$ for odd $p_{\Lambda \Sigma}$. Thus in the first case, the cross-section does not have a $\cos ^{2} \theta$ dependence, which it has in the second case. A refined analysis of the angular distribution can thus decide $p_{\Lambda \Sigma}$. Figure 47 describes the qualitative results of the above discussion. Turning now to the experimental data ${ }^{1}$, we indeed find that $\sigma_{\Lambda}(E)$ rises from the threshold to a peak of about $0.8 \mathrm{mb}$ near $970 \mathrm{MeV}$ when the kinetic energy of the pion is near $970 \mathrm{MeV}$ and then drops again to $0.2-0.3 \mathrm{mb}$. As remarked before the angular distribution is nearly energy independent and is strongly peaked forward.

Captures at rest of polarized $\Sigma^{-}$by protons ${ }^{2}$ can lead to polarized $\Lambda^{0}$, i.e. in

$$
\Sigma^{-}+p \rightarrow \Lambda^{0}+n \text {. }
$$

As for the capture at rest of $K^{-}$by $p$, it mainly takes place from the $S$ orbits. Using the now familiar arguments, we can easily see that the polarization vectors $\boldsymbol{p}_{\Lambda}$ and $\boldsymbol{p}_{\Sigma}$ are identical for even $p_{\Lambda \Sigma}$ while $\boldsymbol{p}_{\Lambda}$ will have a component along its direction of motion for odd $p_{\Lambda \Sigma}$. Thus the angular dependence of $\boldsymbol{p}_{\Lambda}$ can yield $p_{\Lambda \Sigma}$. The main difficulty is the production of polarized $\Sigma^{\mathbf{0}}$ s.

Another possibility is to study the decays of polarized $\Sigma^{03}$ i.e.

$$
\Sigma^{0} \rightarrow \Lambda^{0}+\gamma
$$

1 See J. Steinberger, Int. Conf. on High Energy Physics, CERN, Ed. B. Feretti (1958).

2 A. Pais and S. B. Treiman, Phys. Rev. 109, 1759 (1958).

${ }^{3}$ G. Feldman and T. Fulton, Nuclear Phys. 8,1 (1958). See also: A. P. Balachandran and N. R. Ranganathan, Nuovo Cim. 16, 1142 (1960). 
The transition matrix can be written as

$$
\begin{array}{lll}
T=A \boldsymbol{\sigma} \cdot\left(\boldsymbol{\varepsilon} \times \boldsymbol{p}_{\Lambda 0}\right) & & p_{\Lambda \Sigma}=\text { even } \\
T=A^{\prime}(\boldsymbol{\sigma} \cdot \varepsilon) & p_{\Lambda \Sigma}=\text { odd }
\end{array}
$$

where $\varepsilon$ is the polarization of the $\gamma$-ray and $\boldsymbol{p}_{A^{0}}$ is a unit vector in the direction of the $\Lambda^{0}$ momentum in the rest system of $\Sigma^{0}$. As before, the polarization of $\Lambda^{0}$ can be calculated. We have

$$
\begin{aligned}
\boldsymbol{p}_{\Lambda^{0}} & =-\boldsymbol{p}_{\Sigma}+2 \boldsymbol{\varepsilon} \times \boldsymbol{p}_{\Lambda^{0}}\left\{\boldsymbol{p}_{\Sigma} \cdot\left(\boldsymbol{\varepsilon} \times \boldsymbol{p}_{1^{0}}\right)\right\} ; & & p_{\Lambda \Sigma}=\text { even } \\
& =-\boldsymbol{p}_{\Sigma}+2 \boldsymbol{\varepsilon}\left(\boldsymbol{p}_{\Sigma} \cdot \boldsymbol{\varepsilon}\right) ; & & p_{\Lambda \Sigma}=\text { odd }
\end{aligned}
$$

In the first case, $\boldsymbol{p}_{\Lambda^{0}}$ has a component perpendicular to the photon polarization and in the second case it has only a component parallel to it. Thus for polarized $\Sigma^{0}$ decays, we have a test which can serve to distinguish between the two possibilities.

It has also been noted that the branching ratio for the electromagnetic processes $^{1}$

$$
\begin{aligned}
\Sigma^{0} & \rightarrow \Lambda^{0}+e^{+}+e^{-} \\
& \rightarrow \Lambda^{0}+\gamma
\end{aligned}
$$

depends upon $p_{\Lambda \Sigma}$. Assuming a local hyperon electromagnetic interaction, the ratio to the first order is

$$
\begin{aligned}
& \varrho=\frac{1}{184} \text { for even } p_{\Lambda \Sigma} \\
& \varrho=\frac{1}{165} \text { for odd } p_{\Lambda \Sigma} .
\end{aligned}
$$

Since the values differ by 12 per cent, we can hope to determine $p_{\Lambda \Sigma}$ by an experimental study of $\varrho$. The capture of $\Sigma^{-}$by $\mathrm{He}^{4}$ can also lead to a determination of $p_{\Lambda} \Sigma^{2}$ :

$$
\Sigma \pm+\mathrm{He}^{4} \rightarrow{ }_{\Lambda} \mathrm{He}^{5}+\pi^{ \pm} .
$$

Assuming spin $1 / 2$ for ${ }_{A} \mathrm{He}^{5}$, this problem is essentially the same as the associated production in $\pi^{-}-p$ collision to decide $p_{K Y}$. If $\Sigma^{ \pm}$has a polarization $\boldsymbol{p}_{\Sigma}$ the differential cross-section can be written as

$$
\frac{\mathrm{d} \sigma}{\mathrm{d} \Omega}=\left[\frac{\mathrm{d} \sigma}{\mathrm{d} \Omega}\right]_{0}\left(1 \pm \boldsymbol{p}_{\Sigma} \cdot \boldsymbol{p}_{\Lambda}\right)
$$

where $\boldsymbol{p}$ is the polarization of ${ }_{A} \mathrm{He}^{5}$ and $\left(\frac{\mathrm{d} \sigma}{\mathrm{d} \Omega}\right)_{0}$ is the differential cross-section when $\boldsymbol{p}_{\Sigma^{0}}=0$ i.e. for unpolarized $\Sigma$ 's. As before the plus or minus signs refer to even and odd $p_{\Lambda \Sigma}$ respectively.

1 G. Feinberg, Phys. Rev. 109, 1019 (1958).

2 A. Sirlin and R. Spitzer, Phys. Rev. Lett. 3, 110 (1959). 
Relative parity $p_{x} \Xi p_{x} \Xi$ can be studied directly by the reaction

$$
\boldsymbol{\tau}^{-}+p \rightarrow \Xi^{0}+K^{0}+K^{0} \text {. }
$$

In this process the two final $K$-mesons will always exist in a state of even parity. 'Thus studying the energy dependence of the cross-section and angular distribution we can detcrmine the $p_{N} \Xi$ rather neatly. However very few cascade events have been observed so far to permit any possibility of determining $p_{N} \Xi$ now. Perhaps we can also draw conclusions regarding $p_{N}$ from the study of the absorption in hydrogen, deuterium and helium bubble ehambers of eascade particles. ${ }^{1}$

1 s. B. Treman, Phys. Rev. 113, 355 (1959). 


\section{SYMMETRIES AND STRONG INTERACTIONS}

\section{I NTR O D U C'TI O N}

The CONCEPT of an interaction in quantum field theory is based on the inclusion, in the Lagrangian or the Hamiltonian of the system of particles, terms coupling the free particle fields. The entire Hamiltonian hence consists of two parts, one representing the sum of the uncoupled or free-field terms corresponding to the various fields and the other consisting of the interaction terms. Such a division is not only meaningful but leads to quantitative predictions if perturbative expansions are valid. Since the field variables are expressed in terms of annihilation and creation operators and space-time wave functions, the interaction term in a local field theory will have the same space-time arguments in all the fields contained in it and thus will describe the annihilation and creation of particles at a space-time vertex. In a perturbative expansion it is assumed that only a few vertices are relevant and integration is performed over the space-time arguments of these vertices.

Even if a perturbative approach is not possible as in the case of the strong interactions, a knowledge of the exact form of the interaction Hamiltonian is still useful, at least for a partial understanding of the experimental data and in predicting a few results. The exact form of the interaction Hamiltonian will depend on the space-time and the intrinsic properties of the free fields and also on certain a priori principles of invariances which are believed to hold true in a "reasonable" description of Nature.

In the theory of elementary particles we are hampered by the fact that we do not have field equations for particles other than the electron, photon and neutrino. The $\mu$-meson can be treated as a heavy electron while the nucleons are assumed to be Dirac particles under certain simple conditions. In the case of the strange particles even these assumptions cannot be made. However it is still believed to be possible to devise interaction terms for the strange particles without any detailed assumptions regarding their fields except for the inclusion of their intrinsic attributes like spin, strangeness, isotopic spin and parity to account for the well-known conservation laws. This approach has led to the d'Espagnat-Prentki ${ }^{1}$ Lagrangian for the strong interactions of the elementary particles. But this Lagrangian involves a multiplicity of coupling constants which

1 B. D'Espagnat and J. Prentki, Progress in Elementary Particle Physics and Cosmic Ray Physics, Vol.4, p. 1, North Holland Publishing Company, Amsterdam (1958). This article also contains an extensive bibliography on this subject. See also, P. Roman, Theory of Elementary Particles, North Holland (1960). 
are to be determined from experimental data. As remarked earlier, this is rendered practically impossible in view of our inability to compute the eonsequences of any strong interaction term.

A reduction in the number of coupling constants is thus highly desirable. This is achieved by building into the Lagrangian certain a priori internal symmetries between the interactions of the baryons and the bosons. In such an approach, a hierarchy of strong interactions with different symmetry properties are introduced. It is usually postulated that when the interaction with highest symmetry, usually taken to be the symmetry of the four-dimensional rotation group, is alone present. the baryons and sometimes the bosons form degenerate fields. The observed multiplet structure of the baryons and the bosons are the result of the perturbation of the original symmetry by the interactions of lower symmetry. These ideas have led to quite a few models of strong interactions like the global symmetry model of Sehwinger and Gell-Mann, the cosmic symmetry model of Sakurai and certain other models due to Pais, Salam and Polkinghorne, Dallaporta, Feynman and others.

Such attempts have been stimulated by the possibility of experimentally checking the occurrence of the postulated symmetries. The initial symmetry should be strong enongh so as not to be masked or destroyed completely by the interactions of lower symmetries, in which case it is impossible to conclude whether the initial symmetry is realized or not. Indeed, at present the $K^{-}-p$ interaction data suggest that the global symmetry of the pion couplings to the baryons is not a useful concept, since the $K$-baryon interactions cannot be treated as a perturbation in view of the large cross-section for the absorption process, as pointed out by Dalitz and Tuan. ${ }^{1}$

\section{MO D E LS OF S'TRONG INTERACTIONS}

The d'Espagnat-Prentki scheme ${ }^{2}$

The first complete interaction Hamiltonian describing these strong interactions was due to d'Espagnat and Prentki who made the following plausible assumptions, (a) only Yukawa couplings exist, i.e. the interaction is linear in the boson field, (b) the total charge $Q$ and the total baryon number $N$ are conserved, (c) the interaction is charge independent which implies that the interaction behaves like a scalar under arbitrary rotations in the three-dimensional isospin space and (d) the interactions also conserve strangeness.

On these assumptions, d'Espagnat and Prentki were able to show that the conservation of charge is equivalent to the requirement of invariance under a reflection with respect to the plane perpendieular to the third axis in the isotopic spin space (i.e. the $x_{1}-x_{2}$ plane). To see this we must first study the transformation of an isospinor $\psi$ under a reflection. Let $I$ be the unitary operator which trans-

\footnotetext{
1 R. H. Dalitz and S. F. Tuan, Ann. Phys. 10, 307 (1960).

2 B. D'Espagrat and J. Prextki, loc. cit.
} 
forms $\psi$ to $\psi^{\prime}=I \psi I^{-1}=A \psi$ under inversion. Since we know that $\bar{\psi} \boldsymbol{\tau} \psi$ behaves like a vector under rotations in isospace, we have now

$$
\bar{\psi} A^{-1} \boldsymbol{\tau} A \psi= \pm \bar{\psi} \boldsymbol{\tau} .
$$

The plus or minus sign indicates that $\bar{\psi} \boldsymbol{\tau} \psi$ can either be a pseudo or a proper vector. However we note that there is no $A$ which anticommutes with all the $\tau$ matrices. Further a unit matrix commutes with all the $\boldsymbol{\tau}$ matrices. Thus $\bar{\psi} \boldsymbol{\tau} \psi$ is a pseudovector and $A$ must be of the form $A=k[I]$. If we recall our discussion on the behaviour of spinors under space inversion we note that $k= \pm 1, \pm i$. But the cases $k= \pm 1$ will not correspond to reflections. ${ }^{1}$ Thus under inversion we have

$$
\psi \rightarrow \psi^{\prime}= \pm i \psi .
$$

The two kinds of isospinors are respectively termed the isospinors of the first and the second kind and are denoted by $\xi$ and $\eta$. The bilinear covariants that can be formed from $\xi$ and $\eta$ are given below in the table. In columns 1 and 2 , the two spinors may refer to different fields.

\begin{tabular}{c|c|c|c}
\hline Quantity & 1 & 2 & 3 \\
\hline Isoscalars & $\xi^{\dagger} \xi$ & $\eta^{\dagger} \eta$ & $\tilde{\xi} \tau_{2} \eta$ \\
Isopseudoscalars & $\tilde{\xi} \tau_{2} \xi$ & $\tilde{\eta} \tau_{2} \eta$ & $\xi^{\dagger} \eta$ \\
Isovectors & $\tilde{\xi} \tau_{2} \tau \xi$ & $\tilde{\eta} \tau_{2} \tau \eta$ & $\xi^{\dagger} \tau \eta$ \\
Isopseudovectors & $\xi^{\dagger} \tau \xi$ & $\eta^{\dagger} \tau \eta$ & $\tilde{\xi} \tau_{2} \tau \eta$ \\
\hline
\end{tabular}

If we denote by $u$ the isoparity quantum number, then we define the general formula for any operator $\psi$

with $u=0 \quad$ for isoscalar

$$
I \psi I^{-1}=\psi^{\prime}=( \pm i)^{u} \psi
$$

$= \pm 2$ isopseudoscalar

$= \pm 2$ isovector

$=0$ isopseudovector

$=+1$ for $\xi$

$=-1$ for $\eta$

where $I$ is the inversion operator. Further if we put $I$ in the form

$$
I=\mathrm{e}^{-1 / 2 i \pi U}
$$

then the eigenvalues of $U$ will give us the isofermion numbers if we call $\xi$ the isofermion and $\eta$ the anti-isofermion.

Let us now assume that strong interactions are invariant under both rotations and reflections in isospace. This implies that for the strong interactions both $I$ and $U$ are constants of motion. Since the electric charge is also a constant

1 In the ordinary space inversion, the matrix of transformation is $k \gamma_{4}$ and so even $k= \pm 1$ correspond to reflections. 
of motion, let us assume that we can have a relation between the charge $Q, U$ and $I$ of the form

$$
Q=\alpha I_{3}+\beta U
$$

Applying this relation to the nucleon doublet we find $\alpha=1, \beta=1 / 2$ provided we take the nucleon to be an isospinor of the first kind, i.e.

$$
Q=I_{3}+U / 2 \text {. }
$$

This expression has an elegant geometric interpretation. We recognize that a reflection in the $x_{1} x_{2}$ plane is equivalent to the combined operation of an inversion followed by a rotation of $\pi$ about the third axis in the isospace. The corresponding operator is

$$
R=\mathrm{e}^{-i \pi I_{3}} \mathrm{e}^{-\frac{i \pi U}{2}}=\mathrm{e}^{-i \pi\left(I_{3}+\frac{U}{2}\right)}=\mathrm{e}^{-i \pi Q} .
$$

Thus the invariance under $R$ will automatically lead to charge conservation.

The above expression (7) helps us to fix the isotopic spin properties of the strange particles. The $\Sigma$ particle should only form an isopseudovector, i.e. $U=0$ and not an isovector, for the non-zero value of $U$ (i.e. \pm 2 ) will not yield $Q= \pm 1,0$. We can also conclude that $N$ and $K$ are isospinors of the first kind, $\Lambda$ an isoscalar, $\Xi$ an isospinor of the second kind, and $\boldsymbol{\tau}$ and $\Sigma$ isopseudovectors.

Having determined the transformation properties of the various particles, let us now proceed to construct the interaction Hamiltonian which should be a pure scalar in isospace because of the simultaneous demand of invariance under reflections and rotations in isospace. To conserve parity, the interaction should also be a scalar in the ordinary space. In the following let us assume that we have chosen the correct $\gamma$-matrices (denoted by $\Gamma$ ) to satisfy the latter demand and concentrate our attention on the needs of the first condition.

(a) The $\pi-N$ coupling: This is seen to be ${ }^{1}$

$$
G_{1} i \bar{\psi}_{N} \Gamma \boldsymbol{\tau} \psi_{N} \cdot \boldsymbol{\tau} .
$$

(b) The coupling $\Lambda \Sigma \pi$ : This can be written as

$$
G_{2}\left(\bar{\psi}_{\Lambda} \Gamma \boldsymbol{\psi}_{\Sigma} \cdot \boldsymbol{\tau}\right)+h \cdot c .
$$

It is to be noted that since $\Lambda$ is an isoscalar, it is not possible to form either a pseudovector or spinor with it. Thus there can be no $\Lambda \Lambda \pi$ interactions.

(c) The $\Sigma \Sigma \pi$ coupling: Since all the three fields are isopseudovectors, the isoscalar takes the form

$$
G_{3} i\left(\overline{\boldsymbol{\psi}}_{\Sigma} \Gamma \times \boldsymbol{\psi}_{\Sigma}\right) \cdot \pi
$$

(d) The $\Xi \Xi \pi$ coupling: This case is similar to (a) and hence the isoscalar is

$$
G_{4} i \bar{\psi}_{\Xi} \tau \Gamma \psi_{\Xi} \cdot \pi \text {. }
$$

1 We will be using the particle symbol for the field whenever such a notation is found more convenient. 
Turning now to $K$-baryon couplings we have the following isoscalars ${ }^{1}$ :

(a) $\Lambda N K: F_{1} \bar{\psi}_{N} \Gamma \psi_{\Lambda} K+h \cdot c$

(b) $\Sigma N K: F_{2} \bar{\psi}_{N} \boldsymbol{\tau} K \cdot \Gamma \boldsymbol{\psi}_{\Sigma}+h \cdot c$

(c) $\Lambda \Xi K: \quad F_{3} \bar{\psi}_{\Lambda} \Gamma \tilde{\psi}_{\Xi} \tau_{2} K+h \cdot c$

(d) $\Sigma \Xi K: F_{4} \psi_{\Sigma} \Gamma \tilde{\psi}_{\Xi} \tau_{2} \tau K+h \cdot c$

Thus the total interaction Hamiltonian is

$$
\begin{aligned}
H_{\mathrm{int}}= & H_{\boldsymbol{\pi}}+H_{K} \\
= & G_{1} i \bar{\psi}_{N} \Gamma \boldsymbol{\tau} \psi_{N} \boldsymbol{\pi}+G_{2} i\left(\bar{\psi}_{\Lambda} \Gamma \boldsymbol{\psi}_{\Sigma} \boldsymbol{\pi}+h \cdot c\right) \\
& +i G_{3}\left(\overline{\boldsymbol{\psi}}_{\Sigma} \Gamma \times \boldsymbol{\psi}_{\Sigma}\right) \boldsymbol{\pi}+G_{4} i \bar{\psi}_{\Xi} \Gamma \boldsymbol{\tau} \psi_{\Xi} \boldsymbol{\pi} \\
& +F_{1}\left(\bar{\psi}_{N} \Gamma \psi_{\Lambda} K+h \cdot c\right)+F_{2}\left(\bar{\psi}_{N} \boldsymbol{\tau} K \Gamma \boldsymbol{\psi}_{\Sigma}+h \cdot c\right) \\
& +F_{3}\left(\bar{\psi}_{\Lambda} \Gamma \bar{\psi}_{\Xi} \tau_{2} K+h \cdot c\right) \\
& +F_{4}\left(\bar{\psi}_{\Sigma} \Gamma \bar{\psi}_{\Xi} \tau_{2} \boldsymbol{\tau} K+h \cdot c\right) .
\end{aligned}
$$

$\Gamma$ is $\gamma_{5}$ in the case of $H_{\pi}$ except for $\Sigma \Lambda \pi$. However $\Gamma=1$ or $\gamma_{5}$ in $H_{K}$ depending or the relative parity of $K$-mesons. All the coupling constants, i.e. eight in number, have to be determined from experiments. It is quite obvious that this will be a hopeless task to attempt, because of the variety of the couplings. Also, we cannot treat these interactions as perturbations because of their strength. In view of these difficulties theorists have made another approach to the understanding of the strong interactions by attempting to study the symmetries of the system composed of baryons, pions and $K$-mesons from a priori considerations and using these to construct the interaction Hamiltonian. These symmetries will relate different parts of the system and hence may reduce the number of the coupling constants. All these investigations are based on the common belief that the interactions can be classified according to the degree of symmetry they exhibit. It is further assumed that when the interaction possessing the highest symmetry alone is present, the masses of the baryons and perhaps of the mesons are equal. The observed mass differences are due to the perturbations by interactions having lower symmetry than the initial one. A typical example is the mass difference between the neutron and proton which presumably arises due to the electromagnetic interaction which has no charge symmetry. However the pionnucleon interaction which is charge independent does not distinguish between the neutron and proton. We can show that we need at least three coupling constants to describe the strong interactions. Let us assume that both the $\pi$-and $K$-baryon interactions are highly symmetric, i.e. the pion-baryon and $K$-baryon interactions can be described through two coupling constants. To account for the baryonic mass differences, we need one more interaction which can serve as a perturbation. Since it is difficult to postulate this, attempts have been made assuming symmetry only of either the $\pi$ couplings (global) or the $K$ couplings (cosmic).

$1 \tilde{\psi}$ refers to the transpcsition of the isotopic spin indices only. 
Global and cosmic symmetries

Schwinger made the first attempt at such a theory of strong interactions. ${ }^{1}$ 'The baryons possess three types of charges, the nucleonic charge or baryon number $\Lambda$, hypercharge $U$ and electric charge $Q$. Among the bosons the $K$ 's are characterized by $Q$ and $U$ while the pions possess only the electric charge. Thus we are justified in regarding the nuclconic charge as the source of both the $K$ and $\pi$ interactions, $N$ and $U$ causing the $\boldsymbol{x}$ interactions and $Q$ inducing the electromagnetic interactions.

It is possible to look from a fundamental point of view at the hypercharge $U$ and isotopic spin $I$, by introducing a four-dimensional isospace instead of the usual three-dimensional one. We will be dealing with the rotations in such a fourdimensional isospace later. Let it be assumed for the present that we need four quantum numbers for a complete specification of a state in this space and they are the isotopic spin $I$, its third component $I_{3}$, the hypercharge $U$ and its third component $U_{3}$. The quantum numbers of the baryons are given below.

\begin{tabular}{c|c|c|c}
\hline$U$ & $U_{3}$ & $I$ & Particle \\
\hline$\frac{1}{2}$ & $1 / 2$ & $1 / 2$ & $N\left(\begin{array}{c}n \\
p\end{array}\right)$ \\
$\frac{1}{2}$ & $-1 / 2$ & $1 / 2$ & $\Xi\left(\begin{array}{c}\Xi^{0} \\
\Xi^{-}\end{array}\right)$ \\
0 & 0 & 0 & $\Lambda$ \\
0 & 0 & 1 & $\Sigma\left(\Sigma^{+} \Sigma^{0} \Sigma^{-}\right)$ \\
\hline
\end{tabular}

When all the interactions are switched off, all the baryons are assumed to be degenerate and to possess the same mass. Since the $K$ interaction depends on $U$ and $I$, introduction of this interaction will induce a mass splitting between the two groups $N$ and $\Xi$ and $A$ and $\Sigma$. Turning now to $\pi$ interactions, we can see that the coupling of pions to the baryons arise both from the nucleonic charge, the coupling constant being $g_{\Pi}$, and from the hypercharge, the coupling constant being $G_{I I}$. Since for the nucleons both $U$ and $N$ are of the same sign, the self masses caused by both these couplings add, while for the $\Xi$ these two contributions are of opposite sign as $N$ and $U_{3}$ are of opposite sign. Thus the pion couplings serve to remove the degeneracy between the $N$ and $\Xi$. Schwinger also introduced a $\bar{K} K \pi$ coupling, making use of the parity doublet theory which conserves parity. This coupling introduces a strong $I$ dependent coupling between the nucleons and $K$-mesons. Since we have $A \rightarrow N+\bar{K}$ and $\Sigma \rightarrow N+\bar{K}$ we can see that the pion couplings will also split the $\Sigma$ and $\Lambda$ masses. However this $\bar{K} K \pi$ coupling has lost much of its appeal with the overthrow of parity conservation which rendered the parity doublet theory rather academic. As we shall see

1 J. Schwixger, Phys. Rev. 104, 1164 (1956). 
presently, the $\bar{K} K \pi$ coupling has been revived by introducing an odd relative parity betweeen $K^{+}$and $K^{0}$.

Schwinger ${ }^{1}$ in a recent contribution has studied the problem of the structure of interactions between the elementary particles more fundamentally. We shall content ourselves here with giving the main ideas of the theory without going into the details. The general basis of the theory is that as we proceed from the familiar weak interactions to the electromagnetic interactions and then to others of increasing strength we always reduce the multiplets occuring at each stage to a simpler single particle. Or in other words the inherent multiplicity of this particle is revealed as the weaker interactions of lower symmetry spoil the original higher symmetry.

According to Schwinger the elementary particles are the realizations of the four-dimensional internal symmetry in terms of three dimensions. To see the meaning of this, we note that a space of dimension $v$ will contain $\frac{v(v-1)}{2}$ antisymmetric matrices. All these matrices will have eigenvalues $\pm 1,0$. The eigenvalue 0 is not possible in the two-dimensional case. For $v=3$, we have three antisymmetric matrices which correspond to the angular momentum operator of eigenvalue unity. These matrices give us the $I=1$ representation of three-dimensional rotations. However for $v=4$ we have six antisymmetric matrices which can be interpreted as the transformations of a vector quantity having four components. If we look at this space as $(3+1)$ dimensional, we obtain the $I=1$ and $I=0$ representations of the three-dimensional rotations. But we can form linear combinations of these six antisymmetric matrices as follows: If $I_{i j}$ denotes the antisymmetric matrix corresponding to rotation in the $i j$ plane, we have

$$
\left.\begin{array}{c}
I_{3}=\frac{1}{2}\left(I_{12}+I_{34}\right) \\
K_{3}=\frac{1}{2}\left(I_{12}-I_{34}\right)
\end{array}\right\} .
$$

These $I_{3}$ and $K_{3}$ have eigenvalues $\pm 1 / 2$, i.e. these correspond to spinor representations. In this approach we look upon the four-dimensional rotation as a direct product of two three-dimensional rotations. Thus we arrive at the important result that the six fundamental rotation matrices can be regarded in terms of three dimensions either as the direct product of $I=1$ and $I=0$ representation or as the direct product of two $I=\frac{1}{2}$ representations. It is further natural to suppose that there exist fields which realize these equivalent representations. The distinction between the integral and half-integral $I$ representations arise when the underlying four-dimensional symmetry is reduced to three-dimensional ones.

As a clue to the understanding of this reduction of the symmetry let us turn to the well-known breakdown of the three-dimensional isotopic spin symmetry by the electromagnetic interactions which distinguish the $x_{1}-x_{2}$ plane among the three planes. The remaining two-dimensional symmetry leads to the conservation

1 J. Schwinger, Ann. Phys. 2, 407 (1957). 
of the charge. Hence it is natural to identify $I_{12}$ with $Q$. However in terms of the three-dimensional representation, $Q$ will appear in different ways according to the mode of breaking up the four dimensions to three. For $I=1, Q=I_{12}$. But for $I=1 / 2$ representations, $I_{12}=I_{3}+K_{3}$. Thus in general we can write $Q=I_{3}+\frac{1}{2} Y$, where $Y=0$ if $I$ is integral and $\mathrm{Y}= \pm 1$ if $I=1 / 2$.

The baryons, $K$-mesons and pions are realized as follows:

\begin{tabular}{c|c|c}
\hline$I$ & $Y$ & Particle \\
\hline $1 / 2$ & 1 & $N$ \\
$1 / 2$ & -1 & $\Xi$ \\
1 & 0 & 2 \\
0 & 0 & $A$ \\
$1 / 2$ & 1 & $K^{+}, K^{0}$ \\
$1 / 2$ & -1 & $K^{-}, \bar{K}^{0}$ \\
1 & 0 & $\pi$ \\
0 & 0 & $\sigma(?)$ \\
\hline
\end{tabular}

It might be quite possible that Nature does not realize the $I=(0,1)$ representation for bosons. Notice that the $\pi$ 's can be accounted for by means of a self-dual antisymmetric tensor which possesses only three independent components. This provides a way of realizing the $I=1$ representation without the accompanying $I=0$ representation.

We have thus obtained the highest symmetry realized in nature, i.e. the fourdimensional symmetry of the rotation group. The next task is to split the initial multi-degenerate single fermion field and a single boson field into its components which describe the family of elementary parties as they are observed. This must arise due to a sequence of interactions of successively lower symmetry.

Schwinger has constructed a $\pi$-baryon interaction which is highly symmetric; i.e. which does not distinguish between $I=1 / 2$ and $I=0,1$ representations. ${ }^{1}$ The nucleonic charge or the baryon number of the heavy fermion field arises naturally and the pion field is dynamically dependent on this charge. Such a $\pi$ interaction is said to possess "global symmetry". At this stage the fermion field possesses $4 \times 16$ components. The factor four arises due to space-time properties and the sixteen "internal" components are the direct product of the four components for isotopic spin properties, two components for nucleonic charge properties and two because of the two ways of realizing the three-dimensional isospin space from the four-dimensional one.

This global symmetry of $\pi$-baryon coupling is broken by $K$-meson couplings which possess only three-dimensional symmetry. The degeneracy between the $N$ and $\Xi$ is removed by assuming different parities, i.e. $N$ and $\Xi$ have opposite parities while the degeneracy between $\Sigma$ and $\Lambda$ vanishes because of the different

1 J. SCHWINGeR, loc. cit. 
four-dimensional symmetries possessed by $\pi$ and $K$ interactions with these particles.

These ideas have been also extended to the lepton family. The $\mu$-meson mystery, i.e. its large mass, is supposed to be explained by postulating a new particle $\sigma$ and an interaction between $\sigma$ and the $\mu$-meson. A family of bosons with $I=1$ and with the photon as its neutral member exists. The charged bosons may carry mass, as all the electrically charged members of the lepton family do, and are expected to mediate the weak interactions. If they do, their mass will be very large and the origin of this large mass will again be a problem.

The universal $\pi$-baryon coupling has also been advocated by Gell-Mann on phenomenological grounds. ${ }^{1}$ He has argued from the available data on photoproduction of $K$-mesons that $\pi$-baryon couplings are stronger than $K$-baryon couplings. From this view point it is justifiable to treat the $K$-meson interactions as a perturbation on the initial $\pi$-baryon couplings. It is also natural to presume that the $\pi$-baryon coupling should have an identical form also for all the baryons. This is not possible if we treat $\Sigma$ and $\Lambda$ as an isovector and an isoscalar respectively. So instead, two new isodoublets are introduced besides the nucleon and the cascade particle. The new particles $Y^{0}$ and $Z^{0}$ are defined as

$$
\begin{aligned}
& Y^{0}=\frac{1}{\sqrt{ } 2}\left(\Lambda^{0}-\Sigma^{0}\right) \\
& Z^{0}=\frac{1}{\sqrt{2}}\left(\Lambda^{0}+\Sigma^{0}\right)
\end{aligned}
$$

These new "mixture" particles are linear combinations of the observed $\Lambda^{0}$ and $\Sigma^{0}$ particles. It is assumed that $\left(\Sigma^{+} Y^{0}\right)$ and $\left(Z^{0} \Sigma^{-}\right)$are isodoublets. There is of course freedom to associate either $Y^{0}$ or $Z^{0}$ with $\Sigma^{+}$or $\Sigma^{-}$, a circumstance which has been exploited in some of the models for strong interactions. For convenience of reference in later discussions, let us collect the four doublets below.

$$
N_{1} \equiv\left(\begin{array}{c}
p \\
n
\end{array}\right) ; \quad N_{2} \equiv\left(\begin{array}{c}
\Sigma^{+} \\
Y^{0}
\end{array}\right) ; \quad N_{3}=\left(\begin{array}{c}
Z^{0} \\
\Sigma^{-}
\end{array}\right) ; \quad N_{4}=\left(\begin{array}{c}
\Xi^{0} \\
\Xi^{-}
\end{array}\right)
$$

The $\pi$-baryon interaction takes the form

$$
\begin{aligned}
L_{\pi}=i G_{1} \bar{N}_{1} \boldsymbol{\tau} \gamma_{5} N_{1} \cdot \boldsymbol{\tau} & +i G_{2} \overline{\mathbf{N}}_{2} \boldsymbol{\tau} \gamma_{5} N_{2} \cdot \boldsymbol{\tau}+i G_{3} \overline{\mathbf{N}}_{3} \boldsymbol{\tau} \gamma_{5} N_{3} \cdot \boldsymbol{\tau} \\
& +i G_{4} \bar{N}_{4} \boldsymbol{\tau} \gamma_{5} N_{4} \cdot \boldsymbol{\tau} .
\end{aligned}
$$

Gell-Mann has postulated that $G_{1}=G_{2}=G_{3}=G_{4}$, i.e. the pions interact with all the baryons in the same way and with the same strength. This implies that when no other interaction is present, it is not possible to distinguish between the different baryons by $\pi$-meson interactions, i.e. all the baryons are degenerate when the $\pi$-baryon interaction alone is present. Regarding the $K$-baryon interaction, no assumption is made. This model is useful in the sense that it allows the

1 M. Gell-Mann, Phys. Rev. 106, 1296 (1957). 
$K$-meson interactions to be treated in a perturbative manner. However, we shall see later this global symmetry of $\pi$-baryon coupling has not been borne out by experimental evidence, particularly from $K^{-}-p$ interactions.

Sakurai $^{1}$ has developed a different model of strong interactions in which the $K$-baryon coupling has a higher symmetry than the $\pi$-baryon coupling. He has pointed out that the conclusion that $K$-meson coupling is weaker than $\pi$-meson eoupling is not justified in view of the smaller Compton wavelength of the $K$. meson. He is in disagreement with the view that the stronger couplings are more symmetric than the weaker ones.

Though the pion-baryon interaction allows transfer of electric charge between bosons and fermions yet the interactions are charge independent, while the electromagnetic interactions mediated by neutral quanta, i.e. photons, are not so symmetric. This idea is applied to the $K$-meson interactions which allow a transfer of the hypercharge while the pion interactions do not. Thus it should be expected, if the analogy were correct, that the $K$-meson interactions should be more symmetric than the $\pi$ couplings. Also this high symmetry should be broken by the $\tau$ coupling. The $K$-meson-baryon interactions obtained in this model are said to possess "eosmic symmetry". The interaction is given by

$$
[K]=\sqrt[V]{2} F\left[\bar{N}_{1} \cdot N_{2} K^{0}+\bar{\Gamma}_{I} \cdot N_{3} K^{+} \pm\left(\bar{N}_{4} \cdot N_{2} \bar{K}^{+}-\bar{N}_{4} \cdot N_{3} \bar{K}^{0}\right)\right]+h \cdot c .
$$

In this it has been assumed that $[N, \Lambda, K]$ and $[N, \Sigma, K]$ couplings are of the same sign and similarly also the couplings of $\Lambda$ and $\Sigma$ with the $\Xi$. However we could have chosen them to be of opposite sign. Then the interaction becomes

$$
[K]=/ 2 F\left[\bar{N}_{1} \cdot N_{2}^{\prime} K^{0}+\bar{\nu}_{1} \cdot N_{3}^{\prime} K^{+} \pm\left(\bar{N}_{4} \cdot N_{2}^{\prime} \bar{K}^{+}-\bar{N}_{4} \cdot N_{3}^{\prime} \bar{K}_{0}\right)\right]+h \cdot c
$$

where

$$
N_{2}^{\prime}=\left(\begin{array}{c}
\Sigma^{+} \\
-Z^{0}
\end{array}\right) ; \quad N_{3}^{\prime}=\left(\begin{array}{c}
-Y^{0} \\
\Sigma^{-}
\end{array}\right)
$$

These interactions are obviously charge independent. It can be verified that the interactions are also invariant under the three transformations:

a) $N_{1} \stackrel{\mp}{\rightarrow} N_{4} ; \quad K \rightleftarrows i \tau_{2} K^{*} \equiv \bar{K}$.

b) $\quad N_{2} \rightarrow-N_{3}, \quad N_{3} \rightarrow N_{2} ; \quad\left(\begin{array}{c}K^{+} \\ K^{0}\end{array}\right) \rightarrow\left(\begin{array}{c}K^{0} \\ -K^{+}\end{array}\right) ; \quad\left(\begin{array}{c}\bar{K}^{0} \\ -\bar{K}^{+}\end{array}\right) \rightarrow\left(\begin{array}{c}-\bar{K}^{+} \\ -\bar{K}^{0}\end{array}\right)$,

c) $N_{1} \rightleftarrows N_{2} ; \quad K^{+} \rightleftarrows K^{+} ; \quad N_{4} \rightleftarrows \mp N_{3} ; \quad K^{0} \rightleftarrows \bar{K}^{0}$.

Invariance under (a) simply expresses that $K$-meson couplings are hyperchargesymmetric; while the invariance under (b) expresses that $K$ couplings do not distinguish between the two baryon doublets having zero hypercharge. Invariance under (c) guarantees the hypercharge independence of $K$-meson-baryon interactions, i.e. they are independent of the hypercharge of the baryons.

Since the $K$ couplings exhibit very high symmetry, the baryons are necessarily degenerate when these couplings are present. This symmetry needs to be broken

1 J. J. Sakurai, Phys. Rev. 113, 1679 (1959). 
by the $\pi$-baryon couplings. Sakurai has suggested a plausible method of breakdown of the cosmic symmetry. The electromagnetic interactions while breaking the three-dimensional isotopic symmetry pick out only the +1 eigenstate of $\tau_{3}$, i.e. only the proton has electromagneti cinteraction (ignoring of course the anomalous moment interaction.) In the weak interactions it is $\left(1+\gamma_{5}\right) \psi$ that is retained. A similar idea can be pursued to account for the large mass difference among the baryons, i.e. $N-\Xi$ mass difference. If we add the two symmetric interactions $\left(\bar{N}_{1} \tau N_{1}+\bar{N}_{4} \tau N_{4}\right) \cdot \boldsymbol{\tau}$ and $\left(\bar{N}_{1} \tau N_{1}-\bar{N}_{4} \tau N_{4}\right) \cdot \boldsymbol{\tau}$ the symmetry $N_{1} \rightleftarrows N_{4}$ is broken and in the result we have null $\boldsymbol{\tau}-N_{4}$ coupling. An identical approach to destroy the symmetry between $N_{2}$ and $N_{3}$ is of no avail as we require a triplet and a singlet instead of two doublets. Further since the $\Lambda-\Sigma$ mass difference is rather small, we can perhaps infer that the coupling constant for $[\Lambda \Sigma \pi]$ and $[\Sigma \Sigma \pi]$ must be of the same order. However if we choose the couplings to be of the same sign the original symmetry still persists. So let us choose $G_{\Lambda \Sigma \Pi}=-G_{\Sigma \Sigma \Pi}$. The $\pi$ interaction with the $\Lambda$ and $\Sigma$ can now be written as

$$
\begin{gathered}
\propto[\Lambda \Sigma \pi]+[\Sigma \Sigma \pi]=\bar{\Sigma} \times \Sigma \cdot \pi-\bar{\Lambda} \cdot \Sigma \cdot \pi-\Sigma \cdot \Lambda \cdot \pi \\
\alpha \bar{N}_{2} \boldsymbol{\tau} N_{2} \cdot \pi+\bar{N}_{3} \tau N_{3} \cdot \pi .
\end{gathered}
$$

As regards $U=0$ baryons, the $\pi$-and $K$-meson interactions are symmetric but in a different way. The mass difference arises only when the $\pi$-nucleon interaction is switched on. The pion-baryon interactions in the model discussed here are characterized by two coupling constants. Sakurai has also suggested that the $\pi-N$ coupling will be twice $G_{\Lambda \Sigma \Pi}$ or $G_{\Sigma \Sigma I I}$.

An alternative view regarding the cosmic symmetry of $K$ interactions is possible. ${ }^{1}$ We observe that in the electromagnetic interactions, the total rest mass of the fermion minus the total rest mass of the antifermions is a constant of motion, while in weak interactions this conservation law of fermion mass is violated. Let us take this fact seriously and draw the following analogy between the $\pi$-baryon and the electromagnetic interactions. The $\pi$-baryon interaction which allows a change of fermion charge is also charge independent while the electromagnetic interactions are not so. So correspondingly we can expect interactions which allow a transfer of a mass between fermions should not depend on the mass state of the fermion. Since $K$-mesons couple different baryons, we should naturally expect that $K$-mesons should be symmetrically coupled to all the baryons, i.e. we obtain the cosmic symmetry model of $K$ interactions. Assuming charge independence of $\pi$-baryon interactions, we see that except for $\Sigma-\Lambda-\pi$ coupling, the $\pi$ 's connect the same mass states of the fermions. Our principle suggests that we can perhaps put the $\Sigma-\Lambda-\pi$ coupling equal to zero.

These considerations suggest a model of strong interaction with cosmic symmetry for $K$-baryon interactions while we need the three coupling constants $G_{1}, G_{2}$ and $G_{3}$ for $\pi$-baryon interaction where $G_{1}, G_{2}$ and $G_{3}$ correspond to $G_{1}$,

1 A. Ramakrishnan, A. P. Balachandran, and N. R. Ranganathan, Proc. Ind. Acad. Sci. 53, 1 (1961). 
$G_{2}$ and $G_{3}$ of the d'Espagnat and Prentki Lagrangian. As the model contains four coupling eonstants, it will not lead to any result in contradiction with the experiment (see later our diseussion of Pais' work). To reduce still further the number of the coupling constants, we may draw the following analogy. The symmetry of $\pi$-baryon interaction is broken down by the electromagnetic interaction in a definite way depending on $I_{3}$ of the particle. The $K$-meson interactions also connect different hypercharge states of the baryons and consequently they also exhibit a great symmetry between the baryons having different hypercharge, a result used by Sakurai. We now suggest that this symmetry is broken down by the pion-baryon interactions depending on the value of the hypercharge. Hence we have $G_{1}=-G_{3} ; G_{2}=0$. This implies that the $\pi$ interactions are cylindrically symmetrical about the third axis of the three dimensional hypercharge space. In this model the strong interaction Lagrangian is

$$
\begin{aligned}
L_{1 \mathrm{nt}}= & G\left[\bar{N}_{1} \gamma_{5} \tau N_{1}-\bar{N}_{4} \gamma_{5} \boldsymbol{\tau} N_{4}\right] \pi \\
& +F\left[\left(\bar{N}_{1} N_{2}\right) K^{0}+\left(\bar{N}_{1} N_{3}\right) K^{+}+\left(\bar{N}_{4} N_{2}\right) \bar{K}^{+}\right. \\
& \left.-\left(\bar{N}_{4} N_{\jmath}\right) \bar{K}^{0}+h \cdot c\right],
\end{aligned}
$$

where we have omitted a factor $\Gamma$ in the $K$ interaction which can be 1 or $i \gamma_{5}$ depending on the relative parity of $K$. As such, the model is highly symmetrical and will lead to results in contradiction with the experiments. To remove $\Sigma-\Lambda$ degeneracy, we can set

$$
F_{\Lambda N_{1} K}=F_{\Sigma N_{1} K}=F_{N_{4} \Lambda K}=-F \overline{N_{4} \Sigma K}=F \text {. }
$$

'This implies that we use the two new doublets $N_{2}^{\prime}$ and $N_{3}^{\prime}$ for the interaction of $K$-meson with $\Xi$ where

$$
N_{2}^{\prime}=\left(\begin{array}{c}
\Sigma^{+} \\
-Z^{0}
\end{array}\right) \quad N_{3}^{\prime}=\left(\begin{array}{c}
-Y^{0} \\
\Sigma^{-}
\end{array}\right)
$$

'This model suffers from a serious draw back in that it cannot remove $N-\Xi$ degeneracy.

\section{Doublet approximation}

The approximation of Gell-Mann, introduced by means of the "mixture particles" involves the neglect of the mass difference between the $\Lambda^{\mathbf{0}}$ and $\Sigma^{\mathbf{0}}$. This scheme of grouping the eight baryons into four doublets is called the doublet approximation. We shall digress a little on the symmetries of this scheme, a study of which will be useful for our later discussions. ${ }^{1}$

In Fig. 48 we have placed the baryons at the vertices. The pion, neutral and charged $K$-meson transitions are indicated by drawing horizontal, vertical and diagonal lines connecting the appropriate pairs of baryons.

1 Dallaporta, Nuovo Cim. 7, 200 (1957). See also the lecture notes on "Strong Interactions of Strange Particles and their Symmetries" by N. Dallaporta, Tata Institute of Fundamental Research, Bombay (1959). 
We have treated the pair of baryons connected by pions as isodoublets. It is natural to treat the baryons connected by charged and neutral $K$-mesons also as

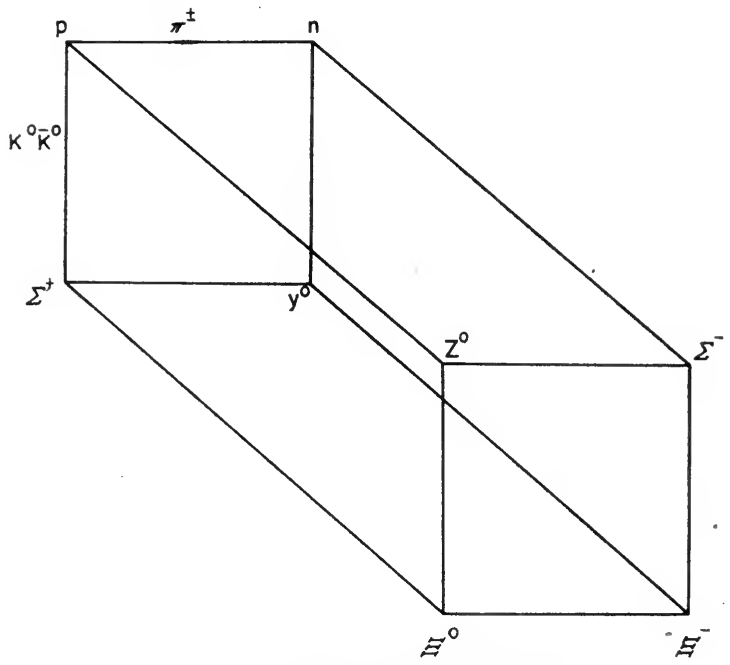

FIG. 48. Doublet approximation.

doublets in a different sense. Accordingly we will call the baryons connected by neutral $K$-mesons as strangeness doublets and distinguish them by the third

\begin{tabular}{l|r|r|r|r|r}
\hline & $Q$ & $U$ & \multicolumn{1}{|c|}{$\tau_{3}$} & $\omega_{3}$ & \multicolumn{1}{c}{$\zeta_{3}$} \\
\hline$p$ & 1 & 1 & $1 / 2$ & $1 / 2$ & $1 / 2$ \\
$n$ & 0 & 1 & $-1 / 2$ & $1 / 2$ & $1 / 2$ \\
$\Sigma^{+}$ & 1 & 0 & $1 / 2$ & $-1 / 2$ & $1 / 2$ \\
$Y^{0}$ & 0 & 0 & $-1 / 2$ & $-1 / 2$ & $1 / 2$ \\
$Z^{0}$ & 0 & 0 & $1 / 2$ & $1 / 2$ & $-1 / 2$ \\
$\Sigma^{-}$ & -1 & 0 & $-1 / 2$ & $1 / 2$ & $-1 / 2$ \\
$\Xi^{0}$ & 0 & -1 & $1 / 2$ & $-1 / 2$ & $-1 / 2$ \\
$\Xi^{-}$ & -1 & -1 & $-1 / 2$ & $-1 / 2$ & $-1 / 2$ \\
$\pi^{0}$ & 0 & 0 & 0 & 0 & 0 \\
$\pi^{+}$ & 1 & 0 & +1 & 0 & 0 \\
$\pi^{-}$ & -1 & 0 & -1 & 0 & 0 \\
$K^{+}$ & 1 & 1 & 0 & 0 & 1 \\
$K^{0}$ & 0 & 1 & 0 & 1 & 0 \\
$\bar{K}^{0}$ & 0 & -1 & 0 & -1 & 0 \\
$K^{-}$ & -1 & -1 & 0 & 0 & -1 \\
\hline
\end{tabular}

component of a strangeness vector $\omega_{3}$, assuming values $+1 / 2$ and $-1 / 2$. For baryons connected by charged $K$-mesons, we shall use a quantum number called isostrangeness spin $\zeta_{3}$ which takes $+1 / 2$ or $-1 / 2$. These quantum numbers of the various baryons are listed below. Assuming that these quantum numbers are conserved it is easy to find the appropriate quantum numbers of the bosons. 
'The most important feature of the table is that $K$ 's are isosinglets, i.e. no longer do $K^{+}, h^{0}$ form an isodoublet. From the table we find that there exist two relations

$$
\begin{aligned}
& Q=\tau_{3}+\zeta_{3} \\
& U=\omega_{3}+\zeta_{3}
\end{aligned}
$$

according to which we should have only three independent conservation laws apart from the law of conservation of baryons. However in the Gell-MannNishijima scheme we have apparently two conservation laws, i.e. conservation of $Q$ and $U$. But it should be noted that there exists an arbitrariness in assuming that $K^{+}$has positive hypercharge and $K^{-}$has negative hypercharge which is removed here because of the three conservation laws.

In this scheme there exists besides the usual charge conjugation two other types of conjugation. The antibaryon is obtained from the baryon by charge c'onjugation which reverses the signs of $Q, U$ and $N$ which implies the reversal of signs of $\tau_{3}, \omega_{3}$, and $\zeta_{3}$. We can also perform this simultaneous reversal of $Q$, $U$ and $N$ in two steps. ${ }^{1}$ (a) Reversal of $N$ and (b) the reversal of $Q$ and $U$. To comprehend the meaning of this we realize that we can go from $p$ to $\bar{p}$ by reaching $\Xi^{-}$through (b) and then $\bar{p}$ through (a), a procedure which may be justified as follows. In the case of electrons, the wave equation satisfied by the positron is obtained by reversing the charge and replacing the wave function by the charge conjugated wave function. However the heavy fermions seem to be characterized also by the baryon number and hence the equation satisfied by them may differ from the Dirac equation of the electron in that it may contain some more terms involving the nucleonic charge $N$. Thus it is plausible that we should obtain the antiparticle wave equation by reversing both the charge and the baryon number. Since $Q$ and $N$ are independent it is possible to reverse them separately and obtain two types of conjugation leading to different particles.

To impart further physical meaning to these ideas, let us imagine that there exists a fundamental baryon $B^{0}$ with $N=+1$ and $Q=U=0$. To get the baryons $N_{1}$ and $N_{4}$, we first "clothe" $B^{0}$ with $K$ 's and $\bar{K}$ 's and then subsequently with $\pi$ 's. On clothing these again with $\bar{K}$ 's and $K$ 's, we obtain $N_{2}$ and $N_{3}$. The procedure is listed below.

$$
\text { I }
$$

$$
\begin{aligned}
B^{0}+K^{+} \rightarrow p^{\prime} ; & B^{0}+K^{0} \rightarrow n^{\prime} \\
B^{0}+\bar{K}^{0} \rightarrow \Xi^{0^{\prime}} ; & B^{0}+K^{-} \rightarrow \Xi^{-\prime} \\
n^{\prime}+\pi^{+} \rightarrow p & p^{\prime}+\pi^{-} \rightarrow n ; \\
\Xi^{-\prime}+\pi^{+} \rightarrow \Xi^{0} ; & \Xi^{0^{\prime}}+\pi^{-} \rightarrow \Xi^{-} \\
p+\bar{K}^{0} \rightarrow \Xi^{0}+K^{+} \rightarrow \Sigma^{+} ; & p+K^{-} \rightarrow \Xi^{0}+K^{0} \rightarrow Z^{0} \\
n+{\overline{K^{0}}}^{0} \rightarrow \Xi^{-}+K^{+} \rightarrow Y^{0} ; & n+K^{-} \rightarrow \Xi^{-}+K^{0} \rightarrow \Sigma^{-}
\end{aligned}
$$

In this picture any baryon possesses a "spinor core" $B^{0}$ (source of the nucleonic charge $\mathrm{N}$ ) and its $Q$ and $U$ arise from the meson cloud. We can define a "spinor conjugation" which reverses the nucleonic charge alone without affecting $Q$ and $U$

\footnotetext{
1 P. Budist, N. Dallaporta and L. Fonda, Nuovo Cim. 9, 316 (1958).
} 
and a "boson conjugation" which reverses only the $Q$ and $U$ of the surrounding meson cloud. It can be seen that the usual charge conjugation is a product of these two conjugations and that the two faces of the cube of Fig. 48, i.e. $p n \Sigma^{+} Z^{0}$ and $Y^{0} \Sigma^{-} \Xi^{0} \Xi^{-}$are related by boson conjugation. Further, since boson conjugation connects $N_{1}$ and $N_{4}$, invariance under it demands the equality of $\pi$ and $K$ couplings with these doublets. Invariance under the spinor conjugation is also guaranteed provided the strong interactions are invariant under charge conjugation. It is necessary in order to split the $N_{1}-N_{4}$ degeneracy to introduce interactions which will violate separate invariance of boson conjugation and spinor conjugation though still invariant under charge conjugation.

Pais was the first to point out that the above schemes of strong interactions with high symmetries are not warranted by the experimental evidence. ${ }^{1}$ For we shall see presently that these interactions forbid reactions which actually do occur. Let us write down the interaction Lagrangian assuming the doublet approximation

$$
\begin{aligned}
L_{\text {int }}= & L_{I I}+L_{K} \\
= & G_{1} \bar{N}_{1} \gamma_{5} \tau \cdot N_{1} \pi+G_{2} \bar{N}_{2} \gamma_{5} \tau \cdot N_{2} \pi \\
& +G_{3} \bar{N}_{3} \gamma_{5} \tau N_{3} \pi+G_{4} \bar{N}_{4} \gamma_{5} \tau N_{4} \pi \\
& +F_{1} \bar{N}_{1} \Gamma N_{2} K^{0}+F_{2} \bar{N}_{1} \Gamma N_{3} K^{+} \\
& F_{3} \bar{N}_{2} \Gamma N_{4} K^{+}-F_{4} \bar{N}_{3} \Gamma N_{4} K^{0}+h \cdot c
\end{aligned}
$$

where $\Gamma=1$ or $i \gamma_{5}$ depending upon the parity of $K^{+}$and $K^{0}$. To satisfy charge independence with the customary isospin assignments to the baryons or, in other words, to obtain the d'Espagnat-Prentki interaction Lagrangian from this, we have to impose the equality of the coupling constants of $\Sigma$ and $\Lambda$ with the $\pi$-and $K$-mesons. Hence we have

$$
G_{2}=G_{3}=G ; \dot{F}_{1}=F_{2}=F_{\mathrm{I}} ; F_{3}=F_{4}=F_{\mathrm{II}} .
$$

It can be shown that if the doublet approximation holds good then the following reactions are forbidden.

$$
\left.\begin{array}{c}
K^{+}+n \rightarrow K^{\circ}+p \\
K^{-}+p \rightarrow \bar{K}^{\circ}+n \\
\pi^{+}+p \rightarrow \Sigma^{+}+K^{+} \\
K^{-}+p \rightarrow \Sigma^{+}+\pi^{-}
\end{array}\right\}
$$

The doublet approximation guarantees the separate conservation of $\tau_{3}, \omega_{3}$ and $\zeta_{3}$ while in none of the above processes are these quantities conserved, even though $Q$ and $U$ are conserved. ${ }^{2}$ We note that the interaction Lagrangian (21) with the condition given by (22) is invariant under the transformation

$$
N_{2} \leftrightarrow N_{3} ; K^{+} \leftrightarrow K^{0} ; \bar{K}^{0} \rightarrow \bar{K}^{+}
$$

1 A. PaIs, Phys. Rev. 110, 574 (1958).

2 Pais uses the quantum numbers $S_{1}$ and $S_{2}$ which can be related to $\omega_{3}$ and $\zeta_{3}$ as

$$
\omega_{3}=S_{2}+\frac{N}{2} ; \quad \zeta_{3}=S_{1}+\frac{N}{2}
$$


$N_{2} \leftrightarrow N_{3}$ implies the transformation $Y^{0} \leftrightarrow \Sigma^{-}$. Let us consider now the reaction

$$
\boldsymbol{\pi}^{-}+p \rightarrow \Sigma^{-}+K^{+} \text {. }
$$

Invariance under these transformations permit us to write

i.e.

$$
\left\langle\Sigma-K^{+} \mid \boldsymbol{\pi} p\right\rangle=-\sqrt{2}\left\langle\Sigma^{0} K^{0} \mid \boldsymbol{\pi} p\right\rangle,
$$

$$
\frac{\mathrm{d} \sigma}{\mathrm{d} \Omega}\left(\Sigma^{-} K^{+}\right)=2 \frac{\mathrm{d} \sigma}{\mathrm{d} \Omega}\left(\Sigma^{0} K^{0}\right) .
$$

This result implies not only the equality of cross-section for $\Sigma_{1}-\Sigma^{0}$ production but also an identical angular distribution of the charged and neutral $\Sigma$ 's. However it is well known that charged hyperons tend to have a forward peaking while the neutral hyperons have a backward peaking. Thus we have shown that the doublet approximation fares very badly and the strong interactions cannot be described with these coupling constants alone.

\section{Pais model ${ }^{1}$}

To overeome these difficulties Pais suggested that the relative parity $p(K)$ between $K^{+}$and $K^{0}$ can be odd. This is possible for under the doublet approximation $K^{+}$and $K^{0}$ belong to different isogroups. If $K^{+}$and $K^{0}$ form an isodoublet, the relative parity has necessarily to be even. Let us also assume that the relative parity of $K^{+}$and $A$ is even. Then the $K$ interactions become

$$
\left[K^{*}\right]=F_{\mathrm{I}}\left[\bar{N}_{1} i \gamma_{5} N_{2} K^{0}+\bar{N}_{1} N_{3} K^{+}\right]+F_{\mathrm{II}}\left[\bar{N}_{2} N_{4} K^{+}-\bar{N}_{3}\left(i \gamma_{5}\right) N_{4} K^{0}\right]+h \cdot c
$$

With this assumption of odd $p(K)$, a significant result emerges, i.e. to preserve charge independence in pion-nucleon interactions the equality of $\Sigma$ and $\Lambda$ couplings with $\pi$ - and $K$-mesons given by (22) becomes a necessary condition. To understand this, let us calculate the contribution of $K$ interactions to the self masses of $p$ and $n$. In the self energy diagrams the intermediate state for $p$ involves $\Sigma^{+}$and $K^{0}$ or $Z^{0}$ and $K^{+}$while for the neutron it will contain a $Y^{0}$ and $K^{0}$ or $\Sigma^{-}$and $K^{+}$. The $K^{+}$ has a scalar interaction and $K^{0}$ has a pseudoscalar interaction. If we denote by $a$ and $b$, the scalar and pseudoscalar matrix elements respectively since the contribution to $p$ and $n$ should be equal due to charge independence, we find

i.e.

$$
F_{I}^{2} a^{2}+F_{I I}^{2} b^{2}=F_{I I}^{2} a^{2}+F_{I}^{2} b^{2},
$$

i.e.

$$
\begin{aligned}
& \left(F_{I}^{2}-F_{I I}^{2}\right)\left(a^{2}-b^{2}\right)=0, \\
& F_{\mathrm{I}}= \pm F_{\mathrm{II}} \text { for } a \neq b .
\end{aligned}
$$

If we assume $p(K)$ to be even we have $a=b$ and thus no such condition emerges in that case.

We have already seen that the relation (22) is not in conformity with experiment. We must introduce an interaction which will violate the separate conservation

1 A. Pais, Phys. Rev. 112, 624 (1958). 
of $\tau_{3}, \omega_{3}$ and $\zeta_{3}$. An interaction of this type was suggested by Pais and involves the $\bar{K} K \pi$ coupling. ${ }^{1}$ It is represented by

$$
G_{K \Pi}\left(\bar{K}^{+} K^{0} \pi^{+}+\bar{K}^{0} K^{+} \pi^{-}\right)
$$

It will conserve parity if $p(K)$ is odd. It can be verified that it does not conserve any of the quantities $\tau_{3}, \omega_{3}$ and $\zeta_{3}$ and is not charge independent. The introduction of this interaction will allow all the previously forbidden reactions.

Through this interaction, some features of the strange particle phenomenon like scattering of $K$-mesons and associated production can be qualitatively understood. It is obvious from the structure of the interaction that while charge exchange scattering involves an exchange of a single pion, the direct scattering involves an exchange of two pions. For charge exchange scattering the virtual $\pi$ gives a factor $\frac{1}{[1+\alpha(1-\cos \theta)]^{2}}$ where $\alpha=2 p^{2} / m_{\pi}^{2}$ with $\boldsymbol{p}$ the momentum of the "incoming" $K^{+}$. This factor implies a forward peaking in the charge exchange cross-section. For heavy nuclei there will be a suppression of this forward peaking due to the Pauli principle. Pais has found that for the best fit with the experimental data on total cross-section, $G_{K \Pi} \sim e$, the unit of electric charge (with $h=c=1$ ). The direct scattering of $K^{+}$can be treated as a $\pi N$ scattering with an effective $K^{2} \pi^{2}$ interaction. In the energy range $100-200 \mathrm{MeV}$, the $\pi-N$ scattering is predominantly through $I=3 / 2$ state and hence it is to be expected that the ratio of $K^{+}+p$ to $K^{+}+n$ scattering will be $9: 1$. Experimentally we find that there is an appreciable difference between the cross-sections for $K^{+}+p$ and $K^{+}+n$ direct scatterings.

This theory affords a plausible explanation of the well-known forward peaking of charged hyperons and backward peaking of neutral hyperons in the centre of mass system. The neutral hyperon production occurs through a $\pi^{-}-p$ scattering followed by $\bar{K} K \pi$ and $K$-baryon interactions, i.e. the scattered $\pi^{-}$emits a. $K^{-}$which is absorbed by $p$ which turns into a $A^{0}$ or $\Sigma^{0}$, or the scattered $p$ can emit a $K^{+}$which absorbs $\pi^{-}$becoming a $K^{0}$. Making use of the fact that $\left(\pi^{-}-p\right)$ scattering shows a very marked forward peaking in the centre of mass system due to shadow scattering, we can understand the backward scattering of neutral hyperons. A similar procedure can explain the forward peaking of $\Sigma^{+}$hyperon, noting that $\Sigma^{+}$production vertex is pseudoscalar. Thus on evaluating the matrix element, this imparts a minus sign and hence gives a forward peaking of $\Sigma^{+}$. It is seen that $\Sigma^{-}$production is not possible through $G_{K I I}$.

The experiments on the angular distribution of $\pi^{-}$arising from the decay of $A^{0}$ or $\Sigma^{-}$produced in $\pi^{-}-p$ collisions indicate that there exists an "up-down" asymmetry about the production plane (defined by the momentum of the in-

1 A. Pars, loc. eit. Such a cubic boson interaction like $\bar{K} K \pi$ is seen to be prohibited if we demand invariance under $\gamma_{5}$ transformation, since this invariance would imply $\Phi \rightarrow-\Phi$ simultaneously with $\psi \rightarrow \gamma_{5} \psi$. See A. Ramakrishnan, A. P. Balachandran and N. R. Ranfanathan, loc. cit. See also G. Baym, Phys. Rev. 117, 886 (1960). 
coming $\pi^{-}$and the momentum of the hyperon) in the case of $\Lambda^{0}$, and no asymmetry in the case of $\Sigma^{-}$. This result can be understood if it is assumed that the produced $A^{0}$ are polarized while the $\Sigma^{-}$are not. ${ }^{1}$ This assumption is justified in a qualitative way assuming $p(K)$ to be odd. The invariant transition matrix elements for the two production processes can be written as

$$
\left.\begin{array}{c}
V_{\Sigma^{-}}=A\left(\sigma_{\Sigma^{-}} \cdot \boldsymbol{p}_{I^{-}}\right)+B\left(\boldsymbol{\sigma}_{\Sigma^{-}} \cdot \boldsymbol{p}_{\Sigma^{-}}\right) \\
V_{A^{\bullet}}=A^{\prime}+i B \sigma_{A^{\prime}} \cdot\left(\boldsymbol{p}_{A^{\bullet}} \times \boldsymbol{p}_{I^{-}}\right)
\end{array}\right\} .
$$

'The difference in structure follows from the fact that while $K^{0}$ is a pseudoscalar, $K^{+}$is a scalar. Assuming that $A, B, A^{\prime}$ and $B^{\prime}$ are real we have maximum polarization for $A^{0}$ production and no polarization in case of $\Sigma^{-}$. We should also expect $\Sigma 0$ to be polarized as much as $\Lambda^{0}$.

This $G_{K I I}$ interaction will destroy charge independence in strange particle reactions, evidence for which is not conclusive. Besides, it can in no way generate the baryon mass spectrum. Indeed, Pais has posed the question whether there exists as yet an unknown mechanism of mass displacement hidden behind the actually observed mass spectrum of the particles.

\section{Salam-Polkinghorne model ${ }^{2}$}

Earlier in this chapter we have studied a model of strong interactions in which we have made use of the symmetries available in a four-dimensional isotopic spin space. We will now refer to some other models of strong interactions of elementary particles. In these theories as in Schwinger's the elementary particles are realized as irreducible representations of the four-dimensional rotation group.

In a four-dimensional real orthogonal space, there are six possible rotations, the infinitesimal generators of which are $4 \times 4$ matrices $I_{\alpha \beta}$ where $\alpha, \beta=1,2,3,4$. 'These matrices can be represented analytically as

$$
\left(I_{\alpha \beta}\right)_{\gamma \delta}=\left(\delta_{\alpha \gamma} \delta_{\beta \delta}-\delta_{\alpha \delta} \delta_{\beta \gamma}\right),
$$

where $\delta_{\alpha \gamma}$ etc. is a Kronecker delta and $\alpha \beta$ define the particular matrix while $\gamma \delta$ define the rows and columns. As mentioned before, it is possible to group these $I_{\alpha \beta}$ 's such that we obtain two distinct three-dimensional rotation groups. To do this. let us define

$$
\begin{aligned}
& I_{i}^{+}=\frac{1}{2}\left(I_{k l}+I_{i 4}\right) \\
& I_{i}^{-}=\frac{1}{2}\left(I_{k l}-I_{i 4}\right) .
\end{aligned}
$$

1 Though this explanation suffices for $\Sigma^{-}$decay, the experimental results on $\Sigma^{+}$decay show that there is a maximum up-down asymmetry in $p+\pi^{0}$ channel while there is no asymmetry in $n+\pi^{+}$channel. Obviously this implies that the $\Sigma^{+}$are polarized when they are produced. A more satisfying explanation is that the decay in $n+\pi^{+}$channel of the $\Sigma^{+}$and the $n+\pi^{-}$ of the $\Sigma^{-}$are parity conserving while the $p+\pi^{0}$ channel is non-parity conserving. This situation is possible provided that $V-A$ interaction is operative in $p+\pi^{0}$ while $V$ or $A$ alone causes the other two channels (see the chapter on "Weak Interactions").

2 A. Salam and J. C. Polkinghorne, Nuovo Cim. 2, 685 (1955). 
where $i, k, l=1,2,3$ and all the other cyclic permutations thereof. We can verify that the following commutation relations hold good.

$$
\left[I_{i}^{+}, I_{k}^{+}\right]=-I_{l}^{+} \quad\left[I_{i}^{-}, I_{k}^{-}\right]=-I_{l}^{-} \quad\left[I_{l}^{+}, I_{k}^{-}\right]=0 .
$$

If we let

$$
I_{i}=-i I_{i}^{+} \quad K_{i}=-i I_{i}^{-}
$$

then

$$
\left[I_{i}, I_{k}\right]=i I_{l} \quad\left[K_{i}, K_{k}\right]=i K_{l} \quad\left[K_{i}, I_{l}\right]=0 .
$$

Since $\boldsymbol{I}$ and $\boldsymbol{K}$ commute and obey commutation relations just like angular momentum operators, $\boldsymbol{I}^{2}$ and $\boldsymbol{K}^{2}$ can be simultaneously diagonalized and the eigenvalues are $0,1 / 2,1 \ldots$ As usual we can assume the third component of $\boldsymbol{I}$ and $\boldsymbol{K}$, i.e. $I_{3}$ and $K_{3}$ to be diagonal. Any representation of these rotations can be labelled by a pair of numbers $i$ and $k$ denoting the lengths of $\boldsymbol{I}^{2}$ and $\boldsymbol{K}^{2}$. The dimensions of the representation will be given by $(2 i+1)(2 k+1)$.

A classification of the elementary particles and their interactions can be now attempted by realizing the particles as certain irreducible representations of the four-dimensional group. Salam and Polkinghorne have discussed the following model. ${ }^{1}$ The $\Lambda^{0}$ hyperon is treated as a scalar, the $\Sigma$ 's as a self-dual antisymmetric tensor (only three independent components) while the nucleons and cascades are treated together as a four vector in this space. The $K$-mesons form a four vector while the $\pi$-mesons form a self-dual antisymmetric tensor. We list below the quantum numbers involved in such a description:

$\left.\begin{array}{c|l|c|c|c|c}\hline \text { Particle } & \multicolumn{1}{|c|}{\begin{array}{c}\text { Description } \\ \text { and symbol. }\end{array}} & I & K & I_{3} & K_{3} \\ \hline \pi^{+} & \begin{array}{l}\text { Pseudoscalar } \\ \pi^{0} \\ \pi^{-}\end{array} & \begin{array}{l}\text { Isoself-dual } \\ \text { tensor. } \\ \varphi_{\alpha \beta}^{\Pi I}\end{array}\end{array}\right\}$

1 A. Salam and J. C. Polkivghorne, loc. cit. 
It is seen that while $I$ and $I_{3}$ give the customary isospin assignments of the multiplets, $K_{3}$ is related to the hypercharge of the d'Espagnat-Prentki scheme discussed earlier by

$$
K_{3}=\frac{1}{2} u \text {. }
$$

We can also note that the charge $Q$ is given by $Q=I_{3}+K_{3}$.

Having classified the elementary particles let us study the symmetries of the interaetions between them. ${ }^{1}$ In accordance with our general belief concerning the symmetries and strengths of the interactions, it is natural that we should expect that the strong interactions are invariant under all the rotations in the four-dimensional space. This implies that $I, K, I_{3}$ and $K_{3}$ are conserved. The selection rules for the strong interactions may be written as

$$
\Delta \boldsymbol{I}=0 ; \quad \Delta \boldsymbol{K}=\mathbf{0} .
$$

Since the electromagnetic interactions discriminate between the members of the multiplets, the selection rules for the electromagnetic interactions will be

$$
\Delta I_{3}=0 ; \quad \Delta K_{3}=0 .
$$

The weak interactions cannot be invariant under separate rotations in the two spaces but due to charge conservation will conserve $I_{3}+K_{3}$. To impart a geometric meaning let us define an $M$ space in which the generators of rotations are given by

$$
M_{i}=I_{i}+K_{i}=I_{k l} .
$$

Setting $L_{i}=-i M_{i}$, it follows that $L_{i}$ satisfy the angular momentum commutation rules. The eigenvalues corresponding to the operator $\boldsymbol{L}^{2}$ are given by $L$ which can take values $0,1 / 2,1 \ldots$ The $M$ space in which $L_{i}^{2}$ are rotation operators form a subspace which is orthogonal to the fourth axis of the isospace. By definition the range of eigenvalues of $L$ is given by

$$
L=I+K, I+K-1 \ldots|I-K| \text {. }
$$

It is now clear that the weak interactions should conserve $M_{3}$, i.e. they should be invariant under rotations about the third axis in this space.

We can now envisage two classes of strong interactions, i.e. very strong (VS) and medium strong (MS). As before, let us demand that the very strong interactions are invariant under all the rotations in the $I$ and $K$ spaces and hence in $M$ space. We will characterize the (MS) interactions by their invariance under all the rotations in $I$ space and also under rotations around the third axis in the $K$ space, the latter being necessary to conserve $Q$. The (MS) interactions are thus not invariant under arbitrary rotations in $K$ space but rotations around the third axis in $K$ space leave the (MS) interactions invariant. The lower symmetry of the (MS) interactions allows us to resolve the $N \Xi$ degeneracy.

1 J. C. Polkinghorne, Nuovo Cim.6, 864 (1957); B. D'Espagnat, J. Prentki and A. Salam, Nucl. Phys. 5, 447 (1958); B. D'Espagnat, and J. Prentki, Nucl.. Phys. 6, 596 (1958). 
The very strong interactions should be scalars in the four-dimensional space and are built up of baryons and pions. The interaction Lagrangian can be written as

where

and

$$
\Omega^{I I}=G_{1} \bar{\psi}_{\alpha}^{N} \gamma_{5} \psi_{\beta}^{N} \varphi_{\alpha \beta}^{I I}+G_{2} \bar{\psi}_{\alpha \beta}^{\Sigma} \gamma_{5} \psi_{\beta \gamma}^{\Sigma} \varphi_{\gamma \alpha}^{I I}+G_{3}\left[\bar{\psi}_{\alpha \beta}^{\Sigma} \gamma_{5} \psi^{A} \varphi_{\alpha \beta}^{I I}+h \cdot c\right] .
$$

Similar expressions hold for $\varphi_{\alpha}^{K}$ and $\varphi_{\alpha \beta}^{I}$ respectively like $\psi_{\alpha}^{N}$ and $\psi_{\alpha \beta}^{\Sigma}$.

In terms of the components, $\Omega^{\pi}$ becomes

$$
\begin{aligned}
\mathfrak{Q}^{I I}= & G_{1} i\left[\bar{\psi}_{N} \gamma_{5} \boldsymbol{\tau} \psi_{N} \boldsymbol{\pi}+\bar{\psi}_{\Xi} \gamma_{5} \boldsymbol{\tau} \psi_{\Xi} \boldsymbol{\pi}\right] \\
& +4 G_{2} i\left[\overline{\boldsymbol{\psi}}_{\Sigma} \gamma_{5} \times \boldsymbol{\psi}_{\Sigma}\right] \boldsymbol{\pi}+4 G_{3} i\left[\overline{\boldsymbol{\psi}}_{\Sigma} \gamma_{5} \psi_{\Lambda} \boldsymbol{\pi}+h \cdot c\right] .
\end{aligned}
$$

Except for a reduction of the coupling constant from four to three due to the $N-\Xi$ degeneracy, the pion interaction is identical with the corresponding part of d'Espagnat-Prentki Lagrangian.

Since the (MS) interactions are invariant under all rotations in the $I$ space and under rotations about the third axis in the $K$ space, it follows that $L^{K}$ must be a scalar in the four-space plus the third component of a vector in the $K$ space. The electromagnetic interactions which are invariant under rotations about the third axis in the usual three dimensional isospace have a similar structure. Let us attempt to formulate the scalar

$$
\begin{aligned}
\mathfrak{\Omega}_{1}^{K}= & F_{1} \bar{\psi}_{\alpha}^{N} \gamma_{5} \psi^{\Lambda} \varphi_{\alpha}^{K}+F_{2} \bar{\psi}_{\alpha}^{N} \gamma_{5} \psi_{\alpha \beta}^{\Sigma} \varphi_{\beta}^{K}+h \cdot c \\
= & F_{1}\left[\bar{\psi}_{N} \gamma_{5} \psi_{\Lambda} \varphi_{K}+i \bar{\psi}_{\Xi} \tau_{2} \varphi_{K}^{\dagger} \gamma_{5} \psi_{\Lambda}+h \cdot c\right] \\
& -F_{2}\left[\bar{\psi}_{N} \tau \varphi_{K} \gamma_{5} \boldsymbol{\psi}_{\Sigma}+i \bar{\psi}_{\Xi} \tau \tau_{2} \varphi_{K}^{\dagger} \gamma_{5} \boldsymbol{\psi}_{\Sigma}+h \cdot c\right] .
\end{aligned}
$$

The third component of a vector in $K$ space is easily formed by inserting between the vectors $\psi_{\alpha}^{N}$ and $\varphi_{\alpha}^{K}$ the third component of the rotation operator in $K$ space. We thus have

$$
\begin{aligned}
\Omega_{2}^{K}= & \frac{1}{2} F_{1}^{\prime}\left(\bar{\psi}_{N} \gamma_{5} \psi_{\Lambda} \varphi_{K}-i \bar{\psi}_{\Xi} \tau_{2} \varphi_{K}^{+} \gamma_{5} \psi_{N}+h \cdot c\right) \\
& -\frac{1}{2} F_{2}^{\prime}\left(\bar{\psi}_{N} \tau \varphi_{K} \gamma_{5} \psi_{\Sigma}-i \bar{\psi}_{\Xi} \tau \tau_{2} \varphi_{K}^{\dagger} \gamma_{5} \psi_{\Sigma}+h \cdot c\right) .
\end{aligned}
$$


$\mathfrak{Q}_{1}^{K}$ is identical with the part of d'Espagnat-Prentki interaction dealing with $K$. meson interaction except that the coupling constants have been reduced to two. On comparing $\mathfrak{I}_{1}^{K}$ and $\mathfrak{\Omega}_{2}^{K}$, the only difference is that in $\mathfrak{Q}_{2}^{K}$ the $N$ and $\Xi$ interact with $K$-mesons with coupling constants having opposite sign. $\mathfrak{Q}_{1}^{K}$ and $\mathfrak{Q}_{2}^{K}$ by themselves alone are not sufficient to resolve the $N-\Xi$ degeneracy since self mass depends on the square of the coupling constants. If however we set the strong $K$ meson Iagrangian as

$$
\mathfrak{Q}^{K}=\mathfrak{Q}_{1}^{K}+\mathfrak{2}_{2}^{K},
$$

then we have four coupling constants and the interaction is identical with that of the d'Espagnat-Prentki Lagrangian. This naturally resolves the $N-\Xi$ degeneracy.

We wish to stress the fact that to resolve $N-\Xi$ degeneracy we must only treat the $K$-meson interaction as medium strong. The choice of very strong $K$ interactions and medium strong $\pi$ interactions will not split the $N-\Xi$ degeneracy. Of course we can still remove the degeneracy arbitrarily by removing the coupling of the pion to $\Xi$ (Sakurai model).

Analogous to the electromagnetic interactions, the (MS) interactions will satisfy the selection rule

$$
|\Delta \boldsymbol{K}|=0,1 \quad \Delta \boldsymbol{I}=0 .
$$

It is possible to discuss a scheme of weak interactions by demanding that they are not only invariant under rotations around the third axis of the $M$ space, but also are invariant under arbitrary rotations in $M$ space. We shall not go into details here except pointing out these $M$ invariant interactions have the property that they can give rise to both $|\Delta \boldsymbol{I}|=1 / 2$ and $3 / 2$ transitions. ${ }^{1}$ Let us consider the decay $\Lambda^{0} \rightarrow p+\pi^{-}$. The initial state has $I=0, K=0$ and $L=0$ while the final state has $I=3 / 2,1 / 2, K=1 / 2$ and $L=2,1,0$. Let us suppose that only weak and (VS) interactions are allowed. Then $\Delta L=0$, which implies that in the final state $I=1 / 2$ or $|\Delta I|=1 / 2$. The $|\Delta I|=3 / 2$ admixture can arise from the (MS) interactions. The (MS) interactions can enter the decay of $\Lambda$ when the particle splits first into a $\bar{K}^{0}$ and $n$ while in the absence of it such a $(\Lambda N K)$ vertex cannot occur. The violation of $|\Delta \boldsymbol{I}|=1 / 2$ rule will not be very strong because of the (MS) interactions. It is to be noted that except for the decay of $K^{+}$into two pions (though slow compared to $K^{0}$ ) all the other non-leptonic decays of strange particles seem to obey $|\Delta \boldsymbol{I}|=1 / 2$ rule perfectly.

A scheme for including leptons in this four isospace has been discussed which is however far from satisfactory. ${ }^{2}$

Pais has also studied the classification of the symmetries of interactions on a formal basis using a four-dimensional isospace. ${ }^{3}$ We shall not here deal with his

${ }^{1}$ It should be remembered that a four vector will split into a three vector plus a scalar, a self dual antisymmetric tensor will be a three vector and a four scalar will become three scalar in the $M$ space.

2 I. SAAVEDra, Nucl. Phys. 10, 6 (1959).

3 A. Pais. Proc. Nat. Acad. Sci.40, 484 (1954). 
first model as it gave rise to many non-observed particles. The second model $^{1}$ is more realistic and is intimately related to the doublet approximation. The quantum numbers for the elementary particles are listed below.

\begin{tabular}{|c|c|c|c|c|}
\hline Particle & $I$ & $I_{3}$ & $K$ & $K_{3}$ \\
\hline$p$ & $1 / 2$ & $1 / 2$ & & 0 \\
\hline$n$ & & $-1 / 2$ & 0 & 0 \\
\hline$\Sigma^{+}$ & $1 / 2$ & $1 / 2$ & $1 / 2$ & $1 / 2$ \\
\hline$Y^{0}$ & & $-1 / 2$ & & $1 / 2$ \\
\hline$Z^{0}$ & $1 / 2$ & $1 / 2$ & $1 / 2$ & $-1 / 2$ \\
\hline$\Sigma^{-}$ & & $-1 / 2$ & & $-1 / 2$ \\
\hline$\Xi^{0}$ & $1 / 2$ & $+1 / 2$ & & 0 \\
\hline$\Xi^{-}$ & & $-1 / 2$ & 0 & 0 \\
\hline$\pi^{+}$ & & 1 & & 0 \\
\hline$\pi^{0}$ & 1 & 0 & 0 & 0 \\
\hline$\pi^{-}$ & & -1 & & 0 \\
\hline$K^{+}$ & 0 & 0 & $1 /$ & $1 / 2$ \\
\hline$K^{0}$ & & 0 & $/ 2$ & $-1 / 2$ \\
\hline $\begin{array}{l}\bar{K}^{0} \\
K^{-}\end{array}$ & 0 & $\begin{array}{l}0 \\
0\end{array}$ & $1 / 2$ & $\begin{array}{r}1 / 2 \\
-1 /\end{array}$ \\
\hline
\end{tabular}

Having assigned representations to the particles, the symmetries of various interactions are assigned. The $\pi$ interactions have the highest symmetry in that they conserve all the quantum numbers $I, I_{3}, K$ and $K_{3}$. Due to the oddness of $p(K)$ the introduction of $K$-meson coupling destroys the equivalence between the states with $K_{3}=+Y_{2}$ and $K_{3}=-Y_{2}$. For the $K$-meson interactions $I, I_{3}$ and $K_{3}$ are conserved but not $K$. As in the Salam-Polkinghorne scheme, the electromagnetic interactions conserve $I_{3}$ and $K_{3}$. The $\bar{K} K \pi$ interactions happen to be the weakest among the class of the strong interactions in that even separate conservation of $I_{3}$ and $K_{3}$ no longer holds good. Only $I_{3}+K_{3}$ is a good quantum number.

We shall now refer to a model of strong interactions in which all the baryons are grouped together by treating them as different "charge" states of a single field which will be described by a 32 -component spinor wave function. ${ }^{2}$ As before, introducing a four-dimensional hypercharge space, we form a $\pi$-baryon interaction which is left invariant under rotations in a three-dimensional subspace, i.e. the isotopic spin space. As in the Salam-Polkinghorne model, the $K$-baryon interaction is a sum of two terms, the first one being a scalar in the four-dimensional space, the second transforming like the third component of a vector in the other three-dimensional subspace. The $N-\Xi$ degeneracy is removed because of the difference in the coupling constants of $K$ with $N$ and $\Xi$. To remove the $\Lambda$ and $\Sigma^{0}$ degeneracy, use is made of the freedom in defining the doublets $N_{2}$ and $N_{3}$, i.e.

1 A. PAIS, Phys. Rev. 112, 624 (1958).

2 J.Tromno, Nuovo Cim. 6, 69 (1957); N. Dallaporta and Tayoda, Nuova Cim. 14, 142 (1959); N. Dallaporta and V. De Santis, Nuovo Cim. 14, 225 (1959); N. Dallaporta and L. K. Pandit, Nuovo Cim. 16, 135 (1960). 
we can exchange $Y^{0}$ and $Z^{0}$. If we add the two Lagrangians, $A$ and $\Sigma$ have different coupling constants with $\pi$ and $K$-mesons. Except for $\Lambda \Sigma \pi$ coupling 'onstant, all the other pion-baryon coupling constants satisfy global symmetry.

\section{Feynman's model ${ }^{1}$}

We will now take up a very speculative model of strong interactions due to Feynman who obtains a relation between the square of the baryon mass $M$ and the quantum numbers like strangeness $S$ and the total isotopic spin $I$ which are conserved in the strong interactions,

$$
M^{2}=a+b I(I+1)-c S .
$$

The three constants are determined from the masses of $p, \Lambda$ and $\Xi^{-}$. The constants so obtained give correctly the masses of the $\Sigma$ hyperons. In this expression we neglect the mass difference within a multiplet. Naturally the question arises whether it is possible for any scheme of strong interactions to produce this formula.

We now recognize that $I(I+1)$ is the eigenvalue of the square of the isotopic spin vector $\boldsymbol{I}$ and hence if the interaction of a particle depends upon $\boldsymbol{I}$, it should yield such a term. Obviously a particle coupled to $\boldsymbol{I}$ should transform like an isotopic vector. Thus it is suggested that the pion (isovector) is coupled to the total $\boldsymbol{I}$.

In the absence of the pion coupling $\Lambda^{0}$ and $\Sigma^{0}$ have the same mass so that the $K$-meson couplings do not destroy this degeneracy. This can be achieved by writing a $K$ interaction Lagrangian which preserves isotopic spin and strangeness and setting $F_{1}=F_{2}=F_{I} ; F_{3}=F_{4}=F_{I I}$. Since $F_{I} \neq F_{I I}$ there will be no longer a degeneracy between the $\Lambda^{0}$ and the $\Sigma^{0}$ in the fourth order. Assuming global symmetry for pion interactions in this model of strong interactions, we have three coupling constants $G, F_{I}$ and $F_{I I}$.

Let us assume that in the absence of these couplings all the baryons have equal masses. In the absence of pion couplings, the contribution of $K$ interactions to the self-masses of $p(n)$ and $\Xi^{-}\left(\Xi^{0}\right)$ is proportional to $F_{I}^{2}$ and $F_{I I}^{2}$ respectively while for other baryons it is proportional to $F_{1}^{2}+F_{1 I}^{2}$. According to this we should expect that the square of the mass of the degenerate $\Sigma$ and $\Lambda$ should be the average of that of the nucleon and the $\Xi$. This is precisely what is expressed by the term $-c S$. Thus we have developed a model of strong couplings which can yield the formula given by (45).

In order to study the symmetry properties of the strong couplings we suppose that the pion couplings are not strong, i.e. the perturbation treatment of the pion couplings is valid. Near resonances involving pion couplings this assumption will break down. The reason for regarding pion coupling as not strong seems to be

${ }^{1}$ R. P. Feysmax (unpublished). Professor Feynman feels that the physicist's problem is not just to make speculative models, but really to find out what is going on in nature. In his opinion, this model is a speculation and almost certainly does not describe the observed reality. However, we wish to include it to demonstrate the gross inadequacy of conventional ideas to answer the challenge of strong interactions and the necessity for new ways of thinking. 
favoured because the $A^{0}-\Sigma^{0}$ mass difference, caused by pion couplings, in this model, is smaller than the other mass difference between the baryons.

In the absence of the pion couplings let us attempt to define a few quantum numbers to describe the symmetry of the system. We see that the $K$-meson couples $p, Z^{0}, \Sigma^{+}, \Xi^{0}$ together and so also $n, Y^{0}, \Sigma^{-}$and $\Xi^{-}$. These two sets are independent. In each set we have two single independent members and a doublet. Let us call these quantum numbers by $A_{3}$ and $B_{3}$, which are listed below.

\begin{tabular}{c|c|c}
\hline & $A_{3}$ & $B_{3}$ \\
\hline$p$ & $1 / 2$ & 0 \\
$\Sigma^{+}$ & $1 / 2$ & $1 / 2$ \\
$Z$ & $1 / 2$ & $-1 / 2$ \\
$\Xi^{0}$ & $1 / 2$ & 0 \\
$n$ & $-1 / 2$ & 0 \\
$\Sigma^{-}$ & $-1 / 2$ & $-1 / 2$ \\
$Y^{0}$ & $-1 / 2$ & $+1 / 2$ \\
$\Xi^{-}$ & $-1 / 2$ & 0 \\
$K^{+}$ & 0 & $1 / 2$ \\
$K^{-}$ & 0 & $-1 / 2$ \\
$K^{0}$ & 0 & $-1 / 2$ \\
$\bar{K}^{0}$ & 0 & $1 / 2$ \\
\hline
\end{tabular}

As before, we can interpret $A_{3}$ and $B_{3}$ as the third component of two three-dimensional vectors obtained from a four dimensional space. $A_{3}$ is identically equal to $\tau_{3}$ introduced in the doublet approximation. The isotopic spin of the particle is given by $\boldsymbol{I}=\boldsymbol{A}+\boldsymbol{B}$. Indeed this relation suggests a method of breaking the symmetry. We can have two pions $\pi_{a}$ and $\pi_{b}$ which depend respectively on $A$ and $B$ alone, the $A_{3}$ or $B_{3}$ values of $\pi_{a}$ and $\pi_{b}$ being $(1,0)$ and $(0,1)$ respectively. It follows now that $\pi_{b}$ connects only $\Sigma^{-}, \Sigma^{+}, Y^{0}$ and $Z^{0}$. A virtual pion can be emitted as $\pi_{a}$ and absorbed as $\pi_{b}$ thus breaking the laws of conservation of $\boldsymbol{A}$ and $\boldsymbol{B}$ separately. If however virtual pion processes are not important, we can expect $\boldsymbol{A}$ and $\boldsymbol{B}$ to be conserved separately. Otherwise only the total $\boldsymbol{A}+\boldsymbol{B}$, i.e. isotopic spin, is conserved.

As an application of this model of the strong interaction let us consider the charge exchange scattering of $K^{+}$. This process is forbidden because of the failure of the conservation of $A_{3}$. However we note that there is large charge exchange scattering in the energy region 200-300 MeV, though at low energy regions there is very little charge exchange scattering. This is at best explained by invoking a resonance involving virtual pions, in which case we have no separate conservation. A similar difficulty exists in explaining $K^{-}-p$ capture reaction.

For the sake of completeness we shall also discuss a model of weak interaction which follows from this scheme of strong interactions. As we have seen earlier the weak interaction can be pictured as an interaction of a current with itself, i.e. $J_{w}^{\dagger} J_{w}$. We also know that $J_{w}$ can be written as

$$
J_{w}=J_{l}+J_{s c}+J_{s u c},
$$


where the terms have the previous meaning. In order that vector coupling need not be renormalized, it was shown that $J_{s c}$ should be coupled to the operator $I_{+}$. In the present case $I_{+}=A_{+}+B_{+}$. For simplicity let as assume $J_{8 c}$ to be coupled to $A_{+}$alone. As long as $A^{+}$is separately conserved, the renormalization of the coupling constant is not required. Thus the form $J_{s c}$ is

$$
J_{s c}=(\bar{p} n)+\left(\overline{\Sigma^{+}} Y\right)+\left(\bar{Z} \Sigma^{-}\right)+\left(\Xi^{0} \Xi^{-}\right) .
$$

It can be verified that $J_{* c}$ has $A_{z}=-1$ and $B_{z}=0$, i.e. its $A$ spin is 1 , and $B$ spin equals zero.

We shall now determine the structure of $J_{z n c}$ by discussing the most mysterious feature of $K$ particle decays, i.e. the slow decay of $K^{+} \rightarrow \pi^{+}+\pi^{0}$ as compared to the $2 . \tau$ decay mode of the neutral $K$-meson. We will so determine the structure of $J_{s n c}$ such that $K^{0}$ decay is allowed, while $K^{+}$is forbidden on neglecting the virtual pions which is an interesting feature of this model. To see this we note that $J_{s \| c}$ can consist of the following terms

\begin{tabular}{c|c|c}
\hline & $A_{3}$ & $B_{3}$ \\
\hline $\bar{p} Y$ & -1 & $1 / 2$ \\
$-(\bar{p} Z)$ & $0, \overline{0}$ & $-1 / 2$ \\
$\left(\bar{n} \Sigma^{-}\right)$ & $0, \overline{0}$ & $-1 / 2$ \\
$(\bar{\Sigma}, n)$ & -1 & $-1 / 2$ \\
\hline
\end{tabular}

where $\overline{0}$ indicates that the term belongs to a triplet of $A=1$. If we retain only terms with $A_{3}=0$ for non-leptonic decays of the strange particles induced by $J_{8 c}^{+} J_{8 n c}+h \cdot c$ we will have the selection rule $\Delta A_{3}= \pm 1$. From the quantum numbers of $K^{0}$ and $\pi^{0}$ we see that the decay $K^{0} \rightarrow \pi^{0}+\pi^{0}$ is forbidden. If we have only $(\bar{p} Y)$, we can verify that the decay $K^{+} \rightarrow \pi_{b}^{+}+\pi_{a}^{0}$ is allowed because of the presence $A=1$ state. But on adding the term $\left(\Sigma^{+} n\right)$, the total coupling of $J_{s c} J_{s n c}^{\dagger}$ has $\Delta A_{3}=0$ with $A=0$ or 2 states only. $K^{+}$is then forbidden to decay into $\pi_{b}^{+}+\pi_{a}^{0}$ and also into states $\pi_{a}^{+}+\pi_{b}^{0}$ and $\pi_{a}^{+}+\pi_{a}^{0}$ because of $\Delta A_{3}=0$. The decay into the state $\pi_{b}^{+}+\pi_{b}^{0}$ is equally prevented. However if we include the terms like $(\bar{p} Z)$ or $\left(n \Sigma^{-}\right)$, these decays become possible. Thus we determine the structure of $J_{\& n e}$ as

$$
J_{s \| c}=(\bar{p} Z)+\left(\overline{\Sigma^{+}} n\right) .
$$

Adding the corresponding terms with $\Xi$ we have

$$
J_{s u c}=(\bar{p} Y)+\left(\bar{\Sigma}^{+} n\right)+\left(\bar{\Xi}^{0} \Sigma^{-}\right)+\left(\bar{Z}_{1} \bar{\Xi}^{-}\right) \text {. }
$$

The main drawback of this is the existence of terms with strangeness -1 and +1 which implies that we can have decays like $\Delta S=2$ for which there is very little evidence as discussed before. The total weak interaction takes a very symmetric and simple form

$$
J=\bar{v}(e+\mu)+\left(\bar{p}+\bar{\Sigma}^{+}\right)(n+Y)+\left(\bar{\Xi}^{0}+\bar{Z}^{0}\right)\left(\Xi^{-}+\Sigma^{-}\right) .
$$

This current with equal coefficients for $J_{8 c}$ and $J_{8 n c}$ will allow a higher branching ratio for the $\beta$-decays of hyperons, i.e. $\Sigma^{+} \rightarrow n+e^{+}+v, A^{0} \rightarrow p+e^{-}+\bar{v}$ than 
so far observed from the experiments. Of course as pointed out earlier, it is not clear whether $J_{s n c}$ has the same coupling constant as the other parts of the total weak interaction current.

We will conclude our discussion of this model with a few remarks on the selection rules. For the leptonic decays with the emission of negative leptons

$$
\Delta A_{3}=+1, \quad \Delta B_{3}=-1 / 2 ; \quad|\Delta \boldsymbol{A}|=1, \quad|\Delta \boldsymbol{B}|=1 / 2 .
$$

For the emission of positively charged leptons we have

$$
\Delta A_{3}=-1, \quad \Delta B_{3}=+1 / 2, \quad|\Delta \boldsymbol{A}|=1 ; \quad|\Delta \boldsymbol{B}|=1 / 2 .
$$

However it forbids $K^{+} \rightarrow \mu^{+}+v$ since $\Delta A_{3}=0$. For non-leptonic decays with $\Delta S= \pm 1$, the selection rules are

$$
\Delta B_{3}=-\frac{1}{2}, \Delta A_{3}=0 ; \quad|\Delta \boldsymbol{B}|=1 / 2 ; \quad|\Delta \boldsymbol{A}|=0 \quad \text { or } \quad 2 .
$$

The rule $\Delta B_{3}=-1 / 2$ allows $K_{1}^{0}$ decay through both $A=0$ or 2 . If we do not allow $A=2$ state, then we have the usual $|\Delta \boldsymbol{I}|=1 / 2$ rule. These rules for the $3 \pi$ decay modes for $K^{+}$and $K_{2}^{0}$ predict that $K_{2}^{0} \rightarrow \pi^{+}+\pi^{-}+\pi^{0}$ is half as fast as $K^{+} \rightarrow 2 \pi^{+}+\pi^{-}$while $B \pi^{0}$ decay of $K_{2}^{0}$ is thrice as fast as $K^{+} \rightarrow 2 \pi^{0}+\pi^{+}$. It is to be noted that there are three channels, i.e. all the three pions are $\pi_{b s}^{\prime}$ with $A=0$ or one $\pi_{b}$ and two $\pi_{a s}^{\prime}$ with $A=0$ or 2 .

We have presented the various "wild ideas" on strong interactions with a view to pointing out the inadequacy of our knowledge about them. Most of the models are incomplete and yet we cannot distinguish between them because of our inability to calculate accurately the consequences of the strong interactions. The search for more symmetries of strong couplings seems to be the only way of understanding the strong interactions. We can safely remark that the laws of strong interaction have not been understood as yet.

\section{COMPOUND MODELS}

When the new particles were being discovered, attempts were made to understand the spectrum of the elementary particles using compound models. In these models some of the elementary particles were treated as fundamental and the rest of the particles were pictured as bound states made up of these constituents. A number of compound models became possible because of the different choices of the fundamental constituents. The general idea underlying the compound models is to use a minimum number of fields. Heisenberg and his collaborators have studied systematically a model of elementary particles using a non-linear spinor equation.

We shall deal here with some of the phenomenological models. Fermi and Yang $^{1}$ first proposed that the pions should be considered as the bound states of

1 E. Fermi and C. N. YANG, Phys. Rev. 76, 1739 (1949). 
a nucleon and an antinucleon. A combination of a nucleon and an antinucleon will yield the following four states composed of a triplet and a singlet, i.e.

$$
\begin{aligned}
& p \bar{n} \rightarrow \pi^{+} \\
& \left.\begin{array}{r}
\frac{1}{\sqrt{ } 2}(p \bar{p}+n \bar{n}) \rightarrow \pi^{0} \\
n \bar{p} \rightarrow \pi^{-}
\end{array}\right\} \text {triplet. } \\
& \frac{1}{\sqrt{ } 2}(p \bar{p}-n \bar{n}) \rightarrow \pi^{0^{\prime}}-\text { singlet }
\end{aligned}
$$

The last particle has not been so far observed though it has been proposed by Nambu and others ${ }^{1}$ in order to explain some anomalous features the electromagnetic structure of the nucleons. Apart from this drawback, the main difficulty of the picture is to explain the large mass difference between $N \bar{N}$ and $\pi$ which has to be accounted for by postulating a very deep attractive potential well between $N$ and $\bar{N}$ to provide for the large binding energy. Indeed the explanation of large value of the binding energy is a problem in all the compound models.

Goldhaber proposed a model by treating the nucleons, the $K$-mesons and their antiparticles as fundamental. ${ }^{2}$ Since the hyperons have strangeness -1 , it is clear that we should have an attractive potential between $\bar{K}$ and the nucleons which is fortunately true, experimentally. The $\Lambda$ and $\Sigma$ hyperons are obtained as

$$
\begin{array}{ll}
\left(p \bar{K}_{0}\right) \rightarrow \Sigma^{+} & \frac{1}{\sqrt{ } 2}\left(p K^{-}+n \bar{K}^{0}\right) \rightarrow \Sigma^{0} \\
\left(n K^{-}\right) \rightarrow \Sigma^{-} & \frac{1}{\sqrt{ } 2}\left(p K^{-}-n \bar{K}^{0}\right) \rightarrow \Lambda^{0}
\end{array}
$$

Sinee both the nucleons and $K$-mesons are isospinors, the isotopic spin assignments of $\Sigma$ and $\Lambda$ also follow. Let us choose an isotopic spin dependent potential of the form

$$
V=-V_{0}+\tau_{1} \cdot \tau_{2} V_{1}
$$

where $0<V_{1}<V_{0}$. Since $\tau_{1} \cdot \tau_{2}=-3$ for $I=0$, and +1 for $I=+1, \Lambda$ has more binding energy than $\Sigma$ and so has a lower mass than $\Sigma$. It is pertinent to point out that such a model of the $\Lambda$ and $\Sigma$ hyperon cannot account for the updown asymmetry of the pions resulting from the decays of the hyperons. For we can picture the decay of the hyperons as resulting from the decay of the $K$-mesons, either through $2 \pi$ or $3 \pi$ modes, the excess of pions being absorbed by the nucleon "core". However no asymmetry will result from the decay of a spinless boson into spinless bosons for the conservation of angular momentum does not allow a mixture of angular momentum partial waves in the final state. In order to explain the very interesting features of the asymmetry in the various channels of $\Sigma$-decay,

1 Y. Nambu, in the Proc. 7th. Rochester Conference, Interscience, New York (1957).

2 S. Goldhaber, Phys. Rev. 101, 433 (1956). See also Proc. 7th Rochester Conference, Interscience, New York (1957). 
Barshay and Schwartz have recently considered $\Sigma$ as a compound state of a $\Lambda$ and $\pi .^{1}$

To obtain the cascade particles of strangeness -2 it is obvious that we should compound the $\Lambda$ on $\Sigma$ with $\bar{K}$ 's. When we do so we obtain two isodoublets and a quadruplet with $I=3 / 2$. Since only one doublet has been observed, i.e. $\left(\Xi^{-}, \Xi^{0}\right)$ the model is faced with serious difficulties. Absence of hyperons with strangeness +1 can be easily explained by means of a repulsive potential between the nucleons and $K$-mesons which also seems to be supported by the experiments on $K^{+}-N$ scattering.

Another very interesting model which has many attractive features and which has recently received attention is that of Sakata which treats $p, n$ and $\Lambda$ as fundamental particles. ${ }^{2}$ We have already referred to the important features of this model from the point of view of universal Fermi interactions. We now give the picture of elementary particles following from this model which is essentially an extension of the Fermi-Yang model with the addition of $\Lambda$ to account for the strange particles. We have

$$
\begin{aligned}
N \bar{\Lambda} \rightarrow K ; & \bar{N} \Lambda \rightarrow \bar{K} \\
(N(\bar{N} \Lambda)) \rightarrow \Sigma ; & (\Lambda(\bar{N} \Lambda)) \rightarrow \Xi .
\end{aligned}
$$

There is a large fluctuation in binding energy as successively a baryon and an antibaryon are added as seen from the masses of $K$ and $\Sigma$ particles. Recently Yamaguchi ${ }^{3}$ has proposed a model of strong interactions on the basis of this model. Thirring ${ }^{4}$ has studied a model of strong interactions employing only three fields as fundamental. These fermion fields are two component spinor fields.

\section{SPACE-TIME PROPERTIES AND INTERNAL SYMMETRIES OF STRONG INTERACTIONS}

It is now well established that while the weak interactions do not conserve parity, the strong interactions are invariant under space reflections. It is rather surprising that such a geometrical invariance should be at all related to the strengths of the interactions involved in a particular phenomenon. However we note that the strongly interacting particles possess internal degrees of freedom such as isospin and strangeness which the leptons do not seem to possess. It is also surprising that the domain of validity of the parity conservation and strangeness conservation coincide. We are thus led to expect an intimate relation between "internal" symmetries and the space-time symmetries. We will here summarize some important investigations based on this approach. We will assume that all

1 S. Barshay and M. Schwartz, Phys. Rev. Lett.4, 618 (1960). See also A. P. Balachandran and N. R. Ranganathan, Nuovo Cim. 18, 1269 (1960); G. Bhamathi, S. Indumathi, A. P. Batachandran and N. G. Deshpande, Nuovo Cim. 19, 190 (1061).

2 S. Sakata, Progr. Theoret. Phys. 16, 686 (1956).

3 Y. Yamaguchi, Progr. Theoret. Phys. Supplement, 11, 37 (1959).

4 W. Thirring, Nucl. Phys. 14, 895 (1959).

EPOR $\quad 23$ 
the interactions of the elementary particles are invariant under $C P$ which seems to hold. 'The following theorems can be proved:

(a) Theorem 1: If $C P$ invariance holds and if the Yukawa coupling is direct then parity must be conserved for charge symmetric interactions of the form $\bar{\Gamma} \boldsymbol{\tau} \boldsymbol{x}^{1}{ }^{1}$

Assuming no parity conservation, the interaction Lagrangian is

$$
\begin{aligned}
L_{\text {int }} & =\bar{N} \boldsymbol{\tau}\left(g_{8}+g_{p} \gamma_{5}\right) N \cdot \boldsymbol{\tau}+\bar{N} \boldsymbol{\tau}\left(g_{8}^{*}-g_{p}^{*} \gamma_{5}\right) N \cdot \boldsymbol{\tau} \\
& =\bar{N} \boldsymbol{\tau}\left(g_{8}^{\prime}+g_{p}^{\prime} \gamma_{5}\right) N \boldsymbol{\tau}
\end{aligned}
$$

where $g_{s}^{\prime}$ is pure real and $g_{p}^{\prime}$ is pure imaginary. In this it is to be noted that the $\boldsymbol{\pi}$ field is self-conjugate, i.e. $\boldsymbol{x} \equiv \boldsymbol{x}^{\dagger}$. If we now demand $C P$ invariance, we find that either $g_{s}^{\prime}$ or $g_{p}^{\prime}$ should vanish, for to have $C P$ invariance all the coupling constants should have the same phase. To observe the role of internal symmetry in this theorem, we note that charge symmetry of the interactions requires that the coupling constants characterizing the $\bar{p} \cdot n \pi^{+}$and the $\bar{n} p \pi^{-}$interactions be the same not only in absolute magnitude but also in phase and sign.

We can also verify that if $C$ invariance holds and if the $\pi-N$ interaction must be of the derivative type, then it must conserve parity and $\pi^{0}$ must be pseudoscalar provided $\pi^{0}$ is even under charge conjugation. If we now further assume charge independence, the charged pions are also pseudoscalar, a result pointed out by Sakurai. ${ }^{2}$ It is to be noted that $\pi^{0}$ is even under $C$, independent of whether $\pi^{0}$ is scalar or pseudoscalar. This will follow provided that $\pi^{0}$ is spinless and we allow the virtual process $\pi^{0} \rightarrow p+\bar{p}$.

These results apply equally to $\Xi \Xi \pi$ and $\Sigma \Sigma \pi$ interactions. However these theorems in no way help us to prove that the $\Sigma \Lambda \pi$ interaction will be parity conserving. To see this, let us write a $C P$ invariant but parity non-conserving and charge independent $\Sigma \Lambda \pi$ interaction, i.e.

$$
L_{\Sigma \Lambda \pi}=\Sigma\left(a+b \gamma_{5}\right) \Lambda \boldsymbol{\pi}+\bar{\Lambda}\left(a-b \gamma_{5}\right) \Sigma \boldsymbol{\pi} .
$$

It is easily seen that charge independence of the interaction does not guarantee the invariance of the Lagrangian under $\Sigma \leftrightarrow \Lambda$ interchange. Thus we cannot obtain any restriction on the structure of the interaction. To obtain such restrictions, we have to introduce the doublet approximation for the $\Sigma-\Lambda$ system and assume $g_{\Sigma \Lambda \pi}=g_{\Sigma \Sigma_{\pi}}$. Then we know from our previous discussions that the $\pi$ interactions with $\Sigma$ and $\Lambda$ have the same form as $\pi-N$ interactions. We can thus obtain parity conservation in $\Sigma \Lambda \pi$ interactions. $^{3}$

Turning now to $K$ interactions, since the $K$ 's connect different baryon multiplets, it is clear that we cannot exchange the member of a doublet with those of a triplet. We can hope to obtain necessary restrictions for parity conservation

1 G. Feinberg, Phys. Rev. 108, 878 (1957). S. N. Gupta, Cam. J. Phys. 35, 1309 (1957); V. G. Solov'ev, J. Expt. Theor. Phys. 33, 537, 796 (1957).

2 J. J. SAKURAI, loc. cit.

${ }^{3}$ G. Feinberg and F. Gürsey, Phys. Rev. 114, 1153 (1953). 
on previous lines only when we group all the baryons into doublets. The above discussions on charge independent $\pi-N$ interactions clearly show that for the cosmic symmetry model of $K$ interactions parity will be conserved provided the interactions are $C P$ invariant since cosmic symmetry provides for hypercharge symmetry.

To discuss the case of $K$ interactions when no such symmetry is assumed, let us write the $C P$ invariant non-derivative Yukawa $K$-baryon interactions as

$$
\begin{aligned}
L_{\mathrm{int}}= & \bar{N}_{1}\left(g_{8}+g_{p} \gamma_{5}\right) N_{2} K^{0}+\bar{N}_{2}\left(g_{8}^{*}-g_{p}^{*} \gamma_{5}\right) N_{1} K^{0 \dagger} \\
& +\bar{N}_{1}\left(g_{8}^{\prime}+g_{p}^{\prime} \gamma_{5}\right) N_{3} K^{+}+N_{3}\left(g_{8}^{* \prime}-g_{p}^{*} \gamma_{5}\right) N_{1} K^{+\dagger} \\
& +\bar{N}_{3}\left(f_{8}+f_{p} \gamma_{5}\right) N_{4} K^{0}+\bar{N}_{4}\left(f_{8}^{*}-f_{p}^{*} \gamma_{5}\right) N_{3} K^{0 \dagger} \\
& +\bar{N}_{2}\left(f_{8}^{\prime}+f_{p}^{\prime} \gamma_{5}\right) N_{4} K^{+}+\bar{N}_{4}\left(f_{8}^{\prime *}-f_{p}^{\prime *} \gamma_{5}\right) N_{2} K^{+\dagger}
\end{aligned}
$$

Considering the first two terms alone in the above expression we find that for the first to be invariant under $C P$ we should have

$$
g_{s}=\eta_{1} g_{s}^{*}, \quad g_{p}=g_{p}^{*} \eta_{1}
$$

where $\eta_{1}$ is the product of the phase factors for the $N_{1}, N_{2}$ and $K$ fields under $C P$, i.e. $\eta_{1}^{*}=\eta_{N_{1}}^{*} \eta_{N_{\mathrm{s}}} \eta_{K^{0}}$. Similar conditions can be obtained for the other terms and the interactions can be written using these relations

$$
\begin{aligned}
L_{\text {int }}= & g_{8}\left(\bar{N}_{1} N_{2} K^{0}+\eta_{1} \bar{N}_{2} N_{1} K^{0 \dagger}\right)+g_{p}\left(\bar{N}_{1} \gamma_{5} N_{2} K^{0}-\eta_{1} \bar{N}_{2} \gamma_{5} N_{1} K^{0 \dagger}\right) \\
& +g_{8}^{\prime}\left(\bar{N}_{1} N_{3} K^{+}+\eta_{1}^{\prime} \bar{N}_{3} N_{1} K^{+\dagger}\right)+g_{p}^{\prime}\left(\bar{N}_{1} \gamma_{5} N_{3} K^{+}-\eta_{1}^{\prime} \bar{N}_{3} \gamma_{5} N_{1} K^{+\dagger}\right) \\
& +f_{8}\left(\bar{N}_{3} N_{4} K^{0}+\eta_{2} \bar{N}_{4} N_{3} K^{0 \dagger}\right)+f_{p}\left(\bar{N}_{3} \gamma_{5} N_{4} K^{0}-\eta_{2} \bar{N}_{4} \gamma_{5} N_{3} K^{0 \dagger}\right) \\
& +f_{8}^{\prime}\left(\bar{N}_{2} N_{4} K^{+}+\eta_{2}^{\prime} \bar{N}_{4} N_{2} K^{+\dagger}\right)+f_{p}^{\prime}\left(\bar{N}_{2} \gamma_{5} N_{4} K^{+}-\eta_{2}^{\prime} \bar{N}_{4} \gamma_{5} N_{2} K^{+\dagger}\right) .
\end{aligned}
$$

To obtain parity conservation for $K^{0}$ interactions, let us define the "charge symmetry" operation

$$
N_{1} \leftrightarrow N_{2} \quad N_{3} \leftrightarrow N_{4} \quad K^{0} \leftrightarrow K^{0 \dagger} .
$$

In order to preserve the terms involving $K^{+}$we see that $K^{+}$should transform as

$$
K^{+} \rightarrow \varepsilon_{+} K^{+}
$$

where $\varepsilon_{+}$is a phase factor with $\left|\varepsilon_{+}\right|=1$. Invariance under (6) implies that

Also

$$
\varepsilon_{+} g_{s}^{\prime}=f_{s}^{\prime} ; \quad \varepsilon_{+} g_{p}^{\prime}=f_{p}^{\prime} ; \quad \eta_{1}^{\prime}=\eta_{2}^{\prime} .
$$

$$
g_{s} \text { or } g_{p}=0, \quad f_{s} \text { or } f_{p}=0, \quad \eta_{1}= \pm 1, \quad \eta_{2}= \pm 1 .
$$

This $K^{\mathbf{0}}$ interaction preserves the parity quantum number. For $K^{+}$interactions to conserve parity, we have the transformation

$$
N_{1} \leftrightarrow N_{3}, \quad N_{2} \leftrightarrow N_{4}, \quad K^{+} \rightarrow K^{+\dagger} \text { with } K^{0} \rightarrow \varepsilon_{0} K^{0} .
$$

It is easy to see as before that this will ensure parity conservation for $K^{+}$interactions. We have not so far assumed any relation between the interactions of $K^{+}$and $K^{0}$ and also properties like the relative parity of $K^{+}$and $K^{0}$. 
Though we have shown that parity conservation exists for strong interactions, we have yet to show why the weak interactions which have no internal symmetry do not have parity conservation. Sakurai ${ }^{1}$ has pointed out that if we impose invariance under $G$ conjugation, for strangeness conserving interaction we have equal mixtures of eigenspinors of $\gamma_{5}$ while for strangeness non-conserving interactions, $G$ conjugation generates a charge non-conserving interaction since $G$ conjugation amounts to inversion in isospin space. We have the interesting suggestion that the origin of parity conservation lies in inversion invariance in isospin space. So far there are no compelling reasons to assign isotopic spin to the leptons, a fact which suggests to us that every non-electromagnetic process involving leptons is parity non-conserving. ${ }^{2}$

\section{Heisenberg's nonlinear theory of elementary particles ${ }^{3}$}

From a survey of elementary particle interactions it is clear that the conventional methods of quantum field theory of representing particles by their corresponding fields and their interactions by suitable products of their field operators have been successful in explaining in some measure the various phenomena, albeit part by part, associated with these particles. These partial successes have convinced the theoretical physicists that there must be a "plan behind the maze"-a plan which must be discerned within the framework of quantum field theory. The search for this plan has led the creators of quantum field theory to believe in a "unified field equation" from which the fields associated with all the particles can be generated.

The mathematical barriers for tackling such a unified equation are very considerable. However the present situation demands new ideas, as in the days just before the birth of quantum theory. Therefore, Heisenberg and his co-workers have recently formulated and developed the idea of such a "unified field equation" and pushed it to the extent of calculating numerical values for the ratios of masses of various known particles and the coupling constants in their interactions. We give here a general outline of their theory to indicate the nature of the attempt rather than provide a detailed and systematic account of a well developed theory.

The basic belief behind this theory is that all elementary particles are stationary (or quasi stationary) states of some substance, matter or energy, since they can be created or transformed into each other in high energy interactions provided such transformations do not violate certain conservation laws. The distinction between elementary and non-elementary particles is just a convenient convention and not fundamental. Chew and Frautschi ${ }^{4}$ have recently suggested how such a "principle of equivalence" may be expressed within the $S$-matrix framework for

1 J. J. SaKURAI, loc. cit.

2 See however A. Ravakrishnax, A. P. Balachandran, N. R. Ranganathan and N. G. Deshpande, Nucl. Phys. 26, 52 (1959).

${ }^{3}$ H. P. DürR et al., Z. Naturforschung, 14a, pp. 441-485 (1959).

+ G. F. Chew and S. C. Frautschi, Phys. Rev. Lett. 7, 304 (1961). 
all strongly interacting particles; if supported by experiment, their ideas might mean that the concept of an elementary particle would lose much of its usefulness (for details, ref. Appendix 14). It is hoped that a unified description of matter is finally bound to be simpler than the description of various particles through their individual fields. Such a unified theory must be a spinor theory so that particles of half integral spins as well as integral spins can be generated out of it.

In his attempt to evolve a unified theory, Heisenberg suggested the following basic non-linear differential equation for matter

$$
\gamma_{\mu} \frac{\partial \psi}{\partial x_{\mu}} \pm l^{2} \gamma_{\mu} \gamma_{5} \psi\left(\bar{\psi} \gamma_{\mu} \gamma_{5} \psi\right)=0
$$

The $\gamma$ 's are the usual gamma matrices and $l$ is a new independent variable of the dimension of length whose significance will be explained shortly. The non-linear term signifies interaction. In this theory it is not meaningful to ask the question how many fields are necessary to represent all the observed particles, or how many particles a given field operator can represent. Only questions regarding the group properties of the field operators and their irreducible constituent parts may be answered satisfactorily. The totality of the matrix elements of the complex operator $\psi$ can be broken up into a series of irreducible sub-parts which transform differently under various transformations and can therefore be regarded as the wave functions describing many particles, stable and unstable. To establish a connection between the field operator $\psi(x)$ and wave functions $\varphi(x)$ we must examine the matrix elements $\varphi(x)$ of $\psi(x)$ (or products of $\psi(x)$ ) between vacuum and one particle states.

We shall now refer to the invariance properties of equation (1) under various transformations.

\section{(a) Transformations of continuous groups}

(1) The fundamental equation (1) is invariant under all transformations of the inhomogeneous Lorentz group. The conservation laws for energy, momentum and angular momentum consequently follow.

(2) It is invariant under the Pauli-Gürsey transformations

$$
\begin{aligned}
& \psi \rightarrow a \psi+b \gamma_{5} C^{-1} \bar{\psi}^{T} \\
& \bar{\psi} \rightarrow a^{*} \bar{\psi}+b^{*} \psi^{T} C \gamma_{5}
\end{aligned}
$$

$\bar{\psi}=\psi^{\dagger} \gamma_{4} ; \quad C \gamma_{\mu} C^{-1}=-\gamma_{\mu}^{T} ; \quad C \gamma_{5} C^{-1}=\gamma_{5}^{T} ; \quad C^{T}=-C ; \quad|a|^{2}+\mid b^{2}=1$.

This group is isomorphic to the group of rotations in a three-dimensional space. Hence, invariance under transformations (2) implies the conservation of a quantum number $I$, analogous to angular momentum in ordinary space. $I$ is the isospin and its third component (by convention) is connected to the charge $Q$ by $Q=I_{3}+l_{Q} / 2$, where $l_{Q}$ is a new quantum number introduced in this description of elementary particles. 
(3) Equation (1) is invariant under the transformations of the Touschek group,

$$
\begin{aligned}
& \psi \rightarrow \mathrm{e}^{-i \alpha \gamma_{b}} \psi \\
& \bar{\psi} \rightarrow \bar{\psi} \mathrm{e}^{-i \alpha \gamma_{b}}
\end{aligned}
$$

and the quantum number associated with it is $I_{N}$ which is connected to the baryon and lepton numbers ( $N$ and $L$ respectively) through another newly introduced quantum number $l_{N}$. The relation is

$$
\begin{aligned}
& N=I_{N}+\frac{l_{N}}{2} \\
& L=I_{N}-\frac{l_{N}}{2} .
\end{aligned}
$$

The two newly introduced quantum numbers $l_{Q}$ and $l_{N}$ are connected to strangeness $S$ by

$$
S=l_{Q}-l_{N}
$$

While $l_{Q}$ and $l_{N}$ may run through any positive or negative integral values, it is found that to explain obscrved facts, it is sufficient if $S$ takes only the values 0 , $\pm 1, \pm 2$. Therefore one more continuous one parameter group is needed to represent $l_{Q}$ or $l_{N}$. Fortunately equation (1) contains another one-parametric continuous group since equation ( 1 ) is invariant under the so-called "scale transformation"

$$
\psi \rightarrow \eta^{3 / 2} \psi(x \eta, l \eta) .
$$

$\psi$ has to be regarded as a function of $x$ and $l$ in order to define this transformation. $l$ plays the role of an independent variable and therefore the totality of solutions of equation (1) for all possible values of $l$ must be considered. Since the scale transformation does not commute with translation, according to the usual interpretation of quantum theory we cannot simultaneously specify the mass eigenvalue of a state and the quantum number corresponding to scale transformation. This, as stressed by Heisenberg, means that only the ratios between the masses of elementary particles can be experimentally tested. In nature there is no unit of mass or length.

The scale transformation can also be given as

$$
O_{\eta} \psi(x, l) O_{\eta}^{-1}=\eta^{3 / 2} \psi(x \eta, l \eta) \text {. }
$$

The operator $O_{\eta}$ corresponding to an infinitesimal scale transformation is

$$
O_{\eta}=\mathrm{e}^{\varepsilon \Lambda}=\left(\mathrm{e}^{\varepsilon}\right)^{\Lambda}=(1+\varepsilon)^{\Lambda}=\eta^{\Lambda}
$$

where $\eta=1+\varepsilon(\varepsilon$ small). The eigenvalues of $\Lambda$ can be used to specify the quantum states. This quantum number must be identified with $l_{N} / 2$ mentioned earlier and for particles with $S=0$ also with $l_{Q} / 2$. Since the non-linear equation (1) contains only odd integral powers of $\psi$ and not arbitrary powers, the matrix elements of $\psi$ should correspond only to transitions producing half integral changes in quantum numbers of $\Lambda$. The greatest physical interest is attached to $\Lambda \Lambda= \pm \frac{1}{2}$ which 
corresponds to the creation and destruction of particles with $\Lambda= \pm \frac{1}{2}$ (The states with half integral eigenvalue of $\Lambda$ are produced by applying an odd number of $\psi$ operators on the vacuum and states with integral eigenvalues by applying an even number of $\psi$ 's).

\section{(b) Discrete groups}

The only clearly understood discrete transformations are $C, P$ and $T$. Since $\psi$ contains another independent variable $l$, we can define another transformation which commutes with all the others and hence is suitable for defining a quantum number.

Whether strangeness can be incorporated into the scheme of a discrete group is yet to be investigated. The usual charge conjugation $C$ and parity $P$ operations do not commute with the transformations of the Pauli-Gürsey and Touschek groups respectively. Therefore these operations cannot be used to define quantum numbers. Heisenberg and his co-workers were able to show that other operations called $C$ and $P$ of the second kind can be defined which commute with these transformations. Thus we can assign only a parity of the second kind along with $I_{N}$. Recently Dürr has succeeded in connecting the parity of the second kind with the operation $l \rightarrow-l$ and in demonstrating its precise conservation.

We have already mentioned that the wave functions $\varphi(x)$ of individual particles can be derived as the matrix elements of the operator $\psi(x)$ (or of products of such operators). It is found that the field equation of particles with non-zero mass (described by $\varphi_{B}(x)$ ) and having the full symmetry of the fundamental field equation cannot be a differential equation of the first order (Dirac equation). However it can be a second order equation like the Klein-Gordon equation for spinor fields. It is well-known that a Klein-Gordon equation for a four-component spinor can be put into the form of a Dirac equation by increasing the components to eight. This is done by introducing the new symbols

$$
\hat{\varphi}_{B}(x)=\frac{-i \gamma_{v} p_{v}}{\chi} \varphi_{B}(x)=-\frac{1}{\chi} \gamma_{v} \frac{\partial \varphi_{B}}{\partial x_{v}} .
$$

Defining an eight component spinor

$$
\Phi=\frac{1}{1 / 2}\left[\begin{array}{l}
\varphi(x) \\
\hat{\varphi}(x)
\end{array}\right]
$$

it can be shown that $\Phi$ satisfies the first order differential equation

$$
\left(I \gamma_{v} \frac{\partial}{\partial x_{v}}+\Sigma_{1} \chi\right) \Phi(x)=0
$$

where $I=\left[\begin{array}{ll}1 & 0 \\ 0 & 1\end{array}\right] ; \Sigma_{1}=\left[\begin{array}{ll}0 & 1 \\ 1 & 0\end{array}\right]$ are eight-by-eight matrices. Transforming $\Phi$ to

$$
\Phi^{\prime}=\frac{1}{\sqrt{2}}\left[\Sigma_{1}+\Sigma_{3}\right] \Phi=\frac{1}{2}\left(\begin{array}{l}
\varphi_{B}+\hat{\varphi}_{B} \\
\varphi_{B}-\hat{\varphi}_{B}
\end{array}\right)
$$


the above differential equation splits up into two Dirac equations

$$
\begin{aligned}
& \left(\gamma_{v} \frac{\partial}{\partial x_{v}}+\gamma\right) \frac{1}{2}\left(\varphi_{B}+\hat{\varphi}_{B}\right)=0 \\
& \left(\gamma_{v} \frac{\partial}{\partial x_{v}}-\chi\right) \frac{1}{2}\left(\varphi_{B}-\hat{\varphi}_{B}\right)=0 .
\end{aligned}
$$

A four component field function which satisfies a Klein-Gordon equation is equivalent to two Dirac equations of four component functions and hence is sufficient for describing two spinor particles (and their antiparticles); that is, a mass doublet. We can identify the two nucleons, proton and neutron, with such a mass doublet. In deducing these wave equations the following requirements have to be considered :

(1) The wave functions of the nucleons shall be positive energy solutions of the Dirac equation

$$
\left(\gamma_{v} \frac{\partial}{\partial x_{v}}+\chi\right) \varphi_{N}=0 .
$$

(2) If the operator $\psi(x)$ (and hence the Klein-Gordon function $\varphi_{B}$ also) be subject to the Pauli-Gursey transformation then the nucleon wave functions $\varphi_{N}$ transform among themselves according to the usual isotopic spin transformations.

(3) If $\varphi_{B}$ is subjected to the Touschek transformation, then $\varphi_{N}$ shall transform as

where

$$
\varphi_{N}(x) \rightarrow \mathrm{e}^{i \alpha I_{N}} \varphi_{N}
$$

$I_{X}=+\frac{1}{2}$ for proton and neutron

$I_{\mathrm{N}}=-\frac{1}{2}$ for antiproton and antineutron.

In order to remove the ambiguity in connecting the Pauli-Gürsey group with the isospin group (which can be done in various ways) the axes of rotation must be specified. Therefore we have

(4) under the gauge transformation $\varphi_{B} \rightarrow e^{i \alpha 3 / 2} \varphi_{B}$ all $\varphi_{N}$ shall transform as under a rotation round the third isotopic axis. From the requirements (1-4) an explicit representation for the nucleons can be given as follows:

$$
\begin{aligned}
\varphi_{p}(x) & =\frac{1}{2}\left(1-\frac{1}{\chi} \gamma_{v} \frac{\partial}{\partial x_{v}}\right) R \varphi_{B}(x) \\
\varphi_{n}(x) & =\frac{1}{2}\left(1-\frac{1}{\chi} \gamma_{v} \frac{\partial}{\partial x_{v}}\right) R \varphi_{B}^{C}(x) \\
& =\frac{1}{2}\left(1-\frac{1}{\chi} \gamma_{v} \frac{\partial}{\partial x_{v}}\right) R \varphi_{B}^{D}(x) \\
\mathscr{f}_{\bar{p}}(x) & =\frac{1}{2}\left(1-\frac{1}{\chi} \gamma_{v} \frac{\partial}{\partial x_{v}}\right) L \varphi_{B}^{C}(x) \\
& =-\frac{1}{2}\left(1-\frac{1}{\chi} \gamma_{v} \frac{\partial}{\partial x_{v}}\right) L \varphi_{B}^{D}(x) \\
\varphi_{\bar{n}}(x) & =\frac{1}{2}\left(1-\frac{1}{\chi} \gamma_{v} \frac{\partial}{\partial x_{v}}\right) L \varphi_{B}(x),
\end{aligned}
$$


where

$$
\left.\begin{array}{rl}
\varphi^{D}(x) & =C^{-1} \gamma_{5} \bar{\varphi}^{T}(x)=D^{-1} \bar{\varphi}^{T}(x) \\
R & =\frac{1+\gamma_{5}}{1} ; \quad L=\frac{1-\gamma_{5}}{2}
\end{array}\right\} .
$$

Because of the presence of the operator $\left(1-\frac{1}{\chi} \gamma_{\nu} \frac{\partial}{\partial \varkappa_{\nu}}\right)$ all wave functions here satisfy the Dirac equation. This can be seen thus. $\varphi_{B}$ satisfies the Klein-Gordon equation so that

Hence

$$
\left(\gamma_{\nu} \frac{\partial}{\partial x_{\nu}}+\chi\right)\left(1-\frac{1}{\chi} \gamma_{\nu} \frac{\partial}{\partial x_{v}}\right) \varphi_{B}=-\frac{1}{\chi}\left(\frac{\partial^{2}}{\partial x_{v}^{2}}-\chi^{2}\right) \varphi_{B}=0 .
$$

$$
\left(\gamma_{v} \frac{\partial}{\partial x_{v}}+\chi\right) \varphi_{N}=0
$$

Thus the nucleon wave function $\varphi_{N}$ transforms like an isospinor when $\varphi_{B}$ is subjected to transformations of the Pauli-Gürsey-Touschek group. To find the transformation of $\varphi_{B}$ which corresponds to parity operation on $\varphi_{N}$ we first note that the usual parity operation

$$
\begin{gathered}
\varphi(r, t) \rightarrow \gamma_{4} \varphi(-r, t) \\
\varphi^{C}(r, t) \rightarrow-\gamma_{4} \varphi(-r, t)
\end{gathered}
$$

does not commute with the Touschek transformation with which the baryonic number is associated. Hence this operation does not also commute with the projection operators in the expressions for nucleon wave functions given above. In fact, it can be shown that the parity operation of the first kind on $\varphi_{B}$ takes $p \rightarrow \bar{n}$.

A suitably commuting parity operation (of the second kind) is defined on the energy shell of the nucleons $\left(p_{v}^{2}=-k^{2}\right)$ as follows

$$
\begin{gathered}
\varphi(r, t) \rightarrow-\frac{1}{\chi} \gamma_{v} \frac{\partial}{\partial x_{\nu}} \gamma_{4} \varphi(-r, t) \\
\varphi^{C}(r, t) \rightarrow \frac{1}{\chi} \gamma_{v} \frac{\partial}{\partial x_{v}} \gamma_{4} \varphi^{C}(-r, t) .
\end{gathered}
$$

This definition assigns opposite intrinsic parities to $\varphi$ and $\varphi^{c}$. If we give opposite intrinsic parities to neutron and proton then the "parity transformation of the second kind" on $\varphi_{B}$ is equivalent to the "parity transformation of the first kind" on nucleon wave functions. But in the usual theory, protons and neutrons are given the same intrinsic parity. (It may be pointed out that the fundamental equation (1) is not invariant under the parity transformation of the second kind if equation (1) is extended to the off the energy shell parts of $\psi(x)$.) But parity of the second kind may, according to Dürr, be connected instead with operation $l \rightarrow-l$ under which equation (1) is invariant. 
'The usual charge conjugation (first kind) on $\varphi_{B}$, does not give charge conjugation for the nucleon wave functions $\varphi_{N}$. The charge conjugation of the second kind defined by

$$
\varphi \rightarrow-\frac{1}{\chi} \gamma_{\nu} \frac{\partial}{\partial x_{v}} C^{-1} \frac{\varphi^{T}}{T}
$$

on the energy shell of the nucleons produces the desired charge conjugation of the nucleons. The fundamental equation (1) is invariant under the "charge conjugation of the second kind" only if this operation is connected with $l \rightarrow-l$ in the way suggested by Dürr. It is interesting to note that the combined operation $C P$ (both of the second kind) is identical with the combined operation $C P$ of the first kind.

For the leptons (in the narrow sense of a massless electron-neutrino doublet) a parity operation and charge conjugation of the second kind cannot be defined, for the operator $\boldsymbol{\sigma} \cdot \boldsymbol{p} /|p|$ which corresponds to the operator $\frac{1}{\chi} \gamma_{\nu} \frac{\partial}{\partial x_{\nu}}$ for the nucleons now commutes with the Touschek transformation. On the other hand, $C, P$ and $T$, consequently also, $C P T$ remain defined which is sufficient for the description of experimental facts (see chapter VI).

The leptons (in the sense mentioned) and the nucleons can be brought into a single system by using the invariance of the family of wave equations with parameter $l$ under the scale transformation.

In the above we have considered at some length the wave functions of the nucleons which form the manifestations of the baryons in the lower approximation. In order to explain the production of the strange particles $\left(\Lambda^{0}\right.$ and $\left.\Sigma\right)$ which have spin $1 / 2$ and integral isospin, it is postulated that the ground state, called the "world" which forms the basis for the existence of elementary particles is highly degenerate in the sense that it has a very high isospin and hence is asymmetric. The usual fully symmetric vacuum can be constructed in this theory if one goes over by the transformation $\psi=\left(1+\gamma_{5}\right) \chi$ into a two component theory in which the interaction vanishes. The mass-difference between the neutron and proton would be understandable if we considered them as the two doublet components of a state, "nucleon + world", for which the resulting isospin can be either parallel or antiparallel with that of the "world" which again carries an arbitrarily high isospin.

On the basis of this interpretation, we can understand the production of the strange particles $\Lambda^{0}$ and $\Sigma$ in the following way. By applying an odd number of field operators on the ground state a particle with half-integral spin is produced. The half-integral isospin which is thereby added to the ground state can however be so coupled to the large isospin of the world that the resultant isospin of the particle manifests itself as having an integral value. An analogy exists for this in the atomic spectrum of the calcium atom. The spin of the valence electron appears not as $1 / 2$ (which would give a doublet spectrum) but coupled with a spin $1 / 2$ of the inner shells so that a triplet spectrum results as actually observed. 
Investigations of the "world" expectation value of a product of two field operators have shown that while it is invariant under the transformation $C P$, it is not invariant under $P$ and $C$ separately which would mean that a single strange particle cannot have a well-defined parity and only a pair of such particles of opposite strangeness may have a parity.

Having outlined the transformation properties of the non-linear equation, we shall briefly refer to the method of deriving mass eigenvalues of elementary particles.

The principle of micro-causality for a linear field theory namely, the vanishing of the commutator (or anticommutator) of the field operators for the space-like separation and its non-vanishing otherwise, is taken over to the non-linear field theory to provide a method of quantization. But the commutator in this case is expected not to contain the $\delta, \delta^{\prime}$ singularities on the light cone occurring in the usual quantum field theory but to exhibit infinite oscillations there; a circumstance which is considered as a consequence of the non-linear interaction and as a condition for the convergence of the theory. If we were to connect the commutators in this general theory with those corresponding to a field with definite mass, by integration over a weight function $\varrho(\xi)$ of the mass, such a $\varrho$ should take negative values also since the $\delta, \delta^{\prime}$ singularities have to be weakened on integrating with respect to $\xi$. This implies the use of an indefinite metric in Hilbert space (i.e. formally negative probabilities) an idea the depth and extent of which is not yet fully realized. The $\varrho$ function is assumed to satisfy the conditions

$$
\begin{aligned}
\int \varrho(\xi) \mathrm{d} \xi & =0 . \\
\int \varrho(\xi) \xi \mathrm{d} \xi & =0 . \\
\int \varrho(\xi) e^{-\xi} \mathrm{d} \xi & =1 . \quad \text { (normalization) }
\end{aligned}
$$

The technique of evaluating the mass eigenvalues will be illustrated here only in the case of fermions. The fundamental differential equation (1) generates an infinite system of coupled integral equations between the so-called " $\tau$ functions". (The matrix elements of the time ordered products of the field operators between the vacuum and a one-particle state) connecting $\tau$ functions of $N$ variables and $N+2$ variables. To obtain an eigenvalue equation, it must be possible to reduce the functions with a large number of variables to $\tau$ functions of a smaller number of variables. Therefore, another set of functions $\varphi$ (uniquely related to the $\tau$ functions) is introduced such that a Tamm-Dancoff approximation of cutting off the system of integral equations at a finite workable stage, i.e. setting $\varphi_{n}$ to zero when $n>N$ is meaningful. The finite system of coupled integral equations so obtained defines an eigenvalue problem. The contraction function to be used when connecting $\tau$ and $\varphi$ functions is the propagator weighted with $\varrho(\xi)$ containing only one mass in an approximation to facilitate the calculation. If in general $\varrho(\xi)$ is allowed to contain more masses, these mass values are obtained as a partly discrete, partly continuous spectrum. For the calculation of the masses 
of the fermions, we start with the function of one variable. Retaining only $\phi$ functions of one variable in the system of equations ('Tamm-Dancoff approximation) and setting $q(x)=\tau(x)$ (by definition) we are led to the differential equation of the form

$$
\gamma_{\mu} P_{\mu} Q\left(-p^{2}\right) \tau(x)=0 \text {. }
$$

The conditions relating to the existence of the solutions lead to the search for the zeroes of $Q\left(-p^{2}\right)$ which give the mass numbers of the elementary particles. $\tau$ functions of an odd number of variables are used for calculating the mass spectrum of fermions and that of an even number of variables for bosons.

A similar calculation of the mass eigenvalues for the strange particles has not been carried out. It was found that an interaction between the isospin of a particle and of the ground state cannot be expressed if one uses only the vacuum expectation values of products of two field operators which enter the calculation of mass eigenvalues and hence strange particles cannot appear as eigenvalues. 'To obtain their mass spectra we have to include the vacuum expectation value of products of at least four field operators of which not much is known (see (hapter V). But the special properties of equation (1) may perhaps make its evaluation easier than in the case of the usual local field theories. 


\section{Part Two \\ COSM I C RAYS}

"... Aetherial Dominations who possess,

Elysian, windless, fortunate abodes,

Beyond Heaven's constellated wilderness."

P. B. Shelley 


\section{PRIMARY COSMIC RADIATION}

\section{PRIMARY COSMIC RADIATION ${ }^{1}$}

THe FIRST essential pre-requisite to any study of cosmic ray phenomena is a detailed and accurate knowledge of the nature and composition of the primary radiation. It is from such a knowledge that we have to develop a theory of the origin of cosmic rays which in turn will throw light on the fundamental nuclear processes in the stellar and interstellar regions. Unless our knowledge of the primary radiation is precise, any theoretical prediction we may make about nuclear interactions of cosmic radiation in its passage through the atmosphere will not be reliable. Hence experimenters have spared no efforts in devising new techniques to push the accuracy of our knowledge to the greatest possible extent. The study of primary cosmic radiation can be broadly divided into three parts: (1) the determination of the mass spectrum of the incident particles, (2) the energy and the spatial distributions of the incident particles and their variation on the surface of the earth and at various levels of the atmosphere, and (3) the temporal variations of the primary component.

The most striking features of cosmic radiation are the near constancy of the mass, energy and spatial distributions at any particular point on the earth and the extreme smallness of the temporal deviations. Thus any theory of cosmic rays and their origin should in the first instance explain this constancy. However it has been found that the slight deviations themselves may serve as leads in speculating upon the origin of cosmic rays. We shall now deal with the three aspects one after the other.

\section{The mass speetrum}

Primary cosmic radiation consists of positively charged particles which have been identified to be nuclei ranging from the lightest (hydrogen) to the heaviest (iron). Their mass spectrum as compared with the relative abundance in the universe is given in the table. The mean number of nuclei arriving per square $\mathrm{cm}$ per unit solid angle per second is approximately 0.1 .

1 S. F. Singer, "The primary cosmic radiation and its time variations", Progress in Elementary Particle and Cosmic Ray Physics, Vol. 4, p. 205 (1958). This article also contains an extensive bibliography. 
The general features of the mass spectrum are:

(i) protons comprise the bulk of the radiation,

(ii) $\alpha$-particles comprise 10 to 15 per cent of the radiation,

(iii) heavy nuclei do occur but constitute only 1 to 2 per cent of the total,

(iv) electrons and photons are almost completely absent and their abundance has an upper limit of 1 per cent and 0.1 per cent respectively.

\begin{tabular}{|c|c|c|c|c|}
\hline \multirow[b]{2}{*}{ Nuclei } & \multicolumn{3}{|c|}{ Primary Cosmic Radiation ${ }^{1}$} & \multirow[b]{2}{*}{$\begin{array}{c}\text { Universal } \\
\text { abundance per } \\
10^{5} \text { hydrogen atoms }\end{array}$} \\
\hline & $\begin{array}{l}\text { Number per } \\
10^{5} \text { protons }\end{array}$ & $\begin{array}{l}\text { As a } \% \text { of } \\
\text { primary } \\
\text { particles }\end{array}$ & $\begin{array}{l}\% \text { of primary } \\
\text { nuclons carried }\end{array}$ & \\
\hline Protons $(p)$ & 100,000 & 91.5 & 69 & 100,000 \\
\hline $\begin{array}{l}\alpha \text { particles } \\
\quad(Z=2)\end{array}$ & 10,000 & 7.8 & 23 & 7700 \\
\hline $\begin{array}{c}L \text {-group } \\
(\mathrm{Li}, \mathrm{Be}, \mathrm{B} \\
Z=3 \text { to } 5)\end{array}$ & $\lesssim 50(?)$ & - & - & $3.6 \times 10^{-4}$ \\
\hline $\begin{array}{c}M \text { group } \\
(\mathrm{C}, \mathrm{N}, \mathrm{O}, \mathrm{F}, Z=6 \text { to } 9)\end{array}$ & $520(400)$ & 0.4 & 4.5 & 80 \\
\hline $\begin{array}{l}H \text {-group } \\
(Z \geqq 10)\end{array}$ & 160 & 0.15 & 3.5 & 30 \\
\hline $\begin{array}{c}\text { Iron } \\
\left(\mathrm{Fe}{ }^{Z=26)}\right.\end{array}$ & 30 & - & - & 1.5 \\
\hline $\begin{array}{c}\text { All nuclei } \\
(Z>30)\end{array}$ & $<1$ & $<0.001$ & - & $10^{-3}$ \\
\hline
\end{tabular}

Regarding the neutron composition of primary radiation the position is not clear. It is natural to expect that the neutrons originate from a near source like the sun since we know that the lifetime of a neutron is very short. But it is possible for very high energy neutrons to have a life long enough due to relativistic time dilatation so that it can be assumed that they may have come from galactic sources.

The accurate determination of the mass spectrum is subject to considerable error owing to the low intensity of the heavy primaries and the uncertainty in the measurement of ionization. The heavy nuclei have a large probability of losing energy by ionization even within the top layer of the atmosphere. Although the presence of $\mathrm{Li}, \mathrm{Be}$ and $\mathrm{B}$ have been identified, still the possibility of heavy nuclei splitting into $\mathrm{Li}, \mathrm{Be}$ and $\mathrm{B}$ by collisions with air molecules remains and this makes the determination of the mass spectrum very uncertain.

1 V. L. Ginzburg, Progress in Elementary Particle and Cosmic Ray Physics 4, 339 (1958). At the recent Kyoto Conference (September 1961), new data was reported by M. M. SHAPIRo et al. The principal changes in the above table will be that the universal abundance of the $L$ and $M$ groups are $6 \times 10^{-4}$ and 150 , respectively, the corresponding cosmic-ray abundance being 110 and 400 , respectively, i. e. the presence of the $\mathrm{Li}$ group is well established. 
An examination of the table leads to the following conclusions:

(a) There is considerable doubt as to the exact composition of light elements, ${ }^{1}$

(b) There is a virtual absence of $L$-flux or flux of light nuclei with less than $0.4 \mathrm{~m}^{-2}$ sterad $^{-1} \mathrm{sec}^{-1}$.

The first thing that strikes us about the charge spectrum is that it nearly corresponds to the abundance of the elements in the universe. But closer examination shows significant differences like the following:

(1) the presence of $\mathrm{Li}, \mathrm{Be}$ and $\mathrm{B}$ in appreciable quantities,

(2) a pronounced peak at iron, and

(3) the $\mathrm{C}: \mathrm{N}: \mathrm{O}$ ratio is inverse to that of cosmic matter which is $1: 2: 6$ according to Suess and Urey. ${ }^{2}$ It is the study of these differences that will reveal information about these high energy particles and their interactions. The explanation of these differences lies in one of the two possibilities:

(i) The original radiation corresponds to universal abundance, the difference arising due to subsequent interactions before reaching the earth.

(ii) The composition of the original radiation is itself quite different from what could be expected from cosmic abundance and the subsequent fragmentation is of such a nature as to yield a spectrum resembling the cosmic abundance with observed deviations. In either case it is necessary to devise a fragmentation theory which is of course more important in the latter.

Adopting the second point of view and assuming that the primary radiation consists essentially of nuclei of metals the other components being the products of fragmentation, we can list the following points in favour of it:

(1) It would explain the observed abundances of $\mathrm{Li}, \mathrm{Be}$ and $\mathrm{B}$,

(2) The peak in the abundance at iron and the natural preponderance of heavy nuclei are explained,

(3) There may be injection mechanisms favouring heavy nuclei.

In this connection it is necessary to stress the importance of not merely the relative abundance of various nuclei irrespective of their energy but also at various energies since as we shall see later, the age of a cosmic ray particle is closely connected with its energy, e.g. in the Fermi theory of cosmic radiation (which will be discussed in great detail in a later chapter) energy increases with age. Hence at the low energy end of the spectrum fewer fragmentation products are to be expected but this is in disagreement with the currently accepted statement that all primary components have similar velocity spectra. This implies that the mean free path of cosmic rays in the galaxy is shorter than the mean free path for nuclear collision and has led to a revision of the Fermi theory by $\operatorname{Rossi}^{3}$ et al.

1 The $L: M$ ratio quoted by Bristol workers is $1: 1$ while a different ratio has been obtained using a different determination of fragmentation probabilities by Gottstein et al.

2 H. C. Urey and Suess, Rev. Mod. Phys. 28, 53(1956).

3 B. Rossi, S. Olbert and P. Morrison, Phys. Rev. 94, 440 (1954). 
A clear cut experiment demonstrating the absence of Li etc. above the atmosphere would be fatal to the hypothesis of the origin of the primary radiation by fragmentation.

\section{Energy spectrum and spatial distribution}

It is very important to know the energy and spatial distribution and the associated quantities like the intensity, energy density and number density of cosmic ray particles of various types.

The energy distribution has two characteristic features:

(1) a low energy cut-off;

(2) a power law spectrum, i.e. the number of particles of type $j$ (irrespective of their direction) between energy $E$ and $E+\mathrm{d} E$ is given by

$$
N_{j}(E) \mathrm{d} E=N_{j}^{\gamma-1}\left(\frac{E}{E_{c}}\right)^{\gamma-1} \frac{\mathrm{d} E}{E}
$$

on the assumption that no particle has energy below $E_{c} . N_{j}$ is the total flux coming from all directions. The number of particles above an energy $E$ is given by

$$
\int_{E_{c}}^{N} N_{j}(E) \mathrm{d} E=N_{j}\left(\frac{E}{E_{c}}\right)^{\gamma-1} .
$$

Considerable theoretical significance cannot be attached to a power law spectrum especially when experimental data requires $\gamma$ to be chosen suitably for different energy ranges and for different types of particles. However, it was in an attempt to explain the power law spectrum that Fermi was led to making a far reaching hypothesis regarding the origin of cosmic radiation. According to present experimental data the following values ${ }^{1}$ for $\gamma$ are found suitable.

\begin{tabular}{c|c}
\hline $\begin{array}{c}\text { Energy range } \\
\text { per nucleon }\end{array}$ & $\begin{array}{c}\text { Value of } \\
\gamma\end{array}$ \\
\hline 0.5 and $1 \mathrm{GeV}$ & $\sim 2$ \\
0.35 and $10-20 \mathrm{GeV}$ & 1.9 to 2.2 \\
$5 \times 10^{10}$ to $10^{12} \mathrm{eV}$ & $\sim 2.5$ \\
$10^{13}$ and $10^{18} \mathrm{eV}$ & 2.7 to 3 \\
above $2 \mathrm{GeV}$ & 2.5 \\
\hline
\end{tabular}

The most striking feature of the spatial distribution is the near isotropy of cosmic radiation, a feature which has to be explained in any theory of its origin. Since cosmic rays are charged particles it is to be expected that their motion should be affected by the magnetic field of the earth. The Lorentz force acting on charged particles in a magnetic field deflects particles which would otherwise

1 V. L. Ginzburg, loc. cit. 


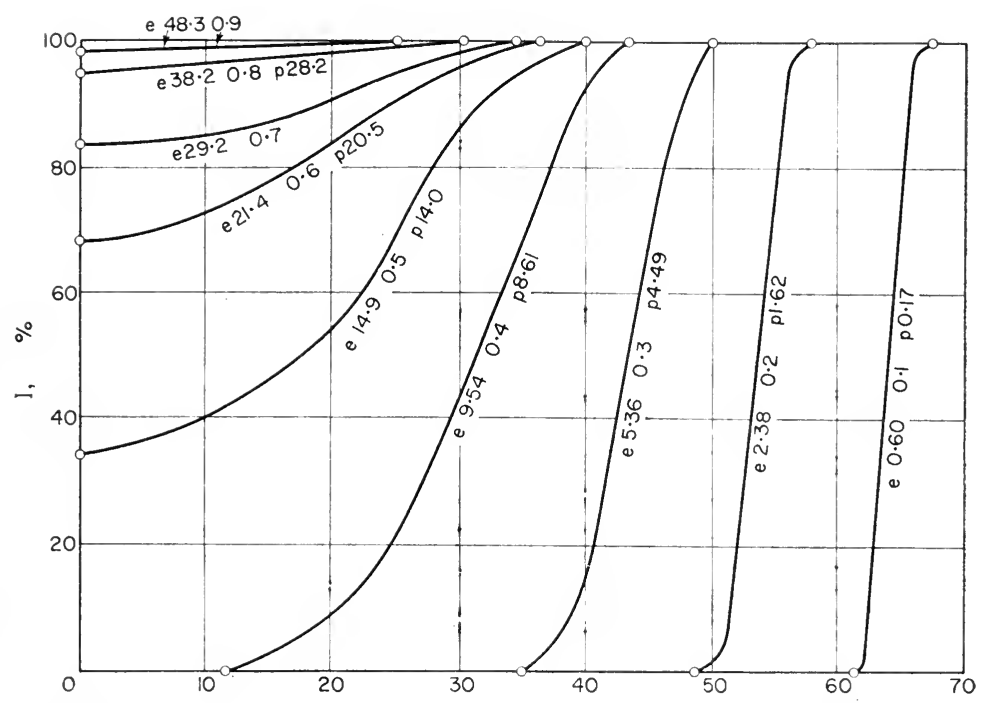

FIG. 49. Dependence of cosmic ray intensity on magnetic latitude.

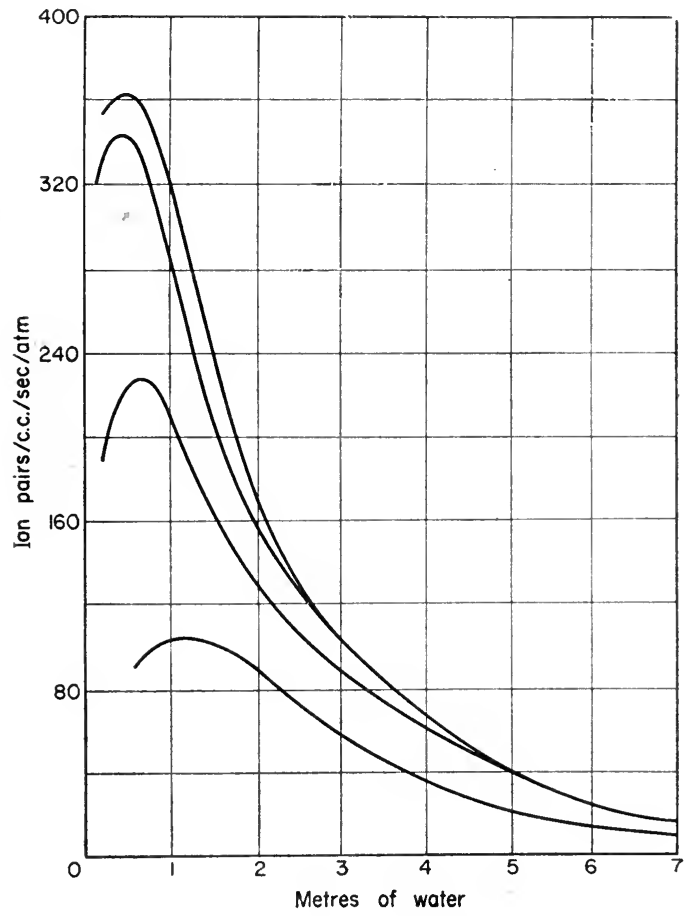

FIG. 50. Cosmic ray ionization as a function of altitude at four different latitudes. 
travel in straight lines. Such deflections should naturally affect the intensity distribution with latitude. One remarkable feature of this geomagnetic effect is that certain directions of arrival are forbidden depending on the latitude of observation and the momentum of the particles while the intensity in the allowed directions is still isotropic and it is only due to the fact that certain directions are forbidden that the intensity varies with latitude.

The variation of cosmic ray intensity with latitude at sea level was first reported by $\mathrm{Clay}^{1}$ and later confirmed by Compton ${ }^{2}$ and co-workers and the variation with geomagnetic latitude was better correlated. All the observations indieated that the cosmic ray intensity does not vary very much above a geomagnetic latitude $50^{\circ} \mathrm{N}$ or $\mathrm{S}$. A decided seasonal effect was also noted and this was believed to be correlated with atmospheric temperature.

The latitude effect is completely explained as will be shown later by studying the motion of a charged cosmic ray particle in the magnetic field of the earth. The graph representing the variation of cosmic ray intensity with latitude is given in Fig. 45.

The fact that the intensity does not vary beyond a certain latitude implies that there is a low energy cut-off and that for particles with energy greater than this, all directions are allowed above a particular latitude. This has been discussed in the chapter on geomagnetic effects.

\section{TIME VARIATION OF COSMIC RAYS ${ }^{3}$}

The subject of time variations of cosmic rays has become important in recent years with the increasing realization that it will give some clue to the origin and production of cosmic radiation, its mode of propagation and the influence of the electromagnetic fields in interplanetary and perhaps interstellar space. It is reasonable to expect that all the known time variations of low energy cosmic rays are mostly due to events in the solar system.

The solar daily variation of cosmic ray intensity exhibits a number of features which are clearly of non-meteorological origin. Moreover many of these relate to radiation outside the influence of the geomagnetic field and provide information concerning the anisotropy of primary radiation. The anisotropy is of a variable character undergoing change from day to day and its twelve-month average characteristics also change markedly from year to year with striking relationships with the eleven and twenty-two year cycles of solar activity.

Because of the obvious difficulty of making continued observations at high altitudes where the nucleonic component predominates, most of the observations are done at sea level or below the earth where the cosmic radiation consists mostly of the mesonic component. It has been found that meteorological factors have

1 J. Clay, Proc. Roy. Acad., Amst. 30, 1115 (1927).

2 A. H. Comptox and R. N. Turner, Phys. Rev. 52, 799 (1937).

3 S. F. Sixger, loc. cit. See also V. Sarabhai and N. W. Nerurkar, Ann. Rev. Nucl. Sci. 6, 1 (1956). 
less effect on the day to day fluctuations of the nucleonic component than on the mesonic component. When all the local atmospheric effects have been taken into account, some effects remain which seem to be truly characteristic of the primary radiation. For instance, the daily variations of the nucleonic component measured at different latitudes and averaged over a large number of days have a peak to peak amplitude of about 1 per cent which is comparable to the amplitudes obtained at sea level, of mesonic intensities.

The time variations of cosmic rays can be classified as follows:

\section{The diurnal variation}

This is due to the observation from the rotating earth of anisotropic radiation. The presence of this anisotropy is not clearly understood and in fact its effect is quite small.

The diurnal variation of the secondary component measured at sea level and at mountain altitudes by means of ionization chambers and counters is less than 1 per cent, the value depending on the angular aperture of the telescope, while that of the neutron component is perhaps three times larger. The diurnal variation seems to be energy dependent, repeats itself for a few days, but may then die out and disappear. It seems to be enhanced during periods of magnetic activity and shows a 27-day recurrence. The phase of maximum of the variation seems to vary in a regular manner during the solar cycle.

Alfven has used these data to put forth a mechanism for acceleration of cosmic rays within the solar system by means of magnetic field variations. ${ }^{1}$ It is first necessary to translate from the maximum phase of the diurnal variation to the direction of the anisotropy outside of the earth's magnetic field. He interprets the resultant anisotropy as due to an outflow or inflow of cosmic rays across the earth's orbit coupled with a tangential component due to the earth's motion; the predominance of the outflow indicates the production of cosmic rays near the sun. A model has been suggested in which the electric field of a solar corpuscular beam modulates the cosmic ray intensity. Another possibility also exists, viz. the field of the rotating earth interacting with the streaming interplanetary gas sets up electric fields which modulate cosmic ray intensity over a restricted range of azimuthal angles.

\section{Variations at the source of cosmic radiation}

These may be sudden spectacular increases as are observed in synchronization with solar flares or variations which show a trend throughout the sun spot cycle which one might call secular variation. These may indicate variations in the output of cosmic rays which fall into two classes of solar origin.

(i) Short term variation due to solar flares: Five major solar flare effects on cosmic rays have been observed before 1956 .

1 H. Alfven, Tellus, 6, 232 (1954). 
(1) 2Sth February 1942, (2) 7th March 1942, (3) 25th July 1946, (4) 19th November 1949 and (5) 23rd February 1956.

On the first four oceasions no increase has been observed at Huancayo near the geomagnetic equator. 'Therefore the primary particles responsible for these increases were thought to be eharged particles with energy $<15 \mathrm{BeV}$, which is the threshold energy below which protons cannot penetrate the earth's magnetic field at the equator. 'The last event was the largest and the produced particles were detected at the geomagnetic equator. Observations on the 1949 flare showed a 5 to 6 -fold increase of neutron intensity as compared to a 12 per cent increase in the ionizing component at the same station. The large relative increase in neutron intensity is expected since primary protons of energy $<10 \mathrm{BeV}$ are much more efficient at producing neutron rather than meson secondaries.

During the 1956 flare, a large increase in intensity occurred simultaneously beyond $25^{\circ}$ latitude. The nucleonic component predominated over the mesonic and the radiation was in general more energy dependent than the normal cosmic ray intensity. There was an average increase of 5.7 per cent near the magnetic equator in India which may be ascribed to a flare of $50 \mathrm{BeV}$ protons coming direct from the sun. As regards the dependence on energy the components below $10 \mathrm{BeV}$ undergo greater variations than those above it. In general, the energy spectrum of the primaries associated with solar flares is much steeper than that of the normal primaries.

It seems likely that the primary particles responsible for these increases are accelerated on or near the sun by some mechanism associated with a flare, although there is a definite time delay between the two phenomena. Maximum cosmic ray intensity seems to occur later than maximum flare intensity by up to an hour and the cosmic ray intensity remains above average several hours after the flare has disappeared.

Geomagnetic analysis, assuming that these particles come from the sun, explains very well the extreme longitude dependence of these increases. From this analysis an upper limit of $\sim 5 \times 10^{32}$ gauss $\mathrm{cm}^{3}$ has been derived for the solar dipole moment. It is significant to observe that the rate of decay of intensity after the maximum of the solar flare increase has been reached depends greatly on the location of the observer. It is therefore thought that such changes in polar regions are due to particles which do not come directly from the sun but have been redirected and temporarily stored by magnetic fields.

Various models for such a magnetic field have been discussed. Attempts have been made to explain the polar increases in terms of deflections by a galactic field. It has been shown that both the geographic distribution and the intensity decay could be explained by assuming the solar flare particles to progress along a weak interstellar field with all possible pitch angles. The reflection of the particles from a heliocentric boundary of radius of about 1.2 astronomical units has been also considered as contributing to the temporal variations.

(ii) Long term variation due to sunspot effects: The long term variations of cosmic ray intensity are associated with sun spot activity and continuous 
observations extend back only to 1937. The general intensity is directly related to the number of sun spots, the change in the twelve month mean intensity being of the order of 4 per cent between the periods of sun spot maximum and minimum. The change was the same both on magnetically quiet as well as on disturbed days. It was found that there was a 50 per cent increase in ionization at $70,000 \mathrm{ft}$ for the sun spot minimum year 1954 as compared to the sun spot maximum year 1937. These changes were much higher at higher altitudes. The intensity in the upper atmosphere in 1940 was found to be much greater than in 1954 .

There were large changes of the twelve month average daily variation of the meson intensity between the years 1953 and 1956 . These can be related to either the eleven or twenty-two year cycle of sun spot activity. The correlation was best during the sun spot minimum indicating that the variations are related to the solar activity in the equatorial belt of the sun. In fact it was later found to be correlated with the activity of the $5303 \AA$ coronal emission.

Associated with the solar cycle intensity variation is the phase shift of the diurnal variation. The shift of the time of maximum diurnal intensity was found to be independent both of the direction of arrival of the particle and the geomagnetic disturbance of the day.

At Huancayo the twelve month mean daily variation exhibited a maximum at noon from 1937 to 1943 and a maximum at early morning in 1952. In the intervening period there was a progressive increase of the early morning maximum and decrease of the noon maximum. During 1945-50, the daily variation exhibited two maxima. Only half the cycle of changes are completed in eleven years, indicating a twenty-two year cycle.

\section{Modulation effects}

These variations are brought about by electromagnetic conditions of short duration. These may either be sudden decreases (Forbush decreases) of cosmic ray intensity often associated with world wide magnetic storms or variations which show a twenty-seven day recurrence relation similar to that of the small magnetic storms and auroral displays and hence related to the rotational period of the sun.

The great world-wide storms have a fairly well defined pattern characterized by a sudden increase in the horizontal field intensity (.5-1 per cent) which lasts for an hour or so followed by a slow recovery over a period of a days.

The Forbush type of decreases are quite striking and in some cases are as large as 10 per cent. While they are often associated with magnetic storms there are many examples where the decreases occur without storms and conversely storms without decreases. This suggests that the correlation between the two is not an immediate one but goes back to a common cause. Alfven ${ }^{1}$ (1946) first suggested that the emission of corpuscular matter from the sun which is responsible for magnetic storms is also responsible for the cosmic ray decrease. In Morrison's ${ }^{2}$

1 H. Alfven, Nature, Lond. 158, 618 (1946).

2 P. Morrison, Phys. Rev. 101, 1397 (1956). 
model clouds of ionized matter containing turbulent magnetic fields but free from cosmic radiation are being continually emitted from the active solar regions. The cosmic rays gradually diffuse into these clouds and when the earth is surrounded by such a cloud, decreases in cosmic ray intensity are observed due to seattering of the primary particles.

Dorman $^{1}$ considers an ionized solar matter beam to carry a frozen magnetic field derived either from the dipole field of the sun or from the local field at its origin. The trapped field gets progressively attenuated with increasing distance from the sun and can also get weakened and randomized due to the onset of turbulence at a certain stage. Changes of cosmic ray intensity and isotropy could occur (1) because of the electric polarization of the beam and (2) due to magnetic deflection of low energy primaries. Modulation of intensity alone indicates presence of beams largely randomized through turbulence.

A systematic analysis of cosmic ray data along with the geomagnetic disturbances has been presented recently by Sarabhai et al. ${ }^{2}$ who observe: "At the present moment no detailed verification of the modulation theories involving cosmic ray beams could be considered to have been achieved; it appears that beams certainly play an important role but it is more than likely that we have other processes as well".

\section{Prehistoric intensity variations}

It has been estimated using deep core samples that the cosmic ray flux has not varied by more than 10-20 per cent over the last 35,000 years; other methods based on meteorites or nuclear disintegrations in the atmosphere have been suggested to allow even longer extrapolation or to cross-check the existing analysis.

1 Dormax, J. Expt. Theoret. Phys. 26, 337 (1953).

2 V. Sarabhai and Satyaprakash, Proc. Ind. Acad. Sci. 51, 84 (1960). 


\section{GEOMAGNETIC EFFECTS}

Since primary cosmic radiation consists essentially of charged particles it is to be expected that the magnetic field of the earth's dipole should influence the direction and intensity of the radiation through the deflection of the particles as they approach the earth. We begin the study with the general mathematical theory of the motion of a charged particle in the field of a magnetic dipole and then deal with the particular considerations of our problem, i.e. the relation between the geomagnetic field and the intensity of cosmic radiation at the surface of the earth.

\section{MOTION OF A CHARGED PARTICLE IN THE FIELD OF A MGNETIC DIPOLE ${ }^{1}$}

\section{Equations of motion}

The theory of the motion of a charged particle in a magnetic field with special reference to that of the earth's dipole was first developed in a series of papers by Stormer and later extended by Lemaitre, Vallarta ${ }^{2}$ and their collaborators.

The equation of motion of a particle of charge $e$ in an electromagnetic field with electric and magnetic components $\boldsymbol{E}$ and $\boldsymbol{H}$ respectively is

$$
\frac{\mathrm{d}}{\mathrm{d} t}(m \dot{\boldsymbol{r}})=e\left(\boldsymbol{E}+\frac{1}{c} \dot{\boldsymbol{r}} \times \boldsymbol{H}\right)
$$

where $m$ is the relativistic mass, $c$ the velocity of light and $\boldsymbol{r}$ the position vector of the particle. In a pure magnetic field, $\boldsymbol{E}$ being zero, the force on the particle is always perpendicular to the direction of motion; the energy and the absolute values of the momentum and velocity and consequently the relativistic mass $m$ are constants. Thus we can write

$$
\frac{\mathrm{d}^{2} \boldsymbol{r}}{\mathrm{d} t^{2}}=\frac{e}{m c}(\dot{\boldsymbol{r}} \times \boldsymbol{H}) .
$$

We shall now assume that the centre of the dipole is situated at the origin

1 Parts of this chapter are based upon the treatment of the subject found in Cosmic Rays, L. Janossy, Oxford.

2 C. Stormer, Z. Astrophys. 1, 237 (1938). G. Lemattre and M. S. Vallarta, Phys. Rev. 43, 87 (1933). 
and its moment $\boldsymbol{M}$ is along the $z$-axis. The field at a point $\boldsymbol{r}$ with Cartesian components $x, y, z$ is given by

$$
\boldsymbol{H}=-\operatorname{grad} \frac{M_{z}}{r^{3}} .
$$

Since the velocity and mass are constants, Stormer simplified the equations of motion by introducing a new independent variable $s$ instead of $t$ where $s=v t$. Thus, if velocities are measured with respect to $s$, all particles have the magnitudes of their "new" velocities $v_{s}$ equal to unity. ${ }^{1}$ Further we normalize $\left(\frac{e M}{c p}\right)^{1 / 2}$ which has the dimension of length to unity by a proper choice of the unit of length and $v$ is measured in terms of these units. The equations of motion in Cartesian coordinates thus reduce to

$$
\begin{aligned}
& \frac{\mathrm{d}^{2} x}{\mathrm{~d} s^{2}}=-\frac{1}{r^{5}}\left[3 y z z^{\prime}-\left(3 z^{2}-r^{2}\right) y^{\prime}\right] \\
& \frac{\mathrm{d}^{2} y}{\mathrm{~d} s^{2}}=-\frac{1}{r^{5}}\left[\left(3 z^{2}-r^{2}\right)\left(x^{\prime}-3 x z z^{\prime}\right)\right] \\
& \frac{\mathrm{d}^{2} z}{\mathrm{~d} s^{2}}=-\frac{1}{r^{5}}\left[3 x z y^{\prime}-3 z y x^{\prime}\right]
\end{aligned}
$$

where

$$
x^{\prime}=\frac{\mathrm{d} x}{\mathrm{~d} s}, \quad y^{\prime}=\frac{\mathrm{d} y}{\mathrm{~d} s} \quad \text { and } \quad z^{\prime}=\frac{\mathrm{d} z}{\mathrm{~d} s} .
$$

We shall now show that when the equations are expressed in cylindrical instead of Cartesian coordinates, the study of the peculiar features of the dynamical problem is made easier.

If $z, Q, \varphi$ are the cylindrical coordinates

$$
\varrho^{2}=r^{2}-z^{2} ; \tan \varphi=y / x .
$$

After simple calculation we obtain

$$
\begin{aligned}
\varrho^{2} \frac{\mathrm{d} \varphi}{\mathrm{d} s} & =2 g-\varrho^{2} / r^{3} \\
\frac{\mathrm{d}}{\mathrm{d} s}\left(r^{2}\right) & =\frac{\mathrm{d}}{\mathrm{d} s}\left(\varrho^{2}+z^{2}\right)=1-\left(\frac{2 g}{\varrho}-\frac{\varrho}{r^{3}}\right)^{2}=Q
\end{aligned}
$$

where $2 g$ is the constant of integration obtained in deriving (6). Its interpretation will be given presently. Since equation (1) involves only $r, \varrho$ and $z$, it represents the motion in the meridional plane ( $\varrho, z$ plane) and can be studied without reference to $\varphi$. The angular velocity of the meridional plane about the $z$ axis (the axis of the dipole), is given by $\frac{\mathrm{d} \varphi}{\mathrm{d} s}$ which is a function of $\varrho$ and $z$.

$1 v$ is a number denoting the magnitude of the velocity. Hence $s$ has the dimension of time but is numerically equal to the length of unity described in time $t$. Though the newly defined velocity is unity for all $v$, any casual reference to velocity and momentum of the particle would mean that they refer to the case when the independent variable is $t$. 
Since $\left|v_{s}\right|^{2}$ is unity, $\frac{1}{2}\left|v_{8}\right|^{2}=1 / 2$ (this is proportional to the kinetic energy for non-relativistic particles) and this term can be resolved into two partsone corresponding to the motion in the meridional plane $(Q / 2)$ and the other to the motion perpendicular to the meridional plane equal to

$$
\frac{1}{2}\left(\varrho \varphi^{\prime}\right)^{2}=1 / 2-Q / 2
$$

the prime denoting differentiation with respect to $s$. This quantity can be fictitiously reckoned to be the potential of the particle when we are considering the motion only in the meridional plane. Janossy calls $-\frac{Q}{2}$ the "potential" but it is more logical to treat (8) as the "fictitious" potential for the obvious reason that in the non-relativistic case the sum of the "fictitious potential" and the kinetic energy in the meridional plane should give the total kinetic energy of the particles the actual potential energy of which in the field of the dipole is zero. Two equations immediately follow on recognizing $\frac{Q}{2}$ to be $\frac{1}{2}\left(\varrho^{\prime 2}+z^{\prime 2}\right)$ in the meridional plane:

$$
\varrho^{\prime \prime}=\frac{1}{2} \frac{\partial Q}{\partial \varrho} ; \quad z^{\prime \prime}=\frac{1}{2} \frac{\partial Q}{\partial z} .
$$

We shall now interpret $2 g$ in physical terms. ${ }^{1} \varrho^{2} \frac{\partial \varphi}{\partial t}=\varrho^{2} \dot{\varphi}$ is proportional to the kinetic angular momentum of the particle. According to relativistic electrodynamics, when the vector potential is not zero in a magnetic field, the total angular momentum is to be distinguished from the kinetic angular momentum. If $L$ is the Lagrangian the particle, $\frac{\partial L}{\partial \dot{\varphi}}$ is the total angular momentum and $\frac{\partial L}{\partial \dot{\varphi}}=\frac{\mathrm{d}}{\mathrm{d} t}[\boldsymbol{A} \cdot \boldsymbol{v}]$ where $\boldsymbol{A}$ is the vector potential and $v$ the velocity. The coefficient of $\boldsymbol{A} \cdot \boldsymbol{v}$ is actually $\frac{e M}{c p}$ which has been normalized to unity. As $r \rightarrow \infty$ the total angular momentum tends to be essentially kinetic. It is the total momentum $2 g$ that is conserved during the motion. If $\lambda$ is the latitude and $\boldsymbol{e}_{\lambda}, \boldsymbol{e}_{\varphi}, \boldsymbol{e}_{r}$ are three mutually perpendicular unit vectors

$$
\begin{aligned}
\boldsymbol{e}_{\varphi} & =\boldsymbol{e}_{\boldsymbol{r}} \times \boldsymbol{e}_{\curlywedge} \\
\boldsymbol{v} & =\dot{r} \boldsymbol{e}_{r}+r \dot{\lambda} \boldsymbol{e}_{\lambda}+r \dot{\varphi} \cos \lambda \boldsymbol{e}_{\varphi} .
\end{aligned}
$$

The vector potential of the magnetic field of the dipole is

$$
\begin{gathered}
\boldsymbol{A}=-\left[\boldsymbol{M} \times \nabla \frac{1}{r}\right]=\left[M \boldsymbol{K} \times \nabla \cdot \frac{1}{r}\right]=\boldsymbol{M} \frac{\cos \lambda}{r^{2}} e_{\varphi} \\
\boldsymbol{H}=[\nabla \times \boldsymbol{A}]=\frac{\boldsymbol{M}\left(\cos \lambda \boldsymbol{e}_{\lambda}-2 \sin \lambda \boldsymbol{e}_{r}\right)}{r^{3}} .
\end{gathered}
$$

1 This is based on the discussion by Fermi, Nuclear Physics, Chicago University Press. 
The relativistic Lagrangian of the particle is given by

$$
\begin{gathered}
L=-m_{0} c^{2} / 1-\beta^{2}+\frac{e}{c} \boldsymbol{A} \cdot \boldsymbol{v} \quad \text { where } \beta=v / c \\
=-m_{0} c^{2} / 1-\beta^{2}+\frac{e M}{c} \frac{\cos ^{2} \lambda}{r} \dot{\varphi} . \\
\frac{\mathrm{d}}{\mathrm{d} t} \frac{\partial L}{\partial \dot{\varphi}}=\frac{\partial L}{\partial \varphi}
\end{gathered}
$$

Since

and $L$ is not an explicit function of $\varphi, \frac{\partial L}{\partial \varphi}=0$ or $\frac{\partial L}{\partial \dot{\varphi}}$ is a constant of motion

$$
\begin{aligned}
p_{q} & =\frac{\partial L}{\partial \varphi}=-M\left(c^{2} \frac{\partial}{\partial v} / 1-\beta^{2}\right) \frac{\partial \dot{v}}{\partial \varphi}+\frac{e M}{c} \frac{\cos ^{2} \lambda}{r} \\
& =p\left\{\frac{r^{2} \cos ^{2} \lambda}{v} \varphi+\frac{e M}{c p} \frac{\cos ^{2} \lambda}{r}\right\} .
\end{aligned}
$$

If we define $\omega$ as the angle which the tangent to the trajectory makes with the east-west direction, then

$$
\cos \omega=\frac{v_{\varphi}}{v}=\frac{r \cos \lambda}{v} \varphi .
$$

Hence

$$
\frac{p_{q}}{p}=r \cos \lambda \cos \omega+\left(\frac{e M}{m v c}\right) \frac{\cos ^{2} \lambda}{r}=2 g .
$$

Thus we have identified $2 g$ to be proportional to the total angular momentum associated with the particle.

\section{STORMER AND MAIN CONES}

We write the equation for $\cos \omega$ as

$$
\cos \omega=\frac{2 g}{r \cos \lambda}-\frac{\cos \lambda}{r^{2}} .
$$

Using Stormer units of length, i.e. setting $\left(\frac{e M}{p c}\right)^{1 / 2}=1$ since $r$ is measured in terms of Stormer units, the radius of the earth $r_{0}(p)$ in these units depends upon the energy of the particle. Thus in studying the trajectory of a particle of momentum $\boldsymbol{p}$ the radius of the earth is assigned a value $r_{0}(p) .^{1}$

Since $\cos \omega$ can lie only between -1 and +1 it is clear that (1) imposes restrictions upon the values of $g$ and $r$. The condition on $\cos \omega$ implies the nonnegative nature of the kinetic energy $Q$ since

so that the two hyperbolae

$$
Q=\left(1-\cos ^{2} \omega\right)
$$

$$
\left.\begin{array}{l}
\cos \omega=+1 \\
\cos \omega=-1
\end{array}\right\}=\frac{2 g}{r \cos \lambda}-\frac{\cos \lambda}{r^{2}}
$$

1 See for example, Meix NeR's lucid treatment in "Cosmic Radiation", edited by W. HeISENBERG (1946), Dover. 
for a given $\lambda$ divide the $r-g$ plane into regions of positive and negative kinetic energy, the latter being forbidden.

A trajectory of a particle in the meridional plane is characterized by the co-ordinates $r$ and $\lambda$ corresponding to a given $g$. In the $r-g$ plane it is represented by a line parallel to the $r$-axis since $g$ is a constant of motion.

Fig. 51 shows the curves corresponding to

$$
\cos \omega= \pm 1
$$

in the $r-g$ plane for a particular $\lambda$. The following geometrical features are immediately evident:

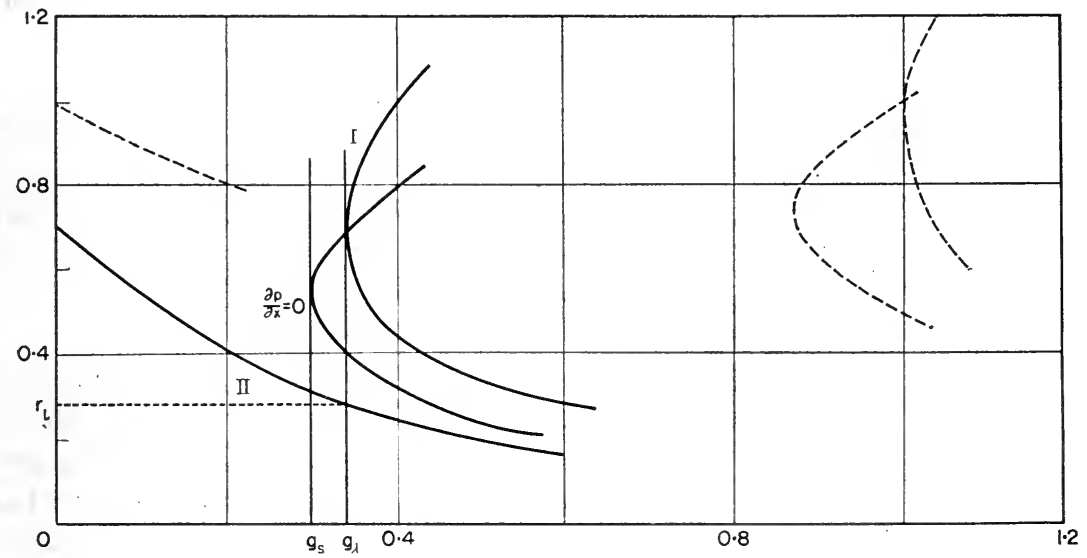

Fig. 51. The curves (I) $\cos \omega=+1$, (II) $\cos \omega=-1$ and $\partial P / \partial x=0$ for a given $\lambda$. Solid curves refer to $\lambda=60^{\circ}$; broken ones for $\lambda=0$.

(i) $g=g_{\lambda}=\cos ^{3 / 2} \lambda$ is a line tangential to the curve $I$ at $r=\cos ^{2 / 2} \lambda$ and cuts the curve $I I$ at $r=r_{\lambda}=(/ / 2-1) \cos ^{1 / 2} \lambda$. As $\lambda$ increases the critical values of $g_{\lambda}$ and $r_{\lambda}$ decrease so that the curve $I$ advances towards the origin. The maximum of this critical value of $g$ as $\lambda$ varies is unity and occurs for $\lambda=0$.

(ii) Any line $g=$ constant $<\cos ^{3 / 2} \lambda$ cuts only the curve $I I$ and in particular $g=0$ cuts the curve $I I$, at $r=\cos ^{2 / 2} \lambda$. The maximum of this $r$ as $\lambda$ varies is unity and occurs for $\lambda=0$.

(iii) For a given $r$ as $\cos \omega$ varies from -1 to $+1,2 g$ varies from $2 g=-r \cos \lambda$ $+\left(\cos ^{2} \lambda\right) / r$ to $2 g=r \cos \lambda+\left(\cos ^{2} \lambda\right) / r$. However we shall now show that this entire range of $g$ values is not permissible if we impose certain conditions on the trajectories.

Now in order that a trajectory (represented by a line parallel to the $r$-axis) may reach an observation point on the surface of the earth denoted by $\left(r_{0}(p), \lambda_{0}\right)$ it must assume values from $+\infty$ up to $r_{0}(p)$. In case it were to assume values less than $r_{0}(p)$ it will lie within the earth and will be forbidden due to the "shadow effect" of the earth. Ignoring this for the moment we shall consider a trajectory of a particle with momentum $\boldsymbol{p}$, to be absolutely forbidden if any part of it 
$\left(r>r_{0}(p)\right)$ lies in the forbidden region for any $\lambda$. Since we do not restrict the $\lambda$ values of the trajectory we require

$$
g \geqq 1
$$

to ensure that the trajectory of a particle with momentum $p$ such that $r_{0}(p)<1$ cuts the forbidden region whatever be the value of $\lambda$. Hence $\cos \omega$ varies from -1 to a value

$$
\cos \omega_{s}\left(\lambda_{0}, p\right)=\frac{2}{r_{0} \cos \lambda_{0}}-\frac{\cos \lambda_{0}}{r_{0}^{2}}
$$

and this corresponds to a cone called the Stormer cone and all directions with $\omega$ between 0 and $\omega_{g}$ are absolutely forbidden. In terms of the momentum $p$ of the particle

$$
\cos \omega_{s}(p)=\frac{2}{\left(p / p_{u}\right)^{1 / 2} \cos \lambda_{0}}-\frac{\cos \lambda_{0}}{\left(p / p_{u}\right)},
$$

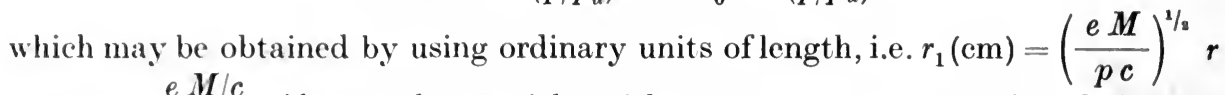
and $p_{u}=\frac{e M / c}{r_{1}^{2}}$. Alternately, particles with momentum $p<p_{0}$ will be forbidden
where

$$
p_{0}=p_{u} \frac{\cos ^{2} \lambda \cos ^{2} \omega}{\left[1+\sqrt{\left.1-\cos ^{3} \lambda \cos \omega_{8}\right]^{2}}\right.}
$$

In defining the above forbidden directions we require $g \geqq 1$ since there is no restriction on the $\lambda$ value of the trajectory. However trajectories with $\lambda \geqq \lambda_{0}$ corresponding to particles with momentum $p$ such that $r_{0}(p)<\cos ^{1 / 2} \lambda_{0}$ are forbidden if $g>\cos ^{3 / 2} \lambda_{0}$, since such trajectories always pass through the forbidden region. Thus $g_{\lambda}=\cos ^{3 / 2} \lambda_{0}$ determines the critical value $\omega_{c}\left(r_{0}, \lambda_{0}\right)$ given by

$$
\cos \omega_{c}\left(\lambda_{0}, r_{0}\right)=\frac{2 \cos ^{1 / 2} \lambda_{0}}{r_{0}}-\frac{\cos \lambda_{0}}{r_{0}^{2}}
$$

and particles with momentum $p$ and $r_{0}(p)<\cos ^{1 / 2} \lambda_{0}$ can approach the observation point $\left(\lambda_{0} r_{0}\right)$ at an angle $\omega$ such that

$$
-1<\cos \omega<\cos \omega_{c}\left(\lambda_{0} r_{0}\right) .
$$

In terms of the momentum of the particle

$$
\cos \omega_{c}\left(\lambda_{0} p_{0}\right)=\frac{2 \cos ^{1 / 2} \lambda_{0}}{\left(p / p_{u}\right)^{1 / 2}}-\frac{\cos \lambda_{0}}{\left(p / p_{u}\right)} .
$$

Another way of representing this relation is to ask for the minimum momentum $p_{0}$ which a particle should have if it were to approach the point at an angle $\omega$. This is given by

Summarizing we have

$$
p_{0}\left(\lambda_{0}, \omega\right)=\frac{\cos ^{2} \omega}{\cos \lambda^{0}(V[1-\cos \omega)]+1^{2}} .
$$

(i) For particles with momentum $\boldsymbol{p}$ where

$$
p>p_{u} \cos \lambda_{0}, \text { i.e. } r_{0}>\cos ^{1 / 2} \lambda_{0}
$$

all directions are allowed for trajectories with $\lambda \geqq \lambda_{0}$. For the equatorial plane $\lambda_{0}=0$. 
(ii) If $r_{0}<(/ 2-1) \cos ^{1 / 2} \lambda_{0}$ all directions will be forbidden for trajectories with $\lambda \leqq \lambda_{0}$. Therefore particles with momentum $p$ where

$$
p<p_{l}^{(0)} \cos \lambda_{0}=p_{u}\left(\gamma^{2}-1\right)^{2} \cos \lambda_{0}
$$

are forbidden in all directions for $\lambda \leqq \lambda_{0}$. For the equatorial plane the critical momentum is $p_{l}^{(0)}$.

(iii) For particles with momentum $p$ such that

$$
(/ 2-1) \cos ^{1 / 2} \lambda_{0}<r_{0}(p)<\cos ^{2 / 2} \lambda_{0}
$$

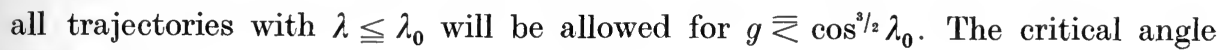
$\omega^{c}\left(\lambda_{0} r_{0}\right)$ is

$$
\cos \omega_{c}\left(\lambda_{0} r_{0}\right)=\frac{2 \cos ^{1 / 2} \lambda_{0}}{r_{0}}-\frac{\cos \lambda_{0}}{r_{0}^{2}}
$$

and all directions as $\cos \omega$ varies from -1 to the above value will be allowed The cone with $\omega$ varying from $\pi$ to $\omega_{c}\left(\right.$ i.e. $\left.\pi-\omega_{c}\right)$ is called the main cone and within the angle $\pi-\omega_{c}$ all directions are allowed for $\lambda \leqq \lambda_{0}$. In terms of momentum $\boldsymbol{p}$ all particles with momentum $p>p(\lambda, \omega)=\frac{\cos ^{2} \omega}{\cos \lambda(V[1-\omega]+1)^{2}}$ can approach
$\left(r_{0}, \lambda_{0}\right)$ at an angle $\omega$.

\section{DEVIATIONS FROM THE STORMER AND MAIN CONES}

We have taken the trajectory of a particle to be forbidden if the kinetic energy at any point on the trajectory is negative, this being the only condition which has till now been imposed. The allowed directions were defined as those complementary to the forbidden. There are two other conditions which have to be satisfied for a direction to be allowed.

(1) Since the equations of motion cannot be explicitly solved, we have to ascertain whether the trajectory of a particle coming in the allowed direction can be traced to infinity. This was first realized by Stormer who demonstrated the existence of periodic orbits which are confined to some bounded space. A rigorous definition of periodic orbits will be given presently. Obviously if we are not able to trace the trajectory in any "allowed" direction to infinity, then that direction is in fact forbidden.

(2) Even if we are able to trace orbits to infinity it will be shown that in some cases certain directions are still forbidden. If a trajectory is such that before reaching $\left(r_{0}, \lambda_{0}\right)$ it assumes values of $r<r_{0}$ it implies that it intersects the earth before reaching the observation point and will hence be forbidden by the shadow effect of the earth. If these two additional conditions are imposed, it is clear that the Stormer and the main cones previously defined provide an inadequate description of the allowed regions. We shall now consider the shadow effect of the earth.

1 T. K. Radha and R. Thunga, Report of the Cosmic Ray Symposium (1960) D.A.E. Govt. of India. 
To consider these refinements it is necessary to introduce a more convenient set of coordinates for the description of allowed and forbidden regions in the meridional plane. The main defect of the polar representation $r-\lambda$ is that the regions for small $r$ are contracted as compared with those for large $r-$ a defect inherent in any polar representation. It is therefore more convenient to plot the trajectory with $\lambda$ as a Cartesian coordinate and define the variable $x$ as

$$
\mathrm{e}^{x}=2 g r .
$$

This is known as the Goursat transformation and $x$ is a measure of the energy. ${ }^{1}$ In addition we define a new independent variable $\sigma$ as

$$
\mathrm{e}^{2 x} \mathrm{~d} \sigma=(2 g)^{3} \mathrm{~d} s .
$$

A simple meaning can be given to this transformation of the independent variable. If we imagine the motion of a particle in a fixed meridional plane (this is not so in the present case), the velocity about the centre of the dipole in the two representations are equal.

Applying these transformations, we have

$$
\frac{\mathrm{d}^{2} x}{\mathrm{~d} \sigma^{2}}=\frac{1}{2} \frac{\partial P}{\partial x} ; \quad \frac{\mathrm{d}^{2} \lambda}{\mathrm{d} \sigma^{2}}=\frac{1}{2} \frac{\partial P}{\partial \lambda}
$$

where

$$
P(x, \lambda)=Q e^{2 x}-\left(e^{-x} \cos \lambda-\frac{1}{\cos \lambda}\right)^{2} ; \quad a=(2 g)^{-4}
$$

and $\quad \cos \omega= \pm r \cos \lambda \frac{\mathrm{d} \varphi}{\mathrm{d} s}=\frac{2 g}{r \cos \lambda}-\frac{\cos \lambda}{r^{2}}=\sin \theta=\left(1-\frac{P}{a} \cdot \mathrm{e}^{-2 x}\right)^{1 / 2}$.

These equations which are equivalent to $(1 \cdot 9)$ are not integrable in a closed form, and for their treatment there has been recourse to general theorems, series expansions and numerical methods.

We shall here make some general remarks upon types of orbits. The boundary lines between the region of positive and negative energy are given in the Goursat representation by those corresponding to $P=0$. In the Goursat representation, the region corresponding to $Q>0$ is that in which $P>0\left(\right.$ since $\left.P=\frac{r^{2}}{4 g^{2}} Q\right)$.

We shall first consider the series of curves corresponding to $P=0$ in the $x$, $\lambda$ plane for various values of $g$. This curve for a given $g$ consists of two distinct unconnected parts $\mathrm{A}$ and $\mathrm{B}$ corresponding to the pair of curves $\cos \omega=+1$ and -1 in the $r-g$ plane. The diagram (Fig. 52) is a two-dimensional $(x, \lambda)$ representation of a three-dimensional surface with $g$ as the third dimension. The characteristics of the curves in the $r-g$ plane discussed before may be identified from this diagram as follows :

1 E. J. Schremp, Phys. Rev. 54, 153, 158 (1937); Phys. Rev. 57, 1061 (1940); C. Stormer, loc. cit.; G. Lemaitre and M. S. Vallarta, Phys. Rev. 49, 719 (1936); 50, 493 (1936). R. A. Huxter, Phys. Rev. 5j), 15, 107, 614 (1939); A. Banos, Math. Phys.18, 211 (1939); L. BonckanRET, Amr. Soc. Sci. Bruxelles A 54, 174, (1937). 
(1) The lower set of curves (A) correspond to the curve $\cos \omega=-1$ in the $r-g$ plane and for a given $g$, the higher the latitude, the smaller the $x$ and correspondingly their $r$ values. This represents exactly the lowering of the curve $\cos \omega=-1$ in the $r-g$ plane as $\lambda$ increases. The curve A shifts to the left as $g$ decreases, i.e. for a given $\lambda$ smaller the $g$ value smaller the $x$ value or larger the $r$ value. It should be noted that while for a given $g, r$ increases as $x$ increases, if we were to decrease $g, r$ increases as $x$ decreases. This is as it should be which can be seen from the curve $\cos \omega=-1$.

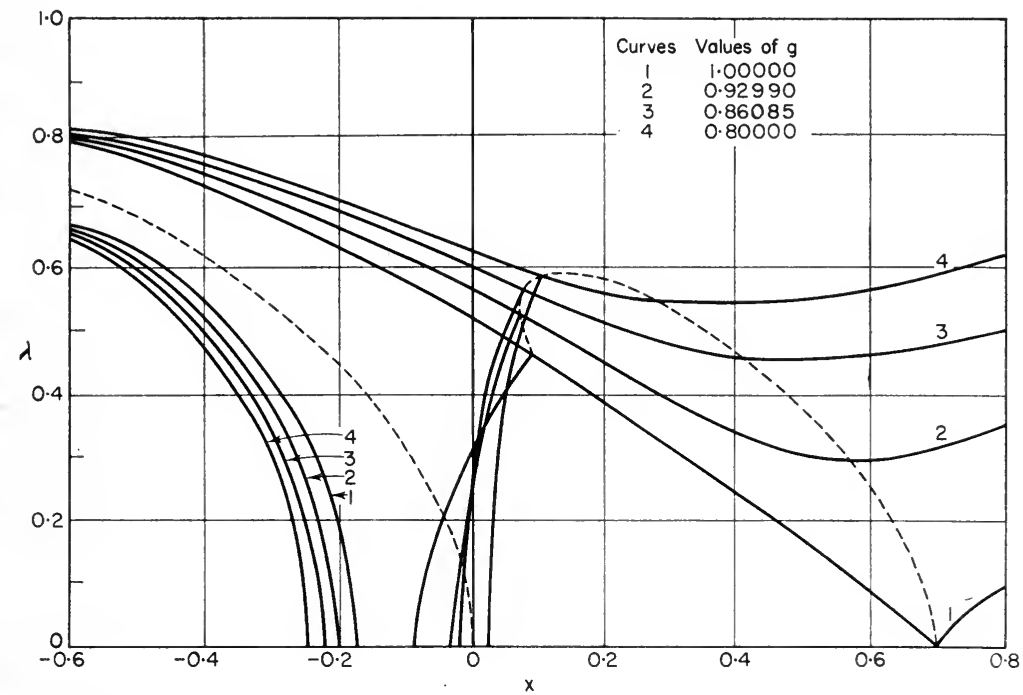

Fig. 52. Curves $P=0$, inner stable and outer unstable periodic orbits for various values of $g$.

(2) The upper set of curves (B) correspond to $\cos \omega=+1$. For a given $\lambda$ and $g$ there are in general two values of $x$ except at the turning point which corresponds to a $\lambda_{0}$ such that $g_{0}=\cos ^{3 / 2} \lambda_{0}$. In the $r-g$ plane, this corresponds to the tangential value $g_{c}$ for $\cos \omega=+1$. The curve for $g=1$ touches the equator $(\lambda=0)$ at $x \sim 0.7$ (i.e. $r=1$ ) and higher the value of $\lambda_{\min }$, lower the $g$ and $x$ values which again confirms the shape of $\cos \omega+1$ as $\lambda$ increases.

We shall now consider the contours $P=$ a constant in the $x-\lambda$ plane for a given $g(=0.89)$, taking $P$ as the third dimension. Remembering that the unit in which $r$ is measured depends upon the particle energy as we have already seen, the position of the earth's surface is defined by $r_{0}(p)$. If we wish to plot any trajectory, we must start with some point with a finite $\left(r_{0}, \lambda_{0}, \varphi\right)$ and given $g$ momentum values and trace the path of the particle "forward" or "backward" by increasing or decreasing $\sigma$. This is the only way of plotting trajectories of particles, since there is no meaning in plotting a trajectory from an infinite distance which is the real starting point for cosmic rays. 
To study the path of a particle, we first take a point $\left(x_{0}, \lambda_{0}\right)$ and assume a certain direction of motion for the particle at this point. If the particle at this point experiences an acceleration to the right and continues to do so for $x>x_{0}$, it can never return to an $x$-value less than $x_{0}$. Thus if $\left(x_{0}, \lambda_{0}\right)$ represents a point on the earth, the trajectory can never return to the earth. Of course we have assumed that the particle is moving exactly in the opposite direction to which the cosmic ray particles will arrive and this is equivalent to our previous statement

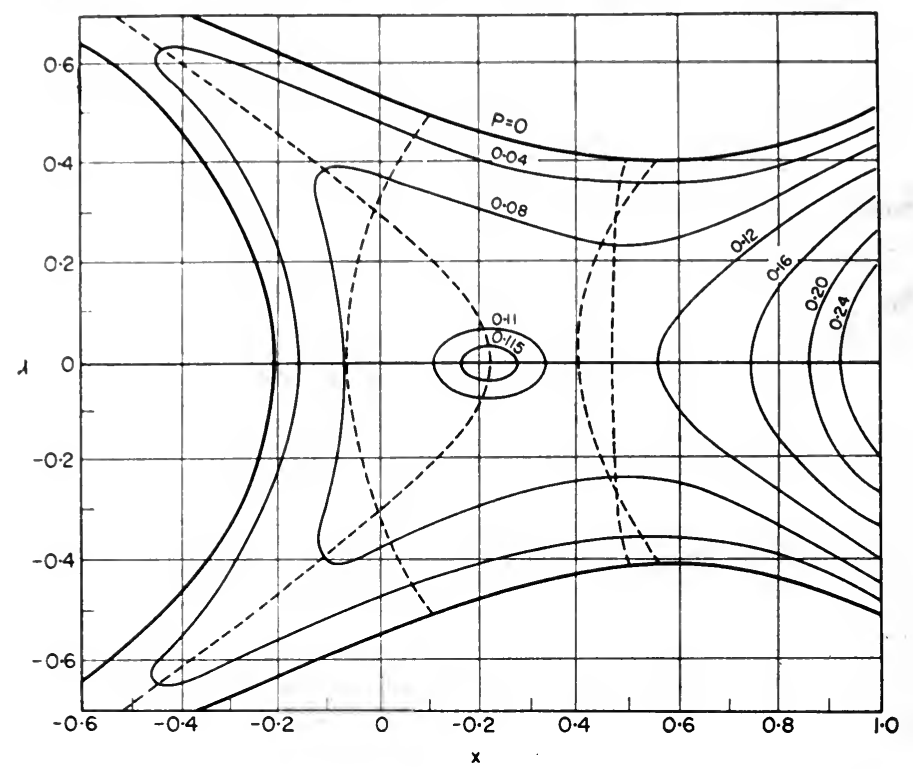

FIG. 53. Contour diagram of the function $P(\varkappa, \lambda)$ for $g=0.89(a=0.10)$.

that a trajectory can be traced forwards or backwards since the equations of motion are not altered by replacing $\sigma$ by $-\sigma$. A particular example of such a point is $x_{0}=0.54$, where if a particle goes towards the right in any arbitrary direction, it can never return to the initial value. Particles arriving from infinity at $x_{0}$ would never have intersected the earth earlier. Also for a given $\lambda$ we can find the value $x_{\max }(\lambda)$ and $x_{\min }(\lambda)$ for which $P$ is a maximum and minimum respectively. The loci of points $x_{\max }(\lambda)$ and $x_{\min }(\lambda)$ as we vary $\lambda$ will be called the curves of maximum and minimum $P$.

In the region between the curves of maximum and minimum $P$, the $x$ component of the acceleration is directed towards the left. In this case for a particle starting out from any $\left(x_{0} \lambda_{0}\right)$ in the region, there is a possibility of returning to its initial value depending upon its momentum and its initial direction. Thus it is clear that the trajectory of a particle which never enters a region of negative acceleration (and of course negative energy) will be certainly allowed and hence will not be subject to the shadow effect of the earth. We shall now obtain the condition for a trajectory to pass only through regions of positive acceleration. 
Since the question of the shadow effect of the earth arises only when a particle experiences a negative acceleration in some region along its trajectory, we now plot the curve $\partial P / \partial x=0$ in the $r-g$ plane for a given $\lambda$. This is given by

$$
2 g=r^{3}+\frac{\cos ^{2} \lambda}{r}
$$

and marks out the region of negative acceleration in the $r-g$ plane, as is seen in the figure. The following geometrical features for a given $\lambda$ are obvious (see Fig. 51). ${ }^{1}$

(i) The two branches correspond to $P_{\max }$ and $P_{\min }$ and the tangential value of $g_{s}$ is $0.8774 \cos ^{3 / 2} \lambda$ at $r_{s}=\frac{\cos ^{1 / 2} \lambda}{1 \cdot 316}$. Thus $g_{s}$ is always less than $g_{\lambda}=\left(\cos ^{3 / 2} \lambda\right)$.

(ii) The curves $\cos \omega=+1$ and $\frac{\partial P}{\partial x}=0$ intersect at $g=\cos ^{8 / 2} \lambda$ and $r=\cos ^{1 / 2} \lambda$, i.e. at $\left(g_{\lambda}, r_{\lambda}\right)$.

(iii) The $\cos \omega$ value on any point on the curve $\frac{\partial P}{\partial x}=0$ is given by

$$
\cos \omega=\sin \theta=r^{2} / \cos \lambda
$$

as obtained by substituting for $2 g$ in the general formula for $\cos \omega$. The basic features of the simple shadow cone may now be qualitatively explained by applying arguments similar to those used in the case of negative energy.

(a) Since the trajectory of a particle is not subject to the shadow effect if it never enters a region of negative acceleration, the critical value of $g$ in analogy with the arguments for the main cone is now given by

$$
g_{s 1}=0.8774 \cos ^{3 / 2} \lambda_{0}
$$

and for particles with $r_{0}<\cos ^{1 / 2} \lambda_{0}$ trajectories with $\lambda \leqq \lambda_{0}$ are always allowed.

(b) However a condition similar to the Stormer condition (i.e. $g=1$ in this case $g_{s 2}=0.8774$ ) has no particular significance since once within a region of negative acceleration there is no simple method of determining the allowed and forbidden directions. This is due to the fact that the trajectory of a particle subject to a negative acceleration is not necessarily forbidden since before the particle velocity becomes zero it may enter a region of positive acceleration and will therefore be allowed. It is this complicated structure which gives rise to the penumbra. Thus we have the following values of $g$ which define the boundary points. (1) $g=1$ which defines the Stormer cone with $\cos \omega=\sin \theta=\frac{2}{r_{0} \cos \lambda_{0}}-\frac{\cos \lambda_{0}}{r_{0}^{2}}$
within which all directions are forbidden.

(2) $g_{0}=\cos ^{3 / 2} \lambda_{0}$ which defines the boundary of the main cone within which all directions are allowed, except those due to the simple shadow cone.

(3) The cone with $g_{s}=0.8774 \cos ^{3 / 2} \lambda_{0}$ which defines a cone within which all directions are certainly allowed.

(4) The cone $\frac{r_{0}^{2}}{\cos \bar{\lambda}_{c}}=\cos \omega$ which is either more or less restrictive than the

\footnotetext{
1 G. Lemaitre and M. S. Vallarta, ibid.
} 
main cone depending upon the $r$ value, i.e. the energy of the particle. For $r_{0}$ values below $r_{l}$

$$
\frac{r_{0}^{2}}{\cos \lambda_{0}}>\frac{2 \cos ^{1 / 2} \lambda_{0}}{r_{0}}-\frac{\cos \lambda_{0}}{r_{0}^{2}}
$$

sinee the line parallel to the $g$ axis cuts the tangent $g=\cos ^{2 / 2} \lambda_{0}$ first and then the eurve $\frac{\partial P}{\partial x}=0$. But for $r_{0}$ between $r_{l}$ and $\cos ^{1 / 3} \lambda_{0}$

$$
\frac{r_{0}^{2}}{\cos \lambda_{0}}<\frac{2 \cos ^{1 / 2} \lambda_{0}}{r_{0}}-\frac{\cos \lambda_{0}}{r_{0}^{2}} .
$$

The shadow effeet can be explained in a slightly different way as follows:

Taking a point on the earth $\left(x_{0}, \lambda_{0}\right)$, the longitude being at present irrelevant (since the situation is symmetrieal with respect to longitude about the dipole)

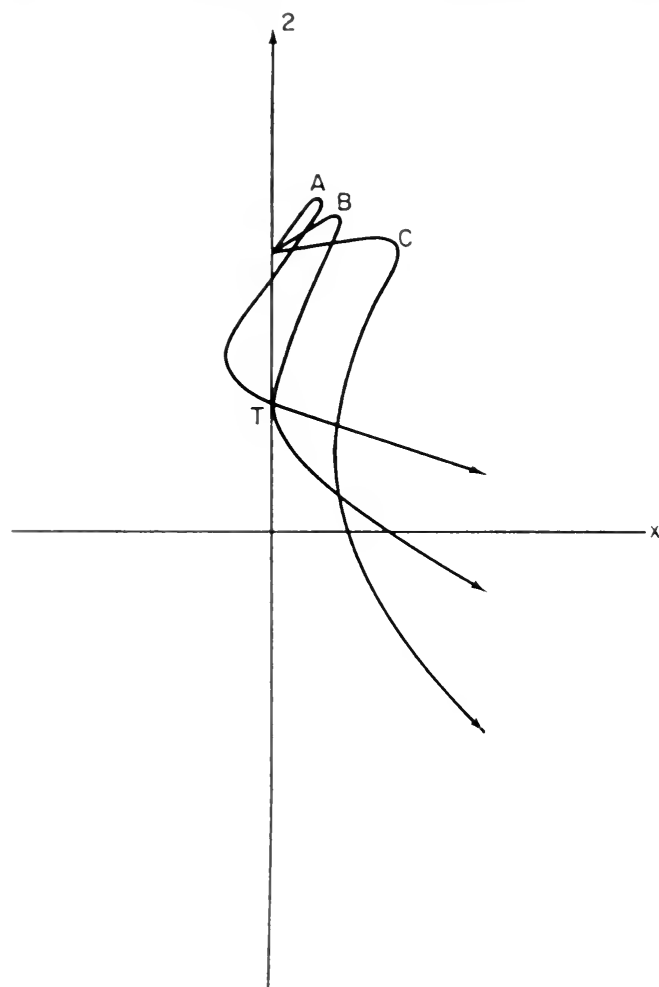

Fig. 54. Simple orbit of the second kind (B). Vertical line represents the earth's surface. Orbit $A$ is in shadow. Orbit $C$, if non-reentrant gives an allowed direction at 0 corresponding to the interior of the main cone; if re-entrant, orbit $\mathrm{C}$ may or may not be in shadow according to the positions of its subsequent minima of $x$.

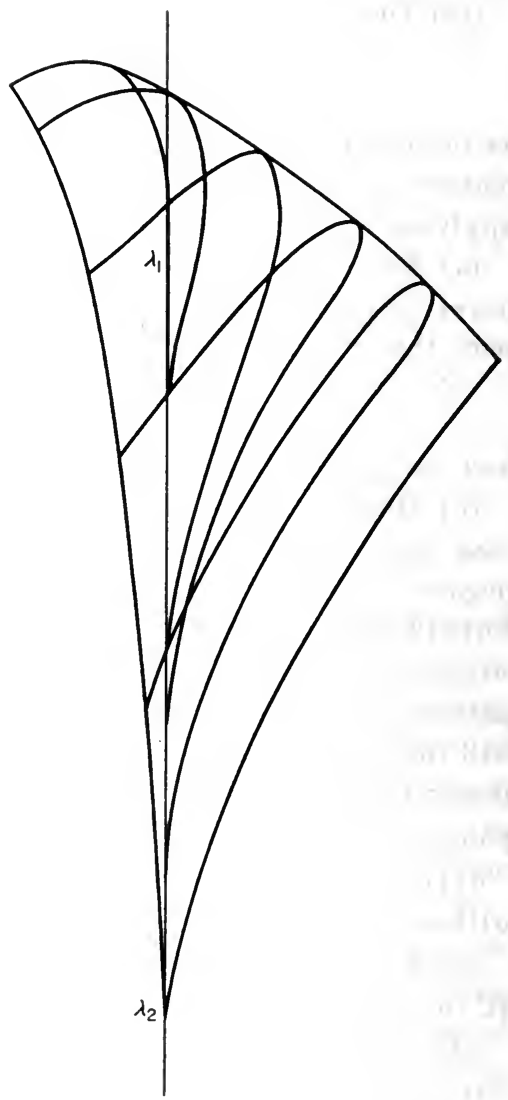

Fig. 55. Family of simple orbits of the second kind, $x=$ constant vertical line represents earth's surface. 
it is only necessary to obtain for each $\left(x_{0}, \lambda_{0}\right)$ the family of all orbits possessing sections $O T$ like that of orbit $B$ in Fig. 54. This is a one parameter family, the parameter being $g$. The angles $\eta, \theta(=90-\omega)\left(\eta\right.$ being defined by $\left.\tan \eta=\frac{\mathrm{d} \dot{\lambda}}{\mathrm{d} x}\right)$ defined by this family provide the simple shadow cone for the observation point $(\lambda, r)$. The orbits in question have been obtained with the aid of Bush differential analyses. To facilitate the finding of points on the earth at which the shadow effect is felt, we adopt the following procedure.

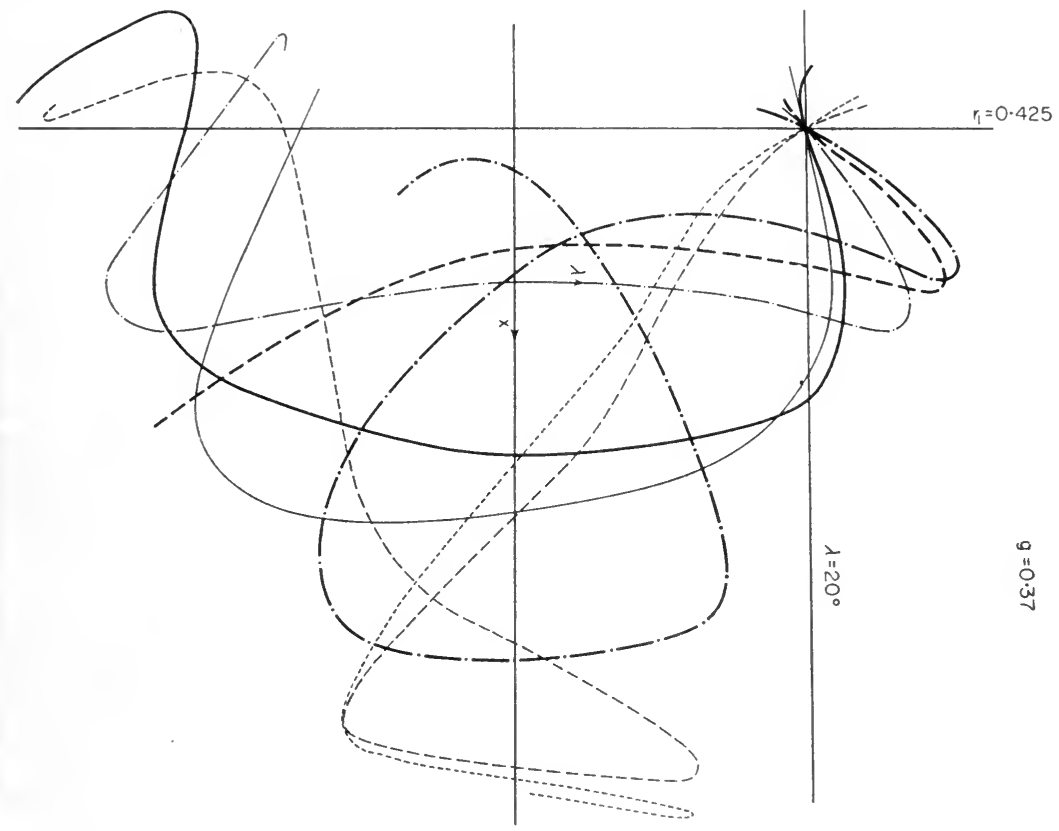

Fig. 56. Family of orbits which start out from one point.

To find the range of $\lambda$, when $x$ is specified as $x_{0}$ when it denotes a point on the earth (the value of $x$ for the earth is determined by the momentum) we study the behaviour of the trajectories which intersect the earth again, as we vary $\lambda$. We have noted that the trajectory stemming from the point $0\left(x_{0}, \lambda_{0}\right)$ touches the line $x=x_{0}$ at $\left(x_{0}, \lambda \uparrow\right)$. No shadow effect will be felt if there exists no $\lambda \uparrow$ corresponding to a given $\lambda_{0}$. We shall now investigate the limits of $\lambda \uparrow$. This is done by studying trajectories which start tangential to $x=x_{0}$ at $\lambda \uparrow$. As we increase $\lambda \uparrow$, we reach a point $\lambda_{1}$ where the trajectory is essentially to the left of $x=x_{\mathrm{c}}$. The point $\left(x_{0}, \lambda_{1}\right)$ represents obviously the point on the contour at which $\frac{\partial P}{\partial x}=0$. The $x$-acceleration of the particles starting tangentially below $\lambda_{1}$ is initially towards the right and above $\lambda_{1}$, towards left. As we decrease $\lambda^{1}$, we reach a point $\lambda_{2}$ such that a trajectory tangential to $x=x_{0}$ reaches the boundary $P=0$ and 
reverses. This is called the self-reversing orbit and at $\lambda_{2}=0$, it can be identified to be the outer principal periodic orbit. To obtain the points $\lambda_{1}$ and $\lambda_{2}$ for various values of $g$, we have drawn the loci of zero $x$-acceleration or $\frac{\partial P}{\partial x}=0$ and zero $x$-velocity which are shown in Fig. 51 .

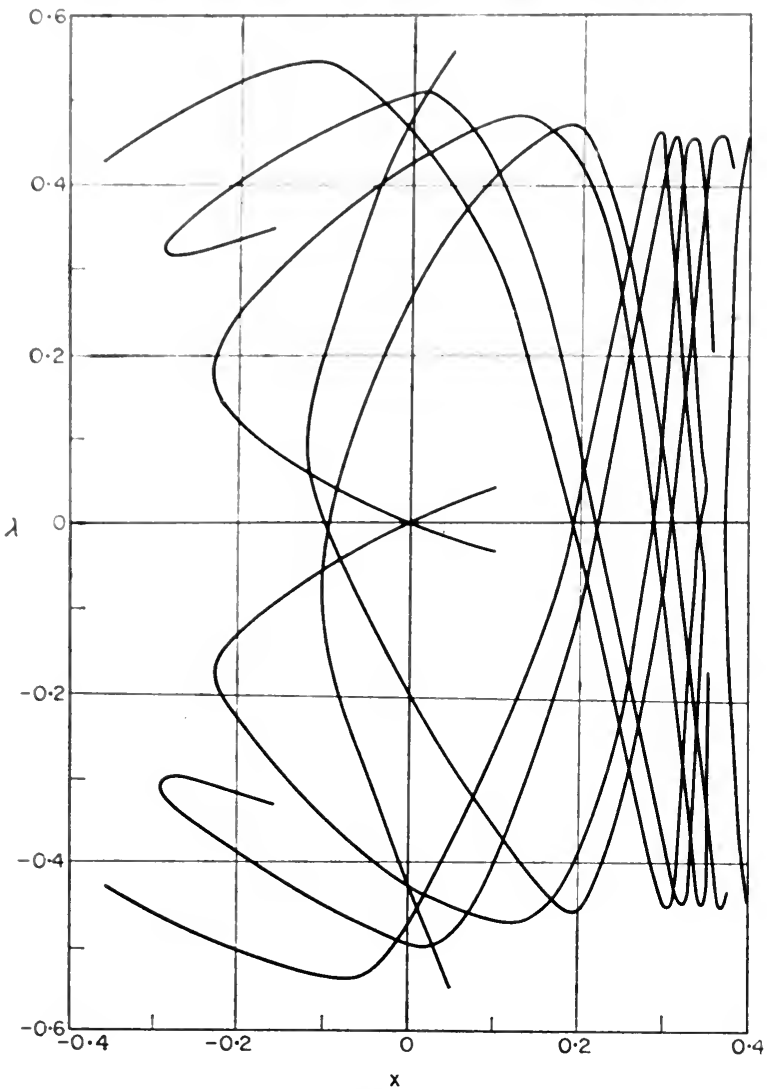

Fig. 57. Family of asymptotic orbits for $g=0.85$.

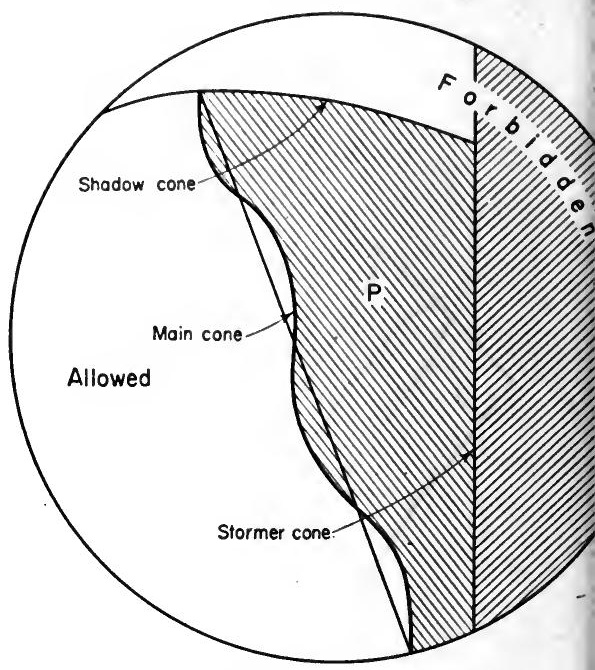

Fig. 58. The observer's hemisphere at latitude $\lambda$ showing schematically the allowed and forbidden directions of incidence for a given rigidity.

Thus the existence of the shadow cone depends upon the possibility of finding a $\lambda \uparrow$. If $\lambda_{1}$ and $\lambda_{2}$ coincide, no shadow effect can be felt on the earth with respect to the particles of that $g$ value which yields the limiting $\lambda$ values. If there exists no self-reversing orbit, i.e. $\left(x, \lambda_{2}\right)$ lies on $P(x, \lambda)$ and if $\lambda_{1}$ and $\lambda_{2}$ coincide the point $\lambda_{2}$ corresponds to zero $x$-velocity and no analytical expression can be found. Since an analytical expression can be found for $\lambda_{1}$ and not for $\lambda_{2}$ we merely find the value of $g$ when $\lambda_{1}$ lies on $P\left(x, \lambda_{1}\right)=0$; we observe that $\lambda_{1}=\lambda_{2}$ is also the point of intersection of $\frac{\mathrm{d}^{2} x}{\mathrm{~d} \sigma^{2}}=0$ and $P=0$.

$$
\frac{\mathrm{d} P}{\mathrm{~d} x}=0 \quad \text { vields } \quad 2 g=r^{3}+\frac{\cos ^{2} \lambda}{r}
$$


and $P=0$ yields

$$
\frac{r^{2}}{4 g^{2}}-\left(\frac{\cos ^{2} \lambda}{2 g r}-1\right)^{2} \sec ^{2} \lambda=0 .
$$

These two curves intersect for $r^{2}=\cos \lambda, g^{2}=r^{3}$.

The above discussion is only to facilitate the finding of points at which the shadow effect can be felt. For each chosen value of $g$ and each chosen value of $x$, we obtain a curve giving $\eta$ as a function of $\lambda$. From these curves, diagrams are then constructed giving $\eta$ as a function of $r$ for each $\lambda$. Once we are in possession of these curves, we are in a position to plot the simple shadow cone at a place of given latitude $\lambda$. The coordinates of a point on the loci are given by $\sin \theta$, $\sin \eta \cos \theta$.

In addition we have a class of orbits which do not extend to infinity at all, which are defined for obvious reasons as periodic orbits. The simplest periodic orbits we can think of are those in the equatorial plane. For example we can show that a particle with a given $g$-value can describe a circle of radius $r=g$ without moving out of the orbit in the equatorial plane. This follows immediately from the definition since $g=r^{2} \frac{\partial \varphi}{\partial s}$. For a circular motion $r \mathrm{~d} \varphi=\mathrm{d} s$ and hence $r=g$. In particular $g=1, r=1$ is a periodic orbit. This periodic orbit can be represented by a point in the $r-\lambda$ plane since the particle is confined to the equatorial plane. A few important features corresponding to $g=1, r=1$ can be mentioned. Examining the equation (1.6) for the motion in the equatorial plane, we find since

$$
\begin{gathered}
z=z^{\prime}=0 \text { and } \varrho=r \\
\varphi=\varphi_{0}=\frac{\int\left(2 g-\frac{1}{r}\right) \frac{\mathrm{d} r}{r^{2}}}{\sqrt{/\left[1-\frac{1}{r^{2}}\left(2 g-\frac{1}{r}\right)^{2}\right]}}
\end{gathered}
$$

so that $\varphi$, the angle described by the meridian plane since the particle started its course from infinity tends to infinity as $r \rightarrow 1$ for $g=1$. This obviously means that the particle spirals round the circle $r=1$ an infinite number of times and so if we ask for the angle described about the meridian plane as $r \rightarrow 1$, it must clearly be infinite. This would mean that if a periodic orbit is described by a particle, any slight displacement would make it describe the asymptotic orbit and it may reach infinity. Thus the orbit $g=1, r=1$ is "unstable". Any periodic orbit is called unstable if there exist asymptotic orbits which can be traced to infinity.

For simplicity we have mentioned a class of periodic orbits in the equatorial plane. Stormer has shown that three-dimensional periodic orbits can also exist and he has derived in particular the two principal periodic orbits whose characteristics are as follows. They reach a point $(x, \lambda)$ at which $P=0$, i.e. the velocities become zero. These two are represented in the contour diagrams. Note that the three-dimensional periodic motion represents an oscillatory motion along curves 1 and II in (Fig. 51). The orbit nearer the dipole is stable while the other is unstable. 


\section{LAT I'TU DE EFEET}

In view of the multiplicity of eonditions imposed and the complicated nature of the orbits, it may seem difficult to determine the variation of the directional intensity of radiation. But on the assumption that the particles are initially isotropic in space, it was realized by Lemaitre and Vallarta that as an immediate consequence of the Liouville theorem in classical dynamics, the spatial distribution remains isotropic in allowed direetions. Hence to compute the intensity of radiation at any latitude we need only know the solid angle corresponding to the total allowed cone.

We shall first state the Liouville theorem in a form which immediately reveals how the initial assumption of isotropy turns out to be fortuitous in the application of the theorem.

Let the motion of a particle be represented by a trajectory in a six-dimensional phase space. If it is represented by a point $P_{0}$ in phase space at time $t=0$, it goes over to $P_{t}$ at $t$. Similarly every point goes over to a corresponding point, the transformation being one to one. If $P_{0}$ is a measurable set of points, then the measure of $P_{0}$ coincides with the measure of $P_{t}$, i.e. the measure of measurable point sets is an invariant under this transformation. If now we have an isotropic distribution of particles, it corresponds to an uniform distribution of points in phase space so that once we assume particles are isotropic in one region of external space, the motion is such that isotropy is maintained in all allowed directions. Thus the total intensity of radiation corresponding to a particular momentum is determined if we know the angle of the allowed cone.

The total density of radiation corresponding to particles of momentum $p$, at a given latitude $\lambda$, is thus given by

$$
I(p, \lambda)=\int_{A(p, \lambda)} I(\theta, p, \lambda) \mathrm{d} \Omega_{\theta}
$$

where $\mathrm{d} \Omega_{\theta}$ is the solid angle around $\theta$ the directional intensity and $A(p, \lambda)$ defines the total allowed cone. The overall intensity for all momenta

$$
I(\lambda)=\int I(p, \lambda) \mathrm{d} p,
$$

and the number density of particles of type $j$ is

$$
N_{j}=\int \frac{I_{j}(\theta, p, \lambda)}{v_{j}} \mathrm{~d} \Omega_{\theta} .
$$

If the distribution of particles is isotropic for all velocities

and the flux of particles of type $j$ is

$$
N_{j}=\frac{4 \pi}{v_{j}} I_{j}
$$

$$
F_{j}=\int I_{j} \cos \theta_{j} \mathrm{~d} \Omega_{\theta}=\tau I_{j}=\frac{1}{4} V_{j} N_{j} .
$$

The calculated values appear to be in fair agreement with the observed ones. 


\section{INTERACTION OF COSMIC RAYS WITH MATTER}

\section{GENERAL DESCRIPTION}

SINCE primary cosmic radiation consists of nuclei ranging in energies from $10^{5}$ to $10^{18} \mathrm{eV}$, the study of cosmic ray phenomena implies the study of the interactions of such fast particles with air nuclei or more generally with matter. This falls broadly into two classes:

(i) Relating to individual collisions between the incident particles and air nuclei, or the secondaries produced in such collisions and the air nuclei. The study is based on the theory of elementary particle interactions according to the existing state of quantum mechanics which Part I deals with in detail.

(ii) Relating to cascade phenomena, i.e. the computation of the cumulative effect of these fundamental particle interactions over finite thicknesses of matter assuming the cross-sections for the individual processes.

We will now deal with these elementary particle interactions with special reference to cosmic rays and the present status of cascade theory. Since the beginning of the study of cosmic rays, cascade phenomena have claimed the attention of both the theorist and the experimenter. For obvious reasons the different features of this phenomenon have been studied separately as for example the longitudinal development of nucleon cascades arising from nuclear interactions, soft cascades from electromagnetic interactions, meson production without reference to other particles and lateral spread ignoring other features. In principle all these features must be imbedded in a cascade generated by such high energy particles for a better understanding of the high energy component of cosmic rays. However a quantitative study of this is a formidable task for two reasons :

(1) Exact cross-sections for all but electromagnetic phenomena are not available,

(2) The inclusion of all the processes makes the problems mathematically intractable and numerical computation almost impossible.

As an introduction to the above study we shall here give a descriptive account of cosmic ray phenomena associated with the passage of a fast primary through the atmosphere.

The phenomena can be described in most general terms as follows: A fast primary nucleon enters the atmosphere at any arbitrary angle to the zenith. It collides with the air nuclei and in a single nuclear collision produces a sheaf of -mesons (charged and neutral), nucleons, sometimes heavier fragments and strange particles. The charged mesons either decay into $\mu$-mesons or interact with air 
nuclei, the interaction between the meson and the nucleus being of the same type as a nucleon-nucleus collision. The neutral meson either interacts with the nucleus, or what is more probable, decays almost immediately into two highly energetic gamma rays which generate the soft caseades. Among the strange particles the charged $K$-mesons, if they have high energies, interact with the nuclei produeing the same type of particles as in a pion-nucleon collision at low energies. The $\mathrm{K}^{+}$-mesons can undergo only scattering by the nucleons and ultimately decay into pions or $\mu$-mesons, the abundant mode being $K_{\mu 2}$ decay. The $K^{-}$-mesons at such low energies can undergo a variety of interactions with nuelei resulting in the production of hyperons and $\pi$-mesons. The $K^{\mathbf{0}}$ particles will decay into two $\pi$-mesons. The hyperons at very high energies reproduce the nuclear collision phenomena while at low energies decay or interact with nuclei forming hyperfragments. The hyperfragments in turn decay into ordinary nuclei with or without emission of pions.

High energy electrons are not only produced in the soft cascades generated by photons but also by the decay of $\mu$-mesons. The $\mu$-mesons which are highly energetic and weakly interacting mostly decay but since the decay time is comparable to the time for traversing the atmosphere they comprise the penetrating components at sea level.

Three additional features have to be ineluded in the deseription of the development of such eascades:

(1) The loss of energy by ionization of charged particles.

(2) The angular distribution of particles produced in any collision (i.e. they need not be eollimated).

(3) Lateral spread due to multiple scattering.

The theories of nucleon-nucleon collisions are in a primitive stage in view of the strong coupling of the meson and nucleon fields and the eonsequent failure of the standard perturbation methods. Even if we do not invoke perturbation theory, there are serious limitations to any field theoretie caleulation for the production of more than one meson in a nucleon-nucleon collision.

Only statistical approaches of doubtful validity in their quantitative estimates are available. As regards the interaction of the strange particles the nature of the coupling is in a speculative stage and the caleulation at such high energies available in the cosmic rays is beyond hope. Ad hoc assumptions have to be made about the particles at the point of production.

The processes deseribed above ean be sehematically represented by the following diagram in which a primary particle (e.g. $p, \alpha$, etc.) enters at $A$ and collides with a nucleus in the atmosphere. The following conventions have been adopted in the diagram:

(i) Particles produced in a single collision are represented along a horizontal line.

(ii) The decay products of a particle are represented by lines diverging from a point. 


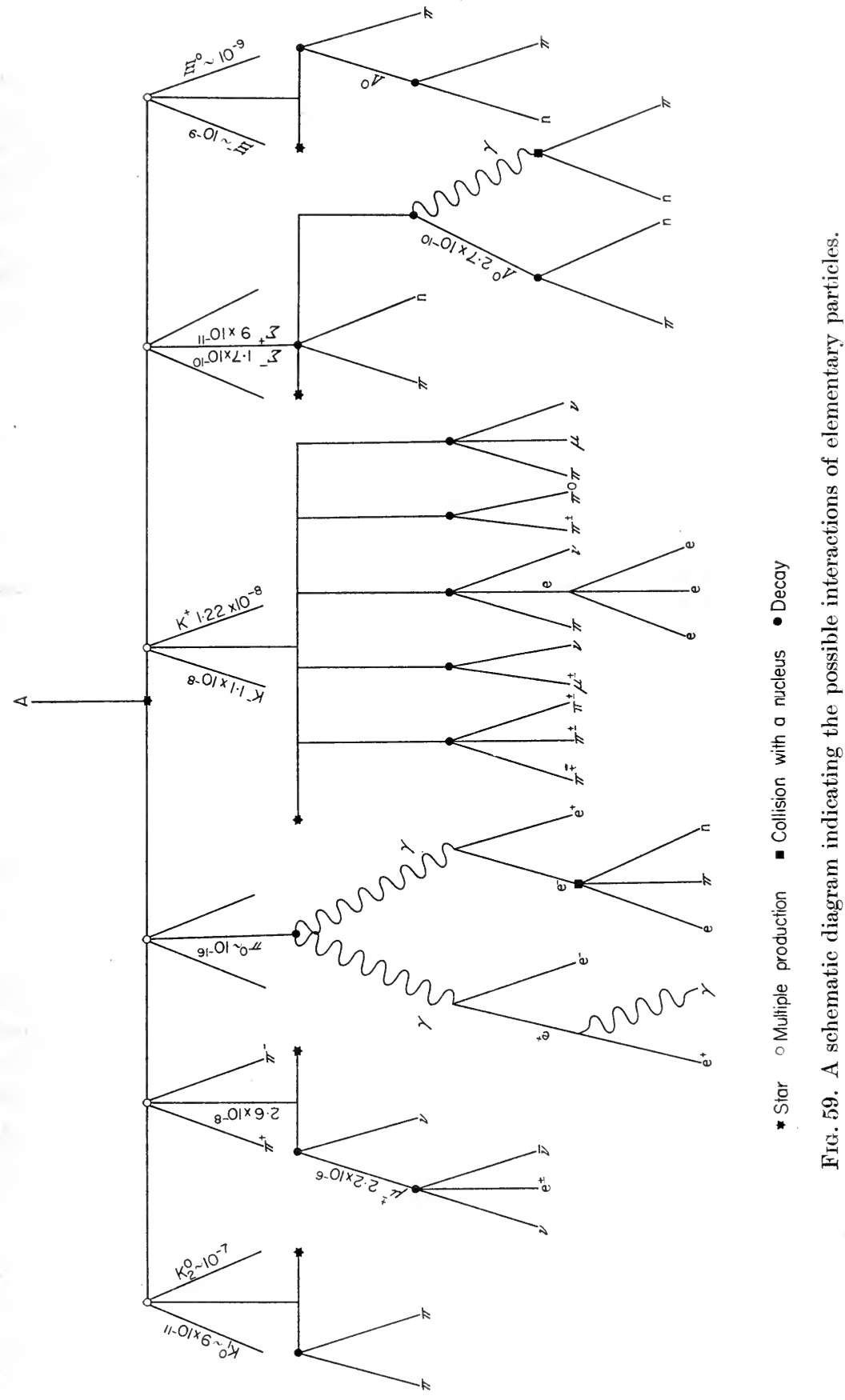




\section{․ $\mu-M$ ESON INTERACTIONS IN COSMIC RAYS ${ }^{1}$}

The $\mu$-meson is one of the secondary particles produced by the passage of cosmic rays through the atmosphere. Historically the $\mu$-meson was discovered earlier than the $\pi$-meson and originally mistaken for the Yukawa particle till the latter was identified to be the $\tau$-meson. The cosmic ray $\mu$-mesons are assumed to originate from the decay of the $\pi$-mesons in flight, the pions being produced by the interaction of the primary nucleonic component with the nuclei of the atmosphere.

The $\mu$-mesons have weak interaction with the nuclei and thus form the penetrating component of cosmic radiation. They lose energy through ionization and

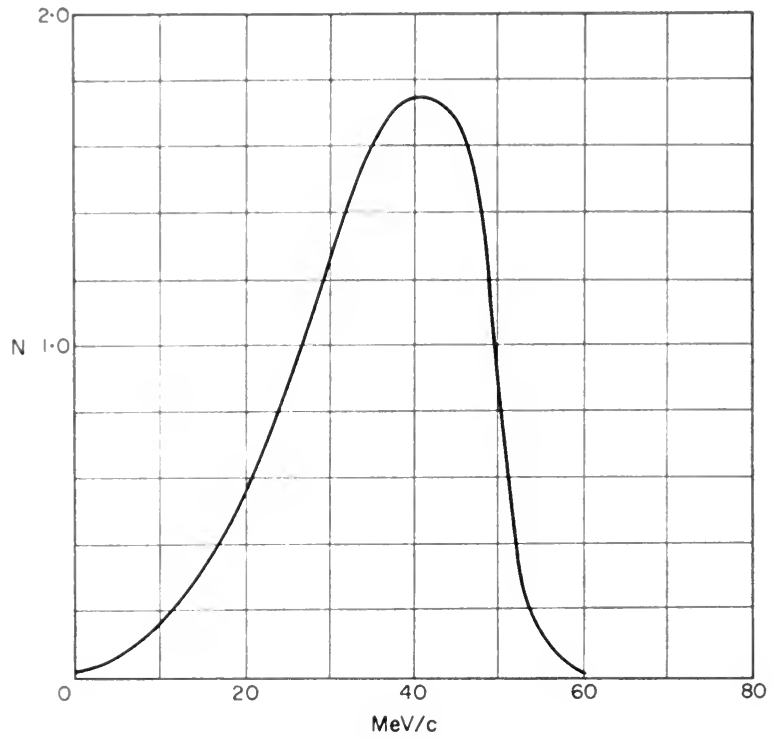

Fig. 60. Spectrum of electrons from the decay of negative $\mu$-mesons.

decay either in flight or at rest according to the scheme $\mu^{ \pm} \rightarrow e^{ \pm}+v+\bar{v}$. How ever the slow negative $\mu$-mesons can be captured to form mesic atoms. Such bound mesons can either decay spontaneously or be captured by the protons in the nucleus according to the scheme $p+\mu^{-} \rightarrow n+\nu$. Such nuclear capture is also called forced decay. The probability of capture is proportional to the square of the amplitude of the $\mu$-meson wave function at the nucleus and the total number of protons. If we assume that the mesic atom is hydrogen-like, the probability $P$ is given by

$$
P \sim Z\left(Z^{3 / 2}\right)^{2} \sim Z^{4} .
$$

1 ". The interaction of $\mu$-mesons with matter", by G. N. Fowler and A. W. WolfEndale, Progress in Cosmic Ray Physics and Elementary Particles, Vol.4, p. 107 (1958). This article also contains extensive references. 
A rough estimate gives the lifetime of the decay to be

$$
\frac{1}{\tau_{\text {capture }}}=\frac{5000(210)^{3}}{\pi} \mathrm{g}^{2} \frac{\mathrm{m}^{3} Z^{4}}{a^{3}} .
$$

The apparent lifetime is given by

$$
\frac{1}{\tau}=\frac{1}{\tau_{\text {decay }}}+\frac{1}{\tau_{\text {eapture }}}
$$

For $Z=11$ the decay and capture lifetimes are equal. The variation of $\mu$-meson with depth is determined solely by the spectrum of the parent mesons, their decay processes and by their electromagnetic interaction with nuclei.

If we consider the $\mu$-meson as a heavy electron with no other distinct characteristics, we have only to replace the mass of the electron $m_{e}$ by that of the meson $m_{\mu}$ in the cross-sections for electrodynamics processes. However this is not true though it is well realized that $\mu$-meson interaction with the nuclei is essentially electromagnetic. Following Fowler et $a l .{ }^{1}$ we shall roughly classify these interactions as classical, i.e. known and non-classical.

\section{Classical electromagnetic interaction : ionization}

The Bethe formula for the loss of energy of a heavy particle of velocity $v$ through ionization is

$$
-\left.\frac{\partial E}{\partial \varkappa}\right|_{\text {ion }}=\frac{4 \pi Z^{2} e^{4} \eta}{v^{3} m_{\mu}}\left(\log \frac{2 v^{2} m_{\mu}}{\bar{I}\left(1-\beta^{2}\right)}-\beta^{2}\right) \mathrm{erg} / \mathrm{cm}
$$

$\eta$ being the number of electrons per $\mathrm{cm}^{2}$ and $I$ the average ionization potential (in ergs) of these electrons in the absorber and $\beta=v / c$. This formula differs from that for ionization due to electrons in the following respects. We have assumed $m_{\mu}$ to be much higher than the electronic mass so that the meson traverses the matter practically undeflected, i.e. the transverse component of the momentum acquired during collision is negligible. The two particles being different, we need not consider exchange effects. Experimentally observed values agree exactly with those given by theory.

\section{Collisions involving free electrons}

The electrons are classed as $\delta$ rays $(10-100 \mathrm{keV})$ or knock-on electrons $(\mathrm{MeV})$ depending on their energy. These processes amount to electron-electron interaction (with a $\mu$ replacing an electron) involving transfer of energy and momentum.

The mechanism of production of $\delta$ rays is as follows. A slow heavy particle ionizes an atom. An electron may be ejected with sufficient energy to cause secondary ionizations and be observable as $\delta$ rays. We then have rays travelling

\footnotetext{
${ }^{1}$ Fowler et al., ibid.
} 
away from the path of the incident non-relativistic particle. If the incident heary particle is relativistic, these rays have a momentum component along its path. The cross-section for $\delta$ rays of energy between $\omega$ and $\omega+\mathrm{d} \omega$ is

$$
N(\omega) \mathrm{d} \omega=\frac{2 \pi N Z r_{0}^{2}}{A p^{2}} m c^{2} \frac{\mathrm{d} w}{w^{2}} \mathrm{~d} x
$$

where $\beta$ is the $\mu$-meson velocity and $r_{0}$ the classical electron radius. Numerical calculations using this formula give approximately $150 \delta$ rays above $20 \mathrm{keV}$ in argon at $52 \mathrm{~g} / \mathrm{cm}^{2}$ density which agrees fairly well with experimental value.

When the energies of the electrons are higher, the spin of the $\mu$-meson should be taken into account while computing the eross-section. Assuming spin $1 / 2$, it amounts to considering the electromagnetic interaction between particles of different masses. We have

where

$$
\sigma\left(E_{\mu} ; E\right) \mathrm{d} E=2 \pi r_{0}^{2} / \beta^{2}\left\{\left(1-\beta^{2} \frac{E}{E_{\max }}\right)+\left(\frac{E}{E_{\mu}}\right)^{2}\right\} \frac{\mathrm{d} E}{E^{2}}
$$

$$
E_{\mathrm{max}}=E_{\mu}^{2} /\left(E_{\mu}+m_{\mu}^{2} c^{2} / 2 m\right)
$$

the maximum energy transferable by a $\mu$-meson of energy $E_{\mu}$. The total observed cross-section of $20 \times 10^{-28} \mathrm{~cm}^{2} /$ electron agrees with the theoretical value of $18.4 \times 10^{-28} \mathrm{~cm}^{2} /$ electron. However, there was an appreciable excess of high energy electrons over that predicted by theory. Attempts were made to explain this by associating an anomalous magnetic moment $\delta$ with the $\mu$. The crosssection in this case reads

$$
\sigma\left(E_{\mu}, \varepsilon\right) \mathrm{d} \varepsilon=\delta^{2} \pi\left(\frac{e^{2}}{m_{\mu}} c^{2}\right)^{2}(1-\varepsilon) \frac{\mathrm{d} \varepsilon}{\varepsilon}
$$

where $\varepsilon=\frac{E}{E_{\mu}}$ while the cross-section for a normal Dirac particle is

$$
\sigma\left(E_{\mu}, \varepsilon\right) \mathrm{d} \varepsilon=2 \pi\left(\frac{e^{2}}{m_{\mu} c^{2}}\right)^{2} \frac{m_{\mu}}{m} \cdot \frac{m_{\mu} c^{2}}{E_{\mu}} \cdot \frac{\mathrm{d} \varepsilon}{\varepsilon^{2}}\left(1-\varepsilon+\frac{\varepsilon^{2}}{2}\right) .
$$

But general experimental evidence seems to rule out a large anomalous magnetic moment. There may be corrections to the cross-section due to emission of real photons, i.e. by Bremsstrahlung.

\section{Photoproduction}

The observed cross-section for photoproduction of $\mu$-meson pairs is much higher than that given by the Bethe-Heitler formula. ${ }^{1}$ To explain the above cross-section it was proposed that the $\mu$ pairs were not all due to photoproduction but some were due to direct production by $\pi^{0}$-mesons. Such a hypothesis follows from the model proposed by Wentzel $^{2}$, in which all pions ean be considered

1 See p. 258, Quantum Theory of Radiation, W. Heitler (Oxford Press) (1954).

2 G. Wextzel, Phys. Rev. 79, 710 (1950). It is to be noticed that according to the recent ideas on weak interactions $\boldsymbol{x}^{0}$ cannot undergo decay into $\mu$ pair as it will involve violation of angular momentum in $\pi^{0}$ rest system. 
composite systems of $\mu$ pairs. However this gave $\mu$ pair production to be nearly 6 per cent of $\pi$ production whereas it should be less than 0.1 per cent for agreement with experiment. Probability of production of $\mu$ pairs by annihilation of electronpositron pairs was considered. This turned out to be $1 / 70$ th the probability of two photon annihilation of a $40 \mathrm{keV}$ positron and an electron at rest.

Anomalous (i.e. not Rutherford) scattering of $\mu$-mesons occurs due to the finite size the nucleus. At large angles there is deviation from Coulomb-scattering by an uniform nucleus. Anomalous scattering corresponds to multiple Moliere-scattering within the nucleus.

As we have already mentioned by non-classical is meant the electromagnetic interactions whose cross-section cannot be directly obtained, like the production of stars and showers. The energy of the $\mu$-meson may be just sufficient to eject a single proton from a nucleus or produce an "evaporation" (cold) star or produce mesons and characteristic showers. All these processes are due to the interaction of the electromagnetic field of the electron with the $\mu$-meson and can be explained on the basis of a "virtual" photon flux.

We shall now give a brief outline of this process. Consider a high energy $\mu$-meson $(v \cong c)$ approaching a heavy stationary nucleus of charge $Z e$. In the rest system of the $\mu$-meson the nucleus moves with a velocity approaching $c$. Hence the electric field $\boldsymbol{E}$ of the nucleus is contracted and the perpendicular magnetic field $\boldsymbol{H}$ is about the same magnitude. Thus the nucleus looks like a plane electromagnetic wave (corresponding to photons of various frequencies) to the $\mu$-meson. These photons undergo Compton-scattering by the $\mu$-meson. Referring back to the laboratory system (the rest-system of the nucleus) these scattered photons appear to have been emitted by the meson. So in all the calculations the $\mu$-meson is effectively replaced by a "virtual" photon flux. By "virtual" we mean an "equivalent" photon beam which has been obtained simply by a Lorentz transformation.

The equivalent photon spectrum is given by

$$
N_{k f} \mathrm{~d} k_{f}=\frac{\alpha}{\pi}\left\{z^{2}\left[K_{0}^{2}(z)-K_{1}^{2}(z)\right]+2 z K_{0}(z) K_{1}(z)\right\} \frac{\mathrm{d} k_{f}}{t}
$$

where $K_{0}, K_{1}$ are the usual Bessel functions; $z=m_{\mu} b_{\min } \frac{k_{f}}{E}, b_{\min }$ being the least impact parameter in this process. For very high energies $E \gg m_{\mu}, b_{\min }=R$ the radius of the region over which electromagnetic interaction is strong. We then have

$$
N_{k} \mathrm{~d} k_{f} \cong \frac{2 \alpha}{K_{f}}\left\{\log \frac{E}{m_{\mu} R k_{f}}-0.38\right\} \mathrm{d} k_{f}
$$

when the perturbed system is a single nucleon $R$ is presumably the Compton wavelength and

$$
N k_{f} \mathrm{~d} k_{f} \cong \frac{2 \alpha}{\pi k_{f}}\left\{\log \frac{E}{k_{f}}-0.38\right\} \mathrm{d} k_{f} .
$$


Thus eross-section for proton production is $2.4 \times 10^{-30} \mathrm{~cm}^{2}$ per nucleon while that for star production is

$$
\sigma_{\mu}=\frac{0.9 \alpha}{\pi} \sigma k_{f}\left[\log \frac{E_{0}}{E}\right]^{2}
$$

where $\varepsilon-150 \mathrm{MeV}$, the minimum photon energy required to produce a star with three prongs.

$E_{0}=$ energy loss up to the depth in question. Prong distribution has also been given.

Highly energetic $\mu$-mesons produce pions and showers. There is also a possibility of $K$-mesons being produced in $\mu$-meson interaction.

\section{THEORIFS OF MUL'TIPLEPRODUCTION}

Experimental evidence from the study of (i) jet showers and large star events in nuelear emulsions and (ii) extensive air showers and in particular the penetrating component in cloud chamber experiments suggested the possibility of the production of more than one particle in a single collision between the nuclei at extremely high energies. Two types of attempts have been made to explain this phenomenon.

It is assumed that only one meson is produced in a single nucleon-nucleon collision but a nucleon when passing through a nucleus produces an internuclear cascade by successive eollisions with the nucleons in the nucleus. For simplicity the collisions inside the nucleus are considered independent and the standard methods of cascade theory are used, the total thickness of "matter" traversed being the diameter of the nucleus. Unlike the case of a cascade in finite thicknesses of matter in the gross, it is not possible to analyse the development of the internuclear cascade at various "thicknesses" but we can only make calculations on the basis that the nucleon has passed through the entire nucleus. In other words the cascade hypothesis is just used to compute ${ }^{2}$ the cross-sections for the production of particles in the various energy ranges in a single encounter between a nucleon and a nucleus.

Alternatively it is assumed that even in a nucleon-nucleon collision multiple production is possible. The same considerations apply to a collision with a nucleus. Since the nucleus is closely packed with nucleons the concept of a cascade due to suceessive collisions loses meaning and the collision has to be considered as a single process.

Recent experiments on collisions by artificially accelerated nucleons clearly show that many mesons can be produced even in a single encounter. Perhaps

1 The theories of Multiple Particle Production by Ziro Koba and Shuji Takagi, Fortschr. der Physik 7, 1(1959). This article also contains quite an extensive bibliography. See also Elementary Particles by Enrico Fermi, Yale University Press. We wish to thank Professor Z. Koba for his lectures while he visited Madras on "Theories of Multiple Production" which elarified many difficulties.

2 For a detailed discussion see chapter XIII. 
very high multiplicities in collisions with nuclei may necessitate a cascade hypothesis with the nucleus though the independence of successive collisions may not be valid.

Several theories based on widely varying assumptions have been proposed by:

(i) Heisenberg, in analogy with multiple Bremsstrahlung,

(ii) Lewis, Oppenheimer and Wouthuysen by a generalization of the BlochNordsieck method in electrodynamics,

(iii) Fermi, on purely statistical and thermodynamic considerations,

(iv) Peaslee and Takagi, on the fire ball and isobar models,

(v) Landau and Pomeranchuk, by an extension of Fermi's theory,

(vi) Sudarshan and Srivastava, by a covariant formulation of the statistical and theory,

(vii) Bhabha.

At present no reliable calculations based on a field theoretic approach is possible because of the strong interactions involved in the production of mesons. We therefore confine ourselves mainly to the theories of Fermi and of Landau and Pomeranchuk, giving only a brief description of some of the other theories.

\section{Heisenberg's theory of multiple production}

The possibility of multiple meson production in a collision of relativistic nucleons was suggested by Heisenberg ${ }^{1}$ soon after Yukawa's formulation of the meson theory of nuclear forces. He extended the concept of Bremsstrahlung in electromagnetic theory to the meson theory as follows. The sudden deflection of an electrically charged particle leads at the moment of deflection to a discrepancy between the electromagnetic fields surrounding the particle before and after the deflection. The difference between the electromagnetic fields gets detached and wanders away as radiation (Bremsstrahlung). The mean number of light quanta $\mathrm{d} n_{\gamma}$ in the frequency interval $\mathrm{d} \omega$ is given by

$$
\mathrm{d} n_{\gamma}=e^{2} \frac{\mathrm{d} \omega}{\omega} .
$$

This leads to the infra-red catastrophe, i.e. the emission of an infinite number of very low energy quanta. In the case of the sudden deflection of a nucleon the corresponding meson spectrum (assuming it to be of the Bremsstrahlung form) is given by

$$
\mathrm{d} n_{\pi} \simeq f^{2} \frac{\mathrm{d} \varepsilon}{\varepsilon}
$$

where $f^{2}$ is the coupling constant between the meson and nucleon and $\varepsilon$ the energy of the meson including its rest mass. While the infra-red catastrophe is avoided by the finite mass of the meson the large value of $\varepsilon$ leads to multiplicities greater than unity even for moderately high energies. Also unlike the electromagnetic

.1 W. Heisenberg, Z. Phys. 101, 533 (1936). See also R. E. Marstak, Meson Physics, McGran Hill, New York (1952).

EPCR 26 
ease the strength of the meson-nucleon interaction increases with energy. This has been explained by Heisenberg as being due to non-linear effects which become significant at high energies causing a turbulent meson mixing of the nucleon wave packet.1 In the course of this mixing the meson spectrum of the nucleon wave packet undergoes a displacement towards low-frequency leading to the creation of many low energy mesons. The qualitative consequences of this model are:

(i) A penetrating shower should contain more low energy than high energy mesons.

(ii) The mean number of mesons produced should increase with the available nucleonic energy.

(iii) The form of the meson spectrum should have very slight dependence on the primary energy.

(iv) The angular distribution of mesons should be isotropic in the centre of mass system in analogy with the isotropic distribution of small turbulences which are discarded by a turbulent fluid.

The production spectrum is given by

$$
n_{\pi}(\bar{\varepsilon}, \bar{\theta}) \mathrm{d} \bar{\varepsilon} \mathrm{d} \bar{\Omega}=k \frac{d \bar{\varepsilon}}{\bar{\varepsilon}^{m}} \mathrm{~d} \bar{\Omega}
$$

where $\bar{\theta}$ is the angle of emission of the meson in the centre of mass system and $k$ and $m$ are constants.

\section{The L.O.W. Method}

The second major attempt to develop a theory of multiple production was that of Lewis, Oppenheimer and Wouthuysen ${ }^{2}$ who adopted the Bloch-Nordsieck method with suitable modifications. The assumptions underlying the BlochNordsieck method ${ }^{3}$ which are found to hold for the electromagnetic case are: (a) the collision time is small compared to the period of the emitted radiation, (b) the recoil effect due to the emitted radiation on the source can be neglected, and (c) only those components of the field which satisfy the assumptions (a) and (b) need be considered. In this method the emitted radiation spectrum is calculated as the difference between the quasi-static fields before and after collision. The total cross-section is unchanged even though collisions are now accompanied by the emission of radiation.

At first sight it appears that the Bloch-Nordsieck method will be invalid due to the increasing strength of the meson-nucleon interaction with energy. However,

${ }^{1}$ W. Heisenberg, Z. Phys. 113, 61 (1939); 126, 569 (1949); 133, 65 (1952). See also G. Wataghin, Acad. Bras. Ciene, 129 (1943).

${ }^{2}$ H. W. Lewis, J. R. Oppenheimer and S. A. Wouthuysen, Phys. Rev. 73, 127 (1948). See also H. Lewis, Proc. 7 th Rochester Conference, IX, p. 1 (1957).

3 F. Bloch and A. Nordsieck, Phys. Rev. 52, 54 (1937). 
strong interactions at high energies do not necessarily imply a completely inelastic collision. If there is only a small energy transfer to the mesons and no large momentum changes of the nucleons the assumptions (a), (b) and (c) can still hold. The changes in the nucleonic charges and spins produce a discrepancy between the initial and final meson fields present around the nucleons which can be treated by the Bloch-Nordsieck method. Neglecting the final state interaction between the nucleons and mesons, the probability $P_{N}$ for emission of $N$ mesons in a nucleon-nucleon collision is

$$
P_{N} \propto J^{N}\left(\frac{f^{2}}{4 \pi}\right)^{N} \frac{\left(E^{2}-p^{2}\right)^{N-1}}{N ![(N-1) !]^{2}} .
$$

The factor $J$ appears because the spin and the isotopic spin of the nucleons have been treated as classical variables. (This is a good approximation if several mesons are emitted such that the spin and isotopic spin average out.) $J$ is of the order of magnitude unity and $E$ and $p$ are the total energy and momentum of the mesons in the centre of mass system. If the mesons are emitted isotropically $p$ is zero. The factor $\left(E^{2}-p^{2}\right)^{N-1}$ appears mainly from a phase-space calculation taking into account the pseudoscalar nature of the meson and a pseudovector coupling for the meson-nucleon interaction. Assuming the value of 0.08 for the renormalized unrationalized pseudovector coupling constant the ratios for the production of 1, 2 and 3 mesons for a centre of mass energy of the two-incident nucleons slightly over $1 \mathrm{BeV}$ are $1.2: 1: 0.45$. This compares well with the experimental value of $0.8: 1: 0.3$ though the theory is not designed for such low multiplicities. At higher energies the agreement with experiment is much nearer and the angular distribution of the mesons is also well accounted for.

\section{Fermi's statistical theory of multiple meson production ${ }^{1}$}

The basic assumptions of Fermi's theory are: (i) The two incident particles coalesce and the energy brought in by them is released within a common region of interaction. The volume $\Omega$ of this region is assumed to be nearly the volume occupied by the meson clouds surrounding the nucleon which for a stationary nucleon is given by

$$
\Omega_{0}=\frac{4 \pi R^{3}}{3} \text {. }
$$

Here $R=\xi / \mu$ where $\xi$ is a coefficient of the order of unity and $\mu$ the meson mass. As the nucleons are in motion their pion clouds in the centre of mass system undergo a Lorentz contraction given by

$$
\Omega=\Omega_{0} \frac{2 M}{W}
$$

where $W$ is the total energy in the centre of mass system and $M$ is the nucleon mass.

1 E. Fermi, Progr. Theoret. Phys. 5, 570 (1950); Phys. Rev. 81, 683 (1951); Phys. Rev. 92, 452 (1953); 93, 1434 (1954). 
(ii) After a time this region of interaction disintegrates into one or the other of the possible final states of the system.

(iii) 'The interactions involved are sufficiently strong and the interaction region survives for a sufficiently long period for all of the possible final states to have become equally excited, i.e. a statistical equilibrium is attained. Hence the probability for a given outcome is proportional to the number of states comprising the outcome.

(iv) The ensemble of the possible final states is to be selected on the basis of conservation laws which are expected to hold during the interaction. Besides the conservation of energy, momentum and charge, the isotopic spin, the baryon number and other similar variables should be subject to appropriate constraints.

The transition rate from an initial state $|i\rangle$ to a final state $|f\rangle$ is given by

$$
\omega=2 \pi|\langle f|H| i\rangle|^{2} \varrho(W)
$$

where $\langle f|H| i\rangle$ is the matrix element of the Hamiltonian connecting the two states and $\varrho(W)$ the density of final states of the system for a total energy $W$. The final states are specified by the numbers and charges of the nucleons and mesons and perhaps by other particles which emerge from the interaction region. To take into account the assumption of equilibrium among the various final states, we consider that the interaction $H$ projects the state $|i\rangle$ uniformly over the space of all the allowed states representing virtual particles confined to the volume $\Omega$. The final state consists of products of free particle wave functions since all the particles are free in the final state. If we normalize these free particle wave functions in a large volume $V$, the $N$ particle final state will contain a factor $(V)^{-1 / 2 N}$ but each of these $N$ particle virtual states is contained within the interaction volume $\Omega$ and hence will have a normalizing factor $(\Omega)^{1 / 2} N$. Thus the matrix element is

$$
\langle f|H| i\rangle=\mathrm{constant}\left(\frac{\Omega}{V}\right)^{1 / 2 N} .
$$

The constant, by the assumption of statistical equilibrium is independent of the final state and can be ignored in the calculations, as we are interested only in the relative abundances of the various final states for a given interaction. If $n$ of the $N$ particles are identical, their wave functions should be completely symmetric (or antisymmetric) in the exchange of the coordinates of the identical particles. In this case the matrix element is given by

$$
\langle f|H| i\rangle=\mathrm{constant}\left(\frac{\Omega}{V}\right)^{\frac{1}{2} N}\left(\frac{1}{n !}\right)^{1 / 2} .
$$

The concept of indistinguishability must be carefully applied in ascribing the statistical weights. For instance, if we treat the positive and negative mesons as different particles, the possible degeneracies should be allowed for by dividing the matrix element by $\left[n^{+} ! n^{-} !\right], n^{+}$and $n^{-}$being the number of positive and negative mesons respectively in the final state. However, if we consider the positive, negative and the neutral meson as the three states of a single particle characterized 
by the total isotopic spin 1 , the matrix element should be divided by $(n !)^{1 / 2}$, $n$ being the total number of mesons present in the final state.

The density of final states $\varrho(W)$ per unit energy for $N$ particles of total energy $W$ will be ${ }^{1}$

$$
\frac{\mathrm{d}}{\mathrm{d} W}\left\{(2 \pi)^{-3 N} \int_{V} \int_{W} \prod_{i=1}^{N} \mathrm{~d}^{3} x_{i} \mathrm{~d}^{3} p_{i}\right\} .
$$

Thus the relative probability that $n$ pions and $s$ nucleons will emerge from a high energy interaction is given by

$$
S(n, s)=\left(\frac{\Omega}{V}\right)^{N} \sum \frac{\mathrm{d}}{\mathrm{d} W}(2 \pi)^{-3 N} \int_{V} \int_{W} \prod_{i=1}^{N} \mathrm{~d}^{3} x_{i} \mathrm{~d}^{3} p_{i}
$$

where $N=n+s$ and the pions are assumed to be distinguishable. The integrations should conform to the constraints imposed upon the system. The conservation of linear momentum reduces the independent variables from $N$ to $N-1$ giving the relative probability $S(n, s)$ as

$$
S(n, s)=\left(\frac{\Omega}{V}\right)^{N-1} \sum \frac{\mathrm{d}}{\mathrm{d} W}(2 \pi)^{-3(N-1)} \int_{V} \int_{W} \prod_{i=1}^{N-1} \mathrm{~d}^{3} x_{i} \mathrm{~d}^{3} p_{i} .
$$

The summation sign indicates that a suitable sum must be taken over the various possible charge and angular momentum states. Neglecting the conservation of angular momentum and integrating over the space variables, we have

$$
S(n, s)=(\Omega)^{N-1}(2 \pi)^{-3(N-1)} \sum \frac{\mathrm{d}}{\mathrm{d} W} V_{W}(n, s)
$$

where $V_{W}$ is the volume of the $3(N-1)$ dimensional momentum space corresponding to the $N-1$ independent particles which is calculated such that the total energy of all the $N$ particle is $\leqq W$.

We have to calculate the quantity

$$
\frac{\mathrm{d}}{\mathrm{d} W} V_{W}=\frac{\mathrm{d}}{\mathrm{d} W}\left\{\prod_{i=1}^{N} \int \mathrm{d}^{3} p_{i}\right\} \delta\left(\sum_{i=1}^{N} p_{i}\right) U\left(W-\sum_{i=1}^{N}\left(p_{i}^{2}+M_{i}^{2}\right)^{1 / 2}\right)
$$

where the integrals are taken over the momentum space of all $N$ particles with masses $M_{i}$. The delta function ensures momentum conservation and $U(x)$ is the step function

$$
\begin{aligned}
& U(x)=1 \quad \text { for } \quad x>0 \\
& =0 \quad \text { for } \quad x<0 .
\end{aligned}
$$

Expressing the delta and step functions in terms of their Fourier representations

$$
\delta(x)=(2 \pi)^{-3} \int_{-\infty}^{\infty} \mathrm{d}^{3} \lambda \mathrm{e}^{i \lambda \cdot x}
$$

1 R. Milburn, Rev. Mod. Phys. 27, 1 (1955). 
and

$$
U(x)=(2 \pi i)^{-1} \int_{-\infty-i \varepsilon}^{\infty+i \varepsilon} \mathrm{d} \alpha^{N} \alpha \mathrm{e}^{i \alpha \cdot W} \quad(\varepsilon>0)
$$

and performing the integration, Lepore and Stuart ${ }^{1}$ deduced an expression for the above quantity in terms of the Hankel functions,

where

$$
\begin{aligned}
\frac{\mathrm{d} V W}{\mathrm{~d} W}= & \frac{2}{(2 \pi)^{3}}\left(2 \pi^{2}\right)^{N} \cdot \prod_{i=1}^{N} M_{i}^{2} \int_{-\infty-i \varepsilon}^{+\infty+i \varepsilon} \mathrm{d} \alpha \alpha^{N} \mathrm{e}^{i \alpha \cdot W} \\
& \int_{0}^{\infty} \frac{\mathrm{d} \lambda \lambda^{2}}{\left(\alpha^{2}-\lambda^{2}\right)^{N}} \prod_{j=1}^{N}\left\{H_{2}^{(2)}\left[M_{j}\left(\alpha^{2}-\lambda^{2}\right)^{1 / 2}\right]\right\}
\end{aligned}
$$

and $H_{1}^{(2)}(z)$ is given by the following relation

$$
\int_{-\infty}^{\infty} \mathrm{d} \theta^{\prime} \cosh \theta^{\prime} \exp \left[-i z\left(\cosh \theta^{\prime}\right)\right]=-\pi H_{1}^{(2)}(z) .
$$

The expression (17) can be integrated over $\lambda$ and $\alpha$ for the case of zero mass particles, i.e. for the case in which all the particles are treated as being extremely relativistic. We obtain in this case, on expanding the Hankel function about the origin and keeping only the first term

$$
\left(\frac{\mathrm{d} V_{W}}{\mathrm{~d} W}\right)_{E \cdot R}=\frac{\pi^{N-1}}{2^{N-1}} \frac{(4 N-4) !(2 N-1)}{[(2 N-1) !]^{2}(3 N-4) !} W^{3 N-4} .
$$

It is interesting to compare this result with that obtained by Fermi $^{2}$ who neglected momentum conservation and calculated the result assuming the condition that the upper bound of the total energy is fixed, i.e.

He obtained

$$
\sum_{i=1}^{N} p_{i} \leqq W
$$

$$
\left(V_{W}\right)_{\text {Fermi }}^{E \cdot R}=\frac{2^{3 N} \pi^{N} W^{3 N}}{(3 N) !} .
$$

On imposing the condition of momentum conservation by reducing the number of particles by unity and taking the energy derivative, we have

$$
\left(\frac{\mathrm{d} V_{W}}{\mathrm{~d} W}\right)_{\mathrm{Fermi}}^{E \cdot R}=\frac{\pi^{N-1}}{(3 N-4) !} 2^{3(N-1)} W^{3 N-4}
$$

which has the same energy dependence as that obtained above, but a different coefficient.

${ }^{1}$ J. V. Lepore and R. N. Stuart, UCRL 2386 Nov. 17 (1953). Also Phys. Rev. 94, 1724 (1954).

2 E. Ferui, loc. cit. 
An exact calculation can also be made for the case of non-relativistic particles of mass $M$ whose kinetic energy is given by $p^{2} / 2 M$. A calculation similar to the above yields

$$
\left(\frac{\mathrm{d} V_{W}}{\mathrm{~d} W}\right)^{N \cdot R}=\frac{2^{\frac{3(8-1)}{2}} \pi^{3(8-1) / 2} M^{3 s / 2}(W-s M)^{\left(\frac{3 s}{2}-5 / 2\right)}}{(s M)^{3 / 2} \Gamma[3(s-1) / 2]} .
$$

If the particles involved have different masses, we make the replacement

$$
s M \rightarrow \sum_{i=1}^{8} M_{i} \quad \text { and } \quad M^{(38 / 2)} \rightarrow \prod_{i=1}^{8}\left(M_{i}\right)^{3 / 2} .
$$

It is assumed that the momentum conservation holds only for the nucleons (treated as non-relativistic particles). The produced mesons are treated as extremely relativistic (their masses are accounted for by taking $(p=\mu)$ for the total energy of the pion) and they can assume any energy consistent with the overall energy conservation. In this case, the expression for $\frac{\mathrm{d} V_{W}}{\mathrm{~d} W}$ is the product of equations (22) and (23), i.e. we have

$$
\left(\frac{\mathrm{d} V_{W}}{\mathrm{~d} W}\right)_{\text {approx. }}=\left[\frac{H^{38 / 2}(2 \pi)^{\frac{3(8-1)}{2}}}{(s M)^{3 / 2}}\right]\left[2^{3 n} \pi^{n}\right] \frac{[W-s M-n \mu]^{(38 / 2)+3 n-5 / 2}}{\Gamma\left[\frac{3(s-1)}{2}+3 n\right]} .
$$

Energy conservation among the increased number of particles has been taken into account through the adoption of a common energy factor and through a suitable change in the argument of the delta function.

Another method is based on a step-by-step calculation which is done as follows: Consider the case of extreme relativistic particles. We may denote the total phase space volume for $n$ such particles by $V(n)$. Obviously $V(1) \equiv 0$ as only one particle is involved. The momentum volume available to a particle of momentum $\boldsymbol{p}$ which together with another particle of momentum $\boldsymbol{p}^{\prime}$ forms a system having a fixed total momentum $\boldsymbol{P}=\boldsymbol{p}+\boldsymbol{p}^{\prime}$ is given by

$$
V_{W, P}=2 \pi \int_{0}^{p(w)} \mathrm{d} p p^{2} \int_{w+w^{\prime} \leqq W} \mathrm{~d}(\cos \theta) .
$$

Here $w$ and $w^{\prime}$ are the energies of the particles and $p^{2}=p^{2}+P^{2}-2 p P \cos \theta$. Hence for fixed $W$ and $P$ the boundary of the region of integration will be specified by a definite equation relating $p$ and $\cos \theta$. Thus, for $V(2)$ we observe that the integration limits are determined by

or

$$
p^{2}+P^{2}-2 p P \cos \theta=p^{\prime 2}=w^{\prime 2}=(W-p)^{2}
$$

which gives

$$
p(\cos \theta)=\frac{W^{2}-P^{2}}{2(W-P \cos \theta)}
$$

$$
V(2)=2 \pi \int_{-1}^{1} \mathrm{~d}(\cos \theta) \frac{1}{3}[p(\cos \theta)]^{3}=\frac{\pi}{6} W\left(W^{2}-P^{2}\right)
$$


or

$$
\frac{\mathrm{d} V(2)}{\mathrm{d} w}=\frac{\pi}{2} W^{2}
$$

which is the same result as given by the general formula.

It is possible to adopt the above treatment to the case of pion-nucleon eollisions resulting in the emission of $n$ pions (considered as massless particles) and a single nucleon of mass $M$ treated relativistically. $V(n)$ is ealeulated as for the $n$ massless particles detailed above and the particle of mass $M$ is added along with the requirement that the total momentum $\boldsymbol{P}$ equals zero. The momentum limit is determined from the equation

or

$$
p^{2}=\left\{W-\left(M^{2}+p^{2}\right)^{1 / 2}\right\}^{2}
$$

$$
p=\frac{W^{2}-M^{2}}{2 w} \text {. }
$$

Then for $n=1$, we have for the momentum volume

$$
V(1 M, 10)=2 \pi \int_{-1}^{1} \mathrm{~d}(\cos \theta) \int_{0}^{p(\cos \theta)} p^{2} \mathrm{~d} p=\frac{\pi}{6}\left[\frac{W^{2}-M^{2}}{W}\right]^{3} .
$$

Since the formulae for other cases are complicated we quote only the energy derivatives for values of $n=1$ and 2

$$
\begin{aligned}
& \frac{\mathrm{d} V}{\mathrm{~d} w}(1 M, 10)=\frac{\pi}{2} W^{2}\left(1-v^{2}\right)\left(1-v^{4}\right) \\
& \frac{\mathrm{d} V}{\mathrm{~d} w}(1 M, 20)=\frac{\pi^{2}}{N} \frac{W^{5}}{2 \cdot 5 !}\left\{\left(1-v^{2}\right)\left[7-43 v^{2}-3 v^{6}+2 v^{8}+128 v^{4} \log \frac{1}{v}\right] \text { etc. }\right\}
\end{aligned}
$$

where $v=\frac{M}{W}$ and represents the relative effect of the particle of mass $M$ over what it would have had as a mass zero particle.

These and other similar formulae for higher values of $n$ permit one to calculate the relative probability for the production of various numbers of mesons in pionnucleon collisions. Taking the centre of mass energy as $1.92 \mathrm{BeV}$ and the nucleon mass as $0.938 \mathrm{BeV}, v=0.487$. Omitting also consideration of the charge states and the identity of particles, the relative probabilities $S(n, 1)$ for the emission of $n$ pions and one nucleon was obtained by Milburn as

$$
\begin{aligned}
& S(1,1)=\frac{\Omega}{8 \pi^{3}} \frac{\pi W^{2}}{2}(0.72) \\
& S(2,1)=\left(\frac{\Omega}{8 \pi^{3}}\right)^{2} \frac{\pi^{2} W^{5}}{2 \cdot 5 !}(1.547) \\
& S(3,1)=\left(\frac{\Omega}{8 \pi^{3}}\right)^{3} \frac{3 \pi^{3} W^{8}}{2 \cdot 8 !}(0.485) .
\end{aligned}
$$


The total energy $W$ in the above equation is expressed in momentum units and the factor in $W$ becomes $\frac{W}{2 \pi}=\frac{W}{M} \frac{M}{2 \pi}=\frac{1}{v \lambda_{\mu}}$ where $\lambda_{\mu}$ equals $\frac{2 \pi}{M}=1.2 \times 10^{-13} \mathrm{~cm}$, the extent of the meson cloud around a nucleon. Taking the nuclear volume $\Omega_{0}$ to be $\frac{4 \pi}{3}\left(\lambda_{\mu}\right)^{3}$ the results in terms of the ratio of the interaction volume $\Omega$ to the nucleon volume $\Omega_{0}$ are obtained as

$$
\begin{aligned}
\frac{S(2,1)}{S(1,1)} & =2.04\left(\frac{\Omega}{\Omega_{0}}\right) \\
\frac{S(3,1)}{S(2,1)} & =0.318\left(\frac{\Omega}{\Omega_{0}}\right) \\
\frac{S(3,1) S(1,1)}{[S(2,1)]^{2}} & =0.156 .
\end{aligned}
$$

It is clear that the interaction volume plays an important role in the determination of meson multiplicity.

For the case of several particles of non-vanishing mass, a similar calculation gives the energy derivative for one nucleon and one pion (with mass) as

$$
\frac{\mathrm{d} V_{W}}{\mathrm{~d} W}(1 M, 1 m)=\frac{\pi W^{2}}{2}(0.714)
$$

taking the pion mass as $0.136 \mathrm{BeV}$ and the nucleon mass as $0.938 \mathrm{BeV}$ for a total energy $1.92 \mathrm{BeV}$ in the centre of mass system. The inclusion of the pion mass has thus reduced the one pion weight as compared to the case of a massless pion, equation (35), by a factor of 0.99 . Hence the ratio of the relative probabilities becomes

$$
S(2,1) / S(1,1)=1.54\left(\frac{\Omega}{\Omega_{0}}\right) .
$$

It was suggested by Fermi that besides charge conservation the conservation of isotopic spin can be imposed upon the interacting pion-nucleon system. The number of pions and nucleons are combined to form a joint system and the total isotopic spin vector is formed in the same way as the total angular momentum of the particles. The resultant isotopic spin is the vector sum of the isotopic spins of the component particles, i.e. $\boldsymbol{T}=\sum_{i} \boldsymbol{T}_{i}$. Conservation of charge and isotopic spin means that $T$ and $T_{z}$ are good quantum numbers during the interaction. We shall hereafter denote a state with total isotopic spin $T$ and charge component $M\left(=T_{z}\right)$ by $(T, M)$. Let us consider the possibility of producing one more pion in a pionnucleon collision. With two pions and one nucleon, the state $(3 / 2,-3 / 2)$ for example can be formed in two ways when the total isotopic spin $T_{2 \pi}$ of the two pions is 2 and 1. In the spirit of Fermi's statistical theory we assume that these two possibilities are equally excited and this degeneracy provides the $(3 / 2,-3 / 2)$ state an overall weight of 2 . To proceed with the calculation we must first relate the various 
two pion states according to their total isotopic spin $T_{2 \pi}$ and the total $Z$ component $M_{2 \pi}$. This relation is given by the set of Clebsch-Gordon coefficients $C\left(1,1 ; M_{1} M_{2} \mid 1,1 ; T_{2 \pi} M_{2 \pi}\right)$ evaluated for $T_{2 \pi}=2,1,0$. The amplitudes are given compactly in an obvious notation

$$
\begin{aligned}
& (2,2)=(++) \\
& (2,1)=(+0) \\
& (2,0) \quad \frac{1}{3}(+-)+\frac{2}{3}(0,0) \\
& (2,-1)=(0,-) \\
& (2,-2)=(--) \\
& (1,1)=(+0) \\
& (1,0)=(+-) \\
& (1,-1)-(+0) \\
& (0,0)=\frac{2}{3}(+-)+\frac{1}{3}(0,0) .
\end{aligned}
$$

The nucleon can be added to these two-pion states by utilizing the coefficients $C\left(T_{2 \pi},{ }^{1 / 2} ; M_{2 \pi} M_{N} \mid T_{2 \pi}{ }^{1} / 2 ; T M\right)$. A system in the state $(3 / 2,-3 / 2)$ will form twopion. one-nucleon states with the relative weights for $(p--)$ being $4 / 5$ and for $\left(n, 0^{-}\right)$being $6 / 5$. The sum of these weights is just 2 representing the overall degeneracy of the $(3 / 2,-3 / 2)$ state. In practice these factors will be nullified by the reduction in the over all weight arising from the indistinguishability of the pions. Similar calculations have been made by Fermi for the products of nucleonnucleon collisions.

In the foregoing, statistical correlations and the conservation of angular momentum have been neglected. The calculations have been simplified by Fermi ${ }^{1}$ by a thermodynamical treatment of the meson-nucleon system in statistical equilibrium. This is specially convenient at high energies $>\left(5 \times 10^{12} \mathrm{eV}\right)$ as relativistic thermodynamics can be applied to both the nucleons and the mesons. Since pions are bosons, Stefan's law for black body radiation holds except for a factor of $3 / 2$ arising from the fact that the pion carries a statistical weight of 3 because of its positive, negative and neutral charges while the photon has a statistical weight of 2 due to its two polarization states. Thus the energy associated with the $\pi$-meson is

$$
u_{\pi}=\frac{\pi^{2}(k T)^{4}}{10}
$$

where $k$ is the Boltzmann constant and $T$ the temperature. The contribution of the nucleons to the energy density is given by

$$
u_{N}=\frac{2 \times 11.364}{\pi^{2}}(k T)^{4} .
$$

1 E. Fermi, loc. cit. 
The factor 2 takes account of the possibility of creation of antinucleons. The temperature of the meson-nucleon system is obtained by equating the total energy in the centre of mass system to the product of the volume $\Omega$ and the sum of the two energy densities. We have

$$
(k T)^{4}=\frac{0.152 W^{2}}{M \Omega_{0}} .
$$

Statistical mechanical arguments also give for the number densities of the pions and the nucleons,

$$
n_{\pi}=0.367(k T)^{3}: n_{N}=0.855(k T)^{3} .
$$

It was shown by Fermi that the angular momentum conservation merely reduces the total number of pions and nucleons by a factor of 0.51 . Hence the total number of nucleons and pions are given respectively by

$$
0.54 \sqrt{\frac{W}{M}} \text { and } 1.3 \sqrt{\frac{W}{m}} \text {. }
$$

Thus this theory predicts the production of comparable numbers of pions nucleons and antinucleons at very high energies.

As mentioned above the effect of angular momentum on the numbers of nucleons and mesons predicted by this model is not very serious but its effect on the angular distribution of the emitted particles is much more critical. The effect is particularly felt for large values of the impact parameter. Since angular momentum must be conserved the outgoing particles must carry away the large amount of angular momentum associated with the two nucleons colliding with a large impact parameter $(l \sim b / \lambda)$. The particles emitted in the direction of either the incident or the target nucleon in the centre of mass system will carry the largest amount of angular momentum. Thus forward and backward emission will be preferred and the angular distribution of the emitted particles will not be isotropic in the centre of mass system.

\section{Some applications of Fermi's theory}

(a) Calculations on Fermi's theory applied to $\pi^{--} p$ events at $1.37 \mathrm{BeV}$ laboratory energy for the $\pi^{-}$is given below. The normalized relative probabilities for three values of interaction volume $\Omega$ (given in terms of the proton volume $\Omega_{0}$ ) are listed assuming that not more than three pions are produced in significant quantities.

Relative production probabilities for 1,2 and 3 pions in $\pi^{-}-p$ collisions at $1.37 \mathrm{BeV}$ (Lab. system)

\begin{tabular}{c|c|c|c}
\hline & $\Omega=\Omega_{0}$ & $\Omega=0.78 \Omega_{0}$ & $\Omega=0.5 \Omega_{0}$ \\
\hline$S(1,1)$ & 0.36 & 0.42 & 0.56 \\
$S(2,1)$ & 0.56 & 0.51 & 0.42 \\
$S(3,1)$ & 0.08 & 0.07 & 0.03 \\
\hline
\end{tabular}


It is of considerable interest to list the distribution of charged secondaries and compare with experimental data.

Distribution of charged secondaries produced in $\pi^{-}-p$ collision

\begin{tabular}{l|c|c|c|c}
\hline $\begin{array}{l}\text { Charged } \\
\text { sccondaries }\end{array}$ & $\Omega=\Omega_{0}$ & $\Omega=0.78 \Omega_{0}$ & $\Omega=0.5 \Omega_{0}$ & Experiment \\
\hline$(\pi+p)$ elastic & 0.27 & 0.32 & 0.45 & 0.11 \\
$\left(\tau^{-}+p\right)$ inelastic & 0.30 & 0.29 & 0.25 & 0.35 \\
$\pi^{+}+\pi^{+}$ & 0.40 & 0.36 & 0.29 & 0.50 \\
$\pi^{+}+2 \pi^{-}+p$ & 0.03 & 0.03 & 0.01 & 0.04 \\
\hline
\end{tabular}

The agreement of theory with experiment is not very good since it predicts many more elastic $\pi^{-}-p$ events than what appear to occur. It becomes worse when nucleon-nucleon collisions are considered. Recent experiments ${ }^{1}$ on meson production in $(n-p)$ and $(p-p)$ collisions at cosmotron energies 0.8, 1.5 and $2.75 \mathrm{BeV}$ have shown a more frequent double pion production than could be accounted for by the simple statistical theory. We give the main results below. ${ }^{2}$

Multiplicity of pion production as a function of beam energy

\begin{tabular}{c|c|c|c}
\hline $\begin{array}{c}\text { Beam } \\
\text { energy }\end{array}$ & $\begin{array}{c}\text { Experimental Ratio } \\
\text { (single }: \text { double }: \text { triple) }\end{array}$ & $\begin{array}{c}\text { Fermi } \\
\text { (single, double, triple) }\end{array}$ & $\begin{array}{c}\text { Kovacs } \\
\text { (single }: \text { double) }\end{array}$ \\
\hline $0.8 \mathrm{BeV}$ & $100: 0: 0$ & $100: 0: 0$ & $100: 0$ \\
$1.5 \mathrm{BeV}$ & $80: 20: 0$ & $94: 6: 0$ & $55: 45$ \\
$2.75 \mathrm{BeV}$ & $36: 48: 16$ & $78: 20: 2$ & $28: 72$ \\
\hline
\end{tabular}

We find that the multiplicities are considerably more than those predicted by Fermi's theory but not so large as those of Kovacs ${ }^{3}$ whose calculation takes into account the strong pion-nucleon final state interaction. Qualitatively Kovacs' results are in rough agreement with experiment.

To explain the multiplicities, Peaslee ${ }^{4}$ has advanced an "isobar model" in which either or both the colliding nucleons become excited and finally, after separation, they decay into one or more pions. The intermediate excited nucleon states were considered to be states of angular momentum $3 / 2$, isotopic spin $3 / 2$ and the excitation energy about $190 \mathrm{MeV}$, corresponding to the resonance peak observed in the $\pi-N$ scattering experiments. Peaslee ${ }^{5}$ found that his model gives large double-pion production.

1 M. M. BLock et al. CERN Symposium (1956).

2 The table is reproduced from the previous reference.

3 J. S. Kovacs, Phys. Rev. 101, 397 (1956)

4 D. Peaslee, Phys. Rev. 94, 1085 (1954); Phys. Rev. 75, 1580 (1954).

5 D. Peaslee, Ibid. 
Pion-nucleon and nucleon-nucleon experiments conducted in the energy range 5-10 $\mathrm{BeV}$ will shed great light upon the problem of multiple meson production. For pion-nucleon case at such energies, Fermi's theory predicts multiplicities of sufficient magnitudes to enable one to determine whether any particular charge state is formed preferentially. It will also be of interest to compare the excited states of nucleons in nucleon-nucleon case following Peaslee's approach with the excited states in pion-nucleon collisions. Conclusions must await the acquisition of experimental data of suitable quality and quantity.

(b) Fermi's theory is quite general and deals with the multiple production of particles in a two-particle collision without any reference to the detailed nature of the interactions except through the masses and generalized parameters like volume and temperature. Hence it can be directly used even in processes involving the production of new particles as for example $\pi$ - and $K$-mesons resulting from the annihilation of nucleon-anti-nucleon pairs. These multiplicities have been calculated by Sudarshan ${ }^{\mathbf{1}}$ on the Fermi and Pomeranchuk-Landau models (which will be discussed presently) for the annihilation of a nucleon pair at rest. In this case, there is no Lorentz contraction of the volume in the centre of mass system and hence there is no anisotropy.

For the Fermi model we consider three separate cases:

(i) All mesons are relativistic,

(ii) All mesons are non-relativistic,

(iii) The $K$-mesons are non-relativistic but the pions are relativistic.

Then we can immediately write down the appropriate densities of states as

$$
\begin{gathered}
\frac{\mathrm{d} V}{\mathrm{~d} W}=\frac{1}{W}\left(\frac{\pi W^{3}}{2}\right)^{n-1} \frac{(4 n-4) !}{(2 n-1) !(2 n-2) !(3 n-4) !} \\
\frac{\mathrm{d} V}{\mathrm{~d} W}=\frac{\left(8 \pi^{3}\right)^{\frac{n-1}{2}} \cdot\left(m_{K}^{n_{K}} m_{\pi}^{n_{\pi}}\right)^{3 / 2}}{\left[n_{K} m_{K}+n_{\pi} m_{\pi}\right]^{3 / 2}} \frac{\left(W-n_{K} m_{K}-n_{\pi} m_{\pi}\right)^{\frac{3 n}{2}}-\frac{5}{2}}{\Gamma\left(\frac{3}{2}\left(n_{K}+n_{\pi}-1\right)\right)} \\
\frac{\mathrm{d} V}{\mathrm{~d} W}=\frac{m_{K}^{3 / 2}\left(2 \pi m_{K}\right)^{3 / 2\left(n_{K}-1\right)}(8 \pi)^{n_{\pi}}}{\left(n_{K} m_{K}\right)^{3 / 2}} \frac{\left(W-n_{K} m_{K}-n_{\pi} m_{\pi}\right)^{3\left(n_{\pi}+\frac{1}{2} n_{K}\right)-\frac{5}{2}}}{\Gamma\left[3_{2}\left(n_{k}+2 n_{\pi}-1\right)\right]}
\end{gathered}
$$

where $n=n_{\pi}+n_{K} ; W=2.0 ; m_{K}=0.520 ; m_{\pi}=0.148$. (The unit of mass is the nucleon mass and all energies are expressed in terms of rest energy of the nucleon.) The relative probabilities are

$$
P\left(n_{\pi}, n_{K}\right)=G\left(0.945 \Omega / \Omega_{0}\right)^{n-1} \frac{\mathrm{d}}{\mathrm{d} W} V\left(n_{\pi} n_{K}\right)
$$

where $\Omega_{0}=\frac{4 \pi}{3}\left(\frac{1}{m_{\pi}}\right)^{3}$, where $\frac{1}{m_{\pi}}=1.4 \times 10^{-13} \mathrm{~cm}$ is the fundamental "volume" in which the annihilation takes place. It is to be noted that $\Omega$ does not involve

1 E. C. G. Sudarshan, Phys. Rev. 103, 258 (1956). 
any Lorentz contraction factor and the factor $\left(0.945 \Omega / \Omega_{0}\right)^{n-1}$ is neglected in the calculations. Using these expressions, the average and root mean square multiplicities of pions and of the total number of mesons have been computed by Sudarshan. ${ }^{1}$ These are given in 'Table 1 .

Table I

(a, b. c correspond to equations $(51 \mathrm{a}, 51 \mathrm{~b}, 51 \mathrm{c}))$

\begin{tabular}{c|c|c|c|c|c|c}
\hline \multirow{2}{*}{ Quantity } & \multicolumn{2}{|c|}{$\mathrm{a}$} & \multicolumn{2}{|c|}{$\mathrm{b}$} & \multicolumn{2}{|c}{$\mathrm{c}$} \\
\cline { 2 - 5 } \cline { 5 - 7 } & $p \bar{p}$ & $n \bar{p}$ & $p \bar{p}$ & $n \bar{p}$ & $p \bar{p}$ & $n \bar{p}$ \\
\hline$n_{\pi}$ & 1.52 & 1.63 & 1.74 & 1.84 & 2.27 & 2.43 \\
$n_{\pi}^{2}{ }^{2 / 2}$ & 1.84 & 1.94 & 2.12 & 2.23 & 2.70 & 3.01 \\
$n_{\pi}+n_{K}$ & 2.81 & 2.90 & 3.27 & 3.34 & 2.72 & 2.84 \\
$\left(n_{\pi}+n_{K}\right)^{2} 1 / 2$ & 2.89 & 2.96 & 3.32 & 3.38 & 2.95 & 3.22 \\
$P_{K}$ & 65 & 64 & 76 & 75 & 23 & 21 \\
\hline
\end{tabular}

The results for antineutron annihilation, namely $\bar{n} n, p \bar{n}$ are identical to the results for $p \bar{p}$ and $n \bar{p}$ respectively.

As has been pointed out earlier, the Fermi theory does not take into account the strong final state interaction of the mesons. The Pomeranchuk-Landau ${ }^{2}$ theory which includes the final state interaction has been applied by Sudarshan ${ }^{3}$ to the present problem. The results of the calculation are given in Table II.

Table II

(Corresponds to equation (51 c))

\begin{tabular}{c|l|l|l|l|l|l}
\hline \multirow{2}{*}{ Quantity } & \multicolumn{2}{|c|}{ Case 1 } & \multicolumn{2}{c|}{ Case 2 } & \multicolumn{2}{c}{ Case 3 } \\
\cline { 2 - 5 } \cline { 5 - 7 } & $p \bar{p}$ & $n \bar{p}$ & $p \bar{p}$ & $n \bar{p}$ & $p \bar{p}$ & $n \bar{p}$ \\
\hline$\left.n_{\pi}\right\rangle$ & 3.54 & 3.55 & 2.94 & 3.03 & 2.74 & 2.84 \\
$\left.\left(n_{\pi}\right)^{2}\right\rangle^{1 / 2}$ & 3.64 & 3.64 & 3.12 & 3.18 & 2.98 & 3.04 \\
$\left.n_{\pi}+n_{K}\right\rangle$ & 3.59 & 3.59 & 3.12 & 3.18 & 3.10 & 3.16 \\
$\left\langle\left(n_{\pi}+n_{K}\right)^{2}\right\rangle^{1 / 2}$ & 3.66 & $3.6 \tilde{y}$ & 3.21 & 3.25 & 3.18 & 3.22 \\
$P_{K}$ & 3 & 2 & 9 & 8 & 18 & 16 \\
\hline
\end{tabular}

Comparison of 'Tables I and II shows that the pion multiplicity of PomeranchukLandau model is nearer to the observed one (which is 3.4) than Fermi's.

The statistical models predict a smaller value (2.2-3.4) for pion multiplicity and a larger number of events involving $K$-emission than the measured values. Koba and Takeda ${ }^{4}$ suggest that some characteristics of nucleon (anti-nucleon)

1 E. C. G. Sudarshax, loc. cit.

2 I. Ya. Poneranchuk and L. Landau. Dokl. Akad. Nauk. SSSR. 78, 889 (1951).

3 E. C. G. Sudarshan, loc. cit.

Z. Koba and G. TAKeda (Preprint). 
structure are being reflected in the transition matrix elements leading to the large pion multiplicity. They divide the pions resulting out of annihilation into two classes:

(i) those coming from the pion cloud and (ii) those produced by the violent annihilation of the core-anticore pair. The essential part of the core annihilation will end within a short period $\sim \frac{1}{2 m_{N}}$ which is smaller than the period in which a pion in the cloud is absorbed and re-emitted by the core nucleon which is of the $\operatorname{order} \frac{1}{w_{\pi}}$. Therefore during the short time in which the core annihilation is completed, the number of pions in the cloud will be constant and these having lost their centres will then be emitted, the number of pions being 2 or 3 on the average. On the other hand during the violent annihilation process, three or more pions will be produced. Koba and Takeda calculate the pions emitted from the cloud on the basis of the static theory and apply the statistical theory to the core annihilation. The mean number of pions emitted from the cloud turns out to be 1.96. If this is added to the usual number given by the statistical theory the agreement with experiment is very good.

\section{Extension of Fermi's theory by Landau and Pomeranchuk ${ }^{1}$}

Quantitative calculations on the basis of Fermi's theory do not agree very well with experimental results, especially those relating to angular and energy distributions. Hence the following improvement of Fermi's theory was suggested by Landau. ${ }^{2}$

At the moment of collision there are produced a number of particles in a volume, the linear dimensions of which are determined by the nature of the nuclear forces and the energy of the incident particles. It must be emphasized that the concept of the number of particles has a meaning only in a limited sense since the density of mesons and nucleons is very large in the nucleus. The mean free path of a particle in such a system is obviously small compared to its dimensions. This system will expand with time and according to Landau the expansion process is hydrodynamic in character as the smallness of the mean free path allows us to view the motion as microscopic, i.e. as the motion of an ideal non-viscous, nonthermal conductive fluid. Since the velocities of the particles are comparable with that of light, the equations are those of relativistic hydrodynamics.

The total number of particles does not remain constant during the hydrodynamical stage of expansion. The number of particles emerging after the collision is determined not by those produced at the moment of collision as was the case in Fermi's theory but at the end of the expansion stage when they fly apart as free particles. This feature was suggested by Pomeranchuk and Landau's

1 I. Ya. Pomeranchuk, Dokl. Akad. Nauk, SSSR. 78, 889 (1951); L. D. Landau, Izv. Akad. Nauk, USSR. 17, 57 (1953).

${ }^{2}$ L. D. LandaU, Ibid. 
theory which essentially eonsists in studying the expansion stage in a quantitative form.

'The end of the expansion stage comes when the mean free path of the particles becomes equal to the linear dimensions of the expanding volume. The order of magnitude of the temperature of the system at this point is given by

$$
T \sim \mu
$$

where $\mu$ is the mass of the meson and the temperature is measured in energy units. It is to be noted that the above relation is independent of the nature of the system, i.e. the energy of the colliding particles. When the temperature is lower than $\mu$, the equilibrium number density falls off exponentially $\mathrm{e}^{-\mu / \boldsymbol{T}}$ as the system "cools". So the mean free path rapidly approaches the linear dimensions of the expanding system. The formula for $T$ is valid even when heavy mesons are generated.

We assume that the equation of state for the compressed matter at a temperature $T \gg \mu$ is

$$
p=\varepsilon / 3
$$

where $p$ and $\varepsilon$ are the pressure and the energy density. Since the number of particles is not given but is defined by the condition of the statistical equilibrium, the chemical potential is given by

$$
\zeta=\varepsilon-T s+p=0
$$

where $s=$ entropy per unit volume. Thus we find

$$
T s=\frac{4}{3} \varepsilon .
$$

Making use of the relation $\mathrm{d} \varepsilon=T \mathrm{~d} s$ for a given volume, we obtain .

$$
s \propto \varepsilon^{3 / 4} ; T \sim \varepsilon^{1 / 4} .
$$

It is clear that the entropy of the system in the various parts will remain constant during the whole of the hydrodynamical expansion, if we assume that the motion of the ideal fluid is performed adiabatically. Dividing the entire system into microscopically small homogeneous portions, let $s_{\alpha}$ be the entropy of one of these parts and $n_{\alpha}$ the number of particles in the $\alpha$ th portion produced at the moment of its "flying away". These times may not coincide for different portions. The ratio $s_{\alpha} / n_{\alpha}$ depends only weakly on the temperature so that we can put

$$
n_{\alpha}=\text { constant } \times s_{\alpha}
$$

where the constant is some universal constant and is found to be of the order of $\mu^{3}$. Thus we find for all the portions

$$
N=\text { constant } \times S
$$

where $N$ is the total number of particles and $S$ is the total entropy of the system. 
Consider now the "head-on" collisions between two protons. Let $E$ ' be the energy of each proton in the centre of mass system of the two particles. If $V$ is the volume in which the energy is distributed, the total entropy of the system $S$ is proportional to $\varepsilon^{3 / 4} V$. Since $\varepsilon=\frac{E^{\prime}}{V}$ we have the result that entropy as well as the number of particles is proportional to $E^{\prime^{3 / 4}} V^{1 / 4}$. To calculate the volume $V$, we note that the transverse dimension of the system is of the order of the range of the nuclear forces, i.e. $\frac{1}{\mu}$ and the longitudinal dimension in the centre of mass system suffers a Lorentz contraction in the ratio $M / E^{\prime}$ and thus the system looks like a strongly flattened disk with its volume given by

$$
V \sim a^{2} M / E^{\prime}
$$

Thus $N$ is proportional to $V E^{\prime}$ or in the laboratory system where one of the protons is at rest and the energy is $E$, we have

$$
N \propto E^{1 / 4} .
$$

This formula agrees with that obtained by Fermi. From dimensional consideration, we can express $N$ as $N=K\left(\frac{E}{2 M}\right)^{1 / 4}$ where $K$ is a constant of order of unity.

Pursuing the above arguments, we can consider the collision of two identical nuclei of mass $A$. The number of particles produced is

$$
N=K A^{3 / 4}\left(\frac{E}{2 M}\right)^{1 / 4} \text {. }
$$

We find from this expression that heavy nuclei are more effective than protons in producing the particles. $K$ is found to be of the order of 2 on a comparison with experimental data.

The angular and energy distribution of the particles produced, can be obtained only by a detailed study of the hydrodynamical motion of the matter in the system. It has already been remarked that relativistic hydrodynamics should be employed since the matter moves with velocity nearly that of light. The relativistic hydrodynamical equations are expressed by the relations

$$
\frac{\partial T^{i k}}{\partial x^{k}}=0
$$

where $T^{i k}$ is the energy-momentum tensor of matter and is given for an ideal fluid by

$$
T^{i k}=p g^{i k}+(\varepsilon+p) u^{i} u^{k}
$$

where $u$ is the four velocity and $g^{11}=g^{22}=g^{33}=1$ and $g^{00}=-1$.

In the centre of mass system at the moment of collision, the system has the form of a very strongly flattened disk. This form is preserved during the hydroEPCR 27 
dynamical expansion. The motion of matter is regarded as one-dimensional along the thickness of the disk ( $x$-axis). Then the equations of motion become

$$
\frac{\partial T^{00}}{\partial t}+\frac{\partial T^{01}}{\partial x}=0 ; \quad \frac{\partial T^{01}}{\partial x}+\frac{\partial T^{11}}{\partial x}=0
$$

where

$$
\begin{aligned}
& T^{00}=\varepsilon\left(u^{0}\right)^{2}+p\left(u^{1}\right)^{2} ; \quad T^{01}=(\varepsilon+p) u^{0} u^{1} . \\
& T^{11}=\varepsilon\left(u^{1}\right)^{2}+p\left(u^{0}\right)^{2} ; \quad\left(u^{0}\right)^{2}-\left(u^{1}\right)^{2}=1
\end{aligned}
$$

The "disk" expands on both sides in the centre of mass system and hence we choose the origin of the coordinate system on the middle plane and observe the motion in the positive direction of the $x$-axis, i.e. $x>0, u^{1}>0$. Denoting the initial thickness by $\Delta$, we consider the case when due to expansion the thickness $t \gg \Delta$. The matter will oceupy a space $0<x<t$. Most of the matter moves with a velocity of the order of light. In the narrow layer $t-x \ll t$, the matter is moving with velocity nearly that of light and it is in this region that most of the energy is concentrated. Introducing a new variable $\mathscr{E}=t-x$ and in the extreme relativistic case we have $u^{0} \simeq u^{1} \equiv u \gg 1$ and $u^{0}-u^{1} \approx 1 / 2 u$. The equations (62) then take the form

$$
\begin{gathered}
\frac{\partial}{\partial t}\left(\varepsilon u^{2}\right)=-\frac{1}{4} \frac{\partial \varepsilon}{\partial \mathscr{E}} \\
\frac{\partial \varepsilon}{\partial t}=-\frac{\partial}{\partial \mathscr{E}}\left(\frac{\varepsilon}{u^{2}}\right) .
\end{gathered}
$$

We shall look for solutions of these equations in the domain of values $t \gg \mathscr{E} \gg \Delta$ and we assume that $u(\mathscr{E}, t)$ shall be of the form $u^{2}=t t / \mathscr{E}$ where $f$ is a slowly varying function of $t$ and $\mathscr{E}$. Finally we obtain

where

$$
\varepsilon=\varepsilon_{0} \mathrm{e}^{\left\{-\frac{4}{3}\left(\eta \div \tau-\gamma^{\prime} \tau\right)\right\}}
$$

$$
\begin{aligned}
\tau & =\log t / \Delta ; \quad \eta=\log \mathscr{E} / \Delta . \\
f & =\frac{1}{2} \sqrt{\frac{\tau}{\eta}} \sim 1 .
\end{aligned}
$$

To discuss the energy and entropy distribution along the thiekness of the "disk" we first note that the energy density is given by $T^{00} \sim \varepsilon u^{2}$. Putting $u^{2} \sim t / \mathscr{E}$ and using the expression (69) we have for energy in a layer $\mathrm{d} \mathscr{E}$

$$
\mathrm{d} E \propto \exp \left[-1 / 3(V \tau-2 V \eta)^{2}\right] \mathrm{d} \eta \text {. }
$$

From this it is seen that the energy distribution is maximum at $\eta=\tau / 4$ and the energy is mainly concentrated in the domain $\mathscr{E} \sim 4 \gamma\left(t \Delta^{3}\right)$. As $s \alpha \varepsilon^{3 / 4}$ we obtain for the entropy in a layer $\mathrm{d} \mathscr{E}$

$$
\mathrm{d} s \propto \exp \left[-\frac{1}{2}(\gamma \tau-\gamma / \eta)^{2}\right] \mathrm{d} \eta .
$$

This has a maximum at $\eta=\tau$ and unlike the energy, this is concentrated in the region $\mathscr{E} \sim t$. 
These solutions will be valid only so long as the angle of flying-away $\theta$, i.e. the angle formed by the trajectory of the given matter element with the $x$-axis is very small. Further if we introduce new variables $\lambda$ and $L$ defined by $\mathscr{E} / a=e^{-\lambda}$; $\frac{\Delta}{a}=\mathrm{e}^{-L}$, the entropy distribution becomes

$$
\mathrm{d} s \propto \mathrm{e}^{V L^{2}-\lambda^{2}} \mathrm{~d} \lambda
$$

and the distribution of the particles produced is given by

$$
\mathrm{d} N=C \mathrm{e}^{/ L^{2}-\lambda^{2}} \mathrm{~d} \lambda
$$

where $C$ is a normalization constant. The angle of flying-away is

$$
\theta \sim \frac{\mathscr{E}}{a}=\mathrm{e}^{-\lambda}
$$

and is a constant with $\mathscr{E}$ for each element and so for each particle. These expressions determine the angular distribution of the generated particles (in the centre of mass system) with a single parameter $\lambda$. The constant $L$ is related to the energy of the colliding particles and is found to be

$$
L=\frac{1}{2} \log \left(\frac{E}{2 M_{A}}\right)
$$

where $M_{A}$ is the mass of particles. The angular distribution given by the expression (75) does not give spherical symmetry as assumed by Fermi but $\frac{\mathrm{d} N}{\mathrm{~d} \theta}$ per unit angle rapidly increases with decreasing $\theta$. The expression (74) can be rewritten for practical purposes in the form

$$
\mathrm{d} N \propto \mathrm{e}^{-\lambda^{2} / 2 L} \mathrm{~d} \lambda .
$$

The angular distribution can be put in the form of a Gaussian distribution if we define $\lambda$ to be $-\log \frac{\tan \theta}{2}$. The actual distribution curve of the particles in the angle $\theta$ must possess comparatively long "tails". on both sides of the maximum. Landau ${ }^{1}$ shows that the energy distribution of particles in the centre of mass system is given by

$$
\mu u^{1} \sim M \exp \left\{-\frac{L}{6}+\lambda+\frac{1}{3} \sqrt{ } L^{2}-\lambda^{2}\right\} .
$$

Finally we have to carry out the transition from the centre of mass system to the laboratory system in which one of the nucleons is at rest. If $\chi$ is the angle of flying away in the laboratory system Landau shows that $\chi=\mathrm{e}^{-\lambda-L} . \lambda$ can take both positive and negative values to account for the particles flying in the

1 L. D. Landau, loc. cit. 
positive and negative directions of $x$ axis in the centre of mass system. Similarly the energy distribution in the laboratory system becomes

$$
\mu u \sim M \exp \left\{\frac{5 L}{6}+\lambda+\frac{1}{3} V L^{2}-\lambda^{2}\right\}
$$

with $\lambda$ having both signs. The coefficients can now be determined making use of the relations

and we obtain

$$
\int \mathrm{d} N=N ; \quad \int u \mu \mathrm{d} N=E
$$

$$
\mu u=\frac{5}{2} \frac{\sqrt{ } 5}{\sqrt{3}} \frac{M}{K} \exp \left[\frac{5 L}{6}+\lambda+\frac{1}{3} \gamma L^{2}-\lambda^{2}\right]
$$

From this expression it is seen that the majority of particles have energy of the order of

$$
M\left(\frac{E}{2 M_{A}}\right)^{\frac{7}{12}}
$$

in the laboratory system. The figure below shows the results using the above expression.

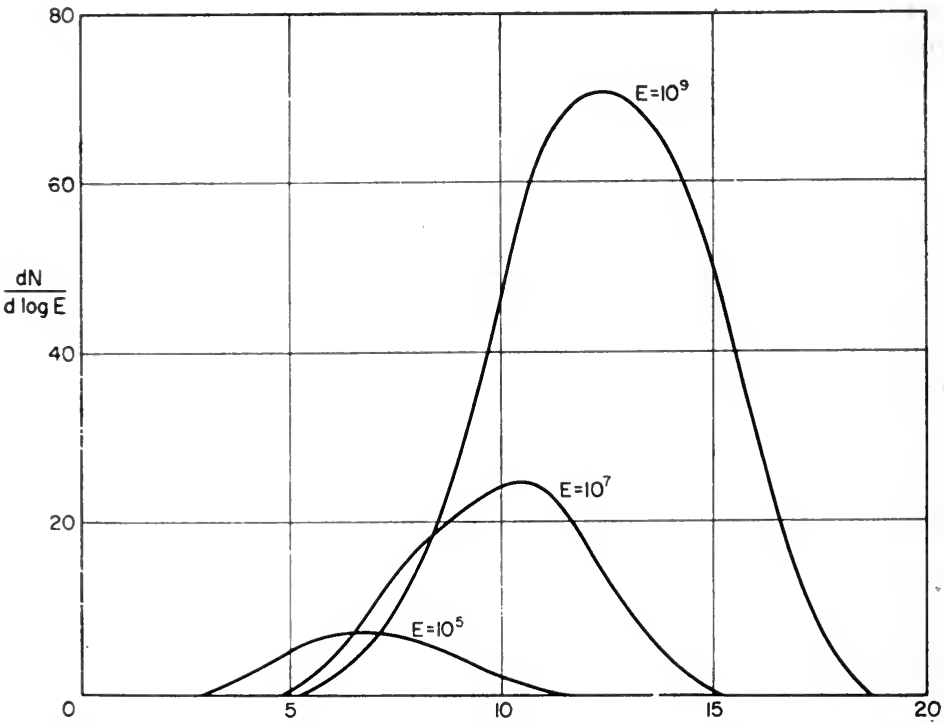

FIG. 61. Differential energy spectrum of secondary particles generated in nuclear interactions at high energies (for various energies of the original particles). Areas under the curves are proportional to the total number of secondary particles (mesons and nucleons).

\section{A covariant formulation of the statistical theory}

We shall now describe a fully covariant formulation of the statistical theory of meson production given by Srivastava and Sudarshan. ${ }^{1}$ The transition probability from an initial state consisting of two nucleons of four-momenta $p_{1}^{\prime}$ and $p_{2}^{\prime}$

${ }^{1}$ G. P. Srivastava and E. C. G. Sudarshan, Phys. Rev. 110, 765 (1958). 
to a final state involving two nucleons of four-momenta $p_{1}$ and $p_{2}$ and pions of four-momenta $q_{1}, q_{2}, \ldots q_{m}$ is given by

$$
\begin{aligned}
W_{m}=\int \mathrm{d}^{4} p_{1} \mathrm{~d}^{4} p_{2} \mathrm{~d}^{4} q_{1} \cdots \mathrm{d}^{4} q \delta^{4}\left(p_{1}+p_{2}+q_{1}+\cdots q_{m}-p_{1}^{\prime}-p_{2}^{\prime}\right) \\
\delta\left(p_{1}^{2}-m^{2}\right) \delta\left(p_{2}^{2}-m^{2}\right) \cdots \delta\left(q_{1}^{2}-\mu^{2}\right) \cdots \delta\left(q_{m}^{2}-\mu^{2}\right) f\left(p p^{\prime} q\right)
\end{aligned}
$$

where $f\left(p p^{\prime} q\right)$ is an invariant function of the nucleon and pion four-momenta which is obtained by averaging over the initial and summing over the final spins of the absolute square of the matrix element. In the statistical theory we replace $f\left(p p^{\prime} q\right)$ by a constant quantity independent of the four-momenta but depending on the number of meson and nucleon lines in the diagram corresponding to the process. From dimensional arguments we have

$$
f\left(p p^{\prime} q\right)=A S(m) K^{-2 m}
$$

where $K$ is a parameter of the dimensions of mass and $A$ a numerical constant. The factor $S(m)$ takes account of the constraints on the final state due to the conservation laws. The Compton wavelength corresponding to the mass $K$ takes the place of the linear dimensions of the Fermi volume. $K$ can be chosen so as to fit the experimental data on a nucleon-nucleon process at any one energy. The way in which $K$ enters the theory shows clearly that it is a covariant constant.

\begin{tabular}{|c|c|c|}
\hline & $\begin{array}{c}\text { Ratio of } \\
\text { single: double: triple } \\
\text { proton energy }= \\
1.5 \mathrm{BeV}\end{array}$ & $\begin{array}{c}\text { Ratio of } \\
\text { single: double: triple } \\
\text { proton energy }= \\
2.75 \mathrm{BeV}\end{array}$ \\
\hline $\begin{aligned} \text { Present theory } K^{-1} & =1.5 \times 10^{-13} \mathrm{~cm} \\
K^{-1} & =2.0 \times 10^{-13} \mathrm{~cm}\end{aligned}$ & $\begin{array}{l}77: 28: 3 \\
77: 50: 1\end{array}$ & $\begin{array}{l}39: 29: 5 \\
39: 51: 17\end{array}$ \\
\hline Experiment & $80: 20: 0$ & $36: 48: 16$ \\
\hline
\end{tabular}

The table below gives a comparison with experiment of the meson multiplicities in proton-proton collisions calculated on the basis of this theory.

\section{Bhabha's theory ${ }^{1}$}

Bhabha's theory of the production of mesons and nucleons in very energetic nucleon-nucleon collisions is based on the distribution of the observed rest mass of the nucleon into the proper mass and the field mass. It is assumed that a fraction $\epsilon$ of the observed mass $M$ of a nucleon is distributed in some arbitrary manner over a volume of radius of the order of the Compton wavelength of the meson, and the rest is concentrated in a sphere of radius $\zeta$, where $\zeta$ is of the order of the Compton wavelength of the nucleon. The possibility of several different types of mesons, viz. $\pi$ and $K$ may be taken into account by representing the distribution of the mass of the field not by a simple exponential but by a superposition of exponentials. Then it is possible to calculate the amount of

1 H. J. Внавна, Proc. Roy. Soc., A 219, 293 (1953). 
energy available in each region and ultimately in the whole volume for shower production, from general considerations using the principle of conservation of energy and momentum. The shower production takes place in three distinct regions and the total energy available may be represented as $E_{f}=2 E_{0}$ where $E_{f}$ corresponds to the region in which the meson fields of the two nucleons overlap and $2 E_{0}$ corresponds to the other two regions which contain the spheres of radius $\zeta$, each of which surrounds one of the initial nucleons. It has been pointed out by Bhabha that the theories of Fermi and Heisenberg are based on the tacit assumption that the entire mass of the nucleon is located in the field and distributed over the volume occupied by the field. In the present theory, the total energy that goes into shower production in any particular encounter is scaled down by a further factor $\in$ in addition to the exponential factor introduced by Heisenberg, except in rare head on collisions. Thus, the partition of the rest mass of a nucleon between its proper mass and field mass becomes a matter of practical interest susceptible of experimental exploration.

One can go further. In Fermi's theory the size of a shower is practically a unique function of the initial energy of the colliding particles. The impact parameter of the collision only alters the number of particles by a factor of two or so. In the Heisenberg theory. the size of a shower is not a unique function of energy and for a given initial energy the size may vary from a shower of one or two particles to that of the maximum size possible for the given energy, depending upon the impact parameter. The present analysis permits us to calculate the size-frequency distribution of showers produced by nucleons of the same energy, if the spatial distribution of the field energy or mass of a nucleon is known, or conversely, to calculate in principle the spatial distribution of the field energy of a nucleon from the frequency-size distribution of meson showers. Thus the study of shower production in high-energy encounters becomes a method of obtaining experimental information on the localization of field energy of a nucleon and on the fundamental problem of the self-energy of nucleons.

\section{Isobar and fireball models ${ }^{1}$}

The angular distribution of secondary particles with very sharp peaks in the forward and backward directions and the hypothesis of the possible existence of a metastable excited state of the nucleon, the isobar, predicted by the early strong coupling meson theories, further strengthened by the resonance in the $\left({ }^{3} / 2,3 / 2\right)$ state in the (low-energy) pion-nucleon interactions, have led to an isobar model for the multiple production of mesons. The other proposed model according to which the collision of two energetic nucleons gives rise to two "fireballs" each of which separates from the nucleons violently and after a very short time disintegrates into a number of mesons has in common with the isobar model the assumption of a hypothetical metastable state in the intermediate stage.

1 Z. Ковa and S. Takagi, Progr. Theoret. Phys. 7, 123 (1952); W. L. Kraushaar and L. J. Marks, Phys. Rev. 93, 326 (1954); G. Cocconi, Phys. Rev. 111, 1699 (1958). 
When two nucleons of momentum $\boldsymbol{p}_{1}$ and $\boldsymbol{p}_{2}$ collide with each other, both momentum and energy have to be conserved. In the centre of mass system, the total momentum is zero before and after the collision. But the magnitude of the individual momenta may be less than the magnitude before collision. The energy associated with the motion and the mass (as obtained from the energy-momentum relation) will also be less than that before collision. This loss in energy goes into the making of the metastable state of a particle with a fictitious mass, $M^{*}$ greater than the nucleon mass. The two "isobars" now fly apart and decay independently into secondary particles. The decay process can be treated by a statistical method though many of the characteristic features of the model do not depend on this approximation. We can assume for the interaction volume of Fermi's theory, a sphere of radius $\frac{1}{\mu}$ in the centre of mass of the isobars which results in a different multiplicity-energy relation from Fermi's model. When the inelasticity is assumed to be much smaller than one, the multiplicity depends but little on the energy and mainly on the inelasticity since if the latter is kept constant, the increase of the incident energy is used up for the increase of the translational motion of the isobars and not to increase the "thermal" energy of the constituent particles. A consequence of this motion of the isobars is an angular distribution with rather sharp peaks, both forward and backward. For inelasticity near unity, this anisotropy is less pronounced in conformity with experiment. If further we take into account angular momentum conservation, the isobars can have high spins and the decay products might have a tendency of being coplanar in the plane determined by $\left(\boldsymbol{p} \times \boldsymbol{p}^{*}\right)$ where $\boldsymbol{p}$ is the incident direction and $\boldsymbol{p}^{*}$ the direction of emission of the isobar. Thus the detection of an azimuthal asymmetry in individual jets, which cannot be accounted for as due to statistical fluctuations, will lend support to the isobar and fireball models.

The isobar model puts too stringent a restriction on the upper limit of multiplicity $N \leqq \frac{\eta}{1-\eta}$ where $\eta$ is much smaller than unity. 


\section{CASCADE PROCESSES}

\section{I NTRO I U CTION}

ThLL now we have considered the various types of interactions possible between a fast incident particle of cosmic radiation and the individual nuclei of matter it traverses. We shall now consider the cumulative effects of such interactions when a single particle enters a finite thickness of matter. This particle collides with air nuclei in the atmosphere and in each collision a number of other particles are produced. These secondary particles in their passage through matter collide with other nuclei and produce further particles. Thus a cascade is formed and one of the main problems in cosmic radiation is to study the nature of this multiplication. In any typical collision, particles of different types at various energies and angles of emergence are produced and the probabilities of the individual collisions are determined by the corresponding quantum mechanical crosssections. The successive collisions are considered independent and cascade multiplication is essentially a statistical phenomenon or in more precise terms a stochastic process which can be studied by methods of classical probability. A comprehensive knowledge of the cross-sections for various processes may in principle be sufficient for developing the theory of such cascades. But this is obviously a difficult task, too comprehensive to serve any purpose. It will be convenient to study cascade development with emphasis on one of the two aspects given below.

\section{Longitudinal development of showers}

We assume that all the particles produced move in the same direction as the incident particle. We ignore the lateral spread of the particles and concern ourselves only with different types and energies as this makes a stochastic analysis possible. We can deal not merely with the mean values of the various quantities involved but also their probability distributions or at least the fluctuations about the mean number.

\section{Lateral spread of showers}

We develop a cascade theory including lateral spread but confine ourselves only to the mean values of the various quantities.

To simplify the treatment and to get a better insight into the physical processes involved it is found more convenient to classify showers according to the types 
of particles involved like soft cascades, nucleon cascades and meson cascades. This is only a matter of convenience and in many high energy interactions all these types are involved.

The literature on cascade theory has grown out of proportion ${ }^{1}$ to the use it can be put to in explaining the increasing mass of experimental data. However in view of its fundamental importance in the mathematical theory of stochastic processes, it is considered worthwhile to discuss cascade theory in detail with particular emphasis on the stochastic aspects and the new methods stimulated by such a study. ${ }^{2}$ It is now possible to present the entire cascade theory in a concise and compact form bringing out the relation between the various methods developed.

\section{O N E - D I M ENSIONAL CASCADES}

\section{General formulation}

We shall first formulate in a general manner a cascade process involving different types of particles. Let $t$ be the one dimensional parameter representing the thickness of matter traversed. We shall assume that at $t=0$ we have a particle of type $i$ with energy $E_{0}$. This particle can be called the primary of the cascade. Our object is to give a statistical description of the numbers of particles and their energies at a thickness $t$ if we assume that a particle of type $i$ and energy $E_{0}$ is replaced in a collision by an aggregate $\left\langle E_{l}^{j}\right\rangle$ which consists of $k_{1}$ particles of type $1, k_{2}$ particles of type $2, \ldots k_{n}$ particles of type $n$. The $k_{j}$ particles of type $j$ are distributed in energy as follows. One of them lies in the range between $E_{1}^{j}$ and $E_{1}^{j}+\mathrm{d} E_{1}^{j}$, the second between $E_{2}^{j}$ and $E_{2}^{j}+\mathrm{d} E_{2}^{j}, \ldots$ the $k_{j}^{-t h}$ between $E_{k_{j}}^{j}$ and $E_{k_{j}}^{j}+\mathrm{d} E_{k_{j}}^{j}$. The probability of its production in $\mathrm{d} t$ is

$$
R^{(i)}\left(\left\langle E_{l}^{j}\right\rangle \mid E_{0}\right) \mathrm{d} t
$$

This cross-section is defined by quantum mechanical laws and represents the probability of a collision of the particle of type $i$ with a nucleus in its passage through matter of thickness $\mathrm{d} t$, the collision being electromagnetic or nuclear. The total cross-section $R^{i}\left(E_{0}\right)$ is obtained by integrating over all energy variables and summing over all values of $k_{j}$ and $j$ and multiplying by $\frac{1}{\prod_{j} k_{j} !}$. This latter

1 For an introduction to the mathematical aspects, see (a) M. S. BARTLETT, An Introduction to Stochastic Processes, Cambridge University Press (1955). For a comprehensive summary of the works of various authors on cascade processes, see BHARUCHA-REID, Markov Processes and Their Applications, McGraw-Hill (1960). The mathematical methods used therein and the relations between them are discussed in detail by Alladi Ramakrishnan in "Probability and Stochastic Processes", in Handbuch der Physik, Vol. 3/2, 578 (1958).

2 See for the summary of the methods, Alladi Ramakrishnan and P. M. Mathews, Progr. Theoret. Phys. 11, 95 (1954).

Messel and his collaborators have made extensive calculations on this subject by carrying through systematically the analytic solution of complicated equations which can be formally written by the use of the new methods. 
factor arises since integration with respect to each of the energy variables over the whole range implies counting an event representing the occurrence of $k_{j}$ particles $k_{1}$ ! times.

We have now to define a function which gives a detailed description of the caseade at thickness $t$. The most comprehensive one is obviously that which defines the probability

$$
\tau\left(\left\langle E_{l}^{j}\right\rangle \mid E_{0} ; t\right) \mathrm{d}\left\langle E_{l}^{j}\right\rangle
$$

that particles of the aggregate $\left\langle E_{l}^{j}\right\rangle$ lie in the corresponding energy ranges and none elsewhere. This function is obviously too difficult to handle. We should be normally content with the function

$$
\pi^{i}\left(\left\langle v_{m}\right\rangle \mid E_{0} ; t\right)
$$

the probability that we have $v_{m}$ particles of type $m(m=1, \ldots, n)$ with energy greater than or equal to $E_{m}$ at $t$ given that at $t=0$ we had only one particle of type $i$ and energy $E_{0}$. The standard method for the study of the development of the cascade is to examine the variation of this function with $t$. This can be done only if we know the individual energies of the particles at $t$ as in the case of the comprehensive $J$ function to be defined later but it is not possible in the case of the $\pi$ function. However we have chosen the $\pi$ function as sufficient for our purpose and it is only recently that methods have been developed to deal with it directly. This method known as the regeneration point method was first introduced by Janossy ${ }^{1}$ in his treatment of electron-photon cascades. We shall here present the corresponding treatment in the general case.

The joint probability that nothing happens to the initial particle of type $i$ and energy $E_{0}$ till $\tau$ and that the aggregate $\left\langle E_{l}^{j}\right\rangle$ is produced in $\mathrm{d} \tau$ is

$$
\mathrm{e}^{\left[-R^{(i)}\left(E_{0}\right) \tau\right]} R^{(i)}\left(\left\langle E_{l}^{i}\right\rangle E_{0}\right) \mathrm{d} \tau .
$$

Somewhere between 0 and $t$ in the interval $(\tau, \tau+\mathrm{d} \tau)$ the initial particle of type $i$ produces an aggregate and the members of this aggregate become in turn primaries of cascades. This aggregate can be formed anywhere between 0 and $t$ and therefore we obtain the integral equation

$$
\begin{aligned}
\pi^{(i)}\left(\left\langle v_{m}\right\rangle \mid E_{0} ; t\right) & =\int_{0}^{t} \int_{\left.<E_{i}\right\rangle} \frac{\mathrm{e}^{-R^{(i)}\left(E_{0}\right) \tau} R^{(i)}\left(\left\langle E_{l}^{j}\right\rangle \mid E_{0}\right)}{\prod_{j} k_{j} !} \\
& \times \sum_{j} \sum_{l} \pi\left(\left\langle v_{m}\right\rangle \mid\left\langle E_{l}^{j}\right\rangle ; t-\tau\right) \mathrm{d}\left\langle E_{l}^{j}\right\rangle \mathrm{d} \tau \\
& +\mathrm{e}^{-R^{(i)}\left(E_{0}\right) \tau} \prod_{j(j \neq i)} \delta\left(v_{j}\right) \delta\left(v_{i}-1\right) H\left(E_{0}-E_{i}\right)
\end{aligned}
$$

where $\delta\left(v_{i}\right)$ is the Kronecker delta and $H(E)$ the Heaviside unit function. The last term merely indicates that nothing happens between 0 and $t$ and we have the initial particle of type $i$ with energy $E_{0}$.

1 L. Jaxossy, Proc. Phys. Soc. A 63, 241 (1950). See also R. Bellman and T. E. Harris, Proc. Nat. Acad. Sci. 43, 601 (1948). 
$\pi\left(\left\langle v_{m}\right\rangle \mid\left\langle E_{l}^{j}\right\rangle ; t-\tau\right)$ is the probability that we have the aggregate $\left\langle v_{m}\right\rangle$ at $t$ given that we started with the aggregate $\left\langle E_{l}^{j}\right\rangle$ at $\tau$. This is a function of the duration $t-\tau$ and not of $t$ and $\tau$ severally since the process is assumed to be homogeneous in $t$. The probability frequency function $\pi\left(\left\langle v_{m}\right\rangle \mid\left\langle E_{l}^{j}\right\rangle ; t-\tau\right)$ can be. decomposed in terms of the probability frequency function with simpler initial conditions, i.e. the one particle initial condition as in $\pi^{i}\left(\left\langle v_{m}\right\rangle \mid E_{0} ; t\right)$ in the following manner if we assume that the particles of the aggregate $\left\langle E_{l}^{j}\right\rangle$ behave independently as primaries of cascade processes,

$$
\pi\left(\left\langle v_{m}\right\rangle \mid\left\langle E_{l}^{j}\right\rangle ; t-\tau\right)=\prod_{l, j} \pi^{(j)}\left(\left\langle v_{m}^{j l}\right\rangle \mid E_{l}^{j} ; t-\tau\right)
$$

where

$$
\sum_{l}\left\langle v_{m}^{j l}\right\rangle=\left\langle v_{m}\right\rangle
$$

$\left\langle v_{m}^{j l}\right\rangle$ being the aggregate of $\nu_{m}^{j l}$ particles with energy greater than $E_{m}, m=1,2, \ldots$ produced by a particle of type $j$ and energy $E_{l}^{j}$.

Equation (6) can be reduced to a differential equation in $t$ by differentiating with respect to $t$. We then have a system of simultaneous integro-differential equations involving summations over $v_{i}$. The sums can be reduced to a product of suitably defined generating functions by the application of convolution theorems. In spite of this the equations are still too general and simplifying assumptions have to be made to obtain physically interesting results.

The simplification consists in (1) limiting the number of types of particles, (2) assuming simplified expressions for the cross-sections and (3) asking not for the distribution function but only for its moments.

If we are interested only in the mean numbers, there is no necessity to introduce the $\pi$ function and thereby obtain the mean. A standard method of studying the variation with $t$ of the mean number of particles is available in this case. In fact when the cascade theory was formulated in 1937 by Bhabha and Heitler ${ }^{1}$ and Carlson and Oppenheimer ${ }^{2}$ they were interested mainly in the mean number and the fluctuation problem was raised only incidentally. For the calculation of moments it is still possible to use the standard method of studying the variation of the statistical distribution of particles with $t$ provided such a distribution is defined by what are known as product densities which are much easier to handle than the $\pi$ functions. We shall first define the product density functions and instead of describing the general method of application, we shall deal with two important processes, i.e. the nucleon and electron-photon cascades.

\section{Method of product density functions ${ }^{3}$}

If the particles are distributed in the $E$-space which is a continuum, a function $f_{n}\left(E_{1}, E_{2}, \ldots, E_{n}\right)$ called the product density of degree $n$ is defined such that $f_{n}\left(E_{1}, E_{2}, \ldots, E_{n}\right) \mathrm{d} E_{1} \mathrm{~d} E_{2} \ldots \mathrm{d} E_{n}$ represents the probability that there is a

1 H. J. Bhabha and W. Heitler, Proc. Roy. Soc. A 159, 432 (1937).

2 J. F. Carlson and J. R. Oppenhermer, Phys. Rev. 51, 220 (1937).

3 Alladr Ramakrishnan, Proc. Camb. Phil. Soc. 46, 595 (1950). 
particle in $\mathrm{d} E_{1}$, a partiele in $\mathrm{d} E_{2} \ldots$, and a particle in $\mathrm{d} E_{n}$ irrespective of the number elsewhere. $f_{n}$ is not a probability density but $f_{n}\left(E_{1}, E_{2}, \ldots, E_{n}\right) \mathrm{d} E_{1} \ldots \mathrm{d} E_{n}$ is a probability magnitude. Therefore it is possible to write equations for $f_{n}$ by studying the behaviour of individual particles at $E_{1}, E_{2}, \ldots, E_{n}$.

If $N(E)$ is the stochastic variable representing the number of particles with energy above $E$ then

$$
\mathscr{E}\left\{[N(E)]^{r}\right\}=\sum_{s=1}^{r} C_{\delta}^{r} \int_{E}^{\infty} \int_{E}^{\infty} \cdots \int_{E}^{\sim} f_{s}\left(E_{1}, E_{2}, \cdots, E_{s}\right) \mathrm{d} E_{1} \mathrm{~d} E_{2} \cdots \mathrm{d} E_{s}
$$

where $\mathscr{E}$ denotes the expectation value and $C_{s}^{r}$ are the coefficients defined by the identity

$$
M^{r}=\sum_{s=1}^{r} C_{s}^{r} M(M-1) \cdots(M-s+1) .
$$

$M$ being a positive integer. The coefficients are obtained by setting $M=1,2, \ldots$

It is clear that for the caleulation of the $n$-th moment of $N(E)$ a knowledge of the product densities of degrees less than or equal to $n$ is necessary. In cosmic ray problems, sinee we are dealing with two types of particles electrons and photons, we define $f_{n}\left(E_{1}, E_{2}, \ldots, E_{n} ; t\right)$ and $g_{n}\left(E_{1}, E_{2}, \ldots, E_{n} ; t\right)$ as product densities of degree $n$ of electrons and photons respectively, and $f g_{n, m}\left(E_{1}, E_{2}, \ldots, E_{n+m} ; t\right)$ as the mixed product density of degree $(n, m)$ of electrons and photons, i.e.

$$
f g_{n, m}\left(E_{1}, E_{2}, \ldots, E_{n+m} ; t\right) \mathrm{d} E_{1} \mathrm{~d} E_{2} \ldots \mathrm{d} E_{n+m}
$$

is the probability that there is an electron in $\mathrm{d} E_{1}$, an electron in $\mathrm{d} E_{2}, \ldots$, an electron in $\mathrm{d} E_{n}$, a photon in $\mathrm{d} E_{n+1}$, a photon in $\mathrm{d} E_{n+2}, \ldots$ and a photon in d $E_{n+m}$.

Since $f_{n} \mathrm{~d} E_{1} \mathrm{~d} E_{2}, \ldots \mathrm{d} E_{n}$ is a probability magnitude, its variation with $t$ can be expressed in terms of the $f, g$ and $f g$ functions. Equations involving product densities of order greater than two are not of immediate interest since the analytical solution of $f_{2}, g_{2}$ and $f g_{1,1}$ is itself a long and tedious process.

Closely allied to the product density functions are the density functions of Janossy ${ }^{1}$ defined in an attempt to treat the fluctuation problem of nucleon cascades. He defined a function $J_{h}\left(E_{1}, E_{2}, \ldots, E_{h}\right) \mathrm{d} E_{1} \mathrm{~d} E_{2} \ldots \mathrm{d} E_{h}$ representing the probability that there is one particle in $\mathrm{d} E_{1}$, one in $\mathrm{d} E_{2}$, and one in $\mathrm{d} E_{h}$ and none in the other energy states. $J_{h}$ is not a probability density. Janossy deals with $J_{h} / h$ ! but it is $J_{h}$ that has a physical significance since the particles are indistinguishable.

The Janossy functions $J_{h}$ are related to the product density functions $f_{h}$ as follows: ${ }^{2}$

$$
\begin{array}{r}
f_{h}\left(E_{1}, E_{2}, \cdots, E_{h}\right)=\sum_{n} \frac{1}{(n-h) !} \int_{E_{n}} \int_{E_{n-1}} \ldots \int_{E_{h+1}} J_{n}\left(E_{1}, E_{2}, \cdots, E_{n}\right) . \\
\mathrm{d} E_{1} \mathrm{~d} E_{2} \cdots \mathrm{d} E_{n} .
\end{array}
$$

1 L. Jaxossy, Proc. Roy. Irish. Acad. A 53, 181 (1950).

2 A detailed account of the connection between $f_{h}$ and $J_{h}$ is given by Alladi RamakrishnaN in Proc. Camb. Phil. Soc. 48, 451 (1952). 
The probability $\pi(n, E)$ that there are $n$ particles above an energy $E$ is given by

$$
\pi(n, E)=\frac{1}{n !} \int_{E}^{\infty} \int_{E}^{\infty} \cdots \int_{E}^{\infty} J_{n}\left(E_{1}, E_{2}, \cdots, E_{n}\right) \mathrm{d} E_{1} \mathrm{~d} E_{2} \cdots \mathrm{d} E_{n} .
$$

Since it is difficult to obtain the solution of $J_{n}$ for any $n$ (in fact it is only possible to obtain the solution for small values of $n$, by iterative methods) we have to content ourselves with the first few moments of the number of particles above a certain energy. To calculate the $p$ th moment, we have to know $J_{n}$ for all values of $n$ while we need only product densities of degree less than or equal to $p$. Hence for the calculation of moments which is the only feasible task we need only the product densities.

We shall first take up the application of the product density technique to the case of the one particle cascade.

\section{Cascades involving only one type of particle ${ }^{1}$}

We shall assume that the cascade consists only of one type of particles, i.e. nucleons. Given the initial energy spectrum of nucleons at $t=0$ and that a nucleon of energy $E$ drops to an interval between $E_{i}$ and $E_{i}+\mathrm{d} E_{i}$ creating a recoil nucleon of energy between $E_{j}$ and $E_{j}+\mathrm{d} E_{j}$ with a probability per unit distance given by $\omega\left(E_{i}, E_{j} \mid E\right) \mathrm{d} E_{i} \mathrm{~d} E_{j}$, our object is to calculate the moments of the distribution of the number of nucleons above a certain energy. Since we cannot distinguish between the recoil nucleon and the incident nucleon, we can define

$$
W\left(E_{i}, E_{j} \mid E\right)=\omega\left(E_{i}, E_{j} \mid E\right)+\omega\left(E_{j}, E_{i} \mid E\right)
$$

where $W\left(E_{i}, E_{j} \mid E\right)$ represents the probability per unit distance that a particle of energy $E$ is replaced by a pair one of which has an energy $E_{i}$ and another $E_{j}$. Let us write the diffusion equation for $f_{n}\left(E_{1}, E_{2}, \ldots, E_{n} ; t\right)$ the product density of degree $n, f_{n}\left(E_{1}, E_{2}, \ldots, E_{n} ; t\right) \mathrm{d} E_{1} \mathrm{~d} E_{2} \ldots \mathrm{d} E_{n}$ representing the probability that there is a nucleon in the interval $\mathrm{d} E_{1}$, a nucleon in $\mathrm{d} E_{2}, \ldots$ and a nucleon in the interval $\mathrm{d} E_{n}$ irrespective of the number of nucleons elsewhere. We have

$$
\begin{aligned}
\frac{\partial f_{n}\left(E_{1}, E_{2}, \cdots, E_{n} ; t\right)}{\partial t} & =-f_{n}\left(E_{1}, E_{2}, \cdots, E_{n} ; t\right) \sum_{i=1}^{n} W\left(E_{i}\right) \\
& +\int_{E} f_{n}\left(E_{1}^{\prime}, E_{2}^{\prime}, \cdots, E_{n-1}^{\prime}, E ; t\right) W\left(E_{i} \mid E\right) \mathrm{d} E \\
& +\int_{E} f_{n-1}\left(E_{1}^{\prime \prime}, E_{2}^{\prime \prime}, \ldots, E_{n-2}^{\prime \prime}, E ; t\right) W\left(E_{i}, E_{j} \mid E\right) \mathrm{d} E
\end{aligned}
$$

1 M. S. Bartlett, An Introduction to Stochastic Processes, Cambridge University Press (1955); Alladi Ramakrishnan. Proc. Camb. Phil. Soc. 48, 451 (1952). 
where

$$
\begin{aligned}
W\left(E_{i}\right) & =\int_{E_{j}} \int_{E_{k}} W\left(E_{j}, E_{k} \mid E_{i}\right) \mathrm{d} E_{j} \mathrm{~d} E_{k} \\
W\left(E_{i} \mid E\right) & =\int_{k_{j}} W\left(E_{i}, E_{j} \mid E\right) \mathrm{d} E_{j}
\end{aligned}
$$

and $E_{k}^{\prime}$ for $k=1,2, \ldots n-1$, represents the values $E_{k}$ for $k=1,2, \ldots, n$ omitting $E_{i}$ and $E_{k}^{\prime \prime}$ indicates omission of two variables. The equation is derived by just considering what happens to each of the nucleons envisaged in the function $f_{n} \cdot{ }^{1}$

Assuming that $W^{\prime}\left(E_{i}, E_{j} \mid E\right) \mathrm{d} E_{i} \mathrm{~d} E_{j}$ ean be expressed in the form $W\left(\varepsilon_{i}, \varepsilon_{j}\right) \mathrm{d} \varepsilon_{i} \mathrm{~d} \varepsilon_{j}$ where $\varepsilon_{i}=\frac{E_{i}}{E}$ and $\varepsilon_{j}=\frac{E_{j}}{E}$, the formal solution of these equations can be obtained by the Mellin transform technique. Setting

$$
\int_{0}^{1} \int_{0}^{1} W\left(\varepsilon_{i}, \varepsilon_{j}\right) \mathrm{d} \varepsilon_{i} \mathrm{~d} \varepsilon_{j}==1
$$

by suitably choosing the unit of $t$ and defining

$$
a\left(s_{i}, s_{j}\right)=\int_{0}^{1} \int_{0}^{1} W\left(\varepsilon_{i}, \varepsilon_{j}\right) \varepsilon_{i}^{8 i-1} \varepsilon_{j}^{8 j-1} \mathrm{~d} \varepsilon_{i} \mathrm{~d} \varepsilon_{j}
$$

$c_{n}\left(s_{1}, s_{2}, \ldots, s_{n} ; t\right)=\int \ldots \int f_{n}\left(E_{1}, E_{2}, \ldots, E_{n} ; t\right) E_{1}^{s_{1}-1} \ldots E_{n}^{s_{n}-1} \mathrm{~d} E_{1} \ldots \mathrm{d} E_{n}$

we obtain the equation

$$
\begin{aligned}
& \frac{\partial}{\partial t} c_{n}\left(s_{1}, s_{2}, \ldots, s_{n} ; t\right)=-n c_{n}\left(s_{1}, s_{2}, \ldots, s_{n} ; t\right)+\sum_{i} c_{n}\left(s_{1}^{\prime}, s_{2}^{\prime}, \ldots, s_{n-1}^{\prime}, s_{i} ; t\right) \\
& \times\left\{a\left(s_{i}, 1\right)+a\left(1, s_{i}\right)\right\}+\sum_{\substack{i \\
i \neq j}} \sum_{\substack{i \\
j}} c_{n-1}\left(s_{1}^{\prime \prime}, s_{2}^{\prime \prime}, \ldots, s_{n-2}^{\prime \prime} ; s_{i}+s_{j}-1 ; t\right) a\left(s_{i}, s_{j}\right) .
\end{aligned}
$$

In particular we solve for $c_{1}$ and $c_{2}$ in the case of a cascade generated by a single particle of energy $E_{0}$ :

$$
\begin{aligned}
c_{1}\left(s_{1} ; t\right) & =E_{0}^{\delta_{1}-1} \mathrm{e}^{-A_{s_{1}} \cdot t} \\
A_{8} & =1-a\left(s_{1}, 1\right)-a\left(1, s_{1}\right) \\
c_{2}\left(s_{1}, s_{2} ; t\right) & =E_{0}^{s_{1}+s_{2}-2}\left\{\frac{a\left(s_{1}, s_{2}\right)+a\left(s_{2}, s_{1}\right)}{A_{s_{1}}+A_{s_{2}}+A_{s_{1}+s_{2}-1}}\right\} \times\left\{\mathrm{e}^{\left.-A_{s_{1}+s_{2}-1}-\mathrm{e}^{-\left(A_{8_{1}}+A_{8_{2}}\right) \cdot t}\right\}}\right.
\end{aligned}
$$

and the mean and mean square numbers of particles above the energy $E$ is given by

$$
\begin{aligned}
\mathscr{E}\{N(E ; t)\} & =\int_{E}^{\infty} f_{1}\left(E_{1} ; t\right) \mathrm{d} E_{1} \\
\mathscr{E}\left\{[N(E ; t)]^{2}\right\} & =\mathscr{E}\{N(E ; t)\}+\int_{E}^{\infty} \int_{E}^{\infty} f_{2}\left(E_{1}, E_{2} ; t\right) \mathrm{d} E_{1} \mathrm{~d} E_{2} .
\end{aligned}
$$

1 A detailed derivation of this is given in the section on electron-photon cascades. 
$f_{1}$ and $f_{2}$ are obtained by inverting $c_{1}$ and $c_{2}$ as follows:

$$
\begin{aligned}
f_{1}(E ; t) & =\frac{1}{2 \pi i} \int_{\gamma-i \infty}^{\gamma+i \infty} c_{1}\left(s_{1}, t\right) E^{-s_{1}} \mathrm{~d} s_{1} \\
f_{2}\left(E_{1}, E_{2} ; t\right) & =\frac{1}{2 \pi i} \iint c_{1}\left(s_{1}, s_{2} ; t\right) E_{1}^{-s_{1}} E_{2}^{-s_{2}} \mathrm{~d} s_{1} \mathrm{~d} s_{2} .
\end{aligned}
$$

We have considered only a process in which the fundamental cross-section is defined for the production of two particles. Quite generally we can define a cross-section ${ }^{1}$

$$
W_{n}\left(E_{1}, E_{2}, \ldots, E_{n} \mid E_{0}\right) \mathrm{d} E_{1} \mathrm{~d} E_{2}, \ldots, \mathrm{d} E_{n} \mathrm{~d} t
$$

that $n$ particles are produced in the ranges $\mathrm{d} E_{1}, \mathrm{~d} E_{2}, \ldots, \mathrm{d} E_{n}$ and none elsewhere by a particle of energy $E_{0}$ in passing through $\mathrm{d} t$. The total cross-section is given by

$$
W=\sum_{n} \frac{1}{n !} \int_{E_{1}} \ldots \int_{E_{n}} W_{n}\left(E_{1}, E_{2}, \ldots, E_{n} \mid E_{0}\right) \mathrm{d} E_{1} \ldots \mathrm{d} E_{n} .
$$

The mean number of particles produced in $\mathrm{d} t$ in the energy interval $\mathrm{d} E_{1}$ is given by

and

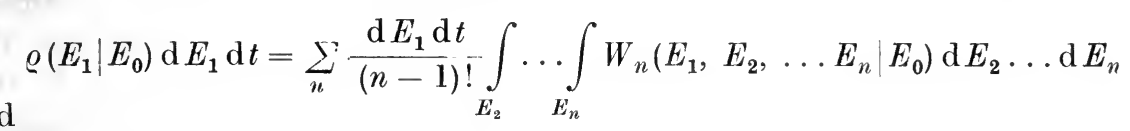

$\varrho\left(E_{0}\right)=\int_{E_{1}} \varrho\left(E_{1} \mid E_{0}\right) \mathrm{d} E_{1}=\sum_{n} \frac{1}{n !} \int_{E_{1}} \ldots \int_{E_{n}} n W_{n}\left(E_{1}, E_{2}, \ldots E_{n} \mid E_{0}\right) \mathrm{d} E_{1} \ldots \mathrm{d} E_{n}$.

From the above equation it is quite obvious that we can have processes in which the fundamental cross-sections $W_{n}$ 's differ and are represented by say $W_{n}^{A}$ and $W_{n}^{B}$ but yield the same $\varrho\left(E_{1} \mid E_{0}\right)$.

For example when

and

$$
\left.\begin{array}{c}
W_{n}^{B}=0 \text { if } n \neq 1 \\
W_{1}^{B} \equiv \varrho\left(E_{1} \mid E_{0}\right)
\end{array}\right\}
$$

the function representing the mean density is also the cross-section for the $\mathrm{r}$ ep lace ment of a particle of energy $E_{0}$ by that of energy $E_{1}$. The equations obeyed by the product densities, including degree one, will however be different since the total cross-section $W$ is different in both the cases, and this $W$ occurs in the equations for product densities. Thus even for writing the equations for mean number it is not enough to know the $\varrho$ for the process but it is also necessary to know the detail ed cross-sections $W_{n}{ }^{2}$

1 Alladi Ramakrishnan and K. Venkatesan, Proc. Cosmic Ray Symposium, D.A.E., Govt. of India (1961).

2 This fact may prove helpful in gleaning the multiplicative nature of the cross-section even from the mean number. 


\section{Electron-photon caseades}

It was in the study of cosmic ray showers consisting of electrons and photons that the easeade theory was first put forward by Carlson and Oppenheimer and independently by Bhabha and Heitler. In their original formulation, Bhabha and Heitler assumed only the two quantum electrodynamic processes, Bremsstrahlung and pair production. In the later treatments of Bhabha and Chakrabarthy $y^{1}$, ionization loss was included. It is customary to assume that $\beta \mathrm{d} t$, where $\beta$ may be a function of $E$, is the deterministic loss in the energy of a particle of energy $E$ in passing through matter of thickness $\mathrm{d} t$. The inclusion of a deterministic loss introduces, as we shall see, a new mathematical feature in the theory.

In view of the comparative completeness of the theory of soft cascades we shall summarize the theory following closely Bhabha and Chakrabarthy. Though considerable refinements have been made later, their formulation describes all the general features of the behaviour of the soft component of cosmic rays. The new refinements do not carry us any further from the point of view of numerical calculations and understanding of the physical phenomena.

Our object in the first instance is to calculate the mean energy spectrum of electrons and photons emerging at thickness $t$ defined by the functions $f_{1}(E ; t)$ and $g_{1}(E ; t)$ such that $f_{1}(E ; t) \mathrm{d} E$ and $g_{1}(E ; t) \mathrm{d} E$ represent the mean number of electrons and photons in the range $d E$ respectively. We assume that the probability that an electron of energy $E$ drops to an energy between $E^{\prime}$ and $E^{\prime}+\mathrm{d} E^{\prime}$ emitting a photon of energy $E-E^{\prime}$ while passing through matter of thickness $\mathrm{d} t$ is

$$
\frac{4 Z^{2}}{137}\left(\frac{e^{2}}{m c^{2}}\right)^{2} \log \left(183 Z^{-1 / 3} R^{(1)}\left(E^{\prime} \mid E\right) \mathrm{d} E^{\prime}\right.
$$

where

$$
R^{(1)}\left(E^{\prime} \mid E\right) \mathrm{d} E^{\prime}=\left\{\frac{E-E^{\prime}}{E}-\left(\frac{4}{3}+\alpha\right)\left(1-\frac{E}{E-E^{\prime}}\right)\right\} \frac{\mathrm{d} E^{\prime}}{E}
$$

and $Z e$ is the charge of the nuclei of the material traversed. It is convenient to choose the characteristic unit of length for the medium traversed by the electron as

$$
\frac{4 Z^{2}}{137}\left(\frac{e^{2}}{m c^{2}}\right)^{2} \log \left(183 Z^{-1 / 3}\right)
$$

so that the differential cross-section is now given by $R^{(\mathbf{1})}$. The only parameter depending on the material traversed is $\alpha$. The above is valid in the case of complete screening of the nucleus by the surrounding atomic electrons. It is to be noted that this cross-section is asymmetric in $E$ and $E-E^{\prime}$. The corresponding probability that a photon gives rise to an electron of energy $E^{\prime}$ and a positron of energy $E-E^{\prime}$ with the same characteristic unit of length is given by

$$
R^{(2)}\left(E^{\prime} \mid E\right) \mathrm{d} E^{\prime}=\left[1-\left(\frac{4}{3}+\alpha\right)\left(\frac{E^{\prime}}{E}-\frac{E^{\prime 2}}{E^{2}}\right)\right] \frac{\mathrm{d} E^{\prime}}{E} .
$$

1 H. J. Bhabha and S. K. Chakrabarthy, Proc. Roy. Soc. A 181, 267 (1943). 
If $f_{1}(E ; t)$ is the density (product density of degree one) of the positive and negative electrons of energy $E$ and $g_{1}(E ; t)$ of the photons of energy $E$ at $t$, they satisfy the equations

$$
\begin{aligned}
\frac{\partial f_{1}}{\partial t}(E ; t)= & -f_{1}(E ; t) \int_{0}^{E} R^{(1)}\left(E^{\prime} \mid E\right) \mathrm{d} E^{\prime} \\
& +\int_{E}^{\infty} f_{1}\left(E^{\prime} ; t\right) R^{(1)}\left(E \mid E^{\prime}\right) \mathrm{d} E^{\prime}+2 \int_{E}^{\infty} g_{1}\left(E^{\prime} ; t\right) R^{(2)}\left(E \mid E^{\prime}\right) \mathrm{d} E^{\prime} \\
& +\frac{\partial}{\partial E}\left\{\beta f_{1}(E ; t)\right\} . \\
\frac{\partial g_{1}}{\partial t}(E ; t)= & -g_{1}(E ; t) \int_{0}^{E} R^{(2)}\left(E^{\prime} \mid E\right) \mathrm{d} E^{\prime} \\
& +\int_{E}^{\infty} f_{1}\left(E^{\prime} ; t\right) R^{(1)}\left(E^{\prime}-E \mid E^{\prime}\right) \mathrm{d} E^{\prime} .
\end{aligned}
$$

The factor 2 in (30) arises because

$$
R^{(2)}\left(E \mid E^{\prime}\right)=R^{(2)}\left(E^{\prime}-E \mid E^{\prime}\right) .
$$

The first term on the right hand side of the equation (30) gives the depletion of the electrons from the range $\mathrm{d} E$ due to radiation; the second, the influx into this range from electrons of higher energy; the third term, the contribution from the photon (by pair creation) while the fourth term is due to ionization loss. This term is obtained as follows: particles in the neighbourhood of the range of width $\Delta\left(1+\frac{\partial \beta}{\partial E} \mathrm{~d} t\right)$ at energy $E+\frac{\partial \beta}{\partial E} \mathrm{~d} t$ after penetration of thickness $\mathrm{d} t$ will have an energy $E$ in the range $\Delta$. Thus the difference between particles in the range of width $\Delta$ in the neighbourhood of $E$ at thicknesses $t$ and $t+\mathrm{d} t$ is given by

$$
f_{1}(E+\beta \mathrm{d} t ; t)\left(1+\frac{\partial \beta}{\partial E} \mathrm{~d} t\right) \Delta-f_{1}(E ; t) \Delta=\left(\beta \frac{\partial f_{1}}{\partial E}+f_{1} \frac{\partial \beta}{\partial E}\right) \Delta \cdot \partial t .
$$

Similarly the terms in the second equation can be interpreted.

Noting that $R^{(1)}\left(E^{\prime} \mid E\right) \mathrm{d} E^{\prime}$ is homogeneous in $E$ and $E^{\prime}$, that is, it can be written as $R^{(1)}(\varepsilon) \mathrm{d} \varepsilon$ where $\varepsilon=\frac{E^{\prime}}{E}$ we can reduce the above equations by the Mellin transform. Defining the Mellin transforms of $f_{1}$ and $g_{1}$ as

$$
\begin{aligned}
& p(s ; t)=\int_{0}^{\infty} E^{s-1} f_{1}(E ; t) \mathrm{d} E \\
& q(s ; t)=\int_{0}^{\infty} E^{\delta-1} g_{1}(E ; t) \mathrm{d} E
\end{aligned}
$$


we find that $p(s ; t)$ and $q(s ; t)$ satisfy the equations

$$
\begin{aligned}
& \frac{c}{\partial t} p(s ; t)=-A_{s} p(s ; t)+B_{8} q(s ; t)-\beta(s-1) p(s-1 ; t) \\
& \frac{\partial}{\partial t} q(s ; t)=C_{s} p(s ; t)-D q(s ; t)
\end{aligned}
$$

where

$$
\begin{aligned}
& A_{g}-\int_{0}^{1}\left\{R^{(1)}(\varepsilon)\left(1-\varepsilon^{(8-1)}\right) \mathrm{d} \varepsilon\right. \\
& =\left(\frac{4}{3}+\alpha\right)\left\{\frac{\mathrm{d}}{\mathrm{d} s} \log \Gamma(s)+\gamma-1+\frac{1}{s}\right\}+\frac{1}{2}-\frac{1}{s(s+1)} \\
& B_{s}=2\left(\frac{1}{s}+\left(\frac{4}{3}+\alpha\right) \frac{1}{(s+1)(s+2)}\right) \\
& C_{s}=\frac{1}{s+1}+\left(\frac{4}{4}+x\right) \frac{1}{s(s+1)} \\
& \text { D) }=\frac{7}{9}+\frac{1}{6} \alpha
\end{aligned}
$$

$\gamma$ being the Euler-Mascheroni constant.

The essential difficulty in solving the differential equation $(36)$ for $p(s ; t)$ is due to the occurrence of the term $p(s-1 ; t)$. Since $s$ is a complex variable, it is not possible to use any familiar iteration procedure. Bhabha and Chakrabarthy have obtained the solution for this equation, first by obtaining $p_{0}(s ; t)$ the solution without ionization loss and therefrom the general solution with $\beta \neq 0$.

Eliminating $q(s ; t)$ from the above equations (36) and (37), we have for $\beta=0$.

$$
\frac{\partial^{2}}{\partial t^{2}} p_{0}(s ; t)=-\left(A_{s}+D\right) \frac{\partial}{\partial t} p_{0}(s ; t)=\left(A_{s} D-B_{8} C_{s}\right) p_{0}(s ; t) \text {. }
$$

This has the solution

$$
p_{0}(s, t)=a_{8} \mathrm{e}^{-\lambda_{s} t}+b_{s} \mathrm{e}^{-\mu_{s} t}
$$

$a_{8}, b_{8}$ being independent of $t$ and $\mu_{8}, \lambda_{8}$ being the roots of the equation

i.e.

$$
\lambda_{8}^{2}-\left(A_{8}+D\right) \lambda_{8}+C\left(A_{8}-B_{8} C_{s}\right)=0,
$$

$$
\begin{aligned}
& \lambda_{8}=\frac{1}{2}\left(A_{s}+D\right)+\frac{1}{2}\left\{\left(A_{8}-D\right)^{2}+4 B_{8} C_{s}\right\}^{1 / 2} \\
& \mu_{8}=\frac{1}{2}\left(A_{8}+D\right)-\frac{1}{2}\left\{\left(A_{s}-D\right)^{2}+4 B_{8} C_{8}\right\}^{1 / 2}
\end{aligned}
$$

and $a_{8}$ and $b_{s}$ are determined by the initial conditions. For a shower initiated by an electron of energy $E_{0}$ we have

$$
\begin{gathered}
f_{1}(E ; 0)=\delta\left(E-E_{0}\right) ; \quad g_{1}(E ; 0)=0 \\
p(s ; 0)=E_{0}^{s-1} ; \quad q(s ; 0)=0 \\
\frac{\partial}{\partial t}\{p(s, t)\}_{t=0}=-A_{8} E_{0}^{8-1} .
\end{gathered}
$$


Imposing the above boundary condition we obtain

where

$$
p_{0}(s, t)=E_{0}^{s-1} \psi_{0}(s, t)
$$

$$
\psi_{0}(s, t)=\left\{\frac{D-\lambda_{s}}{\mu_{s}-\lambda_{s}} \mathrm{e}^{-\lambda_{s} t}+\frac{\mu_{s}-D}{\mu_{s}-\lambda_{s}} \mathrm{e}^{-\mu_{s} t}\right\} .
$$

The solution of (30) which includes ionization loss can be shown to be

$$
f_{1}(E ; t)=\frac{1}{2 \pi i E_{0}} \int_{\substack{\varepsilon \\ \sigma-i \infty}}^{\sigma+i \infty}\left(\frac{E_{0}}{E}\right)\left\{\sum_{n=0}^{\infty}\left(\frac{-\beta}{E}\right)^{n} \frac{\Gamma(s+n)}{\Gamma(s)} \psi_{n}(s ; t)\right\} \mathrm{d} s
$$

where the function $\psi_{n}(s ; t)$ satisfies the recurrence relation

$$
\psi_{n}(s ; t)=\int_{0}^{t} \psi_{0}\left(s+n ; t-t^{\prime}\right) \psi_{n-1}\left(s ; t^{\prime}\right) \mathrm{d} t^{\prime}
$$

which can also be written in the form

$$
\psi_{n}(s ; t)=\int_{0}^{t} \psi_{0}(s ; t) \psi_{n-1}\left(s+1 ; t-t^{\prime}\right) \mathrm{d} t^{\prime}
$$

$\psi_{n}$ and $\psi_{n-1}$ satisfy the equation

$$
\begin{gathered}
\left\{\frac{\partial}{\partial t^{2}}+\left(A_{s+n}+D\right) \frac{\partial}{\partial t}+\left(A_{s+n} D-B_{s+n} C_{s+n}\right)\right\} \psi_{n}(s ; t) \\
=\left(\frac{\partial}{\partial t}+D\right) \psi_{n-1}(s ; t) .
\end{gathered}
$$

The series within the curly brackets in the expression for $f_{1}(E ; t)$ is uniformly and absolutely convergent if $E>\beta t$. It also follows that the integral on the contour $c$ is convergent, so that $f_{1}(E ; t)$ is a well defined function of $E$ and $t$ and hence the summation and integration can be interchanged.

We now introduce the functions

$$
\begin{gathered}
\varphi(s ; t)=\frac{\psi_{1}(s ; t)}{\psi_{0}(s ; t)} \\
h_{n}(s ; t)=\left\{\psi_{0}(s ; t) \frac{\varphi^{n}}{n !}-\psi_{1}(s ; t) \frac{\varphi^{n-1}}{(n-1) !}+\cdots\right\}
\end{gathered}
$$

and write$$
f_{1}\left(E_{1} t\right)=\frac{1}{2 \pi i E_{0}} \int_{\sigma-i \infty}^{\sigma+i \infty}\left(\frac{E_{0}}{E+\beta \varphi}\right)^{s}\left\{\sum_{n=0}^{\infty}\left(\frac{\beta}{E+\beta \varphi}\right)^{n} \frac{\Gamma(s+n)}{\Gamma(s)} h_{n}(s ; t)\right\} \mathrm{d} s .
$$

The solution (55) is an analytic continuation of (49) and the restriction $E>\beta t$ can be dropped, so that (55) is the exact solution of (30) for all $E \geqq 0$. 
'To get the total number of particles with energy greater than $E$ we integrate (55) from $E$ to $E_{0}$ and obtain

$$
\varepsilon\{N(E ; t)\}=\frac{1}{2 \pi i} \int_{\sigma-i \infty}^{\sigma+i \infty} \frac{1}{s-1}\left(\frac{E_{0}}{E+\beta \varphi}\right)^{k-1}\left\{\sum_{\substack{n=0 \\ \Gamma(s+n)}}^{\left.\frac{\beta}{\Gamma+\beta \varphi}\right)^{n}}\right.
$$

This expression can be written as

where

$$
\varepsilon\{N(E ; t)\}=\sum_{n=0}^{\infty} N_{n}(E ; t)
$$

$$
\begin{aligned}
& N_{n}(E ; t)=\frac{1}{2 \pi i} \int_{\sigma-i \infty}^{\sigma+i \infty} \frac{1}{s+n-1}\left(\frac{E_{0}}{\beta}\right)^{s-1} \\
& \times\left(\frac{\beta}{E+\beta \varphi}\right)^{s+n-1} \frac{I^{\prime}(s+n)}{\Gamma(s)} h_{n}(s ; t) \mathrm{d} s .
\end{aligned}
$$

Multiplying equation (30) and (31) by $E$ and integrating with respect to $E$ from 0 to $\infty$ and adding these two equations, we get

$$
\begin{gathered}
\frac{\partial}{\partial t}\left\{\int_{0}^{\infty} E f_{1}(E ; t) \mathrm{d} E+\int_{0}^{\infty} E g_{1}(E ; t) \mathrm{d} E\right\} \\
=-\beta \int_{0}^{\infty} f_{1}(E ; t) \mathrm{d} E \\
=-\beta N(0 ; t) .
\end{gathered}
$$

In other words, the change in the total energy of the shower is entirely due to collision loss. Integrating (57) with respect to $t$ from 0 to $\infty$, we get for a shower initiated by an electron of energy $E_{0}$,

$$
\int_{0}^{t} N(0 ; t) \mathrm{d} t=\frac{E_{0}}{\beta} .
$$

We shall now take up the calculation of the fluctuations about the mean number. For this purpose we have to obtain the diffusion equations for the product densities of degree two and obtain therefrom the mean square number and the deviation from the mean number of particles above a certain energy. ${ }^{1}$ Numerical results, as complete as for mean number, are available only when we neglect the ionization loss. ${ }^{2}$ The equations including ionization loss can be formally written down but then explicit analytical solution has not been obtained till now in a manner suitable for numerical computation.

1 H. J. Bhabea and A. Ramakrishnan, Proc. Ind. Acad. Sci. 32, 141 (1950).

2 A. Ramakrishnay and P. M. Mathews, Progr. Theoret. Phys. 11, 95 (1954). 
Equations for $f_{2}$ and $g_{2}$, the product densities of degree two of electrons and photons and mixed product density $f g_{1,1}$ can be written as follows:

$$
\begin{aligned}
& \frac{\partial f_{2}}{\partial t}\left(E_{1}, E_{2} ; t\right)=-f_{2}\left(E_{1}, E_{2} ; t\right)\left\{\int_{0}^{E_{1}} R^{(1)}\left(E \mid E_{1}\right) \mathrm{d} E+\int_{0}^{E_{2}} R^{(1)}\left(E \mid E_{2}\right) \mathrm{d} E\right\} . \\
& +\int_{E_{1}}^{\infty} f_{2}\left(E, E_{2} ; t\right) R^{(1)}\left(E_{1} \mid E\right) \mathrm{d} E+\int_{E_{2}}^{\infty} f_{2}\left(E_{1}, E ; t\right) R^{(1)}\left(E_{2} \mid E\right) \mathrm{d} E \\
& +2 \int_{E_{2}}^{\infty} f g_{1,1}\left(E_{1}, E ; t\right) R^{(2)}\left(E_{2} \mid E\right) \mathrm{d} E+2 \int_{E_{1}}^{\infty} f g_{1,1}\left(E_{2}, E ; t\right) R^{(2)}\left(E_{1} \mid E\right) \mathrm{d} E \\
& +2 g_{1}\left(E_{1}+E_{2} ; t\right) R^{(2)}\left(E_{1} \mid E_{1}+E_{2}\right) \\
& \frac{\partial f g_{1,1}}{\partial t}\left(E_{1}, E_{2}, t\right)=-f g_{1,1}\left(E_{1}, E_{2} ; t\right)\left\{\int_{0}^{E_{1}} R^{(1)}\left(E \mid E_{1}\right) \mathrm{d} E+\int_{0}^{E_{2}} R^{(2)}\left(E \mid E_{2}\right) \mathrm{d} E\right\} \\
& +\int_{E_{1}}^{\infty} f g_{1,1}\left(E, E_{2} ; t\right) R^{(1)}\left(E_{1} \mid E\right) \mathrm{d} E+\int_{E_{1}}^{\infty} 2 g_{2}\left(E, E_{2} ; t\right) R^{(2)}\left(E_{1} \mid E\right) \mathrm{d} E \\
& +\int_{E_{2}}^{\infty} f_{2}\left(E_{1}, E ; t\right) R^{(1)}\left(E-E_{2} \mid E\right) \mathrm{d} E+f_{1}\left(E_{1}+E_{2} ; t\right) R^{(1)}\left(E_{1} \mid E_{1}+E_{2}\right) \\
& \frac{\partial \dot{f} g_{1,1}}{\partial t}\left(E_{1}, E_{2} ; t\right)=-f g_{1,1}\left(E_{2}, E ; t\right)\left\{\int_{0}^{E_{2}} R^{(1)}\left(E \mid E_{2}\right) \mathrm{d} E+\int_{0}^{E_{1}} R^{(2)}\left(E \mid E_{1}\right) \mathrm{d} E\right\} \\
& +\int_{E_{2}}^{\infty} f g_{1,1}\left(E, E_{1} ; t\right) R^{(1)}\left(E_{2} \mid E\right) \mathrm{d} E+\int_{E_{2}}^{\infty} 2 g_{2}\left(E, E_{1} ; t\right) R^{(1)}\left(E_{2} \mid E\right) \mathrm{d} E \\
& +\int_{E_{1}}^{\infty} f_{2}\left(E_{2}, E ; t\right) R^{(1)}\left(E-E_{1} \mid E\right) \mathrm{d} E+f_{1}\left(E_{1}+E_{2} ; t\right) R^{(1)}\left(E_{2} \mid E_{1}+E_{2}\right) \\
& +\int_{E_{2}}^{\infty} f g_{1,1}\left(E, E_{1} ; t\right) R^{(1)}\left(E-E_{2} \mid E\right) \mathrm{d} E \\
& +\int_{E_{1}}^{\infty} f g_{1,1}\left(E, E_{2} ; t\right) R^{(1)}\left(E-E_{1} \mid E\right) \mathrm{d} E .
\end{aligned}
$$

It is to be noted that $f g_{1,1}\left(E_{1}, E_{2} ; t\right)$ is not equal to $f g_{1,1}\left(E_{2}, E_{1} ; t\right)$. The initial conditions are defined as

$$
f_{1}(E ; 0)=\delta\left(E-E_{0}\right) ; \quad g_{1}(E ; 0)=0,
$$

i.e. at $t=0$ there is only one primary of energy $E_{0}$ and

$$
f_{2}\left(E_{1}, E_{2} ; 0\right) \doteq 0 ; \quad g_{2}\left(E_{1}, E_{2} ; 0\right)=0 ; \quad f g_{1,1}\left(E_{1}, E_{2} ; 0\right)=0 .
$$

The various terms in the equations are written down by the now familiar arguments on studying the individual behaviour of the particles in the various energy 
ranges and estimating their contributions to the product densities. It is noteworthy that the equations for the product density of degree two contain on the right hand side terms involving produet density of degree one. In fact these are the terms that define the correlation between the particles in the two energy ranges.

(i) The term $2 g_{1}\left(E_{1}+E_{2} ; t\right) R^{(2)}\left(E_{1} \mid E_{1}+E_{2}\right)$ in (61) represents the contribution of product density of degree one to the produet density of degree two arising due to the ereation of a pair by a photon of energy $E_{1}+E_{2}$.

(ii) The term $f_{1}\left(E_{1}+E_{2} ; t\right) R^{(1)}\left(E_{1} \mid E_{1}+E_{2}\right)$ in (62) arises duc to the radiation of a photon of energy $E_{2}$ by an electron of energy $E_{1}+E_{2}$ and thus contributing to the mixed product density $f g_{1.1}\left(E_{1}, E_{2}\right)$.

(iii) The term $f_{1}\left(E_{1}+E_{2} ; t\right) R^{(1)}\left(E_{2} \mid E_{1}+E_{2}\right)$ in (63) represents the production of a photon of energy $E_{1}$ radiated by an electron of energy $E_{1}+E_{2}$, thus contributing to the mixed product density $f g_{1,1}\left(E_{2}, E_{1}\right)$.

(iv) The absence of a correlation term in equation (64) does not mean that the photons are uncorrelated since the product density equations are coupled.

If the correlation terms were absent, then the solutions for $f_{2}\left(E_{1}, E_{2} ; t\right)$ could have been written as $f_{2}\left(E_{1}, E_{2} ; t\right)=k f_{1}\left(E_{1} ; t\right) f_{1}\left(E_{2} ; t\right)$

and with the initial conditions the equations will yield the trivial solution zero for densities of all orders.

The above equations have been completely solved using the Mellin transform technique. We define the transforms,

$$
\begin{aligned}
v(r, t) & =\int_{0}^{\infty} E^{r-1} f_{1}(E ; t) \mathrm{d} E ; \quad \gamma(r, t)=\int_{0}^{\infty} E^{r-1} g_{1}(E ; t) \mathrm{d} E \\
\gamma_{2}(r, s, t) & =\int_{0}^{\infty} \mathrm{d} E \int_{0}^{\infty} \mathrm{d} E^{\prime} E^{r-1} E^{\prime-1} f_{2}\left(E, E^{\prime} ; t\right) \\
\gamma^{\prime} \gamma(r, s, t) & =\int_{0}^{\infty} \mathrm{d} E \int_{0}^{\infty} \mathrm{d} E^{\prime} E^{r-1} E^{\prime 8-1} f g_{1,1}\left(E, E^{\prime} ; t\right) \\
\gamma_{2}(r, s, t) & =\int_{0}^{\infty} \mathrm{d} E \int_{0}^{\infty} \mathrm{d} E^{\prime} E^{r-1} E^{\prime 8-1} g_{2}\left(E, E^{\prime} ; t\right) .
\end{aligned}
$$

The diffusion equations can be written in the matrix form as

$$
\frac{\mathrm{d}}{\mathrm{d} t} \varphi^{(2)}(r, s ; t)=\{\tau(r) \times I+I \times \tau(s)\} \varphi^{(2)}(r, s ; t)+\psi(r, s ; t)
$$

where

$$
\phi^{(2)}(r, s ; t)=\left(\begin{array}{c}
\nu_{2}(r ; s ; t) \\
\nu \gamma(r, s ; t) \\
\nu \gamma(s, r ; t) \\
\gamma_{2}(r, s ; t)
\end{array}\right) .
$$

$I$ is a $2 \times 2$ unit matrix, $\psi$ is a column vector defined by

$$
\psi(r, s: t)=\psi_{1}(r, s) E_{0}^{r+8-2} \mathrm{e}^{-\lambda_{r+s-1} t}+\psi_{2}(r, s) E_{0}^{r+s-2} \mathrm{e}^{-\mu_{r+s-1} t}
$$


where

$$
\begin{gathered}
\psi_{1}(r, s)=\frac{1}{\mu_{r+8-2}-\lambda_{r+s-1}}\left[\begin{array}{c}
a_{1}(r, s) C_{r+s-1} \\
a_{2}(r, s)\left(D-\lambda_{r+s-1}\right) \\
a_{2}(s, r)\left(D-\lambda_{r+8-1}\right) \\
0
\end{array}\right] \\
\psi_{2}(r, s)=\frac{1}{\mu_{r+8-1}-\lambda_{r+s-1}}\left[\begin{array}{c}
-a_{1}(r, s) C_{r+s-1} \\
a_{2}(r, s)\left(\mu_{r+8-1}-D\right) \\
a_{2}(s, r)\left(\mu_{r+s-1}-D\right) \\
0
\end{array}\right]
\end{gathered}
$$

and

$$
\begin{gathered}
a_{1}(r, s)=\frac{2 \Gamma(r) \Gamma(s)}{\Gamma(r-s)}\left\{1 \pi\left(\frac{4}{3}+\alpha\right) \frac{r s}{(r+s+1)(r+s)}\right\} \\
a_{2}(r, s)=\frac{\Gamma(r) \Gamma(s)}{\Gamma(r+s)}\left\{\frac{s}{r+s}+\left(\frac{4}{3}+\alpha\right) \frac{r}{s-1}\right\}
\end{gathered}
$$

According to the initial conditions we have

$$
\nu(s ; 0)=E_{0}^{s-1} ; \quad \gamma(s ; 0)=0 .
$$

Hence the solution for the inhomogeneous equation is given by

$$
\varphi^{(2)}(r, s ; t)=\zeta(r, s ; t) \int_{0}^{t} \zeta^{-1}\left(r, s ; t^{\prime}\right) \psi\left(r, s ; t^{\prime}\right) \mathrm{d} t^{\prime}
$$

where $\zeta$ is the solution of the homogeneous equation

$$
\frac{\mathrm{d} \zeta}{\mathrm{d} t}(r, s ; t)=\{\tau(r) \times I+I \times \tau(s)\} \zeta(r, s ; t) .
$$

Corresponding to the two parts $\psi_{1}$ and $\psi_{2}$ of $\psi, \varphi^{(2)}$ splits into $\varphi_{1}^{(2)}$ and $\varphi_{2}^{(2)}$. Thus we find that

$$
\begin{aligned}
& \varphi_{1}^{(2)}(r, s ; t)=E_{0}^{r+s-2} \zeta_{0}(r, s) \eta\left(\lambda_{r+s-1}\right) \zeta_{0}^{-1}(r, s) \psi_{1}(r ; s) \\
& \varphi_{2}^{(2)}(r, s ; t)=E_{0}^{r+s-2} \zeta_{0}(r, s) \eta\left(\mu_{r+8-1}\right) \zeta_{0}^{-1}(r, s) \psi_{2}(r, s)
\end{aligned}
$$

where $\eta(x)$ is the diagonal matrix with the four elements

$$
\frac{\mathrm{e}^{-x t}-\mathrm{e}^{-\left(\lambda_{r}+\lambda_{s}\right) t}}{\lambda_{r}+\lambda_{s}-x}, \frac{\mathrm{e}^{-x t}-\mathrm{e}^{-\left(\lambda_{r}+\mu_{s}\right) t}}{\lambda_{r}+\mu_{s}-x}, \frac{\mathrm{e}^{-x t}-\mathrm{e}^{-\left(\mu_{r}+\lambda_{s}\right) t}}{\mu_{r}+\lambda_{s}-x}, \frac{\mathrm{e}^{-x t}-\mathrm{e}^{-\left(\mu_{r}+\mu_{s}\right) t}}{\mu_{r}+\mu_{s}-x} .
$$

One very important feature of this matrix is that it remains finite even if any one of the denominators of its elements tends to zero.

From the theory of product densities the mean square number of electrons with energy greater than $E$ is given by

$$
\begin{gathered}
\mathscr{E}\left\{N^{2}(E ; t)\right\}=\mathscr{E}\{N(E ; t)\}+\int_{E}^{E_{0}} \int_{E}^{E_{0}} f_{2}\left(E_{1}, E_{2} ; t\right) \mathrm{d} E_{1} \mathrm{~d} E_{2} \\
f_{2}\left(E_{1}, E_{2} ; t\right)=\frac{1}{\left(2 \pi i E_{0}\right)^{2}} \int_{\sigma-i \infty}^{\sigma+i \infty} \int_{\sigma-i \infty}^{\sigma+i \infty} v_{2}(r, s) E_{1}^{-r} E_{2}^{-s} \mathrm{~d} r \mathrm{~d} s .
\end{gathered}
$$


Numerical calculations have been made using the following approximation as has been done even in the case of mean numbers for all but small thicknesses. ${ }^{1}$ Since the terms involving $\mathrm{e}^{-\left(\lambda_{r}+\lambda_{8}\right) t}$ and $\mathrm{e}^{-\lambda_{r+8-1} t}$ exceed those involving $\mu$ in the exponent by an order of magnitude it is found that terms involving $\mu$ can be neglected. Thus we have

$$
\begin{gathered}
\delta\left\{N^{2}(E ; t)\right\}=\frac{\delta\{N(E ; t)\}+\frac{1}{(2 \pi i)^{2}} \int_{\sigma-i \infty}^{\sigma+i \infty} \int_{\sigma-i \infty}^{\sigma+i \infty}\left(\frac{E_{0}}{E}\right)^{r+8-2}}{\frac{G(r, s ; t)}{(r-1)(s-1)} \mathrm{e}^{-\left(\lambda_{r}+\lambda_{s}\right) t} \mathrm{~d} r \mathrm{~d} s}
\end{gathered}
$$

where

$$
\begin{aligned}
& G(r, s ; t)=\frac{G_{1}(r, s)+G_{2}(r, s) \mathrm{e}^{\left(\lambda_{r}+\lambda_{s}-\lambda_{r+s-1}\right) t}}{\lambda_{r}+\lambda_{s}-\lambda_{r+s-1}} \\
& G_{1}(r, s)=\frac{D-\lambda_{r}}{\mu_{r}-\lambda_{r}} \frac{D-\lambda_{8}}{\mu_{8}-\lambda_{8}} \frac{1}{\lambda_{r}+\lambda_{8}-\mu_{r+8-1}}\left\{a_{1}(r, s) C_{r+8-1}\right. \\
& \left.+a_{2}(r, s) \frac{\mu_{8}-D}{C_{s}}\left(D-\lambda_{r}-\lambda_{8}\right)+\frac{a_{2}(s, r) \mu_{r}-D}{C_{r}\left(D-\lambda_{r}-\lambda_{r}\right)}\right\} \\
& G_{2}(r, s)=\left[\left\{D(D-x)\left(x_{r}+x_{8}\right)+\lambda_{r} \mu_{r} x_{8}+\lambda_{8} \mu_{8} x_{r}-x x_{r} x_{8}\right\}\right. \\
& \times\left(a_{1}(r, s) C_{r+8-1}\right)+\frac{\left(D-\lambda_{s}\right)\left(\mu_{s}-D\right)}{C_{8}}\left\{D\left(x_{r}+x_{s}\right)\right. \\
& \left.-\lambda_{r} \mu_{r}+\left(\lambda_{s}-x\right)\left(\mu_{s}-x\right)\right\} a_{2}(r, s)\left(D-\lambda_{r+8-1}\right) \\
& +\frac{\left(D-\lambda_{r}\right)\left(\mu_{r}-D\right)}{C_{r}}\left\{D\left(x_{r}+x_{8}\right)-\lambda_{s} \mu_{s}+\left(\lambda_{r}-x\right)\left(\mu_{r}-x\right)\right\} \\
& \left.a_{2}(s, r)\left(D-\lambda_{r+8-1}\right)\right]\left\{\left(\mu_{r_{+8-1}}-\lambda_{r+8-1}\right)\left(\lambda_{r}+\mu_{8}-x\right)\right. \\
& \left.\left(\lambda_{s}+\mu_{r}-x\right)\left(\mu_{r}+\mu_{s}-x\right)\right\}^{-1}
\end{aligned}
$$

The numerical results are tabulated in the appendix.

\section{Higher moments of the number distribution}

Messel $^{2}$ and his collaborators have in a series of papers made a systematic use of the Janossy ${ }^{3}$ and the product density technique for the calculation of the higher moments of the number distribution of electrons and photons. By defining

1 It is a well-known mathematical fact that under suitable sircumstances the entire distribution function can be obtained from a knowledge of all the moments. The question whether it is possible to obtain a valid approximation from the first few moments is interesting. This has claimed the attention of Messel and Green.

2 A comprehensive list of references is given in a review article by H. Messec in Progress in Cosmic Ray Physics Amsterdam (1954), Vol. 2, Chapter IV.

3 L. Jaxossy, Proc. Roy. Irish Acad. A 53, 181 (1950). 
Mellin transforms for higher density expressions and writing the resulting equations in the matrix form they ${ }^{1}$ have obtained formal and explicit solutions of the equations where ionization loss is neglected. In a subsequent contribution ${ }^{2}$ they have discussed the modifications that are necessary when ionization loss is taken into account. In this case following Bhabha and Chakrabarthy ${ }^{3}$ they have assumed that the higher density functions can be expanded as an infinite series in positive power of $\beta$ and obtained recurrence relations between the transforms of various densities.

\section{Alternative approach to the caseade theory}

Till now we were interested in the probability distribution of the number of particles at a given depth $t$ assuming the shower to be initiated by a particle at $t=0$. However in nuclear emulsions it would be more convenient to estimate the energies of particles at the point of their production rather than the energies of all particles at a particular thickness. This suggested a new mode of approach to the cascade theory.

Instead of dealing with the probability distributions at a particular thickness, we define the function ${ }^{4} \pi^{i}\left(n_{1}, n_{2}, \ldots, n_{m}, E_{1}, \ldots, E_{m} \mid E_{0}, t\right)$ as the probability that $n_{j}$ particles of type $j$ each with energy greater than $E_{j}$ at the point of its production (called primitive energy) are produced between 0 and $t$. Although it may not be possible to determine $\pi$ exactly the moments of $n_{j}$ from $\pi$ can be calculated.

The new product density functions are defined as follows:

$$
F_{k}\left(E_{1}, E_{2}, \ldots, E_{k} ; t_{1}, t_{2}, \ldots, t_{k}\right) \mathrm{d} E_{1} \mathrm{~d} E_{2} \ldots \mathrm{d} E_{k} \mathrm{~d} t_{1} \mathrm{~d} t_{2} \ldots \mathrm{d} t_{k}
$$

is the joint probability that an electron of energy between $E_{1}$ and $E_{1}+\mathrm{d} E_{1}$ is produced between $t_{1}$ and $t_{1}+\mathrm{d} t_{1}$, an electron of energy between $E_{2}$ and $E_{2}+\mathrm{d} E_{2}$ is produced between $t_{2}$ and $t_{2}+\mathrm{d} t_{2}$ and an electron of energy between $E_{k}$ and $E_{k}+\mathrm{d} E_{k}$ is produced between $t_{k}$ and $t_{k}+\mathrm{d} t_{k} \cdot F_{k}$ is the product density of degree $k$ for electrons. Similarly $G_{k}$ product density of degree $k$ of photons and $F G_{l, k}$ the mixed product density of $l$ electrons and $k$ photons can be defined.

The product densities $F_{k}, G_{k}, F G_{l, k-l}$ are over $E$ space as well as $t$ space. Using the properties for the product density functions it is found that if the product densities are integrated over $E_{1}, E_{2} \ldots E_{k}$ over the range $E_{c}$ to $\infty$ they are still product densities of particles produced between $t_{i}$ and $t_{i}+\mathrm{d} t_{i}$ $(i=1,2, \ldots, k)$, the primitive energy of each particle being greater than $E_{c}$. The moments can be obtained from this by integration over the proper range of $t$. For example if we are interested in the $r$-th moment of the number of elec-

1 H. Messel and R. B. Potts, Phys. Rev. 86, 847 (1952).

2 H. Messel and R. B. Potts, Phys. Rev. 87, 759 (1952).

3 H. J. Bhabha and S. K. Chakrabarthy, Phys. Rev. 74, 1352 (1948).

4 A. Ramakrishnan and S. K. Srinivasan, Proc. Ind. Acad. Sci. 44, 263 (1956). 
trons produced between 0 and $t$, the primitive energy of each of the electrons being greater than $E_{c}$, it is given by

$$
\begin{aligned}
\mathscr{E}\left\{N^{r}\left(E_{c}, t\right)\right\}= & \frac{\Gamma}{i} C_{i}^{r} \int_{E_{c}}^{\infty} \mathrm{d} E_{1} \int_{E_{c}}^{\infty} \mathrm{d} E_{2} \ldots \int_{E_{c}}^{\infty} \mathrm{d} E_{i} \\
& \int_{0}^{t} \mathrm{~d} t_{1} \int_{0}^{t} \mathrm{~d} t_{2} \ldots \int_{0}^{t} \mathrm{~d} t_{i} F_{i}\left(E_{1} \ldots E_{i} ; t_{1} \ldots t_{i}\right)
\end{aligned}
$$

$C_{i}^{r}$ being already defined.

Equations for the product densities of degree 1 alone will be given since explicit solutions for degrees greater than 2 are not available and numerical evaluation for even degree 2 is very difficult.

Assuming the usual Bethe-Heitler cross-sections and neglecting ionziation loss the equations for product densities of degree 1 can be written as

$$
\begin{aligned}
& F_{1}^{(i)}\left(E \mid E_{0} ; t\right)=2 \int_{E^{2}}^{E_{0}} g_{1}^{(i)}\left(E^{\prime}, E_{0} ; t\right) R^{(2)}\left(E \mid E^{\prime}\right) \mathrm{d} E^{\prime} . \\
& G_{1}^{(i)}\left(E \mid E_{0} ; t\right)=\int_{E}^{E_{0}} j_{1}^{(i)}\left(E^{\prime} \mid E_{0}, t\right) R^{(1)}\left(E^{\prime}-E \mid E^{\prime}\right) \mathrm{d} E^{\prime}
\end{aligned}
$$

$g_{1}^{(i)}$ and $f_{1}^{(i)}$ are old product densities of degree 1 of photons and electrons respectively. Defining the Mellin transforms $P_{1}^{(i)}\left(s \mid E_{0}, t\right)$ and $Q_{1}^{(i)}\left(s \mid E_{0} ; t\right)$ as

$$
\begin{aligned}
& F_{1}^{(i)}\left(s \mid E_{0} ; t\right)=\int_{0}^{\infty} F_{1}^{(i)}\left(E \mid E_{0} ; t\right) E^{s-1} \mathrm{~d} E \\
& Q_{1}^{(i)}\left(s \mid E_{0} ; t\right)=\int_{0}^{\infty} G_{1}^{(i)}\left(E \mid E_{0} ; t\right) E^{8-1} \mathrm{~d} E
\end{aligned}
$$

we obtain

$$
\begin{aligned}
& P_{1}^{(i)}\left(s \mid E_{0} ; t\right)=\gamma_{1}^{(i)}\left(s \mid E_{0} ; t\right) B_{8} \\
& Q_{1}^{(i)}\left(s \mid E_{0} ; t\right)=v_{1}^{(i)}\left(s \mid E_{0} ; t\right) C_{8}
\end{aligned}
$$

$\gamma_{1}^{(i)}$ and $v_{1}^{(i)}$ being the Mellin transforms of $g_{1}^{(i)}$ and $f_{1}^{(i)}$ respectively.

Similar equations for product densities of degree 2 can also be written down but we do not refer to them since numerical results are not available.

The asymptotic behaviour of cascades for large thicknesses can be explained as follows: It is well-known that the old product densities and all the moments of the number of particles above a certain energy $E_{c}\left(E_{c}>0\right)$ at thickness $t$ tend to zero as $t \rightarrow \infty$. This is obvious from a physical point of view. Since the BetheHeitler cross-sections are homogeneous the total cross-section is independent of the energy of the particle and as such the probability of finding a particle above a certain cnergy $E_{c}$ at $t$ tends to zero as $t \rightarrow \infty$. As the new product densities are expressed as linear combinations of the old densities, for the same reason, the moments of the number of particles produced between zero and $t$ with this primitive energy, being greater than $E_{c}$ tends to a finite limit as $t \rightarrow \infty$. 
The mean numbers of electrons and photons produced between 0 and $t$, the primitive energy of each of them being greater than $E_{c}$, are given by

$$
\begin{aligned}
& \mathscr{E}\left\{\mathscr{N}\left(E_{c} \mid E_{0} ; t\right)\right\}=\frac{1}{2 \pi i} \int_{0}^{t} \mathrm{~d} \tau \int_{\sigma-i \infty}^{\sigma+i \infty} \frac{B_{8} \gamma_{1}^{(1)}\left(s \mid E_{0} ; \tau\right)}{s-1}\left(\frac{E_{0}}{E_{c}}\right)^{8-1} \mathrm{~d} s \\
& \mathscr{E}\left\{\mathscr{M}\left(E_{c} \mid E_{0} ; t\right)\right\}=\frac{1}{2 \pi i} \int_{0}^{t} \mathrm{~d} \tau \int_{\sigma-i \infty}^{\sigma+i \infty} \frac{C_{s} \nu_{1}^{(1)}\left(s \mid E_{0} ; \tau\right)}{s-1}\left(\frac{E_{0}}{E_{c}}\right)^{8-1} \mathrm{~d} s .
\end{aligned}
$$

Inverting the order of integration over $s$ and $\tau$, we have

$$
\begin{aligned}
& \mathscr{E}\left\{\mathscr{N}\left(E_{c} \mid E_{0} ; t\right)\right\}=\frac{1}{2 \pi i} \int_{\sigma-i \infty}^{\sigma+i \infty} \frac{B_{s} C_{s}}{\left(\mu_{s}-\lambda_{s}\right)(s-1)}\left(\frac{E_{0}}{E_{c}}\right)^{s-1} \\
& \quad \times\left[\frac{1-e^{-\lambda_{s} t}}{\lambda_{s}}-\frac{1-\mathrm{e}^{-\mu_{s} t}}{\mu_{s}}\right] \mathrm{d} s \\
& \mathscr{E}\left\{\mathscr{M}\left(E_{c} \mid E_{0} ; t\right)\right\}=\frac{1}{2 \pi i} \int_{\sigma-i \infty}^{\sigma+i \infty} \frac{C_{s}}{(s-1)\left(\mu_{s}-\lambda_{s}\right)}\left(\frac{E_{0}}{E_{c}}\right)^{s-1} \\
& \times\left[\frac{D-\lambda_{s}}{\lambda_{s}}\left(1-\mathrm{e}^{-\lambda_{s} t}\right)+\frac{\mu_{s}-D}{\mu_{s}}\left(1-\mathrm{e}^{-\mu_{s} t}\right)\right] \mathrm{d} s .
\end{aligned}
$$

From this it can be seen that $\mathscr{E}\left\{\mathscr{N}\left(E_{c} \mid E\right)\right\}$ and $\mathscr{E}\left\{\mathscr{M}\left(E_{c} \mid E_{0}\right)\right\}$ tend to a finite limit as $t \rightarrow \infty .^{1}$

\section{Further refinements to cascade theory}

New approach by the regeneration point method

The essential difference between the old and the new approaches to cascade theory, while apparent from the definition of the two types of the product density functions, is strikingly brought out by the equations representing them when we employ the regeneration point technique. ${ }^{2}$ We shall first discuss the simple case of cascade consisting of only one type of particle when its development is defined by the cross-section $R\left(E^{\prime} \mid E\right) \mathrm{d} E^{\prime} \mathrm{d} t$, representing the probability that a particle of energy $E$ drops to an energy interval lying between $E^{\prime}$ and $E^{\prime}+\mathrm{d} E^{\prime}$, producing another particle of energy $E-E^{\prime}$ in traversing a matter of thickness $\mathrm{d} t$.

Let $\pi_{\mathrm{I}}\left(n, E \mid E_{0} ; t\right)$ represent the probability that $n$ particles are at $t$, the energy of each particle being greater than $E$ and $\pi_{\mathrm{II}}\left(n, E \mid E_{0}, t\right)$, the probability that there are produced $n$ particles between 0 and $t$ each having energy greater than $E$ at the point of the production, with the primary energy in both cases being $E_{0}$.

1 S. K. Srinivasan and N. R. Ranganathan, Proc. Ind. Acad. Sci. 45, 69 (1957).

2 Alladi Ramakrishnan and K. Venkatesan, loc. cit. 
This difference in reckoning the energies of the particles in $\pi_{I}$ and $\pi_{\Pi}$ gives rise to a corresponding difference in the summation in the equations satisfied by them.

Consider the first regeneration point $\tau$ where the primary particle of energy $\boldsymbol{E}_{0}$ is replaced by two particles of energy $E^{\prime}$ and $E_{0}-E^{\prime}$ which become in turn independent primaries of subsequent cascades. For $\pi_{1}\left(n, E \mid E_{0}, t\right)$ these two primaries together produce $n$ particles with energy greater than $E$ in a distance $t-\tau$, i.e. there exist at $t, n$ particles with energy greater than $E$. However for $\tau_{\text {II }}\left(n, E \mid E_{0} ; t\right)$ the counting of particles is different as it depends on the energy at the point of production. Since even the primary particle with energy $E_{0}>E$ at $t=0$ forms part of the system of particles with primitive energy greater than $E$ which are produced between 0 and $t$ it is clear that the two independent primaries at $\tau$ need produce together only $n-1$ particles, of course including the two primaries. We can now write down the equations satisfied by $\pi_{I}$ and $\pi_{I I}$

$$
\begin{aligned}
\tau_{\mathrm{I}}\left(n, E E_{0} ; t\right)= & \int_{0}^{t} \mathrm{~d} \tau \mathrm{e}^{-R\left(E_{0}\right) \tau} \int_{E^{\prime}}^{E_{0}} R\left(E^{\prime} \mid E_{0}\right) \mathrm{d} E^{\prime} \\
& \times \sum_{m+m^{\prime}=n} \pi_{1}\left(m, E \mid E^{\prime}, t-\tau\right) \pi_{\mathrm{I}}\left(m^{\prime}, E \mid E_{0}-E^{\prime}, t-\tau\right) \\
& +\mathrm{e}^{-R\left(E_{0}\right) t} \delta(n-1) \pi_{\mathrm{I}}\left(n, E \mid E_{0} ; t\right) \\
\tau_{\mathrm{II}}\left(n, E E_{0} ; t\right)= & \int_{0}^{t} \mathrm{~d} \tau \mathrm{e}^{-R\left(E_{0}\right) \tau} \int_{E}^{E_{0}} R\left(E^{\prime} \mid E_{0}\right) \mathrm{d} E^{\prime} \\
& \times \sum_{m+m^{\prime}=n-1} \pi_{\mathrm{II}}\left(m, E \mid E_{0} ; t-\tau\right) \pi_{\mathrm{II}}\left(m^{\prime}, E \mid E_{0}-E^{\prime} ; t-\tau\right) \\
& +\mathrm{e}^{-R\left(E_{0}\right) t} \delta(n-1) \pi_{\mathrm{II}}\left(n, E \mid E_{0} ; t\right)
\end{aligned}
$$

where

$$
R\left(E_{0}\right)=\int_{0}^{E_{0}} R\left(E^{\prime} \mid E_{0}\right) \mathrm{d} E^{\prime} .
$$

Defining the generating function $G\left(u, E \mid E_{0} ; t\right)$ as

$$
G_{\mathrm{I}, \mathrm{II}}\left(u, E \mid E_{0} ; t\right)=\sum_{n=0}^{\infty} u^{n} \pi_{\mathrm{I}, \mathrm{II}}\left(n, E \mid E_{0} ; t\right)
$$

the integral equations yield the following differential equations with respect to $t$,

$$
\begin{gathered}
\frac{\partial G_{\mathrm{I}}^{\prime}}{\partial t}\left(u, E E_{0} ; t\right)=\int_{E^{\prime}}^{E_{0}}\left\{G_{\mathrm{I}}\left(u, E \mid E^{\prime} ; t\right) G_{\mathrm{I}}\left(u, E \mid E_{0} ;-E^{\prime} ; t-G_{\mathrm{I}}\left(u, E \mid E_{0} ; t\right)\right\}\right. \\
\times R\left(E^{\prime} \mid E_{0}\right) \mathrm{d} E^{\prime} \\
\frac{\partial G_{\mathrm{II}}}{\partial t}\left(u, E \mid E_{0} ; t\right)=\int_{E^{\prime}}^{E_{0}}\left\{u G_{\mathrm{II}}\left(u, E \mid E^{\prime} ; t\right) G_{\mathrm{II}}\left(u, E \mid E_{0}-E^{\prime} ; t\right)-G_{\mathrm{II}}\left(u, E \mid E_{0} ; t\right)\right\} \\
\times R\left(E^{\prime} \mid E_{0}\right) \mathrm{d} E^{\prime} .
\end{gathered}
$$




\section{Correlation with respect to thickness of matter traversed ${ }^{1}$}

Product densities hitherto defined refer to the distribution of particles either in the energy space in the old approach or in the product space of energy and thickness in the new approach. It is interesting to define the product density $\mathscr{F}_{n}\left(E_{1} ; t_{1}, E_{2} ; t_{2}, \ldots, E_{n} ; t_{n} \mid E_{0} ; t_{0}\right)$ corresponding to different thicknesses such that $\mathscr{F}_{n} \mathrm{~d} E_{1} \mathrm{~d} E \ldots \mathrm{d} E_{n}$ represents the probability that there exists a particle in $\mathrm{d} E_{1}$ at $t_{1}$ one in $\mathrm{d} E_{2}$ at $t_{2}$ and one in $\mathrm{d} E_{n}$ at $t_{n}$. This is also the mean of the product of the numbers in the range $\mathrm{d} E_{1}$ at $t_{1}, \mathrm{~d} E_{2}$ at $t_{2}$ and $\mathrm{d} E_{n}$ at $t_{n}$. If we set $t_{1}=t_{2}=\cdots=t_{n}$ it does not just reduce to the product density of degree $n$ corresponding to a particular thickness. The $n$-th order densities corresponding to different thicknesses is connected to all the densities of order $m$ less than or equal to $n$ corresponding to the same thickness. We here establish the connection for the second order. For $t_{2}>t_{1}>t_{0}$,

$$
\begin{aligned}
\mathscr{F}_{2}\left(E_{1} ; t_{1}, E_{2} ; t_{2} \mid E_{0} ; t_{0}\right)= & \int f_{2}\left(E_{1}, E_{2}^{\prime} \mid E_{0} ; t_{1}-t_{0}\right) f_{1}\left(E_{2} \mid E_{2}^{\prime} ; t_{2}-t_{1}\right) \mathrm{d} E_{2}^{\prime} \\
& +f_{1}\left(E_{1} \mid E_{0} ; t_{1}-t_{0}\right) f_{1}\left(E_{2} \mid E_{1} ; t_{2}-t_{1}\right) .
\end{aligned}
$$

This equation is obtained by the following argument. The particle of energy $E_{2}$ at $t_{2}$ must belong to a cascade generated by a particle of energy $E_{2}^{\prime}\left(E_{2}^{\prime} \neq E_{1}\right)$ or by the particle with energy $E_{1}$ at $t_{1}$. The first and second terms correspond to these possibilities respectively. For the case when $f$ is homogeneous in the energy variables, the Mellin transform of $\mathscr{F}_{2}\left(E_{1}, t_{1}, E_{2} ; t_{2} \mid E_{0} ; t_{0}\right)$ is obtained in terms of the Mellin transforms of $f_{2}\left(E_{1}, E_{2} \mid E_{0} ; t_{1}-t_{0}\right)$. and $f_{1}(\varepsilon ; t)$ where $\varepsilon=\frac{E_{2}}{E_{1}}$. It is to be noted that $\mathscr{F}_{2}$ is not symmetric in $t_{1}$ and $t_{2}$ as should be expected from the evolutionary nature of the Markovian process. If we assume that the entire cascade is generated by a single particle of energy $E_{0}$ the second order density at $t_{1}=t_{0}$ is zero and hence the first term vanishes. The expression for $\mathscr{F}$ then reduces to

$$
\mathscr{F}\left(E_{1} ; t_{0}, E_{2} ; t_{2} \mid E_{0} ; t_{0}\right)=\delta\left(E_{1}-E_{0}\right) f_{1}\left(E_{2} \mid E_{1} ; t_{2}-t_{0}\right) .
$$

Defining $N\left(E_{c} ; t_{1}\right)$ and $N\left(E_{c} ; t_{2}\right)$ as the random variables representing the number of particles above energy $E_{c}$ at $t_{1}$ and at $t_{2}$ the expectation value $\mathscr{E}\left\{N\left(E_{c} ; t_{1}\right) N\left(E_{c} ; t_{2}\right)\right\}$ is given by

When $t_{2} \rightarrow t_{1}=t$

$$
\mathscr{E}\left\{N\left(E_{c} ; t_{1}\right) N\left(E_{c} ; t_{2}\right)\right\}=\int_{E_{c}}^{\infty} \int_{E_{c}}^{\infty} \mathscr{F}_{2}\left(E_{1} ; t_{1}, E_{2} ; t_{2} \mid E_{0} ; t_{0}\right) \mathrm{d} E_{1} \mathrm{~d} E_{2} .
$$

and hence

$$
f_{1}\left(E_{2} \mid E_{2}^{\prime} ; t_{2}-t_{1}\right) \text { tends to } \delta\left(E_{2}-E_{2}^{\prime}\right)
$$

$$
\int t_{2}\left(E_{1}, E_{2}^{\prime} \mid E_{0} ; t-t_{0}\right) \delta\left(E_{2}-E_{2}^{\prime}\right) \mathrm{d} E_{2}^{\prime} \quad \text { becomes } \quad f_{2}\left(E_{1}, E_{2} \mid E_{0} ; t-t_{0}\right)
$$

1 Alladi Ramakrishnan and T. K. Radha, Proc. Camb. Phil. Soc. 57, 843 (1961). 
and therefore

$$
\begin{aligned}
& \mathscr{F}_{2}\left(E_{1} ; t_{1}, E_{2}: t_{2} \mid E_{0} ; t_{0}\right) \rightarrow \mathscr{F}_{2}\left(E_{1} ; t, E_{2} ; t \mid E_{0} ; t_{0}\right) \\
& \quad=t_{2}\left(E_{1}, E_{2} ; t \mid E_{0} ; t_{0}\right)+f_{1}\left(E_{1} ; t \mid E_{0} ; t_{0}\right) \delta\left(E_{2}-E_{1}\right),
\end{aligned}
$$

i.e. the density $\mathscr{F}_{2}\left(E_{1} ; t_{1}, E_{2} ; t_{2} \mid E_{0} ; t_{0}\right)$ in the limit $t_{2} \rightarrow t_{1}$ does not just represent the second order density but also includes the first order term with the $\delta$-function as its coefficient. As $t_{2} \rightarrow t_{1}=t$

$$
\begin{gathered}
\mathscr{E}\left\{N\left(E_{c} ; t_{1}\right) N\left(E_{c} ; t_{2}\right)\right\} \rightarrow \\
\int_{E_{c}}^{\infty} \int_{E_{c}}^{\infty} f_{2}\left(E_{1}, E_{2} ; t \mid E_{0} ; t_{0}\right) \mathrm{d} E_{1} \mathrm{~d} E_{2}+\int_{E_{c}}^{\infty} \int_{E_{c}}^{\infty} f_{1}\left(E_{1} ; t \mid E_{0} ; t_{0}\right) \delta\left(E_{1}-E_{2}\right) \mathrm{d} E_{1} \mathrm{~d} E_{2}
\end{gathered}
$$

and tends to $\mathscr{E}\left\{N^{2}\left(E_{c} ; t\right)\right\}$ as it should.

In the case of cascades with many types of particles such as the electron-photon' cascade the second order density $\mathscr{F}$ will be connected not only to the product. density $f_{2}$ of electrons but also to the mixed product density of photons and electrons $f g_{1.1}$ of all orders such that

$$
\begin{aligned}
& \mathscr{F}_{2}^{(1)}\left(E_{1} ; t_{1}, E_{2} ; t_{2} \mid E_{0} ; t_{0}\right) \\
& =\int_{E_{3}}^{\infty} f_{2}^{(1)}\left(E_{1}, E_{2}^{\prime} ; t_{1} \mid E_{0} ; t_{0}\right) f_{1}^{(1)}\left(E_{2} \mid E_{2}^{\prime} ; t_{2}-t_{1}\right) \mathrm{d} E_{2}^{\prime} \\
& \quad+f_{1}^{(1)}\left(E_{1} ; t_{1} \mid E_{0} ; t_{0}\right) f_{1}^{(1)}\left(E_{2} \mid E_{1} ; t_{2}-t_{1}\right) \\
& \quad+\int_{E_{2}}^{\infty} f g_{1,1}^{(1)}\left(E_{1} ; E_{2}^{\prime} ; t_{1} \mid E_{0} ; t_{0}\right) f_{1}^{(2)}\left(E_{2} \mid E_{2}^{\prime} ; t_{2}-t_{1}\right) \mathrm{d} E_{2}^{\prime} .
\end{aligned}
$$

where superscript $i==1$ represents an electron-generated shower and $i=2$ a photon-generated shower.

The equation for the higher density $\mathscr{F}_{n}$ can be formally written down and in this case it involves $\mathscr{F}_{m}$ where $m<n$, and $f g_{l, m}$ such that $l+m \leqq n$.

\section{Polarization phenomena in cascades}

So far in our treatment of the soft cascades, the parameters used to describe the longitudinal development were mainly the thickness of matter traversed and the energies of the different types of particles comprising the cascade. Since now it is possible to study cascades initiated by polarized electrons available from $\mu$-meson decay, the question arises whether it is possible to include the state of polarization of particles in the description of cascade.

The inclusion of polarization brings in its wake a new feature not present in our previous treatments. An electron can exist in a "forward" $(F)$ or "backward" $(B)$ polarization pure state with respect to its direction of motion or in any other state of polarization which can be expressed as a linear combination of $F$ and $B$ states. This feature has to be included in the product density functions which is best achieved by adopting the density matrix formalism. Thus we define 
$\pi_{i j}\left(E \mid E_{0} ; t\right)$ and $\gamma_{i j}\left(E \mid E_{0} ; t\right)$ to be the product density function of degree one for electrons and photons where the labels $i$ and $j$ are $F$ and $B$. It is to be noted that these functions are product densities only in $E$ space and the various values of the suffixes $i$ and $j$ yield the four types of product densities $\pi_{F F}, \pi_{F B}, \pi_{B F}$ and $\pi_{B B}$ for the electrons and similar functions for the photons.

To write down the equations for $\pi_{i j}$ 's we need the matrix elements of the processes rather than the total cross-sections. For in cascades with the inclusion of the state of polarization of the particles, the progeny of a particle depends very much on the state of polarization of the "parent" which will be determined by the relative phases of the production matrix elements for the parents. This feature is essentially due to quantum mechanics and it complicates the cascade equations. However a great simplification is possible if we neglect the angular distribution of particles by integrating over the angles. ${ }^{1}$

Dyson ${ }^{2}$ has studied in detail the variation with depth of the mean of number of longitudinally polarized particles which is defined by

$$
\pi_{L}\left(E \mid E_{0} ; t\right)=\pi_{F^{\prime}}\left(E \mid E_{0} ; t\right)-\pi_{B B}\left(E \mid E_{0} ; t\right) .
$$

The function gives the mean excess of the forward polarized particles over the backward ones. The usual cascade function will be the sum of the $\pi_{F F}$ and $\pi_{B B}$. Equations dealing with polarized cascades according to the new approach can be studied. The equations for the different product densities of degree two to deal with the fluctuation problem can also be formally solved. ${ }^{3}$ But at the present stage of measuring polarization of particles in the cascade showers, these discussions are not of direct experimental interest.

\section{Discussion of numerical results}

Before discussing the results of the cascade theory with and without ionization loss, we note the following important features of the cross-sections for radiation, pair creation and ionization loss.

(i) If we choose the unit of length according to (28) the cross-sections for radiation and pair creation are very nearly independent of the material. Their only dependence on the material is through the term $4 / 3+\alpha$ where $\alpha$ is very small compared to $4 / 3$.

(ii) The mean energy loss per unit characteristic length at very high energies is equal to the energy itself, i.e.

or

$$
\left.\frac{\partial E}{\partial t}\right|_{\text {radiation }} \sim E
$$

1 F. J. Dyson and K. W. Mcvoy, Phys. Rev. 106, 1360 (1957).

2 F. J. Dyson (unpublished.)

3 N. R. Ranganathan and R. Vasudevan, Proc. Phys. Soc. (Lond.) 76, 659 (1960). See also Proceedings of the Summer School of Theoretical Physics, Part II, 261 (1959), Ministry of Scientific and Cultural Affairs, Government of India, New Delhi. 
where $E_{0}$ is the initial energy and $t$ is measured in cascade units. Thus we can also interpret the cascade unit as the distance in which the energy of a particle becomes $\frac{1}{\mathrm{e}}$ times its initial value.

(iii) The ionization loss $\beta$ is taken to be independent of the energy and is proportional to $\frac{1}{Z}$. The critical energy is defined as the energy at which radiation and ionization losses are equal. Hence it follows from (100) that the critical energy $E_{\mathrm{c}}$ is equal to $\beta$. A table of the values of $\beta$ and $l$ for various materials is given below.

\begin{tabular}{l|c|c}
\hline Material & $\beta$ in $\mathrm{MeV}$ & $l$ in $\mathrm{gcm}$ \\
\hline Air & 84.2 & 37.7 \\
Water & 83.8 & 37.1 \\
Carbon & 102 & 44.6 \\
Nitrogen & 89 & 39.4 \\
Oxygen & 78 & 35.3 \\
Lead & 7.8 & 5.83 \\
\hline
\end{tabular}

(iv) The cross-section for Bremsstrahlung becomes infinite as the photon energy tends to zero (infra-red catastrophe) i.e. as $\varepsilon \rightarrow 1$ in $R^{(1)}$. Therefore the total number of photons of energy greater than or equal to zero tends to infinity. Since we have assumed the cross-section for pair creation to be dependent only on the ratio of particle to photon energy the total number of electrons with energy equal to and greater than zero also tends to infinity. However it is meaningless to talk of electrons with energy equal to zero. We should consider only electrons and photons of energy greater than a particular value $E_{i}$, the numbers of

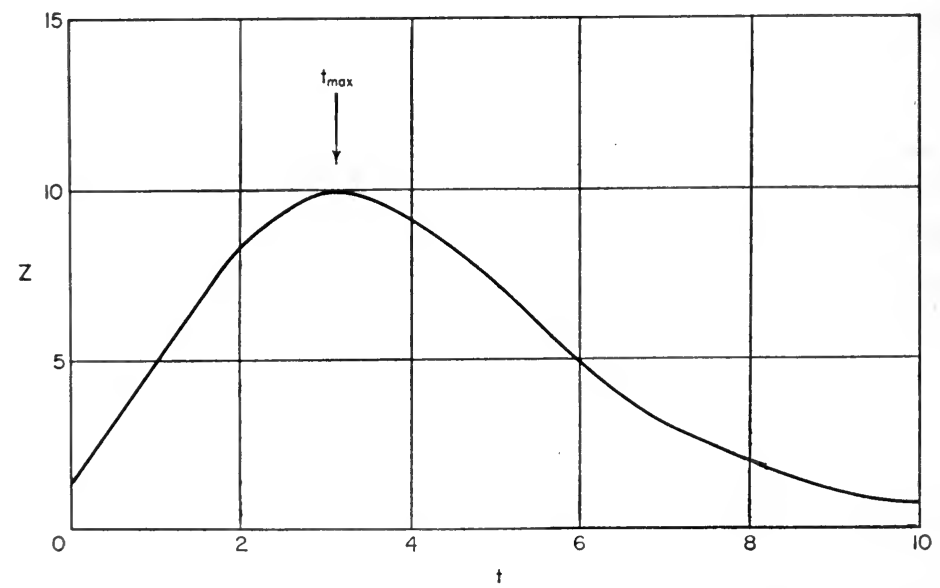

FIG. 62. Total number of electrons as a function of the thickness of air in radiation units $\left(37.7 \mathrm{~g} \cdot \mathrm{cm}^{-2}\right)$ for shower initiated by single photons of various energies. 
which are always finite so long as $E_{i}>0$. Mathematically this is equivalent to saying that although the two terms $\int_{0}^{1} R^{(1)}(\varepsilon) \mathrm{d} \varepsilon$ and $\int_{0}^{1} R^{(1)}(\varepsilon) \varepsilon^{\varepsilon-1} \mathrm{~d} \varepsilon$ are divergent, their difference is always finite. ,

The number of particles $N(E ; t)$ above $E$ varies with the thickness $t$ due to particles entering and leaving the energy interval $(E, E+\mathrm{d} E)$. At the beginning of the cascade, the contribution is always greater than the depletion. In the case of cascades without ionization loss the total number of particles above energy $E$ at a thickness $t$ is given by

$$
N(E ; t)=\frac{1}{2} \frac{1}{\pi i} \int_{\sigma-i \infty}^{\sigma+i \infty}\left(\frac{E_{0}}{E}\right)^{s-1} \frac{1}{s-1}\left\{\frac{D-\lambda}{\mu-\lambda} \mathrm{e}^{-\lambda t}+\frac{\mu-D}{\mu-\lambda} \mathrm{e}^{-\mu t}\right\} \mathrm{d} s .
$$

The maximum of $N$ occurs when

$$
\frac{\partial N}{\partial t}(E, t)=0
$$

which gives the corresponding value $t_{\max }$ to be

$$
t_{\max }(E)=1.01 \log _{\mathrm{e}} \frac{E_{0}}{E}-1.57 \text {. }
$$

The value of $N$ at this point is

$$
N_{\max }(E)=0.137 \frac{E_{0}}{E}\left\{\log \frac{E_{0}}{E}-1.31\right\}^{-1 / 2} .
$$

Fluctuations: The development of a cascade is a multiplicative process from the point of view of stochastic theory and it has become customary to compare it with two stochastic models, the Furry and the Poisson. The first represents the simplest multiplicative and the second the simplest non-multiplicative process. We shall first discuss the principal features of these models before comparing the cascade process with them.

We define a Furry process ${ }^{1}$ as follows: Considering only one type of particle, if we postulate that $\lambda \mathrm{d} t$ is the probability that a particle produces another in thickness $\mathrm{d} t$, then the probability that a new particle is produced in thickness $\mathrm{d} t$ is $n \lambda \mathrm{d} t$ if there are $n$ particles. The probability $\pi(n, t)$ that the stochastic variable $n(t)$ representing the number of particles at thickness $t$ is $n$ satisfies the equation

$$
\frac{\partial \pi}{\partial t}^{(n ; t)}=\lambda[(n-1) \pi(n-1 ; t)-n \pi(n ; t)] .
$$

1 W. H. Furry, Phys. Rev. 52, 569 (1937). See also A. Ramakrishnan's article in Handbuch der Physik, loc. cit.

EPCR 29 
Assuming that at $t=0$, there is only one particle, i.e. $\pi(1,0)=1, \pi(n, 0)=0$ if $n \neq 1$, we obtain the Furry distribution

where

$$
\tau(n ; t)=\frac{1}{\mathscr{E}\{n(t)\}}\left[1-\frac{1}{\mathscr{E}\{n(t)\}}\right]^{n-1}
$$

$$
\delta\{n(t)\}=\mathrm{e}^{\lambda t}
$$

The mean number $\mathcal{E}\{n(t)\}$ increases exponentially and therefore has no maximum. A very interesting feature of this distribution is that as regards the discrete variable $n, \tau$ has a maximum for $n=0$ and falls of monotonically with increasing $n$.

If we define a stochastic variable $m(t)=n(t)$ - 1, i.e. $m(t)$ represents the secondaries, then

and

$$
\mathscr{E}\{m(t)\}=\mathrm{e}^{\lambda t}-1
$$

$$
\sigma^{2}\{m(t)\}=\mathscr{E}\left\{m^{2}(t)\right\}-[\mathscr{E}\{m(t)\}]^{2}=[\mathscr{E}\{m(t)\}]^{2} .
$$

The mean square deviation of $n(t)$ is

$$
\sigma_{F^{\prime}}^{2}=\mathscr{E}\left\{n^{2}(t)\right\}-[\mathscr{E}\{n(t)\}]^{2}=[\mathscr{E}\{n(t)\}]^{2}-\mathscr{E}\{n(t)\} .
$$

If $\mathrm{e}^{\lambda t} \gg 1$, the distinction between $m(t)$ and $n(t)$ is not important and we have

$$
\frac{\sigma_{F}^{2}}{\varepsilon\{n(t)\}^{2}}=1-\frac{1}{\varepsilon\{n(t)\}} \sim 1 \text { if } \mathrm{e}^{\lambda t} \gg 1 \text {. }
$$

In a Poisson process, $\lambda \mathrm{d} t$ is defined as the probability that a new particle is produced in $\mathrm{d} t$ and is independent of the number of the existing particles (nonmultiplicative). Then $\pi(n, t)$ satisfies the equation

$$
\frac{\partial \pi}{\partial t}(n ; t)=[\pi(n-1 ; t)-\pi(n ; t)] \lambda
$$

with $\tau(0 ; 0)=1, \pi(n ; 0)=0, n \neq 0$. This yields the Poisson distribution

$$
\pi(n ; t)=\mathrm{e}^{-\lambda t} \frac{(\lambda t)^{n}}{n !}
$$

and

$$
\begin{aligned}
\mathscr{E}\{n(t)\} & =\lambda t \\
\sigma_{p}^{2} & =\mathscr{E}\{n(t)\} \\
\frac{\sigma_{p}^{2}}{\mathscr{E}\{n(t)\}^{2}} & =\frac{1}{\mathscr{E}\{n(t)\}} \simeq 0 \quad \text { if } \quad t \gg 1,
\end{aligned}
$$

i.e. if we write $\sigma_{p}^{2}=k \sigma_{F}^{2}, k \simeq 0$. It does not however imply as we shall presently see, that if $k \simeq 0$, the process resembles the Poisson process. In comparing any stochastic process with these two simple models we obtain the mean $\mathscr{E}\{n\}$ and the mean square number $\mathscr{E}\left\{n^{2}\right\}$ and express the mean square deviation $\sigma^{2}$ as

$$
\sigma^{2}=k_{p}^{2}=k^{\prime} \sigma_{p}^{2} \text {. }
$$


Such a comparison is meaningful only if $\mathscr{E}\{n\} \gg 1$ (say at least 10). If $k \simeq 1$ we say that the process resembles the Furry type. But if $k \simeq 0$ we cannot conclude that the process is of the Poisson type, since we may still have $k^{\prime} \gg 1$. This happens when $\mathscr{E}\{n(t)\} \gg 1$ such that

$$
\frac{\sigma^{2}}{[\mathscr{E}\{n(t)\}]^{2}} \simeq 0 \quad \text { but } \quad \frac{\sigma^{2}}{\mathscr{E}\{n(t)\}}=k^{\prime} \gg 1 \quad \text { though } \quad k^{\prime} \ll \mathscr{E}\{n(t)\} .
$$

This fact does not seem to have been realised in making comparisons with the Furry and Poisson processes in many treatments of the cascade theory. In comparing the cascade process with a Furry model, it has already been observed that the mean number above a certain energy reaches a maximum in the cascade process while in a Furry type there is no maximum. Therefore it is considered worthwhile comparing the distribution of this number with the Furry distribution as $t$ varies. It is obvious that at thicknesses small compared to $t_{\max }$ the distribution should be of the Furry type provided the mean number is greater than about ten.

If we are considering the total number of particles (above a certain energy) the distribution will be exactly of the Furry type if there were only one type of particle and the cross-section is independent of the energy. In the case of electronphoton cascades we have two types of particles and the computation of the total number is rendered difficult in view of the infra-red catastrophe. However assuming the total cross-section to be finite it can be shown by a simple calculation that the electrons and the photons obey independently an approximately Furry distribution and that they are correlated in a manner such that the electrons and photons together have also a Furry distribution.

In the actual case of the electron-photon cascade we compare the number of particles above a certain energy with the Furry type. The numerical calculations on the fluctuations, i.e. the determination of $k$ and $k^{\prime}$ have been done by Ramakrishnan and Mathews. ${ }^{1}$ An examination of the tables leads to the following conclusions :

(i) For a given $y$, the thicknesses at which

$$
\mathscr{E}\{n\}, \mathscr{E}\left\{n^{2}\right\}, \sigma^{2}, \frac{\sigma^{2}}{\mathscr{E}\{n\}} \text { and } \frac{\sigma^{2}}{[\mathscr{E}\{n\}]^{2}-\mathscr{E}\{n\}}
$$

are maximum are all different as expected.

(ii) The negative values of $\frac{\sigma^{2}}{[\mathscr{E}\{n\}]^{2}-\mathscr{E}\{n\}}$ correspond to negative values $\left[[\mathscr{E}\{n\}]^{2}-\mathscr{E}\{n\}\right]$, i.e. when $\mathscr{E}\{n\}<1$.

(iii) For very large values of $y$ but before the shower maximum the deviation is a finite fraction (about 0.1 to 0.2 times) of the Furry deviation.

(iv) Comparing with the Poisson distribution it is found that $\frac{\sigma^{2}}{\mathscr{E}\{n\}}$ increases with $y$ for a given value of $t$. At the shower maximum the deviation is many

1 A. Ramakrishnan and P. M. Mathews, loc. cit. (1954). 
times the Poisson value (a result mistakenly supposed to be at variance with that of Scott and Uhlenbeck ${ }^{1}$ and Janossy and Messel $^{2}$ ). For example, for $y=8$ the maximum occurs between $t=6$ and $t=8$ and the deviation is about twelve times the Poisson value or 0.1 times the Furry deviation.

In a theory including ionization loss numerical results are available only for the mean numbers and it is naturally interesting to compare the energy spectrum when collision loss is ineluded with that when it is neglected. The exact solution is given by the equation (55) and it is elear that as $\beta$ tends to zero, the solution tends to (49). In the infinite series, two important points have to be noted:

(1) By the definition of $\psi_{n}$ in $(53,54) \psi_{1}(s, t)=0$ and it suffices to take only two terms of the series $\psi_{0}$ and $\psi_{2}$.

(2) $q(s ; t)$ has the following asymptotic forms. For large values of $s$,

$$
\begin{aligned}
q(s ; t) & \simeq \frac{2}{\alpha^{\prime} \log s} & \text { when } & \alpha^{\prime} t>2 \\
& \simeq t & \text { when } & \alpha^{\prime} t<2
\end{aligned}
$$

where

$$
\alpha^{\prime}=\left(\frac{4}{3}+\alpha\right) \text {. }
$$

The actual values of $\varphi(s ; t)$ have been tabulated.

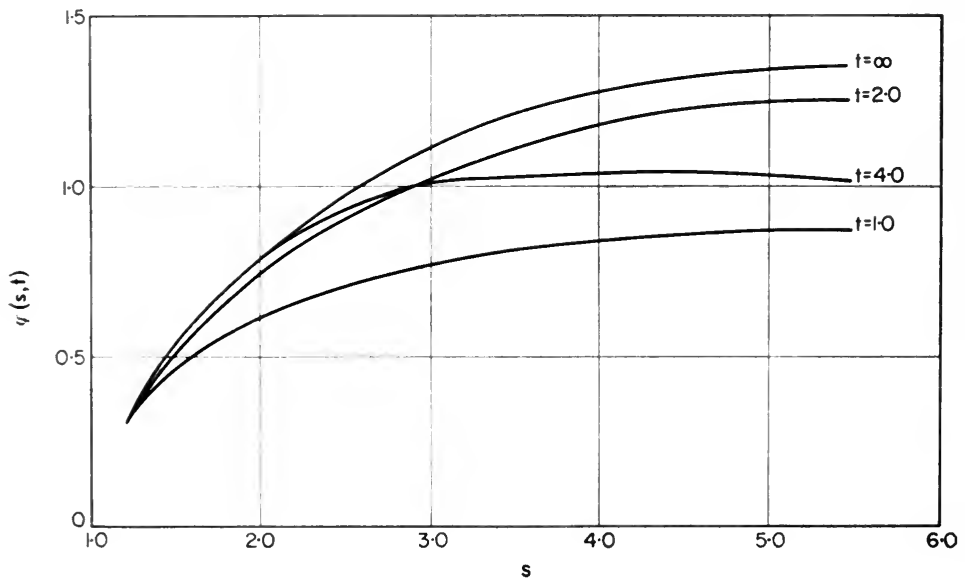

FIG. 63. $\varphi(s, t)$ as functions of $s$.

It is therefore found convenient to discuss the results for small and large thicknesses separately.

1 W. T. Scott and G. E. Uhlenbeck, Phys. Rev. 62, 497 (1942).

2 O. JAxossy and H. Messel, Proc. Phys. Soc. A 63, 1101 (1950). 
(1) Small thicknesses: Correct to the order of $t^{2}$, the solution reduces to

$$
\begin{aligned}
f_{1}(E ; t)= & \frac{1}{2 \pi i E_{\mathrm{J}}} \int_{\sigma-i \infty}^{\sigma+i \infty}\left(-\frac{E_{0}}{E_{0}+\beta t}\right)\left[1-A_{s} t+\frac{1}{2}\left(A_{s}^{2}+B_{s} C_{s}\right) t^{2}\right. \\
& \left.+\frac{\beta}{E_{0}}\left(\frac{s-1}{2}\right)\left(A_{s}-A_{s-1}\right) t^{2}\right] \mathrm{d} s .
\end{aligned}
$$

If we neglect the term with the coefficient $\beta$ in the square bracket, the spectrum is identical with that without ionization loss except that the energy parameter is shifted through $\beta t$, i.e. the density at $E$ when ionization loss is neglected is equal to that at $E-\beta t$ when ionization loss is included which implies that all the electrons lose energy deterministically by $\beta t$. This is obviously incorrect since some of the electrons are created at some intermediate point and hence lose energy less than $\beta t$. The inclusion of the term with coefficient $\beta$ corrects this error. At small thicknesses for initial energy $E_{0} \gg \beta$ there are not enough particles near the critical energy $\beta$. The spectrum in the neighbourhood of $\beta$ is important only for large thicknesses.

(2) Large thicknesses; Numerical results have been obtained by Bhabha and Chakrabarthy $^{1}$ using the method of saddle points corresponding to a given energy $E$ and thickness $t$. Each term in the infinite series for $f_{1}(E, t)$ has its own saddle point but we need only the saddle points $s_{0}$ and $s_{2}$ corresponding to $\psi_{0}$ and $\psi_{\mathbf{2}}$. These vary very little with $E$ and therefore we can take the values corresponding to $E_{0}$. For energies corresponding to $\beta, N_{n}(E, t)$ takes the form

$$
N_{n}(E ; t) \simeq\left\{\frac{\beta \varphi\left(s_{n} ; t\right)}{E+\beta \varphi\left(s_{n} ; t\right)}\right\}^{s_{n}+n-1} N_{n}(t) .
$$

Keeping only the first two terms, this becomes

$$
f_{1}(E ; t) \simeq \text { const } \times\left\{\left(s_{0}-1\right)\left(\frac{\beta \varphi}{E+\beta \varphi}\right)^{s_{0}} N_{0}+\left(s_{0}+1\right)\left(\frac{\beta \varphi}{E+\beta \varphi}\right)^{s_{0}+2} N_{2}\right\} .
$$

Whenever $N_{2} \ll N_{0}$ the spectrum is determined almost entirely by the term $\psi_{0}$ and is of the form $\left(\frac{\beta \varphi}{E+\beta \varphi}\right)^{s_{0}}$, i.e. a modified power law. The values of $\varphi$ are tabulated and are approximately equal to 1 . The value of $s_{0}$ can be obtained from Fig. 60 for any $t$ and $y_{0}$. At the shower maximum $s_{0} \simeq 2$ and before the maximum $s_{0}$ varies from 1.3 to 2 with increasing $t$. This implies that the power in the modified power law increases slowly with thickness corresponding to an increase in the number of electrons at the low energy end. The spectrum becomes nearly an inverse square law (modified) at the shower maximum and the role of the terms $\mathrm{N}_{2}$, $N_{3}$ etc. is to accentuate this tendency since $s_{0}$ and $s_{2}$ are not very different when $\mathrm{N}_{2}$ and $N_{0}$ are comparable. From equation (124) it can be seen that due to the factors $\left(s_{0}-1\right)$ and $\left(s_{0}+1\right)$ the contribution of the second term will be greater

1 H. J. Bhabha and S. K. Chakrabarthy, Phys. Rev. 74, 1352 (1948). 
than the first even if $N_{2}$ is slightly less than $N_{0}$ for energies lower than those determined by

$$
\left(\frac{E+\beta \varphi}{\beta \varphi}\right)^{2} \sim \frac{s_{0}+1}{s_{0}-1} \frac{N_{\mathbf{2}}}{N_{0}} .
$$

It can be actually shown by numerical calculations that the contribution due to $\mathrm{N}_{2}$ for the above range of energies is greater than that of $N_{0}$.

The values of $N_{0}$ and $N_{2}$ for various $y$ and $t$ are given in the appendix.

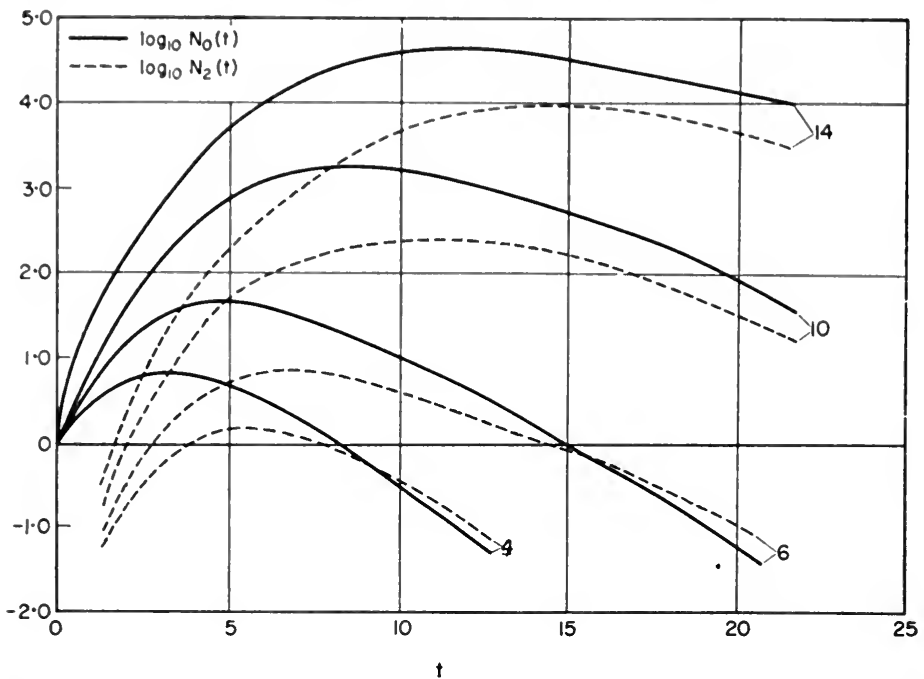

Fig. 64. $N_{0}$ and $N_{2}$ as functions of $t$; numbers on the curves indicate the values of $y_{0}$.

\section{Intranuclear cascades}

In the cascade processes treated till now, the quantum mechanical crosssection per unit thickness of matter for fundamental processes were assumed and the development of the processes with thicknesses was studied as a stochastic process. For the electrodynamic processes the cross-sections are known while for nuclear interactions, especially processes involving multiple production, the cross-sections are derived either by using phenomenological or by quasistatistical arguments. So speculative is such a study that it has been suggested by Heitler and Janossy ${ }^{1}$ that the cross-section for the multiple production of the mesons can be calculated by considering the collision of a fast particle with a nucleus as a cascade phenomenon within the nucleus. In analogy with the thickness of matter we have in this case the nuclear matter within the nucleus and the diameter of the nucleus corresponds to the parameter $t$ at which the cascade distribution is studied. This merely implies that we carry over the mathematics of the cascade theory to the computation of the probability $\pi\left(n \mid E_{0} ; t\right)$ that $n$-mesons are produced

1 W. Heitler and L. JAxossy, Proc. Phys. Soc. A 62, 669 (1949). 
by an incident particle of energy $E_{0}$ in passing through a nucleus of diameter $t$. This yields the cross-section of the process which in turn has to be used in studying the cascade development in matter.

Let $\dot{R}\left(E^{\prime} \mid E\right) \mathrm{d} E^{\prime}$ be the cross-section for the emission of a meson of energy $E-E^{\prime}$, i.e. the incident nucleon drops from an energy $E$ to an energy between $E^{\prime}$ and $E^{\prime}+\mathrm{d} E^{\prime}$. If $\pi\left(n, E \mid E_{0} ; t\right) \mathrm{d} E$ is the joint probability that a nucleon of energy $E_{0}$ (initially at $t=0$ ) after passing through a distance $t$ has undergone $n$ collisions and has an energy between $E$ and $E+\mathrm{d} E$ the diffusion equation can be written as

$$
\begin{aligned}
\frac{\partial \pi}{\partial t}\left(n, E \mid E_{0} ; t\right)= & -\pi\left(n, E \mid E_{0} ; t\right) \int_{0}^{E} R\left(E^{\prime} \mid E\right) \mathrm{d} E^{\prime} \\
& +\int_{E}^{E_{0}} \pi\left(n-1, E^{\prime} \mid E_{0} ; t\right) R\left(E \mid E^{\prime}\right) \mathrm{d} E^{\prime}
\end{aligned}
$$

Heitler and Janossy made the following simplifying assumptions that the mesons in turn do not produce further particles and that $R\left(E^{\prime} \mid E\right)=0$ if $E<E_{c}$.

Defining

$$
\begin{aligned}
\pi^{(1)}\left(n, E \mid E_{0} ; t\right) & \equiv \pi\left(n, E \mid E_{0} ; t\right) \text { for } E<E_{c} \\
\pi^{(2)}\left(n, E \mid E_{0} ; t\right) & \equiv \pi\left(n, E \mid E_{0} ; t\right) \text { for } E>E_{c}
\end{aligned}
$$

the equation breaks up into two parts:

$$
\begin{aligned}
\frac{\partial \pi^{(2)}}{\partial t}\left(n, E \mid E_{0} ; t\right)= & -\pi^{(2)}\left(n, E \mid E_{0} ; t\right) \int_{0}^{E} R\left(E^{\prime} \mid E\right) \mathrm{d} E^{\prime} \\
& +\int_{E}^{E_{0}} \pi^{(2)}\left(n-1, E^{\prime} \mid E_{0} ; t\right) R\left(E \mid E^{\prime}\right) \mathrm{d} E^{\prime} \\
\frac{\partial \pi^{(1)}}{\partial t}\left(n, E \mid E_{0} ; t\right)= & \int_{E_{c}}^{E_{0}} \pi^{(2)}\left(n-1, E^{\prime} \mid E_{0} ; t\right) R\left(E \mid E^{\prime}\right) \mathrm{d} E^{\prime}
\end{aligned}
$$

assuming 1$$
\begin{aligned}
& \text { (i) } R\left(E^{\prime} \mid E\right) \mathrm{d} E^{\prime} \text { is homogeneous and can be expressed as } R\left(\frac{E^{\prime}}{E}\right) \frac{\mathrm{d} E^{\prime}}{E} \text {. } \\
& \text { (ii) the distribution of the initial energy } E_{0} \text { is given by }
\end{aligned}
$$$$
F\left(E_{0}\right)=\gamma\left(\frac{E_{c}}{E_{0}}\right)^{\gamma+1} \frac{\mathrm{d} E_{0}}{E_{c}}
$$

with

$$
\int_{E_{c}}^{\infty} F\left(E_{0}\right) \mathrm{d} E_{0}=1
$$

1 A. Ramakrishnan, Proc. Phys. Soc. A 63, 861 (1950). 
We can obtain $p_{n}$ the probability of the occurrence of $n$-mesons irrespective of the energy state of the primary nucleon. Further, the probability that $n$ collisions have occurred and the energy of the primary is reduced to a value below the critical energy can be defined as

$$
p_{n}^{(1)}=\int_{E_{c}}^{\infty} F^{\prime}\left(E_{0}\right) \mathrm{d} E_{0} \int_{0}^{E_{c}} \pi^{(1)}\left(n, E \mid E_{0} ; t\right) \mathrm{d} E
$$

and $p_{n}^{(2)}$ as the probability that $n$ collisions have occurred and the energy of the primary is still above $E_{c}$,

and

$$
p_{n}^{(2)}=\int_{E_{c}}^{\sim} F\left(E_{0}\right) \mathrm{d} E_{0} \int_{E_{c}}^{E_{0}} \pi^{(2)}\left(n, E \mid E_{0} ; t\right) \mathrm{d} E
$$

$$
p_{n}=p_{n}^{(1)}+p_{n}^{(2)} .
$$

On performing the integration over $E$ and $E_{0}$ we obtain

$$
\begin{aligned}
& \frac{\partial p_{n}^{(2)}}{\partial t}=-p_{n}^{(2)} R+p_{n-1}^{(2)} R_{\gamma+1} \\
& \frac{\partial p_{n}^{(1)}}{\partial t}=-p_{n-1}^{(2)} R-p_{n-1}^{(2)} R_{\gamma+1}
\end{aligned}
$$

where $R$ is the integrated cross-section and

$$
R_{r}=\int_{0}^{E} R\left(\frac{E^{\prime}}{E}\right)\left(\frac{E^{\prime}}{E}\right)^{\gamma+1} \frac{\mathrm{d} E^{\prime}}{E^{\prime}} .
$$

Solving the equations (144) and (145)

$$
\begin{aligned}
& p_{n}^{(2)}=\frac{(R t)^{n}}{n !} \mathrm{e}^{-R \cdot t} R_{\gamma+1}^{n} \\
& p_{n}^{(1)}=\Gamma_{n}(R t) R_{\gamma+1}^{n-1}\left(1-R_{\gamma+1}\right) .
\end{aligned}
$$

It should be noted that integration with respect to the initial energy spectrum has an important mathematical consequence that it has the same effect of reducing the integrals with respect to energy to products as in the case of the Mellin transform, the real exponent $\gamma$ taking the place of the complex variable $s$. Of course in this case we have one obvious simplification that "the spectral transforms themselves represent the original functions" while in the Mellin transform, the inverse has to be taken. In fact in the calculation of mean numbers the integration can be performed over the initial spectrum. This simplification by the integration over initial energy spectrum is not possible for product densities of degree two since such integration over $E_{0}$ will affect both the variables $\varepsilon_{1}=\frac{E_{1}}{E_{0}}$ and $\varepsilon_{2}=\frac{E_{2}}{E_{0}}$ occurring in $f_{2}\left(E_{1}, E_{2} \mid E_{0} ; t\right)$. 


\section{INTERACTIONSAT EXTREMELY HIGH ENERGIES}

The quantitative study of cascade theory has till now been confined to the longitudinal development of electromagnetic cascades. Since there is no upper limit to the possible energy of the particles in the primary cosmic radiation, cascade showers can be initiated by particles of energy much greater than $10^{11} \mathrm{eV}$ and we have to take into account all types of elementary particle interactions as well as the lateral spread of the showers.

Such extensive air showers $(E A S)$ initiated by primaries which are invariably nucleons, consist of mesons, nucleons and strange particles produced in nuclear and electromagnetic collisions at high energies. It has been found experimentally that the particles which arrive at sea level are spread over large areas of the order of hundreds of metres. The lateral spread occurs due to two circumstances (i) multiple Coulomb-scattering, (ii) the angular distribution of particles at the point of production. The lateral spread of the electronic component is mainly due to the former.

It is found convenient to study the longitudinal and lateral developments separately. Even in the study of the longitudinal development, with our inadequate knowledge of the cross-sections for the individual processes it is not possible to study in a quantitative way the particle composition of cascades in finite thicknesses of matter. However it is possible to study the individual reactions in detail in "jet" showers in nuclear emulsion, small thicknesses of which correspond to large distances in air. As regards the lateral spread we shall deal only with the electromagnetic cascades which will give an idea of the nature and magnitude of the problem.

\section{The longitudinal development of "jets"}

We shall outline the general features of the "jet" before referring to the present experimental situation.

(a) The shower axis: We define the axis of a shower as the direction of the primary which of course is isotropically distributed in space (except for the forbidden directions according to the geomagnetic theory). Since the high energy particles are extremely collimated we can speak of the "core" of the shower. The shower axis in the emulsion is obtained by extrapolating the primary track beyond the origin of the jet and is determined as follows: If $\varepsilon_{i}$ is the energy of the particle making an angle $\varphi_{i}$ with the approximate shower axis, a large number of such particles are chosen such that $\sum_{i} \varepsilon_{i} \sin \varphi_{i}$ equals zero. The track closest to the shower axis may be chosen as the reference for all angular measurements.

(b) The nature of the tracks: The charged secondaries are classified into three groups depending on the grain densities of the tracks. The black and grey tracks

1 "The extensive air showers" by K. Greisen, Progress in Cosmic Ray Physics, 3, 3 (1956). Extensive Air Showers by William GalbRaith, Butterworths (1958). 
correspond to the low energy protons and knock-on particles from the target nucleus. The minimum tracks correspond to the shower particles produced in nucleon-nucleon collisions.

(c) The angular distribution: It has been found that most of the secondaries emerge in a narrow cone about the primary axis, a circumstance which can be explained as follows. The secondaries produced in the collision of a stationary and a relativistic nucleon are distributed symmetrically (but for statistical fluctuations) about the two nucleons in the centre of mass system.

The angle $\theta$ at which a particle emerges in the laboratory system is given by

$$
\tan \theta=\frac{r^{\prime} \sin \theta^{\prime}}{\gamma_{c}\left(v^{\prime} \cos \theta^{\prime}+\beta_{c}\right)} \quad \text { with } \quad \gamma_{c}=\frac{1}{\sqrt{1}-\beta_{c}^{2}}
$$

where $v^{\prime}, \theta^{\prime}$ are the velocity and angle of emission in the centre of mass system and $\beta_{c}$ the relative velocity of the two frames. We shall hereafter denote the various quantities in the laboratory $(L)$ and centre of mass $(C)$ system by unprimed and primed variables. It can be shown (see appendix) that to a given angle $\theta$ in $L$ there corresponds two angles $\theta_{1}^{\prime}$ and $\theta_{2}^{\prime}$ in $C$, i.e. there are two velocities $v_{1}$ and $v_{2}$ in $L$ at the angle $\theta$ corresponding to the same velocity $v^{\prime}$ along $\theta_{1}^{\prime}$ and $\theta_{2}^{\prime}$. The correspondence between the various angles in the two systems is as follows:

For a given velocity $v^{\prime}$ there is an angle $\theta_{M}$ such that the two angles $\theta_{1}^{\prime}$ and $\theta_{2}^{\prime}$ coincide and are equal to $\theta_{M}^{\prime} . \theta_{M}$ is the maximum angle of emission which can be assumed in $L$ by a particle with velocity $v^{\prime}$ in $C$. As $\theta^{\prime}$ varies from 0 to $\theta_{M}^{\prime}, \theta$ varies from 0 to $\theta_{M}$ and as $\theta^{\prime}$ increases from $\theta_{M}^{\prime}$ to $\pi, \theta$ decreases from $\theta_{M}^{\prime}$ to 0 . Thus all the particles are emitted within a cone of half angle $\theta_{M}$ in $L$. Consider now the particles emitted in the forward direction in $C$, i.e. $0<\theta^{\prime}<\pi / 2$. In the laboratory system they will be within 0 to $\theta_{F}$ where $\theta_{F}<\theta_{M}$. Let $\theta_{2}^{\prime}=\theta_{c}^{\prime}$ be the angle corresponding to $\theta_{1}^{\prime}=\frac{\pi}{2}$, i.e. particles along $\theta_{1}^{\prime}=\pi / 2$ or $\theta_{2}^{\prime}=\theta_{c}^{\prime}$ will emerge along $\theta_{F}$ in $L$. The particles emitted between $\theta_{1}^{\prime}=\pi / 2$ and $\theta_{M}^{\prime}$ will lie between $\theta_{F}$ and $\theta_{M}$, those between $\theta_{M}^{\prime}$ and $\theta_{c}^{\prime}$ between $\theta_{M}$ and $\theta_{F}$ and those between $\theta_{c}^{\prime}$ and $\pi$ between $\theta_{F}$ and 0 . The particles emitted in the backward direction in the $C$ system fall into two classes. Those with $\frac{\pi}{2} \leqq \theta^{\prime} \leqq \theta_{c}^{\prime}$ will lie between $\theta_{F} \leqq \theta \leqq \theta_{M}$ while those with $\theta_{c}^{\prime} \leqq \theta^{\prime} \leqq \pi$ lie in the same forward cone $0 \leqq \theta \leqq \theta_{F}$ as the particles in the
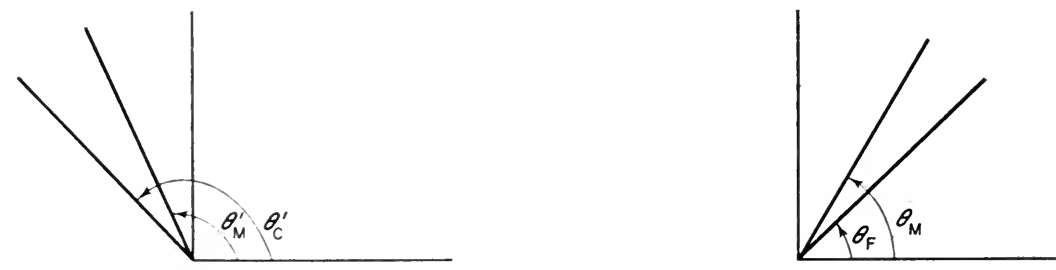

FIG. 65. Correspondence between the angles in the c.m. and laboratory systems. 
forward direction. However these two sets of particles can be distinguished by their velocities since the velocity corresponding to $\theta_{2}^{\prime}$ is always smaller than that for $\theta_{1}^{\prime}$. Experimentally the cones of half angles $\theta_{F}$ and $\theta_{M}$ can be easily identified. The degree of anisotropy in the centre of mass system is reflected on the density of particles within the cone of angle $\theta_{F}$. We summarize below the correspondence between the angles in the two frames.

Centre of mass system

$$
\begin{aligned}
0 & \leqq \theta^{\prime} \leqq \pi / 2 \\
\frac{\pi}{2} \leqq \theta^{\prime} & \leqq \theta_{M}^{\prime} \\
\theta_{M}^{\prime} & \leqq \theta^{\prime} \leqq \theta_{c}^{\prime} \\
\theta_{c}^{\prime} & \leqq \theta^{\prime} \leqq \pi
\end{aligned}
$$

Laboratory system

$$
\begin{gathered}
0 \leqq \theta \leqq \theta_{F} \\
\theta_{F} \leqq \theta \leqq \theta_{M} \\
\theta_{M} \geqq \theta \geqq \theta_{F} \\
\theta_{F} \geqq \theta \geqq \theta
\end{gathered}
$$

The approximate value of $\theta_{F}$ in radians is given by

$$
\theta_{F} \sim \sqrt{\frac{2 M_{p}}{E_{p}}}
$$

where the nucleon mass $M_{p}$ and the primary energy $E_{p}$ are expressed in $\mathrm{BeV}$.

(d) Determination of energies: The energy of the primary particle cannot be measured directly. It is usually estimated from the approximate relation

$$
E_{p} \simeq \frac{2 M_{p}}{\theta_{F}^{2}} \simeq \frac{2}{\theta_{F}^{2}} \quad \mathrm{BeV} .
$$

This relation is valid only for nucleon-nucleon collisions with isotropic distribution of secondaries in the centre of mass system.

In the case of a nucleon-nucleon collision the total energy $W$ is given by

$$
W=2 \gamma_{c}^{2} M_{p} \text {. }
$$

A fairly accurate method of determining the energy is through $\gamma_{c}$. The two methods of obtaining $\gamma_{c}$ are given below.

(i) If $n_{i}$ and $n_{f}$ are the number of forward and backward shower tracks (which can be identified in the laboratory system by separating out the cones) and $\varphi_{i}$ and $\varphi_{b i}$ the angles of the individual tracks in the laboratory system then

$$
\log \gamma_{c}=\frac{1}{2 n_{f}} \sum_{i} \log \tan \varphi_{f i}+\frac{1}{2 n_{b}} \sum_{i} \log \tan \varphi_{t i} .
$$

(ii) If $E$ and $E^{\prime}$ are the energies of the shower particles in the two system and $\varphi_{i}$ the space angles between the track and the forward direction we have

$$
\frac{1}{E}=\frac{1}{2 \gamma_{c} E^{\prime}}+\frac{\gamma_{c}}{2 E^{\prime}} \tan ^{2} \varphi_{i} \text {. }
$$


The plot of $\frac{1}{E}$ against $\tan ^{2} \varphi_{i}$ is a straight line when all the secondaries are emitted with the same energy. The intercept on the $E$ axis is $\frac{1}{2 \gamma_{c} E^{\prime}}$ and the slope is $\gamma_{c} / E^{\prime}$.

(c) The transicerse momentum ${ }^{1}$ : Besides energy, the transverse momentum $p_{\perp}$ of the particle measured in a direction perpendieular to the line of flight of the primary is an important characteristic of the particle. It was first noted by Nishimura that $p_{\perp}$ is almost independent of the energy of the primary, for example, for pions $p_{\perp} \sim 0.5 \mathrm{BeV} / \mathrm{e}$. Sinee the perpendicular component is unaffected by the Lorentz transformation the value of $p_{\perp}$ is identical in the two systems. The average $p_{\perp}$ of the $\gamma$ rays was expected to be half that of the parent pion. However the transverse momentum of the $\gamma$ rays measured in a direction perpendicular to the direction of the pion was found to be very small which can be neglected in comparison with that of the pion. The mean value of $p_{\perp}$ for non-pions is found to be of the order of $1.5 \mathrm{BeV}$.

An approximate method of measuring the energy is to assume the constancy of $p_{\perp}$. The measurement of angular distribution in the centre of mass system will yield the total energy of the secondary particles. The laboratory angular distribution can be easily measured as most of the secondaries are pions.

(f) Inelasticity: The inelasticity $I$ of a process is the fraction of the total kinetic energy of the nucleons in $L$ transferred to the newly created particles. That it. is not possible for two colliding particles to lose all their kinetic energy in $L$ is clear from the following argument. In the $C$-system the total momentum is zero and both the particles can be brought to rest. This corresponds to the maximum transfer of energy to the newly created particles. Observing the collision in the $L$-system the nucleons will have a velocity the magnitude of which is equal to that of the $C$-system. The value of $I_{\max }$ is given by

$$
\frac{\gamma_{c}}{\gamma_{c}+1}
$$

We briefly summarize the various methods of determining $I$.

(1) $I$ is given by the relation

$$
I=\sum_{i} \varepsilon_{i} / \varepsilon
$$

where $\varepsilon_{i}$ and $\varepsilon$ are the energies of the $i$-th and the primary particles respectively. Experiments in air showers indicate that a primary may retain up to 80 per cent of its energy, i.e. $I \sim 0.2$.

(2) $I$ ean also be determined from measuring the ratio $\lambda_{\text {att }} / \lambda_{\text {int }}$ where the attenuation length $\lambda_{\text {att }}$ is defined as the distance in which the number of stable particles above energy $E_{0}$ (with a spectral energy distribution) falls off by a factor $\frac{1}{e}$. The ratio $S=\lambda_{\text {att }} / \lambda_{\text {int }}$ is always $\geqq 1$, the equality holding only when all

1 See S. Hasegawa, K. Niu, Institute for Nuclear Study Reports (1958). 
the interactions result in catastrophic absorption. The extent to which $S$ exceeds unity depends on $I$. Experiments with primaries of energy $\sim 100 \mathrm{BeV}$ yield a value of $I \sim 0.5$ while at $10^{7} \mathrm{BeV}, I \sim 0.3$.

(3) A third method of determining $I$ from individual events is by Daniel ${ }^{1}$ et al. "The quantity

$$
\varepsilon=\frac{n_{s} M}{2} \gamma_{s}\left(1-\beta_{s} \cos \theta\right)=M \bar{\gamma}_{s} /\left(\frac{2 \gamma_{c}}{n_{s}}\right)
$$

was determined for each track in the outer fringe. Here $M$ is the ratio of the rest mass of a secondary to that of a proton, $\varepsilon$ the ratio of the total energy of a secondary to the average energy available for each secondary and $\bar{\gamma}_{s} / 2 \gamma_{c}$ the ratio of the energy of the particle to the total available energy in the centre of mass system. The mean value of $\bar{\varepsilon}$ was obtained as $0.65 \pm 0.20$ for primaries of energy $>100 \mathrm{BeV}$. This is consistent with the view that about a third of the available energy is carried by neutral particles.

(4) Another method of determining $I$ is to measure the maximum angle of emission of secondary particles in the laboratory system. For $\bar{\gamma}_{s}<\gamma_{c}$ there must exist a maximum value of $\theta$ corresponding to $\cos \theta^{\prime}=-\bar{\beta}_{\delta} / \beta_{c}$ such that $\bar{\gamma}_{s} \simeq \gamma_{c} \sin \theta_{\max } . I$ is evaluated by assuming that most of the secondary particles are pions. This method holds only if

(i) all the secondary particles are emitted with the same $\bar{\gamma}_{s}$,

(ii) $\theta_{\max }$ can be measured without statistical error.

However such conditions do not hold in practice. The accurate determination of $I$ requires either the direct measurement of angle and energy or some extra condition such as the constancy of $p_{\perp}$ besides the identity of particles.

\section{The lateral spread of the extensive air showers (EAS)}

As stated earlier we shall deal only with the lateral spread of the electrons in electromagnetic cascades. Similar considerations apply to other types of showers.

In any quantitative study we are interested in the average number of particles in the energy range between $E$ and $E+\mathrm{d} E$ at thickness $t$. Let $t$ be chosen as the $z$-axis and $r$ the radial distance in the $x y$-plane. Let the particle move in a direction making an angle between $\theta$ and $\theta+\mathrm{d} \theta$ with the $z$-axis. $\theta_{x}$ and $\theta_{y}$ are the angles which the projections of the particle direction in the $x z$ - and $y z$-planes make with the $z$-axis. Thus the number of particles can be denoted by $\pi(E, r, \theta ; t) \mathrm{d} E \mathrm{~d} r \mathrm{~d} \theta$ and when there are more than one type of particle, similar functions can be defined for each of them. To obtain these functions their variation with $t$ will have to be studied. We have already discussed the situation in great detail when the distribution in $r$ and $\theta$ have not been taken into account. We include this by defining $\sigma(\varphi) \mathrm{d} \varphi$ to be the probability that an electron is deflected at an angle $(\varphi, \mathrm{d} \varphi)$ with its original direction in travelling a thickness $\mathrm{d} t . \sigma(\varphi)$ is an even

1 R. R. Daniel et al., Bristol Conference on Hyperons and Heavy Mesons (1951). 
function as the scattering should by symmetric about the original direction. For scattering due to a Coulomb-field we take

where

$$
\sigma(q) \mathrm{d} \varphi=\frac{1}{4 \pi \log \left(183 Z^{-1 / 3}\right)}\left(\frac{E_{s}}{E}\right)^{2} \frac{\mathrm{d} \varphi}{\varphi^{4}},
$$

$$
E_{s}=2 \pi(137)^{1 / 2} m_{\mathrm{e}}=21 \mathrm{MeV} .
$$

Then the variation in the number of particles within an interval $\theta$ and $\theta+\mathrm{d} \theta$ caused by seattering is given by:

$$
-\pi(E, r, \theta ; t) \int_{\varphi} \sigma(\varphi) \mathrm{d} \varphi+\int_{\varphi} \sigma(\varphi-\theta) \pi(E, r, \varphi ; t) \mathrm{d} \varphi .
$$

There is also a deterministic lateral (spatial) displacement $\theta \mathrm{d} t$ and this implies that particles with coordinates $r-\theta \mathrm{d} t$ at $t$ move to $r$ at $t+d t$, i.e.

$$
\pi(r, \theta ; t+\mathrm{d} t)=\pi(r-\theta \mathrm{d} t, \theta ; t) .
$$

The change in the number density at $r$ due to variation $\mathrm{d} t$ assuming only this process is given by

$$
\pi(r, \theta, t+\mathrm{d} t)=\pi(r-\theta \mathrm{d} t, \theta ; t)=\pi(r, \theta, t)-\theta \mathrm{d} t \frac{\partial \pi}{\partial r}(r, \theta, t) .
$$

Expanding $\pi(E, r, \theta+\varphi, t)$ as a Taylor series in $\theta$, we obtain (11) as:

$$
\begin{gathered}
-\pi(E, r, \theta ; t) \int \sigma(\varphi) \mathrm{d} \varphi+\left[\int _ { \varphi } \left\{\pi(E, r, \theta ; t)+\varphi \nabla_{\theta} \pi(E, r, \theta ; t)\right.\right. \\
\left.\left.+\frac{\varphi^{2}}{2} \nabla_{\theta}^{2} \pi(E, r, \theta ; t)+\cdots\right\} \sigma(\varphi) \mathrm{d} \varphi\right] .
\end{gathered}
$$

The second term within the square bracket vanishes as $\sigma$ is an even function. At this stage, we make the following approximation. The mean square angle of scattering is given by

$$
\int_{\bar{\varphi}} q^{2} \sigma(\varphi) \mathrm{d} \varphi=\frac{1}{2}\left(\frac{E_{8}}{E}\right)^{2} .
$$

All higher moments of $\varphi$ are neglected. This implies that $\sigma(\varphi)$ rapidly tends to zero as $\varphi$ differs from zero, i.e. the scattering is confined to an extremely small angle. This approximation is necessary since even the cumulative angular deviation $\theta$ is considered small enough such that $\sin \theta=0$, i.e. the path length $t$ traversed is identical with the $z$-coordinate. If this approximation is not made finite values of $\varphi$ will be allowed and therefore $\theta$ can take values greater than $\pi / 2$ which implies the possibility of back scattering. The process will not then be Markovian with respect to $z$ and considerable mathematical difficulties are introduced which are not yet solved even from a formal stochastic point of view. ${ }^{1}$

1 This point does not seem to have been realised by Kamata and Nishimura who believe that the Landau approximation can be improved by replacing $\theta$ by $\sin \theta$. Therefore we are not giving an account of their treatment when the approximation is not made. 
With the above approximation, the diffusion equations for a three-dimensional electron-photon cascade can be written as:

$$
\begin{aligned}
& \frac{\partial f}{\partial t}=-A^{\prime} f+B^{\prime} g+\frac{E_{s}}{4 E^{2}} \nabla_{\theta}^{2} f+\beta-\theta_{x} \frac{\partial f}{\partial x}-\theta_{y} \frac{\partial f}{\partial y} \\
& \frac{\partial g}{\partial f}=C^{\prime} f-D g+\theta_{x} \frac{\partial g}{\partial x}-\theta_{y} \frac{\partial g}{\partial y}
\end{aligned}
$$

$f\left(E, r, \theta \mid E_{0} ; t\right)$ and $g\left(E, r, \theta \mid E_{0} ; t\right)$ are the electron and photon densities respectively and $A^{\prime}, B^{\prime}, C^{\prime}$ and $D$ symbolically refer to the corresponding expressions in the electron-photon cascade equations (30) and (31). If we take the Mellin transform of the $f$ and $g$ functions with respect to $E$ we obtain $A(s), B(s), C(s)$ and $D$ respectively. The above set of equations are referred to as the Landau ${ }^{1}$ equations. It is easily seen that the introduction of the distribution in $r$ and $\theta$ has resulted in the additional term $\frac{E_{s}^{2}}{4 E^{2}} \Delta_{\theta}^{2} f, \theta_{x} \frac{\partial f}{\partial x}$ and $\theta_{y} \frac{\partial f}{\partial y}$ in the diffusion equations. A general solution of these equations has been obtained by Nishimura and Kamata ${ }^{2}$ by an extension of the method due to Bhabha and Chakrabarthy. ${ }^{3}$ To solve these equations we make use of the standard Fourier transform technique and define

$$
\begin{aligned}
& p^{\prime}\left(E, \eta, \xi \mid E_{0} ; t\right)=\frac{1}{4 \pi^{2}} \int_{-\infty}^{\infty} \int_{\theta} f\left(E, r, \theta \mid E_{0} ; t\right) \mathrm{e}^{i \eta \cdot r+i \xi \cdot \theta} \mathrm{d} r \mathrm{~d} \theta \\
& q^{\prime}\left(E, \eta, \xi \mid E_{0} ; t\right)=\frac{1}{4 \pi^{2}} \int_{-\infty}^{\infty} \int_{\theta} g\left(E, r, \theta \mid E_{0} ; t\right) \mathrm{e}^{i \eta \cdot r+i \xi \cdot \theta} \mathrm{d} r \mathrm{~d} \theta
\end{aligned}
$$

$\eta$ and $\xi$ have two components $\eta_{1}, \eta_{2}$ and $\xi_{1}, \xi_{2}$ respectively.

In terms of $p^{\prime}$ and $q^{\prime}$, equations (16) and (17) take the form

$$
\begin{aligned}
& \left(\frac{\partial}{\partial t}-\eta \frac{\partial}{\partial \xi}\right) p^{\prime}=-A^{\prime} p^{\prime}+B^{\prime} q^{\prime}-\frac{E_{s}^{2} \xi^{2}}{4 E^{2}} p^{\prime}+\beta \frac{\partial p^{\prime}}{\partial E} . \\
& \left(\frac{\partial}{\partial t}-\eta \frac{\partial}{\partial \xi}\right) q^{\prime}=C^{\prime} p^{\prime}-C^{\prime} q^{\prime} .
\end{aligned}
$$

Eliminating $q^{\prime}$ between the two equations we have

$$
\begin{gathered}
\left\{\left(\frac{\partial}{\partial t}-\eta \frac{\partial}{\partial \xi}\right)^{2}+\left(A^{\prime}+D\right)\left(\frac{\partial}{\partial t}+\eta \frac{\partial}{\partial \xi}\right)+\left(A^{\prime} D+B^{\prime} C^{\prime}\right)\right\} p^{\prime}\left(E, \eta, \xi \mid E_{0} ; t\right) \\
=\left(\frac{\partial}{\partial t}-\eta \frac{\partial}{\partial \xi}\right)\left(-\frac{E_{s}^{2} \xi^{2}}{4 E^{2}}+\beta \frac{\partial}{\partial E}\right) p^{\prime}\left(E, \eta, \xi \mid E_{0} ; t\right) .
\end{gathered}
$$

1 L. Landau, J. Phys., 3237 (1940). See also G. Moliere's article in Cosmic Radiation, Ed. W. Heisen berg, Dover (1943).

2 J. Kamata and K. Nishimura, Progr. Theoret. Phys. Supp., 6, 135 (1958). This paper contains an extensive reference to the work on the subject.

3 H. J. Bhabha and S. K. Chakrabarthy, Phys. Rev. 74, 1352 (1948). 


\section{Radial structure functions}

The number of particles between $r$ and $r+\mathrm{d} r$ is given by $2 \pi r \mathrm{~d} r f\left(E, r \mid E_{0} ; t\right)$ where

$$
f^{(r)}\left(E, r \mid E_{0} ; t\right)=\int_{-\infty}^{\infty}\left(E, r, \theta \mid E_{0} ; t\right) 2 \pi \theta \mathrm{d} \theta
$$

Substituting for $f$ in the integral in terms of $p^{\prime}\left(E, \eta, \xi \mid E_{0} ; t\right)$ we get

$$
\begin{aligned}
f^{(r)}\left(E, r \mid E_{0} ; t\right) & =\int_{-\infty}^{\infty} E^{-i \eta \cdot r} p^{\prime}\left(E, \eta, 0 \mid E_{0} ; t\right) \mathrm{d} \eta \\
& =\int_{0}^{\infty} p^{(r)}\left(E, \eta, 0 \mid E_{0} ; t\right) J_{0}(\eta r) 2 \pi \eta \mathrm{d} \eta
\end{aligned}
$$

where $J_{0}$ is the Bessel function and $\eta=V\left(\eta_{1}^{2}+\eta_{2}^{2}\right)$ and $r=V\left(x^{2}+y^{2}\right)$.

Thus we need only $p^{\prime}\left(E, \eta, 0 \mid E_{0} ; t\right)$ to obtain the lateral structure function $f^{(r)}\left(E, r \mid E_{0} ; t\right)$. However $p^{\prime}\left(E, \eta, 0 \mid E_{0} ; t\right)$ cannot be obtained merely by setting $\xi$ equal to zero beeause the derivative of $p^{\prime}$ with respect to $\xi$ occurs in the equation. Assuming the vector $\xi$ to be parallel to $\eta$ the equation for $p^{\prime}\left(E, \eta, \xi_{1}, \xi_{2}=0 \mid E_{0} ; t\right)$ is

$$
\begin{gathered}
\left\{\left(\frac{\partial}{\partial t}-\eta \frac{\partial}{\partial \xi_{1}}\right)^{2}+\left(A^{\prime}+D\right)\left(\frac{\partial}{\partial t}-\eta \frac{\partial}{\partial \xi_{1}}\right)+\left(A^{\prime} D-B^{\prime} C^{\prime}\right)\right\} p^{\prime}\left(\xi_{2}=0\right) \\
=\left(\frac{\partial}{\partial t}-\eta \frac{\partial}{\partial \xi_{1}}+D\right)\left(-\frac{4 E_{s}^{2}}{4 E^{2}} \xi_{1}^{2}+\beta \frac{\partial}{\partial E}\right) p^{\prime}\left(\xi_{2}=0\right) .
\end{gathered}
$$

Defining two new variables $\zeta$ and $t$ by $\zeta=\eta\left(\xi-t^{\prime}\right)$ and $t=t^{\prime}$ the equation (25) beeomes

$$
\begin{aligned}
& \left\{\frac{\partial}{\partial t^{\prime 2}}+\left(A^{\prime}+D\right) \frac{\partial}{\partial t^{\prime}}+\left(A^{\prime} D-B^{\prime} C^{\prime}\right)\right\} p^{\prime} \\
& =\left(\frac{\partial}{\partial t^{\prime}}+D\right)\left[+\frac{E_{8}^{2}}{4 E^{2}} \eta^{2}\left(\zeta-t^{\prime}\right)+\beta \frac{\partial}{\partial E}\right] p^{\prime}
\end{aligned}
$$

Defining $F$ as a Mellin transform of $p^{r}$ with respect to $E$ with $s$ as a complex argument, the solution of the above equation in terms of the powers of $\frac{E_{s}^{2} \eta^{2}}{E^{2}}$ and $\beta / E$ is (on writing $t^{\prime}=t$ )

$$
F_{1}\left(s, \eta, 0 E_{0} ; t\right)=\frac{1}{2 \pi} E_{0}^{s}\left\{\sum_{m=n}^{\infty} \sum_{n=0}^{\infty}\left(-\frac{E_{s}^{2} \eta^{2}}{4} \bar{E}^{2}\right)^{m}\left(-\frac{\beta}{E}\right)^{n} \varphi_{m, n}(s, t)\right\}
$$

where $\varphi_{m, n}$ is defined through the equation

$$
\begin{aligned}
& \int \frac{\partial^{2}}{\partial t^{2}}+(A(s+2 m+n)+D) \frac{\partial}{\partial t}+(A(s+2 m+n) D-B(s+2 m+n) \\
& \times C(s+2 m+n)) \Phi_{m, n} \\
& \left.=\left(\frac{\partial}{\partial t}+D\right)\left\{(\zeta-t)^{2} \Phi_{m-1, n}+(s+2 m+n) \Phi_{m, n-1}\right\}\right]
\end{aligned}
$$


with

and $\Phi_{0,0}$ satisfies

$$
\varphi_{m, n}=\underset{(\zeta-t) \rightarrow 0}{\operatorname{Lim}} \Phi_{m, n}
$$

$$
\left[\frac{\partial^{2}}{\partial t^{2}}+\{A(s)+D\} \frac{\partial}{\partial t}+A(s) D-B(s) C(s)\right] \Phi_{0,0}=0
$$

with the boundary conditions

$$
\Phi_{0,0}(s, 0)=1 ;\left.\quad \frac{\partial}{\partial t} \Phi_{0,0}(s, t)\right|_{t=0}=-A(s)
$$

corresponding to a shower initiated by an electron of energy $E_{0} . \Phi_{0,0}$ is given by

where

$$
\Phi_{0,0}=H_{1}(s) \mathrm{e}^{\lambda_{1}(s) t}+H_{2}(s) \mathrm{e}^{\lambda_{2}(s) t}
$$

and

$$
\begin{gathered}
H_{1}(s)=\frac{D+\lambda_{1}(s)}{\lambda_{1}(s)-\lambda_{2}(s)} ; \quad H_{2}(s)=\frac{D+\lambda_{2}(s)}{\lambda_{1}(s)-\lambda_{2}(s)} \\
\lambda_{1}(s)=\frac{1}{2}\left[-(A(s)+D)+\left\{(A(s)-D)^{2}+4 B(s) C(s)\right\}^{1 / 2}\right]
\end{gathered}
$$

$$
\lambda_{2}(s)=\frac{1}{2}\left[-(A(s)+D)-\left\{(A(s)-D)^{2}+4 B(s) C(s)\right\}^{1 / 2}\right] .
$$

The solution of (28) is given by the recurrence formula

$$
\begin{aligned}
\Phi_{m, n}(s, \zeta-t, t)=\int_{0}^{t} \Phi_{0,0}(s, 2 m & \left.+n, t-t^{\prime}\right)\left\{\left(\zeta-t^{\prime}\right)^{2} \Phi_{m-1, n}\left(s, \zeta-t^{\prime}, t^{\prime}\right)\right. \\
& \left.+(s+2 m+n) \Phi_{m, n-1}\left(s, \zeta-t^{\prime}, t^{\prime}\right)\right\} \mathrm{d} t^{\prime} .
\end{aligned}
$$

The solution $p^{(r)}$ has been obtained as a double infinite series involving the summation over the discrete variables $m$ and $n$. Kamata and Nishimura ${ }^{1}$ have expressed this as integrals over the continuous complex variables $u$ and $v$ by first defining the function

and recognizing that

$$
M_{1}(m, n, s, t)=\frac{\varphi_{m, n}(s, t)}{\Gamma(m+1) \Gamma(n+1)}
$$

$$
\begin{aligned}
\sum_{m=n}^{\infty} \sum_{n=0}^{\infty}\left(-\frac{E_{s}^{2} \eta^{2}}{4 E^{2}}\right)^{m}(-\beta / E)^{n} & \varphi_{m, n}(s, t) \\
& =\int_{0}^{\infty} \mathrm{e}^{-\alpha} \frac{E_{s}^{2} \eta^{2}}{4 E^{2}}-\alpha^{\prime} \beta / E F\left(\alpha, \alpha^{\prime}, s, t\right) \mathrm{d} \alpha \mathrm{d} \alpha^{\prime}
\end{aligned}
$$

where $F\left(\alpha, \alpha^{\prime}, s, t\right)$ is a function which has the Mellin transform $M_{1}(u, v, s, t)$. The function $M_{1}(u, v, s, t)$ is obtained by merely replacing $m$ and $n$ by the two complex variables $u$ and $v$ respectively. The double series is uniformly and abso-

1 J. Kamata and K. Nishimura, Ibid. (1958).

EPCR 30 
lutely convergent for $E>\frac{E_{8} \eta t^{2 / 2}}{3}$ and $E>8 \beta t . M_{1}$ satisfies the same equation(28) as $\Phi_{m, n}$. Thus changing the order of integration and inverting we get

$$
\begin{array}{r}
f^{(r)} \frac{1}{\mathrm{~s} \pi^{4} i} \int_{-i \sim}^{i \sim} \mathrm{d} s \mathrm{~d} u \mathrm{~d} v\left(\frac{E_{0}}{E}\right)^{s} \frac{1}{E}\left(\frac{E}{E_{s}}\right)^{2}\left(\frac{\beta}{E}\right)^{v}\left(\frac{E^{2} r^{2}}{E_{s}^{2}}\right)^{-u-1} \\
I^{\prime}(u+1) \Gamma(-v) M_{1}(u, v, s, t) .
\end{array}
$$

This solution for the lateral structure function is valid irrespective of the restrictions on $E$. The lateral structure function for particles above energy $E$ is given $\mathrm{by}$

$$
\begin{aligned}
f^{r}\left(E, r E_{0} ; t\right) & =\int_{E^{\prime}}^{\infty} f^{(r)}\left(E, r \mid E_{0} ; t\right) \mathrm{d} E \\
& -\frac{1}{8 \pi^{4} i} \int_{-i \infty}^{+i \infty} \frac{\mathrm{d} s \mathrm{~d} u \mathrm{~d} v}{s+2 u+v}\left(\frac{E_{0}}{E}\right)^{8}\left(\frac{E}{E_{8}}\right)^{2}\left(\frac{\beta^{2}}{E}\right)^{v}\left(\frac{E_{0} r^{2}}{E_{8}^{2}}\right)^{-u-1} \\
& \times I(u+1) \Gamma(-v) M_{1}(u, v, s, t) .
\end{aligned}
$$

At the limit $E=0$, the integration with respect to $v$ is equal to the residues of the integrands at $v=-s-2 u$ and we obtain the structure function for the total number of particles as

$$
\begin{aligned}
f^{r}\left(0, r E_{0} ; t\right)= & -\frac{1}{4 \pi^{3}} \int_{-i \infty}^{i \infty} \mathrm{d} s \mathrm{~d} u\left(\frac{E_{0}}{\beta}\right)^{8}\left(\frac{\beta}{E_{8}}\right)^{2}\left(\frac{\beta^{2} r^{2}}{E_{s}^{2}}\right)^{-u-1} \Gamma(u+1) \\
& \times \Gamma(s+2 u) M_{1}(u,-s-2 u, s, t) .
\end{aligned}
$$

The total number of electrons is obtained by integrating over $r$ in the above expression

$$
\int_{0}^{\infty} \bar{f}^{(r)}\left(0, r / E_{0} ; t\right) 2 \pi r \mathrm{~d} r=\frac{1}{2 \pi i} \int \mathrm{d} s\left(\frac{E_{0}}{\beta}\right)^{8} \Gamma(s) M_{1}(0,-s, s, t) .
$$

\section{Angular structure functions}

The angular structure function $f^{(\theta)}\left(E, \theta \mid E_{0} ; t\right)$ for electrons is given by

$$
\begin{aligned}
f^{(\theta)}\left(E, \theta \mid E_{0} ; t\right) & =\int_{-\infty}^{+\infty} f\left(E, r, \theta \mid E_{0} ; t\right) 2 \pi r \mathrm{dr} \\
& =\int_{0}^{\infty} p^{\prime}(\theta ; \xi) \tau_{0}(\xi, \theta) 2 \pi \xi \mathrm{d} \xi .
\end{aligned}
$$

The solutions $f^{(\theta)}$ and $f^{(\theta)}$ are obtained in a manner similar to that of $f^{(r)}$ and $f^{(r)}$ These expressions can be obtained by replacing $r$ by $\theta$ and $M_{1}(u, v, s, t)$ by 
$M_{2}(u, v, s, t)$ where $M_{2}$ is a solution of the equation

$$
\begin{array}{r}
\partial^{2} / \partial t^{2}+\left\{A(s+2 u+v)+D\left[\frac{\partial}{\partial t}+A(s+2 u+v) D-B(s+2 u+v)\right.\right. \\
\left.C(s+2 u+v)] M_{2}(u, v, s ; t)\right\} \\
=\left(\frac{\partial}{\partial t}+D\right)\left\{u M_{2}(u-1, v, s ; t)+(s+2 u+v) v M_{2}(u, v-1, s ; t)\right\}
\end{array}
$$

with

$$
M_{2}(0,0, s ; t)=H_{1}(s) \mathrm{e}^{\lambda_{1}(s) t}+H_{2}(s) \mathrm{e}^{\lambda_{2}(s) t} .
$$

It is to be noted that (44) differs from the equation for $M_{1} . M_{1}$ was obtained as a limit while $M_{2}$ is defined directly, i.e.

$$
M_{1}(u, v, s, t)=\operatorname{Lim}_{(\zeta-t) \rightarrow 0} M(u, v, s, t, \zeta-t)
$$

The approximate values of the structure functions have been obtained by the saddle-point integration with respect to $s$.

(1) Approximation $A$, i.e. on neglecting the ionization loss $\beta$ we have

$$
f^{(r)} \sim \frac{1}{r^{2}-\bar{s}-2 / 3} \text { for } \quad 2-\bar{s}+\frac{2}{3}>0
$$

$f^{(r)} \sim$ constant for $2-\bar{s}-\frac{2}{3}<0$ where the saddle point $\bar{s}$ is defined by

$$
\left.\frac{\partial \lambda_{1}}{\partial s}(s) t\right|_{\bar{s}}+\log \frac{E_{0}}{E}+\log \left(\frac{E_{r}}{E_{s}}\right)_{\bar{s}}=0 .
$$

(ii) Approximation $B$, i.e. on taking $\beta$ to be a constant, we have

$$
\begin{gathered}
f^{(r)} \sim \frac{1}{r^{2}-\bar{s}} \text { for } \quad 2-\bar{s}>0 \\
f^{(r)} \sim \quad \text { constant for } \quad 2-\bar{s}<0
\end{gathered}
$$

with

$$
\left.\frac{\partial \lambda_{1}(s) t}{\partial s}\right|_{\bar{s}}+\log \frac{E_{0}}{\beta}+\log \left(\frac{\beta r}{E_{s}}\right)_{\bar{s}}=0 .
$$

At the limit $E_{0} \leftarrow \infty$ the $\bar{s}$ 's defined above coincide with what is termed "the shower age" in the one-dimensional cascade theory.

Attempts have also been made to obtain lateral distribution function curves from empirical considerations. For example, Bethe ${ }^{1}$ has suggested the radial distribution function which represents the Moliere distribution for $s=1$

$$
\varphi(r)=45 r^{-1}(1+4 r) \exp \left(-4 r^{2 / 3}\right)
$$

1 H. A. Bethe, see p. 29, Extensive Air Shouers, W. Galbraith, Butterworths (1958). 
$r$ being measured in units of $R_{1}$, the lateral distance through which an electron of energy $E_{c}$ is scattered in traversing a radiation length. The density of particles at $r$ is then given by

$$
\Delta(r)=N \varphi(r) / R_{1}^{2}
$$

where $N$ is the total number of particles in the shower. Another empirical distribution due to Greisen ${ }^{1}$ is of the form

$$
\varphi(r)=c(\bar{s}) r^{\bar{s}-2}(r+1)^{\vec{s}-4 \cdot 5} .
$$

We have till now diseussed only the lateral distribution of soft showers. For reasons repeatedly mentioned, the corresponding distributions for neutrons and mesons eannot be quantitatively estimated though Rosenthal ${ }^{2}$ has made some calculations using the Fermi model ${ }^{3}$ for high energy collisions. His results are summarized below:

\begin{tabular}{c|r|r|c|c|c}
\hline $\begin{array}{c}\text { Height } \\
\text { above sea level }\end{array}$ & $R_{\text {el-ph }}$ & $R_{N}$ & $R_{\pi}$ & $R_{\mu}$ & $R_{l}$ \\
\hline $3 \mathrm{~km}$ & 100 & 190 & 80 & 400 & 180 meters \\
Sea level & 70 & 190 & 90 & 600 & 200 meters \\
\hline
\end{tabular}

The quantity $R_{\text {el-ph }}$ is the mean square radius of electron based on pure photonelectron cascade theory. The other quantities are derived from nucleon cascade models and refer respectively to $N, \pi, \mu$ and $e$. It is to be noted that the distribution of electrons is larger than that given by the cascade theory and the $\mu$-meson distribution falls off less steeply than that for any of the other components.

The experiments have shown that the $N$ component falls off in a similar manner to that of electrons near the shower axis while for $\mu$-mesons the fall-off is more gradual. For example at $500 \mathrm{~m}$ from the axis $\mu$-mesons constitute half the number of shower particles. In all experiments it has become customary to determine the relative abundance of $\mu$ for the various distances from the shower axis. The lateral distribution $\varrho(r)$ fits the equation

$$
\varrho(r)=r^{-n}
$$

$n=1.6 \pm 0.3$ for showers containing $10^{7}$ particles

$n=2.0 \pm 0.4$ for showers containing $10^{8}$ particles.

Despite the inadequacies and limitations in the treatment of the lateral distribution, considerable experimental work is being done to study the density distribution of the particles in the shower irrespective of their nature. The most important conclusion is that the extensive showers have a single core and the Moliere distribution fairly fits the data. However in view of the suggestion of Lewis, Oppenheimer and Wouthuysen ${ }^{4}$ regarding the multiple production of mesons in

\footnotetext{
1 K. Greisen, Phys. Rev. 75, 1071 (1949).

2 I. L. Rosenthal, J. Expt. Theoret. Phys. 23, 440 (1952).

3 E. Fermi, Progr. Theoret. Phys. 5, 570 (1951); Phys. Rev. 81, 683 (1951).

${ }^{4}$ H. W. Lewis et al., Phys. Rev. 73, 127 (1947).
} 
nuclear collisions at narrow angles, attempts are made to determine whether there are multiple cores corresponding to the sheaf of particles.

Another interesting feature is that the lateral structure is the same for all showers, that is, the particle density is proportional to the total number of particles. This also implies that if the total number of particles has a distribution function, the density of particles has the same distribution at any point.

As has been previously emphasized one of the important aims in the study of extensive showers is the determination of the energy spectrum of the primary particle. Olbert ${ }^{1}$ has made some estimates of the primary energy from the number and the density distribution of the air showers using the Fermi ${ }^{2}$ and Landau ${ }^{3}$ model for nuclear collisions. He obtained a relationship between the total number of particles and energy as:

$$
\begin{aligned}
& N=6 \times 10^{-12} E_{0}^{1.1} \text { using the Fermi model, and } \\
& N=5 \times 10^{-12} E_{0}^{1.6} \text { based on the Landau model }
\end{aligned}
$$

for air showers at sea level. For a shower of $10^{7}$ particles, Olbert's estimate of the primary energy from the first equation would be $3 \times 10^{16} \mathrm{eV}$ and from the second $5 \times 10^{16} \mathrm{eV}$. Thus the primary energy is not sensibly dependent upon the model assumed for the high energy nuclear interactions. Therefore if we are able to determine the number spectra, this yields the initial energy spectra. Using these the following result was obtained by Clarke ${ }^{4}$

$$
n\left(>E_{0}\right)=2.2 \times 10^{-10}\left(\frac{10^{15}}{E_{0}}\right)^{2.13} \mathrm{~cm}^{-2} \mathrm{sec}^{-1} \mathrm{sterad}^{-1}
$$

for the energy region $10^{15} \mathrm{eV}-10^{18} \mathrm{eV}$.

\section{ENERGY BALANCEOF COSMICRADIATION}

In the study of cosmic ray processes we have assumed only the known interactions of elementary particles. Since cosmic radiation consists of extremely high energy particles it is considered worthwhile to investigate whether the dissipation of energy of cosmic rays can be accounted for by assuming only the known interactions or we have to invoke new elementary processes hitherto unknown. For this purpose we shall calculate the energy balance after allowing for the dissipation through known processes. For such a calculation we need:

(a) The composition and intensity of primary radiation at various latitudes (the reference latitude is taken by Puppi to be $50^{\circ} \mathrm{N}$ ). This helps us to calculate the energy flux of cosmic rays as they enter the atmosphere which can be com-

1 S. Olbert, see G. Clark etal., see also S. Olbert and R. Stora, Ann. Phys. 1, 247 (1947).

2 E. Fermi, loc. cit.

3 L. Landau, loc. cit.

4 G. Clarke et al., Nature, Lond. 180, 353, 406 (1957).

5 This section is based on the article, "The energy balance of cosmic radiation" by G. PuppI, Progress in Cosmic Ray Physics, 3, 341 (1956). 
pared with the energy dissipated in the atmosphere and underground by the various components.

(b) The nature and intensity throughout the atmosphere and at sea level of the various seeondary components by the primary radiation,

(c) The exact modes in which the primary radiation generates the secondary components and manner in which the various secondary components transform into each other or are absorbed.

We have described earlier in a qualitative way the nature of inelastic collisions of the primary particle with the nuclei and the consequent secondary products. The charged and neutral pions and any type of heavy meson or hyperon cannot constitute a component with marked intensity anywhere in the atmosphere because of their short mean lifetime. Thus in the study of energy' balance, by secondary particles we mean only $\mu$-mesons, electrons, photons and nucleons. We will now take as the basis of our discussion the information on the behaviour of these components with respect to latitude. We will assume also that the primary component consists mainly of protons and neutrons, neglecting the very small fraction of heavy nuclei.

\section{The $\mu$-mesonic component}

It is clear that we must possess an accurate knowledge of the spectrum of the $\mu$-mesons at some depth so that the behaviour of the $\mu$-mesons with depth will vield the $\pi$-meson spectrum, which is the generation spectrum of the $\mu$-mesons. It will be seen presently that the generation spectrum is an important quantity in computing the energy balance. The $\mu$-meson spectrum at sea-level is one of the best determined quantities in the study of cosmic rays. The present data on the range spectrum of mesons compiled from the underground and the magnetic spectrograph measurements can be converted to yield the differential energy speetrum at $50^{\circ} \mathrm{N}$ shown below.

From this, the total density of muons of any energy is shown to be

$$
0.89 \times 10^{-2} \mathrm{~cm}^{-2} \mathrm{sec}^{-1} \mathrm{sterad}^{-1}
$$

and the total energy contained in the spectrum is given by

$$
38 \mathrm{MeV} \mathrm{cm}^{-2} \mathrm{sec}^{-1} \text { sterad }^{-1} \text {. }
$$

In order to arrive at the generation spectrum of the $\mu$-component, let us assume that all the muons originate from the decay of pions in flight and that these $\pi$-mesons are produced by a primary nucleonic component, the intensity of which decays exponentially, i.e. the production function of $\pi$ 's can be written as follows

$$
g_{\pi}(E ; t)=g_{\pi}(E) \frac{1}{L_{\pi}} \mathrm{e}^{-t / I_{\pi}}
$$

where $g_{\pi}(E)$ represents the generation function of the $\pi$-mesons and $L_{\pi}$ their production length. The diffusion equations for $\pi(E ; t) \mathrm{d} E$ and $\mu(E ; t) \mathrm{d} E$ which 
describe the differential energy spectra of pions and muons are

$$
\begin{aligned}
& \frac{\partial}{\partial t} \mu(E ; t)=\frac{\partial}{\partial \varepsilon}\left(\beta_{\mu} \mu(\varepsilon ; t)\right)-R_{\mu}(\varepsilon) \mu(\varepsilon ; t)+R_{\pi}(K \varepsilon) \pi(K \varepsilon ; t) \\
& \frac{\partial}{\partial t} \pi(E ; t)=\frac{\partial}{\partial E}\left(\beta_{\pi} \pi(E ; t)\right)-\left[\varrho+R_{\pi}(E)\right] \pi(E ; t)
\end{aligned}
$$

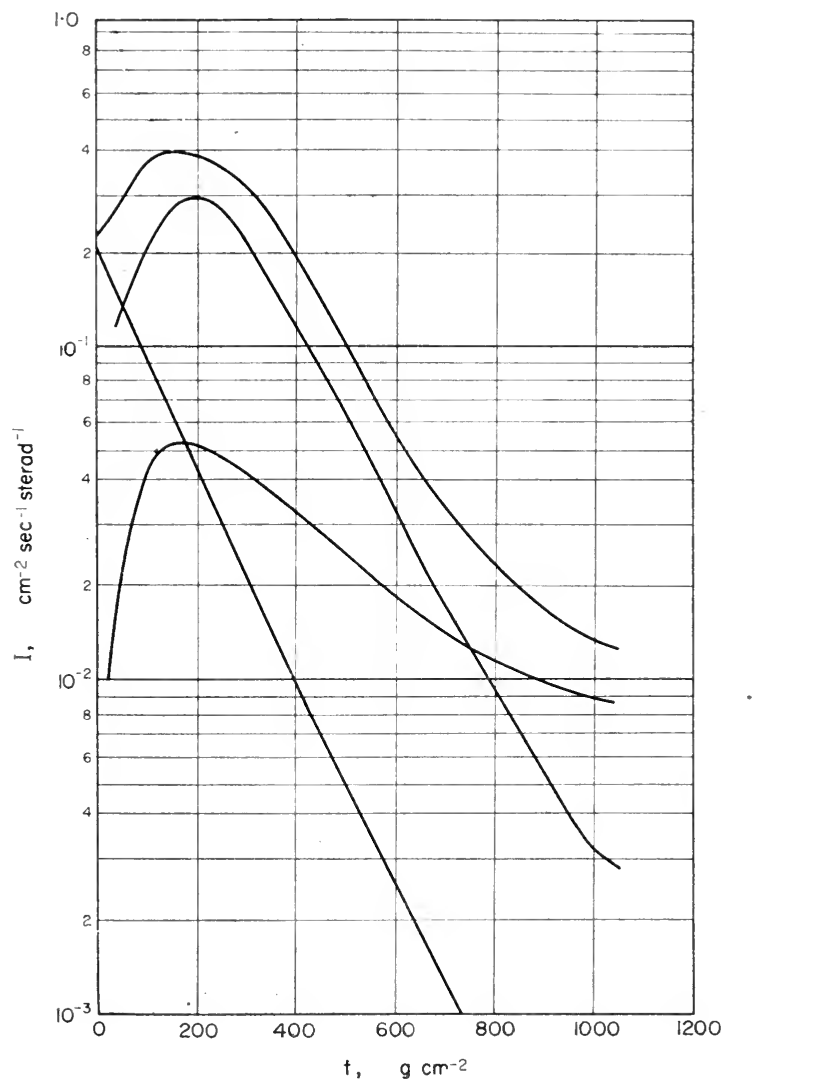

FIG. 66. Height intensity curves for the various components at $30^{\circ} \mathrm{N}$.

where $R_{\mu}(\varepsilon) \mathrm{d} t$ is the probability that the $\mu$-meson decays in thickness $\mathrm{d} t, R_{\pi}(K \varepsilon) \mathrm{d} t$ the probability that a pion of energy $K \varepsilon$ produces a muon of energy $\varepsilon, \varrho$ is the cross-section for the inelastic collision for the pions.

If we confine ourselves to the high energy $\mu$-mesons at sea level (i.e. energy $>1 \mathrm{GeV}$ ) we can assume the ionization losses to be constant. It can be shown that

$$
\mu(E ; t) \approx \frac{\gamma A}{K^{\gamma}} \cdot\left[\frac{L_{\pi}\left(\eta-B_{0} t\right)}{\eta B_{0} t}\right]^{b / \eta} \cdot \frac{B}{B+K_{\eta}} \cdot \frac{1}{\eta^{\gamma}+1}
$$


where $b-m_{\mu} c \tau_{\mu} \cdot H_{0}, B=\frac{m_{x} c}{\tau_{\tau}} H_{0}$ and the parameters $A$ and $\gamma$ can be determined from comparison with spectrum at sea level. We have assumed that it is possible to represent the pion produetion speetrum as follows

$$
g_{\pi}(E)=\frac{\gamma A}{E^{\gamma+1}}
$$

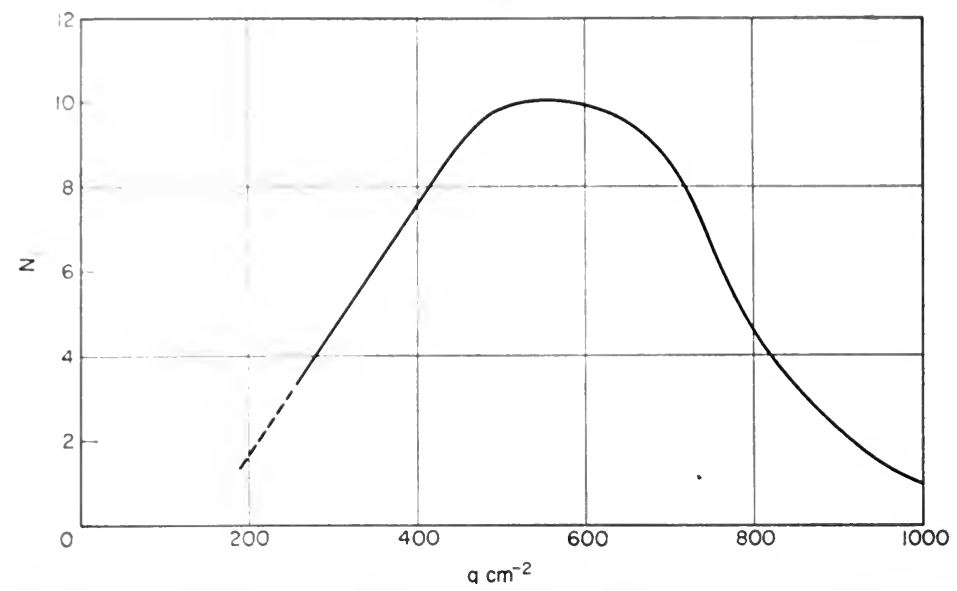

Fig. 67. Number of particles in shower at different altitudes in terms of the number at sea-level.

Thus we conclude that the $\pi$ production spectrum generating mesons of energies $>1 \mathrm{GeV}$ is

$$
g_{\pi}(E)=\frac{23}{E^{2 \cdot 75}}(\mathrm{GeV})^{-1} \mathrm{~cm}^{-2} \mathrm{sec}^{\cdot 1} \mathrm{sterad}^{-1} .
$$

In writing down the last equation we have implicity assumed that $\mu$-mesons originate only from the decay of $\pi$ 's. We will not here consider the influence of the contribution due to the deeay of $K$ particles into $\mu$-mesons. The total energy eontained in the production spectrum at $50^{\circ} \mathrm{N}$ amounts to

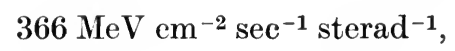

i.e. this amount of energy goes into the production of $\mu$-mesons at $50^{\circ} \mathrm{N}$. On multiplying the original diffusion equation (3) by energy $\varepsilon$ and integrating over all energies and over the whole of the atmosphere we obtain:

$$
\begin{aligned}
\int_{\mu^{2} c}^{\alpha} \varepsilon G(\varepsilon) \mathrm{d} \varepsilon= & \int_{m_{\mu} c^{2}}^{\alpha} \varepsilon \mu\left(\varepsilon ; t_{0}\right) \mathrm{d} \varepsilon+\int_{0}^{t_{0}} \mathrm{~d} t \int_{m_{\mu} c^{2}}^{\alpha} \beta(\varepsilon) \cdot \mu(\varepsilon ; t) \mathrm{d} \varepsilon \\
& +\int_{0}^{t_{0}} \mathrm{~d} t \int_{m_{\mu} c^{2}}^{\alpha} R(\varepsilon) \mu(\varepsilon ; t) \mathrm{d} \varepsilon+\int_{0}^{t_{0}} m_{\mu} c^{2} \beta\left(m_{\mu} c^{2}\right) \mu\left(m_{\mu} c^{2} ; t\right) \mathrm{d} t
\end{aligned}
$$

where it has been assumed that $G(\varepsilon, \lambda)$ is the generating function for the $\mu$-meson irrespective of its production mechanism. While the left hand side 
represents the total energy contained in the generation spectrum, i.e. the total energy transferred to the $\mu$-mesons, the terms on the right hand side represent the various ways in which energy is dissipated in the atmosphere, the first giving the residual energy at sea level, the second referring to the energy loss through ionization and electromagnetic interactions, the third and fourth giving the energy which disappears with the $\mu$-mesons which decay in flight and disintegrate at rest. A part of the energy lost through the decay will be recovered in the electronphoton component. The first term can be estimated from the total energy contained in the $\mu$ spectrum at sea level and this has already been given as

$$
38 \mathrm{MeV} \mathrm{cm} \mathrm{cec}^{-1} \text { sterad }^{-1} \text {. }
$$

To calculate the total energy dissipated in ionization throughout the atmosphere we must know the differential spectra at various altitudes. However it is found that $\bar{\beta}$ which refers to a mean ionization loss over all the energies of the spectrum is a slowly varying function of the altitude which we can estimate from the spectrum of $\mu$-mesons at sea level. Thus we find that the second term is

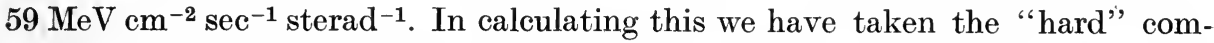
ponent, i.e. radiation filtered through a depth of atmosphere equivalent to a block of lead of thickness 10 to $20 \mathrm{~cm}$ to consist entirely of $\mu$-mesons. Though this assumption is fairly justified in the lower atmosphere, except for the loss of very slow $\mu$-mesons, at higher atmosphere the subtraction of the nucleonic component from the penetrating showers to estimate the $\mu$-mesons is a procedure with considerable uncertainty. In a similar way the third term yields $187 \mathrm{MeV}$ $\mathrm{cm}^{-2} \mathrm{sec}^{-1}$ sterad $^{-1}$. The fourth term is mainly due to very slow mesons and is found to be small of the order of $5 \mathrm{MeV} \mathrm{cm}{ }^{-2} \mathrm{sec}^{-1}$ sterad ${ }^{-1}$. Thus the total energy of the $\mu$-mesons is $298 \mathrm{MeV} \mathrm{cm}{ }^{-2} \mathrm{sec}^{-1}$ sterad $^{-1}$. To get the total energy contained in the pion spectrum, we multiply this quantity by a factor 1.27 and thus obtain $367 \mathrm{~cm}^{-2} \mathrm{sec}^{-1}$ sterad $^{-1}$ as given by the generation function.

The calculations have till now been made on the assumption that all the particles travel in the vertical direction, which is not quite true. In the first instance the $\mu$-mesons undergo multiple Coulomb-scattering, but due to the exponential production in atmosphere, the angular spread is essentially a function of $\mu$-meson energy at the point of production. This means that the effect of multiple scattering can be taken into account by suitably altering the vertical intensity. The relation between $I^{0}$, the intensity in the absence of scattering, and $I$, that effectively measured, is

$$
I \nu=I_{v}^{0}\left(1-m\left\langle\theta^{2}\right\rangle\right)
$$

where $\left\langle\theta^{2}\right\rangle$ is of the order $10^{-2}$. This correction is very small and does not have any appreciable effect on the balance. Another small correction arises from the fact that the $\mu$-mesons emitted from $\pi$ decay may be inclined at an angle to the vertical. For muons of momentum $300 \mathrm{MeV} / \mathrm{c}$ emitted from pions with energy between 300 and $330 \mathrm{MeV}$, the maximum angle of emission is $7.4^{\circ}$ which is of the order of the angle due to multiple scattering. 
Besides these, we must now discuss the correction due to the angular distribution of pions when they are produced. There are two possible limiting cases, viz. a secondary collimated in the direction of the primary and a secondary which is part of an isot ropic distribution. The latter secondaries will contribute to $\mu$-mesons when they are produced by inclined primaries. However due to the lack of information on the production length of pions in high atmosphere, we cannot estimate this important correction in any definite manner. Puppi ${ }^{1}$ concludes that the global energy contained in the spectrum after applying the necessary corrections is $409 \mathrm{MeV} \mathrm{cm} \mathrm{cm}^{-2} \mathrm{sec}^{-1}$ sterad $^{-1}$. He also discusses the presence of positive excess in the vertical muons but concludes that it only reflects the particular composition of primary radiation.

\section{The Eleetron-photon component}

The origin of the electron-photon component is rather well understood and it lies mainly in the bremsstrahlung of fast charged particles like electrons, protons and pions, the decays of muons and the rapid decay of neutral pions into two photons. The photons or electrons generated from these undergo cascade multiplication because of successive bremsstrahlung and pair production processes. The multiplication reaches a limit near $84 \mathrm{MeV}$ in air and after this the particles lose their energy mainly through ionization. Since it is difficult to decide whether the photons or electrons are produced directly by the nucleonic components or as secondaries of secondary particles like the pions, we will evaluate the energy contained in the electrons and photons by noting that the whole electronphoton shower energy must reappear in the form of ionization energy. If we know the total electronic track length, we can evaluate

$$
\int_{m_{c} c^{2}}^{a} \int_{0}^{t_{0}} \beta(E) f(E ; t) \mathrm{d} E \mathrm{~d} t
$$

which will give the total energy lost in ionization where $f(E ; t)$ refers to the differential energy spectrum of the electrons at depth $t$. It has been shown by Rossi ${ }^{2}$ how the total track of electrons of energies greater than a certain limit $(\approx 10 \mathrm{MeV}$ in the present case) can be related to the global energy of the primaries which have generated the cascade, i.e.

$$
E_{0}=3.2 \pi T_{e}
$$

It must be remembered however that part of the electrons come from electromagnetic interactions and decays of muons and hence the energies of these electrons have already been accounted for in the mesonic component. Also it is found that 7 per cent of the hard component produces knock-on electrons, i.e. it contributes $6 \mathrm{MeV} \mathrm{cm} \mathrm{Mec}^{-1}$ sterad $^{-1}$ to the total energy. It has been found by

1 G. PUPPI, ibid.

2 B. Rossi, Rev. Mod. Phys. 20, 537 (1948). See also High Energy Particles, New York, Prentice Hall (1952). 
Puppi ${ }^{1}$ that the contribution through decay is $62 \mathrm{MeV} \mathrm{cm}{ }^{-2} \mathrm{sec}^{-1}$ sterad $^{-1}$, using Michel's form for the spectrum of decay electrons. Since the total electronic track gives $324 \mathrm{MeV} \mathrm{cm}-2 \mathrm{sec}^{-1}$ sterad ${ }^{-1}$, we can conclude that the contribution of neutral pions to the electron-photon component is $256 \mathrm{~cm}^{-2} \mathrm{sec}^{-1} \mathrm{sterad}^{-1}$. In the above we have arrived at the value for total electron-photon energy by means of a difference between the height intensity curves of the total ionizing component and the mesonic component. We have ignored the details regarding the shape of the spectrum of the electron-photon component and its variation with depth. The information that we have regarding the shape of the spectrum is not adequate but recent experiments of Lovati ${ }^{2}$ show that the differential spectrum whether of photons or electrons with an energy greater than $300 \mathrm{MeV}$ at $3500 \mathrm{~m}$ obey a power law with an exponent 25 . From the present available information it can be inferred that the integral spectra of photons and electrons in a vertical direction can be represented by a function of the form

$$
\left(\frac{A}{1+E / B}\right)^{\gamma}
$$

with $1.7 \leqq \gamma \leqq 1.8$ and $50 \leqq B \leqq 150 \mathrm{MeV}$. It is also found that the photonelectron ratio is never less than unity, the ratio at sea-level being unity. This leads to a ratio two at an altitude $3500 \mathrm{~m}$. This constitutes a proof that most of the photons originate in the decay of neutral pions. The knowledge of spectra at various levels coupled with electron-photon ratio at various altitudes will permit a direct calculation of the global energy dissipated in the electron-photon component, instead of the present estimates based on the subtraction method. As for the $\mu$-mesonic component, we have to apply corrections due to the angular spread of electrons due to multiple Coulomb-scattering and the angular correlations between the primaries and neutral pions when they are produced. Due to uncertainties, we prefer not to evaluate such corrections and thus the total neutral pion energy is

$$
256 \mathrm{MeV} \mathrm{cm}{ }^{-2} \mathrm{sec}^{-1} \text { sterad }^{-1} \text {. }
$$

Finally we note that the secondary processes like photo-meson production and production of neutral mesons through interactions of charged mesons give very small corrections which in view of the large uncertainty contained in our estimates can be safely neglected.

\section{The nucleonic eomponent}

We have to discuss the nature of energy dissipation by the nucleonic component in processes different from generation of charged and neutral pions. The total ionization losses of the charged part of nucleonic component can be roughly estimated to be

$$
70 \mathrm{MeV} \mathrm{cm} \text { cm }^{-2} \mathrm{sec}^{-1} \text { sterad }^{-1} \text {. }
$$

1 G. Puppi, ibid.

2 Lovati, see G. Puppi's article, ibid. 
The nucleonic component loses energy by nuclear excitation resulting in the formation of stars. 'The frequency $N$ of nuclear disintegration as function of the number $n$ of the ionizing prongs obey the following relations

$$
N(>n)=N_{0} \mathrm{e}^{-0.4 n} ; \quad N(>n)=N_{0} \mathrm{e}^{-(0.55) n}
$$

at balloon and mountain altitudes respectively. At mountain altitudes the average number of prongs per star is 3. Puppi estimates from the various emulsion data that the global number of disintegration in an atmospheric column of unit section is about $3 \mathrm{~cm}^{-2} \mathrm{sec}^{-1}$. This number should be increased to allow for the undetected disintegrations. It is known that for every nuclear disintegration with $N$ ionizing particles, there are about $1.5 N$ neutrons which implies that the total production of neutrons is $4.5 \mathrm{~cm}^{-2} \mathrm{sec}^{-1}$ in unit atmospheric column. Still we have to correct the above figures by the disintegrations in which only neutrons are emitted and by events which are excluded by the selection device for ionizing particles. The time variation experiments of Simpson ${ }^{1}$ have revealed considerable information on the behaviour of slow neutrons. These slow neutrons originate mainly in low energy stars and they slow down to thermal energy due to collisions with air nuclei when they are captured by nitrogen in the reaction $\mathrm{N}^{\mathbf{1 4}}(n, p) \rightarrow \mathrm{C}^{\mathbf{1 4}}$. 'Though the number of thermal neutrons captured per gram of air at various levels can be estimated, for the energy balance we are interested in the total number of neutrons produced in nuclear disintegrations. A fraction of neutrons escape from the atmosphere and a very large portion does not reach thermal energies. Bethe's ${ }^{2}$ analysis indicates that to justify the number of thermal captures in the atmosphere which is $3.5 \mathrm{~cm}^{-2} \mathrm{sec}^{-1}$ the number of neutrons produced in nuclear disintegrations should be much greater, e.g. the number of neutrons generated in nuclear disintegrations with the kinetic energy of $4 \mathrm{MeV}$ is about

$$
(22 \pm 4) \mathrm{cm}^{-2} \mathrm{sec}^{-1} \text {. }
$$

This remark helps us to interpret the variation with height in atmosphere of neutron capture frequency. We find that the estimates based on the thermal captures do not agree with those obtained from the frequency of disintegration with ionizing particle and in fact the former is greater by a factor of five. We have to conclude that the major source of slow neutrons are events in which only neutrons are emitted. Puppi ${ }^{3}$ estimates that the total energy contribution from all types of nuclear disintegration to the vertical component is $230 \mathrm{MeV} \mathrm{cm} \mathrm{cm}^{-2}$ $\sec ^{-1}$ sterad $^{-1}$. This value is very uncertain as we have not considered the eventual low energy particles such as protons, alpha particles and deuterons.

The above analysis of the vertical beam of cosmic rays at $50^{\circ} \mathrm{N}$ gives a total energy dissipated to be $965 \mathrm{MeV} \mathrm{cm}{ }^{-2} \mathrm{sec}^{-1}$ sterad ${ }^{-1}$, through the three components, i.e. mesons, electron-photon and the nucleonic component. The total incoming primary energy is estimated to be around $1400 \mathrm{MeV} \mathrm{cm} \mathrm{cec}^{-\mathbf{1}} \mathrm{sterad}^{-1}$.

1 J. A. Sinpsox, Phys. Rev. 76, 1750 (1949).

2 H. A. Bethe, C. Forff and G. Placzek, Phys. Rev. 57, 573 (1940).

${ }^{3}$ G. PuppI, ibid. 
In view of the large uncertainties in the estimates on the low energy nucleonic component and nucleonic cascades of other particles the disagreement between the dissipated and primary energy need not disturb us. Thus we can conclude there is not sufficient energy balance after allowing for dissipation through known processes to warrant any assumption of hitherto unknown processes.

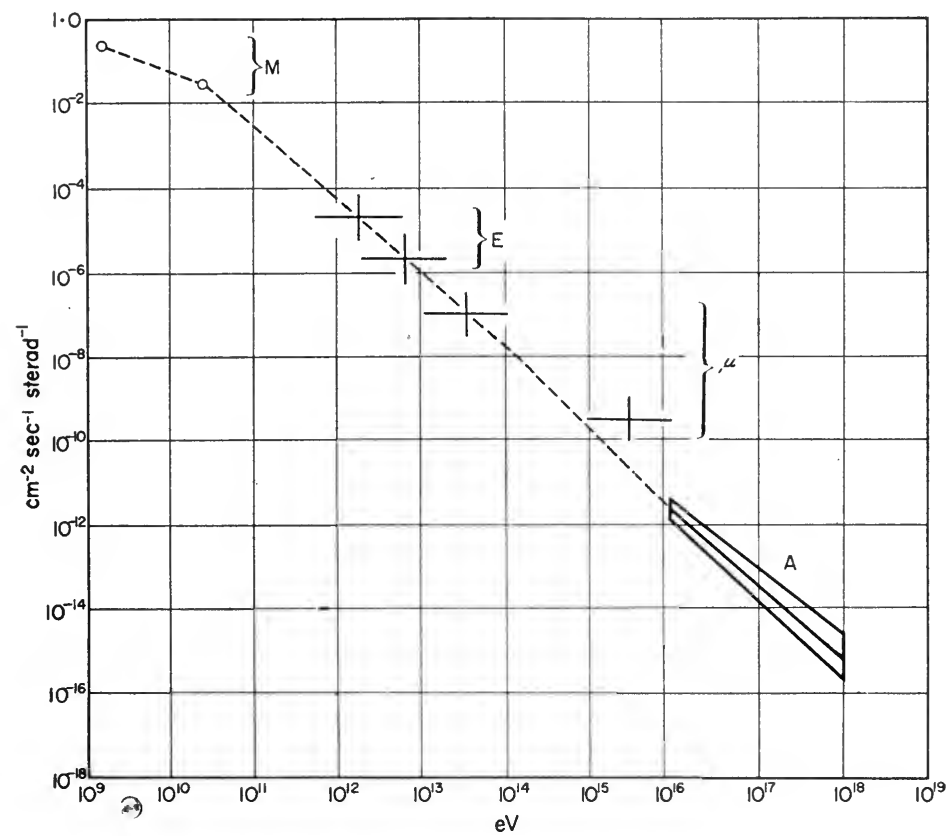

Fig. 68. Energy spectrum of primary cosmic ray particles. 


\section{ORIGIN OF COSMIC RADIATION ${ }^{1}$}

Br FAR the most interesting question about cosmic radiation relates to its origin. 'Till recently the theories of the origin of cosmie radiation were in a speculative stage since very few criteria had to be satisfied with the hitherto available observational data on the primary components of cosmic radiation. However since 1948 our knowledge of the composition of the primary components in the neighbourhood of the earth has become more precise and conclusions about the distribution of cosmic radiation in the galaxy and beyond have become possible from the data on radio-astronomy.

It is convenient to deal with the life history of the cosmic radiation, before it reaches the earth, in two stages.

The first relates to the primitive source of cosmic radiation, that is, where the particles are first produced.

The second relating to the intermediate mechanism through which these particles attain the energy, eomposition and spatial distribution characteristic of the radiation that reaches the earth.

An intermediate mechanism implies the following:

(a) There must be a method by which the injected particles are accelerated to the very high energies found in cosmic rays,

(b) there must be a region of space in which the cosmic rays are trapped or stored for the acceleration mechanism to be effective,

(c) there must be a mechanism of escape which could explain the energy spectrum.

These three factors will naturally depend on the sources of injection. It is obvious that if the primitive source were extragalactic, the storage domain should be the intergalactic space; if the source were galactic, the domain must be intergalactic, and if the source were solar, the domain must be the region in the neighbourhood of the earth and the sun. It would be worthwhile to compare the density of the cosmic rays with the various forms of energy available in the universe before making any postulate about the source of the cosmic rays.

1 See "The Origin of Cosmic Radiation". By V. I. Ginzburg, Progress in Cosmic Ray Physics, 4, 339 (1958).

We are deeply indebted to Professor S. Chandrasekhar for giving us his private notes on the theories of origin of Cosmic Rays. Also we wish to thank Professor Phillip Morrison whose lecture at Madras clarified many difficulties on this subject. 


\begin{tabular}{l|c}
\hline Source & Energy density $U$ in $\mathrm{Me} \mathrm{V}$ \\
\hline Cosmic rays & 1 \\
Solar light & 1 \\
Turbulence in galactic gas & $10-100$ \\
Galactic rotation (systematic) & $10^{+3}$ \\
Mass energy (rest mass in space & $10^{+9}$. \\
and stars-averaged over) & 1 \\
Magnetic energy & \\
\hline
\end{tabular}

The original surmise that the turbulence energy of the universe can be considered as a primitive source was given up since it was realised that the production of the cosmic rays would affect the hydrodynamics of the gas. The rotational energy is also ruled out since there is no known mechanism of converting this energy into the kinetic energy of single protons. The density of cosmic rays being unity the energy of the source must be of much higher order and hence it is natural to trace it to the mass energy.

An extreme postulate was made by certain workers on cosmic radiation that no intermediate mechanism is operative but the cosmic rays are produced with the known characteristics directly in a catastrophic production.

In the theory of catastrophic production, it is assumed that high energy particles are directly produced in some forms of stellar explosions. Originally the following were considered the obvious objections to a catastrophic theory.

(i) Since the energy density of cosmic radiation is comparable to that of starlight, if we believe that the radiation fills the universe, the total energy is comparable to the rest energy of universal matter and it seems untenable that matter is being annihilated at such a rate.

(ii) The radiation is isotropic and it seems difficult to suggest a mechanism by which the particles ejected in stellar explosions can be rendered isotropic.

(iii) Any suggested catastrophic process by its very nature must be ad hoc and its existence cannot be proved. The most powerful argument against catastrophic production is the existence of heavy primaries which have the same energy per nucleon as the protons. It is extremely hard to believe that any catastrophic process which gives a velocity of many billion electron volts per nucleon should not provide enough energy to disrupt the nucleus. But, we shall see that all these objections can be met effectively by introducing an intermediate mechanism involving gradual acceleration. Among other reasons for postulating the existence of an intermediate mechanism the following one stands out as the most important.

Relative Abundances of Elements

\begin{tabular}{c|c|c}
\hline Elements & Cosmic Rays & Sun \\
\hline $\mathrm{H}$ & 1 & 1 \\
$\mathrm{He}$ & 0.1 & 0.01 \\
$Z>2$ & 0.03 & $\simeq 0.001$ \\
Electrons & $<10^{-2}$ & 1.3 \\
\hline
\end{tabular}


An examination of the table of relative abundance of the various elements reveals that there are practically no electrons in the cosmic rays. Since electrons should also be produced in the processes which give rise to the protons the absence of electrons in cosmic rays is explained as follows:

The relativistic eleetrons which we can expect to be produced along with other particles that are actually observed in cosmic rays lose their energy by magnetic bremsstrahlung while getting accelerated by the interstellar magnetic fields. The evidence for this is found in the cosmic radio noise.

The shape of the distribution of the cosmic ray particles with energy is found to be as follows. There is a definite cut-off at the low energy and at about $10^{9} \mathrm{MeV}$ and the spectrum is practically linear over a wide energy range. There is no evidence for a cut-off at the high energy end of the spectrum which is not well defined. But it is natural to expect such a cut-off since the relativistic protons which comprise the major portion of the cosmic radiation can also lose energy by magnetic bremsstrahlung and the cut-off is expected to be at about $10^{20} \mathrm{MeV}$. In view of the wide range of the energy spectrum of the cosmic radiation ranging from $10^{9}$ to $10^{19} \mathrm{eV}$ it is obvious that we should not seek a single region of the universe as a possible primitive source. There is reason to expect that the low energy region of the spectrum is of solar origin, the straight line portion from $10^{9}$ to $10^{19} \mathrm{eV}$ of galactic origin, sources of extragalactic nature giving rise to particles of energy even greater than $10^{19} \mathrm{eV}$. Before discussing the possible sources, we shall give an account of the methods of acceleration.

\section{THEORIES OF INTERMEDIATE MECHANISM}

Gradual acceleration of charged particles can only be realized either by electrostatic or non-static magnetic fields. The possibility of acceleration by an electric field is immediately dispensed with by the following considerations:

The potential difference for producing such high velocities has to be very high, but in regions of high conductivity which do occur in galactic and intergalactic space, it would not be easy to maintain such large fields. A quantitative idea of the dissipation of electrostatic fields is obtained directly by considering Maxwell's equations. If $\sigma$ is the conductivity of the gas, the current $\boldsymbol{j}$ is given by $\sigma \boldsymbol{E}$ and therefore we obtain

$$
\begin{aligned}
\boldsymbol{\nabla} \times \boldsymbol{H} & =\frac{1}{c}\left[\frac{\partial \boldsymbol{E}}{\partial t}+4 \pi \boldsymbol{j}\right] \\
& =\frac{1}{c}\left[\frac{\partial \boldsymbol{E}}{\partial t}+4 \pi \sigma \boldsymbol{E}\right] .
\end{aligned}
$$

"Taking the divergence on both sides,

$$
\nabla \cdot(\nabla \times \boldsymbol{H})=0=\frac{1}{c}\left[\frac{\partial}{\partial t}(\nabla \cdot \boldsymbol{E})+4 \pi \sigma \boldsymbol{\nabla} \cdot \boldsymbol{E}\right],
$$


neglecting the derivative of $\sigma$ and noting that $\nabla \cdot \boldsymbol{E}=4 \pi \varrho$ we obtain

$$
\frac{\partial \varrho}{\partial t}=-4 \pi \sigma Q \quad \text { or } \quad \varrho=\mathrm{e}^{-4 \pi \sigma t}
$$

i.e. the charge distribution decays with a mean lifetime $\frac{1}{4 \pi \sigma}$. In an ionized gas, the conductivity has the value $\sigma=N \lambda e^{2} / m v$ where $N$ is the electron density, $\lambda$ the mean free path, $m$ the mass, $v$ the average velocity and $e$ the electronic charge. Assuming (i) that the interstellar space contains one hydrogen atom per $\mathrm{cm}^{3}$, (ii) that this hydrogen is completely ionized (although actually only a fraction is ionized), and (iii) that the kinetic temperature of the electron is a few degrees absolute, we find $v \sim 10^{6} \mathrm{~cm} \mathrm{sec}^{-1}$ and $\lambda \sim 10^{5} \mathrm{~cm}$ and therefore we get $\sigma=10^{7} \mathrm{sec}^{-1}$. Since the velocity is proportional to $T^{\frac{1}{2}}$ and the mean free path to $T^{2}$, the conductivity increases as $T^{3}$. At higher temperatures we shall therefore expect higher conductivities, say values exceeding $\sigma=10^{15} \mathrm{sec}^{-1}$.

In a completely ionized gas, the product $N \lambda$ is independent of the density and thus the conductivity does not depend on the assumed number of hydrogen atoms per cubic centimetre. Even for incomplete ionization the conductivity will remain practically unchanged as long as the total scattering cross-section of the ions is greater than that of the neutral atom. Actually an ionization as low as one in $10^{11}$ will give the same product $N \lambda$ as we obtained for complete ionization and a low temperature of a few degrees absolute. The low number $10^{-11}$ of electrons per proton could easily arise from even such rare elements as $\mathrm{Cs}$ or $\mathrm{Rb}$. These can be ionized by radiation of $3000 \AA$ and such radiation is surely present throughout the galaxy. Due to ionization of some of the more abundant elements like $\mathrm{Na}$, $\mathrm{K}$ and $\mathrm{Ca}$ which are rather more difficult to ionize, it is believed that the number of electrons per $\mathrm{cm}^{3}$ does not become lower than $10^{-5}$ times the number of protons per $\mathrm{cm}^{3}$ anywhere within the galaxy. Thus we may consider that the lower bound of the conductivity $\sigma$ in the interstellar spaces in the galaxy is of the order of $10^{7} \mathrm{sec}^{-1}$. This of course does not mean that electric fields cannot persist for times longer than $10^{-7} \mathrm{sec}$, but it does mean that extended electrostatic fields must not be expected anywhere within the galaxy.

Therefore two modes of acceleration involving magnetic fields have been proposed :

(i) The betatron mechanism of Swann ${ }^{1}$, (ii) Fermi's ${ }^{2}$ mechanism of energetic collisions of the charged particles with "wandering" magnetized interstellar matter.

\section{Swann's mechanism}

The main merit of this mechanism lies in its simplicity. The method of acceleration can be described in the following terms.

1 W. F. G. Swann, Phys. Rev. 43, 217 (1933).

2 E. Fermi, $A p . J .119$, 1 (1954).

EPCR 31 
Let us assume a magnetic field in the $z$ direction and let it increase with time throughout space. If $N_{r}$ be the magnetic flux in a cylinder of radius $r$, the electric field $E_{\theta}$ produced tangential to the surface of the cylinder is given by

$$
2 \pi r E_{\theta}=-\frac{1}{c} \frac{\partial N_{r}}{\partial t}
$$

If $v$ is the velocity of the particle and $T$ its kinetic energy

$$
\frac{\partial T}{\partial t}=E_{0} v e=\frac{v e}{2 \pi r c} \cdot \frac{\partial N_{r}}{\partial t} ; \quad \frac{m v^{2}}{r}=\frac{H e v}{c} .
$$

If we assume the particle to have relativistic velocities, the kinetic energy is given by the well-known relation

$$
m^{2} v^{2} c^{2}=T^{2}+2 m_{0} c^{2} T .
$$

From these four equations we obtain

$$
\frac{1}{2 \pi} \frac{\partial N_{r}}{\partial t}=r^{2} \frac{\partial H_{r}}{\partial t}
$$

where $H_{r}$ is the magnetic field at the point where the electron is found. Since, by definition, $N=\int_{0}^{r} H \cdot 2 \pi \varrho \mathrm{d} e$ we obtain

$$
\frac{\partial}{\partial t} \int H_{\varrho} \mathrm{d} \varrho=\frac{\partial}{\partial t}\left[H_{r} \cdot r^{2}\right]
$$

This implies that $r H(r)$ is constant for all values of $t$. In other words, it implies the particle moves in a spiral such that the percentage time rate of change of field at all points in its trajectory is the same. From the above theory it is clear that even with $H$ growing up only to $2 \times 10^{-3}$ gauss, provided $r$ is very large $\left(3 \times 10^{10} \mathrm{~cm}\right)$, we obtain kinetic energies of the order of $1.7 \times 10^{10} \mathrm{eV}$.

Two main criticisms have been advanced against this theory:

(1) Whether such changing magnetic fields exist in the universe,

(2) Criticism by Teller ${ }^{1}$ using a relativistic argument which runs as follows: The motion of magnetic lines of force through stationary matter gives rise to an electric field $E=\frac{\boldsymbol{v} \times \boldsymbol{H}}{c}$ where $\boldsymbol{v}$ is the velocity of motion of the magnetic lines of force. The conclusion of the previous paragraph means that in the coordinate system in which the conducting matter is at rest, $\boldsymbol{v}$ is zero and therefore no electric field will be felt in the system in which the conducting gas is at rest.

In the presence of a magnetic field charges may flow freely along the magnetic lines of force. This means that the component of the electric field which is parallel to the magnetic field will disappear in materials of high conductivity within a very short time. If the electric and magnetic fields are perpendicular, two cases

1 E. Teller, Rep. Progr. Phys. 17, 154 (1954). 
should be distinguished according as whether $E$ or $H$ is greater, measured in the C.G.S. system of units. If $E$ is greater a coordinate system can be found moving with the velocity $\frac{c|H|}{|E|}$ in which the magnetic field vanishes but an electric field is present. In this coordinate system, the electric field will again vanish within a very short time and we can therefore exclude the possibility of $|E|$ being greater than $|H|$. If the magnetic field is larger, then a coordinate system can be found moving with a velocity $c \frac{|E|}{|H|}$ in which a magnetic field is present but the electric field vanishes. In this coordinate system, positive and negative ions will move in circles with opposite senses of rotation. Thus in this coordinate system, the ions will not move relative to the lines of force of the magnetic field. Given a sufficient length of time in which ions and neutral material may come to equilibrium, a situation will be established in which no electric field is present and no "wind" is felt in our new coordinate system. In another coordinate system both the electric and magnetic fields will be present, while positive and negative ions will move in cycloids with opposite senses of rotation but equal velocities of translation. The above discussion leads to the conclusion that changing magnetic fields will produce electric fields, but these electric fields will impart a motion $c \frac{|E|}{|H|}$ to the conducting matter as a whole and will not lead to acceleration of individual ions which could be identified with the primary particles of cosmic radiation.

Without referring to the relevance of this criticism to Swann's ${ }^{1}$ theory, Teller's remarks themselves are open to objection.

Since the electric field changes with $t$ the velocity of the moving frame has to be continuously changed, that is, it becomes an accelerated frame to which special relativistic theories do not apply.

Magnetic fields unlike electric fields are conserved for long periods due to the effects of self induction in a conductor of large dimensions like the ionized material in the interstellar space. This is easily seen if we take the curl on both sides of equation (1) and the time derivative of the equation $\nabla \times \boldsymbol{E}=-\frac{1}{c} \frac{\partial \boldsymbol{H}}{\partial t}$. Neglecting the derivative of $\sigma$ and using the fact that $\boldsymbol{\nabla} \cdot \boldsymbol{H}=0$ we obtain

$$
\nabla^{2} \boldsymbol{H}=\frac{1}{c^{2}} \frac{\partial^{2} \boldsymbol{H}}{\partial t^{2}}+\frac{4 \pi \sigma}{c^{2}} \frac{\partial \boldsymbol{H}}{\partial t} .
$$

We can understand the time dependence of $\boldsymbol{H}$ by neglecting one of the three terms of the equation (9)

(1) If $\nabla^{2} \boldsymbol{H}=0, \quad \boldsymbol{H}=\boldsymbol{H}_{0} \mathrm{e}^{-4 \pi \sigma t}$.

This is the magnetic field induced by a decaying electric field with a charge distribution given in equation (3).

1 W. F. G. Swann, Ibid.; E. Fermi, Ibid. 
(2) If $\partial \boldsymbol{H} / \mathrm{c} t \sim 0$, the equation is that of the propagation of electromagnetic radiation.

(3) If $\frac{\partial^{2} \boldsymbol{H}}{\partial t^{2}} \sim 0$ we have a slowly changing or quasistatic magnetic field which is of great interest to us since interstellar magnetic fields are probably of this type. Then the equation reduces to a diffusion equation with diffusion coefficient $4 \pi \sigma$

$c^{2}$

$$
\boldsymbol{V}^{2} \boldsymbol{H}=\frac{4 \pi \sigma}{c^{2}} \frac{\partial \boldsymbol{I}}{\partial i}
$$

If $L$ is a linear dimension within which considerable spatial variation has originally been present, $t \sim \frac{4 \pi \sigma}{c^{2}} L^{2}$ is the time in which the magnetic field is dissipated. Using for $\sigma$ its minimum value $10^{7} \mathrm{sec}^{-1}$ and for $L$ the value $3 \times 10^{10} \mathrm{~cm}$, we find $t=1.2 \times 10^{8} \mathrm{sec}=4$ years. If $L$ is a light day, we obtain $t=3 \times 10^{10}$ years which is greater than the age of the universe. Thus for distances, quite modest on a galactic scale, magnetic fields remain tied to the conducting gas.

It follows therefore that these magnetic fields can constitute a mechanism for trapping cosmic ray particles within the galaxy until the energy density is built up to the required order of magnitude.

The observations of Hiltner and $\mathrm{Hall}^{1}$ of an appreciable polarization of light coming to us from distant stars has been interpreted as due to the orientation of the non-spherical dust grains by a magnetic field. On this interpretation, the polarization gives us some information on the strength and direction of the magnetic field. Hiltner's measurements indicate that in the vicinity of the earth, the magnetic field is approximately parallel to the direction of the spiral arm of the galaxy. This fact suggests that we may perhaps think that the spiral arms are magnetic lines of force. The direction of polarization of starlight indicates further that in our vicinity the magnetic lines of force show irregular deviations from parallelism of the order of $10^{\circ}$. This fact excludes the hypothesis that the lines of force yield completely to the turbulent motions of interstellar matter because then they would be rapidly bent into shapes much more irregular than observed. We are forced to conclude that the field is sufficiently strong to yield only a little to the transverse component of the turbulent motion. Indeed, as was pointed out by Davis ${ }^{2}$, the small deviations from parallelism of the field enable us to estimate the field to be of the order of $10^{-5}$ gauss. Recently Chandrasekhar and Fermi $^{3}$ have re-examined the problem considering in particular the balance between magnetic and gravitational effects in the spiral arm. They conclude that the field intensity is $6 \times 10^{-6}$ gauss. Owing to the turbulence the lines of

1 Hiltwer and Hall, Science 109, 165 (1949); J. S. HALL, Science 109, 166 (1949); J.S. Hall and A. H. Miksell, Ap. J. $\mathbf{5 4 ,} 187$ (1949).

2 L. Davis, Phys. Rev. 100, 1440 (1955), 101, 351 (1956).

3 S. Chandrasekhar (private communication); S. Chandrasekhar and E. Fermi, $\boldsymbol{A} p . J$. $118,113(1953)$. 
force are laterally deformed until the magnetic field increases to the point of forcing a reversal of the motion of the material and pushing back the diffuse matter and imposes on it some kind of very irregular oscillatory motions. It is therefore expected that the lines of force will sway back and forth and also that the field intensity will fluctuate along the same lines of force. Thus we are inclined to believe that the magnetic field constitutes the accelerating mechanism.

\section{Fermi's mechanism of acceleration of particles}

Fermi $^{\mathrm{i}}$ suggested a mechanism of acceleration due to the interaction of cosmic particles with wandering magnetic fields in interstellar spaces. Such fields have remarkable stability, as we found earlier, because of the large dimensions and high conductivity of the interstellar space. Indeed the conductivity is so high that one might describe the magnetic lines of force as attached to the matter and partaking in its streaming motions. On the other hand, the magnetic field itself reacts on the hydrodynamics of interstellar matter which according to Alfven ${ }^{2}$ can pictorially be described by saying that to each line of force we should attach a material density due to the mass of matter to which the line of force is linked. On this point of view, Alfven obtained a simple formula for the velocity of propagation of magneto-elastic waves, $v=H_{0}\left(4 \pi \varrho_{0}\right)^{1 / 2}, H_{0}$ being the intensity of the magnetic field and $\varrho_{0}$ the density of interstellar matter.

Now consider a fast particle moving in such wandering magnetic fields. If the particle is a proton having an energy of a few $\mathrm{BeV}$ it will spiral round the lines of force with the radius of the order of $10^{+12} \mathrm{~cm}$ until it "collides" against an irregularity in the cosmic field and undergoes some kind of irregular motion. On a collision both gain or loss of energy may take place, gain being more probable as is understood by the argument that ultimately statistical equilibrium must be established between the degrees of freedom of the wandering field and the degrees of freedom of the particle. Equipartition may lead to an unbelievably high energy and a limitation to this process is imposed by the enormous times that are required to attain such high energies. A charged particle will be spiralling round a uni-directional magnetic field with the axis of the spiral parallel to the field. We shall give here a dynamical argument to show that there is a gain in the energy of a particle in a collision with a cloud.

If the radius of the spiral is of the order of $10^{12} \mathrm{~cm}$ and the irregularities of the field are of the order of $10^{18} \mathrm{~cm}$, the particle will perform many turns on its spiralling path before encountering an appreciably different field intensity. As the particle approaches the region where the field intensity increases, the pitch of the spiral decreases $\operatorname{since} \sin ^{2} \theta / H$ is a constant where $\theta$ is the angle between the direction of the line of force and the direction of the velocity. The particle is "reflected" at the point where $\sin \theta=1$. If the magnetic field were static, this reflection will not produce any change in the kinetic energy of the particle.

1 E. Fermi, Ibid.

2 H. Alfven, Cosmical Electrodynamics, Oxford (1950). 
However, the particle will gain energy if a region of high field intensity moves towards it and lose energy if the region of high field intensity moves away from it. The net result will be an average gain, primarily for the reason that head-on collisions are more frequent than overtaking collisions because the relative velocity is larger in the former case. 'The amount of energy gained or lost in a collision of the two types deseribed can be estimated by a simple argument using the concepts of special relativity without any reference to the detailed mechanism of collision. In the frame of reference $S^{\prime}$ in which the irregularity of the field against which the collision takes place is at rest, there is no change in the energy of the particle. The change of energy in the original frame $S$ is obtained by the following method: We first transform the initial energy and momentum from $S$ to $S^{\prime}$. In $S^{\prime}$ an elastic collision takes place whereby the momentum changes direction and the energy remains unchanged. Transforming back to $S$, one obtains the final values of energy and momentum.

Let the "irregularity" move with a velocity $v=B c$ and the particle with a velocity $u=\beta c$ and a momentum $P_{x}$ in $S$. In $S^{\prime}$ the momentum $P_{x}^{\prime}$ and energy $E^{\prime}$ is given by

$$
\begin{aligned}
c P_{x}^{\prime} & =\frac{c P_{x}+B E}{\sqrt{1}-B^{2}} \\
E^{\prime} & =\frac{E+B c P_{x}}{\sqrt{1}-B^{2}} .
\end{aligned}
$$

After collision in $S^{\prime}$ the momentum and energy of the particle are $P_{n}^{\prime \prime}$ and $E^{\prime \prime}$ given by

$$
\begin{aligned}
c P_{x}^{\prime \prime} & =-\left(\frac{c P_{x}+B E}{-\sqrt{1-B^{2}}}\right) \\
E^{\prime \prime} & =\frac{E+B c P_{x}}{\sqrt{1-B^{2}} .}
\end{aligned}
$$

Transforming back to the original frame $S$ we obtain $P_{x}^{\prime \prime \prime}$ and $E^{\prime \prime \prime}$ of the particle after collision

$$
\begin{aligned}
c P_{x}^{\prime \prime \prime} & =\frac{c P_{x}^{\prime \prime}-B E^{\prime \prime}}{\sqrt{1}-B^{2}} \\
E^{\prime \prime \prime} & =\frac{E^{\prime \prime}-B c P_{x}^{\prime \prime}}{\sqrt{1-B^{2}}} \\
& =\left\{\frac{E+B c P_{x}}{\left.\sqrt{1-B^{2}}+B \frac{c P_{x}+B E}{\sqrt{1-B^{2}}}\right\} \frac{1}{\sqrt{1-B^{2}}}}\right. \\
& =\left(E+2 B c P_{x}+B^{2} E\right) /\left(1-B^{2}\right) \\
\frac{E^{\prime \prime \prime}}{E} & =\left(1+\frac{2 c P_{x} B}{E}+B^{2}\right) /\left(1-B^{2}\right) .
\end{aligned}
$$

For the particle with velocity $u$ and energy $E$ we have

$$
\begin{aligned}
& c P_{x}=\frac{m c u_{x}}{\sqrt{ } 1-u^{2} / c^{2}} ; \quad E=\frac{m c^{2}}{\sqrt{ } 1-u^{2} / c^{2}} ; \quad \frac{c P_{x}}{E}=\frac{u_{x}}{c}=\beta \cos \theta \\
& \frac{E^{\prime \prime \prime}}{E}=\frac{1+2 B \beta \cos \theta+B^{2}}{1-B^{2}} \text {. }
\end{aligned}
$$


For an overtaking collision we get a similar formula except that the sign of $B$ must be changed

$$
\begin{aligned}
\log \left(\frac{E^{\prime \prime \prime}}{E}\right) & =2 B^{2}+2 B \beta \cos \theta-2 B^{2} \beta^{2} \cos ^{2} \theta \text { (head on collisions) } \\
& =2 B^{2}-2 B \beta \cos \theta-2 B^{2} \beta^{2} \cos ^{2} \theta \text { (overtaking collisions). }
\end{aligned}
$$

Probability for the head on collision is $\frac{\beta \cos \theta+B}{2 \beta \cos \theta}$ and the probability for the overtaking collision $\frac{\beta \cos \theta-B}{2 \beta \cos \theta}$.

$$
\begin{aligned}
& {\left[\begin{array}{rl}
{\left[\log \frac{E^{\prime \prime \prime}}{E}\right]_{A v}=} & \frac{1}{2 \beta \cos \theta}\left[\left(2 B^{2}+2 B \beta \cos \theta-2 B^{2} \beta^{2} \cos ^{2} \theta\right)(\beta \cos \theta+B)\right. \\
& \left.+\left(2 B^{2}-2 \beta B \cos \theta-2 B^{2} \beta^{2} \cos ^{2} \theta\right)(\beta \cos \theta-B)\right]
\end{array}\right.} \\
& =4 B^{2}-2 B^{2} \beta^{2} \cos ^{2} \theta \\
& \text { i.e. } \\
& \qquad \log \left(\frac{E+\mathrm{d} E}{E}\right)=c B^{2} \quad(c \sim 1) .
\end{aligned}
$$

The average gain in energy per collision is of the order of magnitude $\delta(E)=B^{2} E$.

If $E$ is the energy of the particle inclusive of the rest energy the average gain in energy per collision is

$$
\delta E=B^{2} E \text { where } B=\frac{v}{c} \sim 10^{-4} .
$$

A particle starting with non-relativistic energy will therefore after $N$ collisions attain the energy

$$
E=M c^{2} \mathrm{e}^{B^{2} N}, \quad N \text { being very large. }
$$

To explain the energy spectrum, Fermi introduced an absorption process due to collisions with matter of an average density of $10^{-24} \mathrm{~g} / \mathrm{cm}^{3}$ leading to an absorption mean free path $\lambda=7 \times 10^{25} \mathrm{~cm}$. The average lifetime of a cosmic ray particle is, let us say, $T$ which is approximately $2 \times 10^{15} \mathrm{sec} \sim 10$ million years. The cosmic ray particles now present will therefore, on an average, have this age. Some of them will accidentally escape destruction and will be considerably older. Indeed the absorption process can be considered to proceed according to an exponential law. If we assume that the original particles at all times have been supplied at the same rate, we expect the age distribution to be $\mathrm{e}^{-t / \tau} \frac{\mathrm{d} t}{T}$. During this age $t$ the particle has been gaining energy. If we call $\tau$ the time between scattering collisions, the energy acquired by a particle of age $t$ will be

$$
E(t)=M c^{2} \mathrm{e}^{B^{2} t / \tau} .
$$

Hence the probability that the particle has energy between $E$ and $E+\mathrm{d} E$ is given by

$$
\pi(E) \mathrm{d} E=\left(\frac{\tau}{B^{2} t}\right)\left(M c^{2}\right)^{\tau / B^{2} T} \frac{1}{E^{\left(1+\tau / B^{2} T\right)}} \mathrm{d} E
$$


It is gratifying to find that the theory leads naturally to the conclusion that the spectrum of the cosmic radiation obeys an inverse power law. By a comparison of the exponent of this law with the one known from cosmic rays observations, i.e. about 2.5, we find a relationship which permits us to determine the value of $\tau$, the time interval between collisions. We have

$$
2.9=1+\left(\tau / B^{2} T\right)
$$

from which it follows that $\tau=1.9 \mathrm{~B}^{2} T=4 \times 10^{7}$ sec $\sim 1.3$ years. Since the particles travel with approximately the velocity of light, this corresponds to a mean distance between collisions of the order of a light year or about $10^{18} \mathrm{~cm}$. such a collision mean free-path seems to be quite reasonable.

The theory explains quite naturally why no electrons are found in the primary cosmic radiation. This is due to the fact that at all energies the rate of loss of energy by the electron exceeds the gain. At low energies up to about $300 \mathrm{MeV}$ the loss is mainly due to ionization. Above this energy, radiative losses due to acceleration of the electrons in the interstellar magnetic fields play a dominant role. This last energy loss is quite negligible for protons. Also the inverse Compton effect discussed by Feenberg and Primakoff ${ }^{1}$ will contribute to eliminate high energy electrons.

Since Fermi ${ }^{2}$ first propounded this theory there have been changes in the views about the structure of the galactic field which affect the validity of this theory. They are: (1) the galactic field is now considered much more regular than he assumed, (2) the protonic and heavier components have very much the same energy spectrum. This is incompatible with the absorption mechanism of Fermi, since heavy nuclei have larger cross-sections for collision than protons. The difficulty would be removed if we were to introduce an absorption process equally effective for both types, i.e. for example, collision against stars. But simple estimates show that probability of such a collision is vanishingly small. Another means of removing cosmic ray particles is by diffusion outside the galaxy. For such a theory it is necessary to assume that the escape time for diffusion outside the galaxy is appreciably shorter than the nuclear collision time. Since this method of removal is very efficient it was found necessary by Fermi $^{3}$ to improve the acceleration mechanism in a manner so as to make possible the attainment of high energies before escape. This he was able to do by postulating a "jaw mechanism" by which a cosmic ray particle is "caught" between two magnetic discontinuities such that it is repeatedly reflected and gains energy till it escapes from the jaw.

Let $H$ be the average value of the magnetic field and $H_{\max }$ the maximum field along the line of force that may be likely to cause a reflection. If $\theta$ is the angle of the pitch of the spiral where the field average is $H$, reflections will occur for particles having $\theta>\chi$ where $\sin \chi=V \frac{H}{H_{\max }}$. A simple calculation shows that when a particle with $\theta>\%$ is caught in a trap both its energy and angle of pitch will

1 E. Feenberg and H. Prinakoff, Phys. Rev. 73, 449 (1948).

2 E. FerMi, loc. cit.

3 E. Fermi, loc. cit. 
change with time, but the product $E \sin \theta$ remains approximately constant. The particle can escape the trap only when $\theta$ has decreased to such a value that the condition $\theta>\chi$ is no longer fulfilled. In this process, the energy must increase by a factor $\sin \theta / \sin \chi$. This process leads to a sizeable energy gain in a relatively short time. For example if the jaws of the trap are 10 light years apart and move towards each other at $10 \mathrm{~km} / \mathrm{sec}$ the time required for a 10 per cent energy increase is only a few thousand years.

\section{SOURCES OF COSMICRADIATION}

We have till now discussed the accelerating mechanism in quite a general manner. The actual domain of storage and acceleration as we have mentioned already depends on the sources. We shall now take up the discussion of the sources

\begin{tabular}{|c|c|c|c|}
\hline Source & $\begin{array}{c}\mathrm{I} \\
\text { Solar origin }\end{array}$ & $\begin{array}{l}\text { II } \\
\text { Galactic } \\
\text { origin }\end{array}$ & $\begin{array}{l}\text { III } \\
\text { Extragalactic } \\
\text { origin }\end{array}$ \\
\hline $\begin{array}{l}\text { 1. Total energy of the radiation (too high if } \\
\text { extra galactic in its origin). }\end{array}$ & + & & - \\
\hline $\begin{array}{l}\text { 2. Universal occurrence of cosmic radiation. } \\
\text { Other celestial bodies can also produce } \\
\text { these radiation. }\end{array}$ & + & + & + \\
\hline $\begin{array}{l}\text { 3. Absence of Compton-Getting effect. (Due } \\
\text { to this effect an isotropic cosmic radiation } \\
\text { in space will cause higher intensity to be } \\
\text { observed in the apex of the earth than in } \\
\text { the anti-apex.) }\end{array}$ & + & - & - \\
\hline $\begin{array}{l}\text { 4. Correlation of intensity with solar flares } \\
\text { argues for incident radiation from thesun. }\end{array}$ & + & - & - \\
\hline $\begin{array}{l}\text { 5. No day and night effect in the intensity } \\
\text { of these rays precludes the solar origin. }\end{array}$ & - & + & + \\
\hline 6. Presence of any 11 year effect. & + & - & - \\
\hline $\begin{array}{l}\text { 7. The theory of Fermi which requires ex- } \\
\text { tensive magnetic fields and dust clouds } \\
\text { argues only for a galactic origin. }\end{array}$ & - & + & + \\
\hline $\begin{array}{l}\text { 8. Absence of any directional effect found in } \\
\text { the continuous radiation is unfavourable } \\
\text { for the idea of a solar origin. }\end{array}$ & - & + & + \\
\hline $\begin{array}{l}\text { 9. Directional effect of high energy particles } \\
\text { pointing to points in galaxy from which } \\
\text { radio frequency waves arrive, argues for } \\
\text { a galactic origin. }\end{array}$ & - & + & + \\
\hline $\begin{array}{l}\text { 10. Presence of high energy heavy particles } \\
\text { can be produced only in the favourable } \\
\text { conditions obtaining in supernovae and } \\
\text { so is an argument against solar origin. } \\
\text { 11. Presence of } \mathrm{Li}, \mathrm{Be}, \mathrm{B} \text {. }\end{array}$ & - & + & + \\
\hline
\end{tabular}


of cosmie radiation. The three possible origins of cosmic rays are (1) solar origin, (2) galactic origin and (3) extragalactic origin. The table given above due to Clay argues for the greatest possibility for a continuous radiation of galactic origin with the superposed radiation from the sun.

\section{Solar contribution to cosmic radiation}

There is evidence for magnetic fields of a few thousand gauss in the neighbourhood of sun spots which presumably extend above the surface of the sun to considerable heights. At these heights, the energy density due to the magnetic field is apt to be considerably greater than the energy density present as hydrodynamic energy and thermal energy per unit volume when carried by the material, since the density of the material is only $10^{8}$ atoms per $\mathrm{cm}^{3}$. Under these conditions the motion of the ionized material is eompletely determined by the lines of force. The energy density, pressure and inertia of the material react little on the changes of the magnetic fields. If there is any motion or change in the magnetic field due to, say, sun spot activity, the tenuous material of the corona will be swept along by the moving magnetic field. In particular, rapidly moving magnetic fields may accelerate matter in the corona to a sufficiently high velocity and this constitutes the injection mechanism in the Richtmyer-Teller ${ }^{1}$ theory. We now imagine that an ionized gas is accelerated to $10^{8} \mathrm{eV} / \mathrm{sec}$ and a fraction of gas finds itself at the end of the acceleration process in a region of low magnetic intensity. If now Fermi collisions should occur with wandering magnetic fields of small intensity and high velocities, particles of the observed energy can be produced. For this phenomenon to occur it is necessary to postulate a field of $10^{-5}$ gauss confined to the solar system which is necessary to trap the particles in the solar system while they are being energized and to render the distribution isotropic. Observations have shown that the geomagnetic field and other fields in our system deflect the cosmic rays by $1 / 10^{\text {th }}$ of a radian in about ten earth radii (for a typical $10 \mathrm{BeV}$ cosmic ray). The field turns out to be one milligauss or a little less. There is a paucity of cosmic rays below $2 \mathrm{BeV}$ and this cut-off varies with the solar activity cycle of eleven years and is a sign of the influence of the magnetic field around the sun on cosmic rays. Alfven ${ }^{2}$ is in favour of the Richtmyer-Teller ${ }^{3}$ theory and supports the postulate regarding the existence of an extensive magnetic field. The solar dipole is insufficient to cause such a field and Alfven ${ }^{4}$ suggests the following mechanism. Suppose that in an electrically conducting medium with the mass density $\varrho$ there is an external magnetic field $H^{\prime}$. If parts of the medium are put into motion with velocity $v$, the magnetic field $H_{0}$ is superimposed on the initial field. In the case of an infinite electrical conductivity,

1 R. D. Richtyyer and E. Teller, Phys. Rev. 75, 1729 (1949).

2 H. Alfven, loc. cit. (1950).

${ }^{3}$ R. D. Richtwyer and E. Teller, Ibid.

4 Alffven, loc. cit. (1950). 
the average value of $H^{\prime}$ increases until the magnetic energy equals the kinetic energy; $\frac{H^{2}}{8 \pi}=\frac{1}{2} \varrho v^{2}$. When this limit is reached an equipartition of energy is accomplished and the magnetic field energy is converted back into kinetic energy. The state characterizing a magneto hydrodynamic wave is reached. We shall apply these results to interstellar spaces around the solar system. Setting $\varrho=10^{-24} \mathrm{gm} \mathrm{cm}^{-3}$ which is the usually accepted value of interstellar density, we have $H^{\prime}=v(4 \pi \varrho)^{1 / 2}=3.5 \times 10^{-12} v$. A magnetic storm is usually supposed to be produced by a corpuscular beam sent out radially from the sun with a velocity of the order $2 \times 10^{8} \mathrm{~cm}$ per sec. When it passes through the solar magnetic field, the beam will distort the magnetic lines of force and will cause an induced field which will grow up to the order of $H^{\prime}=3.5 \times 10^{-12} \times 2 \times 10^{8}=7 \times 10^{-4}$ gauss. It should also be observed that the beam consists of ionized gas which has a high conductivity. The magnetic field in the region close to the sun where the beam emanates may in part be frozen into the matter of the stream. In this way, the magnetic field is transported outwards. Both these effects produce magnetic fields in the outskirts of the solar dipole field which are much stronger than the dipole field itself. Considerations of the decay of these fields and the total energy necessary to produce fields of an extension and strength sufficient to trap cosmic radiation of varying energy however make it rather dubious whether this effect is sufficient. The solar origin of cosmic rays might seem attractive for the low energy component because only a very small fraction of the sun's energy would be required to maintain the cosmic radiation. On the other hand the solar hypothesis runs into difficulties when trying to account for the cosmic ray particles of high energies. Particles with more than $10^{14} \mathrm{eV}$ per nucleon have been observed, and if such particles are assumed to have been accelerated on the solar surface, fields of 1000 gauss extending over at least $3 \times 10^{8} \mathrm{~cm}$ will be needed to confine them. In the latter phase when these particles circulate in interplanetary space fields of $10^{-5}$ gauss extending over at least $3 \times 10^{16} \mathrm{~cm}$ or $10^{6}$ light seconds will be needed. A further argument against the solar origin for the high energy component is the fact that the additional effects which accompany solar outbursts are considerably less energetic than the steady component of cosmic radiation. Moreover the sun makes very few cosmic ray particles above $10 \mathrm{BeV}$ even in its most violent spasms. It is appropriate to look farther afield for the origin of high energy cosmic rays. The solar disturbances which coincide with the intensity increases in cosmic rays are also associated with the emission of radio waves of a few metres in wavelength. The sun however is not the only celestial body which emits such radio waves. Definite positions in the sky called "radio stars" emit radiation similar to that which is emitted by active spots on the sun. It is reasonable to suggest that the radio stars in addition to emitting radio waves should also produce energetic particles similar to those which the disturbed sun emits. The greater average radio intensity of the stars might give reason to expect a greater flux of cosmic ray particles arriving from them. Thus it seems likely that the high energy component of cosmic radiation is due to phenomena similar 
to solar magnetic aetivities, but actually taking place in distant astronomical objects.

\section{(ialactic sources of cosmic rays ${ }^{1}$}

Conclusions based on the distribution of the electronic component of eosmie radiation at the source by a study of cosmic radio-emission will form a valuable supplement to our knowledge of the composition of the primary cosmic radiation in the vicinity of the earth. Cosmic radio-emission consists of a component from discrete sources (radio-nebulae) and of a general emission of galactic origin containing both thermal and non-thermal components. Evidence for the non-thermal component comes from the fact that if we assume that the effective temperature $T_{\text {If }}$ of thermal radiation cannot exceed the kinetic energy of interstellar gas (which even in the completcly ionized region never exceeds $10^{4}$ degrees) we should

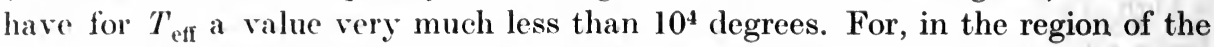
galactic centre or the galactic pole (where the optical thickness of the gas is small) actually $T_{\text {eff }}-2.7 \times 10^{5}$ degrees for $\lambda=16.3 \mathrm{~m}$ and $10^{6}$ degrees for $\lambda=32.8 \mathrm{~m}$ etc. in the region of the galactic centre and $T_{\text {eff }} \approx 7.5 \times 10^{4}$ degrees for $\lambda=16.3 \mathrm{~m}$ in the region of the galactic pole ( $\lambda$ being the wavelength of the radiation). Hence there should be a non-thermal galactic radio-emission, the sourees for which (from the analysis of radio-isophotes) should form a quasi-spherical sub-system of radius $R \approx 3.5 \times 10^{22} \mathrm{~cm}$ which is approximately the spatial distribution of diffuse interstellar gas at a very low concentration $\left(n \leqq 0.1 \mathrm{~cm}^{-3}\right)$.

The non-thermal component ean be well accounted for on the basis of the "magnetic bremsstrahlung hypothesis" according to which this part of the cosmic radio-emission is due to the radiation by relativistic electrons accelerated in interstellar magnetic fields. A relativistic electron of energy $E$ following a helical path in a magnetic field $H$ with a frequency

$$
\omega_{H}=\frac{\epsilon H}{m c} \frac{m c^{2}}{E}
$$

will emit radiation almost exclusively into a narrow cone of aperture $\alpha \approx \frac{m c^{2}}{E} \ll 1$. Hence, if the electron moves in a circle, an observer in the plane of the orbit will observe pulses of radiation of period

$$
\angle t \approx \frac{r \alpha}{c}\left(\frac{m c^{2}}{E} \cdot\right)^{2} \approx \frac{m c}{e H} \cdot\left(\frac{m c^{2}}{E}\right)^{2}
$$

where $r \quad \frac{c}{\omega_{H}}$ is the radius of the orbit and the factor $\left(\frac{m c^{2}}{E}\right)^{2}$ is due to the Doppler effect. If the electron moves helically and the angle between its velocity and the magnetic is not too small, $\theta \gg \alpha \approx m c^{2} / E$ and we have

$$
\Delta t \approx \frac{m c}{e H_{\perp}}-\left(\frac{m c^{2}}{E}\right)^{2}
$$

1 V. I. Gixzburg, loc. cit. 
where $H_{\perp}$ is the component of the magnetic field normal to the direction of motion. The radiation thus consists of a continuous spectrum consisting of harmonics of frequency $\omega_{H}$ with a maximum at the frequency

$$
\omega_{\max } \approx \frac{1}{\Delta t} \approx \frac{\mathrm{e} H_{\perp}}{m c} \cdot\left(\frac{E}{m c^{2}}\right)^{2} .
$$

More detailed calculations of the energy radiated by an electron per second in a frequency range $\mathrm{d} v=\frac{\mathrm{d} \omega}{2 \pi}$ viz., $P(v, E) \mathrm{d} v$ have been done. The expression for $P(\nu, E)$ is of the form

At maximum frequency

$$
P(\nu, E)=\dot{P}(v)=16 \frac{\mathrm{e}^{3} H_{\perp}}{m c^{2}} \cdot p\left(\frac{\omega}{\omega_{m}}\right) .
$$

$$
\begin{aligned}
P\left(v_{\max }\right) & =\frac{1.6 \mathrm{e}^{3} H_{\perp}}{m c^{2}}=2.16 \times 10^{-22} H_{\perp} \mathrm{erg} \mathrm{sec}^{-1} \mathrm{cycle}^{-1} . \\
v_{\max } & =0.5 w_{m} / 2 \pi=1.4 \times 10^{6} H_{\perp}\left(E_{m} / m c^{2}\right)^{2} .
\end{aligned}
$$

The intensity of magnetic bremsstrahlung emission observed at the earth is

$$
I_{v}=\frac{1}{4 \pi} \int P(v, E) N_{e}(E, \boldsymbol{r}) \mathrm{d} E \mathrm{~d} \boldsymbol{r}
$$

where $N_{e}(E, \boldsymbol{r})$ is the differential electron spectrum at the point $\boldsymbol{r}$. If we assume that all the electrons have a common energy $E_{m}$ we have for the maximum intensity of the radiation

$$
\begin{aligned}
I_{v_{\max }}^{\max } & =\frac{P\left(v_{\max }\right)}{4 \pi} N_{e} R \\
& =1.7 \times 10^{-23} H_{\perp} N_{e} R \mathrm{erg} \mathrm{cm}^{-2} \mathrm{sec}^{-1} \mathrm{sterad}^{-1} \mathrm{cycle}^{-1}
\end{aligned}
$$

where $N_{e}$ and $H_{\perp}$ are average values along the line of sight and $R$ is the extent of the emitting region in that direction.

For thermal emission in the direction of the galactic pole

$$
I_{v}=\frac{2 k v^{2}}{c^{2}} T_{\mathrm{eff}}=\frac{2 k}{\lambda^{2}} T_{\mathrm{eff}}
$$

where $T_{\text {eff }}=7.5 \times 10^{4}$ degrees for $\lambda=16.3$. Hence

$$
N_{e} H_{\perp} R=\frac{2 k T_{\text {eff }}}{\lambda^{2} 1.7 \times 10^{-23}}=4.6 \times 10^{5}
$$

As discussed earlier in this chapter, there is reason to believe that the intensity of interstellar magnetic fields is of the order $3 \times 10^{-6}-10^{-5}$ gauss. Using $H_{\perp} \approx 10^{-5}$ gauss and $R \approx 5 \times 10^{22} \mathrm{~cm}$ (for the galactic "corona") we get

$$
N_{e} \approx 10^{-12} \mathrm{~cm}^{-3}
$$

and

$$
E_{m}=\left(\frac{v_{\max }}{1.4 \times 10^{6} H_{\perp}}\right)^{1 / 2} m c^{2}=6 \times 10^{8} \mathrm{eV}
$$


which is in agreement with the data on the electronic component of cosmic radiation near the earth. Thus the magnetic bremsstrahlung hypothesis seems to be in good agreement with experimental facts. Further if we assume a power law spectrum for $N_{e}(E)$ we infer that the emission at $\lambda=16.3 \mathrm{~m}$ is determined mainly by electrons with energies in the range $10^{8}<E<5 \times 10^{9} \mathrm{eV}$ if the value for the exponent $\gamma$ is taken as 2.64 which is in agreement with the data of radio-astronomy.

Thus the above considerations lead to the conelusion that the galaxy can be thought of as a spherical container for cosmic radiation of radius $R=3.5 \times 10^{22} \mathrm{~cm}$ and with walls that are practically perfect reflectors. That the assumption about perfect reflection at the boundary is reasonable can be scen if we consider the approximate equality $\frac{1}{2} \varrho u^{2} \approx \frac{1}{S \pi} H^{2}$ where $\varrho$ is the density of the medium and $u$ its velocity. For the galactic "corona" $\varrho \approx 10^{-25}-10^{-26} \mathrm{~g} \mathrm{~cm}^{2}$ while for intergalactic space, $\varrho \approx 10^{-29}-10^{-30} \mathrm{~g} \mathrm{~cm} \mathrm{~cm}^{2}$ which would give $H \approx 1-3 \times 10^{-7}$ gauss. Thus the properties of the galactic "corona" and the intergalactic medium are very different and the difference in their magnetic field strengths will alone be enough to ensure that the galaxy reflects all particles coming from outside it.

The total energy of cosmic radiation in the galaxy $W_{c \cdot r}$ will be equal to $u_{c \cdot r} \times \frac{4}{3} \pi R^{3}$ where $w_{c \cdot r}=1 \mathrm{eV} \mathrm{cm}^{-3}$ for protons (it will be an order $10^{-2}$ less for electrons). Thus

$$
W_{c \cdot r} \approx 10^{67}-10^{68} \mathrm{eV} \approx 10^{55}-10^{56} \mathrm{ergs} .
$$

The radio-emissions from nebulae such as Cassiopeia-A and the Crab nebula can be similarly considered to be of magnetic bremsstrahlung origin.

A further confirmation for the hypothesis comes from the data on the polarization of the optical spectrum of both galactic (e.g. the Crab) or extragalactic (e.g. Virgo. A) nebulae. Only the magnetic bremsstrahlung mechanism can explain a strong polarization since the emission from relativistic electrons will be strongly polarized.

Thus the radio-astronomical evidence points to a galactic origin of cosmic rays and the presence of cosmic electrons throughout the galaxy is in complete contradiction with theories of an exclusively solar origin of cosmic radiation. We shall now consider some particular examples of possible sources.

Cassiopeia-A and Taurus-A (the Crab nebula) which are, as already mentioned, sources of powerful non-thermal radio-emission are the expanding envelopes formed during the explosions of supernovae of the years 369 and 1054. The envelopes of other supernovae also emit such radiation so that radio-astronomy gives us the important result that the explosions of supernovae lead to an abundant production of relativistic electrons. Calculations (e.g. Oort and Walraven ${ }^{1}$ ) show that the energy spectrum of these electrons ranges from $\gamma \approx 3.5$ to $\gamma \approx 1.5$. The total energy of the electrons varies between $10^{45}-10^{48}$ ergs.

\footnotetext{
1 J. H. Oont and I. Walraven, Bull. Nether. Astron. Inst. 12, 285 (1956).
} 
It is natural to postulate that relativistic electrons are also produced from explosions of novae although these electrons will have energies of a smaller order $\left(10^{42}-10^{44} \mathrm{ergs}\right)$ since the novae have an energy output $10^{3}-10^{4}$ times smaller than that of supernovae. As supernovae do not erupt in our galaxy oftener than once in 30 years $\left(10^{9} \mathrm{sec}\right)$, the rate of generation of relativistic electrons from them will be

$$
U_{e, s n}=\frac{W_{s n}}{\tau_{s n}} \approx \frac{10^{45}-10^{48}}{10^{9}} \approx 10^{36}-10^{39} \mathrm{erg} \mathrm{sec}^{-1} .
$$

For novae, for which the period $\tau_{n}$ is 0.01 year,

$$
U_{\mathrm{e}, n}=3 \times 10^{36}-10^{38} \mathrm{erg} \mathrm{sec}^{-1} .
$$

Now, on the sun, particles of energy of the order of several $\mathrm{BeV}$ are formed on the average at a rate $10^{23}-10^{24} \mathrm{sec}^{-1}$ and in spite of the $10^{11}$ stars of our galactic system emitting radiation of the same power, the rate would be only $10^{32}-10^{33} \mathrm{erg} \sec ^{-1}$. The mechanism of loss of energy by protons and nuclei in the interstellar medium is mainly by nuclear collision. (For protons the lifetime is $T_{p}=6 \times 10^{8}$ years $=1.9 \times 10^{16}$ sec.) Hence assuming that the protons carry a good percentage of the total cosmic ray energy $W_{c \cdot r}$ in the galaxy we get for the rate of loss energy by cosmic rays in the galaxy

$$
U_{c \cdot r}=\frac{W_{c \cdot r}}{T_{p}} \approx 10^{39}-10^{40} \mathrm{erg} \mathrm{sec}^{-1} .
$$

As we saw, the loss of energy by the electrons in interstellar space is mainly by magnetic bremsstrahlung and a calculation on the basis that electrons carry the bulk of the energy of cosmic rays gives

$$
U_{\mathrm{e}}=10^{38}-10^{39} \mathrm{erg} \mathrm{sec}^{-1} \text {. }
$$

The above facts conclusively show that only the supernovae origin of cosmic rays is in consonance with such high rates of energy loss from the galaxy since they are the only possible sources of injection into the galaxy of cosmic electrons at a power of $10^{38}-10^{39} \mathrm{erg} \mathrm{sec}^{-1}$. There is no direct evidence about the transfer of energy to protons and other nuclei during these explosions, but it is extremely probable that energy is transferred to them in the same manner and quantity as to the electrons.

The above conclusions are not dependent on or influenced by the mechanism of particle acceleration which by itself forms an independent and important problem. There are reasons to believe that the Fermi mode of acceleration may not be very effective in interstellar space. In the first place, the movement of the interstellar medium is relatively slow $\left(u \leqq 10^{7} \mathrm{~cm} \mathrm{sec}^{-1}\right)$ and secondly the free path $\lambda$ is very great $\left(\approx 3 \times 10^{20} \mathrm{~cm}\right)$. Now the coefficient $\alpha$ which determines the rate of mean particle energy increase in the equation

$$
\frac{\mathrm{d} E}{\mathrm{~d} t}=\alpha E
$$


is equal to $\frac{u^{2} v}{c^{2} \lambda}$ where $v$ is the velocity of the particle and hence is small in interstellar space. In the envelopes of supernovac, velocities of the order of $1-3 \times 10^{8} \mathrm{~cm} \mathrm{sec}^{-1}$ are to be found and in the early stages of turbulence, $\lambda \approx 10^{13} \mathrm{~cm}$. 'Thus a will have a value very much higher. Assumption of isotropic turbulence in the envelope leads to values of $\alpha \approx 10^{-8}$. Statistical acceleration will be extremely effective in the explosion process at the centres of the supernovae themselves before the envelope has separated from it. Further evidence for this idea comes from the fact that under statistical acceleration the energy reached by a particle is proportional to its mass $(1-22 a)$ and thus protons, e.g. will be accelerated to an energy about 1840 times that of the electrons. (This may be even higher due to magnetic bremsstrahlung losses with the increase of energy of electrons.) Calculations derived on this basis give an energy spectrum which to the accuracy at present available, corresponds to the actual spectrum of cosmic radiation. The value for the total energy of cosmic radiation in the galaxy

$$
W_{c \cdot r}=10^{55}-10^{56} \mathrm{ergs}
$$

ean be accounted for by assuming that the resultant average spectrum of all cosmic radiation is the sum of the spectra of cosmic particles accelerated in the eruptions of about $10^{6-10^{7}}$ supernovae (the total energy of relativistic electrons in supernovae is $\left.10^{45}-10^{48} \mathrm{ergs}\right)$.

The supernovae origin of cosmic radiation is also confirmed by the composition of cosmic radiation near the earth. The estimation of the latter requires the use of the system of equations

$$
\frac{\partial N_{i}}{\partial t}=\nabla\left(D_{i} \nabla N_{i}\right)-\frac{N_{i}}{T_{i}}+\sum_{j} p_{i j} \frac{N_{j}}{T_{j}}+q_{i},
$$

where $N_{i}(\boldsymbol{r}, t)$ is the concentration of nuclei of species $i, q_{i}(\boldsymbol{r}, t)$ is the number of nuclei of the same kind formed at the source per unit volume per unit time, $D_{i}(\boldsymbol{r})$ is the coefficient of diffusion and $T_{i}(r)$ is the lifetime of nuclei of species $i$ until they are broken up by nuclear collision in interstellar space; $p_{i j}$ is the probability of formation of a nucleus of species $i$ as a result of the disintegration of a nucleus of type $j$.

The above system of equations have been solved subject to various assumptions such as

(a) the structure of the galaxy does not change during the lifetime of cosmic particles $\left(-\frac{\partial N_{i}}{\partial t}=0\right)$

(b) the sources are near the galactic plane (as is actually the case for novae and probably so for supernovae).

We present here only the important results which are:

(i) The distance of the source from the earth $r=1.8 \times 10^{22} \mathrm{~cm}$ which is only 1.4 times smaller than the actual distance of the earth from the centre of the galaxy. 
(ii) Assuming a proper distribution of sources together with the diffusion of cosmic particles the relatively small amounts of the elements $\mathrm{Li}$, Be and B near the earth can be explained and further in order to establish a connection with the data on the composition of the radiation in the vicinity of the earth we have only to assume that the relative number of medium and heavy nuclei in the sources are from five to ten times higher than the universal average. Such an assumption is in agreement with recent pictures of the constitution of supernovae. The solutions of the diffusion equations mentioned above determine also the distribution of cosmic radiation in the galaxy and hence can be used to evaluate the degree of anisotropy $\delta$. The result is that one can achieve $\delta<10^{-3}$ in agreement with experiment for particles with $E<10^{5} \mathrm{eV}$ and a value $\delta \leqq 10^{-2}$ for nuclei with $E \approx 10^{18} \mathrm{eV}$.

Thus we may conclude that the picture of the origin of cosmic radiation developed here with the envelopes of novae and supernovae as the sources of cosmic radiation seems to be in consonance with the experimental results on the energy spectrum, the composition and the isotropy of cosmic radiation.

There is always the possibility of considering even extragalactic sources of cosmic radiation but in such a case the storage space must be the intergalactic space and the acceleration must also take place there. Since the storage space is large, very weak magnetic fields are sufficient to confine the particles in it. The extremely high energy spectrum may perhaps be traced to such sources (e.g. M 87). 


\section{APPENDICES}

\section{DIMENSIONS ANI UNITS}

IT Is well-known that the dimensions of various physical quantities could be expressed in terms of a chosen few ( $L, M, T$-length, mass and time in mechanics) which are treated as fundamental dimensions. This choice of fundamental dimensions is quite arbitrary.

In the study of elementary particles it is convenient to choose velocity $V$ and action $A$ as fundamental dimensions, the unit being the velocity of light $c$ and the Planck's constant divided by $2 \pi$, i.e. $\frac{h}{2 \pi}=h$ respectively ${ }^{1}$. This choice is called the system of natural units and is usefully employed in the algebraic calculations of quantum theory so that one need not write the often occurring $c$ and $\hbar$ throughout a calculation.

The definition of this system of units will be complete if another dimension and its unit are specified. This choice is made to suit the problem under investi-

\begin{tabular}{|c|c|c|c|c|c|c|c|c|}
\hline \multirow[b]{2}{*}{$\begin{array}{l}\text { Physical } \\
\text { Quantity }\end{array}$} & \multicolumn{4}{|c|}{ Dimensions } & \multicolumn{4}{|c|}{$\begin{array}{l}\text { Conversion factor } \\
\text { (C. G. S./natural) }\end{array}$} \\
\hline & $\begin{array}{l}\text { in terms } \\
\text { of }(L, M, T)\end{array}$ & $\begin{array}{c}\text { in terms } \\
\text { f }(A, V, M)\end{array}$ & $\begin{array}{l}\text { in terms } \\
\text { f }(A, V, L)\end{array}$ & $\begin{array}{c}\text { in terms } \\
\text { of }(A, V, T)\end{array}$ & $\begin{array}{c}\hbar=c=1 \hbar \\
\mathrm{m}=1 \\
\text { system sy }\end{array}$ & $\begin{array}{l}=c= \\
1 \mathrm{gm} \\
\text { stem s }\end{array}$ & $\begin{array}{r}\hbar=1 \\
c=1 \\
1 \mathrm{~cm} \\
\text { ystem }\end{array}$ & $\begin{array}{l}\hbar=1 \\
c=1 \\
1 \text { sec } \\
\text { system }\end{array}$ \\
\hline Mass & $M$ & $M$ & $A L^{-1} V^{-1}$ & $A V^{2} T^{-1}$ & $m$ & 1 & $\hbar / c$ & $\hbar / c^{2}$ \\
\hline Length & $L$ & $A V^{-1} M^{-1}$ & $L$ & $V T$ & $\begin{array}{c}\hbar / m c \\
\text { (Compton } \\
\text { wave- } \\
\text { length) }\end{array}$ & $\frac{\hbar}{c}$ & 1 & $c$ \\
\hline Time & $T$ & $A V^{-2} H^{-1}$ & $L V^{-1}$ & $T$ & $\hbar / m c^{2}$ & $\frac{\hbar}{c^{2}}$ & $\frac{1}{c^{2}}$ & 1 \\
\hline Velocity & $L T T^{1}$ & $V$ & $V$ & V & $c$ & $c$ & $c$ & $c$ \\
\hline Momentum & $M L T^{\sim 1}$ & $M V$ & $A L^{-1}$ & $A V^{-1} T^{-1}$ & $m c^{2}$ & $c$ & $\hbar$ & $\frac{h}{c}$ \\
\hline Energy & $M L^{-2} T^{-2}$ & $M V^{2}$ & $A L^{-1} V$ & $A T^{-1}$ & $\begin{array}{c}m c^{2} \\
\hbar^{2}\end{array}$ & $\begin{array}{c}c^{2} \\
h^{2}\end{array}$ & $\hbar c$ & $\hbar$ \\
\hline Cross-section & $L^{2}$ & $A^{2} V^{-2} M=2$ & $L^{2}$ & $V^{2} T^{2}$ & $\frac{n^{2}}{m^{2} c^{2}}$ & $\frac{n^{2}}{c^{2}}$ & 1 & $c^{2}$ \\
\hline Charge & $\begin{array}{c}K^{-1 / 2} M^{1 / 2} L^{3 / 2} \\
T^{-1}\end{array}$ & $K^{-\frac{1}{2}}$ & $A^{\frac{1}{2}}$ & $V^{\frac{1}{2}}$ & $\hbar \mathrm{c}$ & $\hbar \mathrm{c}$ & $\hbar \mathrm{c}$ & $\hbar \mathrm{c}$ \\
\hline
\end{tabular}

\footnotetext{
1 In C.G.S. units,

$C=2.99793 \times 10^{10} \mathrm{~cm} . \mathrm{sec} . \quad \hbar=1.05443 \times 10^{-27}$ erg. - sec. $\quad e=4.80286 \times 10^{-10}$ e.s.u. 
gation. This dimension may be taken as mass. Usually in meson theoretical calculations the $\pi$-meson mass is taken as unity, while in a purely quantum electrodynamical calculation, the electron mass could with advantage be taken as unity or as in mechanics one gram could taken as the unit. Some authors take the length or time as the third dimension with one centimetre or one second as the unit respectively.

While comparing with experiments however one has to come back to the C.G.S. system with units of $L M T$ being $1 \mathrm{~cm}, 1 \mathrm{~g}$ and $1 \mathrm{sec}$ respectively and this means a proper replacement of $c$ 's and $\hbar$ 's in the final expressions of a theoretical calculation. This can be achieved by elementary dimensional analysis and the table given above furnishes the various conversion factors.

$L M T$ stand as usual, for Length, Mass and Time while $A$ and $V$ denote Action and Velocity respectively. $A=M L^{2} T^{-1}, V=L T^{-1}$.

In applying the conversion factors to the final expressions (to convert into C.G.S. units) one has to make sure that all the quantities occurring in the expression are already expressed in natural units. For example, in an electro-dynamical calculation the charge $e$ occurring in a final expression for cross-section has to be expressed in natural units before applying the conversion factor for the crosssection.

It is sometimes said that in the natural system of units, all physical quantities have dimensions of powers of $L$ only. This statement refers to the $\hbar=1, c=1$ and $L$ system and is understood if we allow the $h$ 's and $c$ 's in the conversion factors to earry with them their dimensions. In this system we have:

\begin{tabular}{c|c|c}
\hline Physical quantity & Dimension & Unit \\
\hline Mass & $L^{-1}$ & $\hbar c^{-1} \mathrm{~cm}^{-1}$ \\
Time & $L$ & $c^{-1} \mathrm{~cm}$ \\
Velocity & $L^{0}$ (Dimensionless) & $c$ \\
Energy & $L^{-1}$ & $\hbar c \mathrm{~cm}^{-1}$ \\
Action & $L^{0}$ (Dimensionless) & $\hbar$ \\
\hline
\end{tabular}

The $\hbar$ 's and $c$ 's in the last column are treated as dimensionless. From this point of view, all quantities become dimensionless in the $\hbar=1, c=1, m=1$ system, excepting for electromagnetic quantities like charge which carry a dimension in $K$.

\section{COMPOSITIONOF ANGULAR MOMENTA}

\section{Addition of two angular momenta-Clebsch-Gordan coefficients ${ }^{1}$}

In many physical problems there arise situations where two (or more) angular momenta have to be coupled. Examples are the orbital and intrinsic spin angular momenta of a single particle, the angular momenta of the particles of a system or the angular momenta of a particle and the radiation it emits or absorbs.

1 A. R. Edmonds, Angular Momentum in Quantum Mechanics, Princeton University Press (1957). See also Elementary Theory of Angular Momentum by M. E. Rose, John Wiley (1957). 
Consider a system in which $\boldsymbol{J}_{1}$ and $\boldsymbol{J}_{2}$ are two commuting angular momentum operators. The resultant angular momentum is given by

$$
\boldsymbol{J}=\boldsymbol{J}_{1}+\boldsymbol{J}_{2}
$$

The state of the system can be represented in two distinct schemes for there are two sets of four mutually commuting observables

$$
\begin{aligned}
& \text { (A) } J_{1}^{2}, J_{2}^{2}, J_{1 z}, J_{2 z} \\
& \text { (B) } J_{1}^{2}, J_{2}^{2}, J^{2}, J_{z} .
\end{aligned}
$$

The simultaneous eigenfunction in the representation (A) in which $J_{1}^{2}, J_{2}^{2}, J_{1 z}$ and $J_{2 z}$ are diagonal can be written as the direct product $\psi_{j_{1}}^{m_{1}} \psi_{j_{2}}^{m_{2}}$ where $\psi_{j_{1}}^{m_{1}}$ is the simultaneous eigenfunction of $J_{1}^{2}$ and $J_{1 z}$ and $\psi_{j_{2}}^{m_{2}}$ that of $J_{2}^{2}$ and $J_{2 z}$. If the eigenfunctions in the representation (B) in which $J_{1}^{2}, J_{2}^{2}, J^{2}$ and $J_{z}$ are diagonal are written as $\psi_{j}^{m}\left(j_{1} j_{2}\right)$ they can be expanded in terms of the eigenfunctions of the other representation as

$$
\psi_{j}^{m}\left(j_{1} j_{2}\right)=\sum_{m_{1} m_{2}} C\left(j_{1} j_{2} j ; m_{1} m_{2} m\right) \psi_{j_{1}}^{m_{1}} \psi_{j_{2}}^{m_{3}}
$$

where $C\left(j_{1} j_{2} j ; m_{1} m_{2} m\right)$ is called the vector addition or Clebsch-Gordan or Wigner coefficient: Since $\boldsymbol{J}=\boldsymbol{J}_{1}+\boldsymbol{J}_{2}$ the Clebsch-Gordan coefficient vanishes unless $m=m_{1}+m_{2}$. The maximum value which $j$ can assume is obviously the maximum value of $m$ which is $j_{1}+j_{2}$, i.e. the sum of the maximum values of $m_{1}$ and $m_{2}$ respectively. Thus $\psi_{j}^{m}$ is an element of a column vector consisting of $\left(2 j_{1}+1\right)$ $\left(2 j_{2}+1\right)$ elements. Thus the above equation (3) can be written as

$$
\Psi(\mathrm{B})=C \Psi(\mathrm{A})
$$

where $\Psi(\mathrm{A})$ and $\Psi(\mathrm{B})$ are column vectors with typical elements as eigenfunctions in the schemes $\mathrm{A}$ and $\mathrm{B}$ respectively. We must prescribe a method of writing $\Psi(\mathrm{A})$ and $\Psi(\mathrm{B})$ explicitly, and this determines the way in which the matrix $\boldsymbol{C}$ has to be written. The column vector $\Psi(\mathrm{B})$ is written by varying $j$ from $\left|j_{1}-j_{2}\right|$ to $j_{1}+j_{2}$ and exhausting all the values of $m$, i.e. $-j$ to $+j$ for each value of $j$ before going from one value of $j$ to another. $\Psi(\mathrm{A})$ is obtained by running over all the values of $m_{1}$ for a given $m_{2}$ as $m_{2}$ varies from $-j_{2}$ to $+j_{2}$. This scheme fixes the method of labelling the elements of $\boldsymbol{C}$, i.e. $j m$ represent the indices for the column and $m_{1} m_{2}$ for the row. Since $\boldsymbol{C}$ is unitary and if we choose its elements to be real $\boldsymbol{C}^{-1}=\boldsymbol{C}^{\boldsymbol{T}}$ and the inverse transformation is given by

$$
\psi_{j_{1}}^{m_{1}} \psi_{j_{2}}^{m_{2}}=\sum_{j m} C\left(j_{1} j_{2} j ; m_{1} m_{2} m\right) \psi_{j}^{m}
$$

It is to be noted that in this case $j m$ fixes the row. The unitarity condition implies

$$
\begin{aligned}
\sum_{m_{1} m_{2}} C\left(j_{1} j_{2} j m_{1} m_{2} m\right) \cdot C\left(j_{1} j_{2} j^{\prime} ; m_{1} m_{2} m^{\prime}\right) & =\delta_{j j^{\prime}} \delta_{m m^{\prime}} \\
\sum_{j m} C\left(j_{1} j_{2} j ; m_{1} m_{2} m\right) C\left(j_{1} j_{2} j ; m_{1}^{\prime} m_{2}^{\prime} m\right) & =\delta_{m_{1} m_{1}^{\prime}} \delta_{m_{2} m_{2}^{\prime}}
\end{aligned}
$$

with $m=m_{1}+m_{2}$ and $m^{\prime}=m_{1}^{\prime}+m_{2}^{\prime}$. 


\section{Computation of Clebsch-Gordan coefficients ${ }^{1}$}

We can obtain a set of recursion relations which completely determine the Clebsch-Gordan coefficients with the proper phases. Knowing the state $\psi_{j}^{m}$, i.c. all the coefficients $C\left(j_{1} j_{2} j ; m_{1} m_{2} m\right)$ for the given $j$ and $m$ we can obtain the state $\psi_{j}^{m-1}$ by using the well-known relation

where

$$
J_{-} \psi_{j}^{m}=V[(j+m)(j-m+1)] \cdot \psi_{j}^{m-1},
$$

$$
J_{-}=J_{x}-i J_{y}=\left(J_{1 x}-i J_{1 y}\right)+\left(J_{2 x}-i J_{2 y}\right)=J_{1^{-}}+J_{2^{-}} .
$$

Expanding (8) in the scheme $\mathrm{A}$ and using the relations, $J_{-}=J_{1_{-}}+J_{2^{-}}$we obtain

$$
\left(J_{1-}+J_{2-}\right) \sum_{m_{1} m_{2}} \psi_{j_{1}}^{m_{1}} \psi_{j_{2}}^{m_{2}} C\left(j_{1} j_{2} j ; m_{1} m_{2} m\right)
$$

$$
\begin{aligned}
= & \sum_{m_{1} m_{2}} V\left[\left(j_{1}+m_{1}\right)\left(j_{1}-m_{1}-1\right)\right] \psi_{j_{1}}^{m_{1}-1} \psi_{j_{2}}^{m-m_{1}} C\left(j_{1} j_{2} j ; m_{1} m-m_{1} m\right) \\
& \quad+\sum_{m_{1} m_{2}} V\left[\left(j_{2}+m_{2}\right)\left(j_{2}-m_{2}+1\right)\right] \psi_{j_{1}}^{m_{1}} \psi_{j_{2}}^{m_{2}-1} C\left(j_{1} j_{2} j ; m_{1} m-m_{1} m\right) \\
= & \sum_{m_{1} m_{2}} V[(j+m)(j-m+1)] \psi_{j_{1}}^{m_{1}} \psi_{j_{2}}^{m-m_{1}-1} C\left(j_{1} j_{2} j ; m_{1} m-m_{1}-1, m-1\right) .
\end{aligned}
$$

Equating the coefficients of $\psi_{j_{1}}^{m_{1}} \psi_{j_{2}}^{m_{2}}$ on both sides

$$
\begin{gathered}
{[(j+m)(j-m+1)]^{1 / 2} C\left(j_{1} j_{2} j ; m_{1} m_{2} m-1\right)} \\
=\left[\left(j_{1}+m_{1}+1\right)\left(j_{1}-m_{1}\right)\right]^{1 / 2} C\left(j_{1} j_{2} j ; m_{1}+1 m_{2} m\right) \\
+\left[\left(j_{2}+m-m_{1}\right)\left(j_{2}-m+m_{1}+1\right)\right]^{1 / 2} C\left(j_{1} j_{2} j ; m_{1} m_{2}+1 m\right) .
\end{gathered}
$$

This is the recursion relation which steps down the value of $m$. Similarly by applying $J_{+}$to $\psi_{j}^{m}$ we can obtain the following recursion relation which steps up the value of $m$ by unity

$$
\begin{aligned}
& {[(j-m)(j+m+1)]^{1 / 2} C\left(j_{1} j_{2} j ; m_{1} m_{2} m+1\right)} \\
& =\left[\left(j_{1}-m_{1}+1\right)\left(j_{1}+m_{1}\right)\right]^{1 / 2} C\left(j_{1} j_{2} j ; m_{1}-1, m_{2}, m\right) \\
& \quad+\left[\left(j_{2}-m+m_{1}\right)\left(j_{2}+m-m_{1}+1\right)\right]^{1 / 2} C\left(j_{1} j_{2} j ; m_{1} m_{2} m\right) .
\end{aligned}
$$

We can also obtain the recursion relation which steps down the value of $j$. As the derivation is elaborate we give here only the result:

$$
\begin{aligned}
& {\left[m_{1}-m \cdot \frac{j_{1}\left(j_{1}+1\right)-j_{2}\left(j_{2}+1\right)+j(j+1)}{2 j(j+1)}\right] C\left(j_{1} j_{2} j ; m_{1} m_{2} m\right) } \\
&= {\left[\frac{\left(j^{2}-m^{2}\right)\left(j-j_{1}+j_{2}\right)\left(j+j_{1}-j_{2}\right)\left(j_{1}+j_{2}+j+1\right)\left(j_{1}+j_{2}-j+1\right)}{4 j^{2}(2 j-1)(2 j+1)}\right]^{1 / 2} } \\
&+ {\left[\frac{\left[(j+1)^{2}-m^{2}\right]\left(j+1-j_{1}+j_{2}\right)\left(j+1+j_{1}-j_{2}\right)\left(j_{1}+j_{2}+j+2\right)\left(j_{1}+j_{2}-j\right)}{4(j+1)^{2}(2 j+1)(2 j+3)}\right]^{1 / 2} } \\
& C\left(j_{1} j_{2} j+1 ; m_{1} m_{2} m\right) .
\end{aligned}
$$

1 E. U. Condon and G. H. Shortley, "The Theory of Atomic Spectra", Cambridge University Press (1951). 
The recursion relation (13) gives us the state $\psi_{j-1}^{m}$ when we know the state $\psi_{j}^{m}$ and $\psi_{j+1}^{\prime \prime \prime}$.

Starting with the state of highest $j$ and $m$, i.e. $\psi_{j=j_{1}+j_{2}}^{m+j_{1}+j_{2}}$ for whieh there is only one non-vanishing coeffieient namely

$$
C\left(j_{1} j_{2} j=j_{1}+j_{2} ; m_{1}=j_{1}, m_{2}=j_{2} ; m=j_{1}+j_{2}\right)=1
$$

we can obtain all the elements of the matrix $C$ using the recursion formulae (11) and (13). Since the last element, i.e. the element corresponding to the last row and last column is unity we can obtain every element of the last row by using the recursion relation (11). Once the last row is obtained the next row can be determined by the use of the recursion relation (13) and by repeated applications of these relations all the elements of the matrix can be obtained.

The general formula for the $C$-coeffieients was first given by Wigner by the use of group theoretical methods. His result is

$$
\begin{gathered}
C\left(j_{1} j_{2} j: m_{1} m_{2} m\right)=\delta\left(m, m_{1}+m_{2}\right) \\
]_{v}^{\left(j+j_{1}-j_{2}\right) !\left(j-j_{1}+j_{2}\right) !\left(j_{1}+j_{2}-j\right) !(j+m) !(j-m) !(2 j+1)} \\
\left(j+j_{1}+j_{2}+1\right) !\left(j_{1}-m_{1}\right) !\left(j_{1}+m_{1}\right) !\left(j_{2}-m_{2}\right) !\left(j_{2}+m_{2}\right) ! \\
\frac{(-1)^{v+j_{2}+m_{2}}\left(j+j_{2}+m_{1}-v\right) !\left(j_{1}-m_{1}+v\right) !}{\left(j-j_{1}+j_{2}-v\right) !(j+m-v) ! v !\left(v+j_{1}-j_{2}-m\right) !}
\end{gathered}
$$

In this summation $v$ takes all integral values such that none of the arguments for the factorials becomes negative.

A number of derivations of the general formula are now available notable of which are that of Racah and of Schwinger. The general formula is so complex that the calculation of coefficients by its use is as tedious as the direct use of the recursion relations. However it is only rarely necessary to have recourse to these formulae since a knowledge of the general properties of the coefficients will often suffice and convenient algebraic tables are available for small values of $J$.

\section{Symmetry properties of the Clebseh-Gordan coefficients and the Wigner 3-j symbol}

$j_{1}, j_{2}$ and $j$ form a triangle $\Delta\left(j_{1} j_{2} j\right)$. The Clebsch-Gordan coefficients have a geometrical significance and possess symmetries similar to the spatial rotational symmetries of the system concerned. The symmetry relations are

$$
\begin{aligned}
C\left(j_{1} j_{2} j ; m_{1} m_{2} m\right) & =(-1)^{j_{1}+j_{2}-j} C\left(j_{1} j_{2} j ;-m_{1},-m_{2},-m\right) \\
& =(-1)^{j_{1}+j_{2}-j} C\left(j_{2} j_{1} j ; m_{2} m_{1} m\right) \\
& =(-1)^{j_{1}-m_{1}}\left[\frac{2 j+1}{2 j_{2}+1}\right]^{1 / 2} C\left(j_{1} j j_{2} ; m_{1},-m,-m_{2}\right) .
\end{aligned}
$$

The maximum symmetry is obtained for the Wigner's $3-j$ symbol which is defined as

$$
\left[\begin{array}{ccc}
j_{1} & j_{2} & j \\
m_{1} & m_{2}-m
\end{array}\right]=(-1)^{j_{1}-j_{2}+m(2 j+1)^{-1 / 2}} C\left(j_{1} j_{2} j ; m_{1} m_{2} m\right) .
$$


Even permutation of the columns leaves the numerical value unchanged:

$$
\left[\begin{array}{ccc}
j_{1} & j_{2} & j \\
m_{1} & m_{2} & -m
\end{array}\right]=\left[\begin{array}{lrr}
j_{2} & j & j_{1} \\
m_{2} & -m & m_{1}
\end{array}\right]=\left[\begin{array}{rrr}
j & j_{1} & j_{2} \\
-m & m_{1} & m_{2}
\end{array}\right] .
$$

Odd permutation is equivalent to multiplication by $(-1)^{j_{1}+j_{2}+j}$ :

\section{Rotation matrices}

$$
\begin{aligned}
(-1)^{j_{1}+j_{2}+j}\left[\begin{array}{ccc}
j_{1} & j_{2} & j \\
m_{1} & m_{2}-m
\end{array}\right]=\left[\begin{array}{ccc}
j_{2} & j_{1} & j \\
m_{2} & m_{1}-m
\end{array}\right] \\
=\left[\begin{array}{ccc}
j_{1} & j & j_{2} \\
m_{1}-m & m_{2}
\end{array}\right]=\left[\begin{array}{ccc}
j & j_{2} & j_{1} \\
-m & m_{2} & m_{1}
\end{array}\right] .
\end{aligned}
$$

To rotate the axis of quantization, we apply the rotation operator $\Re$, to the wave function $\psi_{j}^{m}$ and obtain a new wave function which is a superposition of different eigenfunctions $\psi_{j}^{m^{\prime}}$

$$
\psi_{j}^{m}\left(\theta_{1}^{\prime} \varphi^{\prime}\right)=\sum_{m^{\prime}} \underset{m m^{\prime}}{D^{j}}(\alpha, \beta, \gamma) \psi_{j}^{m}\left(\theta_{1} \varphi\right)
$$

where $\alpha, \beta, \gamma$ are the Eulerian angles of the rotation $R$. The angles $\theta, \varphi$ go over to $\theta^{\prime}, \varphi^{\prime}$ in the new system. The transformation matrix $D$ has the following properties

$$
\begin{gathered}
\sum_{m^{\prime}} D_{m m^{\prime}}^{j}\left(R_{1}\right) D_{m^{\prime} m_{1}}^{j}\left(R_{2}\right)=D_{m m_{1}}^{j}\left(R_{1} R_{2}\right) \\
D_{m_{0}}^{j}(\alpha, \beta, 0)=\sqrt{\frac{4 \pi}{(2 j+1)}} Y_{j}^{m *}(\beta \alpha) \\
D_{m_{s} 0}^{j}(0, \beta, \gamma)=\sqrt{\frac{(j-m) !}{(j+m) !}} P_{j}^{m}(\cos \beta) \\
D_{\mathbf{0}, 0}^{j}(\alpha, \beta, \gamma)=P_{j}(\cos \beta) \\
D_{\mu m}^{j *}=(-1)^{+\mu-m} D_{-\mu-m}^{j} .
\end{gathered}
$$

Another important result is the possibility of their expansion in what is known as the Clebsch-Gordan series

or

$$
D_{\mu m}^{j}=\sum_{\mu_{1}} \sum_{m_{1}} C\left(j_{1} j_{2} j ; \mu_{1} \mu-\mu_{1}\right) C\left(j_{1} j_{2} j ; m_{1} m-m_{1}\right) D_{\mu_{1} m_{1}}^{j_{1}} D_{\mu-\mu_{1}, m-m_{1}}^{j_{2}}
$$

$$
D_{\mu_{1} m_{1}}^{j_{1}} D_{\mu_{2}, m_{2}}^{j_{2}}=\sum_{j, \mu_{1}+\mu_{2}, m_{1}+m_{2}} C\left(j_{1} j_{2} j ; M_{1} M_{2}\right) C\left(j_{1} j_{2} j ; m_{1} m_{2}\right) D_{\mu_{1}+\mu_{2}, m_{1}-m_{2}}^{j} .
$$

Note that in the above we have dropped the third magnetic quantum number in writing the $C$ coefficient since the relation $\mu=\mu_{1}+\mu_{2}$ automatically fixes its value.

The coupling of three angular momenta, Racah coefficients and the Wigner 6-j symbol

When we consider the coupling of three or more angular momenta, more than one scheme is possible. The relations between different schemes lead to the "recoupling coefficients" otherwise known as the "Racah coefficients". 
The addition of three angular momenta $\boldsymbol{J}_{1}, \boldsymbol{J}_{2}, \boldsymbol{J}_{3}$ can be effected by first forming an intermediate state corresponding to $\boldsymbol{J}^{\prime}=\boldsymbol{J}_{1}+\boldsymbol{J}_{2}$ and then combining . $\boldsymbol{J}^{\prime}$ with $\boldsymbol{J}_{\mathbf{3}}$. Alternatively the intermediate state corresponding to $\boldsymbol{J}^{\prime \prime}=\boldsymbol{J}_{\mathbf{2}}+\boldsymbol{J}_{\mathbf{3}}$ can be formed which when combined with that corresponding to $J_{1}$ yields the final state. The two final states so obtained $\psi_{j m}\left(J^{\prime}\right)$ and $\psi_{j m}\left(J^{\prime \prime}\right)$ are related by a unitary transformation

$$
\psi_{j m}\left(J^{\prime}\right)=\sum_{J^{\prime \prime}} R_{j^{\prime \prime} j^{\prime}}^{j} \psi_{j m}\left(J^{\prime \prime}\right)
$$

The Racah coefficients $W$ are defined by

$$
R_{j^{\prime \prime} j^{\prime}}^{j}=\left[\left(2 j^{\prime \prime}+1\right)\left(2 j^{\prime}+1\right)\right]^{1 / 2} W\left(j_{1} j_{2} j j_{3} ; j^{\prime} j^{\prime \prime}\right) .
$$

Therefore the Racah coefficients relate the two coupling schemes. It should be noted that the phases can be so chosen that they arereal. Using $(a b c d ; e f)$ for $\left(j_{1} j_{2} j j_{3} ; j^{\prime} j^{\prime \prime}\right)$ and $\alpha \beta \gamma \delta$ for the four projections of the angular momenta $a, b, c$ and $d$ on the $z$ axis and noting that $\gamma=\alpha+\beta+\delta$ the following relations can be derived:

$$
\begin{gathered}
\frac{\Sigma}{j}[(2 e+1)(2 f+1)]^{1 / 2} W(a b c d ; e f) C(b d f ; \beta \delta) C(a f c ; \alpha, \beta+\delta) \\
=C(a b e ; \alpha \beta) C(e d c ; \alpha+\beta, \delta)
\end{gathered}
$$

$[(2 e-1)(2 f+1)]^{1 / 2} W(a b c d ; f) C(a f c ; \alpha, \beta+\delta)$

$$
=\sum_{\beta} C(a b d ; \alpha \beta) C(e d c ; \alpha+\beta, \delta) C(b d f ; \beta \delta)
$$

$[(2 e+1)(2 f+1)]^{1 / 2} W(a b c d ; e f)$

$$
=\sum_{x} \sum_{\beta} C(a b e ; \alpha \beta) C(e \mathrm{~d} c ; \alpha+\beta, \delta) C(b d f ; \beta \delta) C(a f c ; \alpha \beta+\delta) .
$$

$W(a b c d ; e f)=0$ unless the triangular conditions $\Delta(a b e), \Delta(e d c), \Delta(b d f)$ and $\mathcal{J}(a f c)$ are satisfied. The Wigner 6-j symbol can be defined by

$$
\left[\begin{array}{l}
j_{1} j_{2} j^{\prime} \\
j_{3} j j^{\prime \prime}
\end{array}\right]=(-1)^{j_{1}+j_{2}+j_{3}+j} W\left(j_{1} j_{2} j j_{3} ; j^{\prime} j^{\prime \prime}\right) .
$$

A cyclic permutation leaves the symbol invariant; so does the interchange of lower and upper arguments of any two columns. There are twenty-four such operations which leave the symbol invariant, which are isomorphic with the symmetry group of a regular tetrahedron with sides $j_{1} j_{2} j_{3} j^{\prime} j^{\prime \prime}$ and $j$. We also have the following relations for the permutation of the arguments of the Racah coefficient.

$$
\begin{gathered}
W(a b c d ; e f)=W(b a d c ; e f)=W(c d a b ; e f)=W(a c b d ; f e) \\
W(a b c d ; e f)=(-1)^{e+f-a-d} W(e b c f ; a d)=(-1)^{e+f-b-c} W(a e f d ; b c) \\
\sum_{e}(2 e+1)(2 f+1) W(a b c d ; e f) W(a b c d ; e g)=\delta_{f g} \\
W(a b c d ; e f)=\frac{(-1)^{f-b-d} \delta_{a b} \delta_{c d}}{[(2 b+1)(2 d+1)]^{1 / 2}} .
\end{gathered}
$$




\section{The 9-j symbol}

Wigner has considered the two modes of composing four angular momenta and established the connection between these two coupling schemes as the relation between the $L-S$ and $J-J$ couplings.

$\left\langle a b(c) a^{\prime} b^{\prime}\left(c^{\prime}\right) d \mid a a^{\prime}(e) b b^{\prime}(f) d\right\rangle$

$$
=\left[(2 e+1)(2 f+1)(2 c+1)\left(2 c^{\prime}+1\right)\right]^{1 / 2}\left\{\begin{array}{lll}
a & a^{\prime} & e \\
b & b^{\prime} & f \\
c & c^{\prime} & d
\end{array}\right\} .
$$

This is invariant under even permutation of columns and rows and under transposition while an odd permutation produces a change of sign

$$
(-1)^{a+a^{\prime}+e+b+b^{\prime}+f+c+c^{\prime}+d} \text {. }
$$

Interchange of the rows with the columns of the $3 \times 3$ matrix leaves the $9-j$ symbol unchanged.

Numerical values for some of the $9-j$ symbols have been tabulated by Sharp et al.

$$
\begin{aligned}
& {\left[(2 c+1)\left(2 c^{\prime}+1\right)(2 e+1)(2 f+1)\right]^{1 / 2}\left\{\begin{array}{lll}
a & a^{\prime} & e \\
b & b^{\prime} & f \\
c & c^{\prime} & d
\end{array}\right\}} \\
& =\sum_{\alpha \beta \alpha^{\prime} \beta^{\prime}} C(a b c ; \alpha \beta \gamma) C\left(a^{\prime} b^{\prime} c^{\prime} ; \alpha^{\prime} \beta^{\prime} \gamma^{\prime}\right)(-1)^{a^{\prime}-\alpha^{\prime}} \\
& \gamma \gamma^{\prime} \varepsilon \varphi C\left(a a^{\prime} e ; \alpha-\alpha^{\prime} \varepsilon\right)(-1)^{b^{\prime}-\beta^{\prime}} C\left(b b^{\prime} f ; \beta-\beta^{\prime} \varphi\right)(-1)^{c^{\prime}-\gamma^{\prime}} \\
& C\left(c c^{\prime} d ; \gamma-\gamma^{\prime} \delta\right) C(e f d ; \varepsilon \varphi \delta) \\
& =\left[(2 e+1)\left(2 c^{\prime}+1\right)(2 f+1)(2 c+1)\right]^{1 / 2} \sum_{k}(2 k+1) W\left(a a^{\prime} d f ; c k\right) \\
& W\left(b f c^{\prime} a^{\prime} ; b^{\prime} k\right) W\left(a b d c^{\prime} ; c k\right) \text {. }
\end{aligned}
$$

\section{ALGEB RA OF THE $\gamma$-MATRICES}

The $\gamma$-matrices occuring in the Dirac equation are defined through their irreducibility and by the anticommutation rules

where

$$
\left\{\gamma_{\mu}, \gamma_{\nu}\right\}=2 \delta_{\mu \nu}
$$

$$
\begin{aligned}
\delta_{\mu \nu} & =0 \quad \text { for } \mu \neq v \\
& =+1 \text { for } \mu=v=4 \\
& =-1 \text { for } \mu=v=1,2,3 .
\end{aligned}
$$

Following are the important properties of the $\gamma$-matrices:

1 R. P. Feynuan, Lectures delivered at the California Institute of Technology (1953). 
(1) If $\gamma_{\mu}$ and $\gamma_{\mu}^{\prime}$ are two irreducible sets of matrices satisfying the above rule (1) then they are related to each other by

$$
\gamma_{\mu}^{\prime}=S \gamma_{\mu} S^{-1}
$$

where the matrix $S$ is unique except for an arbitrary multiplieative factor.

(2) By taking linear combinations of these $\gamma$-matrices we ean form sixteen linearly independent matrices which are

$$
\begin{gathered}
I_{1} \quad 1: I_{2}=\gamma_{! n}(\mu=1,2,3,4) ; I_{3}=\gamma_{\mu} \gamma_{v}(\mu \neq \nu) \\
I_{4}=\gamma_{5} \gamma_{\mu} ; I_{5}=\gamma_{5}=\gamma_{1} \gamma_{2} \gamma_{3} \gamma_{4} .
\end{gathered}
$$

(3) The maximum number of linearly independent matrices of order $n$ is $n^{2}$. Hence from (2) we find that in this ease $n \geqq 4$. We can now give an explicit representation of the $\gamma$-matrices of order 4

$$
\gamma_{i}=\left[\begin{array}{rr}
0 & \sigma_{i} \\
-\sigma_{i} & 0
\end{array}\right] \quad i=1,2,3 ; \quad \gamma_{t}=\left[\begin{array}{rr}
1 & 0 \\
0 & -1
\end{array}\right]
$$

where $\sigma_{i}$ are the $2 \times 2$ Pauli matrices. It follows that

also

$$
\gamma_{i}^{2}=-1 ; \gamma_{i}^{2}=1 \text { and } \gamma_{s}^{2}=-1
$$

$$
\left\{\gamma_{5}, \gamma_{\mu}\right\}==0 \text {. }
$$

(4) If $a$ is a four vector we can define the operator a by

$$
\mathrm{a}=a_{t} \gamma_{t}-a_{x} \gamma_{x}-a_{y} \gamma_{y}-a_{z} \gamma_{z} \text {. }
$$

From this it ean be shown that

where

$$
a b+b a=2 a \cdot b
$$

$$
a \cdot b=a_{\mu} b_{! \iota} .
$$

The following results ean be demonstrated:

$$
\begin{gathered}
\left\{\gamma_{5}, \mathrm{a}\right\}_{+}=0 \\
\gamma_{x} \mathrm{a} \gamma_{x}=\mathrm{a}+2 a_{x} \gamma_{x} ; \quad \gamma_{\mu} \gamma_{\mu}=4 ; \quad \gamma_{\mu} \mathrm{a} \gamma_{\mu}=-2 \mathrm{a} \\
\gamma_{\mu} \mathrm{ab} \gamma_{\mu}=4 a \cdot b ; \quad \gamma_{\mu} \mathrm{abc} \gamma_{\mu}=-2 \mathrm{cba} .
\end{gathered}
$$

\section{THE DENSITY OF STATES ${ }^{1}$}

We are interested in computing the number of states $\varrho\left(E ; \boldsymbol{p}_{1} \boldsymbol{p}_{2} \cdots \boldsymbol{p}_{n}\right)$ available to a system of $n$ particles when its total energy lies between $E$ and $E+\mathrm{d} E$ and the particles have momenta $\boldsymbol{p}_{1}, \boldsymbol{p}_{2}, \ldots, \boldsymbol{p}_{n}$. To do this it is convenient to compute $\varrho(E ; \boldsymbol{p})$ and $\varrho\left(E ; \boldsymbol{p}_{1} \boldsymbol{p}_{2}\right)$ the density of states for one and two particle systems respectively. The generalization to the $n$ particle system follows immediately.

1 Alladi Ramakrishax Handbuch der Physik. III, 524, Springer-Verlag (1959). 
The computation is based on the postulate that the number of states available to a single particle is uniformly distributed in the six-dimensional phase space.

\section{One particle system}

The number of states available for a particle of momentum between $\boldsymbol{k}$ and $\boldsymbol{k}+\mathrm{d} \boldsymbol{k}$ is, by the fundamental assumption of quantum mechanies $\frac{\mathrm{d}^{3} k}{h^{3}}$ or $\frac{\mathrm{d}^{3} k}{(2 \pi)^{3}}$ if $\hbar=1$. The number of states available when $|\boldsymbol{k}|$ lies between $p$ and $p+\mathrm{d} p$ is

$$
p^{2} \mathrm{~d} p /(2 \pi)^{3} \text {. }
$$

Hence the density of states per unit energy interval ${ }^{1}$ is

$$
\varrho\left(E_{p}\right)=\left(\frac{p^{2}}{(2 \pi)^{3}}\right) \times\left(\frac{\mathrm{d} p}{\mathrm{~d} E}\right)=\frac{E p}{(2 \pi)^{3}} \text { since } \frac{\mathrm{d} p}{\mathrm{~d} E}=\frac{E}{p} .
$$

Two particle system

If $p_{1}$ and $p_{2}$ are the momenta and $E_{1}$ and $E_{2}$ the energies of the two particles the total momentum and energy are given by

$$
\begin{aligned}
& \boldsymbol{p}=\boldsymbol{p}_{1}+\boldsymbol{p}_{2} \\
& \boldsymbol{E}=E_{1}+E_{2} .
\end{aligned}
$$

The number of states available in the energy interval $\mathrm{d} E$ is just the number of states available to a particle of momentum between $\boldsymbol{p}_{1}$ and $\boldsymbol{p}_{1}+\mathrm{d} \boldsymbol{p}_{1}$ (the other momentum $\boldsymbol{p}_{2}$ being fixed) i.e.

$$
\frac{1}{(2 \pi)^{3}} p_{1}^{2} \mathrm{~d} p_{1}=\frac{1}{(2 \pi)^{3}} p_{1}^{2}\left(\frac{\mathrm{d} p_{1}}{\mathrm{~d} E}\right) \mathrm{d} E
$$

where $\mathrm{d} p_{1}$ has to be computed corresponding to a given $\mathrm{d} E$. Then $\varrho$ is obtained as

\section{Three particle system}

$$
\varrho\left(E ; \boldsymbol{p}_{1} \boldsymbol{p}_{2}\right)=\frac{E_{1} E_{2} p_{1}^{3}}{(2 \pi)^{3}\left[E p_{1}^{2}-E_{1}\left(\boldsymbol{p} \cdot \boldsymbol{p}_{1}\right)\right]} .
$$

In the case of a three particle system we ask for the density of final states per unit interval of total energy $E$ and per unit interval of the energy of the particle when the two other particles have momenta $\boldsymbol{p}_{1}$ and $\boldsymbol{p}_{2}$ respectively. The momentum $\boldsymbol{p}_{3}$ of the third particle is determined by the momenta of the other two particles. The density of states is obtained by multiplying the density of states $\varrho\left(E ; p_{1}\right)$ corresponding to particle 1 by the density of states $\varrho\left(E-E_{1}\right.$; $p-p_{1}$ ) of the two particle system. Thus we have

$$
\varrho\left(E ; \boldsymbol{p}_{1} \boldsymbol{p}_{2} \boldsymbol{p}_{3}\right)=\frac{1}{(2 \pi)^{6}} \frac{E_{1} E_{2} E_{3} p_{2}^{3} p_{1}}{p_{2}^{2}\left(E-E_{1}\right)-E_{2} \boldsymbol{p}_{2} \cdot\left(\boldsymbol{p}-\boldsymbol{p}_{1}\right)} .
$$

1 In lit erature the term "density of states" is used rather loosely, either to denote the number of states in an infinitesimal interval $\mathrm{d} p$ or the number per unit energy range. We however wish to keep this distinction clear. To obtain the number from the density we multiply by the factor $\frac{p}{E} \mathrm{~d} p$. 
When one of the particles has infinite mass (and hence infinite energy) we have, on setting $E_{3}=m_{3}=\infty$

$$
\varrho\left(E ; \boldsymbol{p}_{1} \boldsymbol{p}_{2} \boldsymbol{p}_{3}\right)=\frac{1}{(2 \pi)^{6}} E_{2} p_{2} p_{1}^{2} \mathrm{~d} p_{1} .
$$

The density of states of a $n$ particle system can be easily obtained by considering it to be composed of two systems, one containing a particle of energy and momentum $E_{1}$ and $\boldsymbol{p}_{1}$ and the other of $(n-1)$ particles of total energy and momentum $\mathbf{E}-\mathbf{E}_{1}$ and $\boldsymbol{p}-\boldsymbol{p}_{1}$, and applying the above rule for composition successively.

\section{RELATIVISTIC TRANSFORMATIONS}

We here summarize some of the standard results relating to relativistic transformations. These have been used in the main part of the book in two contexts:

(1) The first occurs in the theory of elementary particles where it is assumed that the laws of physics are independent of the system of reference in which the phenomena are observed. The physical laws are expressed as equations connecting physical quantities and their variations with coordinates. By invariance under transformations is meant the invariance of such equations and relationships and not of the physical quantities themselves. The systems of reference envisaged here are those which move with uniform velocity relative to one another. ${ }^{1}$

(2) The second context in which we use relativistic transformations is in the study of physical quantities in different coordinate systems for ease in computation. Such cases arise in collision processes involving a finite number of particles.

Let the relative spatial coordinates of two events be denoted by $x_{1}, x_{2}, x_{3}$ and the temporal coordinate by $x_{4}\left(x_{4} \equiv t\right)$ in the coordinate system $S$ and let their corresponding values in the system $S^{\prime}$ moving with a uniform velocity $v$ along the $X$-axis of $S$ be $x_{1}^{\prime}, x_{2}^{\prime}, x_{3}^{\prime}$ and $x_{4}^{\prime}$. These quantities are then related by the equations

$$
\begin{aligned}
& x_{1}^{\prime}=\gamma\left(x_{1}-v t\right)=\gamma\left(x_{1}-\beta x_{4}\right) \\
& x_{2}^{\prime}=x_{2} \\
& x_{3}^{\prime}=x_{3} \\
& x_{4}^{\prime}=\gamma\left(t-v x_{1}\right)=\gamma\left(x_{4}-\beta x_{1}\right)
\end{aligned}
$$

with $\beta=v$ and $\gamma=\left(1-\beta^{2}\right)^{-1 / 2}$.

If the relative velocity is in any direction, i.e. has components $v_{1}, v_{2}, v_{3}$, in the $x, y, z$ directions respectively we have merely to write $v_{1}$ instead of $v$ in the transformation formula for $x_{1}$. The other two coordinates have similar formulae. Any quantity whose components transform according to the above law is called a four-vector. Thus $x_{1}, x_{2}, x_{3}$ and $x_{4}$ form the components of a four-vector. The scalar product of two four-vectors $a$ and $b$ is defined as $a \cdot b=a_{4} b_{4}-a_{1} b_{1}-a_{2} b_{2}-a_{3} b_{3}$.

1 W. Pauli, Theory of Relativity, Pergamon Press (1958). 
This is invariant under the above transformation. In particular the invariant $a_{4}^{2}-a_{1}^{2}-a_{2}^{2}-a_{3}^{2}$ is called the square of the "length" of the four-vector $a$.

More generally if we define $L$ as a $4 \times 4$ matrix which transforms $x$ to $x^{\prime}$, i.e. $x^{\prime}=L x$ such that the quadratic form $x_{4}^{2}-x_{1}^{2}-x_{2}^{2}-x_{3}^{2}=x^{T} A x$ is invariant, then the relation $L^{T} A L=A$ holds. Here $A$ is the matrix

and $x$ is the vector

$$
\left[\begin{array}{rrrr}
-1 & 0 & 0 & 0 \\
0 & -1 & 0 & 0 \\
0 & 0 & -1 & 0 \\
0 & 0 & 0 & 1
\end{array}\right]
$$

$$
\left[\begin{array}{l}
x_{1} \\
x_{2} \\
x_{3} \\
x_{4}
\end{array}\right]
$$

If we had taken the fourth dimension as $i$, then $L$ corresponds to a rotation in four-dimensional space. The transformation given in (1) is a particular case of $L$ with

$$
L=\left[\begin{array}{rrrr}
\gamma & 0 & 0 & -\beta \gamma \\
0 & 1 & 0 & 0 \\
0 & 0 & 1 & 0 \\
-\beta \gamma & 0 & 0 & \gamma
\end{array}\right]
$$

We now give below some examples of four-vectors:

(1) $x, y, z, t$ the space and time coordinates of an event.

(2) $-\frac{\partial}{\partial x},-\frac{\partial}{\partial y},-\frac{\partial}{\partial z}, \frac{\partial}{\partial t}$

(3) $\frac{u_{x}}{\sqrt{\left(1-u^{2}\right)}}, \frac{u_{y}}{V\left(1-u^{2}\right)}, \frac{u_{z}}{V\left(1-u^{2}\right)}, \frac{1}{\sqrt{\left(1-u^{2}\right)}}$

where $u_{x}, u_{y}, u_{z}$ are the components of the velocity of the particle.

(4) $\frac{F_{x}}{\sqrt{ }\left(1-u^{2}\right)}, \frac{F_{y}}{\sqrt{ }\left(1-u^{2}\right)}, \frac{F_{z}}{\sqrt{ }\left(1-u^{2}\right)}, \frac{\mathrm{d} E}{\mathrm{~d} t} \frac{1}{\sqrt{\left(1-u^{2}\right)}}$

where $\boldsymbol{F}$ is the force on the particle and $E$ its energy.

(5) $p_{x}, p_{y}, p_{z}, E$ the momentum and energy of a particle.

(6) $k_{x}, k_{y}, k_{z}, v$ where $k$ is the propagation vector of a wave and $v$ is its frequency.

(7) $j_{x}, j_{y}, j_{z}, \varrho$ the current and charge densities.

(8) $A_{x}, A_{y}, A_{z}, \varphi$ the vector and scalar potentials of an electromagnetic field.

For the sake of completeness we give also the transformations of some closely related physical quantities which however are not four-vectors. 
(1) 'The components of the veloeity $"$ of a particle transform as

$$
u_{r}^{\prime} \quad \begin{gathered}
u_{x}-v \\
\left(1-u_{x} v\right)
\end{gathered} . \quad u_{y}^{\prime}=\frac{u_{v}}{\gamma\left(1-u_{x} v\right)}, \quad u_{z}^{\prime}=\frac{u_{z}}{\gamma\left(1-u_{x} v\right)} .
$$

$(2)$ We shall give the transformation law for acceleration $\dot{\boldsymbol{u}}$ under the limited condition $\|$, i.e. we find the acceleration in the momentary rest frame of the particle. We then have

$$
\begin{aligned}
& \dot{u}_{x}=\dot{u}_{x}^{\prime}\left(1-u^{2}\right)^{3 / s} \\
& \dot{u}_{y}=\dot{u}_{y}^{\prime}\left(1-u^{2}\right) \\
& \dot{u}_{z}=\dot{u}_{z}^{\prime}\left(1-u^{2}\right) .
\end{aligned}
$$

(3) If $\theta$ is the angle made by the velocity $\boldsymbol{u}$ of a particle moving in the $x_{1}-x_{2}$ plane in $S$ with the positive direction of the $x_{1}$-axis, the corresponding angle $\theta^{\prime}$ in $S^{\prime}$ is given by

$$
\begin{aligned}
\tan \theta^{\prime} & =\frac{u \sin \theta}{\gamma(u \cos \theta-v)} \\
\tan \theta & =\frac{u^{\prime} \sin \theta^{\prime}}{\gamma\left(u^{\prime} \cos \theta^{\prime}+v\right)} .
\end{aligned}
$$

In the non-relativistic case, $\gamma=1$ and $u \sin \theta=u^{\prime} \sin \theta^{\prime}$, i.e. the velocity perpendicular to the relative velocity of the frames is unaffected. Even in the relativistic case. it is not affected very much if $\gamma \sim 1$.

There is one interesting result which can be obtained as a corollary to the above transformations and which has applications in collision problems. For a given $u^{\prime}$ in $S^{\prime}$ and $\theta$ in $S$ there are two solutions $\theta_{1}^{\prime}$ and $\theta_{2}^{\prime}$ possible for $\theta^{\prime}$ as given by the equation

$$
\cos \theta^{\prime}=\frac{-\mu \alpha^{2} \pm \sqrt{1-\left(\mu^{2}-1\right) \alpha^{2}}}{\alpha^{2}+1}
$$

where $\alpha=\gamma \tan \theta$ and $\mu=\frac{v}{u^{\prime}}$. This equation is obtained by solving equation (5) for $\cos \theta^{\prime}$. This implies from (7) that there are two values $u_{1}$ and $u_{2}$ for the velocity in the $S$ frame corresponding to these two values of $\theta^{\prime}$ in the $S^{\prime \prime}$ frame. This can be easily demonstrated geometrically in the non-relativistic case. The velocity $u$ in $S$ is just the third side of the triangle which has to be determined given the two sides $v$ and $u^{\prime}$ and the angle $\theta$ between $v$ and $u$. Two triangles are possible if $u^{\prime}<v$; otherwise, only one exists. These cases are shown in figure (69). The arguments are similar in the relativistic case $\mu$ being double valued if $\mu>1$ and single valued if $\mu<1$.

Finally we notice that if $F^{\prime}\left(\theta^{\prime}, E^{\prime}\right) \mathrm{d} \Omega^{\prime} \mathrm{d} E^{\prime}$ denotes the number of particles emitted in the $S^{\prime}$ system between the solid angles $\Omega^{\prime}$ and $\Omega^{\prime}+\mathrm{d} \Omega^{\prime}$ and the energies $E^{\prime}$ and $E^{\prime}+\mathrm{d} E^{\prime}$, the distribution of the particles in the $S$ system is given by

where

$$
F(\theta, E) \mathrm{d}(\cos \theta) \mathrm{d} E=F^{\prime}\left[\theta^{\prime}(\theta, E), E^{\prime}(\theta, E)\right] J \mathrm{~d}(\cos \theta) \mathrm{d} E
$$

$$
J=\frac{\left.\partial j \cos \theta^{\prime}, E^{\prime}\right)}{\partial(\cos \theta, E)} .
$$


If we wish to find the angular distribution $F(\theta) \mathrm{d}(\cos \theta)$ in the $S$ system irrespective of the energy of the new particle, we have

where now

$$
F(\theta) \mathrm{d}(\cos \theta)=2 \pi \int F\left[\theta^{\prime}(\theta, E), E^{\prime}(\theta, E)\right] \cdot J \mathrm{~d}(\cos \theta)
$$

$$
J=\left.\frac{\partial\left(\cos \theta^{\prime}\right)}{\partial(\cos \theta)}\right|_{E^{\prime}} .
$$

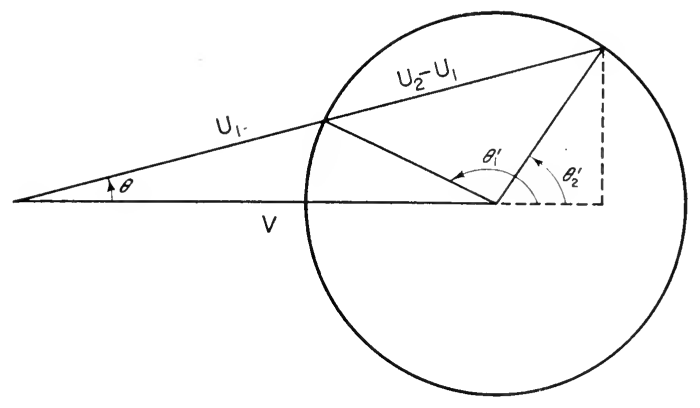

FIG. 69. Geometrical representation of ambiguity in $u$ given $u^{\prime}\left(u^{\prime}<u\right)$.

Application to collision problems

In collision problems it is often found convenient to describe the phenomena in the centre of mass system $S^{\prime}$ of the particles and finally transform back to the laboratory system $S$. In order to carry out these transformations, we have merely to compute the velocity of the centre of mass relative to the laboratory system. The computation of this velocity is done by a consideration of suitable invariant products of four-vectors in the two systems.

Two particle collisions

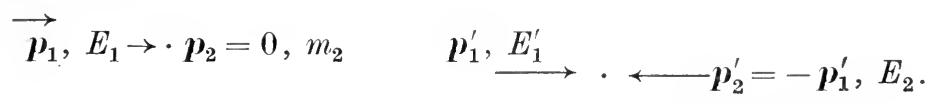

Laboratory system Centre of mass system

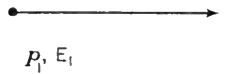

$P_{1}, \mathrm{E}_{1}$

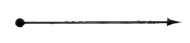

$p_{1}^{\prime}, E_{1}^{\prime}$

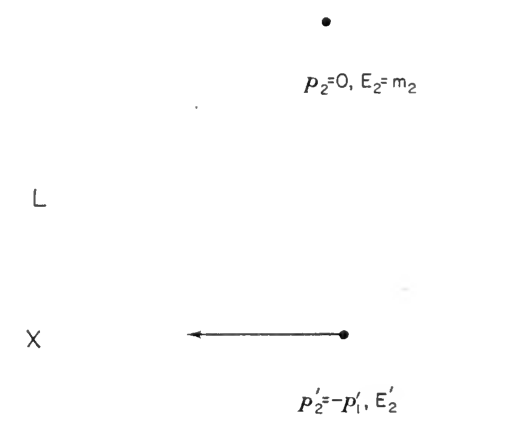

C.M.

FIG. 70. Energies and moments in the laboratory and centre of mass systems. 
Let us consider the collision of two particles of rest masses $m_{1}$ and $m_{2}$ the subscript 2 denoting the particle which is at rest in the $S$ system. Let $\boldsymbol{p}_{1}, E_{1}$ and $\boldsymbol{p}_{2}(=0), E_{2}\left(=m_{2}\right)$ be their corresponding momenta and energies and let $\boldsymbol{p}\left(=\boldsymbol{p}_{1}\right)$ and $E\left(=E_{1}+m_{2}\right)$ denote the total momentum and energy of the system. The corresponding quantities in the $S^{\prime}$ system are denoted by primes. The velocity of $S^{\prime}$ relative to $S$ is denoted by $\beta$. The total energy-momentum four-vector in the two systems are then given by

$$
\begin{aligned}
& S: p_{1} 000 E_{1}+m_{2} \\
& S^{\prime}: 0000
\end{aligned}
$$

Noting that the coordinate system is so chosen that $\boldsymbol{p}_{1}$ is along the $x$-axis, it follows that

or

$$
0 \| \gamma\left[p_{1}-\beta\left(E_{1}+m_{2}\right)\right]
$$

$$
\beta=-\frac{p_{1}}{E_{1}+m_{2}} \text {. }
$$

Knowing $\beta$ all the other quantities can be computed in the $S^{\prime}$ system using the appropriate transformation formulae.

\section{Decay of a single particle into three particles}

Let us consider a particle which decays at rest into three particles of equal mass all moving in the same plane. Taking the laboratory system as the one in which the original particle is at rest, the configuration of decay can be specified in terms of the momentum $\boldsymbol{p}_{1}$ of the decay particle 1 and the momentum $\boldsymbol{q}$ of one of the other two decay particles 2 and 3 in their centre of mass system. In the laboratory the individual momenta of the three particles are $\boldsymbol{p}_{1}, \boldsymbol{p}_{\mathbf{2}}$ and $\boldsymbol{p}_{\mathbf{3}}$ respectively. The total momentum is zero and the total energy is $M=w_{1}+w_{2}+w_{3}$ where $w_{1}, w_{2}$ and $w_{3}$ denote the energies of the particles. Now let $\boldsymbol{p}^{\prime}$ be the momentum of particle 1 in the $2-3$ rest system and $w_{1}^{\prime}$ the corresponding energy. The velocity $v$ of the 2-3 system with respect to the laboratory system is given by

$$
v=\frac{p}{M-w_{1}},
$$

taking the plane of decay to be the $x y$-plane and the $x$-axis in the direction of $\boldsymbol{p}_{1}$. The energy-momentum four-vectors of the individual particles and the whole system in the laboratory and the centre of mass frames of reference can be written as follows:

\begin{tabular}{l|ccccccccccc}
\hline & \multicolumn{3}{|c}{ Laboratory System } & \multicolumn{4}{c|}{$\begin{array}{c}2-3 \text { centre } \\
\text { of mass system }\end{array}$} & $\begin{array}{c}\text { Four- } \\
\text { Vector }\end{array}$ \\
\hline Particle 1 & $p_{1}$ & 0 & 0 & $w_{1}$ & $p_{1}^{\prime}$ & 0 & 0,0, & $w_{1}^{\prime}$ & $(A)$ \\
Particle 2 & $p_{2} \cos \alpha$ & $p_{1} \sin \alpha$ & 0 & $w_{2}$ & $q \cos \theta$, & $q \sin \theta, 0$, & $w^{\prime}$ & $(B)$ \\
Particle 3 & $p_{3} \cos \beta$ & $-p_{2} \sin \beta$ & 0 & $w_{3}$ & $-q \cos \theta$, & $-q \sin \theta$, & 0, & $w^{\prime}$ & $(C)$ \\
The total system & 0 & 0 & 0 & $M$ & $p_{1}^{\prime}$, & 0 &, & 0, & $\left(2 w^{\prime}+w_{1}^{\prime}\right)$ & $(D)$ \\
\hline
\end{tabular}


To determine $q$, we make use of the fact that the scalar product of $A$ and $D$ is invariant. Therefore

But

$$
M w_{1}=\left(2 w^{\prime}+w_{1}^{\prime}\right) w_{1}^{\prime}-p_{1}^{\prime 2}=2 w^{\prime} w_{1}^{\prime}+m^{2} .
$$

Hence

$$
\begin{aligned}
w_{1}^{\prime} & =\left(w_{1}+\frac{p_{1}}{M-w_{1}} p_{1}\right) /\left[1-\left(\frac{p_{1}}{M-w_{1}}\right)^{2}\right]^{1 / 2} \\
& =\left(M w_{1}-m^{2}\right) /\left(M^{2}+m^{2}-2 M w_{1}\right)^{1 / 2} \\
w^{\prime} & =\frac{M w_{1}-m^{2}}{2 w_{1}^{\prime}}=\frac{\left(M^{2}+m^{2}-2 M w_{1}\right)^{1 / 2}}{2} \\
w^{\prime 2} & =q^{2}+m^{2}=\left(M^{2}+m^{2}-2 M w_{1}\right) / 4 .
\end{aligned}
$$

$$
q=\frac{\left(M^{2}-3 m^{2}-2 M w_{1}\right)^{1 / 2}}{2} .
$$

To describe the process fully it is then enough to specify $\omega_{1}=m+t(t$ being the kinetic energy of particle 1 ) and the angle $\theta$ between the vectors $p$ and $q . \theta$ can be calculated from the observed momenta in the following way. Taking the scalar product of $B$ and $D$

$$
M w_{2}=\left(2 w^{\prime}+w_{1}^{\prime}\right) w^{\prime}-p^{\prime} q \cos \theta .
$$

But

$$
p^{\prime}=\left(p_{1}+\frac{p_{1}}{M-w_{1}} w_{1}\right) /\left[1-\frac{p_{1}^{2}}{\left(M-w_{1}\right)^{2}}\right]^{1 / 2}=\frac{M p_{1}}{\left(M^{2}+m^{2}-2 M w_{1}\right)^{1 / 2}} .
$$

Substituting the values of $p^{\prime}$ and $w_{1}^{\prime}$

$$
\begin{aligned}
M w_{2} & =2 w^{\prime 2}+\frac{w^{\prime}\left(M w_{1}-m^{2}\right)-M p q \cos \theta}{\left(M^{2}+m^{2}-2 M w_{1}\right)^{1 / 2}} \\
& =2 w^{\prime 2}+\frac{w^{\prime}\left(M w_{1}-m^{2}\right)-M p q \cos \theta}{2 w^{\prime}} .
\end{aligned}
$$

Similarly from $C$ and $D$, we get

$$
\begin{aligned}
M w_{3} & =2 w^{\prime 2}+\frac{w^{\prime}\left(M w_{1}-m^{2}\right)+M p q \cos \theta}{2 w^{\prime}} \\
M\left(w_{3}-w_{2}\right) & =\frac{2 M p q \cos \theta}{2 w^{\prime}}, \\
\text { i.e. } \quad \cos \theta & =\frac{w_{3}-w_{2}}{p} \frac{w^{\prime}}{q}
\end{aligned}
$$

EPCR 33 
$\cos \theta$ can be expressed also in terms of the measured momenta $\boldsymbol{p}_{1}, \boldsymbol{p}_{\mathbf{2}}, \boldsymbol{p}_{3}$ in the laboratory system as

$$
\begin{aligned}
\cos \theta & =\frac{u_{3}^{2}-u_{2}^{2}}{u_{2}+u_{3}} \frac{\left(M^{2}+m^{2}-2 M w_{1}\right)^{1 / 2}}{2 p_{1} q} \\
& =\frac{p_{3}^{2}-p_{2}^{2}}{2 p q}\left[1-\left(\frac{p_{1}}{M-w_{1}}\right)^{2}\right]^{1 / 2} .
\end{aligned}
$$

\section{I N I RIAN' FUNCTIONS}

We shall now summarize the derivation of the various invariant functions associated with free particle propagators which are expressed in terms of the wave functions. It will be convenient to define these functions first as a sum over the three dimensional momentum $\boldsymbol{p}$ and later express them as integrals. Let $\psi$ be the wave function with one or more components and let us associate with it the propagator $G(2,1)$ given by

$$
G(2,1)=\sum_{p} \psi_{p}(2) \psi_{p}^{\dagger}(1)
$$

where (1) and (2) denote space-time points. If we assume the relativistic relation $E^{2}=p^{2}+m^{2}$ between the energy $E$ and momentum $\boldsymbol{p}, E$ has two roots given by $\pm E_{p}$ where $E_{p}=+\gamma p^{2}+m^{2}$. Then $G(2,1)$ can be written as

$$
\begin{aligned}
G(2,1) & =\sum_{\boldsymbol{p}} \psi_{\boldsymbol{p}}^{(+)}(2) \psi_{\boldsymbol{p}}^{(+) \dagger}(1)+\sum_{\boldsymbol{p}} \psi_{\boldsymbol{p}}^{(-)}(2) \psi_{\boldsymbol{p}}^{(-) \dagger}(1) \\
& =G_{+}(2,1)+G_{-}(2,1),
\end{aligned}
$$

where $G_{+}$and $G_{-}$are the sums corresponding to the positive and negative values of the energy.

We shall define two associated propagators $G^{\prime}$ and $G_{0}$, for reasons which will be apparent presently, as

$$
G^{\prime}(2,1)=G_{+}(2,1)-G_{-}(2,1)
$$

and

$$
\begin{aligned}
& G_{0}(2,1) \equiv G(2,1) \quad \text { for } \quad t_{2}>t_{1} \\
& \equiv 0 \quad \text { for } \quad t_{2}<t_{1} \text {. }
\end{aligned}
$$

From these we ean define

$$
G_{F}(2,1)=G_{0}(2,1)-G_{-}(2,1)=\frac{G^{\prime}(2,1)+\varepsilon\left(t_{2}-t_{1}\right) G(2,1)}{2},
$$

where $\varepsilon\left(t_{2}-t_{1}\right)= \pm 1$ depending on $t_{2}-t_{1} \gtrless 0$.

We now express these functions as integrals

$$
\begin{aligned}
G_{+}(2,1) & =\int \frac{1}{2 E_{p}} \frac{1}{(2 \pi)^{3}} \psi_{\boldsymbol{p}}^{(+)}(2) \psi_{\boldsymbol{p}}^{(+) \dagger}(1) \mathrm{d}^{3} p \\
& =\int \frac{1}{2 E_{p}} \frac{1}{(2 \pi)^{3}} \delta\left(p_{4}-E_{p}\right) \psi_{p}(2) \psi_{p}^{\dagger}(1) \mathrm{d}^{4} p
\end{aligned}
$$

1 G. Kallex, Handbuch der Physik, Vol. 5, Part I, Springer-Verlag (1958). 
where $\psi_{\boldsymbol{p}}^{(+)}$denotes the wave function with positive values of the energy and $\psi_{p}$ the wave function corresponding to the four momentum $p$. Using the contour integral representation for the $\delta$-function, we write

$$
\begin{aligned}
G_{+}(2,1) & =\int_{p} \int_{C_{+}\left(E_{p}\right)} \frac{1}{2 E_{p}} \cdot \frac{1}{2 \pi i} \cdot \frac{1}{\left(2 \pi^{3}\right)} \frac{\psi_{p}(2) \psi_{p}^{\dagger}(1)}{p_{4}-E_{p}} \mathrm{~d}^{4} p \\
& =\frac{-i}{(2 \pi)^{4}} \int_{p} \mathrm{~d}^{3} p \int_{C_{+}\left(E_{F}\right)} \frac{1}{2 E_{p}} \frac{\psi_{p}(2) \psi_{p}^{\dagger}(1)}{p_{4}-E_{p}} \cdot \mathrm{d} p_{4},
\end{aligned}
$$

where $p_{4}$ is treated as a complex variable, and the contour $C_{+}\left(E_{p}\right)$ is an anticlockwise (+) contour round $E_{p}$ (see Fig. 71). Similarly $G_{-}$is given by

$$
\begin{aligned}
G_{-}(2,1) & =\int_{p} \frac{1}{2 E_{p}} \frac{1}{(2 \pi)^{3}} \psi_{p}^{(-)}(2) \psi_{p}^{(-) \dagger}(1) \mathrm{d}^{3} p \\
& =\int_{p} \frac{1}{2 E_{p}} \cdot \frac{1}{(2 \pi)^{3}} \delta\left(p_{4}+E_{p}\right\rangle \psi_{p}(2) \psi_{p}^{\dagger}(1) \mathrm{d}^{3} p \\
& =\int_{C_{+}\left(-E_{p}\right)} \frac{1}{2 E_{p}} \frac{1}{2 \pi i} \frac{1}{(2 \pi)^{3}} \frac{\psi_{p}(2) \psi_{p}^{\dagger}(1)}{p_{4}+E_{p}} \mathrm{~d}^{4} p \\
& =\frac{-i}{(2 \pi)^{4}} \int_{C_{+}\left(-E_{p}\right)} \frac{1}{2 E_{p}} \frac{\psi_{p}(2) \psi_{p}^{\dagger}(1)}{p_{4}+E_{p}} \mathrm{~d}^{4} p,
\end{aligned}
$$

where $C_{+}\left(-E_{p}\right)$ is the anticlockwise contour round $-E_{p}$ (see Fig. 71).

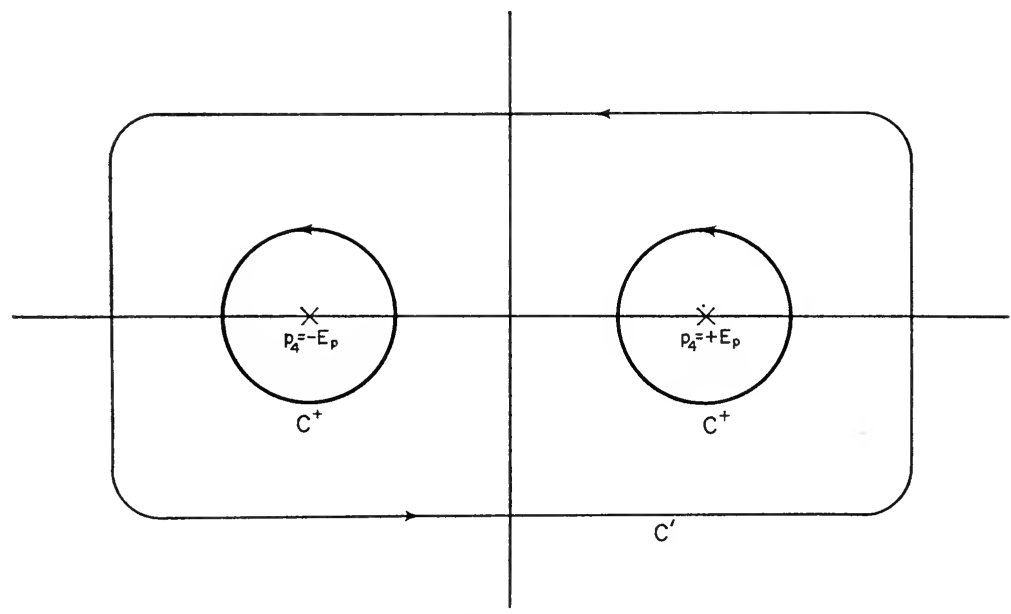

Fig. 71. The contour $C^{\prime}$. 
Noting that

$$
\frac{1}{2 E_{p}}\left\{\frac{1}{p_{4}-E_{p}}-\frac{1}{p_{4}+E_{p}}\right\}=\frac{1}{p_{4}^{2}-E_{p}^{2}}
$$

we can write the functions $G, G^{\prime}$ and $G_{F}$ as integrals of the same function $\frac{\psi_{p}(2) \psi_{p}^{\dagger}(1)}{p_{4}^{2}-E_{p}^{2}}$ over suitably defined contours. Thus

$$
G=G_{+}+G_{-}=\frac{-i}{(2 \pi)^{4}} \int_{C} \frac{\psi_{p}(2) \psi_{p}^{\dagger}(1)}{p_{4}^{2}-E_{p}^{2}} \mathrm{~d}^{4} p
$$

where

$$
\begin{aligned}
C & =C_{+}\left(E_{p}\right)-C_{+}\left(-E_{p}\right) \\
& =C_{+}\left(E_{p}\right)+C_{-}\left(-E_{p}\right),
\end{aligned}
$$

where $C_{+}$and $C_{-}$denote anticlockwise and clockwise contours respectively. Thus $C$ is represented by:

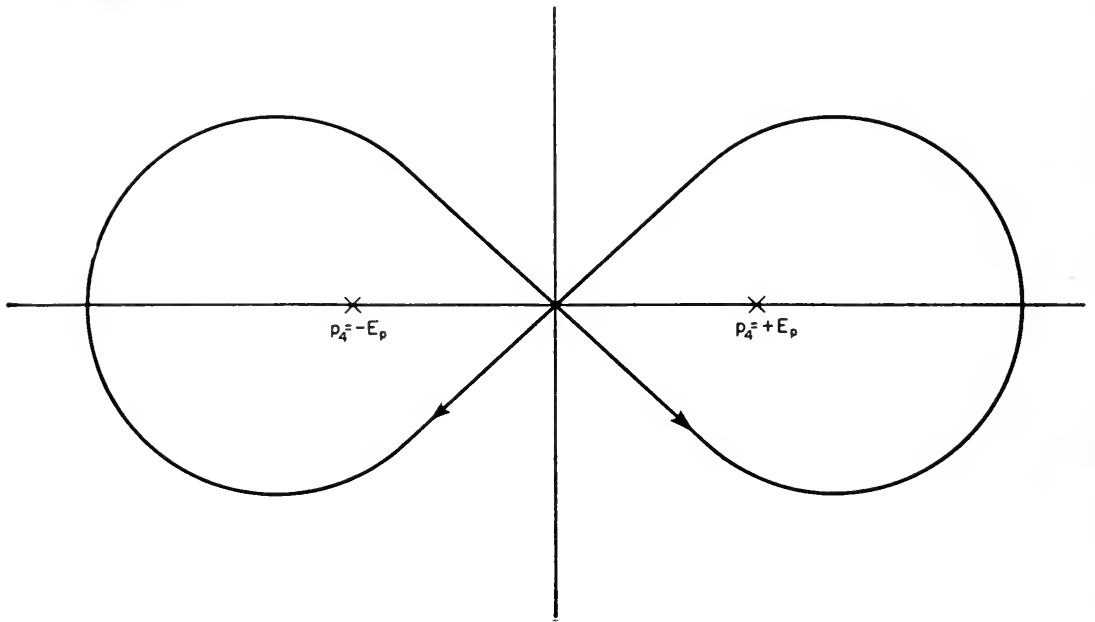

Fig. 72. The contour $C$.

Similarly

$$
G^{\prime}=G_{+}-G_{-}=\frac{-i}{(2 \pi)^{4}} \int_{C^{\prime}} \frac{\psi_{p}(2) \psi_{p}^{\dagger}(1)}{p_{4}^{2}-E_{p}^{2}} \mathrm{~d}^{4} p
$$

where

$$
C^{\prime}=C_{+}\left(E_{p}\right)+C_{+}\left(-E_{p}\right) \text { (see Fig. 71) }
$$

and

$$
\begin{array}{rlll}
G_{F} & =G_{+} & \text {for } & t_{2}>t_{1} \\
& =-G_{-} & \text {for } & t_{2}<t_{1}
\end{array}
$$

that is, the function $\frac{\psi_{p}(2) \psi_{p}^{\dagger}(1)}{p_{4}^{2}-E_{p}^{2}}$ has to be integrated over the contour $C_{+}\left(E_{p}\right)$ 
if $t_{2}>t_{1}$ and over $C_{-}\left(-E_{p}\right)$ if $t_{2}<t_{1}$. This is achieved by taking the contour $C_{F}$ as shown in Fig. 73.

This contour can be closed downwards when $t_{2}>t_{1}$ since $p_{4}$ will then have a large negative imaginary part and hence $\mathrm{e}^{-i p_{4}\left(t_{2}-t_{1}\right)}$ is of the form $\mathrm{e}^{-\alpha\left(t_{2}-t_{1}\right)}$ and

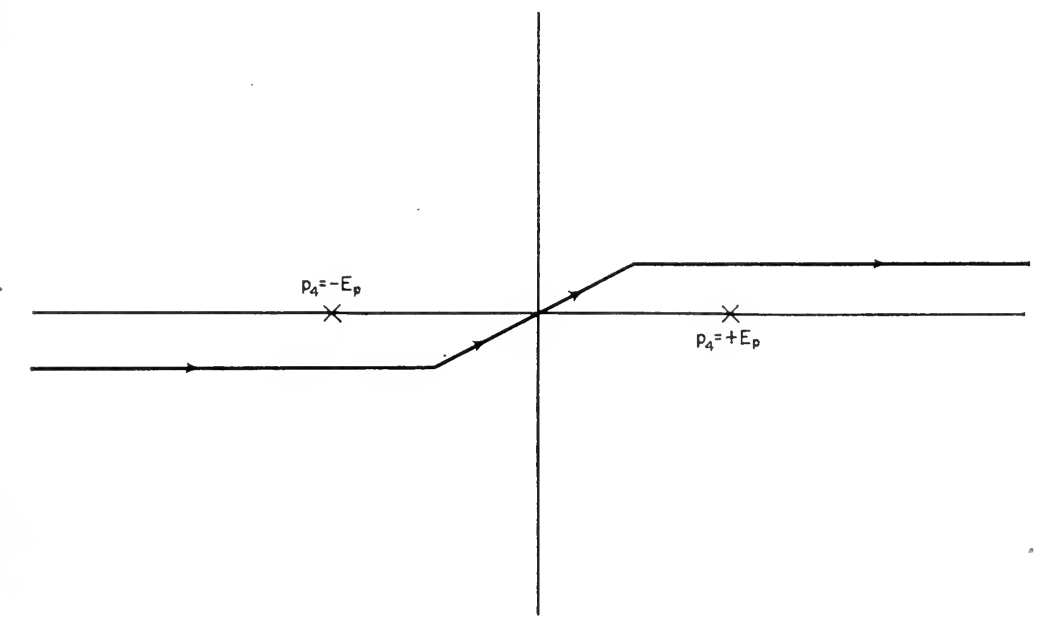

Fig. 73. The contour $C_{F}$.

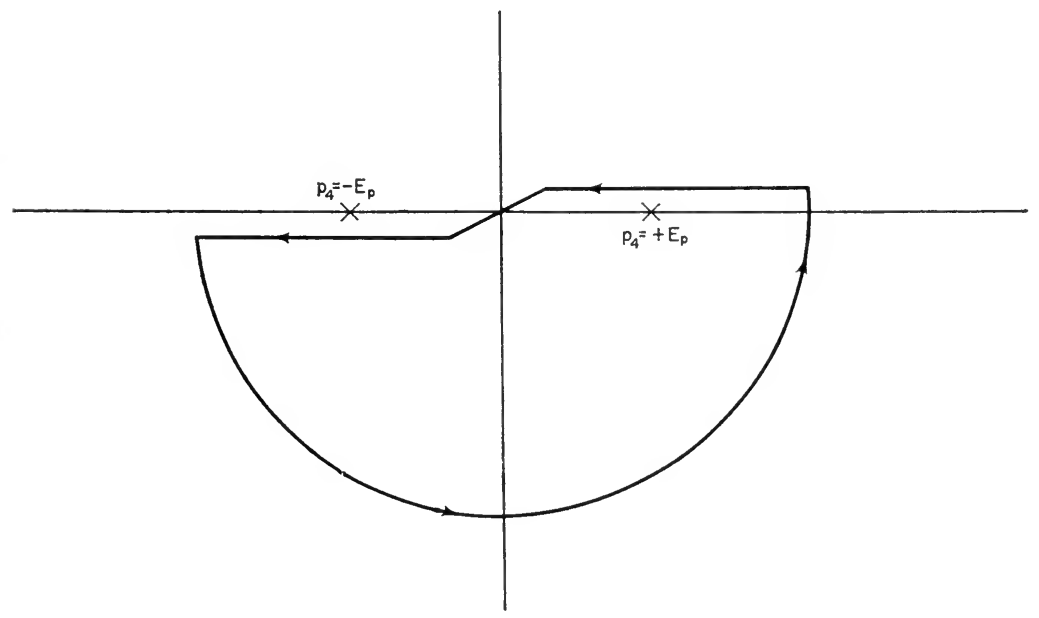

Fig. 74. The contour $-C_{F} \cdot\left(x_{0}+0\right)$.

as $\alpha$ tends to $\infty$, the integrand tends to zero and the closure can be made without any contribution to the integral. The contour being anti clockwise, this gives $G_{+}(2,1)$. In a similar manner, for $t_{2}<t_{1}$, the closure can be made in the upper half plane (see Fig. 75). The contour being anticlockwise, we now obtain $-G_{-}(2,1)$. 
The same result can be obtained by taking a contour along the real axis from $+\infty$ to $-\infty$ and assuming that the singularities occur at $E_{p}-i \varepsilon$ and $-\left(E_{p}-i \varepsilon\right)$

and making $\varepsilon \rightarrow 0$.
If we integrate the function $\frac{\psi_{p}(2) \psi_{p}^{\dagger}(1)}{p_{4}^{2}-E_{p}^{2}}$ over the contour which is a line darallel to the real axis and above the singularities $\pm E_{p}$ we obtain by the above arguments

$$
\begin{aligned}
& G_{R}=G_{+}+G_{-} \text {if } t_{2}>t_{1} \\
& =0 \quad \text { if } t_{2}<t_{1} \text {. }
\end{aligned}
$$

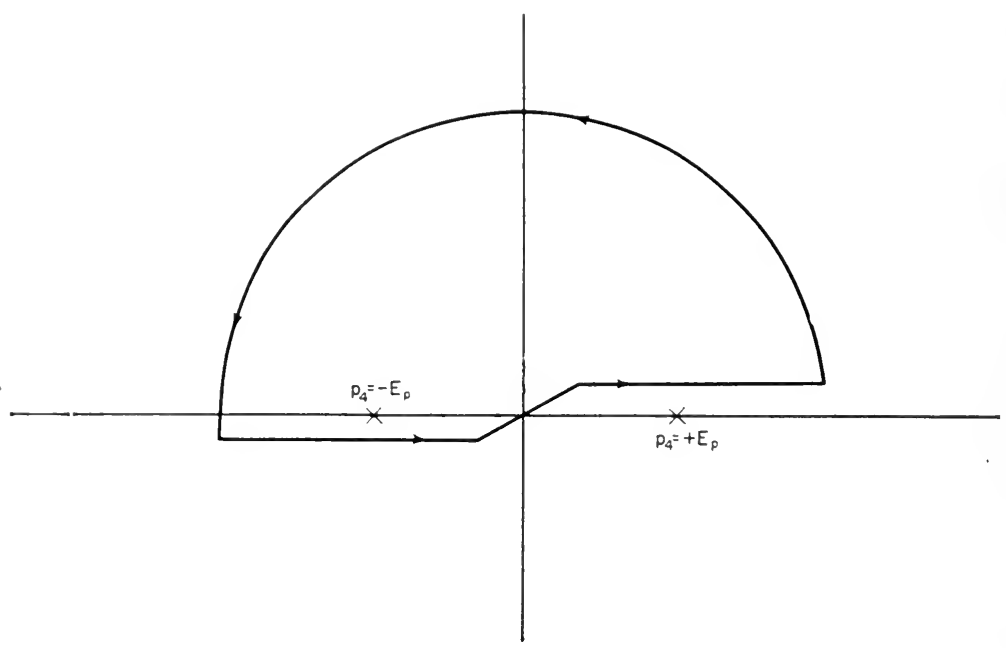

FIG. 75. The contour $C_{F} \cdot\left(x_{0}-0\right)$.

On the other hand, integration over a contour which is a line parallel to the real axis and below the singularities $\pm E_{p}$ yields

$$
\begin{aligned}
& G_{A}=0 \quad \text { if } t_{2}>t_{1} \\
& =-\left(G_{+}+G_{-}\right) \text {if } t_{2}<t_{1} \text {. }
\end{aligned}
$$

Writing $\mathrm{e}^{-i k \cdot x}$ for the wave function $\psi$ we identify the $G$ functions with the known invariant functions as

$$
\begin{aligned}
& G_{+}=i \Delta_{+} ; \quad G_{-}=i \Delta_{-} \\
& G_{F}=\frac{-\Delta_{F}}{2} \\
& G_{R}=-i \Delta_{R} \\
& G_{A}=i \Delta_{A} .
\end{aligned}
$$

On writing the spinors for $\psi$, we identify $G_{F}$ as the Feynman propagator $\boldsymbol{K}_{F}$. It is also customary to define a function $S_{F}$ as

such that

$$
\begin{gathered}
S_{F}=-2 K_{F} \\
S_{F}=(\mathrm{p}+m) \Delta_{F} .
\end{gathered}
$$




\section{QUANTIZATION OF THE ELECTROMAGNETIC FIELD}

When we try to quantize the electromagnetic field, i.e. to obtain an expression for $A_{\mu}(x)$ corresponding to field operators we immediately run into the difficulty that the Lorentz condition $\frac{\partial A_{\mu}(x)}{\partial x_{\mu}}=0$, which is so essential in the classical theory for establishing that the equation for the potentials $\square A_{\mu}(x)=0$ corresponds to the Maxwell equations, cannot be taken over into the quantized theory as an operator identity. For, this would contradict the commutation relations since

$$
\left[\frac{\partial A_{\mu}(x)}{\partial x_{\mu}}, A_{\nu}\left(x^{\prime}\right)\right]=-i \frac{\partial D}{\partial x_{\nu}}\left(x-x^{\prime}\right) \neq 0 .
$$

If on the other hand, we ignore the Lorentz condition, we are led to the result that the energy is not positive definite, which is untenable. The two difficulties are connected with each other and when the Lorentz condition is properly incorporated in the quantized theory, the states of negative energy will not appear as we shall see presently.

Fermi $^{2}$ tried to get over the difficulty in imposing the Lorentz condition as an operator equation by observing that to get the classical electromagnetic theory as a limiting case, it is not necessary that all the Maxwell equations should correspond to operator identities in the quantized theory, but that it is sufficient if the expectation values of the field operators satisfy these equations. He therefore took the equation

$$
\frac{\partial A_{v}}{\partial x_{v}}|\psi\rangle=0
$$

as the "auxiliary" condition in the quantized theory. Using the expansion of $A_{\mu}(x)$ in terms of the creation and annihilation operators of the photon corresponding to the four degrees of freedom, this can be written as

$$
\begin{aligned}
{\left[a^{(3)}(\boldsymbol{k})+i a^{(4)}(\boldsymbol{k})\right]|\psi\rangle } & =0 \\
{\left[a^{*(3)}(\boldsymbol{k})+i a^{*(4)}(\boldsymbol{k})\right]|\psi\rangle } & =0,
\end{aligned}
$$

where $a^{(3)}$ and $a^{*(3)}$ are the annihilation and creation operators respectively of the longitudinal photon and $a^{(4)}$ and $a^{*(4)}$ are the creation and annihilation operators respectively of the scalar photon. (The change in the role of the operators in the case of the scalar photon is connected with the fact that the fourth component of $A_{\mu}(x)$ is an imaginary quantity while the other components are real.) ${ }^{3}$ We see that the auxiliary condition affects only the longitudinal and scalar photons and leaves the transversal degrees of freedom uninfluenced. A possible state vector

1 In this section the fourth component of four vectors is $i$ times the corresponding quantities in the previous chapters. Thus $A_{4}$ here corresponds to $i A_{4}$ of the earlier chapters.

2 E. Fermi, Rev. Mod. Phys. 4, 87 (1932).

3 See, e.g. G. Kallen, "Quanten Electrodynamik" in Handbuch der Physik, Vol. V Part I, p. 187, Springer-Verlag (1958). 
which satisfies the auxiliary condition can be written as a product

$$
|\psi\rangle=\left|\psi_{T}\right\rangle \prod_{\boldsymbol{k}}|\Phi(\boldsymbol{k})\rangle
$$

where $\left|\psi_{T}\right\rangle$ contains only the transversal photons and

$$
\left|\Phi_{k}\right\rangle=\sum_{n^{(3)} n_{n}^{(0)}} \alpha_{n^{(3)} n^{(3)}}\left|n^{(3)}, n^{(4)}\right\rangle=\sum_{n^{(3)} n^{(0)}} \frac{\left[a^{*(3)}\right]^{n^{(3)}}\left[a^{(4)}\right]^{n^{(0)}}}{V n^{(3)} ! n^{(4) !}}|0\rangle,
$$

where $n^{(3)}$ and $n^{(4)}$ are the number of longitudinal and scalar photons respectively, present in the state. Substitution in (3) gives a pair of equations for the consistency of which we must have

$$
\alpha_{n^{(3)}, n^{(3)}}=C \delta_{n^{(3)}, n^{(4)}}(-i)^{n^{(4)}},
$$

where the constant $C$ is to be determined by the normalization condition

$$
\left\langle\Phi_{n} \mid \Phi_{n}\right\rangle=1 .
$$

Fermi's method thus gives a precise prescription for the mixing of the longitudinal and scalar photons which can appear in a physically realizable state. These degrees of freedom are thus eliminated and only the transversal photons are allowed to exist, their energy being equal to the total energy of the electromagnetic field. (The energy of the longitudinal photons cancels exactly with that of the scalar photons.) Thus the total energy of a state is always positive definite.

Though the above method of inclusion of the Lorentz condition looks elegant at first sight there are mathematical difficulties associated with it. Thus for instance, it looks as if the auxiliary condition (2) is in contradiction with the commutation rules, since

$$
\left\langle\psi\left|\left[\frac{\partial A_{\mu}(x)}{\partial x_{\mu}}, A_{v}\left(x^{\prime}\right)\right]\right| \psi\right\rangle=-i \frac{\partial D}{\partial x_{\nu}} D\left(x-x^{\prime}\right) \neq 0,
$$

instead of being zero as we should expect from (2). But this is due to the fact that the vector $\left|\Phi_{k}\right\rangle$ is not normalizable with a finite $C$. Thus (2) would lead to an expression of the form $0 \cdot \infty$ which must be first defined by a limiting procedure. It is found that this can be done only at the cost of choosing infinite gauge functions. ${ }^{1}$

The difficulties connected with the unnormalizable state vectors arise from the fact that in (3), both creation and annihilation operators are present which leads to the solution (4) containing state vectors with an infinite number of particles which cannot be always explicitly normalized. Further the conditions (3) would mean not only that longitudinal and scalar photons are not present but also that such photons cannot be emitted which latter condition is too stringent. Gupta and Bleuler ${ }^{2}$ proposed that the auxiliary condition ( $3 \mathrm{a}$ ) should be modified

\footnotetext{
1 F. Coester, Phys. Rev. 83, 798 (1951); also G. KaLLen, loc. cit. p. 196 and the following.

2 S. N. Gupta, Proc. Phys. Soc. Lond. A 63, 681 (1950); 64, 850 (1951); K. Bleuler, Helv. Phys. Acta 23, 567 (1950).
} 
such that only annihilation operators are present in it. But this cannot be done by merely dropping equation $(3 \mathrm{~b})$, since $(3 \mathrm{a})$ itself contains both creation and annihilation operators. Thus we must choose the following representation for $a^{(4)}$ and $a^{*(4)}$ :

$$
\left\langle n+1\left|a^{*(4)}\right| n\right\rangle=\left\langle n\left|a^{(4)}\right| n+1\right\rangle=\bigvee n+1
$$

thus bringing the roles of the creation and annihilation operators for the scalar photons also in line with those for the other components of the electromagnetic field. But this would violate the reality condition of the $A_{\mu}(x)$, the fourth component also being real.

To obviate this, Gupta introduced the indefinite metric operator $\eta$ originally used by Dirac ${ }^{1}$ in a different connection which has the following properties: The norm of a state vector is defined as

$$
\text { Norm }|\psi\rangle=\langle\psi|\eta| \psi\rangle \text {. }
$$

To ensure the reality of the norm, $\eta$ must be hermitian. Similarly the expectation value of an operator $F$ is defined by

$$
\bar{F}=\langle\psi|\eta F| \psi\rangle \text {. }
$$

With these new definitions the norm of a state vector can also be 0 or negative and a hermitian operator need not necessarily have real eigenvalues which is what we require. For the component of the electromagnetic field we thus impose the conditions

or

$$
\left.\begin{array}{rl}
\left\langle\psi\left|\eta A_{k}(x)\right| \psi\right\rangle & =\left\langle\psi\left|A_{k}^{*}(x) \eta^{*}\right| \psi\right\rangle \\
& =\left\langle\psi\left|A_{k}(x) \eta\right| \psi\right\rangle
\end{array}\right\}
$$

$$
\left[A_{k}(x), \eta\right]=0 ; \quad k=1,2,3
$$

and

$$
\left\langle\psi\left|\eta A_{4}(x)\right| \psi\right\rangle=-\left\langle\psi\left|A_{4}(x) \eta\right| \psi\right\rangle
$$

or

$$
\left\{A_{4}(x), \eta\right\}=0 \text {. }
$$

The auxiliary condition in the Gupta-Bleuler formalism will be

$$
\left[a^{(3)}(\boldsymbol{k})+i a^{(4)}(\boldsymbol{k})\right]|\psi\rangle=0
$$

both the operators appearing being annihilation operators now. In $x$-space, the condition will be

$$
\frac{\partial A_{\mu}^{(+)}(x)}{\partial x_{\mu}}|\psi\rangle=0
$$

where $A_{\mu}^{(+)}(x)$ stands for the negative frequency part of $A_{\mu}(x)$. If we write, as before, an arbitrary state vector as

$$
|\psi\rangle=\left|\psi_{T}\right\rangle \prod_{k}\left|\Phi_{k}\right\rangle
$$

1 P. A. M. Dirac, Proc. Roy. Soc. A 180, 1 (1942). 
then equation (16) contains only a single condition for the quantities $\left|\Phi_{k}\right\rangle$. As the fundamental system of solutions, we can choose

$$
\begin{aligned}
& \left|\Phi^{(0)}\right\rangle=|0,0\rangle \\
& \left.\Phi^{(1)}\right\rangle=|1,0\rangle+i|0,1\rangle \\
& \left.\Phi^{(n)}\right\rangle=\sum_{r=0}^{\prime \prime}(i)^{r} /\left(\begin{array}{l}
n \\
r
\end{array}\right)|n-r, r\rangle
\end{aligned}
$$

with the orthogonality condition

$$
\left\langle\Phi^{(n)}|\eta| \Phi^{n^{\prime}}\right\rangle=0 \quad \text { for } \quad n \neq n^{\prime} .
$$

Also the normalization condition gives

$$
\left\langle\Phi_{n}|\eta| \Phi^{n}\right\rangle=\sum_{r=0}^{\infty}(-1)^{r}\left(\begin{array}{c}
n \\
r
\end{array}\right) \delta_{n 0},
$$

which means that none of the norms is negative and only the state $\left|\Phi^{(0)}\right\rangle$, with no longitudinal or scalar photon, has a norm different from zero. Thus in the Gupta-Bleuler formalism the state vector $\left|\Phi^{(0)}\right\rangle$ and the state vector $\left|\Phi^{(0)}\right\rangle$ $+\sum_{n \neq 0} C^{(n)}(\boldsymbol{k})\left|\Phi^{(n)}(\boldsymbol{k})\right\rangle$ (where $C^{(n)}(\boldsymbol{k})$ are arbitrary coefficients) are equivalent. This ensures that we will always have to do with normalizable state vectors. Further if we evaluate the expectation value of the Hamiltonian in the state $|\psi\rangle$, it is found to be equal to the energy of the transverse photons alone and independent of the mixing of the longitudinal and scalar photons.

We thus see that the Gupta-Bleuler method which uses all the four components of the electromagnetic field on the same footing and thus always exhibits the relativistic invariance explicitly provides a satisfactory way of quantizing the electromagnetic field.

\section{A RE-EXAMINATION OF THE EQUIVALENCE OF THE FEYNMANANDS-MATRIX FORMALISMS}

It is well-known that the results of quantum mechanics are meaningful only under a probabilistic interpretation of the squares of the modulus of complex quantities like the wave functions or matrix elements. The principle of superposition however applies only to the complex amplitudes occurring in the theory and not to the positive definite quantities, the squares of their moduli, as in classical probability theory. It has been a long held belief of the author that as long as this principle is safeguarded, the methods of stochastic theory should find direct application in the study of quantum mechanical systems evolving with time. ${ }^{1}$ This belief was encouraged by the possibility that the Feynman formalism can be viewed from a new angle by suitably imbedding the methods of inverse probability

1 A semi-expository paper entitled "Stochastic methods in quantum mechanics" is to be published shortly. 
into the study of a time-dependent electrodynamic system. The faith in this approach was further strengthened by the "deduction of the well-known expressions for field operators through perturbation expansions of older quantum mechanics". It was felt therefore that the equivalence of the Feynman and field theoretic methods must be proved in a direct and transparent manner without recourse to complex algebraic methods and notation hitherto used. Such a proof is now presented ${ }^{1}$ and in order to realise the simplicity of the new approach in comparison with the older methods, it is found necessary to re-examine in detail the subtle variations in the meaning of the terms such as "intermediate states", "virtual particles", "kernel functions", "propagators" and "perturbations". We also need some simple lemmas from matrix and probability theories. Thus we divide this appendix into four parts :

(i) Mathematical preliminaries dealing with lemmas in matrix and inverse probability theories,

(ii) A new viewpoint regarding the kernel function formalism of Feynman,

(iii) Interaction term in field theory,

(iv) Derivation of the Feynman expansion from the $S$-matrix formalism.

\section{Mathematical preliminaries}

Let $\boldsymbol{\pi}(t)$ be a column vector of $n$ components $\pi(i ; t)$ with $i=1,2, \ldots, n$ varying with $t$ according to the equation

$$
\frac{\partial \boldsymbol{\pi}}{\partial t}^{(t)}=[A(t)] \boldsymbol{\pi}(t),
$$

where $[A(t)]$ is a matrix function of $t$. By a solution of the equation we mean the determination of $\boldsymbol{\pi}\left(t_{2}\right)$ given $\boldsymbol{\pi}\left(t_{1}\right)$ when $t_{2}-t_{1}$ is finite. From the infinitesimal transformation

$$
\boldsymbol{\pi}(t+\Delta)=\{1+[A(t)] \Delta\} \boldsymbol{\pi}(t)+0\left(\Delta^{2}\right)
$$

$\Delta$ being positive, the solution $\boldsymbol{\pi}\left(t_{2}\right)$ for $t_{2}>t_{1}$ is obtained on iteration, as

$$
\boldsymbol{\pi}\left(t_{2}\right)=U_{A}\left(t_{2}, t_{1}\right) \boldsymbol{\pi}\left(t_{1}\right)
$$

where $U_{A}\left(t_{2}, t_{1}\right)$ has the integral expansion

$$
\begin{gathered}
1+\int_{t_{1}}^{t_{2}} A\left(\tau_{1}\right) \mathrm{d} \tau_{1}+\int_{t_{1}}^{t_{2}} \int_{t_{1}}^{\tau_{2}} A\left(\tau_{2}\right) A\left(\tau_{1}\right) \mathrm{d} \tau_{2} \mathrm{~d} \tau_{1}+\cdots \\
\cdots+\int_{t_{1}}^{t_{2}} \int_{t_{1}}^{\tau_{n}} \cdots \int_{t_{1}}^{\tau_{2}} A\left(\tau_{n}\right) \ldots A\left(\tau_{1}\right) \mathrm{d} \tau_{n} \ldots \mathrm{d} \tau_{1}
\end{gathered}
$$

and the time variables in the integrand of the $n$-th order are such that

$$
t_{2}<\tau_{n}<\tau_{n-1} \cdots<\tau_{1}<t_{1} .
$$

1 An inchoate form of this proof was suggested earlier by the author in association with N. R. Ranganathan and R. Vasudevan. But at that time a unique prescription for ordering the operators in the interaction term was not available and the validity of applying stochastic methods was not clear. 
However (2) is also valid when $\Delta$ is negative so that $\pi\left(t_{2}\right)$ when $t_{2}<t_{1}$ can still be obtained in terms of $\pi\left(t_{1}\right)$, i.e. we trace baek the evolution of $\pi(t)$ from $t_{1}$ to $t_{2}{ }^{1}$ In this case, we have

$$
\begin{array}{r}
U_{A}\left(t_{2}, t_{1}\right)=1+(-1) \int_{t_{2}}^{t_{1}} A\left(\tau_{1}\right) \mathrm{d} \tau_{1}+(-1)^{2} \int_{t_{2}}^{t_{1}} \int_{\tau_{2}}^{t_{2}} A\left(\tau_{2}\right) A\left(\tau_{1}\right) \mathrm{d} \tau_{2} \mathrm{~d} \tau_{1}+\cdots \\
\cdots+(-1)^{n} \int_{t_{2}}^{t_{1}} \int_{\tau_{n}}^{t_{1}} \ldots \int_{\tau_{2}}^{t_{1}} A\left(\tau_{n}\right) A\left(\tau_{n-1}\right) \ldots A\left(\tau_{1}\right) \mathrm{d} \tau_{n} \ldots \mathrm{d} \tau_{1}
\end{array}
$$

where

$$
t_{2}<\tau_{n}<\tau_{n-1} \cdots<\tau_{1}<t_{1} \text {. }
$$

It is to be noted that the integration in this expansion is from $t_{2}$ or $\tau_{i}$ to $t_{1}$. If we wish to retain the same form for $U_{A}\left(t_{2}, t_{1}\right)$ in both cases we can drop the faetor $(-1)^{n}$ in the above expansion and integrate from $t_{1}$ to $t_{2}$ and order the variables $\tau_{n}, \tau_{n-1}, \ldots, \tau_{1}$ in the direction $t_{1}$ to $t_{2}$ which implies that

and

$$
t_{1}<\tau_{1}<\tau_{2} \cdots<\tau_{n}<t_{2} \text { if } t_{2}<t_{1}
$$

$$
t_{2}>\tau_{n}>\tau_{n-1} \cdots>\tau_{1}>t_{1} \text { if } t_{2}>t_{1} .
$$

However if we wish to have the upper limit greater than the lower limit we have to use the form (5). The factor $(-1)$ may be ascribed to $A(\tau)$, i.e. we replace every $A(\tau)$ in (4) by $-A(\tau)$ and alter the limit of integration to obtain (5).

We now consider the equation

$$
\frac{\partial \boldsymbol{x}}{\partial t}^{(t)}=[A+B(t)] \boldsymbol{x}(t) .
$$

The solution for $t_{2}>t_{1}$ is

where

$$
\boldsymbol{\pi}\left(t_{2}\right)=U\left(t_{2}, t_{1}\right) \boldsymbol{x}\left(t_{1}\right)
$$

$$
\begin{aligned}
& U\left(t_{2}, t_{1}\right)=U_{A}\left(t_{2}, t_{1}\right)+\int_{t_{1}}^{t_{2}} U_{A}\left(t_{2}, \tau_{1}\right) B\left(\tau_{1}\right) U_{A}\left(\tau_{1}, t_{1}\right) \mathrm{d} \tau_{1}+\cdots \\
& \cdots+\iint \cdots \int U_{A}\left(t_{2}, \tau_{n}\right) B\left(\tau_{n}\right) U_{A}\left(\tau_{n}, \tau_{n-1}\right) \ldots U_{A}\left(\tau_{2},\left\{\tau_{1}\right) B\left(\tau_{1}\right) U_{A}\left(\tau_{1}, t_{1}\right) \mathrm{d} \tau_{n} \ldots \mathrm{d} \tau_{1} .\right.
\end{aligned}
$$

For $t_{2}<t_{1}$ we merely replace $B(\tau)$ by $-B(\tau)$ and integrate from $t_{2}$ to $t_{1}$. There is however no need to replace $U_{A}$ by $-U_{A}$ since for $t_{2}>t_{1}$ the expansion of $U_{A}\left(\tau_{k}, \tau_{k-1}\right)$ with $\tau_{k}<\tau_{k-1}$ itself in the integrand implies the change of $A$ to $-A$. In the above expansion we have treated $B$ as a perturbation on $A$ and $U_{A}$ is the transformation funetion in the absence of the perturbation $B$. In quantum

1 This is the basis for dealing with questions relating to inverse probability in stochastic process where $\boldsymbol{\pi}(t)$ is a probability vector and $[A(t)]$ a stochastic matrix: see for example, Alladi Ramakrishnan, "Probability and Stochastic Processes", Handbuch der Physik, Vol. III, Springer-Verlag (1959). 
mechanics $B$ corresponds to $H_{\text {int }}$ and $A$ to $H_{0}$. If $A$ is time-independent and the eigenvectors of $A$ are $u_{1}, u_{2}, \ldots, u_{n}$ with eigenvalues $\lambda_{1}, \lambda_{2}, \ldots, \lambda_{n}$ then $U_{A}\left(t_{2}, t_{1}\right)=\mathrm{e}^{A\left(t_{1}-t_{2}\right)}$ can be recognized to be

$$
\sum_{m} u_{m} \bar{u}_{m} \mathrm{e}^{\lambda_{m}\left(t_{2}-t_{1}\right)}
$$

\section{A new viewpoint regarding the kernel function formalism of Feynman}

We shall now apply the results of the previous section to the quantum electrodynamics involving an electron. In chapter II we arrived at a perturbation expansion for the matrix element $b_{f i}$ in the case of the non-relativistic Schrödinger equation as

$$
\begin{gathered}
b_{f i}\left(t_{1}, t_{0}\right)=\delta_{f i}+\int_{t_{0}}^{t} \mathrm{~d} t_{1} \int_{-\infty}^{\infty} \mathrm{d}^{3} x_{1} \bar{\psi}_{f}(1) H^{\prime}(1) \psi_{i}(1)+\cdots \\
+\int_{t_{0}}^{t} \mathrm{~d} t_{n} \int_{t_{0}}^{t_{n}} \mathrm{~d} t_{n-1} \ldots \int_{t_{0}}^{t_{2}} \mathrm{~d} t_{1} \int_{-\infty}^{+\infty} \mathrm{d}^{3} x_{n} \ldots \int_{-\infty}^{+\infty} \mathrm{d}^{3} x_{1} \\
\sum_{\left\langle m_{k}\right\rangle}\left[\bar{\psi}_{f}(n) H^{\prime}(n) \psi_{m_{n-1}}(n) \bar{\psi}_{m_{n-1}}(n-1) H^{\prime}(n-1) \ldots \bar{\psi}_{m_{1}}(1) H^{\prime}(1) \psi_{i}(1)\right],
\end{gathered}
$$

where $H^{\prime}(k)$ is the perturbation at $k, f$ and $i$ refer to the final and initial states and $m_{k}$ refers to the intermediate states, the sum $\sum_{\left\langle m_{k}\right\rangle}$ running over all $m_{k}$. While the integration over each space variable extends from $-\infty$ to $+\infty$, the time integration is, of course, ordered. The term

$$
\sum_{m_{k}} \psi_{m_{k}}(k+1) \bar{\psi}_{m_{k}}(k)
$$

occurring in the integrand is identified to be the kernel $K(k+1, k)$. In extending the above result to the Dirac equation, it was shown that a modified kernel was necessary. We shall now view this modification from a new angle. We postulate that in the scattering of a Dirac particle if a transition occurs from a positive energy state $\psi_{m_{k-1}}^{(k)}$ at $t_{k}$ to a positive energy state $\psi_{m_{k}}$ at $t_{k}{ }^{\circ}+\Delta$, we assume that this state is propagated to $t_{k+1}$ with $t_{k+1}>t_{k}$. If on the other hand $\psi_{m_{k}}(k)$ is a negative energy state, we assume that it is the result of a transition due to a perturbation acting at vertex $(k+1)$ with $t_{k+1}<t_{k}$ in the time interval between $t_{k+1}-\Delta$ and $t_{k}$. We therefore trace back the state from $t_{k}$ to $t_{k+1}$ and then to $t_{k+1}-\Delta$, i.e. to a state before the perturbation. ${ }^{1}$ If this state is still a state of negative energy, we continue to trace it back and if it has positive energy we study its evolution forward in time. When we trace back to $t_{k+1}-\Delta$ we replace

1 No interesting consequence follows if we consider it to be the result of a perturbation at $t_{k}$ since that has been considered in the transition from $\psi_{m_{k-1}}$ to $\psi_{m_{k}}$. What we are doing is immediately justifiable if we interpret the procedure in the Dirac hole theory. A transition to a negative energy state implies the filling of the hole which must be in existence at that time. We then ask when was this hole created? 
the perturbation $H^{\prime}(k+1)$ by $-H^{\prime}(k+1)$. Thus we use the kernel defined as

and

$$
K(k+1, k)=\sum_{E>0} \psi(k+1) \bar{\psi}(k) \text { for } t_{k+1}>t_{k}
$$

$$
=\sum_{k<0} \psi(k+1) \bar{\psi}(k) \text { for } \quad t_{k+1}<t_{k}
$$

and attach the positive or negative sign to the perturbation at $t_{k+1}$ according as $E$ is positive or negative. On shifting the negative sign to the negative energy part. of the kernel we obtain the Feynman kernel.

The above considerations would be very formal except for the fact that it leads to a unique preseription for writing the interaction term in the language of quantum field theory.

\section{Interaction term in quantum field theory}

It has long been realised that since the interaction is written as a product of field operators characterized by the same space-time point, there is an ambiguity in "time ordering" of such operators, which has to be resolved before applying algebraic methods based on Wick's theorem. In quantum electrodynamics we have

where

$$
H_{\mathrm{int}}=\bar{\psi} \gamma_{\mu} \psi A_{\mu}
$$

$$
\psi=\sum_{p}\left[b_{p} u(p) \mathrm{e}^{i p \cdot x}+d_{p}^{\dagger} v(p) \mathrm{e}^{-i p \cdot x}\right]
$$

where $u(p), v(p)$ are the electron and positron spinors respectively and $v(p, E)=u(-p,-E)$ when $E$ is positive. For convenience in notation $\psi$ can be written as

$$
\psi(k)=\sum_{p}\left[\hat{b}_{p}(k)+\hat{d}_{p}^{\dagger}(k)\right],
$$

where $\hat{b}_{p}(k)$ denotes that the usual spinor and the exponential terms corresponding to the space-time vertex $(k)$ are attached to the operator $b_{p}$. Considering only fermion operators and ignoring the photon operator $A_{\mu}$ for the present, the bilinear term is

$$
\begin{aligned}
\bar{\psi} \psi & =\sum_{p}\left[\hat{b}_{p}^{\dagger}+\hat{d}_{p}\right] \sum_{p^{\prime}}\left[\hat{b}_{p^{\prime}}+\hat{d}_{p^{\prime}}^{\dagger}\right] \\
& =\sum_{p p^{\prime}}\left[\hat{b}_{p}^{\dagger} \hat{b}_{p^{\prime}}+\hat{b}_{p}^{\dagger} \hat{d}_{p^{\prime}}^{\dagger}+\hat{d}_{p} \hat{d}_{p^{\prime}}^{\dagger}+\hat{d}_{p} \hat{b}_{p^{\prime}}\right] .
\end{aligned}
$$

The operators correspond to the same space-time point and since we know that at any vertex one of the four fundamental processes can occur, the operator should occur in pairs but not necessarily in the order prescribed by $\bar{\psi} \psi$. In fact we should provide a unique prescription for the choice of the correct order and this is done if we apply the arguments of the previous section.

The process of pair annihilation at $t$ is interpreted as the transition of a positive energy electron at $t$ to a negative energy state at $t+\Delta$, the perturbation 
acting in the interval $\Delta$ and hence the electron destruction operator should be placed to the right of the positron destruction operator $d_{p}$ in the interaction term. In the case of pair creation in the interval between $t-\Delta$ and $t$, we trace the negative energy state of the electron at $t$ back to a positive energy state at $t-\Delta$ so that in this case $d_{p}^{\dagger}$ is placed to the left of $b_{p^{\prime}}^{\dagger}$. For electron and positron scattering the creation operators are naturally placed to the left of the annihilation operators. On the basis of the above arguments, we postulate that the interaction term at a space-time point $k$ should read (ignoring boson operators and $\boldsymbol{\gamma}$-matrices)

$$
\begin{aligned}
H_{\text {int }}(k)= & \sum_{\substack{p_{i} \\
i=2,3,3}} \sum_{p_{i}^{\prime}}\left[\hat{b}_{p_{1}^{\prime}}^{\dagger}(k) \hat{b}_{p_{1}}(k)+\hat{d}_{p_{2}^{\prime}}^{\dagger}(k) \hat{d}_{p_{2}}(k)\right. \\
& \left.+\hat{d}_{p_{3}^{\prime}}(k) \hat{b}_{p_{3}}(k)+\hat{d}_{p_{4}^{\prime}}^{\dagger}(k) \hat{b}_{p_{4}}^{\dagger}(k)\right] \\
= & \sum_{\langle p\rangle} \sum_{\alpha}[k]
\end{aligned}
$$

where the bracket $[k]$ contains one of the four types of terms $\hat{b}^{\dagger} b, \hat{d}^{\dagger} \hat{d}, \hat{d}^{\dagger} \hat{b}^{\dagger}$ $+d^{\dagger} b^{\dagger}$ and the sum over $\alpha$ indicates the sum over all the four such terms. The sum over $\langle p\rangle$ means a sum over the momenta $p_{i}, p_{i}^{\prime}$. This interaction term differs from (15) in that the positron creation operator $d^{\dagger}$ stands to the left of $d$ or $b^{\dagger}$.

\section{Derivation of the Feynman expansion from the $\boldsymbol{S}$-matrix formalism}

On the basis of the above interaction term, it is possible to establish the equivalence between Feynman and field theoretic perturbation formalisms in a rigorous and straightforward manner. The integrand of the $n$-th order term in the $S$-matrix expansion (ignoring the photon operators for the present) can be written as

$$
H^{(n)}=\sum_{\langle p\rangle} \sum_{\alpha}[n][n-1] \ldots[1]
$$

where the time variables are ordered,

$$
\text { i.e. } t_{n}>t_{n-1} \cdots>t_{2}>t_{1} \text {. }
$$

Considering only electron scattering let us take the matrix element of $H^{(n)}$ between initial and final one particle states, say electrons of momenta $\boldsymbol{p}_{1}$ and $\boldsymbol{p}_{2}$ respectively

$$
{ }_{0}\left\langle b_{p_{2}}\left|H^{(n)}\right| b_{p_{1}}^{\dagger}\right\rangle_{0},
$$

where no integration over space and time variables is as yet performed. We shall now show how this reduces to the integrand of the Feynman expansion in an $n$-th order process. The extension to any other electrodynamic process follows without any change in principle. We have ignored for the moment the photon operator $A_{\mu}$ the inclusion of which will be discussed presently.

We are interested only in the terms in $H^{(n)}$ which give a non-vanishing contribution to the matrix element. We shall call the product

$$
[n][n-1] \ldots[1]
$$


a typical term ${ }^{1}$ of $H^{(n)}$. We now adopt the following procedure in rearranging the terms. Since the initial electron must disappear, some bracket, say [i] should contain $\hat{b}_{p_{1}}$ and we move this bracket to the left of $b_{p_{1}}^{+}$. This does not involve a change in sign since we move the bracket as a whole and not a single operator. This bracket [i] will in addition contain a $\hat{b}_{p^{\prime}}^{\dagger}$ or $\hat{d}_{p^{\prime}}$ to its left. If there is a $\hat{b}_{p^{\prime}}^{+}$ then another bracket say [j] with $t_{j}>t_{i}$ should again contain a $\hat{b}_{p}$, and we move $[j]$ to the left of $[i]$. The arrangement would look like

where

$$
[n] \ldots[j+1][j-1] \ldots[i+1][i-1] \ldots[j][i] b_{p_{1}^{\prime}}^{\dagger}>_{0}
$$

or

$$
\begin{aligned}
{\left.[j][i] b_{p_{1}}^{\dagger}\right\rangle_{0} } & \left.=\left[\hat{b}_{p_{2}^{\prime}}^{\dagger}(j) \hat{b}_{p_{1}^{\prime}}(j)\right]\left[\hat{b}_{p_{1}^{\prime}}^{\dagger}(i) \hat{b}_{p_{1}}(i)\right] b_{p_{1}}^{\dagger}\right\rangle_{0} \\
& \left.=\left[\hat{d}_{p_{2}^{\prime}}(j) \hat{b}_{p_{1}^{\prime}}(j)\right]\left[\hat{b}_{p_{1}^{\prime}}^{\dagger}(i) \hat{b}_{p_{1}}(i)\right] b_{p_{1}}^{\dagger}\right\rangle_{0} .
\end{aligned}
$$

In either case we have the term

$$
\begin{aligned}
& \sum_{p_{1}^{\prime}} \hat{b}_{p_{1}^{\prime}}(j) \hat{b}_{p_{1}^{\prime}}^{\dagger}(j) \quad \text { with } \quad t_{j}>t_{i} \\
= & \sum_{p_{1}^{\prime}} u\left(p_{1}^{\prime}\right) \bar{u}\left(p_{1}^{\prime}\right) \mathrm{e}^{-i p_{1}^{\prime}\left(x_{j}-x_{i}\right)}\left(b_{p_{1}^{\prime}} b_{p_{1}^{\prime}}^{\dagger}\right) .
\end{aligned}
$$

Since $b_{p_{1}} b_{p_{1}}^{\dagger}$ is equal to unity we recognize the above to be the Feynman kernel for $t_{j}>t_{i}$.

If on the other hand [i] contains the destruction operator of a positron $\hat{d}_{p_{1}^{\prime}}(i)$ to the left of $\hat{b}_{p_{1}}(i)$ there should be another bracket [k] with $t_{k}<t_{i}$ which must contain $d_{p_{1}^{\prime}}^{\dagger}(k)$ since the positron should have existed before it could be annihilated. We now shift $[k]$ to the left of $[i]$ so that the arrangement now reads

where

$$
\left.[n] \ldots[i+1][i-1] \ldots[k+1][k-1] \ldots[k][i] b_{p_{1}}^{\dagger}\right\rangle_{0}
$$

or

$$
\begin{aligned}
{[k][i] } & =\left[\hat{d}_{p_{1}^{\prime}}^{\dagger}(k) \hat{d}_{p_{2}^{\prime}}(k)\right]\left[d_{p_{1}^{\prime}}(i) \hat{b}_{p_{1}}(i)\right] \\
& =-\left[\hat{d}_{p_{2}^{\prime}}(k) \hat{d}_{p_{1}^{\prime}}^{\dagger}(k)\right]\left[\hat{d}_{p_{1}^{\prime}}(i) \hat{b}_{p_{1}}(i)\right]
\end{aligned}
$$

$$
\left[\hat{d}_{p_{1}^{\prime}}^{\dagger}(k) \hat{b}_{p_{2}^{\prime}}^{\dagger}(k)\right]\left[\hat{d}_{p_{1}^{\prime}}(i) \hat{b}_{p_{1}}(i)\right]=-\left[\hat{b}_{p_{2}^{\prime}}^{\dagger}(k) \hat{d}_{p_{1}^{\prime}}^{\dagger}(k)\right]\left[\hat{d}_{p_{1}^{\prime}}(i) \hat{b}_{p_{1}}(i)\right] \text {. }
$$

In either case we have the term

On summing over the $p_{1}^{\prime}$ we get

$$
\hat{d}_{p_{1}^{\prime}}^{\dagger}(k) \hat{d_{p_{1}^{\prime}}}(i)
$$

$$
\begin{aligned}
& -\sum_{E>0} v\left(p_{1}^{\prime}\right) \bar{v}\left(p_{1}^{\prime}\right) \mathrm{e}^{-i p_{1}^{\prime} \cdot\left(x_{k}-x_{i}\right)}\left(d_{p_{1}^{\prime}}^{\dagger} d_{p_{1}^{\prime}}\right) \\
& -\sum_{E<0} v\left(p_{1}^{\prime}\right) \bar{v}\left(p_{1}^{\prime}\right) \mathrm{e}^{-i p_{1}^{\prime} \cdot\left(x_{k}-x_{i}\right)}\left(d_{p_{1}^{\prime}}^{\dagger} d_{p_{1}^{\prime}}\right) \quad \text { with } \quad t_{k}<t_{i},
\end{aligned}
$$

${ }^{1}$ In stochastic theory we speak of a "typical realization" or "typical realizable sequence of events" and probabilities are assigned to each such sequence. Here integration over space and time implies a superposition of these amplitudes. The integral amplitude vanishes unless there is energy momentum conservation. But that is no reason why we should not speak of the amplitude for a typical sequence of events corresponding to $n$ space-time points.

For a detailed comparison of the concept of realization in quantum mechanics and classical probability theory, see Alladi Ramakrishnan and N. R. Ranganathan, T. Math. Mech. In press. 
which is the Feynman kernel for the propagation of the negative energy state since $d_{p_{\mathbf{1}}^{\dagger}}^{\dagger} d_{p_{1}^{\prime}}$ is unity.

The procedure can be repeated and since we have uniquely defined the mode of arrangement for the fermion operators in a manner such that the operators are ordered in the Feynman sequence we obtain the integrand of the Feynman. expansion. In this procedure we note that if both the creation and destruction operators for a certain momentum and energy are contained in the interaction terms, they contribute to the kernel. Inside the interaction terms, we look only for destruction operators corresponding to the initial state and creation operators for the final state. We wish to point out that our method differs from the hitherto available algebraic methods in two important respects.

(1) We do not just move each operator separately but the operators corresponding to one vertex together, and

(2) We insist that $d^{\dagger}$ should always be to the left of $d$ or $b^{+}$at any vertex.

We now point out at the risk of a pedagogic explanation that $b_{p}^{\dagger}(1)$ represents creation of a real particle of momentum at the space-time point (1). A typical term therefore represents the creation of a real particle at every space-time point. The question of energy momentum conservation does not arise when there is no integration over space and time, since a bracket merely contains a product of wave functions of real particle states. If the integral is to be non-vanishing, energy conservation must of course be satisfied. Thus our rearrangement has resulted in obtaining the integrand in the Feynman formalism and not the integrated matrix element. In the kernel we have a product of two terms, summed over all $p$ or the sum can be replaced by an integral as

$$
\int \frac{1}{2 E_{p}} u(p) \vec{u}(p) \mathrm{e}^{-i p \cdot\left(x_{2}-x_{1}\right)} \mathrm{d}^{3} p .
$$

The kernel can also be treated as an integral over four-dimensional momentum $p$ (not corresponding to any real particle) weighted by $\frac{1}{p^{2}-m^{2}}$

$$
K(2,1)=\frac{u(p) \vec{u}(p) \mathrm{e}^{-i p \cdot\left(x_{2}-x_{1}\right)}}{p^{2}-m^{2}} \mathrm{~d}^{4} p .
$$

Thus the kernel can be regarded as a sum over wave functions with such four momenta with amplitude $\frac{1}{p^{2}-m^{2}}$. Such a dual explanation is not possible when we take the field operators singly and not in pairs.

We would however prefer to think of the kernel as representing the sum over the momenta of real particles. When we now integrate, say over space-time points, the kernel connecting (1) and (2) is transformed by the product of two exponential functions like $\mathrm{e}^{i p \cdot\left(x_{1}-x_{2}\right)}$ and $\mathrm{e}^{i q \cdot\left(x_{2}-x_{1}\right)}$ where $p$ is the four momentum of a real electron and $q$ that of a photon. This is equivalent to taking the transform with momentum arguments $p+q$ which represents a virtual electron EPCR $\quad 34$ 
since the energy-momentum relationship is not satisfied for $p+q$. In momentum space we speak of the propagator $\frac{1}{p+q-m}$ of a virtual particle, but there is no meaning in speaking of kernel functions in configuration space of a virtual particle. It is convenient to regard the sum over with real particle wave functions and the "intermediate" states in the integrand as real particle states. ${ }^{1}$

The introduction of the boson operators does not introduce any difficulty in this argument since these operators commute with each other. Once the Feynman arrangement has been obtained we need only to impose the restriction that in a typical term only annihilation operators corresponding to incident bosons and creation operators corresponding to emergent bosons, should occur.

Since we have given a unique prescription for ordering the operators, these considerations can be extended without any change of principle to the case when the incident and emergent system consists of more than one particle.

In getting (26) we have ignored the contribution from the $\delta$-function when $d_{p}$ crossed $d_{p}^{\dagger}$ while moving $d_{p}$ to the extreme right. If we wish to take into consideration this contribution we should use the ordering of the operators as in the usual interaction $\bar{\psi} \gamma_{\mu} \psi$. We shall now illustrate this for the case which leads to the negative energy part of the Feynman kernel ${ }^{2}$ since this alone involves a difference in procedure between the two methods.

If a bracket [i], which now contains bilinear fermion operators in the order determined by $\bar{\psi} \psi$, contains a $b_{p_{1}}$ with a $d_{p^{\prime}}$ attached to its left, we obtain

$$
[n], \ldots[i+1] d_{p^{\prime}}(i)[i-1] \ldots[1] b_{p_{1}} b_{p_{1}}^{\dagger}>_{0}
$$

where we have shifted $b_{p_{1}}$ (the operators being detached from their wave functions) through the intervening brackets, which results in no change of sign since none of the brackets contains either a $b_{p_{1}}$ or a $b_{p_{1}}^{\dagger}$. As before we argue that there should be another bracket $[k]$ with $t_{k}<t_{i}$ which should contain a $d_{p^{\prime}}^{\dagger}$ and shifting to its left we have

or

$$
[n] \ldots[i+1][i-1] \ldots d_{p^{\prime}}(i)\left[b_{p^{\prime \prime}}^{\dagger}(k) d_{p^{\prime}}^{\dagger}(k)\right] \ldots>_{0}
$$

$$
\left.[n] \ldots[i+1][i-1] \ldots d_{p^{\prime}}(i)\left[d_{p^{\prime \prime}}(k) d_{p^{\prime}}^{\dagger}(k)\right] \ldots\right\rangle_{0}
$$

In either case we obtain

$$
\left.-d_{p^{\prime}}(i) d_{p^{\prime}}^{\dagger}(k)\right\rangle_{0}
$$

As far as the wave functions attached to the operators are concerned, we have

$$
\begin{aligned}
& {\left[\bar{v}_{p^{\prime}}(i) u_{p_{1}}(i)\right]\left[\bar{u}_{p^{\prime \prime}}(k) \bar{v}_{p^{\prime}}(k)\right]} \\
& \equiv \bar{u}_{p^{\prime \prime}}(k) v_{p^{\prime}}(k) \bar{v}_{p^{\prime}}(i) u_{p_{1}}(i)
\end{aligned}
$$

1 'This of course should be distinguished from the the real intermediate states in the matrix element which correspond to the singularities in the propagator in momentum representation.

2 The proof of equivalence using the usual interaction has been done in collaboration with R. Thunga and T. K. Radha. 
and with the negative sign obtained from the commutation of the operators we get

$$
\begin{aligned}
& -\sum_{p^{\prime}} v_{p^{\prime}}(k) \bar{v}_{p^{\prime}}(i) \mathrm{e}^{-i p^{\prime}\left(x_{k}-x_{i}\right)} \\
= & -\sum_{p^{\prime}} u_{-E}(k) \bar{u}_{-E}(i) \mathrm{e}^{-i p^{\prime}\left(x_{k}-x_{i}\right)} \quad \text { with } t_{k}<t_{i},
\end{aligned}
$$

which gives an element of the negative energy part of the Feynman kernel.

\section{ON THE CONNECTION BETWEEN SPIN AND STATISTICS}

We give below the proof of Luders and Zumino ${ }^{1}$ on the connection between spin and statistics. The theorem on the connection between spin and statistics states that particles with integral spin obey Bose statistics and particles with half-integral spin obey Fermi statistics. Fields corresponding to particles with integral spin are to be quantized with minus commutation relations and those corresponding to particles with half-integral spin are to be quantized with plus commutation (anti-commutation) relations. The connection between spin and statistics was first proved by Pauli for non-interacting fields. ${ }^{2}$

\section{Hermitian spin-zero field}

Let $\varphi(x)$ be a hermitian spin zero field in the Heisenberg representation. We first postulate:

I. The theory is invariant with respect to the proper inhomogenous Lorentz group.

It follows that the expectation value $\langle\varphi(x) \varphi(y)\rangle_{0}$ between physical vacuum states is an invariant function of the difference four-vector

Therefore

$$
\xi_{\mu}=x_{\mu}-y_{\mu} \text {. }
$$

$$
\langle\varphi(x) \varphi(y)\rangle_{0}=f(\xi)
$$

where $f(\xi)$ depends only upon $\xi_{\mu} \xi_{\mu}$ if $\xi$ is space-like whereas if it is time-like, $f(\xi)$ can also depend upon whether $\xi_{\mu}$ points into the future or the past light cone. It follows from (2) that

$$
\langle[\varphi(x), \varphi(y)]\rangle_{0}=0 ; \quad \xi \text { (space-like). }
$$

We further postulate:

II. Two operators of the same field at points separated by a space-like interval either commute or anti-commute.

We have therefore only to show that the assumption

$$
\left\langle[\varphi(x), \varphi(y)]_{+}\right\rangle_{0}=0 \quad(\xi \text { space-like })
$$

1 G. Luders and B. Zumino, Phys. Rev. 110, 1450 (1958).

${ }^{2}$ W. Pauli, Phys. Rev. 58, 716 (1940). See also J. Schwinger, Phys. Rev. S2, 914 (1951); 91, 713 (1953). F. J. Belinfante, Physica 6, 870 (1939); W. Pauli and F. J. Belinfante, Physica 7, 177 (1940); N. Burgoyne, Nuovo Cim. 8, 607 (1958). 
leads to a contradiction. Equations (3) and (4) give

We also postulate:

$$
\langle q(x) q(y)\rangle_{0}=0 \quad(\xi \text { space-like }) .
$$

1II. The vacuum is the state of lowest energy. It follows by the method of analytic continuation used by Hall and Wightman ${ }^{1}$ that equation (5) holds, not only for space-like $\xi$, but for all $\xi$.

Our fourth postulate is:

IV. The metric of the Hilbert space is positive definite.

It follows from (5) that

$$
\varphi(x) \Omega=0
$$

where $\Omega$ is the physical vacuum. We finally postulate:

V. The vacuum is not identically annihilated by a field.

Since equation (6) contradicts this postulate, Eq. (4) is untenable. Postulate V seems essential in order that a Hilbert space can be constructed.

\section{Hermitian spin one-half field}

Let $\psi_{a}(x)$ be a Majorana spin one-half field so that

$$
\bar{\psi}_{\alpha}(x)=\psi_{\beta}(x) C_{\beta x}
$$

where $C$ is the $4 \times 4$ charge conjugation matrix. We consider

$$
\left.\left\langle\psi_{\beta}^{*}(x) \psi_{\beta}(y)\right\rangle_{0}=C_{\alpha \delta} \gamma_{4 \delta \beta}<\psi_{\alpha}(x) \psi_{\beta}(y)\right\rangle_{0}
$$

where the star denotes hermitian conjugation in Hilbert space. Let

The matrix $\eta$ is symmetric:

$$
C_{a \delta} \lambda_{4 \delta \beta}=\eta_{x \beta} \text {. }
$$

$$
\eta_{x \beta}=\eta_{\beta} .
$$

Since (8) is the fourth component of a four vector, it follows from postulate I that

$$
\eta_{x \beta}\left\langle\psi_{\alpha}(x) \psi_{\beta}(y)\right\rangle_{0}=\xi_{4} g(\xi)
$$

where $g(\xi)$ is an invariant function of $\xi_{\mu}$. From (10) and (11), it follows that

$$
\eta_{x \beta}\left\langle\left[\psi_{\alpha}(x), \psi_{\beta}(y)\right]_{+}\right\rangle_{0}=0 \quad(\xi \text { space-like }) .
$$

As before, because of postulate II, we have to show that the assumption

$$
\eta_{x \beta}\left\langle\left[\psi_{x}(x), \psi_{\beta}(y)\right]\right\rangle_{0}=0 \quad(\xi \text { space-like })
$$

leads to a contradiction. From (12) and (13), we get

$$
\eta_{\alpha \beta}\left\langle\psi_{\alpha}(x) \psi_{\beta}(y)\right\rangle_{0}=0 \quad \text { ( } \xi \text { space-like) }
$$

1 D. Hall and A.S. Wightman, Kgl. Danske Vidensk Selsk. 31, No. 5 (1957). 
From postulate III, it follows that (14) holds for all $\xi$. Because of equation (8) and postulate IV, it follows that

This contradicts postulate $V$.

$$
\psi_{\alpha}(x) \Omega=0
$$

\section{Non-hermitian fields}

We assume that non-hermitian fields are reasonable concepts only if they admit a gauge transformation of the first kind:

$$
\varphi(x) \rightarrow \varphi(x) \mathrm{e}^{i \alpha} ; \quad \varphi^{*}(x) \rightarrow \varphi^{*}(x) \mathrm{e}^{-i \alpha},
$$

similarly for spinor fields etc. The generating operator for the transformation may represent some conserved quantity like electric charge, baryon number, strangeness (in strong and electromagnetic interactions) or lepton charge (if it is conserved). We first consider a spin-zero field $\varphi(x)$. From the above postulate, it follows that

$$
\langle\varphi(x) \varphi(y)\rangle_{0}=\left\langle\varphi^{*}(x) \varphi^{*}(y)\right\rangle_{0}=0 .
$$

We have to show that the assumption

$$
\left\langle\left[\varphi^{*}(x), \varphi(y)\right]_{+}\right\rangle_{0}=0 \quad(\xi \text { space-like })
$$

leads to a contradiction. From postulate I and equation (18) it follows that

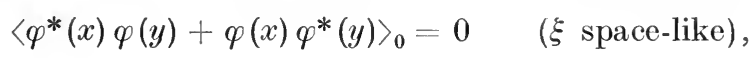

Using equation (17), we obtain

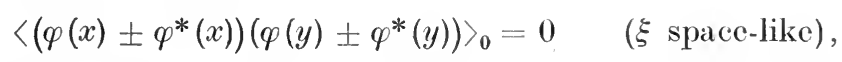

where both the plus or both the minus signs are to be taken together. Postulate III makes equation (20) generally valid. Since both $\varphi(x)+\varphi^{*}(x)$ and $i\left(\varphi(x)-\varphi^{*}(x)\right)$ are hermitian fields, postulate IV leads to

$$
\varphi(x) \Omega=\varphi^{*}(x) \Omega=0
$$

which contradicts postulate $\mathrm{V}$.

For spin one-half fields, the argument is quite similar. The analogue of equation (17) is

$$
\left\langle\psi_{\alpha}(x) \psi_{\beta}(y)\right\rangle_{0}=\left\langle\bar{\psi}_{a}(x) \bar{\psi}_{\beta}(y)\right\rangle_{0}=0 .
$$

The assumption to be disproved is

$$
\left\langle\left[\psi_{\alpha}^{*}(x), \psi_{\alpha}(y)\right]\right\rangle_{0}=0 \quad(\xi \text { space-like }) .
$$

Since the left-hand side transforms like the fourth-component of a four-vector, we have

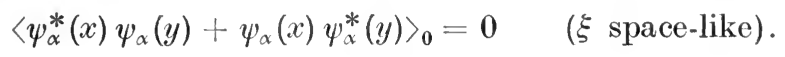

Equation (20) is to be replaced by

$$
\eta_{\alpha \beta}\left\langle\left[\psi_{\alpha}(x) \pm \zeta_{\alpha \gamma} \psi_{\gamma}^{*}(x)\right\rfloor\left[\psi_{\beta}(y) \pm \zeta_{\beta \delta} \psi_{\delta}^{*}(y)\right]\right\rangle_{0}=0,
$$


where the matrix $\zeta$ is the inverse of $\eta$ and $\zeta_{\alpha \beta}^{*}=\eta_{\alpha \beta}$. Since $\psi_{\alpha}(x)+\zeta_{\alpha \gamma} \psi_{\gamma}^{*}(x)$ and $i\left(\psi_{2}(x)-\zeta_{\alpha \gamma} \psi_{*}^{*}(x)\right)$ are Majorana fields, we have

$$
\psi_{2}(x) \Omega=\bar{\psi}_{a}(x) \Omega=0
$$

which eontradicts postulate $\mathrm{V}$.

\section{PHASE SHIF'A NALYSIS OF SCATTERING}

We have had occasion to deal with phase shifts in various chapters of the book. To define this quantity and find its relationship with the scattering crosssection, it is easiest to study the quantum mechanical scattering from a central potential, the Schrödinger equation for which can be written as

$$
-\frac{h^{2}}{2 \mu} V^{2} u+V u=E u
$$

where $\mu$ is the reduced mass of the system of scatter and the scattered particle. The general solution of this equation is of the form

$$
u\left(r, \theta=\sum_{l=0}^{\infty} R_{l}(r) P_{l}(\cos \theta)\right.
$$

where $P_{l}$ is the Legendre polynomial of order $l$. (Because of the symmetry about the polar axis, there is no dependence on the azimuthal angle.) Assuming that $V$ can be neglected for $r$ greater than some distance $a$, the most general form of $R_{l}(r)$ that is real is

$$
R_{l}(r)=A_{l}\left[\cos \delta_{l} j_{l}(k r)-\sin \delta_{l} n_{l}(k r)\right]
$$

where $\delta_{l}$ is real and $j_{l}$ and $n_{l}$ are the spherical Bessel and Neumann functions respectively. $\delta_{l}$ is the phase shift of the $l$-th partial wave since it is the difference of phase between the asymptotic forms of the actual radial function $R_{l}(r)$ and the radial function $j_{l}(k r)$ when the scattering potential is absent.

$$
R_{l}(r) \underset{r \rightarrow D}{\rightarrow}(k r)^{-1} \sin \left(k r-\frac{1}{2} l \pi+\delta_{l}\right) .
$$

Now we can represent the asymptotic form of $\mu(r, \theta)$ also in the form

$$
u(r, \theta) \underset{r \rightarrow \infty}{\rightarrow} \mathrm{e}^{i k z}+\frac{f(\theta)}{r} \mathrm{e}^{i k r}
$$

where the first term on the right represents the incident plane wave corresponding to a particle moving in the positive $z$-direction and the second term to the scattered wave which can be only outgoing, as the scattered particles can move only away from the scatter. Now we have

$$
\mathrm{e}^{i k z}=\mathrm{e}^{i k r \cos \theta}=\sum_{l=0}^{\infty}(2 l+1) i^{l} j_{l}(k r) P_{l}(\cos \theta) .
$$


Substituting the asymptotic form of this in '(5) and equating it to the asymptotic form of (2), we obtain on equating the coefficients of $\mathrm{e}^{i k r}$ and $\mathrm{e}^{-i k r}$ on the two sides of the equation

$$
\begin{gathered}
2 i k f(\theta)+\sum_{l=0}^{\infty}(2 l+1) i_{l} \mathrm{e}^{-\frac{1}{2} i \pi l} P_{l}(\cos \theta)=\sum_{l=0}^{\infty} A_{l} \mathrm{e}^{i\left(\delta_{l} l \frac{1}{2} l \pi\right)} P_{l}(\cos \theta) \\
\sum_{l=0}^{\infty}(2 l+) i^{l} \mathrm{e}^{\frac{1}{2} i l r} P_{l}(\cos \theta)=\sum_{l=0}^{\infty} A_{l} \mathrm{e}^{-\left(\delta_{l}-\frac{1}{2} l \pi\right)} P_{l}(\cos \theta)
\end{gathered}
$$

Since these equations are true for all values of $\theta$ and equation $(7 \mathrm{~b})$ becomes on using the orthogonality of the Legendre polynomials

$$
A_{l}=(2 l+1) i^{l} \mathrm{e}^{i \delta_{l}} .
$$

Substituting this in (7a) we obtain the expression for the scattering amplitude, viz.

$$
f(\theta)=(2 i k)^{-1} \sum_{l=0}^{\infty}(2 l+1)\left(\mathrm{e}^{2 i \delta_{l}}-1\right) P_{l}(\cos \theta)
$$

The differential cross-section is given by

$$
\sigma(\theta)=|f(\theta)|^{2}=\frac{1}{k^{2}}(2 l+1) \mathrm{e}^{i \delta_{l}} \sin \delta_{l}\left|P_{l}(\cos \theta)\right|^{2}
$$

and the total cross-section by

$$
\sigma=\frac{4 \pi}{k^{2}} \sum_{l=0}^{\infty}(2 l+1) \sin ^{2} \delta_{l} .
$$

We thus see that the phase shifts completely determine the scattering, the cross-section vanishing when each of the $\delta_{l}$ is $0^{\circ}$ or $180^{\circ}$. In this sense we can consider the $S$-matrix as the phase shift operator with eigenvalue $\mathrm{e}^{2 i \delta}$ when $\delta$ is real, i.e. for pure scattering processes. (For absorptive processes the phase shift will be complex.) Hence the eigenvalue of the $T$-matrix will correspondingly be $\mathrm{e}^{i \delta} \sin \delta$ while the reaction matrix $k$, which is connected with the $S$-matrix by the relation

$$
S=\frac{1+i k}{1-i k}
$$

and has the eigenvalue $\tan \delta$. Thus we can talk of the scattering eigenphases of a system.

If we compare the expression (9) for $\theta=0$ (i.e. forward scattering) with (11), we obtain the relation

$$
\sigma=\frac{4 \pi}{k} \operatorname{Im} j(0),
$$

which is called the optical theorem or the Bohr-Peierls-Placzek ${ }^{1}$ relation.

1 N. Bohr et al., Nature, Lond. 144, 200 (1939). 
For large angular momenta when the classical approximation is applicable, we can write the impact parameter $\mathscr{f}$ in terms of $l$ as

$$
\mathscr{T} \sim \frac{\lambda l}{2 \pi}
$$

where $\lambda$ is the do Broglie wavelength. The smallest impact parameter is given by $l$ o. If the range of interaction is less than $\lambda / \mathbf{2} \pi$ then a particle with an angular momentum other than zero hardly experiences scattering at all. The wave function for a particle with angular momentum $l$ becomes zero like $r^{l}$ at the origin. Henee the probability of finding a particle with $l>0$ in the region of interactions is very small if the range of action of the forces is much less than $\lambda / 2 \pi$. Thus the method of partial wave analysis should be particularly suitable for low energy nuclear scattering since the nuclear forces are of short range.

It ean be demonstrated that the sign of the phase shift is related to the nature of the potential. A repulsive potential makes the phase shift negative while an attractive potential leads to a positive phase shift.

1 See, e.g. L. I. Schiff. Quantum Mechanics, MeGraw-Hill, New York (1949). 


\section{NUMERICAL RESULTS ON CASCADE THEORY}

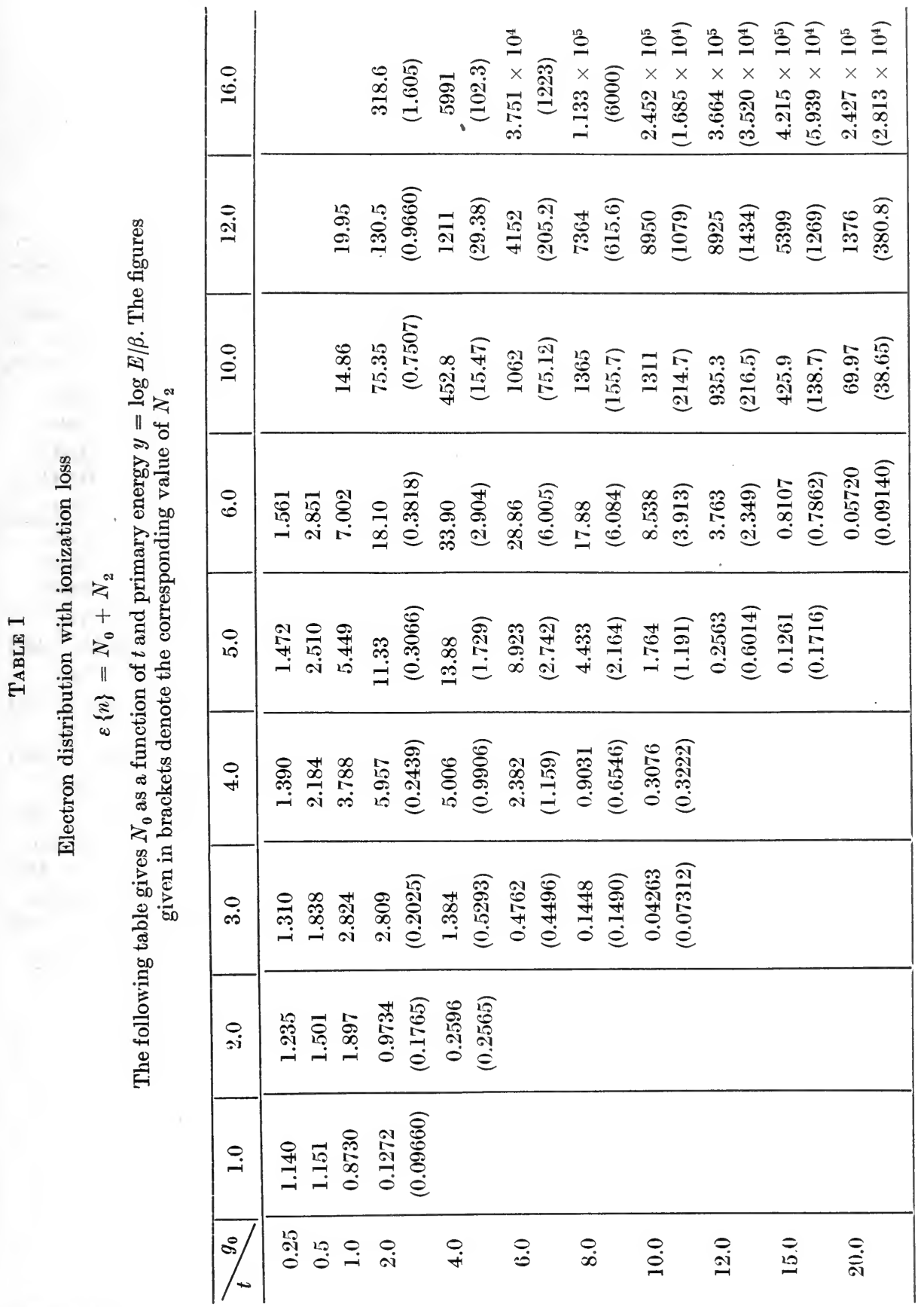




\section{TABLE II}

Electron distribution (Electron primary)

$$
\varepsilon\{n\}
$$

$[\varepsilon\{n\}]^{2}$ is given in brackets

\begin{tabular}{|c|c|c|c|c|c|c|c|}
\hline$y^{t}$ & 2 & 3 & 4 & 5 & 6 & 7 & 8 \\
\hline 3 & $\begin{array}{l}1.98 \\
3.93\end{array}$ & $\begin{array}{c}1.69 \\
(2.87)\end{array}$ & $\begin{array}{l}1.21 \\
(1.45)\end{array}$ & $\begin{array}{l}.779 \\
(.606)\end{array}$ & $\begin{array}{l}.475 \\
(.225)\end{array}$ & $\begin{array}{l}.280 \\
(.0782)\end{array}$ & $\begin{array}{l}.160 \\
(.0256)\end{array}$ \\
\hline 4 & $\begin{array}{r}3.90) \\
(15.2)\end{array}$ & $\begin{array}{l}(4.34) \\
(18.8)\end{array}$ & $\begin{array}{c}3.85 \\
(14.9)\end{array}$ & $\begin{array}{c}2.98 \\
(8.90)\end{array}$ & $\begin{array}{c}2.11 \\
(4.47)\end{array}$ & $\begin{array}{c}1.41 \\
(1.98)\end{array}$ & $\begin{array}{c}.897 \\
(.805)\end{array}$ \\
\hline i & $\begin{array}{r}6.87 \\
(47.3\end{array}$ & $\begin{array}{c}9.47 \\
(89.8)\end{array}$ & $\begin{array}{r}10.2 \\
(103)\end{array}$ & $\begin{array}{c}9.27 \\
(86.0)\end{array}$ & $\begin{array}{c}7.55 \\
(56.9)\end{array}$ & $\begin{array}{c}5.67 \\
(32.1)\end{array}:$ & $\begin{array}{c}4.01 \\
(16.1)\end{array}$ \\
\hline 6 & $\begin{array}{l}11.2) \\
(125)\end{array}$ & $\begin{array}{r}18.6 \\
(345)\end{array}$ & $\begin{array}{r}23.4 \\
(549)\end{array}$ & $\begin{array}{r}24.7 \\
(611)\end{array}$ & $\begin{array}{r}22.9 \\
(524)\end{array}$ & $\begin{array}{r}19.3 \\
(372)\end{array}$ & $\begin{array}{r}15.1 \\
(228)\end{array}$ \\
\hline 7 & & $\begin{array}{c}33.3 \\
(1110)\end{array}$ & $\begin{array}{c}48.8 \\
(2380)\end{array}$ & $\begin{array}{l}58.8 \\
(3450)\end{array}$ & $\begin{array}{l}61.3 \\
(3760)\end{array}$ & $\begin{array}{c}57.5 \\
(3310)\end{array}$ & $\begin{array}{c}49.6 \\
(2460)\end{array}$ \\
\hline 8 & & $\begin{array}{c}56.4 \\
(3180)\end{array}$ & $\begin{array}{c}94.2 \\
(8870)\end{array}$ & $\begin{array}{c}128 \\
\left(1.63 \times 10^{4}\right)\end{array}$ & $\begin{array}{c}149 \\
\left(2.22 \times 10^{4}\right)\end{array}$ & $\begin{array}{c}154 \\
\left(2.38 \times 10^{4}\right)\end{array}$ & $\begin{array}{c}146 \\
\left(2.13 \times 10^{4}\right)\end{array}$ \\
\hline 9 & & & $\begin{array}{c}171 \\
\left(2.92 \times 10^{4}\right)\end{array}$ & $\begin{array}{c}259 \\
\left(6.71 \times 10^{4}\right)\end{array}$ & $\begin{array}{c}.334 \\
\left(1.12 \times 10^{5}\right)\end{array}$ & $\begin{array}{c}381 \\
\left(1.45 \times 10^{5}\right)\end{array}$ & $\begin{array}{c}393 \\
\left(1.54 \times 10^{5}\right)\end{array}$ \\
\hline 10 & & & $\begin{array}{c}296 \\
\left(8.73 \times 10^{4}\right)\end{array}$ & $\begin{array}{c}496 \\
\left(2.46 \times 10^{5}\right)\end{array}$ & $\begin{array}{c}703 \\
\left(4.94 \times 10^{5}\right)\end{array}$ & $\begin{array}{c}874 \\
\left(7.64 \times 10^{5}\right)\end{array}$ & $\begin{array}{c}979 \\
\left(9.58 \times 10^{5}\right)\end{array}$ \\
\hline I2 & & & & $\begin{array}{c}1580 \\
\left(2.51 \times 10^{6}\right)\end{array}$ & $\begin{array}{c}2660 \\
\left(7.10 \times 10^{6}\right)\end{array}$ & $\begin{array}{c}3890 \\
\left(1.51 \times 10^{7}\right)\end{array}$ & $\begin{array}{c}5070 \\
\left(2.57 \times 10^{7}\right)\end{array}$ \\
\hline 14 & & & & & $\begin{array}{c}8620 \\
\left(7.42 \times 10^{7}\right)\end{array}$ & $\begin{array}{c}14500 \\
\left(2.11 \times 10^{8}\right)\end{array}$ & $\begin{array}{c}21700 \\
\left(4.70 \times 10^{8}\right)\end{array}$ \\
\hline 16 & & & & & & $\begin{array}{c}47400 \\
\left(2.25 \times 10^{9}\right)\end{array}$ & $\begin{array}{c}80100 \\
\left(6.41 \times 10^{9}\right)\end{array}$ \\
\hline
\end{tabular}




\section{TABLE III}

Electron distribution (Electron primary)

$$
\varepsilon\left\{n^{2}\right\}
$$

$\sigma^{2}$ is given in brackets

\begin{tabular}{|c|c|c|c|c|c|c|c|}
\hline$y^{t}$ & 2 & 3 & 4 & 5 & 6 & 7 & 8 \\
\hline 3 & $\begin{array}{c}6.48 \\
(2.55)\end{array}$ & $\begin{array}{c}4.49 \\
(1.62)\end{array}$ & $\begin{array}{c}2.57 \\
(1.12)\end{array}$ & $\begin{array}{l}1.37 \\
(.76)\end{array}$ & $\begin{array}{l}.740 \\
(.515)\end{array}$ & $\begin{array}{l}.400 \\
(.322)\end{array}$ & $\begin{array}{l}.216 \\
(.190)\end{array}$ \\
\hline 4 & $\begin{array}{l}21.6 \\
(6.4)\end{array}$ & $\begin{array}{l}25.4 \\
(6.6)\end{array}$ & $\begin{array}{l}18.6 \\
(3.7)\end{array}$ & $\begin{array}{l}12.1 \\
(3.2)\end{array}$ & $\begin{array}{c}6.89 \\
(2.42)\end{array}$ & $\begin{array}{c}3.76 \\
(1.78)\end{array}$ & $\begin{array}{c}2.05 \\
(1.24)\end{array}$ \\
\hline 5 & $\begin{array}{c}63.1 \\
(15.8)\end{array}$ & $\begin{array}{l}114 \\
(24)\end{array}$ & $\begin{array}{l}126 \\
(23)\end{array}$ & $\begin{array}{l}101 \\
(15)\end{array}$ & $\begin{array}{c}67.9 \\
(11.0)\end{array}$ & $\begin{array}{l}40.6 \\
(8.5)\end{array}$ & $\begin{array}{l}22.8 \\
(6.7)\end{array}$ \\
\hline 6 & $\begin{array}{l}163 \\
(38)\end{array}$ & $\begin{array}{l}424 \\
(79)\end{array}$ & $\begin{array}{c}650 \\
(101)\end{array}$ & $\begin{array}{l}698 \\
(87)\end{array}$ & $\begin{array}{l}584 \\
(60)\end{array}$ & $\begin{array}{l}414 \\
(42)\end{array}$ & $\begin{array}{l}261 \\
(33)\end{array}$ \\
\hline 7 & & $\begin{array}{l}1360 \\
(250)\end{array}$ & $\begin{array}{l}2800 \\
(420)\end{array}$ & $\begin{array}{l}3910 \\
(460)\end{array}$ & $\begin{array}{l}4140 \\
(380)\end{array}$ & $\begin{array}{l}3570 \\
(260)\end{array}$ & $\begin{array}{l}2640 \\
(180)\end{array}$ \\
\hline 8 & & $\begin{array}{c}3900 \\
(720)\end{array}$ & $\begin{array}{c}1.04 \times 10^{4} \\
\left(.15 \times 10^{4}\right)\end{array}$ & $\begin{array}{l}1.86 \times 10^{4} \\
\left(.23 \times 10^{4}\right)\end{array}$ & $\begin{array}{l}2.45 \times 10^{4} \\
\left(.23 \times 10^{4}\right)\end{array}$ & $\begin{array}{c}2.57 \times 10^{4} \\
\left(.19 \times 10^{4}\right)\end{array}$ & $\begin{array}{l}2.26 \times 10^{4} \\
\left(.13 \times 10^{4}\right)\end{array}$ \\
\hline 9 & & & $\begin{array}{l}3.45 \times 10^{4} \\
\left(.53 \times 10^{4}\right)\end{array}$ & $\begin{array}{c}7.67 \times 10^{4} \\
\left(.96 \times 10^{4}\right)\end{array}$ & $\begin{array}{l}1.24 \times 10^{5} \\
\left(.12 \times 10^{5}\right)\end{array}$ & $\begin{array}{l}1.57 \times 10^{5} \\
\left(.12 \times 10^{5}\right)\end{array}$ & $\begin{array}{l}1.64 \times 10^{5} \\
\left(.10 \times 10^{5}\right)\end{array}$ \\
\hline 10 & & & $\begin{array}{l}1.03 \times 10^{5} \\
\left(.16 \times 10^{5}\right)\end{array}$ & $\begin{array}{c}2.82 \times 10^{5} \\
\left(.36 \times 10^{5}\right)\end{array}$ & $\begin{array}{c}5.53 \times 10^{5} \\
\left(.59 \times 10^{5}\right)\end{array}$ & $\begin{array}{c}8.36 \times 10^{5} \\
\left(.72 \times 10^{5}\right)\end{array}$ & $\begin{array}{l}1.03 \times 10^{6} \\
\left(.07 \times 10^{6}\right)\end{array}$ \\
\hline 12 & & & & $\begin{array}{l}2.91 \times 10^{6} \\
\left(.40 \times 10^{6}\right)\end{array}$ & $\begin{array}{l}8.05 \times 10^{6} \\
\left(.95 \times 10^{6}\right)\end{array}$ & $\begin{array}{l}1.68 \times 10^{7} \\
\left(.17 \times 10^{7}\right)\end{array}$ & $\begin{array}{l}2.80 \times 10^{7} \\
\left(.23 \times 10^{7}\right)\end{array}$ \\
\hline 14 & & & & & $\begin{array}{c}8.53 \times 10^{7} \\
\left.\left.1.11 \times 10^{7}\right)\right)\end{array}$ & $\begin{array}{l}2.37 \times 10^{8} \\
\left(.26 \times 10^{8}\right)\end{array}$ & $\begin{array}{l}5.19 \times 10^{8} \\
\left(.49 \times 10^{8}\right)\end{array}$ \\
\hline 16 & & & & & & $\begin{array}{c}2.56 \times 10^{9} \\
\left(.31 \times 10^{9}\right)\end{array}$ & $\begin{array}{c}7.16 \times 10^{9} \\
\left(.75 \times 10^{9}\right)\end{array}$ \\
\hline
\end{tabular}




\section{TABLE IV}

Electron distribution (Electron primary)

$$
\begin{gathered}
\frac{\sigma^{2}}{[\varepsilon\{n\}]^{2}-\varepsilon\{n\}} \\
\frac{\sigma^{2}}{\varepsilon\{n\}} \text { is given in brackets }
\end{gathered}
$$

\begin{tabular}{c|c|c|c|c|c|c|c}
\hline$y$ & 2 & 3 & 4 & 5 & 6 & 7 & 8 \\
\hline 3 & 1.3 & 1.4 & 4.7 & -4.4 & -2.1 & -1.6 & -1.4 \\
& $(1.3)$ & $(.96)$ & $(.93)$ & $(.98)$ & $(1.1)$ & $(1.2)$ & $(1.2)$ \\
4 & .57 & .46 & .34 & .54 & 1.0 & 3.1 & -1.3 \\
& $(1.6)$ & $(1.5)$ & $(.96)$ & $(1.1)$ & $(1.1)$ & $(1.3)$ & $(1.4)$ \\
5 & .39 & .30 & .25 & .20 & .22 & .32 & .55 \\
& $(2.3)$ & $(2.5)$ & $(2.2)$ & $(1.6)$ & $(1.5)$ & $(1.5)$ & $(1.7)$ \\
6 & .33 & .24 & .19 & .15 & .12 & .12 & .15 \\
& $(3.4)$ & $(4.2)$ & $(4.3)$ & $(3.5)$ & $(2.6)$ & $(2.2)$ & $(2.2)$ \\
7 & & .23 & .18 & .14 & .10 & .080 & .075 \\
& & $(7.5)$ & $(8.6)$ & $(7.8)$ & $(6.2)$ & $(4.5)$ & $(3.6)$ \\
8 & & .23 & .17 & .14 & .10 & .081 & .061 \\
& & $(13)$ & $(16)$ & $(18)$ & $(15)$ & $(12)$ & $(8.9)$ \\
9 & & & .18 & .14 & .11 & .083 & .064 \\
& & & $(31)$ & $(37)$ & $(36)$ & $(31)$ & $(25)$ \\
10 & & .18 & .15 & .12 & .094 & .073 \\
& & & $(54)$ & $(73)$ & $(84)$ & $(82)$ & $(72)$ \\
12 & & & .16 & .13 & .11 & .089 \\
& & & & $(250)$ & $(360)$ & $(440)$ & $(450)$ \\
14 & & & & & .15 & .12 & .10 \\
& & & & & $(1300)$ & $(1800)$ & $(2300)$ \\
16 & & & & & .14 & .12 \\
& & & & & & $(6500)$ & $(9400)$ \\
\hline
\end{tabular}




\section{TABLe V}

Photon distribution (Electron primary)

$$
\varepsilon\left\{n^{2}\right\}
$$

$\sigma^{2}$ is given in brackets

\begin{tabular}{|c|c|c|c|c|c|c|c|}
\hline $\mathrm{t}^{y}$ & 2 & 3 & 4 & 5 & 6 & 7 & 8 \\
\hline 3 & $\begin{array}{c}7.07 \\
(1.30)\end{array}$ & $\begin{array}{c}5.62 \\
(1.26)\end{array}$ & $\begin{array}{c}3.82 \\
(1.18)\end{array}$ & $\begin{array}{l}2.19 \\
(.94)\end{array}$ & $\begin{array}{l}1.34 \\
(.81)\end{array}$ & $\begin{array}{c}.653 \\
(.450)\end{array}$ & $\begin{array}{c}.364 \\
(.291)\end{array}$ \\
\hline 4 & $\begin{array}{l}27.0 \\
(3.2)\end{array}$ & $\begin{array}{l}33.1 \\
(3.7)\end{array}$ & $\begin{array}{l}28.4 \\
(3.7)\end{array}$ & $\begin{array}{c}19.8 \\
(3.6)\end{array}$ & $\begin{array}{l}11.6 \\
(2.8)\end{array}$ & $\begin{array}{c}6.59 \\
(2.29)\end{array}$ & $\begin{array}{c}3.60 \\
(1.70)\end{array}$ \\
\hline 5 & $\begin{array}{c}90.1 \\
(10.4)\end{array}$ & $\begin{array}{c}156 \\
(13.9)\end{array}$ & $\begin{array}{l}180 \\
(14)\end{array}$ & $\begin{array}{l}157 \\
(12)\end{array}$ & $\begin{array}{l}113 \\
(11)\end{array}$ & $\begin{array}{l}73.0 \\
(9.9)\end{array}$ & $\begin{array}{r}41.9 \\
(8.6)\end{array}$ \\
\hline 6 & $\begin{array}{r}280 \\
(54)\end{array}$ & $\begin{array}{l}623 \\
(62)\end{array}$ & $\begin{array}{c}906 \\
(62)\end{array}$ & $\begin{array}{l}1050 \\
(55)\end{array}$ & $\begin{array}{l}933 \\
(43)\end{array}$ & $\begin{array}{l}703 \\
(39)\end{array}$ & $\begin{array}{r}471 \\
(32)\end{array}$ \\
\hline 7 & & $\begin{array}{l}2400 \\
(500)\end{array}$ & $\begin{array}{l}4209 \\
(320)\end{array}$ & $\begin{array}{l}5890 \\
(310)\end{array}$ & $\begin{array}{l}6430 \\
(250)\end{array}$ & $\begin{array}{l}5790 \\
(160)\end{array}$ & $\begin{array}{l}4510 \\
(140)\end{array}$ \\
\hline 8 & & & $\begin{array}{c}162 \times 10^{2} \\
(1320)\end{array}$ & $\begin{array}{c}282 \times 10^{2} \\
\left(17 \times 10^{2}\right)\end{array}$ & $\begin{array}{c}373 \times 10^{2} \\
\left(12 \times 10^{2}\right)\end{array}$ & $\begin{array}{c}405 \times 10^{2} \\
(1000)\end{array}$ & $\begin{array}{c}373 \times 10^{2} \\
(900)\end{array}$ \\
\hline 9 & & & $\begin{array}{c}557 \times 10^{2} \\
(4930)\end{array}$ & $\begin{array}{c}119 \times 10^{3} \\
\left(80 \times 10^{2}\right)\end{array}$ & $\begin{array}{l}191 \times 10^{3} \\
\left(9 \times 10^{3}\right)\end{array}$ & $\begin{array}{c}245 \times 10^{3} \\
\left(8 \times 10^{3}\right)\end{array}$ & $\begin{array}{l}261 \times 10^{3} \\
\left(5 \times 10^{3}\right)\end{array}$ \\
\hline 10 & & & $\begin{array}{c}174 \times 10^{3} \\
\left(168 \times 10^{2}\right)\end{array}$ & $\begin{array}{r}450 \times 10^{3} \\
\left(35 \times 10^{3}\right)\end{array}$ & $\begin{array}{c}860 \times 10^{3} \\
\left(49 \times 10^{3}\right)\end{array}$ & $\begin{array}{l}130 \times 10^{4} \\
\left(5 \times 10^{4}\right)\end{array}$ & $\begin{array}{l}159 \times 10^{4} \\
\left(2 \times 10^{4}\right)\end{array}$ \\
\hline 12 & & & & $\begin{array}{r}495 \times 10^{4} \\
\left(47 \times 10^{4}\right)\end{array}$ & $\begin{array}{r}131 \times 10^{5} \\
\left(14 \times 10^{5}\right)\end{array}$ & $\begin{array}{r}266 \times 10^{5} \\
\left(17 \times 10^{5}\right)\end{array}$ & $\begin{array}{r}438 \times 10^{5} \\
\left(17 \times 10^{5}\right)\end{array}$ \\
\hline 14 & & & & & $\begin{array}{c}145 \times 10^{6} \\
\left(15 \times 10^{6}\right)\end{array}$ & $\begin{array}{c}389 \times 10^{6} \\
\left(29 \times 10^{6}\right)\end{array}$ & $\begin{array}{c}831 \times 10^{6} \\
\left(47 \times 10^{6}\right)\end{array}$ \\
\hline 16 & & & & & & $\begin{array}{l}438 \times 10^{7} \\
\left(39 \times 10^{7}\right)\end{array}$ & $\begin{array}{l}118 \times 10^{8} \\
\left(8 \times 10^{8}\right)\end{array}$ \\
\hline
\end{tabular}




\section{TABLE VI}

Photon distribution (Electron primary)

$$
\varepsilon\{n\}
$$

$[\varepsilon\{n\}]^{2}$ is given in brackets

\begin{tabular}{|c|c|c|c|c|c|c|c|}
\hline$t^{y}$ & 2 & 3 & 4 & 5 & 6 & 7 & 8 \\
\hline 3 & $\begin{array}{c}2.40 \\
(5.77)\end{array}$ & $\begin{array}{c}2.09 \\
(4.36)\end{array}$ & $\begin{array}{c}1.62 \\
(2.63)\end{array}$ & $\begin{array}{c}1.12 \\
(1.25)\end{array}$ & $\begin{array}{l}.729 \\
(.532)\end{array}$ & $\begin{array}{l}.450 \\
(.203)\end{array}$ & $\begin{array}{l}.271 \\
(.0732)\end{array}$ \\
\hline 4 & $\begin{array}{l}4.88 \\
(23.8)\end{array}$ & $\begin{array}{c}5.42 \\
(29.4)\end{array}$ & $\begin{array}{c}4.97 \\
(24.7)\end{array}$ & $\begin{array}{c}4.02 \\
(16.2)\end{array}$ & $\begin{array}{c}2.98 \\
(8.87)\end{array}$ & $\begin{array}{c}2.08 \\
(4.31)\end{array}$ & $\begin{array}{c}1.38 \\
(1.90)\end{array}$ \\
\hline 5 & $\begin{array}{c}8.93 \\
(79.7)\end{array}$ & $\begin{array}{r}11.9 \\
(142)\end{array}$ & $\begin{array}{r}12.9 \\
(166)\end{array}$ & $\begin{array}{r}12.0 \\
(145)\end{array}$ & $\begin{array}{r}10.1 \\
(102)\end{array}$ & $\begin{array}{c}7.88 \\
(62.0)\end{array}$ & $\begin{array}{c}5.77 \\
(33.3)\end{array}$ \\
\hline 6 & $\begin{array}{r}15.0 \\
(226)\end{array}$ & $\begin{array}{r}23.7 \\
(562)\end{array}$ & $\begin{array}{r}29.7 \\
(880)\end{array}$ & $\begin{array}{r}31.6 \\
(996)\end{array}$ & $\begin{array}{r}29.8 \\
(890)\end{array}$ & $\begin{array}{r}25.8 \\
(664)\end{array}$ & $\begin{array}{r}20.9 \\
(439)\end{array}$ \\
\hline 7 & & $\begin{array}{c}43.6 \\
(1900)\end{array}$ & $\begin{array}{c}62.3 \\
(3880)\end{array}$ & $\begin{array}{c}74.7 \\
(5580)\end{array}$ & $\begin{array}{c}78.6 \\
(6180)\end{array}$ & $\begin{array}{c}75.0 \\
(5630)\end{array}$ & $\begin{array}{c}66.1 \\
(4370)\end{array}$ \\
\hline 8 & & & $\begin{array}{c}122 \\
\left(149 \times 10^{2}\right)\end{array}$ & $\begin{array}{c}163 \\
\left(265 \times 10^{2}\right)\end{array}$ & $\begin{array}{c}190 \\
\left(369 \times 10^{2}\right)\end{array}$ & $\begin{array}{c}199 \\
\left(395 \times 10^{2}\right)\end{array}$ & $\begin{array}{c}191 \\
\left(364 \times 10^{2}\right)\end{array}$ \\
\hline 9 & & & $\begin{array}{c}225 \\
\left(508 \times 10^{2}\right)\end{array}$ & $\begin{array}{c}333 \\
\left(111 \times 10^{3}\right)\end{array}$ & $\begin{array}{c}426 \\
\left(182 \times 10^{3}\right)\end{array}$ & $\begin{array}{c}487 \\
\left(237 \times 10^{3}\right)\end{array}$ & $\begin{array}{c}507 \\
\left(257 \times 10^{3}\right)\end{array}$ \\
\hline 10 & & & $\begin{array}{c}397 \\
\left(158 \times 10^{3}\right)\end{array}$ & $\begin{array}{c}644 \\
\left(415 \times 10^{3}\right)\end{array}$ & $\begin{array}{c}900 \\
\left(811 \times 10^{3}\right)\end{array}$ & $\begin{array}{c}1120 \\
\left(124 \times 10^{4}\right)\end{array}$ & $\begin{array}{c}1250 \\
\left(157 \times 10^{4}\right)\end{array}$ \\
\hline 12 & & & & $\begin{array}{c}2120 \\
\left(448 \times 10^{4}\right)\end{array}$ & $\begin{array}{c}3420 \\
\left(117 \times 10^{5}\right)\end{array}$ & $\begin{array}{c}4990 \\
\left(249 \times 10^{5}\right)\end{array}$ & $\begin{array}{c}6490 \\
\left(422 \times 10^{5}\right)\end{array}$ \\
\hline 14 & & & & & $\begin{array}{c}114 \times 10^{2} \\
\left(131 \times 10^{6}\right)\end{array}$ & $\begin{array}{c}190 \times 10^{2} \\
\left(360 \times 10^{6}\right)\end{array}$ & $\begin{array}{c}280 \times 10^{2} \\
\left(785 \times 10^{6}\right)\end{array}$ \\
\hline 16 & & & & & & $\mid \begin{array}{c}632 \times 10^{2} \\
\left(399 \times 10^{7}\right)\end{array}$ & $\begin{array}{c}105 \times 10^{3} \\
\left(110 \times 10^{8}\right)\end{array}$ \\
\hline
\end{tabular}




\section{Table VII}

Photon distribution (Electron primary)

$$
\begin{gathered}
\frac{\sigma^{2}}{[\varepsilon\{n\}]^{2}-\varepsilon\{n\}} \\
\frac{\sigma^{2}}{\varepsilon\{n\}} \text { is given in brackets }
\end{gathered}
$$

\begin{tabular}{|c|c|c|c|c|c|c|c|}
\hline$t^{y}$ & 2 & 3 & 4 & 5 & 6 & 7 & 8 \\
\hline 3 & $\begin{array}{c}.39 \\
(.54)\end{array}$ & $\begin{array}{c}.56 \\
(.61)\end{array}$ & $\begin{array}{r}1.2 \\
(.73)\end{array}$ & $\begin{array}{c}7.0 \\
(.84)\end{array}$ & $\begin{aligned}-4.1 & (1.1)\end{aligned}$ & $\begin{array}{r}-1.8 \\
(1.0)\end{array}$ & $\begin{array}{c}-1.5 \\
(1.1)\end{array}$ \\
\hline 4 & $\begin{array}{l}.17 \\
(.65)\end{array}$ & $\begin{array}{l}.15 \\
(.68)\end{array}$ & $\begin{array}{l}.19 \\
(.74)\end{array}$ & $\begin{array}{l}.30 \\
(.91)\end{array}$ & $\begin{array}{c}.47 \\
(.92)\end{array}$ & $\begin{array}{c}1.0 \\
(1.1)\end{array}$ & $\begin{array}{c}3.3 \\
(1.2)\end{array}$ \\
\hline 5 & $\begin{array}{l}.15 \\
(1.2)\end{array}$ & $\begin{array}{l}.11 \\
(1.2)\end{array}$ & $\begin{array}{l}.091 \\
(1.1)\end{array}$ & $\begin{array}{c}.088 \\
(.97)\end{array}$ & $\begin{array}{c}.12 \\
(1.0)\end{array}$ & $\begin{array}{r}.18 \\
(1.3)\end{array}$ & $\begin{array}{l}.31 \\
(1.5)\end{array}$ \\
\hline 6 & $\begin{array}{c}.26 \\
(3.6)\end{array}$ & $\begin{array}{c}.11 \\
(2.6)\end{array}$ & $\begin{array}{l}.076 \\
(2.1)\end{array}$ & $\begin{array}{r}.057 \\
(1.7)\end{array}$ & $\begin{array}{c}0.50 \\
(1.4)\end{array}$ & $\begin{array}{r}.060 \\
(1.5)\end{array}$ & $\begin{array}{r}.077 \\
(1.5)\end{array}$ \\
\hline 7 & & $\begin{array}{r}.27 \\
(11)\end{array}$ & $\begin{array}{l}.084 \\
(5.1)\end{array}$ & $\begin{array}{c}.055 \\
(4.1)\end{array}$ & $\begin{array}{l}.040 \\
(3.1)\end{array}$ & $\begin{array}{l}.029 \\
(2.2)\end{array}$ & $\begin{array}{l}0.032 \\
(2.1)\end{array}$ \\
\hline 8 & & & $\begin{array}{l}.089 \\
\text { (11) }\end{array}$ & $\begin{array}{l}.064 \\
(10)\end{array}$ & $\begin{array}{c}.034 \\
(6.5)\end{array}$ & $\begin{array}{l}.026 \\
(5.1)\end{array}$ & $\begin{array}{r}.026 \\
(4.9)\end{array}$ \\
\hline 9 & & & & $\begin{array}{l}.072 \\
(24)\end{array}$ & $\begin{array}{l}.051 \\
(22)\end{array}$ & $\begin{array}{l}.036 \\
(17)\end{array}$ & $\begin{array}{r}.018 \\
(8.9)\end{array}$ \\
\hline 10 & & & & $\begin{array}{l}.085 \\
(55)\end{array}$ & $\begin{array}{l}.060 \\
(54)\end{array}$ & $\begin{array}{l}0.43 \\
(48)\end{array}$ & $\begin{array}{l}.010 \\
(13)\end{array}$ \\
\hline 12 & & & & $\begin{array}{l}.10 \\
(220)\end{array}$ & $\begin{array}{r}.071 \\
(250)\end{array}$ & $\begin{array}{l}.067 \\
(340)\end{array}$ & $\begin{array}{r}.039 \\
(250)\end{array}$ \\
\hline 14 & & & & & $\begin{array}{c}.11 \\
\left(13 \times 10^{2}\right)\end{array}$ & $\begin{array}{c}.081 \\
\left(15 \times 10^{2}\right)\end{array}$ & $\begin{array}{c}.059 \\
\left(17 \times 10^{2}\right)\end{array}$ \\
\hline 16 & & & & . & & $\begin{array}{c}.097 \\
\left(61 \times 10^{2}\right)\end{array}$ & $\begin{array}{c}.078 \\
\left(79 \times 10^{2}\right)\end{array}$ \\
\hline
\end{tabular}




\section{TABLE VIII}

Electron distribution: New approach (Electron primary)

$$
\varepsilon\{N\}
$$

$\varepsilon\{n\}$ is given in brackets

\begin{tabular}{c|c|c|c|c|c|c|c|c}
\hline$y$ & 4 & 5 & 6 & 7 & 8 & 9 & 10 & 11 \\
\hline 0.5 & .524 & .750 & .994 & 1.521 & 1.521 & 1.801 & 2.092 & 2.394 \\
& $(1.139)$ & $(1.338)$ & $(1.553)$ & $(1.781)$ & $(2.019)$ & $(2.268)$ & $(2.527)$ & $(2.796)$ \\
0.6 & .729 & 1.053 & 1.407 & 1.786 & 2.188 & 2.612 & 3.057 & 3.526 \\
& $(1.252)$ & $(1.530)$ & $(1.836)$ & $(2.163)$ & $(2.512)$ & $(2.880)$ & $(3.269)$ & $(3.678)$ \\
0.7 & .961 & 1.400 & 1.888 & 2.418 & 2.988 & 3.598 & 4.248 & 4.940 \\
& $(1.381)$ & $(1.751)$ & $(2.162)$ & $(2.611)$ & $(3.096)$ & $(3.616)$ & $(4.173)$ & $(4.767)$ \\
0.8 & 1.216 & 1.790 & 2.437 & 3.151 & 3.931 & 4.777 & 5.693 & 6.679 \\
& $(1.524)$ & $(1.995)$ & $(2.530)$ & $(3.123)$ & $(3.773)$ & $(4.483)$ & $(5.254)$ & $(6.088)$ \\
0.9 & 1.492 & 2.220 & 3.054 & 3.989 & 5.027 & 6.170 & 7.423 & 8.792 \\
& $(1.677)$ & $(2.261)$ & $(2.936)$ & $(4.548)$ & $(4.458)$ & $(5.491)$ & $(6.530)$ & $(7.670)$ \\
1.0 & 1.788 & 2.690 & 3.740 & 4.938 & 6.288 & 7.797 & 9.745 & 11.330 \\
& $(1.837)$ & $(2.546)$ & $(3.378)$ & $(4.336)$ & $(5.424)$ & $(6.649)$ & $(8.020)$ & $(9.546)$ \\
1.1 & 2.103 & 3.199 & 4.497 & 6.003 & 7.726 & 9.682 & 11.887 & 14.360 \\
& $(2.003)$ & $(2.846)$ & $(3.856)$ & $(5.039)$ & $(6.406)$ & $(7.970)$ & $(.9747)$ & $(11.752)$ \\
1.2 & 2.433 & 4.735 & 5.326 & 7.189 & 9.356 & 11.851 & 14.703 & 17.940 \\
& $(2.172)$ & $(3.159)$ & $(4.366)$ & $(5.806)$ & $(7.498)$ & $(9.466)$ & $(11.733)$ & $(14.328)$ \\
1.3 & 2.779 & 4.327 & 6.226 & 8.502 & 11.189 & 14.329 & 17.965 & 22.145 \\
& $(2.343)$ & $(3.485)$ & $(4.908)$ & $(6.638)$ & $(8.705)$ & $(11.147)$ & $(14.002)$ & $(17.310)$ \\
\hline
\end{tabular}




\section{Table IX}

Electron distribution: New approach (photon primary)

$$
\varepsilon\{N\}
$$

$\varepsilon\{n\}$ is given in brackets

\begin{tabular}{c|c|c|c|c|c|c|c|c}
\hline$y$ & 4 & 5 & 6 & 7 & 8 & 9 & 10 & 11 \\
\hline 0.5 & .710 & .766 & .822 & .879 & .938 & 1.000 & 1.063 & 1.128 \\
& $(.682)$ & $(.746)$ & $(.806)$ & $(.865)$ & $(.925)$ & $(.987)$ & $(1.050)$ & $(1.115)$ \\
0.6 & .063 & .951 & 1.044 & 1.141 & 1.243 & 1.349 & 1.459 & 1.573 \\
& $(.817)$ & $(.917)$ & $(1.015)$ & $(1.114)$ & $(1.216)$ & $(1.323)$ & $(1.432)$ & $(1.546)$ \\
0.7 & 1.025 & 1.157 & 1.298 & 1.450 & 1.611 & 1.781 & 1.959 & 2.146 \\
& $(.955)$ & $(1.102)$ & $(1.249)$ & $(1.403)$ & $(1.564)$ & $(1.733)$ & $(1.910)$ & $(2.095)$ \\
0.8 & 1.198 & 1.384 & 1.590 & 1.813 & 2.054 & 2.311 & 2.584 & 2.872 \\
& $(1.097)$ & $(1.300)$ & $(1.511)$ & $(1.736)$ & $(1.975)$ & $(2.229)$ & $(2.499)$ & $(2.784)$ \\
0.9 & 1.382 & 1.636 & 1.922 & 2.237 & 2.582 & 2.954 & 3.354 & 3.781 \\
& $(1.243)$ & $(1.513)$ & $(1.803)$ & $(2.117)$ & $(2.457)$ & $(2.823)$ & $(3.216)$ & $(3.637)$ \\
1.0 & 1.579 & 1.914 & 2.298 & 2.729 & 3.206 & 3.727 & 4.293 & 4.906 \\
& $(1.391)$ & $(1.741)$ & $(2.125)$ & $(2.550)$ & $(3.016)$ & $(3.526)$ & $(4.080)$ & $(4.680)$ \\
1.1 & 1.788 & 2.219 & 2.722 & 3.295 & 3.935 & 4.645 & 5.426 & 6.281 \\
& $(1.541)$ & $(1.982)$ & $(2.479)$ & $(3.037)$ & $(3.659)$ & $(4.349)$ & $(5.109)$ & $(5.492)$ \\
1.2 & 2.011 & 2.553 & 3.196 & 3.939 & 4.782 & 5.728 & 6.781 & 7.947 \\
& $(1.694)$ & $(2.237)$ & $(2.864)$ & $(3.580)$ & $(4.392)$ & $(5.304)$ & $(6.322)$ & $(7.452)$ \\
1.3 & 2.246 & 2.916 & 3.723 & 4.668 & 5.756 & 6.992 & 8.385 & 9.947 \\
& $(1.847)$ & $(2.505)$ & $(3.280)$ & $(4.182)$ & $(5.220)$ & $(6.403)$ & $(7.741)$ & $(9.243)$ \\
\hline
\end{tabular}




\section{RESONANCE STATES OF STRONGLY INTERACTING PARTICLES}

\section{(1) The $x-x$ resonance}

The existence of this resonance was first suspected from a study of the isovector part of the nucleon electromagnetic form factor. ${ }^{1}$ Subsequently an experimental study ${ }^{2}$ of the reactions

$$
\begin{aligned}
\pi^{-}+p & \rightarrow \pi^{-}+\pi^{0}+p, \\
& \rightarrow \pi^{-}+\pi^{+}+n
\end{aligned}
$$

has revealed the existence of this resonance via a peak in the $Q$-value distribution of the two pions. The mass of this resonance is $765 \mathrm{MeV}$ with a half-width of $75 \mathrm{MeV}$. Experiments also reveal that the quantum numbers of the resonance are $I=1$ and $J=1$.

(2) The $3 x$ resonances

(a) Experiments ${ }^{3}$ tend to indicate a spectrum anomaly of $H e^{3}$ produced in the collision of protons with deuterium in the form of a peak in the region corresponding to double pion production. The isotopic spin of the particle which this suggests has been measured to be $I=0$. It is also suggested ${ }^{4}$ that the particle has $J=1$ since it has never been observed in $K$-decay. Thus it seems to be a particle of the nature of a $3 \pi$-bound state.

(b) Evidence has recently been reported $^{5}$ for an $I=0, J=1$ resonance (which is distinct from the $3 \pi$ bound state discussed in (a)) in a study of the effective mass distribution of triplets of pions in the reaction

$$
\bar{p}+p \rightarrow \pi^{+}+\pi^{+}+\pi^{-}+\pi^{-}
$$

The mass of the resonance is $787 \mathrm{MeV}$ with a half-width $\Gamma / 2<15 \mathrm{MeV}$ corresponding to a life-time $\tau>4 \times 10^{-23}$ sec.

(3) The $\boldsymbol{K}-\boldsymbol{x}$ resonance

Recently a study of the reactions

$$
\begin{aligned}
K^{-}+p & \rightarrow K^{-}+\pi^{0}+p \\
& \rightarrow \overline{K^{0}}+\pi^{-}+p
\end{aligned}
$$

has revealed the existence of a $K-\pi$ resonance. ${ }^{6}$ The branching ratio at resonance for the two channels (a) and (b) is predicted to be 2 and $1 / 2$ for $I=1 / 2$ and $3 / 2$

1 W. P. Frazer and J. R. Fulco, Phys. Rev., 117, 1609 (1960).

2 Erwix et al., Phys. Rev., Letters, 6, 628 (1961).

${ }^{3}$ A. Abashiax, N. E. Booth and K. M. Crowe, Phys. Rev. Letters, 5, 258 (1960); Rev. Mod. Phys., 33, 393 (1961).

+ G. F. Chew, Rev. Mod. Phys., 33, 394, (1961).

5 Maglić et al., Phys. Rev. Letters, 7, 178 (1961); Phys. Rev. 125, 687 (1962).

${ }_{6}$ Alstox et al., Phys. Rev. Letters, 6, 300 (1961). 
states respectively. The observed ratio is $1.4 \pm 0.4$ indicating the $I=1 / 2$ state for the resonance. The resonance has a mass of $885 \pm 3 \mathrm{MeV}$ with a full width at half-maximum of $16 \mathrm{MeV}$. The spin of the resonance is not as yet known although the isotropic angular distribution of the $K$ and $\pi$ from the decay of the resonance suggests $J=0$ or 1 .

\section{(4) The $\Lambda-\pi$ resonance}

This resonance was first observed in the $15 \mathrm{in}$. Berkeley hydrogen bubble chamber in the reaction

$$
K^{-}+p \rightarrow \Lambda^{0}+\pi^{+}+\pi^{-}
$$

for incident $K^{-}$-momentum of $1.15 \mathrm{BeV} / \mathrm{c}^{1}$ The resonance has been christened $Y_{1}^{*}$ and clearly has isotopic spin 1 . The mass of the resonance is $1385 \mathrm{MeV}$ with a half-width of about $25 \mathrm{MeV}$. The helium chamber collaboration group ${ }^{1}$ have also observed it in the reaction

$$
K^{-}+H e^{4} \rightarrow \Lambda^{0}+\pi^{-}+H e^{3} .
$$

Their analysis of the angular distribution of the $Y_{1}^{*}$ decay products suggests $J=1 / 2$ for the resonance. This inference however needs further corroboration. $\dagger$

(5) The $\Sigma-\pi$ resonance

This resonance has been observed in the study of the reactions ${ }^{2}$

$$
\begin{aligned}
K^{-}+p & \rightarrow \Sigma^{+}+\pi^{-}+\pi^{+}+\pi^{-}, \\
& \rightarrow \Sigma^{-}+\pi^{+}+\pi^{+}+\pi^{-}, \\
& \rightarrow \Sigma^{0}+\pi^{0}+\pi^{+}+\pi^{-}, \\
& \rightarrow \Lambda^{0}+\pi^{0}+\pi^{+}+\pi^{-} .
\end{aligned}
$$

The $Q$-value of the final $\Sigma-\pi$ system shows a peaking at about $1400 \mathrm{MeV}$ indicative of a resonance. The corresponding distribution of $Q(\Lambda \pi)$ shows no such behaviour suggesting that the observed $\Sigma-\pi$ resonance is something distinct from $Y_{1}^{*}$. (Notice that $Y_{1}^{*}$ cannot decay into $\Sigma^{0}+\pi^{0}$ since $\Sigma^{0}+\pi^{0}$ has no $I=1$ component.) The relative number of events with $\Sigma^{0} \pi^{0}$ in the final state compared to those with $\Sigma^{+} \pi^{-}$and $\Sigma^{-} \pi^{+}$in the final state near resonance suggests $I=0$ for the resonance which has therefore been called $Y_{0}^{*}$. The mass of the resonance is about $1400 \mathrm{MeV}$ with a half-width of about $20 \mathrm{MeV}$. The spin of the resonance is not yet known.

1 Alston et al., Phys. Rev. Letters, 5, 520 (1960); Berge et al., Phys. Rev. Letters, 6, 557 (1961); Dahl et al., Phys. Rev. Letters, 6, 142 (1961); Helium Chamber Collaboration Group, Nuovo Cimento, 20, 724 (1961); M. H. Alston and M. Ferro-Luzzi, Rev. Mod. Phys., 33, 416, (1961).

2 Alston et al., Phys. Rev. Letters, 6, 698 (1961).

$\dagger$ There is now evidence that $J \geqq 3 / 2$ for the $Y_{1}^{*}$; ref. R. P. Euy et al., Phys. Rev. Lett. 7 , 461 (1961). 


\section{(6) The $\bar{K}-N$ resonance}

A resonance in the $K^{-}-p$ system with a mass of $1810 \mathrm{MeV}$ has been reported recently. The eross-section curve for $K^{-}-n$ scattering reveals no corresponding structure indicating that the resonance is in the $I=0$ state.

There also seems to be some evidence for a resonance in the $K^{--}-p$ system at a centre-of-mass energy of about $95 \mathrm{MeV}$.

\section{GAUGE THEORIES OF ELEMEN'TARY PAR'TICLES}

While Heisenberg's theory attempts to be very comprehensive in its scope and is a departure from the conventional field theories, there is still a belief that a dynamical theory of elementary particles is possible within the present framework. Of particular interest in this connection are the attempts of GellMann², Neeman ${ }^{3}$, Sakurai ${ }^{4}$ and Salam and Ward ${ }^{5}$ which are in a sense sequels to the work of Yang and Mills. ${ }^{6}$

Actually all these stem from the well-known principle in electrodynamics (already referred to in Chapter XX) which states that the existence of the electromagnetic field is a necessary consequence of demanding the invariance of the Lagrangian under gauge transformations of the first kind on the electron field:

$$
\psi(x)=\mathrm{e}^{i e \Lambda(x)} \psi(x) .
$$

With constant $1,(1)$ merely expresses the fact that charge is conserved in these interactions. When $A$ is a function of $x$, the transformation acquires a deep and beautiful significance. The free Lagrangian

$$
L=-\bar{\psi}\left(i \gamma_{\mu} \delta_{\mu}+m\right) \psi
$$

(where we have written $\gamma_{\mu} \delta_{\mu}$ for $\nabla$ ) is no longer invariant under (1) and we are constrained to add to it a term

$$
L_{\mathrm{int}}=-e \bar{\psi} \gamma_{\mu} \psi A_{\mu}
$$

where $A_{\mu}$ is a new field which may be identified with the electromagnetic field and which transforms as

$$
A_{\mu}(x) \rightarrow A_{\mu}(x)+\frac{\partial \Lambda(x)}{\partial x_{\mu}}
$$

when $\psi(x)$ transforms as in (1). The transformation (4) is called a gauge transformation of the second kind and implies that the electromagnetic field is massless. In this sense then, the existence of the electromagnetic field is a consequence of

1 L. T. Kerth, Rev. Mod. Phys., 33, 389, (1961).

M. Gell-Mann, Phys. Rev. (to be published).

${ }^{3}$ Y. Neemax, Nuclear Physics, 26, 222 (1961).

4 J. J. Sakurai, Ann. Phys., 11, l (1960).

5 A. Salani and J. C. WARD, Nuovo Cimento, 19, 165 (1961); 20, 419 (1961).

'C. N. YAXG and R. L. Milis, Phys. Rev., 96, 191 (1954). 
the conservation of charge. It is to be emphasized that invariance under (1) implies that the phase of the charged field can be altered from one space time point to another arbitrarily, i.e. the local phase of the field is not a quantity of significance. Thus when (1) is a symmetry of the system, the notion of a local field acquires a deeper significance than what is conventionally attributed to it.

It may be recognized that what has been done here to generate the electromagnetic field is to replace the ordinary derivative $\delta_{\mu}$ in the free Lagrangian by the covariant derivative ${ }^{1} \delta_{\mu}-i e A_{\mu}$ corresponding to the space-time dependent transformation (1). A leading attempt to extend this idea to other conservation laws as well was that of Yang and Mills who by the localization of isotopic spin rotations "derived" the existence of a triplet of vector mesons coupled to the isotopic vector current. Sakurai subsequently tried to build a theory of strong interactions by localization of the gauges belonging to the three conservation laws peculiar to these interactions, viz. baryon number, hypercharge and isotopic spin. In the latter theory, it is essential that there be only two fundamental elementary fields apart from the vector mesons. The chief difficulty in these theories is that they give vector mesons of zero mass in analogy with electromagnetism (except possibly for those associated with isotopic spin rotations) ${ }^{2}$ for which no experimental evidence exists.

Theories generated by such gauge transformations have their interaction forms almost uniquely fixed. Thus if the symmetry property is expressed by ${ }^{3}$

$$
\Psi^{\prime}=[1+i \boldsymbol{I} \alpha] \Psi
$$

where $\boldsymbol{I}$ is the generator of the transformation, the covariant derivative is

$$
\nabla_{\mu}=\partial_{\mu}-i \boldsymbol{I} \boldsymbol{A}_{\mu}
$$

Thus the problem of finding the correct interactions is reduced to one of finding the correct symmetry properties. For instance, let us assume that a scalar $\sigma$ particle exists and write the kinetic energy part of the free Lagrangian as

where

$$
L_{K E}=\bar{N}_{L} i \gamma_{\mu} \delta_{\mu} N_{L}+\bar{N}_{R} i \gamma_{\mu} \delta_{\mu} N_{R}+\frac{1}{2}\left(\delta_{\mu} \boldsymbol{\pi}\right)^{2}+\frac{1}{2}\left(\delta_{\mu} \sigma\right)^{2}
$$

$$
N_{L}=\frac{1}{2}\left(1+\gamma_{5}\right) N ; \quad N_{R}=\frac{1}{2}\left(1-\gamma_{5}\right) N
$$

and $N$ and $\boldsymbol{\pi}$ are the nucleon and pion fields. The widest possible symmetries of this Lagrangian are those contained in the four-dimensional rotation group with $\left(N_{L}, N_{R}\right)$ and $(\boldsymbol{\pi}, \sigma)$ forming four vectors (cf. F. Gursey's work discussed in Chapter 7, Sec. 3). Correspondingly we have six vector fields $\boldsymbol{U}$ and $\boldsymbol{V}$ in the covariant derivatives and

$$
\begin{aligned}
L_{\mathrm{int}}= & {\left[\bar{N} i \gamma_{\mu} \gamma_{5} \boldsymbol{\tau} N+\left(\partial_{\mu} \sigma\right) \boldsymbol{\pi}-\sigma\left(\partial_{\mu} \boldsymbol{\pi}\right)\right] \boldsymbol{V}_{\mu} } \\
& +\left[\bar{N} i \gamma_{\mu} \boldsymbol{\tau} N-\frac{1}{2}\left(\partial_{\mu} \boldsymbol{\pi} \times \boldsymbol{\pi}-\boldsymbol{\tau} \times \partial_{\mu} \boldsymbol{\tau}\right)\right] \boldsymbol{U}_{\mu} \\
& +\frac{1}{2}\left(\boldsymbol{\pi} \times \boldsymbol{U}_{\mu}+\sigma \boldsymbol{V}_{\mu}\right)^{2}+\frac{1}{2}\left(\boldsymbol{\pi} \cdot \boldsymbol{V}_{\mu}\right)^{2}
\end{aligned}
$$

\footnotetext{
1 For the definition of covariant derivative see, for example, A. Ernstern, The Meaning of Relativity, Methuen (1946), p. $69^{\circ}$.

2 A. Salam, Rev. Mod. Phys., 33, 426 (1961).
} 
W: notice that the fermion mass term is not invariant under this group, which is a very unsatisfactory feature. There has also been a detailed investigation of other symmetry groups in this connection. We present below Salam and Ward's considerations (ref. 5, p. 548) on the unitary group in three dimensions where the elementary fields are taken to be $p, n$ and $\Lambda$ as in the Sakata model. Let $x=\left[\begin{array}{c}p \\ n \\ 1\end{array}\right]$. 'The kinetic energy part of the Lagrangian then reads

$$
L_{K E}=\bar{x} i \gamma_{\mu} \partial_{\mu} x
$$

'The unitary transformation is

$$
x \rightarrow[1+i H] x
$$

where $H=x_{\varrho} H_{Q}$ and $\alpha_{Q}^{\prime} s$ are infinitesimal. There are eight spurless matrices $H_{Q}$ corresponding to the fact that this is an eight-parameter group. A representation of $H$ is generated by

$$
H^{\prime}=x \bar{x}=\left[\begin{array}{lll}
p \bar{p} & p \bar{n} & n \bar{\Lambda} \\
n \bar{p} & n \bar{n} & n \bar{\Lambda} \\
\Lambda \bar{p} & \Lambda \bar{n} & \Lambda \bar{\Lambda}
\end{array}\right]
$$

This is not irreducible since it has non-vanishing spur. The matrix

$$
H=H^{\prime}-\frac{1}{3} \quad \text { Spur } \quad H^{\prime} \times I
$$

is therefore irreducible. The covariant derivative is defined through

$$
\nabla_{\mu}=\partial_{\mu}-i H_{\mu}
$$

where $H_{\mu}$ is obtained from $H$ by the identifications of $\frac{p \bar{p}+n \bar{n}-2 \Lambda \bar{\Lambda}}{\gamma^{2}}$ with a vector meson $\varrho_{\mu}^{\circ}$ with $I=0, \frac{p \vec{p}-n \bar{n}}{\gamma^{2}}$ with the neutral component $\pi_{\mu}^{\circ}$ of a triplet of vector mesons etc. Thus

$$
H_{\mu}=\left[\begin{array}{ccc}
\frac{1}{1^{6}} \varrho_{\mu}^{\circ}+\frac{1}{1^{2}} \pi_{\mu}^{\circ} & \pi_{\mu}^{+} & K_{\mu}^{+} \\
\pi_{\mu}^{-} & \frac{1}{\gamma^{6}} \varrho_{\mu}^{\circ}-\frac{1}{\gamma^{2}} \pi_{\mu}^{\circ} & K_{\mu}^{\circ} \\
K_{\mu}^{-} & K_{\mu}^{\circ} & -\frac{2}{\gamma^{6}} \varrho_{\mu}^{\circ}
\end{array}\right]
$$

where $\pi_{\mu}$ and $K_{\mu}$ are vector mesons with $I$-spin and strangeness identical with those of $\pi$ and $K$ and $\varrho_{\mu}^{\circ}$ has $I=0$. The Sakata Lagrangian now reads

$$
\begin{aligned}
L= & \bar{x} i \gamma_{\mu} \delta_{\mu} x+\bar{x} \gamma_{\mu} H_{\mu} x+\frac{S p}{3}\left[\delta_{\mu} H_{\nu}-\delta_{\nu} H_{\mu}\right]^{2}+ \\
& + \text { mass terms for } p, n, \Lambda \text { and } H \text { fields. }
\end{aligned}
$$


The $\pi_{\mu}$ 's occurring here correspond to the Yang-Mills triplet of bosons (ref. 5, p. 548) and may be the $I=1, J=1, \pi-\pi$ resonance which has been recently observed. ${ }^{1}$ Similarly the $K_{\mu}$ and $\varrho_{\mu}$ may also be the observed $K-\pi^{2}$ and $3 \pi^{3}$ resonances.

Pseudovector currents may be generated by considering transformations on $x_{L}=\frac{1}{2}\left(1+\gamma_{5}\right) x$ and $x_{R}=\frac{1}{2}\left(1-\gamma_{5}\right) x$ separately:

$$
x_{L} \rightarrow\left(1+i H_{1}\right) x_{L}, \quad x_{R} \rightarrow\left(1-i H_{2}\right) x_{R}
$$

since $H_{\mu}=\frac{1}{2}\left(H_{1 \mu}+H_{2 \mu}\right)$ and $H_{\mu}^{\prime}=\frac{1}{2}\left(H_{1 \mu}-H_{2 \mu}\right)$ are vector and pseudovector respectively. It is possible that the components of the divergence $\frac{\partial H_{\mu}}{\partial x_{\mu}^{\prime}}$ which is not zero because of the presence of mass terms in the Lagrangian, may be the $\pi$ and $K$ fields themselves.

Weak interactions can also be considered in this scheme. An irreducible representation of the unitary group is provided by

$$
B=\left[\begin{array}{ccc}
-\sqrt{2} b^{\circ} & b & b \\
b^{*} & \frac{1}{\sqrt{2}} b^{\circ} & \frac{1}{\gamma^{2}} b^{\circ} \\
b^{*} & \frac{1}{\sqrt{2}} b^{\circ} & \frac{1}{\gamma^{2}} b^{\circ}
\end{array}\right]
$$

where $b^{0}$ is real. A gauge transformation of the type $x_{L} \rightarrow(1+i B) x_{L}$ would lead to a weak interaction of the type

$$
\begin{aligned}
L_{\mathrm{int}}=\left[\bar{p}_{L} \gamma_{\mu} n_{L}+\bar{p}_{L} \gamma_{\mu} \Lambda_{L}\right] B_{\mu}^{+}+ \\
\quad+\frac{1}{\gamma^{2}}\left[-2 \bar{p}_{L} \gamma_{\mu} n_{L}+\bar{n}_{L} \gamma_{\mu} n_{L}+\bar{\Lambda}_{L} \gamma_{\mu} \Lambda_{L}+\bar{n}_{L} \gamma_{\mu} \Lambda_{L}+\bar{\Lambda}_{L} \gamma_{\mu} n_{L}\right] B_{\mu}^{\circ}
\end{aligned}
$$

Analogous to $\left[\begin{array}{l}p \\ n \\ \Lambda\end{array}\right]$, we may consider the triplet $\left[\begin{array}{l}v \\ e \\ \mu\end{array}\right]$ for leptons to define
the gauge.

Other considerations of $a^{\prime}$ similar nature are those of Neemann ${ }^{1}$ and of Gell-Mann ${ }^{2}$ referred to before. From the latter's considerations, there emerges a theory of weak interactions and a picture of strongly interacting particles which is rather unified. Since the basic fields are those of the Sakata model, the theory inevitably leads to the well-known $|\Delta \boldsymbol{I}|=\frac{1}{2}$ and $\frac{\Delta Q}{\Delta S}=+2$ rules for strangenessnon-conserving currents in weak interactions. Any evidence that the latter is wrong may then suggest that the group has to be widened still further to take these experimental facts into account.

1 Erwin et al., Phys. Rev. Letters, 6, 628 (1961).

2 Alston et al., Phys. Rev. Letters, 6, 300 (1961).

3 A. Abashian, N. E. Booth and K. M. Crowe, Phys. Rev. Letters, 5, 258 (1960); Rev. Mod. Phys., 33, 393 (1961). 


\section{4. 'T HE PRINCIPLE OF EQUIVALENCE FOR ALL STRONGLY INTERACTING PARTICLES}

The principle of equivalence ${ }^{1}$ states that the correct theory should not allow us to decide which particles are elementary.

The mathematical framework in which this principle has been expressed by Chew and Frautschi uses the analytic continuation ${ }^{2}$ of the $S$-matrix as a function of the complex energy $E$ and angular momentum $J$, which has been shown to be possible for potential scattering and for any amplitude obeying the Mandel. stam representation.

In the union of the complex $J$ and $E$ planes, the locus $\alpha(E)$ of a single pole in the $J$ plane (as $E$ varies) is an analytic function of $E$. If $\operatorname{Re} \alpha(E)$ passes through a positive integer or zero, with $\frac{\mathrm{d}}{\mathrm{d} E}(\operatorname{Re} \alpha)>0$, then for this integer, there occurs a physical resonance or bound state. To each locus $\alpha_{i}(E)$, therefore, corresponds a family of poles with different $(J, E)$; these poles are called "Regge poles". The principle of equivalence may be satisfied by postulating that all the poles of the $S$-matrix are Regge poles; this is an extension to angular momentum of the "principle of maximum analyticity."

Poles corresponding to elementary particles can arise if, for certain internal quantum numbers, the relativistic continuation in $J$ is restricted to a smaller region than that for the non-relativistic case and the region $\operatorname{Re} J<J_{\min }(E), J_{\min }$ $(E)>0$, is excluded from the domain of the $J, E$ space into which the $S$-matrix can be continued.

The question whether or not all poles of the $S$-matrix are Regge poles, which will decide whether or not the conventional (Lagrangian) field theory is correct, may be decided experimentally. Regarding $\alpha(E)$ as $\alpha(s), s=E^{2}$, one may either (i) work in positive $s$ and look for families of particles (corresponding to families of Regge poles), or (ii) work in negative $s$ and trace out $\alpha(s)$ for a range of $s$ from the asymptotic energy variation of the backward peak in the crossed channel. The width and tail of the backward peak vary differently according as the scattering is controlled by a Regge pole or an elementary particle pole.

The quantum numbers of the vacuum inevitably receive special emphasis; a proposal is that all forward diffraction peaks may be controlled by a Regge pole with the internal quantum numbers of the vacuum.

Better high-energy experiments may decide whether the principle of maximum analyticity in $J$ is valid.

1 G. F. Chew and S. C. Frautschi, Phys. Rev., Letters, 7, 304 (1961); 8, 41 (1962).

2 T. RegGe, Nuovo Cimento, 14, 951 (1959); Nouvo Cimento, 18, 947 (1960). 


\section{AUTHOR INDEX}

\section{$\mathbf{A}$}

Abashian, A. 546

ADAIR, R. K. 248, 295, 310, 320

AKHIEZER, A. I. 38

ALDER, K. 255

Alfven, H. 373, 375, 485, 490

Allen, J. S. 258, 262

ALston, M. H. 547

Alvarez, L. 236

Amati, D. 306, 309, 310, 312, 313, 316

Ambler, E. 253, 259

Anderson, H. L. 177, 274

\section{B}

Balachandran, A. P. 281, 311, 322, 335, $341,353,356$

BaNos, A. 383

BARANGer, M. 107

BargmanN, V. 206

BarshaY, S. 319, 353

BARTlett, M. S. 425,429

BAYM, G. 341

BAZ, A. I. 320

Belinfante, F. J. 144, 531

Bellman, R. 426

Berestetsky, V. B. 38

BERLEY,D. 296

BerLyet, D. 277

Bernstein, J. 282

Bethe, H. A. 22, 30, 107, 112, 113, 176, 178, $189,467,476$

Внавна, Н. J. 421, 427, 432, 436, 441, 453, 463

Bhamathi, G. 95, 353

Bharucha-Reid, A. T. 425

BILENKII, S. M. 312

BinceR, A. M. 258

Blatt, J. B. 182

BLeUleR, K. 520

Blin-Stoyle, R. J. 273

BLOCH, F. 109, 402

BLOCK, M. M. 412

Bludman, S. A. 277

Bogoliviov, N. N. 112, 140, 206, 210

BoLDT 264, 311

EPCR 36
BONCKANRET 383

BonsigNoRI, F. 186

Воотн, Е. Т. 239

BraunbeCK, W. 109

Breit, G. 320

Bremmermann 206

Brown, L. M. 230, 270

Brueckner, K. A. 176

BUdini, P. 338

Burgoyne, N. 531

Burgx, M. T. 255, 259, 273

\section{C}

CAPPS, R. H. 310, 311

CARLson, J. F. 427

CASE, K. M. 38, 241, 263

CASSELS, J. M. 274

Cassen, B. 30

Castillejo, L. 193

Ceolin, C. 312

Chakrabarthy, S. K. 432, 441, 453, 463, 478

Chamberlatn, O. 247

Chandrasekhar, S. 484

Chew, G. F. 167, 180, 183, 186, 189, 190, 194, $213,220,317,552$

Chinowski, W. 239

Clarke, G. 469

Clay, J. 372

Coester, F. 101, 156, 520

Colliers, G. B. 296

Compton, A. H. 372

Condon, E. U. 30, 501

CooL, R. L. 264, 266, 276, 296

Costa, G. 312

Cowan, C. L. 263

Crawford, F. S. 230, 244, 264, 290

Culligan, G. 261

Curtis, R. B. 254, 255, 256

Cutkosky, R. E. 196

D

DAHL, O. 296

DALitz, R. H. 221, 231, 234, 235, 277, 289, $290,299,300,304,305,314,317,326$

Dallaporta, N. $267,326,336,338,347$ 
DAxcorf, S. MI. IsO)

DANIEL, R. R. 461

DAxYsz, M. 297

DAIIDON, W. C. 179

DAVIES, R. 262, 263

DAVIS, H. F. 277

DAvis, L. 484

DAY. 'T. B. 315,316

DAYHOFF, E.S. 107

DrRado. I. 186

De Santis, V. 347

Deshpaxde, N. G. $281,353,356$

D'Espacsat, B. 226, 265, 325, 326, 344

Devtsch, M. 259

Drrac, P. A. M. 9, 21, 521

DORMAX 376

Dowss, B. 299. 300, 317

DürR, H. P. 356, 359, 361, 362

Drsox, F. J. 97, 109, 111, 183, 207, 258, 447

$\mathbf{E}$

Edex, R. J. 213

EDyoxds, A. R. 499

Einstein, A. 549

Eisler, F. 264, 295

ERWIN 546

\section{$\mathbf{F}$}

FABRI, E. 234

Fazzini, T. 274

Feenberg, E. 488

Feisberg, G. 277, 323, 354

FeINBERg, V. Ya. 207

FELD, B. T. 312

Feldmax, D. 139

Feldyax, G. 159, 319, 322

FERII, E. 113, 178, 179, 351, 379, 400, 401, $403,406,409,410,413,468,469,481,483$, $484,485,488,519$

Ferretti, B. 211, 274, 285, 289, 294, 322

Ferro-Luzzi, M. 547

Feshbach, H. 19

Feymuax, R. P. 21, 25, 52, 54, 65, 74, 97, $104,107,269,270,271,274,275,277,326$, 348,505

Fierz, M. 109

Finkelsteis, R. 274

Foldy, L. L. 30

FoxDA, L. 316, 338

Ford, G. W. 258

ForfF, C. 476

Fowler, G. N. 396, 397
Fowlek, P. H. 230

Frauenfelder, H. 258

Frautschi, S. F. 552

Frazer, W. R. 186, 546

French, J. B. 107

Fried, H. M. 107

FRY, W. F. 24

Fubini, S. 282

FujII, A. 297, 308

Fulco, J. R. 186, 546

Fulton, T. 319, 322

FURRY, W. H. 154, 449

\section{G}

Galbraith, W. 457, 467

GAMBA, G. 275

Gartenhaus, S. 197

GARWIN, R. L. 260

Gatto, R. 237, 264, 265, 273

Gell-MaNN, M. 158, 187, 221, 225, 237, 240, $243,244,265,267,269,270,271,273,275$, $277,282,326,333,336,548$

Gershtein, S. S. 271

GiNZBURG, V. L. 368, 370, 478

GLASER, D. A. 244

GLASHOW, S. L. 275, 319

GLAUBER, R. J. 109

GOEBEL, C. 314

GOLDBERGER, M. L. 179, 180, 202, 211, 273, $275,277,282$

GOLDHABER, M. 258, 270

GOLDHABER, S. 352

GooD, M. L. 241

Gottstein, K. 268

Green 440

Greisen, K. 457, 468

Grodzins, L. 257, 258, 270

GUHST, S. P. 255

GunN, J. C. 107

GuPTA, S. N. 354, 520

GüRSEY, F. 150, 277, 354

H

HaAs, R. 248

Hall, D. 532

HaLl, J. S. 206, 484

Hamilton, J. 156

HARRIS, G. 235

HARRIS, T. E. 426

HaSEgaWA, S. 460

HaYward, R. W. 253

HEINBERG, M. 258 
Heisenberg, W. 120, 128, 167, 175, 351, 356, $380,401,402,463$

Heitler, W. 53, 54, 74, 180, 181, 182, 398, $427,454,455$

Hiltwer 484

de Hoffmann, F. 22, 30, 112, 113, 176, 189 Hofstadter, R. 273

Hoppes, D. D. 253

Hough, P. V. C. 86

Hudson, R. P. 253

HUNTER, R. A. 384

Hurst, C. A. 198

\section{I}

IGI, K. 314,315

IMPEDUGLIA, T. 239, 274

INDUMATHI, S. 95, 353

ITABASHT, K. 172

IWAO, S. 299, 302

\section{J}

JACKSON, J. D. 254, 255, 256, 290

Janossy, L. 377, 379, 426, 428, 440, 452, 454,455

JАUCH, J. М. 87, 97, 99, 104, 109, 112

\section{$\mathbf{K}$}

KäLlen, G. 133, 198, 514, 519, 520

Kamata, J. 462, 463, 465

KaPLON, M. F. 175,285

Karplus, R. 105, 107, 205, 304

Kawaguchi, M. 265, 309

KEMmer, N. 143, 156, 183

Kerth, L. T. 548

KibbLe, T. W. B. 213

Kinoshita, T. 261, 277

KLEIN, A. 107

КовА, Z. $400,414,422$

KonopInskI, E. 261

Kotani, T. 256, 348

KovaCs, J. S. 412

Kramers, H. A. 204

KraUshaAR, W. L. 422

KrolL, N. M. 105, 107, 196

Kronig, R. 204

L

LAMB, W. E. JR. 107

LANDAU, L. 36, 253, 255, 257, 401, 413, 414, $415,419,463,469$
LANDE, K. 239

LATTES, C. M. G. 168

LEDERMAN, L. M. 239

LEE, T. D. 36, 166, 224, 230, 231, 236, 237, $243,246,249,250,252,253,257,259,261$, $264,282,283,303$

Lehmann, H. 187, 198, 207, 208, 210, 212, 219

LEIPUNER, L. B. 248

LEITNER, J. 275, 315

Lemaitre, G. 377, 384, 387, 392

LEPORE, J. V. 406

I.EWIS, H. 402

LEwIS, H. W. 401, 402, 468

LEwIs, R. R. 254, 255, 256

LIOUVILLE 392

Lovati 475

Low, F. E. 180, 183, 186, 187, 190, 194

LudERs, G. 159, 246, 250, 531

\section{II}

Maglí, B. C. 546

Mahmoud, H. M. 261

Mandelstam, S. 186, 213, 220, 317

Marks, I. J. 422

MaRSHAK, R. E. 172, 197, 224, 234, 245, 269, $275,297,308$

MaRX, G. 270

Mathews, P. M. 425, 436, 451

Matthews, P. T. 9, 101, 143, 158, 159, 314

McLennan, J. A. 263

McVoy, K. W. 258, 447

MeIXNer 380

Melissinos, A. C. 294

Messel, H. 425, 440, 441, 452

Miksell, A. H. 484

Milburn, R. 405

Mrlus, R. L. 162, 548

Minami 179

Miyazawa, H. 197

Moliere, G. 463

Moravcsik, M. J. 309, 318

Morita, M. 254, 256

Morita, R. S. 254, 256

Morpurgo, G. 109

Morrison, P. 297, 369, 376

Mullin, C. J. 258

Nakano, T. 225

NAMBU, Y. 352

NeEman, Y. 548 
Nerurkar. N. W. 372

Nishijima, K. 225, 265, 278

Nishimura, K. 462, 463, 465

Niv, K. 460

Nordsifek, A. 109. 402

\section{$\mathbf{0}$}

Oннме, R. 206, 243, 249, 252

OKuBo, S. 245, 250

OKus', L. B. 272, 275, 316. 320

Olbert, S. 369, 469

Oort, J. H. 494

OPPENheIMER, J. R. 401; 402, 427

Orear. J. 177, 178, 235, 236

\section{P}

PAGE, L. A. 255, 258

PAIS, A. 236, 237, 239, 240, 241, 243, 316, $317,322,326,339,340,341,342,346,347$

Pandit, L. K. 347

PAULI, W. 3, 16, 19, 97, 109, 159, 175, 180 246, 263, 508, 531

Peaslee, D. 401, 412, 413

Peierls, R. F. 171

Peng, H. W. 156, 180

Perkiss, D. H. 230

Petermane, A. 198

Pevsner, A. 317

Piccioni, O. 239, 241

Placzek, G. 476

Pniewski, J. 297

Poisson 449

Polkinghorne, J. C. 143, 326, 342, 343, 344

Pomeranchuk, I. YA. 316, 401, 413, 414, 415

Pontecorvo, B. 275

PotTs, R. B. 441

Poweld, C. F. 230

Prentki, J. 226, 265, 325, 326, 344

Primakoff, H. 488

Puppi, G. 266, 469, 474, 475, 476

Pursey, D. L. 143, 263

\section{$\mathbf{R}$}

\section{RACAH, G. 502}

RADHA, T. K. $89,383,445,530$

RAMAKRISHNAN, A. 52, 65, 89, 95, 281, 335, $341,356,425,427,428,429,431,436,441$, $443,445,449,451,455,506,524,528$

RaNGaNathaN, N. R. 311, 322, 335, 341, 353, $356,443,447,523,528$

Ravenhall, D. G. 290, 307
REGGE, T. 552

RETHERFORD, R. E. 107

RiChTMYER, R. D. 490

Rohrlich, F. 87, 97, 99, 104, 109, 112

Rose, M. E. 499

Rosenfeld, A. H. 221, 243, 244, 265, 269, 290

Rosenthal, I. L. 468

Ross, M. H. 158

Rossi, B. 369, 474

Ruderman, M. A. 196, 205, 304

Russee, J. E. 316

RYNDin, R. M. 312

$\mathbf{S}$

SAAVEDRA, I. 346

SACHS, R. G. 241

SAKATA, S. 353

SAKURAI, J. J. 230, 269, 308, 326, 334, 354, 356,548

Salam, A. 36, 111, 158, 243, 257, 314, 326, $342,343,344,548$

Salzman, F. 193

SALZMAN, G. 193

SARABHAI, V. 372, 376

SATYAPRAKASH 376

SCHIFF, L. I. 536

SCHOPPER, H. 259

SCHREMP, E. J. 384

SCHRÖDINGER, E. 19

SCHWARTZ, M. 353

SCHWEBER, S. S. 22, 112, 113

SChWINGER, J. 99, 105, 107, 140, 154, 159, $326,330,331,332,342,531$

ScOTT, W. T. 452

SEBE, T. 182

SÉGRÈ, E. 247

SELLERI, F. 186

Serber, R. 241

SERPE, J. 263

SHAPIRO, M. M. 222

SHaW, G. L. 158

Shirkov, D. V. 112, 140, 206, 210

Shortley, G. H. 501

SIGNELL, P. S. 197

Srmpson, J. A. 476

SINGER, S. F. 367, 372

Sirlin, A. 261, 316, 323

SNow, G. A. 222, 315

Solov'Ev, V. G. 354

SOMMERFELD 85

SPITZER, R. 316, 323

Srinivasan, S. K. 186, 196, 441, 443 
Srivastava, G. P. 401,420

STECH, B. 255

STEINBERGER, J. 294, 322

STORA, R. 469

StORMer, C. 377, 380, 384

STUART, R. N. 406

SUCHER, J. 315

Sudarshan, E. C. G. 224, 234, 245, 269, 276, $294,302,401,413,414,420$

Suess 369

SUNYAR, A. W. 258, 270

SwanN, W. F. G. 481, 483

SyMANZIK, K. 187, 198, 206

\section{$\mathbf{T}$}

Taffara, N. 312

Takagi, S. 400, 401, 422

TAKEDA, G. 158, 414

TAMM, I. 180

TANNER, N. 248

TAYODA 347

TAYLOR, J. G. 309, 318, 319

TAYLOR, O.S. 206, 235

Teller, E. 482, 490

TeUCher, M. 235

Thirring, W. 109, 198, 235, 282, 353

THUNGA, R. 89, 383, 530

Tiomno, J. 150, 266, 347

ToLL, J. S. 202

TOUSCHEK, B. 109

Tremman, S. B. 237, 241, 254, 255, 273, 274, $275,277,282,315,316,322,324$

TriebWasser, S. 107

TRIPP, R. D. 247, 292

Tuan, S. F. 290, 314, 326

TURner, R. N. 371

\section{$\mathbf{U}$}

UHLENBECK, G. E. 452

URETSKY, J. L. 309, 318

UREY, H. C. 369

UTIYAMa, R. 162

\section{V}

VALLARTA, M. S. 377, 384, 387, 392

VASUdeVAN, R. 52, 65, 447, 523
Venkatesan, K. 186, 196, 281, 431, 443

Villars, F. 19, 97

Vitale, B. 306, 309, 310, 312, 313, 316

\section{W}

WALRAVEN, I. 494

WAPSTRA, A. H. 259

WARD, J. C. 105, 243, 548

WATAGHIN, G. 402

WATSON, K. M. 158, 311

WeINBerg, S. 166, 245, 273

Weinmann, E. 109

WEISSKOPF, V. F. 19, 107, 180

Wentzel, G. 14, 99, 112, 144, 167, 175, 243, 398

WEYL, H. 36

WheEler, J. A. 266

Wick, G. C. 131, 156, 183, 306

Wiegand, C. 247

Wrghtman, A. S. 156, 206, 306, 532

Wigner, E. P. 154, 156, 180, 306, 320

Wilkinson, D. H. 248

WinTer, A. 255

WINZELER, H. 235

Wolfendale, A. W. 396

Wolfenstein, L. 307

Wouthuysen, S. A. 401, 402

Wu, C. S. 253, 254, 259

WYLD, JR. H. W. 254, 255, 274, 290

\section{$\mathbf{Y}$}

YaMAGUChI, Y. 353

$Y_{A N G}$, C. N. $36,139,150,154,162,166,178$, $179,224,231,237,243,246,249,250,252$, $253,257,259,261,264,282,283,303,351$, 548

Yennie, D. R. 107

YPSILANTIS, T. 247

YUKAWA, H. 33, 167

\section{$\mathbf{Z}$}

Zachariasen, F. 196

ZEL'Dovich, J. B. 271

ZimmermanN, W. 187, 198

Zumino, B. 250, 531 


\section{SUBJEC'T INDEX}

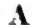

Absence of negative energy states 207

Acceleration of particles, mechanism of Fermi 485

Adjoint wave function 23

Air showers 400

Angular distributions of colliding particles 394

Angular momentum $16,144,147$

in classical mechanics 18

composition of 499

eigenfunctions 44

intrinsic 16

of photon 42, 44

operator 16

in quantum mechanics 18

Angular structure functions 466

Annihilation 79,122

of particles 13

operator 115

Anomalous magnetic moment 105

Anomalous scattering of $\mu$-mesons 399

Anticommutation rules 124

Antiparticle 13, 151

creation operator 121

anti-neutrino 36

Associated production 223

Asymptotic condition 199, 207

Attribute

dynamical 3

intrinsic 3

Axial vector 146

\section{B}

Bare mass 98

Baryon gauge 147

Bessel functions 47

Beta decay 153, 248, 259

Betatron mechanism of Swann 481

Bethe formula 397

Bethe-Heitler formula 398

Bhabha's theory of the photoproduction of mesons 421

Bilinear interaction term 121

Bloch-Nordsieck method 403
Bohr magneton 17

Bohr radius 85

Born approximation 85

Boson conjugation 339

Bound state wave function 107

'Bra' vector 10

Breit frame 209

Bremsstrahlung 74, 81, 88, 398

\section{C}

Canonical energy-momentum tensor 144

Canonical momenta 137

Canonically conjugate variables 6

Capture of mesons 157

Cascade process 393,424

Cascade theory 441, 443

Cassiopeia-A 494

"Catastrophic" term 163

"Catastrophic" transition 175

Causality 199, 207

Charge 12

conjugation $37,147,151,252,362$

density 19

gauge 162, 226

independence of interaction 165

independence of nuclear forces 32

renormalization $99,103,105$

symmetry 166

wave-function 32

Charged fermion field 137

Chew-Low

cut-off theory 194

formalism 180,183

method 196

Chronological operator 127

Classical

current 137

Hamiltonian 17

physics 3

Clebsch-Gordon coefficients 45, 229, 499, 501

Collision process 53

Collision theory 7, 116, 139

Commutation relations $17,113,124$

Commutator 137

Complementarity principle 4, 11 
Complex

field variables 126

fields 152

scalar fields 149, 161

Compound models 351

Compton Effect 74, 90

Compton scattering 87

Concept

of handedness 28

of hole 29

of interaction 12

of spin 18

Cones, cosmic radiation 380

Configuration representation 113

Configuration space $3,8,9$

wave function in 41

Conserved quantities 147

of energy 56

of parity 231

of probability 11

Constant of motion 147

Continuous transformations 147,160

Contraction 130

Cosmic

couplings 329

radiation, Fermi theory 369

symmetry 330,334

Coulomb

field $7,35,85,88$

interaction 251

wave-function 108

Coupling 132

constant 212

constant in weak interactions 172,273 in strong interactions 175

of three angular momenta 503

Covariant formulation of the statistical theory 420

CPT test for invariance 247

CPT Theorem 158, 249

Crab nebula 494

Creation

operator $115,119,121$

of pairs 74

of particles 13, 66

"Crossing" theorem 190

Cross-section, Compton scattering 90

Current 7, 19, 129, 135

-current interaction 275

term 163

\section{D}

Damping, radiation 180

Decay

experiments 256

of hypernuclei 303

of $K$-particles 230,237

of a single particle 512

Delta rays 397

Density

Hamiltonian 132

Lagrangian 132

matrix 12

of states 64,506

Detailed balance 156

Deviation, mean 8

from stormer cones 384

Diagrams

Feynman for lamb shift 108

self energy for bound electron 108

vacuum polarization 108

Difference equation 15

Dimension of an integral 109

Dimensions and units 498

Dirac

description of neutrino 262

electron 65

equation $21,23,35,37,64$

equation for neutrino 249

field 161

Hamiltonian 37

hole theory 29

matrices 123

Direct couplings 132

Discrete transformations 147

Dispersion relations 140, 193, 201, 203

Diurnal variation of cosmic radiation 372

Divergences, removal of 109

Doppler effect 169

Doublet approximation 336,339

Dynamical variable $5,9,112$

Dyson's representation 210

\section{E}

Eigenfunction 6

angular momentum 44

complete set 10

parity 45

Elastic scattering 157

Electron 17

-electron interaction $\mathbf{7 4 , 9 0}$

-photon cascade 432

-photon component 474 


\section{Electron}

-photon interaction 122

propagator 107

scattering 122

self energy 97

Elementary length 212

Elementary particle 2.21

Energetic collisions with magnetized interstellar matter 481

Energy

balance of cosmic radiation 469

determination $\mathbf{4 5 9}$

of electromagnetic field 40

loss by fast particles 75

loss by ionization 394

-momentum tensor 144,147

shell 60

spectrum of primary cosmic ray particles 477

Electric field 48

Electric state $46,48,51$

Elect romagnetic

energy 40

field $17,33,38,150,162,519$

interactions 223

processes 74

tensor 106

Equation for free particle 24

Equation of motion 5, 144

for charged particle in a magnetic field 377

d'Espagnat-Prentki interaction 339

d'Espagnat-Prentki scheme 326

Euler-Mascheroni constant 434

Evaporation 399

Exclusion principle 13, 29

Expectation value, vacuum 126

External photon line 101

\section{$\mathbf{F}$}

Fermi

coupling constant 273

interaction 168,266

Lagrangian 136

mechanism for acceleration of particles $\mathbf{4 8 5}$

particles 90

phase shifts 179

theory of cosmic radiation 369

Fermion fields 123, 137

Feynman

diagram 82, 108, 140

expansion 123

formalism 54, 112

graphs 69

interpretation 13
Feynman

kernel 64

model 348

notation 23

perturbation expansion 71

rules 74

S-matrix formalism 522

Fields 3, 152

angular momentum of photon 43

Coulomb 7, 85

Dirac 161

electromagnetic 162

gravitational 3

operator 112,124

potential 5

pseudoscalar 164

scalar 161

screening 85

self 3

spinor 150,161

Field Theory 12, 57, 112, 116

perturbation expansion 117

propagators 139

Fierz transformation 259

Fireball model 422

Forbidden directions 383

Forbush decreases of cosmic ray intensity $\mathbf{3 7 5}$

Form factor, Sommerfeld 88

Four dimensional isospace 346

Four-dimensional rotation group 342

Four-momentum vector 147

Fourier relationship 8

Four-vector 47

Free particle Hamiltonian 12

Free particle wave equation 24

Furry process 449

Furry theorem 154

G

Gamma matrices 23, 505

Gamow-Teller coupling constant 273

Gamow-Teller selection rule 253

Galactic sources of cosmic rays 492

Gauge 147

invariance 49,100

transformation 100,147

Gauss theorem 144

Gell-Mann-Low Theorem 187

Geomagnetic effects and cosmic radiation $\mathbf{3 7 7}$

Global couplings 329

Global symmetry 330

G operator 166

Goursat transformation 384 
Graphs, Feynman 69

Gravitational fields 3

Gravitational interactions 223

Gupta-Bleuler method 522

Gyromagnetic ratio 17

\section{$\mathbf{H}$}

Hamiltonian 6, 12, 17, 21, 113

beta decay 248

density 132

Dirac 37

free particle 12

interaction 142

Kemmer 165, 183

time dependent 52

Handedness 28

Heaviside unit function 42

Heavy particle number 147

Heisenberg

non-linear theory of elementary particles 356

operators 135,200

representation $133,139,143,187$

Helicity 36

of neutrino 258

Hermiticity 11

Hilbert space metric 532

Hole 29

Homogeneous Lorentz transformation 125

Hydrodynamical motion 417

Hydrogen atom 7

Hypercharge gauge 226

Hyperons 294

decay 303

\section{I}

Impact parameter 85

Improper graph 110

"in" and "out" fields 138

Incoming field 136

Incoming particles 136

Inelasticity 460

Infinitesimal rotation operator 43

Infra-red divergence 109

Interactions 14, 113

bilinear term 121

charge independence 165

cosmic rays with matter 393

current 195

Hamiltonian 142, 248
Interactions

at extremely high energies 457

electron-electron 74

Fermi 168, 266

fermion with electromagnetic field 137

Lagrangian 136

representation of field operator 124

representation in field theory $53,118,132$, 526

Yukawa 165

Intermediate mechanisms 480

Intermediate states 58

Internal photon line 101

Internal symmetries of strong interactions 353

Intranuclear cascades 454

Intrinsic

angular momentum 16

attributes 3

parity 150

variation, matrix of 146

Invariance 145

under $C, P, T 247$

under translation 125

Invariant functions 514

Ionisation 75,397

Irreducible graph 110

Isobar model 422

Isoboson character 226

Isofermion character 226

Isoscalars 329

Isospace 346

Isostrangeness spin 337

Isotopic space 147

Isotopic spin formalism 12, 30, 33, 223

\section{$\mathbf{J}$}

Jaw mechanism 488

Jet showers 400, 457

\section{$\mathbf{K}$}

$\bar{K} K \pi$ coupling 341

$K^{-}$-nuclear potential 291

$K$-particle decay 230

interactions 285

Kemmer Hamiltonian 165, 183

Kemmer interaction 186

Kernel Functions 52, 54, 63, 90, 97

Ket vector 9

Klein-Gordon equation 18, 21, 33 
Klein-Nishina formula 78

Knock-on electrons 397

Kroll-Ruderman theorem 196

\section{I}

Lagrangian 6,145

density 132,161

Lamb shift 105,107

A $\Sigma_{\pi}$-coupling $32 \mathrm{~s}$

Landau approximation 462

Latitude effect and cosmic rays 392

Least action 144

Left handed particle 28

Limit of a propagator 128

Liouville theorem 392

Local interaction 132

Long term variation in cosmic ray intensity 374

Longitudinal polarization 28

Longitudinal vector 45

Lorentz

condition 49

group 38

invariance 249

transformation 13, 23, 37, 125, 145, 207

Low equation 189

L.O.W. method 402

\section{M}

Magnetic moment, anomalous 105

Magnctie

Bremsstrahlung 75, 492

field 48

moment, anomalous 105

moment of electron 17, 105

moment of neutron 30

moment of proton 30

state $46,48,50$

Majorana description of neutrino 262

Mandelstam representation 186, 213

Mass

bare 98

observed 98

renormalisation 105

Mass-spectrum of primary cosmic radiation 367

Matrix

elements 69,123

density 12

gamma 23

of intrinsic variation 146
Matrix

Pauli spin 20

reaction 157

S. 139

trace 12

unitary 139

Maxwell's equations 38

Mean square deviation 450

Mean value

dynamical variable 5,12

momentum 5

Mellin transform $\mathbf{4 6 5}$

Mesic atoms 396

Meson 33, 34

current 195

decay 303

field 34,165

photo-production 157

radiative capture 157

scattering 176

vector 101

wave function 33

Microscopic causality 199

Mixed $T$ product 131

"Mixture" particles 336

Models of strong interactions 326

Modulation effects of cosmic ray intensity 375

Moliere multiple scattering 399

Moller scattering 259

Momentum

cut-off factor 189

-energy tensor of matter 417

operator 43

of photon field 41

space 3,9

transfer 209

Momentum representation wave mechanics of electron 65 wave mechanics of photon in 39

Motion of a charged particle in the field of a magnetic dipole 377

$M$ space 344

Multiple meson production 403

production of particles 400

scattering 394, 399

$\mu$-meson interactions 396

$\mu$-mesonic component 470

\section{$\mathbf{N}$}

Negative energy

particle 13

photon 13

solutions 29 
Negative energy

states $29,67,121,207$

Negative helicity 36

Negative meson 34

Neutral field 124, 152

$K$ decay 237

meson 34

meson vector 101

Neutrino 35, 36, 249, 262

helicity 258

Neutron 29

Non-conservation of parity 248

Non-Hermitian field 161, 533

Non-invariance under $T$ in decay experiments 256

Non-linear theory of particles $\mathbf{3 5 6}$

Non-mesonic decay 303

Normal product operator $127,129,131$

Normalization $10,41,68,95$

Nuclear

forces 32

magneton 30

potential 291

Nucleon 30

current 195

density 34

-nucleon collisions 394

"spread" 189

\section{$\mathbf{0}$}

Observed mass 98

Occupation number space 114

Occupation numbers 112

One meson Low equation 193

Operators 9

angular momentum 16

annihilation and creation $115,119,121$

calculus 12,114

in field theory 133

G 166

infinitesimal rotation 43

momentum 43

parity 46

spin 43,163

Orbital angular momentum 44

Orbits for cosmic ray particles 384

Origin of cosmic radiation 478

Outgoing state 136

\section{$\mathbf{P}$}

Pair annihilation 74,79

Pair creation $66,74,86,122$
Pais model 340

Parity 46, 147, 149, 247, 305

conservation 231

doublet 237

eigenfunctions 45

operator 46

of the second kind 359

Particle of spin $1 / 217$

Pauli equation 17,21

relativistic 35

Pauli-Gürsey transformations 357, 360

Pauli-Gürsey-Touschek group 361

Pauli-Lüders theorem 159

Pauli principle 13, 29, 66, 90, 94

Pauli spin matrix 20

Period orbits 391

Perturbation

expansions 52

expansions for Schrödinger equation 116

Feynman expansion 71

Kernel 97

theory 54, 65

Phase shift 157

analysis of scattering $\mathbf{5 3 4}$

Fermi 179

Yang 179

"Photoelectric" transition 175

Photon 13

angular momentum and spin 42

external line 101

field 35

internal line 101

momentum of field 41

negative energy of 13

probability density 41

propagator 91, 99, 102

self-energy 99

spin 44

wave mechanies 38

Photoproduction

of mesons 174, 176, 194, 398, 421

Physical vacuum 143

$\pi$-baryon coupling 333

$\pi$-meson 167

$\pi-\mu$ decay 274

$\pi-N$ coupling 328

Pion

-nucleon coupling 212

-nucleon scattering 186

physics 167

Plane wave potentials 51

Plane wave solutions 41

Poisson bracket 11 
Poisson process 450

Polar vector 146,150

Polarisation 28, 44, 80 phenomena in cascades 447 vacuum 104

Pomeranchuk-Landau model of multiple particle production 413

P-operator 128

$P$ ordering 119

Positive energy states 13

helicity 36 meson 34

Positron 66 scattering 122

Potentials expansion of 48 sealar 17,33 vector 17

Prehistoric intensity variations in cosmic ray intensities 376

Primary cosmic radiation 367

Primitive graph 109

Principle of complementarity 4, 11 of detailed balance 156 of invariance 145 of least action 144 of Pauli 13, 29, 32 of superposition $4,9,11$ of uncertainty 8

Probability conservation 11 density 22 density of photon 41

Product density functions 427

Production of hyperons 294

Propagators 139, 142 electron 107 photon 91, 93, 99, 102

Proper graph 110

Proton 29

Pseudoscalar fields 149, 152

Pseudoscalar function 146

Puppi's triangle 266

\section{Q}

Quantized charged fermion field 137

Quantized electromagnetic field 519

Quantum electrodynamies 52

Quantum Field Theory 112

non-perturbative 198
$\mathbf{R}$

Racah coefficients 503

Radial functions 45

Radiation damping 180

Radiation zone 48

Radiative capture of mesons 157

Radiative transition 81

Radical structure functions $\mathbf{4 6 4}$

Radius, Bohr 85

Rayleigh-Thomson scattering 78

Reaction matrix 157

Real scalar field 124

Reducible graph 110

Reflection, strong 158

Reflection, time 154, 159

Regeneration point method 426,443

Regularization of integrals 96

Relative abundance of elements $\mathbf{3 6 7}$

Relative parity between strange particles 305

Relativistic

hydrodynamical equations 417 invariant interaction term 132 thermodynamics 410

transformations 508

Relativity 14

Removal of divergences 109

Renormalization 95, 97, 103

Renormalized charge 99, 103

Resonances 170, 190

Reversal, Wigner's time 154

Right handed particle 28

Rotation

group 342

in isotopic spin space 147

matrices 503

operator 43

S

Saddle points, method of 453

Sakata's model 272

Salam-Polkinghorne model 342

Scalar

fields $149,152,161$

function 146

potential $17,33,48$

Scale transformation 358

Scattering 534

amplitudes 206

Compton 87

elastic 157

meson-nucleon 176 
Scattering

pion-nucleon 186

Rayleigh-Thomson 78

theory 7

Schrödinger equation $3,6,38,113,155$

for particle of spin $1 / 217$

perturbation expansion 116

for photon in momentum space 39

Schrödinger representation $53,118,123,133$

Schwinger time reflection 159

Screening of Coulomb field 85, 88

Secular variations of cosmic radiation 373

Selection rule, Gamow-Teller 253

Self action 99

Self energy 12, 95, 97, 99, 110

Self field 3

Shadow effect of the earth and the cosmic radiation 386

Showers 424, 457

$\Sigma$-interactions 296

$\Sigma \Sigma \pi$-coupling 328

Single particle wave equation 14

Singularities 109

Skeleton of a graph 110

Slater determinant 13

$S$-matrix formalism $120,128,131,139,188$, 219,522

Solar contribution to cosmic radiation 490

Solution, stationary 7

Sommerfeld form factor 88

Source

of cosmic radiation 489

distribution function 34

variations of cosmic radiation 373

Space

configuration 3

inversion 147

-like separation 126

momentum 3

reflection 147

rotation 147

Space-time properties of strong interactions 353

Spatial distribution of cosmic radiation 370

Spectral condition 208

Spherical vector function $43,45,51$

Spherical wave solution 51

Spherically symmetric solution 33

Spin 3, 7, 12, 14, 16, 22, 147

and statistics 531

operator 43,163

of photon 42,44
Spin

vector of strangeness 337

wave functions 43

-zero field 531

Spinless field̦ 152

Spinor field 150, 152, 161

"Spread" of the nucleon 189

Standard representation 23

State vector 112,135

Static Coulomb field 35

Stationary solutions 7

Statistical

approaches to nucleon-nucleon collisions 394

mechanics 156

theory of multiple meson production 403

Stefan's law 410

Stochastic variable 450

Stormer cones 380,387

Strange particles 221, 285

Strangeness 223

conservation 237

spin vector 337

Strong

coupling 175

reflection 158

time reflection 154

Strong interactions 223, 325

space-time properties 353

and strange particles 285

symmetries 325

Substitution law 87, 213

Sunspot effects and long term variation in cosmic ray intensity 374

Superposition principle 4, 9, 11

Swann's betatron mechanism 481

Symmetries and strong interactions 325

Symmetrized functions 113

Symmetry

charge .166

Clebsch-Gordon coefficients 502

\section{$\mathbf{T}$}

Table of types transformations 147

transformations properties of scalar and spinor fields 160

Tamm-Dancoff method 180, 182, 188, 363

Taurus-A 494

Tensor, of electromagnetic field 106

Tests for invariance under $C, P, T 247$ 
Tetrahedron representation of the fourfermion interaction $\mathbf{2 6 s}$

Thomas-Fermi model 85

E..7 coupling $32 \mathrm{~s}$

Time

dependent s'chrödinger equation 155

inversion 147,149

reflection 154

reversal 147

variation of cosmic radiation 371

$T$-operator 128

$T$-matrix $18 \mathrm{~s}$

$\tau^{+}-\theta^{+}$puzzle 231

Total

angular momentum 45

energy 209

isotopic spin 30

Touschek group 357,360

Trajectories of cosmic rays 385

Transform 8

Transformations 143

contact 11

continuous 147

discrete 147

Fierz 259

gauge 100,147

Lorentz 12

matrix 37

properties of operators under Lorentz transformation 207

scalar and spinor fields 160

Table of types 147

under space-time rotation 18

unitary 11

Transition

probability 64

radiative 81

racuum-vacuum 141

Translation in four dimensional space 147

Translational invariance of operator 135

Transversality condition 40,49

Transverse polarization 28

Transverse spherical vectors 46

"True" vacuum 143

Two component theory of $\beta$-decay 257 component wave-functions 36

formalism 30

and the neutrino 259

Two particle collisions 511

Two photon pair annihilation 74

Typical realization 528
$\mathbf{U}$

Ultra-violet divergence 109

Uncertainty principle 8

Unified field equation 356

Unit function of Heaviside $\mathbf{4 2}$

Unitary

matrix 139

transformation 11,147

Universal Fermi interaction 231, 266

Unstable periodic orbits 391

\section{$\mathbf{v}$}

Vacuum 119, 143

expectation value 126,128

polarisation 104

processes 103

state 149

-vacuum transitions 141

Variables

canonically conjugate 6,9

complex field 126

dynamical $5,9,112$

Variations at source of cosmic radiation 373

Vector

axial 146

bra 10

field 43

four-momentum 147

ket 9

matrix equation 53

meson 101

polar 146,150

potential 17,48

state 112,115

Vertex part 104, 110

"Virtual" photon 399

W

Wave function 3

adjoint 23

bound state 107

charge 32

in configuration space $8,41,113$

Coulomb 108

neutrino 29

neutron 29

photon 38

proton 29 
Wave function renormalization 105 single particle 3,14 spin 43 total angular momentum 45 transform 8 two component 36

Wave Mechanics, basic postulates 3 Wave Mechanics of photon 38

Weak coupling 172 interactions 223, 228, 246, 277 magnetism in $\beta$-decay 273 time reflection 154
Wick's chronological operator 127

Wick's theorem 126, 130

Wigner

$j$ symbols 502,505

coefficients 234

time reversal 154

"World" basis for existence of elementary particles 362

\section{$\mathbf{Y}$}

Yang phase shifts 179

Yukawa interaction 142, 151, 165, 183

Yukawa theory 192 
• 


QC

174

.1

R28
Ramakrishnan, Alladi

Elementary particles and cosmic rays

\section{PLEASE DO NOT REMOVE CARDS OR SLIPS FROM THIS POCKET}

ERINDALE COLLEGE LIBRARY 
